NBSIR 82-2550

\title{
Stopping Powers and Ranges of Electrons and Positrons
}

U.S. DEPARTMENT OF COMMERCE

National Bureau of Standards

Washington, DC 20234

August 1982

Prepared for:

Office of Standard Reference Data

National Bureau of Standards

Washington, DC 20234

Office of Health and Environmental Research

Department of Energy

Washington, DC 20545

- $Q C$ ifice of Naval Research

100

lington, Virginia 22217 

NBSIR 82-2550

\section{Stopping Powers and Ranges of Electrons and Positrons}

U.S. DEPARTMENT OF COMMERCE

National Bureau of Standards

Washington, DC 20234

August 1982

Prepared for:

Office of Standard Reference Data

National Bureau of Standards

Washington, DC 20234

Office of Health and Environmental Research

Department of Energy

Washington, DC 20545

Office of Naval Research

Arlington, Virginia 22217 

NBSIR $82-2550$

Stopping Powers and Ranges of

Electrons and Positrons

M. J. Berger and S. M. Seltzer

U.S. DEPARTMENT OF COMMERCE

National Bureau of Standards

Washington, DC 20234

August 1982

Prepared for:

Office of Standard Reference Data

National Bureau of Standards

Washington, DC 20234

Office of Health and Environmental Research

Department of Energy

Washington, DC 20545

Office of Naval Research

Arlington, Virginia 22217

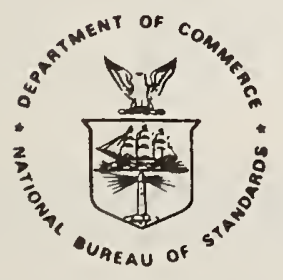

U.S. DEPARTMENT OF COMMERCE, Malcolm Baldrige, Secretary NATIONAL BUREAU OF STANDARDS, Ernest Ambler, Director 



\title{
STOPPING POWERS AND RANGES OF ELECTRONS AND POSITRONS*
}

\author{
M. J. Berger and S. M. Seltzer \\ Center for Radiation Research \\ National Bureau of Standards \\ Washington, D.C. 20234
}

\begin{abstract}
Tables of stopping powers and related data are given for electrons in 25 elements and 46 mixtures and compounds, and for positrons in 8 materials. The tables include: (1) collision stopping powers (ionization and excitation losses); (2) radiative stopping powers (bremsstrahlung losses); (3) total stopping powers; (4) ranges (rectified pathlengths computed in the continuousslowing-down approximation); (5) radiation yields (fraction of initial electron energy converted to bremsstrahlung in the course of slowing down); and (6) the logarithmic derivatives of all these quantities with respect to the mean excitation energy of the medium (the key parameter of the Bethe stopping power formula). The results are tabulated at 81 energies between $1000 \mathrm{MeV}$ and $10 \mathrm{keV}$. Collision stopping powers for electrons in materials of low atomic number are given also for energies down to $1 \mathrm{keV}$. The principal new ingredients in the preparation of the tables are: (1) improved values of the mean excitation energies for elements and compounds, derived from stopping-power and range measurements and from semi-empirical oscillatorstrength distributions and dielectric-response functions; (2) densityeffect corrections evaluated according to the method of Sternheimer, using up-to-date input parameters; and (3) use of new theoretical cross sections of Pratt and Tseng for electron-nucleus bremsstrahlung and of Haug for electron-electron bremsstrahlung.
\end{abstract}

Key words: Collision stopping power, electrons, positrons, radiation yield, radiative stopping power, range.

* These tables were prepared as input for a report on stopping power to be written by a committee sponsored by the International Commission on Radiation Units and Measurements (ICRU). The ICRU sponsors of this committee are A. Allisy and R. S. Caswe11. The committee members are H. H. Andersen, M. J. Berger (chairman), H. Bichsel, J. A. Dennis, M. Inokuti, D. Powers, and J. E. Turner. Consultants to the committee are S. M. Seltzer and R. M. Sternheimer. All of the above have made important contributions to this work. It should be emphasized that this report is a draft submitted to the ICRU, and may be revised before being included in an ICRU document.

We would like to acknowledge the financial support for this work provided by the NBS Office of Standard Reference Data, the Office of Health and Environmental Research of the Department of Energy, and the Office of Naval Research.

Last but not least we would like to thank Mrs. Gloria Wiersma for her editorial help in the preparation of the manuscript. 

ABSTRACT . . . . . . . . . . . . . . . . . . . . . . . i

1. INTRODUCTION . . . . . . . . . . . . . . . . . . . . . . . . 1

1.1. Purpose and scope

1.2. Background

1.3. New features

2. FORMULAS FOR THE COLLISION STOPPING POWER . . . . . . . . . . . . . . 3

2.1. General Formulas

2.2. Stopping-power formulas for heavy charged particles

2.3. Stopping-power formulas for electrons and positrons

3. METHODS FOR ESTIMATING MEAN EXCITATION ENERGIES . • • . . . . . . . • . . . 7

3.1. Use of oscillator-strength and dielectric data

3.2. Use of stopping-power and range data

3.2.1. Bichsel's shell corrections

3.2.2. Comparisons of Bichsel's and Bonderup's she11 correction

4. SELECTION OF MEAN EXCITATION ENERGIES FOR ELEMENTS . . . . . . • . . . . . 12

5. SELECTION OF MEAN EXCITATION ENERGIES FOR COMPOUNDS . . . . . . . . . . . 13

6. DENSITY EFFECT . . . . . . . . . . . . . . . . . . . . 16

6.1. General equations

6.2. Sternheimer's mode1

6.3. Numerical evaluation

6.4. Complications for inhomogeneous media

7. RESTRICTED COLLISION STOPPING POWER . . . . . . . . . . . . . . . 19

8. ELECTRON COLLISION STOPPING POWERS AT LOW ENERGIES . . . . . . . . . . . . 20

8.1. Calculations for gases

8.2. Calculations for solids and liquids

8.3. Comparison of stopping powers

9. RADIATIVE STOPPING POWER . . . . . . . . . . . . . . . . . 23

9.1. Electron-nucleus bremsstrahlung

9.1.1. High-energy region

9.1 .2 . Low-energy region

9.1.3. Intermediate energy region

9.2. Electron-electron bremsstrahlung

9.2.1. High-energy region

9.2.2. Low-energy region

9.2 .3 . Intermediate energy region

9.3. Accuracy and comparison with experiments

10. RANGES AND RADIATION YIELDS . . . . . . . . . . . . . . . . . . 26

11. MISCELLANEOUS COMPARISONS . . . . . . . . . . . . . . . . . . . . 27

11.1. Positron-electron differences

11.2. Comparison of calculated and experimental stopping powers

11.3. Comparisons with previous calculations

REFERENCES . . . . . . . . . . . . . . . . 29

TABLES TO ACCOMPANY TEXT . . . . . . . . . . . . . . . . . . . 41

FIGURES . . . . . . . . . . . . . . . . . . . 66 66

MAIN STOPPING-POWER AND RANGE TABLES . . . . . . . . . . . . . . . . . . 79 



\section{INTRODUCTION}

1.1. Purpose and scope. In radiation physics, chemistry, biology, and medicine, it is often important to have accurate information about the stopping power of various media for charged particles, that is, the average rate at which the charged particles lose energy along their tracks. The purpose of this report is to supply up-to-date stopping-power information, with emphasis on the requirements of biomedical dosimetry. The contents of this report are the following: (a) In Sections 2 to 6 , topics are reviewed which are pertinent to the evaluation of stopping powers for any charged particle within the framework of the Bethe theory. ${ }^{1}$ These include shell corrections, the determination of mean excitation energies from experimental data, the use of the Bragg additivity rule for compounds, and the density-effect correction. Recommended values of mean excitation energies are given in Table 4.3 for elemental substances and in Table 5.5 for compounds and mixtures. (b) In Sections 7 to 11 , topics are reviewed which are pertinent mainly or entirely to electrons. These include the radiative stopping power due to the emission of bremsstrahlung, and the information on electron collision stopping power at energies below $10 \mathrm{keV}$ where the Bethe theory is no longer fully applicable. (c) In Section 12, electron stoppingpower tables are presented for 25 elements and 46 compounds and mixtures, covering the energy region from $10 \mathrm{keV}$ to $1000 \mathrm{MeV}$. These tables also include the range (rectified pathlength) and the radiation yield (fraction of electron kinetic energy converted to bremsstrahlung as the electrons slow down to rest), both computed in the continuous-slowing-down approximation. ${ }^{2}$ Such data are also given for positrons in a few materials.

1.2. Background. For electrons it is customary to separate the total stopping power into two components: (a) the collision stopping power, which is the average energy loss per unit pathlength due to inelastic Coulomb collisions with bound atomic electrons of the medium resulting in ionization and excitation; (b) the radiative stopping power, which is the average energy loss per unit pathlength due to the emission of bremsitrahlung in the electric field of the atomic nucleus and of the atomic electrons. ${ }^{3}$ The separation of the electron stopping power into two components is useful for two reasons. First, the methods used for the evaluation of the two components are quite different. Second, the energy going into the ionization and excitation of atoms is absorbed in the medium rather close to the electron track, whereas most of the energy lost in the form of bremsstrahlung travels far from the track before being absorbed. This distinction is important when attention

${ }^{1}$ The results obtained will be applied to the tabulation of stopping powers for heavy charged particles in a future report.

${ }^{2}$ In this approximation, energy-loss fluctuations are disregarded, and the rate of energy loss at any point along the track is assumed to be equal to the stopping power.

${ }^{3}$ The nomenclature "collision stopping power" and "radiative stopping power" is that adopted by the International Commission on Radiation Units and Measurements (ICRU, 1980).

In the literature, the collision stopping power is often referred to as stopping power, with the adjective "collision" omitted, especially in circumstances where the radiative stopping power is negligible. The collision stopping power is sometimes also called "ionization loss." Numerically, but not conceptually, the collision stopping power is identical with the "linear energy transfer" (more precisely, the unrestricted linear energy transfer LET $_{\infty}$ ) often used in radiobiology (see, e.g., ICRU, 1970).

The excitations contributing to the collision stopping power include not only electronic excitations but also vibrational and rotational excitations of molecules; however, the latter two processes are relatively unimportant above the threshold energy for electronic excitation. Charged particles also lose some energy in elastic collisions with atoms. The transfer of recoil energy to atoms in such events is proportional to the ratio of the mass of the incident particle to the mass of the atom. This mode of energy loss is therefore unimportant for electrons except at extremely low energies where the cross sections for electronic, vibrational, and rotational energy losses become very small. For incident heavy particles, however, elastic collisions with atoms constitute a significant mode of energy loss, and give rise to a so-called "nuclear stopping power" which will be discussed in a future report on heavy charged particles. 
is focussed on the energy "imparted locally" to the medium along the track rather than on the energy lost by the incident electron. Actually, even a part of the energy lost in ionizing collisions is converted to kinetic energy of secondary electrons, and is thus carried some distance away from the primary electron track. In order to estimate energy imparted 1 ocally in a crude but simple manner, it is therefore useful to introduce a restricted collision stopping power defined as the average energy loss per unit pathlength due to excitation events and due to ionization events in which the energy transferred to secondary electrons is smaller than some chosen limit. Some data on restricted stopping powers are also given in this report.

Even though electron stopping powers and ranges are widely used, they are rarely measured and must be obtained from stopping-power theory. All previous tables as well as the tables in this report contain collision stopping powers for electrons at energies above $10 \mathrm{keV}$ evaluated according to the theory of Bethe $(1930,1932,1933)$. The energy of $10 \mathrm{keV}$ is a commonly accepted lower limit for the applicability of the theory. The principal non-trivial quantity describing the properties of the medium in Bethe's stopping-power formula is the mean excitation energy, which is a logarithmic average of the excitation energies of the medium weighted by the corresponding oscillator strengths. Except for elements with very low atomic number $\mathrm{Z}$, the mean excitation energies in $\mathrm{eV}$ are approximately equal to $10 \cdot \mathrm{Z}$. Accurate $a b$ initio calculations of mean excitation energies are possible at present only for simple atomic gases. For most materials it is necessary to determine mean excitation energies from experimental data. Another important quantity in the stopping-power formula, not contained in Bethe's original theory, is the density-effect correction, which takes into account the reduction of the collision stopping power due to the polarization of the medium by relativistic charged particles (Fermi, 1940). A11 of the tabulations including the present one have relied on the method of Sternheimer (1952) for the evaluation of the density-effect correction.

The first extensive electron stopping-power and range tables were calculated by Nelms, first without and later with the density-effect correction (Ne1ms, 1956, 1958). The tables of Berger and Seltzer (1964, 1966) included not only the collision stopping power but also the radiative stopping power. The latter was evaluated by a combination of bremsstrahlung cross sections given by Bethe and Heitler (1934) and empirical corrections recommended by Koch and Motz (1959). Pages et al. (1972) in their tables used the same mean excitation energies and bremsstrahlung cross sections as those of Berger and Seltzer, but evaluated the density-effect correction with somewhat different input parameters.

\subsection{New features. The principal new aspects of this work are the following:}

(a) A careful review has been made of the mean excitation energies derived from the analysis of stopping-power and range measurements, and from semi-empirical dipole oscillator-strength distributions for gases or dielectric-response functions for liquids and solids.

(b) In the extraction of mean excitation energies from measured stopping powers and ranges, use has been made of empirical shell corrections recently deve1oped by Bichse1 (unpublished) as an extension of his earlier work (Bichse1, 1961, $1963,1972)$.

(c) For compounds for which no direct experimental information is available, the mean excitation energies have been calculated as weighted sums of the mean excitation energies of the atomic constituents (Bragg additivity). The required mean excitation energies of the constituents have been adjusted to take into account, at least approximately, the effects of chemical binding and the physical state of aggregation.

(d) The Main Tables indicate the sensitivity of the electron collision stopping power, range, and bremsstrahlung yield to a change of the mean excitation energy. This enables the reader to make the appropriate adjustments if he prefers values of the mean excitation energy different from those adopted in this report.

(e) The density-effect correction has been re-evaluated according to the dispersion model of Sternheimer (1952). Rather than using Sternheimer's earlier results or the universal fit given by Sternheimer and Peierls (1971), we have evaluated the density effect using as input the mean excitation energies adopted in this report and values of the binding energies for atomic subshells from Carlson $(1975)$. 
(f) The radiative stopping power has been calculated with improved theoretical bremsstrahlung cross sections. For bremsstrahlung in the field of the atomic nucleus, cross sections were obtained as follows: (i) At energies up to $2 \mathrm{MeV}$, recent theoretical results were used that are based on the solution of the Dirac equation and numerical evaluation of the pertinent matrix elements (Tseng and Pratt, 1971; Pratt et al., 1977). (ii) Above $50 \mathrm{MeV}$, use was made of cross sections in the high-energy approximation (Davies, Bethe, and Maximon, 1954; 01sen, 1955), evaluated with improved form-factor screening corrections derived from Hartree-Fock wave functions. (iii) In the energy region from 2 to $50 \mathrm{MeV}$, it has been found possible to construct reliable cross sections by interpolating with respect to electron energy, using the accurate low- and high-energy theoretical results as anchor points. The less important process of bremsstrahlung in the field of the atomic electrons was taken into account according to the theory of Haug (1975) augmented by a screening correction.

\section{FORMULAS FOR THE COLLISION STOPPING POWER}

2.1. General formulas. In this section various formulas of the Bethe theory and its elaborations will be stated briefly without derivation. For a detailed discussion of stopping-power theory the reader is referred to the many excellent reviews in the literature, e.g., Bohr (1948), Bethe and Ashkin (1953), Uehling (1954), Fano (1963), Inokuti (1971), Jackson (1975), Sigmund (1975), and Ahlen (1980).

The linear collision stopping power, with dimensions of energy/length, will be denoted as - $(\mathrm{dE} / \mathrm{dx})_{\mathrm{col}}$ or $\mathrm{S}_{\mathrm{col}}$. We shall find it convenient to consider also the corresponding mass collision stopping power, $S_{c o l} / \rho$, where $\rho$ is the density of the medium. The change from linear to mass stopping power largely removes the dependence on the density, except for a residual dependence due to the density-effect correction. With $\mathrm{S}_{\mathrm{col}}$ in $\mathrm{MeV} / \mathrm{cm}$ and $\rho$ in $\mathrm{g} / \mathrm{cm}^{3}, \mathrm{~S}_{\mathrm{col}} / \rho$ has units of $\mathrm{MeV} /\left(\mathrm{g} / \mathrm{cm}^{2}\right)$.

The collision stopping power is due to energy transfers from the incident particle to bound atomic electrons. We denote by do/dW the cross section (per atomic electron) for inelastic collisions resulting in an energy transfer of magnitude $W$. The mass collision stopping power can then be expressed as

$$
\frac{1}{\rho} S_{\operatorname{col} 1}=\frac{N_{a} Z}{A} \int W \frac{d \sigma}{d W} d W
$$

The leading factor, $\mathrm{N}_{\mathrm{a}} \mathrm{Z} / \mathrm{A}$, represents the number of atomic electrons per gram of the medium. $\mathrm{N}_{\mathrm{a}}=6.022045 \times 10^{2} \mathrm{~mol}^{-1}$ is Avogadro's number, and $\mathrm{Z}$ and $\mathrm{A}$ are the atomic number and atomic weight.

Following the formulation of Uehling (1954), we now discuss the results of Bethe's evaluation of the stopping-power expression (2.1). These results are applicable to electrons and positrons, mesons, protons, alpha particles and to fullystripped heavy ions. The energy transfers $W$ to atomic electrons in inelastic collisions are divided into two classes, depending on whether they are smaller or larger than some value $W_{C}$. which must satisfy two conditions: (a) $W_{C}$ must be large compared to the binding energies of the atomic electrons of the stopping medium. (b) The impact parameters associated with energy losses smaller than $W_{c}$ must be large compared to atomic dimensions. The mass collision stopping power is expressed as the sum of two components,

$$
\frac{1}{\rho} S_{C O 1}=\frac{1}{\rho} S_{C O 1}\left(W<W_{C}\right)+\frac{1}{\rho} S_{C O 1}\left(W>W_{C}\right)
$$

The main result of the Bethe theory, applicable to electrons and heavy charged particles, is that

$$
\frac{1}{\rho} S_{c o 1}\left(W<W_{c}\right)=\frac{2 \pi N_{a} r_{e}^{2} m c^{2}}{\beta^{2}} \frac{z}{A} z^{2}\left[\ln \frac{2 m c^{2} \beta^{2} W_{c}}{\left(1-\beta^{2}\right) I^{2}}-\beta^{2}\right],
$$


where $N_{a}$ is Avogadro's number, $r_{e}$ is the classical electron radius, mc ${ }^{2}$ is the electron rest energy, $\beta$ is the velocity of the incident particle (projectile) divided by the velocity of light, $z$ is the projectile charge in units of the charge of the electron, $Z$ and $A$ are the atomic number and atomic weight of the target atoms, and I is the mean excitation energy. Note that (Particle Data Group, 1980)

$$
\begin{aligned}
2 \pi \mathrm{N}_{\mathrm{a}} \mathrm{e}^{2} \mathrm{mc}^{2} & =(2 \pi)\left(6.022045 \times 10^{23} \mathrm{~mol}^{-1}\right)\left(7.940775 \times 10^{-26} \mathrm{~cm}^{2}\right)(0.5110034 \mathrm{MeV}) \\
& =0.153536 \mathrm{MeV} \mathrm{cm} \mathrm{mol}^{-1} .
\end{aligned}
$$

Equation (2.3) is valid when the velocity of the projectile is large compared to the velocities of the atomic electrons. Applied to the electrons in the $\mathrm{K}$ shell this implies the requirement that $(2 / 137 \beta)<<1$. The stopping-power component due to close collisions is evaluated as if the atomic electrons were free and at rest:

$$
\frac{1}{\rho} S_{c o 1}\left(W>W_{c}\right)=N_{a} \frac{Z}{A} \int_{W_{c}}^{W_{m}} W \frac{d \sigma}{d W} d W,
$$

where do/dW is now the differential cross section for energy transfer $W$ in a collision with a free electron, and where

$$
W_{m}=2 \tau(\tau+2) \mathrm{mc}^{2} /\left[1+2(\tau+1)(\mathrm{m} / \mathrm{M})+(\mathrm{m} / \mathrm{M})^{2}\right]
$$

is the largest possible energy transfer, with $\tau$ the kinetic energy of the projectile in units of its rest mass, and $\mathrm{m} / \mathrm{M}$ the ratio of the electron mass to that of the projectile.

2.2. Stopping-power formula for heavy charged particles. Formulas for heavy charged particles are given here because they are needed for the analysis of experimental data to obtain mean excitation energies. When $m \ll M$ Eq (2.5) can be approximated by

$$
W_{m}=2 \tau(\tau+2) m c^{2}=\frac{2 m c^{2} \beta^{2}}{1-\beta^{2}} .
$$

The differential scattering cross section is (Ueh1ing, 1954; Fano, 1963)

$$
\mathrm{d} \sigma=\frac{2 \pi \mathrm{r}_{\mathrm{e}}^{2} \mathrm{mc}}{\beta^{2}} z^{2} \frac{\mathrm{d} w}{\mathrm{~W}^{2}}\left(1-\beta^{2} w / w_{m}\right) .
$$

Combining Eqs (2.2) to (2.7), one obtains the result

$$
\frac{1}{\rho} S_{\mathrm{col}}=\frac{4 \pi \mathrm{N}_{\mathrm{a}} \mathrm{r}^{2} \mathrm{mc}^{2}}{\beta^{2}} \frac{z}{\mathrm{~A}} \mathrm{z}^{2}\left[\ln \frac{2 \mathrm{mc}^{2} \beta^{2}}{\left(1-\beta^{2}\right) \mathrm{I}}-\beta^{2}\right] .
$$

As expected for a consistent treatment, the quantity $W_{C}$ which separates hard and soft collisions does not appear explicitly in Eq (2.8).

The accuracy of the stopping-power formula is improved by the addition of the following corrections: 
(1) A shell correction, ${ }^{4}$ which compensates for the fact that the projectile velocity is not necessarily large compared to the velocity of the target electrons;

(2) A density-effect correction which accounts for the reduction of the stopping power due to the polarization of the medium; and

(3) Corrections which represent departures from the first Born approximation.

Following Lindhard (1976), Andersen et al. (1977) and Ritchie and Brandt (1978), the collision stopping-power formula is written as

$$
\frac{1}{\rho} S_{c o l}=\frac{4 \pi N_{a} r_{e}^{2} m c^{2}}{\beta^{2}} \frac{z}{A} z^{2} L(\beta),
$$

where L( $\beta)$, the stopping number per atomic electron, is expressed as the sum of three terms,

$$
L(\beta)=L_{0}+z L_{1}+z^{2} L_{2}
$$

The first term,

$$
L_{0}(\beta)=\ln \left(\frac{2 m c^{2} \beta^{2}}{1-\beta^{2}}\right)-\beta^{2}-\ln I-\frac{C}{Z}-\frac{\delta}{2},
$$

adds to $\mathrm{Eq}(2.8)$ a shell correction, $\mathrm{C} / \mathrm{Z}$, and a density-effect correction $\delta / 2$. These corrections will be discussed further in Sections 3.2 and 6 , respectively.

The second and third terms in Eq (2.10) extend the treatment beyond the first Born approximation. The term $\mathrm{zL}_{\mathrm{l}}$ is often referred to as the " ${ }^{3}$ correction." Because of the appearance of an odd power of $z$, the stopping power for particles with positive and negative charge will differ, and this was in fact first observed by Barkas et al. (1956). For this reason, following Lindhard (1976), we shall refer to the $\mathrm{zL}_{1}$ term as the Barkas correction. It was first calculated by Ashley, Ritchie, and Brandt (1972, 1973) in a semi-classical approximation, using a harmonicoscillator model and assuming that only distant collisions contribute to the effect. The assumed minimum impact parameter (approximately equal to the orbital radius of the atomic electrons) is not precisely specified by the theory, and its value must be adjusted on the basis of experimental stopping-power data. Other derivations of the Barkas correction have been given by Jackson and McCarthy (1972) who used a somewhat different quantum-mechanical cut-off parameter, by Hill and Merzbacher (1974) who carried out a quantum-mechanical perturbation calculation for distant collisions, and by Lindhard (1976) on the basis of his free-electron-gas model. These theories lead to results essentially equivalent to those of Ashley, Ritchie, and Brandt, especially in view of the adjustable value of the minimum impact parameter. We shali in this report use the theoretical results of Ashley et al., as given by them in terms of a numerical function $F$ which is defined such that

$$
z L_{1}=\frac{z Z \alpha^{3}}{\beta^{3}} F(b \alpha \sqrt{Z} / \beta)
$$

where $\alpha=1 / 137.03604$ is the fine-structure constant. The multiplier $b$ in the argument of $F$ is related to the choice of the minimum impact parameter, and usually has a value between 1 and 2 (see, e.g., Table 3.4 ).

\footnotetext{
"The name "shell correction" arose because the correction was calculated separately for interactions with atomic electrons in different shells, starting with the treatment of K-shell electrons by Livingston and Bethe (1937).
} 
The correction term $z^{2} \mathrm{~L}_{2}$ in $\mathrm{Eq}(2.10)$ is contained in an extension of Bethe's stopping-power formula due to Bloch (1933), and will therefore be called the Bloch correction. Bloch's formula is based on a quantum-mechanical impact-parameter method that takes into account, approximately, the perturbation of the wave functions of the atomic electrons due to the incident particle. The correction has the form

$$
z^{2} L_{2}=\psi(1)-\operatorname{Re} \psi(1+i y)
$$

where $y=z \alpha / \beta$ and where $\psi$ is the logarithmic derivative of the gamma function. Using properties of this function (see, e.g., p. 259 in Abramowitz and Stegun, 1964) one can transform Eq (2.13) into

$$
z^{2} L_{2}=-y^{2} \sum_{n=1}^{\infty}\left[n\left(n^{2}+y^{2}\right)\right]^{-1}
$$

For very small $y$, the Bloch correction is negligible. For $y>1$, the value of $z^{2} L_{2}$ can be shown to approach - 0.577 - $\ell_{n} y$. When this asymptotic value is added to the stopping number (square brackets in Eq (2.8)), the resulting stopping formula is the same as the classical formula of Bohr (1913).

2.3. Stopping-power formulas for electrons and positrons. For electrons, 1arge energy transfers to atomic electrons (considered as free) are governed by the M $\phi 11$ er (1932) cross section,

$$
\mathrm{d} \sigma=\frac{2 \pi \mathrm{r}_{\mathrm{e}}^{2} m c^{2}}{\beta^{2}} \frac{\mathrm{dW}}{\mathrm{W}^{2}}\left[1+\frac{\mathrm{W}^{2}}{(\mathrm{~T}-\mathrm{W})^{2}}+\frac{\tau^{2}}{(\tau+1)^{2}} \frac{\mathrm{W}}{\mathrm{T}}^{2}-\frac{(2 \tau+1)}{(\tau+1)^{2}} \frac{\mathrm{W}}{(\mathrm{T}-\mathrm{W})}\right]
$$

where $\tau=\mathrm{T} / \mathrm{mc}^{2}$ is the kinetic energy of the incident electron in units of its rest mass. The M $\phi 1 l$ er cross section, valid when $137 \beta \gg 1$, takes into account relativity and spin effects as well as exchange effects associated with the indistinguishability of the incident and target electrons. By convention, the collision stopping-power pertains to the faster of the two electrons emerging from the collision. The maximum possible energy transfer $W_{m}$, while equal to $T$ according to $E q(2.5)$, is therefore taken to be equal to $\mathrm{T} / 2$. With the M $\phi 11$ er cross section, and using Eqs (2.1), $(2.2)$, and (2.4), one obtains the following formulas for the electron mass collision stopping power (Rohrlich and Carlson, 1953; Uehling, 1954):

$$
\frac{1}{\rho} S_{c o l}=\frac{2 \pi N_{a} r_{e}^{2} m c^{2}}{\beta^{2}} \frac{Z}{A}\left[\ln (T / I)^{2}+\ln (1+\tau / 2)+F^{-}(\tau)-\delta\right],
$$

where

$$
F^{-}(\tau)=\left(1-\beta^{2}\right)\left[1+\tau^{2} / 8-(2 \tau+1) \ln 2\right]
$$

One half times the quantity in square brackets in Eq (2.16) is the stopping number per atomic electron, $L(\beta)$, and is analogous to the stopping number $L(\beta)$ for protons in Eq (2.9). ${ }^{5}$ For positrons, energy transfers larger than $W_{C}$ are treated by the Bhabha (1936) cross section (given by Eq (10) in Uehling, 1954). There are no exchange effects, and the maximum possible energy transfer is $W_{m}=T$. The mass collision stopping-power formula for positrons is similar to that for electrons, except that $\mathrm{F}^{-}$is replaced by

${ }^{5}$ The factor $1 / 2$ arises because it is conventional to use $2 \pi$ for electrons and $4 \pi$ for heavy charged particles in the leading factor of the stopping-power formula. 
Some remarks are necessary regarding the absence of shell corrections from the electron stopping-power formula, Eq (2:16). For heavy particles, such corrections have been developed on the basis of the assumption that the projectile particle can be considered equivalent to a perturbing potential whose center moves with constant velocity. This assumption, while satisfied for protons down to rather low velocities, is much less applicable to electrons, so that there is no sound theoretical basis for extending the available shell-correction theory to electrons.

Corrections to the Bethe theory, analogous to shell corrections for stopping power, have been discussed by Inokuti (1971) in regard to excitation cross sections. He indicates that these corrections contain an additive term proportional to the ratio of electron to projectile mass, m/M, such that the corrections can be expected to be significantly larger for electrons than for protons. It seems likely that this will also be the case for stopping-power shell corrections.

We have attempted to make a rough estimate of the possible error in the calculated electron stopping power due to the omission of a shell correction term, assuming, arbitrarily, that the error is twice as large as the reduction of the proton stopping power for protons with the same velocity. At an electron energy of $100 \mathrm{keV}$, the error is then estimated to be $\sim 0.3 \%$ for $\mathrm{H}_{2} \mathrm{O}, \sim 0.7 \%$ for $\mathrm{Al}, \sim 1.3 \%$ for $\mathrm{Cu}, \sim 2 \%$ for $\mathrm{Ag}$, and $\sim 3 \%$ for $\mathrm{Au}$. At $10 \mathrm{keV}$, the corresponding error estimates are $\sim 2 \%$ for $\mathrm{H}_{2} \mathrm{O}, \sim 4 \%$ for $\mathrm{Al}, \sim 9 \%$ for $\mathrm{Cu}, \sim 12 \%$ for $\mathrm{Ag}$, and $\sim 21 \%$ for Au. The use of Eq (2.16) is questionable at energies below $10 \mathrm{keV}$. However, for low $-\mathrm{Z}$ materials such as water, air, or plastics, the evidence presented later in Section 8 suggests that the errors may amount to $\sim 3 \%$ at $5 \mathrm{keV}, \sim 7 \%$ at $2 \mathrm{keV}$, and 10 to $15 \%$ at $1 \mathrm{keV}$.

\section{METHODS FOR ESTIMATING MEAN EXCITATION ENERGIES}

In order to obtain accurate values of the mean excitation energy $I$, it is necessary to take into account the specific electronic structure of the atom, molecule, or solid of interest. Accurate $a b$ initio calculations of I are available for some atomic gases. For other materials one must rely on semi-empirical methods for determining I, using data available from two sources: stopping-power and range measurements, usually for protons and alpha particles; and dielectric-response functions (for solids) and oscillator-strength distributions (for gases), which can often be obtained from cross sections for the interaction of photons with the medium of interest.

Only moderate accuracy of the mean excitation energy is required for the determination of the electron collision stopping power. Let $\Delta \mathrm{S}_{\mathrm{col}}$ be the uncertainty of $\mathrm{S}_{\mathrm{col}}$ corresponding to an uncertainty $\Delta \mathrm{I}$ of $\mathrm{I}$. From Eq (2.16) it can be seen that at low energies, where the density-effect correction is negligible,

$\Delta \mathrm{S}_{\mathrm{col}} / \mathrm{S}_{\mathrm{CO}}=-(\Delta \mathrm{I} / \mathrm{I}) / \mathrm{L}$, where the stopping number L ranges in value from $\gtrsim 3$ at $10 \mathrm{keV}$ to $\sim 15$ at $1000 \mathrm{MeV}$. At high energies the I-dependence of the density-effect correction is such as to reduce the I-dependence of the collision stopping power further; in fact, in the limit of extremely high energies the collision stopping power becomes independent of I (see Section 6). Figure 3.1 gives the ratio of logarithmic derivatives - $\left(\mathrm{dS}_{\mathrm{Col}} / \mathrm{S}_{\mathrm{col}}\right) /(\mathrm{dI} / \mathrm{I})$ as a function of electron kinetic energy, for a few materials. The value of this ratio decreases with increasing electron energy. The bends in the curves for water at $\sim 0.5 \mathrm{MeV}$, and for air at $\sim 25 \mathrm{MeV}$, are due to the sudden onset of the density-effect correction for nonconducting media.

3.1. Use of oscillator-strength and dielectric data. The use of such data is growing in importance; they are becoming more plentiful and allow the determination of I-values as accurately as from the best stopping-power measurements. The accuracy results in part from the fact that the oscillator-strength distributions are subject to various sum rules which act as constraints in the evaluation of experimental data and provide connections to other measurable physical quantities. The theory underlying the use of oscillator-strength and dielectric data has been reviewed by Fano (1963) and Inokuti and Turner (1978).

For gases, the mean excitation energy can be obtained from the expression 


$$
\ln I=\int_{0}^{\infty} \frac{d f}{d E} \ln E d E / \int_{0}^{\infty} \frac{d f}{d E} d E,
$$

where $\mathrm{df} / \mathrm{dE}$ is the density of optical dipole oscillator strength per unit energy of excitation above the ground state.

A variety of methods has been used to evaluate Eq (3.1). For a dilute gas of free atoms, systematic calculations of oscillator-strength distributions and I-values, based on the use of Hartree-Slater central potentials and a single-electron model, have been carried out by Dehmer et az. (1975) for atomic numbers $Z$ up to 18 , and have been continued by Inokuti et al. (1981) for $Z$ up to 38 . Critical evaluations of experimental oscillator-strength distributions for atomic and molecular gases, based on the use of photo-electric cross sections and inelastic electron scattering cross sections, have been obtained by various authors, for example, Zeiss et al. $(1975 ; 1977 a, b ; 1980)$. Another semi-empirical method, denoted here as M(n) fit, makes use of the moments $M(n)=f^{\infty} \mathrm{df} / \mathrm{dE} E^{\mathrm{n}} \mathrm{dE}$ of the oscillator-strength distribu-

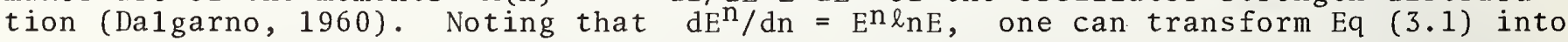

$$
\ln I=\frac{1}{M(0)}\left[\frac{\mathrm{dM}(\mathrm{n})}{\mathrm{dn}}\right]_{n=0}
$$

According to the Thomas-Kuhn sum rule, $M(0)=Z$. The moments $M(2), M(1)$, and $M(-1)$ are calculated theoretically, and $M(-2)$ is obtained from experimental polarizability data. By fitting these moments, an analytical function $M(n)$ is obtained which is then differentiated to get $\ln I$.

I-values for 9 gases, obtained according to Eq (3.1) by various theoretical and semi-empirical methods, are given in Table 3.1, which is an adaptation and slight extension of Table $V$ in Dehmer et al. (1975). The most accurate I-values are those derived with the use of semi-empirical oscillator-strength distributions.

The values of the ratio I/Z obtained in the systematic calculations of Dehmer et al. (1975) and Inokuti et al. (1981) show a periodic variation with $Z$, which is interpreted as due to atomic shell structure. Quite similar variations are found when I-values are calculated on the basis of the local-plasma approximation of Lindhard and Scharff (1953). According to this model the mean excitation energy is obtained from the equation

$$
\ln I=(1 / Z) \int_{0}^{\infty} 4 \pi r^{2} n_{0}(r) \ln \left(\gamma h \omega_{0}\right) d r,
$$

where

$$
\omega_{0}=\left(4 \pi e^{2} n_{0}(r) / m\right)^{\frac{1}{2}}
$$

is the plasma frequency corresponding to a electron density $n_{0}(r)$ at a distance $r$ from the nucleus, $\hbar$ is Planck's constant divided by $2 \pi$, and $\gamma$ is a free parameter for which the value $\sqrt{2}$ is recommended. As pointed out by Dehmer et al. $(1975)$, the use of Eqs (3.3) and (3.4) is equivalent to assuming an approximate oscillatorstrength distribution

$$
\frac{d f}{d E}=\left\langle\delta\left(E-\gamma \hbar \omega_{0}(r)\right)\right\rangle,
$$

where the brackets denote the ground state expectation value and $\delta$ is a delta function. Expression (3.3) was evaluated by Bichsel and Laulainer (1971), by Chu and Powers (1972), and by Ziegler (1980), with electron density distributions derived from Hartree-Slater wave functions. Ziegler has also extended the calculations to solids, using solid-state wave functions. 
Figure 3.2 shows the irregular $Z$-dependence of $I / Z$ as calculated by Dehmer et $a l$., and by Inokuti et al., from theoretical oscillator-strength distributions, and by Chu and Powers and by Ziegler according to the local-plasma approximation. In order to make the two types of results comparable, the local-plasma results are given for $\gamma=1$. As will be shown later in Section 4, the Z-dependence of the experimental $I / Z$ ratios has similar irregularities. Moreover, the calculated and experimental $\mathrm{I} / \mathrm{Z}$ ratios can be brought into rather good agreement with $\gamma \sim 1.3$.

The use of optical dipole oscillator-strength distributions is justified only for dilute gases for which there is only a weak correlation between the positions of the electrons in the medium. For condensed materials the mean excitation energy can be obtained from an alternative equation, in terms of the dielectric-response function $\epsilon(\omega)$ of the medium (Fano, 1956, 1963):

$$
\ln I=\left(2 / \pi \omega_{p}^{2}\right) \int_{0}^{\infty} \mathrm{d} \omega \omega \operatorname{Im}[-1 / \epsilon(\omega)] \ln (\hbar \omega),
$$

where $\omega_{p}$ is the plasma frequency. The corresponding plasma energy ${ }^{6}$ is

$$
\hbar \omega_{p}=\left(4 \pi \hbar^{2} e^{2} n_{e} / m\right)^{\frac{1}{2}}=28.816(\rho Z / A)^{\frac{1}{2}} \mathrm{eV},
$$

where $\mathrm{n}$ is the total number of electrons per unit volume, and $e$ is the charge of the electron.

The dielectric-response function is complex-valued; for non-magnetic materials, its real and imaginary parts, $\epsilon_{1}(\omega)$ and $\epsilon_{2}(\omega)$, can be expressed in terms of the real and imaginary parts, $n(\omega)$ and $k(\omega)$, of the refractive index:

$$
\left.\begin{array}{l}
\epsilon_{1}(\omega)=n^{2}(\omega)-\kappa^{2}(\omega) \\
\epsilon_{2}(\omega)=2 n(\omega) \kappa(\omega)
\end{array}\right\} .
$$

It is therefore possible to obtain $\epsilon(\omega)$ from optical data, and just as in the case of oscillator-strength distributions there are sum rules which serve as consistency checks for experimental data.

The dielectric-response function for aluminum has been evaluated by Shiles et $a$ l. (1980) by a dispersion analysis involving the use of the reflectance, ellipsometric and transmission optical data from many experiments, and also the use of electron energy-1oss data. These authors obtained an I-value of $165.7 \pm 1.0 \mathrm{eV}$ for aluminum. Mean excitation energies for various compounds, obtained in a similar manner (but usually with less abundant optical data) will be discussed in Section 5 .

3.2. Use of stopping-power and range data. There is an abundant experimental literature on stopping powers and ranges for protons and alpha particles (see, e.g., Andersen and Ziegler, 1977; Ziegler, 1977; Andersen, 1977). The extraction of mean excitation energies from such data would be a routine matter if it were not for the fact that the stopping-power theory contains correction terms which are not always known with the accuracy desirable. From measured stopping-power data, the mean excitation energy can be determined by inverting Eq (2.9):

$$
\ln I=\ln \left(\frac{2 m c^{2} \beta^{2}}{1-\beta^{2}}\right)-\beta^{2}-x-\left(\frac{S_{c o l}}{\rho}\right)_{\operatorname{expt}} /\left(\frac{4 \pi N_{a} r_{e}^{2} m c^{2}}{\beta^{2}} \frac{z}{A} z^{2}\right),
$$

\footnotetext{
${ }^{6}$ The plasma energy specified in terms of Eq (3.7) is a nominal value calculated with the electron density for all atomic electrons. It is larger than the plasma energy used to describe collective excitations (plasmons) which is calculated with the density of participating electrons.
} 
where

$$
x=\frac{C}{Z}+\frac{\delta}{2}-z L_{1}-z^{2} L_{2}
$$

is the total correction, combining the shell correction, density-effect correction, Barkas correction, and Bloch correction.

Let $\Delta \mathrm{S}_{\mathrm{CO}}$ denote the uncertainty of the measured value of $\mathrm{S}_{\mathrm{Col}}$, and let $\Delta \mathrm{x}$ denote the uncertainty of the correction term $x$. Assuming that the two uncertainties can be combined quadratically, the overall uncertainty of the estimated I-value is

$$
\Delta I=I \cdot\left[\left(\Delta S_{c o 1} / S_{c o 1}\right)^{2} L_{e x p}^{2}+(\Delta x)^{2}\right]^{\frac{1}{2}}
$$

where Lexp is the experimental stopping number (1ast term in Eq (3.9)). When range data are used to determine I-values, the error analysis is more complicated, and must take into account the uncertainty of the experimental range value as well as the uncertainties of the correction term $x$ at all energies up to the initial projectile energy.

In the evaluation of the correction $x$ we have followed a procedure suggested by $H$. Bichsel, earlier versions of which have been described by Bichsel (1961, 1963, 1972). Included in the procedure are the evaluation of the Barkas correction according to Ashley, Ritchie, and Brandt $(1972,1973)$, the Bloch correction according to Eq (2.14), and the shell correction by the method outlined below.

3.2.1. Bichsel's shell corrections. The total shell correction $C$ is the sum of the contributions $C_{K}, C_{L}, \ldots$ from the different atomic shells. Theoretical values calculated with hydrogenic wave functions are available for $\mathrm{C}_{K}$ (Walske, 1952; Khandelwal, 1968), for $C_{L}$ (Walske, 1956; Bichse1, 1967; Khandelwal, 1968), and for $\mathrm{C}_{\mathrm{M}}$ (Khandelwal and Merzbacher, 1966). The error due to the use of hydrogenic wave functions is expected to be relatively minor for the $K$ shell, more serious for the $L$ shell (particularly for atomic numbers $Z \lesssim 30$ ) and probably even greater for the M she11.

Extension of the calculations to higher she11s, with use of improved wave functions, is possible but would be very laborious. Bichsel instead adopts a semiempirical scaling procedure with parameters that are determined by experimental stopping-power data. He assumes that the dependence of $C_{M}$ on the particle velocity is similar to that of $C_{L}$, except for scale factors, and also extends this assumption to the higher shells. The Walske result for the $L$ shell, $C_{L}\left(\theta_{L}, \eta_{L}\right)$, depends on the atomic number through the parameter $\theta_{L}$ and on the particle energy through the quantity

$$
\eta_{L}=\left(\beta / \alpha Z^{*}\right)^{2}
$$

where $Z^{*}=Z-4.15$ is the effective nuclear charge for the $L$ shell. The M-shell correction is calculated from the scaling relation

$$
C_{M}=V_{M} C_{L}\left(\theta_{L}, H_{M}{ }^{\eta}\right),
$$

where $V_{M}$ is equal to $1 / 8$ times the number of electrons in the $M$ shell, and where $\mathrm{H}_{\mathrm{M}}$ is an adjustable parameter. Analogous scaling relations are used for the $\mathrm{N}^{\mathrm{M}}$ she 11 and for a combined $0-\mathrm{P}$ shell.?

${ }^{7}$ For $z<10$, Eq (3.13) is also applied to the incompletely filled L she11, with $V_{L}$ as defined above and $H_{L}=1$. For low atomic numbers, the effective charge $Z^{*}$ for the $L$ shell has in this work been set equal to $Z$ - d, with values of d taken from Clementi and Raimondi (1963).

$$
\text { For } \begin{aligned}
& Z=3 \\
& \mathrm{~d}=1.72,2.09,2.48,2.82,3.16,3.53,3.84,4.24 . \\
& \hline
\end{aligned}
$$


The scaling parameters $H_{n}$ (for $n=M, N$, and $0-P$ ), the parameter $b$ of the Ashley-Ritchie-Brandt theory for the Barkas correction, and the mean excitation $I$ were determined by Bichsel in a two-stage procedure. First he chose values of I, $\mathrm{b}$, and the $\mathrm{H}_{\mathrm{n}}$ 's by trial and error for a small number of key elements so as to obtain the best possible agreement between calculated and measured stopping powers and ranges for protons and alpha particles, taking into account all available data. The parameters thus chosen for $\mathrm{C}, \mathrm{Al}, \mathrm{Cu}, \mathrm{Ag}$, and $\mathrm{Au}$ are given in Table 3.2. They are of course not unique, but provide a good fit to the data. Table 3.3 gives illustrative values, for the same elements, of the shell corrections, Barkas corrections, Bloch corrections, and the stopping number per electron. The relative contributions of the various corrections to the proton stopping number for gold are indicated in Fig. 3.3 where it can be seen that at energies above $500 \mathrm{MeV}$ the densityeffect correction is more important than the shell correction.

The second stage of Bichsel's procedure consists of interpolating the parameters $\mathrm{H}_{\mathrm{n}}$ vs. $\mathrm{Z}$, to obtain values for all elements. His interpolation scheme is given in Table 3.4 which also lists recommended b-values for all elements. The shell corrections thus obtained exhibit small irregularities as functions of $Z$, especially at 1 ow proton energies. Before being used in the present work they were smoothed graphically. The smoothed shell corrections of Bichsel, plotted vs. $Z$, are shown in Fig. 3.4 for various proton energies from $2 \mathrm{MeV}$ to $100 \mathrm{MeV}$. Bichsel's shell corrections as functions of the proton energy are given in Fig. 3.5 for various elements.

3.2.2. Comparison of Bichsel's and Bonderup's shell correction. Theoretical shell corrections have been derived by Bonderup (1967) on the basis of the statistical gas model of Lindhard and Scharff (1953) and Lindhard and Winther (1964). The theoretical she11 corrections, plotted against atomic number, are shown in Fig. 3.4 and can be seen to agree rather well with Bichsel's empirical shell corrections for atomic numbers $Z$ up to $\sim 50$. With increasing $Z$ the Bonderup corrections continue to increase whereas those of Bichsel reach a peak and then decrease. ${ }^{8}$

To investigate the influence of the adopted shell corrections we now consider the high-energy proton range measurements of Barkas and von Friesen (1961) and the low- and intermediate-energy stopping-power measurements of Burkig and MacKenzie (1957), Sørensen and Andersen (1973) and Ishiwari et al. (1979) for the elements Cu, $\mathrm{Pb}$, and $\mathrm{U}$.

The proton energy in the Barkas-von Friesen experiment had the nominal value of $750 \mathrm{MeV}$ but was uncertain by one or two MeV. Using the measured proton range value of $273.29 \mathrm{~g} / \mathrm{cm}^{2}$ in aluminum, and using the accurate value $\mathrm{I}_{\mathrm{Al}}=166 \mathrm{eV}$ from Shiles et al. (1980), one estimates the beam energy to have been $749.0 \mathrm{MeV}$. With this energy one obtains the I-values shown in Table 3.5, which are estimated to have an uncertainty of $\sim 10 \mathrm{eV}$. The use of Bonderup's rather then Bichsel's she11 corrections would lower the I-values for $\mathrm{Pb}$ and $U$ by a few eV but leave that for $\mathrm{Cu}$ unchanged. It is interesting to note that the omission of the density-effect correction would have a much greater influence on I than the change of shell corrections.

Table 3.6 compares I-values deduced from medium-energy as well as high-energy experiments. It can be seen that for copper the same I-value is obtained (within the estimated uncertainties) regardless of the type of shell correction that is used. For lead and uranium, however, the use of the Bonderup shell correction leads to estimated I-values for lead and uranium that are $\sim 10$ percent lower for the mediumenergy expeximents than for the high-energy experiment. With Bichsel's shell corrections, approximately the same I-value is obtained regardless of energy. Inasmuch as the mean excitation energy is a material constant independent of the projectile energy, we have adopted Bichsel's shell corrections as providing the better representation of the experimental situation. 9

${ }^{8} \mathrm{~A}$ similar turning over of the curve of shell correction $v s$. atomic number is also characteristic of the empirical shell corrections given by Andersen and Ziegler (1977) which lie between the Bonderup and Bichsel shell corrections.

${ }^{9}$ It may be noted that Andersen and Nielsen (1981) also find that the Bonderup shell correction for protons in gold at energies from 2 to $7 \mathrm{MeV}$ is 1 arger than the empirical shel1 correction which they deduce from their stopping-power measurements. These authors suggest that the discrepancy may be due to the non-relativistic treatment of inner-shell electrons in Bonderup's theory. 


\section{SELECTION OF MEAN EXCITATION ENERGIES FOR ELEMENTS}

Using Eq (3.9) with shell corrections from Bichsel, the Barkas correction according to Ashley, Ritchie, and Brandt, and the Bloch correction, we have extracted I-values for a large number of elements from 22 experiments. The data are mainly for protons with energies $\gtrsim 5 \mathrm{MeV}$, so that the value of the correction term $\mathrm{x}=\mathrm{C} / \mathrm{Z}+\delta / 2-z \mathrm{~L}_{1}-z^{\widetilde{2}} \mathrm{~L}_{2}$ is in general small compared to the stopping number (see Table 3.3). The measurements that were analyzed are listed in Table 4.1. The deduced I-values and their uncertainties are given in Table 4.2. The overall uncertainties have been calculated according to Eq (3.11), combining quadratically (a) the experimental uncertainties, and (b) an assumed 10-percent uncertainty of the correction term $x$.

Several of the experiments listed in Tab1e 4.1 provide stopping-power ratios relative to a reference material. Burkig and Mackenzie (1957) and Nakano et al. (1963) measured stopping-power ratios relative to aluminum. We have interpreted these by assuming the stopping-power for aluminum to be that calculated with an I-value of $166 \mathrm{eV}$ as obtained by Shiles et al. (1980) from dielectric data. The experiments of Bakker and Segrè (1951) and of Thompson (1952) give stopping-power ratios with respect to copper, which we have analyzed assuming an I-value of $322 \mathrm{eV}$ for copper consistent with various other experimental results. The experiment of Nordin and Henkelman (1979) gives stopping-power ratios relative to liquid water, which have been analyzed assuming for water an I-value of $75 \mathrm{eV}$ derived by Ritchie et al. (1978) and Ashley (1982a) from dielectric data. The measurement uncertainties entered into Eq (3.11) have been augmented to include the uncertainties of the calculated stopping powers of the reference materials.

The experimental results of Thompson were also analyzed relative to water instead of copper, and those of Nordin and Henkelman relative to aluminum instead of water. This changed the deduced I-values by only a fraction of one percent, and an average of the values with the two reference materials was taken. The I-values from the experiment of Bakker and Segrè, when analyzed with respect to aluminum instead of copper, changed significant1y, and both sets of I-values are given in Table 4.2.

The final choices of mean excitation for elements were made as follows.

(a) For the gases $\mathrm{H}_{2}$, He, $\mathrm{N}_{2}, \mathrm{O}_{2}$, and $\mathrm{Ne}$, the information from semi-empirical dipole oscillator-strength distributions was judged to be more accurate than that from stopping-power data, and I-values were selected from those given in Table 3.1 . (b) For aluminum, the value $I=166 \mathrm{eV}$ was used as derived by Shiles et al. from dielectric data; this value is also supported by the results of several stoppingpower measurements. (c) For other elements, I-values were selected by taking into account the results from stopping-power and range experiments collected in Table 4.2. The available information is in most cases good enough to specify I-values accurate to a few percent.

We have also estimated I-values for elements for which no experimental information is available. Following Andersen and Ziegler (1977) we have done this by imitating the local $Z$-dependence as predicted by the results of calculations done in the local-plasma approximation (see Fig. 3.2). The following interpolation scheme was used. Denoting calculated I-values by $I_{C}(Z)$, experimental values by $I(Z)$ and interpolated values by $I_{\text {int }}(Z)$, we have assumed that

$$
I_{\text {int }}(Z)=I_{c}(Z)\left|\frac{I\left(z_{1}\right)}{I_{c}\left(z_{1}\right)} \frac{z_{2}-z}{z_{2}-z_{1}}+\frac{I\left(z_{2}\right)}{I_{c}\left(z_{2}\right)} \frac{Z-Z_{1}}{z_{2}-z_{1}}\right| \text {, }
$$

where $Z_{1}$ and $Z_{2}$ are the closest atomic numbers, with experimental I-values, that bracket the atomic number $Z$ of interest. Either $Z, Z_{1}$, and $Z_{2}$ pertain to gases, in which case the required values of $I_{C}$ are taken from the results of Chu and Powers (1972) obtained with free-atom wave functions, or $Z, Z_{1}$, and $Z_{2}$ pertain to solids in which case the values of $I_{C}$ are taken from the results of Ziegler (1980) obtained with solid-state wave functions (the version indicated by ziegler as first-order solid with continuous interstitials). 
The value $I=700 \mathrm{eV}$ listed in Table 3.1 for radon, obtained by Bell and Dalgarno (1965) by a "M(n) fit" (see Section 3.1), is expected to be inaccurate because of a numerical error in the moment $M(-1)$ and because of neglected relativistic effects. The adopted value $I=794 \mathrm{eV}$ was estimated by extrapolating the ratios $I(Z) / I_{C}(Z)$ for rare gases to $Z=86$.

Table 4.3 gives the adopted I-values, and the corresponding ratios I/Z, for all elements. The estimated uncertainties of the I-values are given only for experimentally-based I-values. These uncertainties are intended to take into account both the uncertainties of individual experimental results and the scatter of the results from different experiments. ${ }^{10}$ The interpolated I-values are enclosed in parentheses. To indicate the irregular $Z$-dependence of the adopted mean excitation energies, a plot of $I / Z$ vs. $Z$ is shown in Fig. 4.1.

As a check on the acceptability of the adopted $I$-values and correction terms to the stopping-power formula, we demonstrate in Figs. (4.2) to (4.5) that close fits are obtained to a large body of experimental stopping-power data. Comparisons are made in terms of the ratio of the experimental to the calculated stopping power. Figure 4.2, taken from Bichsel and Porter (1982), pertains to protons and alpha particles in $\mathrm{N}_{2}$ and $\mathrm{O}_{2}$. Figure 4.3 pertains to proton stopping power at energies up to $20 \mathrm{MeV}$ in $\mathrm{Al}, \mathrm{Cu}, \mathrm{Ag}, \mathrm{Au}, \mathrm{Pb}$, and $\mathrm{U}$. Figures 4.4 and 4.5 pertain to the proton stopping-power measurements of Ishiwari et al. (1979) at $6.5 \mathrm{MeV}$, and those of Burkig and MacKenzie (1957) at $19.8 \mathrm{MeV}$, in many elements.

For a few elements, the I-values adopted in this report are compared in Table 4.4 with I-values recommended in eleven earlier papers.

\section{SELECTION OF MEAN EXCITATION ENERGIES FOR COMPOUNDS}

The experimental information on I-values for compounds is steadily increasing. I-values for many gases have been determined by Zeiss et al. (1975, 1977a,b), Thomas and Meath (1977), and Jhanwar et al. (1981) from semi-empirical dipole oscillatorstrength distributions. For liquid water and for various plastics, I-values have been obtained by Ritchie et al. (1978), Ashley (1979) and Painter et az. (1980) from dielectric data. I-values for a large number of condensed compounds, obtained under uniform conditions, can be deduced from an experiment by Thompson (1952) who measured the pathlengths traveled by protons while slowing down from $340 \mathrm{MeV}$ to $200 \mathrm{MeV}$ in many organic liquids, in water, and in a few solids. Thompson showed that the ratios of these pathlengths to the pathlength in a reference material (copper) can be interpreted as reciprocals of the corresponding stopping-power ratios at an intermediate energy. We have re-analyzed Thompson's data, applying small multiplescattering corrections according to a procedure of Bichsel (1954) and shell corrections for copper, to obtain stopping-power ratios relative to copper at $267.5 \mathrm{MeV}$.

There are many compounds of interest for which one needs to estimate I-values in the absence of direct experimental information. It has been known since the early work of Bragg and Kleeman (1905) that the collision stopping-power of a compound can be approximated by the weighted sum of the stopping powers of the atomic constituents of the compound. For the mass collision stopping power $S_{c o l} / p$, the additivity rule takes the form

$$
\frac{1}{\rho} S_{c o 1}=\sum_{j} w_{j} \frac{1}{\rho} S_{c o 1}
$$

where $w_{j}$ is the fraction by weight of the $j^{\prime}$ th atomic constituent. This additivity rule is equivalent to replacing, in the stopping-power formula Eq $(2.16)$, the quantities $\mathrm{Z} / \mathrm{A}, \mathrm{I}$, and $\delta$ by

${ }^{10}$ The uncertainties of the I-values in Table 4.3 are figures of merit, arrived at by subjective judgements, and with a meaning that is not easily defined. One possible interpretation would be the following. If, in the future, the measurement accuracy and theoretical analysis are sufficiently improved so that I-values can be determined with an accuracy an order of magnitude better than at present, we expect that for at least 90 percent of the cases in Table 4.3 the future I-values will lie within the limits of uncertainty given in this report. 


$$
\begin{gathered}
z / A=\sum_{j} w_{j}\left(z_{j} / A_{j}\right), \\
\ell n I=\left[\sum_{j} w_{j}\left(z_{j} / A_{j}\right) \ln I_{j}\right] /\langle Z / A\rangle, \\
\delta=\left[\sum_{j} w_{j}\left(z_{j} / A_{j}\right) \delta_{j}\right] /\langle Z / A\rangle,
\end{gathered}
$$

where $Z_{j}, A_{j}, I_{j}$, and $\delta_{j}$ pertain to the $j^{\prime}$ th constituent. It should be noted that $\langle Z \% A\rangle$ is equal to the total number of electrons in the molecule divided by the molecular weight. The use of $\langle\delta\rangle$ calculated from Eq (5.4) is in general not a good approximation, and it is preferable to calculate the density-effect correction directly for the compound of interest (see Section 6.3).

The application of this additivity rule requires the choice of appropriate values for the mean excitation energies of the constituents. The simplest procedure, often used in the past, is to take the same I-values for the atomic constituents of a compound as for the corresponding elemental substances. This introduces some error because of the neglect of molecular binding effects. An additional error may be incurred when elemental I-values for gases (e.g., oxygen) are applied to the constituents of solid compounds.

The accuracy of the additivity rule can be improved by assigning I-values to the constituents which are not unique but take on various values depending on the type of compound and on the physical state of aggregation of the medium. When carried to an extreme this is equivalent to abandoning additivity and treating each compound separately. However, it is possible to find simple rules for assigning I-values to atomic constituents with which the I-values for a large number of compounds can be represented satisfactorily. We have adopted such an assignment scheme which is given in Table 5.1.

A more elaborate assignment scheme was developed earlier by Thompson (1952) who interpreted his experimental stopping powers for various compounds in terms of the stopping powers (and I-values) of atomic constituents in various chemical environments. We have extracted I-values from Thompson's stopping powers for atomic constituents, and have thus obtained the up-dated Thompson assignment scheme given in Table 5.2. In this scheme, different I-values are assigned to constituents depending on the type of chemical bond involved. Thompson's conclusions as to the influence of bond types on the I-values of atomic constituents in organic liquids are quite plausible. However, the available data base is not sufficient to pin these distinctions down with great precision, and there is a need for further experiments similar to Thompson's.

Mean excitation energies for organic solids and other compounds have also been estimated by Brandt $(1956,1958,1960)$ on the basis of experimental data and theoretical considerations. Brandt (1960) used as an initial approximation free-atom I-values for atomic constituents according to a formula of Jensen (1937), modified these values to take into account the valence states (aromatic, aliphatic, ...) of atomic constituents, and then assumed Bragg additivity. Brandt also applied a "lowenergy density-effect" correction which raised the mean excitation energy for the compound as a whole. His valence-state corrections and density-effect corrections were obtained from theoretical polarization data for atoms and experimental molarrefractivity data for molecular compounds. As will be shown later in the comparison in Table 5.6, Brandt's theoretical I-values for low-Z compounds are not very different from those adopted in the present work. However, his I-values for compounds containing high-z constituents tend to be too low, a fact already noted by Brandt with respect to photographic emulsion.

Table 5.3 lists the presently available experimental I-values, and their origin, for 13 gases, 27 liquids, and 14 solids. The table also gives the differences between these I-values and corresponding Bragg-additivity I-values obtained with our adopted assignment scheme and that of Thompson. In most cases these differences are smaller than the estimated experimental uncertainties. There are large discrepancies 
in only two cases, for liquid dichloroethane and for paraffin wax. ${ }^{11}$ For gases our assignment scheme works particularly well. For liquids it is slightly inferior to Thompson's scheme, but for solids it is somewhat better.

Thompson's scheme includes more chemical detail than ours but on the whole works no better and is more complicated to apply. We have therefore used our scheme (Table 5.1) for the prediction of I-values in the absence of experimental data. For compounds with the constituents $\mathrm{H}, \mathrm{C}, \mathrm{N}$, and $\mathrm{O}$ (and perhaps also $\mathrm{F}$ and $\mathrm{Cl}$ ) such predictions have a sound empirical basis. One would expect that the errors associated with such predictions will have the same order of magnitude as the differences between experimental and Bragg-additivity I-values in Table 5.3. The situation is less clear in regard to condensed materials with atomic constituents other than those listed above. Our assignment scheme uses for these other constituents I-values that are 13 percent larger than the corresponding I-values for condensed elemental substances as given in Table 4.3. This increase was found to be required to obtain a good approximation to the rather accurately known experimental I-values for $\mathrm{Al}_{2} \mathrm{O}_{3}, \mathrm{SiO}_{2}$, and photographic emulsion, and is also consistent with the less certain experimental data for $\mathrm{LiF}$ and $\mathrm{CaF}_{2}$. In the absence of other information we have assumed that the 13 -percent increase should also be applied to the constituents of other compounds such as sodium iodide and cesium iodide.

Certain composite materials, for example tissue-equivalent gases, pyrex glass, and human tissues, have constituent molecules whose I-values are known independently from experiments. In order to take advantage of this information, we have treated such composite materials via a Bragg rule for the combination of atomic and molecular constituents. For human tissues, a prominent constituent with a known I-value is water. In Table 5.4, the water content and I-values for various types of tissue are given.

Table 5.5 lists the adopted mean excitation energies, densities and elemental compositions for the 46 compounds and mixtures for which electron stopping-power tables are given in this report. The symbol + indicates I-values derived directly from experimental data or I-values for mixtures (such as air) all of whose constituents have experimentally determined I-values. The symbol ++ indicates I-values derived by applying the additivity rule to molecular constituents one or more of which have experimentally determined I-values. In all other cases, the I-values were obtained from the additivity rule, Eq (5.3), with constituent I-values from Table 5.1.

A letter grade (A, B, or C) is given next to each I-value in Table 5.5, to indicate the relative quality of these data. The assignment of these grades was based on the information contained in Table 5.3 , but also involved subjective judgements. For the most part the following guidelines were followed. Direct experimental I-values were given the grade A unless the experimental uncertainty was greater than 5 percent, in which case they were given the grade B. I-values for low-Z gas compounds were given the grade A. I-values for condensed compounds were given the grade $B$ if the constituents consisted predominantly of the elements $H$, $\mathrm{C}, \mathrm{N}, \mathrm{O}, \mathrm{F}$, or $\mathrm{Cl}$. The grade $\mathrm{C}$ was given to I-values for condensed compounds which contain mainly other constituents whose I-values were adjusted upward by the 13-percent rule. We would assign an estimated uncertainty ${ }^{2}$ of 5 percent with grade $A, 5$ to 10 percent with grade $B$, and 10 to 15 percent with grade C. In order to indicate how the recommended I-values for compounds have changed over the years, a comparison with previously recommended values is given in Table 5.6.

For electrons, the dependence of the collision stopping power on the state of aggregation can be expressed completely through the mean excitation energy, provided the Bethe stopping-power theory (without shell corrections) is applicable. This dependence is indicated in Table 5.7 for a few elements and compounds. It should be noted that the values for molecular gases, liquids and solids are empirical, whereas those for "atomic gases" are from theoretical oscillator-strength distributions.

${ }^{11}$ Because the experimental I-value for paraffin wax is inconsistent with those for other straight-chain hydrocarbons (n-pentane, n-hexane, n-heptane, and polyethylene), we have adopted the I-value of $55.9 \mathrm{eV}$, using the Bragg-additivity values according to Table 5.1. This is the only case in which the Bragg-additivity value was adopted in preference to the experimental value.

${ }^{12}$ Concerning the meaning that might be attached to the estimated uncertainties, see footnote 10 . 


\section{DENSITY EFFECT}

6.1. General equations. The passage of a charged particle through a medium results in the polarization of atoms in the medium, and this polarization in turn screens the electric field acting on the particle so as to reduce the stopping power. This reduction is particularly strong in dense media, and is therefore called the density effect. The greater the particle velocity, the greater is the density effect because, by virtue of the Lorentz contraction, distant collisions become more important. In fact, at very high energies the density-effect correction to the stopping power is significant even in dilute gases.

The density effect was first predicted by Swann (1938) and calculated by Fermi (1940). The results of such a calculation depend on the model used for representing the dielectric properties of the medium. Whereas Fermi used only one dispersion frequency, more realistic models were developed by Wick (1943), Halpern and Hal1 (1948), and Sternheimer (1952). Sternheimer introduced a procedure for making the dielectric model consistent with the (experimentally known) mean excitation energy for the medium, which is essential for obtaining accurate results.

As shown by Fano (1956, 1963), the density-effect correction can be expressed as follows in terms of the dielectric-response function $\epsilon(\omega)$ of the medium:

$$
\delta=\left(2 / \pi \omega_{p}^{2}\right) \int_{0}^{\infty} \omega \mathrm{d} \omega \operatorname{Im}[-1 / \epsilon(\omega)] \ln \left(1+\ell^{2} / \omega^{2}\right)-\left(\ell / \omega_{p}\right)^{2}\left(1-\beta^{2}\right),
$$

where $\omega_{p}$ is the plasma frequency, proportional to the square root of the density, given by $\mathrm{Eq}$ (3.7). The quantity $l$ is defined, as a function of $\beta$, as the root of the equation

$$
1-B^{2} \epsilon(i \ell)=0 .
$$

It can be shown that in the limit $\beta \rightarrow 1$

$$
\delta \rightarrow \ln \left[\left(\hbar \omega_{p}\right)^{2} /\left(1-\beta^{2}\right) I^{2}\right]-1
$$

When this result is inserted into the stopping-power Eqs (2.9) or (2.16), the mean excitation energy I disappears from the final result. Thus in the limit of very high energies, the stopping power depends on the properties of the medium only through the plasma energy $\hbar \omega_{p}$, and therefore only on the density and the ratio $\mathrm{Z} / \mathrm{A}$.

In the solution of Eq (6.2), a difference arises between conducting and nonconducting media. For conductors, the dielectric-response function is such that the equation has a root for any value of $\beta$. The density effect is then present, though sma11, even at arbitrarily low energies. For insulators, however, a root exists only above a minimum value of $\beta_{0}=\epsilon(0)^{-\frac{1}{2}}$, which is a property of the material, and there is no density effect for $B<\beta_{0}$.

The most accurate method of evaluating the density-effect correction is to use semi-empirical dielectric-response functions in Eqs (6.1) and (6.2). This has recently been done by Inokuti and Smith (1982) for aluminum, and by Ashley (1982b) for water. Reliable and complete dielectric-response functions for other materials are scarce, and in general one must rely on Sternheimer's method, which - - although more approximate - - will be shown below to give results in very good agreement with those of Inokuti and Smith and of Ashley.

6.2. Sternheimer's mode1. As discussed by Fano (1963), Sternheimer's mode1 can be related to Eqs (6.1) and (6.2) as follows. In Eq (6.1) one sets $\epsilon=1 /\left(1-\alpha_{L}\right)$, where

$$
\alpha_{L}(\omega)=\left(\hbar \omega_{p}\right)^{2} \sum_{n=1}^{N} \frac{f_{n L}}{E_{n L}-(\hbar \omega)^{2}-i \gamma_{L} \hbar \omega}
$$


is the longitudinal polarizability expressed in terms of the energy levels $\mathrm{E}_{\mathrm{nL}}$ of single atoms and the corresponding oscillator strengths $f_{n L}$. In the limit in which the damping constant $\gamma_{L} \rightarrow 0$, the resulting expression for $\delta$ becomes

$$
\delta=\sum_{n=1}^{N} f_{n L} \ln \left[1+(h \ell)^{2} / E_{n L}^{2}\right]-\left(\ell / \omega_{p}\right)^{2}\left(1-\beta^{2}\right) .
$$

In $\mathrm{Eq}(6.2)$, one sets $\epsilon=1+\alpha_{\mathrm{T}}$, where $\alpha_{\mathrm{T}}$ is the transverse polarizability and is given by an expression similar to Eq (6.4) but with different energy levels $\mathrm{E}_{\mathrm{nT}}$, oscillator strengths $f_{\mathrm{nT}}$, and damping term $\gamma_{\mathrm{T}}$. In the limit $\gamma_{\mathrm{T}} \rightarrow 0$, Eq $(6.2)$ is then transformed into

$$
\alpha_{T}(i \ell)=\left(h \omega_{p}\right)^{2} \sum_{n=1}^{N} \frac{f_{n T}}{E_{n T}^{2}+(\hbar \ell)^{2}}=\frac{1}{\beta^{2}}-1 .
$$

The following approximations are now made:

a. The distinction between the longitudinal and transverse oscillator strengths $f_{n L}$ and $f_{n T}$ is disregarded, and both are approximated by $f_{n}$, the fraction of electrons in the n'th atomic shell.

b. The energy levels are assumed to be

$$
\begin{aligned}
& E_{n T}=\mu_{S t} E_{n}, \\
& E_{n L}=\left[\mu_{S t}^{2} E_{n}^{2}+f_{n}\left(\hbar \omega_{p}\right)^{2}\right]^{\frac{1}{2}},
\end{aligned}
$$

where the $E_{n}$ 's are atomic energy levels. The quantity $\mu_{S t}$ is the Sternheimer factor and is given a value such that the equation

$$
\sum_{n=1}^{N} f_{n} \ln \left[\mu_{S t}^{2} E_{n}^{2}+f_{n}\left(\hbar \omega_{p}\right)^{2}\right]^{\frac{1}{2}}=\ln I
$$

is satisfied. The dielectric model is then consistent with the mean excitation energy I (as known from experimental data), and the density-effect correction $\delta$ calculated according to $\mathrm{Eq} \mathrm{(6.5)} \mathrm{goes} \mathrm{into} \mathrm{the} \mathrm{correct} \mathrm{asymptotic} \mathrm{limit,} \mathrm{Eq} \mathrm{(6.3),}$ for very high particle energies. The values of $\mu$ St typically range from 1.5 to 2.5 .

For conductors, the electrons in the outermost shell, $\mathrm{n}=\mathrm{N}$, are considered to be conduction electrons, and the corresponding binding energy $E_{N}$ is set equal to zero. This has the consequence that Eq (6.6) has a solution for arbitrarily small values of $\beta$. For insulators, on the other hand, Eq (6.6) has a solution only for velocities greater than $\beta_{0}$ given by

$$
\frac{1}{\beta_{0}^{2}}-1=\sum_{n=1}^{N} \frac{f_{n}}{\mu_{S t}^{2} E_{n}^{2} /\left(\hbar \omega_{p}\right)^{2}}
$$

As a final refinement, which makes little difference except for liquid $\mathrm{H}_{2}$, a Lorentz-Lorenz correction is applied (Sternheimer, 1952), through the replacement of $\mathrm{f}_{\mathrm{n}}\left(\hbar \omega_{\mathrm{p}}\right)^{2}$ in Eqs $(6.8)$ and $(6.9)$ by $\lambda_{n} \mathrm{f}_{\mathrm{n}}\left(\mathrm{h} \omega_{\mathrm{p}}\right)^{2}$, where $\lambda_{N}=1$ for conductors, and $\lambda_{n}=2 / 3$ in all other cases.

6.3 Numerical evaluation. The evaluation is done by first solving Eq (6.9) for $\mu_{S t}$, then Eq (6.6) for $\ell$ as a function of $B$, and substituting the value of $\ell$ into Eq (6.5). Such calculations using I-values current at the time, were carried 
out by Sternheimer (1952, 1956, 1966, 1967) and Sternheimer and Peier1s (1971) for many materials. The results were reported in terms of a useful approximation formula for $\delta$. In the present work we have systematically re-evaluated $\delta$ using the I-values adopted in the present report and atomic binding energies from Appendix 1 of Carlson (1975). The number of conduction electrons for metals and semi-conductors was taken to be equal to the lowest valence number. Compounds have been treated as insulators. For compounds, the sums with respect to $n$ in the various equations were extended to include all atoms in the compound. Departures from simple Bragg additivity were taken into account by using the appropriate density and mean excitation energy for the compound. We have used the direct numerical output of the calculations rather than an analytical approximation formula when computing the stopping-power tables.13 These values of $\delta$ are listed in the Main Tables.

Values of $\delta$ for a few media, calculated by the method of Sternheimer described above, are shown in Fig. 6.1. When expressed as functions of the particle kinetic energy in units of the rest mass, these results are applicable to any charged particle. Table 6.1 gives illustrative results regarding the percent reduction of the electron collision stopping power due to the density effect.

Figure 6.2 shows the difference between the $\delta$-value of Inokuti and Smith (1982) and the corresponding Sternheimer value for aluminum, and Fig. 6.3 shows the difference between the $\delta$-value of Ashley $(1982 \mathrm{~b})$ and the corresponding Sternheimer value for water. In both cases the differences are positive at some energies and negative at others. For aluminum the absolute value of the difference is always smaller than $\sim 0.04$, and for water it is always smaller than $\sim 0.09$. Figure 6.4 shows the percent amount by which the electron collision stopping power is changed when the more exact density-effect corrections of Inokuti and Smith or of Ashley are replaced by Sternheimer's corrections. It can be seen that the absolute percent difference is smaller than 0.2 percent for aluminum and smaller than 0.5 percent for water. The very satisfactory agreement in the cases of aluminum and water gives one confidence that Sternheimer's results will be accurate in genera1. ${ }^{14}$

6.4. Complications for inhomogeneous media. The theory for the density effect is designed for media that are homogeneous and isotropic. In some cases of practical interest these conditions are not met. An important case is that of graphite, a porous material consisting of somewhat loosely packed graphite crystallites arranged in a layered structure with a dielectric-response function that is a directiondependent tensor (Raether, 1980). The crystallite density is $2.265 \mathrm{~g} / \mathrm{cm}^{2}$, whereas the bulk density may range from 1.5 to $1.9 \mathrm{~g} / \mathrm{cm}^{2}$, depending on the method by which the graphite is manufactured. Typical densities for reactor-grade graphite are 1.7 to $1.8 \mathrm{~g} / \mathrm{cm}^{3}$. The porosity structure is complicated (Gmelin, 1968), including "micropores" ranging in diameter from 2 to 60 nanometers, and "macropores" ranging in diameter up to 20 micrometers. It is not clear what density value should be used in a simple theory which neglects all these complications. In the stopping-power tables we give results for densities of 1.7 and $2.265 \mathrm{~g} / \mathrm{cm}^{3}$. At energies above a few $\mathrm{MeV}$, the use of the higher density would decrease the collision stopping power by more than one percent.

Another case of interest is photographic emulsion. The standard emulsion considered here consists of $10 \mathrm{w}-\mathrm{z}$ gel (17.4\% by weight, average density $1.29 \mathrm{~g} / \mathrm{cm}^{3}$ ) and silver halides $\left(82.6 \%\right.$ by weight, average density $\left.6.47 \mathrm{~g} / \mathrm{cm}^{3}\right)$. Two extreme approaches can be considered: (a) The density effect can be calculated as if the emulsion were homogeneous, with the bulk density $3.185 \mathrm{~g} / \mathrm{cm}^{3}$; this is what was done to produce the tabulated results. (b) The emulsion can be considered to be inhomogeneous, and the density effect can be calculated separately for the two components, gel and silver halides, with the proper density for each. The use of method (b) instead of (a) would lower the collision stopping power by $0.2 \%$ at $1 \mathrm{MeV}, 0.4 \%$ at $10 \mathrm{MeV}, 0.8 \%$ at $100 \mathrm{MeV}$ and $0.7 \%$ at $1000 \mathrm{MeV}$.

Similar considerations can also be applied to A-150 tissue-equivalent plastic which consists of an inhomogeneous mixture of polyethylene, nylon, carbon black and calcium fluoride (Smathers et al., 1977). In this case the use of method (b) instead of (a) would lower the collision stopping power by no more than $0.1 \%$.

\footnotetext{
${ }^{13}$ Updated parameters for Sternheimer's approximation formula will be given in a forthcoming paper (Sternheimer, Seltzer, and Berger, 1982).

${ }^{14}$ The evidence from high-energy charged-particle penetration data also confirms the accuracy of Sternheimer's method (see, e.g., Crispin and Fowler, 1970).
} 


\section{RESTRICTED COLLISION STOPPING POWER}

In radiation dosimetry and in radiobiological modeling (track-structure theory) one may want to know the fraction of the energy lost by an electron that is absorbed "locally" in the medium in the vicinity of the electron track. To obtain this fraction accurately one must carry out transport calculations that take into account (a) the initial energy spectrum and angular distribution of the secondary electrons set in motion in ionization events, and (b) the penetration, diffusion and slowing down of the secondary electrons. A simple, approximate answer can be obtained in terms of the fraction $\mathrm{L}(\mathrm{T}, \Delta) / \mathrm{S}_{\mathrm{Col}}(\mathrm{T})$, where $\mathrm{L}(\mathrm{T}, \Delta)$ is the restricted collision stopping power. (The symbol L $(\mathrm{T}, \Delta)$ used here should not be confused with the stopping number $L$ discussed in Sections 2 and 3.) This quantity is defined as the mean energy loss per unit pathlength due to collisions involving energy transfers W, from the incident electron to the medium, that are smaller than some chosen cut-off $\Delta$. The kinetic energy of the secondary electrons from ionization events is then also smaller than $\Delta$, and the range for an electron of energy $\Delta$ roughly specifies the region around the track of the incident electron within which energy is absorbed "1ocally". The significance of the concept of restricted stopping power, and possible extensions and generalizations, are discussed in an ICRU report on linear energy transfer (ICRU, 1970).

An application of restricted collision stopping powers occurs in the Bragg-Gray theory of cavity ionization as formulated by Spencer and Attix (1955), in which the cut-off energy $\Delta$ serves to specify the dimension of the cavity. Another application occurs in the Monte Carlo simulation of electron tracks. In such a calculation, energy-loss straggling can be taken into account approximately, with reduced computational effort, by limiting random sampling to large energy transfers, which are rare, and by treating the numerous small energy transfers in the continuousslowing-down approximation with use of a restricted collision stopping power.

In order to obtain an expression for the restricted collision stopping power, one must replace the maximum energy transfer $W_{m}$ in the integral in Eq (2.4) by $\Delta$. The results for electrons and positrons (indicated by superscripts - and + ) are:

$$
\frac{1}{\rho} L^{ \pm}(T, \Delta)=\frac{2 \pi N_{a} r_{e}^{2} m c^{2}}{\beta^{2}} \frac{Z}{A}\left[\ln (T / I)^{2}+\ln (1+\tau / 2)+G^{ \pm}(\tau, n)-\delta\right] .
$$

Equation (7.1) is similar to $\mathrm{Eq}(2.16)$, and the various symbols have the same meaning, except that the functions $\mathrm{F}^{ \pm}(\tau)$ are replaced by $\mathrm{G}^{ \pm}(\tau, n)$. For electrons,

$$
\begin{aligned}
G^{-}(\tau, n) & =-1-\beta^{2}+\ln [4(1-n) n]+(1-\eta)^{-1} \\
& +\left(1-\beta^{2}\right)\left[\tau^{2} \eta^{2} / 2+(2 \tau+1) \ln (1-\eta)\right]
\end{aligned}
$$

where $n=\Delta / T$ is the fractional energy cut-off. For positrons

$$
\begin{aligned}
G^{+}(\tau, n) & =\ln 4 n-\beta^{2}\left[1+\left(2-u^{2}\right) n-\left(3+u^{2}\right)(u \tau / 2) n^{2}\right. \\
& \left.+(1+u \tau)\left(u^{2} \tau^{2} / 3\right) n^{3}-\left(u^{3} \tau^{3} / 4\right) n^{4}\right],
\end{aligned}
$$

where $u=(\tau+2)^{-1}$. We note that $\mathrm{G}^{-}(\tau, 1 / 2)+\mathrm{F}^{-}(\tau)$ and that $\mathrm{G}^{+}(\tau, 1)=\mathrm{F}^{+}(\tau)$, so that $\mathrm{L}^{-}(\mathrm{T}, \mathrm{T} / 2)=\mathrm{S}_{\mathrm{col}}^{-}(\mathrm{T})$ and $\mathrm{L}^{+}(\mathrm{T}, \mathrm{T})=\mathrm{S}_{\mathrm{Co} 1}^{+}(\mathrm{T})$.

A condition for the validity of $\mathrm{Eq} \mathrm{(7.1)} \mathrm{is} \mathrm{that} \mathrm{the} \mathrm{cut-off} \mathrm{energy} \Delta$ be larger than the binding energies of the atomic electrons in the target material. For the K- and L-shell electrons of high-Z materials this condition is difficult to satisfy for $\Delta$-values of practical interest. Tables 7.1 and 7.2 give the ratios of the restricted to the total collision stopping power for electrons and positrons in seven substances, for cut-off energies $\Delta=100,10$ and $1 \mathrm{keV}$. The results shown are limited to cases for which $\Delta$ is larger than the L-shell binding energy and at least 
comparable with the $\mathrm{K}$-shell binding energy. The results for $\mathrm{Pb}$ for $\Delta=100 \mathrm{keV}$, for $\mathrm{Ag}$ and $\mathrm{Cu}$ for $\Delta=10 \mathrm{keV}$, and for Al for $\Delta=1 \mathrm{keV}$ have been included for completeness, but may be inaccurate.

Excluded from the definition of restricted stopping power used above is the reduction of the collision stopping power due to the escape of Auger electrons, which may occur subsequent to inner-shell ionization. For water, graphite, and air, the results in Tables 7.1 and 7.2 are not affected by this possibility, because the $\mathrm{K}$-shell binding energies of these materials are $\sim 1 / 2 \mathrm{keV}$. However, for these materials it would not be accurate to use Eq (7.1) with a cut-off energy as low as $100 \mathrm{eV}$, as is sometimes done in radiobiological calculations.

\section{ELECTRON COLLISION STOPPING POWERS AT LOW ENERGIES}

There is an energy below which the concept of an electron collision stopping power loses its usefulness. The lower the electron kinetic energy, the larger is the fraction of the energy lost, on the average, in a single inelastic collision. For example, it can be shown (Paretzke and Berger, 1978) that the average fractional energy loss in a collision with a water molecule in vapor is 0.5 percent at $10 \mathrm{keV}$, 3.6 percent at $1 \mathrm{keV}, 6.4$ percent at $0.5 \mathrm{keV}$, and 22 percent at $0.1 \mathrm{keV}$. The continuous-slowing-down approximation, $i . e$., the use of a stopping power to describe the gradual energy loss along the electron track, ceases to be meaningful at energies below several hundred eV. The evaluations of low-energy electron stopping powers found in the literature have often extended down to lower energies (typically $20 \mathrm{eV}$ ), but such results have significance mainly as a summary description of low-energy energy-loss cross sections.

8.1. Calculations for gases. There have been considerable advances in recent years in the knowledge of electron-impact ionization and excitation cross sections at energies from $10 \mathrm{keV}$ down to a few $\mathrm{eV}$. The need for such data has arisen in two contexts: in the evaluation of semi-empirical oscillator-strength distributions such as those referred to in Table 3.1 , and in the calculation of electron energy degradation spectra which take into account the slowing down of electrons and the buildup of successive generations of secondary electrons from ionization events. The often incomplete and sparse experimental data have been supplemented by estimated cross sections obtained by theoretical modeling, the adopted cross sections have been subjected to consistency checks in the form of sum rules, and comprehensive sets of cross sections have been assembled for a number of gases. This approach, and the results obtained, have been described by Green and Miller (1974), Fano (1975), and Inokuti, Douthat, and Rau (1976). For atmospheric gases, cross sections have been given by Jackman et al. (1977) where references to earlier work of Green and co-workers can also be found. From knowledge of the total cross sections for ionization and excitation, and the ionization cross section differential in the energy transferred to secondary electrons, one can readily construct collision stopping powers according to Eq (2.1).

8.2. Calculations for solids and liquids. Almost all of these calculations have been based on the approach of Lindhard (1954), Fano (1956), and Ritchie (1959). The stopping properties of the medium are expressed in terms of a complex-valued dielectric-response function $\epsilon(K, \omega)$ that depends on the momentum transfer $\hbar K$ and energy transfer $\hbar \omega$. The collision stopping power is proportional to a double integral, with respect to $K$ and $\omega$, over the quantity $(\omega / K) \operatorname{Im}[-1 / \epsilon(K, \omega)]$.

In the so-called statistical model, the dielectric-response function is calculated according to the local-plasma approximation (free electron gas model) of Lindhard (1954), which has already been mentioned in Section 3 in connection with the evaluation of the mean excitation energy. The dielectric-response function has been calculated by Ashley, Ritchie and collaborators using a variety of models, adapted to conductors, semi-conductors and insulators, that take into account single-electron excitations as well as collective excitations (plasmons). Whenever possible, experimental optical data were used to obtain the dielectric-response function in the limit of zero momentum transfer, $\epsilon(0, \omega) .15$ It was still necessary, however, to obtain $\epsilon(K, \omega)$ for non-zero $K$ through theoretical extrapolation, guided by the requirement that very large energy transfers should be described by the Rutherford cross section, or by the Mфlier cross section when exchange is taken into account. Usually the

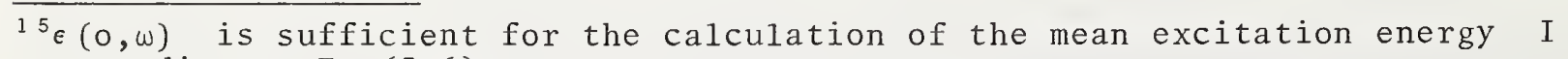
according to $\mathrm{Eq}(3.6)$. 
contribution to the stopping power from inner-shell electrons has been evaluated with the use of theoretical atomic generalized oscillator strengths, which is justified on the basis that the wave functions of the inner-shell electrons are insensitive to the state of aggregation of the medium.

Tables of electron collision stopping powers have been given by Ashley et $a$. (1975) for $\mathrm{Al}$ and $\mathrm{Al}_{2} \mathrm{O}_{3}$; Ashley et al. (1976a) and Tung et al. (1979) for Al, Si, Ni, $\mathrm{Cu}, \mathrm{Ag}$ and $\mathrm{Au}$; Ashley et al. (1976b) for Ge and GaAs; Tung et al. (1976) for Si and $\mathrm{SiO}_{2}$; Ashley et al. (1978) for polystyrene; Ritchie et al. (1978) for water; Ashley et al. (1979) for Al; Painter et al. (1980) for polyethylene; Ashley (1980) for various organic solids, Ashley and Anderson (1981) for $\mathrm{SiO}_{2}$; and Ashley (1982a) for water. Other calculations based on the use of Lindhard's statistical model have been reported by Sugiyama (1976).

8.3. Comparison of stopping powers. The case of water is especially interesting because comparisons can be made between stopping powers from different authors for water vapor as well as liquid water. In the left-hand panel of Fig. 8.1, for water vapor, curve 1 is from Paretzke and Berger (1978), 16 curve 2 is from Green, 17 and curve 3 is from the Bethe theory, Eq (2.16), with $I=71.6 \mathrm{eV}$. Curves 1 and 2 both 1 ie below the Bethe curve 3 , but the differences are smaller than $10 \%$ down to $\sim 400 \mathrm{eV}$. Curves 1 and 2 agree rather closely down to $\sim 150 \mathrm{eV}$, but diverge at lower energies. The main reason for this divergence lies with the total ionization cross section which Green took from the experiment of Schutten et al. (1966) whereas Berger relied also on those of Märk and Egger (1976) below $150 \mathrm{eV}$.

In the right panel in Fig. 8.1, for liquid water, curve 4 is from Kutcher and Green (1976), curve 5 is from Ritchie et al. (1978), curve 6 from Ashley (1982a), and curve 7 is from the Bethe theory with $I=75 \mathrm{eV}$. The calculations leading to curves 4, 5, and 6 all take into account collective excitations (plasmons) and use the experimental optical data of Heller et al. (1974) on uv absorption in liquid water. Ashley constructed the dielectric function for liquid water from an insulator model fixed by the available optical data, and treated ionization from the K shell by theoretical generalized oscillator strengths. As explained by Ashley, the main difference between his calculation and the earlier work of Ritchie et al. consists of an improved treatment of exchange effects, which results in a lowering of the peak of the stopping-power curve at $\sim 120 \mathrm{eV}$ by 25 percent. The formalism for obtaining the collision stopping power in terms of the dielectric response functions is a Bornapproximation theory. The uncertainties resulting from the use of this approximation could easily be 10 percent at $100 \mathrm{eV}$ and even greater at lower energies. The peak of the stopping-power curve 6 of Ashley is 13 percent above the corresponding value for water vapor (curve 1). It is not clear whether this difference is significant, because it lies within the combined limits of uncertainties for the liquid and vapor results.

Figure 8.2 compares collision stopping powers in $\mathrm{H}_{2}$ gas calculated by Green ${ }^{17}$ and by Spencer and $\mathrm{Pal}$ (1978). Also shown are curves obtained with the Bethe formula for $\mathrm{H}_{2}$ gas $(\mathrm{I}=19.2 \mathrm{eV})$ and for liquid hydrogen $(\mathrm{I}=21.8 \mathrm{eV})$. The results of Green and of Spencer and $\mathrm{Pal}$ are in good agreement down to $\sim 30 \mathrm{eV}$; both of these results deviate significantly from the Bethe theory only below $\sim 200 \mathrm{eV}$. Also shown in Fig. 8.2 is a stopping-power curve for air which was constructed from the results of Green ${ }^{17}$ for $\mathrm{N}_{2}, \mathrm{O}_{2}$, Ar, and $\mathrm{CO}_{2}$. Again there is good agreement with the Bethe formula down to $200 \mathrm{eV}$. An earlier stopping-power curve for air, calculated by Green and Peterson (1968), coincides with curve 5 in Fig. 8.2 down to $1 \mathrm{keV}$, and is 10 percent lower at $0.1 \mathrm{keV}$. Using the Green-Peterson stopping powers and taking into account multiple elastic-scattering deflections, depth-dose curves have been calculated for an air medium irradiated by $12-\mathrm{keV}$ and $5-\mathrm{keV}$ electrons (Berger et al., 1970). These results are in good agreement with measurements in air by Grü (1957) and in nitrogen by Cohn and Caledonia (1970).

\footnotetext{
${ }^{16}$ Curve 1 is the result of a calculation by Berger given in Fig. 4 of Paretzke and Berger (1978). That figure also shows stopping-power curves obtained independently by Paretzke, and by olivero, Stagat and Green (1972). These curves are all quite similar to curves 1 and 2 in Fig. 8.2 at energies down to $\sim 150 \mathrm{eV}$.

${ }^{17}$ Green has calculated (and communicated to us in January 1978) collision stopping powers from $15 \mathrm{keV}$ down to a few eV, based on the cross sections given in Jackman et ar. (1977) and on cross sections for other gases compiled by his group. In the case of water vapor, Green's results are an updated version of stopping powers (energy-loss functions) given earlier by 0livero, Stagat, and Green (1972).
} 
Figure 8.3 shows stopping-power results of Ashley (1982c) for polyethylene, and results of Ashley et al. (1978) for polystyrene obtained from dielectric-response functions constructed with the use of optical data for the valence electrons. Similar results for silicon dioxide, from Ashley and Anderson (1981), are shown in Fig. 8.4.

Figure 8.5 compares stopping-power curves for aluminum and gold from the Bethe theory with calculations of Ashley et al. (1976a) obtained with a statistical model of the dielectric-response function, without differentiating between inner- and outer-shell electrons. Also shown is a stopping-power curve for aluminum calculated by Ashley et al. (1979) with further refinements. These refinements include the limitation of the dielectric-response function modeling to conduction electrons, consideration of damping and core polarizability, and the use of atomic generalized oscillator strengths from Hartree-Slater wave functions for inner-shell electrons. It can be seen that the differences between the simple and refined calculations for aluminum are not very great.

other comparisons and analyses of low-energy stopping-power data can be found in Iskef et aZ. (1980), and in Waibel and Grosswendt (1980).

An experimental investigation of electron stopping power in air and collodion ${ }^{28}$ was carried out by Cole (1969). For collodion the experiment consisted of determining the foil thicknesses for which the number transmitted was 5 percent of that in the incident beam. For air the experiment consisted of measuring characteristic depths such that only 1 percent of the ionization in air occurred at greater depths. Cole considered these foil thicknesses and characteristic depths as "ranges". With this somewhat arbitrary definition of ranges he fitted his data for collodion and air by a single polynomial curve of range $v s$. incident electron energy (with a stated accuracy of 10 percent) and obtained the stopping power by differentiating this curve with respect to energy.

Cole's results suggest that, in the case of air and collodion, gas-solid stoppingpower differences, if any, are small. This is consistent with the later findings of Schou (1979) and Sørensen and Schou (1978), based on the analysis of their electron penetration measurements in Iiquid nitrogen at 1 to $3 \mathrm{keV}$, that the stopping powers in liquid are at most a few percent higher than the stopping powers in gas.

The results of Cole are compared in Table 8.1 with the stopping powers for cellulose nitrate from the Bethe theory, and for air from the Bethe theory and from the calculation of Green. ${ }^{7}$ There is close agreement between experimental and theoretical results down to $\sim 400 \mathrm{eV}$. Below this energy the experimental values are higher, by 19 percent at $100 \mathrm{eV}$ and much more at lower energies.

The significance of Cole's results is obscured by two uncertainties. First, a considerable error must have resulted from the numerical differentiation of his range-energy curve, especially at low energies. Second, his analysis neglected the effect of multiple-scattering angular deflections which make the pathlength traveled by electrons greater than the depth of penetration. Thus multiple scattering would tend to make the apparent stopping power determined by Cole larger than the true stopping power, by an amount that is expected to increase as the electron energy decreases.

The comparisons in Figs. 8.1 to 8.5 indicate that the differences between the stopping-power values from the Bethe theory and those from more elaborate low-energy treatments are often rather small, even at energies where the conditions of applicability of the Bethe theory are no longer satisfied. We therefore give in Table 8.2 a set of stopping-power values for selected materials covering the energy region from $10 \mathrm{keV}$ to $1 \mathrm{keV}$. We estimate that at $1 \mathrm{keV}$ the departures of the correct stopping powers from the tabulated results will be no greater than $\sim(I / 7)$ percent, where I is the mean excitation energy in $\mathrm{eV}$.

${ }^{18}$ Collodion is a plastic prepared as a suspension of cellulose nitrate in ether and alcohol. The estimated mean excitation energy of cellulose nitrate is $87.0 \mathrm{eV}$, a value rather close to that for air, $85.7 \mathrm{eV}$. 


\section{RADIATIVE STOPPING POWER}

The mass radiative stopping power can be expressed in terms of bremsstrahlung cross sections as

$$
\begin{aligned}
-\frac{1}{\rho}\left(\frac{\mathrm{dE}}{\mathrm{dx}}\right)_{\mathrm{rad}} & =\frac{1}{\rho} S_{\mathrm{rad}}(\mathrm{T})= \\
& =\frac{N_{a}}{\mathrm{~A}}\left[\int_{0}^{\mathrm{T}} \mathrm{k} \frac{\mathrm{d} \sigma_{\mathrm{n}}}{\mathrm{dk}} \mathrm{dk}+2 \int_{0}^{T^{\prime}} \mathrm{k} \frac{\mathrm{d} \sigma_{e}}{\mathrm{dk}} \mathrm{dk}\right],
\end{aligned}
$$

where $d \sigma_{n} / d k$ is the differential cross section for the emission of a photon of energy $k$ due to the interaction of the electron with the screened Coulomb field of the atomic nucleus, and $\mathrm{d} \sigma_{e} / \mathrm{dk}$ is the corresponding cross section due to the Coulomb interaction with one of the atomic electrons. The upper limit of the energy of the photons that can be emitted in electron-electron interactions is

$$
T^{\prime}=m c^{2} T\left[T+2 m c^{2}-\beta\left(T+m c^{2}\right)\right]^{-1} .
$$

It is convenient to introduce dimensionless, scaled, radiative energy-loss cross sections

$$
\phi_{\mathrm{rad}}^{(\mathrm{n})}=\left(\alpha \mathrm{r}_{\mathrm{e}}^{2} z^{2}\right)^{-1} \int_{0}^{\mathrm{T}}(k / E) \frac{\mathrm{d} \sigma}{\mathrm{dk}} \mathrm{dk}
$$

and

$$
\phi_{\mathrm{rad}}^{(e)}=\left(\alpha \mathrm{r}_{\mathrm{e}}^{2}\right)^{-1} \int_{0}^{\mathrm{T}^{\prime}}(k / E) \frac{\mathrm{d} \sigma_{e}}{\mathrm{dk}} \mathrm{dk},
$$

where $\alpha$ is the fine-structure constant, and $E=T+m c^{2}$ is the total energy of the electron. In terms of these quantities the radiative stopping power can be written as

$$
\frac{1}{\rho} S_{\mathrm{rad}}(\mathrm{T})=\frac{\mathrm{N}_{a}}{\mathrm{~A}} \alpha \mathrm{r}_{\mathrm{e}}^{2} \mathrm{E} \mathrm{Z}^{2} \phi_{\mathrm{rad}}^{(\mathrm{n})}(\mathrm{T})\left[1+(1 / \mathrm{Z}) \phi_{\mathrm{rad}}^{(\mathrm{e})}(\mathrm{T}) / \phi_{\mathrm{rad}}^{(\mathrm{n})}(\mathrm{T})\right] .
$$

The ratio $\phi_{\text {rad }}^{(e)} / \phi_{\text {rad }}^{(n)}$ has in previous work usually been assumed to be unity. As will be shown below, this ratio is actually slightly higher than 1 at high energies, falls to $\sim 0.5$ at $700 \mathrm{keV}$, and tends to vanish at low energies. ${ }^{19}$

For compounds we assume that additivity is a good approximation, and set

$$
\frac{1}{\rho} S_{\mathrm{rad}}(T)=\sum_{j} w_{j} \frac{1}{\rho_{j}} S_{\mathrm{rad}, j}(\mathrm{~T})
$$

where $w_{j}$ is the fraction by weight of the j'th constituent.

\footnotetext{
${ }^{19}$ According to Joseph and Rohrlich (1958), the vanishing at very low energies is due to the lack of an electric dipole moment for the electron-electron system.
} 


\subsection{Electron-nucleus bremsstrahlung}

9.1.1. High-energy region. For $\mathrm{T} \geqslant 50 \mathrm{MeV}$, the bremsstrahlung cross section was evaluated from the analytical expressions of Davies, Bethe, and Maximon (1954), and 01sen (1955). This formula corrects the Bethe (1934) Born-approximation result through the inclusion of a Coulomb correction $f(Z)$ derived with the use of Sommerfeld-Maue wave functions. The formula is based on the high-energy approximation, $i . e .$, the assumption that the energies of the electron both before and after the bremsstrahlung event are large compared to the electron rest energy. The cross section has the form

$$
k \frac{d \sigma_{n}}{d k}=4 \alpha r_{e}^{2} z^{2} \sum_{i=1}^{2} g_{i}(E, k)\left[e_{i}+\int_{q_{0}}^{1} h_{i}(q)[1-F(q, z)]^{2} d q-f(z)\right]
$$

where $q$ is the momentum transfer and

$$
\mathrm{q}_{0}=m c^{2} \mathrm{k} /[2 \mathrm{E}(\mathrm{E}-\mathrm{k})]
$$

the minimum momentum transfer (with both in units of mc). The Coulomb correction is given by

$$
f(z)=(\alpha z)^{2} \sum_{n=1}^{\infty}\left[n\left(n^{2}+\alpha^{2} z^{2}\right)\right]^{-1}
$$

The other quantities in Eq (9.7) are defined as follows:

$$
\begin{aligned}
& \mathrm{g}_{1}=1+(E-\mathrm{k})^{2} / \mathrm{E}^{2} \\
& \mathrm{e}_{1}=1 \\
& \mathrm{~h}_{1}=\left(\mathrm{q}-\mathrm{q}_{0}\right)^{2} / \mathrm{q}^{3}
\end{aligned}
$$$$
g_{2}=-2(E-k) / 3 E
$$$$
e_{2}=5 / 6
$$$$
\begin{aligned}
h_{2} & =q^{3}-6 q_{0}^{2} q \ln \left(q / q_{0}\right) \\
& +3 q_{0}^{2} q-4 q_{0}^{3} / q^{4}
\end{aligned}
$$

$F(q, Z)$ is the atomic form factor, normalized such that $F(0, Z)=1$. For $1 \leqslant Z \leqslant 6$ the atomic form factor was taken from the non-relativistic calculations of Hubbell et al. (1975) which include electron-correlation effects; for $Z>6$ it was taken from the work of Hubbel1 and $\emptyset$ verb $\phi$ (1979) which is relativistic but omits correlation effects.

9.1.2. Low-energy region. For $\mathrm{T} \leqslant 2 \mathrm{MeV}$, use was made of work by Pratt et al. (1977) which constitutes a significant advance over the Born-approximation theory of Bethe and Heitler. In this work, the bremsstrahlung process is treated as a singleelectron transition in a self-consistent screened central potential. Electron wave functions are obtained in partial wave-function expansions through the numerica 1 solution of the Dirac equation, and the matrix elements for the bremsstrahlung cross section are evaluated numerically from the wave functions. The required amount of computation is very large, especially in view of the large number of partial waves which have to be included, so that results have been obtained so far only for a limited number of materials and energies. The theory underlying the computations and initial numerical results were given by Tseng and Pratt (1971). Further numerical results for electrons with energies from 1 to $500 \mathrm{keV}$ were published by Lee et al. (1976), and for 1- and 2-MeV electrons by Kissel and MacCallum (1977). An extensive set of data for all elements $2 \leqslant z \leqslant 92$, including bremsstrahlung cross sections differential in photon energy as well as the total energy-weighted cross section was prepared by Pratt et al. (1977) through elaborate interpolation procedures. A few 
exploratory calculations, for $5-$ and $10-\mathrm{MeV}$ electrons in $\mathrm{Al}$ and $\mathrm{U}$, have recently been published by Tseng and Pratt (1979) which involve the calculation of selected terms in the partial wave expansions and interpolation between them. We have used the tables of Pratt et al. (1977) to obtain $\phi$ (n) and have derived values for $Z=1$ and for $Z=93$ to 100 by extrapolation.

9.1.3. Intermediate energy region. For $\mathrm{T}$ between 2 and $50 \mathrm{MeV}$, the scaled radiative energy-loss cross section has only a mild dependence on $Z$ and on the electron energy T. Being anchored down firmly below $2 \mathrm{MeV}$ and above $50 \mathrm{MeV}$, the curve of $\phi_{\text {rad }}(\mathrm{T})$ vs. $\mathrm{T}$ in the gap region can readily be obtained by interpolation. We have done this using a cubic-spline least-squares algorithm of Powell (1967). Typical results of this interpolation for $Z=1,6,13,29,47$ and 79 are shown in Fig. 9.1. The results of the interpolation have been found to be quite insensitive to the choice of the upper cut-off energy for the gap region. A shift from 50 to $100 \mathrm{MeV}$ would change the integrated cross section in the gap region by less than 1 percent, and a shift down to $20 \mathrm{MeV}$ would change it less than 3 percent.

In four cases ( $\mathrm{Al}$ and $\mathrm{U}$ at 5 and $10 \mathrm{MeV}$ ) the radiative energy-loss cross sections can also be derived directly from the differential bremsstrahlung cross sections of Tseng and Pratt (1979), and are found to agree to within 1-2 percent with our interpolated results.

\subsection{Electron-electron bremsstrahlung}

9.2.1. High-energy region. For $\mathrm{T} \geqslant 50 \mathrm{MeV}$, a combination of three crosssection formulas was used. The first is the Bethe-Heitler (1934) Born-approximation result which disregards screening and was derived in the high-energy approximation:

$$
\mathrm{k}\left(\frac{\mathrm{d} \sigma_{\mathrm{e}}}{\mathrm{dk}}\right)_{B H}=4 \alpha \mathrm{r}_{\mathrm{e}}^{2}\left[\mathrm{~g}_{1}(\mathrm{E}, \mathrm{k})+\mathrm{g}_{2}(\mathrm{E}, \mathrm{k})\right]\left[\ln \left(1 / \mathrm{q}_{\mathrm{o}}\right)-1 / 2\right],
$$

where $g_{1}, g_{2}$, and $q_{0}$ have the same meaning as in Eqs (9.7) to (9.10). The second formula used is that of Wheeler and Lamb (1939), also based on the first Born and high-energy approximations, which treats screening with use of the incoherent scattering function $S(q, Z)$ :

$$
k\left(\frac{d \sigma_{e}}{d k}\right)_{W L}=4 \alpha r_{e}^{2} \sum_{i=1}^{2} g_{i}(E, k)\left[e_{i}+\int_{q_{o}}^{1} h_{i}(q) s(q, z) d q\right] \text {. }
$$

It can be seen that this equation can be obtained from Eq (9.7) by setting $Z=1$ and replacing $[1-F(q, z)]^{2}$ by $S(q, z)$. In Eq $(9.12), S(q, z)$ is normalized such that $S(\infty, Z)=1$. We have taken the incoherent scattering function from Hubbell et al. (1975). The third cross section used is one derived by Haug (1975) in lowest order perturbation theory, without consideration of screening effects, but treating recoil and exchange effects accurately. Haug also considered the Coulomb correction (departure from Born approximation) but found it unimportant at high energies. Haug's calculation is expressed in complicated formulas (his Eqs 2.15, A1 and A2) which are too lengthy to be reproduced here. We have used numerical values given in Haug's paper.

Taking Haug's cross section as initial approximation, we have assumed that the electron-electron differential bremsstrahlung cross section is

$$
\frac{d \sigma_{e}}{d k}=\left(\frac{d \sigma_{e}}{d k}\right)_{H G}+\left[\left(\frac{d \sigma_{e}}{d k}\right)_{W L}-\left(\frac{d \sigma_{e}}{d k}\right)_{B H}\right] \text {, }
$$

where the first term incorporates an exchange correction and the terms in square brackets constitute a screening correction. This treatment can be justified on the basis that the two corrections are almost independent of each other, with exchange affecting mainly large momentum transfers and screening mainly small momentum transfers. 
9.2.2. Low-energy region. For $\mathrm{T} \leqslant 2 \mathrm{MeV}$, we have assumed that

$$
\frac{\phi_{\mathrm{rad}}^{(\mathrm{e})}}{\phi_{\mathrm{rad}}^{(\mathrm{n})}}=\int_{0}^{\mathrm{T}^{\prime}} \mathrm{dk} k\left(\frac{\mathrm{d} \sigma_{\mathrm{e}}}{\mathrm{dk}}\right)_{H G} / \int_{0}^{\mathrm{T}} \mathrm{dk} \mathrm{f}_{E} \mathrm{k}\left(\frac{\mathrm{d} \sigma_{\mathrm{e}}}{\mathrm{dk}}\right)_{\text {BHSR }},
$$

where $\left(\mathrm{d} \sigma_{\mathrm{e}} / \mathrm{dk}\right)_{H G}$ is again Haug's cross section with exchange and no screening, and where $(\mathrm{d} \sigma \mathrm{e} / \mathrm{dk})$ BHSR is the cross section without screening derived by Bethe and Heitler (1934), Sauter (1934), and Racah (1934) in the Born approximation but without invoking the high-energy approximation. The so-called Elwert factor

$$
f_{E}=\beta[1-\exp (-2 \pi \alpha Z / \beta)] / \beta^{\prime}\left[1-\exp \left(-2 \pi \alpha Z / \beta^{\prime}\right)\right],
$$

which depends on the electron velocities $\beta$ and $\beta^{\prime}$ before and after the collision, is an approximate Coulomb correction due to Elwert (1939). For the electron-electron bremsstrahlung cross section in the numerator of $\mathrm{Eq}(9.14)$ a Coulomb correction was not considered necessary. Equation (9.14) is valid to the extent that screening does not change the ratio of radiative energy-loss cross sections significantly.

9.2.3. Intermediate energy region. With $\phi_{\text {rad }}^{(e)}$ determined for $T \leqslant 2 \mathrm{MeV}$ and for $T \geqslant 50 \mathrm{MeV}$, the quantity $\phi_{\mathrm{rad}}^{(\mathrm{n})}(\mathrm{T})\left[1+(1 / \mathrm{Z}) \phi_{\mathrm{rad}}^{(\mathrm{e})}(\mathrm{T}) / \phi_{\mathrm{rad}}^{(\mathrm{n})}(\mathrm{T})\right]$ was obtained in the gap region, 2 to $50 \mathrm{MeV}$, by the same interpolation procedure previously used in Section 9.1 .3 for $\phi_{\mathrm{rad}}^{(\mathrm{n})}(\mathrm{T})$. This completed the evaluation of $\phi_{\mathrm{rad}}^{(\mathrm{e})}(\mathrm{T})$ and of the total radiative stopping power according to $\mathrm{Eq}(9.5)$.

The ratio $\phi_{\mathrm{rad}}^{(\mathrm{e})}(\mathrm{T}) / \phi_{\mathrm{r}}(\mathrm{n})(\mathrm{T})$ is shown in Fig. 9.2 for hydrogen, carbon and gold as a function of electron energy.

9.3. Accuracy and comparison with experiments. Pratt et al. (1977) estimate the uncertainty of their differential bremsstrahlung cross sections to be no greater than 10 percent. It seems plausible that the radiative stopping power, obtained as an integral over these cross sections, has a smaller uncertainty, perhaps 5 percent. Comparisons are made in Fig. 9.3 between calculated radiative stopping powers in five materials at energies up to $2.5 \mathrm{MeV}$, and corresponding results derived from bremsstrahlung measurements. There is good agreement, within the limits of experimental error, with the results of Aiginger (1966), Rester and Dance 20 (1967) and Rester and Edmonson (1972); the theoretical results are lower than those from the earlier measurements of Motz (1955) and Motz and Placious (1958).21

The high-energy theory of Davies, Bethe, and Maximon (1954) and 01sen (1955) provides the cross section not only for bremsstrahlung but also for the closely related process of pair production. Whereas there is a scarcity of experimental bremsstrahlung measurements above $2.5 \mathrm{MeV}$, there are sufficient data to assess the accuracy of the pair production cross section. According to Hubbell et al. (1980), the theoretical pair production cross sections above $\sim 50 \mathrm{MeV}$ are confirmed by experiments to within 1-2 percent.

In the transition region between $2 \mathrm{MeV}$ and $50 \mathrm{MeV}$ we expect our interpolated results to have an accuracy of $3-5$ percent.

\section{RANGES AND RADIATION YIELDS}

The ranges and radiation yields given in this report were obtained in the continuous-slowing-down approximation (c.s.d.a.). In this approximation energy-1oss fluctuations are neglected, and electrons are assumed to lose energy continuously along their track, with a mean energy loss per unit pathlength given by the stopping power. The c.s.d.a. range (in units of $\mathrm{g} \mathrm{cm}^{-2}$ ) is evaluated from the expression

\footnotetext{
${ }^{20} \mathrm{Also}$ private communication from D. Rester (1967).

${ }^{21}$ Rester and Edmonson (1972) suggest that an incomplete background correction in the experiment of Motz accounts for this discrepancy.
} 


$$
r_{o}\left(T_{o} \rightarrow T_{f}\right)=\rho \int_{T_{f}}^{T}\left[S_{c o 1}(T)+S_{r a d}(T)\right]^{-1} d T
$$

and represents the average pathlength ${ }^{2}$ traveled by an electron as it slows down from an initial energy $\mathrm{T}_{0}$ to a final energy $\mathrm{T}_{f}$. The choice of $\mathrm{T}_{f}$ should, in principle, be adapted to the purpose for which the range is to be used. For example, if one wanted to know how far electrons can travel while they are capable of ionizing atoms of the medium, it would be appropriate to set $T_{f}$ equal to the lowest atomic ionization potential.

The stopping powers needed for the evaluation of $r_{0}$ from $E q$ (10.1) are avai1able from the Bethe theory only down to some intermediate energy $T_{i}$, and the part of the integral from $\mathrm{T}=\mathrm{T}_{i}$ to $\mathrm{T}_{\mathrm{f}}$ must be obtained by an approximate estimate. Fortunately this residual range is relatively small so that a simple approximation is sufficient. We have followed Nelms (1956) in assuming that the integrand $\left[\mathrm{S}_{\mathrm{col}}(\mathrm{T})+\mathrm{S}_{\mathrm{rad}}(\mathrm{T})\right]^{-1}$ is zero at $\mathrm{T}=0$ and can be interpolated linearly to its value at $T=T_{i}$. The range is then given by

$$
\mathrm{r}_{0}\left(\mathrm{~T}_{0} \rightarrow \mathrm{T}_{f}\right)=\mathrm{r}_{0}\left(\mathrm{~T}_{0} \rightarrow \mathrm{T}_{i}\right)+\rho\left[\mathrm{S}_{\mathrm{co} 1}\left(\mathrm{~T}_{\mathrm{i}}\right)+\mathrm{S}_{\mathrm{rad}}\left(\mathrm{T}_{\mathrm{i}}\right)\right]^{-1} \int_{\mathrm{T}_{f}}^{\mathrm{T}_{i}}\left(\mathrm{~T} / \mathrm{T}_{i}\right) \mathrm{dT},
$$

where the first term is calculated according to Eq (10.1), and the second term is the residual range. In the range tables we give results obtained with $\mathrm{T}_{i}=1 \mathrm{keV}$ and $\mathrm{T}_{\mathrm{f}}=0$.

For some materials it is possible to avoid the crude approximation used above for residual ranges below $1 \mathrm{keV}$, and to rely instead on estimated low-energy stopping powers. We have made such calculations for water vapor, polystyrene, aluminum and gold, using data from Figs. 8.1, 8.3, and 8.5. As shown in Table 10.1, the ranges $\mathrm{r}_{\mathrm{O}}\left(\mathrm{T}_{\mathrm{O}} \rightarrow 0\right)$ from $\mathrm{Eq}(10.2)$, for initial energies $\mathrm{T}_{\mathrm{O}}=10 \mathrm{keV}$, actually are quite close numerically to the more accurate ranges calculated with a final energy $\mathrm{T}_{\mathrm{f}} \cong 10 \mathrm{eV}$.

The radiation yield (also called bremsstrahlung efficiency) is the fraction of the initial energy $\mathrm{T}_{0}$ of an electron that is converted to bremsstrahlung energy as the electron slows down to rest. The c.s.d.a. yield is given by

$$
Y\left(T_{0}\right)=\frac{1}{T_{0}} \int_{0}^{T_{0}}\left[S_{c o 1}(T)+S_{r a d}(T)\right]^{-1} S_{r a d}(T) d T .
$$

In the evaluation of this expression one is again faced with the difficulty that neither $\mathrm{S}_{\mathrm{col}}$ nor $\mathrm{S}_{\mathrm{rad}}$ is known accurately below an intermediate energy $\mathrm{T}_{\mathrm{i}}=1 \mathrm{keV}$. We have made the assumption that $\mathrm{S}_{\mathrm{rad}}\left(\mathrm{S}_{\mathrm{col}}+\mathrm{S}_{\mathrm{rad}}\right)^{-1}$ is zero at $\mathrm{T}=0$ and increases linearly to its value at ${ }_{T}^{2}=T_{i}$.

\section{MISCELLANEOUS COMPARISONS}

11.1 Positron-electron differences. For the collision stopping power and range, differences arise because of the use of the Bhabha cross section instead of the Mфller cross sections for large energy transfers. Table 11.1 shows ratios of positron stopping powers to electron stopping powers, and ratios of positron ranges

\footnotetext{
${ }^{22}$ This is actually only an approximation, but a very close one. For example, we have determined through Monte Carlo transport calculations that in a water medium the actual mean pathlength down to a final energy $\mathrm{T}_{f}=12.6 \mathrm{eV}$ is $\sim 1$ percent greater than $\mathrm{r}_{0}\left(\mathrm{~T}_{0} \rightarrow \mathrm{T}_{f}\right)$ for $\mathrm{T}_{\mathrm{o}} \gtrsim 10 \mathrm{keV}$.
} 
to electron ranges, for a representative set of materials. ${ }^{2}{ }^{3}$ Table 11.2 gives the ratio of the restricted collision stopping power for positrons to that for electrons in water.

In our tabulations, the radiative stopping power for positrons has been assumed to be the same as that for electrons, which is a good approximation at energies above, say, $10 \mathrm{MeV}$. However, it should be mentioned that exploratory calculations by Feng et al. (1981), employing the same method as that previously used by them for electrons, indicate significant differences between positrons and electrons in regard to the differential bremsstrahlung cross sections in oxygen and uranium at 500,50 , and $10 \mathrm{keV}$.

11.2. Comparison of calculated and experimental stopping powers. There is available only a limited amount of experimental data pertaining to the total stopping power (collision plus radiative). Comparisons with measurements by Paul and Reich (1950) at 2.8 and $4.7 \mathrm{MeV}$ and by ziegler (1958) at $32 \mathrm{MeV}$ are given in Table 11.3. The experimental and calculated results agree, within the limits of experimental uncertainty, at 2.8 and $32 \mathrm{MeV}$, but not at $4.7 \mathrm{MeV}$. Table 11.4 presents comparisons with stopping-power ratios (relative to Be and $A l$ ) measured by Westermark (1961) and by Hereford (1948). Experimental and theoretical values agree within the limits of experimental uncertainty.

In Figs. 11.1 and 11.2 collision stopping powers measured by Kalil et az. (1959) and Ishigure et al. (1978) in aluminum and by Ziemer et az. (1959) in copper are compared with results from the Bethe theory. These figures show Fano plots, i.e., plots of stopping power $v s$. energy in a scaled representation in which the Bethe formula gives a straight line. The experimental results of Kalil et al. for aluminum between $10 \mathrm{keV}$ and $1 \mathrm{MeV}$, and those of Ziemer et al. for copper between $200 \mathrm{keV}$ and $1 \mathrm{MeV}$ cluster around the theoretical straight lines, but the dispersion of the experimental points is considerable.

11.3. Comparisons with previous calculations. Differences between the stopping powers, ranges, and radiation yields from this report and those from earlier tables by Berger and Seltzer $(1964,1966)$ and Pages et al. (1972) are shown in Table.11.5 for a few materials. Whereas the differences in regard to the collision stopping power amount to only a few percent, the differences in regard to the radiative stopping power and radiation yield are much larger, especially at energies below $\sim 1 \mathrm{MeV}$, due to the change from Bethe-Heitler bremsstrahlung cross sections to the more accurate results of pratt et al. (1977).

${ }^{23}$ The positron ranges used to compute the range ratios in Table 11.1 pertain to the case in which the positron slows down to rest before being annihilated. If one included the possibility of positron annihilation in flight, the positron ranges would be slightly decreased. From results given by Heitler (1947) one can conclude that the shortening of the positron range in lead would amount to 1.7 percent at $0.511 \mathrm{MeV}, 4.2$ percent at $5.11 \mathrm{MeV}, 4.0$ percent at $51.1 \mathrm{MeV}$, and 3.1 percent at $511 \mathrm{MeV}$. 


\section{REFERENCES}

Abramowitz, M. and Stegun, I. (1964). Handbook of Mathematical Functions, National Bureau of Standards, Applied Mathematics Se ries-55.

Ahlen, S. P. (1980). "Theoretical and experimental aspects of the energy loss of relativistic heavily ionizing particles," Rev. Mod. Phys. 52, 121.

Aiginger, H. (1966). "Elektron-Bremsstrahlungs-Wirkungsquerschnitte von $180-$ und 300-keV Elektronen," Z. f. Phys. 197, 8.

Andersen, H. H. (1977). Bibliography and Index of Experimental Range and Stopping Power Data, Pergamon Press, New York.

Andersen, H. H., Bak, J. F., Knudsen, H. and Nielsen, B. R. (1977). "Stopping power of $\mathrm{Al}, \mathrm{Cu}$, and $\mathrm{Au}$ for MeV hydrogen, helium and lithium ions. $\mathrm{Z}_{1}^{3}$ and $\mathrm{Z}_{1}$ proportional deviations from the Bethe formula," Phys. Rev. A16, 1929.

Andersen, H. H., Hanke, C. C., Simonsen, H., Sørensen, H. and Vajda, P. (1968). "Stopping power of the elements $A=20$ through $Z=30$ for $5-12-\mathrm{MeV}$ protons and deuterons," Phys. Rev. $175,389$.

Andersen, H. H., Hanke, C. C., Sørensen, H. and Vajda, P. (1967). "Stopping power of $\mathrm{Be}, \mathrm{Al}, \mathrm{Cu}, \mathrm{Ag}, \mathrm{Pt}$ and $\mathrm{Au}$ for 5-12-MeV protons and deuterons," Phys. Rev. 153, 338 .

Andersen, H. H. and Nielsen, B. R. (1981). "The stopping power of gold in the Bethe region," Nuc1. Instr. Meth. 191, 475.

Andersen, H. H., Simonsen, H. Sфrensen, H. and Vajda, P. (1969). "Stopping power of $\mathrm{Zr}, \mathrm{Gd}$ and $\mathrm{Ta}$ for 5-12-MeV protons and deuterons. Further evidence of an oscillatory behavior of the excitation potential," Phys. Rev. 186, 372.

Andersen, H. H. and Ziegler, J. F. (1977). Hydrogen: Stopping Powers and Ranges in A11 Elements, Pergamon Press, New York.

Ashley, J. C. (1979). "Mean excitation energy of polystyrene," Phys Rev. B19, 5429.

Ashley, J. C. (1980). "Inelastic interactions of low-energy electrons with organic solids: Simple formulae for mean free paths and stopping powers," IEEE Trans. Nuc1. Sci. Vo1. NS-27, 1454.

Ashley, J. C. (1982a). "Stopping power of liquid water for low-energy electrons," Radiat. Res. $\underline{89}, 25$.

Ashley, J. C. (1982b). "Density effect in liquid water," Radiat. Res. $\underline{89}, 32$.

Ashley, J. C. (1982c). "Energy losses and inelastic mean free paths of low-energy electrons in polyethylene," Radiat. Res. 90, 433.

Ashley, J. C. and Anderson, V. E. (1981). "Interaction of low-energy electrons with silicon dioxide," J. Electron Spectroscopy and Related Phenomena, 24, 127.

Ashley, J. C., Ritchie, R. H. and Brandt, W. (1972). "Z $Z_{1}^{3}$ effect in the stopping power of matter for charged particles," Phys. Rev. B5, 2393.

Ashley, J. C., Ritchie, R. H. and Brandt, W. (1973). " $\mathrm{Z}_{1}^{3}$ dependent stopping power and range contributions," Phys. Rev. A8, 2402.

Ashley, J. C., Tung, C. J. and Ritchie, R. H. (1975). "Electron interaction cross sections in $\mathrm{Al}$ and $\mathrm{Al}_{2} \mathrm{O}_{3}$; calculations of mean free paths, stopping powers, and electron slowing-down spectra," IEEE Trans. Nucl. Sci. Vol. NS-22, 2533.

Ashley, J. C., Tung, C. J., Ritchie, R. H. and Anderson, V. E. (1976a). "Calculations of mean free paths and stopping powers of low energy electrons $(<10 \mathrm{keV})$ in solids using a statistical model," IEEE Trans. Nucl. Sci. Vo1. NS-23, 1833. 
Ashley, J. C., Tung, C. J. and Ritchie, R. H. (1976b). "Inverse mean free path, stopping power, csda range and straggling in Ge and GaAs for electrons of energy $10 \mathrm{keV}, "$ Rome Air Development Center Report RADC-TR-76-350.

Ashley, J. C., Tung, C. J. and Ritchie, R. H. (1978). "Inelastic interactions of electrons with polystyrene: calculations of mean free paths, stopping powers, and csda ranges," IEEE Trans. Nuc1. Sci. Vo1. NS-25, 1566.

Ashley, J. C., Tung, C. J. and Ritchie, R. H. (1979). "Electron inelastic mean free paths and energy losses in solids, 1. Aluminum metal," Surface Sci. 11 409.

Bader, M., Pixley, R. E., Moser, F. J. and Whaling, W. (1956). "Stopping cross sections of solids for protons, 50-600 keV," Phys. Rev. 103, 32 .

Bakker, C. J. and Segrè, E. (1951). "Stopping power and energy loss for ion pair production for $340-\mathrm{MeV}$ protons," Phys. Rev. $81,489$.

Barkas, W. H., Barrett, P. H., Cuer, P., Heckman, H., Smith, F. M. and Ticho, H. K. (1958). "The range-energy relation in emulsion," Nuovo Cimento $\underline{8}, 186$.

Barkas, W. H., Birnbaum, W. and Smith, F. M. (1956). "Mass ratio method applied to the measurement of L-meson masses and the energy balance in pion decay," Phys. Rev. 101, 778 (1956).

Barkas, W. H. and von Friesen, S. (1961). "High-velocity range and energy-loss measurements in $\mathrm{Al}, \mathrm{Cu}, \mathrm{Pb}, \mathrm{U}$ and emulsion," Nuovo Cimento (Supp1) $19,41$.

Barkas, W. H. (1963). Nuclear Research Emulsions, Vo1. 1, Academic Press, New York.

Bel1, K. L. and Kingston, A. E. (1967). "Some properties of atomic helium," Proc. Phys. Soc. (London), $\underline{90}, 901$.

Bel1, R. J. and Dalgarno, A. (1965). "Stopping powers and straggling in neon, argon and krypton," Proc. Phys. Soc. (London), $\underline{86}, 375$.

Be11, R. J. and Da1garno, A. (1966). "Stopping powers and straggling in the rare gases," Proc. Phys. Soc. (London), $\underline{89}, 55$.

Berger, M. J. and Seltzer, S. M. (1964). "Tables of energy losses and ranges of electrons and positrons," pp. 205-268, NAS-NRC pub1.1133, National Academy of Sciences-National Research Council, Washington; also NASA Publication SP-3012, National Aeronautics and Space Administration (1964).

Berger, M. J. and Seltzer, S. M. (1966). "Additional stopping power and range tables for protons, mesons and electrons," NASA SP-3036, National Aero-nautics and Space Administration.

Berger, M. J., Seltzer, S. M. and Maeda, K. (1970). "Energy deposition by auroral electrons in the atmosphere," J. Atmos. Terr. Phys. $\underline{32}, 1015$.

Besenbacher, F., Andersen, H. H., Hvelplung, P. and Knudsen, H. (1979). "Stopping power of swift hydrogen and helium ions in gases," Mat. Fys. Medd. Vid. Selsk. 40 No. 3 .

Bethe, H. (1930). "Zur Theorie des Durchgangs schneller Korpuskularstrahlen durch Materie," Ann. Phys. $\underline{5} 325$.

Bethe, H. (1932). "Bremsformel für Elektronen relativistischer Geschwindigkeit," Z. f. Phys. $\underline{76}, 293$.

Bethe, H. (1933). "Quantenmechanik der Ein- und Zwei-Elektronenprobleme," pp. 273560, Handbuch der Physik, Vol. 24/1, Springer, Berlin.

Bethe, H. A. (1934). "The influence of screening on the creation and stopping of electrons," Proc. Cambr. Philos. Soc. $\underline{30}, 524$.

Bethe, H. A. and Ashkin, J. (1953). "Passage of radiations through matter," Vol. I, Experimental Nuclear Physics (ed. E. Segrè), Wiley, New York. 
Bethe, H. and Heitler, W. (1934). "On the stopping of fast particles and on the creation of positive electrons," Proc. Roy. Soc. Al46, 83.

Bhabha, H. J. (1936). "The scattering of positrons by electrons with exchange on Dirac's theory of the positron," Proc. Roy. Soc. A154, 195.

Bichsel, H. (1961). "Higher shell corrections in stopping power," University of Southern California, Physics Dept. Technical Report No. 3.

Bichse1, H. (1963). "Passage of charged particles through matter," pp. 8-20 to 8-47, American Institute of Physics Handbook, 2nd Edition, American Institute of Physics.

Bichsel, H. (1967). "The L-shell correction in stopping power," University of Southern California Report, USC-136-120.

Bichse1, H. (1968). "Charged particle interactions," p. 157, Radiation Dosimetry, Vol. 1, 2nd Edition (F. H. Attix, W. C. Roesch, eds.), Academic Press, New York.

Bichse1, H. (1972). "Passage of charged particles through matter," pp. 8-142 to 8-189, American Institute of Physics Handbook, 3rd Edition, American Institute of Physics.

Bichsel, H. (1978). "Physical data used in charged particle dosimetry," AAPM Charged Particle Beam Task Group Workshop, Los Alamos Scientific Laboratory.

Bichsel, H. and Hilko, R. H. (1980). "Measurement of the energy loss of alpha particles in carbon dioxide," Helv. Phys. Acta. $\underline{53}, 655$.

Bichse1, H. and Laulainen, N. S. (1971). "Mean excitation energies of stopping power for a toms," Bull. Am. Phys. Soc. 16, 842 .

Bichsel, H. and Porter, L. E. (1982). "The stopping power of protons and alpha particles in $\mathrm{H}_{2}$, He, $\mathrm{N}_{2}, \mathrm{O}_{2}, \mathrm{CH}_{4}$ and air," Phys. Rev. A 25,2455 .

Bichsel, H. and Tschalär, C. (1965). "Range difference measurements for protons in $\mathrm{C}$, Al, sapphire, Si, quartz, Ge and Ag," Bull. Am. Phys. Soc. II, 723.

Bloch, F. (1933). "Zur Bremsung rasch bewegter Teilchen beim Durchgang durch Materie," Ann. Phys. $16,285$.

Bohr, N. (1913). "On the theory of the decrease of velocity of moving electrified particles on passing through matter," Phil. Mag. 25, 10.

Bohr, N. (1948). "The penetration of atomic particles through matter," Mat. Fys. Medd. Dan. Vid. Selsk. 18 , (8).

Bonderup, E. (1967). "Stopping of swift protons evaluated from statistical atomic model," Mat. Fys. Medd. Dan. Vid. Selsk. 35, No. 17.

Bourland, P. D., Chu, W. K. and Powers, D. (1971). "Stopping cross section of gases for alpha particles from 0.3 to $2.0 \mathrm{MeV}$," Phys. Rev. B3 , 3625.

Bragg, W. H. and Kleeman, R. (1905). "On the alpha particles of radium and their loss of range in passing through various atoms and molecules," Phil. Mag. $\underline{10}$, 318 .

Brandt, W. (1956). "Stopping power and valence states," Phys. Rev. $104,691$.

Brandt, W. (1958). "Survey of stopping power," Health Phys. 1 , 11.

Brandt, W. (1960). "Energy loss and range of charged particles in compounds," Research Report, Radiation Physics Laboratory, E. I. du Pont and Co., Wilmington, Del.

Brolley, J. E., Jr. and Ribe, F. L. (1955). "Energy loss by 8.86-MeV deuterons and 4.43-MeV protons," Phys. Rev. 98, 1112. 
Brown, L. M. (1950). "Asymptotic expression for the stopping power of K electrons," Phys. Rev. 79297.

Burkig, V. C. and Mackenzie, K. R. (1957). "Stopping power of some metallic elements for $19.8 \mathrm{MeV}$ protons," Phys. Rev. 106, 848.

Carlson, T. A. (1975). Photoelectron and Auger spectroscopy, Plenum Press, New York and London.

Chan, Y. M. and Dalgarno, A. (1965a). "The stopping powers of atoms and molecules," Proc. Roy. Soc. A285, 457.

Chan, Y. M. and Dalgarno, A. (1965b). "The dipole spectrum and properties of helium," Proc. Phys. Soc. (London), $\underline{86}, 777$.

Chilton, A. B., Cooper, J. N. and Harris, J. C. (1954). "The stopping power of various elements for protons of energies from 400 to $1050 \mathrm{keV}$," Phys. Rev. $\underline{93}$, 413.

Chu, W. K. and Powers, D. (1972). "Calculation of mean excitation energy for all elements," Phys. Lett. 40A, 23.

Clementi, E. and Raimondi, D. L. (1963). "Atomic screening constants from SCF functions," J. Chem. Phys. 38, 2686.

Cohn, A. and Caledonia, G. (1970). "Spatial distribution of fluorescent radiation emission caused by an electron beam." J. App1. Phys. 41, 3767.

Cole, A. (1969). "Absorption of $20-\mathrm{eV}$ to $50,000-\mathrm{eV}$ electron beams in air and plastic," Radiat. Res. 38, 7.

Condensed Chemical Dictionary (1977). 9th Edition (revised by G. G. Hawley) Van Nostrand Reinhold Co., New York.

CRC Handbook of Chemistry and Physics (1979). 60th Edition (R. C. Weast, ed.) CRC Press, Boca Raton, F1orida.

Crispin, A. and Fowler, G. N. (1970). "Density effect in the ionization energy 1oss of fast charged particles in matter," Rev. Mod. Phys. 42290.

Dalgarno, A. (1960). "The stopping powers of atoms," Proc. Phys. Soc. (London) $\underline{76}$, 422 .

Dalgarno, A., Degges, T. and Williams, D. A. (1967). "Dipole properties of molecular nitrogen," Proc. Phys. Soc. (London), $\underline{92}, 291$.

Dalton, P. and Turner, J. E. (1968). "New evaluation of mean excitation energies for use in radiation dosimetry," Hea1th Phys. 15, 257.

Davies, H., Bethe, H. A. and Maximon, L. C. (1954). "Theory of bremsstrah1ung and pair production. II. Integral cross section for pair production," Phys. Rev. $\underline{93}$, 788 .

Dehmer, J. L., Inokuti, M. and Saxon, R. P. (1975). "Systematics of moments of dipole oscillator-strength distributions for atoms for the first and second row," Phys. Rev. A12, 102 .

Eggarter, E. (1975). "Comprehensive optical and collision data for radiation action, I I. Ar," J. Chem. Phys. 62, 833.

Elwert, G. (1939). "Verschärfte Berechnung der Intensität und Polarisation im kontinuierlichen Röntgenspektrum," Ann. Phys. 34, 178.

Fano, U. (1956). "Atomic theory of electromagnetic interactions in dense materials," Phys. Rev. 103, 1202 .

Fano, U. (1963). "Penetration of protons, alpha particles and mesons," Ann. Rev. Nuc1. Sci. 13, 1 . 
Fano, U. (1975). "Platzman's analysis of the delivery of radiation energy to molecules," Radiat. Res. 64, 217.

Fano, U. and Turner, J. E. (1964). "Contributions to the theory of shell corrections," NAS-NRC Pub1. 1133, 49.

Feng, I. J., Pratt, R. H. and Tseng, H. K. (1981) . "Positron bremsstrah1ung," Pitt-256, U. of Pittsburg.

Fermi, E. (1940). "The ionization loss of energy in gases and in condensed materials," Phys. Rev. $\underline{57} 485$.

Ford, A. L. and Browne, J. C. (1973). "Direct-resolvent-operator computations on the hydrogen-molecule dynamic polarizability, Rayleigh, and Raman scattering." Phys. Rev. 7A, 418 .

Garbincius, P. H. and Hyman, L. G. (1970) . "Range-energy relation in hydrogen," Phys. Rev. A, 2, 1834 .

Garcia, J. D. (1966). "Radiative corrections to the energies of atoms and molecules," Phys. Rev. $147,66$.

Gerhart, D. E. (1975). "Comprehensive optical and collision data for radiation action. I. $\mathrm{H}_{2}$," J. Chem. Phys. 62, 821 .

Gmelins Handbuch der Anorganischen Chemie (1968). Kohlenstoff, Teil B-Lieferung 2, 8 th Edition, Verlag Chemie, Weinheim.

Goodman, L. J. (1969). "A modified tissue equivalent 1iquid," Health Phys. $16,763$.

Goodman, L. J. (1978). "Density and composition uniformity of A-150 tissue-equivalent plastic," Phys. Biol. Med. $\underline{23}, 753$.

Green, A. E. S. and Miller, J. H. (1974). "Atomic and molecular effects in the physical state," Physical Mechanisms in Radiation Biology (R. D. Cooper and R. W. Wood, eds.), Report CONF-721001, U.S. Atomic Energy Commission, pp. 68-111.

Green, A.E. S. and Peterson, L. R. (1968). "Energy loss function for electrons and protons in planetary gases," J. Geophys. Res. $\underline{73}, 233$.

Greene, D., Major, J. and Law, J. (1973). "The G-value for the ferrous sulphate dosimeter for $14-\mathrm{MeV}$ neutrons," Phys. Med. Biol. 18, 369.

Grün, A. E. (1957). "Lumineszenz-photometrische Messungen der Energieabsorption in Strahlungsfeld von Elektronenquellen. Eindimensionaler Fall in Luft," $z$. $f$. Naturf. $12 \mathrm{a}, 89$.

Halpern, O. and Ha11, H. (1948). "The ionization loss of energy of fast charged particles in gases and condensed bodies," Phys Rev. $\underline{73} 477$.

Hanke, C. C. and Bichsel, H. (1970). "Precision energy loss measurements for natural alpha particles in argon," Mat. Fys. Medd. Dan. Vid. Selsk. 38, No. 3 .

Hanke, C. and Laursen, J. (1978). "Stopping cross section of alpha particles for 1.0 to $8.5 \mathrm{MeV}$ in $\mathrm{H}_{2}$, He, $\mathrm{N}_{2}, \mathrm{O}_{2}$, Ne, $\mathrm{Kr}$ and Xe," Nucl. Instr. Meth. $\underline{151}, 253$.

Haug, E. (1975). "Bremsstrahlung and pair production in the field of free electrons," Z. f. Naturforsch. 30a, 1099 .

Heitler, W. (1947). The Quantum Theory of Radiation, 2nd Edition, Oxford University Press, London.

Heller, J. M., Hamm, R. N., Birkhoff, R. D. and Painter, L. R. (1974). "Collective oscillation in liquid water," J. Chem. Phys. 60, 3483.

Hereford, F. L. (1948). "The specific primary ionization and energy loss of fast electrons in matter," Phys. Rev. $74,574$. 
Hill, K. W. and Merzbacher, E. (1974). "Polarization in distant Coulomb collisions of charged particles with atoms," Phys. Rev. A $\underline{9}, 156$.

Holden, N. E. (1979). "Atomic weights of the elements," (prepared for Commission on Atomic Weights, IUPAP), Pure and Appl. Chem. 51, 405.

Hoyer, U. and Wäffler, H. (1971). "Der atomare Bremsquerschnitt von $\mathrm{H}_{2}, \mathrm{D}_{2}, \mathrm{He}, \mathrm{N}_{2}$, and A für $\alpha$-Teilchen im Umladungsgebiet," $Z$. Naturf. 26a, 592.

Hubbel1, J.H. (1969). "Photon cross sections, attenuation coefficients, and energy absorption coefficients from $10 \mathrm{keV}$ to $100 \mathrm{GeV}, "$ NSRDS-NBS 29.

Hubbell, J.H., Veigele, W. J., Briggs, E. A., Brown, R. T., Cromer, D. T. and Howerton, R. J. (1975). "Atomic form factors, incoherent scattering functions, and photon scattering cross sections," J. Phys. and Chem. Reference Data $\underline{4}, 471$; errata in 6 , 615 (1977).

Hubbe11, J. H. and $\emptyset$ verb $\phi$, I. (1979). "Relativistic atomic form factors and photon coherent scattering cross sections," J. Phys. and Chem. Reference Data $\underline{8}, 69$.

Hubbell, J. H., Gimm, H. A. and $\emptyset$ verb $\phi$, I. (1980). "Pair, triplet, and total atomic cross sections for $1-\mathrm{MeV}$ to $100-\mathrm{GeV}$ photons in elements $\mathrm{A}=1$ to $100, " \mathrm{~J}$. Phys. and Chem. Reference Data $\underline{9}, 1023$.

ICRP (1975). "Report of the task group on reference man," International Commission of Radiological Protection, Pergamon Press, New York.

ICRU (1964). ICRU Report 10b, "Physical aspects of irradiation. Recommendations of the International Commission on Radiological Units and Measurements," published as National Bureau of Standards Handbook 85.

ICRU (1970). ICRU Report 16, "Linear energy transfer," International Commission on Radiation Units and Measurements, Washington, D.C.

ICRU (1977). ICRU Report 26, "Neutron dosimetry for biology and medicine," International Commission on Radiation Units and Measurements, Washington, D.C.

ICRU (1980). ICRU Report 33, "Radiation quantities and units," International Commission on Radiation Units and Measurements, Washington, D.C.

Inokuti, M. (1971). "Inelastic collisions of fast charged particles with atoms and molecules - the Bethe theory revisited," Rev. Mod. Phys. 43 297.

Inokuti, M., Dehmer, J. L., Baer, T. and Hanson, J. D. (1981). "Oscillator-strength moments, stopping powers, and total inelastic scattering cross sections of all atoms through strontium," Phys. Rev. A $\underline{23}, 95$.

Inokuti, M., Douthat, D. A. and Rau, A. R. P. (1976). "Degradation spectra and ionization yields of electrons in gases," pg. 977, Proc. Fifth Symposium on Microdosimetry, EUR $5452 \mathrm{~d}-\mathrm{e}-\mathrm{f}$, Euratom.

Inokuti, M. and Smith, D. Y. (1982). "Fermi density effect on the stopping power of metallic aluminum," Phys. Rev. B 25, 61.

Inokuti, M. and Turner, J. E. (1978). "Mean excitation energies for stopping power as derived from oscillator-strength distributions," pg. 675, Proc. Sixth Symposium on Microdosimetry, Vol. I, Harwood Academic Publishers, Ltd., New York.

Ishigure, N., Mori, C. and Watanabe, T. (1978). "Electron stopping power in aluminum in the energy region from 2 to $10.9 \mathrm{keV}, " \mathrm{~J}$. Phys. Soc. Japan $44,973$.

Ishiwari, R., Shiomi, N. and Sakamoto, N. (1979). "Stopping powers of Be, Al, Ti, $\mathrm{V}, \mathrm{Fe}$, Co, $\mathrm{Ni}, \mathrm{Cu}, \mathrm{Zn}$, Mo, $\mathrm{Rh}, \mathrm{Ag}, \mathrm{Sn}, \mathrm{Ta}, \mathrm{Pt}$ and Au for $6.75 \mathrm{MeV}$ protons," Phys. Letters $75 \mathrm{~A}, 112$.

Iskef, H., Thwaites, D. I. and Watt, D. E. (1980). "Stopping power of low energy electrons (<10 keV)," p. 201, Proc. Seventh Symposium on Microdosimethy, Vo1. I, Harweed Academic Publishers, Ltd., New York. 
Jackman, C. H., Garvey, R. H. and Green, A. E. S. (1977). "Electron impact on atmospheric gases. I. Updated cross sections," J. Geophys. Res 82, 5081.

Jackson, J. D. (1975). Classical Electro-Dynamics, 2nd Edition, Wiley, New York.

Jackson, J. D. and McCarthy, R. L. (1972). " $\mathrm{Z}^{3}$ corrections to energy loss and range," Phys. Rev. B6, 4131 .

Janni, J. F. (1966). "Calculations of energy loss, range, pathlength, straggling, multiple scattering, and the probability of inelastic nuclear collisions for 0.1 to $1000-\mathrm{MeV}$ protons," AFWL-TR-65-150, Air Force Weapons Laboratory.

Janni, J. F. (1980). "A comprehensive set of adjusted ionization potentials," Trans. Amer. Nuc1. Soc. 34653 .

Jensen, H. (1937). "Eigenschwingungen eines Fermi-Gases und Anwendung auf die Blochsche Bremsformel für schnelle Teilchen," Z. f. Phys. 106, 620 .

Jhanwar, B. L., Meath, W. J. and MacDonald, J. C. F. (1981). "Dipole oscillator strength distributions and sums for $\mathrm{C}_{2} \mathrm{H}_{6}, \mathrm{C}_{3} \mathrm{H}_{\mathrm{n}}, \mathrm{n}-\mathrm{C}_{4} \mathrm{H}_{10}, \mathrm{n}-\mathrm{C}_{5} \mathrm{H}_{12}, \mathrm{n}-\mathrm{C}_{6} \mathrm{H}_{14}$, $\mathrm{n}-\mathrm{C}_{7} \mathrm{H}_{16}$, and $\mathrm{n}-\mathrm{C}_{8} \mathrm{H}_{18}$," Can. J. Phys. $\underline{59}$, 185 .

Joseph J. and Rohrlich, F. (1958). "Pair production and bremsstrahlung in the field of free and bound electrons," Rev. Mod. Phys. 30, 354 .

Kalil, F., Stone, W. G., Hubbel1, Jr., H. H. and Birkhoff, R. D. (1959). "Stopping power of thin aluminum foils for 12 to $127 \mathrm{keV}$ electrons," Oak Ridge National Laboratory Report ORNL-2731.

Kerr, G. D., Hain, L. M., Underwood, N. and Walthers, A. W. (1966). "Molecular stopping cross sections of air, $\mathrm{N}_{2}, \mathrm{Kr}, \mathrm{CO}_{2}$ and $\mathrm{CH}_{4}$ for alpha particles," Health Phys. 12, 1475 .

Khandelwal, G. S. and Merzbacher, E. (1966). "Stopping power of M electrons," Phys. Rev. $144,349$.

Khandelwal, G. S. (1968). "Shell corrections for K- and L-electrons," Nucl. Phys. A $\underline{116}, 97$.

Kissel, L. and MacCa11um, G. (1977). "Benchmark bremsstrahlung energy spectrum data for 1 and $2 \mathrm{MeV}, "$ PITT-179, U. of Pittsburgh.

Koch, H. W. and Motz, J. W. (1959). "Bremsstrahlung cross-section formulas and related data," Rev. Mod. Phys. $31,920$.

Kutcher, G. J. and Green, A. E. S. (1976). "A model for energy deposition in liquid water," Radiat. Res. $67,408$.

Langhoff, P. W. and Yates, A. C. (1972). "Moment theory bounds on the mean energies of stopping, straggling, and molecular excitation," J. Phys. B: Atom. Molec. Phys. 5,1071 .

Langley, R. A. (1975). "Stopping cross sections for helium and hydrogen in $\mathrm{H}_{2}, \mathrm{~N}_{2}$, $\mathrm{O}_{2}$ and $\mathrm{H}_{2} \mathrm{~S}(0.3-2.5 \mathrm{MeV})$," Phys. Rev. $\mathrm{B}, \underline{12}, 3575$.

Lee, C. M., Kissel, L. and Pratt, R. H. (1976). "Electron bremsstrahlung spectrum," $1-500 \mathrm{keV}$, Phys. Rev. A 13,1714 .

Lindhard, J. (1954). "On the properties of a gas of charged particles," Mat. Fys. Medd. Dan. Vid. Selsk. 28,1 .

Lindhard, J. (1976). "The Barkas effect, or $z_{1}^{3}-z_{1}^{4}$ corrections to stopping of swift charged particles," Nuc1. Instr. Meth. $13 \frac{1}{2}, 1$.

Lindhard, J. and Scharff, M. (1953). "Energy loss in matter by fast particles of low charge," Mat. Fys. Medd. Dan. Vid. Selsk. 34 , No. 15. 
Lindhard, J. and Winther, A. (1964). "Stopping power of electron gas and equipartition rule," Mat. Fys. Medd. Dan. Vid. Se1sk. 34, No. 4.

Livingston, M. S. and Bethe, H. A. (1937). "Nuclear physics," Rev. Mod. Phys. 9 282 .

Märk, T. D. and Egger, F. (1976). "Cross section for single ionization of $\mathrm{H}_{2} \mathrm{O}$ and $\mathrm{D}_{2} \mathrm{O}$ by electron impact from threshold up to $170 \mathrm{eV}$,"Int. J. Mass Spectrometry and Phys. 20,89 .

Martin, F. W. and Northcliffe, L. C. (1962). "Energy loss and effective charge of $\mathrm{He}, \mathrm{C}$, and Ar ions below $10 \mathrm{MeV} / \mathrm{amu}$ in gases," Phys. Rev. 128, 1166.

McGuire, E. J. (19.71). "Inelastic scattering of electrons and protons by the elements He to Ne," Phys. Rev. A3, 267.

Miller, W. F. (1956). "A theoretical study of excitation and ionization by electrons in helium and the mean energy per ion pair," Ph.D. Dissertation, Purdue University, Lafayette, Ind.

Mф1ler, C. (1932). "Zur Theorie des Durchgangs schneller Elektronen durch Materie," Ann. Phys. 14, 568 .

Motz, J. W. (1955). "Bremsstrahlung differential cross-section measurements for 0.5 and 1.0-MeV electrons," Phys. Rev. 100, 1560 .

Motz, J. W. and Placious, R. C. (1958). "Bremsstrahlung cross-section measurements for $50-\mathrm{keV}$ electrons," Phys. Rev. 109, 235.

Nakano, G. H., MacKenzie, K. R. and Bichsel, H. (1963). "Relative stopping power of some metallic elements for $28.7 \mathrm{MeV}$ protons," Phys. Rev. 132, 291.

NAS-NRC (1964). Studies in Penetration of Charged Particles in Matter, Committee on Nuclear Science, Subcomittee on Penetration of Charged Particles, Pub1. 1133, Washington, D.C.

NCRP (1961). "Stopping powers for use with cavity chambers," National Council on Radiation Protection, published as National Bureau of Standards Handbook 79.

Nelms, A. T. (1956). "Energy loss and range of electrons and positrons," National Bureau of Standards Circular 577.

Nelms, A. T. (1958). "Energy loss and range of electrons and positrons," Supplement to National Bureau of Standards Circular 577.

Nordin, J. A. and Henkelman, R. M. (1979). "Measurement of stopping power ratios for $60-\mathrm{MeV}$ positive or negative pions," Phys. Med. Bio1. 24, 781 .

Olivero, J., Stagat, R. W. and Green, A. E. S. (1972). "Electron deposition water vapor, with atmospheric applications," J. Geophys. Res. $\underline{77}, 4797$.

Olsen, H. (1955). "Outgoing and ingoing waves in final states and bremsstrahlung," Phys. Rev. $\underline{99}, 1335$.

Pages, L, Bertel, E., Joffre, H. and Sklavenitis, L. (1972). "Energy loss, range and bremstrahlung yield for $10-\mathrm{keV}$ to $100-\mathrm{MeV}$ electrons in various elements and chemical compounds," Atomic Data $\underline{4}, 1$.

Painter, L. R., Arakawa, E. T., Williams, W. and Ashley, J. C. (1980). "Optical properties of polyethylene: measurement and applications," Radiat. Res. $83,1$.

Paretzke, H. G. and Berger, M. J. (1978). "Stopping power and energy degradation for electrons in water vapor," pg. 749, Proc. Sixth Symposium on Microdosimetry, Vol. II, Harwood Academic Publishers, New York.

Particle Data Group (1980). "Review of particle properties," Rev. Mod. Phys. 52 , S1. 
Paul, W. and Reich, H. (1950). "Energieverlust schneller Elektronen in Be, $\mathrm{C}, \mathrm{H}_{2} \mathrm{O}$, Fe und $\mathrm{Pb}, " \mathrm{Z}$. Phys. $127,429$.

Porter, L. E., Naylor, H. and Duder, J. C. (1978). "Stopping power of polystyrene for 2.2 to $5.9-\mathrm{MeV}$ protons," Nucl. Instr. Meth. $\underline{155}, 25$.

Powell, M. J. D. (1967). "Curve fitting by cubic splines," Atomic Energy Research Establishment (Harwe11, England) Report TP 307.

Pratt, R. H., Tseng, H. K., Lee, C. M., Kissel, L., MacCa1lum, C. and Riley, M. (1977). "Bremstrahlung energy spectra from electrons of kinetic energy $1 \mathrm{keV} \leqslant \mathrm{T}, 2000 \mathrm{keV}$ incident on neutral atoms $2 \leqslant Z \leqslant 92 \mathrm{keV}$," Atomic Data and Nuclear Data Tables 20, 175 ; errata in 26,477 (1981).

Racah, G. (1934). "Sopra l'Irradiazione Nell'Urto di Particelle Veloci," Nuovo Cimento 11,461 .

Raether, H. (1980). Excitation of Plasmons and Interband Transitions by Electrons, Springer, Berlin-Heidelberg-New York.

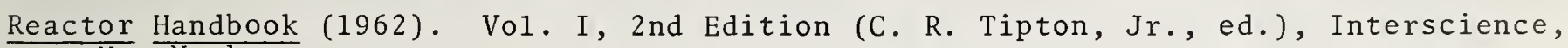
New York.

Rester, D. H. and Dance, W. E. (1967). "Bremsstrahlung cross-section measurements at incident electron energies of $1.0,1.7$ and $2.5 \mathrm{MeV}, "$ Phys. Rev. 161,85 .

Rester, D. H. and Edmonson, N. (1972). "Bremsstrahlung cross-section measurements at an incident electron energy of $0.2 \mathrm{MeV}$ on $\mathrm{Al}, \mathrm{Cu}$ and $\mathrm{Au}, "$ Phys. Rev. $\underline{\mathrm{A} 6}, 1405$.

Reynolds, H. K., Dunbar, D. N. F., Wenzel, W. A., and Whaling, W. (1953). "The stopping cross section of gases for protons, 30-600 keV," Phys. Rev. 92, 742.

Ritchie, R. H. (1959). "Interaction of charged particles with a degenerate FermiDirac gas," Phys. Rev. 114, 644 .

Ritchie, R. H. and Brandt, W. (1978). "Projectile charge dependence of stopping powers," Phys. Rev. A17, 2102.

Ritchie, R. H., Hamm, R. N., Turner, J. E. and Wright, H. A. (1978). "The interaction of swift electrons with liquid water," pg. 345, Proc. Sixth Symposium on Microdosimetry, Vo1. I. Harwood Academic Publishers, Ltd., New York.

Rohrlich, F. and Carlson, B. C. (1953). "Positron-electron differences in energy loss and multiple scattering," Phys. Rev. $\underline{93}, 38$.

Rossi, H. H. and Failla, G. (1956). "Tissue-equivalent ionization chambers," Nucleonics 14 (2) 32 .

Rotondi, E. (1968). "Energy loss of alpha particles in tissue," Radiat. Res. $\underline{33}, 1$.

Sauter, F. (1934). "Über die Bremsstrahlung schneller Elektronen," Ann. Phys. 20, 404 .

Saxon, R. P. (1973). "Total cross sections of inelastic scattering of charged particles by atoms and molecules. VII. Neon atom," Phys. Rev. A8, 839 .

Schou, J. (1979). "Studies on keV and eV electrons," Ris $\phi$ National Laboratory Report R-391.

Schutten, J., DeHeer, A. J., Mustafa, H. R., Boerboom, A. J. H. and Kistemaker, J. (1966): "Gross and partial ionization cross sections for electrons in water vapor in the energy range 0.1-20 keV," J. Chem. Phys. 44, 3924.

Shiles, E., Sasaki, T., Inokuti, M. and Smith, D. Y. (1980). "Self-consistency and sum-rule tests in the Kramers-Kronig analysis of optical data: applications to aluminum," Phys. Rev B 22, 1612. 
Shimamura, I., Watanabe, T., Natori, M. and Matsuzawa, M. (1973). "Progress Report VI, Research group on atoms and molecules," Japan (unpublished).

Sigmund, P. (1975). "Energy loss of charged particles in solids," pp. 3-117, Radiation Damage Processes in Materials (C. H. Dupuy, ed.) Noordhoff, Leyden.

Smathers, J. B., Otte, V. A., Smith, A. R., Almond, P. R., Attix, F. H. Spokas, J. J., Quam, W. M. and Goodman, L. J. (1977). "Composition of A-150 tissue-equivalent plastic," Med. Phys. $\underline{4}, 74$.

Sфrensen, H. and Andersen, H. H. (1973). "Stopping Power of Al, $\mathrm{Cu}, \mathrm{Ag}, \mathrm{Au}, \mathrm{Pb}$ and $\mathrm{U}$ for 5-18-MeV protons and deuterons," Phys. Rev. B $\underline{8}, 1854$.

Sørensen, H. and Schou, J. (1978). "Interaction between solid nitrogen and 1-3-keV electrons," J. App1. Phys. 49, 5311.

Spencer, L. V. and Attix, F. H. (1955). "A theory of cavity ionization," Radiat. Res. $\underline{3}, 239$.

Spencer, L. V. and Pal, R. (1978). "Numerical solution of the electron slowing down equation for molecular hydrogen," National Bureau of Standards Report NBSIR 781523 .

Srdoč, D. (1970). "Experimental technique of measurement of microscopic energy distribution in irradiated matter using Rossi counters," Radiat. Res. 43, 302.

Sternheimer, R. M. (1952). "The density effect for the ionization loss in various materia1s," Phys. Rev. $\underline{88}, 851$.

Sternheimer, R. M. (1956). "Density effect for the ionization loss in various materials," Phys. Rev. 103, 511.

Sternheimer, R. M. (1966). "Density effect for the ionization loss of charged particles," Phys. Rev. 145, 247.

Sternheimer, R. M. (1967). "Density effect for the ionization loss of charged particles," Phys. Rev. 164, 349.

Sternheimer, R. M. and Peierls, R. F. (1971). "General expression for the density effect for the ionization loss of charged particles," Phys. Rev. B $\underline{3}, 3681$.

Sternheimer, R. M., Seltzer, S. M. and Berger, M. J. (1982). "Density effect for the ionization loss of charged particles," to be published.

Streitweiser, A., Jr. and Heathcock, C. H. (1976). Introduction to Organic Chemistry, MacMillan, New York.

Sugiyama, H. (1976). "Energy loss and range of electrons below $10 \mathrm{keV}$," Japanese J. of App1. Phys. 15, 1779 .

Swann, W. F. G. (1938). "Theory of energy loss of high energy particles," J. Franklin Inst. 226,598 .

Swint, J. B., Prior, R. M. and Ramirez, J. J. (1970). "Energy 1oss of protons in gases," Nuc1. Instr. Meth. $80,134$.

Thomas, G. F. and Meath, W. J. (1977). "Dipole spectrum, sums and properties of ground-state methane and their relation to the molar refractivity and dispersion energy constant," Molec. Phys. 34, 113.

Thompson, T. J. (1952). "Effect of chemical structure on stopping powers for highenergy protons," Report UCRL-1910, University of California Radiation Laboratory.

Tschalär, C. and Bichsel, H. (1968). "Mean excitation potential of light compounds," Phys. Rev. 175, 476.

Tseng, H. K. and Pratt, R. H. (1971). "Exact screened calculations of atomic-field bremsstrahlung," Phys. Rev. A3 , 100. 
Tseng, H. K. and Pratt, R. H. (1979). "Electron bremsstrahlung energy spectra above $2 \mathrm{MeV}, "$ Phys. Rev. A $19,1525$.

Tung, C. J., Ashley, J. C., Anderson, V. E. and Ritchie, R. H. (1976). "Inverse mean free path, stopping power, csda range and straggling in silicon and silicon dioxide for electrons of energy $\leqslant 10 \mathrm{keV}$," Rome Air Development Center, Report RADC-TR- $76-125$.

Tung, C. J., Ashley, J. C. and Ritchie, R. H. (1979). "Range of low-energy electrons in solids," IEEE Trans. Nuc1. Sci., Vol. NS-26, 4874,

Turner, J. E., Roecklein, P. D. and Vora, R. B. (1970). "Mean excitation energies for chemical elements," Health Phys. 18, 159.

Uehling, E. A. (1954). "Penetration of heavy charged particles in matter," Annual Rev. Nucl. Sci. $4,315$.

Victor, G. A. and Dalgarno, A. (1969). "Dipole properties of molecular hydrogen," J. Chem. Phys. $\underline{50}, 2535$.

Waibel, E. and Grosswendt, B. (1980). "Stopping power and energy range relation for low-energy electrons in nitrogen and methane," p. 267, Proc. Seventh Symposium on Microdosimetry, Vol. I, Harwood Academic Publishers, Ltd., New York.

Walske, M. C. (1952). "The stopping power of K electrons," Phys. Rev. $\underline{88,} 1283$.

Walske, M. C. (1956). "Stopping power of L-electrons," Phys. Rev. 101, 940.

Wenzel, W. A. and Whaling, W. (1952). "The stopping cross section of $\mathrm{D}_{2} \mathrm{O}$ ice," Phys. $\operatorname{Rev} .87,499$.

Westermark, T. (1961). "Some measurements on energy loss of $2.8 \mathrm{MeV}$ electrons in light elements and compounds," Ark. Kemi 17, 101.

Wheeler, J. A. and Lamb, W. E. (1939). "Influence of atomic electrons on radiation and pair production," Phys. Rev. 55, 858; errata in $\underline{101}, 1836$ (1956).

White, D. R. (1978). "Tissue substitutes in experimental radiation physics," Med. Phys. 467.

Wick, G. C. (1943). "Sul frenamento delle particelle veloci," Nuovo Cimento, $1,302$.

Wolke, R. L., Bishop, W. N., Eibhler, E., Johnson, N. R. and O'Kelley, G. D. (1963). "Ranges and stopping cross sections of low-energy tritons," Phys. Rev. 129, 2591 .

Yates, A. C. and Langhoff, P. W. (1970). "Bounds for oscillator-strength sums from approximate quadratures," Phys. Rev. Lett. 25, 1317.

Zeiss, G. D., Meath, W. J., MacDonald, J. C. F. and Dawson, D. J. (1975). "Dipole spectrum of water vapor and its relation to energy loss of fast charged particles," Radiat. Res. 63, 64 .

Zeiss, G. D., Meath, W. J., MacDonald, J. C. F. and Dawson, D. J. (1977a). "Dipole oscillator strength distributions, sums and some related properties for $L i, N$, $\mathrm{O}, \mathrm{H}_{2}, \mathrm{~N}_{2}, \mathrm{O}_{2}, \mathrm{NH}_{3}, \mathrm{H}_{2} \mathrm{O}$, NO and $\mathrm{N}_{2} \mathrm{O}$," Can. J. Phys. 55,2080 .

Zeiss, G. D., Meath, W. J., MacDona1d, J. C. F. and Dawson, D. J. (1977b). "Accurate evaluation of stopping and straggling mean excitation energies for $\mathrm{N}, \mathrm{O}, \mathrm{H}_{2}, \mathrm{~N}_{2}$, $\mathrm{O}_{2}, \mathrm{NO}, \mathrm{NH}_{3}, \mathrm{H}_{2} \mathrm{O}$, and $\mathrm{N}_{2} \mathrm{O}$ using dipole oscillator strength distributions. A test of the validity of ${ }^{2}$ Bragg's rule," Radiat. Res. $70,284$.

Zeiss, G. D., Meath, W. J., MacDonald, J. C. F. and Dawson, D. J. (-1980). "On the Additivity of atomic and molecular dipole properties and dispersion energies using $\mathrm{H}, \mathrm{N}, \mathrm{O}, \mathrm{H}_{2}, \mathrm{~N}_{2}, \mathrm{O}_{2}, \mathrm{NO}, \mathrm{N}_{2} \mathrm{O}, \mathrm{NH}_{3}$ and $\mathrm{H}_{2} \mathrm{O}$ as models," Molec. Phys. 39 , 
Ziegler, B. (1958). "Energieverlust und Energiestreuung schneller Elektronen in Be, $\mathrm{C}$ und $\mathrm{Al}, " \mathrm{Z}$ Phys. $151,556$.

Ziegler, J. F. (1977). Helium: Stopping Powers and Ranges in a11 Elementa1 Matter, Pergamon Press, New York.

Ziegler, J. F. (1980). "The stopping and ranges of ions in matter," Vo1. 5, Handbook of Stopping Cross Sections for Energetic Ions in a11 Elements, Pergamon Press, New York.

Ziemer, P. L., Johnson, R. M. and Birkhoff, R. D. (1959). "Measurement of stopping power of copper by calorimetric methods," Oak Ridge National Laboratory Report ORNL- 2775 .

Zrelov, V. P., Kruglov, S. P., Mus, K. F., Savel'ev, V. D. and Sulek, P. (1974). "Determination of the average excitation I of copper ion from the ionization range of $660-\mathrm{MeV}$ protons," Yad. Fiz. 19, 1246; Sov. J. Nuc1. Phys. 19, 653. 


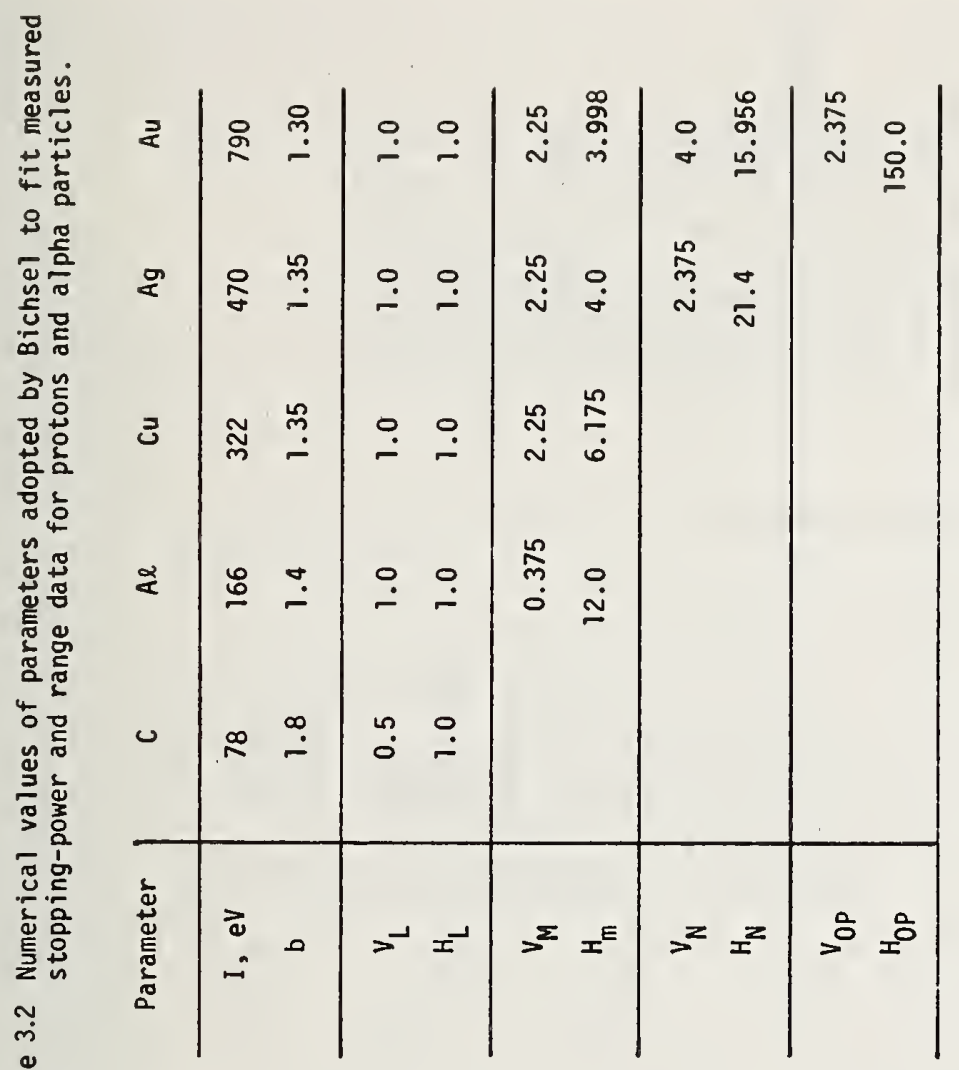

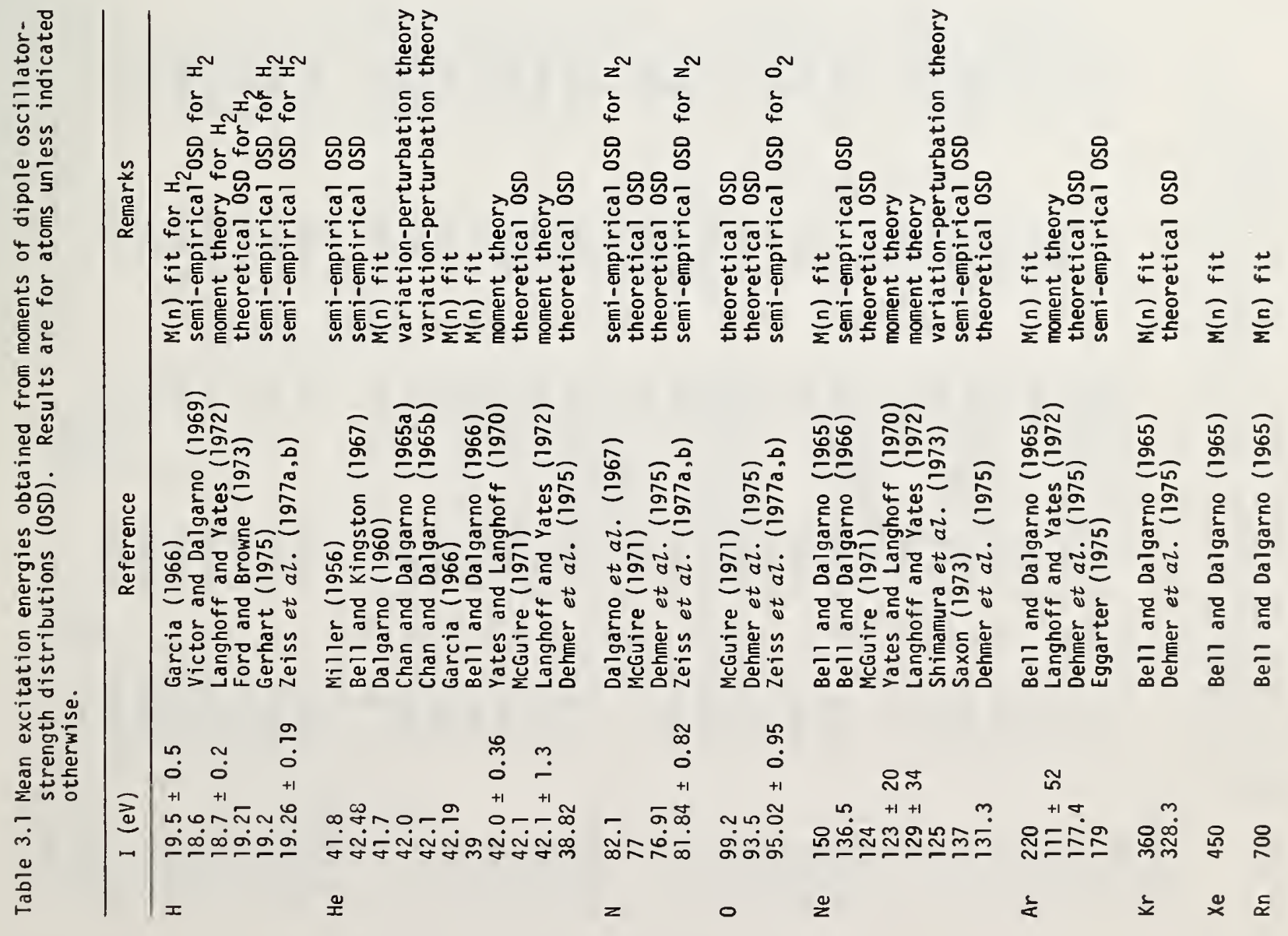




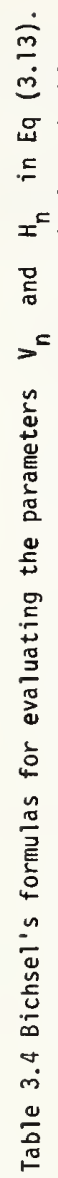

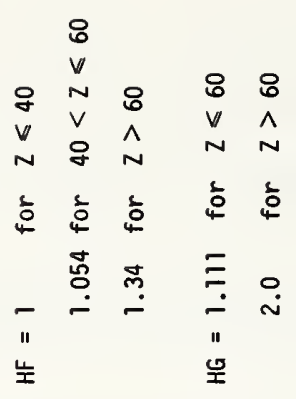

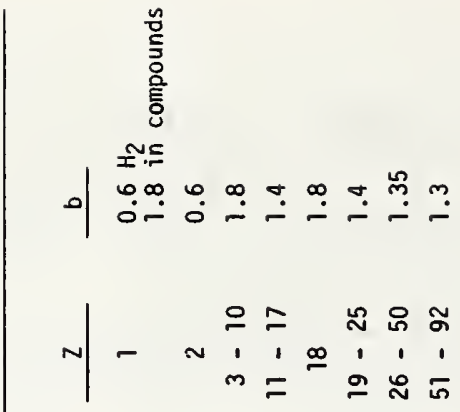

气े

政

㝳

욜

范苍

党

ㅎํㅇ

茫

范范

온

$\backsim \stackrel{\bar{d}}{\square}$

i

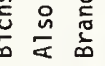

$\dot{0}$

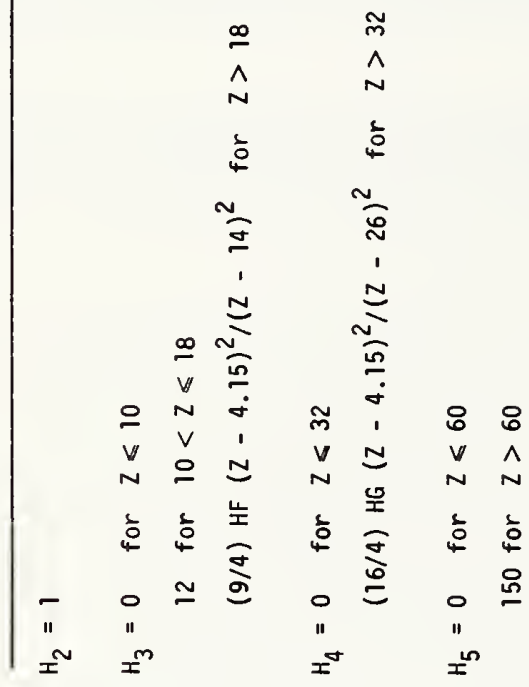

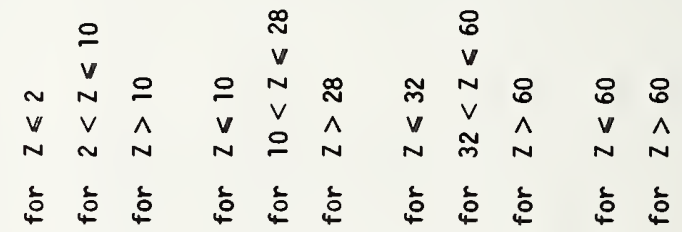

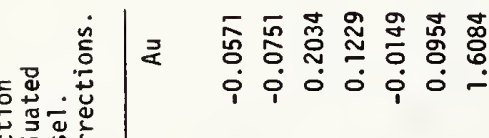

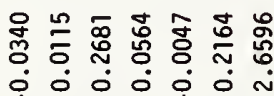

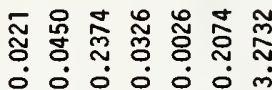

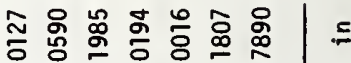

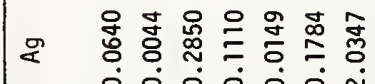

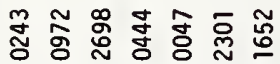

$\stackrel{\infty}{ล}$

$\stackrel{\infty}{\infty} \stackrel{\infty}{\frac{2}{2}}$

$\stackrel{\infty}{\stackrel{\infty}{\infty}}$

$\stackrel{\infty}{\stackrel{\infty}{\Delta}}$

० $N-0 \stackrel{\infty}{\infty}$

",

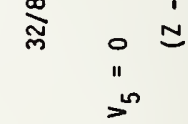

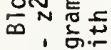

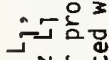

NN范

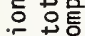

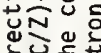

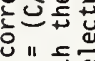

in $x$

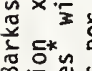

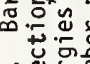

ㄴํㅇ 㝴

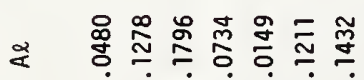

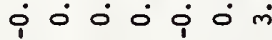

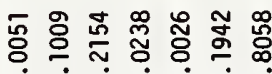

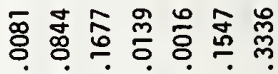

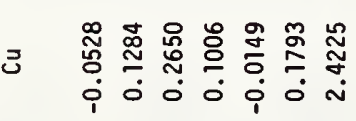

产 总

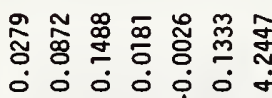

啹空 冚

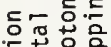

ist

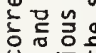

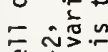

sta

竚

듬

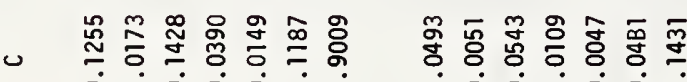

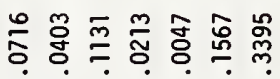

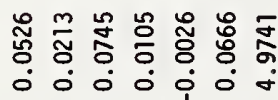

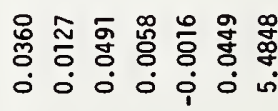

○ 000 i

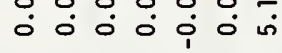

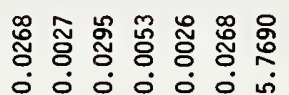

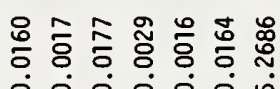

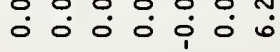

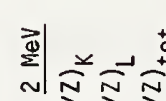

i.

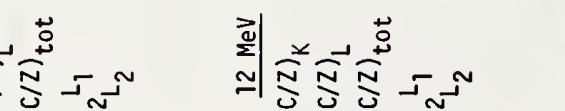

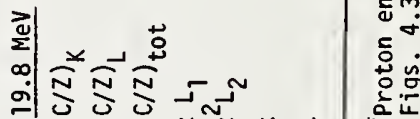


Table 3.5 Analysis of range measurements of Barkas and von Friesen (1961). I-values are evaluated assuming that $I_{A l}=166 \mathrm{eV}$, which implies a proton energy of $749.0 \mathrm{MeV}$. The experimental proton ranges were $314.9 \mathrm{~g} / \mathrm{cm}^{2}$ for $\mathrm{Cu}, 415.6 \mathrm{~g} / \mathrm{cm}^{2}$ for $\mathrm{Pb}$, and $432.5 \mathrm{~g} / \mathrm{cm}^{2}$ for $U$, and have an estimated uncertainty of $0.1 \%$. The corresponding uncertainties of the I-values are $3 \mathrm{eV}$ for $\mathrm{Cu}$ and $6 \mathrm{eV}$ for $\mathrm{Pb}$ and $\mathrm{U}$.

\begin{tabular}{l|ccc} 
& \multicolumn{3}{|c}{$\mathrm{I}, \mathrm{eV}$} \\
\cline { 2 - 4 } Method of Analysis & $\mathrm{Cu}$ & $\mathrm{Pb}$ & $\mathrm{U}$ \\
\hline $\begin{array}{l}\text { Bichsel shell correction, } \\
\text { with density-effect correction }\end{array}$ & 314.3 & 821.2 & 889.2 \\
$\begin{array}{l}\text { Bichsel shell correction, } \\
\text { without density-effect correction }\end{array}$ & 334.6 & 832.4 & 920.2 \\
$\begin{array}{l}\text { Bonderup shell correction, } \\
\text { with density-effect correction }\end{array}$ & 314.6 & 818.3 & 884.8
\end{tabular}

Table 3.6. Comparison of mean excitation energies I extracted from proton stoppingpower and range measurements with the use of Bichsel's and Bonderup's shell corrections. The estimated uncertainties of the I-values are those resulting from the uncertainties of the measurements. The measurements are from the experiments listed in Table 4.1 .

$\mathrm{I}(\mathrm{eV})$, derived with use of

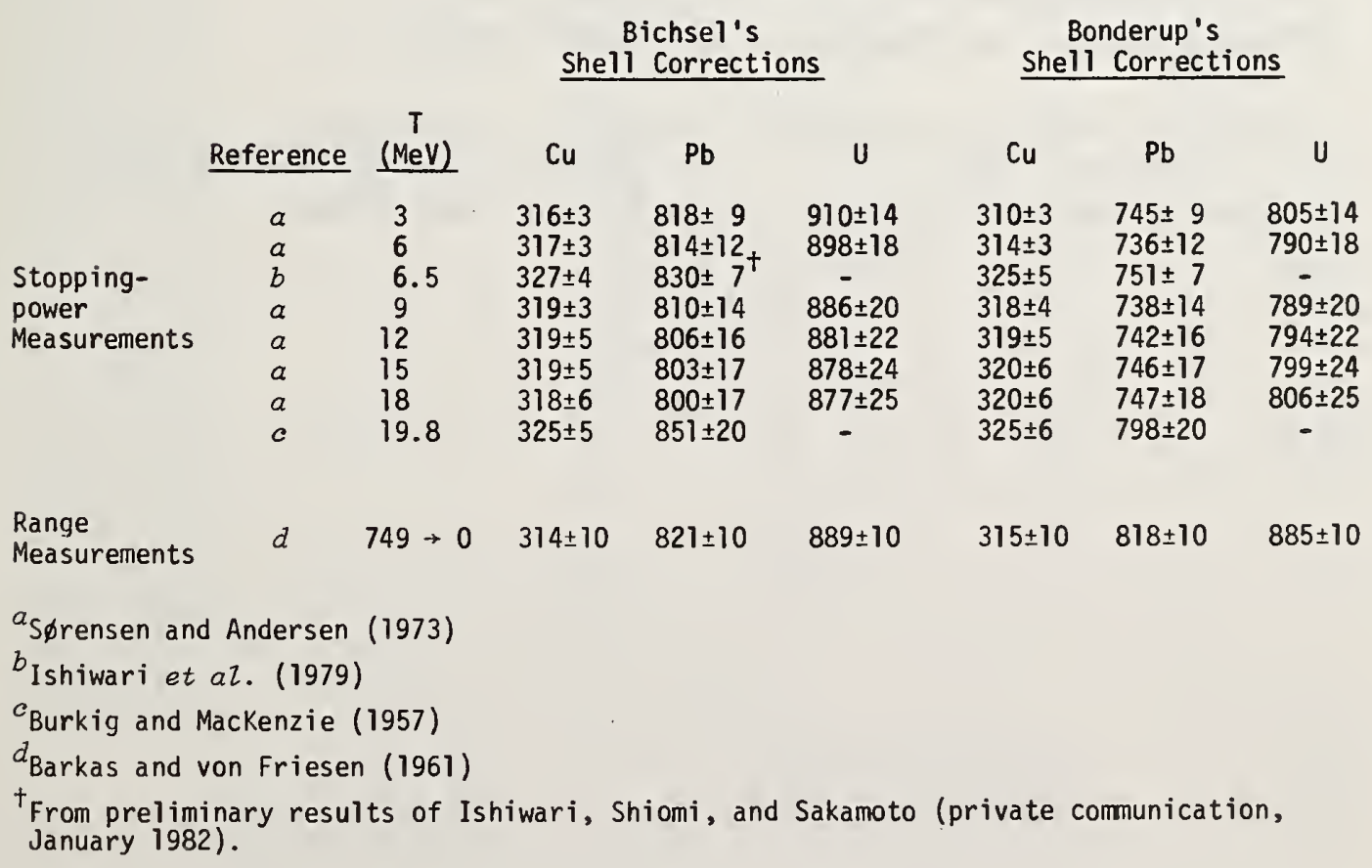


Table 4.1 List of stopping-power and range experiments from which mean excitation energies were deduced. $S$ indicates stopping-power, $R$ indicates range.

Abbrev.

Bak 51

Tho 52

Bro 55

Bur 57

Bar 61

Mar 62

Nak 63

Bic 65

Tsc 68

And 67

And 68

And 69

Gar 70

Swi 70

Han 70

Sør 73

Zre 74

And 77

Nor 79

Ish 79

Bes 79

And 81
Reference

Bakker and Segre (1951)

Thompson (1952)

Brolley and Ribe (1955)

Burkig and Mackenzie (1957)

Barkas and von Friesen (1961)

Martin and Northcliffe (1962)

Nakano, Mackenzie, and Bichsel (1963)

Bichsel and Tschalär (1967)

Tschalär and Bichsel (1968)

Andersen, Hanke, Sørensen, and Vajda (1967)

Andersen, Hanke, Simonsen, Sørensen, and Vajda (1968)

Andersen, Simonsen, Sørenson, and Vajda (1969)

Garbincius and Hyman (1970)

Swint, Prior, and Ramirez (1970)

Hanke and Bichsel (1970)

Sørensen and Andersen (1973)

Zrelov, Kruglov, Mus, Savel'ev, and Sulek (1974)

Andersen, Bak, Knudsen, and Nielsen (1977)

Nordin and Henkelman (1979)

Ishiwari, Shiomi, and Sakamoto (1979)

Besenbacher, Andersen, Hvelplund, and Knudsen (1979)

Andersen and Nielsen (1981)
Type of Data

$\mathrm{S}$ rel. to $\mathrm{Cu}, 300-\mathrm{MeV}$ protons

Partial proton range (rel. to $\mathrm{Cu}$ ), $340-200 \mathrm{MeV}$

S, $4.45 \mathrm{MeV}$

$\mathrm{S}$ rel. to $\mathrm{Al}, 19.8-\mathrm{MeV}$ protons

$R, 750-\mathrm{MeV}$ protons

S, 4 to $40-\mathrm{MeV}$ alpha particles

$\mathrm{S}$ rel. to $\mathrm{Al}, 28.7-\mathrm{MeV}$ protons

$R$, 3- to 30-MeV protons

$R, 3-$ to $30-\mathrm{MeV}$ protons

$S$, 4.5- to 12-MeV protons

$\mathrm{S}, 5-$ to $12-\mathrm{MeV}$ protons

S, 5- to 12-MeV protons

$R, 12-$ to $40-\mathrm{MeV}$ protons

$S, 0.4-$ to 3.4-MeV protons

S, 1- to 9-MeV alpha particles

$\mathrm{S}, 5-$ to $18-\mathrm{MeV}$ protons

$R, 600-M e V$ protons

S, 0.8- to 7.2-MeV protons

S, 60-MeV pions

S, 6.5-MeV protons

S, 40-keV to 1-MeV protons, $100-\mathrm{keV}$ to $1.2-\mathrm{MeV}$ alpha particles

S, 0.8- to 7.2-MeV protons 


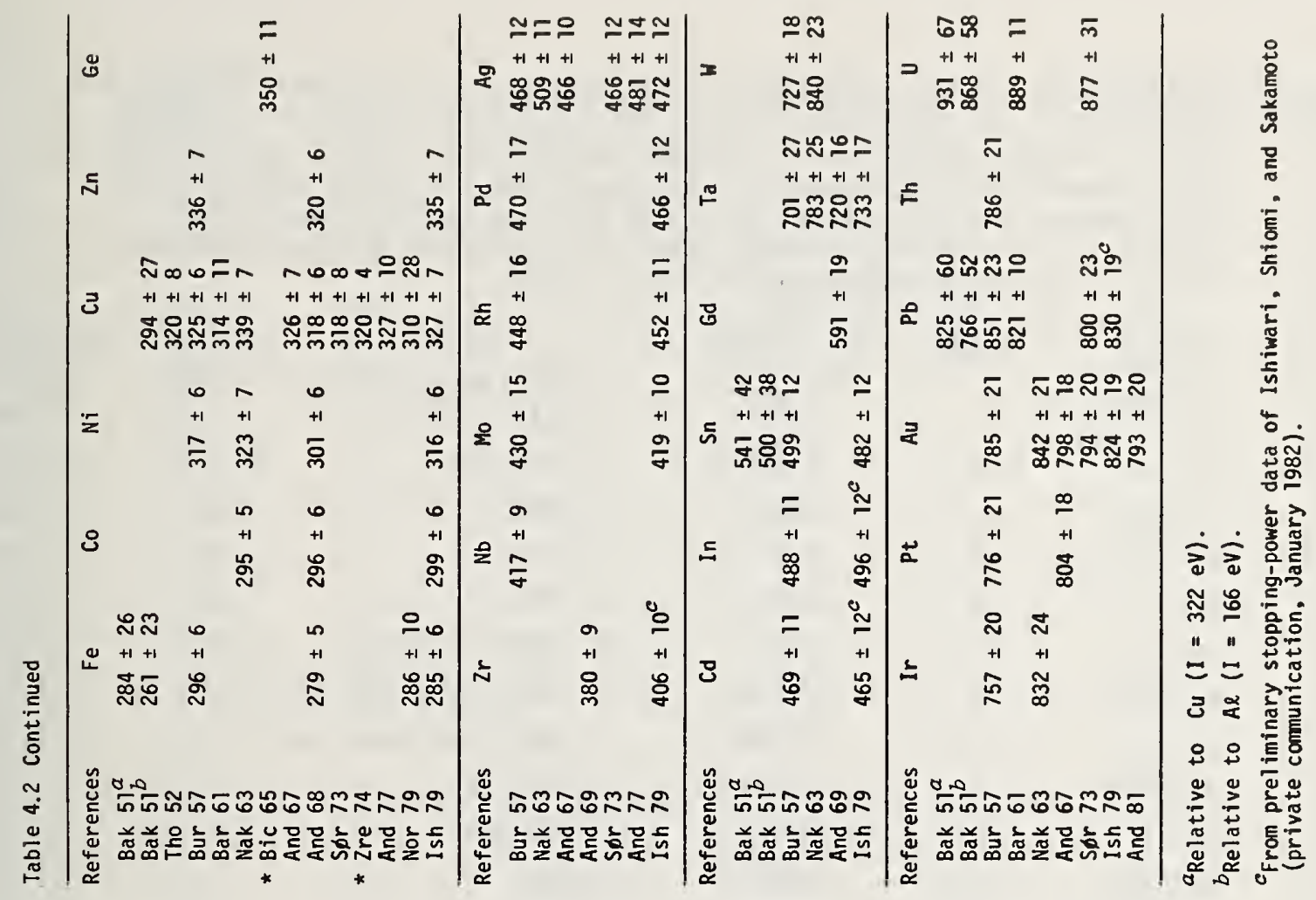

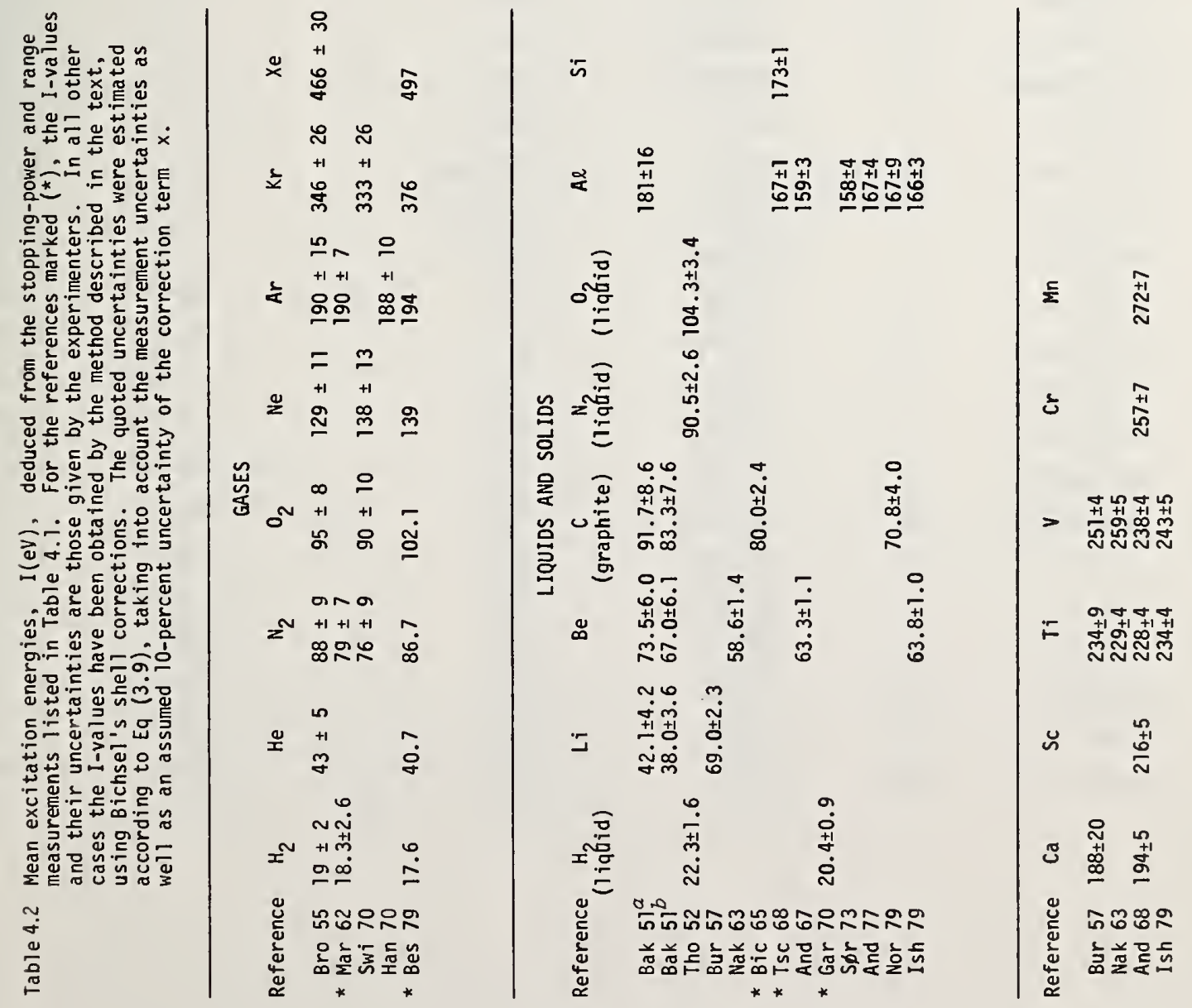


Table 4.3 Atomic number, weight, and mean excitation energies for elemental substances. Unless noted otherwise, the I-values are for substances in the condensed phase. The uncertainties $\Delta I$ attempt to take into account the uncertainties of the underlying measurements, the errors inherent in the analysis of the measurements, and the dispersion of the I-values derived from various sources. I-values in parentheses have been estimated by interpolation of $\mathrm{I} / \mathrm{Z}$ vs. $Z$, or by extrapolation for $Z>92$.

\begin{tabular}{|c|c|c|c|c|c|c|}
\hline$\underline{z}$ & Element & Symbol & $\mathrm{A}^{a} \mathrm{~g} / \mathrm{mol}$ & $\underline{I, e V}$ & $\Delta \mathrm{I}, \mathrm{eV}$ & $\underline{I / Z, \mathrm{eV}}$ \\
\hline 1 & hydrogen & $H$ & 1.0079 & $\begin{array}{l}19.2 \text { molecular gas } \\
21.8 \text { liquid }\end{array}$ & $\begin{array}{l}0.4 \\
1.6\end{array}$ & $\begin{array}{l}19.2 \\
21.8\end{array}$ \\
\hline 2 & helium & He & 4.00260 & 41.8 gas & 0.8 & 20.9 \\
\hline 3 & lithium & Li & 6.941 & 40.0 & 5 & 13.3 \\
\hline 4 & beryllium & $\mathrm{Be}$ & 9.01218 & 63.7 & 3 & 15.9 \\
\hline 5 & boron & B & 10.81 & 76.0 & 8 & 15.2 \\
\hline 6 & carbon & C & 12.011 & 78.0 graphite & 7 & 13.0 \\
\hline 7 & nitrogen & N & 14.0067 & 82.0 molecular gas & 2 & 11.7 \\
\hline 8 & oxygen & 0 & 15.9994 & 95.0 molecular gas & 2 & 11.9 \\
\hline 9 & fluorine & $F$ & 18.998403 & (115) gas & & 11.5. \\
\hline 10 & neon & $\mathrm{Ne}$ & 20.179 & 137 gas & 4 & 13.7 \\
\hline 11 & sodium & $\mathrm{Na}$ & 22.98977 & $(149)$ & & 13.6 \\
\hline 12 & magnesium & $\mathrm{Mg}$ & 24.305 & $(156)$ & & 13.0 \\
\hline 13 & a luminum & Al & 26.98154 & 166 & 2 & 12.8 \\
\hline 14 & silicon & Si & 28.0855 & 173 & 3 & 12.4 \\
\hline 15 & phosphorus & $P$ & 30.97376 & (173) & & 11.5 \\
\hline 16 & sulfur & $s$ & 32.06 & $(180)$ & & 11.3 \\
\hline 17 & chlorine & $\mathrm{Cl}$ & 35.453 & (174) gas & & 10.2 \\
\hline 18 & argon & Ar & 39.948 & 188 gas & 10 & 10.4 \\
\hline 19 & potassium & K & 39.0983 & $(190)$ & & 10.0 \\
\hline 20 & calcium & $\mathrm{Ca}$ & 40.08 & 191 & 8 & 9.6 \\
\hline 21 & scandium & Sc & 44.9559 & 216 & 8 & 10.3 \\
\hline 22 & titanium & $\mathrm{Ti}$ & 47.88 & 233 & 5 & 10.6 \\
\hline 23 & vanadium & v & 50.9415 & 245 & 7 & 10.7 \\
\hline 24 & chromium & $\mathrm{Cr}$ & 51.996 & 257 & 10 & 10.7 \\
\hline 25 & manganese & $M n$ & 54.9380 & 272 & 10 & 10.9 \\
\hline 26 & iron & $\mathrm{Fe}$ & 55.847 & 286 & 9 & 11.0 \\
\hline 27 & cobalt & Co & 58.9332 & 297 & 9 & 11.0 \\
\hline 28 & nickel & $\mathrm{Ni}$ & 58.69 & 311 & 10 & 11.1 \\
\hline 29 & copper & $\mathrm{Cu}$ & 63.546 & 322 & 10 & 11.1 \\
\hline 30 & zinc & $\mathrm{Zn}$ & 65.38 & 330 & 10 & 11.0 \\
\hline 31 & gallium & Ga & 69.72 & (334) & & 10.8 \\
\hline
\end{tabular}


Table 4.3 Continued

\begin{tabular}{|c|c|c|c|c|c|c|}
\hline$\underline{z}$ & El ement & Symbol & $\mathrm{A}{ }^{a} \mathrm{~g} / \mathrm{mol}$ & I, eV & $\Delta I, \mathrm{eV}$ & $I / Z, \mathrm{eV}$ \\
\hline 32 & germanium & Ge & 72.59 & 350 & 11 & 10.4 \\
\hline 33 & arsenic & As & 74.9216 & $(347)$ & & 10.5 \\
\hline 34 & selenium & $\mathrm{Se}$ & 78.96 & $(348)$ & & 10.2 \\
\hline 35 & bromine & $\mathrm{Br}$ & 79.904 & $\begin{array}{l}\text { (343) gas } \\
(357) \text { condensed }\end{array}$ & & $\begin{array}{r}9.8 \\
10.2\end{array}$ \\
\hline 36 & krypton & $\mathrm{kr}$ & 83.80 & 352 gas & 25 & 9.8 \\
\hline 37 & rubidium & $\mathrm{Rb}$ & 85.4678 & $(363)$ & & 9.8 \\
\hline 38 & strontium & $\mathrm{Sr}$ & 87.62 & $(366)$ & & 9.6 \\
\hline 39 & yttrium & $Y$ & 88.9059 & $(379)$ & 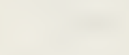 & 9.7 \\
\hline 40 & zirconium & $Z r$ & 91.22 & 393 & 15 & 9.8 \\
\hline 41 & niobium & $\mathrm{Nb}$ & 92.9064 & 417 & 15 & 10.2 \\
\hline 42 & molybdenum & Mo & 95.94 & 424 & 15 & 10.1 \\
\hline 43 & technetium & ${ }^{98}{ }_{\mathrm{TC}}$ & 97.907 & $(428)$ & & 10.0 \\
\hline 44 & ruthenium & $\mathrm{Ru}$ & 101.07 & $(441)$ & & 10.0 \\
\hline 45 & rhodium & $\mathrm{Rh}$ & 102.9055 & 449 & 20 & 10.0 \\
\hline 46 & palladium & $P d$ & 106.42 & 470 & 20 & 10.2 \\
\hline 47 & silver & $\mathrm{Ag}$ & 107.868 & 470 & 10 & 10.0 \\
\hline 48 & cadmium & $\mathrm{Cd}$ & 112.41 & 469 & 20 & 9.8 \\
\hline 49 & indium & In & 114.82 & 488 & 20 & 10.0 \\
\hline 50 & tin & Sn & 118.69 & 488 & 15 & 9.8 \\
\hline 51 & antimony & $\mathrm{Sb}$ & 121.75 & $(487)$ & & 9.5 \\
\hline 52 & tellurium & $\mathrm{Te}$ & 127.60 & $(485)$ & & 9.3 \\
\hline 53 & iodine & I & 126.9045 & $\begin{array}{l}\text { (474) gas } \\
(491) \text { condensed }\end{array}$ & & $\begin{array}{l}8.9 \\
9.3\end{array}$ \\
\hline 54 & xenon & $\mathrm{Xe}$ & 131.29 & 482 gas & 30 & 8.9 \\
\hline 55 & cesium & Cs & 132.9054 & $(488)$ & & 8.9 \\
\hline 56 & barium & $\mathrm{Ba}$ & 137.33 & (491) & & 8.8 \\
\hline 57 & lanthanum & La & 138.9055 & $(501)$ & & 8.8 \\
\hline 58 & cerium & $\mathrm{Ce}$ & 140.12 & $(523)$ & & 8.8 \\
\hline 59 & praseodymium & $\mathrm{Pr}$ & 140.9077 & $(535)$ & & 9.1 \\
\hline 60 & neodymium & $\mathrm{Nd}$ & 144.24 & $(546)$ & & 9.1 \\
\hline 61 & promethium & ${ }^{145} \mathrm{Pm}$ & 144.913 & $(560)$ & & 9.2 \\
\hline 62 & samarium & Sm & 150.36 & $(574)$ & & 9.3 \\
\hline 63 & europium & Eu & 151.96 & $(580)$ & & 9.2 \\
\hline 64 & gadol inium & Gd & 157.25 & 591 & 20 & 9.2 \\
\hline 65 & terbium & Tb & 158.9254 & $(614)$ & & 9.4 \\
\hline 66 & dysprosium & Dy & 162.50 & $(628)$ & & 9.5 \\
\hline 67 & holmium & Ho & 164.9304 & $(650)$ & & 9.7 \\
\hline
\end{tabular}


Table 4.3 Continued

\begin{tabular}{|c|c|c|c|c|c|c|}
\hline$\underline{Z}$ & Element & Symbol & $\underline{A},{ }^{a} \mathrm{~g} / \mathrm{mol}$ & $\underline{\mathrm{I}, \mathrm{eV}}$ & $\Delta \mathrm{I}, \mathrm{eV}$ & $\mathrm{I} / \mathrm{Z}, \mathrm{eV}$ \\
\hline 68 & erbium & Er & 167.26 & $(658)$ & & 9.7 \\
\hline 69 & thul ium & $\mathrm{Tm}$ & 168.9342 & $(674)$ & & 9.8 \\
\hline 70 & ytterbium & $Y_{b}$ & 173.04 & $(684)$ & & 9.8 \\
\hline 71 & lutetium & Lu & 174.967 & (694) & & 9.8 \\
\hline 72 & hafnium & Hf & 178.49 & $(705)$ & & 9.8 \\
\hline 73 & tantalum & $\mathrm{Ta}$ & 180.9479 & 718 & 30 & 9.8 \\
\hline 74 & tungsten & W & 183.85 & 727 & 30 & 9.8 \\
\hline 75 & rhenium & $\operatorname{Re}$ & 186.207 & $(736)$ & & 9.8 \\
\hline 76 & osmium & Os & 190.2 & $(746)$ & & 9.8 \\
\hline 77 & iridium & Ir & 192.22 & 757 & 30 & 9.8 \\
\hline 78 & platinum & Pt & 195.08 & 790 & 30 & 10.1 \\
\hline 79 & gold & $\mathrm{Au}$ & 196.9665 & 790 & 30 & 10.0 \\
\hline 80 & mercury & $\mathrm{Hg}$ & 200.59 & $(800)$ & & 10.0 \\
\hline 81 & thallium & $\mathrm{Tl}$ & 204.383 & $(810)$ & & 10.0 \\
\hline 82 & lead & $\mathrm{Pb}$ & 207.2 & 823 & 30 & 10.0 \\
\hline 83 & bismuth & $\mathrm{Bi}$ & 208.9804 & $(823)$ & & 9.9 \\
\hline 84 & polonium & ${ }^{209} P_{0}$ & 208.982 & $(830)$ & & 9.9 \\
\hline 85 & astatine & $210_{\text {At }}$ & 209.987 & $(825)$ & & 9.7 \\
\hline 86 & radon & ${ }^{222} \mathrm{Rn}$ & 222.018 & (794) gas & & 9.2 \\
\hline 87 & francium & ${ }^{223} \mathrm{Fr}$ & 223.020 & $(827)$ & & 9.5 \\
\hline 88 & radium & $\mathrm{Ra}$ & 226.0254 & $(826)$ & & 9.4 \\
\hline 89 & actinium & $A C$ & 227.0278 & $(841)$ & & 9.4 \\
\hline 90 & thorium & Th & 232.0381 & $(847)$ & & 9.4 \\
\hline 91 & protactinium & $\mathrm{Pa}$ & 231.0359 & $(878)$ & & 9.6 \\
\hline 92 & uranium & U & 238.0289 & 890 & 30 & 9.7 \\
\hline 93 & neptunium & $N p$ & 237.0482 & $(902)$ & & 9.7 \\
\hline 94 & plutonium & ${ }^{239} \mathrm{Pu}$ & 239.052 & $(921)$ & & 9.8 \\
\hline 95 & americium & $243 \mathrm{Am}$ & 243.061 & $(934)$ & & 9.8 \\
\hline 96 & curium & ${ }^{247} \mathrm{Cm}$ & 247.070 & (939) & & 9.8 \\
\hline 97 & berkelium & ${ }^{247} \mathrm{BK}$ & 247.070 & $(952)$ & & 9.8 \\
\hline 98 & californium & ${ }^{251} \mathrm{Cf}$ & 251.080 & $(966)$ & & 9.9 \\
\hline 99 & einsteinium & 252 Es & 252.083 & $(980)$ & & 9.9 \\
\hline 100 & fermium & ${ }^{257} \mathrm{Fm}$ & 257.095 & $(994)$ & & 9.9 \\
\hline
\end{tabular}




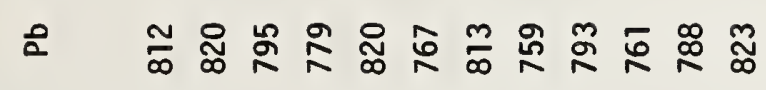

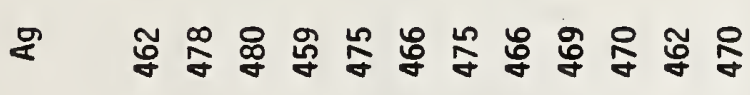

उ

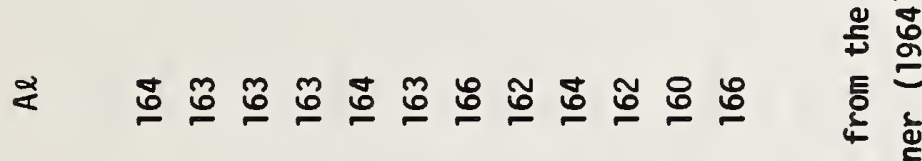

要

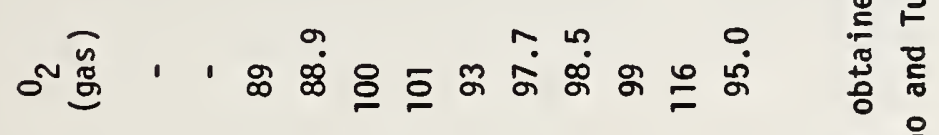

产

高

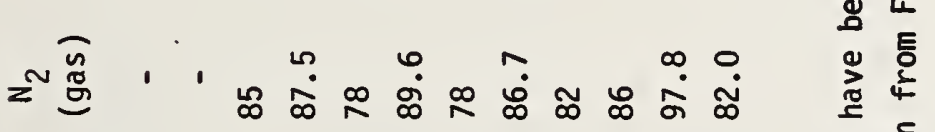

离

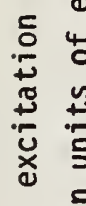

窟

施

高 品

尊

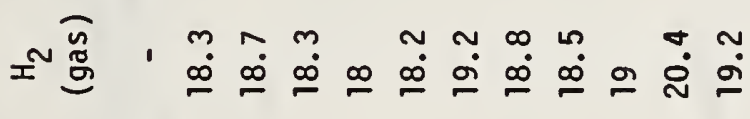

㺃

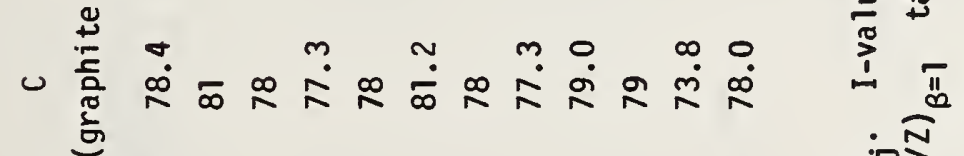

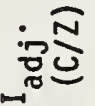

字

龸

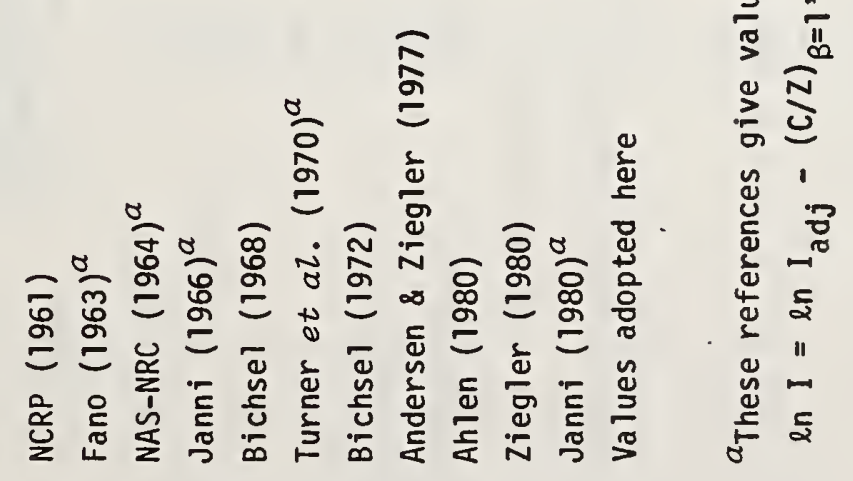


:

造经施。

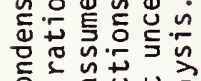

0.

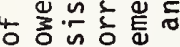

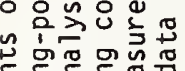

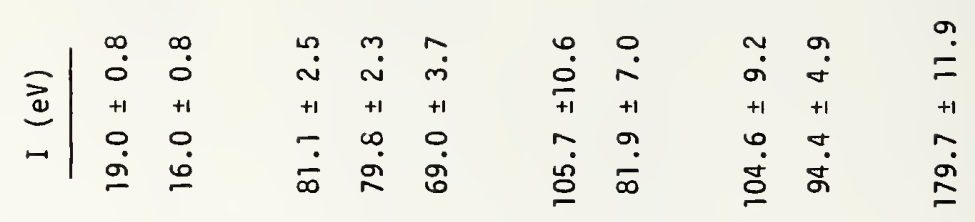

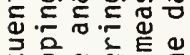

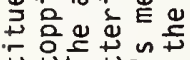

出出若吃

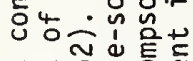

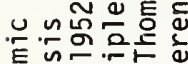

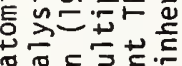

๙

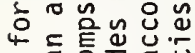

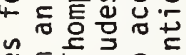

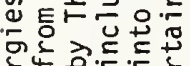

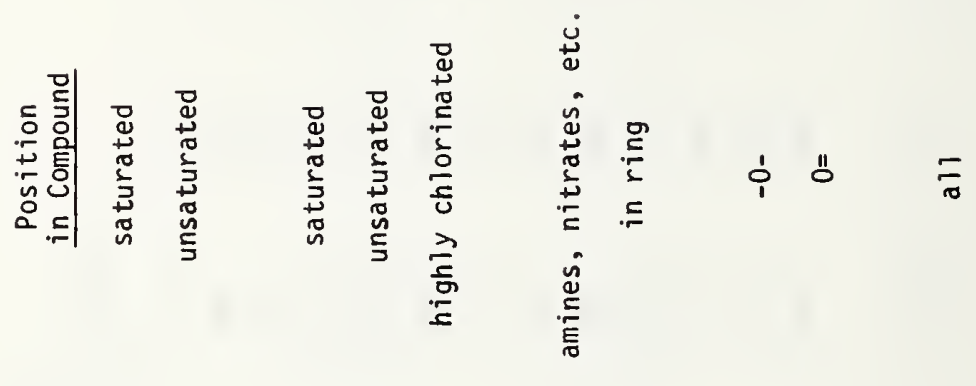

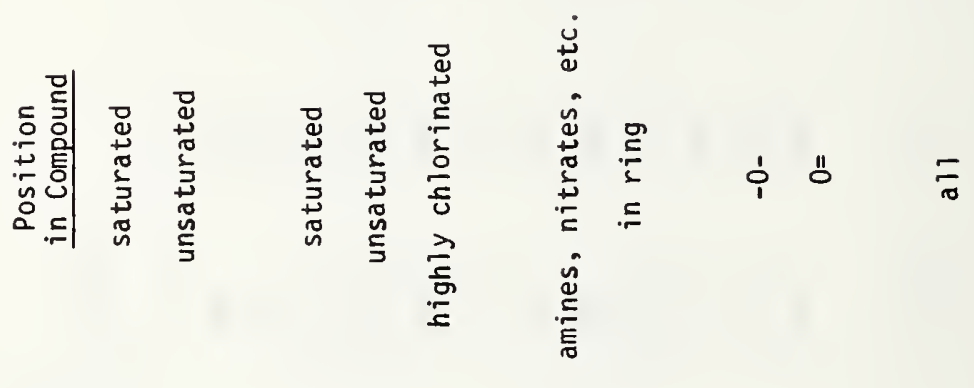

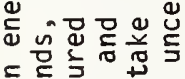

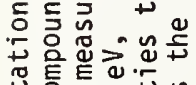

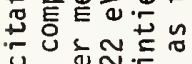

ชิ

ร

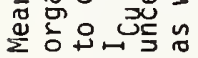

กั

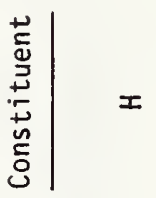

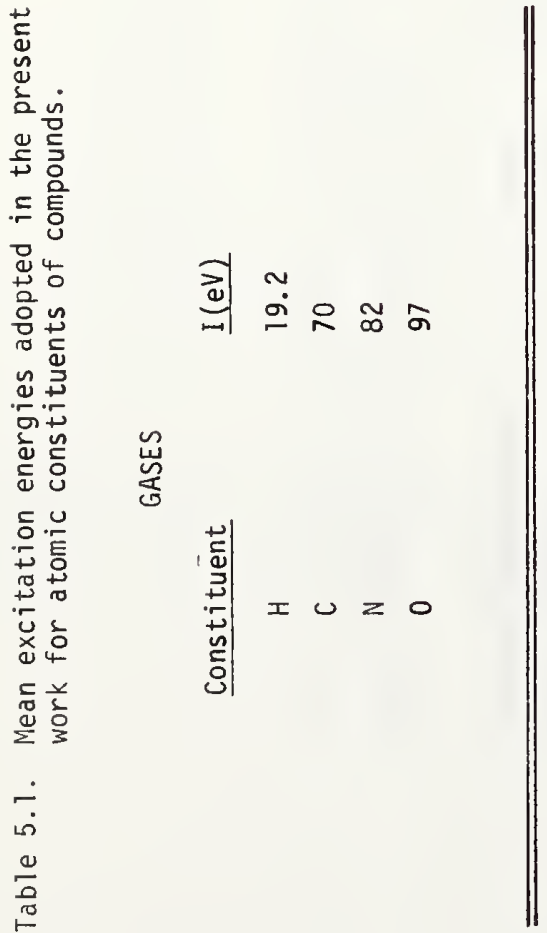

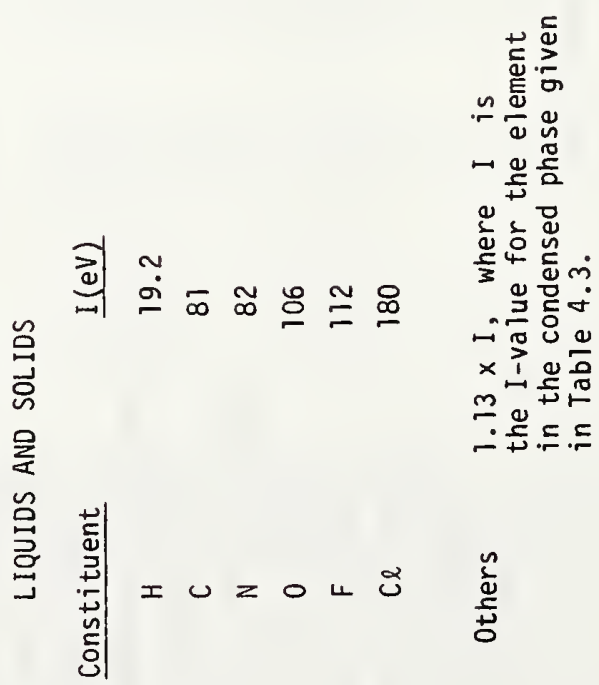




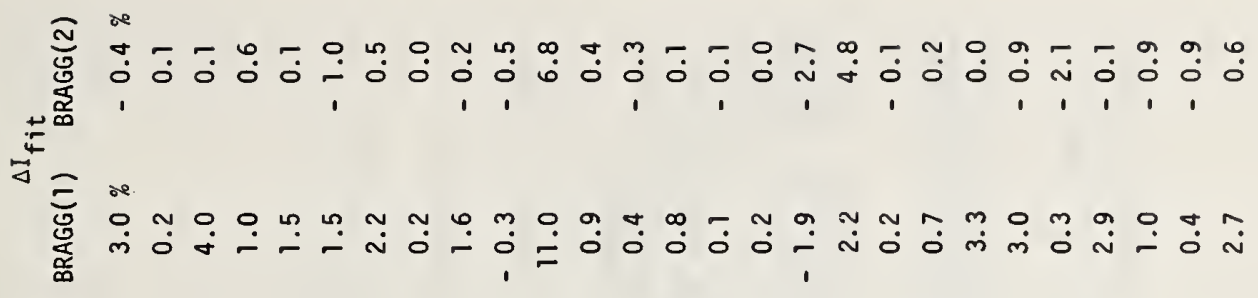

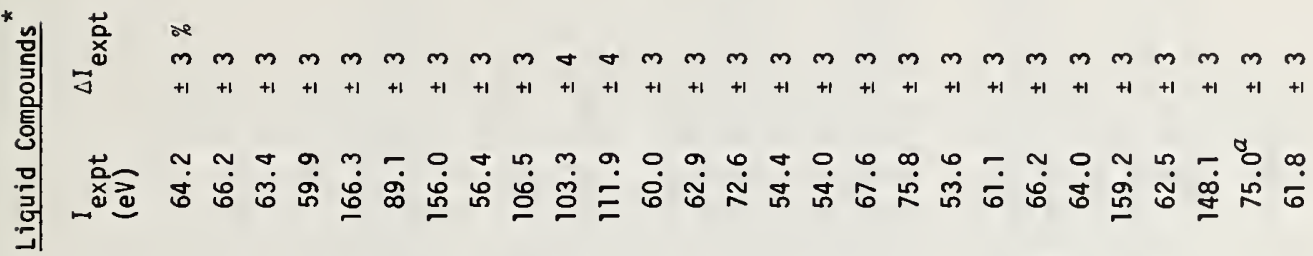

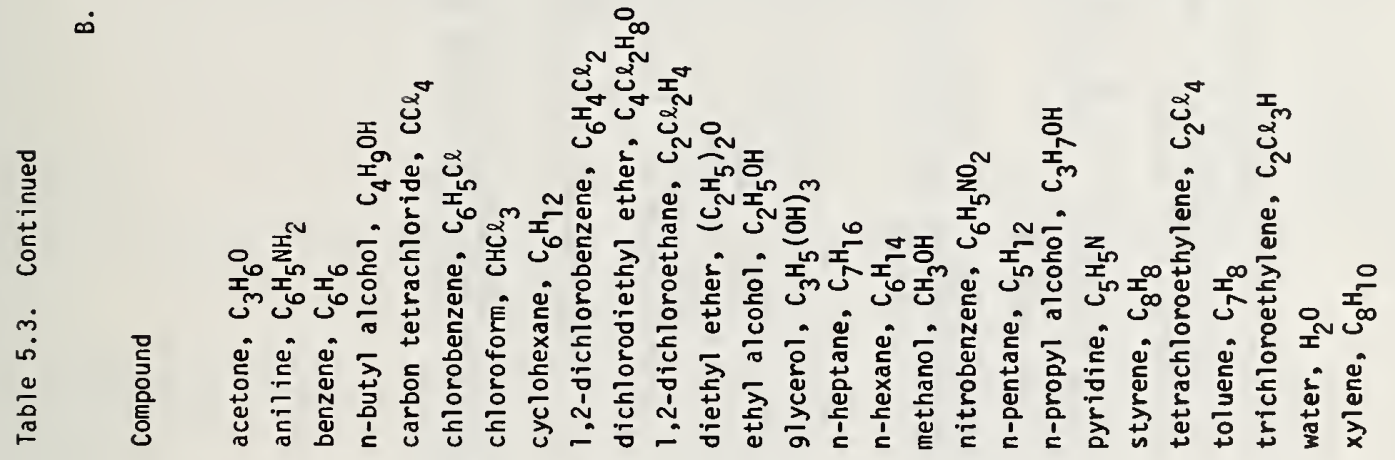

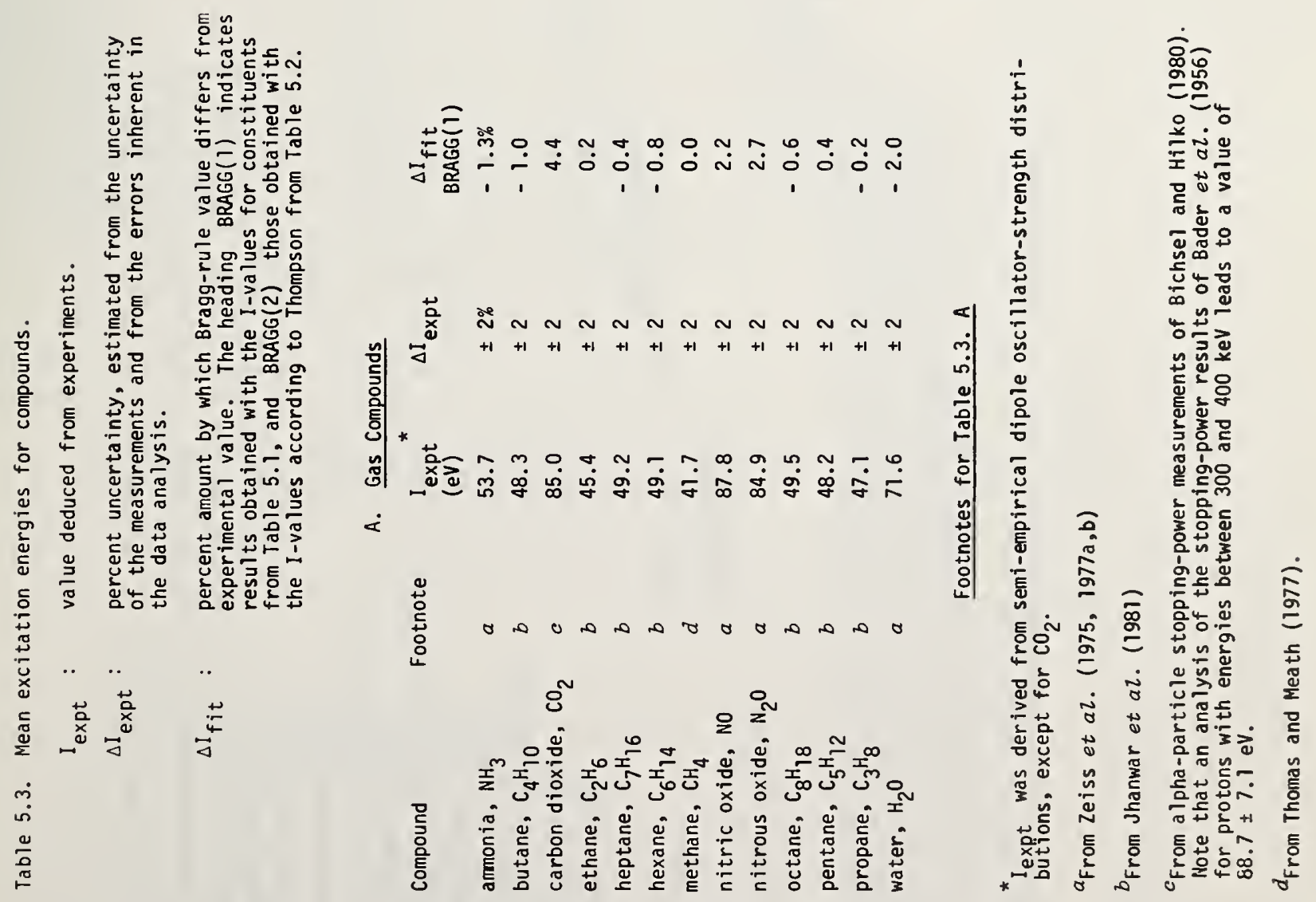



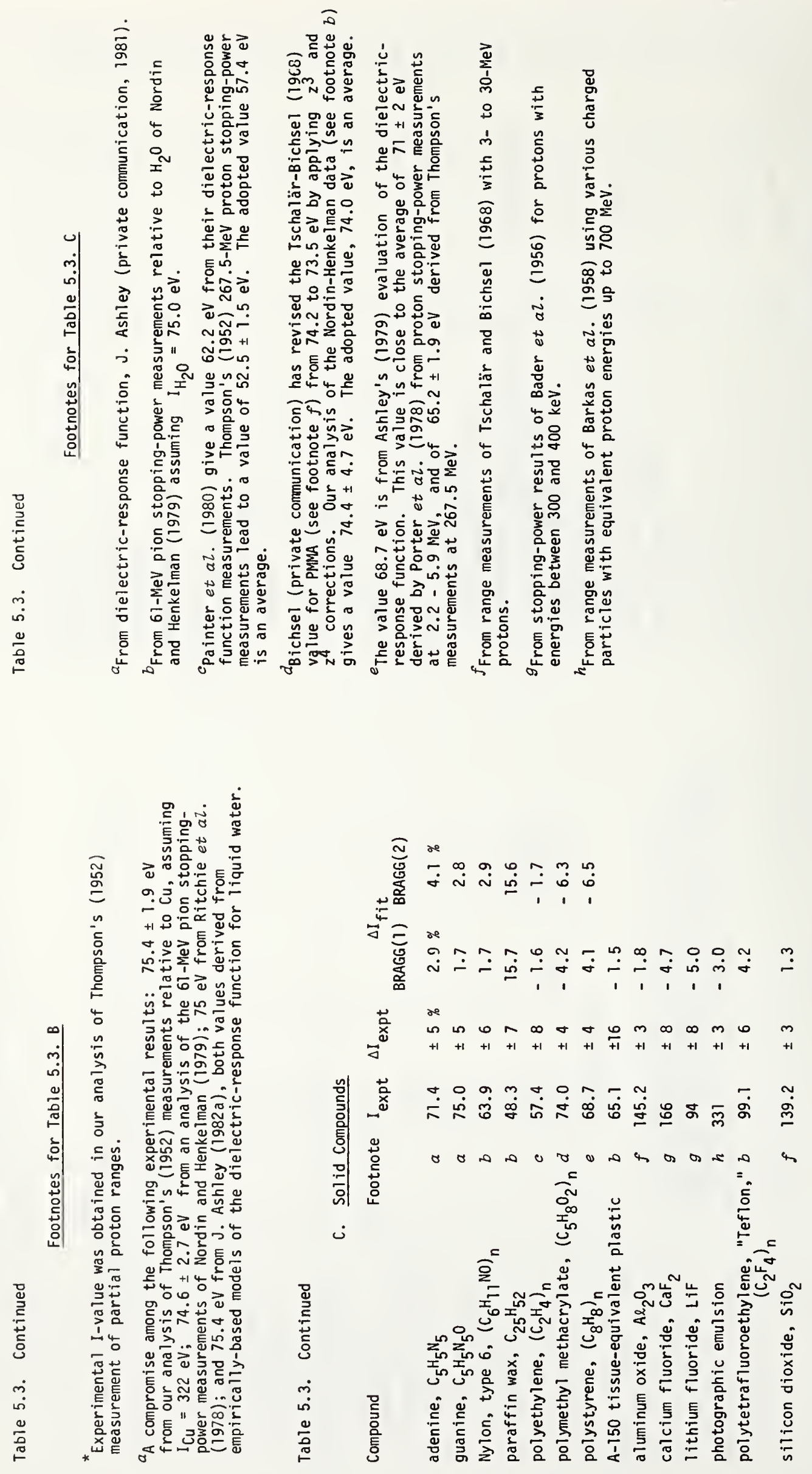
Table 5.4. Mean excitation energies, water content and other properties of selected human tissues. For the tissues labeled "ICRP" the compositions, densities and water content were taken from Tables 105 and 108 of ICRP (1975). For the tissues labeled "ICRU" the composition was taken from ICRU (1964); because this reference does not give other properties, the densities and water content were assumed to be the same as for corresponding ICRP soft or bone tissue.

\begin{tabular}{|c|c|c|c|c|}
\hline Substance & $(e \stackrel{I}{e V})$ & $\begin{array}{l}\text { Density } \\
\left(\mathrm{g} / \mathrm{cm}^{3}\right)\end{array}$ & $\langle Z / A\rangle$ & $\begin{array}{l}\text { Water Content } \\
\text { ( } \% \text { by weight) }\end{array}$ \\
\hline $\mathrm{H}_{2} \mathrm{O}$ & 75.0 & 1.00 & 0.55509 & \\
\hline ICRP adipose tissue & 63.2 & 0.92 & 0.55847 & 15.3 \\
\hline ICRP skin & 72.6 & 1.10 & 0.54933 & 61.5 \\
\hline ICRP brain & 73.3 & 1.03 & 0.55423 & 78.6 \\
\hline ICRP testes & 75.0 & 1.04 & 0.55108 & 80.0 \\
\hline ICRP blood & 75.1 & 1.06 & 0.54995 & 80.0 \\
\hline ICRP lung & 75.2 & 1.05 & 0.54965 & 78.0 \\
\hline ICRP skeletal muscle & 75.3 & 1.04 & 0.54938 & 78.6 \\
\hline ICRP cortical bone & 106.7 & 1.85 & 0.52130 & 15.0 \\
\hline ICRU striated muscle & 74.6 & 1.04 & 0.55005 & 78.6 \\
\hline ICRU compact bone & 92.1 & 1.85 & 0.53010 & 15.0 \\
\hline
\end{tabular}


Table 5.5. Recommended mean excitation energies I for compounds and mixtures of the indicated composition.

Material

A-150 TISSUE-EQUIVAlENT PLASTIC ${ }^{a}$

ACETYLENE, $\mathrm{C}_{2} \mathrm{H}_{2}$

AOIPOSE TISSUE (ICRP) $b$

AIR, DRY (NEAR SEA LEVEL) $c$

ALUMINUM OXIDE, $\mathrm{Al}_{2} \mathrm{O}_{3}$

B-100 BONE-EQUIVALENT PLASTIC $d$

BONE, COMPACT (ICRU) ${ }^{e}$

BONE, CORTICAL (ICRP) $b$

C-552 AIR-EQUIVALENT PLASTIC $f$

CALCIUM FLUORIDE, $\mathrm{CaF}_{2}$

CARBON DIOXIOE, $\mathrm{CO}_{2}$

CEllulose Nitrate, ${ }^{u} \mathrm{C}_{6} \mathrm{H}_{7 .} 7^{\mathrm{O}} 9.6^{\mathrm{N}} 2.3$

CERIC SULFATE OOSIMETER SOLUTION 9

CESIUM IODIDE, CSI

ETHYLENE, $\mathrm{C}_{2} \mathrm{H}_{4}$

FERROUS SULFATE DOSIMETER SOLUTION ${ }^{h}$

GLASS, BOROSILICATE ("PYREX", CORNING 7740$)^{i}$.

"XAPTON" POLYIMIOE FILM, $\left(\mathrm{C}_{22} \mathrm{H}_{10} \mathrm{~N}_{2} \mathrm{O}_{5}\right)_{n}$

LITHIUM FLUORIDE, LIF

LITHIUM TETRABORATE, $\mathrm{Li}_{2} \mathrm{~B}_{4} \mathrm{O}_{7}$

METHANE, $\mathrm{CH}_{4}$

MUSCLE, SKELETAL (ICRP) $b$

MUSCLE, STRIATED (ICRU)e

$0.550051 \quad 1.040 E+00$

MUSCLE-EQUIVALENT LIQUID, WITH SUCROSE MUSCLE-EQUIVALENT LIQUID, WITHOUT SUCROSE ${ }^{k}$ NYLON. TYPE 6 AND TYPE $6 / 6,\left(\mathrm{C}_{6} \mathrm{H}_{11}{ }^{\text {ON) }}\right)_{n}$ PARAFFIN WAX, $\mathrm{C}_{25} \mathrm{H}_{52}$,

PHOTOGRAPHIC EMULSION 2

PLASTIC SCINTILLATOR (UINYLTOLUENE BASED) $m$ POLYCARBONATE, " ${ }^{\text {MAKROLON", }}\left(\mathrm{C}_{16} \mathrm{H}_{14} \mathrm{O}_{3}\right)_{\mathrm{n}}$

POLYETHYLENE, $\left(\mathrm{C}_{2} \mathrm{H}_{4}\right)_{n}$

$0.548281 \quad 1.110 E+00$

$0.550136 \quad 1.070 E+00$

$0.547902 \quad 1.140 E+00$

$0.5727489 .300 E-01$

$0.4545323 .815 E+00$

$0.541415 \quad 1.032 E+00$

$0.526968 \quad 1.200 E+00$

$0.5703379 .400 E-01$

POLYETHYLENE TEREPTHALATE, ${ }^{{ }_{\text {MMYLAR }}\left(\mathrm{C}_{10} \mathrm{H}_{8} \mathrm{O}_{4}\right)_{\mathrm{n}} 0.520370 \quad 1.400 E+00}$

POLYMETHYL METHACRYLATE, $P\left(\mathrm{C}_{5} \mathrm{H}_{8} \mathrm{O}_{2}\right)_{n}$

POLYPROPYLEIE, $\left(\mathrm{C}_{3} \mathrm{H}_{5}\right)_{\mathrm{n}}$

POLYSTYRENE, $9\left(\mathrm{C}_{8} \mathrm{H}_{8}\right)_{n}$

POLYTETRAFLUOROETHYLENE, "rTEFLON", $\left(\mathrm{C}_{2} F_{4}\right)_{n}$

POLYUINYL CHLORIDE, $\left(\mathrm{C}_{2} \mathrm{H}_{3} \mathrm{Cl}\right)_{\mathrm{n}}$

PROPANE, $\mathrm{C}_{3} \mathrm{H}_{8}$

SILICON DIOXIDE, $\mathrm{S}_{2}$

SODIUM IOOIDE, NaI

STILBENE, $\mathrm{C}_{14}{ }^{\mathrm{H}} 12$

TISSUE-EQUIVALENT GAS (METHANE BASED) 8

TISSUE-EQUIVALENT GAS (PROPANE BASED) $t$

TOLUENE, $\mathrm{C}_{7} \mathrm{H}_{8}$

WATER, LIQUID, $\mathrm{H}_{2} \mathrm{O}$

WATER VAPOR, $\mathrm{H}_{2} \mathrm{O}$

$0.555087 \quad 1.000 E+00$

$0.555087 \quad 7.562 E-04$

Composition
(constituent $Z$ : fraction by weight)

$\stackrel{\text { I }}{\ddagger}$ (อV) $G r^{\xi}$

$65.1+B$

1: 0.101327

$58.2 \mathrm{~A}$

$63.2 *+B$

11: 0.119477

11: 0.000500 17: 0.001190

$85.7+A$

$6: 0.000124$

8: 0.470749

$145.2+A$

$85.9++B$

$1: 0.06547$

9: 0.0654711

$91.9++B$

1:: 0.063984

$1: 0.047234$ 12: 0.002200
$30: 0.000100$

$106.4+48$

$86.8+* B$

1: 0.024680

$166.0 * B \quad 9=0.486659$

$85.0+A$

6: 0.272916

$87.0 \quad B$

$1: 0.029216$

$76.7++A$

1: 0.107596

553.1 C 53: 0.488451

$50.7 \mathrm{~A}$

$76.4++A$

$1: 0.143711$

$1: 0.108259$

$134.0+4 A \quad$ 5: 0.040061
$14: 0.377220$

79.6 B $1: 0.026362$

$94.0+B \quad 3: 0.267585$

94.6 C $3=0.082085$

$41.7+A \quad 1: 0.251306$

$75.3^{++A}$

$1: 0.100637$

11: 0.000750

17: 0.000790

74.7+tA $12^{\circ} 0.101997$ 11: 0.000800

$74.3+* A \quad 1: 0.098234$

$74.2+4 \quad 1=0.101969$

$63.9+A \quad 1=0.097976$

55.9 B $\quad 1: 0.148605$

$331.0+A \quad 1: 0.014100$

64.7 B $1: 0.085000$

$73.1 \quad B \quad 1: 0.055491$

$57.4+8 \quad 1=0.143711$

78.7 B $1: 0.041959$

$74.0+A \quad 1: 0.080538$

59.2 B $1: 0.122698$

$68.7+A \quad 1: 0.077418$

$99.1+A \quad 6=0.240183$

108.2 B $1: 0.048380$

$47.1+A \quad 1: 0.182855$

$139.2+A \quad 8: 0.532565$

452.0 C $11: 0.153373$

67.7 B 1: 0.067101

$61.2+A \quad 1: 0.101869$

$59.5++A \quad 1=0.102672$

$62.5+A \quad 1: 0.087510$

$75.0+A \quad 1=0.111894$

$71.6+A \quad 1: 0.111894$
6: 0.775501

$6: 0.922582$

6: 0.637240

0.000020 9: 0.000320

$7: 0.755267$

$13: 0.529251$

6: 0.536945

$6: 0.278000$

5: 0.070000

6: 0.144330 15: 0.104970

$6: 0.501610$

20: 0.513341

8: 0.727084

6: 0.271296

$7: 0.000800$

55: 0.511549

6: 0.856289

7: 0.000027

$8=0.539564$

19: 0.539564

6: 0.691133

$9: 0.732415$

5: 0.255680

$6=0.748694$

12: 0.000190 19: 0.000190

6: 0.123000 $12: 0.000200$

6: 0.156214

6: 0.120058

6: 0.636856

680.851395

6: 0.072261

35: 0.349103

6: 0.915000

$6: 0.755751$

$6: 0.856289$

6: 0.625017

$6=0.599848$

$6: 0.877302$

$6: 0.922582$

9: 0.759817

6: 0.384360

6: 0.817145

14: 0.467435

53: 0.846627

6: 0.932899

$6: 0.456179$

$6: 0.568940$

$6: 0.912490$

8: 0.888106

8: 0.888106
$7: 0.035057 \quad 8: 0.052316$

7: $0.007970 \quad 8: 0.232333$ 5: 0.000160 16: 0.000730 20: 0.000020 26: 0.000020

8: $0.231781 \quad 18: 0.012827$

$7: 0.021500$

$8: 0.032035$

7: 0.027000 8: 0.410016 6: $0.002000 \quad 20: 0.147000$ $\begin{array}{rl}7: & 0.041990 \\ 6: 0.003150 & 20: 0.446096\end{array}$ $8: 0.004527 \quad 9: 0.465209$

$7: 0.121276 \quad 8: 0.578212$ 8: $0.874976 \quad 16: 0.014627$

8: 0.878636

$1: 0.023191$

$7: 0.073270$

$8: 0.209255$

8: 0.662235

7: 0.02768 20:0.000030 16:0.002410

7: $0.035000 \quad 8: 0.729003$ 15: $0.002000 \quad 16: 0.005000$

7: 0.035451

$8: 0.710100$

$7: 0.035451 \quad 8: 0.742522$

7: 0.123779 8: 0.141389

77: $0.019320 \quad 8: 0.066101$

$8: 0.188758$

$8=0.333025$

$8: 0.319614$

17: 0.567260
7: $0.035172 \quad 8: 0.406780$

7: $0.035022 \quad 8: 0.293366$ 


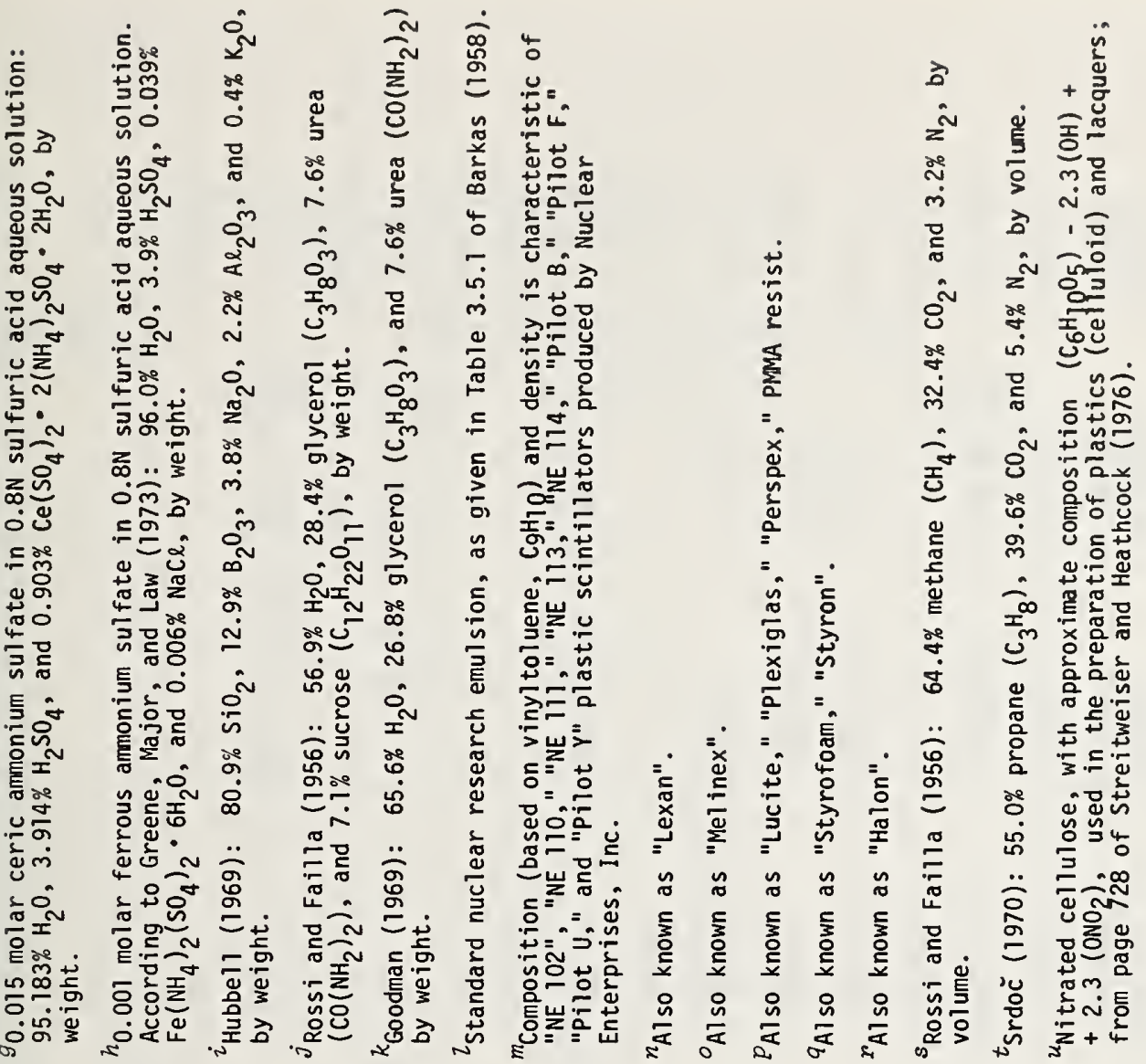

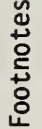

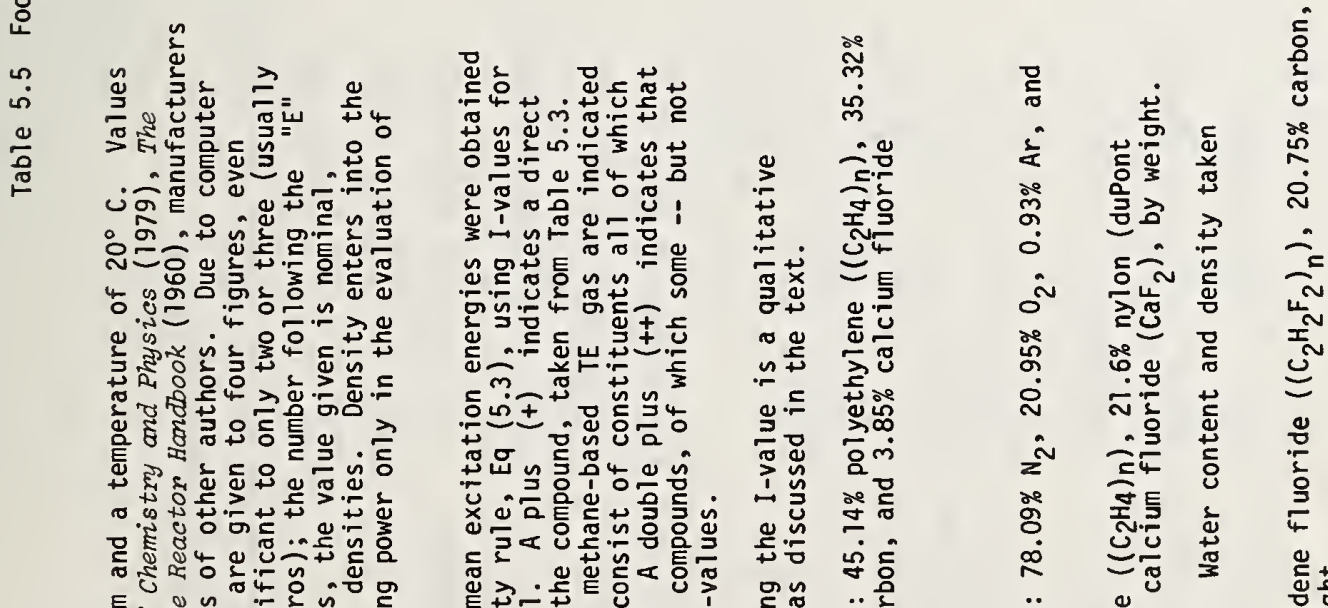

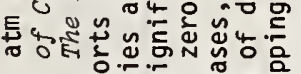

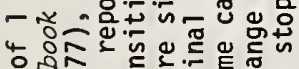

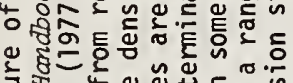

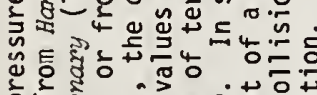

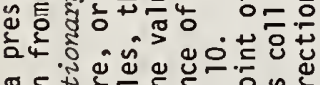

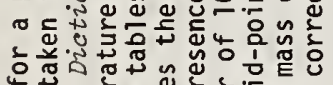

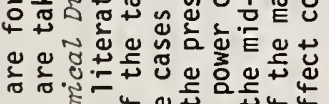

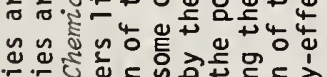

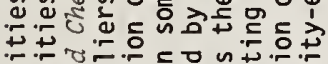

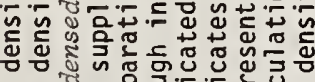

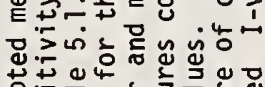

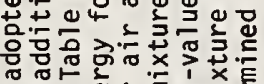

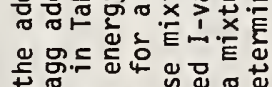

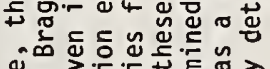

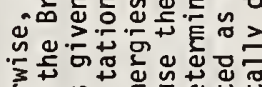

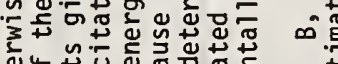

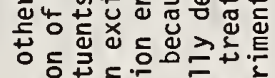

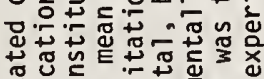

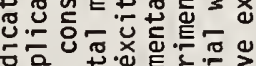

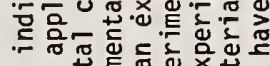

0. 웡

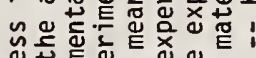

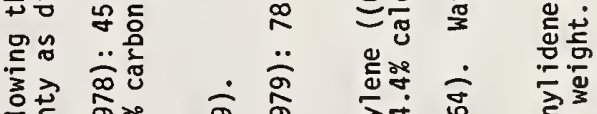

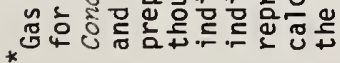

बे

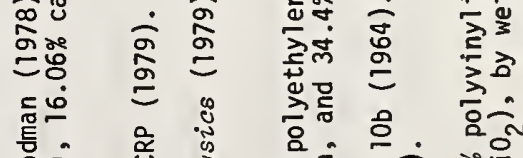

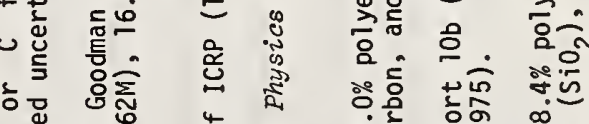

总芯

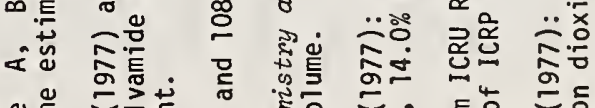

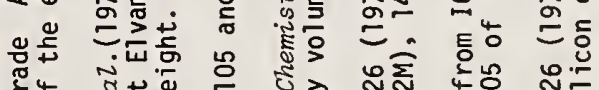

宊

๘

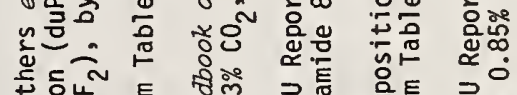

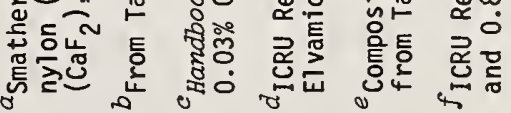




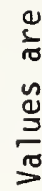

蒙兽

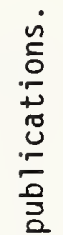

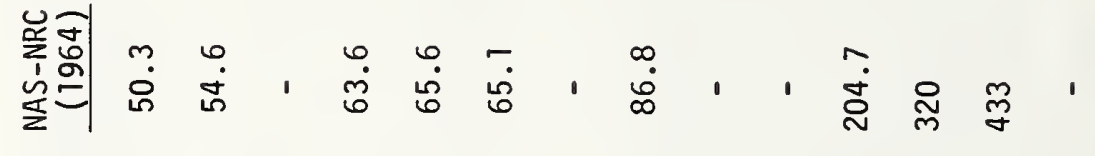

$\frac{\substack{3 \\ \frac{0}{3}}}{\frac{1}{3}}$

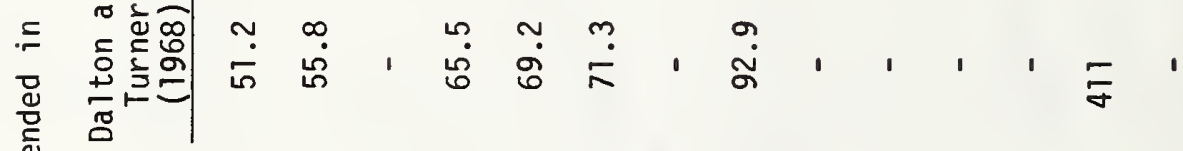

흥

¿ू

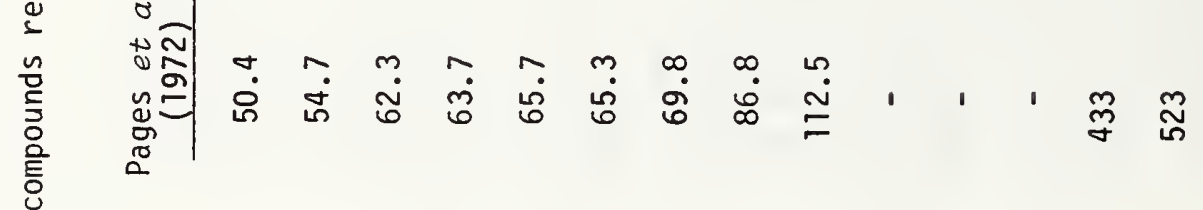

ธั

$\frac{\mathscr{a}}{0}$

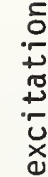

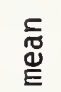

4.

वे

.

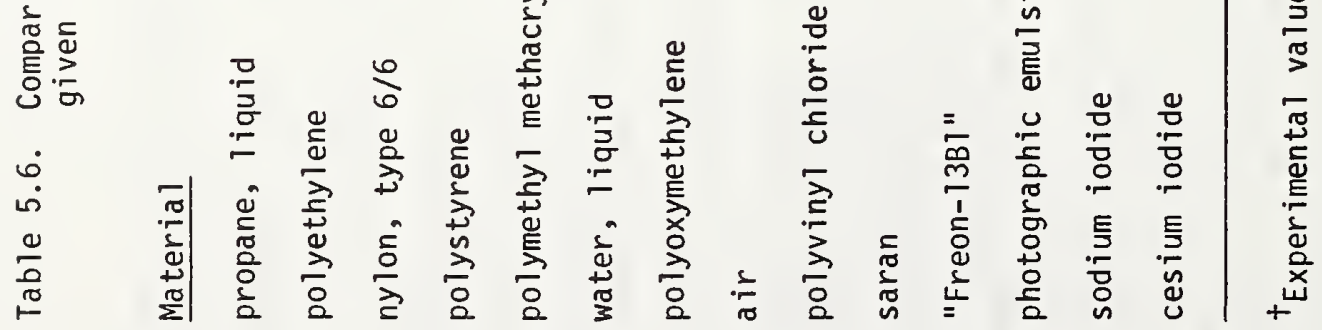

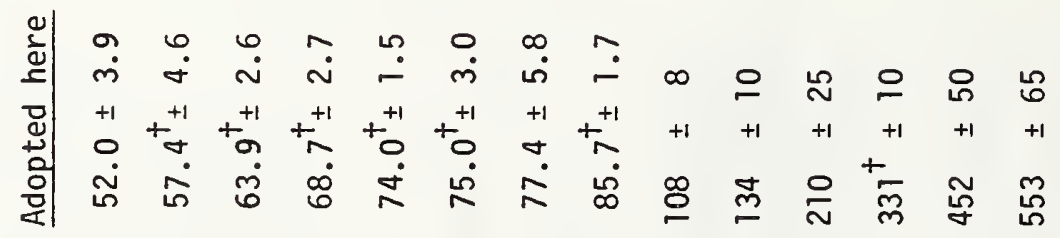

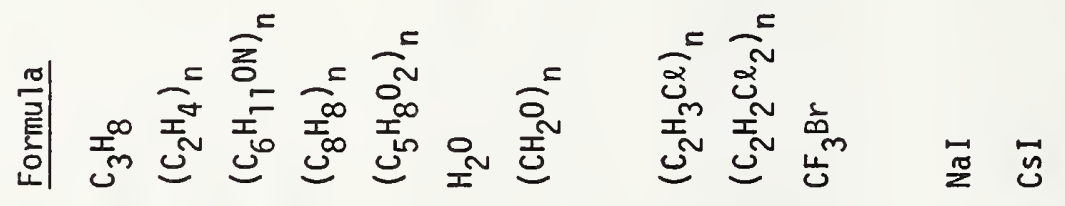

in

$\frac{0}{\circ}$

$\stackrel{\Xi}{\mathscr{N}}$ 
Table 5.7. Dependence of the mean excitation energy on the state of aggregation of the medium.

Substance

Mean Excitation Energy I (ev)

\begin{tabular}{|c|c|c|c|c|c|c|}
\hline $\begin{array}{l}\text { Atomic } \\
\text { gas } \\
\text { (a) }\end{array}$ & $\begin{array}{l}\text { Molecu]ar } \\
\text { gas } \\
\text { (b) }\end{array}$ & $\underset{(c)}{\text { Liquide }}$ & $\begin{array}{l}\text { Sol idd } \\
\text { (d) }\end{array}$ & $(b) /(a)$ & $\begin{array}{l}\text { Ratios } \\
\text { (c)/(b) }\end{array}$ & $(d) /(a)$ \\
\hline 15.0 & 19.2 & 21.8 & & 1.28 & 1.14 & \\
\hline 62.0 & & & 78 & & & 1.26 \\
\hline 76.9 & 82.0 & 90.5 & & 1.07 & 1.10 & \\
\hline 93.5 & 95.0 & 104.3 & & 1.02 & 1.10 & \\
\hline 124 & & & 166 & & & 1.34 \\
\hline 132 & & & 173 & & & 1.32 \\
\hline 182 & & & 233 & & & 1.28 \\
\hline 226 & & & 286 & & & 1.26 \\
\hline 274 & & & 322 & & & 1.18 \\
\hline 292 & & & 350 & & & 1.20 \\
\hline & 71.6 & 75.0 & & & 1.05 & \\
\hline & 47.1 & 52.0 & & & 1.10 & \\
\hline & 48.2 & 53.6 & & & 1.11 & \\
\hline & 49.1 & 54.0 & & & 1.10 & \\
\hline & 49.2 & 54.4 & & & 1.11 & \\
\hline
\end{tabular}

aTheoretica 1: from Brown (1950) for $\mathrm{H}$; from Dehmer et al. (1975) for C, $N$, $0, \mathrm{Al}$, and $\mathrm{Si}$; and from Inokuti et al. (1981) for $\mathrm{Ti}, \mathrm{Fe}, \mathrm{Cu}$, and $\mathrm{Ge}$.

${ }^{b}$ From Tables $3.1,5.3$, and 5.5 .

${ }^{c}$ From Tables 4.2 and 5.3 .

$d_{\text {From Table } 5.5 .}$ 


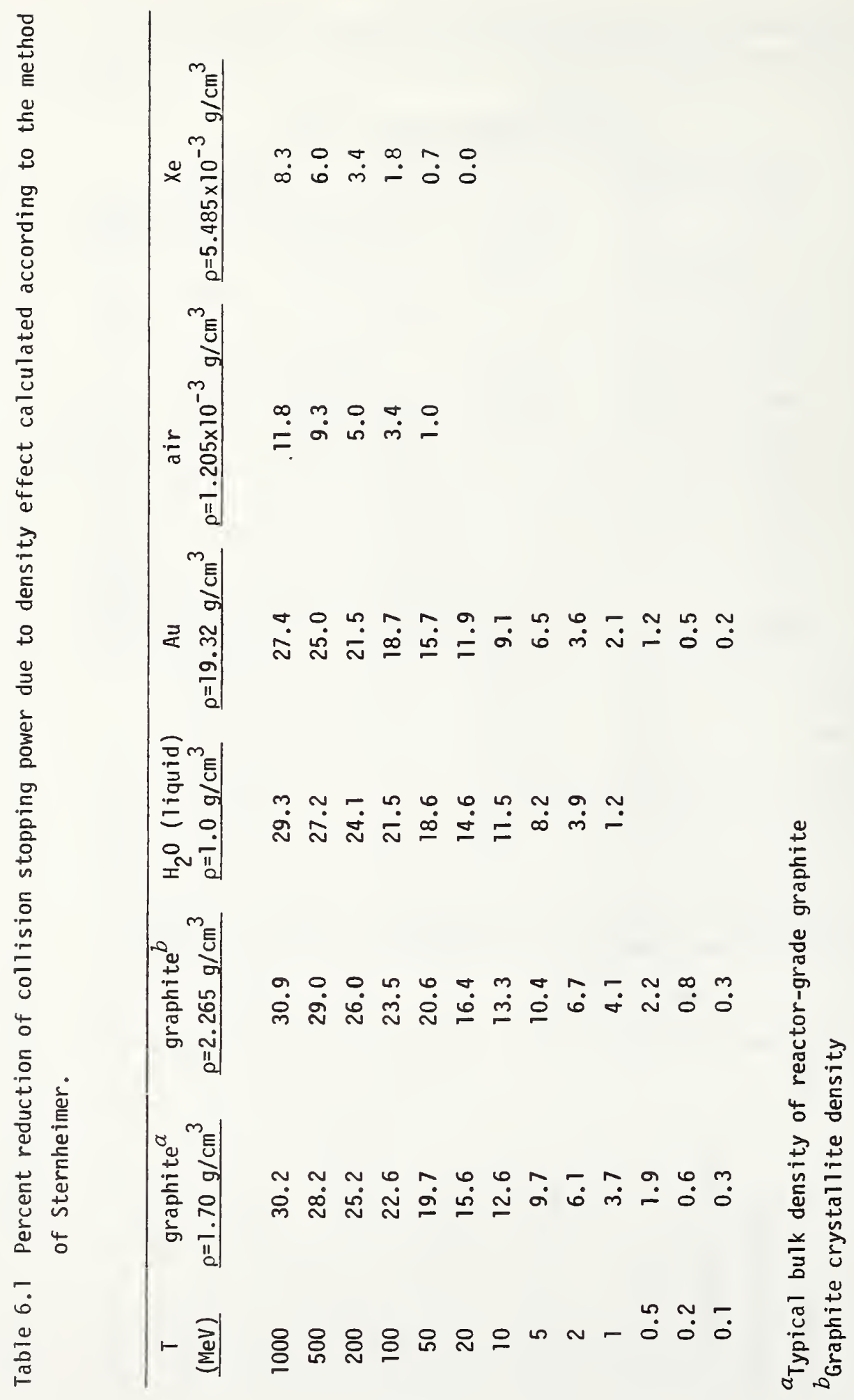




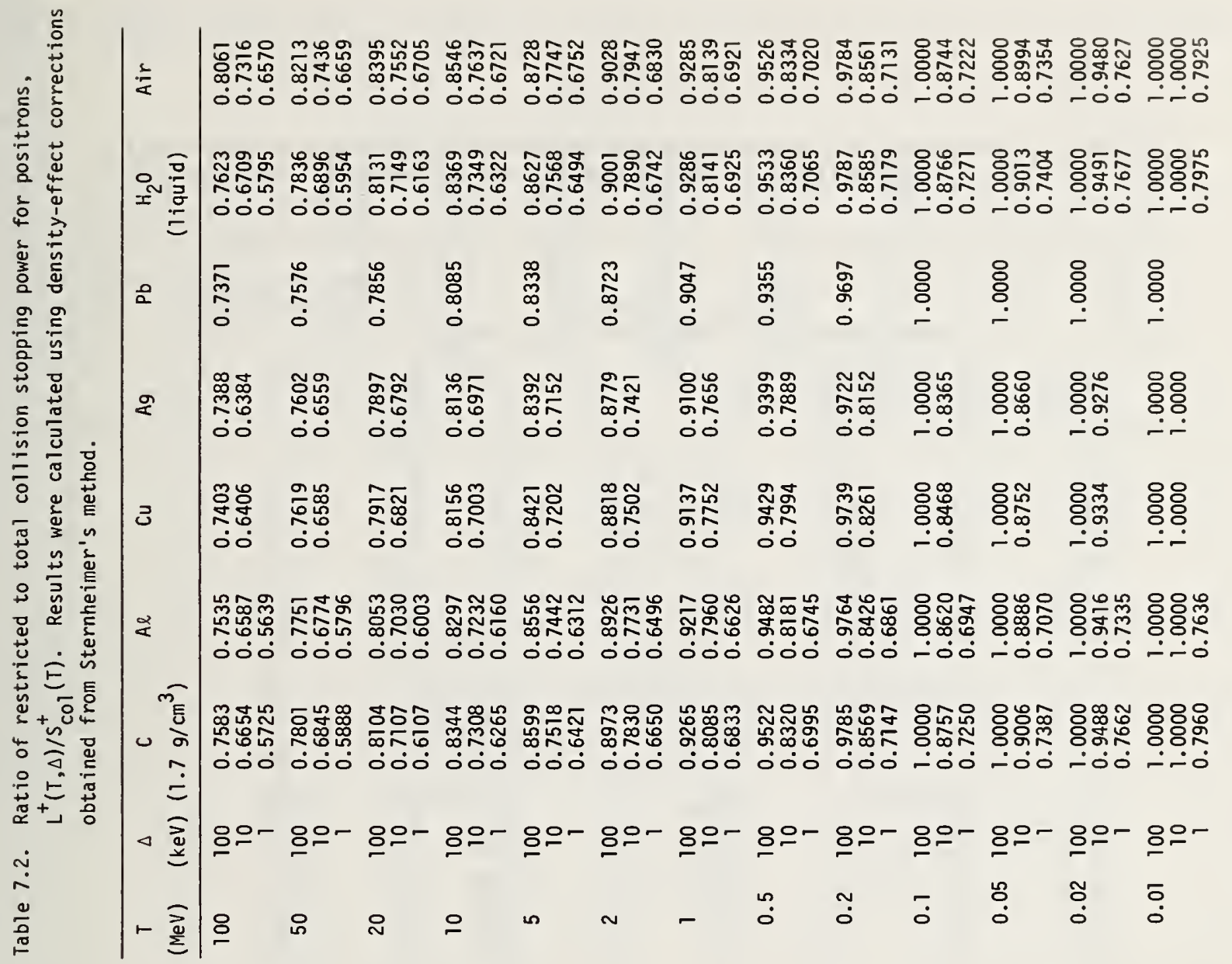

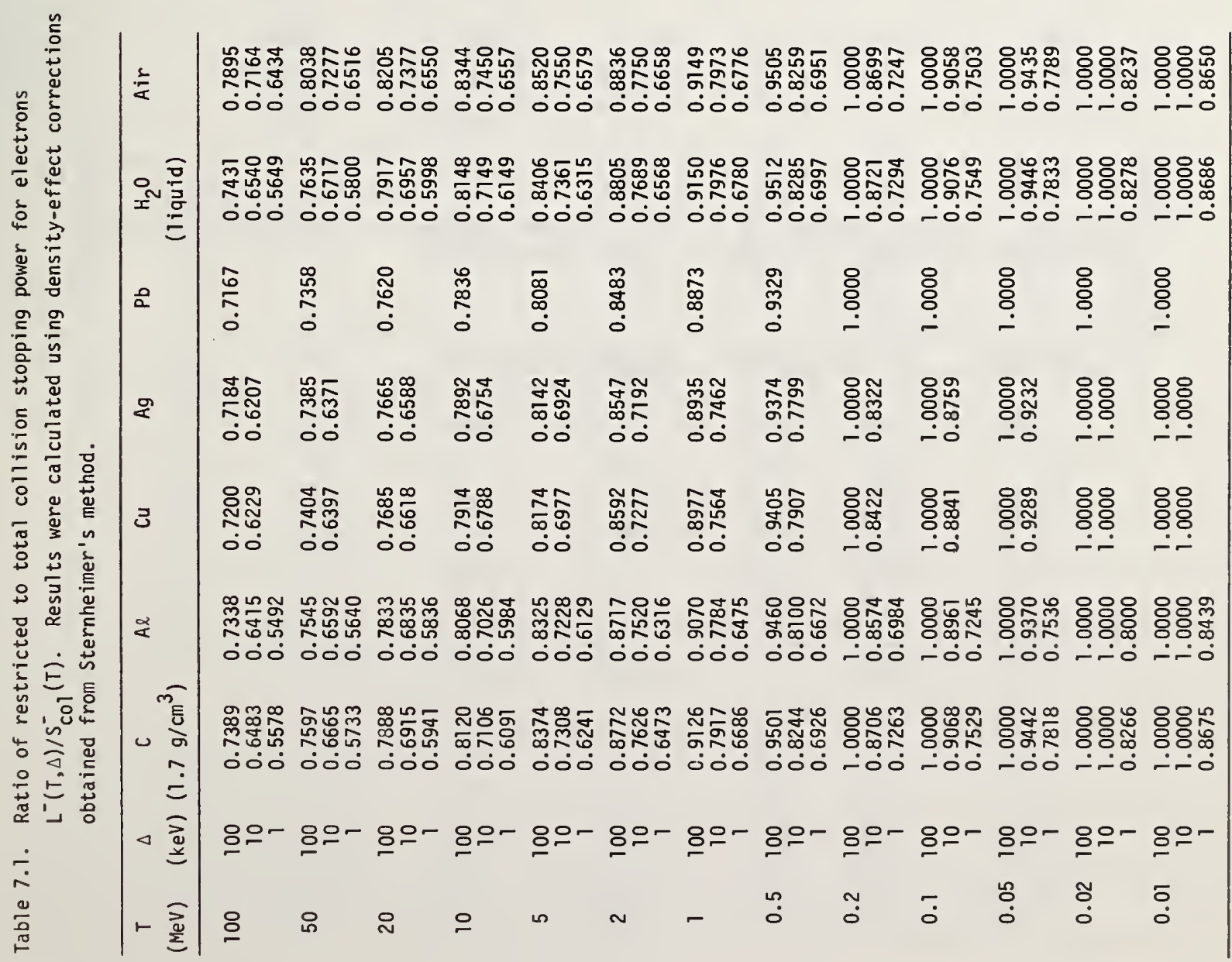


Table 8.1 Comparison of experimental and calculated collision stopping powers in air and collodion. Results are given in units of $\operatorname{MeV} /\left(\mathrm{g} \mathrm{cm}^{-2}\right)$.

\begin{tabular}{|c|c|c|c|c|}
\hline \multirow[b]{2}{*}{$\begin{array}{c}\top \\
(k e V)\end{array}$} & \multicolumn{3}{|c|}{ Calculated Results } & \multirow{2}{*}{$\begin{array}{l}\text { Experimental Results } \\
\text { of Cole (1969) } \\
\text { collodion \& airc }\end{array}$} \\
\hline & $\begin{array}{c}\text { cellulose } \\
\text { nitrate }\end{array}$ & air ${ }^{a}$ & $a i r^{b}$ & \\
\hline 100 & 3.74 & 3.63 & & \\
\hline 60 & 5.26 & 5.11 & & 5.3 \\
\hline 40 & 7.04 & 6.85 & & 7.1 \\
\hline 20 & 11.9 & 11.6 & & 12.0 \\
\hline 10 & 20.3 & 19.8 & 20.4 & 20 \\
\hline 6 & 30.0 & 29.2 & 30.1 & 29 \\
\hline 4 & 40.6 & 39.5 & 40.5 & 39 \\
\hline 2 & 66.8 & 65.0 & 65.8 & 65 \\
\hline 1 & 105 & 103 & 97.9 & 106 \\
\hline 0.6 & 141 & 137 & 139 & 145 \\
\hline 0.4 & 170 & 166 & 170 & 170 \\
\hline 0.2 & & & 216 & 230 \\
\hline 0.1 & & & 218 & 260 \\
\hline 0.06 & & & 170 & 260 \\
\hline 0.04 & & & 104 & 140 \\
\hline 0.02 & & & 26.0 & 110 \\
\hline
\end{tabular}

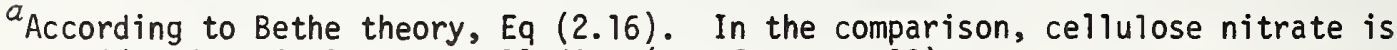
considered equivalent to collodion (see footnote 18).

${ }^{b}$ Green (see footnote 17 ).

${ }^{c}$ Cole's results are the same for collodion and air. 
Table 8.2 Collision stopping power, in $\mathrm{MeV} /\left(\mathrm{g} \mathrm{cm}^{-2}\right)$, for low- 2 materials, calculated according to the Bethe formula, Eq (2.16), at energies from 10 to $1 \mathrm{keV}$.

\begin{tabular}{|c|c|c|c|c|c|c|c|c|c|c|c|}
\hline $\begin{array}{c}\mathrm{T} \\
(\mathrm{keV})\end{array}$ & $\mathrm{H}_{2}$ & $\mathrm{He}$ & $\mathrm{Be}$ & \multicolumn{2}{|c|}{$\underset{\text { graphite }}{C}$} & $\mathrm{~N}_{2}$ & $\mathrm{Ne}$ & \multicolumn{2}{|l|}{$\mathrm{Al}$} & $\mathrm{Si}$ & Ar \\
\hline \multirow{5}{*}{$\begin{array}{r}10 \\
8 \\
6 \\
5 \\
4 \\
3 \\
2 \\
1.5 \\
1 \\
\end{array}$} & $\begin{array}{l}51.3 \\
61.5 \\
77.8 \\
90.2 \\
108 \\
136 \\
188 \\
235 \\
321\end{array}$ & $\begin{array}{r}22.7 \\
27.1 \\
34.0 \\
39.2 \\
46.7 \\
58.3 \\
79.2 \\
98.0 \\
131\end{array}$ & $\begin{array}{r}18.6 \\
22.2 \\
27.7 \\
31.9 \\
37.8 \\
46.9 \\
63.0 \\
77.2 \\
102\end{array}$ & \multicolumn{2}{|c|}{$\begin{array}{c}20.1 \\
23.9 \\
29.8 \\
34.3 \\
40.5 \\
50.1 \\
66.9 \\
81.6 \\
106\end{array}$} & $\begin{array}{r}20.0 \\
23.7 \\
29.5 \\
33.9 \\
40.0 \\
49.4 \\
66.0 \\
80.3 \\
104\end{array}$ & \multicolumn{2}{|l|}{$\begin{array}{l}17.7 \\
20.9 \\
25.9 \\
29.5 \\
34.6 \\
42.3 \\
55.4 \\
66.2 \\
83.4\end{array}$} & $\begin{array}{l}16.5 \\
19.4 \\
23.9 \\
27.2 \\
31.8 \\
38.7 \\
50.2 \\
59.6 \\
73.8\end{array}$ & $\begin{array}{l}16.9 \\
19.9 \\
24.5 \\
27.9 \\
32.5 \\
39.5 \\
51.1 \\
60.5 \\
74.8\end{array}$ & $\begin{array}{l}15.0 \\
17.6 \\
21.6 \\
24.6 \\
28.7 \\
34.7 \\
44.7 \\
52.7 \\
64.7\end{array}$ \\
\hline & $\begin{array}{c}{ }^{\top} \\
\text { (keV) }\end{array}$ & $\begin{array}{c}\text { A-150 } \\
\text { plastic }\end{array}$ & acetylene & $\begin{array}{l}\text { adipose } \\
\text { tissue }\end{array}$ & Air & $\mathrm{Al}_{2} \mathrm{O}_{3}$ & $\begin{array}{c}\text { B-100 } \\
\text { plastic }\end{array}$ & $\begin{array}{l}\text { bone } \\
\text { (ICRU) }\end{array}$ & $\begin{array}{l}\text { bone } \\
\text { (ICRP) }\end{array}$ & $\begin{array}{c}C-552 \\
\text { plastic }\end{array}$ & \\
\hline & $\begin{array}{c}10 \\
8 \\
6 \\
5 \\
4 \\
3 \\
2 \\
1.5 \\
1\end{array}$ & $\begin{array}{r}22.9 \\
27.3 \\
34.1 \\
39.2 \\
46.5 \\
57.6 \\
77.5 \\
94.9 \\
125\end{array}$ & $\begin{array}{c}23.0 \\
27.3 \\
34.2 \\
39.4 \\
46.7 \\
58.0 \\
78.2 \\
96.1 \\
127\end{array}$ & $\begin{array}{r}23.5 \\
27.9 \\
34.9 \\
40.2 \\
47.6 \\
59.1 \\
79.5 \\
97.4 \\
128\end{array}$ & $\begin{array}{c}19.8 \\
23.5 \\
29.2 \\
33.5 \\
39.5 \\
48.8 \\
65.0 \\
79.0 \\
103\end{array}$ & $\begin{array}{l}17.3 \\
20.4 \\
25.2 \\
28.8 \\
33.7 \\
41.1 \\
53.7 \\
64.1 \\
80.3\end{array}$ & $\begin{array}{c}20.9 \\
24.8 \\
30.8 \\
35.4 \\
41.8 \\
51.5 \\
68.7 \\
83.5 \\
108\end{array}$ & $\begin{array}{c}20.7 \\
24.5 \\
30.5 \\
35.0 \\
41.2 \\
50.8 \\
67.5 \\
81.9 \\
106\end{array}$ & $\begin{array}{l}19.7 \\
23.3 \\
29.0 \\
33.2 \\
39.0 \\
47.9 \\
63.4 \\
76.5 \\
98.0\end{array}$ & $\begin{array}{c}19.7 \\
23.4 \\
29.1 \\
33.4 \\
39.5 \\
48.7 \\
64.8 \\
78.8 \\
102\end{array}$ & \\
\hline & $\begin{array}{c}{ }^{\top} \\
\text { (keV) }\end{array}$ & $\mathrm{CaF}_{2}$ & $\mathrm{CO}_{2}$ & $\begin{array}{l}\text { cellulose } \\
\text { nitrate }\end{array}$ & $\begin{array}{l}\text { ceric } \\
\text { sulfate } \\
\text { solution }\end{array}$ & ethylene & $\begin{array}{l}\text { ferrous } \\
\text { sulfate } \\
\text { solution }\end{array}$ & $\begin{array}{c}\text { glass } \\
\text { (Pyrex) }\end{array}$ & Kapton & LiF & \\
\hline & $\begin{array}{l}10 \\
8 \\
6 \\
5 \\
4 \\
3 \\
2 \\
1.5 \\
1\end{array}$ & $\begin{array}{l}16.7 \\
19.6 \\
24.2 \\
27.5 \\
32.2 \\
39.1 \\
50.7 \\
60.2 \\
74.6\end{array}$ & $\begin{array}{c}19.6 \\
23.3 \\
29.0 \\
33.3 \\
39.3 \\
48.4 \\
64.4 \\
78.2 \\
101\end{array}$ & $\begin{array}{c}20.3 \\
24.1 \\
30.0 \\
34.4 \\
40.6 \\
50.1 \\
66.8 \\
81.2 \\
105\end{array}$ & $\begin{array}{l}22.4 \\
26.6 \\
33.1 \\
38.0 \\
45.0 \\
55.6 \\
74.4 \\
90.7 \\
118\end{array}$ & $\begin{array}{l}25.0 \\
29.8 \\
37.3 \\
43.0 \\
51.1 \\
63.7 \\
86.1 \\
106 \\
141\end{array}$ & $\begin{array}{c}22.4 \\
26.6 \\
33.2 \\
38.1 \\
45.1 \\
55.8 \\
74.6 \\
90.9 \\
119\end{array}$ & $\begin{array}{l}17.9 \\
21.1 \\
26.1 \\
29.8 \\
34.9 \\
42.7 \\
55.9 \\
67.0 \\
84.5\end{array}$ & $\begin{array}{c}20.6 \\
24.5 \\
30.5 \\
35.0 \\
41.4 \\
51.2 \\
68.3 \\
83.3 \\
108\end{array}$ & $\begin{array}{l}18.0 \\
21.3 \\
26.5 \\
30.4 \\
35.8 \\
44.1 \\
58.6 \\
71.0 \\
91.6\end{array}$ & \\
\hline$\stackrel{T}{\mathrm{keV}})$ & methane & $\begin{array}{l}\text { muscle } \\
\text { (ICRP) }\end{array}$ & $\begin{array}{l}\text { muscle } \\
\text { (ICRU) }\end{array}$ & $\begin{array}{r}\text { ME } \\
\text { (with }\end{array}$ & $\begin{array}{l}\text { liquid } \\
\text { sucrose) }\end{array}$ & $\begin{array}{l}\text { ME liquid } \\
\text { (without } \\
\text { sucrose) }\end{array}$ & $\begin{array}{l}\text { nylon } \\
\text { type } 6\end{array}$ & $\begin{array}{l}\text { paraffin } \\
\text { wax }\end{array}$ & $\begin{array}{r}p \\
\text { scint }\end{array}$ & $\begin{array}{l}\text { lastic } \\
\text { tillator }\end{array}$ & $\begin{array}{c}\text { poly- } \\
\text { carbonate }\end{array}$ \\
\hline $\begin{array}{l}10 \\
8 \\
6 \\
5 \\
4 \\
3 \\
2 \\
1.5 \\
1\end{array}$ & $\begin{array}{c}28.3 \\
33.8 \\
42.4 \\
49.0 \\
58.3 \\
72.8 \\
98.9 \\
122 \\
163\end{array}$ & $\begin{array}{r}22.3 \\
26.5 \\
33.1 \\
38.0 \\
44.9 \\
55.6 \\
74.4 \\
90.7 \\
118\end{array}$ & $\begin{array}{r}22.4 \\
26.6 \\
33.2 \\
38.1 \\
45.1 \\
55.8 \\
74.6 \\
91.1 \\
119\end{array}$ & $\begin{array}{r}2 \\
2 \\
3 \\
3 \\
4 \\
5 \\
7 \\
9 \\
11\end{array}$ & $\begin{array}{l}22.3 \\
26.5 \\
33.1 \\
38.0 \\
45.0 \\
55.7 \\
74.5 \\
91.0 \\
19\end{array}$ & $\begin{array}{r}22.4 \\
26.6 \\
33.2 \\
38.2 \\
45.2 \\
55.9 \\
74.8 \\
91.3 \\
119\end{array}$ & $\begin{array}{c}23.0 \\
27.4 \\
34.2 \\
39.3 \\
46.6 \\
57.8 \\
77.7 \\
95.2 \\
125\end{array}$ & $\begin{array}{c}25.3 \\
30.2 \\
37.9 \\
43.7 \\
51.9 \\
64.6 \\
87.6 \\
108 \\
143\end{array}$ & & $\begin{array}{l}22.7 \\
27.0 \\
33.7 \\
38.7 \\
45.9 \\
56.9 \\
76.5 \\
93.7 \\
23\end{array}$ & $\begin{array}{c}21.5 \\
25.6 \\
31.9 \\
36.7 \\
43.4 \\
53.7 \\
71.9 \\
87.8 \\
115\end{array}$ \\
\hline & $\stackrel{\stackrel{T}{(k e V)}}{ }$ & $\begin{array}{l}\text { poly- } \\
\text { ethylene }\end{array}$ & PMMA & $\begin{array}{r}p \\
\text { pro }\end{array}$ & $\begin{array}{l}\text { poly- } \\
\text { opylene }\end{array}$ & $\begin{array}{l}\text { poly- } \\
\text { styrene }\end{array}$ & Teflon & PVC & & ropane & \\
\hline & $\begin{array}{r}10 \\
8 \\
6 \\
5 \\
4 \\
3 \\
2 \\
1.5 \\
1\end{array}$ & $\begin{array}{c}24.4 \\
29.1 \\
36.4 \\
41.9 \\
49.7 \\
61.8 \\
83.3 \\
102 \\
135\end{array}$ & $\begin{array}{c}22.0 \\
26.1 \\
32.6 \\
37.4 \\
44.3 \\
54.8 \\
73.4 \\
89.6 \\
117\end{array}$ & & $\begin{array}{l}23.8 \\
28.4 \\
35.5 \\
40.9 \\
48.5 \\
60.2 \\
81.1 \\
99.5 \\
31\end{array}$ & $\begin{array}{r}22.2 \\
26.4 \\
33.0 \\
38.0 \\
44.9 \\
55.7 \\
74.7 \\
91.4 \\
120\end{array}$ & $\begin{array}{l}18.4 \\
21.8 \\
27.1 \\
31.1 \\
36.6 \\
45.1 \\
59.8 \\
72.3 \\
93.0\end{array}$ & $\begin{array}{l}19.3 \\
22.9 \\
28.4 \\
32.4 \\
38.2 \\
46.9 \\
62.0 \\
74.8 \\
95.7\end{array}$ & & $\begin{array}{l}26.2 \\
31.2 \\
39.2 \\
45.2 \\
53.7 \\
66.9 \\
90.7 \\
12 \\
49\end{array}$ & \\
\hline & $\begin{array}{c}T^{\top} \\
(\mathrm{keV})\end{array}$ & $\mathrm{SiO}_{2}$ & stilbene & & $\begin{array}{l}\text { E gas } \\
\text { ethane } \\
\text { ased) }\end{array}$ & $\begin{array}{l}\text { TE gas } \\
\text { (propane } \\
\text { based) }\end{array}$ & toluene & $\begin{array}{c}\text { water } \\
\text { (1iquid) }\end{array}$ & & $\begin{array}{l}\text { water } \\
\text { vapor }\end{array}$ & \\
\hline & $\begin{array}{r}10 \\
8 \\
6 \\
5 \\
4 \\
3 \\
2 \\
1.5 \\
1\end{array}$ & $\begin{array}{l}17.8 \\
21.0 \\
26.0 \\
29.6 \\
34.7 \\
42.4 \\
55.5 \\
66.3 \\
83.4\end{array}$ & $\begin{array}{c}22.1 \\
26.3 \\
32.8 \\
37.7 \\
44.7 \\
55.4 \\
74.3 \\
90.9 \\
119\end{array}$ & & $\begin{array}{l}23.2 \\
27.6 \\
34.5 \\
39.6 \\
47.0 \\
58.3 \\
78.5 \\
96.2 \\
27\end{array}$ & $\begin{array}{r}23.3 \\
27.8 \\
34.7 \\
40.0 \\
47.4 \\
58.9 \\
79.3 \\
97.2 \\
128\end{array}$ & $\begin{array}{c}22.9 \\
27.2 \\
34.0 \\
39.1 \\
46.4 \\
57.6 \\
77.4 \\
94.9 \\
125\end{array}$ & $\begin{array}{c}22.6 \\
26.8 \\
33.4 \\
38.4 \\
45.4 \\
56.2 \\
75.2 \\
91.8 \\
120\end{array}$ & & $\begin{array}{l}22.8 \\
27.1 \\
33.8 \\
38.8 \\
45.9 \\
56.9 \\
76.2 \\
93.1 \\
22\end{array}$ & \\
\hline
\end{tabular}




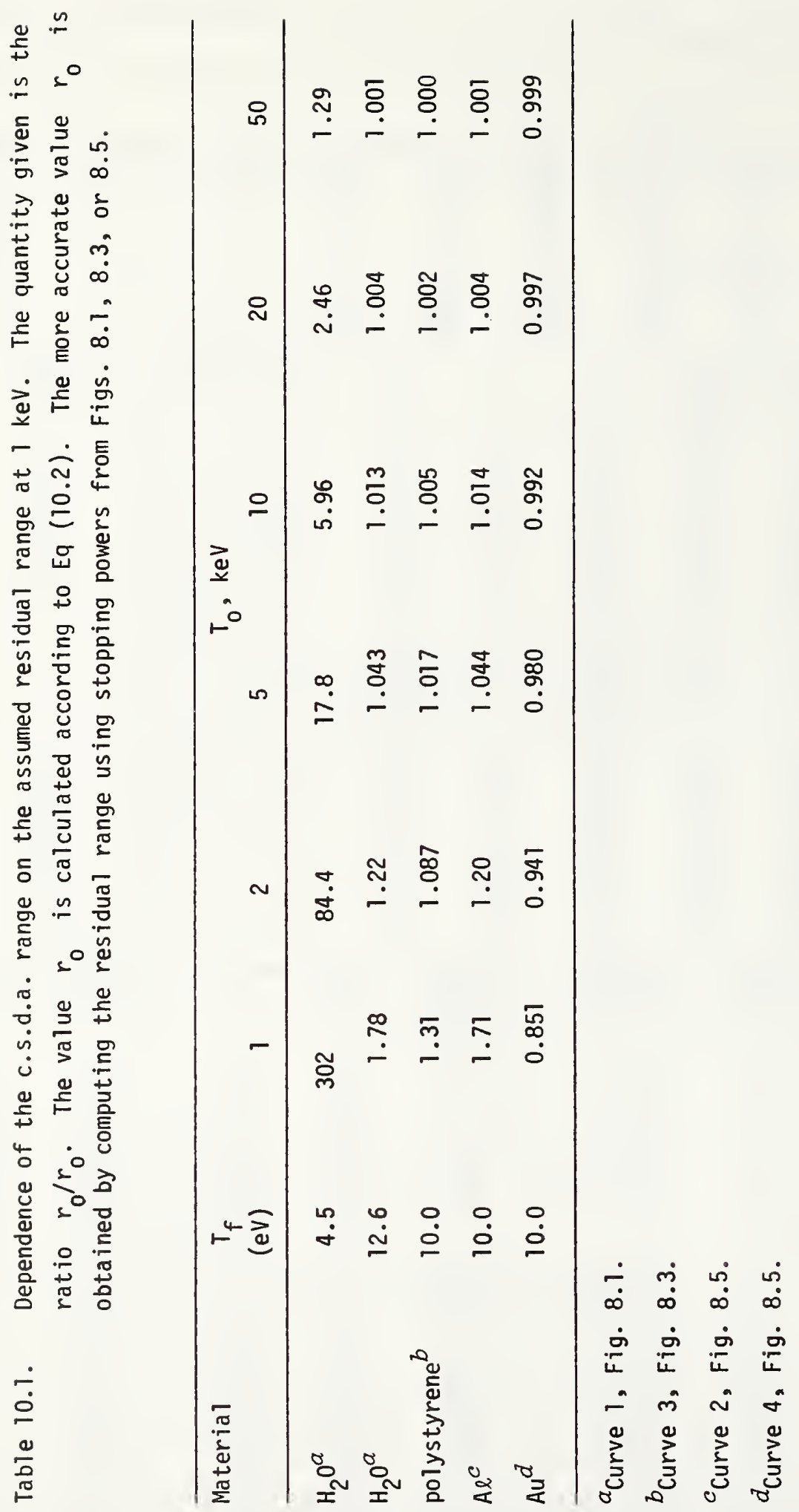




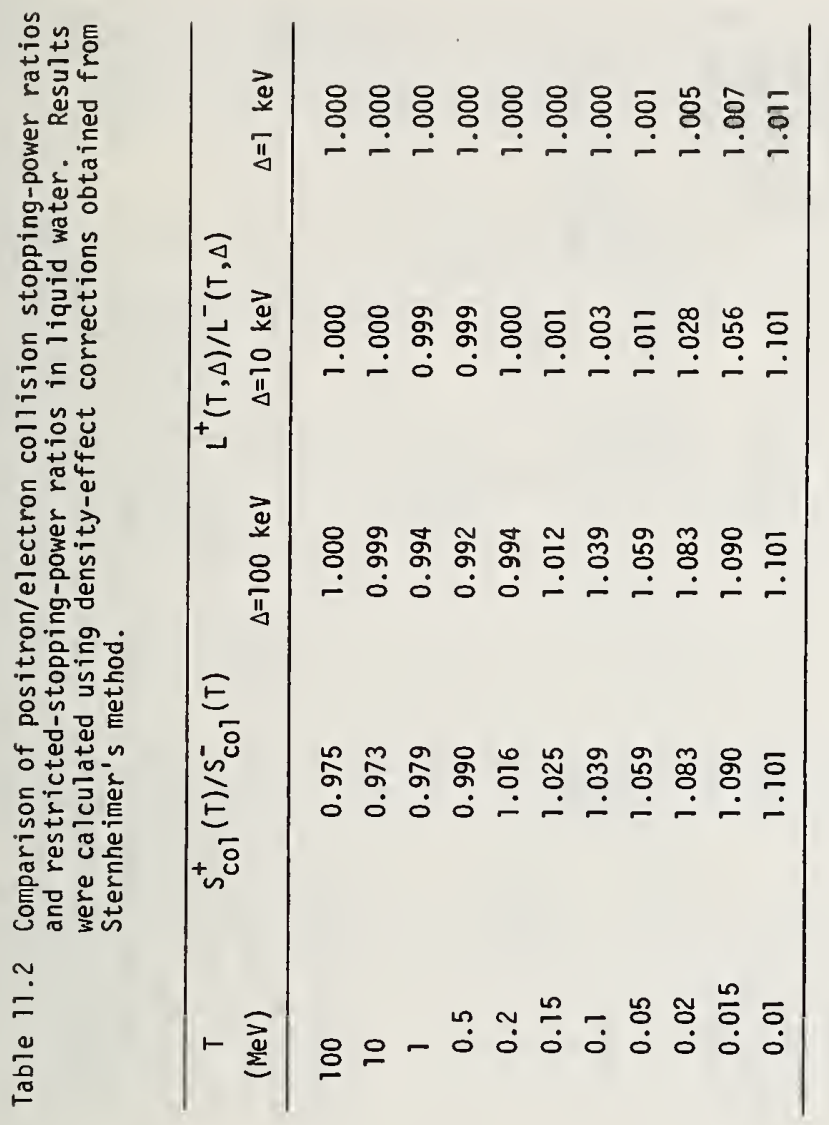

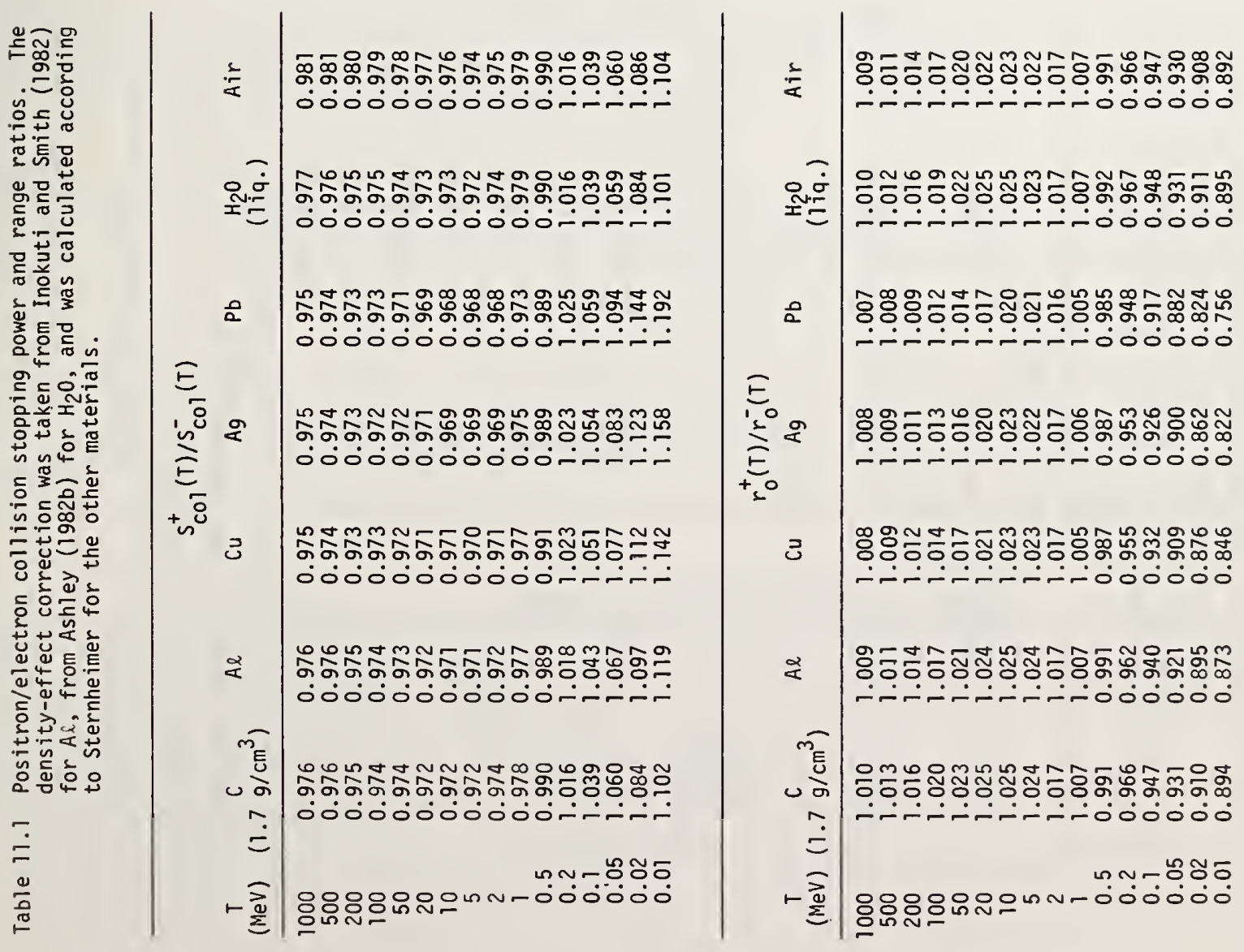




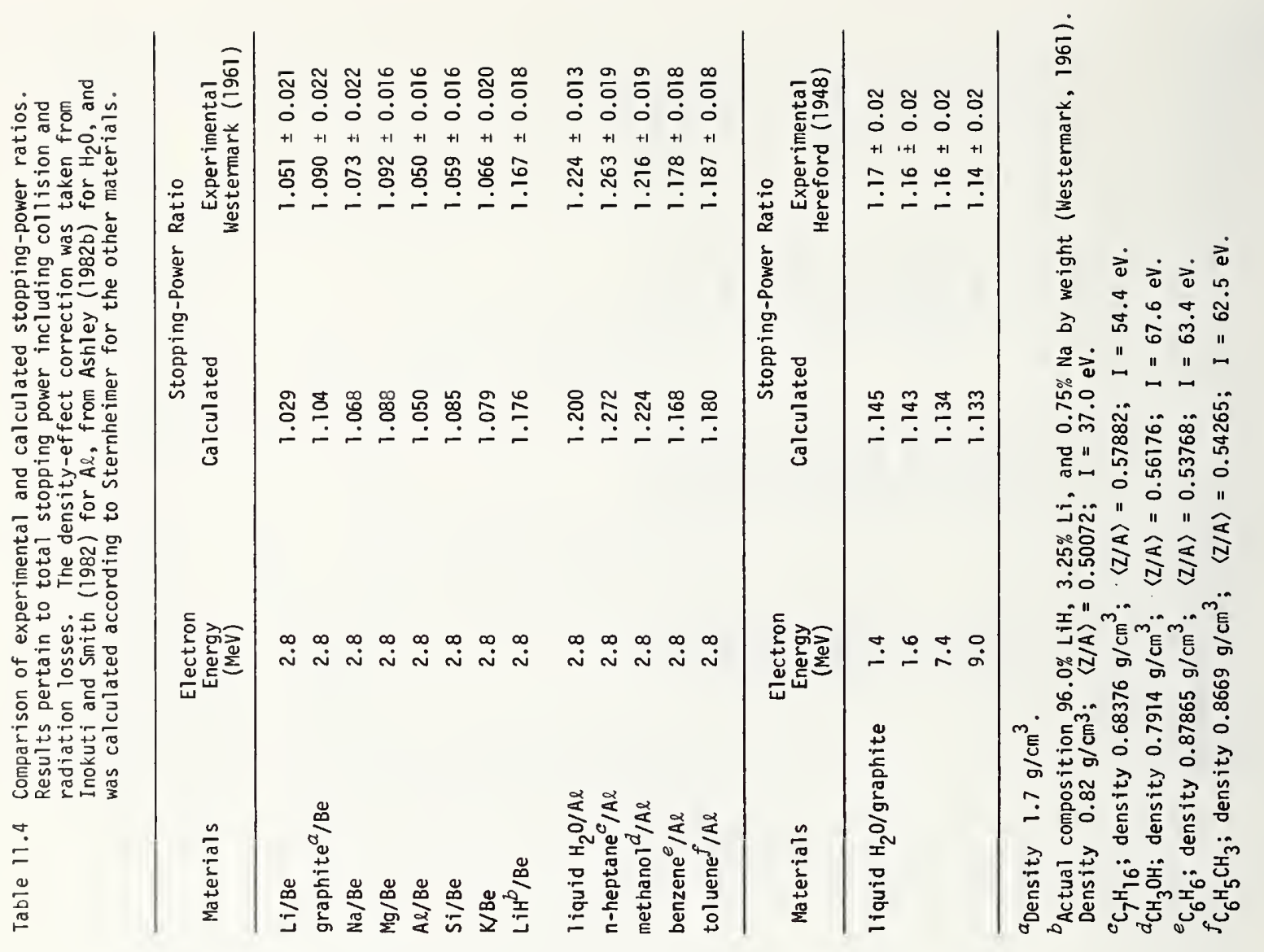

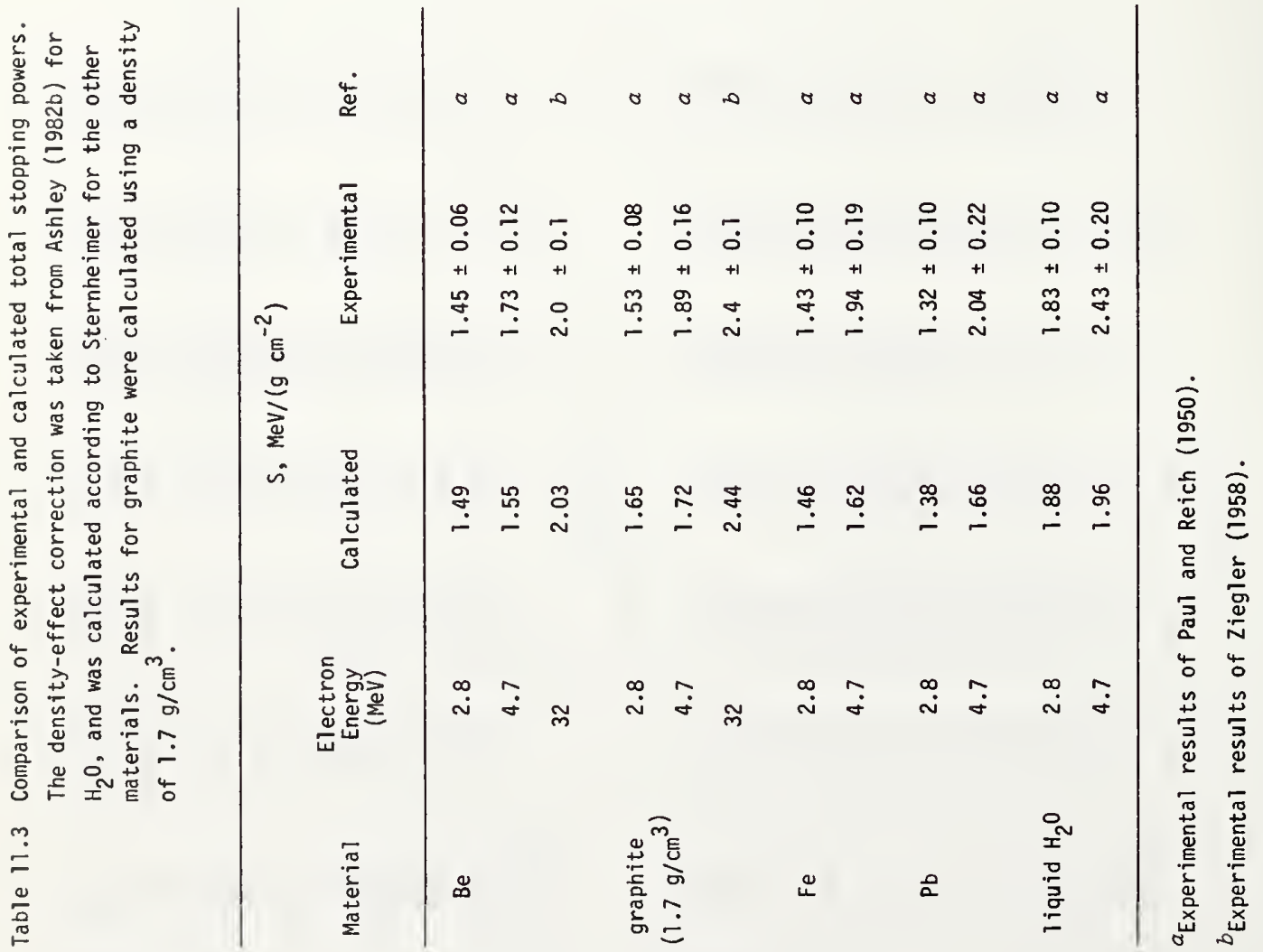


Table 11.5 Comparison of data given in earlier electron stopping-power and range tables with results of the present work. The comparisons are presented in terms of the percent deviations of the results given by Berger and Seltzer (1964) and of Pages et al. (1972) from the results calculated here, for the collision stopping power, radiative stopping power, total stopping power, csda range and radiation yield.

Percent Deviations for Water ${ }^{a}$ (1 iquid)

\begin{tabular}{|c|c|c|c|c|c|c|c|c|c|c|}
\hline \multirow{3}{*}{$(\stackrel{\mathrm{MeV}}{\mathrm{\top}})$} & \multicolumn{6}{|c|}{ Stopping Power } & \multirow{2}{*}{\multicolumn{2}{|c|}{$\begin{array}{r}\begin{array}{c}\text { csda } \\
\text { Range }\end{array} \\
r_{0}(T)\end{array}$}} & \multirow{2}{*}{\multicolumn{2}{|c|}{$\begin{array}{l}\text { Radiation } \\
\text { Yield } \\
Y(T)\end{array}$}} \\
\hline & \multicolumn{2}{|c|}{$\begin{array}{l}\text { Collision } \\
\frac{1}{\rho} S_{\operatorname{col}}(T)\end{array}$} & \multicolumn{2}{|c|}{$\begin{array}{l}\text { Radiative } \\
\frac{1}{\rho} S_{r a d}(T)\end{array}$} & \multicolumn{2}{|c|}{$\begin{array}{l}\text { Total } \\
\frac{1}{\rho} S(T)\end{array}$} & & & & \\
\hline & B\&S & Pages & B\&S & Pages & B\&S & Pages & $B \& S$ & Pages & B\&S & Pages \\
\hline 0.01 & 2.8 & 2.8 & 30.0 & 29.1 & 2.8 & 2.7 & -3.1 & -14.3 & 32.3 & 15.3 \\
\hline 0.1 & 2.0 & 1.9 & 22.6 & 25.6 & 2.0 & 1.9 & -2.2 & -2.3 & 18.7 & 20.3 \\
\hline 1 & 1.7 & 1.6 & 34.9 & 38.1 & 1.9 & 1.9 & -1.9 & -1.9 & 35.5 & 39.2 \\
\hline 10 & 1.2 & 2.7 & 0.8 & -0.6 & 1.2 & 2.5 & -1.7 & -2.2 & 2.4 & 1.0 \\
\hline 100 & 0.0 & 5.1 & -1.3 & 2.3 & -0.6 & 3.6 & -0.2 & -2.8 & -0.8 & -2.2 \\
\hline 1000 & 0.0 & & -1.9 & & -1.7 & & 0.8 & & -0.3 & \\
\hline
\end{tabular}

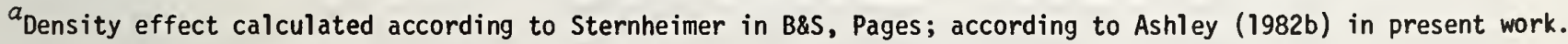

Percent Deviations for Air

\begin{tabular}{c|rr|rr|rr|rr|rr}
\hline 0.01 & -0.3 & -0.3 & 28.6 & 27.6 & -0.3 & -0.3 & 0.3 & -11.9 & 35.2 & 16.7 \\
0.1 & -0.2 & -0.2 & 21.0 & 24.2 & -0.1 & -0.2 & 0.2 & 0.0 & 20.0 & 21.6 \\
1 & -0.1 & -0.2 & 34.3 & 38.3 & 0.1 & 0.1 & 0.0 & -0.1 & 37.4 & 41.5 \\
10 & -0.1 & -0.1 & 0.8 & -0.7 & 0.0 & -0.2 & -0.1 & 0.0 & 4.1 & 3.4 \\
100 & 0.7 & -1.3 & -1.3 & 2.2 & -0.3 & 0.4 & 0.2 & 0.6 & -0.9 & 1.3 \\
1000 & 0.0 & & -1.9 & & -1.8 & & 0.8 & & -0.4 & \\
\hline
\end{tabular}

Percent Deviations for Aluminum ${ }^{b}$

\begin{tabular}{|c|c|c|c|c|c|c|c|c|c|c|}
\hline \multirow{3}{*}{$\left(\mathrm{MeV}^{\top}\right)$} & \multicolumn{6}{|c|}{ Stopping Power } & \multirow{2}{*}{\multicolumn{2}{|c|}{$\begin{array}{c}\begin{array}{c}\text { csda } \\
\text { Range }\end{array} \\
r_{0}(T)\end{array}$}} & \multirow{2}{*}{\multicolumn{2}{|c|}{$\begin{array}{l}\begin{array}{c}\text { Radiation } \\
\text { Yield }\end{array} \\
Y(T)\end{array}$}} \\
\hline & \multicolumn{2}{|c|}{$\begin{array}{l}\text { Collision } \\
\frac{1}{\rho} S_{C o l}(T)\end{array}$} & \multicolumn{2}{|c|}{$\begin{array}{l}\text { Radiative } \\
\frac{1}{\rho} \mathrm{S}_{\mathrm{rad}}(\mathrm{T})\end{array}$} & \multicolumn{2}{|c|}{$\begin{array}{l}\text { Total } \\
\frac{1}{\rho} S(T)\end{array}$} & & & & \\
\hline & B\&S & Pages & B\&S & Pages & B\&S & Pages & B\&S & Pages & B\&S & Pages \\
\hline 0.01 & 0.5 & -0.6 & 31.1 & 30.0 & 0.5 & -0.6 & -0.6 & -13.8 & 40.8 & 22.0 \\
\hline 0.1 & 0.4 & -0.5 & 21.8 & 24.1 & 0.4 & -0.5 & -0.4 & 0.2 & 18.4 & 21.0 \\
\hline 1 & 0.5 & -0.6 & 38.4 & 41.2 & 1.0 & 0.0 & -0.9 & 0.1 & 39.6 & 44.2 \\
\hline 10 & -0.1 & 0.0 & 0.4 & -1.2 & 0.0 & -0.2 & -0.3 & -0.1 & 3.6 & 2.6 \\
\hline 100 & 0.2 & 0.4 & -0.1 & 2.8 & 0.0 & 2.0 & 0.0 & -0.2 & -0.2 & 0.2 \\
\hline 1000 & 0.1 & & -0.3 & & -0.3 & & 0.0 & & 0.0 & \\
\hline
\end{tabular}

${ }^{b}$ Density effect calculated according to Sternheimer in B\&S, Pages; according to Inokuti and Smith in present work.

Percent Deviations for Gold

\begin{tabular}{c|rr|rr|rr|rr|rr}
\hline 0.01 & -0.3 & -0.4 & 115.3 & 104.7 & 0.0 & -0.1 & 0.3 & -27.3 & 176.7 & 108.7 \\
0.1 & 0.0 & -0.2 & 32.9 & 35.2 & 0.7 & 0.6 & -0.4 & -1.1 & 42.2 & 43.4 \\
1 & 1.4 & 0.0 & 27.3 & 31.3 & 4.3 & 3.5 & -3.0 & -2.5 & 26.9 & 31.0 \\
10 & 1.6 & 0.3 & 5.6 & 1.3 & 3.5 & 0.8 & -4.3 & -1.2 & 6.2 & 2.1 \\
100 & 0.6 & 0.5 & 1.3 & 2.3 & 1.2 & 2.2 & -2.2 & 1.4 & 0.3 & -0.5 \\
1000 & 0.1 & & 1.0 & & 1.0 & & -1.7 & & 0.0 & \\
\hline
\end{tabular}




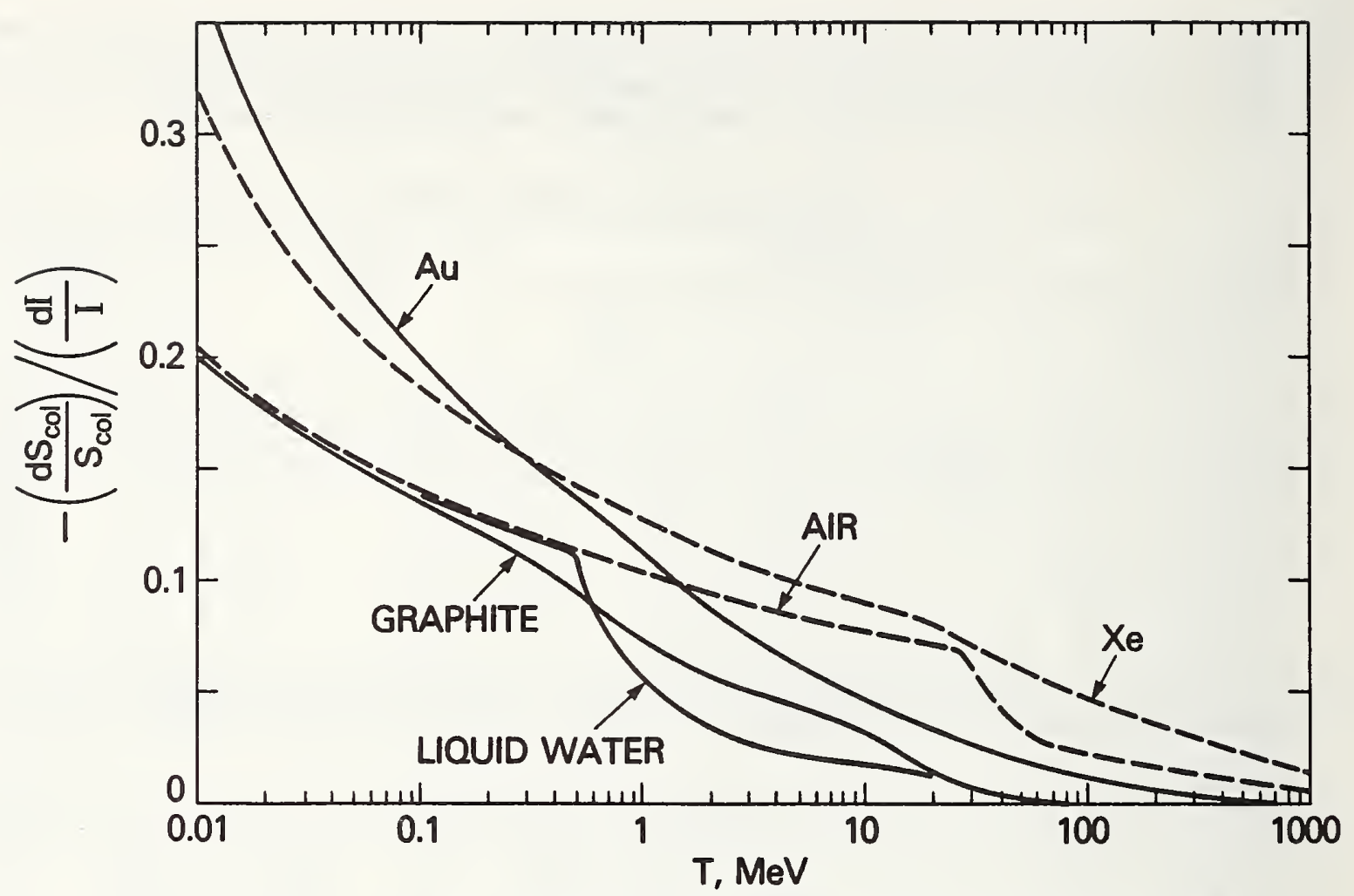

Fig. 3.1. Percent increase (decrease) of the collision stopping power for electrons resulting from a 1 -percent decrease (increase) of the mean excitation energy.

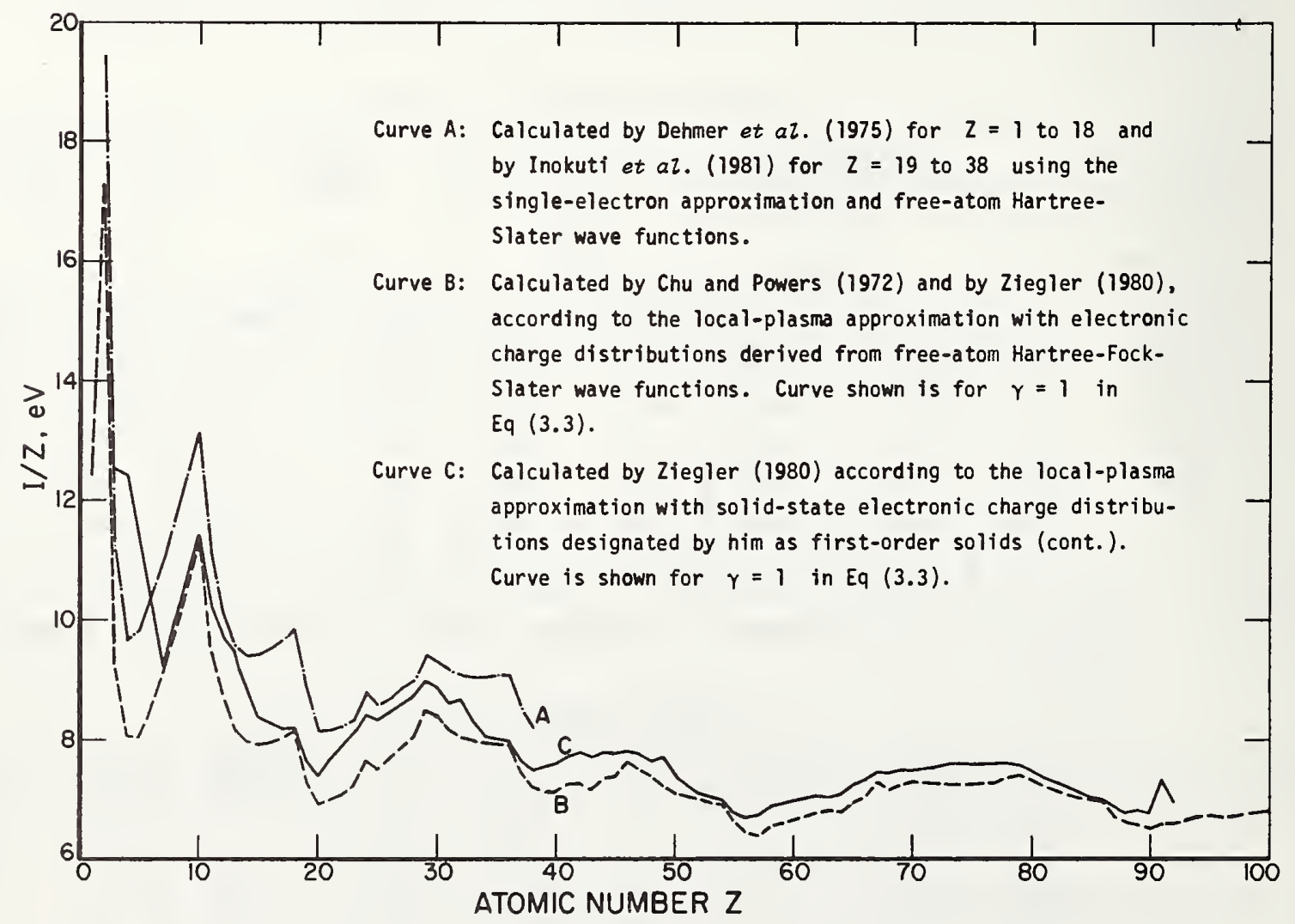

Fig. 3.2. Theoretical mean excitation energies for elements. 

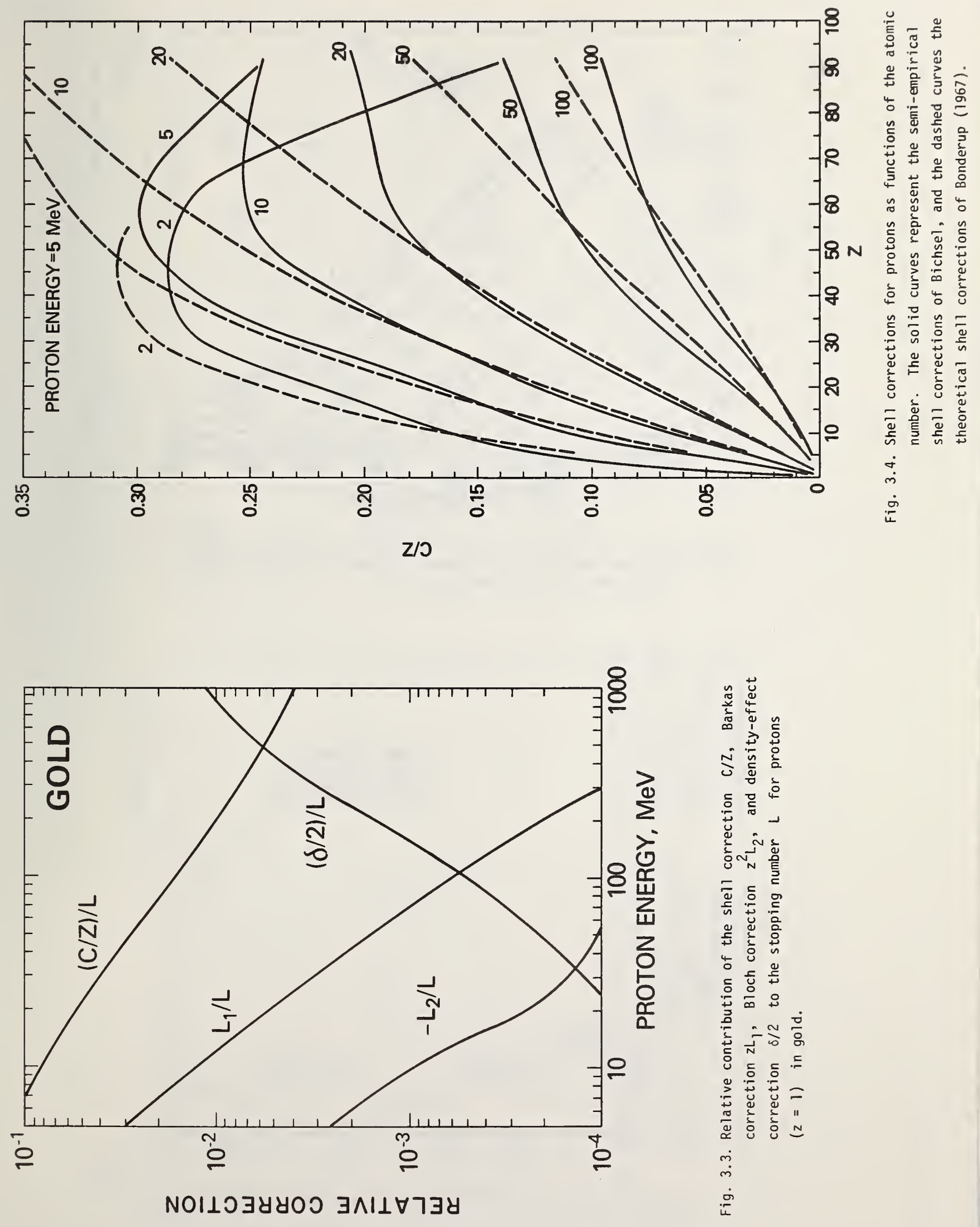


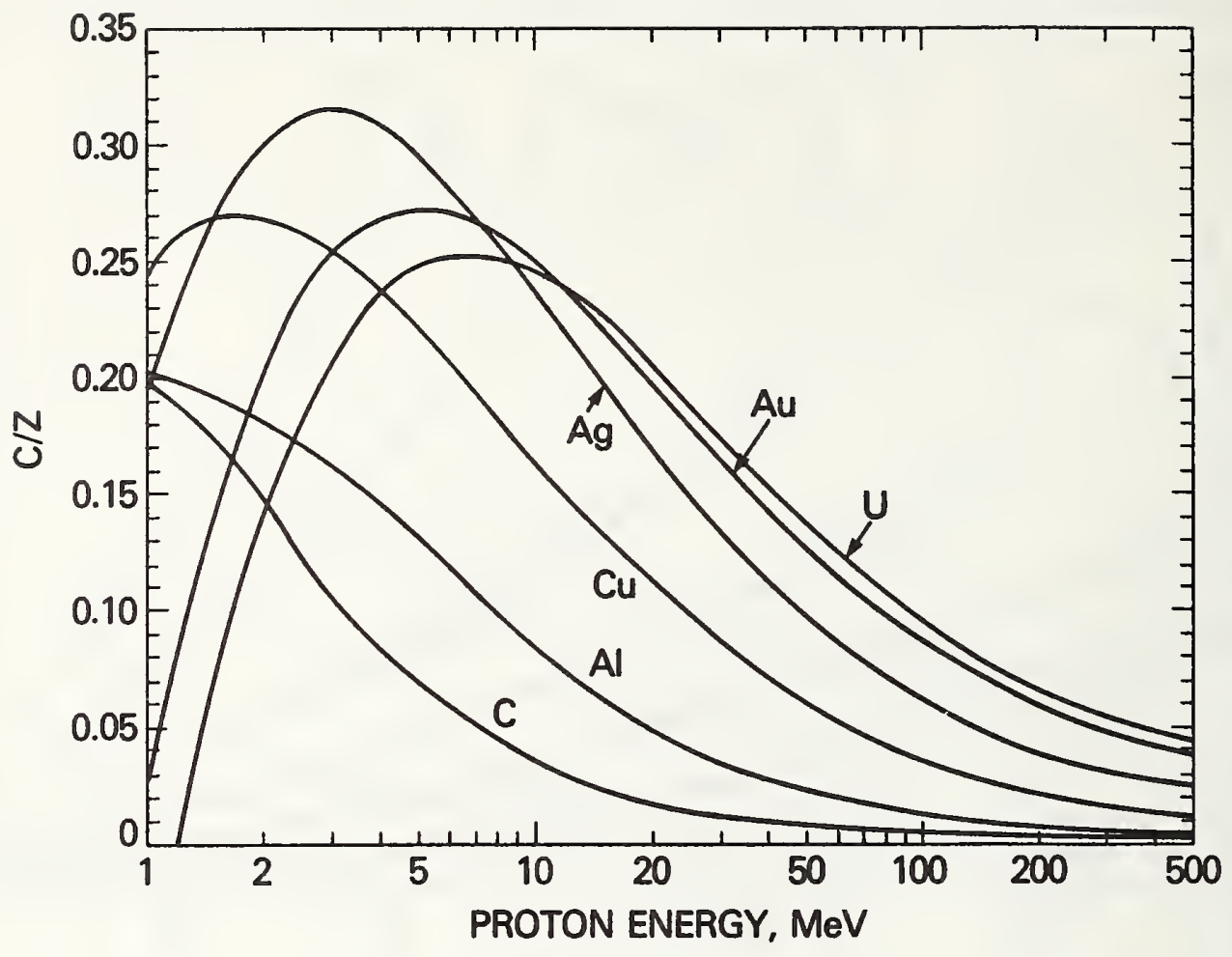

Fig. 3.5. Semi-empirical shell corrections of Bichsel for selected elements, as functions of the proton energy.

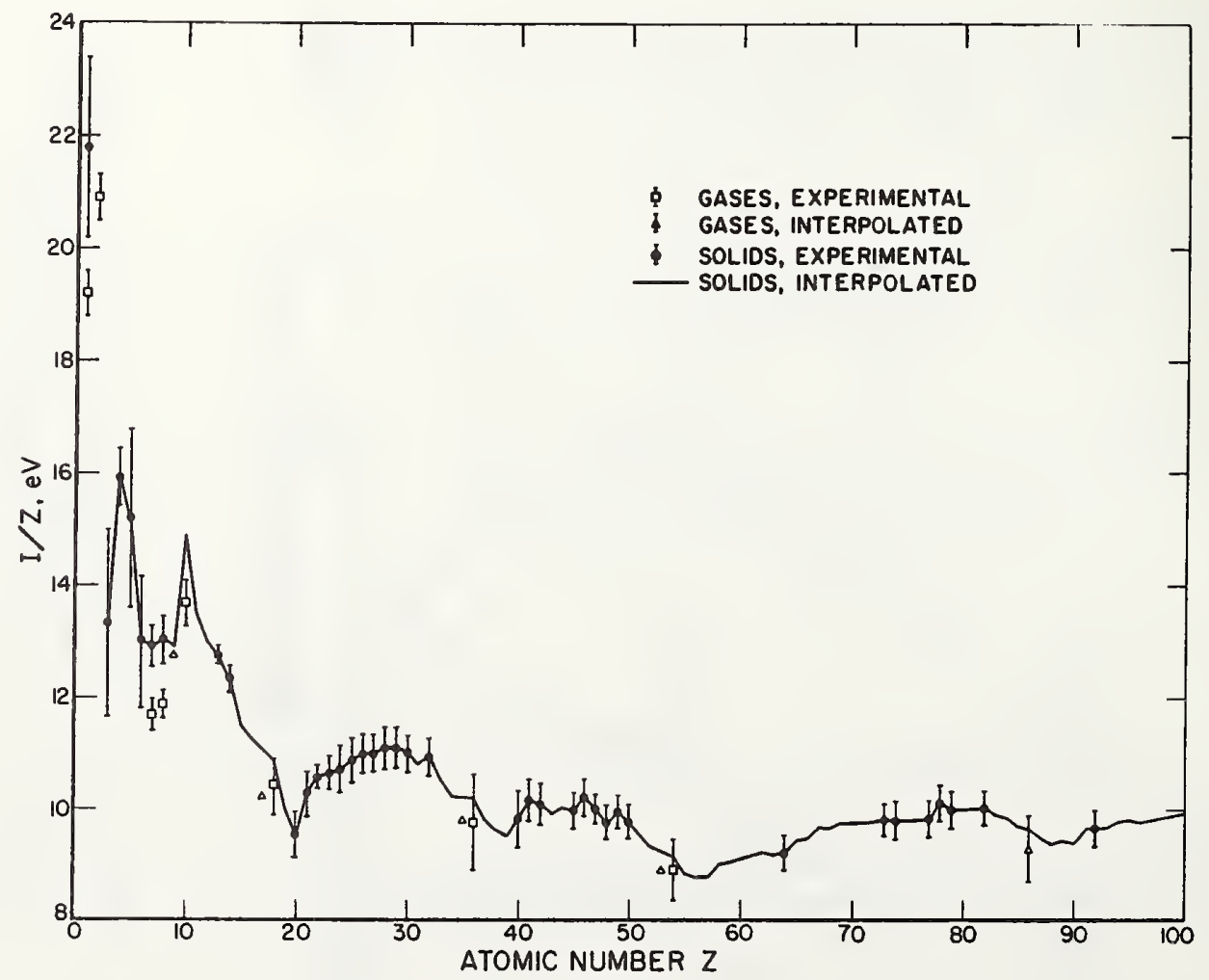

Fig. 4.7. Ratio of the mean excitation energy $I$ to the atomic number $Z$ for elements. 

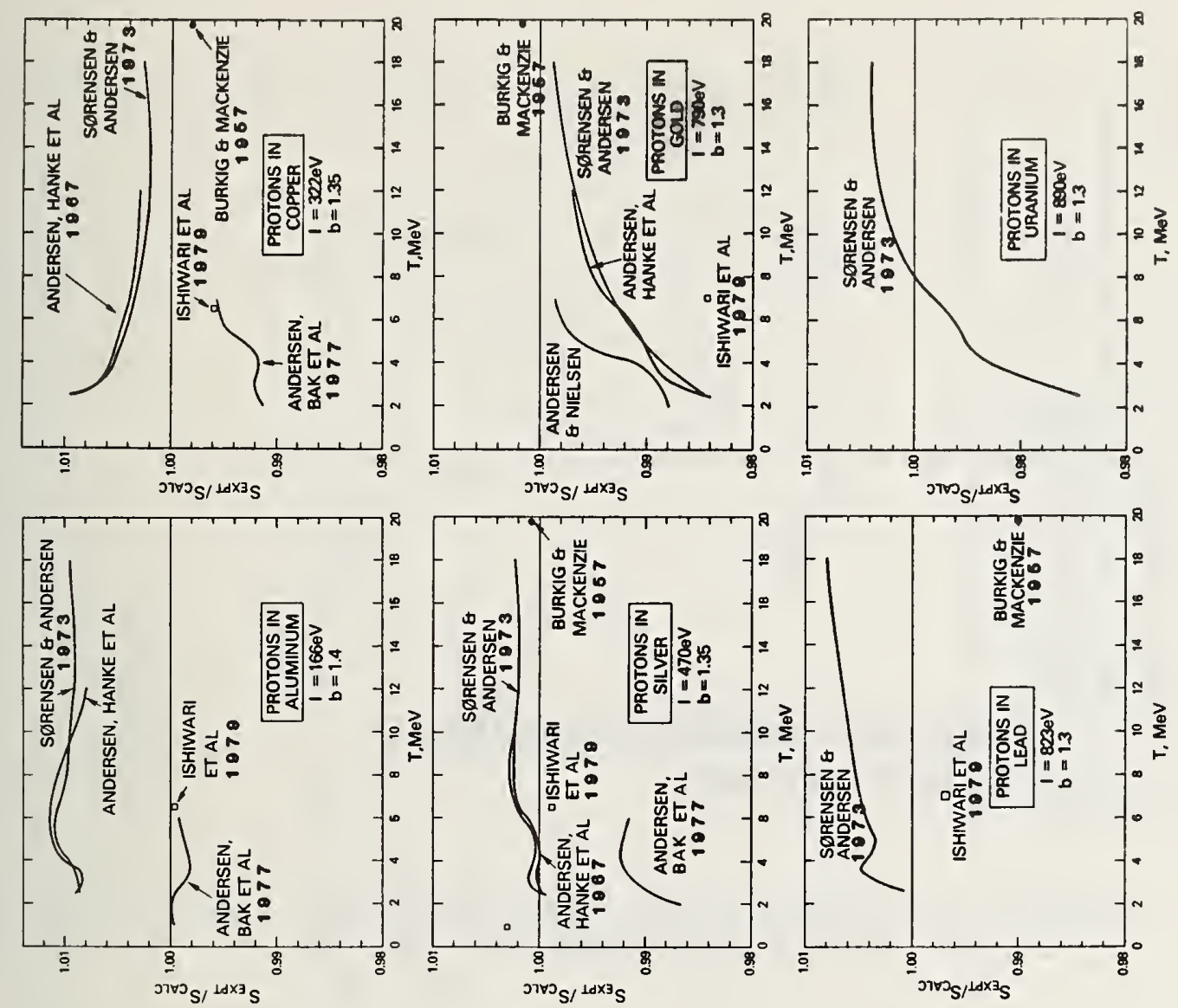

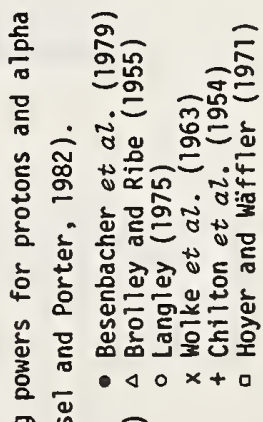

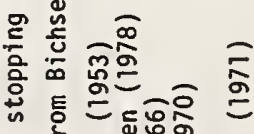

记

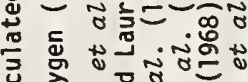

ช

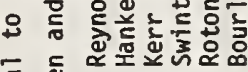

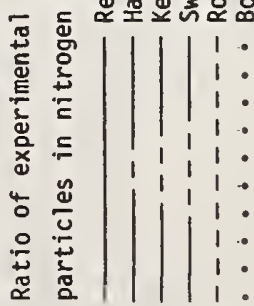

i்

$\dot{\square}$

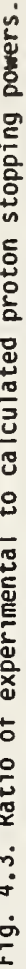
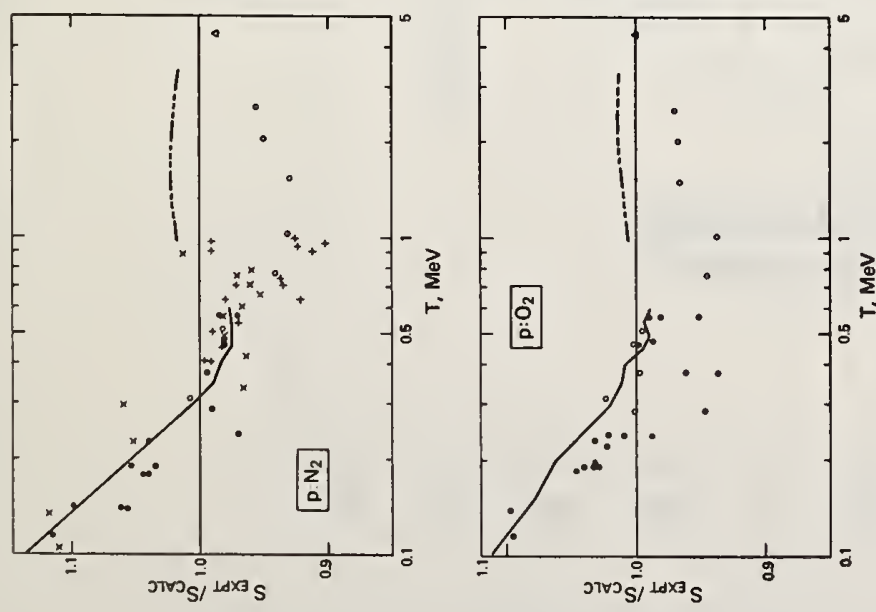


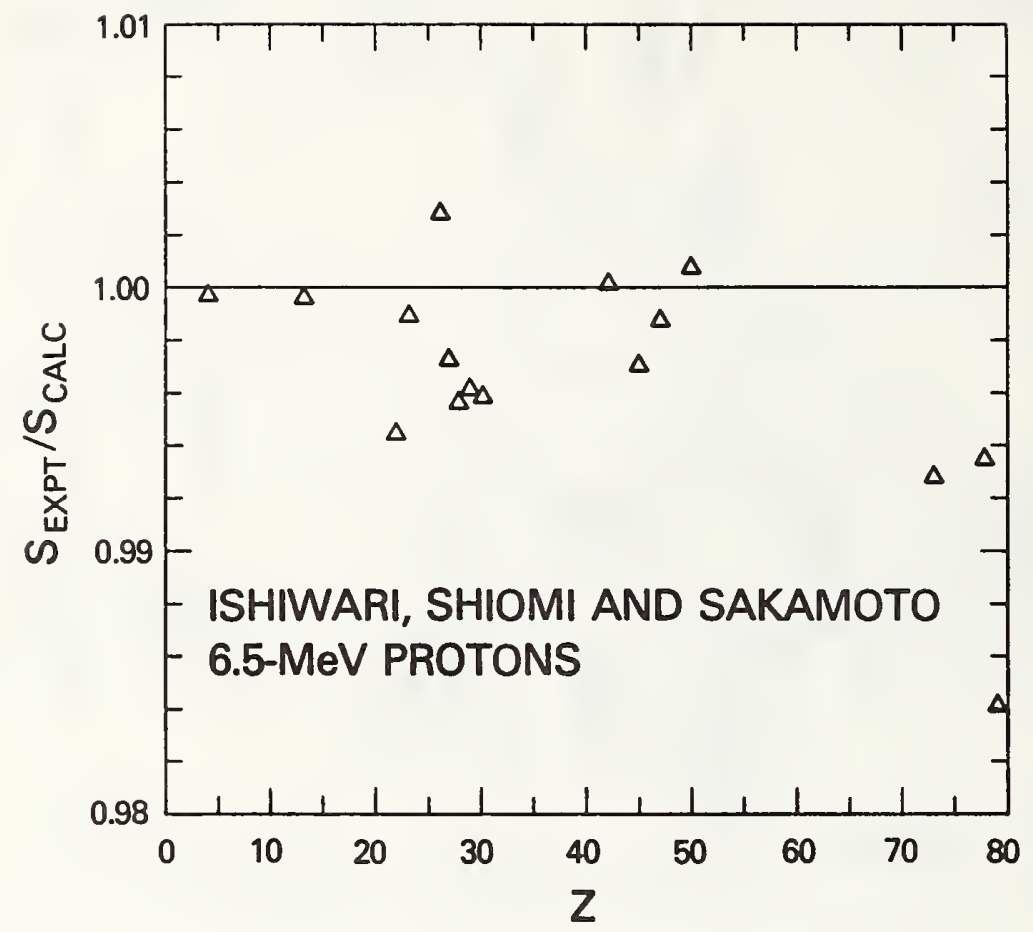

Fig. 4.4. Ratio of experimental to calculated proton stopping powers at $6.5 \mathrm{MeV}$. Experimental data are from Ishiwari (1979).

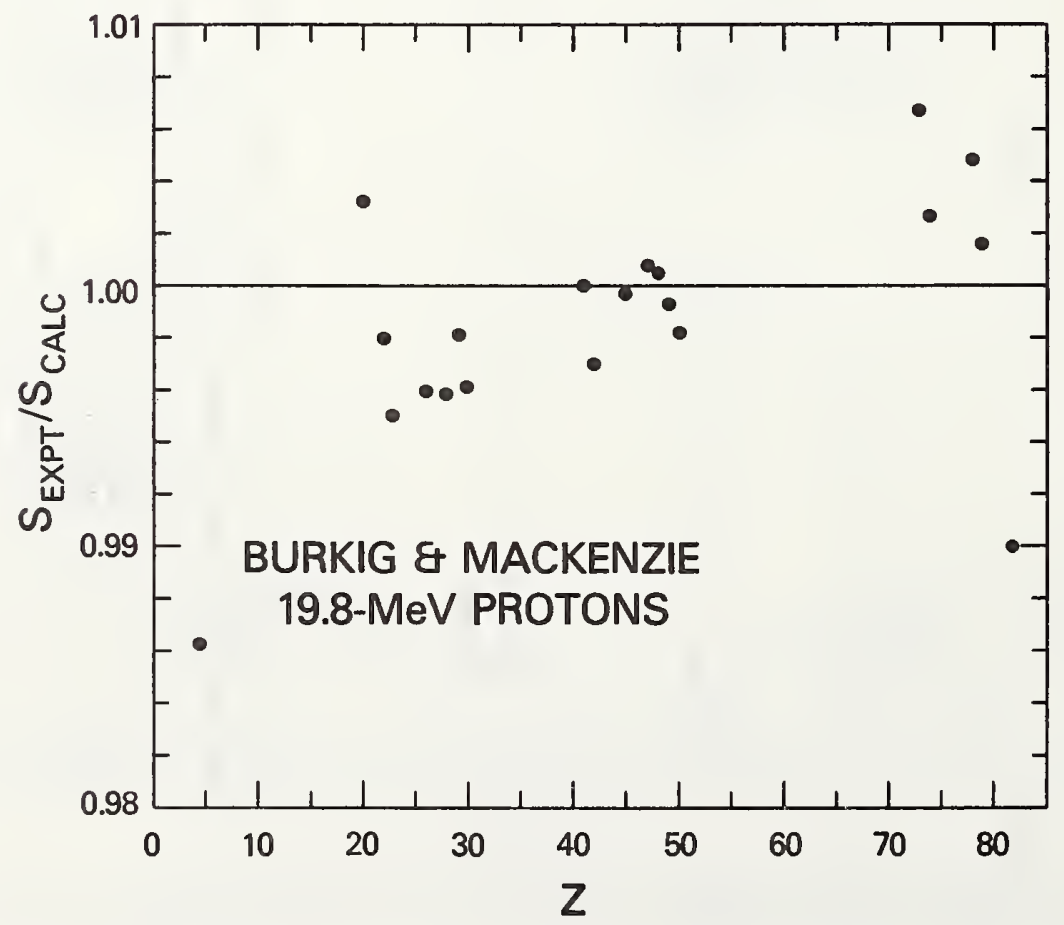

Fig. 4.5. Ratio of experimental to calculated proton stopping powers at $19.8 \mathrm{MeV}$. Experimental data are from Burkig and Mackenzie (1957). 


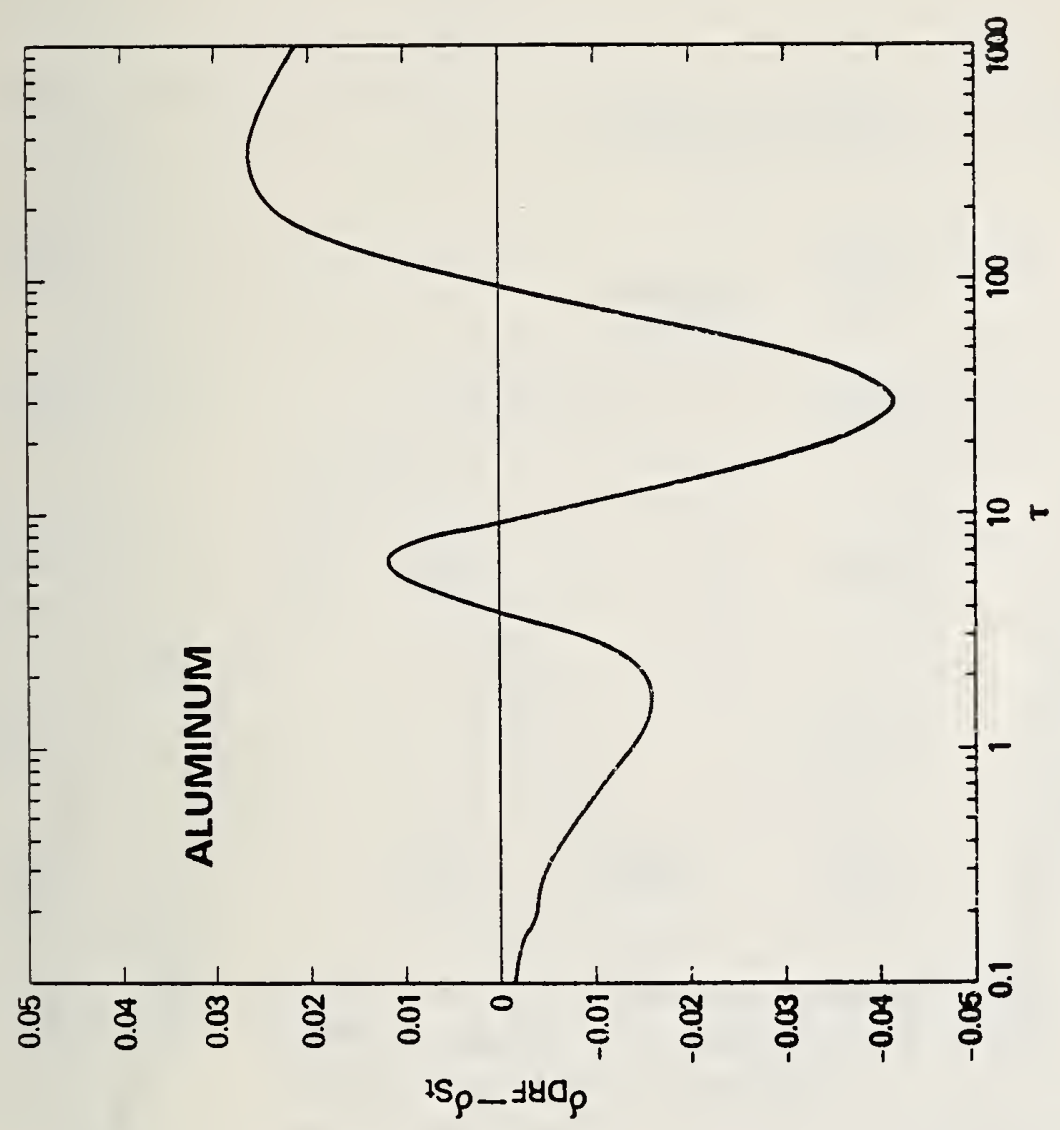

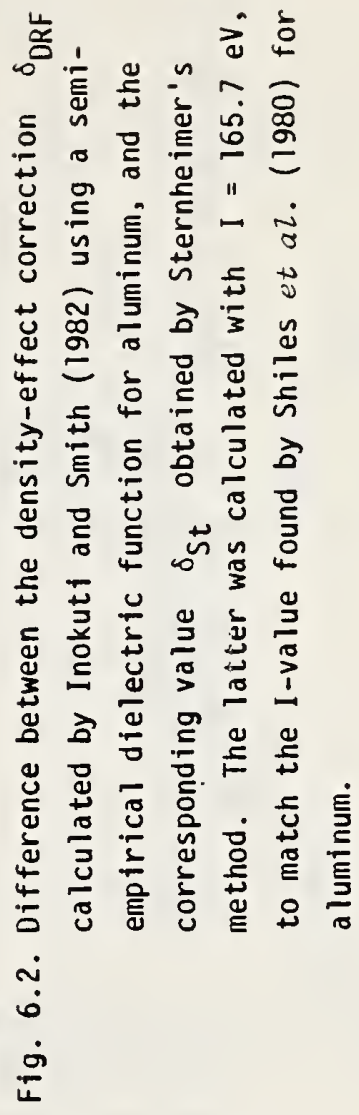
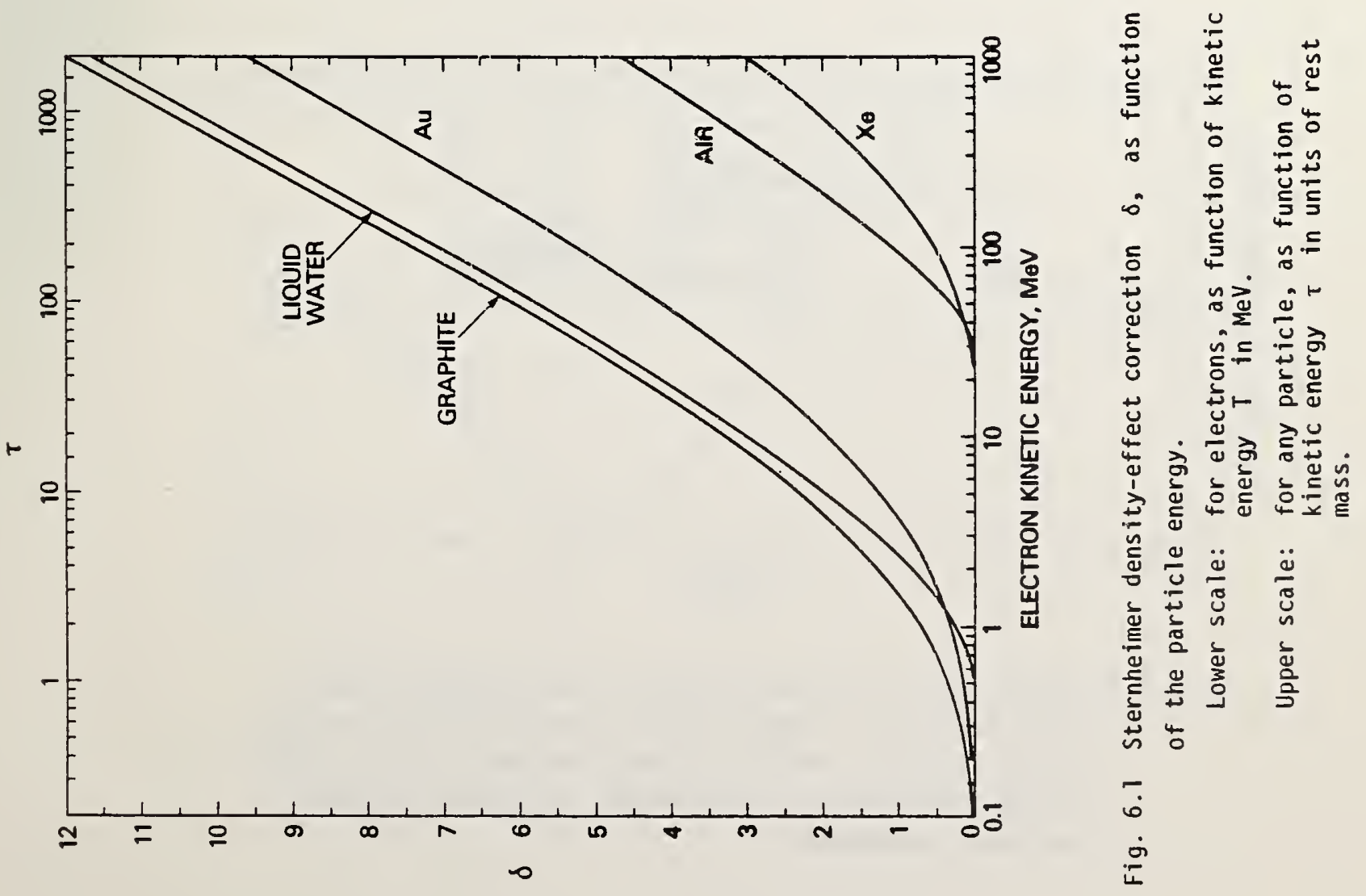


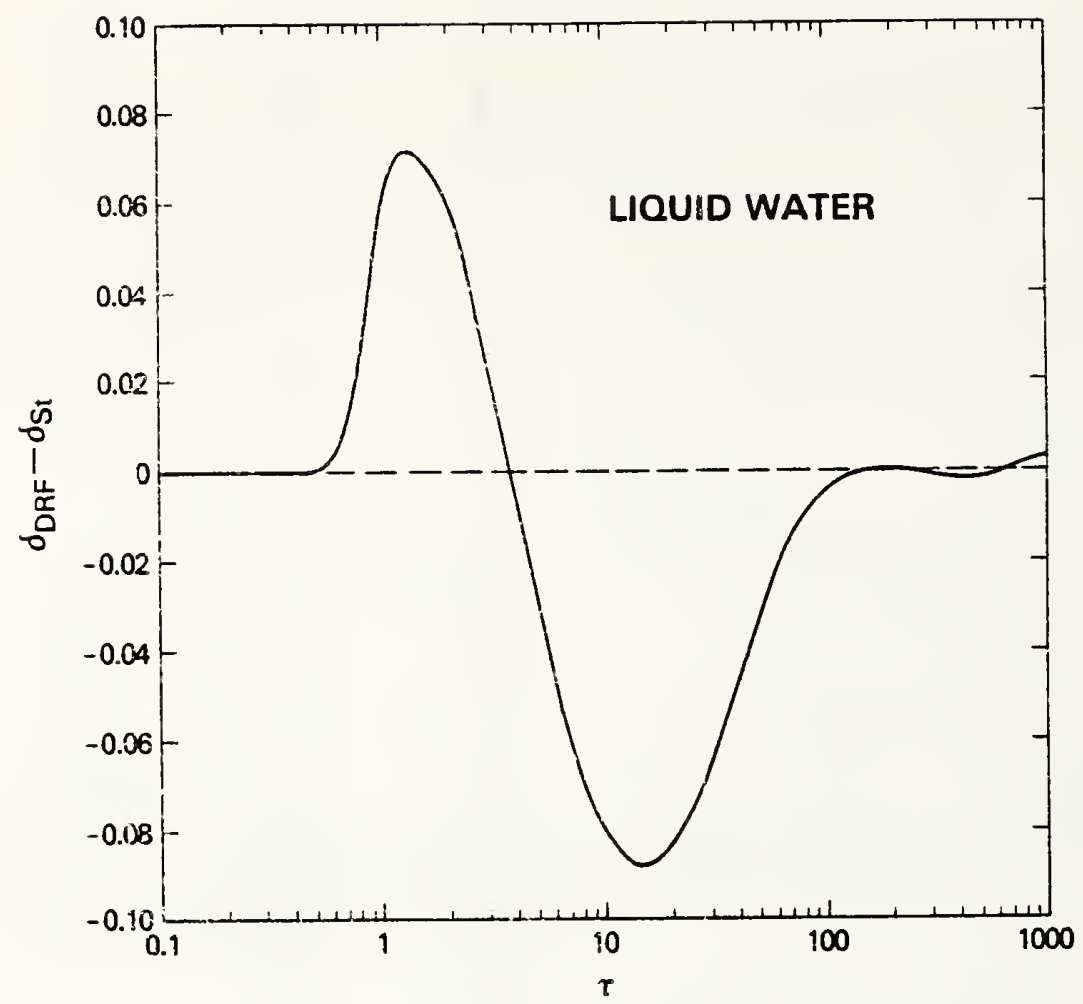

Fig. 6.3. Difference between the density-effect correction $\delta_{D R F}$ calculated by Ashley (i982b) using a semi-empirical dielectric function for water, and the corresponding value ist obtained by Sternheimer's method. The iatter was calculated with $I=75.4 \mathrm{eV}$, to match the I-value for water found by Ashiey.

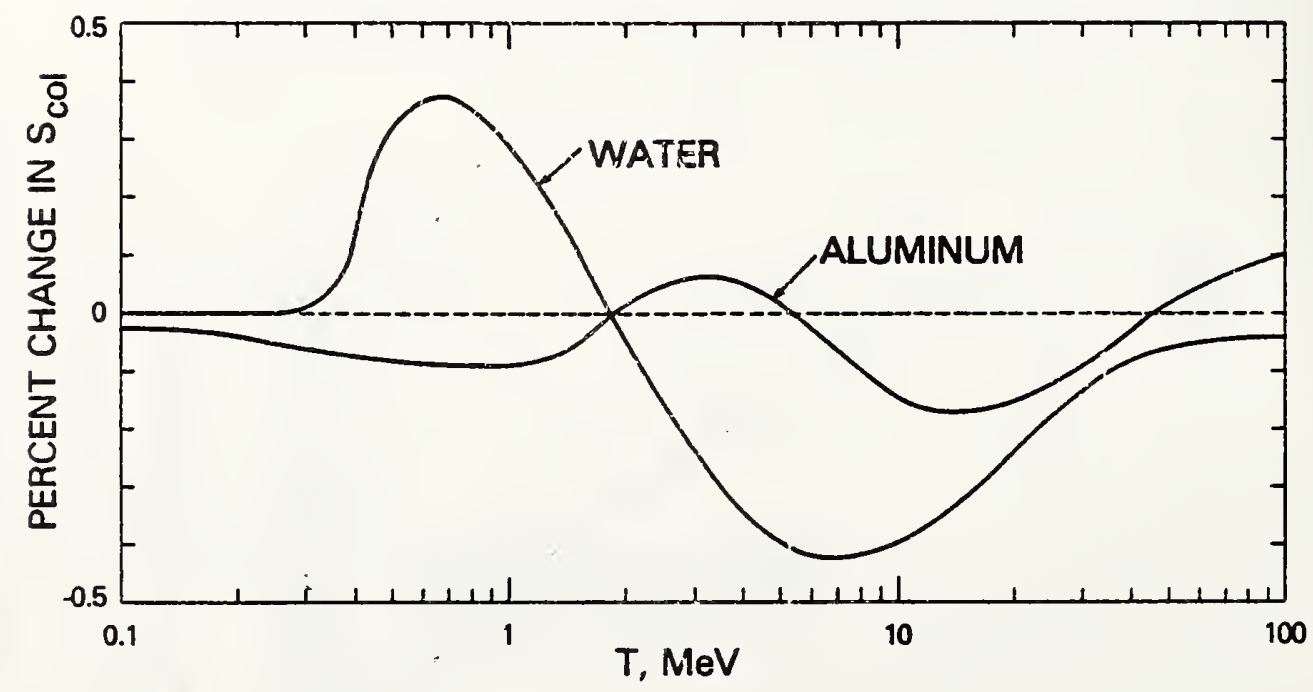

Fig. 6.4. Percent amount by which the electron collision stopping power in aluminum and water is changed, when the density-effect corrections of Inokuti and Smith (1982) for aluminum and that of Ashley (1982b) for water are replaced by density-effect corrections calculated according to Sternheimer. 


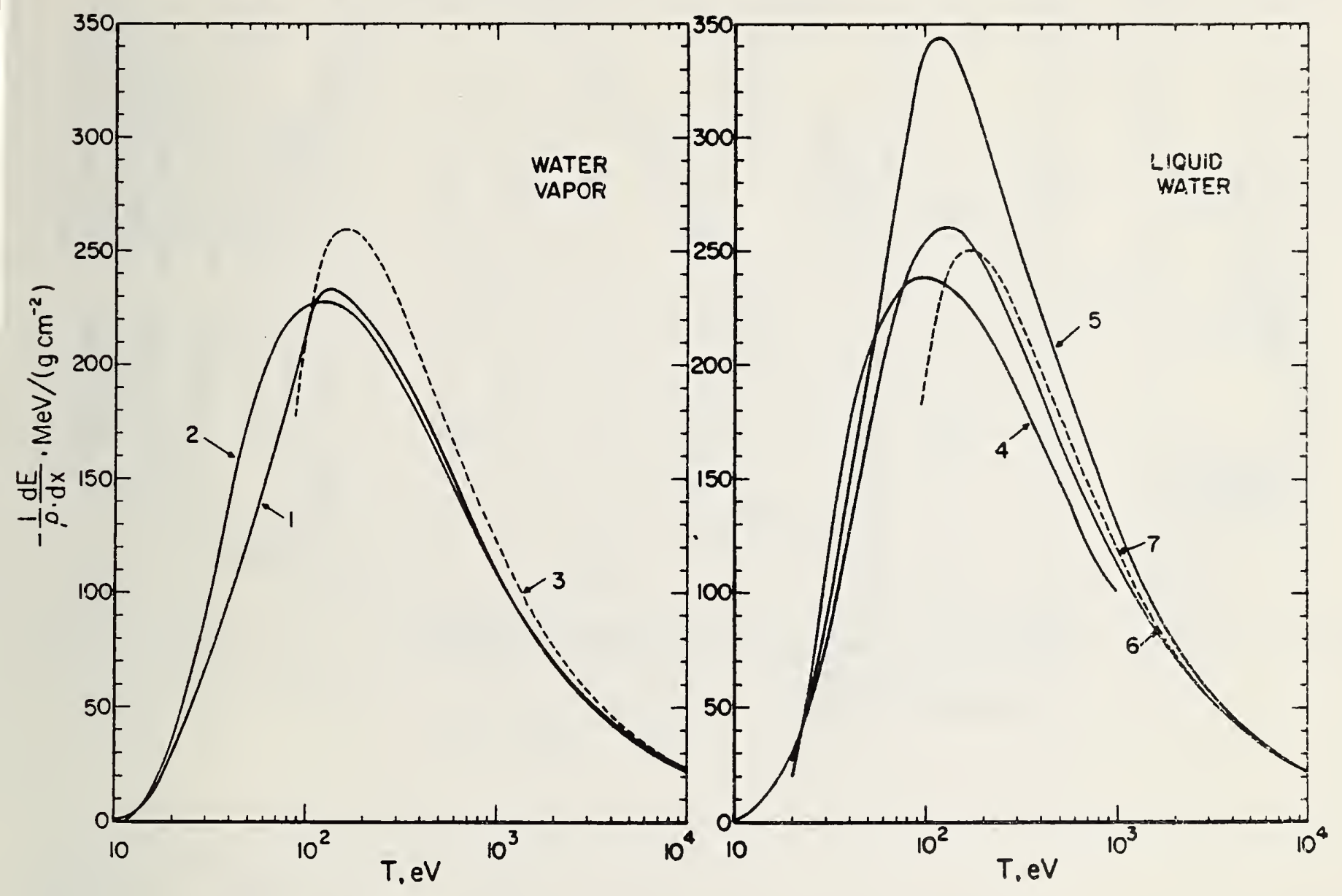

Fig. 8.1. Calculated electron collision stopping power below $10 \mathrm{keV}$ in water.

$\begin{array}{cll}\text { Curve } 1 & \text { water vapor } & \text { Berger (see footnote 16) } \\ 2 & \text { water vapor } & \text { Green (see footnote 17) } \\ 3 & \text { water vapor } & \text { Bethe formula, Eq (2.16), with I } 1=71.6 \mathrm{eV} \\ 4 & \text { liquid water } & \text { Kutcher and Green (1976) } \\ 5 & \text { liquid water } & \text { Ritchie et al. (1978) } \\ 6 & \text { liquid water } & \text { Ashley (1982a) } \\ 7 & \text { liquid water } & \text { Bethe formula, Eq (2.16), with I } I=75.0 \mathrm{eV} .\end{array}$



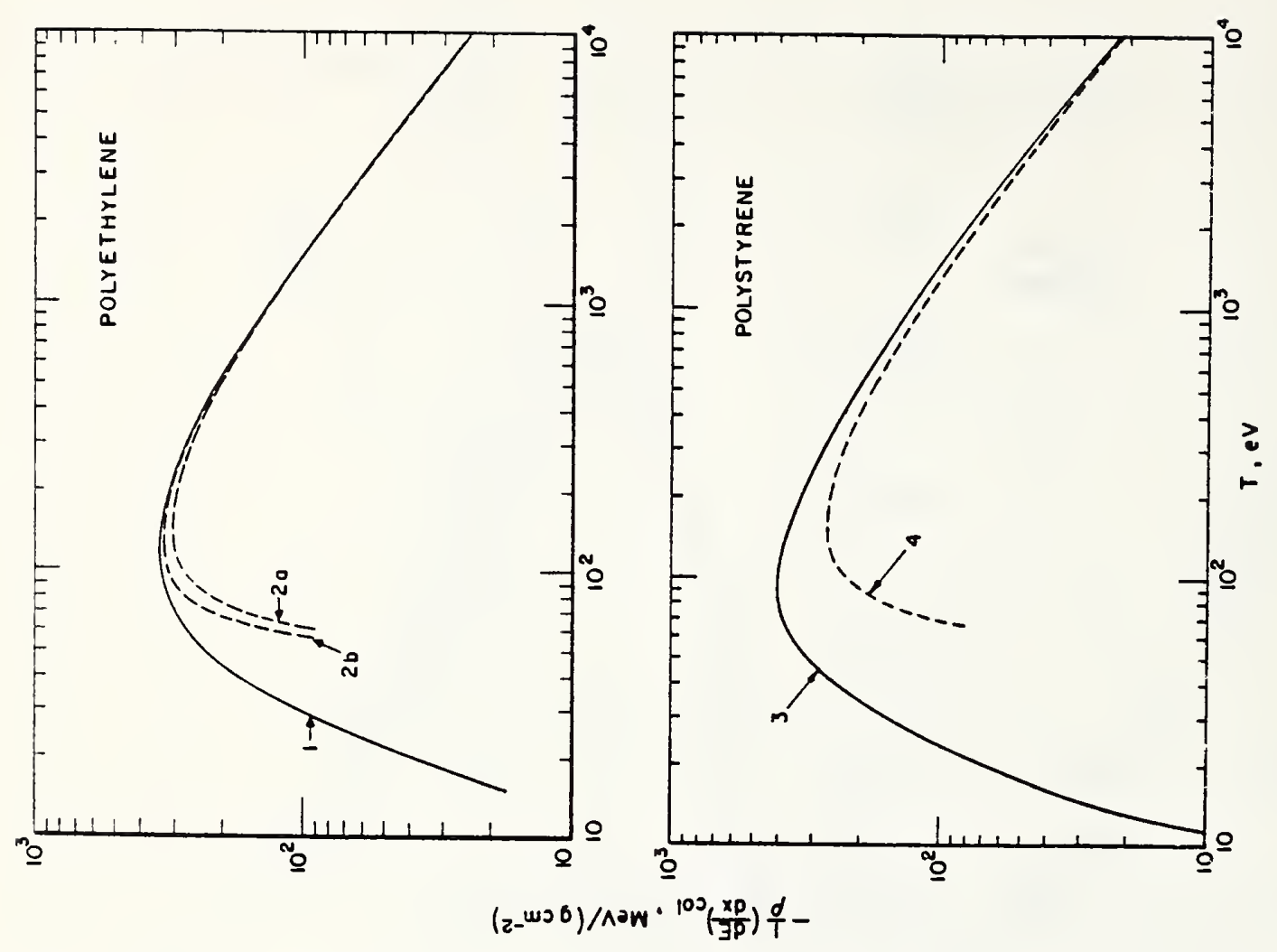

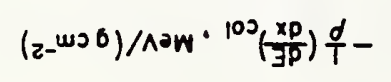
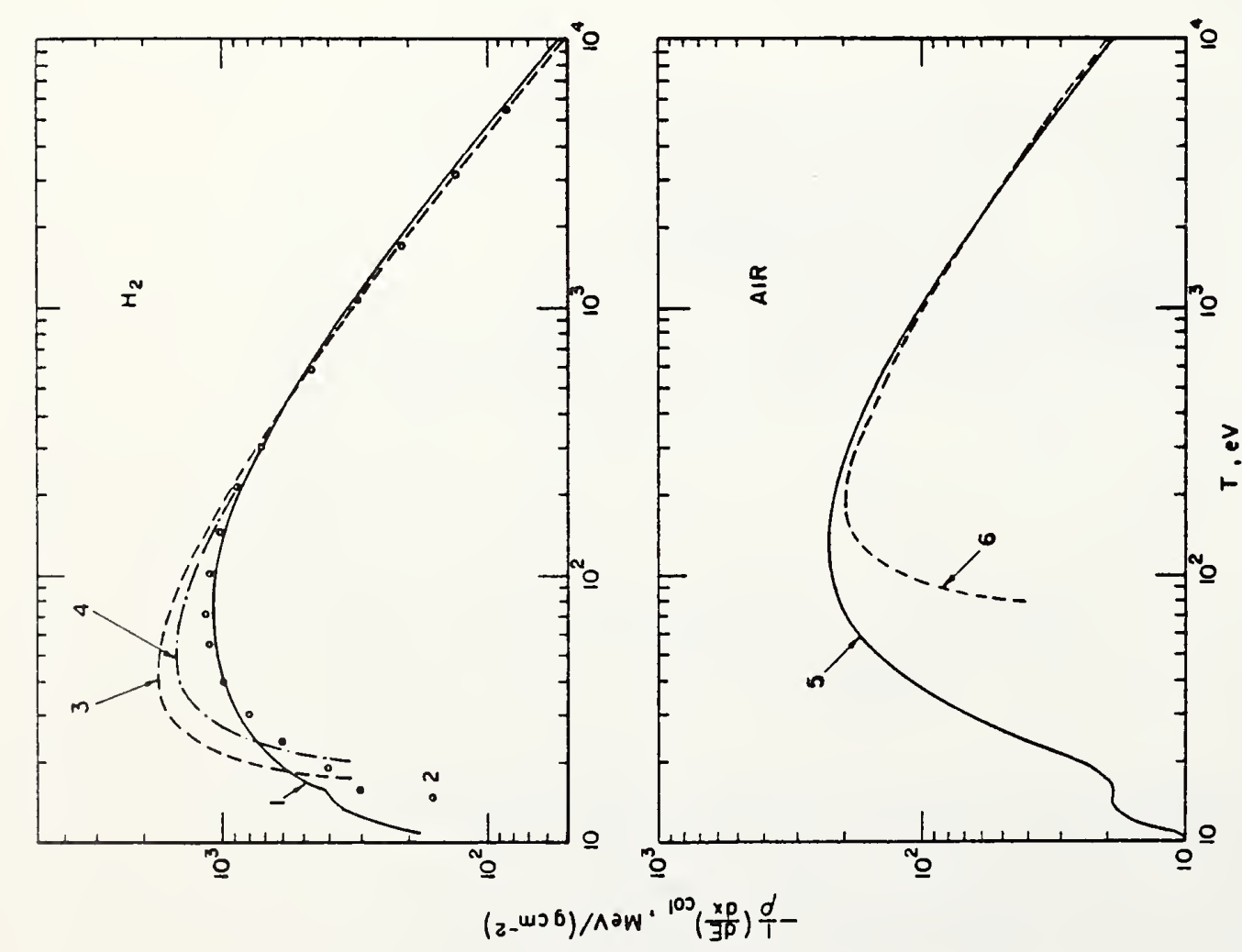
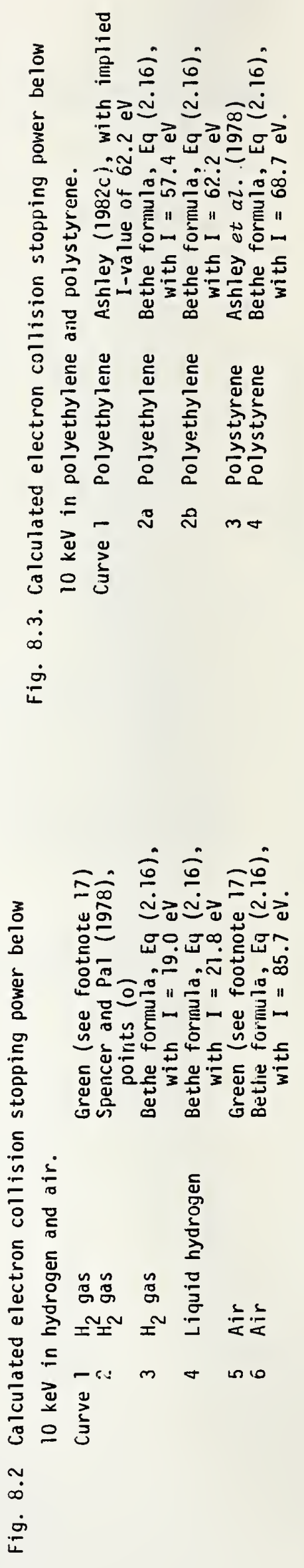

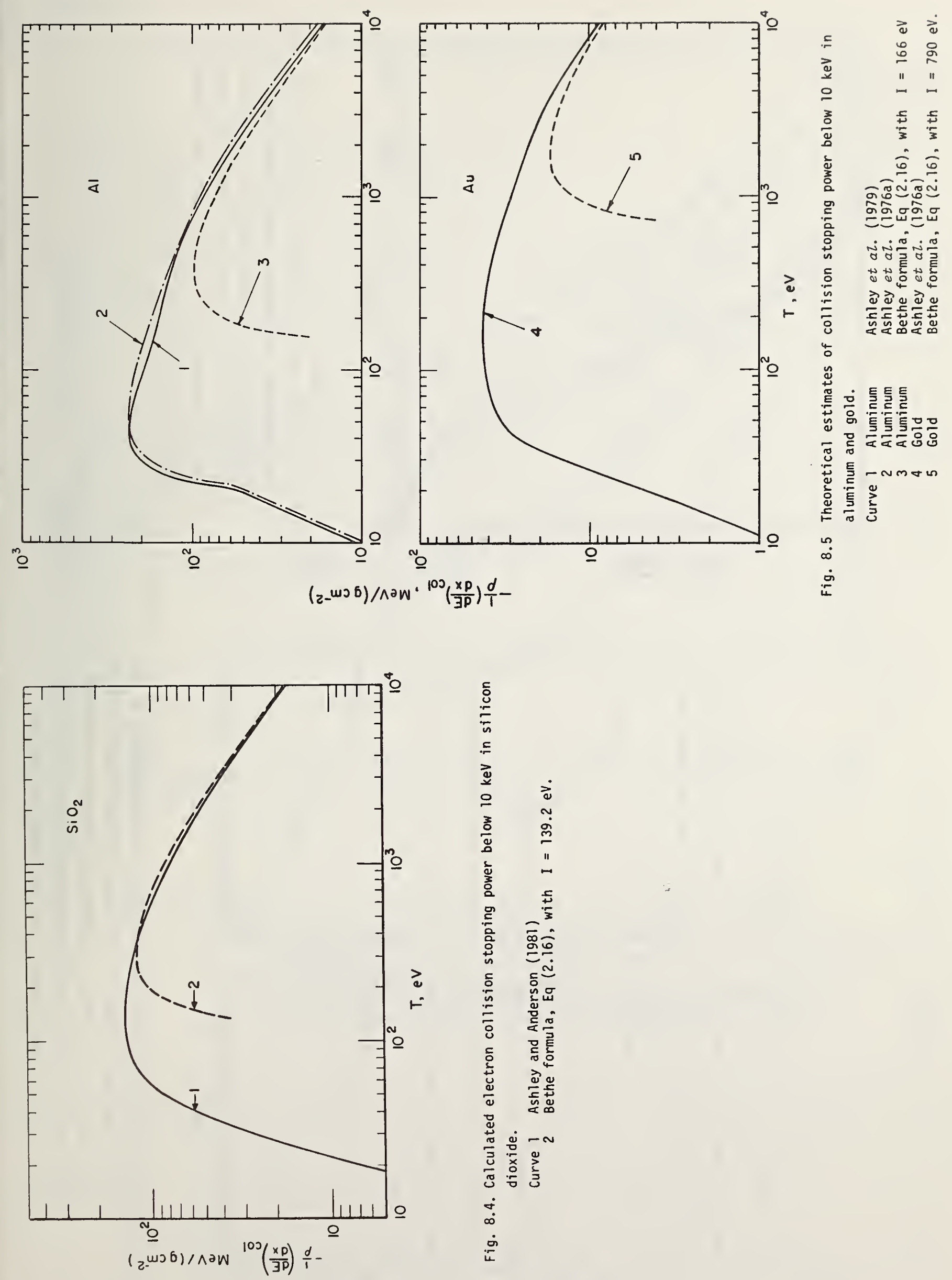

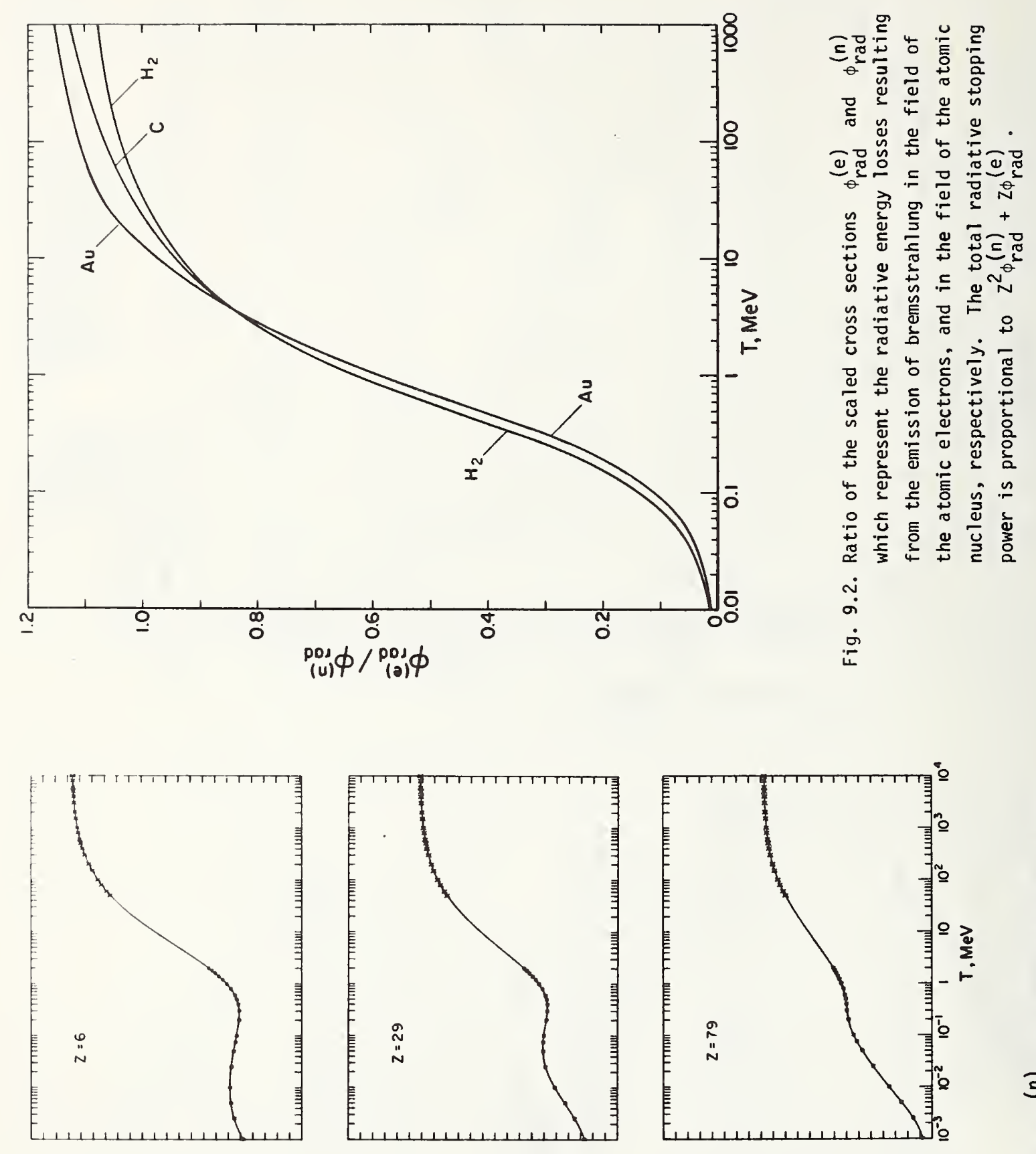

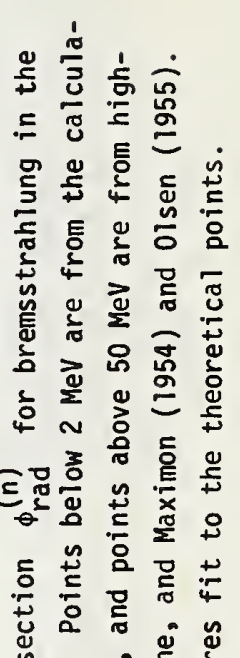
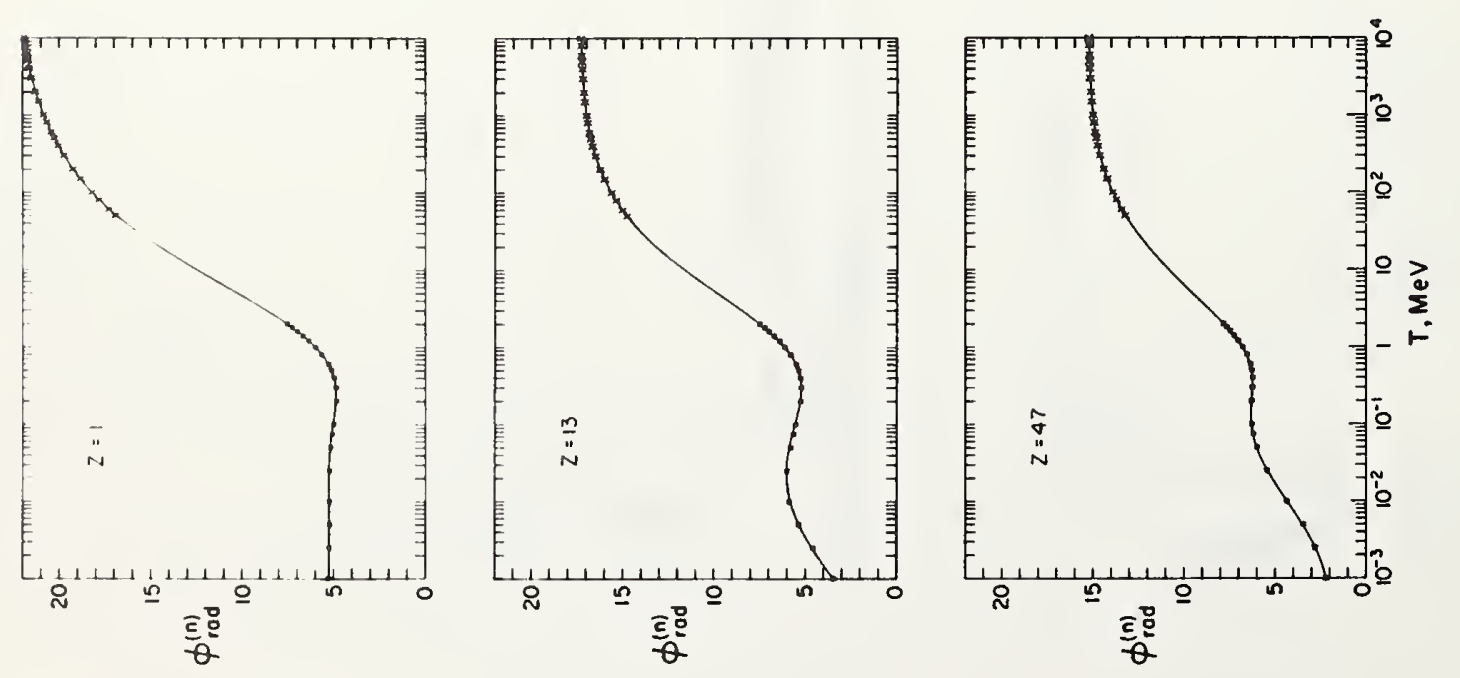

is

若

บั

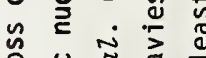

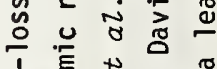

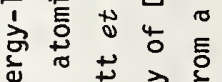

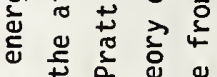

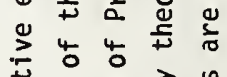

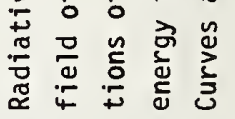

$\dot{\circ}$

i) 


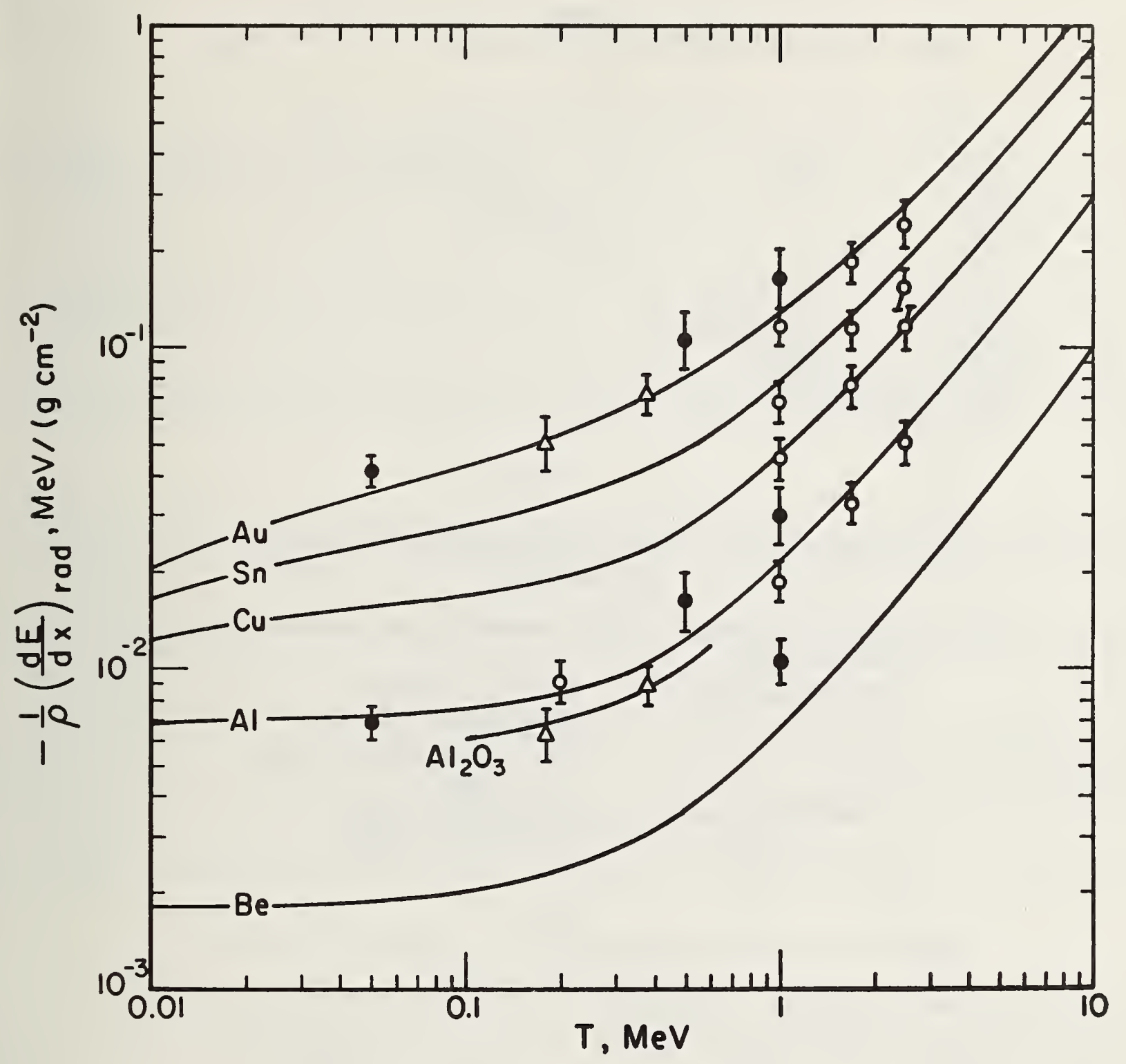

Fig. 9.3. Comparison of theoretical radiative stopping power, calculated according to Eq (9.1), with experimental results.

- Motz (1955) and Motz and Placious (1958), for Be, Al, and Au.

$\triangle$ Aiginger (1966), for $\mathrm{Al}_{2} \mathrm{O}_{3}$ and $\mathrm{Au}$.

- Rester and Dance (1967), Rester and Edmonson (1972) and Rester (private communication) for $\mathrm{Al}, \mathrm{Cu}, \mathrm{Sn}$, and $\mathrm{Au}$. 


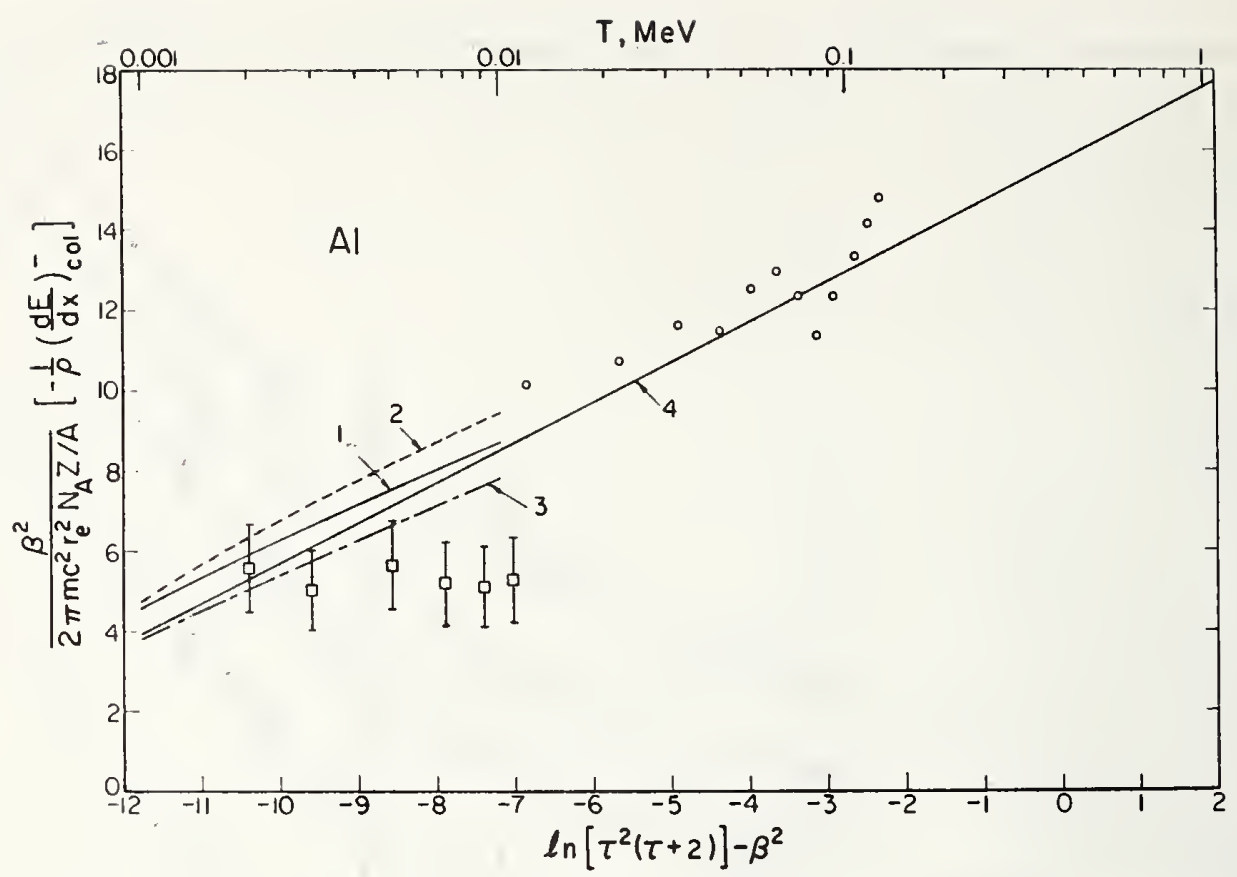

Fig. 11.1 Comparison of theoretical and experimental values of the stopping power in aluminum.

Theoretical results: 1 Ashley et al. (1979)

2 Ashley et ar. (1976a)

3 Ashley et al. (1975)

4 Bethe formula, Eq (2.16), with

$$
\mathrm{I}=166 \mathrm{eV}
$$

Experimental results: 0 Kalil et al. (1959)

Ishigure et al. (1978)

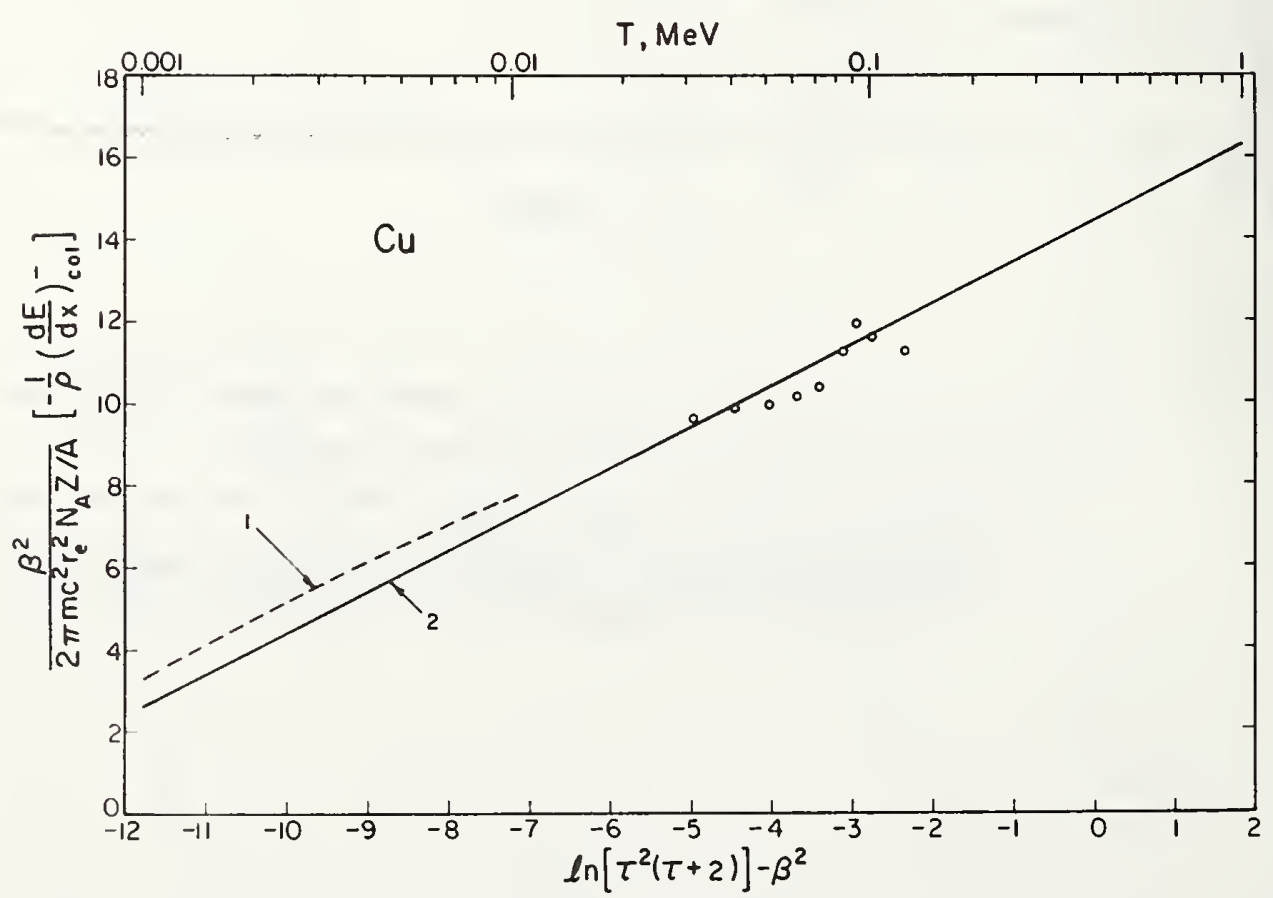

Fig. 11.2 Comparison of theoretical and experimental values of the stopping power in copper.

Theoretical results: $\begin{array}{ll} & \text { Ashley et al. (1976a) } \\ 2 & \text { Bethe formula, Eq (2.16), with }\end{array}$

Experimental results: ○ Ziemer et al. (1959) 


\section{Arrangement of Tables}

A. Electrons in elemental substances. Data are given for electrons in the following elemental substances, in order of increasing atomic number:

$\mathrm{H}_{2}$, lle, Be, C(graphite), $\mathrm{N}_{2}, \mathrm{O}_{2}, \mathrm{Ne}, \mathrm{Al}, \mathrm{Si}, \mathrm{Ar}, \mathrm{Ti}, \mathrm{Fe}, \mathrm{Cu}, \mathrm{Ge}, \mathrm{Kr}, \mathrm{Mo}, \mathrm{Ag}$, $\mathrm{Sn}, \mathrm{Xe}, \mathrm{Gd}, \mathrm{W}, \mathrm{Pt}, \mathrm{Au}, \mathrm{Pb}, \mathrm{U}$.

B. Electrons in mixtures and compounds. Data are given for electrons in mixtures and compounds, arranged in the same sequence in which they are 1 isted in Tab1e 5.5.

C. Positrons in selected materials. Data are given for positrons in graphite, aluminum, copper, silver, lead, air, polymethylmethacrylate, and water.

\section{Content of Tables}

The name of the material, the mean excitation energy I (in eV) and the density $\rho$ (in $\mathrm{g} / \mathrm{cm}^{3}$ ) are given at the head of each table. For gases, the densities are for a pressure of 1 atmosphere $(101.325 \mathrm{kPa})$ and a temperature of $20^{\circ}{ }^{\circ} \mathrm{C}$.

The principal information in each table includes the mass collision stopping power, 1; $\mathrm{\rho} \mathrm{S}_{\mathrm{col}}$, the mass radiative stopping power, $1 / \rho \mathrm{S}_{\mathrm{rad}}$, and the total mass stopping power, $1 / \rho \mathrm{S}_{\mathrm{col}}+1 / \rho \mathrm{S}_{\mathrm{rad}}$ all in units of $\mathrm{MeV} /\left(\mathrm{g} \mathrm{cm}^{-2}\right)$; the c.s.d.a. range, $r_{0}$, in units of $\mathrm{g} \mathrm{cm}^{-2}$; and the radiation yield, $Y$.

The auxiliary information given includes the density-effect correction $\delta$, and the ratio d(log )/d(log I), where the blank space stands for collision stopping power, c.s.d.a. range or radiation yield. This ratio of logarithmic derivatives can be interpreted as the percent change in the respective quantities that would result from a 1 percent change of the mean excitation energy.

Stopping powers, ranges, and yields are expressed in exponential notation, with the number that follows the letter $E$ indicating powers of 10 .

\section{Accuracy}

Above $100 \mathrm{keV}$, the uncertainty of the collision stopping power is estimated to be 1 to 2 percent; between $100 \mathrm{keV}$ and $10 \mathrm{keV}$, the uncertainty is expected to be 2 to 3 percent in $10 \mathrm{w}-Z$ materials, and 5 to 10 percent in high-z materials. The uncertainty of the radiative stopping powers is estimated to be $\sim 2$ percent above $50 \mathrm{MeV}, 2$ to 5 percent between 50 and $2 \mathrm{MeV}$, and $\sim 5$ percent below $2 \mathrm{MeV}$.

\section{Note on Treatment of Density Effect}

The standard procedure used for all materials was to evaluate the density-effect correction to the collision stopping power according to the method of Sternheimer. Electron results using the density-effect correction of Inokuti and Smith (1982) for aluminum, and the density-effect of Ashley (1982b) for water are also given; these are indicated by an asterisk $\left({ }^{*}\right)$ at the head of the tables.

When comparisons are made, e.g., in terms of stopping-power ratios, between the stopping power for water and that for other materials such as tissues, tissuecquivalent materials, ferrous sulfate dosimeter solution or plastics, it is recommended that water results with the Sternheimer density-effect correction be used for the sake of consistency.

For graphite two tables are given: one for a graphite crystallite density of $2.265 \mathrm{~g} / \mathrm{cm}^{3}$, and the other for a typical bulk density of $1.7 \mathrm{~g} / \mathrm{cm}^{3}$. It is recommended that these results be interpolated to the appropriate bulk density for the graphite of interest. 


\begin{tabular}{|c|c|c|c|c|c|c|c|c|c|}
\hline ENERGY & $\begin{array}{l}\text { COLLISION } \\
\mathrm{MeV} \mathrm{cm}^{2} / \mathrm{g}\end{array}$ & $\begin{array}{l}\text { OPPING POWE } \\
\text { RADIATIVE } \\
\text { MeV cm } 2 / \mathrm{g}\end{array}$ & $\begin{array}{c}\text { TOTAL } \\
\mathrm{MeV} \mathrm{cm}^{2} / \mathrm{g}\end{array}$ & $\begin{array}{l}\text { CSDA } \\
\text { RANGE }\end{array}$ & ON & $\begin{array}{l}\text { NS.EFF. } \\
\text { CORR. } \\
\text { DELTA) }\end{array}$ & $\begin{array}{l}d(1 \\
\operatorname{coLL} \\
\text { LOSS }\end{array}$ & $\begin{array}{l}2 / d(1 \\
\text { SDA } \\
\text { A }\end{array}$ & $\begin{array}{l}\text { I ) } \\
\text { RAD } \\
\text { IELD }\end{array}$ \\
\hline $\begin{array}{l}0.0100 \\
0.0125 \\
0.0150 \\
0.0175 \\
0.0200 \\
0.0250 \\
0.0300 \\
0.0350\end{array}$ & $\begin{array}{l}5.125 \mathrm{E}+01 \\
4.271 \mathrm{E}+01 \\
3.682 \mathrm{E}+01 \\
3.249 \mathrm{E}+01 \\
2.917 \mathrm{E}+01 \\
2.439 \mathrm{E}+01 \\
2.110 \mathrm{E}+01 \\
1.870 \mathrm{E}+01\end{array}$ & $\begin{array}{l}9.7 \\
9.88 \\
9.9 \\
1.0 \\
1.0 \\
1.0 \\
1.0\end{array}$ & $\begin{array}{l}4.27 \\
3.68 \\
3.29 \\
2.91 \\
2.43 \\
2.11 \\
1.87\end{array}$ & $\begin{array}{l}1.07 \\
1.61 \\
2.28 \\
2.97 \\
3.78 \\
5.68 \\
7.87 \\
1.0\end{array}$ & $\begin{array}{l}029 E-05 \\
242 E-05 \\
450 E-05 \\
654 E-05 \\
854 E-05 \\
246 E-05 \\
628 E-05 \\
003 E-05\end{array}$ & $\begin{array}{l}0.0 \\
0.0 \\
0.0 \\
0.0 \\
0.0 \\
0.0 \\
0.0 \\
0.0\end{array}$ & & $\begin{array}{l}0.172 \\
0.166 \\
0.161 \\
0.158 \\
0.154 \\
0.149 \\
0.145 \\
0.142\end{array}$ & $\begin{array}{l}0.172 \\
0.166 \\
0.161 \\
0.157 \\
0.154 \\
0.149 \\
0.145 \\
0.142\end{array}$ \\
\hline $\begin{array}{l}0.0400 \\
0.0450 \\
0.0500 \\
0.0550 \\
0.0600 \\
0.0700 \\
0.0800 \\
0.0900\end{array}$ & $\begin{array}{l}1.687 E+01 \\
1.542 E+01 \\
1.424 E+01 \\
1.327 E+01 \\
1.245 E+01 \\
1.114 E+01 \\
1.015 E+01 \\
9.367 E+00\end{array}$ & $\begin{array}{l}1.061 \mathrm{E}-03 \\
1.074 \mathrm{E}-03 \\
1.088 \mathrm{E}-03 \\
1.101 \mathrm{E}-03 \\
1.113 \mathrm{E}-03 \\
1.138 \mathrm{E}-03 \\
1.164 \mathrm{E}-03 \\
1.190 \mathrm{E}-03\end{array}$ & $\begin{array}{l}1.687 E+01 \\
1.542 E+01 \\
1.424 E+01 \\
1.327 E+01 \\
1.245 E+01 \\
1.114 E+01 \\
1.015 E+01 \\
9.368 E+00\end{array}$ & $\begin{array}{l}1.322 \mathrm{E}-03 \\
1.632 \mathrm{E}-03 \\
1.970 \mathrm{E}-03 \\
2.334 \mathrm{E}-03 \\
2.724 \mathrm{E}-03 \\
3.575 \mathrm{E}-03 \\
4.517 \mathrm{E}-03 \\
5.543 \mathrm{E}-03\end{array}$ & $\begin{array}{l}E-05 \\
E-05 \\
E-05 \\
E-05 \\
E-05 \\
E-05 \\
E-05 \\
E-05\end{array}$ & $\begin{array}{l}0.0 \\
0.0 \\
0.0 \\
0.0 \\
0.0 \\
0.0 \\
0.0 \\
0.0\end{array}$ & $\begin{array}{l}-0.129 \\
-0.127 \\
-0.126 \\
-0.124 \\
-0.123 \\
-0.121 \\
-0.119 \\
-0.117\end{array}$ & & $\begin{array}{l}0.139 \\
0.137 \\
0.135 \\
0.134 \\
0.132 \\
0.130 \\
0.127 \\
0.126\end{array}$ \\
\hline $\begin{array}{l}0.1000 \\
0.1250 \\
0.1500 \\
0.1750 \\
0.2000 \\
0.2500 \\
0.3000 \\
0.3500\end{array}$ & $\begin{array}{l}8.737 \mathrm{E}+00 \\
7.590 \mathrm{E}+00 \\
6.819 \mathrm{E}+00 \\
6.266 \mathrm{E}+00 \\
5.851 \mathrm{E}+00 \\
5.275 \mathrm{E}+00 \\
4.898 \mathrm{E}+00 \\
4.635 \mathrm{E}+00\end{array}$ & $\begin{array}{l}1.2 \\
1.3 \\
1.4 \\
1.5 \\
1.6 \\
1.8 \\
2.0\end{array}$ & $\begin{array}{l}8.7 \\
7.5 \\
6.8 \\
6.2 \\
5.8 \\
5.2 \\
4.8 \\
4.6\end{array}$ & $\begin{array}{l}6.6 \\
9.7 \\
1.3 \\
1.7 \\
2.1 \\
3.0 \\
4.0 \\
5.0\end{array}$ & $\begin{array}{l}7 . \\
9: \\
1: \\
1: \\
1 . \\
1 . \\
1 .\end{array}$ & $\begin{array}{l}0.0 \\
0.0 \\
0.0 \\
0.0 \\
0.0 \\
0.0 \\
0.0 \\
0.0\end{array}$ & & & $\begin{array}{l}0.124 \\
0.121 \\
0.118 \\
0.1116 \\
0.1115 \\
0.112 \\
0.1110 \\
0.108\end{array}$ \\
\hline $\begin{array}{l}0.4000 \\
0.4500 \\
0.5000 \\
0.5500 \\
0.6000 \\
0.70000 \\
0.8000 \\
0.9000\end{array}$ & $\begin{array}{l}4.445 E+00 \\
4.302 E+00 \\
4.193 E+00 \\
4.109 E+00 \\
4.042 E+00 \\
3.945 E+00 \\
3.883 E+00 \\
3.842 E+00\end{array}$ & $\begin{array}{l}2.232 \mathrm{E}-0 \\
2.436 \mathrm{E}-0 \\
2.648 \mathrm{E}-0 \\
2.869 \mathrm{E}-0 \\
3.096 \mathrm{E}-0 \\
3.573 \mathrm{E}-0 \\
4.076 \mathrm{E}-0 \\
4.603 \mathrm{E}-0\end{array}$ & $\begin{array}{l}4.447 E+00 \\
4.305 E+00 \\
4.196 E+00 \\
4.111 E+00 \\
4.045 E+00 \\
3.949 E+00 \\
3.887 E+00 \\
3.847 E+00\end{array}$ & $\begin{array}{l}6.159 \mathrm{E}-02 \\
7.303 \mathrm{E}-02 \\
8.480 \mathrm{E}-02 \\
9.684 \mathrm{E}-02 \\
1.091 \mathrm{E}-01 \\
1.341 \mathrm{E}-01 \\
1.597 \mathrm{E}-01 \\
1.856 \mathrm{E}-01\end{array}$ & $\begin{array}{l}2.577 \mathrm{E}-04 \\
2.884 \mathrm{E}-04 \\
3.194 \mathrm{E}-04 \\
3.508 \mathrm{E}-04 \\
3.825 \mathrm{E}-04 \\
4.471 \mathrm{E}-04 \\
5.133 \mathrm{E}-04 \\
5.809 \mathrm{E}-04\end{array}$ & $\begin{array}{l}0.0 \\
0.0 \\
0.0 \\
0.0 \\
0.0 \\
0.0 \\
0.0 \\
0.0\end{array}$ & $\begin{array}{l}-0.100 \\
-0.099 \\
-0.098 \\
-0.097 \\
-0.096 \\
-0.094 \\
-0.093 \\
-0.091\end{array}$ & & $\begin{array}{l}0.106 \\
0.105 \\
0.103 \\
0.102 \\
0.1001 \\
0.1000 \\
0.098 \\
0.097\end{array}$ \\
\hline $\begin{array}{l}1.0000 \\
1.2500 \\
1.5000 \\
1.7500 \\
2.0000 \\
2.5000 \\
3.0000 \\
3.5000\end{array}$ & $\begin{array}{l}3.816 E+00 \\
3.787 E+00 \\
3.788 E+00 \\
3.802 E+00 \\
3.823 E+00 \\
3.873 E+00 \\
3.924 E+00 \\
3.973 E+00\end{array}$ & $\begin{array}{l}5.152 \mathrm{E}-03 \\
6.614 \mathrm{E}-03 \\
8.190 \mathrm{E}-03 \\
9.862 \mathrm{E}-03 \\
1.162 \mathrm{E}-02 \\
1.534 \mathrm{E}-02 \\
1.931 \mathrm{E}-02 \\
2.348 \mathrm{E}-02\end{array}$ & $\begin{array}{l}3.821 \mathrm{E}+00 \\
3.794 \mathrm{E}+00 \\
3.796 \mathrm{E}+00 \\
3.812 \mathrm{E}+00 \\
3.835 \mathrm{E}+00 \\
3.888 \mathrm{E}+00 \\
3.943 \mathrm{E}+00 \\
3.997 \mathrm{E}+00\end{array}$ & $\begin{array}{l}2.117 E-01 \\
2.774 E-01 \\
3.433 E-01 \\
4.090 E-01 \\
4.744 E-01 \\
6.039 E-01 \\
7.316 E-01 \\
8.575 E-01\end{array}$ & $\begin{array}{l}6.501 \mathrm{E}-04 \\
8.289 \mathrm{E}-04 \\
1.016 \mathrm{E}-03 \\
1.209 \mathrm{E}-03 \\
1.409 \mathrm{E}-03 \\
1.824 \mathrm{E}-03 \\
2.257 \mathrm{E}-03 \\
2.703 \mathrm{E}-03\end{array}$ & $\begin{array}{l}0.0 \\
0.0 \\
0.0 \\
0.0 \\
0.0 \\
0.0 \\
0.0 \\
0.0\end{array}$ & $\begin{array}{l}-0.09 \\
-0.08 \\
-0.08 \\
-0.08 \\
-0.08 \\
-0.08 \\
-0.07 \\
-0.07\end{array}$ & & $\begin{array}{l}0.096 \\
0.093 \\
0.091 \\
0.089 \\
0.088 \\
0.086 \\
0.084 \\
0.082\end{array}$ \\
\hline $\begin{array}{l}4.0000 \\
4.5000 \\
5.0000 \\
5.5000 \\
6.0000 \\
7.0000 \\
8.0000 \\
9.0000\end{array}$ & $\begin{array}{l}4.020 E+00 \\
4.063 E+00 \\
4.103 E+00 \\
4.140 E+00 \\
4.175 E+00 \\
4.239 E+00 \\
4.295 E+00 \\
4.345 E+00\end{array}$ & $\begin{array}{l}2.782 \mathrm{E}-02 \\
3.230 \mathrm{E}-02 \\
3.693 \mathrm{E}-02 \\
4.166 \mathrm{E}-02 \\
4.651 \mathrm{E}-02 \\
5.647 \mathrm{E}-02 \\
6.675 \mathrm{E}-02 \\
7.731 \mathrm{E}-02\end{array}$ & $\begin{array}{l}4.047 E+00 \\
4.095 E+00 \\
4.140 E+00 \\
4.182 E+00 \\
4.222 E+00 \\
4.295 E+00 \\
4.361 E+00 \\
4.422 E+00\end{array}$ & $\begin{array}{l}9.818 \mathrm{E}-01 \\
1.105 \mathrm{E}+00 \\
1.226 \mathrm{E}+00 \\
1.346 \mathrm{E}+00 \\
1.465 \mathrm{E}+00 \\
1.700 \mathrm{E}+00 \\
1.931 \mathrm{E}+00 \\
2.159 \mathrm{E}+00\end{array}$ & $\begin{array}{l}3.162 \mathrm{E}-03 \\
3.631 \mathrm{E}-03 \\
4.108 \mathrm{E}-03 \\
4.593 \mathrm{E}-03 \\
5.084 \mathrm{E}-03 \\
6.083 \mathrm{E}-03 \\
7.101 \mathrm{E}-03 \\
8.133 \mathrm{E}-03\end{array}$ & $\begin{array}{r}0.0 \\
0.0 \\
0.0 \\
0.0 \\
-0.0 \\
0.0 \\
0.0 \\
0.0\end{array}$ & & & $\begin{array}{l}0.081 \\
0.080 \\
0.079 \\
0.078 \\
0.077 \\
0.076 \\
0.074 \\
0.073\end{array}$ \\
\hline $\begin{array}{l}10.0000 \\
12.5000 \\
15.0000 \\
17.5000 \\
20.0000 \\
25.0000 \\
30.0000 \\
35.0000\end{array}$ & $\begin{array}{l}4.391 E+00 \\
4.488 E+00 \\
4.569 E+00 \\
4.638 E+00 \\
4.698 E+00 \\
4.799 E+00 \\
4.881 E+00 \\
4.951 E+00\end{array}$ & $\begin{array}{l}1.159 \mathrm{E}-0 \\
1.448 \mathrm{E}-0 \\
1.744 \mathrm{E}-0 \\
2.046 \mathrm{E}-0 \\
2.667 \mathrm{E}-0 \\
3.305 \mathrm{E}-0 \\
3.955 \mathrm{E}-0\end{array}$ & $\begin{array}{l}4.479 E+00 \\
4.604 E+00 \\
4.714 E+00 \\
4.813 E+00 \\
4.903 E+00 \\
5.065 E+00 \\
5.212 E+00 \\
5.347 E+00\end{array}$ & $\begin{array}{l}2.383 E+00 \\
2.934 E+00 \\
3.470 E+00 \\
3.995 E+00 \\
4.5100+00 \\
5.513 E+00 \\
6.485 E+00 \\
7.432 E+00\end{array}$ & $\begin{array}{l}9.177 \mathrm{E}-0 \\
1.183 \mathrm{E}-0 \\
1.451 \mathrm{E}-0 \\
1.722 \mathrm{E}-0 \\
1.994 \mathrm{E}-0 \\
2.540 \mathrm{E}-0 \\
3.084 \mathrm{E}-0 \\
3.625 \mathrm{E}-0\end{array}$ & $\begin{array}{l}0.0 \\
0.0 \\
0.0 \\
0.0 \\
0.0 \\
0.0 \\
0.0 \\
0.0\end{array}$ & & & \\
\hline $\begin{array}{l}40.0000 \\
45.0000 \\
50.0000 \\
55.0000 \\
60.0000 \\
70.0000 \\
80.0000 \\
90.0000\end{array}$ & $\begin{array}{l}5.055 E+00 \\
5.091 E+00 \\
5.120 E+00 \\
5.144 E+00 \\
5.183 E+00 \\
5.213 E+00 \\
5.238 E+00\end{array}$ & $\begin{array}{l}5.28 \\
5.95 \\
6.64 \\
7.32 \\
8.71 \\
1.01 \\
1.15\end{array}$ & $\begin{array}{l}1 E+00 \\
3 E+00 \\
6 E+00 \\
4 E+00 \\
6 E+00 \\
4 E+00 \\
5 E+00 \\
1 E+00\end{array}$ & $\begin{array}{l}9.2 \\
1.0 \\
1.1 \\
1.1 \\
1.3 \\
1.5 \\
1.6\end{array}$ & $\begin{array}{l}4 . \\
5 \\
5 \\
6 \\
7 \\
8 . \\
9\end{array}$ & $\begin{array}{l}9.0 \\
1.5 \\
2.4 \\
3.4 \\
5.5 \\
7.5 \\
9.4\end{array}$ & & & \\
\hline $\begin{array}{l}125.0000 \\
150.0000 \\
175.00000 \\
200.0000 \\
250.00000\end{array}$ & $\begin{array}{l}5.30 \\
5.33 \\
5.35 \\
5.38 \\
5.41 \\
5.44 \\
5.47\end{array}$ & $\begin{array}{l}1.6 \\
2.0 \\
2.3 \\
2.7 \\
3.4 \\
4.2\end{array}$ & $\begin{array}{l}4 E+00 \\
5 E+00 \\
0 E+00 \\
2 E+00 \\
3 E+00 \\
3 E+00 \\
2 E+00 \\
7 E+01\end{array}$ & $\begin{array}{l}1 \\
1 \\
1 \\
1 \\
1 \\
1 \\
1\end{array}$ & $\begin{array}{l}1 . \\
1: \\
1: \\
1 . \\
2 .\end{array}$ & $\begin{array}{l}2.396 \mathrm{E}+ \\
2.828 \mathrm{E}+ \\
3.186 \mathrm{E}+ \\
3.489 \mathrm{E}+\end{array}$ & & & \\
\hline $\begin{array}{l}0.0000 \\
0.0000 \\
0.0000 \\
0.0000 \\
0.0000 \\
0.0000 \\
0.0000\end{array}$ & $\begin{array}{l}5.491 E+00 \\
5.509 E+00 \\
5.526 E+00 \\
5.540 E+00 \\
5.554 E+00 \\
5.577 E+00 \\
5.598 E+00 \\
5.616 E+00\end{array}$ & $\begin{array}{l}5.760 E+00 \\
6.522 E+00 \\
7.286 E+00 \\
8.053 E+00 \\
8.821 E+00 \\
1.036 E+01 \\
1.191 E+01 \\
1.346 E+01\end{array}$ & $\begin{array}{l}125 E+01 \\
203 E+01 \\
281 E+01 \\
359 E+01 \\
438 E+01 \\
594 E+01 \\
751 E+01 \\
908 E+01\end{array}$ & $\begin{array}{l}279 E+01 \\
709 E+01 \\
111 E+01 \\
490 E+01 \\
848 E+01 \\
508 E+01 \\
107 E+01 \\
654 E+01\end{array}$ & $\begin{array}{l}3.34 \\
3.56 \\
3.77 \\
3.96 \\
4.29 \\
4.59 \\
4.85\end{array}$ & $\begin{array}{l}3.987 E+00 \\
4.196 E+00 \\
4.386 E+00 \\
4.559 E+00 \\
4.866 E+00 \\
5.132 E+00 \\
5.367 E+00\end{array}$ & $\begin{array}{l}1 \\
0 \\
0 \\
0 \\
0 \\
0 \\
0 \\
0\end{array}$ & $\begin{array}{l}5 \\
4 \\
3 \\
2 \\
2 \\
1 \\
0 \\
9\end{array}$ & $\begin{array}{l}0.002 \\
0.002 \\
0.002 \\
0.002 \\
0.001 \\
0.001 \\
0.001\end{array}$ \\
\hline (2) & $632 \mathrm{E}+00$ & $.502 E+01$ & $.065 E+01$ & $157 E+01$ & $5.084 \mathrm{E}-01$ & $5.577 E+00$ & -0.000 & 0.009 & 0.001 \\
\hline
\end{tabular}


ELECTRONS IN HELIUM

$I=41.8 \mathrm{eV} \quad$ DENSITY $=1.663 \mathrm{E}-04 \mathrm{~g} / \mathrm{cm}^{3}\left(20^{\circ} \mathrm{C}\right)$

\begin{tabular}{|c|c|c|c|c|c|c|c|c|c|}
\hline ENERGY & $\begin{array}{l}\text { COLLISION } \\
\mathrm{MeV} \mathrm{cm}^{2} / \mathrm{g}\end{array}$ & $\begin{array}{l}\text { DPPING POWE } \\
\text { RADIATIVE } \\
\text { MeV } \mathrm{cm}^{2} / \mathrm{g}\end{array}$ & $\begin{array}{c}\text { TOTAL } \\
\mathrm{MeV} \mathrm{cm}^{2} / \mathrm{g}\end{array}$ & $\begin{array}{l}\text { CSDA } \\
\text { RANGE } \\
\mathrm{g} / \mathrm{Cm}^{2}\end{array}$ & $\begin{array}{l}\text { RADIATION } \\
\text { YIELD }\end{array}$ & $\begin{array}{c}\text { DENS.EFF. } \\
\text { CORR. } \\
\text { (DELTA) }\end{array}$ & $\begin{array}{l}d(1 \\
\operatorname{coLL} \\
\text { LOSS }\end{array}$ & $\begin{array}{l}\text { )/d(1 } \\
\text { CSDA }\end{array}$ & $\begin{array}{l}\text { II ) } \\
\text { RAD } \\
\text { YIELD }\end{array}$ \\
\hline $\begin{array}{l}0.01100 \\
0.0125 \\
0.0150 \\
0.0175 \\
0.0200 \\
0.0250 \\
0.0300 \\
0.0350\end{array}$ & $\begin{array}{l}2.267 \mathrm{E}+01 \\
1.898 \mathrm{E}+01 \\
1.642 \mathrm{E}+01 \\
1.453 \mathrm{E}+01 \\
1.307 \mathrm{E}+01 \\
1.097 \mathrm{E}+01 \\
9.521 \mathrm{E}+00 \\
8.457 \mathrm{E}+00\end{array}$ & $\begin{array}{l}9.885 \mathrm{E}-04 \\
9.941 \mathrm{E}-04 \\
9.995 \mathrm{E}-04 \\
1.005 \mathrm{E}-03 \\
1.010 \mathrm{E}-03 \\
1.020 \mathrm{E}-03 \\
1.029 \mathrm{E}-03 \\
1.039 \mathrm{E}-03\end{array}$ & $\begin{array}{l}2.267 E+01 \\
1.898 E+01 \\
1.642 E+01 \\
1.453 E+01 \\
1.307 E+01 \\
1.097 E+01 \\
9.522 E+00 \\
8.458 E+00\end{array}$ & $\begin{array}{l}2.467 E-04 \\
3.678 \mathrm{E}-04 \\
5.098 \mathrm{E}-04 \\
6.720 \mathrm{E}-04 \\
8.537 \mathrm{E}-04 \\
1.273 \mathrm{E}-03 \\
1.764 \mathrm{E}-03 \\
2.322 \mathrm{E}-03\end{array}$ & $\begin{array}{l}2.412 E-05 \\
2.890 E-05 \\
3.352 E-05 \\
3.802 E-05 \\
4.242 E-05 \\
5.096 E-05 \\
5.923 E-05 \\
6.727 E-05\end{array}$ & $\begin{array}{l}0.0 \\
0.0 \\
0.0 \\
0.0 \\
0.0 \\
0.0 \\
0.0 \\
0.0\end{array}$ & $\begin{array}{l}-0.178 \\
-0.171 \\
-0.166 \\
-0.162 \\
-0.159 \\
-0.154 \\
-0.150 \\
-0.146\end{array}$ & $\begin{array}{l}0.200 \\
0.191 \\
0.185 \\
0.180 \\
0.176 \\
0.169 \\
0.164 \\
0.160\end{array}$ & $\begin{array}{l}0.200 \\
0.191 \\
0.185 \\
0.180 \\
0.176 \\
0.169 \\
0.164 \\
0.160\end{array}$ \\
\hline $\begin{array}{l}0.0400 \\
0.0450 \\
0.0500 \\
0.0550 \\
0.0600 \\
0.0700 \\
0.0800 \\
0.0900\end{array}$ & $\begin{array}{l}7.642 E+00 \\
6.996 E+00 \\
6.471 E+00 \\
6.035 E+00 \\
5.669 E+00 \\
5.084 E+00 \\
4.638 E+00 \\
4.287 E+00\end{array}$ & $\begin{array}{l}1.048 \mathrm{E}-03 \\
1.057 \mathrm{E}-03 \\
1.066 \mathrm{E}-03 \\
1.075 \mathrm{E}-03 \\
1.084 \mathrm{E}-03 \\
1.101 \mathrm{E}-03 \\
1.119 \mathrm{E}-03 \\
1.138 \mathrm{E}-03\end{array}$ & $\begin{array}{l}7.643 \mathrm{E}+00 \\
6.997 \mathrm{E}+00 \\
6.472 \mathrm{E}+00 \\
6.037 \mathrm{E}+00 \\
5.670 \mathrm{E}+00 \\
5.085 \mathrm{E}+00 \\
4.639 \mathrm{E}+00 \\
4.288 \mathrm{E}+00\end{array}$ & $\begin{array}{l}2.945 \mathrm{E}-03 \\
3.630 \mathrm{E}-03 \\
4.374 \mathrm{E}-03 \\
5.174 \mathrm{E}-03 \\
6.029 \mathrm{E}-03 \\
7.896 \mathrm{E}-03 \\
9.958 \mathrm{E}-03 \\
1.220 \mathrm{E}-02\end{array}$ & $\begin{array}{l}7.510 \mathrm{E}-05 \\
8.277 \mathrm{E}-05 \\
9.029 \mathrm{E}-05 \\
9.766 \mathrm{E}-05 \\
1.049 \mathrm{E}-04 \\
1.191 \mathrm{E}-04 \\
1.328 \mathrm{E}-04 \\
1.462 \mathrm{E}-04\end{array}$ & $\begin{array}{l}0.0 \\
0.0 \\
0.0 \\
0.0 \\
0.0 \\
0.0 \\
0.0 \\
0.0\end{array}$ & $\begin{array}{l}-0.144 \\
-0.141 \\
-0.139 \\
-0.138 \\
-0.136 \\
-0.133 \\
-0.131 \\
-0.129\end{array}$ & $\begin{array}{l}0.157 \\
0.154 \\
0.152 \\
0.150 \\
0.148 \\
0.145 \\
0.142 \\
0.140\end{array}$ & $\begin{array}{l}0.157 \\
0.154 \\
0.152 \\
0.150 \\
0.148 \\
0.144 \\
0.142 \\
0.140\end{array}$ \\
\hline $\begin{array}{l}0.1000 \\
0.1250 \\
0.1500 \\
0.1750 \\
0.2000 \\
0.2500 \\
0.3000 \\
0.3500\end{array}$ & $\begin{array}{l}4.003 E+00 \\
3.486 E+00 \\
3.137 E+00 \\
2.887 E+00 \\
2.700 E+00 \\
2.439 E+00 \\
2.269 E+00 \\
2.150 E+00\end{array}$ & $\begin{array}{l}1.157 \mathrm{E}-03 \\
1.207 \mathrm{E}-03 \\
1.260 \mathrm{E}-03 \\
1.316 \mathrm{E}-03 \\
1.375 \mathrm{E}-03 \\
1.499 \mathrm{E}-03 \\
1.632 \mathrm{E}-03 \\
1.774 \mathrm{E}-03\end{array}$ & $\begin{array}{l}4.004 E+00 \\
3.487 E+00 \\
3.139 E+00 \\
2.889 E+00 \\
2.701 E+00 \\
2.441 E+00 \\
2.270 E+00 \\
2.152 E+00\end{array}$ & $\begin{array}{l}1.462 \mathrm{E}-02 \\
2.134 \mathrm{E}-02 \\
2.891 \mathrm{E}-02 \\
3.723 \mathrm{E}-02 \\
4.619 \mathrm{E}-02 \\
6.573 \mathrm{E}-02 \\
8.702 \mathrm{E}-02 \\
1.097 \mathrm{E}-01\end{array}$ & $\begin{array}{l}1.593 \mathrm{E}-04 \\
1.910 \mathrm{E}-04 \\
2.215 \mathrm{E}-04 \\
2.511 \mathrm{E}-04 \\
2.800 \mathrm{E}-04 \\
3.363 \mathrm{E}-04 \\
3.914 \mathrm{E}-04 \\
4.457 \mathrm{E}-04\end{array}$ & $\begin{array}{l}0.0 \\
0.0 \\
0.0 \\
0.0 \\
0.0 \\
0.0 \\
0.0 \\
0.0\end{array}$ & $\begin{array}{l}-0.128 \\
-0.124 \\
-0.122 \\
-0.119 \\
-0.118 \\
-0.115 \\
-0.112 \\
-0.110\end{array}$ & $\begin{array}{l}0.138 \\
0.134 \\
0.131 \\
0.129 \\
0.127 \\
0.124 \\
0.121 \\
0.119\end{array}$ & $\begin{array}{l}0.138 \\
0.134 \\
0.131 \\
0.128 \\
0.126 \\
0.123 \\
0.120 \\
0.118\end{array}$ \\
\hline $\begin{array}{l}0.4000 \\
0.4500 \\
0.5000 \\
0.5500 \\
0.6000 \\
0.7000 \\
0.8000 \\
0.9000\end{array}$ & $\begin{array}{l}2.064 E+00 \\
2.000 E+00 \\
1.952 E+00 \\
1.914 E+00 \\
1.884 E+00 \\
1.842 E+00 \\
1.815 E+00 \\
1.798 E+00\end{array}$ & $\begin{array}{l}1.924 \mathrm{E}-03 \\
2.081 \mathrm{E}-03 \\
2.245 \mathrm{E}-03 \\
2.416 \mathrm{E}-03 \\
2.592 \mathrm{E}-03 \\
2.961 \mathrm{E}-03 \\
3.350 \mathrm{E}-03 \\
3.757 \mathrm{E}-03\end{array}$ & $\begin{array}{l}2.066 E+00 \\
2.002 E+00 \\
1.954 E+00 \\
1.916 E+00 \\
1.887 E+00 \\
1.845 E+00 \\
1.818 E+00 \\
1.801 E+00\end{array}$ & $\begin{array}{l}1.334 \mathrm{E}-01 \\
1.580 \mathrm{E}-01 \\
1.833 \mathrm{E}-01 \\
2.092 \mathrm{E}-01 \\
2.355 \mathrm{E}-01 \\
2.891 \mathrm{E}-01 \\
3.437 \mathrm{E}-01 \\
3.990 \mathrm{E}-01\end{array}$ & $\begin{array}{l}4.997 E-04 \\
5.536 E-04 \\
6.076 E-04 \\
6.619 E-04 \\
7.165 E-04 \\
8.269 E-04 \\
9.389 E-04 \\
1.053 E-03\end{array}$ & $\begin{array}{l}0.0 \\
0.0 \\
0.0 \\
0.0 \\
0.0 \\
0.0 \\
0.0 \\
0.0\end{array}$ & $\begin{array}{l}-0.109 \\
-0.107 \\
-0.106 \\
-0.104 \\
-0.103 \\
-0.101 \\
-0.100 \\
-0.098\end{array}$ & $\begin{array}{l}0.1117 \\
0.116 \\
0.114 \\
0.113 \\
0.112 \\
0.1110 \\
0.109 \\
0.107\end{array}$ & $\begin{array}{l}0.116 \\
0.114 \\
0.1113 \\
0.112 \\
0.110 \\
0.108 \\
0.106 \\
0.105\end{array}$ \\
\hline $\begin{array}{l}1.0000 \\
1.2500 \\
1.5000 \\
1.7500 \\
2.0000 \\
2.5000 \\
3.0000 \\
3.5000\end{array}$ & $\begin{array}{l}1.787 E+00 \\
1.777 E+00 \\
1.780 E+00 \\
1.789 E+00 \\
1.801 E+00 \\
1.827 E+00 \\
1.854 E+00 \\
1.880 E+00\end{array}$ & $\begin{array}{l}4.180 \mathrm{E}-03 \\
5.306 \mathrm{E}-03 \\
6.515 \mathrm{E}-03 \\
7.793 \mathrm{E}-03 \\
9.132 \mathrm{E}-03 \\
1.196 \mathrm{E}-02 \\
1.495 \mathrm{E}-02 \\
1.809 \mathrm{E}-02\end{array}$ & $\begin{array}{l}1.791 E+00 \\
1.782 E+00 \\
1.787 E+00 \\
1.797 E+00 \\
1.810 E+00 \\
1.839 E+00 \\
1.869 E+00 \\
1.898 E+00\end{array}$ & $\begin{array}{l}4.547 E-01 \\
5.947 E-01 \\
7.349 E-01 \\
8.744 E-01 \\
1.013 E+00 \\
1.287 E+00 \\
1.557 E+00 \\
1.822 E+00\end{array}$ & $\begin{array}{l}1.168 \mathrm{E}-03 \\
1.465 \mathrm{E}-03 \\
1.773 \mathrm{E}-03 \\
2.089 \mathrm{E}-03 \\
2.414 \mathrm{E}-03 \\
3.085 \mathrm{E}-03 \\
3.779 \mathrm{E}-03 \\
4.491 \mathrm{E}-03\end{array}$ & $\begin{array}{l}0.0 \\
0.0 \\
0.0 \\
0.0 \\
0.0 \\
0.0 \\
0.0 \\
0.0\end{array}$ & $\begin{array}{l}-0.097 \\
-0.094 \\
-0.092 \\
-0.090 \\
-0.089 \\
-0.087 \\
-0.085 \\
-0.083\end{array}$ & $\begin{array}{l}0.106 \\
0.104 \\
0.102 \\
0.100 \\
0.098 \\
0.096 \\
0.094 \\
0.093\end{array}$ & $\begin{array}{l}0.103 \\
0.101 \\
0.098 \\
0.096 \\
0.095 \\
0.092 \\
0.090 \\
0.088\end{array}$ \\
\hline $\begin{array}{l}4.0000 \\
4.5000 \\
5.0000 \\
5.5000 \\
6.0000 \\
7.0000 \\
8.00000 \\
9.00000\end{array}$ & $\begin{array}{l}1.903 E+00 \\
1.925 E+00 \\
1.946 E+00 \\
1.965 E+00 \\
1.983 E+00 \\
2.015 E+00 \\
2.043 E+00 \\
2.069 E+00\end{array}$ & $\begin{array}{l}2.134 \mathrm{E}-02 \\
2.469 \mathrm{E}-02 \\
2.813 \mathrm{E}-02 \\
3.165 \mathrm{E}-02 \\
3.523 \mathrm{E}-02 \\
4.260 \mathrm{E}-02 \\
5.018 \mathrm{E}-02 \\
5.793 \mathrm{E}-02\end{array}$ & $\begin{array}{l}1.925 \mathrm{E}+00 \\
1.950 \mathrm{E}+00 \\
1.974 \mathrm{E}+00 \\
1.997 \mathrm{E}+00 \\
2.018 \mathrm{E}+00 \\
2.057 \mathrm{E}+00 \\
2.093 \mathrm{E}+00 \\
2.126 \mathrm{E}+00\end{array}$ & $\begin{array}{l}2.084 E+00 \\
2.342 E+00 \\
2.597 E+00 \\
2.848 E+00 \\
3.098 E+00 \\
3.588 E+00 \\
4.070 E+00 \\
4.544 E+00\end{array}$ & $\begin{array}{l}5.218 \mathrm{E}-03 \\
5.957 \mathrm{E}-03 \\
6.706 \mathrm{E}-03 \\
7.465 \mathrm{E}-03 \\
8.231 \mathrm{E}-03 \\
9.781 \mathrm{E}-03 \\
1.135 \mathrm{E}-02 \\
1.293 \mathrm{E}-02\end{array}$ & $\begin{array}{l}0.0 \\
0.0 \\
0.0 \\
0.0 \\
0.0 \\
0.0 \\
0.0 \\
0.0\end{array}$ & $\begin{array}{l}-0.082 \\
-0.081 \\
-0.080 \\
-0.079 \\
-0.078 \\
-0.077 \\
-0.075 \\
-0.074\end{array}$ & $\begin{array}{l}0.091 \\
0.090 \\
0.089 \\
0.088 \\
0.087 \\
0.088 \\
0.084 \\
0.083\end{array}$ & $\begin{array}{l}0.086 \\
0.085 \\
0.084 \\
0.083 \\
0.082 \\
0.080 \\
0.079 \\
0.077\end{array}$ \\
\hline $\begin{array}{l}10.0000 \\
12.5000 \\
15.0000 \\
17.5000 \\
20.0000 \\
25.0000 \\
30.0000 \\
35.0000\end{array}$ & $\begin{array}{l}2.092 E+00 \\
2.141 E+00 \\
2.182 E+00 \\
2.216 E+00 \\
2.247 E+00 \\
2.297 E+00 \\
2.339 E+00 \\
2.374 E+00\end{array}$ & $\begin{array}{l}6.584 \mathrm{E}-02 \\
8.617 \mathrm{E}-02 \\
1.071 \mathrm{E}-01 \\
1.286 \mathrm{E}-01 \\
1.505 \mathrm{E}-01 \\
1.952 \mathrm{E}-01 \\
2.410 \mathrm{E}-01 \\
2.875 \mathrm{E}-01\end{array}$ & $\begin{array}{l}2.157 \mathrm{E}+00 \\
2.227 \mathrm{E}+00 \\
2.289 \mathrm{E}+00 \\
2.345 \mathrm{E}+00 \\
2.397 \mathrm{E}+00 \\
2.493 \mathrm{E}+00 \\
2.580 \mathrm{E}+00 \\
2.662 \mathrm{E}+00\end{array}$ & $\begin{array}{l}5.011 E+00 \\
6.151 E+00 \\
7.258 E+00 \\
8.337 E+00 \\
9.391 E+00 \\
1.144 E+01 \\
1.341 E+01 \\
1.531 E+01\end{array}$ & $\begin{array}{l}1.453 \mathrm{E}-02 \\
1.855 \mathrm{E}-02 \\
2.258 \mathrm{E}-02 \\
2.662 \mathrm{E}-02 \\
3.064 \mathrm{E}-02 \\
3.863 \mathrm{E}-02 \\
4.651 \mathrm{E}-02 \\
5.426 \mathrm{E}-02\end{array}$ & $\begin{array}{l}0.0 \\
0.0 \\
0.0 \\
0.0 \\
0.0 \\
0.0 \\
0.0 \\
0.0\end{array}$ & $\begin{array}{l}-0.074 \\
-0.072 \\
-0.071 \\
-0.069 \\
-0.068 \\
-0.067 \\
-0.066 \\
-0.065\end{array}$ & $\begin{array}{l}0.082 \\
0.080 \\
0.078 \\
0.077 \\
0.075 \\
0.073 \\
0.071 \\
0.070\end{array}$ & $\begin{array}{l}0.076 \\
0.074 \\
0.072 \\
0.071 \\
0.069 \\
0.067 \\
0.065 \\
0.063\end{array}$ \\
\hline $\begin{array}{l}40.0000 \\
45.0000 \\
50.0000 \\
55.0000 \\
60.0000 \\
70.0000 \\
80.0000 \\
90.0000\end{array}$ & $\begin{array}{l}2.405 E+00 \\
2.432 E+00 \\
2.456 E+00 \\
2.478 E+00 \\
2.498 E+00 \\
2.533 E+00 \\
2.564 E+00 \\
2.589 E+00\end{array}$ & $\begin{array}{l}3.346 \mathrm{E}-01 \\
3.822 \mathrm{E}-01 \\
4.303 \mathrm{E}-01 \\
4.786 \mathrm{E}-01 \\
5.272 \mathrm{E}-01 \\
6.252 \mathrm{E}-01 \\
7.239 \mathrm{E}-01 \\
8.231 \mathrm{E}-01\end{array}$ & $\begin{array}{l}2.739 \mathrm{E}+00 \\
2.814 \mathrm{E}+00 \\
2.886 \mathrm{E}+00 \\
2.956 \mathrm{E}+00 \\
3.025 \mathrm{E}+00 \\
3.158 \mathrm{E}+00 \\
3.288 \mathrm{E}+00 \\
3.412 \mathrm{E}+00\end{array}$ & $\begin{array}{l}1.717 E+01 \\
1.897 E+01 \\
2.072 E+01 \\
2.243 E+01 \\
2.410 E+01 \\
2.734 E+01 \\
3.044 E+01 \\
3.343 E+01\end{array}$ & $\begin{array}{l}6.187 \mathrm{E}-02 \\
6.933 \mathrm{E}-02 \\
7.665 \mathrm{E}-02 \\
8.382 \mathrm{E}-02 \\
9.085 \mathrm{E}-02 \\
1.045 \mathrm{E}-01 \\
1.176 \mathrm{E}-01 \\
1.301 \mathrm{E}-01\end{array}$ & $\begin{array}{l}0.0 \\
0.0 \\
0.0 \\
0.0 \\
0.0 \\
0.0 \\
0.0 \\
2.142 \mathrm{E}-02\end{array}$ & $\begin{array}{l}-0.064 \\
-0.063 \\
-0.063 \\
-0.062 \\
-0.062 \\
-0.061 \\
-0.060 \\
-0.048\end{array}$ & $\begin{array}{l}0.068 \\
0.067 \\
0.066 \\
0.065 \\
0.064 \\
0.062 \\
0.061 \\
0.059\end{array}$ & $\begin{array}{l}0.062 \\
0.060 \\
0.059 \\
0.058 \\
0.057 \\
0.055 \\
0.054 \\
0.051\end{array}$ \\
\hline $\begin{array}{l}.0000 \\
.00000 \\
.0000 \\
.0000 \\
.0000 \\
.00000 \\
.00000 \\
.00000\end{array}$ & $\begin{array}{l}2.609 E+00 \\
2.644 E+00 \\
2.668 E+00 \\
2.686 E+00 \\
2.700 E+00 \\
2.722 E+00 \\
2.738 E+00 \\
2.752 E+00\end{array}$ & $\begin{array}{l}9.22 \\
1.12 \\
1.42 \\
1.68 \\
1.93 \\
2.45 \\
2.98 \\
3.48\end{array}$ & $\begin{array}{l}3.532 E+00 \\
3.818 E+00 \\
4.095 E+00 \\
4.367 E+00 \\
4.637 E+00 \\
5.173 E+00 \\
5.705 E+00 \\
6.237 E+00\end{array}$ & $\begin{array}{l}.631 E+01 \\
.311 E+01 \\
.943 E+01 \\
.534 E+01 \\
.090 E+01 \\
.110 E+01 \\
.030 E+01 \\
.868 E+01\end{array}$ & $\begin{array}{l}01 \\
01 \\
01 \\
01 \\
01 \\
01 \\
01 \\
01 \\
01\end{array}$ & $\begin{array}{l}7.841 E-02 \\
2.879 E-01 \\
5.234 E-01 \\
7.535 E-01 \\
9.696 \mathrm{E}-01 \\
1.356 \mathrm{E}+00 \\
1.688 \mathrm{E}+00 \\
1.976 \mathrm{E}+00\end{array}$ & $\begin{array}{l}-0.039 \\
-0.024 \\
-0.017 \\
-0.012 \\
-0.009 \\
-0.006 \\
-0.004 \\
-0.003\end{array}$ & $\begin{array}{l}0.057 \\
0.051 \\
0.047 \\
0.043 \\
0.039 \\
0.034 \\
0.031 \\
0.028\end{array}$ & $\begin{array}{l}0.048 \\
0.039 \\
0.032 \\
0.027 \\
0.023 \\
0.017 \\
0.013 \\
0.011\end{array}$ \\
\hline $\begin{array}{l}.0000 \\
.0000 \\
.0000 \\
.0000 \\
.0000 \\
.0000\end{array}$ & $\begin{array}{l}2.763 E+00 \\
2.773 E+00 \\
2.781 E+00 \\
2.789 E+00 \\
2.796 E+00 \\
2.808 E+00 \\
2.818 E+00 \\
2.828 E+00\end{array}$ & $\begin{array}{l}4.527 \mathrm{E}+ \\
5.049 \mathrm{E}+ \\
5.573 \mathrm{Et} \\
6.097 \mathrm{E} \\
7.147 \mathrm{E} \\
8.199 \mathrm{E}+ \\
9.253 \mathrm{E}\end{array}$ & $\begin{array}{l}E+00 \\
E+00 \\
E+00 \\
E+00 \\
E+00 \\
E+01 \\
E+01\end{array}$ & $\begin{array}{l}02 \\
02 \\
02 \\
02 \\
02 \\
02 \\
02 \\
02\end{array}$ & $\begin{array}{l}4.27 \\
4.48 \\
4.67 \\
5.00 \\
5.29\end{array}$ & $\begin{array}{l}2.457 \mathrm{E} \\
2.661 \mathrm{E} \\
2.847 \mathrm{E} \\
3.017 \mathrm{E} \\
3.321 \mathrm{E} \\
3.584 \mathrm{E}\end{array}$ & $\begin{array}{l}-0.002 \\
-0.002 \\
-0.002 \\
-0.001 \\
-0.001 \\
-0.001 \\
-0.001 \\
-0.001\end{array}$ & $\begin{array}{l}0.026 \\
0.024 \\
0.023 \\
0.022 \\
0.021 \\
0.019 \\
0.018 \\
0.017\end{array}$ & $\begin{array}{l}0.009 \\
0.007 \\
0.006 \\
0.006 \\
0.005 \\
0.004 \\
0.003 \\
0.003\end{array}$ \\
\hline .0000 & $2.836 E+00$ & $1.031 E+01$ & $1.314 E+01$ & $1.588 E+02$ & $5.768 E-01$ & $4.027 \mathrm{E}+00$ & -0.000 & 0.016 & 0.003 \\
\hline
\end{tabular}


ELECTRONS IN BERYLLIUM

$I=63.7 \mathrm{eV} \quad$ DENSITY $=1.848 \mathrm{E}+00 \mathrm{~g} / \mathrm{Cm}^{3}$

\begin{tabular}{|c|c|c|c|c|c|c|c|c|c|}
\hline NERGY & $\begin{array}{l}\text { ST } \\
\text { COLLISION } \\
\mathrm{MeV} \mathrm{cm}^{2} / \mathrm{g}\end{array}$ & 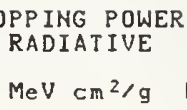 & 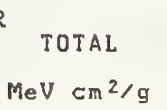 & $\begin{array}{l}\text { CSDA } \\
\text { RANGE } \\
\mathrm{g} / \mathrm{cm}^{2}\end{array}$ & $\begin{array}{l}\text { DIATION } \\
\text { YIELD }\end{array}$ & $\begin{array}{l}\text { ENS.EFF. } \\
\text { CORR. } \\
\text { (DELTA) }\end{array}$ & $\begin{array}{l}d(1 \\
\text { CoLL } \\
\text { LOSS }\end{array}$ & SDA & $\begin{array}{l}\text { I ) } \\
\text { RAD } \\
\text { YIELD }\end{array}$ \\
\hline $\begin{array}{l}0.0100 \\
0.0125 \\
0.0150 \\
0.0175 \\
0.0200 \\
0.0250 \\
0.0300 \\
0.0350\end{array}$ & $\begin{array}{l}1.862 E+01 \\
1.564 E+01 \\
1.356 E+01 \\
1.202 E+01 \\
1.083 E+01 \\
9.113 E+00 \\
7.923 E+00 \\
7.047 E+00\end{array}$ & $\begin{array}{l}1.815 \\
1.819 \\
1.823 \\
1.827 \\
1.831 \\
1.840 \\
1.849 \\
1.859\end{array}$ & $\begin{array}{l}1.863 E+01 \\
1.564 E+01 \\
1.356 E+01 \\
1.202 E+01 \\
1.084 E+01 \\
9.115 E+00 \\
7.925 E+00 \\
7.049 E+00\end{array}$ & $\begin{array}{l}033 \mathrm{E}-04 \\
504 \mathrm{E}-04 \\
226 \mathrm{E}-04 \\
187 \mathrm{E}-04 \\
038 \mathrm{E}-03 \\
544 \mathrm{E}-03 \\
134 \mathrm{E}-03 \\
804 \mathrm{E}-03\end{array}$ & $\begin{array}{l}437 \mathrm{E}-05 \\
488 \mathrm{E}-05 \\
496 \mathrm{E}-05 \\
472 \mathrm{E}-05 \\
419 \mathrm{E}-05 \\
125 \mathrm{E}-04 \\
300 \mathrm{E}-04 \\
.469 \mathrm{E}-04\end{array}$ & $\begin{array}{l}5.100 \mathrm{E}-04 \\
7.492 \mathrm{E}-04 \\
1.031 \mathrm{E}-03 \\
1.354 \mathrm{E}-03 \\
1.718 \mathrm{E}-03 \\
2.564 \mathrm{E}-03 \\
3.560 \mathrm{E}-03 \\
4.701 \mathrm{E}-03\end{array}$ & $\begin{array}{l}-0.193 \\
-0.185 \\
-0.179 \\
-0.174 \\
-0.170 \\
-0.164 \\
-0.159 \\
-0.156\end{array}$ & $\begin{array}{l}0.219 \\
0.209 \\
0.201 \\
0.195 \\
0.190 \\
0.183 \\
0.177 \\
0.172\end{array}$ & $\begin{array}{l}0.218 \\
0.208 \\
0.201 \\
0.195 \\
0.190 \\
0.182 \\
0.177 \\
0.172\end{array}$ \\
\hline $\begin{array}{l}0.0400 \\
0.0450 \\
0.0500 \\
0.0550 \\
0.0600 \\
0.0700 \\
0.0800 \\
0.0900\end{array}$ & $\begin{array}{l}6.375 \mathrm{E}+00 \\
5.841 \mathrm{E}+00 \\
5.407 \mathrm{E}+00 \\
5.047 \mathrm{E}+00 \\
4.743 \mathrm{E}+00 \\
4.257 \mathrm{E}+00 \\
3.887 \mathrm{E}+00 \\
3.594 \mathrm{E}+00\end{array}$ & $\begin{array}{l}1.870 \mathrm{E}-03 \\
1.880 \mathrm{E}-03 \\
1.891 \mathrm{E}-03 \\
1.902 \mathrm{E}-03 \\
1.914 \mathrm{E}-03 \\
1.937 \mathrm{E}-03 \\
1.961 \mathrm{E}-03 \\
1.987 \mathrm{E}-03\end{array}$ & $\begin{array}{l}6.376 \mathrm{E}+00 \\
5.843 \mathrm{E}+00 \\
5.409 \mathrm{E}+00 \\
5.049 \mathrm{E}+00 \\
4.745 \mathrm{E}+00 \\
4.259 \mathrm{E}+00 \\
3.889 \mathrm{E}+00 \\
3.596 \mathrm{E}+00\end{array}$ & $\begin{array}{l}E-03 \\
E-03 \\
E-03 \\
E-03 \\
E-03 \\
E-03 \\
E-03 \\
E-02 \\
E-02\end{array}$ & $\begin{array}{l}1.634 \mathrm{E}-04 \\
1.794 \mathrm{E}-04 \\
1.951 \mathrm{E}-04 \\
2.104 \mathrm{E}-04 \\
2.253 \mathrm{E}-04 \\
2.545 \mathrm{E}-04 \\
2.826 \mathrm{E}-04 \\
3.100 \mathrm{E}-04\end{array}$ & $\begin{array}{l}5.978 \mathrm{E}-03 \\
7.387 \mathrm{E}-03 \\
8.920 \mathrm{E}-03 \\
1.057 \mathrm{E}-02 \\
1.234 \mathrm{E}-02 \\
1.619 \mathrm{E}-02 \\
2.043 \mathrm{E}-02 \\
2.504 \mathrm{E}-02\end{array}$ & $\begin{array}{l}-0.153 \\
-0.150 \\
-0.148 \\
-0.146 \\
-0.144 \\
-0.141 \\
-0.139 \\
-0.137\end{array}$ & $\begin{array}{l}0.168 \\
0.165 \\
0.162 \\
0.160 \\
0.158 \\
0.154 \\
0.151 \\
0.149\end{array}$ & $\begin{array}{l}0.168 \\
0.165 \\
0.162 \\
0.160 \\
0.158 \\
0.154 \\
0.151 \\
0.148\end{array}$ \\
\hline $\begin{array}{l}0.1000 \\
0.1250 \\
0.1500 \\
0.1750 \\
0.2000 \\
0.2500 \\
0.3000 \\
0.3500\end{array}$ & $\begin{array}{l}3.358 E+00 \\
2.926 E+00 \\
2.634 E+00 \\
2.424 E+00 \\
2.266 E+00 \\
2.046 E+00 \\
1.901 E+00 \\
1.800 E+00\end{array}$ & $\begin{array}{l}2.014 \mathrm{E}-03 \\
2.085 \mathrm{E}-03 \\
2.160 \mathrm{E}-03 \\
2.241 \mathrm{E}-03 \\
2.326 \mathrm{E}-03 \\
2.507 \mathrm{E}-03 \\
2.704 \mathrm{E}-03 \\
2.914 \mathrm{E}-03\end{array}$ & $\begin{array}{l}3.360 \mathrm{E}+00 \\
2.928 \mathrm{E}+00 \\
2.636 \mathrm{E}+00 \\
2.426 \mathrm{E}+00 \\
2.269 \mathrm{E}+00 \\
2.049 \mathrm{E}+00 \\
1.904 \mathrm{E}+00 \\
1.803 \mathrm{E}+00\end{array}$ & $\begin{array}{l}1.749 \mathrm{E}-02 \\
2.549 \mathrm{E}-02 \\
3.451 \mathrm{E}-02 \\
4.442 \mathrm{E}-02 \\
5.508 \mathrm{E}-02 \\
7.836 \mathrm{E}-02 \\
1.037 \mathrm{E}-01 \\
1.308 \mathrm{E}-01\end{array}$ & $\begin{array}{l}3.366 \mathrm{E}-04 \\
4.005 \mathrm{E}-04 \\
4.614 \mathrm{E}-04 \\
5.200 \mathrm{E}-04 \\
5.769 \mathrm{E}-04 \\
6.865 \mathrm{E}-04 \\
7.924 \mathrm{E}-04 \\
8.961 \mathrm{E}-04\end{array}$ & $\begin{array}{l}2.997 \mathrm{E}-02 \\
4.354 \mathrm{E}-02 \\
5.858 \mathrm{E}-02 \\
7.478 \mathrm{E}-02 \\
9.188 \mathrm{E}-02 \\
1.280 \mathrm{E}-01 \\
1.658 \mathrm{E}-01 \\
2.043 \mathrm{E}-01\end{array}$ & $\begin{array}{l}-0.135 \\
-0.131 \\
-0.128 \\
-0.126 \\
-0.124 \\
-0.120 \\
-0.1118 \\
-0.115\end{array}$ & $\begin{array}{l}0.147 \\
0.142 \\
0.139 \\
0.136 \\
0.134 \\
0.130 \\
0.127 \\
0.125\end{array}$ & $\begin{array}{l}0.146 \\
0.142 \\
0.138 \\
0.136 \\
0.133 \\
0.129 \\
0.127 \\
0.124\end{array}$ \\
\hline $\begin{array}{l}0.4000 \\
0.4500 \\
0.5000 \\
0.5500 \\
0.6000 \\
0.7000 \\
0.8000\end{array}$ & $\begin{array}{l}1.726 E+00 \\
1.670 E+00 \\
1.627 E+00 \\
1.593 E+00 \\
1.566 E+00 \\
1.527 E+00 \\
1.501 E+00 \\
1.483 E+00\end{array}$ & $\begin{array}{l}3.372 \mathrm{E}-03 \\
3.618 \mathrm{E}-03 \\
3.875 \mathrm{E}-03 \\
4.139 \mathrm{E}-03 \\
4.693 \mathrm{E}-03 \\
5.277 \mathrm{E}-03 \\
5.886 \mathrm{E}-03\end{array}$ & $\begin{array}{l}1.729 E+00 \\
1.673 E+00 \\
1.631 E+00 \\
1.597 E+00 \\
1.571 E+00 \\
1.532 E+00 \\
1.506 E+00 \\
1.489 E+00\end{array}$ & $\begin{array}{l}1.591 \mathrm{E}-01 \\
1.885 \mathrm{E}-01 \\
2.188 \mathrm{E}-01 \\
2.498 \mathrm{E}-01 \\
2.814 \mathrm{E}-01 \\
3.459 \mathrm{E}-01 \\
4.118 \mathrm{E}-01 \\
4.786 \mathrm{E}-01\end{array}$ & $\begin{array}{l}9.985 \mathrm{E}-04 \\
1.100 \mathrm{E}-03 \\
1.202 \mathrm{E}-03 \\
1.304 \mathrm{E}-03 \\
1.406 \mathrm{E}-03 \\
1.612 \mathrm{E}-03 \\
1.821 \mathrm{E}-03 \\
2.033 \mathrm{E}-03\end{array}$ & $\begin{array}{l}2.432 \mathrm{E}-0 \\
2.819 \mathrm{E}-0 \\
3.204 \mathrm{E}-0 \\
3.583 \mathrm{E}-0 \\
3.956 \mathrm{E}-0 \\
4.682 \mathrm{E}-0 \\
5.379 \mathrm{E}-0 \\
6.047 \mathrm{E}-0\end{array}$ & $\begin{array}{l}-0.114 \\
-0.112 \\
-0.110 \\
-0.109 \\
-0.108 \\
-0.105 \\
-0.103 \\
-0.101\end{array}$ & $\begin{array}{l}0.123 \\
0.122 \\
0.120 \\
0.119 \\
0.118 \\
0.115 \\
0.113 \\
0.112\end{array}$ & $\begin{array}{l}0.122 \\
0.120 \\
0.118 \\
0.117 \\
0.1116 \\
0.1113 \\
0.1111 \\
0.109\end{array}$ \\
\hline $\begin{array}{l}1.0000 \\
1.2500 \\
1.5000 \\
1.7500 \\
2.0000 \\
2.5000 \\
3.0000 \\
3.5000\end{array}$ & $\begin{array}{l}1.471 E+00 \\
1.455 E+00 \\
1.450 E+00 \\
1.451 E+00 \\
1.455 E+00 \\
1.466 E+00 \\
1.477 E+00 \\
1.489 E+00\end{array}$ & $\begin{array}{l}6.520 \mathrm{E}-03 \\
8.201 \mathrm{E}-03 \\
1.000 \mathrm{E}-02 \\
1.190 \mathrm{E}-02 \\
1.388 \mathrm{E}-02 \\
1.806 \mathrm{E}-02 \\
2.247 \mathrm{E}-02 \\
2.707 \mathrm{E}-02\end{array}$ & $\begin{array}{l}1.477 E+00 \\
1.463 \mathrm{E}+00 \\
1.460 \mathrm{E}+00 \\
1.463 \mathrm{E}+00 \\
1.469 \mathrm{E}+00 \\
1.484 \mathrm{E}+00 \\
1.500 \mathrm{E}+00 \\
1.516 \mathrm{E}+00\end{array}$ & $\begin{array}{l}8.873 \mathrm{E}-01 \\
1.058 \mathrm{E}+00 \\
1.229 \mathrm{E}+00 \\
1.568 \mathrm{E}+00 \\
1.903 \mathrm{E}+00 \\
2.234 \mathrm{E}+00\end{array}$ & $\begin{array}{l}2.248 \mathrm{E}-03 \\
2.799 \mathrm{E}-03 \\
3.370 \mathrm{E}-03 \\
3.958 \mathrm{E}-03 \\
4.562 \mathrm{E}-03 \\
5.810 \mathrm{E}-03 \\
7.104 \mathrm{E}-03 \\
8.434 \mathrm{E}-03\end{array}$ & $\begin{array}{l}6.687 \mathrm{E}-01 \\
8.178 \mathrm{E}-01 \\
9.534 \mathrm{E}-01 \\
1.078 \mathrm{E}+00 \\
1.194 \mathrm{E}+00 \\
1.405 \mathrm{E}+00 \\
1.596 \mathrm{E}+00 \\
1.772 \mathrm{E}+00\end{array}$ & $\begin{array}{l}-0.099 \\
-0.095 \\
-0.090 \\
-0.086 \\
-0.082 \\
-0.074 \\
-0.066 \\
-0.059\end{array}$ & $\begin{array}{l}0.110 \\
0.107 \\
0.104 \\
0.101 \\
0.099 \\
0.094 \\
0.090 \\
0.086\end{array}$ & $\begin{array}{l}0.107 \\
0.103 \\
0.100 \\
0.096 \\
0.093 \\
0.087 \\
0.081 \\
0.076\end{array}$ \\
\hline $\begin{array}{l}4.0000 \\
4.5000 \\
5.0000 \\
5.5000 \\
6.0000 \\
7.0000 \\
8.0000 \\
9.0000\end{array}$ & $\begin{array}{l}1.499 \mathrm{E}+00 \\
1.508 \mathrm{E}+00 \\
1.517 \mathrm{E}+00 \\
1.525 \mathrm{E}+00 \\
1.532 \mathrm{E}+00 \\
1.544 \mathrm{E}+00 \\
1.555 \mathrm{E}+00 \\
1.564 \mathrm{E}+00\end{array}$ & $\begin{array}{l}3.182 \mathrm{E}-02 \\
3.672 \mathrm{E}-02 \\
4.174 \mathrm{E}-02 \\
4.687 \mathrm{E}-02 \\
5.2111 \mathrm{E}-02 \\
6.283 \mathrm{E}-02 \\
7.386 \mathrm{E}-02 \\
8.514 \mathrm{E}-02\end{array}$ & $\begin{array}{l}1.531 \mathrm{E}+00 \\
1.545 \mathrm{E}+00 \\
1.559 \mathrm{E}+00 \\
1.572 \mathrm{E}+00 \\
1.584 \mathrm{E}+00 \\
1.607 \mathrm{E}+00 \\
1.628 \mathrm{E}+00 \\
1.649 \mathrm{E}+00\end{array}$ & $\begin{array}{l}2.56 \\
2.88 \\
3.21 \\
3.52 \\
3.84 \\
4.47 \\
5.09 \\
5.70\end{array}$ & $\begin{array}{l}9.7 \\
1.1 \\
1.2 \\
1.4 \\
1.5 \\
1.8 \\
2.1 \\
2.4\end{array}$ & $\begin{array}{l}2.2 \\
2.3 \\
2.5 \\
2.7 \\
2.9 \\
3.1\end{array}$ & $\begin{array}{l}-0.0 \\
-0.0 \\
-0.0 \\
-0.0 \\
-0.0 \\
-0.0 \\
-0.0 \\
-0.0\end{array}$ & $\begin{array}{l}2 \\
8 \\
4 \\
1 \\
7 \\
2 \\
7 \\
3\end{array}$ & $\begin{array}{l}.070 \\
.065 \\
.061 \\
.056 \\
.052 \\
.045 \\
.039 \\
.034\end{array}$ \\
\hline $\begin{array}{l}10.0000 \\
12.5000 \\
15.0000 \\
17.5000 \\
20.0000 \\
25.00000 \\
30.0000 \\
35.0000\end{array}$ & $\begin{array}{l}1.572 E+00 \\
1.589 E+00 \\
1.602 E+00 \\
1.613 E+00 \\
1.623 E+00 \\
1.639 E+00 \\
1.652 E+00 \\
1.663 E+00\end{array}$ & $\begin{array}{l}1.262 \mathrm{E}-01 \\
1.566 \mathrm{E}-01 \\
1.878 \mathrm{E}-01 \\
2.194 \mathrm{E}-01 \\
2.840 \mathrm{E}-01 \\
3.499 \mathrm{E}-01 \\
4.166 \mathrm{E}-01\end{array}$ & $\begin{array}{l}1.669 \mathrm{E}+00 \\
1.715 \mathrm{E}+00 \\
1.759 \mathrm{E}+00 \\
1.801 \mathrm{E}+00 \\
1.842 \mathrm{E}+00 \\
1.923 \mathrm{E}+00 \\
2.002 \mathrm{E}+00 \\
2.079 \mathrm{E}+00\end{array}$ & $1.965 \mathrm{~L}+01$ & $\begin{array}{l}3.509 \mathrm{E}-0 \\
4.280 \mathrm{E}-0 \\
5.050 \mathrm{E}-0 \\
5.815 \mathrm{E}-0 \\
7.323 \mathrm{E}-0 \\
8.792 \mathrm{E}-0 \\
1.022 \mathrm{E}-0\end{array}$ & $\begin{array}{l}5.051 E+0 \\
5.404 E+0 \\
5.705 E+0\end{array}$ & $\begin{array}{l}-0.01 \\
-0.00 \\
-0.00 \\
-0.00 \\
-0.00 \\
-0.00 \\
-0.00 \\
-0.00\end{array}$ & $\begin{array}{l}0.049 \\
0.042 \\
0.036 \\
0.032 \\
0.029 \\
0.024 \\
0.021 \\
0.018\end{array}$ & \\
\hline $\begin{array}{l}45.0000 \\
50.0000 \\
55.0000 \\
60.0000 \\
70.0000 \\
80.0000 \\
90.0000\end{array}$ & $\begin{array}{l}1.680 \mathrm{E}+ \\
1.687 \mathrm{E}+ \\
1.694 \mathrm{E}+ \\
1.700 \mathrm{E}+ \\
1.711 \mathrm{Et} \\
1.720 \mathrm{E}+ \\
1.728 \mathrm{E}+\end{array}$ & $\begin{array}{l}6.2 \\
6.9 \\
7.5\end{array}$ & $\begin{array}{l}2.2 \\
2.3 \\
2.3 \\
2.4 \\
2.6 \\
2.7 \\
2.9\end{array}$ & $\begin{array}{l}2.2 \\
2.4 \\
2.6 \\
2.8 \\
3.0 \\
3.4 \\
3.8 \\
4.1\end{array}$ & $\begin{array}{l}1: \\
1: \\
1: \\
2 .\end{array}$ & $\begin{array}{l}6: \\
6: \\
6:\end{array}$ & $\begin{array}{l}-0.001 \\
-0.001 \\
-0.001 \\
-0.001 \\
-0.001 \\
-0.000 \\
-0.000 \\
-0.000\end{array}$ & $\begin{array}{l}.0115 \\
.014 \\
.013 \\
.012 \\
011 \\
011 \\
.010 \\
.009\end{array}$ & \\
\hline $\begin{array}{l}00 \\
00 \\
00\end{array}$ & $\begin{array}{l}1.7 \\
1.7 \\
1.7 \\
1.7 \\
1.7\end{array}$ & $\begin{array}{l}1.6 \\
2.0 \\
2.4 \\
2.7 \\
3.5\end{array}$ & $\begin{array}{l}3.436 E+00 \\
3.811 E+00 \\
4.186 E+00 \\
4.561 E+00 \\
5.314 E+00 \\
6.067 E+00 \\
6.821 E+00\end{array}$ & $\begin{array}{l}1 \\
1 \\
1 \\
1 \\
1 \\
1\end{array}$ & & 9. & & & \\
\hline $\begin{array}{l}0 \\
0 \\
0\end{array}$ & $\begin{array}{l}1.8 \\
1.8 \\
1.8 \\
1.8 \\
1.8 \\
1.8 \\
1.8\end{array}$ & $\begin{array}{l}5.746 E+00 \\
6.494 E+00 \\
7.242 E+00 \\
7.993 E+00 \\
8.744 E+00 \\
1.025 E+01 \\
1.176 E+01 \\
1.327 E+01\end{array}$ & $\begin{array}{l}7.576 E+00 \\
8.332 E+00 \\
9.088 E+00 \\
9.845 E+00 \\
1.060 E+01 \\
1.212 E+01 \\
1.364 E+01 \\
1.515 E+01\end{array}$ & $\begin{array}{l}055 \mathrm{E}+02 \\
118 \mathrm{E}+02 \\
175 \mathrm{E}+02 \\
228 \mathrm{E}+02 \\
277 \mathrm{E}+02 \\
365 \mathrm{E}+02 \\
443 \mathrm{E}+02 \\
513 \mathrm{E}+02\end{array}$ & $\begin{array}{l}5 \\
5 \\
5\end{array}$ & $\begin{array}{l}1.193 \mathrm{E}+0 \\
1.216 \mathrm{E}+0\end{array}$ & $\begin{array}{l}-0 . \\
-0 \\
-0 \\
-0 \\
-0 \\
-0 . \\
-0 . \\
-0 .\end{array}$ & $\begin{array}{l}.004 \\
.003 \\
.003 \\
.003 \\
.003 \\
.003 \\
.003 \\
.003\end{array}$ & $\begin{array}{l}0.000 \\
0.000 \\
0.000 \\
0.000 \\
0.000 \\
0.000 \\
0.000 \\
0.000\end{array}$ \\
\hline & $.893 E+00$ & $.478 E+01$ & $1.667 E+01$ & $.575 E+02$ & $7.204 E-01$ & .237 & 0.000 & 0.002 & 0.000 \\
\hline
\end{tabular}


ELECTRONS IN CARBON (GRAPHITE)

$I=78.0 \mathrm{eV} \quad$ DENSITY $=2.265 \mathrm{E}+00 \mathrm{~g} / \mathrm{cm}^{3}$

\begin{tabular}{|c|c|c|c|c|c|c|c|c|c|}
\hline AERGY & $\begin{array}{l}\text { COLLISION } \\
\mathrm{MeV} \mathrm{cm}^{2} / \mathrm{g}\end{array}$ & $\begin{array}{l}\text { OPP ING POWER } \\
\text { RADIATIVE } \\
\mathrm{MeV} \mathrm{cm} 2 / \mathrm{g}\end{array}$ & $\begin{array}{c}\text { TOTAL } \\
\mathrm{MeV} \mathrm{cm} 2 / g\end{array}$ & $\begin{array}{l}\text { CSDA } \\
\text { RANGE }\end{array}$ & DIATION & $\begin{array}{l}\text { DENS.EFF. } \\
\text { CORR . } \\
\text { (DELTA) }\end{array}$ & $\begin{array}{l}\text { de } \\
\text { coLL } \\
\text { LOSS }\end{array}$ & $\begin{array}{l}1 / d(1 \\
\text { SDA } \\
\text { ANGE }\end{array}$ & $\begin{array}{l}\circ \text { I I ) } \\
\text { RAD } \\
\text { YIELD }\end{array}$ \\
\hline $\begin{array}{l}0.0100 \\
0.0125 \\
0.0150 \\
0.0175 \\
0.0200 \\
0.0250 \\
0.0300 \\
0.0350\end{array}$ & $\begin{array}{l}2.014 E+01 \\
1.694 E+01 \\
1.471 E+01 \\
1.305 E+01 \\
1.177 E+01 \\
9.911 E+00 \\
8.624 E+00 \\
7.677 E+00\end{array}$ & $\begin{array}{l}3.16 \\
3.16 \\
3.17 \\
3.17 \\
3.18 \\
3.19 \\
3.20\end{array}$ & $\begin{array}{l}2.0 \\
1.6 \\
1.4 \\
1.3 \\
1.9 \\
9.9 \\
8.6 \\
7.6\end{array}$ & $\begin{array}{l}E-04 \\
E-04 \\
E-04 \\
E-04 \\
E-04 \\
E-03 \\
E-03 \\
E-03\end{array}$ & $\begin{array}{l}665 E-05 \\
036 E-04 \\
199 E-04 \\
.355 E-04 \\
506 E-04 \\
796 E-04 \\
074 E-04 \\
.340 E-04\end{array}$ & $\begin{array}{l}2.766 \mathrm{E}-03 \\
3.543 \mathrm{E}-03 \\
4.353 \mathrm{E}-03 \\
5.195 \mathrm{E}-03 \\
6.068 \mathrm{E}-03 \\
7.907 \mathrm{E}-03 \\
9.864 \mathrm{E}-03 \\
1.194 \mathrm{E}-02\end{array}$ & $\begin{array}{l}-0.200 \\
-0.191 \\
-0.185 \\
-0.179 \\
-0.175 \\
-0.168 \\
-0.163 \\
-0.159\end{array}$ & $\begin{array}{l}0.229 \\
0.218 \\
0.210 \\
0.203 \\
0.198 \\
0.189 \\
0.183 \\
0.177\end{array}$ & $\begin{array}{l}0.228 \\
0.217 \\
0.209 \\
0.202 \\
0.197 \\
0.189 \\
0.182 \\
0.177\end{array}$ \\
\hline $\begin{array}{l}0.0400 \\
0.0450 \\
0.0500 \\
0.0550 \\
0.0600 \\
0.0700 \\
0.0800 \\
0.0900\end{array}$ & $\begin{array}{l}6.948 E+00 \\
6.370 E+00 \\
5.899 E+00 \\
5.508 E+00 \\
5.177 E+00 \\
4.650 E+00 \\
4.247 E+00 \\
3.929 E+00\end{array}$ & $\begin{array}{l}3.215 \mathrm{E}-03 \\
3.228 \mathrm{E}-03 \\
3.241 \mathrm{E}-03 \\
3.255 \mathrm{E}-03 \\
3.270 \mathrm{E}-03 \\
3.303 \mathrm{E}-03 \\
3.337 \mathrm{E}-03 \\
3.375 \mathrm{E}-03\end{array}$ & $\begin{array}{l}6.951 E+00 \\
6.373 E+00 \\
5.902 E+00 \\
5.511 E+00 \\
5.181 E+00 \\
4.653 E+00 \\
4.250 E+00 \\
3.932 E+00\end{array}$ & $\begin{array}{l}.268 \mathrm{E}-03 \\
.020 \mathrm{E}-03 \\
.836 \mathrm{E}-03 \\
.713 \mathrm{E}-03 \\
.650 \mathrm{E}-03 \\
.691 \mathrm{E}-03 \\
.094 \mathrm{E}-02 \\
.339 \mathrm{E}-02\end{array}$ & $\begin{array}{l}2.597 \mathrm{E}-04 \\
2.847 \mathrm{E}-04 \\
3.090 \mathrm{E}-04 \\
3.328 \mathrm{E}-04 \\
3.559 \mathrm{E}-04 \\
4.009 \mathrm{E}-04 \\
4.443 \mathrm{E}-04 \\
4.862 \mathrm{E}-04\end{array}$ & $\begin{array}{l}1.41 \\
1.64 \\
1.87 \\
2.12 \\
2.38 \\
2.92 \\
3.50 \\
4.10\end{array}$ & $\begin{array}{l}-0.156 \\
-0.153 \\
-0.150 \\
-0.148 \\
-0.145 \\
-0.142 \\
-0.139 \\
-0.136\end{array}$ & $\begin{array}{l}0.173 \\
0.170 \\
0.166 \\
0.164 \\
0.161 \\
0.157 \\
0.154 \\
0.151\end{array}$ & $\begin{array}{l}0.173 \\
0.169 \\
0.166 \\
0.163 \\
0.161 \\
0.157 \\
0.153 \\
0.150\end{array}$ \\
\hline $\begin{array}{l}0.1000 \\
0.1250 \\
0.1500 \\
0.1750 \\
0.2000 \\
0.2500 \\
0.3000 \\
0.3500\end{array}$ & $\begin{array}{l}3.671 E+00 \\
3.201 E+00 \\
2.883 E+00 \\
2.654 E+00 \\
2.482 E+00 \\
2.241 E+00 \\
2.083 E+00 \\
1.972 E+00\end{array}$ & $\begin{array}{l}3.414 \\
3.523 \\
3.640 \\
3.764 \\
3.896 \\
4.178 \\
4.48 \\
4.820\end{array}$ & $\begin{array}{l}3.675 \mathrm{E}+00 \\
3.205 \mathrm{E}+00 \\
2.887 \mathrm{E}+00 \\
2.658 \mathrm{E}+00 \\
2.486 \mathrm{E}+00 \\
2.245 \mathrm{E}+00 \\
2.087 \mathrm{E}+00 \\
1.977 \mathrm{E}+00\end{array}$ & $\begin{array}{l}E-02 \\
E-02 \\
E-02 \\
E-02 \\
E-02 \\
E-02 \\
E-02 \\
E-01\end{array}$ & $\begin{array}{l}.270 \mathrm{E}-04 \\
.246 \mathrm{E}-04 \\
.173 \mathrm{E}-04 \\
.061 \mathrm{E}-04 \\
.919 \mathrm{E}-04 \\
.056 \mathrm{E}-03 \\
.215 \mathrm{E}-03 \\
.369 \mathrm{E}-03\end{array}$ & $\begin{array}{l}4.738 \mathrm{E}-02 \\
6.429 \mathrm{E}-02 \\
8.253 \mathrm{E}-02 \\
1.019 \mathrm{E}-01 \\
1.221 \mathrm{E}-01 \\
1.644 \mathrm{E}-01 \\
2.086 \mathrm{E}-01 \\
2.537 \mathrm{E}-01\end{array}$ & $\begin{array}{l}-0.133 \\
-0.128 \\
-0.124 \\
-0.120 \\
-0.116 \\
-0.110 \\
-0.105 \\
-0.100\end{array}$ & $\begin{array}{l}0.148 \\
0.142 \\
0.138 \\
0.134 \\
0.131 \\
0.126 \\
0.121 \\
0.117\end{array}$ & $\begin{array}{l}0.148 \\
0.142 \\
0.137 \\
0.134 \\
0.130 \\
0.125 \\
0.120 \\
0.116\end{array}$ \\
\hline $\begin{array}{l}0.4000 \\
0.4500 \\
0.5000 \\
0.5500 \\
0.6000 \\
0.7000 \\
0.8000 \\
0.9000\end{array}$ & $\begin{array}{l}1.89 \\
1.83 \\
1.78 \\
1.74 \\
1.71 \\
1.67 \\
1.64\end{array}$ & $\begin{array}{l}5.17 \\
5.5 \\
5.9 \\
6.3 \\
6.75 \\
7.6 \\
8.5\end{array}$ & $\begin{array}{l}1.896 E+00 \\
1.835 E+00 \\
1.788 E+00 \\
1.752 E+00 \\
1.722 E+00 \\
1.680 E+00 \\
1.651 E+00 \\
1.632 E+00\end{array}$ & $\begin{array}{l}1.452 \mathrm{E}-01 \\
1.721 \mathrm{E}-01 \\
1.997 \mathrm{E}-01 \\
2.279 \mathrm{E}-01 \\
2.567 \mathrm{E}-01 \\
3.156 \mathrm{E}-01 \\
3.757 \mathrm{E}-01 \\
4.366 \mathrm{E}-01\end{array}$ & $\begin{array}{l}1.671 \\
1.821 \\
1.971 \\
2.121 \\
2.423 \\
2.728 \\
3.037\end{array}$ & $\begin{array}{l}2.99 \\
3.44 \\
3.89 \\
4.34 \\
4.78 \\
5.64 \\
6.47 \\
7.27\end{array}$ & $\begin{array}{l}-0.096 \\
-0.092 \\
-0.089 \\
-0.086 \\
-0.084 \\
-0.079 \\
-0.076 \\
-0.073\end{array}$ & $\begin{array}{l}0.1114 \\
0.111 \\
0.108 \\
0.105 \\
0.103 \\
0.099 \\
0.095 \\
0.092\end{array}$ & $\begin{array}{l}0.112 \\
0.108 \\
0.1105 \\
0.102 \\
0.100 \\
0.095 \\
0.091 \\
0.088\end{array}$ \\
\hline $\begin{array}{l}1.0000 \\
1.2500 \\
1.5000 \\
1.7500 \\
2.0000 \\
2.5000 \\
3.0000 \\
3.5000\end{array}$ & $\begin{array}{l}1.609 E+00 \\
1.590 E+00 \\
1.584 E+00 \\
1.584 E+00 \\
1.587 E+00 \\
1.598 E+00 \\
1.611 E+00 \\
1.623 E+00\end{array}$ & $\begin{array}{l}1.053 E-02 \\
1.318 E-02 \\
1.602 E-02 \\
1.901 E-02 \\
2.213 E-02 \\
2.870 E-02 \\
3.561 \mathrm{E}-02 \\
4.281 \mathrm{E}-02\end{array}$ & $\begin{array}{l}1.619 \mathrm{E}+c \\
1.603 \mathrm{E}+c \\
1.600 \mathrm{E}+C \\
1.603 \mathrm{E}+c \\
1.609 \mathrm{E}+c \\
1.627 \mathrm{E}+c \\
1.646 \mathrm{E}+c \\
1.666 \mathrm{E}+c\end{array}$ & $\begin{array}{l}8.0 \\
9.6 \\
1.1 \\
1.4 \\
1.7\end{array}$ & $\begin{array}{l}4 . \\
4: \\
5: \\
6 . \\
1 .\end{array}$ & $\begin{array}{l}8.0 \\
9.8 \\
1.9 \\
1.2 \\
1.9 \\
1.6 \\
1.8 \\
2.0\end{array}$ & $\begin{array}{l}-0.0 \\
-0.0 \\
-0.0 \\
-0.0 \\
-0.0 \\
-0.0 \\
-0.0 \\
-0.0\end{array}$ & $\begin{array}{l}0.0 \\
0.0 \\
0.0 \\
0.0 \\
0.0 \\
0.0 \\
0.0\end{array}$ & $\begin{array}{l}0.085 \\
0.078 \\
0.074 \\
0.070 \\
0.067 \\
0.062 \\
0.059 \\
0.056\end{array}$ \\
\hline $\begin{array}{l}4.0000 \\
4.5000 \\
5.0000 \\
5.5000 \\
6.0000 \\
7.0000 \\
8.0000 \\
9.0000\end{array}$ & $\begin{array}{l}1.64 \\
1.65 \\
1.66 \\
1.67 \\
1.68 \\
1.70 \\
1.71\end{array}$ & $\begin{array}{l}5.792 E-02 \\
6.576 \mathrm{E}-02 \\
7.378 \mathrm{E}-02 \\
8.193 \mathrm{E}-02 \\
9.865 \mathrm{E}-02 \\
1.158 \mathrm{E}-01 \\
1.334 \mathrm{E}-01\end{array}$ & $\begin{array}{l}1.686 E+00 \\
1.705 E+00 \\
1.723 E+00 \\
1.741 E+00 \\
1.758 E+00 \\
1.791 E+00 \\
1.823 E+00 \\
1.853 E+00\end{array}$ & $\begin{array}{l}2.631 E+00 \\
2.923 E+00 \\
3.211 E+00 \\
3.497 E+00 \\
4.061 E+00 \\
4.614 E+00 \\
5.158 E+00\end{array}$ & $\begin{array}{l}1.419 \mathrm{E}- \\
1.616 \mathrm{E}- \\
1.815 \mathrm{E}- \\
2.016 \mathrm{E}- \\
2.219 \mathrm{E}- \\
2.628 \mathrm{E}- \\
3.041 \mathrm{E}- \\
3.456 \mathrm{E}-\end{array}$ & $\begin{array}{l}2.340 \mathrm{E} \\
2.474 \mathrm{E} \\
2.598 \mathrm{E} \\
2.713 \mathrm{E} \\
2.924 \mathrm{E} \\
3.114 \mathrm{E} \\
3.287 \mathrm{E}\end{array}$ & $\begin{array}{l}-0.0 \\
-0.0 \\
-0.0 \\
-0.0 \\
-0.0 \\
-0.0 \\
-0.0 \\
-0.0\end{array}$ & $\begin{array}{l}0 . \\
0 . \\
0 . \\
0 . \\
0 . \\
0 .\end{array}$ & $\begin{array}{l}0.054 \\
0.052 \\
0.050 \\
0.048 \\
0.047 \\
0.044 \\
0.041 \\
0.039\end{array}$ \\
\hline $\begin{array}{l}10.0000 \\
12.50000 \\
15.0000 \\
17.5000 \\
20.0000 \\
25.00000 \\
30.00000 \\
35.0000\end{array}$ & $\begin{array}{l}1.730 E+00 \\
1.753 E+00 \\
1.770 E+00 \\
1.785 E+00 \\
1.797 E+00 \\
1.816 E+00 \\
1.832 E+00 \\
1.845 E+00\end{array}$ & $\begin{array}{l}1.5 \\
1.9 \\
2.4 \\
2.92 \\
3.4 \\
4.41 \\
5.4 \\
6.4\end{array}$ & $\begin{array}{l}1.881 E+00 \\
1.950 \mathrm{E}+00 \\
2.015 \mathrm{E}+00 \\
2.077 \mathrm{E}+00 \\
2.139 \mathrm{E}+00 \\
2.258 \mathrm{E}+00 \\
2.375 \mathrm{E}+00 \\
2.491 \mathrm{E}+00\end{array}$ & $\begin{array}{l}5.694 E+00 \\
6.999 E+00 \\
8.260 E+00 \\
9.482 E+00 \\
1.067 E+01 \\
1.294 E+01 \\
1.510 E+01 \\
1.716 E+01\end{array}$ & $\begin{array}{l}3.872 \mathrm{E}-02 \\
4.914 \mathrm{E}-02 \\
5.949 \mathrm{E}-02 \\
6.973 \mathrm{E}-02 \\
7.981 \mathrm{E}-02 \\
9.943 \mathrm{E}-02 \\
1.183 \mathrm{E}-01 \\
1.363 \mathrm{E}-01\end{array}$ & $\begin{array}{l}3.4 \\
3.7 \\
4.1 \\
4.3 \\
4.6\end{array}$ & $\begin{array}{l}-0 . \\
-0 . \\
-0 . \\
-0 \\
-0 . \\
-0 . \\
-0 . \\
-0\end{array}$ & $\begin{array}{l}0 . \\
0 . \\
0 . \\
0 . \\
0 . \\
0 . \\
0 . \\
0 .\end{array}$ & $\begin{array}{l}0.036 \\
0.031 \\
0.027 \\
0.023 \\
0.020 \\
0.016 \\
0.012 \\
0.010\end{array}$ \\
\hline $\begin{array}{l}40.00000 \\
45.00000 \\
50.00000 \\
55.0000 \\
60.0000 \\
70.0000 \\
80.0000 \\
90.0000\end{array}$ & $\begin{array}{l}1.856 \\
1.865 \\
1.874 \\
1.881 \\
1.888 \\
1.900 \\
1.91 \\
1.920\end{array}$ & $\begin{array}{l}8.5 \\
9.8 \\
1.2 \\
1.2 \\
1.2 \\
1.8\end{array}$ & $\begin{array}{l}2.606 \mathrm{E}+00 \\
2.721 \mathrm{E}+00 \\
2.835 \mathrm{E}+00 \\
2.949 \mathrm{E}+00 \\
3.063 \mathrm{E}+00 \\
3.291 \mathrm{E}+00 \\
3.519 \mathrm{E}+00 \\
3.746 \mathrm{E}+00\end{array}$ & $\begin{array}{l}1.91 \\
2.10 \\
2.28 \\
2.45 \\
2.61 \\
2.93 \\
3.22 \\
3.50\end{array}$ & $\begin{array}{l}1.5 \\
1.8 \\
1.8 \\
2.8 \\
2.1 \\
2.8 \\
2.8 \\
2.8\end{array}$ & $\begin{array}{l}6 . \\
6 . \\
6 . \\
6 . \\
7 . \\
7 .\end{array}$ & $\begin{array}{l}-0 \\
-0 \\
-0 \\
-0 \\
-0 \\
-0 \\
-0 \\
-0\end{array}$ & & $\begin{array}{l}08 \\
07 \\
06 \\
05 \\
05 \\
04 \\
03 \\
03\end{array}$ \\
\hline $\begin{array}{l}.0000 \\
.0000 \\
.0000 \\
.0000 \\
.0000 \\
.0000 \\
.0000\end{array}$ & $\begin{array}{l}1.9 \\
1.9 \\
1.9 \\
1.9 \\
1.9 \\
2.9 \\
2.0\end{array}$ & $\begin{array}{l}2 . \\
2 . \\
3 . \\
3 . \\
4 . \\
5 . \\
6 . \\
7 .\end{array}$ & $\begin{array}{l}3 . \\
4 . \\
5: \\
5: \\
6.2 \\
7.5 \\
8 .\end{array}$ & $\begin{array}{l}1 \\
1 \\
1 \\
1 \\
1 \\
1 \\
1 \\
1\end{array}$ & & $\begin{array}{l}0 \\
0 \\
0 \\
0 \\
0 \\
0 \\
0\end{array}$ & & & \\
\hline $\begin{array}{l}0.0000 \\
0.0000 \\
0.0000 \\
0.0000 \\
0.0000 \\
0.0000 \\
0.0000 \\
0.0000\end{array}$ & $\begin{array}{l}2.035 \mathrm{E}+00 \\
2.044 \mathrm{E}+00 \\
2.052 \mathrm{E}+00 \\
2.060 \mathrm{E}+00 \\
2.066 \mathrm{E}+00 \\
2.078 \mathrm{E}+00 \\
2.088 \mathrm{E}+00 \\
2.097 \mathrm{E}+00\end{array}$ & $\begin{array}{l}8.820 E+00 \\
9.964 E+00 \\
1.111 E+01 \\
1.226 E+01 \\
1.340 E+01 \\
1.570 E+01 \\
1.801 E+01 \\
2.031 E+01\end{array}$ & $\begin{array}{l}1.085 \mathrm{E}+01 \\
1.201 \mathrm{E}+01 \\
1.316 \mathrm{E}+01 \\
1.432 \mathrm{E}+01 \\
1.547 \mathrm{E}+01 \\
1.778 \mathrm{E}+01 \\
2.010 \mathrm{E}+01 \\
2.241 \mathrm{E}+01\end{array}$ & $\begin{array}{l}1.081 E+02 \\
1.128 E+02\end{array}$ & $\begin{array}{l}0.533 \\
6.539 \\
6.718 \mathrm{E} \\
6.876 \mathrm{E} \\
7.144 \mathrm{E} \\
7.363 \mathrm{E} \\
7.546 \mathrm{E}\end{array}$ & $\begin{array}{l}1.046 E+01 \\
1.070 E+01 \\
1.091 E+01 \\
1.110 E+01 \\
1.127 E+01 \\
1.158 E+01 \\
1.185 E+01 \\
1.208 E+01\end{array}$ & $\begin{array}{l}0.000 \\
0.000 \\
0.000 \\
0.000 \\
0.000 \\
0.000 \\
0.000 \\
0.000\end{array}$ & $\begin{array}{l}0.005 \\
0.005 \\
0.005 \\
0.004 \\
0.004 \\
0.004 \\
0.004 \\
0.004\end{array}$ & $\begin{array}{l}0.000 \\
0.000 \\
0.000 \\
0.0000 \\
0.000 \\
0.000 \\
0.000\end{array}$ \\
\hline 0000 & $.106 E+00$ & $.262 E+01$ & $2.473 E+01$ & $1.171 E+02$ & $7.703 E-01$ & $1.229 \mathrm{E}+01$ & -0.000 & 0.004 & 0.000 \\
\hline
\end{tabular}




\begin{tabular}{|c|c|c|c|c|c|c|c|c|c|}
\hline $\mathrm{MeV}$ & $\begin{array}{l}\text { COLLISION } \\
\mathrm{MeV} \mathrm{cm}^{2} / \mathrm{g}\end{array}$ & ADI A T IVE & $\begin{array}{l}\text { TOTAL } \\
\mathrm{V} \mathrm{cm}^{2} / \mathrm{g}\end{array}$ & $\begin{array}{l}\text { CSDA } \\
\text { RANGE } \\
g / \mathrm{cm}^{2}\end{array}$ & N & $\begin{array}{l}\text { EFF } \\
\text { RR. } \\
(T A)\end{array}$ & COLL & 5 & $\begin{array}{l}\text { I) } \\
\text { RAD } \\
\text { IELD }\end{array}$ \\
\hline $\begin{array}{l}0.0100 \\
0.0125 \\
0.0150 \\
0.0175 \\
0.0200 \\
0.0250 \\
0.0300 \\
0.0350\end{array}$ & $\begin{array}{l}1.6 \\
1.4 \\
1.3 \\
1.1 \\
9.9 \\
8.6\end{array}$ & $\begin{array}{l}3.1 \\
3.1 \\
3.1 \\
3.1 \\
3.1 \\
3.1 \\
3.2\end{array}$ & $\begin{array}{l}014 E+01 \\
695 E+01 \\
471 E+01 \\
305 E+01 \\
177 E+01 \\
916 E+00 \\
629 E+00 \\
682 E+00\end{array}$ & $\begin{array}{l}2.82 \\
4.17 \\
5.76 \\
7.57 \\
9.59\end{array}$ & $\begin{array}{l}1 . \\
1 . \\
1 . \\
1 .\end{array}$ & $\begin{array}{l}E-03 \\
E-03 \\
E-03 \\
E-03 \\
E-03 \\
E-03 \\
E-03 \\
E-03\end{array}$ & $\begin{array}{l}-0.200 \\
-0.191 \\
-0.185 \\
-0.180 \\
-0.176 \\
-0.169 \\
-0.164 \\
-0.160\end{array}$ & $\begin{array}{l}9 \\
8 \\
0 \\
3 \\
8 \\
9 \\
3 \\
8\end{array}$ & $\begin{array}{l}0.228 \\
0.217 \\
0.209 \\
0.202 \\
0.197 \\
0.189 \\
0.183 \\
0.177\end{array}$ \\
\hline $\begin{array}{l}0.0400 \\
0.0450 \\
0.0500 \\
0.0550 \\
0.0600 \\
0.0700 \\
0.0800 \\
0.0900\end{array}$ & $\begin{array}{l}6 \cdot 3 \\
5 \cdot 9 \\
5 \cdot 5 \\
5 \cdot 1 \\
4 \cdot 6 \\
4 \cdot 2\end{array}$ & $\begin{array}{l}-03 \\
-03 \\
-03 \\
-03 \\
-03 \\
-03 \\
-03 \\
-03\end{array}$ & $\begin{array}{l}+00 \\
+00 \\
+00 \\
+00 \\
+00 \\
+00 \\
+00 \\
+00\end{array}$ & $\begin{array}{l}-03 \\
-03 \\
-03 \\
-03 \\
03 \\
03 \\
03 \\
02 \\
02\end{array}$ & $\begin{array}{l}2 . \\
3 . \\
3 . \\
3 . \\
4 . \\
4 .\end{array}$ & $\begin{array}{l}-02 \\
-02 \\
-02 \\
-02 \\
-02 \\
-02 \\
-02 \\
-02\end{array}$ & $\begin{array}{l}-0 \\
-0 \\
-0 \\
-0 \\
-0 \\
-0 \\
-0\end{array}$ & & $\begin{array}{l}73 \\
70 \\
67 \\
64 \\
62 \\
58 \\
54 \\
51\end{array}$ \\
\hline $\begin{array}{l}0.1000 \\
0.1250 \\
0.1500 \\
0.1750 \\
0.2000 \\
0.2500 \\
0.3000 \\
0.3500\end{array}$ & $\begin{array}{l}3.2 \\
2.8 \\
2.6 \\
2.4 \\
2.2 \\
2.0\end{array}$ & $\begin{array}{l}3.4 \\
3.5 \\
3.6 \\
3.7 \\
3.8 \\
4.1 \\
4.4 \\
4.8\end{array}$ & $\begin{array}{ll}0 & 0 \\
0 & 0 \\
0 & 0 \\
0 & 0 \\
0 & 0 \\
0 & 0 \\
0 & 0 \\
0 & 0\end{array}$ & $\begin{array}{l}.02 \\
022 \\
02 \\
02 \\
02 \\
02 \\
02 \\
02 \\
02 \\
01\end{array}$ & $\begin{array}{l}6.2 \\
7 . \\
8 . \\
8 . \\
1.2 \\
1.2 \\
1.2\end{array}$ & $\begin{array}{l}-02 \\
-02 \\
-02 \\
-02 \\
-02 \\
-01 \\
-01 \\
-01 \\
-01\end{array}$ & $\begin{array}{l}-0 . \\
-0 . \\
-0 . \\
-0 . \\
-0 . \\
-0 . \\
-0 . \\
-0 .\end{array}$ & & $\begin{array}{l}49 \\
43 \\
39 \\
36 \\
33 \\
28 \\
23 \\
20\end{array}$ \\
\hline $\begin{array}{l}0.4000 \\
0.4500 \\
0.5000 \\
0.5500 \\
0.6000 \\
0.7000 \\
0.8000 \\
0.9000\end{array}$ & $\begin{array}{l}+00 \\
+00 \\
+00 \\
+00 \\
+00 \\
+00\end{array}$ & $\begin{array}{l}.03 \\
.03 \\
03 \\
03 \\
03 \\
03 \\
03 \\
03 \\
03\end{array}$ & $\begin{array}{l}0 \\
0 \\
0 \\
0 \\
0 \\
0 \\
0 \\
0\end{array}$ & $\left\{\begin{array}{l}1 \\
1 \\
1 \\
1 \\
1 \\
1 \\
1\end{array}\right.$ & $\begin{array}{l}3 \\
3 \\
3 \\
3 \\
3 \\
3 \\
3\end{array}$ & $\begin{array}{ll}-0 & 1 \\
-0 & 1 \\
-0 & 1 \\
-0 & 1 \\
-0 & 1 \\
-0 & 1 \\
-0 & 1 \\
-0 & 1\end{array}$ & $\begin{array}{l}-0 . \\
-0 . \\
-0 . \\
-0 . \\
-0 . \\
-0 . \\
-0 . \\
-0 .\end{array}$ & $\begin{array}{l}5 \\
3 \\
0\end{array}$ & $\begin{array}{l}16 \\
13 \\
10 \\
8 \\
05 \\
01 \\
96\end{array}$ \\
\hline $\begin{array}{l}1.0000 \\
1.2500 \\
1.5000 \\
1.7500 \\
2.0000 \\
2.5000 \\
3.0000 \\
3.5000\end{array}$ & 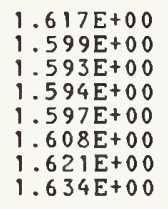 & $\begin{array}{l}02 \\
02 \\
02 \\
02 \\
02 \\
02 \\
02 \\
02\end{array}$ & $\begin{array}{lll}0 & 0 \\
0 & 0 \\
0 & 0 \\
0 & 0 \\
0 & 0 \\
0 & 0 \\
0 & 0 \\
0 & 0\end{array}$ & $\begin{array}{lll}0 & 1 \\
0 & 1 \\
0 & 1 \\
0 & 1 \\
0 & 0 \\
0 & 0 \\
0 & 0\end{array}$ & $\begin{array}{l}3 \\
3 \\
3 \\
3 \\
3 \\
3 \\
2 \\
2\end{array}$ & $\begin{array}{l}01 \\
01 \\
01 \\
00 \\
00 \\
00 \\
00 \\
00 \\
00\end{array}$ & $\begin{array}{l}-0 . \\
-0 . \\
-0 . \\
-0 . \\
-0 . \\
-0 . \\
-0 . \\
-0 .\end{array}$ & & $\begin{array}{l}90 \\
83 \\
78 \\
73 \\
70 \\
65 \\
61\end{array}$ \\
\hline $\begin{array}{l}4.0000 \\
4.5000 \\
5.0000 \\
5.5000 \\
6.0000 \\
7.0000 \\
8.0000 \\
9.0000\end{array}$ & $\begin{array}{l}+00 \\
+00 \\
+00 \\
+00 \\
+00 \\
+00 \\
+00 \\
+00\end{array}$ & $\begin{array}{l}02 \\
02 \\
02 \\
02 \\
02 \\
02 \\
02 \\
01 \\
01 \\
01\end{array}$ & $\begin{array}{l}+00 \\
+00 \\
+00 \\
+00 \\
+00 \\
+00 \\
+00\end{array}$ & $\begin{array}{l}+00 \\
+00 \\
+00 \\
+00 \\
+00 \\
+00 \\
+00\end{array}$ & $\begin{array}{l}02 \\
02 \\
02 \\
02 \\
02 \\
02 \\
02\end{array}$ & $\begin{array}{l}0 \\
0 \\
0 \\
0 \\
0 \\
0 \\
0 \\
0\end{array}$ & $\begin{array}{l}-0 . \\
-0 . \\
-0 . \\
-0 . \\
-0 . \\
-0 . \\
-0 . \\
-0 .\end{array}$ & & $\begin{array}{l}55 \\
53 \\
51 \\
50 \\
48 \\
46 \\
43\end{array}$ \\
\hline $\begin{array}{l}10.0000 \\
12.5000 \\
15.0000 \\
17.5000 \\
20.0000 \\
25.0000\end{array}$ & $\begin{array}{l}.745 E+00 \\
.769 E+00 \\
.787 E+00 \\
.803 E+00 \\
.816 E+00 \\
.836 E+00 \\
.852 E+00 \\
.865 E \div 00\end{array}$ & $\begin{array}{l}1.5 \\
1.9 \\
2.4 \\
2.9 \\
3.4 \\
4.4 \\
5.4 \\
6.4\end{array}$ & $\begin{array}{ll}0 & 0 \\
0 & 0 \\
0 & 0 \\
0 & 0 \\
0 & 0 \\
0 & 0 \\
0 & 0 \\
00\end{array}$ & $\begin{array}{ll}0 & 0 \\
0 & 0 \\
0 & 0 \\
0 & 0 \\
0 & 1 \\
0 & 1 \\
0 & 1 \\
0 & 1\end{array}$ & $\begin{array}{l}2 \\
2 \\
2 \\
2 \\
2 \\
2 \\
1 \\
1\end{array}$ & $\begin{array}{l}0 \\
0 \\
0 \\
0 \\
0 \\
0 \\
0 \\
0\end{array}$ & $\begin{array}{l}-0 . \\
-0 . \\
-0 . \\
-0 . \\
-0 . \\
-0 . \\
-0 . \\
-0 .\end{array}$ & & \\
\hline $\begin{array}{l}.0000 \\
.0000 \\
.00000 \\
.0000 \\
.00000\end{array}$ & $\begin{array}{l}+00 \\
+00 \\
+00 \\
+00 \\
+00 \\
+00 \\
+00\end{array}$ & $\begin{array}{l}8 \\
9 \\
1 \\
1 \\
1 \\
1 \\
1\end{array}$ & & $\begin{array}{l}1 \\
1 \\
1 \\
1 \\
1\end{array}$ & & $\begin{array}{l}0 \\
0 \\
0 \\
0 \\
0 \\
0 \\
0\end{array}$ & $\begin{array}{l}-0 \\
-0 \\
-0 \\
-0 \\
-0 \\
-0 \\
-0\end{array}$ & & \\
\hline $\begin{array}{l}.00 \\
.00 \\
.00 \\
.00 \\
.00\end{array}$ & $\begin{array}{ll}+ & 0 \\
+ & 0 \\
+ & 0 \\
+ & 0 \\
+0 & 0 \\
+0 & 0 \\
+0 & 0 \\
+0 & 0\end{array}$ & $\begin{array}{l}2.7 \\
3.7 \\
4.2 \\
5.4 \\
6.5 \\
7.6\end{array}$ & $\begin{array}{l}0 \\
0 \\
0 \\
0 \\
0 \\
0 \\
0\end{array}$ & 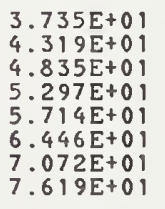 & $\begin{array}{l}1 \\
1 \\
1 \\
1 \\
1 \\
1\end{array}$ & $\begin{array}{l}0 \\
0 \\
0 \\
0 \\
0 \\
0\end{array}$ & $\begin{array}{l}-0 \\
-0 \\
-0 \\
-0\end{array}$ & & \\
\hline $\begin{array}{l}0.0000 \\
0.00000 \\
0.0000 \\
0.0000\end{array}$ & $\begin{array}{l}.066 E+00 \\
.074 E+00 \\
082 E+00 \\
088 E+00 \\
0100 E+00 \\
100 \\
110 E+00 \\
120 E+00\end{array}$ & $\begin{array}{l}9.9 \\
1.1 \\
1.2 \\
1.3 \\
1.5 \\
1.8 \\
2.0\end{array}$ & $\begin{array}{l}3 E+01 \\
3 E+01 \\
4 E+01 \\
9 E+01 \\
0 E+01 \\
2 E+01 \\
3 E+01\end{array}$ & - 1 & $\begin{array}{l}1 \\
1 \\
1 \\
1 \\
1\end{array}$ & $\begin{array}{l}1 \\
1 \\
1 \\
1 \\
1 \\
1 \\
1 \\
1\end{array}$ & $\begin{array}{l}0 \\
0 \\
0 \\
0 \\
0 \\
0 \\
0 \\
0\end{array}$ & $\begin{array}{l}6 \\
5 \\
5 \\
5 \\
5 \\
5 \\
4\end{array}$ & $\begin{array}{l}1 \\
0 \\
0 \\
0 \\
0 \\
0 \\
0\end{array}$ \\
\hline & $E+00$ & $2 E+$ & +0 & $6 \mathrm{Et}$ & $7.687 \mathrm{E}-01$ & & -0.000 & 0.004 & \\
\hline
\end{tabular}




\begin{tabular}{|c|c|c|c|c|c|c|c|c|c|}
\hline $\mathrm{MeV}$ & 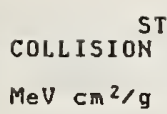 & RADIATIVE & $\begin{array}{c}\text { TOTAL } \\
\mathrm{MeV} \mathrm{cm}^{2} / \mathrm{g}\end{array}$ & $\begin{array}{l}\text { CSDA } \\
\text { RANGE } \\
\mathrm{g} / \mathrm{cm}^{2}\end{array}$ & $\mathrm{BN}$ & $\begin{array}{l}\text { ENS.EFF. } \\
\text { CORR. } \\
\text { DELTA) }\end{array}$ & $\begin{array}{l}d(1 \\
\text { COLL } \\
\text { LOSS }\end{array}$ & $\begin{array}{l}) / d(1 \\
\text { CSDA }\end{array}$ & $\begin{array}{l}\text { I) } \\
\text { RAD } \\
\text { IELD }\end{array}$ \\
\hline $\begin{array}{l}0.0100 \\
0.0125 \\
0.0150 \\
0.0175 \\
0.0200 \\
0.0250 \\
0.0300 \\
0.0350\end{array}$ & 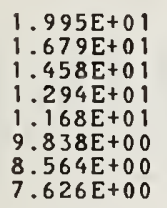 & $\begin{array}{l}3.711 E-03 \\
3.729 E-03 \\
3.740 E-03 \\
3.747 E-03 \\
3.753 E-03 \\
3.762 E-03 \\
3.770 E-03\end{array}$ & $\begin{array}{l}96 E+01 \\
80 E+01 \\
59 E+01 \\
95 E+01 \\
68 E+01 \\
42 E+00 \\
68 E+00 \\
29 E+00\end{array}$ & $\begin{array}{l}2.85 \\
4.22 \\
5.82 \\
7.64 \\
9.68 \\
1.43 \\
1.98 \\
2.60\end{array}$ & $\begin{array}{l}1.023 E-04 \\
1.227 E-04 \\
1.421 E-04 \\
1.608 E-04 \\
1.789 E-04 \\
2.135 E-04 \\
2.465 E-04 \\
2.781 E-04\end{array}$ & $\begin{array}{l}0.0 \\
0.0 \\
0.0 \\
0.0 \\
0.0 \\
0.0 \\
0.0 \\
0.0\end{array}$ & $\begin{array}{l}-0 . \\
-0 . \\
-0 . \\
-0 . \\
-0 . \\
-0 . \\
-0 . \\
-0 .\end{array}$ & $\begin{array}{l}0.232 \\
0.221 \\
0.213 \\
0.206 \\
0.200 \\
0.192 \\
0.185 \\
0.180\end{array}$ & $\begin{array}{l}0.230 \\
0.220 \\
0.211 \\
0.205 \\
0.200 \\
0.191 \\
0.185 \\
0.180\end{array}$ \\
\hline $\begin{array}{l}0.0400 \\
0.0450 \\
0.0500 \\
0.0550 \\
0.0600 \\
0.0700 \\
0.0800 \\
0.0900\end{array}$ & $\begin{array}{l}6.904 E+00 \\
6.331 E+00 \\
5.865 E+00 \\
5.477 E+00 \\
5.150 E+00 \\
4.628 E+00 \\
4.229 E+00 \\
3.914 E+00\end{array}$ & $\begin{array}{l}3.790 E-03 \\
3.803 E-03 \\
3.816 E-03 \\
3.831 E-03 \\
3.846 E-03 \\
3.881 E-03 \\
3.920 E-03 \\
3.961 E-03\end{array}$ & $\begin{array}{l}8 E+00 \\
5 E+00 \\
8 E+00 \\
1 E+00 \\
4 E+00 \\
2 E+00 \\
3 E+00 \\
8 E+00\end{array}$ & $\begin{array}{l}3.25 \\
4.04 \\
4.87 \\
5.75 \\
6.65 \\
8.7 \\
1.10 \\
1.36\end{array}$ & $\begin{array}{l}3.0 \\
3.3 \\
3.6 \\
3.9 \\
4.2 \\
4.7 \\
5.2 \\
5.7\end{array}$ & $\begin{array}{l}0.0 \\
0.0 \\
0.0 \\
0.0 \\
0.0 \\
0.0 \\
0.0 \\
0.0\end{array}$ & $\begin{array}{l}-0.1 \\
-0.1 \\
-0.1 \\
-0.1 \\
-0.1 \\
-0.1 \\
-0 . \\
-0 .\end{array}$ & $\begin{array}{l}0.176 \\
0.173 \\
0.170 \\
0.167 \\
0.165 \\
0.161 \\
0.158 \\
0.155\end{array}$ & $\begin{array}{l}0.176 \\
0.172 \\
0.169 \\
0.167 \\
0.164 \\
0.160 \\
0.157 \\
0.155\end{array}$ \\
\hline $\begin{array}{l}0.1000 \\
0.1250 \\
0.1500 \\
0.1750 \\
0.2000 \\
0.2500 \\
0.3000 \\
0.3500\end{array}$ & $\begin{array}{l}3.660 E+00 \\
3.195 E+00 \\
2.881 E+00 \\
2.655 E+00 \\
2.486 E+00 \\
2.251 E+00 \\
2.097 E+00 \\
1.991 E+00\end{array}$ & $\begin{array}{l}4.005 E-03 \\
4.127 E-03 \\
4.259 E-03 \\
4.400 E-03 \\
4.550 E-03 \\
4.874 E-03 \\
5.227 E-03 \\
5.606 E-03\end{array}$ & $\begin{array}{ll}0 & 0 \\
0 & 0 \\
0 & 0 \\
0 & 0 \\
0 & 0 \\
0 & 0 \\
0 & 0 \\
0 & 0 \\
0 & 0\end{array}$ & $\begin{array}{l}-02 \\
-02 \\
-02 \\
-02 \\
-02 \\
-02 \\
-02 \\
-01\end{array}$ & $\begin{array}{l}6.2 \\
7.3 \\
8.4 \\
9.4 \\
1.0 \\
1.2 \\
1.4 \\
1.5\end{array}$ & $\begin{array}{l}0.0 \\
0.0 \\
0.0 \\
0.0 \\
0.0 \\
0.0 \\
0.0 \\
0.0\end{array}$ & $\begin{array}{l}-0.1 \\
-0.1 \\
-0.1 \\
-0.1 \\
-0.1 \\
-0.1 \\
-0.1 \\
-0.1\end{array}$ & $\begin{array}{l}0 . \\
0 . \\
0 . \\
0 . \\
0 . \\
0 . \\
0 . \\
0 .\end{array}$ & $\begin{array}{l}0.152 \\
0.147 \\
0.144 \\
0.141 \\
0.138 \\
0.134 \\
0.131 \\
0.128\end{array}$ \\
\hline $\begin{array}{l}0.4000 \\
0.4500 \\
0.5000 \\
0.5500 \\
0.6000 \\
0.7000 \\
0.8000 \\
0.9000\end{array}$ & $\begin{array}{l}1.914 E+00 \\
1.857 E+00 \\
1.813 E+00 \\
1.779 E+00 \\
1.753 E+00 \\
1.716 E+00 \\
1.693 E+00 \\
1.679 E+00\end{array}$ & $\begin{array}{l}6.4 \\
6.8 \\
7.3 \\
7.8 \\
8.8 \\
9.8 \\
1.0\end{array}$ & $\begin{array}{ll}0 & 0 \\
0 & 0 \\
0 & 0 \\
0 & 0 \\
0 & 0 \\
0 & 0 \\
0 & 0 \\
0 & 0\end{array}$ & $\begin{array}{ll}-0 & 1 \\
-0 & 1 \\
-0 & 1 \\
-0 & 1 \\
-0 & 1 \\
-0 & 1 \\
-0 & 1 \\
-0 & 1\end{array}$ & $\begin{array}{l}1.76 \\
1.93 \\
2.10 \\
2.27 \\
2.438 \\
2.77 \\
3.10 \\
3.44\end{array}$ & $\begin{array}{l}0.0 \\
0.0 \\
0.0 \\
0.0 \\
0.0 \\
0.0 \\
0.0 \\
0.0\end{array}$ & $\begin{array}{l}-0 . \\
-0 . \\
-0 . \\
-0 . \\
-0 . \\
-0 . \\
-0 . \\
-0 .\end{array}$ & $\begin{array}{l}0.1 \\
0.1 \\
0.1 \\
0.1 \\
0.1 \\
0.1 \\
0.1 \\
0.1\end{array}$ & $\begin{array}{l}0.126 \\
0.124 \\
0.122 \\
0.121 \\
0.1119 \\
0.1117 \\
0.1115 \\
0.1113\end{array}$ \\
\hline $\begin{array}{l}1.0000 \\
1.2500 \\
1.5000 \\
1.7500 \\
2.0000 \\
2.5000 \\
3.0000 \\
3.5000\end{array}$ & $\begin{array}{l}1.670 E+00 \\
1.665 E+00 \\
1.670 E+00 \\
1.681 E+00 \\
1.693 E+00 \\
1.721 E+00 \\
1.749 E+00 \\
1.775 E+00\end{array}$ & $\begin{array}{l}1.21 \\
1.51 \\
1.84 \\
2.18 \\
2.54 \\
3.29 \\
4.07 \\
4.89\end{array}$ & $\begin{array}{l}1.683 \mathrm{E}+00 \\
1.680 \mathrm{E}+00 \\
1.688 \mathrm{E}+00 \\
1.702 \mathrm{E}+00 \\
1.719 \mathrm{E}+00 \\
1.754 \mathrm{E}+00 \\
1.790 \mathrm{E}+00 \\
1.824 \mathrm{E}+00\end{array}$ & $\begin{array}{l}4.883 E-01 \\
6.371 E-01 \\
7.856 E-01 \\
9.331 E-01 \\
1.079 E+00 \\
1.367 E+00 \\
1.649 E+00 \\
1.926 E+00\end{array}$ & $\begin{array}{l}3.788 \mathrm{E}-0 \\
4.655 \mathrm{E}-0 \\
5.541 \mathrm{E}-0 \\
6.444 \mathrm{E}-0 \\
7.364 \mathrm{E}-0 \\
9.243 \mathrm{E}-0 \\
1.116 \mathrm{E}-0 \\
1.311 \mathrm{E}-0\end{array}$ & $\begin{array}{l}0.0 \\
0.0 \\
0.0 \\
0.0 \\
0.0 \\
0.0 \\
0.0 \\
0.0\end{array}$ & $\begin{array}{l}-0.10 \\
-0.10 \\
-0.098 \\
-0.09 \\
-0.09 \\
-0.09 \\
-0.09 \\
-0.08\end{array}$ & $\begin{array}{l}0.1 \\
0.1 \\
0.1 \\
0.1 \\
0.1 \\
0.1 \\
0.1 \\
0.0\end{array}$ & $\begin{array}{l}0.111 \\
0.108 \\
0.105 \\
0.103 \\
0.101 \\
0.097 \\
0.095 \\
0.093\end{array}$ \\
\hline $\begin{array}{l}4.0000 \\
4.5000 \\
5.0000 \\
5.5000 \\
6.0000 \\
7.0000 \\
8.0000 \\
9.0000\end{array}$ & $\begin{array}{l}1.799 E+00 \\
1.821 E+00 \\
1.842 E+00 \\
1.861 E+00 \\
1.879 E+00 \\
1.911 E+00 \\
1.940 E+00 \\
1.965 E+00\end{array}$ & $\begin{array}{l}5.747 \mathrm{E}-02 \\
6.620 \mathrm{E}-02 \\
7.512 \mathrm{E}-02 \\
8.423 \mathrm{E}-02 \\
9.352 \mathrm{E}-02 \\
1.125 \mathrm{E}-01 \\
1.320 \mathrm{E}-01 \\
1.520 \mathrm{E}-01\end{array}$ & $\begin{array}{l}+00 \\
+00 \\
+00 \\
+00 \\
+00 \\
+00 \\
+00 \\
+00\end{array}$ & $\begin{array}{l}E+00 \\
E+00 \\
E+00 \\
E+00 \\
E+00 \\
E+00 \\
E+00 \\
E+00\end{array}$ & $\begin{array}{l}1.509 \\
1.708 \\
1.909 \\
2.110 \\
2.312 \\
2.718 \\
3.124 \\
3.530\end{array}$ & $\begin{array}{l}0.0 \\
0.0 \\
0.0 \\
0.0 \\
0.0 \\
0.0 \\
0.0 \\
0.0\end{array}$ & $\begin{array}{l}-0 . \\
-0 . \\
-0 . \\
-0 . \\
-0 . \\
-0 . \\
-0 . \\
-0 .\end{array}$ & $\begin{array}{l}0 . \\
0 . \\
0 . \\
0 . \\
0 . \\
0 . \\
0 .\end{array}$ & $\begin{array}{l}0.091 \\
0.089 \\
0.088 \\
0.086 \\
0.085 \\
0.083 \\
0.081 \\
0.080\end{array}$ \\
\hline $\begin{array}{l}10.0000 \\
12.5000 \\
15.0000 \\
17.5000 \\
20.0000 \\
25.0000 \\
30.0000 \\
35.0000\end{array}$ & $\begin{array}{l}1.988 E+00 \\
2.038 E+00 \\
2.079 E+00 \\
2.113 E+00 \\
2.144 E+00 \\
2.194 E+00 \\
2.235 E+00 \\
2.266 E+00\end{array}$ & $\begin{array}{l}2.2 \\
2.7 \\
3.3 \\
3.8 \\
5.0 \\
6.1 \\
7.3\end{array}$ & $\begin{array}{l}+\infty \\
+\infty \\
+00 \\
+00 \\
+00 \\
+00 \\
+00 \\
+00 \\
+00 \\
+00\end{array}$ & 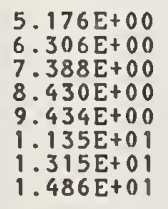 & $\begin{array}{l}-02 \\
-02 \\
-02 \\
-02 \\
-02 \\
-02 \\
-02 \\
-01 \\
-01\end{array}$ & $\begin{array}{l}0.0 \\
0.0 \\
0.0 \\
0.0 \\
0.0 \\
0.0 \\
9.661 \mathrm{E}-03 \\
6.591 \mathrm{E}-02\end{array}$ & $\begin{array}{l}-0 . \\
-0 . \\
-0 . \\
-0 . \\
-0 . \\
-0 . \\
-0 . \\
-0 .\end{array}$ & $\begin{array}{l}0 . \\
0 . \\
0 . \\
0 . \\
0 . \\
0 . \\
0 .\end{array}$ & $\begin{array}{l}75 \\
72 \\
70 \\
68 \\
65 \\
62\end{array}$ \\
\hline $\begin{array}{l}40.0000 \\
45.0000 \\
50.0000 \\
55.0000 \\
60.0000 \\
70.0000 \\
80.0000 \\
90.0000\end{array}$ & $\begin{array}{l}2.310 \mathrm{E}+ \\
2.327 \mathrm{E}+ \\
2.342 \mathrm{E}+ \\
2.355 \mathrm{E}+ \\
2.377 \mathrm{Et}\end{array}$ & $\begin{array}{l}9 . \\
1 . \\
1 . \\
1 . \\
1 . \\
1 . \\
2 .\end{array}$ & & $\begin{array}{l}1 \\
1 \\
1 \\
1 \\
1\end{array}$ & $\begin{array}{l}1 \\
1 \\
1 \\
1 \\
1 \\
1 \\
1\end{array}$ & $\begin{array}{l}1.4 \\
2.3 \\
3.3 \\
4.2 \\
5.2 \\
6.9 \\
8.5 \\
1.0\end{array}$ & $\begin{array}{l}-0 \\
-0 \\
-0 \\
-0 \\
-0 \\
-0 \\
-0 \\
-0\end{array}$ & & $\begin{array}{l}47 \\
44 \\
40 \\
37 \\
32 \\
29\end{array}$ \\
\hline $\begin{array}{l}.0000 \\
.0000 \\
.0000 \\
.00000 \\
.00000\end{array}$ & $\begin{array}{l}E+00 \\
E+00 \\
E+00 \\
E+00 \\
E+00 \\
E+00 \\
E+00 \\
E+00\end{array}$ & $\begin{array}{l}2 \\
3 \\
4 \\
4 \\
6 \\
7 \\
8\end{array}$ & & & & $\begin{array}{l}1 . \\
1 . \\
1 . \\
1 . \\
2 . \\
2 .\end{array}$ & $\begin{array}{l}-0 \\
-0 \\
-0 \\
-0 \\
-0 \\
-0 \\
-0\end{array}$ & & $\begin{array}{l}0 \\
0 \\
0 \\
0 \\
0 \\
0\end{array}$ \\
\hline $\begin{array}{l}0.0000 \\
0.00000 \\
0.00000 \\
0.00000 \\
0.00000\end{array}$ & $\begin{array}{l}2.590 E+00 \\
2.603 E+00 \\
2.614 E+00 \\
2.624 E+00 \\
2.633 E+00 \\
2.648 E+00 \\
2.660 E+00 \\
2.671 E+00\end{array}$ & $\begin{array}{l}124 E+01 \\
253 E+01 \\
383 E+01 \\
512 E+01 \\
771 E+01 \\
031 E+01 \\
290 E+01\end{array}$ & $\begin{array}{l}1 \\
1 \\
1 \\
1\end{array}$ & $\begin{array}{rl} & 01 \\
+ & 01 \\
+ & 1 \\
+01 \\
0 & 1 \\
0 & 1 \\
01 \\
+01\end{array}$ & $\begin{array}{l}1 \\
1 \\
1 \\
1 \\
1 \\
1 \\
1\end{array}$ & 0 & $\begin{array}{l}3 \\
2 \\
1 \\
0 \\
9 \\
8 \\
6 \\
5\end{array}$ & $\begin{array}{l}0 . \\
0 . \\
0 . \\
0 . \\
0 .\end{array}$ & 0 \\
\hline & $81 E+00$ & $550 E+0$ & $18 E+01$ & $04 E+0$ & $34 E-$ & $07 E$ & 0.005 & 01 & \\
\hline
\end{tabular}




\begin{tabular}{|c|c|c|c|c|c|c|c|c|c|}
\hline ENERGY & $\begin{array}{l}\text { COLLISION } \\
\mathrm{MeV} \mathrm{cm}^{2} / \mathrm{g}\end{array}$ & $\begin{array}{l}\text { OPPING POWE } \\
\text { RADIATIVE } \\
\text { MeV } \mathrm{cm}^{2} / \mathrm{g}\end{array}$ & $\begin{array}{c}\text { TOTAL } \\
\mathrm{MeV} \mathrm{cm}^{2} / \mathrm{g}\end{array}$ & $\begin{array}{l}\text { CSDA } \\
\text { RANGE } \\
\mathrm{g}^{\prime} \mathrm{Cm}^{2}\end{array}$ & $\begin{array}{l}\text { ADIATION } \\
\text { YIELD }\end{array}$ & $\begin{array}{l}\text { EHS.EFF. } \\
\text { CORR. } \\
\text { (DELTA) }\end{array}$ & $\begin{array}{l}\text { d( }] \\
\text { coll } \\
\text { Loss }\end{array}$ & $\begin{array}{l}) / d(d \\
\text { CSDA } \\
\text { RANGE }\end{array}$ & $\begin{array}{l}\text { I ) } \\
\text { RAD } \\
\text { IELD }\end{array}$ \\
\hline $\begin{array}{l}0.0100 \\
0.0125 \\
0.0150 \\
0.0175 \\
0.0200 \\
0.0250 \\
0.0300 \\
0.0350\end{array}$ & $\begin{array}{l}1.937 E+01 \\
1.632 E+01 \\
1.419 E+01 \\
1.260 E+01 \\
1.138 E+01 \\
9.595 E+00 \\
8.359 E+00 \\
7.447 E+00\end{array}$ & $\begin{array}{l}4.267 E-03 \\
4.298 E-03 \\
4.316 E-03 \\
4.328 E-03 \\
4.336 E-03 \\
4.347 E-03 \\
4.356 E-03 \\
4.365 E-03\end{array}$ & $\begin{array}{l}1.937 E+01 \\
1.633 E+01 \\
1.419 E+01 \\
1.261 E+01 \\
1.138 E+01 \\
9.600 E+00 \\
8.363 E+00 \\
7.452 E+00\end{array}$ & $\begin{array}{l}2.950 E-04 \\
4.362 E-04 \\
6.009 E-04 \\
7.882 E-04 \\
9.973 E-04 \\
1.478 E-03 \\
2.037 E-03 \\
2.672 E-03\end{array}$ & $\begin{array}{l}1.207 \mathrm{E}-04 \\
1.449 \mathrm{E}-04 \\
1.681 \mathrm{E}-04 \\
1.903 \mathrm{E}-04 \\
2.118 \mathrm{E}-04 \\
2.529 \mathrm{E}-04 \\
2.919 \mathrm{E}-04 \\
3.293 \mathrm{E}-04\end{array}$ & $\begin{array}{l}0.0 \\
0.0 \\
0.0 \\
0.0 \\
0.0 \\
0.0 \\
0.0 \\
0.0\end{array}$ & $\begin{array}{l}-0.209 \\
-0.199 \\
-0.193 \\
-0.187 \\
-0.183 \\
-0.176 \\
-0.170 \\
-0.166\end{array}$ & $\begin{array}{l}0 . \\
0 . \\
0 . \\
0 . \\
0 . \\
0 . \\
0 .\end{array}$ & $\begin{array}{l}0.239 \\
0.227 \\
0.218 \\
0.211 \\
0.206 \\
0.197 \\
0.190 \\
0.185\end{array}$ \\
\hline $\begin{array}{l}0.0400 \\
0.0450 \\
0.0500 \\
0.0550 \\
0.0600 \\
0.0700 \\
0.0800 \\
0.0900\end{array}$ & $\begin{array}{l}6.746 E+00 \\
6.189 E+00 \\
5.735 E+00 \\
5.358 E+00 \\
5.039 E+00 \\
4.530 E+00 \\
4.142 E+00 \\
3.835 E+00\end{array}$ & $\begin{array}{l}4.38 \\
4.40 \\
4.41 \\
4.43 \\
4.47 \\
4.51 \\
4.55\end{array}$ & $\begin{array}{l}0 E+00 \\
3 E+00 \\
9 E+00 \\
2 E+00 \\
4 E+00 \\
5 E+00 \\
6 E+00 \\
9 E+00\end{array}$ & $\begin{array}{l}3.3 \\
4.1 \\
4.9 \\
5.8 \\
6.8 \\
8.9 \\
1.1 \\
1.3\end{array}$ & $\begin{array}{l}E-04 \\
-04 \\
-04 \\
-04 \\
-04 \\
-04 \\
-04 \\
-04\end{array}$ & $\begin{array}{l}0.0 \\
0.0 \\
0.0 \\
0.0 \\
0.0 \\
0.0 \\
0.0 \\
0.0\end{array}$ & $\begin{array}{l}-0.1 \\
-0.1 \\
-0.1 \\
-0.1 \\
-0.1 \\
-0.1 \\
-0.1 \\
-0.1\end{array}$ & $\begin{array}{l}0 . \\
0 . \\
0 . \\
0 . \\
0 . \\
0 . \\
0 .\end{array}$ & $\begin{array}{l}0.180 \\
0.177 \\
0.174 \\
0.171 \\
0.169 \\
0.164 \\
0.161 \\
0.158\end{array}$ \\
\hline $\begin{array}{l}0.1000 \\
0.1250 \\
0.1500 \\
0.1750 \\
0.2000 \\
0.2500 \\
0.3000 \\
0.3500\end{array}$ & $\begin{array}{l}3.586 E+00 \\
3.133 E+00 \\
2.826 E+00 \\
2.606 E+00 \\
2.441 E+00 \\
2.211 E+00 \\
2.061 E+00 \\
1.957 E+00\end{array}$ & $\begin{array}{l}4.7 \\
4.8 \\
5.0 \\
5.2 \\
5.5 \\
5.9 \\
6.4\end{array}$ & $\begin{array}{l}E+00 \\
E+00 \\
E+00 \\
E+00 \\
E+00 \\
E+00 \\
E+00 \\
E+00\end{array}$ & $\begin{array}{l}1.6 \\
2.3 \\
3.2 \\
4.1 \\
5.1 \\
7.3 \\
9.6 \\
1.2\end{array}$ & $\begin{array}{l}7.3 \\
8.6 \\
9.9 \\
1.1 \\
1.2 \\
1.4 \\
1.6 \\
1.8\end{array}$ & $\begin{array}{l}0.0 \\
0.0 \\
0.0 \\
0.0 \\
0.0 \\
0.0 \\
0.0 \\
0.0\end{array}$ & $\begin{array}{l}-0 . \\
-0 . \\
-0 . \\
-0 . \\
-0 . \\
-0 . \\
-0 . \\
-0 .\end{array}$ & $\begin{array}{l}0 . \\
0 . \\
0 . \\
0 . \\
0 . \\
0 . \\
0 .\end{array}$ & $\begin{array}{l}56 \\
51 \\
47 \\
44 \\
41 \\
37 \\
34 \\
31\end{array}$ \\
\hline $\begin{array}{l}0.4000 \\
0.4500 \\
0.5000 \\
0.5500 \\
0.6000 \\
0.7000 \\
0.8000 \\
0.9000\end{array}$ & $\begin{array}{l}1.882 E+00 \\
1.826 E+00 \\
1.784 E+00 \\
1.751 E+00 \\
1.725 E+00 \\
1.690 E+00 \\
1.667 E+00 \\
1.654 E+00\end{array}$ & $\begin{array}{l}7.33 \\
7.83 \\
8.36 \\
8.90 \\
1.00 \\
1.12 \\
1.24\end{array}$ & $\begin{array}{l}+00 \\
+00 \\
+00 \\
+00 \\
+00 \\
+00 \\
+00 \\
+00 \\
+00 \\
+00\end{array}$ & $\begin{array}{l}1.4 \\
1.7 \\
2.0 \\
2.2 \\
2.5 \\
3.1 \\
3.7 \\
4.3\end{array}$ & $\begin{array}{l}2.0 \\
2.2 \\
2.4 \\
2.6 \\
2.8 \\
3.2 \\
3.6 \\
3.9\end{array}$ & $\begin{array}{l}0.0 \\
0.0 \\
0.0 \\
0.0 \\
0.0 \\
0.0 \\
0.0 \\
0.0\end{array}$ & $\begin{array}{l}-0 \\
-0 \\
-0 \\
-0 \\
-0 \\
-0 \\
-0 \\
-0\end{array}$ & $\begin{array}{l}0.1 \\
0.1 \\
0.1 \\
0.1 \\
0.1 \\
0.1 \\
0.1\end{array}$ & $\begin{array}{l}8 \\
6 \\
5 \\
3 \\
2 \\
9 \\
7 \\
5\end{array}$ \\
\hline $\begin{array}{l}1.0000 \\
1.2500 \\
1.5000 \\
1.7500 \\
2.0000 \\
2.5000 \\
3.0000 \\
3.5000\end{array}$ & $\begin{array}{l}1.646 E+00 \\
1.641 E+00 \\
1.647 E+00 \\
1.658 E+00 \\
1.671 E+00 \\
1.699 E+00 \\
1.727 E+00 \\
1.753 E+00\end{array}$ & $\begin{array}{l}1.718 \\
2.084 \\
2.468 \\
2.869 \\
3.711 \\
4.598\end{array}$ & $\begin{array}{l}E+00 \\
E+00 \\
E+00 \\
E+00 \\
E+00 \\
E+00 \\
E+00 \\
E+00\end{array}$ & $\begin{array}{l}4.9 \\
6.4 \\
7.9 \\
9.4 \\
1.0 \\
1.3 \\
1.6 \\
1.9\end{array}$ & $\begin{array}{l}4.3 \\
5.3 \\
6.3 \\
7.4 \\
8.4 \\
1.0 \\
1.2 \\
1.4\end{array}$ & $\begin{array}{l}0.0 \\
0.0 \\
0.0 \\
0.0 \\
0.0 \\
0.0 \\
0.0 \\
0.0\end{array}$ & $\begin{array}{l}-0.10 \\
-0.10 \\
-0.10 \\
-0.0 \\
-0.0 \\
-0.0 \\
-0.0 \\
-0.08\end{array}$ & $\begin{array}{l}0.1116 \\
0.1113 \\
0.1111 \\
0.1108 \\
0.107 \\
0.104 \\
0.1101 \\
0.099\end{array}$ & $\begin{array}{l}0.113 \\
0.110 \\
0.107 \\
0.104 \\
0.102 \\
0.099 \\
0.096 \\
0.094\end{array}$ \\
\hline $\begin{array}{l}4.00000 \\
4.5000 \\
5.0000 \\
5.5000 \\
6.00000 \\
7.00000 \\
8.0000 \\
9.0000\end{array}$ & $\begin{array}{l}1.777 E+00 \\
1.799 E+00 \\
1.820 E+00 \\
1.839 E+00 \\
1.857 E+00 \\
1.889 E+00 \\
1.918 E+00 \\
1.944 E+00\end{array}$ & $\begin{array}{l}7.448 \\
8.449 \\
9.470 \\
1.051 \\
1.264 \\
1.482\end{array}$ & $\begin{array}{l}1.842 E+00 \\
1.874 E+00 \\
1.905 E+00 \\
1.934 E+00 \\
1.962 E+00 \\
2.016 E+00 \\
2.066 E+00 \\
2.114 E+00\end{array}$ & $\begin{array}{l}2.224 \mathrm{E}+00 \\
2.493 \mathrm{E}+00 \\
2.758 \mathrm{E}+00 \\
3.018 \mathrm{E}+00 \\
3.275 \mathrm{E}+00 \\
3.777 \mathrm{E}+00 \\
4.267 \mathrm{E}+00 \\
4.746 \mathrm{E}+00\end{array}$ & $\begin{array}{l}1.720 \mathrm{E}-02 \\
1.945 \mathrm{E}-02 \\
2.171 \mathrm{E}-02 \\
2.398 \mathrm{E}-02 \\
2.626 \mathrm{E}-02 \\
3.081 \mathrm{E}-02 \\
3.536 \mathrm{E}-02 \\
3.990 \mathrm{E}-02\end{array}$ & $\begin{array}{l}0.0 \\
0.0 \\
0.0 \\
0.0 \\
0.0 \\
0.0 \\
0.0 \\
0.0\end{array}$ & $\begin{array}{l}-0.088 \\
-0.086 \\
-0.085 \\
-0.084 \\
-0.083 \\
-0.082 \\
-0.080 \\
-0.079\end{array}$ & $\begin{array}{l}98 \\
96 \\
95 \\
94 \\
92 \\
91 \\
39 \\
37\end{array}$ & $\begin{array}{l}0.0 \\
0.0 \\
0.0 \\
0.0 \\
0.0 \\
0.0\end{array}$ \\
\hline $\begin{array}{l}10.0000 \\
12.5000 \\
15.0000 \\
17.5000 \\
20.0000 \\
25.0000 \\
30.0000 \\
35.0000\end{array}$ & $\begin{array}{l}1.967 E+00 \\
2.016 E+00 \\
2.057 E+00 \\
2.092 E+00 \\
2.122 E+00 \\
2.173 E+00 \\
2.214 E+00 \\
2.246 E+00\end{array}$ & $\begin{array}{l}2.514 \\
3.112 \\
3.723 \\
4.343 \\
5.606 \\
6.890\end{array}$ & $\begin{array}{l}E+00 \\
E+00 \\
E+00 \\
E+00 \\
E+00 \\
E+00 \\
E+00 \\
E+00\end{array}$ & $\begin{array}{l}5.2 \\
6.3 \\
7.4 \\
8.4 \\
9.4 \\
1.1 \\
1.3 \\
1.4\end{array}$ & $\begin{array}{l}5.5 \\
6.6 \\
7.7 \\
8.7 \\
1.0 \\
1.2 \\
1.2\end{array}$ & $\begin{array}{l}0.0 \\
0.0 \\
0.0 \\
0.0 \\
0.0 \\
0.0 \\
2.989 E-0 \\
4.425 E-0\end{array}$ & $\begin{array}{l}-0.078 \\
-0.076 \\
-0.075 \\
-0.074 \\
-0.073 \\
-0.071 \\
-0.065 \\
-0.054\end{array}$ & $\begin{array}{l}0.086 \\
0.083 \\
0.081 \\
0.079 \\
0.077 \\
0.074 \\
0.071 \\
0.068\end{array}$ & $\begin{array}{l}0 \\
0 . \\
0 \\
0 . \\
0 .\end{array}$ \\
\hline $\begin{array}{l}45.0000 \\
50.0000 \\
55.0000 \\
60.0000 \\
70.0000 \\
80.0000\end{array}$ & $\begin{array}{l}2.29 \\
2.31 \\
2.32 \\
2.33 \\
2.36 \\
2.37\end{array}$ & $\begin{array}{l}1.08 \\
1.2 \\
1.3 \\
1.48 \\
1.7\end{array}$ & $\begin{array}{l}3 \\
3 \\
3 \\
3 \\
4 \\
4\end{array}$ & $\begin{array}{l}1.7 \\
1.9 \\
2.0 \\
2.2 \\
2.4\end{array}$ & $\begin{array}{l}1.7 \\
1.5 \\
2 . \\
2.2 \\
2.9\end{array}$ & $\begin{array}{l}1.9 \\
2.8 \\
3.6 \\
4.5 \\
6.2 \\
7.8 \\
9.2\end{array}$ & $\begin{array}{l}-0 \\
-0 \\
-0 \\
-0 \\
-0 \\
-0 \\
-0 \\
-0\end{array}$ & $\begin{array}{l}0 . \\
0 . \\
0 . \\
0 . \\
0 .\end{array}$ & \\
\hline $\begin{array}{llll}0 & 0 & 0 & 0 \\
0 & 0 & 0 & 0 \\
0 & 0 & 0 & 0 \\
0 & 0 & 0 & 0 \\
0 & 0 & 0 & 0\end{array}$ & $\begin{array}{l}2.409 E+00 \\
2.437 E+00 \\
2.460 E+00 \\
2.478 E+00 \\
2.494 E+00 \\
2.520 E+00 \\
2.541 E+00 \\
2.558 E+00\end{array}$ & $\begin{array}{l}3.2 \\
3.9 \\
4.6 \\
5.3 \\
6.7 \\
8.2 \\
9.6\end{array}$ & $\begin{array}{ll}+ & 0 \\
+ & 0 \\
+0 & 0 \\
+0 & 0 \\
+0 & 0 \\
+0 & 0 \\
+0 & 1 \\
+0 & 1\end{array}$ & $\begin{array}{l}3 . \\
4 . \\
4 . \\
4 . \\
5 . \\
5 . \\
6 .\end{array}$ & 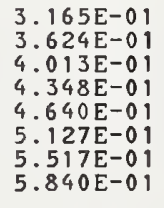 & $\begin{array}{l}1.3 \\
1.8 \\
1.8 \\
2.8 \\
2.8 \\
2.8\end{array}$ & $\begin{array}{l}-0 \\
-0 \\
-0 \\
-0 \\
-0 \\
-0 \\
-0\end{array}$ & $\begin{array}{l}0 \\
0 \\
0 . \\
0 \\
0 . \\
0 . \\
0 .\end{array}$ & \\
\hline $\begin{array}{llll}0 & 0 & 0 & 0 \\
0 & 0 & 0 & 0 \\
0 & 0 & 0 & 0 \\
0 & 0 & 0 & 0 \\
0 & 0 & 0 & 0 \\
0 & 0 & 0 & 0 \\
0 & 0 & 0 & 0\end{array}$ & $\begin{array}{l}2.573 E+00 \\
2.586 E+00 \\
2.597 E+00 \\
2.608 E+00 \\
2.617 E+00 \\
2.633 E+00 \\
2.646 E+00 \\
2.658 E+00\end{array}$ & $\begin{array}{l}1.105 \mathrm{E} \\
1.248 \mathrm{E} \\
1.391 \mathrm{E} \\
1.534 \mathrm{E} \\
1.678 \mathrm{E} \\
1.965 \mathrm{E} \\
2.252 \mathrm{E} \\
2.540 \mathrm{E}\end{array}$ & $\begin{array}{l}62 E+01 \\
07 E+01 \\
51 E+01 \\
95 E+01 \\
39 E+01 \\
28 E+01 \\
17 E+01 \\
05 E+01\end{array}$ & $\begin{array}{l}01 \\
01 \\
01 \\
01 \\
01 \\
01 \\
01 \\
01 \\
01\end{array}$ & $\begin{array}{l}E-01 \\
B E-01 \\
3 E-01 \\
4 E-01 \\
1 E-01 \\
B E-01 \\
4 E-01 \\
1 E-01 \\
4 E-01\end{array}$ & $\begin{array}{l}0 \\
0 \\
0 \\
0 \\
0 \\
0\end{array}$ & $\begin{array}{l}-0 \\
-0 \\
-0 \\
-0 \\
-0 \\
-0 \\
-0\end{array}$ & $\begin{array}{l}0 . \\
0 . \\
0 . \\
0 . \\
0 . \\
0 . \\
0 . \\
0 .\end{array}$ & $\begin{array}{l}07 \\
06 \\
06 \\
05 \\
05 \\
04 \\
04 \\
04\end{array}$ \\
\hline & $.668 E+00$ & $.827 E+01$ & $094 E+01$ & $454 E+01$ & $699 E-01$ & $.603 E+0$ & -0.007 & 0.017 & .0 \\
\hline
\end{tabular}




\begin{tabular}{|c|c|c|c|c|c|c|c|c|c|}
\hline NERGY & $\begin{array}{l}\text { COLLISION } \\
\mathrm{MeV} \mathrm{cm}^{2} / \mathrm{g}\end{array}$ & $\begin{array}{l}\text { OPP ING POWE } \\
\text { RADIATIVE } \\
\mathrm{MeV} \mathrm{cm}^{2} / \mathrm{g}\end{array}$ & $\begin{array}{c}\text { TOTAL } \\
\text { MoV } \mathrm{cm}^{2} / \mathrm{g}\end{array}$ & $\begin{array}{l}\text { CSDA } \\
\text { RANGE } \\
\mathrm{g} / \mathrm{cm}^{2}\end{array}$ & $\begin{array}{l}\text { RADIATION } \\
\text { YIELD }\end{array}$ & $\begin{array}{c}\text { DENS . EFF } \\
\text { CORR. } \\
\text { (DELTA) }\end{array}$ & $\begin{array}{l}d(I \\
\text { coLL } \\
\text { LOSS }\end{array}$ & $\begin{array}{l}) / \mathrm{d}(1 \\
\text { CSDA } \\
\text { RANGE }\end{array}$ & $\begin{array}{l}\text { II ) } \\
\text { RAD } \\
\text { YIELD }\end{array}$ \\
\hline $\begin{array}{l}0.0100 \\
0.0125 \\
0.0150 \\
0.0175 \\
0.0200 \\
0.0250 \\
0.0300 \\
0.0350\end{array}$ & $\begin{array}{l}1.773 E+01 \\
1.500 E+01 \\
1.307 E+01 \\
1.164 E+01 \\
1.052 E+01 \\
8.898 E+00 \\
7.768 E+00 \\
6.932 E+00\end{array}$ & $\begin{array}{l}5.273 E-03 \\
5.340 E-03 \\
5.384 E-03 \\
5.416 E-03 \\
5.439 E-03 \\
5.470 E-03 \\
5.492 E-03 \\
5.510 E-03\end{array}$ & $\begin{array}{l}1.774 E+01 \\
1.500 E+01 \\
1.308 E+01 \\
1.164 E+01 \\
1.053 E+01 \\
8.904 E+00 \\
7.773 E+00 \\
6.938 E+00\end{array}$ & $\begin{array}{l}3.265 \mathrm{E}-04 \\
4.804 \mathrm{E}-04 \\
6.595 \mathrm{E}-04 \\
8.625 \mathrm{E}-04 \\
1.089 \mathrm{E}-03 \\
1.607 \mathrm{E}-03 \\
2.210 \mathrm{E}-03 \\
2.892 \mathrm{E}-03\end{array}$ & $\begin{array}{l}1.622 E-04 \\
1.951 E-04 \\
2.266 E-04 \\
2.569 E-04 \\
2.862 E-04 \\
3.422 E-04 \\
3.953 E-04 \\
4.461 E-04\end{array}$ & $\begin{array}{l}0.0 \\
0.0 \\
0.0 \\
0.0 \\
0.0 \\
0.0 \\
0.0 \\
0.0\end{array}$ & $\begin{array}{l}-0.226 \\
-0.215 \\
-0.207 \\
-0.201 \\
-0.196 \\
-0.188 \\
-0.182 \\
-0.177\end{array}$ & $\begin{array}{l}0.266 \\
0.251 \\
0.240 \\
0.232 \\
0.225 \\
0.214 \\
0.206 \\
0.200\end{array}$ & $\begin{array}{l}0.262 \\
0.248 \\
0.237 \\
0.229 \\
0.223 \\
0.212 \\
0.204 \\
0.198\end{array}$ \\
\hline $\begin{array}{l}0.0400 \\
0.0450 \\
0.0500 \\
0.0550 \\
0.0600 \\
0.0700 \\
0.0800 \\
0.0900\end{array}$ & $\begin{array}{l}6.288 E+00 \\
5.775 E+00 \\
5.357 E+00 \\
5.009 E+00 \\
4.715 E+00 \\
4.244 E+00 \\
3.884 E+00 \\
3.599 E+00\end{array}$ & $\begin{array}{l}6 E-03 \\
3 E-03 \\
9 E-03 \\
7 E-03 \\
5 E-03 \\
4 E-03 \\
9 E-03 \\
7 E-03\end{array}$ & $\begin{array}{l}93 E+00 \\
31 E+00 \\
2 E+00 \\
14 E+00 \\
20 E+00 \\
50 E+00 \\
90 E+00 \\
5 E+00\end{array}$ & $\begin{array}{l}4 \cdot 4 \\
5.3 \\
6.3 \\
7.3 \\
9.6 \\
1.2 \\
1.4\end{array}$ & $\begin{array}{l}4.949 E-04 \\
5.420 E-04 \\
5.876 E-04 \\
6.319 E-04 \\
6.750 E-04 \\
7.580 E-04 \\
8.374 E-04 \\
9.138 E-04\end{array}$ & $\begin{array}{l}0.0 \\
0.0 \\
0.0 \\
0.0 \\
0.0 \\
0.0 \\
0.0 \\
0.0\end{array}$ & $\begin{array}{l}-0.173 \\
-0.170 \\
-0.167 \\
-0.164 \\
-0.162 \\
-0.158 \\
-0.155 \\
-0.153\end{array}$ & $\begin{array}{l}0.194 \\
0.190 \\
0.186 \\
0.183 \\
0.180 \\
0.176 \\
0.172 \\
0.169\end{array}$ & $\begin{array}{l}0.193 \\
0.189 \\
0.186 \\
0.183 \\
0.180 \\
0.175 \\
0.171 \\
0.168\end{array}$ \\
\hline $\begin{array}{l}0.1000 \\
0.1250 \\
0.1500 \\
0.1750 \\
0.2000 \\
0.2500 \\
0.3000 \\
0.3500\end{array}$ & $\begin{array}{l}3.369 E+00 \\
2.948 E+00 \\
2.663 E+00 \\
2.458 E+00 \\
2.304 E+00 \\
2.090 E+00 \\
1.950 E+00 \\
1.854 E+00\end{array}$ & $\begin{array}{l}5.78 \\
5.93 \\
6.10 \\
6.29 \\
6.50 \\
6.94 \\
7.43 \\
7.95\end{array}$ & $\begin{array}{l}3.375 E+00 \\
2.954 E+00 \\
2.669 E+00 \\
2.464 E+00 \\
2.310 E+00 \\
2.097 E+00 \\
1.958 E+00 \\
1.862 E+00\end{array}$ & $\begin{array}{l}1.762 \\
2.557 \\
3.449 \\
4.426 \\
5.475 \\
7.754 \\
1.023 \\
1.285\end{array}$ & $\begin{array}{l}5 E-04 \\
3 E-03 \\
7 E-03 \\
4 E-03 \\
4 E-03 \\
0 E-03 \\
2 E-03 \\
6 E-03\end{array}$ & $\begin{array}{l}0.0 \\
0.0 \\
0.0 \\
0.0 \\
0.0 \\
0.0 \\
0.0 \\
0.0\end{array}$ & $\begin{array}{l}-0.1 \\
-0.1 \\
-0.1 \\
-0.1 \\
-0.1 \\
-0.1 \\
-0.1 \\
-0.1\end{array}$ & $\begin{array}{l}0.166 \\
0.160 \\
0.156 \\
0.152 \\
0.150 \\
0.145 \\
0.142 \\
0.139\end{array}$ & $\begin{array}{l}0.165 \\
0.160 \\
0.155 \\
0.152 \\
0.149 \\
0.144 \\
0.140 \\
0.137\end{array}$ \\
\hline $\begin{array}{l}0.4000 \\
0.4500 \\
0.5000 \\
0.5500 \\
0.6000 \\
0.7000 \\
0.8000 \\
0.9000\end{array}$ & $\begin{array}{l}1.784 E+00 \\
1.732 E+00 \\
1.693 E+00 \\
1.663 E+00 \\
1.639 E+00 \\
1.607 E+00 \\
1.587 E+00 \\
1.575 E+00\end{array}$ & $\begin{array}{l}9.09 \\
9.70 \\
1.03 \\
1.10 \\
1.23 \\
1.38 \\
1.53\end{array}$ & $\begin{array}{l}1.792 \mathrm{E}+00 \\
1.741 \mathrm{E}+00 \\
1.703 \mathrm{E}+00 \\
1.673 \mathrm{E}+00 \\
1.650 \mathrm{E}+00 \\
1.619 \mathrm{E}+00 \\
1.601 \mathrm{E}+00 \\
1.590 \mathrm{E}+00\end{array}$ & $\begin{array}{l}1.5 \\
1.8 \\
2.1 \\
2.4 \\
2.7 \\
3.3 \\
3.9 \\
4.5\end{array}$ & $\begin{array}{l}3 E-03 \\
5 E-03 \\
5 E-03 \\
3 E-03 \\
9 E-03 \\
0 E-03 \\
1 E-03 \\
4 E-03\end{array}$ & $\begin{array}{l}0.0 \\
0.0 \\
0.0 \\
0.0 \\
0.0 \\
0.0 \\
0.0 \\
0.0\end{array}$ & $\begin{array}{l}-0.1 \\
-0.1 \\
-0.1 \\
-0.1 \\
-0.1 \\
-0.1 \\
-0.1 \\
-0.1\end{array}$ & $\begin{array}{l}0.1 \\
0.1 \\
0.1 \\
0.1 \\
0.1 \\
0.1 \\
0.1 \\
0.1\end{array}$ & $\begin{array}{l}0.135 \\
0.133 \\
0.131 \\
0.129 \\
0.127 \\
0.124 \\
0.122 \\
0.120\end{array}$ \\
\hline $\begin{array}{l}1.0000 \\
1.2500 \\
1.5000 \\
1.7500 \\
2.0000 \\
2.5000 \\
3.0000 \\
3.5000\end{array}$ & $\begin{array}{l}1.568 E+00 \\
1.565 E+00 \\
1.572 E+00 \\
1.584 E+00 \\
1.598 E+00 \\
1.626 E+00 \\
1.654 E+00 \\
1.681 E+00\end{array}$ & $\begin{array}{l}1.686 \mathrm{E}-02 \\
2.100 \mathrm{E}-02 \\
2.542 \mathrm{E}-02 \\
3.008 \mathrm{E}-02 \\
3.493 \mathrm{E}-02 \\
4.513 \mathrm{E}-02 \\
5.586 \mathrm{E}-02 \\
6.701 \mathrm{E}-02\end{array}$ & $\begin{array}{l}1.585 E+00 \\
1.586 E+00 \\
1.598 E+00 \\
1.614 E+00 \\
1.633 E+00 \\
1.672 E+00 \\
1.710 E+00 \\
1.748 E+00\end{array}$ & $\begin{array}{l}5.221 E-01 \\
6.799 E-01 \\
8.370 E-01 \\
9.927 E-01 \\
1.147 E+00 \\
1.449 E+00 \\
1.745 E+00 \\
2.034 E+00\end{array}$ & $\begin{array}{l}5.678 E-03 \\
6.929 E-03 \\
8.202 E-03 \\
9.497 E-03 \\
1.081 E-02 \\
1.349 E-02 \\
1.621 E-02 \\
1.897 E-02\end{array}$ & $\begin{array}{l}0.0 \\
0.0 \\
0.0 \\
0.0 \\
0.0 \\
0.0 \\
0.0 \\
0.0\end{array}$ & $\begin{array}{l}-0.110 \\
-0.106 \\
-0.104 \\
-0.101 \\
-0.099 \\
-0.096 \\
-0.094 \\
-0.092\end{array}$ & $\begin{array}{l}0.121 \\
0.118 \\
0.1115 \\
0.1113 \\
0.1111 \\
0.108 \\
0.105 \\
0.103\end{array}$ & $\begin{array}{l}0.1118 \\
0.1114 \\
0.1111 \\
0.1108 \\
0.1106 \\
0.102 \\
0.099 \\
0.097\end{array}$ \\
\hline $\begin{array}{l}4.0000 \\
4.5000 \\
5.0000 \\
5.5000 \\
6.0000 \\
7.0000 \\
8.0000 \\
9.0000\end{array}$ & $\begin{array}{l}1.705 E+00 \\
1.727 E+00 \\
1.748 E+00 \\
1.767 E+00 \\
1.785 E+00 \\
1.817 E+00 \\
1.845 E+00 \\
1.870 E+00\end{array}$ & $\begin{array}{l}9.0 \\
1.02 \\
1.1 \\
1.2 \\
1.5 \\
1.7 \\
2.0\end{array}$ & $\begin{array}{l}1.783 E+00 \\
1.817 E+00 \\
1.850 E+00 \\
1.882 E+00 \\
1.912 E+00 \\
1.970 E+00 \\
2.024 E+00 \\
2.076 E+00\end{array}$ & $\begin{array}{l}2.317 E+00 \\
2.595 E+00 \\
2.868 E+00 \\
3.136 E+00 \\
3.399 E+00 \\
3.915 E+00 \\
4.415 E+00 \\
4.903 E+00\end{array}$ & $\begin{array}{l}2.17 \\
2.45 \\
2.73 \\
3.01 \\
3.29 \\
3.85 \\
4.41 \\
4.96\end{array}$ & $\begin{array}{l}0.0 \\
0.0 \\
0.0 \\
0.0 \\
0.0 \\
0.0 \\
0.0 \\
0.0\end{array}$ & $\begin{array}{l}-0.091 \\
-0.089 \\
-0.088 \\
-0.087 \\
-0.086 \\
-0.084 \\
-0.083 \\
-0.082\end{array}$ & $\begin{array}{l}0.101 \\
0.099 \\
0.098 \\
0.097 \\
0.095 \\
0.093 \\
0.091 \\
0.090\end{array}$ & $\begin{array}{l}0.094 \\
0.093 \\
0.091 \\
0.089 \\
0.088 \\
0.086 \\
0.083 \\
0.082\end{array}$ \\
\hline $\begin{array}{l}.0000 \\
.5000 \\
.0000 \\
.5000 \\
.0000 \\
.0000 \\
.0000 \\
5.0000\end{array}$ & $\begin{array}{l}1.893 E+00 \\
1.942 E+00 \\
1.983 E+00 \\
2.017 E+00 \\
2.047 E+00 \\
2.098 E+00 \\
2.139 E+00 \\
2.174 E+00\end{array}$ & $\begin{array}{l}2.330 \mathrm{E}-01 \\
3.026 \mathrm{E}-01 \\
3.739 \mathrm{E}-01 \\
4.466 \mathrm{E}-01 \\
5.204 \mathrm{E}-01 \\
6.703 \mathrm{E}-01 \\
8.226 \mathrm{E}-01 \\
9.766 \mathrm{E}-01\end{array}$ & $\begin{array}{l}2.126 E+00 \\
2.245 E+00 \\
2.357 E+00 \\
2.464 E+00 \\
2.568 E+00 \\
2.768 E+00 \\
2.962 E+00 \\
3.151 E+00\end{array}$ & $\begin{array}{l}5.379 E+00 \\
6.523 \mathrm{E}+00 \\
7.609 \mathrm{E}+00 \\
8.646 \mathrm{E}+00 \\
9.640 \mathrm{E}+00 \\
1.151 \mathrm{E}+01 \\
1.326 \mathrm{E}+01 \\
1.490 \mathrm{E}+01\end{array}$ & $\begin{array}{l}5.510 \mathrm{E}-02 \\
6.854 \mathrm{E}-02 \\
8.159 \mathrm{E}-02 \\
9.423 \mathrm{E}-02 \\
1.065 \mathrm{E}-01 \\
1.297 \mathrm{E}-01 \\
1.515 \mathrm{E}-01 \\
1.719 \mathrm{E}-01\end{array}$ & $\begin{array}{l}0.0 \\
0.0 \\
0.0 \\
0.0 \\
0.0 \\
0.0 \\
0.0 \\
0.0\end{array}$ & $\begin{array}{l}-0.081 \\
-0.079 \\
-0.077 \\
-0.076 \\
-0.074 \\
-0.073 \\
-0.071 \\
-0.070\end{array}$ & $\begin{array}{l}0 . \\
0 . \\
0 . \\
0 . \\
0 . \\
0 . \\
0 . \\
0 .\end{array}$ & $\begin{array}{l}0.080 \\
0.076 \\
0.073 \\
0.071 \\
0.068 \\
0.065 \\
0.061 \\
0.059\end{array}$ \\
\hline $\begin{array}{l}.0000 \\
.0000 \\
.0000 \\
.0000 \\
.0000 \\
.0000 \\
.0000 \\
.0000\end{array}$ & $\begin{array}{l}2.204 E+00 \\
2.231 E+00 \\
2.255 E+00 \\
2.277 E+00 \\
2.297 E+00 \\
2.329 E+00 \\
2.355 E+00 \\
2.376 E+00\end{array}$ & $\begin{array}{l}1.2 \\
1.4 \\
1.6 \\
1.7 \\
2.0 \\
2.4 \\
2.7\end{array}$ & $\begin{array}{l}3.336 E+00 \\
3.520 E+00 \\
3.701 E+00 \\
3.881 E+00 \\
4.060 E+00 \\
4.413 E+00 \\
4.761 E+00 \\
5.105 E+00\end{array}$ & $\begin{array}{l}1.6 \\
1.7 \\
1.9 \\
2.0 \\
2.1 \\
2.4\end{array}$ & 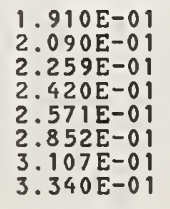 & $\begin{array}{l}0.0 \\
0.0 \\
0.0 \\
0.0 \\
0.0 \\
2.873 \mathrm{E}-02 \\
9.312 \mathrm{E}-02 \\
1.741 \mathrm{E}-01\end{array}$ & $\begin{array}{l}-0 . \\
-0 . \\
-0 . \\
-0 . \\
-0 . \\
-0 . \\
-0 . \\
-0 .\end{array}$ & & $\begin{array}{l}0 . \\
0 . \\
0 . \\
0 . \\
0 . \\
0 . \\
0 .\end{array}$ \\
\hline $\begin{array}{l}.0000 \\
.0000 \\
.0000 \\
.0000 \\
.0000 \\
.00000\end{array}$ & $\begin{array}{l}2.427 E+00 \\
2.452 E+00 \\
2.472 E+00 \\
2.488 E+00 \\
2.515 E+00 \\
2.536 E+00 \\
2.554 E+00\end{array}$ & $\begin{array}{l}3.8 \\
4.6 \\
5.5 \\
6.3 \\
8.0 \\
9.6 \\
1.1\end{array}$ & $\begin{array}{l}6.2 \\
7.1 \\
7.5 \\
8.8 \\
1.9 \\
1.2 \\
1.3\end{array}$ & & & $\begin{array}{l}2.6 \\
4.88 \\
7.0 \\
9.0 \\
1.0 \\
1.4 \\
1.6 \\
1.9\end{array}$ & $\begin{array}{l}-0 \\
-0 \\
-0 \\
-0 \\
-0 \\
-0 \\
-0 \\
-0\end{array}$ & & \\
\hline $\begin{array}{l}0000 \\
0000 \\
0000 \\
00000 \\
0000\end{array}$ & $\begin{array}{l}2.581 E+00 \\
2.581 \mathrm{E}+00 \\
2.593 \mathrm{E}+00 \\
2.603 \mathrm{E}+00 \\
2.612 \mathrm{E}+00 \\
2.628 \mathrm{E}+00 \\
2.642 \mathrm{E}+00 \\
2.655 \mathrm{E}+00\end{array}$ & $\begin{array}{l}1.303 \\
1.471 \\
1.63 \\
1.808 \\
1.976 \\
2.31 \\
2.65 \\
2.98\end{array}$ & $\begin{array}{l}.729 E+01 \\
.899 E+01 \\
.068 E+01 \\
.237 E+01 \\
.576 E+01 \\
.915 E+01 \\
.254 E+01\end{array}$ & $\begin{array}{l}1 \\
1 \\
1 \\
1\end{array}$ & $\begin{array}{l}6 \\
7 \\
7 \\
7 \\
7\end{array}$ & $\begin{array}{l}2.114 \mathrm{Et} \\
2.298 \mathrm{E}+ \\
2.464 \mathrm{Et} \\
2.616 \mathrm{Et} \\
2.755 \mathrm{E}+ \\
3.003 \mathrm{E}+ \\
3.220 \mathrm{E}+ \\
3.413 \mathrm{E} t\end{array}$ & $\begin{array}{l}3 \\
3 \\
2 \\
2 \\
2 \\
1 \\
1 \\
0\end{array}$ & $\begin{array}{l}8 \\
7 \\
6 \\
5 \\
4 \\
3 \\
2 \\
1\end{array}$ & $\begin{array}{l}9 \\
8 \\
7 \\
7 \\
6 \\
5 \\
5 \\
4\end{array}$ \\
\hline & $5 \mathrm{E}$ & 0 & $94 E+01$ & $03 E+0$ & 371 & $587 \mathrm{E}+$ & 0.010 & 020 & \\
\hline
\end{tabular}




\begin{tabular}{|c|c|c|c|c|c|c|c|c|c|}
\hline $\mathrm{MeV}$ & $\begin{array}{l}\text { COLLISION } \\
\mathrm{MeV} \mathrm{cm} 2 / \mathrm{g}\end{array}$ & 2 & TOTAL & $\begin{array}{l}\text { CSDA } \\
\text { RAHGE }\end{array}$ & N & $\begin{array}{l}\text { ENS.EFF. } \\
\text { CORR. } \\
\text { (DELTA) }\end{array}$ & $\begin{array}{l}d(1 \\
\text { COLL } \\
\text { LOSS }\end{array}$ & CSDA & $\begin{array}{l}\text { I) } \\
\text { RAD } \\
\text { IELD }\end{array}$ \\
\hline $\begin{array}{l}0.0100 \\
0.0125 \\
0.0150 \\
0.0175 \\
0.0200 \\
0.0250 \\
0.0300 \\
0.0350\end{array}$ & $\begin{array}{l}1.649 E+01 \\
1.398 E+01 \\
1.220 E+01 \\
1.088 E+01 \\
9.844 E+00 \\
8.338 E+00 \\
7.287 E+00 \\
6.509 E+00\end{array}$ & $\begin{array}{l}6.7 \\
6.8 \\
6.9 \\
7.0 \\
7.0\end{array}$ & $\begin{array}{l}1.3 \\
1.2 \\
1.0 \\
9.8 \\
8.3 \\
7.2 \\
6.5\end{array}$ & $\begin{array}{l}4 \\
4 \\
4 \\
4 \\
3 \\
3 \\
3 \\
3\end{array}$ & & $\begin{array}{l}E-04 \\
E-04 \\
E-04 \\
-04 \\
-03 \\
-03 \\
-03 \\
-03 \\
-03\end{array}$ & & & $\begin{array}{l}0.275 \\
0.260 \\
0.248 \\
0.239 \\
0.232 \\
0.221 \\
0.212 \\
0.206\end{array}$ \\
\hline $\begin{array}{l}0.0400 \\
0.0450 \\
0.0500 \\
0.0550 \\
0.0600 \\
0.0700 \\
0.0800 \\
0.0900\end{array}$ & $\begin{array}{l}+00 \\
+00 \\
+00 \\
+00 \\
+00 \\
+00 \\
+00 \\
+00\end{array}$ & $\begin{array}{l}03 \\
03 \\
03 \\
03 \\
03 \\
03 \\
03 \\
03 \\
03\end{array}$ & $\begin{array}{l}5.4 \\
5.0 \\
4.7 \\
4.4 \\
4.0 \\
3.6 \\
3.4\end{array}$ & $\begin{array}{l}.03 \\
03 \\
03 \\
03 \\
03 \\
.02 \\
.02 \\
.02\end{array}$ & & $\begin{array}{l}4 . \\
5 . \\
6 . \\
8 . \\
1 . \\
1 .\end{array}$ & & & $\begin{array}{l}.201 \\
.196 \\
.192 \\
.189 \\
.186 \\
.181 \\
.177 \\
.173\end{array}$ \\
\hline $\begin{array}{l}0.1000 \\
0.1250 \\
0.1500 \\
0.1750 \\
0.2000 \\
0.2500 \\
0.3000 \\
0.3500\end{array}$ & $\begin{array}{l}3.17 \\
2.78 \\
2.51 \\
2.32 \\
2.17 \\
1.97 \\
1.83\end{array}$ & $\begin{array}{l}03 \\
03 \\
03 \\
03 \\
03 \\
03 \\
03\end{array}$ & $\begin{array}{l}3.1 \\
2.7 \\
2.5 \\
2.3 \\
2.1 \\
1.9 \\
1.8 \\
1.7\end{array}$ & $\begin{array}{l}02 \\
02 \\
02 \\
02 \\
02 \\
02 \\
02 \\
01\end{array}$ & $\begin{array}{l}03 \\
03 \\
03 \\
03 \\
03 \\
03 \\
03\end{array}$ & $\begin{array}{l}2.9 \\
3.6 \\
4.5\end{array}$ & & & $\begin{array}{l}170 \\
164 \\
160 \\
156 \\
153 \\
148 \\
144 \\
141\end{array}$ \\
\hline $\begin{array}{l}0.4000 \\
0.4500 \\
0.5000 \\
0.5500 \\
0.6000 \\
0.7000 \\
0.8000\end{array}$ & $\begin{array}{l}1.5 \\
1.5 \\
1.5 \\
1.5\end{array}$ & $\begin{array}{l}0 \\
1: \\
1 .\end{array}$ & $\begin{array}{l}1.6 \\
1.6 \\
1.5 \\
1.5 \\
1.5 \\
1.5 \\
1.4\end{array}$ & $\begin{array}{l}1 \\
1 \\
1 \\
1 \\
1 \\
1\end{array}$ & $\begin{array}{l}4 . \\
4 . \\
5 .\end{array}$ & $\begin{array}{l}1 \cdot 3 \\
1.5 \\
1.7 \\
1.2 \\
2.3 \\
2.6 \\
3.0\end{array}$ & & & $\begin{array}{l}35 \\
33 \\
31 \\
30 \\
26 \\
24\end{array}$ \\
\hline $\begin{array}{l}1.0000 \\
1.2500 \\
1.5000 \\
1.7500 \\
2.0000 \\
2.5000 \\
3.0000 \\
3.5000\end{array}$ & $\begin{array}{l}100 \\
00 \\
00 \\
00 \\
00 \\
00 \\
00 \\
0 \\
00 \\
00\end{array}$ & $\begin{array}{l}2.6 \\
3.1 \\
3.7 \\
4 . \\
5.6 \\
6 .\end{array}$ & $\begin{array}{l}1.5 \\
1.5 \\
1.5 \\
1.5 \\
1.5 \\
1.5\end{array}$ & $\begin{array}{l}1 \\
1 \\
0 \\
0\end{array}$ & & $\begin{array}{l}4.1 \\
4.8 \\
5.6 \\
6.3 \\
7.7 \\
9.1 \\
1.0\end{array}$ & & & 5 \\
\hline $\begin{array}{l}4.0000 \\
4.5000 \\
5.0000 \\
5.5000 \\
6.0000 \\
7.0000 \\
8.0000 \\
9.0000\end{array}$ & $\begin{array}{l}+\infty 0 \\
+\infty 0 \\
+00 \\
+00 \\
+00 \\
+00\end{array}$ & $\begin{array}{l}1.2 \\
1.4 \\
1.5\end{array}$ & $\begin{array}{l}1.8 \\
1.8 \\
1.8 \\
1.8\end{array}$ & $\begin{array}{l}0 \\
0 \\
0 \\
0 \\
0\end{array}$ & $\begin{array}{l}2.918 \mathrm{E}-02 \\
3.296 \mathrm{E}-02 \\
3.675 \mathrm{E}-02 \\
4.055 \mathrm{E}-02 \\
4.436 \mathrm{E}-02 \\
5.197 \mathrm{E}-02 \\
5.955 \mathrm{E}-02 \\
6.708 \mathrm{E}-02\end{array}$ & $\begin{array}{l}1.311 \mathrm{E}+ \\
1.433 \mathrm{E}+ \\
1.550 \mathrm{E}+ \\
1.661 \mathrm{E}+ \\
1.868 \mathrm{E}+ \\
2.055 \mathrm{E}+ \\
2.226 \mathrm{E}+\end{array}$ & & & 2 \\
\hline $\begin{array}{l}10.0000 \\
12.5000 \\
15.0000 \\
17.5000 \\
20.0000 \\
25.0000\end{array}$ & & $\begin{array}{l}3 \\
4 \\
5 \\
6 \\
8 \\
1 \\
1\end{array}$ & $\begin{array}{l}2.029 \\
2.134 \\
2.237 \\
2.340 \\
2.544 \\
2.746 \\
2.947\end{array}$ & $\begin{array}{l}0 \\
0 \\
0\end{array}$ & & $\begin{array}{l}2.7 \\
3.0 \\
3.2 \\
3.4 \\
3.8 \\
4.1 \\
4.4\end{array}$ & & & \\
\hline $\begin{array}{l}40.0000 \\
45.0000 \\
50.0000 \\
55.0000 \\
60.0000 \\
70.0000 \\
80.0000\end{array}$ & $\begin{array}{l}0 \\
0 \\
0 \\
0 \\
0 \\
0 \\
0\end{array}$ & & $\begin{array}{l}3 \\
3 \\
3 \\
3 \\
4 \\
4 \\
5\end{array}$ & i & $\begin{array}{l}1 \\
1 \\
1 \\
1 \\
1 \\
1 \\
1 \\
1\end{array}$ & $\begin{array}{l}0 \\
0 \\
0 \\
0 \\
0 \\
0 \\
0\end{array}$ & & & \\
\hline $\begin{array}{l}0.00000 \\
5.0000 \\
0.00000 \\
0.0000\end{array}$ & $\begin{array}{l}1.87 \\
1.89 \\
1.90\end{array}$ & $\begin{array}{l}3 \\
7\end{array}$ & $\begin{array}{l}8 \\
9 \\
1\end{array}$ & & $\begin{array}{l}5 . \\
5 .\end{array}$ & $\begin{array}{l}7 . \\
7 . \\
8\end{array}$ & & & \\
\hline $\begin{array}{l}.00 \\
.00\end{array}$ & 0 & 1 & 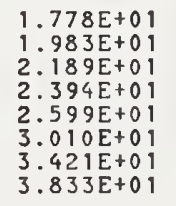 & $\begin{array}{l}1 \\
1 \\
1 \\
1 \\
1 \\
1 \\
1\end{array}$ & 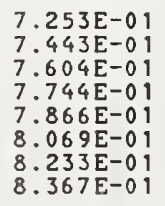 & $\begin{array}{l}E+00 \\
E+00 \\
E+00 \\
E+00 \\
E+00 \\
E+01 \\
E+01 \\
E+01\end{array}$ & $\begin{array}{l}0 \\
0 \\
0 \\
0 \\
0 \\
0 \\
0\end{array}$ & $\begin{array}{l}0.008 \\
0.008 \\
0.008 \\
0.007 \\
0.007 \\
0.007 \\
0.007\end{array}$ & $\begin{array}{l}0.001 \\
0.001 \\
0.001 \\
0.001 \\
0.001 \\
0.001 \\
0.001 \\
0.000\end{array}$ \\
\hline & $E+00$ & 101 & $\therefore+01$ & $+c$ & $481 E-01$ & $.092 E+01$ & 000 & 0.006 & 0.000 \\
\hline
\end{tabular}


ELECTRONS IN ALUMINUM (*)

$I=166.0 \mathrm{eV} \quad$ DENSITY $=2.699 \mathrm{E}+00 \mathrm{~g} / \mathrm{cm}^{3}$

\begin{tabular}{|c|c|c|c|c|c|c|c|c|c|}
\hline Me & $\begin{array}{l}\text { COLLISION } \\
\mathrm{MeV} \mathrm{Cm}^{2} / \mathrm{g}\end{array}$ & $\mathrm{MeV} \mathrm{cm}^{2} / \mathrm{g}$ & $\begin{array}{c}\text { TOTAL } \\
\mathrm{MeV} \mathrm{cm}^{2} / \mathrm{g}\end{array}$ & $\begin{array}{l}\text { CSDA } \\
\text { RANGE } \\
\mathrm{g} / \mathrm{Cm}^{2}\end{array}$ & $N$ & $\begin{array}{l}\mathrm{FF} \text {. } \\
\text { i) }\end{array}$ & $\begin{array}{l}d c \\
\operatorname{col} L\end{array}$ & 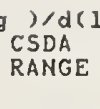 & $\begin{array}{l}\text { I ) } \\
\text { RAD } \\
\text { YIELD }\end{array}$ \\
\hline $\begin{array}{l}0.0100 \\
0.0125 \\
0.0150 \\
0.0175 \\
0.0200 \\
0.0250 \\
0.0300 \\
0.0350\end{array}$ & $\begin{array}{l}1.3 \\
1.2 \\
1.0 \\
9.8 \\
8.3 \\
7.2\end{array}$ & $\begin{array}{l}6.7 \\
6.7 \\
6.8 \\
6.9 \\
7.0\end{array}$ & $\begin{array}{l}1 . \\
1 . \\
1 . \\
9 . \\
8 .\end{array}$ & $\begin{array}{l}5.1 \\
7.1 \\
9.2 \\
1.1 \\
1.7\end{array}$ & $\begin{array}{l}2 . \\
2 . \\
3 . \\
3 . \\
3 . \\
4 . \\
5 .\end{array}$ & $\begin{array}{l}56 E-04 \\
66 E-04 \\
53 E-04 \\
13 E-04 \\
47 E-04 \\
25 E-04 \\
76 E-03\end{array}$ & $\begin{array}{l}-0.2 \\
-0.2 \\
-0.2 \\
-0.2 \\
-0.2 \\
-0 . \\
-0 . \\
-0 .\end{array}$ & $\begin{array}{l}1 \\
5 \\
2 \\
3 \\
5 \\
4 \\
5\end{array}$ & $\begin{array}{l}0.275 \\
0.260 \\
0.248 \\
0.239 \\
0.232 \\
0.221 \\
0.212 \\
0.206\end{array}$ \\
\hline $\begin{array}{l}0.0400 \\
0.0450 \\
0.0500 \\
0.0550 \\
0.0600 \\
0.0700 \\
0.0800 \\
0.0900\end{array}$ & $\begin{array}{ll}0 & 0 \\
0 & 0 \\
0 & 0 \\
0 & 0 \\
0 & 0 \\
0 & 0 \\
0 & 0 \\
0 & 0\end{array}$ & $\begin{array}{l}-03 \\
-03 \\
-03 \\
-03 \\
-03 \\
03 \\
-03 \\
-03\end{array}$ & $\begin{array}{l}5 . \\
5 . \\
4 . \\
4 .\end{array}$ & $\begin{array}{l}4.7 \\
5.7 \\
6.7 \\
7.8 \\
1.0\end{array}$ & $\begin{array}{l}-04 \\
-04 \\
-04 \\
-04 \\
-04 \\
-03 \\
-03 \\
-03\end{array}$ & -03 & $\begin{array}{l}-0.1 \\
-0.1 \\
-0.1 \\
-0.1 \\
-0.1 \\
-0.1 \\
-0.1 \\
-0.1\end{array}$ & $\begin{array}{l}0 \\
0 \\
0 \\
0 \\
0 \\
0\end{array}$ & $\begin{array}{l}0.201 \\
0.196 \\
0.192 \\
0.189 \\
0.186 \\
0.181 \\
0.177\end{array}$ \\
\hline $\begin{array}{l}0.1000 \\
0.1250 \\
0.1500 \\
0.1750 \\
0.2000 \\
0.2500 \\
0.3000 \\
0.3500\end{array}$ & 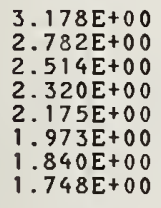 & $\begin{array}{l}.03 \\
.03 \\
.03 \\
.03 \\
.03 \\
.02\end{array}$ & $\begin{array}{l}2 . \\
2 . \\
2 . \\
2 . \\
1 . \\
1 . \\
1 .\end{array}$ & $\begin{array}{l}2.7 \\
3.6 \\
4.6 \\
5.8 \\
8.2 \\
1.0 \\
1.3\end{array}$ & $\begin{array}{l}-03 \\
-03 \\
-03 \\
-03 \\
-03 \\
-03 \\
-03 \\
-03\end{array}$ & $\begin{array}{l}1.1 \\
1.7 \\
2.4 \\
3.1 \\
3.8 \\
5.4\end{array}$ & $\begin{array}{l}-0.1 \\
-0.1 \\
-0.1 \\
-0.1 \\
-0.1 \\
-0.1 \\
-0.1 \\
-0.1\end{array}$ & $\begin{array}{l}0 \\
0 \\
0 \\
0 \\
0 . \\
0 .\end{array}$ & $\begin{array}{l}0.170 \\
0.164 \\
0.160 \\
0.156 \\
0.153 \\
0.148 \\
0.144\end{array}$ \\
\hline $\begin{array}{l}0.4000 \\
0.4500 \\
0.5000 \\
0.5500 \\
0.6000 \\
0.7000 \\
0.8000 \\
0.9000\end{array}$ & $\begin{array}{l}1.681 E+00 \\
1.631 E+00 \\
1.594 E+00 \\
1.564 E+00 \\
1.541 E+00 \\
1.508 E+00 \\
1.487 E+00 \\
1.474 E+00\end{array}$ & $\begin{array}{l}-02 \\
-02 \\
-02 \\
-02 \\
-02 \\
-02 \\
-02 \\
-02\end{array}$ & $\begin{array}{l}0 \\
00 \\
+00 \\
+00 \\
00 \\
00 \\
00 \\
00\end{array}$ & $\begin{array}{l}1.6 \\
1.9 \\
2.2 \\
2.5 \\
2.8 \\
3.5 \\
4.2\end{array}$ & $\begin{array}{l}-03 \\
-03 \\
-03 \\
-03 \\
-03 \\
-03 \\
-03 \\
-03\end{array}$ & $\begin{array}{l}1 . \\
1 . \\
1 . \\
1 .\end{array}$ & $\begin{array}{l}-0.1 \\
-0.1 \\
-0.1 \\
-0.1 \\
-0.1 \\
-0.1 \\
-0.1 \\
-0.1\end{array}$ & & $\begin{array}{l}0.138 \\
0.135 \\
0.133 \\
0.131 \\
0.130 \\
0.126 \\
0.124\end{array}$ \\
\hline $\begin{array}{l}1.0000 \\
1.2500 \\
1.5000 \\
1.7500 \\
2.0000 \\
2.5000 \\
3.0000 \\
3.5000\end{array}$ & $\begin{array}{l}1.458 \\
1.46 \\
1.46 \\
1.47 \\
1.49\end{array}$ & $\begin{array}{l}2.6 \\
3.1 \\
3.7 \\
4.3 \\
5.6 \\
6.9\end{array}$ & $\begin{array}{l}0 \\
0 \\
0 \\
0 \\
0 \\
0 \\
0 \\
0\end{array}$ & $\begin{array}{l}7.2 \\
8.9 \\
1.0 \\
1.2 \\
1.5\end{array}$ & $\begin{array}{l}.03 \\
.03 \\
.02 \\
02 \\
.02 \\
02 \\
02 \\
02\end{array}$ & $\begin{array}{l}4 . \\
4 . \\
5 . \\
6 . \\
7 . \\
9 .\end{array}$ & $\begin{array}{l}-0.1 \\
-0.1 \\
-0.0 \\
-0.8 \\
-0.0 \\
-0.8 \\
-0.0 \\
-0 .\end{array}$ & $\begin{array}{l}2 \\
8 \\
4 \\
1 \\
8 \\
2 \\
6 \\
1\end{array}$ & $\begin{array}{l}0 . \\
0 . \\
0 . \\
0 . \\
0 . \\
0 . \\
0 .\end{array}$ \\
\hline $\begin{array}{l}4.00000 \\
4.5000 \\
5.0000 \\
5.5000 \\
6.0000 \\
7.0000 \\
8.0000 \\
9.0000\end{array}$ & $\begin{array}{l}9 E+00 \\
2 E+00 \\
3 E+00 \\
4 E+00 \\
3 E+00 \\
0 E+00 \\
4 E+00 \\
7 E+00\end{array}$ & $\begin{array}{l}1.1 \\
1.2 \\
1.4 \\
1.5 \\
1.8 \\
2.2\end{array}$ & $\begin{array}{ll}0 & 0 \\
0 & 0 \\
0 & 0 \\
0 & 0 \\
0 & 0 \\
0 & 0 \\
0 & 0 \\
0 & 0\end{array}$ & $\begin{array}{l}2 \\
3 \\
3 \\
3 \\
4\end{array}$ & $\begin{array}{l}02 \\
02 \\
02 \\
02 \\
02 \\
02 \\
02 \\
02 \\
02\end{array}$ & & $\begin{array}{l}-0 . \\
-0 . \\
-0 . \\
-0 . \\
-0 . \\
-0 . \\
-0 . \\
-0 .\end{array}$ & & $\begin{array}{l}72 \\
57 \\
62 \\
58 \\
54 \\
48\end{array}$ \\
\hline $\begin{array}{l}.0000 \\
.5000 \\
.0000 \\
.5000 \\
.0000 \\
.0000\end{array}$ & $\begin{array}{l}+00 \\
+00 \\
+00 \\
+00 \\
+00 \\
+00 \\
+00 \\
+00\end{array}$ & $\begin{array}{l}3 . \\
4 . \\
5 . \\
6 . \\
8 . \\
1 .\end{array}$ & $\begin{array}{l}0 \\
0 \\
0 \\
0 \\
0 \\
0 \\
0\end{array}$ & 1.0 & $\begin{array}{l}.02 \\
02 \\
01 \\
01 \\
01 \\
01 \\
01 \\
01 \\
01 \\
01 \\
01\end{array}$ & $\begin{array}{l}2 \\
2 \\
3 \\
3\end{array}$ & $\begin{array}{l}-0.0 \\
-0.0 \\
-0.0 \\
-0.0 \\
-0.0 \\
-0.0\end{array}$ & & \\
\hline $\begin{array}{l}.0 \\
5.0 \\
0.0\end{array}$ & 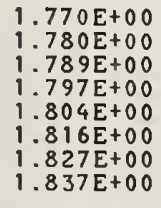 & 1. & & & & & $\begin{array}{l}0 \\
9 \\
9 \\
8 \\
6 \\
5\end{array}$ & & \\
\hline & & & & & & & & & \\
\hline 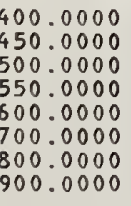 & $\begin{array}{l}0 \\
0 \\
0 \\
0 \\
0 \\
0\end{array}$ & $\begin{array}{l}1 \\
1 \\
1 \\
1 \\
1 \\
1\end{array}$ & & & $\begin{array}{l}1 \\
1 \\
1 \\
1 \\
1 \\
1 \\
1\end{array}$ & & $\begin{array}{l}0 \\
0 \\
0 \\
0 \\
0 \\
0 \\
0 \\
0 \\
0\end{array}$ & & $\begin{array}{l}01 \\
01 \\
01 \\
01\end{array}$ \\
\hline & +00 & 01 & $4 E+01$ & $0 E+0$ & $3.482 \mathrm{E}-01$ & $1.094 \mathrm{~L}$ & .00 & 00 & \\
\hline
\end{tabular}




\begin{tabular}{|c|c|c|c|c|c|c|c|c|c|}
\hline $\mathrm{MeV}$ & $\begin{array}{l}\text { COLLISION } \\
\mathrm{MeV} \mathrm{Cm}^{2} / \mathrm{g}\end{array}$ & $\mathrm{eV} \mathrm{cm}^{2} / \mathrm{g}$ & $\mathrm{MeV} \mathrm{cm}^{2} / \mathrm{g}$ & CS & IN & $\begin{array}{l}\text { NS.EFF. } \\
\text { CORR. } \\
\text { DELTA) }\end{array}$ & coi & $\begin{array}{l}3 / d x( \\
C S D A\end{array}$ & I ) \\
\hline $\begin{array}{l}0.0100 \\
0.0125 \\
0.0150 \\
0.0175 \\
0.0200 \\
0.0250 \\
0.0300\end{array}$ & $\begin{array}{l}1.4 \\
1.2 \\
1.1 \\
1.0 \\
8.5 \\
7.4\end{array}$ & $\begin{array}{l}7.4 \\
7.5 \\
7.6 \\
7.7 \\
7.8 \\
7.8\end{array}$ & $\begin{array}{l}1 \\
1 \\
1\end{array}$ & $\begin{array}{l}04 \\
04 \\
04 \\
04 \\
03 \\
03 \\
03 \\
03\end{array}$ & $\begin{array}{l}289 \mathrm{E}-04 \\
780 \mathrm{E}-04 \\
252 \mathrm{E}-04 \\
709 \mathrm{E}-04 \\
151 \mathrm{E}-04 \\
000 \mathrm{E}-04 \\
807 \mathrm{E}-04 \\
579 \mathrm{E}-04\end{array}$ & $\begin{array}{l}E-03 \\
E-03 \\
E-03 \\
E-03 \\
E-03 \\
E-03 \\
E-03\end{array}$ & & & $\begin{array}{l}0.278 \\
0.262 \\
0.251 \\
0.241 \\
0.234 \\
0.223 \\
0.214\end{array}$ \\
\hline $\begin{array}{l}0.0400 \\
0.0450 \\
0.0500 \\
0.0550 \\
0.0600 \\
0.0700 \\
0.0800 \\
0.0900\end{array}$ & $\begin{array}{lll}0 & & 0 \\
0 & 0 & 0 \\
0 & 0 \\
0 & 0\end{array}$ & $\begin{array}{l}8.0 \\
8.0 \\
8.0 \\
8.1 \\
8.1 \\
8.2\end{array}$ & $\begin{array}{l}4.8 \\
4.5 \\
4.1 \\
3.7 \\
3.4\end{array}$ & $\begin{array}{l}:-03 \\
:-03 \\
-03 \\
-03 \\
-03 \\
-03 \\
-03 \\
-02 \\
-02 \\
-02\end{array}$ & $\begin{array}{l}-04 \\
-04 \\
-04 \\
-04 \\
-03 \\
-03 \\
-03 \\
-03\end{array}$ & $\begin{array}{l}-03 \\
-03 \\
-03 \\
-03 \\
-03\end{array}$ & & & $\begin{array}{l}0.202 \\
0.197 \\
0.193 \\
0.190 \\
0.187 \\
0.182 \\
0.177\end{array}$ \\
\hline $\begin{array}{l}0.1000 \\
0.1250 \\
0.1500 \\
0.1750 \\
0.2000 \\
0.2500 \\
0.3000\end{array}$ & $\begin{array}{l}+00 \\
+00 \\
+00 \\
+00 \\
+00 \\
+00 \\
+00 \\
+00\end{array}$ & $\begin{array}{l}8 . \\
9 . \\
9 \\
9 \\
1 .\end{array}$ & $\begin{array}{l}2.3 \\
2.2 \\
1.0\end{array}$ & $\begin{array}{l}3.3 \\
4.5 \\
5.6 \\
7.9\end{array}$ & $\begin{array}{l}1.9 \\
2.2 \\
2.4 \\
2.8\end{array}$ & $\begin{array}{l}1.8 \\
2.5 \\
3.2 \\
4.0 \\
4.8 \\
6.5 \\
8.4\end{array}$ & $\begin{array}{l}-0 \\
-0 \\
-0 \\
-0 \\
-0 \\
-0 \\
-0 \\
-0\end{array}$ & $\begin{array}{l}71 \\
65 \\
60 \\
56 \\
53 \\
48 \\
43 \\
40\end{array}$ & $\begin{array}{l}0.171 \\
0.164 \\
0.159 \\
0.155 \\
0.152 \\
0.147 \\
0.142\end{array}$ \\
\hline $\begin{array}{l}0.4000 \\
0.4500 \\
0.5000 \\
0.5500 \\
0.6000 \\
0.7000 \\
0.8000\end{array}$ & $\begin{array}{l}.729 E+00 \\
.677 E+00 \\
.638 E+00 \\
.608 E+00 \\
.585 E+00 \\
.551 E+00 \\
.529 E+00 \\
.516 E+00\end{array}$ & $\begin{array}{l}1.290 \mathrm{E}-0 \\
1.374 \mathrm{E}-0 \\
1.461 \mathrm{E}-0 \\
1.551 \mathrm{E}-0 \\
1.740 \mathrm{E}-0 \\
1.938 \mathrm{E}-0 \\
2.145 \mathrm{E}-0\end{array}$ & $\begin{array}{l}1.690 E+0 \\
1.652 E+0 \\
1.623 E+0 \\
1.600 E+0 \\
1.568 E+0 \\
1.549 E+0 \\
1.537 E+0\end{array}$ & $\begin{array}{l}1 \\
1 \\
1 \\
1 \\
1 \\
1\end{array}$ & & $\begin{array}{l}1.2 \\
1.4 \\
1.5 \\
1.7 \\
1.9 \\
2.3 \\
2.7 \\
3.0\end{array}$ & & & $\begin{array}{l}0.1 \\
0.1 \\
0.1 \\
0.1 \\
0.1\end{array}$ \\
\hline $\begin{array}{l}1.0000 \\
1.2500 \\
1.5000 \\
1.7500 \\
2.0000 \\
2.5000 \\
3.0000 \\
3.5000\end{array}$ & $\begin{array}{l}+\infty \\
+\infty \\
+00 \\
+00 \\
+00 \\
+00 \\
+00 \\
+00 \\
+00 \\
+\infty\end{array}$ & & $\begin{array}{ll}0 & 0 \\
0 & 0 \\
0 & 0 \\
0 & 0 \\
0 & 0\end{array}$ & 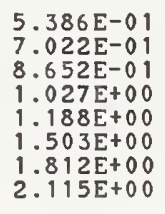 & & $\begin{array}{l}3.4 \\
4.2 \\
5.0 \\
5.7 \\
6.4\end{array}$ & & & $\begin{array}{l}0 \\
0\end{array}$ \\
\hline $\begin{array}{l}4.0000 \\
4.5000 \\
5.0000 \\
5.5000 \\
6.0000 \\
7.0000 \\
8.0000 \\
9.0000\end{array}$ & $\begin{array}{l}1.00 \\
1.62 \\
1.63 \\
1.65\end{array}$ & $\begin{array}{l}1.2 \\
1.3 \\
1.5 \\
1.7 \\
2.0\end{array}$ & $\begin{array}{l}1 . \\
1 . \\
1 . \\
1 . \\
1 .\end{array}$ & $\begin{array}{l}0 \\
0 \\
0 \\
0\end{array}$ & & $\begin{array}{l}1.7 \\
1.5\end{array}$ & & & \\
\hline $\begin{array}{l}15.0 \\
17.5 \\
20.0 \\
25.0\end{array}$ & & $\begin{array}{l}4 \\
5 \\
6 \\
7\end{array}$ & $\begin{array}{l}2 . \\
2 . \\
2 .\end{array}$ & 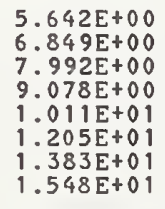 & & 4. $200 \mathrm{DT}$ & & & \\
\hline $\begin{array}{l}45.0 \\
50.0 \\
55.0 \\
60.0 \\
70.0 \\
80.0 \\
90.0\end{array}$ & $\begin{array}{l}1.0 \\
1.8 \\
1.8 \\
1.8 \\
1.88 \\
1.9 \\
1.9\end{array}$ & $\begin{array}{l}1.9 \\
2.1 \\
2.3 \\
2.7\end{array}$ & & & & $\begin{array}{l}b \\
b \\
0 \\
0\end{array}$ & & & \\
\hline $\begin{array}{l}.0 \\
.0 \\
.0\end{array}$ & $\begin{array}{l}1 \\
1 \\
1 \\
1 \\
2\end{array}$ & $\begin{array}{l}8 \\
1\end{array}$ & & & & $\begin{array}{l}0 \\
0 \\
0 \\
0 \\
0 \\
0 \\
0\end{array}$ & & & \\
\hline $\begin{array}{l}0 \\
0 \\
0 \\
0 \\
0 \\
0 \\
0\end{array}$ & $\begin{array}{l}2.038 \mathrm{E}+00 \\
2.046 \mathrm{E}+00 \\
2.053 \mathrm{E}+00 \\
2.060 \mathrm{E}+00 \\
2.072 \mathrm{E}+00 \\
2.082 \mathrm{E}+00 \\
2.091 \mathrm{E}+00\end{array}$ & 2.1 & $\begin{array}{l}1 \\
1 \\
1 \\
1 \\
1\end{array}$ & $\begin{array}{l}9 E+01 \\
2 E+0 \\
1 E+01 \\
9 E+01 \\
2 E+01 \\
7 E+01 \\
0 E+01 \\
1 E+01\end{array}$ & $8.433 \mathrm{E}$ & $\begin{array}{l}9.131 \mathrm{E}+00 \\
9.341 \mathrm{E}+00 \\
9.531 \mathrm{E}+00 \\
9.705 \mathrm{E}+00 \\
1.001 \mathrm{E}+01 \\
1.028 \mathrm{E}+01 \\
1.051 \mathrm{E}+01\end{array}$ & & & \\
\hline & $099 \mathrm{E}+00$ & $.459 E+01$ & $669 E+01$ & $567 \mathrm{E}+01$ & $.542 \mathrm{E}-01$ & $.072 E+01$ & 0.000 & 0.007 & 000 \\
\hline
\end{tabular}




\begin{tabular}{|c|c|c|c|c|c|c|c|c|c|}
\hline ENERGY & COLLISIOI & $\begin{array}{l}\text { PING POW } \\
\text { ADIATIVE }\end{array}$ & TOTAL & $\begin{array}{l}\text { CSDA } \\
\text { RAHGE }\end{array}$ & $\begin{array}{l}\text { RADIATION } \\
\text { YIELD }\end{array}$ & $\begin{array}{c}\text { DENS . EFF . } \\
\text { CORR } \\
\text { (DELTA) }\end{array}$ & $\begin{array}{l}d(1 \\
\text { COLL } \\
\text { LOSS }\end{array}$ & $\begin{array}{l}) / d(1 \\
\text { CSDA } \\
\text { RANGE }\end{array}$ & $\begin{array}{l}\text { RI ) } \\
\text { RAD } \\
\text { YIELD }\end{array}$ \\
\hline $\mathrm{MeV}$ & $\mathrm{MeV} \mathrm{cm}^{2} / \mathrm{g}$ & $\mathrm{MeV} \mathrm{cm} 2 / \mathrm{g}$ & $\mathrm{MeV} \mathrm{cm}^{2} / \mathrm{g}$ & $\mathrm{g} / \mathrm{cm}^{2}$ & & & & & \\
\hline $\begin{array}{l}0.01100 \\
0.0125 \\
0.0150 \\
0.0175 \\
0.0200 \\
0.0250 \\
0.0300 \\
0.0350\end{array}$ & $\begin{array}{l}1.497 E+01 \\
1.271 E+01 \\
1.110 E+01 \\
9.907 E+00 \\
8.974 E+00 \\
7.610 E+00 \\
6.657 E+00 \\
5.950 E+00\end{array}$ & $\begin{array}{l}8.167 E-03 \\
8.444 E-03 \\
8.647 E-03 \\
8.803 E-03 \\
8.926 E-03 \\
9.107 E-03 \\
9.237 E-03 \\
9.334 E-03\end{array}$ & $\begin{array}{l}1.498 E+01 \\
1.271 E+01 \\
1.111 E+01 \\
9.916 E+00 \\
8.983 E+00 \\
7.619 E+00 \\
6.666 E+00 \\
5.959 E+00\end{array}$ & $\begin{array}{l}3.921 E-04 \\
5.740 E-04 \\
7.849 E-04 \\
1.024 E-03 \\
1.289 E-03 \\
1.896 E-03 \\
2.599 E-03 \\
3.394 E-03\end{array}$ & $\begin{array}{l}2.846 E-04 \\
3.487 E-04 \\
4.108 E-04 \\
4.712 \mathrm{E}-04 \\
5.299 \mathrm{E}-04 \\
6.430 \mathrm{E}-04 \\
7.511 \mathrm{E}-04 \\
8.547 \mathrm{E}-04\end{array}$ & $\begin{array}{l}0.0 \\
0.0 \\
0.0 \\
0.0 \\
0.0 \\
0.0 \\
0.0 \\
0.0\end{array}$ & $\begin{array}{l}-0.243 \\
-0.231 \\
-0.222 \\
-0.215 \\
-0.209 \\
-0.200 \\
-0.193 \\
-0.187\end{array}$ & $\begin{array}{l}0.292 \\
0.274 \\
0.261 \\
0.251 \\
0.243 \\
0.230 \\
0.221 \\
0.214\end{array}$ & $\begin{array}{l}0.284 \\
0.268 \\
0.255 \\
0.246 \\
0.238 \\
0.226 \\
0.218 \\
0.211\end{array}$ \\
\hline $\begin{array}{l}0.0400 \\
0.0450 \\
0.0500 \\
0.0550 \\
0.0600 \\
0.0700 \\
0.0800 \\
0.0900\end{array}$ & $\begin{array}{l}5.404 E+00 \\
4.969 E+00 \\
4.614 E+00 \\
4.317 E+00 \\
4.067 E+00 \\
3.666 E+00 \\
3.358 E+00 \\
3.115 E+00\end{array}$ & $\begin{array}{l}9.415 E-03 \\
9.482 E-03 \\
9.540 E-03 \\
9.593 E-03 \\
9.642 E-03 \\
9.733 E-03 \\
9.821 E-03 \\
9.909 E-03\end{array}$ & $\begin{array}{l}5.414 E+00 \\
4.978 E+00 \\
4.623 E+00 \\
4.327 E+00 \\
4.077 E+00 \\
3.675 E+00 \\
3.368 E+00 \\
3.125 E+00\end{array}$ & $\begin{array}{l}4.276 E-03 \\
5.240 E-03 \\
6.283 E-03 \\
7.402 E-03 \\
8.593 E-03 \\
1.118 E-02 \\
1.403 E-02 \\
1.712 E-02\end{array}$ & $\begin{array}{l}9.546 \mathrm{E}-04 \\
1.051 \mathrm{E}-03 \\
1.144 \mathrm{E}-03 \\
1.235 \mathrm{E}-03 \\
1.323 \mathrm{E}-03 \\
1.492 \mathrm{E}-03 \\
1.654 \mathrm{E}-03 \\
1.808 \mathrm{E}-03\end{array}$ & $\begin{array}{l}0.0 \\
0.0 \\
0.0 \\
0.0 \\
0.0 \\
0.0 \\
0.0 \\
0.0\end{array}$ & $\begin{array}{l}-0.183 \\
-0.179 \\
-0.176 \\
-0.173 \\
-0.171 \\
-0.167 \\
-0.163 \\
-0.160\end{array}$ & $\begin{array}{l}0.208 \\
0.203 \\
0.199 \\
0.195 \\
0.192 \\
0.186 \\
0.182 \\
0.178\end{array}$ & $\begin{array}{l}0.205 \\
0.200 \\
0.196 \\
0.193 \\
0.190 \\
0.185 \\
0.180 \\
0.177\end{array}$ \\
\hline $\begin{array}{l}0.1000 \\
0.1250 \\
0.1500 \\
0.1750 \\
0.2000 \\
0.2500 \\
0.3000 \\
0.3500\end{array}$ & $\begin{array}{l}2.918 E+00 \\
2.557 E+00 \\
2.312 E+00 \\
2.136 E+00 \\
2.004 E+00 \\
1.821 E+00 \\
1.701 E+00 \\
1.618 E+00\end{array}$ & $\begin{array}{l}1.000 \mathrm{E}-02 \\
1.025 \mathrm{E}-02 \\
1.052 \mathrm{E}-02 \\
1.081 \mathrm{E}-02 \\
1.113 \mathrm{E}-02 \\
1.182 \mathrm{E}-02 \\
1.258 \mathrm{E}-02 \\
1.340 \mathrm{E}-02\end{array}$ & $\begin{array}{l}2.928 E+00 \\
2.567 E+00 \\
2.323 E+00 \\
2.147 E+00 \\
2.015 E+00 \\
1.832 E+00 \\
1.713 E+00 \\
1.631 E+00\end{array}$ & $\begin{array}{l}2.042 E-02 \\
2.958 E-02 \\
3.985 E-02 \\
5.106 E-02 \\
6.309 E-02 \\
8.920 E-02 \\
1.175 E-01 \\
1.474 E-01\end{array}$ & $\begin{array}{l}1.957 E-03 \\
2.307 E-03 \\
2.633 E-03 \\
2.940 E-03 \\
3.233 E-03 \\
3.784 E-03 \\
4.303 E-03 \\
4.799 E-03\end{array}$ & $\begin{array}{l}0.0 \\
0.0 \\
0.0 \\
0.0 \\
0.0 \\
0.0 \\
0.0 \\
0.0\end{array}$ & $\begin{array}{l}-0.158 \\
-0.153 \\
-0.149 \\
-0.146 \\
-0.143 \\
-0.139 \\
-0.135 \\
-0.132\end{array}$ & $\begin{array}{l}0.175 \\
0.169 \\
0.164 \\
0.160 \\
0.157 \\
0.152 \\
0.148 \\
0.145\end{array}$ & $\begin{array}{l}0.174 \\
0.168 \\
0.163 \\
0.159 \\
0.156 \\
0.151 \\
0.147 \\
0.143\end{array}$ \\
\hline $\begin{array}{l}0.4000 \\
0.4500 \\
0.5000 \\
0.5500 \\
0.6000 \\
0.7000 \\
0.8000 \\
0.9000\end{array}$ & $\begin{array}{l}1.558 E+00 \\
1.514 E+00 \\
1.480 E+00 \\
1.455 E+00 \\
1.435 E+00 \\
1.408 E+00 \\
1.391 E+00 \\
1.381 E+00\end{array}$ & $\begin{array}{l}1.427 \mathrm{E}-02 \\
1.519 \mathrm{E}-02 \\
1.616 \mathrm{E}-02 \\
1.716 \mathrm{E}-02 \\
1.820 \mathrm{E}-02 \\
2.036 \mathrm{E}-02 \\
2.264 \mathrm{E}-02 \\
2.502 \mathrm{E}-02\end{array}$ & $\begin{array}{l}1.572 E+00 \\
1.529 E+00 \\
1.497 E+00 \\
1.472 E+00 \\
1.453 E+00 \\
1.428 E+00 \\
1.414 E+00 \\
1.406 E+00\end{array}$ & $\begin{array}{l}1.787 \mathrm{E}-01 \\
2.109 \mathrm{E}-01 \\
2.440 \mathrm{E}-01 \\
2.777 \mathrm{E}-01 \\
3.119 \mathrm{E}-01 \\
3.814 \mathrm{E}-01 \\
4.518 \mathrm{E}-01 \\
5.227 \mathrm{E}-01\end{array}$ & $\begin{array}{l}5.280 E-03 \\
5.749 E-03 \\
6.211 E-03 \\
6.667 E-03 \\
7.119 E-03 \\
8.015 E-03 \\
8.905 E-03 \\
9.794 E-03\end{array}$ & $\begin{array}{l}0.0 \\
0.0 \\
0.0 \\
0.0 \\
0.0 \\
0.0 \\
0.0 \\
0.0\end{array}$ & $\begin{array}{l}-0.130 \\
-0.128 \\
-0.126 \\
-0.124 \\
-0.122 \\
-0.120 \\
-0.117 \\
-0.115\end{array}$ & $\begin{array}{l}0.142 \\
0.140 \\
0.138 \\
0.136 \\
0.135 \\
0.132 \\
0.129 \\
0.127\end{array}$ & $\begin{array}{l}0.141 \\
0.138 \\
0.136 \\
0.134 \\
0.132 \\
0.129 \\
0.127 \\
0.124\end{array}$ \\
\hline $\begin{array}{l}1.0000 \\
1.2500 \\
1.5000 \\
1.7500 \\
2.0000 \\
2.5000 \\
3.0000 \\
3.5000\end{array}$ & $\begin{array}{l}1.376 E+00 \\
1.375 E+00 \\
1.383 E+00 \\
1.394 E+00 \\
1.407 E+00 \\
1.434 E+00 \\
1.460 E+00 \\
1.484 E+00\end{array}$ & $\begin{array}{l}2.748 E-02 \\
3.399 E-02 \\
4.094 E-02 \\
4.822 E-02 \\
5.581 E-02 \\
7.168 E-02 \\
8.831 E-02 \\
1.056 E-01\end{array}$ & $\begin{array}{l}1.404 E+00 \\
1.409 E+00 \\
1.424 E+00 \\
1.442 E+00 \\
1.463 E+00 \\
1.505 E+00 \\
1.548 E+00 \\
1.589 E+00\end{array}$ & $\begin{array}{l}5.939 E-01 \\
7.718 E-01 \\
9.483 E-01 \\
1.123 E+00 \\
1.295 E+00 \\
1.632 E+00 \\
1.959 E+00 \\
2.278 E+00\end{array}$ & $\begin{array}{l}1.068 E-02 \\
1.291 E-02 \\
1.517 E-02 \\
1.744 E-02 \\
1.973 E-02 \\
2.436 E-02 \\
2.903 E-02 \\
3.370 E-02\end{array}$ & $\begin{array}{l}0.0 \\
0.0 \\
0.0 \\
0.0 \\
0.0 \\
0.0 \\
0.0 \\
0.0\end{array}$ & $\begin{array}{l}-0.1114 \\
-0.110 \\
-0.107 \\
-0.105 \\
-0.103 \\
-0.099 \\
-0.097 \\
-0.095\end{array}$ & $\begin{array}{l}0.126 \\
0.122 \\
0.1119 \\
0.1116 \\
0.1114 \\
0.1111 \\
0.1108 \\
0.105\end{array}$ & $\begin{array}{l}0.122 \\
0.118 \\
0.114 \\
0.1111 \\
0.109 \\
0.104 \\
0.101 \\
0.098\end{array}$ \\
\hline $\begin{array}{l}4.0000 \\
4.5000 \\
5.0000 \\
5.5000 \\
6.0000 \\
7.0000 \\
8.0000 \\
9.0000\end{array}$ & $\begin{array}{l}1.506 E+00 \\
1.526 E+00 \\
1.545 E+00 \\
1.562 E+00 \\
1.579 E+00 \\
1.608 E+00 \\
1.634 E+00 \\
1.657 E+00\end{array}$ & $\begin{array}{l}1.233 E-01 \\
1.415 E-01 \\
1.600 E-01 \\
1.789 E-01 \\
1.981 E-01 \\
2.373 E-01 \\
2.773 E-01 \\
3.181 E-01\end{array}$ & $\begin{array}{l}1.629 \mathrm{E}+00 \\
1.668 \mathrm{E}+00 \\
1.705 \mathrm{E}+00 \\
1.741 \mathrm{E}+00 \\
1.777 \mathrm{E}+00 \\
1.845 \mathrm{E}+00 \\
1.911 \mathrm{E}+00 \\
1.975 \mathrm{E}+00\end{array}$ & $\begin{array}{l}2.589 E+00 \\
2.892 E+00 \\
3.189 E+00 \\
3.479 E+00 \\
3.763 E+00 \\
4.315 E+00 \\
4.848 E+00 \\
5.363 E+00\end{array}$ & $\begin{array}{l}3.837 \mathrm{E}-02 \\
4.303 \mathrm{E}-02 \\
4.766 \mathrm{E}-02 \\
5.227 \mathrm{E}-02 \\
5.684 \mathrm{E}-02 \\
6.588 \mathrm{E}-02 \\
7.476 \mathrm{E}-02 \\
8.347 \mathrm{E}-02\end{array}$ & $\begin{array}{l}0.0 \\
0.0 \\
0.0 \\
0.0 \\
0.0 \\
0.0 \\
0.0 \\
0.0\end{array}$ & $\begin{array}{l}-0.093 \\
-0.092 \\
-0.090 \\
-0.089 \\
-0.088 \\
-0.087 \\
-0.085 \\
-0.084\end{array}$ & $\begin{array}{l}0.103 \\
0.101 \\
0.099 \\
0.098 \\
0.096 \\
0.094 \\
0.092 \\
0.090\end{array}$ & $\begin{array}{l}0.096 \\
0.093 \\
0.091 \\
0.090 \\
0.088 \\
0.085 \\
0.083 \\
0.080\end{array}$ \\
\hline $\begin{array}{l}10.00000 \\
12.5000 \\
15.0000 \\
17.5000 \\
20.0000 \\
25.0000 \\
30.0000 \\
35.0000\end{array}$ & $\begin{array}{l}1.678 E+00 \\
1.722 E+00 \\
1.759 E+00 \\
1.790 E+00 \\
1.818 E+00 \\
1.864 E+00 \\
1.901 E+00 \\
1.931 E+00\end{array}$ & $\begin{array}{l}3.595 E-01 \\
4.651 E-01 \\
5.733 E-01 \\
6.833 E-01 \\
7.949 E-01 \\
1.021 E+00 \\
1.251 E+00 \\
1.484 E+00\end{array}$ & $\begin{array}{l}2.037 E+00 \\
2.187 E+00 \\
2.332 E+00 \\
2.474 E+00 \\
2.613 E+00 \\
2.885 E+00 \\
3.152 E+00 \\
3.415 E+00\end{array}$ & $\begin{array}{l}5.861 E+00 \\
7.045 E+00 \\
8.151 E+00 \\
9.192 E+00 \\
1.018 E+01 \\
1.200 E+01 \\
1.365 E+01 \\
1.518 E+01\end{array}$ & $\begin{array}{l}9.200 \mathrm{E}-02 \\
1.126 \mathrm{E}-01 \\
1.320 \mathrm{E}-01 \\
1.505 \mathrm{E}-01 \\
1.680 \mathrm{E}-01 \\
2.004 \mathrm{E}-01 \\
2.296 \mathrm{E}-01 \\
2.563 \mathrm{E}-01\end{array}$ & $\begin{array}{l}0.0 \\
0.0 \\
0.0 \\
0.0 \\
0.0 \\
0.0 \\
6.376 E-04 \\
2.311 E-02\end{array}$ & $\begin{array}{l}-0.083 \\
-0.081 \\
-0.079 \\
-0.077 \\
-0.076 \\
-0.074 \\
-0.071 \\
-0.063\end{array}$ & $\begin{array}{l}0.088 \\
0.084 \\
0.081 \\
0.079 \\
0.076 \\
0.072 \\
0.069 \\
0.066\end{array}$ & $\begin{array}{l}0.078 \\
0.074 \\
0.070 \\
0.067 \\
0.064 \\
0.060 \\
0.056 \\
0.052\end{array}$ \\
\hline $\begin{array}{l}40.00000 \\
45.0000 \\
50.0000 \\
55.0000 \\
60.0000 \\
70.0000 \\
80.0000 \\
90.0000\end{array}$ & $\begin{array}{l}1.956 E+00 \\
1.977 E+00 \\
1.995 E+00 \\
2.011 E+00 \\
2.025 E+00 \\
2.050 E+00 \\
2.071 E+00 \\
2.089 E+00\end{array}$ & $\begin{array}{l}1.718 E+00 \\
1.954 E+00 \\
2.192 E+00 \\
2.430 E+00 \\
2.670 E+00 \\
3.152 E+00 \\
3.637 E+00 \\
4.125 E+00\end{array}$ & $\begin{array}{l}3.674 E+00 \\
3.931 E+00 \\
4.187 E+00 \\
4.441 E+00 \\
4.695 E+00 \\
5.202 E+00 \\
5.708 E+00 \\
6.214 E+00\end{array}$ & $\begin{array}{l}1.659 \mathrm{E}+01 \\
1.790 \mathrm{E}+01 \\
1.913 \mathrm{E}+01 \\
2.029 \mathrm{E}+01 \\
2.139 \mathrm{E}+01 \\
2.341 \mathrm{E}+01 \\
2.525 \mathrm{E}+01 \\
2.692 \mathrm{E}+01\end{array}$ & $\begin{array}{l}2.807 E-01 \\
3.031 E-01 \\
3.238 E-01 \\
3.431 E-01 \\
3.610 E-01 \\
3.934 E-01 \\
4.220 E-01 \\
4.474 E-01\end{array}$ & $\begin{array}{l}6.443 E-02 \\
1.144 E-01 \\
1.680 E-01 \\
2.226 E-01 \\
2.769 E-01 \\
3.820 E-01 \\
4.807 E-01 \\
5.729 E-01\end{array}$ & $\begin{array}{l}-0.057 \\
-0.053 \\
-0.050 \\
-0.048 \\
-0.046 \\
-0.043 \\
-0.041 \\
-0.040\end{array}$ & $\begin{array}{l}0.063 \\
0.061 \\
0.059 \\
0.056 \\
0.055 \\
0.052 \\
0.049 \\
0.047\end{array}$ & $\begin{array}{l}0.048 \\
0.045 \\
0.042 \\
0.039 \\
0.037 \\
0.033 \\
0.030 \\
0.027\end{array}$ \\
\hline $\begin{array}{l}00.0000 \\
25.0000 \\
50.0000 \\
75.0000 \\
00.0000 \\
50.0000 \\
00.0000 \\
50.0000\end{array}$ & $\begin{array}{l}2.105 E+00 \\
2.138 E+00 \\
2.164 E+00 \\
2.185 E+00 \\
2.204 E+00 \\
2.234 E+00 \\
2.257 E+00 \\
2.275 E+00\end{array}$ & $\begin{array}{l}4.614 E+00 \\
5.845 E+00 \\
7.083 E+00 \\
8.326 E+00 \\
9.573 E+00 \\
1.208 E+01 \\
1.459 E+01 \\
1.711 E+01\end{array}$ & $\begin{array}{l}6.719 E+00 \\
7.982 E+00 \\
9.247 E+00 \\
1.051 E+01 \\
1.178 E+01 \\
1.431 E+01 \\
1.685 E+01 \\
1.938 E+01\end{array}$ & $\begin{array}{l}2.847 E+01 \\
3.188 E+01 \\
3.479 E+01 \\
3.732 E+01 \\
3.957 E+01 \\
4.341 E+01 \\
4.663 E+01 \\
4.940 E+01\end{array}$ & $\begin{array}{l}4.702 E-01 \\
5.183 E-01 \\
5.569 E-01 \\
5.887 E-01 \\
6.155 E-01 \\
6.583 E-01 \\
6.911 E-01 \\
7.174 E-01\end{array}$ & $\begin{array}{l}6.588 E-01 \\
8.510 E-01 \\
1.018 E+00 \\
1.166 E+00 \\
1.301 E+00 \\
1.541 E+00 \\
1.754 E+00 \\
1.947 E+00\end{array}$ & $\begin{array}{l}-0.038 \\
-0.036 \\
-0.034 \\
-0.032 \\
-0.030 \\
-0.027 \\
-0.024 \\
-0.021\end{array}$ & $\begin{array}{l}0.045 \\
0.041 \\
0.039 \\
0.036 \\
0.035 \\
0.032 \\
0.030 \\
0.029\end{array}$ & $\begin{array}{l}0.025 \\
0.021 \\
0.018 \\
0.016 \\
0.015 \\
0.012 \\
0.010 \\
0.009\end{array}$ \\
\hline $\begin{array}{l}0.00000 \\
50.00000 \\
0.00000 \\
50.00000 \\
0.00000 \\
0.00000 \\
0.00000 \\
0.00000\end{array}$ & $\begin{array}{l}2.291 E+00 \\
2.304 E+00 \\
2.315 E+00 \\
2.325 E+00 \\
2.334 E+00 \\
2.349 E+00 \\
2.361 E+00 \\
2.372 E+00\end{array}$ & $\begin{array}{l}1.963 \mathrm{E}+01 \\
2.216 \mathrm{E}+01 \\
2.469 \mathrm{E}+01 \\
2.722 \mathrm{E}+01 \\
2.975 \mathrm{E}+01 \\
3.483 \mathrm{E}+01 \\
3.990 \mathrm{E}+01 \\
4.499 \mathrm{E}+01\end{array}$ & $\begin{array}{l}2.192 \mathrm{E}+01 \\
2.446 \mathrm{E}+01 \\
2.700 \mathrm{E}+01 \\
2.954 \mathrm{E}+01 \\
3.209 \mathrm{E}+01 \\
3.718 \mathrm{E}+01 \\
4.227 \mathrm{E}+01 \\
4.736 \mathrm{E}+01\end{array}$ & $\begin{array}{l}5.182 E+01 \\
5.398 E+01 \\
5.592 E+01 \\
5.769 E+01 \\
5.932 E+01 \\
6.221 E+01 \\
6.473 E+01 \\
6.696 E+01\end{array}$ & $\begin{array}{l}7.389 E-01 \\
7.569 E-01 \\
7.722 E-01 \\
7.854 E-01 \\
7.970 E-01 \\
8.163 E-01 \\
8.319 E-01 \\
8.447 E-01\end{array}$ & $\begin{array}{l}2.125 \mathrm{E}+00 \\
2.290 \mathrm{E}+00 \\
2.444 \mathrm{E}+00 \\
2.587 \mathrm{E}+00 \\
2.721 \mathrm{E}+00 \\
2.966 \mathrm{E}+00 \\
3.184 \mathrm{E}+00 \\
3.380 \mathrm{E}+00\end{array}$ & $\begin{array}{l}-0.019 \\
-0.017 \\
-0.016 \\
-0.0114 \\
-0.0113 \\
-0.011 \\
-0.0110 \\
-0.009\end{array}$ & $\begin{array}{l}0.027 \\
0.026 \\
0.026 \\
0.025 \\
0.024 \\
0.023 \\
0.022 \\
0.021\end{array}$ & $\begin{array}{l}0.008 \\
0.007 \\
0.007 \\
0.006 \\
0.006 \\
0.005 \\
0.004 \\
0.004\end{array}$ \\
\hline 0.0000 & $2.382 E+00$ & $5.007 \mathrm{E}+01$ & $5.245 E+01$ & $6.897 \mathrm{E}+01$ & $.554 E-01$ & $3.558 \mathrm{E}+00$ & 0.009 & 0.021 & 3 \\
\hline
\end{tabular}




\begin{tabular}{|c|c|c|c|c|c|c|c|c|c|}
\hline ENERGY & 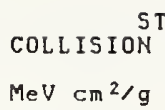 & $\begin{array}{l}\text { OPPING POWE } \\
\text { RADIAT IVE } \\
\text { MeV } \mathrm{cm}^{2} / \mathrm{g}\end{array}$ & $\begin{array}{c}\text { TOTAL } \\
\mathrm{MeV} \mathrm{cm}^{2} / \mathrm{g}\end{array}$ & $\begin{array}{l}\text { CSDA } \\
\text { RANGE }\end{array}$ & $\begin{array}{l}\text { ADIATION } \\
\text { YIELD }\end{array}$ & $\begin{array}{l}\text { DENS.EFF. } \\
\text { CORR } \\
\text { (DELTA) }\end{array}$ & $\begin{array}{l}d(1 \\
\text { COLL } \\
\text { LOSS }\end{array}$ & $\begin{array}{l}) / d(1 \\
\text { CSDA } \\
\text { RANGE }\end{array}$ & $\begin{array}{l}\text { gI ) } \\
\text { RAD } \\
\text { YIELD }\end{array}$ \\
\hline $\begin{array}{l}0.0100 \\
0.0125 \\
0.0150 \\
0.0175 \\
0.0200 \\
0.0250 \\
0.0300 \\
0.0350\end{array}$ & $\begin{array}{l}1.447 E+01 \\
1.231 E+01 \\
1.078 E+01 \\
9.634 E+00 \\
8.737 E+00 \\
7.424 E+00 \\
6.503 E+00 \\
5.819 E+00\end{array}$ & $\begin{array}{l}9.835 \mathrm{E}-03 \\
1.025 \mathrm{E}-02 \\
1.056 \mathrm{E}-02 \\
1.080 \mathrm{E}-02 \\
1.099 \mathrm{E}-02 \\
1.129 \mathrm{E}-02 \\
1.150 \mathrm{E}-02 \\
1.167 \mathrm{E}-02\end{array}$ & $\begin{array}{l}1.447 E+01 \\
1.232 E+01 \\
1.079 E+01 \\
9.644 E+00 \\
8.748 E+00 \\
7.435 E+00 \\
6.514 E+00 \\
5.831 E+00\end{array}$ & $\begin{array}{l}.104 E-04 \\
.983 E-04 \\
.157 E-04 \\
.061 E-03 \\
.334 E-03 \\
.956 E-03 \\
.677 E-03 \\
.490 E-03\end{array}$ & $\begin{array}{l}E-04 \\
E-04 \\
E-04 \\
E-04 \\
E-04 \\
E-04 \\
E-04 \\
E-03\end{array}$ & $\begin{array}{l}-03 \\
-03 \\
-03 \\
-03 \\
-03 \\
-03 \\
-03 \\
-03\end{array}$ & $\begin{array}{l}-0.256 \\
-0.242 \\
-0.232 \\
-0.224 \\
-0.218 \\
-0.208 \\
-0.200 \\
-0.194\end{array}$ & $\begin{array}{l}0.313 \\
0.293 \\
0.278 \\
0.266 \\
0.257 \\
0.243 \\
0.232 \\
0.224\end{array}$ & $\begin{array}{l}0.303 \\
0.284 \\
0.270 \\
0.259 \\
0.250 \\
0.237 \\
0.227 \\
0.220\end{array}$ \\
\hline $\begin{array}{l}0.0400 \\
0.0450 \\
0.0500 \\
0.0550 \\
0.0600 \\
0.0700 \\
0.0800 \\
0.0900\end{array}$ & $\begin{array}{l}5.290 E+00 \\
4.867 E+00 \\
4.522 E+00 \\
4.234 E+00 \\
3.990 E+00 \\
3.598 E+00 \\
3.298 E+00 \\
3.060 E+00\end{array}$ & $\begin{array}{l}1.180 \mathrm{E}-02 \\
1.192 \mathrm{E}-02 \\
1.202 \mathrm{E}-02 \\
1.210 \mathrm{E}-02 \\
1.218 \mathrm{E}-02 \\
1.233 \mathrm{E}-02 \\
1.246 \mathrm{E}-02 \\
1.259 \mathrm{E}-02\end{array}$ & $\begin{array}{l}5.3 \\
4.8 \\
4.5 \\
4.2 \\
4.0 \\
3.6 \\
3.3 \\
3.0\end{array}$ & $\begin{array}{l}E-03 \\
E-03 \\
E-03 \\
E-03 \\
E-03 \\
E-02 \\
E-02 \\
E-02\end{array}$ & $\begin{array}{l}210 \mathrm{E}-03 \\
335 \mathrm{E}-03 \\
456 \mathrm{E}-03 \\
574 \mathrm{E}-03 \\
689 \mathrm{E}-03 \\
909 \mathrm{E}-03 \\
119 \mathrm{E}-03 \\
321 \mathrm{E}-03\end{array}$ & $\begin{array}{l}9.578 \mathrm{E}-03 \\
1.103 \mathrm{E}-02 \\
1.253 \mathrm{E}-02 \\
1.407 \mathrm{E}-02 \\
1.567 \mathrm{E}-02 \\
1.899 \mathrm{E}-02 \\
2.247 \mathrm{E}-02 \\
2.611 \mathrm{E}-02\end{array}$ & $\begin{array}{l}-0.189 \\
-0.185 \\
-0.181 \\
-0.178 \\
-0.175 \\
-0.170 \\
-0.166 \\
-0.163\end{array}$ & $\begin{array}{l}0.217 \\
0.212 \\
0.207 \\
0.203 \\
0.199 \\
0.193 \\
0.188 \\
0.183\end{array}$ & $\begin{array}{l}0.214 \\
0.208 \\
0.204 \\
0.200 \\
0.196 \\
0.191 \\
0.186 \\
0.182\end{array}$ \\
\hline $\begin{array}{l}0.1000 \\
0.1250 \\
0.1500 \\
0.1750 \\
0.2000 \\
0.2500 \\
0.3000 \\
0.3500\end{array}$ & $\begin{array}{l}2.867 E+00 \\
2.514 E+00 \\
2.274 E+00 \\
2.101 E+00 \\
1.971 E+00 \\
1.789 E+00 \\
1.670 E+00 \\
1.588 E+00\end{array}$ & $\begin{array}{l}1.272 \mathrm{E}-02 \\
1.306 \mathrm{E}-02 \\
1.341 \mathrm{E}-02 \\
1.379 \mathrm{E}-02 \\
1.419 \mathrm{E}-02 \\
1.506 \mathrm{E}-02 \\
1.600 \mathrm{E}-02 \\
1.702 \mathrm{E}-02\end{array}$ & $\begin{array}{l}2.880 \mathrm{E}+00 \\
2.527 \mathrm{E}+00 \\
2.287 \mathrm{E}+00 \\
2.115 \mathrm{E}+00 \\
1.985 \mathrm{E}+00 \\
1.804 \mathrm{E}+00 \\
1.686 \mathrm{E}+00 \\
1.605 \mathrm{E}+00\end{array}$ & $\begin{array}{l}2.083 \mathrm{E}-02 \\
3.013 \mathrm{E}-02 \\
4.056 \mathrm{E}-02 \\
5.195 \mathrm{E}-02 \\
6.416 \mathrm{E}-02 \\
9.067 \mathrm{E}-02 \\
1.194 \mathrm{E}-01 \\
1.498 \mathrm{E}-01\end{array}$ & $\begin{array}{l}2.514 \mathrm{E}-03 \\
2.971 \mathrm{E}-03 \\
3.396 \mathrm{E}-03 \\
3.796 \mathrm{E}-03 \\
4.176 \mathrm{E}-03 \\
4.892 \mathrm{E}-03 \\
5.563 \mathrm{E}-03 \\
6.204 \mathrm{E}-03\end{array}$ & $\begin{array}{l}2.987 \mathrm{E}-02 \\
3.978 \mathrm{E}-02 \\
5.023 \mathrm{E}-02 \\
6.105 \mathrm{E}-02 \\
7.212 \mathrm{E}-02 \\
9.467 \mathrm{E}-02 \\
1.174 \mathrm{E}-01 \\
1.402 \mathrm{E}-01\end{array}$ & $\begin{array}{l}-0.160 \\
-0.153 \\
-0.148 \\
-0.144 \\
-0.140 \\
-0.134 \\
-0.129 \\
-0.125\end{array}$ & $\begin{array}{l}0.180 \\
0.172 \\
0.166 \\
0.162 \\
0.158 \\
0.151 \\
0.146 \\
0.142\end{array}$ & $\begin{array}{l}0.178 \\
0.171 \\
0.165 \\
0.160 \\
0.156 \\
0.150 \\
0.145 \\
0.140\end{array}$ \\
\hline $\begin{array}{l}0.4000 \\
0.4500 \\
0.5000 \\
0.5500 \\
0.6000 \\
0.7000 \\
0.8000 \\
0.9000\end{array}$ & $\begin{array}{l}1.52 \\
1.48 \\
1.44 \\
1.42 \\
1.40 \\
1.37 \\
1.35 \\
1.34\end{array}$ & $\begin{array}{l}1.925 \mathrm{E}-02 \\
2.044 \mathrm{E}-02 \\
2.169 \mathrm{E}-02 \\
2.297 \mathrm{E}-02 \\
2.566 \mathrm{E}-02 \\
2.848 \mathrm{E}-02 \\
3.141 \mathrm{E}-02\end{array}$ & $\begin{array}{l}1.546 E+00 \\
1.502 E+00 \\
1.470 E+00 \\
1.445 E+00 \\
1.425 E+00 \\
1.399 E+00 \\
1.383 E+00 \\
1.374 E+00\end{array}$ & $\begin{array}{l}1.816 \mathrm{E}-01 \\
2.144 \mathrm{E}-01 \\
2.481 \mathrm{E}-01 \\
2.824 \mathrm{E}-01 \\
3.173 \mathrm{E}-01 \\
3.881 \mathrm{E}-01 \\
4.601 \mathrm{E}-01 \\
5.326 \mathrm{E}-01\end{array}$ & $\begin{array}{l}6.824 \mathrm{E}-03 \\
7.428 \mathrm{E}-03 \\
8.021 \mathrm{E}-03 \\
8.607 \mathrm{E}-03 \\
9.186 \mathrm{E}-03 \\
1.034 \mathrm{E}-02 \\
1.148 \mathrm{E}-02 \\
1.261 \mathrm{E}-02\end{array}$ & $\begin{array}{l}1.629 \mathrm{E}-01 \\
1.855 \mathrm{E}-01 \\
2.080 \mathrm{E}-01 \\
2.304 \mathrm{E}-01 \\
2.526 \mathrm{E}-01 \\
2.969 \mathrm{E}-01 \\
3.407 \mathrm{E}-01 \\
3.841 \mathrm{E}-01\end{array}$ & $\begin{array}{l}-0.1 \\
-0.1 \\
-0.1 \\
-0.1 \\
-0.1 \\
-0 . \\
-0 . \\
-0 .\end{array}$ & $\begin{array}{l}0 . \\
0 . \\
0 . \\
0 . \\
0 . \\
0 .\end{array}$ & $\begin{array}{l}0.136 \\
0.133 \\
0.130 \\
0.127 \\
0.124 \\
0.120 \\
0.1116 \\
0.112\end{array}$ \\
\hline $\begin{array}{l}1.0000 \\
1.2500 \\
1.5000 \\
1.7500 \\
2.0000 \\
2.5000 \\
3.0000 \\
3.5000\end{array}$ & $\begin{array}{l}1.335 E+00 \\
1.328 E+00 \\
1.330 E+00 \\
1.336 E+00 \\
1.343 E+00 \\
1.359 E+00 \\
1.376 E+00 \\
1.391 E+00\end{array}$ & $\begin{array}{l}3.446 \mathrm{E}-02 \\
4.250 \mathrm{E}-02 \\
5.107 \mathrm{E}-02 \\
6.006 \mathrm{E}-02 \\
6.940 \mathrm{E}-02 \\
8.894 \mathrm{E}-02 \\
1.094 \mathrm{E}-01 \\
1.306 \mathrm{E}-01\end{array}$ & $\begin{array}{l}1.370 E+00 \\
1.371 E+00 \\
1.381 E+00 \\
1.396 E+00 \\
1.412 E+00 \\
1.448 E+00 \\
1.485 E+00 \\
1.521 E+00\end{array}$ & $\begin{array}{l}6.055 \mathrm{E}-01 \\
7.881 \mathrm{E}-01 \\
9.699 \mathrm{E}-01 \\
1.150 \mathrm{E}+00 \\
1.328 \mathrm{E}+00 \\
1.678 \mathrm{E}+00 \\
2.019 \mathrm{E}+00 \\
2.351 \mathrm{E}+00\end{array}$ & $\begin{array}{l}1.375 \mathrm{E}-02 \\
1.662 \mathrm{E}-02 \\
1.951 \mathrm{E}-02 \\
2.244 \mathrm{E}-02 \\
2.539 \mathrm{E}-02 \\
3.137 \mathrm{E}-02 \\
3.740 \mathrm{E}-02 \\
4.345 \mathrm{E}-02\end{array}$ & $\begin{array}{l}4.270 \mathrm{E}-01 \\
5.317 \mathrm{E}-01 \\
6.321 \mathrm{E}-01 \\
7.277 \mathrm{E}-01 \\
8.183 \mathrm{E}-01 \\
9.855 \mathrm{E}-01 \\
1.136 \mathrm{E}+00 \\
1.271 \mathrm{E}+00\end{array}$ & $\begin{array}{l}-0.092 \\
-0.084 \\
-0.078 \\
-0.073 \\
-0.070 \\
-0.064 \\
-0.060 \\
-0.057\end{array}$ & $\begin{array}{l}0.1 \\
0.1 \\
0.0 \\
0.0 \\
0.0 \\
0.0 \\
0.0\end{array}$ & $\begin{array}{l}0.108 \\
0.100 \\
0.094 \\
0.089 \\
0.084 \\
0.076 \\
0.071 \\
0.066\end{array}$ \\
\hline $\begin{array}{l}4.0000 \\
4.5000 \\
5.0000 \\
5.5000 \\
6.0000 \\
7.0000 \\
8.00000 \\
9.0000\end{array}$ & $\begin{array}{l}1.405 E+00 \\
1.418 E+00 \\
1.430 E+00 \\
1.441 E+00 \\
1.451 E+00 \\
1.469 E+00 \\
1.485 E+00 \\
1.498 E+00\end{array}$ & $\begin{array}{l}1.523 \mathrm{E}-01 \\
1.746 \mathrm{E}-01 \\
1.974 \mathrm{E}-01 \\
2.205 \mathrm{E}-01 \\
2.440 \mathrm{E}-01 \\
2.919 \mathrm{E}-01 \\
3.408 \mathrm{E}-01 \\
3.906 \mathrm{E}-01\end{array}$ & $\begin{array}{l}1.557 \mathrm{E}+00 \\
1.593 \mathrm{E}+00 \\
1.627 \mathrm{E}+00 \\
1.661 \mathrm{E}+00 \\
1.695 \mathrm{E}+00 \\
1.761 \mathrm{E}+00 \\
1.825 \mathrm{E}+00 \\
1.889 \mathrm{E}+00\end{array}$ & $\begin{array}{l}2.676 \mathrm{E}+00 \\
2.993 \mathrm{E}+00 \\
3.304 \mathrm{E}+00 \\
3.608 \mathrm{E}+00 \\
3.906 \mathrm{E}+00 \\
4.485 \mathrm{E}+00 \\
5.042 \mathrm{E}+00 \\
5.581 \mathrm{E}+00\end{array}$ & $\begin{array}{l}4.949 \mathrm{E}-02 \\
5.552 \mathrm{E}-02 \\
6.152 \mathrm{E}-02 \\
6.747 \mathrm{E}-02 \\
7.337 \mathrm{E}-02 \\
8.502 \mathrm{E}-02 \\
9.643 \mathrm{E}-02 \\
1.076 \mathrm{E}-01\end{array}$ & $\begin{array}{l}1.39 \\
1.50 \\
1.61 \\
1.71 \\
1.80 \\
1.97 \\
2.12 \\
2.27\end{array}$ & $\begin{array}{l}-0.054 \\
-0.052 \\
-0.050 \\
-0.048 \\
-0.047 \\
-0.044 \\
-0.041 \\
-0.038\end{array}$ & $\begin{array}{l}0.0 \\
0.0 \\
0.0 \\
0.0 \\
0.0 \\
0.0\end{array}$ & $\begin{array}{l}0.063 \\
0.059 \\
0.057 \\
0.054 \\
0.052 \\
0.049 \\
0.045 \\
0.043\end{array}$ \\
\hline $\begin{array}{l}10.0000 \\
12.5000 \\
15.0000 \\
17.5000 \\
20.0000 \\
25.0000 \\
30.0000\end{array}$ & $\begin{array}{l}1.510 E+00 \\
1.535 E+00 \\
1.555 E+00 \\
1.571 E+00 \\
1.584 E+00 \\
1.606 E+00 \\
1.622 E+00 \\
1.636 E+00\end{array}$ & $\begin{array}{l}5.701 \mathrm{E}-01 \\
7.019 \mathrm{E}-01 \\
8.361 \mathrm{E}-01 \\
9.721 \mathrm{E}-01 \\
1.248 \mathrm{E}+00 \\
1.528 \mathrm{E}+00 \\
1.811 \mathrm{E}+00\end{array}$ & $\begin{array}{l}2.105 E+00 \\
2.257 E+00 \\
2.407 E+00 \\
2.556 E+00 \\
2.854 E+00 \\
3.150 E+00 \\
3.447 E+00\end{array}$ & $\begin{array}{l}7.335 \mathrm{E}+00 \\
8.481 \mathrm{E}+00 \\
9.554 \mathrm{E}+00 \\
1.056 \mathrm{E}+01 \\
1.241 \mathrm{E}+01 \\
1.408 \mathrm{E}+01 \\
1.560 \mathrm{E}+01\end{array}$ & $\begin{array}{l}1.445 \mathrm{E}-01 \\
1.690 \mathrm{E}-01 \\
1.919 \mathrm{E}-01 \\
2.134 \mathrm{E}-01 \\
2.527 \mathrm{E}-01 \\
2.875 \mathrm{E}-01 \\
3.187 \mathrm{E}-01\end{array}$ & $\begin{array}{l}2.402 E+0 \\
2.696 \mathrm{E}+0 \\
2.953 \mathrm{E}+0 \\
3.182 \mathrm{E}+0 \\
3.388 \mathrm{E}+0 \\
3.746 \mathrm{E}+0 \\
4.050 \mathrm{E}+0 \\
4.313 \mathrm{E}+0\end{array}$ & $\begin{array}{l}-0.036 \\
-0.0330 \\
-0.026 \\
-0.023 \\
-0.020 \\
-0.016 \\
-0.014 \\
-0.013\end{array}$ & & $\begin{array}{l}0 . \\
0 . \\
0 . \\
0 .\end{array}$ \\
\hline $\begin{array}{l}45.0000 \\
50.0000 \\
55.0000 \\
60.0000 \\
70.0000 \\
80.0000 \\
90.0000\end{array}$ & $\begin{array}{l}1.648 E+00 \\
1.658 E+00 \\
1.667 E+00 \\
1.675 E+00 \\
1.683 E+00 \\
1.698 E+00 \\
1.707 E+00 \\
1.716 E+00\end{array}$ & $\begin{array}{l}2.05 \\
2.38 \\
2.67 \\
2.98 \\
3.25 \\
3.8 \\
4.43 \\
5.02\end{array}$ & $\begin{array}{l}4.0 \\
4.3 \\
4.6 \\
4.9 \\
5.5 \\
6.1 \\
6.7\end{array}$ & $\begin{array}{l}+01 \\
+01 \\
+01 \\
+01 \\
+01 \\
+01 \\
+01 \\
+01\end{array}$ & $\begin{array}{l}3.7 \\
3.7 \\
4.1 \\
4.3 \\
4.7 \\
5 .\end{array}$ & $\begin{array}{l}4.7 \\
4.2 \\
5.7 \\
5.2 \\
5.9 \\
6 .\end{array}$ & $\begin{array}{l}-0 \\
-0 \\
-0 \\
-0 \\
-0 \\
-0 \\
-0\end{array}$ & & $\begin{array}{l}0.010 \\
0.009 \\
0.009 \\
0.007 \\
0.006 \\
0.006\end{array}$ \\
\hline $\begin{array}{l}00000 \\
0000 \\
0000 \\
0000 \\
0000\end{array}$ & $\begin{array}{l}1.75 \\
1.76 \\
1.77 \\
1.79 \\
1.80 \\
1.81\end{array}$ & $\begin{array}{l}7 . \\
8 . \\
1 \\
1 \\
1 . \\
1 . \\
2 .\end{array}$ & $\begin{array}{l}7.348 E+00 \\
8.864 E+00 \\
1.039 E+01 \\
1.191 E+01 \\
1.344 E+01 \\
1.651 E+01 \\
1.958 E+01 \\
2.266 E+01\end{array}$ & $\begin{array}{l}+01 \\
+01 \\
+01 \\
+01 \\
+01 \\
+01 \\
+01 \\
+01 \\
+01\end{array}$ & $\begin{array}{l}5 \cdot 9 \\
6.3 \\
6.6 \\
6.8 \\
7.2 \\
7.5\end{array}$ & 8 & $\begin{array}{l}-0 \\
-0 \\
-0 \\
-0 \\
-0 \\
-0 \\
-0 \\
-0\end{array}$ & $\begin{array}{l}0 . \\
0 . \\
0 . \\
0 . \\
0 .\end{array}$ & $\begin{array}{l}0.005 \\
0.004 \\
0.003 \\
0.003 \\
0.003 \\
0.002 \\
0.002 \\
0.001\end{array}$ \\
\hline $\begin{array}{l}0.0000 \\
0.0000 \\
0.0000 \\
0.0000 \\
0.0000 \\
0.0000\end{array}$ & $\begin{array}{l}1.828 E+00 \\
1.837 E+00 \\
1.844 E+00 \\
1.851 E+00 \\
1.857 E+00 \\
1.868 E+00 \\
1.878 E+00 \\
1.886 E+00\end{array}$ & $\begin{array}{l}2.391 E+01 \\
2.698 E+01 \\
3.006 E+01 \\
3.315 E+01 \\
3.523 E+01 \\
4.241 E+01 \\
4.859 E+01 \\
5.478 E+01\end{array}$ & $\begin{array}{l}2.574 E+01 \\
2.882 E+01 \\
3.191 E+01 \\
3.500 E+01 \\
3.809 E+01 \\
4.428 E+01 \\
5.047 E+01 \\
5.666 E+01\end{array}$ & $\begin{array}{l}871 \mathrm{E}+01 \\
.055 \mathrm{E}+01 \\
219 \mathrm{E}+01 \\
369 \mathrm{E}+01 \\
.506 \mathrm{E}+01 \\
.749 \mathrm{E}+01 \\
960 \mathrm{E}+01 \\
.147 \mathrm{E}+01\end{array}$ & $\begin{array}{l}7.982 \mathrm{E}- \\
8.113 \mathrm{E} \\
8.241 \mathrm{E} \\
8.351 \mathrm{E}- \\
8.446 \mathrm{E} \\
8.603 \mathrm{E} \\
8.728 \mathrm{E}- \\
8.831 \mathrm{E}-\end{array}$ & $\begin{array}{l}8.891 \mathrm{E}+00 \\
9.125 \mathrm{E}+00 \\
9.334 \mathrm{E}+00 \\
9.524 \mathrm{E}+00 \\
9.697 \mathrm{E}+00 \\
1.000 \mathrm{E}+01 \\
1.027 \mathrm{E}+01 \\
1.051 \mathrm{E}+01\end{array}$ & $\begin{array}{l}-0.001 \\
-0.0001 \\
-0.001 \\
-0.000 \\
-0.000 \\
-0.000 \\
-0.000 \\
-0.000\end{array}$ & $\begin{array}{l}0.010 \\
0.010 \\
0.009 \\
0.009 \\
0.009 \\
0.009 \\
0.008\end{array}$ & $\begin{array}{l}0.001 \\
0.001 \\
0.001 \\
0.001 \\
0.001 \\
0.001 \\
0.001 \\
0.001\end{array}$ \\
\hline & $.893 E+00$ & $6.097 E+01$ & $6.286 E+01$ & $6.315 E+01$ & $8.916 E-01$ & $1.072 E+01$ & -0.000 & 0.008 & .001 \\
\hline
\end{tabular}


ELECTRONS IN IRON

$I=286.0 \mathrm{eV} \quad$ DENSITY $=7.874 \mathrm{E}+00 \mathrm{~g} / \mathrm{cm}^{3}$

\begin{tabular}{|c|c|c|c|c|c|c|c|c|c|}
\hline \multirow{3}{*}{$\begin{array}{l}\text { ENERGY } \\
\text { MeV }\end{array}$} & \multicolumn{3}{|c|}{ STOPPING POWER } & \multirow{3}{*}{$\begin{array}{l}\text { CSDA } \\
\text { RANGE } \\
g / \mathrm{Cm}^{2}\end{array}$} & \multirow{3}{*}{$\begin{array}{l}\text { RADIATION } \\
\text { YIELD }\end{array}$} & \multirow{3}{*}{$\begin{array}{c}\text { DENS.EFF. } \\
\text { CORR } \\
\text { (DELTA) }\end{array}$} & \multicolumn{3}{|c|}{$d(\log ) / d(\log I$} \\
\hline & & ATI & TOTAL & & & & & & RAD \\
\hline & $n^{2} / 9$ & eV & 1 & & & & & & \\
\hline $\begin{array}{l}0.0100 \\
0.0125 \\
0.0150 \\
0.0175 \\
0.0200 \\
0.0250 \\
0.0300\end{array}$ & $\begin{array}{l}1.3 \\
1.1 \\
1.0 \\
9.3 \\
8.4 \\
7.1 \\
6.3 \\
5.6\end{array}$ & $\begin{array}{l}1.2 \\
1.2 \\
1.2 \\
1.2 \\
1.2\end{array}$ & $\begin{array}{l}1 . \\
1 . \\
1: \\
9 . \\
8 . \\
7 . \\
6 . \\
5 .\end{array}$ & $\begin{array}{l}4.329 E-04 \\
6.284 E-04 \\
8.539 E-04 \\
1.108 E-03 \\
1.390 E-03 \\
2.032 E-03 \\
2.774 E-03\end{array}$ & & $\begin{array}{l}E-03 \\
E-03 \\
E-03 \\
E-03 \\
E-03 \\
E-03 \\
E-03 \\
E-03\end{array}$ & $\begin{array}{l}-0.270 \\
-0.255 \\
-0.244 \\
-0.235 \\
-0.228 \\
-0.217 \\
-0.209 \\
-0.202\end{array}$ & & $\begin{array}{l}.324 \\
.302 \\
.286 \\
.274 \\
.264 \\
.249 \\
.239 \\
230\end{array}$ \\
\hline $\begin{array}{l}0.0400 \\
0.0450 \\
0.0500 \\
0.0550 \\
0.0600 \\
0.0700 \\
0.0800 \\
0.0900\end{array}$ & $\begin{array}{l}5.14 \\
4.74 \\
4.40 \\
4.12 \\
3.89 \\
3.51 \\
3.22 \\
2.99\end{array}$ & $\begin{array}{l}1.4 \\
1.4 \\
1.4 \\
1.4\end{array}$ & $\begin{array}{l}5 . \\
4 . \\
4 . \\
4 . \\
3 . \\
3 . \\
3 . \\
3 .\end{array}$ & $\begin{array}{l}4.536 \\
5.546 \\
6.637 \\
7.806 \\
9.050 \\
1.175 \\
1.471\end{array}$ & $\begin{array}{l}-03 \\
-03 \\
-03 \\
-03 \\
-03 \\
-03 \\
-03 \\
-03\end{array}$ & $\begin{array}{l}1.2 \\
1.4 \\
1.6 \\
1.9 \\
2.1 \\
2.6\end{array}$ & $\begin{array}{l}-0 . \\
-0 . \\
-0 . \\
-0 . \\
-0 \\
-0 . \\
-0 . \\
-0 .\end{array}$ & & $\begin{array}{l}223 \\
217 \\
212 \\
208 \\
204 \\
198 \\
192 \\
07\end{array}$ \\
\hline $\begin{array}{l}0.1000 \\
0.1250 \\
0.1500 \\
0.1750 \\
0.2000 \\
0.2500 \\
0.3000 \\
0.3500\end{array}$ & $\begin{array}{l}E+00 \\
E+00 \\
E+00 \\
E+00 \\
E+00 \\
E+00 \\
E+00 \\
E+00\end{array}$ & $\begin{array}{l}1 . \\
1: \\
1: \\
1:\end{array}$ & $\begin{array}{l}2.8 \\
2.4 \\
2.2 \\
2.0 \\
1.9 \\
1.7 \\
1.6 \\
1.5\end{array}$ & $\begin{array}{l}3.0 \\
4.1 \\
5.3 \\
6.5 \\
9.2 \\
1.2 \\
1.5\end{array}$ & & $\begin{array}{l}4.4 \\
6.0 \\
7.7 \\
9.5 \\
1.1 \\
1.4 \\
1.7\end{array}$ & $\begin{array}{l}-0.16 \\
-0.15 \\
-0.14 \\
-0.14 \\
-0.13 \\
-0.13 \\
-0.12 \\
-0.12\end{array}$ & & $\begin{array}{l}0.183 \\
0.175 \\
0.168 \\
0.162 \\
0.158 \\
0.151 \\
0.145 \\
0.140\end{array}$ \\
\hline $\begin{array}{l}0.4000 \\
0.4500 \\
0.5000 \\
0.5500 \\
0.6000 \\
0.7000 \\
0.8000 \\
0.9000\end{array}$ & $\begin{array}{l}+\infty 00 \\
++00 \\
+00 \\
+00 \\
+00 \\
+00 \\
+00 \\
+00\end{array}$ & $\begin{array}{l}2 . \\
2 . \\
2 . \\
3 .\end{array}$ & $\begin{array}{l}1 . \\
1 . \\
1: \\
1 . \\
1 .\end{array}$ & & & $\begin{array}{l}2.5 \\
2.7 \\
3.2 \\
3.3 \\
3.6 \\
4.9 \\
4.6\end{array}$ & $\begin{array}{l}-0 \\
-0 \\
-0 \\
-0\end{array}$ & & $\begin{array}{l}36 \\
33 \\
29 \\
27 \\
24 \\
20 \\
16 \\
12\end{array}$ \\
\hline $\begin{array}{l}1.0000 \\
1.2500 \\
1.5000 \\
1.7500 \\
2.0000 \\
2.5000 \\
3.0000 \\
3.5000\end{array}$ & $\begin{array}{l}+00 \\
+00 \\
+00 \\
+00 \\
+00 \\
+00 \\
+00 \\
+00 \\
+00\end{array}$ & $\begin{array}{l}6.13 \\
7.20 \\
8.31 \\
1.06 \\
1.30 \\
1.55\end{array}$ & $\begin{array}{l}1.365 E+00 \\
1.382 E+00 \\
1.400 E+00 \\
1.440 E+00 \\
1.480 E+00 \\
1.520 E+00\end{array}$ & & & & $\begin{array}{l}-0.0 \\
-0.0 \\
-0.0 \\
-0.0 \\
-0.0 \\
-0.0\end{array}$ & & $\begin{array}{l}109 \\
02 \\
96 \\
91 \\
86 \\
79 \\
73 \\
68\end{array}$ \\
\hline $\begin{array}{l}4.00 \\
4.50 \\
5.00 \\
5.50 \\
6.00 \\
7.00 \\
8.00\end{array}$ & $\begin{array}{l}0 \\
0 \\
0 \\
0 \\
0 \\
0\end{array}$ & $\begin{array}{l}2 . \\
2 . \\
2 . \\
2 . \\
3 . \\
4 .\end{array}$ & & 年 & & $\begin{array}{l}1.6 \\
1.7 \\
1.8 \\
1.9 \\
2.0 \\
2.2 \\
2.3 \\
2.5\end{array}$ & & & \\
\hline $\begin{array}{l}15.0 \\
17.5 \\
20.0 \\
25.0 \\
30.0\end{array}$ & 1 & $\begin{array}{l}8 . \\
9 \\
1 .\end{array}$ & & & & & & & \\
\hline $\begin{array}{l}40.0 \\
45.0 \\
50.0 \\
55.0 \\
60.0 \\
70.0\end{array}$ & 1 & 3. & & & & & & & \\
\hline 1 & 1 & & & & & & & & \\
\hline $\begin{array}{l}4 \\
5 \\
5 \\
6\end{array}$ & $871 E+00$ & $.394 E+01$ & $801 E+01$ & & & & $\begin{array}{l}1 \\
1 \\
1 \\
1 \\
1 \\
0 \\
0\end{array}$ & & $\begin{array}{llll}0 & 0 & 1 \\
0 & 0 & 1 \\
0 & 0 & 1 \\
0 & 0 & 1 \\
0 & 0 & 1 \\
0 & 0 & 1 \\
0 & 0 & 1\end{array}$ \\
\hline $0.0 \mathrm{c}$ & $1.878 E+00$ & $7.116 E+01$ & $.304 E+01$ & $5.678 E+01$ & $9.040 E-01$ & $1.087 E+01$ & -0.000 & 0.009 & 0.001 \\
\hline
\end{tabular}




\begin{tabular}{|c|c|c|c|c|c|c|c|c|c|}
\hline $\mathrm{MeV}$ & 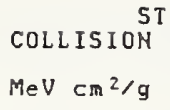 & $\mathrm{eV} \mathrm{cm}^{2} / \mathrm{g}$ & $\begin{array}{c}\text { TOTAL } \\
\mathrm{MeV} \mathrm{cm}^{2} / \mathrm{g}\end{array}$ & $\begin{array}{l}\text { CSDA } \\
\text { RANGE } \\
\mathrm{g} / \mathrm{cm}^{2}\end{array}$ & DN & $\begin{array}{l}\text { ENS.EFF. } \\
\text { CORR. } \\
\text { (DELTA) }\end{array}$ & $\begin{array}{l}d(1 \\
\operatorname{coLL} \\
\text { LOSS }\end{array}$ & CSDA & I ) \\
\hline $\begin{array}{l}0.0100 \\
0.0125 \\
0.0150 \\
0.0175 \\
0.0200 \\
0.0250 \\
0.0300 \\
0.0350\end{array}$ & $\begin{array}{l}1.318 \\
1.127 \\
9.904 \\
8.874 \\
8.066 \\
6.877\end{array}$ & $\begin{array}{l}1.3 \\
1.3 \\
1.3 \\
1.4 \\
1.4\end{array}$ & $\begin{array}{l}1 . \\
9 . \\
8 . \\
8 . \\
6 . \\
6 . \\
5 .\end{array}$ & $\begin{array}{l}4 \\
3 \\
3 \\
3 \\
3 \\
3 \\
3\end{array}$ & & $\begin{array}{l}3 \\
3 \\
3 \\
3 \\
3 \\
3 \\
3\end{array}$ & & & $\begin{array}{l}37 \\
314 \\
297 \\
284 \\
273 \\
57 \\
46\end{array}$ \\
\hline $\begin{array}{l}0.0400 \\
0.0450 \\
0.0500 \\
0.0550 \\
0.0600 \\
0.0700 \\
0.0800 \\
0.0900\end{array}$ & $\begin{array}{l}931 E+00 \\
544 E+00 \\
226 E+00 \\
961 E+00 \\
736 E+00 \\
375 E+00 \\
098 E+00 \\
877 E+00\end{array}$ & $\begin{array}{l}1 . \\
1.5 \\
1.5 \\
1:\end{array}$ & & & & $\begin{array}{l}4 \\
2 \\
2\end{array}$ & $\begin{array}{l}-0.202 \\
-0.197 \\
-0.193 \\
-0.189 \\
-0.186 \\
-0.180 \\
-0.175 \\
-0.171\end{array}$ & & 230 \\
\hline $\begin{array}{l}0.1000 \\
0.1250 \\
0.1500 \\
0.1750 \\
0.2000 \\
0.2500 \\
0.3000 \\
0.3500\end{array}$ & $\begin{array}{l}2.698 \mathrm{E}+00 \\
2.370 \mathrm{E}+00 \\
2.146 \mathrm{E}+00 \\
1.984 \mathrm{E}+00 \\
1.861 \mathrm{E}+00 \\
1.691 \mathrm{E}+00 \\
1.579 \mathrm{E}+00 \\
1.501 \mathrm{E}+00\end{array}$ & $\begin{array}{l}1.710 \mathrm{E}-02 \\
1.763 \mathrm{E}-02 \\
1.816 \mathrm{E}-02 \\
1.870 \mathrm{E}-02 \\
1.926 \mathrm{E}-02 \\
2.045 \mathrm{E}-02 \\
2.172 \mathrm{E}-02 \\
2.307 \mathrm{E}-02\end{array}$ & $\begin{array}{l}2.715 E+00 \\
2.387 E+00 \\
2.164 E+00 \\
2.002 E+00 \\
1.881 E+00 \\
1.711 E+00 \\
1.601 E+00 \\
1.524 E+00\end{array}$ & & & $\begin{array}{l}2 \\
2 \\
2\end{array}$ & $\begin{array}{l}-0.167 \\
-0.157 \\
-0.149 \\
-0.141 \\
-0.135 \\
-0.127 \\
-0.121 \\
-0.116\end{array}$ & & $\begin{array}{l}0.172 \\
0.165 \\
0.159 \\
0.150 \\
0.143\end{array}$ \\
\hline $\begin{array}{l}0.4000 \\
0.4500 \\
0.5000 \\
0.5500 \\
0.6000 \\
0.7000 \\
0.8000 \\
0.9000\end{array}$ & $\begin{array}{l}1.444 E+00 \\
1.402 E+00 \\
1.370 E+00 \\
1.345 E+00 \\
1.326 E+00 \\
1.298 E+00 \\
1.281 E+00 \\
1.270 E+00\end{array}$ & $\begin{array}{l}2.450 \mathrm{E}-02 \\
2.600 \mathrm{E}-02 \\
2.757 \mathrm{E}-02 \\
2.919 \mathrm{E}-02 \\
3.087 \mathrm{E}-02 \\
3.437 \mathrm{E}-02 \\
3.803 \mathrm{E}-02 \\
4.185 \mathrm{E}-02\end{array}$ & $\begin{array}{l}1.469 E+00 \\
1.428 E+00 \\
1.398 E+00 \\
1.375 E+00 \\
1.357 E+00 \\
1.333 E+00 \\
1.319 E+00 \\
1.312 E+00\end{array}$ & $\begin{array}{l}1 \\
1 \\
1 \\
1 \\
1 \\
1\end{array}$ & $\begin{array}{l}9.741 \mathrm{E}-03 \\
1.060 \mathrm{E}-02 \\
1.143 \mathrm{E}-02 \\
1.226 \mathrm{E}-02 \\
1.307 \mathrm{E}-02 \\
1.467 \mathrm{E}-02\end{array}$ & $\begin{array}{l}2.302 \mathrm{E}-01 \\
2.635 \mathrm{E}-01 \\
2.958 \mathrm{E}-01 \\
3.273 \mathrm{E}-01 \\
3.581 \mathrm{E}-01 \\
4.173 \mathrm{E}-01 \\
4.739 \mathrm{E}-01\end{array}$ & $\begin{array}{l}-0.11 \\
-0.10 \\
-0.10 \\
-0.10 \\
-0.10 \\
-0.09 \\
-0.00\end{array}$ & & $\begin{array}{l}0.124 \\
0.121 \\
0.118 \\
0.113 \\
0.110\end{array}$ \\
\hline $\begin{array}{l}1.0000 \\
1.2500 \\
1.5000 \\
1.7500 \\
2.0000 \\
2.5000 \\
3.0000 \\
3.5000\end{array}$ & $\begin{array}{l}3 E+00 \\
5 E+00 \\
9 E+00 \\
5 E+00 \\
3 E+00 \\
9 E+00 \\
5 E+00 \\
20 E+00\end{array}$ & $\begin{array}{l}5 . \\
6 . \\
7 . \\
9 . \\
1 . \\
1 . \\
1 .\end{array}$ & $\begin{array}{l}1.3 \\
1.3 \\
1.3 \\
1.3 \\
1.3 \\
1.4 \\
1.4 \\
1.4\end{array}$ & & $\begin{array}{l}2 . \\
2 . \\
3 \\
3 \\
5\end{array}$ & $\begin{array}{l}5 . \\
7 . \\
8 . \\
9 \\
1: \\
1: \\
1: \\
1 .\end{array}$ & $\begin{array}{l}-0 \\
-0 \\
-0 \\
-0 \\
-0 \\
-0 \\
-0\end{array}$ & & \\
\hline $\begin{array}{l}4.0000 \\
4.5000 \\
5.0000 \\
5.5000 \\
6.0000 \\
7.0000 \\
8.0000\end{array}$ & $\begin{array}{l}1.3 \\
1.3 \\
1.3 \\
1.3 \\
1.3 \\
1.4\end{array}$ & $\begin{array}{l}1.976 \mathrm{E}-01 \\
2.261 \mathrm{E}-01 \\
2.552 \mathrm{E}-01 \\
2.847 \mathrm{E}-01 \\
3.146 \mathrm{E}-01 \\
3.756 \mathrm{E}-01 \\
4.378 \mathrm{E}-01 \\
5.009 \mathrm{E}-01\end{array}$ & $\begin{array}{l}1.573 \mathrm{E}+0 \\
1.613 \mathrm{E}+0 \\
1.653 \mathrm{E}+0 \\
1.693 \mathrm{E}+0 \\
1.771 \mathrm{E}+0 \\
1.849 \mathrm{E}+0 \\
1.925 \mathrm{E}+0\end{array}$ & . & $\begin{array}{l}7 . \\
8 . \\
8 . \\
9 . \\
1 .\end{array}$ & $\begin{array}{l}1.6 \\
1.7 \\
1.8 \\
1.9 \\
2.0 \\
2.2 \\
2.3 \\
2.5\end{array}$ & $\begin{array}{l}-0 \\
-0 \\
-0 \\
-0 \\
-0 \\
-0 \\
-0 \\
-0\end{array}$ & & \\
\hline $\begin{array}{l}15.0000 \\
17.5000 \\
20.0000 \\
25.0000\end{array}$ & $\begin{array}{l}1.46 \\
1.48 \\
1.49 \\
1.51 \\
1.53 \\
1.55 \\
1.57\end{array}$ & $\begin{array}{l}5 . \\
7 . \\
8 . \\
1 . \\
1 . \\
1 .\end{array}$ & $\begin{array}{l}2.1 \\
2.3 \\
2.5 \\
2.7 \\
3.1\end{array}$ & $\begin{array}{l}0 \\
0 \\
1 \\
1 \\
1\end{array}$ & $\begin{array}{l}1.526 \mathrm{E}-0 \\
1.837 \mathrm{E}-0 \\
2.122 \mathrm{E}-0 \\
2.385 \mathrm{E}-0 \\
2.628 \mathrm{E}-0 \\
3.061 \mathrm{E}-0\end{array}$ & $\begin{array}{l}2 . \\
3 \\
3\end{array}$ & & & $\begin{array}{l}0 . \\
0 . \\
0 .\end{array}$ \\
\hline $\begin{array}{l}50.0000 \\
55.0000 \\
60.0000 \\
70.0000 \\
80.0000 \\
90.0000\end{array}$ & $\begin{array}{l}1.5 \\
1.6 \\
1.6 \\
1.6 \\
1.6 \\
1.6 \\
1.6\end{array}$ & $\begin{array}{l}3 \\
3 \\
3 \\
4 \\
4\end{array}$ & $\begin{array}{l}4 . \\
4 . \\
5 . \\
5 . \\
6 .\end{array}$ & & & & & & \\
\hline $\begin{array}{l}0.0 \\
5.0 \\
0.0 \\
0.0\end{array}$ & $\begin{array}{l}1.6 \\
1.7 \\
1.7 \\
1.7 \\
1.7 \\
1.7\end{array}$ & & $\begin{array}{l}1 . \\
1 . \\
1 . \\
2\end{array}$ & & $\begin{array}{l}6 \\
6 \\
7 \\
7\end{array}$ & & & & \\
\hline $\begin{array}{l}.00 \\
.00 \\
.00\end{array}$ & $\begin{array}{l}1.808 \mathrm{E}+00 \\
1.818 \mathrm{E}+00 \\
1.826 \mathrm{E}+00\end{array}$ & $\begin{array}{l}4 \\
4 \\
5\end{array}$ & $\begin{array}{l}3.9 \\
4.9 \\
4.7 \\
5.4\end{array}$ & 4 & 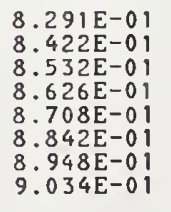 & 1. & $\begin{array}{l}2 \\
1 \\
1 \\
1 \\
1 \\
1\end{array}$ & $\begin{array}{l}2 \\
1 \\
1 \\
1 \\
0 \\
0\end{array}$ & $\begin{array}{ll}0 & 01 \\
0 & 01 \\
0 & 01 \\
0 & 01 \\
0 & 01 \\
0 & 01\end{array}$ \\
\hline .0 & $.833 E+00$ & $.629 E+01$ & $7.813 E+01$ & $441 E+01$ & $9.106 \mathrm{E}-01$ & $074 \mathrm{E}+01$ & -0.000 & .009 & \\
\hline
\end{tabular}


ELECTRONS IN GERMANIUM

$I=350.0 \mathrm{eV} \quad$ DENSITY $=5.323 \mathrm{E}+00 \mathrm{~g} / \mathrm{cm}^{3}$

\begin{tabular}{|c|c|c|c|c|c|c|c|c|c|}
\hline $\mathrm{MeV}$ & $\begin{array}{l}\text { COLLISION } \\
\mathrm{MeV} \mathrm{cm}^{2} / \mathrm{g}\end{array}$ & $\mathrm{eV} \mathrm{cm}^{2} / \mathrm{g}$ & $\begin{array}{c}\text { TOTAL } \\
\mathrm{MeV} \mathrm{cm}^{2} / \mathrm{g}\end{array}$ & $\begin{array}{l}\text { CS } \\
\text { RA }\end{array}$ & N & $\begin{array}{l}E F F . \\
R \text { TA) }\end{array}$ & $\begin{array}{l}\text { CO } \\
\text { LO }\end{array}$ & 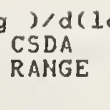 & $\begin{array}{l}\text { gI ) } \\
\text { RAD } \\
\text { YIELD }\end{array}$ \\
\hline $\begin{array}{l}0.01100 \\
0.0125 \\
0.01150 \\
0.0175 \\
0.0200 \\
0.0250 \\
0.0300\end{array}$ & $\begin{array}{l}1.0 \\
9.3 \\
8.4 \\
7.6 \\
6.5 \\
5.7\end{array}$ & $\begin{array}{l}1: \\
1: \\
1:\end{array}$ & & & & $\begin{array}{l}5 E-04 \\
2 E-04 \\
2 E-04 \\
4 E-04 \\
8 E-03 \\
4 E-03 \\
3 E-03\end{array}$ & $\begin{array}{l}-0.286 \\
-0.269 \\
-0.257 \\
-0.247 \\
-0.240 \\
-0.228 \\
-0.219 \\
-0.212\end{array}$ & & $\begin{array}{l}0.347 \\
0.322 \\
0.304 \\
0.291 \\
0.280 \\
0.263 \\
0.251 \\
0.242\end{array}$ \\
\hline $\begin{array}{l}0.0400 \\
0.0450 \\
0.0500 \\
0.0550 \\
0.0600 \\
0.0700 \\
0.0800\end{array}$ & $\begin{array}{l}4.0 \\
3.7 \\
3.5 \\
3.2\end{array}$ & $\begin{array}{l}1: \\
1: \\
1: \\
1:\end{array}$ & $\begin{array}{l}4 . \\
3 . \\
3 \\
3\end{array}$ & $\begin{array}{l}.03 \\
.03 \\
.03 \\
.03 \\
.03 \\
.02\end{array}$ & $\begin{array}{l}-03 \\
:-03 \\
-03 \\
-03 \\
-03 \\
-03 \\
-03 \\
-03\end{array}$ & $\begin{array}{l}2.6 \\
3.0 \\
3.5 \\
4.0 \\
4.5\end{array}$ & $\begin{array}{l}-0.206 \\
-0.201 \\
-0.197 \\
-0.194 \\
-0.191 \\
-0.185\end{array}$ & & $\begin{array}{l}.235 \\
.228 \\
.223 \\
.218 \\
.214 \\
.208 \\
.202 \\
\end{array}$ \\
\hline $\begin{array}{l}0.1000 \\
0.1250 \\
0.1500 \\
0.1750 \\
0.2000 \\
0.2500 \\
0.3000\end{array}$ & $\begin{array}{l}+00 \\
+00 \\
+00 \\
+00 \\
+00 \\
+00 \\
+00 \\
+00\end{array}$ & $\begin{array}{l}1 . \\
1 . \\
2 . \\
2 . \\
2 . \\
2 . \\
2 .\end{array}$ & $\begin{array}{l}2.8 \\
2.8 \\
1.8 \\
1.8 \\
1.8 \\
1.2\end{array}$ & $\begin{array}{l}02 \\
02 \\
02 \\
02 \\
02 \\
02 \\
01\end{array}$ & $\begin{array}{l}4.7 \\
5.4 \\
6.0 \\
6.6 \\
7.8 \\
8.9\end{array}$ & $\begin{array}{l}1.9 \\
2.3 \\
3.2 \\
4.2\end{array}$ & $\begin{array}{l}- \\
- \\
- \\
- \\
- \\
- \\
- \\
-\end{array}$ & $\begin{array}{l}8 \\
8 \\
2 \\
7 \\
3 \\
6 \\
1 \\
6\end{array}$ & $\begin{array}{l}94 \\
86 \\
80 \\
75 \\
71 \\
64 \\
59 \\
54\end{array}$ \\
\hline $\begin{array}{l}0.4000 \\
0.4500 \\
0.5000 \\
0.5500 \\
0.6000 \\
0.7000 \\
0.8000\end{array}$ & $\begin{array}{l}395 E+00 \\
357 E+00 \\
328 E+00 \\
305 E+00 \\
288 E+00 \\
263 E+00 \\
248 E+00 \\
239 E+00\end{array}$ & $\begin{array}{l}2.802 \mathrm{E}-02 \\
2.971 \mathrm{E}-02 \\
3.146 \mathrm{E}-02 \\
3.326 \mathrm{E}-02 \\
3.702 \mathrm{E}-02 \\
4.095 \mathrm{E}-02 \\
4.504 \mathrm{E}-02\end{array}$ & i: & & $\begin{array}{l}2 \\
2 \\
2 \\
2 \\
2\end{array}$ & $\begin{array}{l}6.3 \\
7.5 \\
8.5\end{array}$ & & & \\
\hline $\begin{array}{l}1.0000 \\
1.2500 \\
1.5000 \\
1.7500 \\
2.0000 \\
2.5000 \\
3.0000 \\
3.5000\end{array}$ & $\begin{array}{l}.234 E+00 \\
.231 E+00 \\
.235 E+00 \\
.242 E+00 \\
.250 E+00 \\
.268 E+00 \\
.284 E+00 \\
.300 E+00\end{array}$ & $\begin{array}{l}4.926 \\
6.036 \\
7.213 \\
8.442 \\
9.714 \\
1.236 \\
1.512 \\
1.797\end{array}$ & $\begin{array}{l}1.307 E+00 \\
1.326 E+00 \\
1.347 E+00 \\
1.391 E+00 \\
1.436 E+00 \\
1.480 E+00\end{array}$ & $\begin{array}{ll}0 & 1 \\
0 & 1 \\
0 & 0 \\
0 & 0 \\
0 & 0 \\
0 & 0\end{array}$ & $\begin{array}{l}2.5 \\
2.9 \\
3.4 \\
3.8 \\
4.6\end{array}$ & $\begin{array}{l}2.28 \\
3.08 \\
3.8 \\
4.6 \\
5.4\end{array}$ & & & $\begin{array}{l}1 \\
3 \\
7 \\
1 \\
2 \\
5\end{array}$ \\
\hline $\begin{array}{l}4.0000 \\
4.5000 \\
5.0000 \\
5.5000 \\
6.0000 \\
7.0000 \\
8.0000 \\
9.0000\end{array}$ & $\begin{array}{l}1.3 \\
1.3 \\
1.3 \\
1.3 \\
1.3 \\
1.3\end{array}$ & $\begin{array}{l}2.3 \\
2.6 \\
2.5 \\
3.3 \\
3.5 \\
4\end{array}$ & $\begin{array}{l}1.6 \\
1.6 \\
1.6 \\
1.7 \\
1.8 \\
1.9\end{array}$ & & & 1. & & & \\
\hline $\begin{array}{l}12.5000 \\
15.0000 \\
17.5000 \\
20.0000 \\
25.0000\end{array}$ & $\begin{array}{l}1.4 \\
1.4 \\
1.4 \\
1.5 \\
1.5\end{array}$ & 9. & $\begin{array}{l}2 . \\
2 . \\
2 .\end{array}$ & & 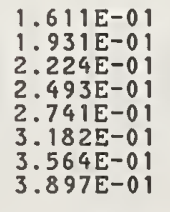 & & & & \\
\hline $\begin{array}{l}40.00 \\
45.00 \\
50.00 \\
55.00 \\
60.00 \\
70.00 \\
80.00 \\
90.00\end{array}$ & $\begin{array}{l}1.5 \\
1.5\end{array}$ & $\begin{array}{l}3.3 \\
4.3\end{array}$ & $\begin{array}{l}5 . \\
5 \\
5\end{array}$ & & & & & & \\
\hline $\begin{array}{l}5.0 \\
0.8 \\
0.8\end{array}$ & & & & & & & & & \\
\hline $\begin{array}{l}550.00 \\
500.00 \\
550.00 \\
600.00 \\
700.00\end{array}$ & $1.801 E+00$ & $\begin{array}{l}3 \\
4 \\
4\end{array}$ & $.379 t+01$ & $\begin{array}{l}1 \\
1 \\
1 \\
1\end{array}$ & 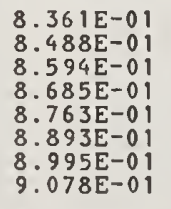 & $.815 E+00$ & & & \\
\hline & $1.808 E+00$ & $8.011 E+01$ & $8.192 E+01$ & $.276 E+01$ & $9.147 \mathrm{E}-01$ & $002 E+01$ & -0.00 & 0.010 & 0.001 \\
\hline
\end{tabular}


ELECTRONS IN KRYPTON

$I=352.0 \mathrm{eV} \quad$ DENSITY $=3.478 \mathrm{E}-03 \mathrm{~g} / \mathrm{cm}^{3}\left(20^{\circ} \mathrm{C}\right)$

\begin{tabular}{|c|c|c|c|c|c|c|c|c|c|}
\hline ENERGY & 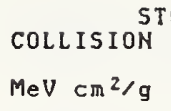 & 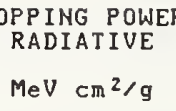 & $\begin{array}{c}\text { TOTAL } \\
\mathrm{MeV} \mathrm{cm}^{2} / \mathrm{g}\end{array}$ & $\begin{array}{l}\text { CSDA } \\
\text { RANGE } \\
\mathrm{g} / \mathrm{cm}^{2}\end{array}$ & $\begin{array}{l}\text { DIATION } \\
\text { YIELD }\end{array}$ & $\begin{array}{c}\text { DENS.EFF. } \\
\text { CORR. } \\
\text { (DELTA) }\end{array}$ & $\begin{array}{l}d(1 \\
\text { COLL } \\
\text { LOSS }\end{array}$ & $\begin{array}{l}\text { CSDA } \\
\text { RANGE }\end{array}$ & $\begin{array}{l}\text { gI ) } \\
\text { RAD } \\
\text { YIELD }\end{array}$ \\
\hline $\begin{array}{l}0.0100 \\
0.0125 \\
0.0150 \\
0.0175 \\
0.0200 \\
0.0250 \\
0.0300 \\
0.0350\end{array}$ & $\begin{array}{l}1.210 E+01 \\
1.036 E+01 \\
9.116 E+00 \\
8.175 E+00 \\
7.437 E+00 \\
6.347 E+00 \\
5.579 E+00 \\
5.006 E+00\end{array}$ & $\begin{array}{l}1.344 \mathrm{E}-02 \\
1.427 \mathrm{E}-02 \\
1.491 \mathrm{E}-02 \\
1.543 \mathrm{E}-02 \\
1.586 \mathrm{E}-02 \\
1.654 \mathrm{E}-02 \\
1.707 \mathrm{E}-02 \\
1.750 \mathrm{E}-02\end{array}$ & $\begin{array}{l}1.211 E+01 \\
1.038 E+01 \\
9.131 E+00 \\
8.191 E+00 \\
7.452 E+00 \\
6.364 E+00 \\
5.596 E+00 \\
5.024 E+00\end{array}$ & $\begin{array}{l}5.046 \mathrm{E}-04 \\
7.285 \mathrm{E}-04 \\
9.860 \mathrm{E}-04 \\
1.276 \mathrm{E}-03 \\
1.596 \mathrm{E}-03 \\
2.325 \mathrm{E}-03 \\
3.165 \mathrm{E}-03 \\
4.110 \mathrm{E}-03\end{array}$ & $\begin{array}{l}5.575 \mathrm{E}-04 \\
6.946 \mathrm{E}-04 \\
8.296 \mathrm{E}-04 \\
9.624 \mathrm{E}-04 \\
1.093 \mathrm{E}-03 \\
1.348 \mathrm{E}-03 \\
1.594 \mathrm{E}-03 \\
1.833 \mathrm{E}-03\end{array}$ & $\begin{array}{l}0.0 \\
0.0 \\
0.0 \\
0.0 \\
0.0 \\
0.0 \\
0.0 \\
0.0\end{array}$ & $\begin{array}{l}-0.287 \\
-0.270 \\
-0.257 \\
-0.248 \\
-0.240 \\
-0.228 \\
-0.219 \\
-0.212\end{array}$ & $\begin{array}{l}0.368 \\
0.340 \\
0.320 \\
0.305 \\
0.292 \\
0.274 \\
0.260 \\
0.250\end{array}$ & $\begin{array}{l}0.347 \\
0.322 \\
0.304 \\
0.291 \\
0.280 \\
0.263 \\
0.251 \\
0.242\end{array}$ \\
\hline $\begin{array}{l}0.0400 \\
0.0450 \\
0.0500 \\
0.0550 \\
0.0600 \\
0.0700 \\
0.0800 \\
0.0900\end{array}$ & $\begin{array}{l}4.561 E+00 \\
4.205 E+00 \\
3.913 E+00 \\
3.669 E+00 \\
3.462 E+00 \\
3.129 E+00 \\
2.874 E+00 \\
2.671 E+00\end{array}$ & $\begin{array}{l}1.785 \mathrm{E}-02 \\
1.816 \mathrm{E}-02 \\
1.844 \mathrm{E}-02 \\
1.869 \mathrm{E}-02 \\
1.891 \mathrm{E}-02 \\
1.932 \mathrm{E}-02 \\
1.969 \mathrm{E}-02 \\
2.003 \mathrm{E}-02\end{array}$ & $\begin{array}{l}4.579 E+00 \\
4.223 E+00 \\
3.931 E+00 \\
3.688 E+00 \\
3.481 E+00 \\
3.149 E+00 \\
2.893 E+00 \\
2.691 E+00\end{array}$ & $\begin{array}{l}5.154 \mathrm{E}-03 \\
6.292 \mathrm{E}-03 \\
7.521 \mathrm{E}-03 \\
8.835 \mathrm{E}-03 \\
1.023 \mathrm{E}-02 \\
1.326 \mathrm{E}-02 \\
1.658 \mathrm{E}-02 \\
2.016 \mathrm{E}-02\end{array}$ & $\begin{array}{l}2.065 \mathrm{E}-03 \\
2.292 \mathrm{E}-03 \\
2.512 \mathrm{E}-03 \\
2.727 \mathrm{E}-03 \\
2.938 \mathrm{E}-03 \\
3.345 \mathrm{E}-03 \\
3.736 \mathrm{E}-03 \\
4.113 \mathrm{E}-03\end{array}$ & $\begin{array}{l}0.0 \\
0.0 \\
0.0 \\
0.0 \\
0.0 \\
0.0 \\
0.0 \\
0.0\end{array}$ & $\begin{array}{l}-0.207 \\
-0.202 \\
-0.198 \\
-0.195 \\
-0.191 \\
-0.186 \\
-0.182 \\
-0.178\end{array}$ & $\begin{array}{l}0.242 \\
0.235 \\
0.229 \\
0.224 \\
0.219 \\
0.212 \\
0.206 \\
0.201\end{array}$ & $\begin{array}{l}0.235 \\
0.228 \\
0.223 \\
0.219 \\
0.215 \\
0.208 \\
0.203 \\
0.198\end{array}$ \\
\hline $\begin{array}{l}0.1000 \\
0.1250 \\
0.1500 \\
0.1750 \\
0.2000 \\
0.2500 \\
0.3000 \\
0.3500\end{array}$ & $\begin{array}{l}2.506 E+00 \\
2.204 E+00 \\
1.999 E+00 \\
1.851 E+00 \\
1.740 E+00 \\
1.585 E+00 \\
1.484 E+00 \\
1.415 E+00\end{array}$ & $\begin{array}{l}2.035 \mathrm{E}-02 \\
2.111 \mathrm{E}-02 \\
2.182 \mathrm{E}-02 \\
2.252 \mathrm{E}-02 \\
2.322 \mathrm{E}-02 \\
2.465 \mathrm{E}-02 \\
2.617 \mathrm{E}-02 \\
2.777 \mathrm{E}-02\end{array}$ & $\begin{array}{l}2.527 \mathrm{E}+00 \\
2.225 \mathrm{E}+00 \\
2.021 \mathrm{E}+00 \\
1.873 \mathrm{E}+00 \\
1.763 \mathrm{E}+00 \\
1.610 \mathrm{E}+00 \\
1.510 \mathrm{E}+00 \\
1.442 \mathrm{E}+00\end{array}$ & $\begin{array}{l}.400 \mathrm{E}-02 \\
.459 \mathrm{E}-02 \\
.640 \mathrm{E}-02 \\
.928 \mathrm{E}-02 \\
.305 \mathrm{E}-02 \\
.028 \mathrm{E}-01 \\
.350 \mathrm{E}-01 \\
.689 \mathrm{E}-01\end{array}$ & $\begin{array}{l}4.4 \\
5.3 \\
6.1 \\
6.8 \\
7.6 \\
8.9 \\
1.0 \\
1.1\end{array}$ & $\begin{array}{l}0.0 \\
0.0 \\
0.0 \\
0.0 \\
0.0 \\
0.0 \\
0.0 \\
0.0\end{array}$ & $\begin{array}{l}-0.175 \\
-0.169 \\
-0.164 \\
-0.160 \\
-0.157 \\
-0.152 \\
-0.147 \\
-0.144\end{array}$ & $\begin{array}{l}0.197 \\
0.189 \\
0.183 \\
0.178 \\
0.174 \\
0.168 \\
0.163 \\
0.159\end{array}$ & $\begin{array}{l}0.194 \\
0.186 \\
0.180 \\
0.176 \\
0.172 \\
0.165 \\
0.160 \\
0.156\end{array}$ \\
\hline $\begin{array}{l}0.4000 \\
0.4500 \\
0.5000 \\
0.5500 \\
0.6000 \\
0.7000 \\
0.8000 \\
0.9000\end{array}$ & $\begin{array}{l}1.365 E+00 \\
1.328 E+00 \\
1.300 E+00 \\
1.279 E+00 \\
1.263 E+00 \\
1.241 E+00 \\
1.229 E+00 \\
1.222 E+00\end{array}$ & $\begin{array}{l}2.947 \mathrm{E}-02 \\
3.125 \mathrm{E}-02 \\
3.311 \mathrm{E}-02 \\
3.504 \mathrm{E}-02 \\
3.703 \mathrm{E}-02 \\
4.116 \mathrm{E}-02 \\
4.547 \mathrm{E}-02 \\
4.996 \mathrm{E}-02\end{array}$ & $\begin{array}{l}1.394 E+00 \\
1.359 E+00 \\
1.333 E+00 \\
1.314 E+00 \\
1.300 E+00 \\
1.283 E+00 \\
1.274 E+00 \\
1.272 E+00\end{array}$ & 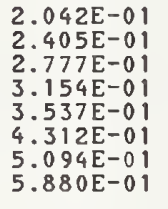 & $\begin{array}{l}1.2 \\
1.3 \\
1.4 \\
1.5 \\
1.6 \\
1.8 \\
2.0 \\
2.2\end{array}$ & $\begin{array}{l}0.0 \\
0.0 \\
0.0 \\
0.0 \\
0.0 \\
0.0 \\
0.0 \\
0.0\end{array}$ & $\begin{array}{l}-0.141 \\
-0.139 \\
-0.136 \\
-0.134 \\
-0.133 \\
-0.129 \\
-0.127 \\
-0.124\end{array}$ & $\begin{array}{l}0.155 \\
0.153 \\
0.150 \\
0.148 \\
0.146 \\
0.143 \\
0.140 \\
0.137\end{array}$ & $\begin{array}{l}0.153 \\
0.150 \\
0.147 \\
0.145 \\
0.143 \\
0.139 \\
0.136 \\
0.133\end{array}$ \\
\hline $\begin{array}{l}1.0000 \\
1.2500 \\
1.5000 \\
1.7500 \\
2.0000 \\
2.5000 \\
3.0000 \\
3.5000\end{array}$ & $\begin{array}{l}1.219 \mathrm{E}+00 \\
1.221 \mathrm{E}+00 \\
1.230 \mathrm{E}+00 \\
1.242 \mathrm{E}+00 \\
1.255 \mathrm{E}+00 \\
1.282 \mathrm{E}+00 \\
1.307 \mathrm{E}+00 \\
1.330 \mathrm{E}+00\end{array}$ & $\begin{array}{l}5.459 \mathrm{E}-02 \\
6.673 \mathrm{E}-02 \\
7.957 \mathrm{E}-02 \\
9.297 \mathrm{E}-02 \\
1.068 \mathrm{E}-01 \\
1.356 \mathrm{E}-01 \\
1.656 \mathrm{E}-01 \\
1.965 \mathrm{E}-01\end{array}$ & $\begin{array}{l}1.273 \mathrm{E}+00 \\
1.288 \mathrm{E}+00 \\
1.310 \mathrm{E}+00 \\
1.335 \mathrm{E}+00 \\
1.362 \mathrm{E}+00 \\
1.417 \mathrm{E}+00 \\
1.473 \mathrm{E}+00 \\
1.527 \mathrm{E}+00\end{array}$ & $\begin{array}{l}6.666 \mathrm{E}-01 \\
8.620 \mathrm{E}-01 \\
1.055 \mathrm{E}+00 \\
1.244 \mathrm{E}+00 \\
1.429 \mathrm{E}+00 \\
1.789 \mathrm{E}+00 \\
2.135 \mathrm{E}+00 \\
2.468 \mathrm{E}+00\end{array}$ & $\begin{array}{l}2.421 \mathrm{E}-02 \\
2.884 \mathrm{E}-02 \\
3.342 \mathrm{E}-02 \\
3.796 \mathrm{E}-02 \\
4.247 \mathrm{E}-02 \\
5.139 \mathrm{E}-02 \\
6.018 \mathrm{E}-02 \\
6.882 \mathrm{E}-02\end{array}$ & $\begin{array}{l}0.0 \\
0.0 \\
0.0 \\
0.0 \\
0.0 \\
0.0 \\
0.0 \\
0.0\end{array}$ & $\begin{array}{l}-0.122 \\
-0.118 \\
-0.1115 \\
-0.112 \\
-0.110 \\
-0.106 \\
-0.103 \\
-0.101\end{array}$ & $\begin{array}{l}0.135 \\
0.130 \\
0.127 \\
0.123 \\
0.121 \\
0.116 \\
0.113 \\
0.110\end{array}$ & $\begin{array}{l}0.131 \\
0.125 \\
0.121 \\
0.1117 \\
0.1114 \\
0.109 \\
0.104 \\
0.101\end{array}$ \\
\hline $\begin{array}{l}4.0000 \\
4.5000 \\
5.0000 \\
5.5000 \\
6.0000 \\
7.0000 \\
8.0000 \\
9.0000\end{array}$ & $\begin{array}{l}1.352 E+00 \\
1.371 E+00 \\
1.389 E+00 \\
1.406 E+00 \\
1.422 E+00 \\
1.450 E+00 \\
1.474 E+00 \\
1.497 E+00\end{array}$ & $\begin{array}{l}2.282 \mathrm{E}-01 \\
2.605 \mathrm{E}-01 \\
2.933 \mathrm{E}-01 \\
3.267 \mathrm{E}-01 \\
3.605 \mathrm{E}-01 \\
4.293 \mathrm{E}-01 \\
4.995 \mathrm{E}-01 \\
5.708 \mathrm{E}-01\end{array}$ & $\begin{array}{l}1.580 E+00 \\
1.632 E+00 \\
1.683 E+00 \\
1.733 E+00 \\
1.782 E+00 \\
1.879 E+00 \\
1.974 E+00 \\
2.067 E+00\end{array}$ & $\begin{array}{l}2.790 \mathrm{E}+00 \\
3.102 \mathrm{E}+00 \\
3.403 \mathrm{E}+00 \\
3.696 \mathrm{E}+00 \\
3.981 \mathrm{E}+00 \\
4.527 \mathrm{E}+00 \\
5.046 \mathrm{E}+00 \\
5.541 \mathrm{E}+00\end{array}$ & $\begin{array}{l}7.729 \mathrm{E}-02 \\
8.560 \mathrm{E}-02 \\
9.374 \mathrm{E}-02 \\
1.017 \mathrm{E}-01 \\
1.095 \mathrm{E}-01 \\
1.247 \mathrm{E}-01 \\
1.392 \mathrm{E}-01 \\
1.531 \mathrm{E}-01\end{array}$ & $\begin{array}{l}0.0 \\
0.0 \\
0.0 \\
0.0 \\
0.0 \\
0.0 \\
0.0 \\
0.0\end{array}$ & $\begin{array}{l}-0.099 \\
-0.097 \\
-0.096 \\
-0.095 \\
-0.093 \\
-0.092 \\
-0.090 \\
-0.089\end{array}$ & $\begin{array}{l}0.107 \\
0.104 \\
0.102 \\
0.100 \\
0.099 \\
0.095 \\
0.093 \\
0.090\end{array}$ & $\begin{array}{l}0.098 \\
0.095 \\
0.092 \\
0.090 \\
0.088 \\
0.084 \\
0.081 \\
0.078\end{array}$ \\
\hline $\begin{array}{l}10.0000 \\
12.5000 \\
15.0000 \\
17.5000 \\
20.00000 \\
25.00000 \\
30.0000 \\
35.0000\end{array}$ & $\begin{array}{l}1.516 E+00 \\
1.559 E+00 \\
1.594 E+00 \\
1.624 E+00 \\
1.650 E+00 \\
1.694 E+00 \\
1.729 E+00 \\
1.758 E+00\end{array}$ & $\begin{array}{l}6.430 E-01 \\
8.269 E-01 \\
1.015 E+00 \\
1.206 E+00 \\
1.399 E+00 \\
1.792 E+00 \\
2.191 E+00 \\
2.595 E+00\end{array}$ & $\begin{array}{l}2.159 E+00 \\
2.386 E+00 \\
2.609 E+00 \\
2.830 E+00 \\
3.050 E+00 \\
3.436 E+00 \\
3.9211 E+00 \\
4.353 E+00\end{array}$ & $\begin{array}{l}6.014 E+00 \\
7.115 E+00 \\
8.116 E+00 \\
9.036 E+00 \\
9.887 E+00 \\
1.142 E+01 \\
1.277 E+01 \\
1.398 E+01\end{array}$ & $\begin{array}{l}1.665 \mathrm{E}-01 \\
1.978 \mathrm{E}-01 \\
2.262 \mathrm{E}-01 \\
2.521 \mathrm{E}-01 \\
2.760 \mathrm{E}-01 \\
3.183 \mathrm{E}-01 \\
3.548 \mathrm{E}-01 \\
3.867 \mathrm{E}-01\end{array}$ & $\begin{array}{l}0.0 \\
0.0 \\
0.0 \\
0.0 \\
0.0 \\
0.0 \\
7.431 \mathrm{E}-03 \\
3.031 \mathrm{E}-02\end{array}$ & $\begin{array}{l}-0.08 \\
-0.08 \\
-0.08 \\
-0.08 \\
-0.08 \\
-0.07 \\
-0.07 \\
-0.06\end{array}$ & $\begin{array}{l}0.088 \\
0.084 \\
0.080 \\
0.077 \\
0.074 \\
0.069 \\
0.066 \\
0.063\end{array}$ & $\begin{array}{l}0.070 \\
0.065 \\
0.061 \\
0.058 \\
0.053 \\
0.048 \\
0.044\end{array}$ \\
\hline $\begin{array}{l}40.0000 \\
45.0000 \\
50.0000 \\
55.0000 \\
60.0000 \\
70.0000 \\
80.00000 \\
90.0000\end{array}$ & $\begin{array}{l}1.782 E+00 \\
1.803 E+00 \\
1.822 E+00 \\
1.838 E+00 \\
1.853 E+00 \\
1.879 E+00 \\
1.901 E+00 \\
1.920 E+00\end{array}$ & $\begin{array}{l}3.002 E+00 \\
3.413 E+00 \\
3.825 E+00 \\
4.240 E+00 \\
4.656 E+00 \\
5.493 E+00 \\
6.334 E+00 \\
7.179 E+00\end{array}$ & $\begin{array}{l}4.785 E+00 \\
5.216 E+00 \\
5.647 E+00 \\
6.078 E+00 \\
6.509 E+00 \\
7.372 E+00 \\
8.235 E+00 \\
9.098 E+00\end{array}$ & $\begin{array}{l}1.508 E+01 \\
1.608 E+01 \\
1.700 E+01 \\
1.785 E+01 \\
1.865 E+01 \\
2.009 E+01 \\
2.137 E+01 \\
2.253 E+01\end{array}$ & $\begin{array}{l}4.14 \\
4.40 \\
4.62 \\
4.83 \\
5.01 \\
5.36 \\
5.62 \\
5.86\end{array}$ & $\begin{array}{l}6.04 \\
9.39 \\
1.28 \\
1.64 \\
1.99 \\
2.70 \\
3.38 \\
4.06\end{array}$ & $\begin{array}{l}-0.064 \\
-0.062 \\
-0.060 \\
-0.058 \\
-0.056 \\
-0.053 \\
-0.050 \\
-0.048\end{array}$ & $\begin{array}{l}0.060 \\
0.058 \\
0.056 \\
0.054 \\
0.052 \\
0.050 \\
0.047 \\
0.046\end{array}$ & $\begin{array}{l}0 . \\
0 . \\
0 \\
0 . \\
0 . \\
0 . \\
0 .\end{array}$ \\
\hline $\begin{array}{l}.0000 \\
.0000 \\
.0000 \\
.0000 \\
.0000 \\
.0000 \\
.0000 \\
.0000\end{array}$ & $\begin{array}{l}1.936 E+00 \\
1.970 E+00 \\
1.996 E+00 \\
2.017 E+00 \\
2.034 E+00 \\
2.062 E+00 \\
2.084 E+00 \\
2.102 E+00\end{array}$ & $\begin{array}{l}1.015 \mathrm{E}+01 \\
1.229 \mathrm{E}+01 \\
1.444 \mathrm{E}+01 \\
1.659 \mathrm{E}+01 \\
2.091 \mathrm{E}+01 \\
2.524 \mathrm{E}+01 \\
2.958 \mathrm{E}+01\end{array}$ & $\begin{array}{l}9.963 E+00 \\
1.212 E+01 \\
1.429 E+01 \\
1.646 E+01 \\
1.863 E+01 \\
2.297 E+01 \\
2.732 E+01 \\
3.168 E+01\end{array}$ & $\begin{array}{l}2.358 E+01 \\
2.585 E+01 \\
2.774 E+01 \\
2.937 E+01 \\
3.080 E+01 \\
3.321 E+01 \\
3.521 E+01 \\
3.690 E+01\end{array}$ & $\begin{array}{l}6.5 \\
6.8 \\
7.1 \\
7.3 \\
7.6 \\
7.9 \\
8.1\end{array}$ & $\begin{array}{l}4.721 E-01 \\
6.316 E-01 \\
7.830 E-01 \\
9.258 E-01 \\
1.060 E+00 \\
1.304 E+00 \\
1.521 E+00 \\
1.714 E+00\end{array}$ & $\begin{array}{l}-0 \\
-0 \\
-0 \\
-0 \\
-0 \\
-0 \\
-0 \\
-0\end{array}$ & $\begin{array}{l}44 \\
41 \\
38 \\
36 \\
35 \\
33 \\
31 \\
30\end{array}$ & $\begin{array}{l}0 . \\
0.0 \\
0.0 \\
0.0 \\
0.0 \\
0 .\end{array}$ \\
\hline $\begin{array}{l}.0000 \\
.0000 \\
.0000 \\
.0000 \\
.0000 \\
.0000 \\
.0000 \\
.0000\end{array}$ & $\begin{array}{l}2.117 E+00 \\
2.130 E+00 \\
2.141 E+00 \\
2.151 E+00 \\
2.160 E+00 \\
2.176 E+00 \\
2.190 E+00 \\
2.201 E+00\end{array}$ & $\begin{array}{l}3.392 E+01 \\
3.827 E+01 \\
4.262 E+01 \\
4.698 E+01 \\
5.134 E+01 \\
6.007 E+01 \\
6.881 E+01 \\
7.755 E+01\end{array}$ & $\begin{array}{l}3.604 E+01 \\
4.040 E+01 \\
4.476 E+01 \\
4.913 E+01 \\
5.350 E+01 \\
6.225 E+01 \\
7.100 E+01 \\
7.975 E+01\end{array}$ & $\begin{array}{l}3.838 E+01 \\
3.969 E+01 \\
4.087 E+01 \\
4.193 E+01 \\
4.291 E+01 \\
4.464 E+01 \\
4.614 E+01 \\
4.747 E+01\end{array}$ & $\begin{array}{l}8.266 E-01 \\
8.397 \mathrm{E}-01 \\
8.507 \mathrm{E}-01 \\
8.601 \mathrm{E}-01 \\
8.683 \mathrm{E}-01 \\
8.817 \mathrm{E}-01 \\
8.924 \mathrm{E}-01 \\
9.011 \mathrm{E}-01\end{array}$ & $\begin{array}{l}1.887 E+00 \\
2.045 E+00 \\
2.189 E+00 \\
2.322 E+00 \\
2.445 E+00 \\
2.667 E+00 \\
2.864 E+00 \\
3.042 E+00\end{array}$ & $\begin{array}{l}-0.021 \\
-0.020 \\
-0.019 \\
-0.018 \\
-0.018 \\
-0.016 \\
-0.015 \\
-0.015\end{array}$ & $\begin{array}{l}0.029 \\
0.028 \\
0.027 \\
0.026 \\
0.026 \\
0.025 \\
0.024 \\
0.023\end{array}$ & $\begin{array}{l}0.006 \\
0.005 \\
0.005 \\
0.005 \\
0.004 \\
0.003 \\
0.003\end{array}$ \\
\hline & $11 \mathrm{E}+00$ & $630 E+01$ & $851 E+01$ & $66 E+01$ & $9.084 E-01$ & $3.203 E+00$ & -0.014 & 0.023 & .003 \\
\hline
\end{tabular}


ELECTRONS IN MOLYBDENUM

$I=424.0 \mathrm{eV} \quad$ DENSITY $=1.022 \mathrm{E}+01 \mathrm{~g} / \mathrm{cm}^{3}$

\begin{tabular}{|c|c|c|c|c|c|c|c|c|c|}
\hline$Y$ & LISION & PO & TOTAL & $\begin{array}{l}\text { CSDA } \\
\text { RANGE }\end{array}$ & DIATION & $\begin{array}{l}\text { DENS.EFF. } \\
\text { CORR. } \\
\text { (DELTA) }\end{array}$ & $\begin{array}{r}d c \\
\operatorname{coLL} \\
05 s\end{array}$ & SD & $\begin{array}{l}\text { I ) } \\
\text { RAD } \\
\text { YIELD }\end{array}$ \\
\hline $\mathrm{MeV}$ & $\mathrm{MeV} \mathrm{cm}^{2} / \mathrm{g}$ & $\mathrm{MeV} \mathrm{cm}^{2} / \mathrm{g}$ & $\mathrm{MeV} \mathrm{cm}^{2} / \mathrm{g}$ & $\mathrm{g} / \mathrm{cm}^{2}$ & & & & & \\
\hline $\begin{array}{l}0.0100 \\
0.0125 \\
0.01150 \\
0.0175 \\
0.0200 \\
0.0250 \\
0.0300 \\
0.0350\end{array}$ & $\begin{array}{l}1.167 \\
1.003 \\
8.843 \\
7.945 \\
7.238 \\
6.192 \\
5.452 \\
4.898\end{array}$ & $\begin{array}{l}02 \\
02 \\
02 \\
02 \\
02 \\
02 \\
02 \\
02 \\
02\end{array}$ & $\begin{array}{l}68 E+01 \\
04 E+01 \\
60 E+00 \\
63 E+00 \\
56 E+00 \\
11 E+00 \\
72 E+00 \\
18 E+00\end{array}$ & $\begin{array}{l}-04 \\
-04 \\
-03 \\
-03 \\
-03 \\
-03 \\
-03 \\
-03\end{array}$ & $\begin{array}{l}E-04 \\
E-04 \\
E-04 \\
E-03 \\
E-03 \\
E-03 \\
E-03 \\
E-03\end{array}$ & $\begin{array}{l}7.953 \mathrm{E}-04 \\
1.029 \mathrm{E}-03 \\
1.275 \mathrm{E}-03 \\
1.535 \mathrm{E}-03 \\
1.807 \mathrm{E}-03 \\
2.389 \mathrm{E}-03 \\
3.017 \mathrm{E}-03 \\
3.692 \mathrm{E}-03\end{array}$ & $\begin{array}{l}-0.303 \\
-0.284 \\
-0.270 \\
-0.260 \\
-0.251 \\
-0.238 \\
-0.228 \\
-0.221\end{array}$ & $\begin{array}{l}0.402 \\
0.369 \\
0.345 \\
0.327 \\
0.312 \\
0.291 \\
0.275 \\
0.263\end{array}$ & $\begin{array}{l}0.373 \\
0.344 \\
0.323 \\
0.308 \\
0.295 \\
0.277 \\
0.264 \\
0.253\end{array}$ \\
\hline $\begin{array}{l}0.0400 \\
0.0450 \\
0.0500 \\
0.0550 \\
0.0600 \\
0.0700 \\
0.0800 \\
0.0900\end{array}$ & $\begin{array}{l}4.467 E+00 \\
4.122 E+00 \\
3.838 E+00 \\
3.601 E+00 \\
3.400 E+00 \\
3.076 E+00 \\
2.826 E+00 \\
2.628 E+00\end{array}$ & $\begin{array}{l}2.099 \mathrm{E}-02 \\
2.142 \mathrm{E}-02 \\
2.181 \mathrm{E}-02 \\
2.216 \mathrm{E}-02 \\
2.247 \mathrm{E}-02 \\
2.305 \mathrm{E}-02 \\
2.357 \mathrm{E}-02 \\
2.404 \mathrm{E}-02\end{array}$ & $\begin{array}{l}4.488 E+00 \\
4.143 \mathrm{E}+00 \\
3.860 \mathrm{E}+00 \\
3.623 \mathrm{E}+00 \\
3.422 \mathrm{E}+00 \\
3.099 \mathrm{E}+00 \\
2.850 \mathrm{E}+00 \\
2.652 \mathrm{E}+00\end{array}$ & $\begin{array}{l}296 \mathrm{E}-03 \\
457 \mathrm{E}-03 \\
708 \mathrm{E}-03 \\
046 \mathrm{E}-03 \\
047 \mathrm{E}-02 \\
354 \mathrm{E}-02 \\
691 \mathrm{E}-02 \\
055 \mathrm{E}-02\end{array}$ & $\begin{array}{l}.458 \mathrm{E}-03 \\
.732 \mathrm{E}-03 \\
.000 \mathrm{E}-03 \\
.262 \mathrm{E}-03 \\
.519 \mathrm{E}-03 \\
.017 \mathrm{E}-03 \\
.497 \mathrm{E}-03 \\
.961 \mathrm{E}-03\end{array}$ & $\begin{array}{l}4.410 \mathrm{E}-03 \\
5.169 \mathrm{E}-03 \\
5.969 \mathrm{E}-03 \\
6.807 \mathrm{E}-03 \\
7.681 \mathrm{E}-03 \\
9.535 \mathrm{E}-03 \\
1.152 \mathrm{E}-02 \\
1.362 \mathrm{E}-02\end{array}$ & $\begin{array}{l}-0.214 \\
-0.203 \\
-0.205 \\
-0.201 \\
-0.198 \\
-0.192 \\
-0.187 \\
-0.183\end{array}$ & $\begin{array}{l}0.254 \\
0.246 \\
0.240 \\
0.234 \\
0.229 \\
0.221 \\
0.214 \\
0.209\end{array}$ & $\begin{array}{l}0.245 \\
0.238 \\
0.232 \\
0.227 \\
0.223 \\
0.216 \\
0.2110 \\
0.204\end{array}$ \\
\hline $\begin{array}{l}0.1000 \\
0.1250 \\
0.1500 \\
0.1750 \\
0.2000 \\
0.2500 \\
0.3000 \\
0.3500\end{array}$ & $\begin{array}{l}2.467 E+00 \\
2.171 E+00 \\
1.970 E+00 \\
1.825 E+00 \\
1.715 E+00 \\
1.563 E+00 \\
1.463 E+00 \\
1.394 E+00\end{array}$ & $\begin{array}{l}2.551 \mathrm{E}-02 \\
2.646 \mathrm{E}-02 \\
2.737 \mathrm{E}-02 \\
2.826 \mathrm{E}-02 \\
3.005 \mathrm{E}-02 \\
3.192 \mathrm{E}-02 \\
3.388 \mathrm{E}-02\end{array}$ & $\begin{array}{l}2.492 E+00 \\
2.197 E+00 \\
1.996 E+00 \\
1.852 E+00 \\
1.743 E+00 \\
1.593 E+00 \\
1.495 E+00 \\
1.427 E+00\end{array}$ & $\begin{array}{l}.445 \mathrm{E}-02 \\
.517 \mathrm{E}-02 \\
.714 \mathrm{E}-02 \\
.016 \mathrm{E}-02 \\
.409 \mathrm{E}-02 \\
.042 \mathrm{E}-01\end{array}$ & $\begin{array}{l}5.409 \mathrm{E}-03 \\
6.474 \mathrm{E}-03 \\
7.469 \mathrm{E}-03 \\
8.406 \mathrm{E}-03 \\
9.292 \mathrm{E}-03 \\
1.095 \mathrm{E}-02 \\
1.248 \mathrm{E}-02 \\
1.391 \mathrm{E}-02\end{array}$ & $\begin{array}{l}1.584 \mathrm{E}-02 \\
2.180 \mathrm{E}-02 \\
2.830 \mathrm{E}-02 \\
3.523 \mathrm{E}-02 \\
4.252 \mathrm{E}-02 \\
5.795 \mathrm{E}-02 \\
7.422 \mathrm{E}-02 \\
9.109 \mathrm{E}-02\end{array}$ & $\begin{array}{l}-0.180 \\
-0.173 \\
-0.167 \\
-0.163 \\
-0.159 \\
-0.153 \\
-0.147 \\
-0.143\end{array}$ & $\begin{array}{l}0.204 \\
0.195 \\
0.188 \\
0.183 \\
0.178 \\
0.171 \\
0.165 \\
0.160\end{array}$ & $\begin{array}{l}0.200 \\
0.192 \\
0.185 \\
0.180 \\
0.175 \\
0.168 \\
0.162 \\
0.157\end{array}$ \\
\hline $\begin{array}{l}0.4000 \\
0.4500 \\
0.5000 \\
0.5500 \\
0.6000 \\
0.7000 \\
0.8000 \\
0.9000\end{array}$ & $\begin{array}{l}1.279 \\
1.257 \\
1.240 \\
1.217 \\
1.203 \\
1.194\end{array}$ & $\begin{array}{l}3.5 \\
3.8 \\
4.0 \\
4.2 \\
4.5 \\
4.9 \\
5.5 \\
6.0\end{array}$ & $\begin{array}{l}1.3 \\
1.3 \\
1.3 \\
1.3 \\
1.2 \\
1.2 \\
1.2 \\
1.2\end{array}$ & $\begin{array}{l}2.4 \\
2.8 \\
3.1 \\
3.5 \\
4.3 \\
5.1 \\
5.9\end{array}$ & $\begin{array}{l}1.6 \\
1.7 \\
1.9 \\
2.0 \\
2.2 \\
2.5 \\
2.7\end{array}$ & $\begin{array}{l}01 \\
0 \\
0 \\
01 \\
01 \\
01 \\
01 \\
01 \\
01 \\
01\end{array}$ & $\begin{array}{l}-0 \\
-0 \\
-0 \\
-0 \\
-0 \\
-0 \\
-0 \\
-0\end{array}$ & $\begin{array}{l}50 \\
53 \\
50 \\
47 \\
44 \\
40 \\
36 \\
33\end{array}$ & $\begin{array}{l}0.153 \\
0.150 \\
0.146 \\
0.143 \\
0.141 \\
0.136 \\
0.131 \\
0.127\end{array}$ \\
\hline $\begin{array}{l}1.0000 \\
1.2500 \\
1.5000 \\
1.7500 \\
2.0000 \\
2.5000 \\
3.0000 \\
3.5000\end{array}$ & $\begin{array}{l}1.18 \\
1.19 \\
1.20 \\
1.20 \\
1.22 \\
1.24 \\
1.26\end{array}$ & $\begin{array}{l}6.5 \\
8.0 \\
9.5 \\
1.1 \\
1.2 \\
1.6 \\
1.9\end{array}$ & $\begin{array}{l}1.268 E+0 \\
1.288 E+0 \\
1.311 E+0 \\
1.337 E+0 \\
1.389 E+0 \\
1.442 E+0 \\
1.495 E+0\end{array}$ & $\begin{array}{l}8.731 E-0 \\
1.069 E+0 \\
1.261 E+0 \\
1.450 E+0 \\
1.817 E+0 \\
2.170 E+0 \\
2.511 E+0\end{array}$ & $\begin{array}{l}3.538 \mathrm{E}-0 \\
4.095 \mathrm{E}-0 \\
4.646 \mathrm{E}-0 \\
5.193 \mathrm{E}-0 \\
6.272 \mathrm{E}-0 \\
7.333 \mathrm{E}-0 \\
8.373 \mathrm{E}-0\end{array}$ & $\begin{array}{l}3.198 \mathrm{E}-0 \\
4.028 \mathrm{E}-0 \\
4.816 \mathrm{E}-0 \\
5.563 \mathrm{E}-0 \\
6.275 \mathrm{E}-0 \\
7.606 \mathrm{E}-0 \\
8.838 \mathrm{E}-0 \\
9.994 \mathrm{E}-0\end{array}$ & $\begin{array}{l}-0.11 \\
-0.1 \\
-0.0 \\
-0.0 \\
-0.08 \\
-0.08 \\
-0.0 \\
-0.0\end{array}$ & $\begin{array}{l}0.1 \\
0.1 \\
0.1 \\
0.1 \\
0.1 \\
0.1 \\
0.0 \\
0.0\end{array}$ & $\begin{array}{l}0.124 \\
0.116 \\
0.110 \\
0.104 \\
0.099 \\
0.091 \\
0.085\end{array}$ \\
\hline $\begin{array}{l}4.0000 \\
4.5000 \\
5.0000 \\
5.5000 \\
6.0000 \\
7.0000 \\
8.0000 \\
9.0000\end{array}$ & $\begin{array}{l}1.277 E+00 \\
1.290 E+00 \\
1.302 E+00 \\
1.313 E+00 \\
1.322 E+00 \\
1.340 E+00 \\
1.355 E+00 \\
1.367 E+00\end{array}$ & $\begin{array}{l}2.699 \mathrm{E}-01 \\
3.077 \mathrm{E}-01 \\
3.461 \mathrm{E}-01 \\
3.851 \mathrm{E}-01 \\
4.246 \mathrm{E}-01 \\
5.049 \mathrm{E}-01 \\
5.867 \mathrm{E}-01 \\
6.697 \mathrm{E}-01\end{array}$ & $\begin{array}{l}1.547 E+0 \\
1.598 E+0 \\
1.648 E+0 \\
1.698 E+0 \\
1.747 E+0 \\
1.845 E+0 \\
1.941 E+0 \\
2.037 E+0\end{array}$ & $\begin{array}{l}2.8 \\
3.1 \\
3.5 \\
3.7 \\
4 .\end{array}$ & $\begin{array}{l}02 \\
021 \\
01 \\
01 \\
01 \\
01 \\
01 \\
01\end{array}$ & $\begin{array}{l}0 \\
0 \\
0 \\
0 \\
0\end{array}$ & $\begin{array}{l}-0.06 \\
-0.06 \\
-0.05 \\
-0.05 \\
-0.05 \\
-0.04 \\
-0.04 \\
-0.03\end{array}$ & & $\begin{array}{l}70 \\
66 \\
62 \\
59\end{array}$ \\
\hline $\begin{array}{l}10.0000 \\
12.5000 \\
15.0000 \\
17.5000 \\
20.0000 \\
25.0000 \\
30.0000 \\
35.0000\end{array}$ & $\begin{array}{l}1.379 E+00 \\
1.402 E+00 \\
1.421 E+00 \\
1.437 E+00 \\
1.450 E+00 \\
1.471 E+00 \\
1.488 E+00 \\
1.502 E+00\end{array}$ & $\begin{array}{l}7.538 \mathrm{E}-01 \\
9.680 \mathrm{E}-01 \\
1.187 \mathrm{E}+00 \\
1.409 \mathrm{E}+00 \\
1.634 \mathrm{E}+00 \\
2.091 \mathrm{E}+00 \\
2.555 \mathrm{E}+00 \\
3.024 \mathrm{E}+00\end{array}$ & $\begin{array}{l}2.133 \mathrm{E} \\
2.370 \mathrm{E} \\
2.608 \mathrm{E} \\
2.845 \mathrm{E} \\
3.084 \mathrm{E} \\
3.562 \mathrm{E} \\
4.043 \mathrm{E} \\
4.526 \mathrm{E}\end{array}$ & & $\begin{array}{l}1.999 \mathrm{E}-01 \\
2.362 \mathrm{E}-01 \\
2.689 \mathrm{E}-01 \\
2.984 \mathrm{E}-01 \\
3.252 \mathrm{E}-01 \\
3.721 \mathrm{E}-01 \\
4.118 \mathrm{E}-01 \\
4.459 \mathrm{E}-01\end{array}$ & $\begin{array}{l}0 \\
0 \\
0 \\
0 \\
0\end{array}$ & $\begin{array}{l}-0.037 \\
-0.032 \\
-0.029 \\
-0.027 \\
-0.025 \\
-0.022 \\
-0.019 \\
-0.017\end{array}$ & & $\begin{array}{l}0.027 \\
0.024 \\
0.020 \\
0.017 \\
0.015\end{array}$ \\
\hline $\begin{array}{l}50.0 \\
55.0 \\
60.0\end{array}$ & $\begin{array}{l}1.52 \\
1.5 \\
1.5 \\
1.5 \\
1.5 \\
1.5\end{array}$ & $\begin{array}{l}3.9 \\
4.4 \\
4.9 \\
5.4 \\
6.3\end{array}$ & $\begin{array}{l}5.011 \mathrm{E}+c \\
5.498 \mathrm{E}+\mathrm{C} \\
5.987 \mathrm{E}+\mathrm{C} \\
6.477 \mathrm{E}+\mathrm{C} \\
6.968 \mathrm{E}+\mathrm{C} \\
7.953 \mathrm{E}+\mathrm{C} \\
8.942 \mathrm{E}+ \\
9.933 \mathrm{E}+\mathrm{C}\end{array}$ & & & & & & \\
\hline .0 & $\begin{array}{l}1.61 \\
1.63 \\
1.64 \\
1.65 \\
1.67 \\
1.68\end{array}$ & $\begin{array}{l}1.430 E+0 \\
1.680 E+0 \\
1.931 E+0 \\
2.433 E+0 \\
2.936 E+0 \\
3.441 E+0\end{array}$ & $\begin{array}{l}1.592 \mathrm{E}+01 \\
1.843 \mathrm{E}+01 \\
2.095 \mathrm{E}+01 \\
2.598 \mathrm{E}+01 \\
3.103 \mathrm{E}+01 \\
3.609 \mathrm{E}+01\end{array}$ & $\begin{array}{l}1 \\
1 \\
1 \\
1 \\
1 \\
1 \\
1 \\
1\end{array}$ & $\begin{array}{l}1 \\
1 \\
1 \\
1 \\
1 \\
1\end{array}$ & $\begin{array}{ll} & 0 \\
0 & 0 \\
0 & 0 \\
0 & 0 \\
0 & 0 \\
0 & 0 \\
0 & 0\end{array}$ & & & \\
\hline $\begin{array}{l}.000 \\
.000 \\
.000 \\
.000 \\
000\end{array}$ & $\begin{array}{l}1 \\
1 \\
1 \\
1 \\
1 \\
1\end{array}$ & $\begin{array}{l}4.4 \\
4.9 \\
5.4 \\
5.9 \\
6.9 \\
8.0\end{array}$ & $\begin{array}{l}6 \\
7 .\end{array}$ & $\begin{array}{l}1 \\
1 \\
1 \\
1 \\
1\end{array}$ & $\begin{array}{l}1 \\
1 \\
1 \\
1 \\
1 \\
1 \\
1 \\
1\end{array}$ & $\begin{array}{l}0 \\
0 \\
0 \\
0 \\
0 \\
0\end{array}$ & $\begin{array}{l}-0 . \\
-0 . \\
-0 . \\
-0 . \\
-0 . \\
-0 .\end{array}$ & $\begin{array}{l}3 \\
3 \\
3 \\
2 \\
2 \\
2 \\
1 \\
1\end{array}$ & $\begin{array}{l}0.001 \\
0.001 \\
0.001 \\
0.001 \\
0.001 \\
0.001 \\
0.001\end{array}$ \\
\hline 0.0000 & $.753 E+00$ & $1.004 E+02$ & $.021 E+02$ & $4.509 E+01$ & $9.297 E-01$ & $.028 E+01$ & -0.000 & 0.011 & 0.001 \\
\hline
\end{tabular}


ELECTRONS IN SILVER

$I=470.0 \mathrm{eV} \quad$ DENSITY $=1.050 \mathrm{E}+01 \mathrm{~g} / \mathrm{cm}^{3}$

\begin{tabular}{|c|c|c|c|c|c|c|c|c|c|}
\hline \multirow{3}{*}{$\begin{array}{l}\text { ENERGY } \\
\text { MeV }\end{array}$} & \multirow{2}{*}{\multicolumn{3}{|c|}{$\begin{array}{l}\text { STOPPING POWER } \\
\text { COLLISION RADIATIVE }\end{array}$}} & \multirow{2}{*}{$\begin{array}{l}\text { CSDA } \\
\text { RANGE }\end{array}$} & \multirow{3}{*}{$\begin{array}{l}\text { RADIATION } \\
\text { YIELD }\end{array}$} & \multirow{3}{*}{$\begin{array}{l}\text { DENS.EFF. } \\
\text { CORR. } \\
\text { (DELTA) }\end{array}$} & \multicolumn{3}{|c|}{$d(\log ) / d(\log I$} \\
\hline & & & & & & & Coli & CSDA & $\begin{array}{l}\text { RAD } \\
\text { YIELD }\end{array}$ \\
\hline & $\mathrm{MeV} \mathrm{cm}^{2} / \mathrm{g}$ & $\mathrm{MeV} \mathrm{cm} 2 / \mathrm{g}$ & $\mathrm{MeV} \mathrm{cm} 2 / \mathrm{g}$ & $\mathrm{g} / \mathrm{cm}^{2}$ & & & & & \\
\hline $\begin{array}{l}0.0100 \\
0.0125 \\
0.0150 \\
0.0175 \\
0.0200 \\
0.0250 \\
0.0300 \\
0.0350\end{array}$ & $\begin{array}{l}1.125 \mathrm{E}+01 \\
9.687 \mathrm{E}+00 \\
8.556 \mathrm{E}+00 \\
7.696 \mathrm{E}+00 \\
7.017 \mathrm{E}+00 \\
6.011 \mathrm{E}+00 \\
5.297 \mathrm{E}+00 \\
4.763 \mathrm{E}+00\end{array}$ & $\begin{array}{l}1.634 \mathrm{E}-02 \\
1.754 \mathrm{E}-02 \\
1.849 \mathrm{E}-02 \\
1.927 \mathrm{E}-02 \\
1.992 \mathrm{E}-02 \\
2.099 \mathrm{E}-02 \\
2.184 \mathrm{E}-02 \\
2.254 \mathrm{E}-02\end{array}$ & $\begin{array}{l}1.127 E+01 \\
9.704 E+00 \\
8.574 E+00 \\
7.715 E+00 \\
7.037 E+00 \\
6.032 E+00 \\
5.319 E+00 \\
4.786 E+00\end{array}$ & $\begin{array}{l}5.577 \mathrm{E}-04 \\
7.976 \mathrm{E}-04 \\
1.072 \mathrm{E}-03 \\
1.380 \mathrm{E}-03 \\
1.720 \mathrm{E}-03 \\
2.490 \mathrm{E}-03 \\
3.375 \mathrm{E}-03 \\
4.368 \mathrm{E}-03\end{array}$ & $\begin{array}{l}7.226 \mathrm{E}-04 \\
9.040 \mathrm{E}-04 \\
1.084 \mathrm{E}-03 \\
1.261 \mathrm{E}-03 \\
1.437 \mathrm{E}-03 \\
1.781 \mathrm{E}-03 \\
2.117 \mathrm{E}-03 \\
2.444 \mathrm{E}-03\end{array}$ & $\begin{array}{l}1.126 \mathrm{E}-03 \\
1.445 \mathrm{E}-03 \\
1.781 \mathrm{E}-03 \\
2.135 \mathrm{E}-03 \\
2.508 \mathrm{E}-03 \\
3.312 \mathrm{E}-03 \\
4.203 \mathrm{E}-03 \\
5.190 \mathrm{E}-03\end{array}$ & $\begin{array}{l}-0.313 \\
-0.292 \\
-0.278 \\
-0.267 \\
-0.258 \\
-0.244 \\
-0.233 \\
-0.225\end{array}$ & $\begin{array}{l}0.424 \\
0.387 \\
0.361 \\
0.341 \\
0.325 \\
0.302 \\
0.285 \\
0.272\end{array}$ & $\begin{array}{l}0.389 \\
0.357 \\
0.335 \\
0.318 \\
0.305 \\
0.285 \\
0.271 \\
0.260\end{array}$ \\
\hline $\begin{array}{l}0.0400 \\
0.0450 \\
0.0500 \\
0.0550 \\
0.0600 \\
0.0700 \\
0.0800 \\
0.0900\end{array}$ & $\begin{array}{l}4.347 E+00 \\
4.012 E+00 \\
3.738 E+00 \\
3.508 E+00 \\
3.313 E+00 \\
2.999 E+00 \\
2.756 E+00 \\
2.563 E+00\end{array}$ & $\begin{array}{l}2.314 \mathrm{E}-02 \\
2.367 \mathrm{E}-02 \\
2.414 \mathrm{E}-02 \\
2.458 \mathrm{E}-02 \\
2.497 \mathrm{E}-02 \\
2.569 \mathrm{E}-02 \\
2.634 \mathrm{E}-02 \\
2.693 \mathrm{E}-02\end{array}$ & $\begin{array}{l}4.370 E+00 \\
4.036 E+00 \\
3.762 E+00 \\
3.533 E+00 \\
3.338 E+00 \\
3.024 E+00 \\
2.782 E+00 \\
2.590 E+00\end{array}$ & $\begin{array}{l}5.46 \\
6.65 \\
7.93 \\
9.31 \\
1.07 \\
1.35 \\
1.73 \\
2.11\end{array}$ & $\begin{array}{l}2.764 \mathrm{E}-03 \\
3.077 \mathrm{E}-03 \\
3.383 \mathrm{E}-03 \\
3.684 \mathrm{E}-03 \\
3.979 \mathrm{E}-03 \\
4.552 \mathrm{E}-03 \\
5.106 \mathrm{E}-03 \\
5.643 \mathrm{E}-03\end{array}$ & $\begin{array}{l}6.284 \mathrm{E}-03 \\
7.495 \mathrm{E}-03 \\
8.836 \mathrm{E}-03 \\
1.032 \mathrm{E}-02 \\
1.196 \mathrm{E}-02 \\
1.573 \mathrm{E}-02 \\
2.022 \mathrm{E}-02 \\
2.543 \mathrm{E}-02\end{array}$ & $\begin{array}{l}-0.219 \\
-0.213 \\
-0.208 \\
-0.204 \\
-0.200 \\
-0.193 \\
-0.186 \\
-0.181\end{array}$ & $\begin{array}{l}0.262 \\
0.253 \\
0.246 \\
0.240 \\
0.235 \\
0.225 \\
0.218 \\
0.211\end{array}$ & $\begin{array}{l}0.251 \\
0.244 \\
0.237 \\
0.232 \\
0.227 \\
0.219 \\
0.212 \\
0.206\end{array}$ \\
\hline $\begin{array}{l}0.1000 \\
0.1250 \\
0.1500 \\
0.1750 \\
0.2000 \\
0.2500 \\
0.3000 \\
0.3500\end{array}$ & $\begin{array}{l}2.406 E+00 \\
2.117 \mathrm{E}+00 \\
1.920 \mathrm{E}+00 \\
1.778 \mathrm{E}+00 \\
1.671 \mathrm{E}+00 \\
1.522 \mathrm{E}+00 \\
1.424 \mathrm{E}+00 \\
1.356 \mathrm{E}+00\end{array}$ & $\begin{array}{l}2.748 \mathrm{E}-02 \\
2.875 \mathrm{E}-02 \\
2.990 \mathrm{E}-02 \\
3.098 \mathrm{E}-02 \\
3.203 \mathrm{E}-02 \\
3.413 \mathrm{E}-02 \\
3.628 \mathrm{E}-02 \\
3.852 \mathrm{E}-02\end{array}$ & $\begin{array}{l}2.434 E+00 \\
2.146 E+00 \\
1.950 E+00 \\
1.809 E+00 \\
1.703 E+00 \\
1.556 E+00 \\
1.460 E+00 \\
1.395 E+00\end{array}$ & $\begin{array}{l}2.509 \mathrm{E}-02 \\
3.607 \mathrm{E}-02 \\
4.832 \mathrm{E}-02 \\
6.165 \mathrm{E}-02 \\
7.591 \mathrm{E}-02 \\
1.067 \mathrm{E}-01 \\
1.400 \mathrm{E}-01 \\
1.750 \mathrm{E}-01\end{array}$ & $\begin{array}{l}6.163 \mathrm{E}-03 \\
7.402 \mathrm{E}-03 \\
8.564 \mathrm{E}-03 \\
9.661 \mathrm{E}-03 \\
1.070 \mathrm{E}-02 \\
1.264 \mathrm{E}-02 \\
1.443 \mathrm{E}-02 \\
1.612 \mathrm{E}-02\end{array}$ & $\begin{array}{l}3.125 \mathrm{E}-02 \\
4.754 \mathrm{E}-02 \\
6.453 \mathrm{E}-02 \\
8.111 \mathrm{E}-02 \\
9.702 \mathrm{E}-02 \\
1.270 \mathrm{E}-01 \\
1.550 \mathrm{E}-01 \\
1.816 \mathrm{E}-01\end{array}$ & $\begin{array}{l}-0.176 \\
-0.166 \\
-0.159 \\
-0.154 \\
-0.149 \\
-0.143 \\
-0.138 \\
-0.133\end{array}$ & $\begin{array}{l}0.206 \\
0.194 \\
0.186 \\
0.179 \\
0.173 \\
0.164 \\
0.158 \\
0.153\end{array}$ & $\begin{array}{l}0.201 \\
0.190 \\
0.181 \\
0.175 \\
0.169 \\
0.161 \\
0.154 \\
0.149\end{array}$ \\
\hline $\begin{array}{l}0.4000 \\
0.4500 \\
0.5000 \\
0.5500 \\
0.6000 \\
0.7000 \\
0.8000 \\
0.9000\end{array}$ & $\begin{array}{l}1.308 E+00 \\
1.271 E+00 \\
1.244 E+00 \\
1.223 E+00 \\
1.207 E+00 \\
1.184 E+00 \\
1.170 E+00 \\
1.162 E+00\end{array}$ & $\begin{array}{l}4.085 \mathrm{E}-02 \\
4.328 \mathrm{E}-02 \\
4.580 \mathrm{E}-02 \\
4.840 \mathrm{E}-02 \\
5.107 \mathrm{E}-02 \\
5.659 \mathrm{E}-02 \\
6.233 \mathrm{E}-02 \\
6.827 \mathrm{E}-02\end{array}$ & $\begin{array}{l}1.348 E+00 \\
1.315 E+00 \\
1.290 E+00 \\
1.271 E+00 \\
1.258 E+00 \\
1.241 E+00 \\
1.233 E+00 \\
1.230 E+00\end{array}$ & $\begin{array}{l}2.115 \mathrm{E}-01 \\
2.491 \mathrm{E}-01 \\
2.875 \mathrm{E}-01 \\
3.266 \mathrm{E}-01 \\
3.661 \mathrm{E}-01 \\
4.462 \mathrm{E}-01 \\
5.271 \mathrm{E}-01 \\
6.084 \mathrm{E}-01\end{array}$ & $\begin{array}{l}1.772 \mathrm{E}-02 \\
1.927 \mathrm{E}-02 \\
2.076 \mathrm{E}-02 \\
2.222 \mathrm{E}-02 \\
2.365 \mathrm{E}-02 \\
2.643 \mathrm{E}-02 \\
2.914 \mathrm{E}-02 \\
3.179 \mathrm{E}-02\end{array}$ & $\begin{array}{l}2.070 \mathrm{E}-01 \\
2.315 \mathrm{E}-01 \\
2.552 \mathrm{E}-01 \\
2.783 \mathrm{E}-01 \\
3.007 \mathrm{E}-01 \\
3.438 \mathrm{E}-01 \\
3.851 \mathrm{E}-01 \\
4.246 \mathrm{E}-01\end{array}$ & $\begin{array}{l}-0.130 \\
-0.126 \\
-0.123 \\
-0.121 \\
-0.119 \\
-0.114 \\
-0.111 \\
-0.108\end{array}$ & $\begin{array}{l}0.148 \\
0.145 \\
0.141 \\
0.139 \\
0.136 \\
0.132 \\
0.128 \\
0.125\end{array}$ & $\begin{array}{l}0.145 \\
0.141 \\
0.137 \\
0.135 \\
0.132 \\
0.127 \\
0.123 \\
0.119\end{array}$ \\
\hline $\begin{array}{l}1.0000 \\
1.2500 \\
1.5000 \\
1.7500 \\
2.0000 \\
2.5000 \\
3.0000 \\
3.5000\end{array}$ & $\begin{array}{l}1.158 E+00 \\
1.156 E+00 \\
1.161 E+00 \\
1.169 E+00 \\
1.178 E+00 \\
1.197 E+00 \\
1.215 E+00 \\
1.232 E+00\end{array}$ & $\begin{array}{l}7.439 \mathrm{E}-02 \\
9.038 \mathrm{E}-02 \\
1.072 \mathrm{E}-01 \\
1.247 \mathrm{E}-01 \\
1.428 \mathrm{E}-01 \\
1.802 \mathrm{E}-01 \\
2.190 \mathrm{E}-01 \\
2.589 \mathrm{E}-01\end{array}$ & $\begin{array}{l}1.232 E+00 \\
1.246 E+00 \\
1.268 E+00 \\
1.294 E+00 \\
1.321 E+00 \\
1.377 E+00 \\
1.434 E+00 \\
1.491 E+00\end{array}$ & $\begin{array}{l}6.896 E-01 \\
8.915 E-01 \\
1.090 E+00 \\
1.286 E+00 \\
1.477 E+00 \\
1.848 E+00 \\
2.203 E+00 \\
2.545 E+00\end{array}$ & $\begin{array}{l}3.441 \mathrm{E}-02 \\
4.082 \mathrm{E}-02 \\
4.711 \mathrm{E}-02 \\
5.330 \mathrm{E}-02 \\
5.942 \mathrm{E}-02 \\
7.144 \mathrm{E}-02 \\
8.317 \mathrm{E}-02 \\
9.461 \mathrm{E}-02\end{array}$ & $\begin{array}{l}4.62 \\
5.52 \\
6.34 \\
7.11 \\
7.83 \\
9.15 \\
1.03 \\
1.14\end{array}$ & $\begin{array}{l}-0.105 \\
-0.099 \\
-0.094 \\
-0.090 \\
-0.086 \\
-0.081 \\
-0.076 \\
-0.072\end{array}$ & $\begin{array}{l}0.122 \\
0.116 \\
0.111 \\
0.107 \\
0.103 \\
0.097 \\
0.092 \\
0.088\end{array}$ & $\begin{array}{l}0.116 \\
0.109 \\
0.103 \\
0.099 \\
0.094 \\
0.087 \\
0.082 \\
0.077\end{array}$ \\
\hline $\begin{array}{l}4.0000 \\
4.5000 \\
5.0000 \\
5.5000 \\
6.0000 \\
7.0000 \\
8.0000 \\
9.0000\end{array}$ & $\begin{array}{l}1.248 \\
1.26 \\
1.27 \\
1.286 \\
1.296 \\
1.31 \\
1.33\end{array}$ & $\begin{array}{l}2.9 \\
3.4 \\
3.8 \\
4.2 \\
4.6 \\
5.5 \\
6.4\end{array}$ & $\begin{array}{l}1.547 E+00 \\
1.603 E+00 \\
1.658 E+00 \\
1.712 E+00 \\
1.766 E+00 \\
1.873 E+00 \\
1.978 E+00 \\
2.083 E+00\end{array}$ & $\begin{array}{l}2.874 E+00 \\
3.192 E+00 \\
3.499 E+00 \\
3.795 E+00 \\
4.083 E+00 \\
4.633 E+00 \\
5.152 E+00 \\
5.645 E+00\end{array}$ & $\begin{array}{l}1.057 E-01 \\
1.166 E-01 \\
1.271 E-01 \\
1.374 E-01 \\
1.474 E-01 \\
1.667 E-01 \\
1.849 E-01 \\
2.023 E-01\end{array}$ & $\begin{array}{l}1.243 E+00 \\
1.337 E+00 \\
1.425 E+00 \\
1.508 E+00 \\
1.587 E+00 \\
1.735 E+00 \\
1.873 E+00 \\
2.001 E+00\end{array}$ & $\begin{array}{l}-0.069 \\
-0.066 \\
-0.063 \\
-0.060 \\
-0.058 \\
-0.054 \\
-0.050 \\
-0.046\end{array}$ & & $\begin{array}{l}0.073 \\
0.069 \\
0.066 \\
0.063 \\
0.060 \\
0.055 \\
0.051 \\
0.047\end{array}$ \\
\hline $\begin{array}{l}12.5000 \\
15.0000 \\
17.5000 \\
20.0000 \\
25.0000 \\
30.0000 \\
35.0000\end{array}$ & $\begin{array}{l}1.38 \\
1.40 \\
1.41 \\
1.43 \\
1.45 \\
1.47\end{array}$ & $\begin{array}{l}1.0 \\
1.3 \\
1.5 \\
1.7 \\
2.2 \\
2.8\end{array}$ & $\begin{array}{l}2.188 E+0 \\
2.447 E+0 \\
2.707 E+0 \\
2.966 E+0 \\
3.226 E+0 \\
3.748 E+0 \\
4.272 E+0 \\
4.800 E+0\end{array}$ & $\begin{array}{l}6.113 E+00 \\
7.193 E+00 \\
8.164 E+00 \\
9.046 E+00 \\
9.854 E+00 \\
1.129 E+01 \\
1.254 E+01 \\
1.364 E+01\end{array}$ & $\begin{array}{l}2.567 \mathrm{E}-01 \\
2.904 \mathrm{E}-01 \\
3.207 \mathrm{E}-01 \\
3.480 \mathrm{E}-01 \\
3.954 \mathrm{E}-01 \\
4.353 \mathrm{E}-01 \\
4.693 \mathrm{E}-01\end{array}$ & $\begin{array}{l}2 . \\
2 . \\
2 . \\
2.8 \\
3.2 \\
3 . \\
3 . \\
3 .\end{array}$ & $\begin{array}{l}-0 . \\
-0 . \\
-0 . \\
-0 . \\
-0 . \\
-0 . \\
-0 . \\
-0 .\end{array}$ & $\begin{array}{l}0 . \\
0 . \\
0: \\
0 . \\
0 . \\
0 .\end{array}$ & \\
\hline $\begin{array}{l}40.0000 \\
45.0000 \\
50.0000 \\
55.0000 \\
60.0000 \\
70.0000 \\
80.0000 \\
90.0000\end{array}$ & $\begin{array}{l}1.519 \mathrm{E}+00 \\
1.528 \mathrm{E}+00 \\
1.535 \mathrm{E}+00 \\
1.548 \mathrm{E}+00 \\
1.559 \mathrm{E}+00 \\
1.569 \mathrm{E}+00\end{array}$ & $\begin{array}{l}4.352 \mathrm{E} \\
4.876 \mathrm{E} \\
5.403 \mathrm{E} \\
5.932 \mathrm{E} \\
6.995 \mathrm{E} \\
8.065 \mathrm{E} \\
9.139 \mathrm{E}\end{array}$ & $\begin{array}{l}5.330 E+0 \\
5.862 E+0 \\
6.395 E+0 \\
6.930 E+0 \\
7.467 E+0 \\
8.543 E+0 \\
9.624 E+0 \\
1.071 E+0\end{array}$ & $\begin{array}{l}1.463 E+01 \\
1.553 E+01 \\
1.634 E+01 \\
1.709 E+01 \\
1.779 E+01 \\
1.904 E+01 \\
2.014 E+01 \\
2.113 E+01\end{array}$ & $\begin{array}{l}1 \\
1 \\
1 \\
1 \\
1 \\
1 \\
1\end{array}$ & $\begin{array}{l}4 . \\
4 . \\
4 . \\
4 . \\
4 . \\
5 \\
5\end{array}$ & $\begin{array}{l}-0 \\
-0 \\
-0 \\
-0 \\
-0 \\
-0 \\
-0\end{array}$ & & \\
\hline $\begin{array}{l}.0000 \\
.0000 \\
.0000 \\
.0000\end{array}$ & $\begin{array}{l}520 E+00 \\
530 E+00 \\
546 E+00 \\
559 E+00 \\
570 E+00\end{array}$ & $\begin{array}{l}1.5 \\
1.8 \\
2.1 \\
2.6 \\
3.2 \\
3.7\end{array}$ & $\begin{array}{l}1 . \\
1 . \\
2 . \\
2 . \\
2 . \\
3 . \\
3 .\end{array}$ & $\begin{array}{l}1 \\
1 \\
1 \\
1 \\
1 \\
1 \\
1 \\
1\end{array}$ & & $\begin{array}{l}5 . \\
6 . \\
6 . \\
6 . \\
6 . \\
7 . \\
7 .\end{array}$ & $\begin{array}{l}-0 \\
-0 \\
-0 \\
-0 \\
-0 \\
-0 \\
-0\end{array}$ & & $\begin{array}{l}0.006 \\
0.005 \\
0.004 \\
0.003 \\
0.003 \\
0.002 \\
0.002 \\
0.002\end{array}$ \\
\hline 0.0000 & $\begin{array}{l}1.695 \mathrm{E}+00 \\
1.702 \mathrm{E}+00 \\
1.708 \mathrm{E}+00 \\
1.718 \mathrm{E}+00 \\
1.727 \mathrm{E}+00 \\
1.735 \mathrm{E}+00\end{array}$ & $\begin{array}{l}4.316 E+01 \\
4.869 E+01 \\
5.422 E+01 \\
5.976 E+01 \\
6.531 E+01 \\
7.641 E+01 \\
8.751 E+01 \\
9.863 E+01\end{array}$ & $\begin{array}{l}4.483 E+01 \\
5.037 E+01 \\
5.592 E+01 \\
6.147 E+01 \\
6.702 E+01 \\
7.813 E+01 \\
8.924 E+01 \\
1.004 E+02\end{array}$ & $\begin{array}{l}.416 E+01 \\
.521 E+01 \\
.616 E+01 \\
.701 E+01 \\
.779 E+01 \\
.917 E+01 \\
.036 E+01 \\
.142 E+01\end{array}$ & $\begin{array}{l}8.720 \mathrm{E}-01 \\
8.823 \mathrm{E}-01 \\
8.908 \mathrm{E}-01 \\
8.981 \mathrm{E}-01 \\
9.044 \mathrm{E}-01 \\
9.147 \mathrm{E}-01 \\
9.228 \mathrm{E}-01 \\
9.293 \mathrm{E}-01\end{array}$ & $9.894 E+00$ & $\begin{array}{l}-0.002 \\
-0.002 \\
-0.002 \\
-0.001 \\
-0.001 \\
-0.001 \\
-0.001\end{array}$ & $\begin{array}{l}0 . \\
0 . \\
0 . \\
0 .\end{array}$ & $\begin{array}{l}0.001 \\
0.00 \\
0.001 \\
0.001 \\
0.001 \\
0.001 \\
0.001 \\
0.001\end{array}$ \\
\hline & $1.743 E+00$ & $1.098 E+02$ & $1.115 E+02$ & $4.237 E+01$ & $9.347 \mathrm{E}-01$ & $1.010 E+01$ & -0.001 & 0.012 & 0.001 \\
\hline
\end{tabular}




\begin{tabular}{|c|c|c|c|c|c|c|c|c|c|}
\hline NERGY & $\begin{array}{l}\text { COLLISION } \\
\mathrm{MeV} \mathrm{Cm}^{2} / \mathrm{g}\end{array}$ & $\begin{array}{l}\text { OPPING POW } \\
\text { RADIATIVE } \\
\text { MeV } \mathrm{cm}^{2} / \mathrm{g}\end{array}$ & $\begin{array}{l}\text { TOTAL } \\
\text { OV } \mathrm{cm}^{2} / \mathrm{g}\end{array}$ & $\begin{array}{l}\text { CSDA } \\
\text { RANGE } \\
\mathrm{g} / \mathrm{Cm}^{2}\end{array}$ & $\begin{array}{l}\text { DIATION } \\
\text { YIELD }\end{array}$ & $\begin{array}{l}\text { DENS.EFF. } \\
\text { CORR } \\
\text { (DELTA) }\end{array}$ & $\begin{array}{l}d(1) \\
\operatorname{coLL} \\
\text { LOSS }\end{array}$ & $\begin{array}{l}\text { SDA } \\
\text { ANGE }\end{array}$ & $\begin{array}{l}\text { II) } \\
\text { RAD } \\
\text { YIELD }\end{array}$ \\
\hline $\begin{array}{l}0.0100 \\
0.0125 \\
0.0150 \\
0.0175 \\
0.0200 \\
0.0250 \\
0.0300 \\
0.0350\end{array}$ & $\begin{array}{l}1.075 \mathrm{E}+01 \\
9.263 \mathrm{E}+00 \\
8.186 \mathrm{E}+00 \\
7.366 \mathrm{E}+00 \\
6.719 \mathrm{E}+00 \\
5.759 \mathrm{E}+00 \\
5.078 \mathrm{E}+00 \\
4.567 \mathrm{E}+00\end{array}$ & $\begin{array}{l}1.645 \mathrm{E}-02 \\
1.769 \mathrm{E}-02 \\
1.869 \mathrm{E}-02 \\
1.951 \mathrm{E}-02 \\
2.021 \mathrm{E}-02 \\
2.134 \mathrm{E}-02 \\
2.224 \mathrm{E}-02 \\
2.299 \mathrm{E}-02\end{array}$ & $\begin{array}{l}1.0 \\
9.2 \\
8.2 \\
7.3 \\
6.7 \\
5.7 \\
5.1 \\
4.5\end{array}$ & $\begin{array}{l}8.3 \\
1.1 \\
1.4 \\
1.8 \\
2.6 \\
3.5 \\
4.5\end{array}$ & $\begin{array}{l}4 \\
4 \\
3 \\
3 \\
3 \\
3 \\
3 \\
3\end{array}$ & $\begin{array}{l}-04 \\
-04 \\
-03 \\
-03 \\
-03 \\
-03 \\
-03 \\
-03 \\
-03\end{array}$ & $\begin{array}{l}-0.316 \\
-0.296 \\
-0.281 \\
-0.269 \\
-0.260 \\
-0.246 \\
-0.236 \\
-0.228\end{array}$ & $\begin{array}{l}34 \\
95 \\
67 \\
47 \\
30 \\
06 \\
89 \\
76\end{array}$ & $\begin{array}{l}0.395 \\
0.362 \\
0.339 \\
0.322 \\
0.308 \\
0.288 \\
0.274 \\
0.263\end{array}$ \\
\hline $\begin{array}{l}0.0400 \\
0.0450 \\
0.0500 \\
0.0550 \\
0.0600 \\
0.0700 \\
0.0800 \\
0.0900\end{array}$ & $\begin{array}{l}4.169 E+00 \\
3.850 E+00 \\
3.587 E+00 \\
3.367 E+00 \\
3.181 E+00 \\
2.880 E+00 \\
2.649 E+00 \\
2.465 E+00\end{array}$ & $\begin{array}{l}2.364 \mathrm{E}-02 \\
2.422 \mathrm{E}-02 \\
2.473 \mathrm{E}-02 \\
2.520 \mathrm{E}-02 \\
2.564 \mathrm{E}-02 \\
2.642 \mathrm{E}-02 \\
2.713 \mathrm{E}-02 \\
2.778 \mathrm{E}-02\end{array}$ & $\begin{array}{l}4.193 E+00 \\
3.874 E+00 \\
3.612 E+00 \\
3.393 E+00 \\
3.206 E+00 \\
2.907 E+00 \\
2.676 E+00 \\
2.492 E+00\end{array}$ & $\begin{array}{l}5.7 \\
6.9 \\
8.2 \\
9.7 \\
1.1 \\
1.4 \\
1.8 \\
2.1\end{array}$ & $\begin{array}{l}03 \\
03 \\
03 \\
03 \\
03 \\
03 \\
03 \\
03\end{array}$ & $\begin{array}{l}4.3 \\
4.9 \\
5 \cdot 4 \\
6.6 \\
7.9 \\
9.2\end{array}$ & $\begin{array}{l}-0.221 \\
-0.216 \\
-0.211 \\
-0.207 \\
-0.203 \\
-0.197 \\
-0.192 \\
-0.188\end{array}$ & & $\begin{array}{l}0.254 \\
0.246 \\
0.240 \\
0.235 \\
0.230 \\
0.222 \\
0.215 \\
0.210\end{array}$ \\
\hline $\begin{array}{l}0.1000 \\
0.1250 \\
0.1500 \\
0.1750 \\
0.2000 \\
0.2500 \\
0.3000 \\
0.3500\end{array}$ & $\begin{array}{l}2.315 E+00 \\
2.039 E+00 \\
1.852 E+00 \\
1.717 E+00 \\
1.615 E+00 \\
1.473 E+00 \\
1.380 E+00 \\
1.316 E+00\end{array}$ & $\begin{array}{l}2.838 \mathrm{E}-02 \\
2.975 \mathrm{E}-02 \\
3.100 \mathrm{E}-02 \\
3.216 \mathrm{E}-02 \\
3.328 \mathrm{E}-02 \\
3.550 \mathrm{E}-02 \\
3.776 \mathrm{E}-02 \\
4.010 \mathrm{E}-02\end{array}$ & $\begin{array}{l}2.343 E+00 \\
2.069 E+00 \\
1.883 E+00 \\
1.749 E+00 \\
1.648 E+00 \\
1.508 E+00 \\
1.418 E+00 \\
1.356 E+00\end{array}$ & $\begin{array}{l}2.612 E-02 \\
3.752 E-02 \\
5.021 E-02 \\
6.401 E-02 \\
7.876 E-02 \\
1.106 E-01 \\
1.448 E-01 \\
1.809 E-01\end{array}$ & $\begin{array}{l}E-03 \\
E-03 \\
E-03 \\
-02 \\
-02 \\
-02 \\
-02 \\
E-02 \\
\varepsilon-02\end{array}$ & $\begin{array}{l}-02 \\
-02 \\
-02 \\
-02 \\
-02 \\
-02 \\
-02 \\
-02 \\
-02 \\
-02\end{array}$ & $\begin{array}{l}-0.1 \\
-0.1 \\
-0.1 \\
-0.1 \\
-0.1 \\
-0.1 \\
-0.1 \\
-0.1\end{array}$ & & $\begin{array}{l}0.205 \\
0.196 \\
0.189 \\
0.183 \\
0.178 \\
0.171 \\
0.164 \\
0.159\end{array}$ \\
\hline $\begin{array}{l}0.4000 \\
0.4500 \\
0.5000 \\
0.5500 \\
0.6000 \\
0.7000 \\
0.8000 \\
0.9000\end{array}$ & $\begin{array}{l}1.269 E+00 \\
1.235 E+00 \\
1.209 E+00 \\
1.189 E+00 \\
1.174 E+00 \\
1.152 E+00 \\
1.140 E+00 \\
1.132 E+00\end{array}$ & $\begin{array}{l}4.5 \\
4.7 \\
5.0 \\
5.3 \\
5.8 \\
6.4\end{array}$ & $\begin{array}{l}1.312 E+00 \\
1.280 E+00 \\
1.257 E+00 \\
1.239 E+00 \\
1.227 E+00 \\
1.211 E+00 \\
1.204 E+00 \\
1.203 E+00\end{array}$ & $\begin{array}{l}2.185 \\
2.571 \\
2.965 \\
3.366 \\
3.772 \\
4.593 \\
5.421 \\
6.252\end{array}$ & $\begin{array}{l}2.2 \\
2.3 \\
2.5 \\
2.8\end{array}$ & $\begin{array}{l}7.8 \\
9: 3 \\
1: 9 \\
1: 0 \\
1: \\
2.9\end{array}$ & $\begin{array}{l}-0 . \\
-0 . \\
-0 . \\
-0 \\
-0 . \\
-0 .\end{array}$ & & $\begin{array}{l}0.154 \\
0.150 \\
0.146 \\
0.142 \\
0.139 \\
0.133 \\
0.128 \\
0.124\end{array}$ \\
\hline $\begin{array}{l}1.0000 \\
1.2500 \\
1.5000 \\
1.7500 \\
2.0000 \\
2.5000 \\
3.0000 \\
3.5000\end{array}$ & $\begin{array}{l}00 \\
00 \\
00 \\
00 \\
00 \\
00 \\
00 \\
000 \\
00\end{array}$ & $\begin{array}{l}9.3 \\
1.1 \\
1.2 \\
1.4 \\
1.8 \\
2.8\end{array}$ & $\begin{array}{l}1.205 \mathrm{E} \\
1.220 \mathrm{E} \\
1.243 \mathrm{E} \\
1.269 \mathrm{E} \\
1.297 \mathrm{E} \\
1.354 \mathrm{E} \\
1.412 \mathrm{E} \\
1.469 \mathrm{E}\end{array}$ & $\begin{array}{l}7.083 \mathrm{E}-01 \\
9.146 \mathrm{E}-01 \\
1.118 \mathrm{E}+00 \\
1.317 \mathrm{E}+00 \\
1.512 \mathrm{E}+00 \\
1.889 \mathrm{E}+00 \\
2.251 \mathrm{E}+00 \\
2.598 \mathrm{E}+00\end{array}$ & $\begin{array}{l}2 \\
2 \\
2 \\
2 \\
2 \\
2 \\
2\end{array}$ & $\begin{array}{l}2 . \\
3 . \\
4: \\
4 . \\
5 . \\
6 . \\
7 .\end{array}$ & $\begin{array}{l}-0 . \\
-0 . \\
-0 . \\
-0 . \\
-0 . \\
-0 . \\
-0 . \\
-0 .\end{array}$ & & $\begin{array}{l}0.087 \\
0.080\end{array}$ \\
\hline $\begin{array}{l}4.0000 \\
4.5000 \\
5.0000 \\
5.5000 \\
6.0000 \\
7.0000 \\
8.0000 \\
9.0000\end{array}$ & $\begin{array}{l}1.218 E+00 \\
1.232 E+00 \\
1.244 E+00 \\
1.256 E+00 \\
1.266 E+00 \\
1.285 E+00 \\
1.301 E+00 \\
1.315 E+00\end{array}$ & $\begin{array}{l}3.9 \\
4.3 \\
4.8\end{array}$ & $\begin{array}{l}1.637 E+00 \\
1.692 E+00 \\
1.747 E+00 \\
1.856 E+00 \\
1.963 E+00 \\
2.070 E+00\end{array}$ & $\begin{array}{l}0 \\
0 \\
0 \\
0 \\
0 \\
0\end{array}$ & $\begin{array}{l}.108 \mathrm{E}-01 \\
.220 \mathrm{E}-01 \\
.329 \mathrm{E}-01 \\
.434 \mathrm{E}-01 \\
.537 \mathrm{E}-01 \\
.734 \mathrm{E}-01 \\
.920 \mathrm{E}-01 \\
.097 \mathrm{E}-01\end{array}$ & $\begin{array}{l}9.857 E-01 \\
1.075 E+00 \\
1.158 E+00 \\
1.236 E+00 \\
1.311 E+00 \\
1.445 E+00 \\
1.576 E+00 \\
1.695 E+00\end{array}$ & $\begin{array}{l}-0.06 \\
-0.06 \\
-0.06 \\
-0.06 \\
-0.05 \\
-0.05 \\
-0.05 \\
-0.04\end{array}$ & $\begin{array}{l}0.084 \\
0.081 \\
0.078 \\
0.076 \\
0.073 \\
0.069 \\
0.066 \\
0.063\end{array}$ & $\begin{array}{l}0.06 \\
0.0 \\
0.05 \\
0.05 \\
0.05 \\
0.02\end{array}$ \\
\hline $\begin{array}{l}10.0000 \\
12.5000 \\
15.0000 \\
17.5000 \\
20.0000 \\
25.0000 \\
30.0000 \\
35.0000\end{array}$ & $\begin{array}{l}.328 E+00 \\
.353 E+00 \\
.374 E+00 \\
.390 E+00 \\
.404 E+00 \\
.426 E+00 \\
.444 E+00 \\
.459 E+00\end{array}$ & $\begin{array}{l}1.331 E+00 \\
1.578 E+00 \\
1.829 E+00 \\
2.338 E+00 \\
2.854 E+00 \\
3.376 E+00\end{array}$ & $\begin{array}{l}2.176 E+00 \\
2.441 E+00 \\
2.705 E+00 \\
2.969 E+00 \\
3.233 E+00 \\
3.764 E+00 \\
4.298 E+00 \\
4.834 E+00\end{array}$ & $\begin{array}{l}6.202 E+00 \\
7.286 E+00 \\
8.258 E+00 \\
9.140 E+00 \\
9.947 E+00 \\
1.138 E+01 \\
1.262 E+01 \\
1.372 E+01\end{array}$ & $\begin{array}{l}2.9 \\
3.2 \\
3.5 \\
4.0 \\
4.4 \\
4.7\end{array}$ & $\begin{array}{l}1.805 E+00 \\
2.057 E+00 \\
2.279 E+00 \\
2.478 E+00 \\
2.658 E+00 \\
2.974 E+00 \\
3.244 E+00 \\
3.480 E+00\end{array}$ & $\begin{array}{l}-0.0 \\
-0.0 \\
-0.0 \\
-0.0 \\
-0.0 \\
-0.0 \\
-0.0 \\
-0.0\end{array}$ & & $\begin{array}{l}0.044 \\
0.038 \\
0.033 \\
0.029 \\
0.026 \\
0.022 \\
0.019 \\
0.016\end{array}$ \\
\hline $\begin{array}{l}40.00000 \\
45.0000 \\
50.0000 \\
55.0000 \\
60.0000 \\
70.0000 \\
80.0000 \\
90.0000\end{array}$ & $\begin{array}{l}+00 \\
+00 \\
+00 \\
+00 \\
+00 \\
+00 \\
+00 \\
+00\end{array}$ & $\begin{array}{l}4.966 E+00 \\
5.503 E+00 \\
6.041 E+00 \\
7.124 E+00 \\
8.213 E+00 \\
9.307 E+00\end{array}$ & $\begin{array}{l}5.373 E+00 \\
5.914 E+00 \\
6.457 E+00 \\
7.002 E+00 \\
7.547 E+00 \\
8.643 E+00 \\
9.743 E+00 \\
1.085 E+01\end{array}$ & & $\begin{array}{l}E-0 \\
E-0 \\
E-0 \\
E=1 \\
E-01 \\
E-01 \\
E-01 \\
E-01 \\
E-01\end{array}$ & $\begin{array}{l}3.689 E+00 \\
3.878 E+00 \\
4.049 E+00 \\
4.206 E+00 \\
4.351 E+00 \\
4.612 E+00 \\
4.843 E+00 \\
5.050 E+00\end{array}$ & $\begin{array}{l}-0.0 \\
-0.1 \\
-0.8 \\
-0.2 \\
-0.0 \\
-0.8 \\
-0.8 \\
-0 .\end{array}$ & & $\begin{array}{l}0.008 \\
0.007 \\
0.007\end{array}$ \\
\hline $\begin{array}{l}.0000 \\
.0000 \\
0000\end{array}$ & $\begin{array}{l}79 E+00 \\
90 E+00 \\
00 E+00 \\
16 E+00 \\
29 E+00 \\
39 E+00\end{array}$ & $\begin{array}{l}+01 \\
+01 \\
+01 \\
+01 \\
+01\end{array}$ & $\begin{array}{l}1.195 E+01 \\
1.473 E+01 \\
1.751 E+01 \\
2.031 E+01 \\
2.310 E+01 \\
2.871 E+01 \\
3.433 E+01 \\
3.996 E+01\end{array}$ & $3.279 t+0$ & 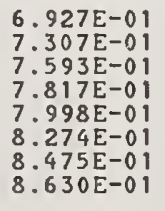 & $\begin{array}{l}5 . \\
5: \\
5: \\
6 . \\
6 . \\
6 . \\
7 . \\
7 .\end{array}$ & & & \\
\hline .00 & $1.703 E+00$ & $\begin{array}{l}1 \\
1 \\
1 \\
1 \\
2\end{array}$ & $\begin{array}{l}1 \\
1 \\
1 \\
1 \\
1 \\
1 \\
1 \\
2\end{array}$ & $\begin{array}{l}.500 \mathrm{E}+01 \\
.592 \mathrm{E}+01 \\
.676 \mathrm{E}+01 \\
.753 \mathrm{E}+01 \\
.888 \mathrm{E}+01 \\
.006 \mathrm{E}+01 \\
.110 \mathrm{E}+01\end{array}$ & $\begin{array}{l}854 \mathrm{E}-01 \\
938 \mathrm{E}-01 \\
009 \mathrm{E}-01 \\
070 \mathrm{E}-01 \\
170 \mathrm{E}-01 \\
249 \mathrm{E}-01 \\
313 \mathrm{E}-01\end{array}$ & $\begin{array}{l}7.839 E+00 \\
8.067 E+00 \\
8.272 E+00 \\
8.458 E+00 \\
8.629 E+00 \\
8.931 E+00 \\
9.194 E+00 \\
9.427 E+00\end{array}$ & $\begin{array}{l}-0.002 \\
-0.002 \\
-0.002 \\
-0.002 \\
-0.001 \\
-0.001 \\
-0.001\end{array}$ & & $\begin{array}{l}0.002 \\
0.001 \\
0.001 \\
0.001 \\
0.001 \\
0.001 \\
0.001 \\
0.001\end{array}$ \\
\hline 000 & $710 E+00$ & $1.118 E+02$ & $1.135 E+02$ & $4.202 E+01$ & $9.366 \mathrm{E}-01$ & $9.635 E+00$ & -0.001 & 0.012 & 0.001 \\
\hline
\end{tabular}




\begin{tabular}{|c|c|c|c|c|c|c|c|c|c|}
\hline ENERGY & $\begin{array}{l}\text { COLLISION } \\
\mathrm{MeV} \mathrm{cm} 2 / \mathrm{g}\end{array}$ & $\begin{array}{l}\text { OPPING POWE } \\
\text { RADIATIVE } \\
\mathrm{MeV} \mathrm{cm} 2 / \mathrm{g}\end{array}$ & $\begin{array}{c}\text { TOTAL } \\
\mathrm{MeV} \mathrm{cm}^{2} / \mathrm{g}\end{array}$ & $\begin{array}{l}\text { CSDA } \\
\text { RANGE } \\
\mathrm{g}^{\prime} \mathrm{cm}^{2}\end{array}$ & $\begin{array}{l}\text { ADIATION } \\
\text { YIELD }\end{array}$ & $\begin{array}{c}\text { DENS.EFF. } \\
\text { CORR. } \\
\text { (DELTA) }\end{array}$ & $\begin{array}{l}\text { d ( } 1 \\
\text { coLL } \\
\text { LOSS }\end{array}$ & )$/ d(1$ & $\begin{array}{l}\text { (I) } \\
\text { RAD } \\
\text { YIELD }\end{array}$ \\
\hline $\begin{array}{l}0.0100 \\
0.0125 \\
0.0150 \\
0.0175 \\
0.0200 \\
0.0250 \\
0.0300 \\
0.0350\end{array}$ & $\begin{array}{l}1.054 E+01 \\
9.078 E+00 \\
8.022 E+00 \\
7.218 E+00 \\
6.583 E+00 \\
5.641 E+00 \\
4.974 E+00 \\
4.473 E+00\end{array}$ & $\begin{array}{l}1.686 \mathrm{E}-02 \\
1.820 \mathrm{E}-02 \\
1.927 \mathrm{E}-02 \\
2.016 \mathrm{E}-02 \\
2.092 \mathrm{E}-02 \\
2.216 \mathrm{E}-02 \\
2.315 \mathrm{E}-02 \\
2.399 \mathrm{E}-02\end{array}$ & $\begin{array}{l}1.055 E+01 \\
9.097 E+00 \\
8.041 E+00 \\
7.238 E+00 \\
6.604 E+00 \\
5.664 E+00 \\
4.997 E+00 \\
4.497 E+00\end{array}$ & $\begin{array}{l}5.971 \mathrm{E}-04 \\
8.531 \mathrm{E}-04 \\
1.146 \mathrm{E}-03 \\
1.474 \mathrm{E}-03 \\
1.836 \mathrm{E}-03 \\
2.657 \mathrm{E}-03 \\
3.600 \mathrm{E}-03\end{array}$ & $\begin{array}{l}7.837 \mathrm{E}-04 \\
9.869 \mathrm{E}-04 \\
1.189 \mathrm{E}-03 \\
1.389 \mathrm{E}-03 \\
1.588 \mathrm{E}-03 \\
1.979 \mathrm{E}-03 \\
2.361 \mathrm{E}-03 \\
2.736 \mathrm{E}-03\end{array}$ & $\begin{array}{l}0.0 \\
0.0 \\
0.0 \\
0.0 \\
0.0 \\
0.0 \\
0.0 \\
0.0\end{array}$ & $\begin{array}{l}-0.315 \\
-0.295 \\
-0.280 \\
-0.269 \\
-0.260 \\
-0.246 \\
-0.236 \\
-0.228\end{array}$ & $\begin{array}{l}0.431 \\
0.393 \\
0.365 \\
0.345 \\
0.329 \\
0.305 \\
0.288 \\
0.275\end{array}$ & $\begin{array}{l}0.391 \\
0.359 \\
0.337 \\
0.320 \\
0.307 \\
0.287 \\
0.272 \\
0.261\end{array}$ \\
\hline $\begin{array}{l}0.0400 \\
0.0450 \\
0.0500 \\
0.0550 \\
0.0600 \\
0.0700 \\
0.0800 \\
0.0900\end{array}$ & $\begin{array}{l}4.083 E+00 \\
3.770 E+00 \\
3.513 E+00 \\
3.298 E+00 \\
3.115 E+00 \\
2.821 E+00 \\
2.594 E+00 \\
2.414 E+00\end{array}$ & $\begin{array}{l}2.5 \\
2.5 \\
2.6 \\
2.6 \\
2.7 \\
2.8 \\
2.9\end{array}$ & $\begin{array}{l}4.108 E+00 \\
3.796 E+00 \\
3.539 E+00 \\
3.324 E+00 \\
3.142 E+00 \\
2.849 E+00 \\
2.623 E+00 \\
2.443 E+00\end{array}$ & $\begin{array}{l}5.8 \\
7.0 \\
8.4 \\
9.9 \\
1.1 \\
1.4 \\
1.8 \\
2.2\end{array}$ & $\begin{array}{l}-03 \\
-03 \\
-03 \\
-03 \\
-03 \\
-03 \\
-03 \\
-03\end{array}$ & $\begin{array}{l}0.0 \\
0.0 \\
0.0 \\
0.0 \\
0.0 \\
0.0 \\
0.0 \\
0.0\end{array}$ & $\begin{array}{l}-0.221 \\
-0.216 \\
-0.211 \\
-0.207 \\
-0.204 \\
-0.198 \\
-0.193 \\
-0.189\end{array}$ & $\begin{array}{l}0.264 \\
0.256 \\
0.249 \\
0.243 \\
0.237 \\
0.229 \\
0.222 \\
0.216\end{array}$ & $\begin{array}{l}0.253 \\
0.245 \\
0.239 \\
0.234 \\
0.229 \\
0.222 \\
0.215 \\
0.210\end{array}$ \\
\hline $\begin{array}{l}0.1000 \\
0.1250 \\
0.1500 \\
0.1750 \\
0.2000 \\
0.2500 \\
0.3000 \\
0.3500\end{array}$ & $\begin{array}{l}2.267 \mathrm{E}+00 \\
1.998 \mathrm{E}+00 \\
1.815 \mathrm{E}+00 \\
1.683 \mathrm{E}+00 \\
1.584 \mathrm{E}+00 \\
1.445 \mathrm{E}+00 \\
1.355 \mathrm{E}+00 \\
1.293 \mathrm{E}+00\end{array}$ & $\begin{array}{l}6 E-02 \\
1 E-02 \\
0 E-02 \\
9 E-02 \\
4 E-02 \\
7 E-02 \\
2 E-02 \\
5 E-02\end{array}$ & $\begin{array}{l}E+00 \\
E+00 \\
E+00 \\
E+00 \\
E+00 \\
E+00 \\
E+00 \\
E+00\end{array}$ & 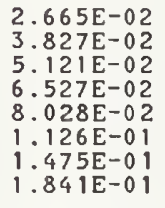 & $\begin{array}{l}-03 \\
-03 \\
-03 \\
-02 \\
-02 \\
-02 \\
-02 \\
-02 \\
-02\end{array}$ & $\begin{array}{l}0.0 \\
0.0 \\
0.0 \\
0.0 \\
0.0 \\
0.0 \\
0.0 \\
0.0\end{array}$ & $\begin{array}{l}-0.1 \\
-0.1 \\
-0.1 \\
-0.1 \\
-0.1 \\
-0.1 \\
-0.1 \\
-0.1\end{array}$ & $\begin{array}{l}0.2 \\
0.2 \\
0.1 \\
0.1 \\
0.1 \\
0.1 \\
0.1 \\
0.1\end{array}$ & $\begin{array}{l}0.206 \\
0.197 \\
0.190 \\
0.184 \\
0.180 \\
0.173 \\
0.167 \\
0.163\end{array}$ \\
\hline $\begin{array}{l}0.4000 \\
0.4500 \\
0.5000 \\
0.5500 \\
0.6000 \\
0.7000 \\
0.8000 \\
0.9000\end{array}$ & $\begin{array}{l}1.249 E+00 \\
1.216 E+00 \\
1.192 E+00 \\
1.173 E+00 \\
1.159 E+00 \\
1.140 E+00 \\
1.130 E+00 \\
1.124 E+00\end{array}$ & $\begin{array}{l}4.555 \mathrm{E}-02 \\
4.824 \mathrm{E}-02 \\
5.103 \mathrm{E}-02 \\
5.389 \mathrm{E}-02 \\
5.681 \mathrm{E}-02 \\
6.285 \mathrm{E}-02 \\
6.910 \mathrm{E}-02\end{array}$ & $\begin{array}{l}1.294 \mathrm{E}+00 \\
1.264 \mathrm{E}+00 \\
1.243 \mathrm{E}+00 \\
1.227 \mathrm{E}+00 \\
1.216 \mathrm{E}+00 \\
1.203 \mathrm{E}+00 \\
1.199 \mathrm{E}+00 \\
1.200 \mathrm{E}+00\end{array}$ & $\begin{array}{l}2.222 \mathrm{E}-01 \\
2.613 \mathrm{E}-01 \\
3.012 \mathrm{E}-01 \\
3.417 \mathrm{E}-01 \\
3.826 \mathrm{E}-01 \\
4.654 \mathrm{E}-01 \\
5.487 \mathrm{E}-01 \\
6.321 \mathrm{E}-01\end{array}$ & $\begin{array}{l}2.0 \\
2.2 \\
2.4 \\
2.5 \\
2.7 \\
3.0 \\
3.3 \\
3.6\end{array}$ & $\begin{array}{l}0.0 \\
0.0 \\
0.0 \\
0.0 \\
0.0 \\
0.0 \\
0.0 \\
0.0\end{array}$ & $\begin{array}{l}-0.148 \\
-0.145 \\
-0.142 \\
-0.140 \\
-0.138 \\
-0.135 \\
-0.132 \\
-0.129\end{array}$ & $\begin{array}{l}0.162 \\
0.159 \\
0.156 \\
0.154 \\
0.152 \\
0.148 \\
0.144 \\
0.142\end{array}$ & $\begin{array}{l}0.159 \\
0.156 \\
0.153 \\
0.150 \\
0.148 \\
0.144 \\
0.140 \\
0.137\end{array}$ \\
\hline $\begin{array}{l}1.0000 \\
1.2500 \\
1.5000 \\
1.7500 \\
2.0000 \\
2.5000 \\
3.0000 \\
3.5000\end{array}$ & $\begin{array}{l}1.122 \mathrm{E}+00 \\
1.126 \mathrm{E}+00 \\
1.135 \mathrm{E}+00 \\
1.147 \mathrm{E}+00 \\
1.160 \mathrm{E}+00 \\
1.186 \mathrm{E}+00 \\
1.211 \mathrm{E}+00 \\
1.233 \mathrm{E}+00\end{array}$ & $\begin{array}{l}8.217 \mathrm{E}-02 \\
9.943 \mathrm{E}-02 \\
1.176 \mathrm{E}-01 \\
1.364 \mathrm{E}-01 \\
1.557 \mathrm{E}-01 \\
1.958 \mathrm{E}-01 \\
2.373 \mathrm{E}-01 \\
2.798 \mathrm{E}-01\end{array}$ & $\begin{array}{l}E+00 \\
E+00 \\
E+00 \\
E+00 \\
E+00 \\
E+00 \\
E+00 \\
E+00\end{array}$ & $\begin{array}{l}3 E-01 \\
3 E-01 \\
3 E+00 \\
0 E+00 \\
3 E+00 \\
3 E+00 \\
7 E+00 \\
4 E+00\end{array}$ & $\begin{array}{l}3.9 \\
4.6 \\
5.3 \\
6.0 \\
6.6 \\
7.9\end{array}$ & $\begin{array}{l}0.0 \\
0.0 \\
0.0 \\
0.0 \\
0.0 \\
0.0 \\
0.0 \\
0.0\end{array}$ & $\begin{array}{l}-0.1 \\
-0.1 \\
-0.1 \\
-0.1 \\
-0.1 \\
-0.1 \\
-0.1 \\
-0.1\end{array}$ & $\begin{array}{l}0.1 \\
0.1 \\
0.1 \\
0.1 \\
0.1 \\
0.1 \\
0.1 \\
0.1\end{array}$ & $\begin{array}{l}0.134 \\
0.128 \\
0.123 \\
0.1199 \\
0.115 \\
0.109 \\
0.104 \\
0.100\end{array}$ \\
\hline $\begin{array}{l}4.0000 \\
4.5000 \\
5.0000 \\
5.5000 \\
6.0000 \\
7.0000 \\
8.0000 \\
9.0000\end{array}$ & $\begin{array}{l}1.254 E+00 \\
1.273 E+00 \\
1.290 E+00 \\
1.306 E+00 \\
1.321 E+00 \\
1.348 E+00 \\
1.372 E+00 \\
1.393 E+00\end{array}$ & $\begin{array}{l}3.233 E-01 \\
3.675 E-01 \\
4.124 E-01 \\
4.580 E-01 \\
5.040 E-01 \\
5.976 E-01 \\
6.927 E-01 \\
7.893 E-01\end{array}$ & $\begin{array}{l}1.577 E+00 \\
1.640 E+00 \\
1.703 E+00 \\
1.764 E+00 \\
1.825 E+00 \\
1.946 E+00 \\
2.065 E+00 \\
2.182 E+00\end{array}$ & $\begin{array}{l}2.898 \mathrm{E}+00 \\
3.209 \mathrm{E}+00 \\
3.508 \mathrm{E}+00 \\
3.796 \mathrm{E}+00 \\
4.075 \mathrm{E}+00 \\
4.606 \mathrm{E}+00 \\
5.104 \mathrm{E}+00 \\
5.575 \mathrm{E}+00\end{array}$ & $\begin{array}{l}1.148 \mathrm{E}-01 \\
1.259 \mathrm{E}-01 \\
1.366 \mathrm{E}-01 \\
1.470 \mathrm{E}-01 \\
1.571 \mathrm{E}-01 \\
1.764 \mathrm{E}-01 \\
1.945 \mathrm{E}-01 \\
2.117 \mathrm{E}-01\end{array}$ & $\begin{array}{l}0.0 \\
0.0 \\
0.0 \\
0.0 \\
0.0 \\
0.0 \\
0.0 \\
0.0\end{array}$ & $\begin{array}{l}-0.102 \\
-0.100 \\
-0.099 \\
-0.097 \\
-0.096 \\
-0.094 \\
-0.092 \\
-0.091\end{array}$ & $\begin{array}{l}0.107 \\
0.105 \\
0.102 \\
0.100 \\
0.098 \\
0.094 \\
0.091 \\
0.089\end{array}$ & $\begin{array}{l}0.097 \\
0.093 \\
0.090 \\
0.088 \\
0.085 \\
0.081 \\
0.077 \\
0.074\end{array}$ \\
\hline $\begin{array}{l}10.0000 \\
12.5000 \\
15.0000 \\
17.5000 \\
20.0000 \\
25.0000 \\
30.0000 \\
35.0000\end{array}$ & $\begin{array}{l}1.412 E+00 \\
1.453 E+00 \\
1.487 E+00 \\
1.515 E+00 \\
1.540 E+00 \\
1.581 E+00 \\
1.613 E+00 \\
1.640 E+00\end{array}$ & $\begin{array}{l}8.870 E-01 \\
1.136 E+00 \\
1.389 E+00 \\
1.647 E+00 \\
1.907 E+00 \\
2.436 E+00 \\
2.973 E+00 \\
3.517 E+00\end{array}$ & $\begin{array}{l}2.299 \mathrm{E}+00 \\
2.589 \mathrm{E}+00 \\
2.876 \mathrm{E}+00 \\
3.162 \mathrm{E}+00 \\
3.447 \mathrm{E}+00 \\
4.017 \mathrm{E}+00 \\
4.586 \mathrm{E}+00 \\
5.156 \mathrm{E}+00\end{array}$ & $\begin{array}{l}6.022 \mathrm{E}+00 \\
7.046 \mathrm{E}+00 \\
7.962 \mathrm{E}+00 \\
8.790 \mathrm{E}+00 \\
9.547 \mathrm{E}+00 \\
1.089 \mathrm{E}+01 \\
1.205 \mathrm{E}+01 \\
1.308 \mathrm{E}+01\end{array}$ & $\begin{array}{l}2.279 \mathrm{E}- \\
2.649 \mathrm{E}- \\
2.977 \mathrm{E}- \\
3.269 \mathrm{E}- \\
3.532 \mathrm{E}- \\
3.988 \mathrm{E}- \\
4.370 \mathrm{E}- \\
4.697 \mathrm{E}-\end{array}$ & $\begin{array}{l}0.0 \\
0.0 \\
0.0 \\
0.0 \\
2.396 \mathrm{E}-03 \\
2.422 \mathrm{E}-02 \\
5.614 \mathrm{E}-02 \\
9.173 \mathrm{E}-02\end{array}$ & $\begin{array}{l}-0.0 \\
-0.0 \\
-0.0 \\
-0.0 \\
-0.0 \\
-0.0 \\
-0.0 \\
-0.0\end{array}$ & $\begin{array}{l}0 . \\
0 . \\
0 . \\
0 . \\
0 . \\
0 . \\
0 . \\
0 .\end{array}$ & $\begin{array}{l}0.071 \\
0.065 \\
0.060 \\
0.056 \\
0.053 \\
0.047 \\
0.042 \\
0.038\end{array}$ \\
\hline $\begin{array}{l}45.00000 \\
50.00000 \\
55.00000 \\
60.00000 \\
70.00000 \\
80.00000 \\
90.00000\end{array}$ & $\begin{array}{l}1.662 \mathrm{E}+00 \\
1.682 \mathrm{E}+00 \\
1.700 \mathrm{E}+00 \\
1.716 \mathrm{E}+00 \\
1.730 \mathrm{E}+00 \\
1.754 \mathrm{E}+00 \\
1.775 \mathrm{E}+00 \\
1.793 \mathrm{E}+00\end{array}$ & $\begin{array}{l}64 \mathrm{E}+00 \\
16 \mathrm{E}+00 \\
71 \mathrm{E}+00 \\
30 \mathrm{E}+00 \\
90 \mathrm{E}+00 \\
17 \mathrm{E}+00 \\
51 \mathrm{E}+00 \\
89 \mathrm{E}+00\end{array}$ & $\begin{array}{l}5 . \\
6 . \\
6 . \\
7 . \\
8 . \\
9 . \\
1 . \\
1 .\end{array}$ & $\begin{array}{l}1 \\
1 \\
1 \\
1 \\
1 \\
1 \\
1\end{array}$ & $\begin{array}{l}5 . \\
5 \\
5 \\
5\end{array}$ & $\begin{array}{l}1.2 \\
1.6 \\
2.0 \\
2.4 \\
2.7 \\
3.5 \\
4.2 \\
4.9\end{array}$ & $\begin{array}{l}-0 . \\
-0 . \\
-0 . \\
-0 . \\
-0 . \\
-0 . \\
-0 .\end{array}$ & & $\begin{array}{l}0.0 \\
0.0 \\
0.0 \\
0.0 \\
0.0 \\
0.0\end{array}$ \\
\hline $\begin{array}{l}.0000 \\
.0000 \\
.0000 \\
.0000 \\
.0000 \\
.0000\end{array}$ & $\begin{array}{l}1.841 E+00 \\
1.867 E+00 \\
1.888 E+00 \\
1.905 E+00 \\
1.934 E+00 \\
1.957 E+00 \\
1.976 E+00\end{array}$ & $\begin{array}{l}1.949 E+01 \\
2.239 E+01 \\
2.821 E+01 \\
3.405 E+01 \\
3.990 E+01\end{array}$ & $\begin{array}{l}+E+01 \\
E+01 \\
E+01 \\
E+01 \\
E+01 \\
5 E+01 \\
1 E+01 \\
3 E+01\end{array}$ & $\begin{array}{l}E+01 \\
E+0 \\
E+01 \\
E+01 \\
E+01 \\
E+01 \\
E+01\end{array}$ & 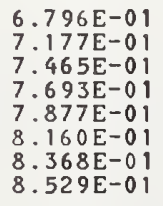 & $\begin{array}{l}5 . \\
7 . \\
8.5 \\
9.8 \\
1.2 \\
1.3 \\
1.5\end{array}$ & $\begin{array}{l}-0 . \\
-0 . \\
-0 . \\
-0 . \\
-0 . \\
-0 . \\
-0 . \\
-0 .\end{array}$ & $\begin{array}{l}0 . \\
0 . \\
0 . \\
0 . \\
0 . \\
0 .\end{array}$ & $\begin{array}{l}18 \\
15 \\
13 \\
11 \\
10 \\
09 \\
07 \\
06\end{array}$ \\
\hline $\begin{array}{l}.0000 \\
.0000 \\
.00000 \\
.00000\end{array}$ & $\begin{array}{l}1.992 \mathrm{E}+00 \\
2.005 \mathrm{E}+00 \\
2.017 \mathrm{E}+00 \\
2.027 \mathrm{E}+00 \\
2.036 \mathrm{E}+00 \\
2.052 \mathrm{E}+00 \\
2.066 \mathrm{E}+00 \\
2.077 \mathrm{E}+00\end{array}$ & $\begin{array}{l}5.750 E+01 \\
6.337 E+01 \\
6.926 E+01 \\
8.102 E+01 \\
9.280 E+01 \\
1.046 E+02\end{array}$ & $\begin{array}{l}5 E+01 \\
3 E+01 \\
2 E+01 \\
0 E+01 \\
9 E+01 \\
8 E+01 \\
7 E+01 \\
7 E+02\end{array}$ & $\begin{array}{l}.225 \mathrm{E}+01 \\
.324 \mathrm{E}+01 \\
.412 \mathrm{E}+01 \\
.492 \mathrm{E}+01 \\
.566 \mathrm{E}+01 \\
.695 \mathrm{E}+01 \\
.808 \mathrm{E}+01 \\
.907 \mathrm{E}+01\end{array}$ & $\begin{array}{l}8.7 \\
8.8 \\
8.9 \\
8.9 \\
9.0 \\
9.1 \\
9.2\end{array}$ & $\begin{array}{l}1 . \\
1 . \\
2 . \\
2 . \\
2 . \\
2 . \\
2 . \\
2 .\end{array}$ & $\begin{array}{l}-0 . \\
-0 . \\
-0 . \\
-0 . \\
-0 . \\
-0 . \\
-0 .\end{array}$ & $\begin{array}{l}0 . \\
0 . \\
0 . \\
0 . \\
0 . \\
0 . \\
0 .\end{array}$ & $\begin{array}{l}0.006 \\
0.005 \\
0.005 \\
0.004 \\
0.004 \\
0.003 \\
0.003 \\
0.003\end{array}$ \\
\hline & & & & & & & 0. & 023 & 0.0 \\
\hline
\end{tabular}


ELECTRONS IN GADOLINIUM

$I=591.0 \mathrm{eV} \quad$ DENSITY $=7.900 \mathrm{E}+00 \mathrm{~g} / \mathrm{cm}^{3}$

\begin{tabular}{|c|c|c|c|c|c|c|c|c|c|}
\hline ENERGY & 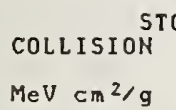 & $\mathrm{MeV} c \mathrm{~m}^{2} / \mathrm{g}$ & $\begin{array}{c}\text { TOTAL } \\
\mathrm{MeV} \mathrm{cm}^{2} / \mathrm{g}\end{array}$ & $\begin{array}{l}\text { CSDA } \\
\text { RANGE }\end{array}$ & $\begin{array}{l}\text { DIATION } \\
\text { YIELD }\end{array}$ & $\begin{array}{l}\text { ENS. EFF. } \\
\text { CORR. } \\
\text { (DELTA) }\end{array}$ & $\begin{array}{l}d(1 \\
\operatorname{coL} L \\
\text { LOSS }\end{array}$ & $\begin{array}{l}\text { S'd } 1 \\
\text { SDA } \\
\text { ANGE }\end{array}$ & $\begin{array}{l}\text { I ) } \\
\text { RAD } \\
\text { YIELD }\end{array}$ \\
\hline $\begin{array}{l}0.0100 \\
0.0125 \\
0.0150 \\
0.0175 \\
0.0200 \\
0.0250 \\
0.0300 \\
0.0350\end{array}$ & $\begin{array}{l}9.753 E+00 \\
8.440 E+00 \\
7.481 E+00 \\
6.747 E+00 \\
6.165 E+00 \\
5.299 E+00 \\
4.681 E+00 \\
4.216 E+00\end{array}$ & $\begin{array}{l}1.847 \\
2.008 \\
2.140 \\
2.250 \\
2.345 \\
2.501 \\
2.628\end{array}$ & $\begin{array}{l}9.771 \\
8.460 \\
7.502 \\
6.769 \\
6.189 \\
5.324 \\
4.707 \\
4.244\end{array}$ & $\begin{array}{l}7 E-04 \\
35 E-04 \\
3 E-03 \\
4 E-03 \\
1 E-03 \\
6 E-03 \\
7 E-03 \\
8 E-03\end{array}$ & $\begin{array}{l}223 \mathrm{E}-04 \\
164 \mathrm{E}-03 \\
406 \mathrm{E}-03 \\
646 \mathrm{E}-03 \\
885 \mathrm{E}-03 \\
357 \mathrm{E}-03 \\
821 \mathrm{E}-03 \\
.278 \mathrm{E}-03\end{array}$ & $\begin{array}{l}2.078 \mathrm{E}-03 \\
2.671 \mathrm{E}-03 \\
3.295 \mathrm{E}-03 \\
3.950 \mathrm{E}-03 \\
4.637 \mathrm{E}-03 \\
6.106 \mathrm{E}-03 \\
7.705 \mathrm{E}-03 \\
9.430 \mathrm{E}-03\end{array}$ & $\begin{array}{l}-0.337 \\
-0.313 \\
-0.297 \\
-0.284 \\
-0.273 \\
-0.258 \\
-0.246 \\
-0.237\end{array}$ & $\begin{array}{l}0.491 \\
0.442 \\
0.407 \\
0.381 \\
0.361 \\
0.331 \\
0.310 \\
0.295\end{array}$ & $\begin{array}{l}0.430 \\
0.391 \\
0.364 \\
0.344 \\
0.328 \\
0.305 \\
0.288 \\
0.276\end{array}$ \\
\hline $\begin{array}{l}0.0400 \\
0.0450 \\
0.0500 \\
0.0550 \\
0.0600 \\
0.0700 \\
0.0800 \\
0.0900\end{array}$ & $\begin{array}{l}3.853 E+00 \\
3.561 E+00 \\
3.321 E+00 \\
3.120 E+00 \\
2.948 E+00 \\
2.672 E+00 \\
2.459 E+00 \\
2.289 E+00\end{array}$ & $\begin{array}{l}2.8 \\
2.9 \\
2.9 \\
3.0 \\
3.1 \\
3.2 \\
3.3 \\
3.4\end{array}$ & $\begin{array}{l}3.882 E+00 \\
3.590 E+00 \\
3.351 E+00 \\
3.150 E+00 \\
2.980 E+00 \\
2.705 E+00 \\
2.492 E+00 \\
2.324 E+00\end{array}$ & $\begin{array}{l}221 E-03 \\
562 E-03 \\
005 E-03 \\
054 E-02 \\
218 E-02 \\
571 E-02\end{array}$ & $\begin{array}{l}.727 \mathrm{E}-03 \\
.170 \mathrm{E}-03 \\
.605 \mathrm{E}-03 \\
.035 \mathrm{E}-03 \\
.458 \mathrm{E}-03 \\
.288 \mathrm{E}-03 \\
.097 \mathrm{E}-03 \\
.885 \mathrm{E}-03\end{array}$ & $\begin{array}{l}1.128 \mathrm{E}-02 \\
1.324 \mathrm{E}-02 \\
1.530 \mathrm{E}-02 \\
1.746 \mathrm{E}-02 \\
1.970 \mathrm{E}-02 \\
2.439 \mathrm{E}-02 \\
2.926 \mathrm{E}-02 \\
3.424 \mathrm{E}-02\end{array}$ & $\begin{array}{l}-0.230 \\
-0.223 \\
-0.218 \\
-0.213 \\
-0.209 \\
-0.202 \\
-0.197 \\
-0.192\end{array}$ & $\begin{array}{l}.282 \\
.272 \\
.263 \\
.256 \\
.250 \\
.239 \\
.231 \\
.224\end{array}$ & $\begin{array}{l}0.266 \\
0.257 \\
0.250 \\
0.244 \\
0.239 \\
0.230 \\
0.222 \\
0.216\end{array}$ \\
\hline $\begin{array}{l}0.1000 \\
0.1250 \\
0.1500 \\
0.1750 \\
0.2000 \\
0.2500 \\
0.3000 \\
0.3500\end{array}$ & $\begin{array}{l}2.151 E+00 \\
1.836 E+00 \\
1.723 E+00 \\
1.597 E+00 \\
1.503 E+00 \\
1.371 E+00 \\
1.285 E+00\end{array}$ & $\begin{array}{l}3 . \\
3 . \\
4 . \\
4 . \\
4 . \\
5 .\end{array}$ & $\begin{array}{l}E+00 \\
E+00 \\
E+00 \\
E+00 \\
E+00 \\
E+00 \\
E+00 \\
E+00\end{array}$ & 2 & 1. & $\begin{array}{l}E-02 \\
E-02 \\
E-02 \\
E-02 \\
E-02 \\
E-01 \\
E-01 \\
E-01\end{array}$ & $\begin{array}{l}-0.187 \\
-0.179 \\
-0.173 \\
-0.168 \\
-0.163 \\
-0.156 \\
-0.151 \\
-0.146\end{array}$ & $\begin{array}{l}1 \\
5 \\
7 \\
0 \\
4\end{array}$ & $\begin{array}{l}0.211 \\
0.200 \\
0.192 \\
0.186 \\
0.180 \\
0.172 \\
0.165 \\
0.160\end{array}$ \\
\hline $\begin{array}{l}0.4000 \\
0.4500 \\
0.5000 \\
0.5500 \\
0.6000 \\
0.7000 \\
0.8000 \\
0.9000\end{array}$ & $\begin{array}{l}183 E+00 \\
51 E+00 \\
27 E+00 \\
109 E+00 \\
095 E+00 \\
375 E+00 \\
064 E+00 \\
057 E+00\end{array}$ & $\begin{array}{l}5.875 E-02 \\
6.211 E-02 \\
6.553 \mathrm{E}-02 \\
6.903 \mathrm{E}-02 \\
7.619 \mathrm{E}-02 \\
8.356 \mathrm{E}-02 \\
9.112 \mathrm{E}-02\end{array}$ & $\begin{array}{l}1.189 E+00 \\
1.174 E+00 \\
1.164 E+00 \\
1.152 E+00 \\
1.148 E+00 \\
1.149 E+00\end{array}$ & $\begin{array}{l}3.1 \\
3.5 \\
4.0 \\
4.8\end{array}$ & & 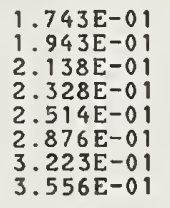 & $\begin{array}{l}-0.142 \\
-0.138 \\
-0.135 \\
-0.132 \\
-0.130 \\
-0.125 \\
-0.121 \\
-0.118\end{array}$ & & $\begin{array}{l}.155 \\
.151 \\
.148 \\
.145 \\
.142 \\
.137 \\
.132 \\
.128\end{array}$ \\
\hline $\begin{array}{l}1.0000 \\
1.2500 \\
1.5000 \\
1.7500 \\
2.0000 \\
2.5000 \\
3.0000 \\
3.5000\end{array}$ & $\begin{array}{l}1.054 E+00 \\
1.054 E+00 \\
1.060 E+00 \\
1.069 E+00 \\
1.079 E+00 \\
1.098 E+00 \\
1.116 E+00 \\
1.133 E+00\end{array}$ & $\begin{array}{l}9.888 \mathrm{E}-02 \\
1.190 \mathrm{E}-01 \\
1.399 \mathrm{E}-01 \\
1.616 \mathrm{E}-01 \\
1.839 \mathrm{E}-01 \\
2.299 \mathrm{E}-01 \\
2.773 \mathrm{E}-01\end{array}$ & $\begin{array}{l}1: \\
1: \\
1:\end{array}$ & $\begin{array}{l}1 \\
1 \\
0 \\
0 \\
0\end{array}$ & & $\begin{array}{l}3.878 \mathrm{E}-01 \\
4.637 \mathrm{E}-01 \\
5.337 \mathrm{E}-01 \\
5.989 \mathrm{E}-01 \\
6.600 \mathrm{E}-01 \\
7.725 \mathrm{E}-01 \\
8.746 \mathrm{E}-01 \\
9.689 \mathrm{E}-01\end{array}$ & & & $\begin{array}{l}0.125 \\
0.1117 \\
0.1111 \\
0.106 \\
0.101 \\
0.094 \\
0.087 \\
0.082\end{array}$ \\
\hline $\begin{array}{l}4.0000 \\
4.5000 \\
5.0000 \\
5.5000 \\
6.0000 \\
7.0000 \\
8.0000\end{array}$ & $\begin{array}{l}1.148 E+00 \\
1.162 E+00 \\
1.174 E+00 \\
1.186 E+00 \\
1.196 E+00 \\
1.215 E+00 \\
1.231 E+00 \\
1.245 E+00\end{array}$ & $\begin{array}{l}3.754 \mathrm{E}-01 \\
4.258 \mathrm{E}-01 \\
4.770 \mathrm{E}-01 \\
5.288 \mathrm{E}-01 \\
5.811 \mathrm{E}-01 \\
6.874 \mathrm{E}-01 \\
7.954 \mathrm{E}-01 \\
9.049 \mathrm{E}-01\end{array}$ & $\begin{array}{l}1.651 \mathrm{E}+0 \\
1.715 \mathrm{E}+0 \\
1.777 \mathrm{E}+0 \\
1.902 \mathrm{E}+0 \\
2.026 \mathrm{E}+0 \\
2.150 \mathrm{E}+0\end{array}$ & . & $\begin{array}{l}1 . \\
1 . \\
1 .\end{array}$ & $\begin{array}{l}0 \\
0 \\
0 \\
0 \\
0 \\
0\end{array}$ & $\begin{array}{l}-0.07 \\
-0.07 \\
-0.07 \\
-0.06 \\
-0.06 \\
-0.06 \\
-0.05 \\
-0.05\end{array}$ & & $\begin{array}{l}0.078 \\
0.074 \\
0.070 \\
0.067 \\
0.064 \\
0.059 \\
0.054 \\
0.051\end{array}$ \\
\hline $\begin{array}{l}10.0000 \\
12.5000 \\
15.0000 \\
17.5000 \\
20.0000 \\
25.0000 \\
30.0000 \\
35.0000\end{array}$ & $\begin{array}{l}1.257 E+00 \\
1.284 E+00 \\
1.304 E+00 \\
1.322 E+00 \\
1.337 E+00 \\
1.360 E+00 \\
1.379 E+00 \\
1.394 E+00\end{array}$ & $\begin{array}{l}1.016 E+00 \\
1.298 E+00 \\
1.585 E+00 \\
1.877 E+00 \\
2.172 E+00 \\
2.771 E+00 \\
3.379 E+00 \\
3.994 E+00\end{array}$ & $\begin{array}{l}2 . \\
3 . \\
3 . \\
4 . \\
5 .\end{array}$ & $\begin{array}{l}8.1 \\
8.9 \\
9.7\end{array}$ & $\begin{array}{l}3 \\
3 \\
3 \\
4 \\
4 \\
4 \\
5\end{array}$ & $\begin{array}{l}0 \\
0 \\
0 \\
0\end{array}$ & $\begin{array}{l}-0.0 \\
-0.0 \\
-0.0 \\
-0.0 \\
-0.0 \\
-0.0\end{array}$ & & \\
\hline $\begin{array}{l}40.0000 \\
45.0000 \\
50.0000 \\
55.0000 \\
60.0000 \\
70.0000 \\
80.0000 \\
90.0000\end{array}$ & & $\begin{array}{l}4 \\
5 \\
5 \\
6 \\
7 \\
8 \\
9\end{array}$ & & & & & & & \\
\hline $\begin{array}{l}25.0000 \\
50.0000 \\
75.0000 \\
00.0000 \\
50.0000 \\
00.0000 \\
50.0000\end{array}$ & $\begin{array}{l}1.518 E+00 \\
1.530 E+00 \\
1.540 E+00 \\
1.556 E+00 \\
1.568 E+00 \\
1.579 E+00\end{array}$ & $\begin{array}{l}1.2 \\
2.2 \\
2 . \\
3 . \\
3 .\end{array}$ & $\begin{array}{l}1 \\
1 \\
1 \\
1 \\
1 \\
1\end{array}$ & 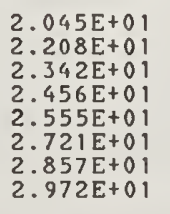 & & & & & \\
\hline $\begin{array}{l}0.0000 \\
0.00000 \\
0.0000 \\
0.0000 \\
0.0000 \\
0.0000 \\
0.0000\end{array}$ & $\begin{array}{l}1.603 E+00 \\
1.610 E+00 \\
1.615 E+00 \\
1.626 E+00 \\
1.634 E+00 \\
1.642 E+00\end{array}$ & $\begin{array}{l}6.0 \\
7.5 \\
7.8 \\
9.1\end{array}$ & $\begin{array}{l}6 . \\
7 . \\
8 . \\
9\end{array}$ & $3.680 \mathrm{E}+01$ & $9.415 \mathrm{E}-0$ & $\begin{array}{l}7.756 \mathrm{E}+00 \\
7.958 \mathrm{E}+00 \\
8.142 \mathrm{E}+00 \\
8.311 \mathrm{E}+00 \\
8.610 \mathrm{E}+00 \\
8.871 \mathrm{E}+00 \\
9.101 \mathrm{E}+00\end{array}$ & $\begin{array}{l}4 \\
4 \\
3 \\
3 \\
3 \\
2 \\
2 \\
2\end{array}$ & & $\begin{array}{l}.002 \\
.002 \\
.001 \\
.001 \\
.001 \\
.001 \\
.001\end{array}$ \\
\hline 0 & $1.649 E+00$ & $1.319 E+02$ & $1.335 E+02$ & $3.758 \mathrm{E}+01$ & $9.460 \mathrm{E}-01$ & $9.308 E+00$ & -0.002 & 0.015 & 0.001 \\
\hline
\end{tabular}




\begin{tabular}{|c|c|c|c|c|c|c|c|c|c|}
\hline \multirow{2}{*}{$\begin{array}{l}\text { ENERGY } \\
\text { MeV }\end{array}$} & \multicolumn{3}{|c|}{$\begin{array}{l}\text { 5TOPPING POWER } \\
\text { Y RADIATIVE }\end{array}$} & \multirow{2}{*}{$\begin{array}{l}\text { CSDA } \\
\text { RANGE } \\
g / \mathrm{cm}^{2}\end{array}$} & \multirow{2}{*}{$\begin{array}{l}\text { RADIATION } \\
\text { YIELD }\end{array}$} & \multirow{2}{*}{$\begin{array}{l}\text { DENS.EFF. } \\
\text { CORR } \\
\text { (DELTA) }\end{array}$} & \multicolumn{3}{|c|}{$d(\log ) / d(\log I)$} \\
\hline & $\mathrm{MeV} \mathrm{cm}^{2} / \mathrm{g}$ & $\mathrm{eV} \mathrm{cm}^{2} / \mathrm{g}$ & $\begin{array}{l}\text { TOTAL } \\
\mathrm{MeV} \mathrm{cm}^{2} / \mathrm{g}\end{array}$ & & & & & & D \\
\hline $\begin{array}{l}0.01100 \\
0.0125 \\
0.0150 \\
0.0175 \\
0.0200 \\
0.0250 \\
0.0300 \\
0.0350\end{array}$ & $\begin{array}{l}8.974 E+00 \\
7.806 E+00 \\
6.945 E+00 \\
6.281 E+00 \\
5.753 E+00 \\
4.961 E+00\end{array}$ & $\begin{array}{l}2.1 \\
2.3 \\
2.4 \\
2.5 \\
2.7 \\
2 .\end{array}$ & $\begin{array}{l}8 . \\
7 . \\
6 . \\
6 \\
5: \\
4 . \\
4 . \\
3 .\end{array}$ & & & $\begin{array}{l}-04 \\
-03 \\
-03 \\
-03 \\
-03 \\
-03 \\
-03 \\
-03\end{array}$ & $\begin{array}{l}2 \\
5 \\
6 \\
2 \\
0 \\
3 \\
0 \\
0\end{array}$ & & $\begin{array}{l}.487 \\
.435 \\
.401 \\
.376 \\
.357 \\
.329 \\
.309 \\
.294\end{array}$ \\
\hline $\begin{array}{l}0.0400 \\
0.0450 \\
0.0500 \\
0.0550 \\
0.0600 \\
0.0700 \\
0.0800 \\
0.0900\end{array}$ & $\begin{array}{l}.631 E+00 \\
.360 E+00 \\
.137 E+00 \\
.950 E+00 \\
.791 E+00 \\
.533 E+00 \\
.335 E+00 \\
176 E+00\end{array}$ & $\begin{array}{l}3 . \\
3 . \\
3 \\
3 . \\
3 . \\
3 . \\
3 .\end{array}$ & $\begin{array}{l}3.6 \\
3.3 \\
3.1 \\
2.9 \\
2.8 \\
2.5 \\
2.3 \\
2.2\end{array}$ & & & $\begin{array}{l}3 \\
3 \\
3 \\
3\end{array}$ & $\begin{array}{l}-0.2 \\
-0 . \\
-0 . \\
-0 . \\
-0 . \\
-0 . \\
-0 .\end{array}$ & & $\begin{array}{l}0.283 \\
0.273 \\
0.265 \\
0.258 \\
0.252 \\
0.243 \\
0.235\end{array}$ \\
\hline $\begin{array}{l}0.1000 \\
0.1250 \\
0.1500 \\
0.1750 \\
0.2000 \\
0.2500 \\
0.3000 \\
0.3500\end{array}$ & $\begin{array}{l}.047 E+00 \\
.808 E+00 \\
.646 E+00 \\
.528 E+00 \\
.439 E+00 \\
.315 E+00 \\
.234 E+00 \\
.178 E+00\end{array}$ & $\begin{array}{l}4 . \\
4 . \\
4 . \\
4 . \\
5 . \\
5 . \\
5 .\end{array}$ & & & $\begin{array}{l}-02 \\
-02 \\
-02 \\
-02 \\
-02\end{array}$ & $\begin{array}{l}2.7 \\
3.4 \\
4.1\end{array}$ & $\begin{array}{l}-0.1 \\
-0.1 \\
-0.1 \\
-0.1 \\
-0.1\end{array}$ & & $\begin{array}{l}0.222 \\
0.211 \\
0.202 \\
0.196 \\
0.190\end{array}$ \\
\hline $\begin{array}{l}0.4000 \\
0.4500 \\
0.5000 \\
0.5500 \\
0.6000 \\
0.7000 \\
0.8000 \\
0.9000\end{array}$ & $\begin{array}{l}+00 \\
+00 \\
+00 \\
+00 \\
+00 \\
+00 \\
+00 \\
+00\end{array}$ & $\begin{array}{l}6 . \\
6: \\
7 \\
7: \\
8: \\
8 . \\
9 . \\
1 .\end{array}$ & $\begin{array}{l}1.136 E+0 \\
1.126 E+0 \\
1.124 E+0 \\
1.126 E+0\end{array}$ & & $\begin{array}{l}3 . \\
4\end{array}$ & 1.1 & $\begin{array}{l}-0.1 \\
-0.1 \\
-0.1 \\
-0.1 \\
-0.1\end{array}$ & & 0. \\
\hline $\begin{array}{l}1.00 \\
1.25 \\
1.50 \\
1.75 \\
2.00 \\
2.50 \\
3.00\end{array}$ & $\begin{array}{l}1.016 E+00 \\
1.016 E+00 \\
1.021 E+00 \\
1.029 E+00 \\
1.037 E+00 \\
1.055 E+00 \\
1.072 E+00 \\
1.087 E+00\end{array}$ & & 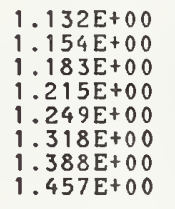 & & & 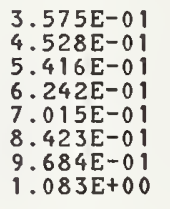 & $\begin{array}{l}-0 . \\
-0 . \\
-0 .\end{array}$ & & $\cdot$. \\
\hline $\begin{array}{l}4.0000 \\
4.5000 \\
5.0000 \\
5.5000 \\
6.0000 \\
7.0000 \\
8.0000 \\
9.0000\end{array}$ & 1. & $\begin{array}{l}4 \\
5 \\
5 \\
6 \\
7 \\
8 \\
1\end{array}$ & $\begin{array}{l}0 \\
0 \\
0 \\
0\end{array}$ & 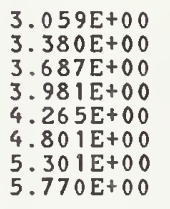 & $\begin{array}{l}1 . \\
1 . \\
2 . \\
2 . \\
2 .\end{array}$ & 1. & & & 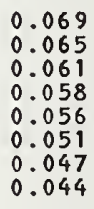 \\
\hline $\begin{array}{l}10.0000 \\
12.5000 \\
15.0000 \\
17.5000 \\
20.0000\end{array}$ & 1 & $\begin{array}{l}1.7 \\
2.0 \\
2.4 \\
3.0\end{array}$ & 3. & 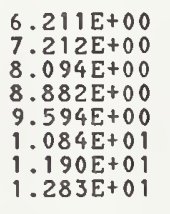 & $\begin{array}{l}3 . \\
3 . \\
4 .\end{array}$ & & & & 0. \\
\hline $\begin{array}{l}40.0000 \\
45.0000 \\
50.0000 \\
55.0000 \\
60.0000 \\
70.0000\end{array}$ & $\begin{array}{l}1 . \\
1: \\
1: \\
1 . \\
1 .\end{array}$ & $\begin{array}{l}5 . \\
6 . \\
7 . \\
9\end{array}$ & $\begin{array}{l}7 . \\
8 . \\
9 \\
1 . \\
1\end{array}$ & & & & & & \\
\hline $\begin{array}{l}0 \\
0 \\
0 \\
0 \\
0\end{array}$ & $\begin{array}{l}1 \\
1 \\
1 \\
1 \\
1\end{array}$ & & & & & & & & \\
\hline 0.0000 & $.570 \mathrm{E}+00$ & $\begin{array}{l}911 E+01 \\
.645 E+01 \\
.011 E+02 \\
.158 E+02 \\
.305 E+02\end{array}$ & 8. & 40 & 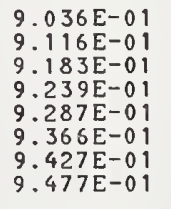 & 8. & & $\begin{array}{l}16 \\
6 \\
5 \\
5 \\
5 \\
5 \\
4\end{array}$ & $\begin{array}{ll}0 & 2 \\
0 & 1 \\
0 & 1 \\
0 & 1 \\
0 & 1 \\
01 \\
01\end{array}$ \\
\hline & $.576 E+00$ & $.452 E+02$ & $.468 E+02$ & $.524 E+01$ & $9.518 \mathrm{E}-01$ & $9.771 E+00$ & -0.001 & .014 & 0.001 \\
\hline
\end{tabular}




\begin{tabular}{|c|c|c|c|c|c|c|c|c|c|}
\hline $\mathrm{MeV}$ & $\begin{array}{l}\text { COLLISION } \\
\mathrm{MeV} \mathrm{Cm}^{2} / \mathrm{g}\end{array}$ & $\begin{array}{l}\text { RADIATIVE } \\
\mathrm{MeV} \mathrm{cm} 2 / \mathrm{g}\end{array}$ & $\begin{array}{c}\text { TOTAL } \\
\mathrm{MeV} \mathrm{cm}^{2} / \mathrm{g}\end{array}$ & $\begin{array}{l}\text { CSDA } \\
\text { RANGE } \\
g / \mathrm{cm}^{2}\end{array}$ & $\mathbf{N}$ & $\begin{array}{l}\text { ERF. } \\
\text { RRA } \\
\text { LTA) }\end{array}$ & $\begin{array}{l}d(1 \\
\operatorname{coLL} \\
\text { LOSS }\end{array}$ & SDA & $\begin{array}{l}\text { I) } \\
\text { RAD } \\
\text { IELD }\end{array}$ \\
\hline $\begin{array}{l}0.0100 \\
0.0125 \\
0.0150 \\
0.0175 \\
0.0200 \\
0.0250 \\
0.0300 \\
0.0350\end{array}$ & $\begin{array}{lll}0 & 0 \\
0 & 0 \\
0 & 0 \\
0 & 0 \\
0 & 0 \\
0 & 0 \\
0 & 0 \\
0 & 0\end{array}$ & $\begin{array}{l}2.2 \\
2.3 \\
2.5 \\
2.6 \\
2.8 \\
3.0\end{array}$ & $\begin{array}{l}7 . \\
6 . \\
6 \\
5 \\
4 .\end{array}$ & $\begin{array}{l}-04 \\
-03 \\
-03 \\
-03 \\
-03 \\
-03 \\
-03\end{array}$ & $\begin{array}{l}144 \mathrm{E}-03 \\
441 \mathrm{E}-03 \\
739 \mathrm{E}-03 \\
037 \mathrm{E}-03 \\
334 \mathrm{E}-03 \\
923 \mathrm{E}-03 \\
507 \mathrm{E}-03 \\
084 \mathrm{E}-03\end{array}$ & $\begin{array}{l}1.084 E-03 \\
1.393 E-03 \\
1.717 E-03 \\
2.059 E-03 \\
2.418 E-03 \\
3.190 E-03 \\
4.039 E-03 \\
4.967 E-03\end{array}$ & $\begin{array}{l}3 \\
5 \\
5 \\
0 \\
7 \\
9 \\
6 \\
6\end{array}$ & & $\begin{array}{l}0.520 \\
0.460 \\
0.421 \\
0.393 \\
0.371 \\
0.340 \\
0.319 \\
0.303\end{array}$ \\
\hline $\begin{array}{l}0.0400 \\
0.0450 \\
0.0500 \\
0.0550 \\
0.0600 \\
0.0700 \\
0.0800 \\
0.0900\end{array}$ & $\begin{array}{l}3.2 \\
3.0 \\
2.8 \\
2.7 \\
2.4 \\
2.2\end{array}$ & $\begin{array}{l}3.3 \\
3.4 \\
3.5 \\
3.6 \\
3.8 \\
4.0\end{array}$ & $\begin{array}{l}3 . \\
3 . \\
2 . \\
2 . \\
2 . \\
2 .\end{array}$ & $\begin{array}{l}8 \\
9 \\
1 \\
1 \\
1 \\
2\end{array}$ & $\begin{array}{l}03 \\
03 \\
03 \\
03 \\
03 \\
03 \\
03 \\
02\end{array}$ & $\begin{array}{l}E-03 \\
E-03 \\
E-03 \\
E-03 \\
E-02 \\
E-02 \\
E-02\end{array}$ & $\begin{array}{l}-0.2 \\
-0.2 \\
-0.2 \\
-0.2 \\
-0.2 \\
-0.2 \\
-0.2 \\
-0.2\end{array}$ & $\begin{array}{l}23 \\
09 \\
97 \\
87 \\
79 \\
65 \\
54 \\
46\end{array}$ & $\begin{array}{l}0.290 \\
0.280 \\
0.272 \\
0.264 \\
0.258 \\
0.247 \\
0.239 \\
0.232\end{array}$ \\
\hline $\begin{array}{l}0.10 \\
0.12 \\
0.15 \\
0.17 \\
0.20 \\
0.25 \\
0.30 \\
0.35\end{array}$ & $\begin{array}{l}1.7 \\
1.6 \\
1.4 \\
1.4 \\
1.2 \\
1.2\end{array}$ & $\begin{array}{l}4 . \\
4.8 \\
5 . \\
5 .\end{array}$ & & $\begin{array}{l}4 \\
7\end{array}$ & $\frac{2}{2}$ & $\begin{array}{l}2 \cdot 3 \\
3 \cdot 3 \\
4 \cdot 2 \\
5 \cdot 1 \\
6 \cdot 0 \\
7 \cdot 8 \\
9.5 \\
1.9\end{array}$ & $\begin{array}{l}-0.2 \\
-0.1 \\
-0.1 \\
-0.1 \\
-0.1 \\
-0.1 \\
-0.1 \\
-0.1\end{array}$ & $\begin{array}{l}8 \\
4 \\
3 \\
5 \\
8 \\
8 \\
0 \\
4\end{array}$ & $\begin{array}{l}0.225 \\
0.213 \\
0.204 \\
0.197 \\
0.191 \\
0.181 \\
0.174 \\
0.168\end{array}$ \\
\hline $\begin{array}{l}0.40 \\
0.45 \\
0.50 \\
0.55 \\
0.60 \\
0.70 \\
0.80 \\
0.90\end{array}$ & $\begin{array}{l}1 . \\
1 . \\
1 . \\
1 .\end{array}$ & $\begin{array}{l}2 \\
2 \\
2 \\
2 \\
2 \\
1\end{array}$ & & & $\begin{array}{l}2 \\
2 \\
2 \\
2 \\
2 \\
2 \\
2 \\
2\end{array}$ & $\begin{array}{l}1.3 \\
1.4 \\
1.6 \\
1.8 \\
1.8 \\
2.3\end{array}$ & $\begin{array}{l}-0 . \\
-0 . \\
-0 . \\
-0 . \\
-0 . \\
-0 .\end{array}$ & & $\begin{array}{l}0 . \\
0 . \\
0 . \\
0 . \\
0 . \\
0 . \\
0 . \\
0 .\end{array}$ \\
\hline $\begin{array}{l}1.0000 \\
1.2500 \\
1.5000 \\
1.7500 \\
2.0000 \\
2.5000 \\
3.0000 \\
3.5000\end{array}$ & $\begin{array}{l}995 E-01 \\
000 E+00 \\
007 E+00 \\
015 E+00 \\
024 E+00 \\
042 E+00 \\
059 E+00 \\
074 E+00\end{array}$ & $\begin{array}{l}1 . \\
1 . \\
2 .\end{array}$ & & & & $\begin{array}{l}3 . \\
4 . \\
4 . \\
5 . \\
6 .\end{array}$ & $\begin{array}{l}-0.105 \\
-0.098 \\
-0.093 \\
-0.088 \\
-0.081 \\
-0.076 \\
-0.072\end{array}$ & $\begin{array}{l}4 \\
6 \\
9 \\
3 \\
9 \\
1 \\
5 \\
0\end{array}$ & $\begin{array}{l}.126 \\
.117 \\
.109 \\
.103 \\
.097 \\
.088 \\
.081\end{array}$ \\
\hline $\begin{array}{l}4.00000 \\
4.5000 \\
5.0000 \\
5.5000 \\
6.0000 \\
7.0000 \\
8.0000 \\
9.0000\end{array}$ & $\begin{array}{l}1.1 \\
1.1 \\
1.1 \\
1.1 \\
1.1 \\
1.1 \\
1.1\end{array}$ & $\begin{array}{l}5 . \\
5 . \\
6 . \\
6 . \\
7 . \\
9 .\end{array}$ & $\begin{array}{l}1.601 E+00 \\
1.671 E+00 \\
1.740 E+00 \\
1.810 E+00 \\
1.948 E+00 \\
2.086 E+00 \\
2.224 E+00\end{array}$ & & $\begin{array}{l}.705 \mathrm{E}-01 \\
.850 \mathrm{E}-01 \\
.989 \mathrm{E}-01 \\
.121 \mathrm{E}-01 \\
.248 \mathrm{E}-01 \\
.488 \mathrm{E}-01 \\
.710 \mathrm{E}-01 \\
.916 \mathrm{E}-01\end{array}$ & $\begin{array}{l}1.2 \\
1: 2 \\
1: 2 \\
1.2\end{array}$ & $\begin{array}{l}-0.06 \\
-0.06 \\
-0.06 \\
-0.05 \\
-0.05 \\
-0.05 \\
-0.04\end{array}$ & $\begin{array}{l}5 \\
2 \\
9 \\
6 \\
3 \\
9 \\
5\end{array}$ & $\begin{array}{l}0 . \\
0 . \\
0 . \\
0 . \\
0 . \\
0 . \\
0 .\end{array}$ \\
\hline $\begin{array}{l}2.5000 \\
5.0000 \\
7.5000 \\
0.0000 \\
5.0000\end{array}$ & $\begin{array}{l}1.2 \\
1.2 \\
1.2 \\
1.2 \\
1.2 \\
1.3 \\
1.3\end{array}$ & $\begin{array}{l}1 . \\
2 . \\
2 . \\
3 . \\
3 . \\
4 .\end{array}$ & & & $\begin{array}{ll}0 & 1 \\
0 & 1 \\
0 & 1 \\
0 & 1 \\
0 & 1 \\
0 & 1 \\
0 & 1 \\
0 & 1\end{array}$ & & $\begin{array}{l}-0 . \\
-0 . \\
-0 . \\
-0 . \\
-0 .\end{array}$ & & $\begin{array}{l}0.035 \\
0.031 \\
0.027 \\
0.025 \\
0.021 \\
0.018 \\
0.016\end{array}$ \\
\hline $\begin{array}{l}45.0000 \\
50.0000 \\
55.0000 \\
60.0000 \\
70.0000\end{array}$ & $\begin{array}{l}1 \\
1 \\
1 \\
1 \\
1 \\
1\end{array}$ & 6 & & & & & $\begin{array}{l}-0 \\
-0 \\
-0 \\
-0 \\
-0 \\
-0 \\
-0\end{array}$ & $\begin{array}{l}4 \\
2 \\
1 \\
0 \\
9 \\
8 \\
6 \\
5\end{array}$ & \\
\hline 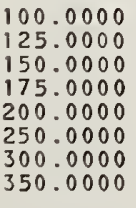 & $\begin{array}{l}1 \\
1 \\
1 \\
1 \\
1 \\
1\end{array}$ & $\begin{array}{l}1 \\
1 \\
1 \\
1 \\
1 \\
\end{array}$ & & & & & $\begin{array}{l}-0 \\
-0 \\
-0 \\
-0 \\
-0 \\
-0 \\
-0\end{array}$ & & $\begin{array}{l}05 \\
04 \\
03 \\
03 \\
02 \\
02 \\
02\end{array}$ \\
\hline $\begin{array}{l}.0000 \\
.0000 \\
.0000 \\
.0000\end{array}$ & $\begin{array}{l}1.5 \\
1.5 \\
1.5 \\
1.5 \\
1.5 \\
1.5 \\
1.5\end{array}$ & $\begin{array}{l}1 \\
1 \\
1 \\
1 \\
2 \\
2 \\
2\end{array}$ & $\begin{array}{l}+01 \\
+01 \\
+01 \\
+01 \\
+02 \\
+02 \\
+02\end{array}$ & $\begin{array}{l}1 \\
1 \\
1 \\
1 \\
1\end{array}$ & $\begin{array}{ll}0 & 1 \\
0 & 1 \\
0 & 1 \\
0 & 1 \\
0 & 1 \\
0 & 1 \\
0 & 1\end{array}$ & & $\begin{array}{l}3 \\
3 \\
2 \\
2 \\
2 \\
2 \\
2\end{array}$ & $\begin{array}{l}17 \\
16 \\
16 \\
16 \\
15 \\
15 \\
14 \\
14\end{array}$ & $\begin{array}{ll}0 & 2 \\
0 & 1 \\
0 & 1 \\
0 & 1 \\
0 & 1 \\
0 & 1 \\
0 & 1 \\
01\end{array}$ \\
\hline & $50 E+0$ & 02 & $15 E+02$ & $48 E+0$ & $534 E-01$ & 0 & 0.001 & 014 & \\
\hline
\end{tabular}




\begin{tabular}{|c|c|c|c|c|c|c|c|c|c|}
\hline \multirow{2}{*}{$\begin{array}{l}\text { ENERGY } \\
\text { MeV }\end{array}$} & \multicolumn{3}{|c|}{$\begin{array}{l}\text { STOPPING POWER } \\
\text { RADIATIVE }\end{array}$} & \multirow{2}{*}{$\begin{array}{l}\text { CSDA } \\
\text { RANGE } \\
g / \mathrm{cm}^{2}\end{array}$} & \multirow{2}{*}{$\begin{array}{l}\text { RADIATION } \\
\text { YIELD }\end{array}$} & \multirow{2}{*}{$\begin{array}{l}\text { DENS.EFF . } \\
\text { CORR. } \\
\text { (DELTA) }\end{array}$} & \multirow{2}{*}{$\begin{array}{l}\text { delo } \\
\operatorname{coll} \\
\text { LOSS }\end{array}$} & \multirow{2}{*}{$\begin{array}{l}\text { J)/d I } \\
\text { CSDA } \\
\text { RANGE }\end{array}$} & \multirow{2}{*}{$\begin{array}{l}\circ \text { Og I ) } \\
\text { RAD } \\
\text { YIELD }\end{array}$} \\
\hline & 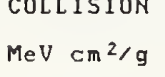 & $\begin{array}{l}\text { RADIATIVE } \\
\mathrm{MeV} \mathrm{cm}^{2} / \mathrm{g}\end{array}$ & $\begin{array}{c}\text { TOTAL } \\
\mathrm{MeV} \mathrm{cm}^{2} / \mathrm{g}\end{array}$ & & & & & & \\
\hline $\begin{array}{l}0.0100 \\
0.0125 \\
0.0150 \\
0.0175 \\
0.0200 \\
0.0250 \\
0.0300 \\
0.0350\end{array}$ & $\begin{array}{l}673 E+00 \\
562 E+00 \\
739 E+00 \\
103 E+00 \\
595 E+00 \\
832 E+00 \\
284 E+00 \\
870 E+00\end{array}$ & $\begin{array}{l}2.2 \\
2.4 \\
2.5 \\
2.6 \\
2.8 \\
3.0 \\
3.1\end{array}$ & $\begin{array}{l}8 . \\
7 . \\
6: \\
6: \\
5: \\
4: \\
3 .\end{array}$ & $\begin{array}{l}917 \mathrm{E}-04 \\
101 \mathrm{E}-03 \\
450 \mathrm{E}-03\end{array}$ & $\begin{array}{l}1.150 E-03 \\
1.449 E-03 \\
1.749 E-03 \\
2.050 E-03 \\
2.349 E-03 \\
2.945 E-03 \\
3.534 E-03 \\
4.117 E-03\end{array}$ & $\begin{array}{l}6.025 \mathrm{E}-04 \\
7.709 \mathrm{E}-04 \\
9.470 \mathrm{E}-04 \\
1.131 \mathrm{E}-03 \\
1.323 \mathrm{E}-03 \\
1.735 \mathrm{E}-03 \\
2.183 \mathrm{E}-03 \\
2.673 \mathrm{E}-03\end{array}$ & $\begin{array}{l}-0.373 \\
-0.345 \\
-0.325 \\
-0.310 \\
-0.298 \\
-0.279 \\
-0.266 \\
-0.256\end{array}$ & $\begin{array}{l}665 \\
579 \\
520 \\
476 \\
444 \\
397 \\
365 \\
341\end{array}$ & $\begin{array}{l}0.519 \\
0.460 \\
0.421 \\
0.392 \\
0.371 \\
0.340 \\
0.3199 \\
0.303\end{array}$ \\
\hline $\begin{array}{l}0.0400 \\
0.0450 \\
0.0500 \\
0.0550 \\
0.0600 \\
0.0700 \\
0.0800 \\
0.0900\end{array}$ & $\begin{array}{l}3.545 \mathrm{E}+00 \\
3.283 \mathrm{E}+00 \\
3.067 \mathrm{E}+00 \\
2.885 \mathrm{E}+00 \\
2.730 \mathrm{E}+00 \\
2.480 \mathrm{E}+00 \\
2.286 \mathrm{E}+00 \\
2.132 \mathrm{E}+00\end{array}$ & $\begin{array}{l}3.321 \mathrm{E}-02 \\
3.439 \mathrm{E}-02 \\
3.547 \mathrm{E}-02 \\
3.647 \mathrm{E}-02 \\
3.741 \mathrm{E}-02 \\
3.914 \mathrm{E}-02 \\
4.070 \mathrm{E}-02 \\
4.214 \mathrm{E}-02\end{array}$ & $\begin{array}{l}3.1 \\
2.9\end{array}$ & $\begin{array}{l}-03 \\
-03 \\
-03 \\
-03 \\
-02 \\
-02 \\
-02 \\
-02\end{array}$ & $\begin{array}{l}4.6 \\
5.2 \\
5.8 \\
6.3 \\
6.9 \\
8.0 \\
9.0 \\
1.0\end{array}$ & $\begin{array}{l}3.208 \mathrm{E}-03 \\
3.791 \mathrm{E}-03 \\
4.427 \mathrm{E}-03 \\
5.121 \mathrm{E}-03 \\
5.877 \mathrm{E}-03 \\
7.597 \mathrm{E}-03 \\
9.624 \mathrm{E}-03 \\
1.199 \mathrm{E}-02\end{array}$ & $\begin{array}{l}-0.248 \\
-0.241 \\
-0.235 \\
-0.230 \\
-0.225 \\
-0.217 \\
-0.211 \\
-0.205\end{array}$ & $\begin{array}{l}.324 \\
.309 \\
.298 \\
.288 \\
.279 \\
.266 \\
.255 \\
.246\end{array}$ & $\begin{array}{l}0.291 \\
0.280 \\
0.272 \\
0.265 \\
0.258 \\
0.248 \\
0.239 \\
0.232\end{array}$ \\
\hline $\begin{array}{l}0.1000 \\
0.1250 \\
0.1500 \\
0.1750 \\
0.2000 \\
0.2500 \\
0.3000 \\
0.3500\end{array}$ & $\begin{array}{l}2.006 E+00 \\
1.773 E+00 \\
1.614 E+00 \\
1.499 E+00 \\
1.412 E+00 \\
1.291 E+00 \\
1.212 E+00 \\
1.157 E+00\end{array}$ & $\begin{array}{l}4.348 \mathrm{E}-02 \\
4.651 \mathrm{E}-02 \\
4.920 \mathrm{E}-02 \\
5.164 \mathrm{E}-02 \\
5.395 \mathrm{E}-02 \\
5.833 \mathrm{E}-02 \\
6.256 \mathrm{E}-02 \\
6.677 \mathrm{E}-02\end{array}$ & $\begin{array}{l}2.049 E+00 \\
1.819 E+00 \\
1.663 E+00 \\
1.550 E+00 \\
1.466 E+00 \\
1.349 E+00 \\
1.274 E+00 \\
1.224 E+00\end{array}$ & $\begin{array}{l}3.044 \mathrm{E}-02 \\
4.343 \mathrm{E}-02 \\
5.784 \mathrm{E}-02 \\
7.343 \mathrm{E}-02 \\
9.004 \mathrm{E}-02 \\
1.257 \mathrm{E}-01 \\
1.639 \mathrm{E}-01 \\
2.040 \mathrm{E}-01\end{array}$ & $\begin{array}{l}1.114 \mathrm{E}-02 \\
1.360 \mathrm{E}-02 \\
1.593 \mathrm{E}-02 \\
1.815 \mathrm{E}-02 \\
2.027 \mathrm{E}-02 \\
2.423 \mathrm{E}-02 \\
2.789 \mathrm{E}-02 \\
3.132 \mathrm{E}-02\end{array}$ & $\begin{array}{l}1.471 \mathrm{E}-02 \\
2.288 \mathrm{E}-02 \\
3.231 \mathrm{E}-02 \\
4.212 \mathrm{E}-02 \\
5.187 \mathrm{E}-02 \\
7.074 \mathrm{E}-02 \\
8.883 \mathrm{E}-02 \\
1.063 \mathrm{E}-01\end{array}$ & $\begin{array}{l}-0.200 \\
-0.189 \\
-0.181 \\
-0.174 \\
-0.169 \\
-0.161 \\
-0.155 \\
-0.150\end{array}$ & $\begin{array}{l}0.239 \\
0.224 \\
0.213 \\
0.204 \\
0.197 \\
0.186 \\
0.178 \\
0.171\end{array}$ & $\begin{array}{l}0.226 \\
0.213 \\
0.203 \\
0.196 \\
0.189 \\
0.179 \\
0.171 \\
0.165\end{array}$ \\
\hline $\begin{array}{l}0.4000 \\
0.4500 \\
0.5000 \\
0.5500 \\
0.6000 \\
0.7000 \\
0.8000 \\
0.9000\end{array}$ & $\begin{array}{l}1.118 \mathrm{E}+00 \\
1.089 \mathrm{E}+00 \\
1.067 \mathrm{E}+00 \\
1.051 \mathrm{E}+00 \\
1.038 \mathrm{E}+00 \\
1.021 \mathrm{E}+00 \\
1.011 \mathrm{E}+00 \\
1.006 \mathrm{E}+00\end{array}$ & $\begin{array}{l}7.5 \\
7.5 \\
8.3 \\
8.8 \\
9.7 \\
1.8 \\
1.1\end{array}$ & $\begin{array}{l}1.189 E+00 \\
1.164 E+00 \\
1.147 E+00 \\
1.135 E+00 \\
1.127 E+00 \\
1.119 E+00 \\
1.118 E+00 \\
1.122 E+00\end{array}$ & 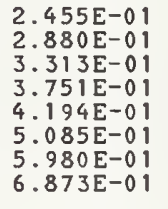 & & $\begin{array}{l}1.2 \\
1.4 \\
1.5 \\
1.7 \\
1.8 \\
2.8\end{array}$ & & $\begin{array}{l}7 \\
7 \\
4 \\
0 \\
5\end{array}$ & $\begin{array}{l}0.160 \\
0.155 \\
0.151 \\
0.147 \\
0.144 \\
0.138 \\
0.133 \\
0.128\end{array}$ \\
\hline $\begin{array}{l}1.0000 \\
1.2500 \\
1.5000 \\
1.7500 \\
2.0000 \\
2.5000 \\
3.0000 \\
3.5000\end{array}$ & $\begin{array}{l}004 E+00 \\
005 E+00 \\
011 E+00 \\
020 E+00 \\
029 E+00 \\
048 E+00 \\
065 E+00 \\
080 E+00\end{array}$ & $\begin{array}{l}1.5 \\
1.7 \\
1.5 \\
2.2 \\
3.7\end{array}$ & $\begin{array}{l}1.186 \mathrm{E}+0 \\
1.220 \mathrm{E}+0 \\
1.255 \mathrm{E}+0 \\
1.327 \mathrm{E}+0 \\
1.400 \mathrm{E}+0 \\
1.472 \mathrm{E}+0\end{array}$ & $\begin{array}{l}2.006 \mathrm{E}+0 \\
2.373 \mathrm{E}+0 \\
2.721 \mathrm{E}+0\end{array}$ & & $\begin{array}{l}1 \\
1 \\
1 \\
1 \\
1 \\
1\end{array}$ & $\begin{array}{l}-0.1 \\
-0.1 \\
-0.0 \\
-0.0 \\
-0.0 \\
-0.0 \\
-0.0 \\
-0.0\end{array}$ & $\begin{array}{l}124 \\
118 \\
112 \\
107 \\
100 \\
094 \\
089\end{array}$ & $\begin{array}{l}0 . \\
0 . \\
0 . \\
0 . \\
0 . \\
0 . \\
0 .\end{array}$ \\
\hline $\begin{array}{l}4.0000 \\
4.5000 \\
5.0000 \\
5.5000 \\
6.0000 \\
7.0000 \\
8.0000 \\
9.0000\end{array}$ & $\begin{array}{l}1.095 E+00 \\
1.107 E+00 \\
1.119 E+00 \\
1.130 E+00 \\
1.139 E+00 \\
1.157 E+00 \\
1.172 E+00 \\
1.185 E+00\end{array}$ & $\begin{array}{l}5.6 \\
6.2 \\
6.8\end{array}$ & $\begin{array}{l}1 . \\
1 . \\
1: \\
1: \\
1 . \\
1 . \\
2 . \\
2 .\end{array}$ & & & $\left\{\begin{array}{l}0 \\
0 \\
0 \\
0 \\
0\end{array}\right.$ & $\begin{array}{l}-0.067 \\
-0.064 \\
-0.062 \\
-0.059 \\
-0.057 \\
-0.054 \\
-0.051 \\
-0.048\end{array}$ & $\begin{array}{l}0 . \\
0 . \\
0 . \\
0 . \\
0 .\end{array}$ & $\begin{array}{l}0.069 \\
0.065 \\
0.061 \\
0.058 \\
0.055 \\
0.050 \\
0.046 \\
0.043\end{array}$ \\
\hline $\begin{array}{l}10.0000 \\
12.5000 \\
15.0000 \\
17.5000 \\
20.0000 \\
25.0000 \\
30.0000 \\
35.0000\end{array}$ & $\begin{array}{l}1.196 E+00 \\
1.221 E+00 \\
1.240 E+00 \\
1.256 E+00 \\
1.270 E+00 \\
1.292 E+00 \\
1.310 E+00 \\
1.325 E+00\end{array}$ & $\begin{array}{l}1.188 \mathrm{E}+00 \\
1.513 \mathrm{E}+00 \\
1.843 \mathrm{E}+00 \\
2.179 \mathrm{E}+00 \\
2.518 \mathrm{E}+00 \\
3.207 \mathrm{E}+00 \\
3.907 \mathrm{E}+00 \\
4.615 \mathrm{E}+00\end{array}$ & $\begin{array}{l}2.733 E+00 \\
3.083 E+00 \\
3.435 E+00 \\
3.788 E+00 \\
4.500 E+00 \\
5.217 E+00 \\
5.939 E+00\end{array}$ & & & $\begin{array}{l}0 \\
0 \\
0 \\
0 \\
0\end{array}$ & & & \\
\hline $\begin{array}{l}0 \\
0 \\
0 \\
0 \\
0 \\
0 \\
0\end{array}$ & $\begin{array}{l}1.3 \\
1.3 \\
1.3 \\
1: 3 \\
1.3\end{array}$ & $\begin{array}{l}0 \\
0 \\
0 \\
0 \\
0\end{array}$ & & & & & $\begin{array}{l}-0 \\
-0 \\
-0 \\
-0 \\
-0 \\
-0 \\
-0\end{array}$ & & \\
\hline 0.0000 & $\begin{array}{l}1.455 E+00 \\
1.464 E+00 \\
1.480 E+00 \\
1.492 E+00 \\
1.503 E+00\end{array}$ & $\begin{array}{l}2 . \\
3 . \\
3 .\end{array}$ & $\begin{array}{l}2 \\
2 \\
3 \\
3 \\
4 \\
5\end{array}$ & $\begin{array}{l}1 \\
1 \\
1 \\
1 \\
1 \\
1\end{array}$ & & $\begin{array}{l}0 \\
0 \\
0\end{array}$ & & & \\
\hline $\begin{array}{l}.0000 \\
.0000 \\
.0000 \\
.0000 \\
.0000\end{array}$ & $\begin{array}{l}1.52 \\
1.53 \\
1.53 \\
1.52 \\
1.55\end{array}$ & $\begin{array}{l}7 . \\
8 . \\
9 \\
1 \\
1 .\end{array}$ & $\begin{array}{l}6 \\
7 \\
8 \\
9 \\
1 \\
1 \\
1\end{array}$ & $\begin{array}{l}3.265 \mathrm{E}+ \\
3.342 \mathrm{E}+\end{array}$ & $\begin{array}{l}9.449 \mathrm{E}-01 \\
9.497 \mathrm{E}-01\end{array}$ & $\begin{array}{l}0 \\
0 \\
0\end{array}$ & $\begin{array}{l}3 \\
3 \\
3 \\
3 \\
2 \\
2 \\
2 \\
2\end{array}$ & $\begin{array}{l}0.0116 \\
0.016 \\
0.016 \\
0.015 \\
0.015 \\
0.015 \\
0.014 \\
0.014\end{array}$ & $\begin{array}{l}0.002 \\
0.001 \\
0.001 \\
0.001 \\
0.001 \\
0.001 \\
0.001 \\
0.001\end{array}$ \\
\hline .0 & $.571 E+00$ & $1.518 E+02$ & $1.533 E+02$ & $.410 E+01$ & $9.536 E-01$ & $605 E+00$ & -0.001 & 0.014 & .001 \\
\hline
\end{tabular}




\begin{tabular}{|c|c|c|c|c|c|c|c|c|c|}
\hline \multirow{2}{*}{$\begin{array}{l}\text { ENERGY } \\
\text { MeV }\end{array}$} & \multicolumn{3}{|c|}{$\begin{array}{l}\text { STOPPING POWER } \\
\text { N RADIATIVE }\end{array}$} & \multirow{2}{*}{$\begin{array}{l}\text { CSDA } \\
\text { RANGE } \\
\mathrm{g} / \mathrm{cm}^{2}\end{array}$} & \multirow[t]{2}{*}{$\begin{array}{l}\text { RADIATION } \\
\text { YIELD }\end{array}$} & \multirow[t]{2}{*}{$\begin{array}{l}\text { DEYS. EFF. } \\
\text { CORR. } \\
\text { (DELTA) }\end{array}$} & \multirow{2}{*}{\multicolumn{3}{|c|}{$\begin{array}{l}d(\log ) / d(\log I) \\
\text { COLL } \operatorname{cSDA} \text { RAD } \\
\text { LOSS RANGE YIELD }\end{array}$}} \\
\hline & $g$ & 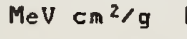 & $c m^{2} / g$ & & & & & & \\
\hline $\begin{array}{l}0.010 \\
0.012 \\
0.015 \\
0.017 \\
0.020 \\
0.025 \\
0.030\end{array}$ & $\begin{array}{l}8.4 \\
7.3 \\
6.5 \\
5.9 \\
5.4 \\
4.7\end{array}$ & $\begin{array}{l}2.2 \\
2.4 \\
2.5 \\
2.6 \\
2.9 \\
3.0 \\
3\end{array}$ & $\begin{array}{l}7.3 \\
6.5 \\
5.9 \\
5.4 \\
4.7\end{array}$ & $\begin{array}{l}4 \\
3 \\
3 \\
3 \\
3 \\
3 \\
3 \\
3\end{array}$ & $\begin{array}{l}3 \\
3 \\
3 \\
3 \\
3 \\
3 \\
3 \\
3\end{array}$ & $\begin{array}{l}4 \\
4 \\
4 \\
4 \\
3 \\
3 \\
3\end{array}$ & & & $\begin{array}{l}0.539 \\
0.475 \\
0.432 \\
0.402 \\
0.379 \\
0.347 \\
0.324 \\
0.308\end{array}$ \\
\hline $\begin{array}{l}0.0400 \\
0.0450 \\
0.0500 \\
0.0550 \\
0.0600 \\
0.0700 \\
0.0800\end{array}$ & $\begin{array}{l}2.9 \\
2.8 \\
2.6 \\
2.4 \\
2.2\end{array}$ & $\begin{array}{l}3.8 \\
3.7 \\
3.8 \\
3.8 \\
4 . \\
4 .\end{array}$ & $\begin{array}{l}3.034 E+00 \\
2.858 E+00 \\
2.708 E+00 \\
2.466 E+00 \\
2.279 E+00 \\
2.130 E+00\end{array}$ & & & .41 & & & $\begin{array}{l}0.295 \\
0.284 \\
0.276 \\
0.268 \\
0.262 \\
0.251 \\
0.242 \\
0.235\end{array}$ \\
\hline $\begin{array}{l}0.1000 \\
0.1250 \\
0.1500 \\
0.1750 \\
0.2000 \\
0.2500 \\
0.3000\end{array}$ & $\begin{array}{l}1.96,4 E+00 \\
1.733 E+00 \\
1.583 E+00 \\
1.471 E+00 \\
1.387 E+00 \\
1.269 E+00 \\
1.193 E+00 \\
1.140 E+00\end{array}$ & $\begin{array}{l}5 \cdot 0 \\
5 \cdot 3 \\
5 \cdot 5\end{array}$ & $\begin{array}{l}1.6 \\
1.5 \\
1.9 \\
1.3 \\
1.2\end{array}$ & $\begin{array}{l}2 \\
2 \\
2 \\
1 \\
1\end{array}$ & & 7.39 & & & $\begin{array}{l}0.229 \\
0.217 \\
0.208 \\
0.201 \\
0.195 \\
0.186 \\
0.178 \\
0.172\end{array}$ \\
\hline $\begin{array}{l}0.4000 \\
0.4500 \\
0.5000 \\
0.5500 \\
0.6000 \\
0.7000 \\
0.8000 \\
0.9000\end{array}$ & $\begin{array}{l}1.102 E+00 \\
1.074 E+00 \\
1.053 E+00 \\
1.037 E+00 \\
1.026 E+00 \\
1.009 E+00 \\
1.000 E+00 \\
9.957 E-01\end{array}$ & $\begin{array}{l}8.2 \\
8.6 \\
9.1 \\
1.0 \\
1.0 \\
1.1\end{array}$ & $\begin{array}{l}175 E+00 \\
152 E+00 \\
135 E+00 \\
124 E+00 \\
117 E+00 \\
1110 E+00 \\
110 E+00 \\
115 E+00\end{array}$ & $\begin{array}{l}494 \mathrm{E}-01 \\
924 \mathrm{E}-01 \\
361 \mathrm{E}-01 \\
804 \mathrm{E}-01 \\
250 \mathrm{E}-01 \\
149 \mathrm{E}-01\end{array}$ & $\begin{array}{l}2 \\
2 \\
2 \\
2 \\
2 \\
2 \\
2 \\
2\end{array}$ & $\begin{array}{l}7 . \\
8: \\
9\end{array}$ & & & $\begin{array}{l}0.166 \\
0.162 \\
0.157 \\
0.154 \\
0.150 \\
0.144 \\
0.139\end{array}$ \\
\hline $\begin{array}{l}1.0000 \\
1.2500 \\
1.5000 \\
1.7500 \\
2.0000 \\
2.5000 \\
3.0000 \\
3.5000\end{array}$ & $\begin{array}{l}1 \\
1 \\
0 \\
0 \\
0 \\
0\end{array}$ & $\begin{array}{l}1 \\
1\end{array}$ & $\begin{array}{l}1 . \\
1.1 \\
1.2 \\
1: 2 \\
1: 3 \\
1: 2\end{array}$ & & $\begin{array}{l}9: \\
1: \\
1:\end{array}$ & $\begin{array}{l}1.809 E-0 \\
2.337 \mathrm{E}-0 \\
2.854 \mathrm{E}-0 \\
3.360 \mathrm{E}-0 \\
3.855 \mathrm{E}-0 \\
4.817 \mathrm{E}-0 \\
5.743 \mathrm{E}-0 \\
6.631 \mathrm{E}-0\end{array}$ & & & \\
\hline $\begin{array}{l}4.0000 \\
4.5000 \\
5.0000 \\
5.5000 \\
6.0000 \\
7.0000 \\
8.0000 \\
9.0000\end{array}$ & $\begin{array}{l}0 \\
0 \\
0 \\
0 \\
0\end{array}$ & 然 & i: & 然 & & & & & $\begin{array}{l}0.070 \\
0.066 \\
0.063 \\
0.060 \\
0.054 \\
0.050 \\
0.046\end{array}$ \\
\hline $\begin{array}{l}15.00 \\
17.50 \\
20.00 \\
25.00\end{array}$ & $\begin{array}{l}1 \\
1 \\
1 \\
1 \\
1 \\
1\end{array}$ & 2. & 3. & & & & & & \\
\hline $\begin{array}{l}0 \\
0 \\
0 \\
0\end{array}$ & $\begin{array}{l}1 \\
1 \\
1 \\
1\end{array}$ & & & & & & & & \\
\hline 0 & $\begin{array}{l}1 \\
1 \\
1 \\
1 \\
1\end{array}$ & 2. & 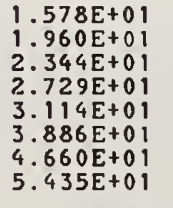 & & & & & & \\
\hline $\begin{array}{l}5 \\
6 \\
7\end{array}$ & $5 / 6 \mathrm{E}+00$ & $\begin{array}{l}8 \\
9 \\
1 \\
1\end{array}$ & & & & & & & 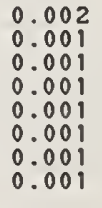 \\
\hline & $583 E+00$ & $.539 E+02$ & $.555 E+02$ & $373 E+0$ & $.539 E-01$ & $986 E+0$ & 0.002 & .015 & 0.001 \\
\hline
\end{tabular}




\begin{tabular}{|c|c|c|c|c|c|c|c|c|c|}
\hline $\mathrm{MeV}$ & $\mathrm{MeV} \mathrm{cm}^{2} / \mathrm{g}$ & $\mathrm{MeV} \mathrm{cm} 2 / \mathrm{g}$ & $\begin{array}{l}\text { TOTAL } \\
\mathrm{eV} \mathrm{cm}^{2} / \mathrm{g}\end{array}$ & $\begin{array}{l}\text { CSDA } \\
\text { RANGE } \\
\mathrm{g} / \mathrm{Cm}^{2}\end{array}$ & $Y$ & $\begin{array}{l}\text { DENS.EFF. } \\
\text { CORR } \\
\text { (DELTA) }\end{array}$ & $\begin{array}{l}d(1 \\
\operatorname{coLL} \\
\operatorname{LOSS}\end{array}$ & $\begin{array}{l}\text { SDA } \\
\text { ANGE }\end{array}$ & $\begin{array}{l}\text { (I ) } \\
\text { RAD } \\
\text { YIEL D }\end{array}$ \\
\hline $\begin{array}{l}0.0100 \\
0.0125 \\
0.0150 \\
0.0175 \\
0.0200 \\
0.0250 \\
0.0300 \\
0.0350\end{array}$ & $\begin{array}{l}6.241 \\
5.662 \\
5.198 \\
4.499 \\
3\end{array}$ & $\begin{array}{l}2.35 \\
2.51 \\
2.67 \\
2.82 \\
3.06 \\
3.27 \\
3.45\end{array}$ & $\begin{array}{l}5.6 \\
5.2 \\
4.5 \\
4.0\end{array}$ & $\begin{array}{l}-04 \\
-03 \\
-03 \\
-03 \\
-03 \\
-03 \\
-03 \\
-03\end{array}$ & $\begin{array}{l}1 . \\
1 . \\
2 . \\
3 . \\
4 .\end{array}$ & $\begin{array}{l}2 . \\
2 . \\
3 . \\
4 . \\
5 . \\
6 . \\
7 .\end{array}$ & $\begin{array}{l}-0 \\
-0 \\
-0 \\
-0 \\
-0 \\
-0 \\
-0\end{array}$ & $\begin{array}{l}8 \\
9 \\
1 \\
9 \\
1 \\
5 \\
1 \\
0\end{array}$ & $\begin{array}{l}0.589 \\
0.510 \\
0.459 \\
0.424 \\
0.397 \\
0.360 \\
0.335 \\
0.317\end{array}$ \\
\hline $\begin{array}{l}0.0400 \\
0.0450 \\
0.0500 \\
0.0550 \\
0.0600 \\
0.0700 \\
0.0800 \\
0.0900\end{array}$ & $\begin{array}{l}313 E+00 \\
070 E+00 \\
870 E+00 \\
701 E+00 \\
557 E+00 \\
325 E+00 \\
144 E+00 \\
001 E+00\end{array}$ & $\begin{array}{l}3.8 \\
4.0 \\
4.1 \\
4.3 \\
4.5\end{array}$ & $\begin{array}{l}2.9 \\
2.7 \\
2.5 \\
2.3 \\
2.1 \\
2.0\end{array}$ & $\begin{array}{l}0.2 \\
1.0 \\
1.2 \\
1.2 \\
1.8 \\
2.2 \\
2.7\end{array}$ & & $\begin{array}{l}9.2 \\
1.2 \\
1.2 \\
1.2 \\
1.5 \\
1.8 \\
2.8 \\
2.8\end{array}$ & & & $\begin{array}{l}0.281 \\
0.273 \\
0.266 \\
0.255 \\
0.246 \\
0.238\end{array}$ \\
\hline $\begin{array}{l}0.1000 \\
0.1250 \\
0.1500 \\
0.1750 \\
0.2000 \\
0.2500 \\
0.3000 \\
0.3500\end{array}$ & $\begin{array}{l}1.833 \mathrm{E}+00 \\
1.666 \mathrm{E}+00 \\
1.518 \mathrm{E}+00 \\
1.411 \mathrm{E}+00 \\
1.330 \mathrm{0}+00 \\
1.218 \mathrm{E}+00 \\
1.144 \mathrm{E}+00 \\
1.033 \mathrm{E}+00\end{array}$ & $\begin{array}{l}5.2 \\
5.5 \\
5.8 \\
6.1 \\
6.7 \\
7.2\end{array}$ & $\begin{array}{l}.932 E+00 \\
.719 E+00 \\
.574 E+00 \\
.470 E+00 \\
.392 E+00 \\
.285 E+00 \\
.217 E+00\end{array}$ & $\begin{array}{l}49 E-02 \\
26 E-02 \\
49 E-02 \\
95 E-02 \\
44 E-02 \\
29 E-01\end{array}$ & $\begin{array}{l}.308 \mathrm{E}-02 \\
.605 \mathrm{E}-02 \\
.888 \mathrm{E}-02 \\
.159 \mathrm{E}-02 \\
.417 \mathrm{E}-02 \\
.903 \mathrm{E}-02\end{array}$ & $\begin{array}{l}2 . \\
3 . \\
4 . \\
5 \\
5 . \\
7 .\end{array}$ & $\begin{array}{l}-0 . \\
-0 . \\
-0 \\
-0 \\
-0 \\
-0 \\
-0 \\
-0 \\
-0\end{array}$ & & \\
\hline $\begin{array}{l}0.4000 \\
0.4500 \\
0.5000 \\
0.5500 \\
0.6000 \\
0.7000 \\
0.8000 \\
0.9000\end{array}$ & $\begin{array}{l}057 E+00 \\
031 E+00 \\
011 E+00 \\
956 E-01 \\
842 E-01 \\
690 E-01 \\
604 E-01 \\
560 E=01\end{array}$ & $294 \mathrm{E}-02$ & $\begin{array}{ll}0 & 0 \\
0 & 0 \\
0 & 0 \\
0 & 0 \\
0 & 0 \\
0 & 0\end{array}$ & $\left\{\begin{array}{l}1 \\
1 \\
1 \\
1\end{array}\right.$ & $\begin{array}{l}2 \\
2 \\
2 \\
2 \\
2 \\
2 \\
2\end{array}$ & $\begin{array}{l}1 . \\
1: \\
1: \\
1: \\
1 .\end{array}$ & $\begin{array}{l}-0 \\
-0 \\
-0 \\
-0 \\
-0 \\
-0 \\
-0 \\
-0\end{array}$ & & \\
\hline $\begin{array}{l}1.0000 \\
1.2500 \\
1.5000 \\
1.7500 \\
2.0000 \\
2.5000 \\
3.0000 \\
3.5000\end{array}$ & $\begin{array}{l}1 \\
1 \\
1 \\
0 \\
0\end{array}$ & $\begin{array}{l}2 \\
2 \\
3\end{array}$ & $\begin{array}{l}0 \\
0 \\
0 \\
0 \\
0 \\
0\end{array}$ & $\begin{array}{l}1 \\
0 \\
0 \\
0 \\
0 \\
0\end{array}$ & & i & & & \\
\hline $\begin{array}{l}4.0000 \\
4.5000 \\
5.0000 \\
5.5000 \\
6.0000 \\
7.0000 \\
8.0000 \\
9.0000\end{array}$ & $\begin{array}{l}1.07 \\
1.09 \\
1.10\end{array}$ & $\begin{array}{l}6.2 \\
6.8 \\
7.5 \\
8.8\end{array}$ & $\begin{array}{l}1 \\
0 \\
0 \\
0 \\
0 \\
0\end{array}$ & $\begin{array}{l}0 \\
0 \\
0 \\
0 \\
0\end{array}$ & $\begin{array}{l}i \\
\vdots \\
i \\
i\end{array}$ & 1 & $\begin{array}{l}-0 \\
-0 \\
-0 \\
-0 \\
-0 \\
-0 \\
-0 \\
-0\end{array}$ & & \\
\hline $\begin{array}{l}10.0000 \\
12.5000 \\
15.0000 \\
17.5000 \\
20.0000 \\
25.0000 \\
30.0000 \\
35.0000\end{array}$ & 8 & 2 & $\begin{array}{l}0 \\
0 \\
0 \\
0 \\
0 \\
0\end{array}$ & $\begin{array}{l}0 \\
0 \\
0 \\
0 \\
1\end{array}$ & & $\begin{array}{l}1 \\
1 \\
2 \\
2 \\
2 . \\
2 . \\
3 \\
3\end{array}$ & & & \\
\hline $\begin{array}{l}50.0 \\
55.0 \\
60.0 \\
70.0 \\
80.0\end{array}$ & 80 & $\begin{array}{l}33 E+00 \\
05 E+00 \\
82 E+00 \\
63 E+00 \\
47 E+00 \\
43 E+01 \\
01 E+01 \\
61 E+01\end{array}$ & $\begin{array}{l}0 \\
0 \\
0 \\
1\end{array}$ & & & & & & \\
\hline $\begin{array}{l}0 \\
0 \\
0 \\
0 \\
0\end{array}$ & & & & & & & & & \\
\hline $\begin{array}{l}0 \\
0 \\
0 \\
0 \\
0 \\
0 \\
0 \\
0\end{array}$ & $\$ 11 E+00$ & $\begin{array}{l}1 \\
2 \\
2 \\
2\end{array}$ & $\begin{array}{l}1 \\
1 \\
2 \\
2\end{array}$ & $\begin{array}{c}i \\
1 \\
i\end{array}$ & $\begin{array}{l}1 \\
1 \\
1 \\
1 \\
1 \\
1 \\
1\end{array}$ & $\begin{array}{l}8.8 \\
9.1\end{array}$ & $\begin{array}{l}-0 \\
-0 \\
-0 \\
-0 \\
-0 \\
-0 \\
-0 \\
-0\end{array}$ & & $\begin{array}{l}01 \\
0 \\
01 \\
01 \\
01 \\
01 \\
01\end{array}$ \\
\hline & $.517 E+00$ & $633 E+02$ & 02 & $E+01$ & $5 E-01$ & 0 & 001 & 15 & \\
\hline
\end{tabular}




\begin{tabular}{|c|c|c|c|c|c|c|c|c|c|}
\hline ENERGY & $\begin{array}{l}\text { COLLISION } \\
\text { MoV } \mathrm{cm}^{2} / \mathrm{g}\end{array}$ & $\begin{array}{l}\text { RADIATIVE } \\
\text { MOV } \mathrm{cm}^{2} / \mathrm{g}\end{array}$ & $\begin{array}{c}\text { TOTAL } \\
\text { MOV } \mathrm{cm}^{2} / \mathrm{g}\end{array}$ & $\begin{array}{l}\text { CSDA } \\
\text { RANGE } \\
\mathrm{g} / \mathrm{cm}^{2}\end{array}$ & $\begin{array}{l}\text { ADI ATION } \\
\text { YIELD }\end{array}$ & $\begin{array}{c}\text { DENS EFF . } \\
\text { CORR. } \\
\text { (DELTA) }\end{array}$ & $\begin{array}{l}\text { d( } 108 \\
\text { coLL } \\
\text { LOSS }\end{array}$ & $\begin{array}{l}\text { JOd ( } 1 \\
\text { SDA } \\
\text { SANGE }\end{array}$ & $\begin{array}{l}\text { g I ) } \\
\text { RAD } \\
\text { YIELD }\end{array}$ \\
\hline $\begin{array}{l}0.0100 \\
0.0125 \\
0.0150 \\
0.0175 \\
0.0200 \\
0.0250 \\
0.0300 \\
0.0350\end{array}$ & $\begin{array}{l}2.294 E+01 \\
1.927 E+01 \\
1.671 E+01 \\
1.482 E+01 \\
1.335 E+01 \\
1.124 E+01 \\
9.769 E+00 \\
8.691 E+00\end{array}$ & $\begin{array}{l}3.156 E-03 \\
3.174 E-03 \\
3.188 E-03 \\
3.197 E-03 \\
3.205 E-03 \\
3.219 E-03 \\
3.232 E-03\end{array}$ & $\begin{array}{l}2.295 \mathrm{E}+01 \\
1.927 \mathrm{E}+01 \\
1.671 \mathrm{E}+01 \\
1.482 \mathrm{E}+01 \\
1.336 \mathrm{E}+01 \\
1.124 \mathrm{E}+01 \\
9.772 \mathrm{E}+00\end{array}$ & $\begin{array}{l}2.46 \\
3.65 \\
5.05 \\
6.64 \\
8.42 \\
1.25 \\
1.73\end{array}$ & $\begin{array}{l}7.529 E-05 \\
9.048 E-05 \\
1.050 E-04 \\
1.191 E-04 \\
1.327 E-04 \\
1.588 E-04 \\
1.838 E-04 \\
2.079 E-04\end{array}$ & $\begin{array}{l}0.0 \\
0.0 \\
0.0 \\
0.0 \\
0.0 \\
0.0 \\
0.0 \\
0.0\end{array}$ & $\begin{array}{l}-0.193 \\
-0.186 \\
-0.180 \\
-0.175 \\
-0.179 \\
-0.165 \\
-0.160 \\
-0.156\end{array}$ & $\begin{array}{l}0.220 \\
0.210 \\
0.202 \\
0.196 \\
0.191 \\
0.183 \\
0.178 \\
0.173\end{array}$ & $\begin{array}{l}0.219 \\
0.209 \\
0.201 \\
0.195 \\
0.190 \\
0.183 \\
0.177\end{array}$ \\
\hline $\begin{array}{l}0.0400 \\
0.0450 \\
0.0500 \\
0.0550 \\
0.0600 \\
0.0700 \\
0.0800 \\
0.0900\end{array}$ & $\begin{array}{l}7.2 \\
6.6 \\
6.2 \\
5.8 \\
5.2 \\
4.8\end{array}$ & $\begin{array}{l}3 \cdot 2 \\
3 \cdot 2 \\
3 \cdot 3 \\
3 \cdot 3 \\
3 \cdot 3 \\
3 \cdot 3\end{array}$ & $\begin{array}{l}+00 \\
+00 \\
+00 \\
+00 \\
+00 \\
+00 \\
+00 \\
+00\end{array}$ & $\begin{array}{l}3.5 \\
4.2 \\
5.8 \\
7.8 \\
9.8\end{array}$ & $\begin{array}{l}2 . \\
2 . \\
2 . \\
3 . \\
3 . \\
3 .\end{array}$ & $\begin{array}{l}0.0 \\
0.0 \\
0.0 \\
0.0 \\
0.0 \\
0.0 \\
0.0 \\
0.0\end{array}$ & $\begin{array}{l}-0 \\
-0 \\
-0 \\
-0 \\
-0 \\
-0 \\
-0 \\
-0\end{array}$ & $\begin{array}{l}9 \\
6 \\
3 \\
1 \\
9 \\
5 \\
2 \\
9\end{array}$ & $\begin{array}{l}0 . \\
0 . \\
0 \\
0 . \\
0 \\
0 . \\
0 .\end{array}$ \\
\hline $\begin{array}{l}0.1000 \\
0.1250 \\
0.1500 \\
0.1750 \\
0.2000 \\
0.2500 \\
0.3000 \\
0.3500\end{array}$ & $\begin{array}{l}3.6 \\
3.2 \\
3.0 \\
2.8 \\
2.5 \\
2.3\end{array}$ & $\begin{array}{l}3.5 \\
3.6 \\
3.8 \\
3.9 \\
4.2 \\
4.5\end{array}$ & $\begin{array}{l}3 . \\
3 \\
3 \\
2 \\
2 . \\
2 .\end{array}$ & $\begin{array}{l}2 \\
2 \\
2 \\
2 \\
2 \\
2 \\
2 \\
1\end{array}$ & $\begin{array}{l}5 . \\
6 . \\
7 . \\
8 . \\
9 . \\
1 .\end{array}$ & $\begin{array}{l}0.0 \\
0.0 \\
0.0 \\
0.0 \\
0.0 \\
0.0 \\
0.0\end{array}$ & $\begin{array}{l}-0 \\
-0 \\
-0 \\
-0 \\
-0 \\
-0 \\
-0 \\
-0\end{array}$ & & $\begin{array}{l}0.142 \\
0.139 \\
0.136 \\
0.134 \\
0.130 \\
0.127 \\
0.124\end{array}$ \\
\hline $\begin{array}{l}0.4000 \\
0.4500 \\
0.5000 \\
0.5500 \\
0.6000 \\
0.7000 \\
0.8000 \\
0.9000\end{array}$ & $\begin{array}{l}6 E+00 \\
6 E+00 \\
3 E+00 \\
1 E+00 \\
7 E+00 \\
8 E+00 \\
4 E+00 \\
1 E+00\end{array}$ & $\begin{array}{l}5.2 \\
5.6 \\
6.0 \\
6.4 \\
6.8 \\
7.7 \\
8.7\end{array}$ & & $\begin{array}{l}1 \\
1 \\
1 \\
1 \\
1 \\
1\end{array}$ & $\begin{array}{l}1 . \\
1 . \\
1 . \\
2 . \\
2 .\end{array}$ & $\begin{array}{l}2.9 \\
6.2 \\
9.7 \\
1.3 \\
1.7 \\
2.4 \\
3.2 \\
3.9\end{array}$ & $\begin{array}{l}-0 . \\
-0 . \\
-0 . \\
-0 . \\
-0 . \\
-0 . \\
-0 . \\
-0 .\end{array}$ & & $\begin{array}{l}0.118 \\
0.111 \\
0.106 \\
0.101 \\
0.096 \\
0.088 \\
0.082 \\
0.076\end{array}$ \\
\hline $\begin{array}{l}1.0000 \\
1.2500 \\
1.5000 \\
1.7500 \\
2.0000 \\
2.5000 \\
3.0000 \\
3.5000\end{array}$ & $\begin{array}{l}1.834 E+00 \\
1.812 E+00 \\
1.803 E+00 \\
1.802 E+00 \\
1.804 E+00 \\
1.814 E+00 \\
1.827 E+00 \\
1.839 E+00\end{array}$ & $\begin{array}{l}1.071 \mathrm{E}-02 \\
1.341 \mathrm{E}-02 \\
1.630 \mathrm{E}-02 \\
1.934 \mathrm{E}-02 \\
2.252 \mathrm{E}-02 \\
2.920 \mathrm{E}-02 \\
3.625 \mathrm{E}-02 \\
4.358 \mathrm{E}-02\end{array}$ & $\begin{array}{l}1.845 E+00 \\
1.825 E+00 \\
1.819 E+00 \\
1.821 E+00 \\
1.827 E+00 \\
1.843 E+00 \\
1.863 E+00 \\
1.883 E+00\end{array}$ & $\begin{array}{ll}E-0 & 1 \\
E-0 & 1 \\
E-0 & 1 \\
E-0 & 1 \\
E-0 & 1 \\
E+0 & 0 \\
E+0 & 0 \\
E+0 & 0\end{array}$ & $\begin{array}{l}3.706 \mathrm{E} \\
4.447 \mathrm{E} \\
5.209 \mathrm{E} \\
5.992 \mathrm{E} \\
7.609 \mathrm{E} \\
9.281 \mathrm{E} \\
1.100 \mathrm{E}\end{array}$ & $\begin{array}{l}4.712 \mathrm{E}-0 \\
6.472 \mathrm{E}-0 \\
8.100 \mathrm{E}-0 \\
9.601 \mathrm{E}-0 \\
1.099 \mathrm{E}+0 \\
1.346 \mathrm{E}+0 \\
1.561 \mathrm{E}+0 \\
1.751 \mathrm{E}+0\end{array}$ & $\begin{array}{l}-0.049 \\
-0.043 \\
-0.039 \\
-0.036 \\
-0.034 \\
-0.031 \\
-0.029 \\
-0.028\end{array}$ & $\begin{array}{l}0.0 \\
0.0 \\
0.0 \\
0.0 \\
0.0 \\
0.0 \\
0.0\end{array}$ & $\begin{array}{l}0.071 \\
0.062 \\
0.056 \\
0.051 \\
0.047 \\
0.041 \\
0.038 \\
0.035\end{array}$ \\
\hline $\begin{array}{l}4.0000 \\
4.5000 \\
5.0000 \\
5.5000 \\
6.0000 \\
7.0000 \\
8.0000 \\
9.0000\end{array}$ & $\begin{array}{l}2 E+00 \\
3 E+00 \\
4 E+00 \\
4 E+00 \\
4 E+00 \\
1 E+00 \\
6 E+00 \\
9 E+00\end{array}$ & $\begin{array}{l}5.116 \mathrm{E}-02 \\
5.896 \mathrm{E}-02 \\
6.695 \mathrm{E}-02 \\
7.511 \mathrm{E}-02 \\
8.341 \mathrm{E}-02 \\
1.004 \mathrm{E}-01 \\
1.179 \mathrm{E}-01 \\
1.358 \mathrm{E}-01\end{array}$ & $\begin{array}{l}+00 \\
+00 \\
+00 \\
+00 \\
+00 \\
+00 \\
+00 \\
+00\end{array}$ & $\begin{array}{l}2.320 E+00 \\
2.579 E+00 \\
2.836 E+00 \\
3.090 E+00 \\
3.591 E+00 \\
4.084 E+00 \\
4.570 E+00\end{array}$ & $\begin{array}{l}1 . \\
1 . \\
1 . \\
1 . \\
2 . \\
2 . \\
2 .\end{array}$ & $\begin{array}{l}1.922 \mathrm{E}+0 \\
2.077 \mathrm{E}+0 \\
2.218 \mathrm{E}+0 \\
2.349 \mathrm{E}+0 \\
2.470 \mathrm{E}+0 \\
2.691 \mathrm{E}+0 \\
2.887 \mathrm{E}+0 \\
3.064 \mathrm{E}+0\end{array}$ & $\begin{array}{l}-0.026 \\
-0.025 \\
-0.024 \\
-0.024 \\
-0.023 \\
-0.021 \\
-0.020 \\
-0.018\end{array}$ & $\begin{array}{l}0.043 \\
0.041 \\
0.039 \\
0.038 \\
0.036 \\
0.034 \\
0.032 \\
0.031\end{array}$ & $\begin{array}{l}0.033 \\
0.031 \\
0.030 \\
0.029 \\
0.027 \\
0.026 \\
0.024 \\
0.023\end{array}$ \\
\hline $\begin{array}{l}10.0000 \\
12.5000 \\
15.0000 \\
17.5000 \\
20.0000 \\
25.0000 \\
30.0000 \\
35.0000\end{array}$ & $\begin{array}{l}975 E+00 \\
95 E+00 \\
10 E+00 \\
24 E+00 \\
46 E+00 \\
63 E+00 \\
77 E+00\end{array}$ & $\begin{array}{l}2.007 \\
2.488 \\
2.979 \\
3.478 \\
4.496 \\
5.532 \\
6.582\end{array}$ & $\begin{array}{l}2.176 E+00 \\
2.243 E+00 \\
2.308 E+00 \\
2.372 E+00 \\
2.495 E+00 \\
2.616 E+00 \\
2.735 E+00\end{array}$ & $\begin{array}{l}0 \\
0 \\
0 \\
0 \\
0 \\
1 \\
1 \\
1\end{array}$ & $\begin{array}{l}4 . \\
5 . \\
6 . \\
7 . \\
9 . \\
1 .\end{array}$ & $\begin{array}{l}3.2 \\
3.5 \\
3.8 \\
4.1 \\
4.3 \\
4.7 \\
5.1 \\
5.4\end{array}$ & $\begin{array}{l}-0 . \\
-0 . \\
-0 . \\
-0 . \\
-0 . \\
-0 . \\
-0 . \\
-0 .\end{array}$ & $\begin{array}{l}9 \\
7 \\
4 \\
2 \\
1 \\
8 \\
6 \\
4\end{array}$ & $\begin{array}{l}21 \\
19 \\
16 \\
14 \\
13 \\
10 \\
08 \\
07\end{array}$ \\
\hline $\begin{array}{l}40.0000 \\
45.0000 \\
50.0000 \\
55.0000 \\
60.0000 \\
70.00000 \\
80.0000 \\
90.0000\end{array}$ & $\begin{array}{l}E+00 \\
E+00 \\
E+00 \\
E+00 \\
E+00 \\
E+00 \\
E+00 \\
E+00\end{array}$ & $\begin{array}{l}8 \\
9 \\
1 \\
1 \\
1 \\
1 \\
1\end{array}$ & & $\begin{array}{ll}0 & 1 \\
0 & 1 \\
0 & 1 \\
0 & 1 \\
0 & 1 \\
0 & 1 \\
0 & 1 \\
0 & 1\end{array}$ & $\begin{array}{l}1 . \\
1 . \\
1 . \\
1 . \\
2 .\end{array}$ & $\begin{array}{l}5 . \\
5.8 \\
6 . \\
6 . \\
6 . \\
6 . \\
7 . \\
7 .\end{array}$ & $\begin{array}{l}-0 . \\
-0 . \\
-0 . \\
-0 . \\
-0 . \\
-0 . \\
-0 . \\
-0 .\end{array}$ & $\begin{array}{l}2 \\
1 \\
1 \\
0 \\
9 \\
8 \\
8\end{array}$ & $\begin{array}{l}06 \\
05 \\
04 \\
04 \\
03 \\
03 \\
02 \\
02\end{array}$ \\
\hline $\begin{array}{l}.0000 \\
.0000 \\
.0000 \\
.0000 \\
.0000 \\
.0000 \\
0000\end{array}$ & $\begin{array}{l}0 \\
0 \\
0 \\
0 \\
0 \\
0 \\
0 \\
0\end{array}$ & $\begin{array}{l}0 \\
0 \\
0 \\
0 \\
0 \\
0 \\
0 \\
0\end{array}$ & & & & $\begin{array}{l}7 . \\
7 . \\
8 . \\
8 . \\
8 . \\
9 .\end{array}$ & $\begin{array}{l}-0 \\
-0 \\
-0 \\
-0 \\
-0 \\
-0\end{array}$ & & \\
\hline $\begin{array}{l}50.00000 \\
00.0000 \\
50.0000 \\
00.00000 \\
00.0000 \\
00.0000 \\
00.0000\end{array}$ & $\begin{array}{l}298 E+00 \\
307 E+00 \\
315 E+00 \\
322 E+00 \\
335 E+00 \\
346 E+00 \\
356 E+00\end{array}$ & $\begin{array}{l}1.016 E+01 \\
1.132 E+01 \\
1.242 E+01 \\
1.367 E+01 \\
1.601 E+01 \\
1.836 E+01 \\
2.072 E+01\end{array}$ & $\therefore 307 t+01$ & $\begin{array}{l}1 \\
1 \\
1 \\
1 \\
2\end{array}$ & 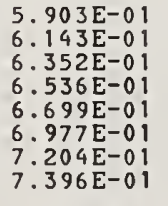 & $\begin{array}{l}1 \\
1 \\
1 \\
1 \\
1\end{array}$ & $\begin{array}{l}-0 . \\
-0 . \\
-0 . \\
-0 . \\
-0 . \\
-0 .\end{array}$ & $\begin{array}{l}03 \\
03 \\
03 \\
03 \\
03 \\
03 \\
02 \\
02\end{array}$ & $\begin{array}{l}0.000 \\
0.000 \\
0.000 \\
0.000 \\
0.0000 \\
0.0000 \\
0.0000 \\
0.000\end{array}$ \\
\hline 000 & $65 E+00$ & $307 E+01$ & $544 E+01$ & $04 E+0$ & $59 \mathrm{E}-0$ & $205 \mathrm{E}+$ & -0.000 & .002 & .000 \\
\hline
\end{tabular}




\begin{tabular}{|c|c|c|c|c|c|c|c|c|c|}
\hline ENERGY & $\begin{array}{l}\text { COLLISION } \\
\text { MeV cm } \mathrm{cm}^{2} / \mathrm{g}\end{array}$ & $\begin{array}{l}\text { PP ING POWE } \\
\text { RADIATIVE } \\
\text { MeV } \mathrm{cm}^{2} / \mathrm{g}\end{array}$ & $\begin{array}{c}\text { TOTAL } \\
\mathrm{MeV} \mathrm{cm}^{2} / \mathrm{g}\end{array}$ & $\begin{array}{l}\text { CSDA } \\
\text { RANGE } \\
\mathrm{g} / \mathrm{Cm}^{2}\end{array}$ & DIA & $\begin{array}{l}\text { ENS.EFF. } \\
\text { CORR. } \\
\text { (DELTA) }\end{array}$ & $\begin{array}{l}\text { d( }) \\
\text { coll } \\
\text { Loss }\end{array}$ & $\begin{array}{l}\text { S } \\
\text { SDA }\end{array}$ & $\begin{array}{l}\text { gI ) } \\
\text { RAD } \\
\text { YIELD }\end{array}$ \\
\hline $\begin{array}{l}0.0100 \\
0.0125 \\
0.01150 \\
0.0175 \\
0.0200 \\
0.0250 \\
0.0300 \\
0.0350\end{array}$ & $\begin{array}{l}2.296 \mathrm{E}+01 \\
1.927 \mathrm{E}+01 \\
1.670 \mathrm{E}+01 \\
1.480 \mathrm{E}+01 \\
1.333 \mathrm{E}+01 \\
1.121 \mathrm{E}+01 \\
9.739 \mathrm{E}+00 \\
8.661 \mathrm{E}+00\end{array}$ & $\begin{array}{l}2.982 \mathrm{E}-03 \\
2.992 \mathrm{E}-03 \\
2.999 \mathrm{E}-03 \\
3.004 \mathrm{E}-03 \\
3.008 \mathrm{E}-03 \\
3.017 \mathrm{E}-03 \\
3.027 \mathrm{E}-03 \\
3.037 \mathrm{E}-03\end{array}$ & $\begin{array}{l}2.29 \\
1.92 \\
1.67 \\
1.48 \\
1.33 \\
1.12 \\
9.74 \\
8.66\end{array}$ & $\begin{array}{l}2.455 \mathrm{E}-04 \\
3.649 \mathrm{E}-04 \\
5.047 \mathrm{E}-04 \\
6.641 \mathrm{E}-04 \\
8.423 \mathrm{E}-04 \\
1.253 \mathrm{E}-03 \\
1.733 \mathrm{E}-03 \\
2.279 \mathrm{E}-03\end{array}$ & $\begin{array}{l}7.143 E-05 \\
8.568 E-05 \\
9.932 E-05 \\
1.125 E-04 \\
1.252 E-04 \\
1.497 E-04 \\
1.731 E-04 \\
1.956 E-04\end{array}$ & $\begin{array}{l}0.0 \\
0.0 \\
0.0 \\
0.0 \\
0.0 \\
0.0 \\
0.0 \\
0.0\end{array}$ & $\begin{array}{l}-0.189 \\
-0.182 \\
-0.176 \\
-0.171 \\
-0.168 \\
-0.162 \\
-0.157 \\
-0.154\end{array}$ & $\begin{array}{l}0.214 \\
0.205 \\
0.198 \\
0.192 \\
0.187 \\
0.180 \\
0.174 \\
0.170\end{array}$ & $\begin{array}{l}0.213 \\
0.204 \\
0.197 \\
0.191 \\
0.187 \\
0.179 \\
0.174 \\
0.169\end{array}$ \\
\hline $\begin{array}{l}0.0400 \\
0.0450 \\
0.0500 \\
0.0550 \\
0.0600 \\
0.0700 \\
0.0800 \\
0.0900\end{array}$ & $\begin{array}{l}7.833 \mathrm{E}+00 \\
7.176 \mathrm{E}+00 \\
6.642 \mathrm{E}+00 \\
6.199 \mathrm{E}+00 \\
5.825 \mathrm{E}+00 \\
5.229 \mathrm{E}+00 \\
4.774 \mathrm{E}+00 \\
4.416 \mathrm{E}+00\end{array}$ & $\begin{array}{l}3.048 \mathrm{E}-03 \\
3.061 \mathrm{E}-03 \\
3.074 \mathrm{E}-03 \\
3.088 \mathrm{E}-03 \\
3.103 \mathrm{E}-03 \\
3.135 \mathrm{E}-03 \\
3.169 \mathrm{E}-03 \\
3.206 \mathrm{E}-03\end{array}$ & $\begin{array}{l}7.836 E+00 \\
7.179 E+00 \\
6.645 E+00 \\
6.202 E+00 \\
5.829 E+00 \\
5.232 E+00 \\
4.777 E+00 \\
4.419 E+00\end{array}$ & $\begin{array}{l}2.886 \mathrm{E}-03 \\
3.554 \mathrm{E}-03 \\
4.279 \mathrm{E}-03 \\
5.058 \mathrm{E}-03 \\
5.890 \mathrm{E}-03 \\
7.705 \mathrm{E}-03 \\
9.708 \mathrm{E}-03 \\
1.189 \mathrm{E}-02\end{array}$ & $\begin{array}{l}2.174 E-04 \\
2.385 \mathrm{E}-04 \\
2.591 \mathrm{E}-04 \\
2.792 \mathrm{E}-04 \\
2.989 \mathrm{E}-04 \\
3.371 \mathrm{E}-04 \\
3.739 \mathrm{E}-04 \\
4.095 \mathrm{E}-04\end{array}$ & $\begin{array}{l}0.0 \\
0.0 \\
0.0 \\
0.0 \\
0.0 \\
0.0 \\
0.0 \\
0.0\end{array}$ & $\begin{array}{l}-0.151 \\
-0.148 \\
-0.146 \\
-0.144 \\
-0.142 \\
-0.140 \\
-0.137 \\
-0.135\end{array}$ & $\begin{array}{l}0.166 \\
0.163 \\
0.160 \\
0.158 \\
0.156 \\
0.152 \\
0.149 \\
0.147\end{array}$ & $\begin{array}{l}0.166 \\
0.163 \\
0.160 \\
0.158 \\
0.155 \\
0.152 \\
0.149 \\
0.147\end{array}$ \\
\hline $\begin{array}{l}0.1000 \\
0.1250 \\
0.1500 \\
0.1750 \\
0.2000 \\
0.2500 \\
0.3000 \\
0.3500\end{array}$ & $\begin{array}{l}4.126 E+00 \\
3.597 E+00 \\
3.240 E+00 \\
2.984 E+00 \\
2.792 E+00 \\
2.525 E+00 \\
2.351 E+00 \\
2.229 E+00\end{array}$ & $\begin{array}{l}3.244 \mathrm{E}-03 \\
3.350 \mathrm{E}-03 \\
3.463 \mathrm{E}-03 \\
3.584 \mathrm{E}-03 \\
3.711 \mathrm{E}-03 \\
3.985 \mathrm{E}-03 \\
4.284 \mathrm{E}-03 \\
4.604 \mathrm{E}-03\end{array}$ & $\begin{array}{l}4.129 E+00 \\
3.600 E+00 \\
3.244 E+00 \\
2.988 E+00 \\
2.796 E+00 \\
2.529 E+00 \\
2.355 E+00 \\
2.234 E+00\end{array}$ & $\begin{array}{l}1.423 \mathrm{E}-02 \\
2.074 \mathrm{E}-02 \\
2.808 \mathrm{E}-02 \\
3.612 \mathrm{E}-02 \\
4.478 \mathrm{E}-02 \\
6.365 \mathrm{E}-02 \\
8.418 \mathrm{E}-02 \\
1.060 \mathrm{E}-01\end{array}$ & $\begin{array}{l}4.441 \mathrm{E}-04 \\
5.270 \mathrm{E}-04 \\
6.058 \mathrm{E}-04 \\
6.812 \mathrm{E}-04 \\
7.540 \mathrm{E}-04 \\
8.937 \mathrm{E}-04 \\
1.028 \mathrm{E}-03 \\
1.158 \mathrm{E}-03\end{array}$ & $\begin{array}{l}0.0 \\
0.0 \\
0.0 \\
0.0 \\
0.0 \\
0.0 \\
0.0 \\
0.0\end{array}$ & $\begin{array}{l}-0.133 \\
-0.130 \\
-0.127 \\
-0.124 \\
-0.122 \\
-0.119 \\
-0.117 \\
-0.114\end{array}$ & $\begin{array}{l}0.145 \\
0.141 \\
0.137 \\
0.135 \\
0.132 \\
0.129 \\
0.126 \\
0.124\end{array}$ & $\begin{array}{l}0.144 \\
0.140 \\
0.137 \\
0.134 \\
0.132 \\
0.128 \\
0.125 \\
0.123\end{array}$ \\
\hline $\begin{array}{l}0.4000 \\
0.4500 \\
0.5000 \\
0.5500 \\
0.6000 \\
0.7000 \\
0.8000 \\
0.9000\end{array}$ & $\begin{array}{l}2.142 E+00 \\
2.076 E+00 \\
2.027 E+00 \\
1.988 E+00 \\
1.958 E+00 \\
1.915 E+00 \\
1.888 E+00 \\
1.871 E+00\end{array}$ & $\begin{array}{l}4.945 \mathrm{E}-03 \\
5.304 \mathrm{E}-03 \\
5.680 \mathrm{E}-03 \\
6.071 \mathrm{E}-03 \\
6.475 \mathrm{E}-03 \\
7.322 \mathrm{E}-03 \\
8.212 \mathrm{E}-03 \\
9.142 \mathrm{E}-03\end{array}$ & $\begin{array}{l}2.147 E+00 \\
2.082 E+00 \\
2.032 E+00 \\
1.994 E+00 \\
1.965 E+00 \\
1.923 E+00 \\
1.897 E+00 \\
1.881 E+00\end{array}$ & $\begin{array}{l}1.289 \mathrm{E}-01 \\
1.525 \mathrm{E}-01 \\
1.769 \mathrm{E}-01 \\
2.017 \mathrm{E}-01 \\
2.270 \mathrm{E}-01 \\
2.785 \mathrm{E}-01 \\
3.309 \mathrm{E}-01 \\
3.838 \mathrm{E}-01\end{array}$ & $\begin{array}{l}1.286 \mathrm{E}-03 \\
1.413 \mathrm{E}-03 \\
1.539 \mathrm{E}-03 \\
1.664 \mathrm{E}-03 \\
1.790 \mathrm{E}-03 \\
2.041 \mathrm{E}-03 \\
2.295 \mathrm{E}-03 \\
2.550 \mathrm{E}-03\end{array}$ & $\begin{array}{l}0.0 \\
0.0 \\
0.0 \\
0.0 \\
0.0 \\
0.0 \\
0.0 \\
0.0\end{array}$ & $\begin{array}{l}-0.113 \\
-0.1111 \\
-0.1100 \\
-0.108 \\
-0.107 \\
-0.105 \\
-0.103 \\
-0.102\end{array}$ & $\begin{array}{l}0.122 \\
0.120 \\
0.119 \\
0.118 \\
0.1116 \\
0.1114 \\
0.1113 \\
0.1111\end{array}$ & $\begin{array}{l}0.121 \\
0.1119 \\
0.1117 \\
0.1116 \\
0.1115 \\
0.1112 \\
0.1110 \\
0.109\end{array}$ \\
\hline $\begin{array}{l}1.0000 \\
1.2500 \\
1.5000 \\
1.7500 \\
2.0000 \\
2.5000 \\
3.0000 \\
3.5000\end{array}$ & $\begin{array}{l}1.861 E+00 \\
1.853 \mathrm{E}+00 \\
1.857 \mathrm{E}+00 \\
1.868 \mathrm{E}+00 \\
1.881 \mathrm{E}+00 \\
1.910 \mathrm{E}+00 \\
1.939 \mathrm{E}+00 \\
1.967 \mathrm{E}+00\end{array}$ & $\begin{array}{l}1.011 \mathrm{E}-02 \\
1.267 \mathrm{E}-02 \\
1.541 \mathrm{E}-02 \\
1.830 \mathrm{E}-02 \\
2.132 \mathrm{E}-02 \\
2.766 \mathrm{E}-02 \\
3.435 \mathrm{E}-02 \\
4.132 \mathrm{E}-02\end{array}$ & $\begin{array}{l}1.871 E+00 \\
1.865 E+00 \\
1.873 E+00 \\
1.886 E+00 \\
1.902 E+00 \\
1.938 E+00 \\
1.974 E+00 \\
2.008 E+00\end{array}$ & $\begin{array}{l}4.372 E-01 \\
5.711 E-01 \\
7.049 E-01 \\
8.380 E-01 \\
9.699 E-01 \\
1.230 E+00 \\
1.486 E+00 \\
1.737 E+00\end{array}$ & $\begin{array}{l}2.808 \mathrm{E}-03 \\
3.466 \mathrm{E}-03 \\
4.139 \mathrm{E}-03 \\
4.829 \mathrm{E}-03 \\
5.532 \mathrm{E}-03 \\
6.973 \mathrm{E}-03 \\
8.450 \mathrm{E}-03 \\
9.955 \mathrm{E}-03\end{array}$ & $\begin{array}{l}0.0 \\
0.0 \\
0.0 \\
0.0 \\
0.0 \\
0.0 \\
0.0 \\
0.0\end{array}$ & $\begin{array}{l}-0.100 \\
-0.097 \\
-0.095 \\
-0.093 \\
-0.092 \\
-0.089 \\
-0.087 \\
-0.085\end{array}$ & $\begin{array}{l}0.110 \\
0.107 \\
0.105 \\
0.103 \\
0.102 \\
0.099 \\
0.097 \\
0.095\end{array}$ & $\begin{array}{l}0.107 \\
0.104 \\
0.101 \\
0.099 \\
0.097 \\
0.094 \\
0.092 \\
0.090\end{array}$ \\
\hline $\begin{array}{l}4.0000 \\
4.5000 \\
5.0000 \\
5.5000 \\
6.0000 \\
7.0000 \\
8.0000 \\
9.0000\end{array}$ & $\begin{array}{l}1.993 E+00 \\
2.017 E+00 \\
2.039 E+00 \\
2.059 E+00 \\
2.078 E+00 \\
2.113 E+00 \\
2.144 E+00 \\
2.171 E+00\end{array}$ & $\begin{array}{l}4.852 \mathrm{E}-02 \\
5.593 \mathrm{E}-02 \\
6.353 \mathrm{E}-02 \\
7.129 \mathrm{E}-02 \\
7.919 \mathrm{E}-02 \\
9.539 \mathrm{E}-02 \\
1.120 \mathrm{E}-01 \\
1.290 \mathrm{E}-01\end{array}$ & $\begin{array}{l}2.041 E+00 \\
2.073 E+00 \\
2.102 E+00 \\
2.131 E+00 \\
2.158 E+00 \\
2.208 E+00 \\
2.256 E+00 \\
2.300 E+00\end{array}$ & $\begin{array}{l}1.984 E+00 \\
2.227 E+00 \\
2.467 E+00 \\
2.703 E+00 \\
2.936 E+00 \\
3.394 E+00 \\
3.842 E+00 \\
4.281 E+00\end{array}$ & $\begin{array}{l}1.148 \mathrm{E}-02 \\
1.303 \mathrm{E}-02 \\
1.458 \mathrm{E}-02 \\
1.615 \mathrm{E}-02 \\
1.773 \mathrm{E}-02 \\
2.090 \mathrm{E}-02 \\
2.409 \mathrm{E}-02 \\
2.729 \mathrm{E}-02\end{array}$ & $\begin{array}{l}0.0 \\
0.0 \\
0.0 \\
0.0 \\
0.0 \\
0.0 \\
0.0 \\
0.0\end{array}$ & $\begin{array}{l}-0 . \\
-0 . \\
-0 \\
-0 . \\
-0 \\
-0 . \\
-0 . \\
-0 .\end{array}$ & & $\begin{array}{l}0.088 \\
0.087 \\
0.085 \\
0.084 \\
0.083 \\
0.081 \\
0.080 \\
0.078\end{array}$ \\
\hline $\begin{array}{l}10.0000 \\
12.5000 \\
15.0000 \\
17.5000 \\
20.0000 \\
25.0000 \\
30.0000 \\
35.0000\end{array}$ & $\begin{array}{l}2.196 E+00 \\
2.249 E+00 \\
2.293 E+00 \\
2.330 E+00 \\
2.363 E+00 \\
2.413 E+00 \\
2.448 E+00 \\
2.476 E+00\end{array}$ & $\begin{array}{l}1.464 \mathrm{E}-01 \\
1.909 \mathrm{E}-01 \\
2.367 \mathrm{E}-01 \\
2.835 \mathrm{E}-01 \\
3.311 \mathrm{E}-01 \\
4.282 \mathrm{E}-01 \\
5.270 \mathrm{E}-01 \\
6.271 \mathrm{E}-01\end{array}$ & $\begin{array}{l}2.342 E+0 \\
2.440 E+0 \\
2.530 E+0 \\
2.614 E+0 \\
2.694 E+0 \\
2.841 E+0 \\
2.975 E+0 \\
3.103 E+0\end{array}$ & $\begin{array}{l}4.712 E+00 \\
5.757 E+00 \\
6.763 E+00 \\
7.735 E+00 \\
8.677 E+00 \\
1.048 E+01 \\
1.220 E+01 \\
1.385 E+01\end{array}$ & $\begin{array}{l}3.049 E-02 \\
3.847 \mathrm{E}-02 \\
4.638 \mathrm{E}-02 \\
5.419 \mathrm{E}-02 \\
6.188 \mathrm{E}-02 \\
7.689 \mathrm{E}-02 \\
9.142 \mathrm{E}-02 \\
1.055 \mathrm{E}-01\end{array}$ & $\begin{array}{l}0.0 \\
0.0 \\
0.0 \\
0.0 \\
2.424 \mathrm{E}-05 \\
5.648 \mathrm{E}-02 \\
1.676 \mathrm{E}-01 \\
2.951 \mathrm{E}-01\end{array}$ & $\begin{array}{l}-0.0 \\
-0.0 \\
-0.0 \\
-0.0 \\
-0.0 \\
-0.0 \\
-0 . \\
-0.0\end{array}$ & $\begin{array}{l}83 \\
81 \\
79 \\
77 \\
75 \\
71 \\
57 \\
53\end{array}$ & $\begin{array}{l}0.077 \\
0.074 \\
0.072 \\
0.070 \\
0.068 \\
0.062 \\
0.055 \\
0.049\end{array}$ \\
\hline $\begin{array}{l}40.0000 \\
45.0000 \\
50.0000 \\
55.0000 \\
60.0000 \\
70.0000 \\
80.0000 \\
90.0000\end{array}$ & $\begin{array}{l}2.498 E+00 \\
2.517 E+00 \\
2.533 E+00 \\
2.547 E+00 \\
2.560 E+00 \\
2.581 E+00 \\
2.600 E+00 \\
2.616 E+00\end{array}$ & $\begin{array}{l}7.284 E-01 \\
8.306 E-01 \\
9.334 E-01 \\
1.037 E+00 \\
1.141 E+00 \\
1.351 E+00 \\
1.562 E+00 \\
1.774 E+00\end{array}$ & $\begin{array}{l}3.347 E+0 \\
3.466 E+0 \\
3.584 E+0 \\
3.7011 E+0 \\
3.932 E+0 \\
4.162 E+0 \\
4.390 E+0\end{array}$ & $\begin{array}{l}1.5 \\
2.1 \\
2.3 \\
2.8 \\
2.8\end{array}$ & $\begin{array}{l}01 \\
01 \\
01 \\
01 \\
01 \\
01 \\
01 \\
01\end{array}$ & $\begin{array}{l}4.2 \\
5.5 \\
6.8 \\
7.8 \\
1.8 \\
1.8 \\
1.2\end{array}$ & $\begin{array}{l}-0 . \\
-0 . \\
-0 . \\
-0 \\
-0 . \\
-0 . \\
-0 . \\
-0\end{array}$ & & $\begin{array}{l}0.040 \\
0.036 \\
0.034 \\
0.031 \\
0.027 \\
0.025 \\
0.022\end{array}$ \\
\hline $\begin{array}{l}0.0000 \\
5.0000 \\
0.0000 \\
5.0000 \\
0.0000 \\
0.0000 \\
0.0000 \\
0.0000\end{array}$ & $\begin{array}{l}2.6 \\
2.6 \\
2.6 \\
2.7 \\
2.7 \\
2.7 \\
2.7 \\
2.7\end{array}$ & $\begin{array}{l}2.5 \\
3.0 \\
3.6 \\
4.1 \\
5.2 \\
6.3 \\
7.4\end{array}$ & $\begin{array}{l}5 \\
5 \\
6 \\
6 \\
8 \\
9 \\
1\end{array}$ & & $\begin{array}{l}1 \\
1 \\
1 \\
1 \\
1 \\
1 \\
1\end{array}$ & $\begin{array}{l}1 \\
1 \\
2 \\
2\end{array}$ & $\begin{array}{l}-0 \\
-0 \\
-0 \\
-0 \\
-0 \\
-0 \\
-0 \\
-0\end{array}$ & & $\left\{\begin{array}{l}20 \\
17 \\
15 \\
12 \\
10 \\
09 \\
08\end{array}\right.$ \\
\hline $\begin{array}{l}0.0000 \\
0.0000 \\
0.0000 \\
0.0000 \\
0.0000 \\
0.0000 \\
0.0000 \\
0.0000\end{array}$ & $\begin{array}{l}2.822 E+00 \\
2.832 E+00 \\
2.840 E+00 \\
2.855 E+00 \\
2.867 E+00 \\
2.878 E+00\end{array}$ & $\begin{array}{l}1.081 E+01 \\
1.193 E+01 \\
1.305 E+01 \\
1.529 E+01 \\
1.754 E+01 \\
1.978 E+01\end{array}$ & $\begin{array}{l}1.251 \mathrm{E}+01 \\
1.364 \mathrm{E}+01 \\
1.476 \mathrm{E}+01 \\
1.589 \mathrm{E}+01 \\
1.815 \mathrm{E}+01 \\
2.040 \mathrm{E}+01 \\
2.266 \mathrm{E}+01\end{array}$ & $\begin{array}{l}7.504 E+01 \\
7.887 E+01 \\
8.239 E+01 \\
8.566 E+01 \\
9.154 E+01 \\
9.674 E+01 \\
1.014 E+02\end{array}$ & $\begin{array}{l}5.403 \mathrm{E}-01 \\
5.653 \mathrm{E}-01 \\
5.872 \mathrm{E}-01 \\
6.066 \mathrm{E}-01 \\
6.240 \mathrm{E}-01 \\
6.537 \mathrm{E}-01 \\
6.785 \mathrm{E}-01 \\
6.993 \mathrm{E}-01\end{array}$ & $\begin{array}{l}4.237 E+00 \\
4.395 E+00 \\
4.680 E+00 \\
4.930 E+00 \\
5.154 E+00\end{array}$ & $\begin{array}{l}-0.0 \\
-0.0 \\
-0.0 \\
-0.0 \\
-0.0 \\
-0.0\end{array}$ & $\begin{array}{l}19 \\
18 \\
17 \\
16 \\
15 \\
15 \\
14\end{array}$ & $\begin{array}{l}0.006 \\
0.005 \\
0.005 \\
0.004 \\
0.004 \\
0.003 \\
0.003\end{array}$ \\
\hline .0000 & $2.887 E+00$ & $2.203 E+01$ & $2.492 E+01$ & $.056 E+02$ & $.173 E-01$ & $5.356 E+00$ & -0.002 & 0.013 & 0.003 \\
\hline
\end{tabular}




\begin{tabular}{|c|c|c|c|c|c|c|c|c|c|}
\hline NERGY & $\begin{array}{l}\text { COLLISION } \\
\text { MeV } \mathrm{cm}^{2} / \mathrm{g}\end{array}$ & $\mathrm{MeV} \mathrm{cm}^{2} / \mathrm{g}$ & $\begin{array}{c}\text { TOTAL } \\
\mathrm{MeV} \mathrm{cm}^{2} / \mathrm{g}\end{array}$ & $\begin{array}{l}\text { CSDA } \\
\text { RANGE } \\
\mathrm{g} / \mathrm{Cm}^{2}\end{array}$ & DIAT & $\begin{array}{l}\text { WS.EFF. } \\
\text { CORR. } \\
\text { DELTA) }\end{array}$ & $\begin{array}{l}\text { del } \\
\text { coLL } \\
\text { LOSS }\end{array}$ & $\begin{array}{l}\text { CSDA } \\
\text { RANGE }\end{array}$ & $\begin{array}{l}\text { I) } \\
\text { RAD } \\
\text { IELD }\end{array}$ \\
\hline $\begin{array}{l}0.0100 \\
0.0125 \\
0.0150 \\
0.0175 \\
0.0200 \\
0.0250 \\
0.0300 \\
0.0350\end{array}$ & $\begin{array}{l}1.705 \\
1.515 \\
1.365 \\
1.148 \\
9.984 \\
8.881\end{array}$ & $\begin{array}{l}3.1 \\
3.1 \\
3.1 \\
3.2 \\
3.2 \\
3.2 \\
3.2 \\
3.2\end{array}$ & $\begin{array}{l}347 E+01 \\
971 E+01 \\
709 E+01 \\
515 E+01 \\
365 E+01 \\
149 E+01 \\
987 E+00 \\
884 E+00\end{array}$ & $\begin{array}{l}2.40 \\
3.57 \\
4.94 \\
6.45 \\
8.23 \\
1.22 \\
1.69\end{array}$ & $\begin{array}{l}7.39 \\
8.88 \\
1.03 \\
1.16 \\
1.30 \\
1.55 \\
1.80\end{array}$ & & $\begin{array}{l}2 \\
4 \\
9 \\
4 \\
0 \\
4 \\
9 \\
6\end{array}$ & $\begin{array}{l}0.218 \\
0.209 \\
0.201 \\
0.195 \\
0.190 \\
0.182 \\
0.177 \\
0.172\end{array}$ & $\begin{array}{l}.217 \\
.207 \\
.200 \\
.194 \\
.189 \\
.182 \\
.176\end{array}$ \\
\hline $\begin{array}{l}0.0400 \\
0.0450 \\
0.0500 \\
0.0550 \\
0.0600 \\
0.0700 \\
0.0800 \\
0.0900\end{array}$ & $\begin{array}{l}8.034 E+00 \\
7.362 E+00 \\
6.816 E+00 \\
6.362 E+00 \\
5.979 E+00 \\
5.369 E+00 \\
4.903 E+00 \\
4.535 E+00\end{array}$ & $\begin{array}{l}3.249 \mathrm{E}-03 \\
3.262 \mathrm{E}-03 \\
3.275 \mathrm{E}-03 \\
3.290 \mathrm{E}-03 \\
3.305 \mathrm{E}-03 \\
3.338 \mathrm{E}-03 \\
3.373 \mathrm{E}-03 \\
3.411 \mathrm{E}-03\end{array}$ & $\begin{array}{l}8.037 E+00 \\
7.365 E+00 \\
6.819 E+00 \\
6.365 E+00 \\
5.983 E+00 \\
5.372 E+00 \\
4.906 E+00 \\
4.539 E+00\end{array}$ & $\begin{array}{l}2.818 \mathrm{E}-03 \\
3.468 \mathrm{E}-03 \\
4.175 \mathrm{E}-03 \\
4.934 \mathrm{E}-03 \\
5.745 \mathrm{E}-03 \\
7.513 \mathrm{E}-03 \\
9.464 \mathrm{E}-03 \\
1.159 \mathrm{E}-02\end{array}$ & $\begin{array}{l}2.260 \mathrm{E}-04 \\
2.480 \mathrm{E}-04 \\
2.693 \mathrm{E}-04 \\
2.902 \mathrm{E}-04 \\
3.106 \mathrm{E}-04 \\
3.501 \mathrm{E}-04 \\
3.881 \mathrm{E}-04 \\
4.250 \mathrm{E}-04\end{array}$ & $\begin{array}{l}0 \\
0 \\
0 \\
0 \\
0 \\
0 \\
0 \\
0\end{array}$ & $\begin{array}{l}-0.153 \\
-0.150 \\
-0.148 \\
-0.146 \\
-0.144 \\
-0.141 \\
-0.139 \\
-0.137\end{array}$ & $\begin{array}{l}0.168 \\
0.165 \\
0.162 \\
0.160 \\
0.158 \\
0.154 \\
0.151 \\
0.149\end{array}$ & .168 \\
\hline $\begin{array}{l}0.1000 \\
0.1250 \\
0.1500 \\
0.1750 \\
0.2000 \\
0.2500 \\
0.3000 \\
0.3500\end{array}$ & $\begin{array}{l}4.238 E+00 \\
3.696 E+00 \\
3.330 E+00 \\
3.068 E+00 \\
2.871 E+00 \\
2.597 E+00 \\
2.418 E+00 \\
2.294 E+00\end{array}$ & $\begin{array}{l}5.5 \\
3.6 \\
3.8 \\
3.9 \\
4.2 \\
4.5\end{array}$ & $\begin{array}{l}3.3 \\
3.0 \\
2.8 \\
2.6 \\
2.4\end{array}$ & $\begin{array}{l}2.7 \\
3.5 \\
4.3\end{array}$ & $\begin{array}{l}4.608 \mathrm{E}-04 \\
5.464 \mathrm{E}-04 \\
6.277 \mathrm{E}-04 \\
7.055 \mathrm{E}-04 \\
7.806 \mathrm{E}-04 \\
9.244 \mathrm{E}-04 \\
1.062 \mathrm{E}-03 \\
1.196 \mathrm{E}-03\end{array}$ & $\begin{array}{l}0.0 \\
0.0 \\
0.0 \\
0.0 \\
0.0 \\
0.0 \\
0.0 \\
0.0\end{array}$ & & & $\begin{array}{l}30 \\
36 \\
33 \\
30 \\
27\end{array}$ \\
\hline $\begin{array}{l}0.4000 \\
0.4500 \\
0.5000 \\
0.5500 \\
0.6000 \\
0.7000 \\
0.8000 \\
0.9000\end{array}$ & $\begin{array}{l}2.204 E+00 \\
2.135 \mathrm{E}+00 \\
2.081 \mathrm{E}+00 \\
2.039 \mathrm{E}+00 \\
2.005 \mathrm{E}+00 \\
1.954 \mathrm{E}+00 \\
1.921 \mathrm{E}+00 \\
1.897 \mathrm{E}+00\end{array}$ & $\begin{array}{l}5.244 \mathrm{E}-03 \\
5.623 \mathrm{E}-03 \\
6.020 \mathrm{E}-03 \\
6.433 \mathrm{E}-03 \\
6.860 \mathrm{E}-03 \\
7.753 \mathrm{E}-03 \\
8.692 \mathrm{E}-03 \\
9.674 \mathrm{E}-03\end{array}$ & $\begin{array}{l}2.209 \mathrm{E}+00 \\
2.141 \mathrm{E}+00 \\
2.087 \mathrm{E}+00 \\
2.045 \mathrm{E}+00 \\
2.011 \mathrm{E}+00 \\
1.962 \mathrm{E}+00 \\
1.929 \mathrm{E}+00 \\
1.907 \mathrm{E}+00\end{array}$ & $\begin{array}{l}1.7 \\
1.9 \\
2.2 \\
2.7\end{array}$ & $\begin{array}{l}1.328 \mathrm{E}-03 \\
1.458 \mathrm{E}-03 \\
1.588 \mathrm{E}-03 \\
1.718 \mathrm{E}-03 \\
1.848 \mathrm{E}-03 \\
2.109 \mathrm{E}-03\end{array}$ & $\begin{array}{l}0.0 \\
1.471 \mathrm{E}-02 \\
4.184 \mathrm{E}-02 \\
7.141 \mathrm{E}-02 \\
1.028 \mathrm{E}-01 \\
1.691 \mathrm{E}-01 \\
2.381 \mathrm{E}-01 \\
3.080 \mathrm{E}-01\end{array}$ & & & $\begin{array}{l}7 \\
3 \\
4\end{array}$ \\
\hline $\begin{array}{l}1.0000 \\
1.2500 \\
1.5000 \\
1.7500 \\
2.0000 \\
2.5000 \\
3.0000 \\
3.5000\end{array}$ & $\begin{array}{l}E+00 \\
E+00 \\
E+00 \\
E+00 \\
E+00 \\
E+00 \\
E+00 \\
E+00\end{array}$ & $\begin{array}{l}1.6 \\
1.9 \\
2.2 \\
2.9 \\
3.6 \\
4.3\end{array}$ & $\begin{array}{l}1.891 \mathrm{E}+00 \\
1.871 \mathrm{E}+00 \\
1.865 \mathrm{E}+00 \\
1.867 \mathrm{E}+00 \\
1.873 \mathrm{E}+00 \\
1.889 \mathrm{E}+00 \\
1.908 \mathrm{E}+00 \\
1.928 \mathrm{E}+00\end{array}$ & $\begin{array}{l}9.6 \\
1.2\end{array}$ & $\begin{array}{l}5 . \\
5.8 \\
7.8\end{array}$ & & $\begin{array}{l}-0.051 \\
-0.044 \\
-0.039 \\
-0.036 \\
-0.033 \\
-0.030 \\
-0.028 \\
-0.026\end{array}$ & & $\begin{array}{l}0.052 \\
0.048 \\
0.042 \\
0.037 \\
0.034\end{array}$ \\
\hline $\begin{array}{l}4.0000 \\
4.5000 \\
5.0000 \\
5.5000 \\
6.0000 \\
7.0000 \\
8.0000 \\
9.0000\end{array}$ & $\begin{array}{l}00 \\
00 \\
00 \\
00 \\
00 \\
00 \\
00 \\
00\end{array}$ & $\begin{array}{l}6 . \\
7 . \\
8 . \\
1 . \\
1 . \\
1 .\end{array}$ & $\begin{array}{l}E+00 \\
E+00 \\
E+00 \\
E+00 \\
E+00 \\
E+00 \\
E+00 \\
E+00\end{array}$ & 4.46 & $\begin{array}{l}1.245 \mathrm{E}-0 \\
1.419 \mathrm{E}-0 \\
1.596 \mathrm{E}-0 \\
1.774 \mathrm{E}-0 \\
1.955 \mathrm{E}-0 \\
2.319 \mathrm{E}-0 \\
2.688 \mathrm{E}-0 \\
3.059 \mathrm{E}-0\end{array}$ & $\begin{array}{l}1 . \\
1 . \\
2 . \\
2 . \\
2 . \\
2 . \\
2 . \\
2 .\end{array}$ & $\begin{array}{l}-0 \\
-0 \\
-0 \\
-0 \\
-0 \\
-0 \\
-0\end{array}$ & & $\begin{array}{l}0.0 \\
0.0 \\
0.0 \\
0.0\end{array}$ \\
\hline $\begin{array}{l}10.0000 \\
12.5000 \\
15.0000 \\
17.5000 \\
20.0000 \\
25.0000 \\
30.0000 \\
35.0000\end{array}$ & $\begin{array}{l}2.04 \\
2.05 \\
2.07 \\
2.09 \\
2.11 \\
2.12\end{array}$ & $\begin{array}{l}2.4 \\
2.4 \\
2.9 \\
3.4 \\
4.5 \\
5.5\end{array}$ & $\begin{array}{l}2.291 \mathrm{E}+00 \\
2.357 \mathrm{E}+00 \\
2.421 \mathrm{E}+00 \\
2.546 \mathrm{E}+00 \\
2.668 \mathrm{E}+00 \\
2.788 \mathrm{E}+00\end{array}$ & 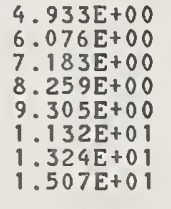 & $\begin{array}{l}3.432 \mathrm{E}-02 \\
4.368 \mathrm{E}-02 \\
5.300 \mathrm{E}-02 \\
6.225 \mathrm{E}-02 \\
7.138 \mathrm{E}-02 \\
8.923 \mathrm{E}-02 \\
1.065 \mathrm{E}-01 \\
1.230 \mathrm{E}-01\end{array}$ & $\begin{array}{l}3.137 \mathrm{E}+00 \\
3.491 \mathrm{E}+00 \\
3.790 \mathrm{E}+00 \\
4.050 \mathrm{E}+00 \\
4.282 \mathrm{E}+00 \\
4.679 \mathrm{E}+00 \\
5.012 \mathrm{E}+00 \\
5.299 \mathrm{E}+00\end{array}$ & $\begin{array}{l}-0.0 \\
-0.0 \\
-0.0 \\
-0.0 \\
-0.0 \\
-0.0 \\
-0.0 \\
-0.0\end{array}$ & & $\begin{array}{l}0 . \\
0 . \\
0 . \\
0 . \\
0 . \\
0 .\end{array}$ \\
\hline $\begin{array}{l}40.0000 \\
45.0000 \\
50.0000 \\
55.0000 \\
60.0000 \\
70.0000 \\
80.0000 \\
90.0000\end{array}$ & $\begin{array}{l}2.13 \\
2.16 \\
2.17 \\
2.17 \\
2.19 \\
2.20 \\
2.21\end{array}$ & $\begin{array}{l}9 . \\
1 \\
1 \\
1 \\
1 \\
1\end{array}$ & $\begin{array}{l}3 . \\
3 . \\
3\end{array}$ & $\begin{array}{l}1 \\
1 \\
1 \\
1 \\
1\end{array}$ & $\begin{array}{l}1.2 \\
1 .: 2 \\
1.8 \\
1.8 \\
2.2 \\
2.2 \\
2 .\end{array}$ & $\begin{array}{l}5 \cdot 7 \\
5 \cdot 9 \\
6 \cdot 1 \\
6 \cdot 3 \\
6 \cdot 6 \\
6 \cdot 8 \\
7.1\end{array}$ & & & \\
\hline $\begin{array}{l}0 \\
0 \\
0 \\
0\end{array}$ & $\begin{array}{l}2.2 \\
2.2 \\
2.2 \\
2.3 \\
2.3 \\
2.3\end{array}$ & & & $\begin{array}{l}3.366 E+01 \\
3.910 E+01 \\
4.392 E+01 \\
4.825 E+01 \\
5.219 E+01 \\
5.911 E+01 \\
6.507 E+01 \\
7.029 E+01\end{array}$ & $\begin{array}{l}4 \\
5\end{array}$ & 9. & & & \\
\hline $\begin{array}{l}0.00000 \\
0.00000 \\
0.00000 \\
0.00000 \\
0.00000 \\
0.00000\end{array}$ & $\begin{array}{l}2.353 E+00 \\
2.362 E+00 \\
2.370 E+00 \\
2.378 E+00 \\
2.391 E+00 \\
2.402 E+00 \\
2.413 E+00\end{array}$ & $\begin{array}{l}1.135 E+01 \\
1.253 E+01 \\
1.370 E+01 \\
1.605 E+01 \\
1.841 E+01 \\
2.077 E+01\end{array}$ & $\begin{array}{l}1.253 E+01 \\
1.371 E+01 \\
1.490 E+01 \\
1.608 E+01 \\
1.844 E+01 \\
2.081 E+01 \\
2.318 E+01\end{array}$ & $.051 E+02$ & $1.3625-01$ & $\begin{array}{l}1.054 E+01 \\
1.073 E+01 \\
1.090 E+01 \\
1.121 E+01 \\
1.148 E+01 \\
1.171 E+01\end{array}$ & & $\begin{array}{l}0.003 \\
0.003 \\
0.003 \\
0.003 \\
0.003 \\
0.003 \\
0.002\end{array}$ & \\
\hline & $422 E+00$ & $313 E+01$ & $55 E+0$ & $92 \mathrm{E}+0$ & $26 E-c$ & $.192 E+0$ & 0.000 & .002 & 000 \\
\hline
\end{tabular}


$I=85.7 \circ \mathrm{V} \quad$ DENSITY $=1.205 \mathrm{E}-03 \mathrm{~g} / \mathrm{cm}^{3}\left(20^{\circ} \mathrm{C}\right)$

\begin{tabular}{|c|c|c|c|c|c|c|c|c|c|}
\hline $\mathrm{MeV}$ & $\begin{array}{l}\text { ST } \\
\text { COLLISION } \\
\text { MeV } \mathrm{cm}^{2} / \mathrm{g}\end{array}$ & $\begin{array}{l}\text { RADIATIVE } \\
\mathrm{MeV} \mathrm{cm} 2 / \mathrm{g}\end{array}$ & $\begin{array}{c}\text { TOTAL } \\
\mathrm{MeV} \mathrm{cm}^{2} / \mathrm{g}\end{array}$ & $\begin{array}{l}\text { CSDA } \\
\text { RANGE } \\
\mathrm{g} / \mathrm{cm}^{2}\end{array}$ & $\mathrm{ON}$ & $\begin{array}{c}\text { DENS. EFF. } \\
\text { CORR. } \\
\text { (DELTA) }\end{array}$ & $\begin{array}{l}\text { de } 1 \\
\text { cold } \\
\text { Loss }\end{array}$ & $\begin{array}{l}\text { SDA } 1 \\
\text { SNGE }\end{array}$ & $\begin{array}{l}I) \\
\text { RAD } \\
\text { IELD }\end{array}$ \\
\hline $\begin{array}{l}0.0100 \\
0.0125 \\
0.0150 \\
0.0175 \\
0.0200 \\
0.0250 \\
0.0300 \\
0.0350\end{array}$ & $\begin{array}{l}1.975 E+01 \\
1.663 E+01 \\
1.445 E+01 \\
1.283 E+01 \\
1.157 E+01 \\
9.753 E+00 \\
8.492 E+00 \\
7.563 E+00\end{array}$ & $\begin{array}{l}3.897 E-03 \\
3.921 E-03 \\
3.937 E-03 \\
3.946 E-03 \\
3.954 E-03 \\
3.966 E-03 \\
3.976 E-03 \\
3.986 E-03\end{array}$ & $\begin{array}{l}1.976 E+01 \\
1.663 E+01 \\
1.445 E+01 \\
1.283 E+01 \\
1.158 E+01 \\
9.757 E+00 \\
8.496 E+00 \\
7.567 E+00\end{array}$ & $\begin{array}{l}2.88 \\
4.26 \\
5.88 \\
7.72 \\
9.78 \\
1.45 \\
2.00 \\
2.62\end{array}$ & $\begin{array}{l}1.082 \mathrm{E}-04 \\
1.299 \mathrm{E}-04 \\
1.506 \mathrm{E}-04 \\
1.706 \mathrm{E}-04 \\
1.898 \mathrm{E}-04 \\
2.267 \mathrm{E}-04 \\
2.618 \mathrm{E}-04 \\
2.955 \mathrm{E}-04\end{array}$ & $\begin{array}{l}0.0 \\
0.0 \\
0.0 \\
0.0 \\
0.0 \\
0.0 \\
0.0 \\
0.0\end{array}$ & $\begin{array}{l}-0.2 \\
-0.1 \\
-0.1 \\
-0.1 \\
-0.1 \\
-0.1 \\
-0.1 \\
-0.1\end{array}$ & $\begin{array}{l}0.235 \\
0.223 \\
0.215 \\
0.208 \\
0.202 \\
0.194 \\
0.187 \\
0.182\end{array}$ & $\begin{array}{l}0.233 \\
0.222 \\
0.213 \\
0.207 \\
0.201 \\
0.193 \\
0.186 \\
0.181\end{array}$ \\
\hline $\begin{array}{l}0.0400 \\
0.0450 \\
0.0500 \\
0.0550 \\
0.0600 \\
0.0700 \\
0.0800 \\
0.0900\end{array}$ & $\begin{array}{l}6.84 \\
6.28 \\
5.81 \\
5.4 \\
5.11 \\
4.5 \\
4.1\end{array}$ & $\begin{array}{l}3.9 \\
4.0 \\
4.0 \\
4.0 \\
4.0 \\
4.0 \\
4.1 \\
4.1\end{array}$ & $\begin{array}{l}6.852 E+00 \\
6.285 E+00 \\
5.823 E+00 \\
5.439 E+00 \\
5.115 E+00 \\
4.597 E+00 \\
4.202 E+00 \\
3.890 E+00\end{array}$ & $\begin{array}{l}3.32 \\
4.08 \\
4.9 \\
5.80 \\
6.7 \\
8.8 \\
1.1 \\
1.3\end{array}$ & $\begin{array}{l}3 . \\
3 . \\
4 . \\
4 . \\
5 . \\
5 .\end{array}$ & $\begin{array}{l}0.0 \\
0.0 \\
0.0 \\
0.0 \\
0.0 \\
0.0 \\
0.0 \\
0.0\end{array}$ & $\begin{array}{l}-0.1 \\
-0.1 \\
-0.1 \\
-0.1 \\
-0.1 \\
-0.1 \\
-0.1 \\
-0.1\end{array}$ & $\begin{array}{l}0 . \\
0 . \\
0 . \\
0 . \\
0 . \\
0 . \\
0 .\end{array}$ & $\begin{array}{l}0.177 \\
0.174 \\
0.171 \\
0.168 \\
0.166 \\
0.162 \\
0.158 \\
0.156\end{array}$ \\
\hline $\begin{array}{l}0.1000 \\
0.1250 \\
0.1500 \\
0.1750 \\
0.2000 \\
0.2500 \\
0.3000 \\
0.3500\end{array}$ & $\begin{array}{l}E+00 \\
E+00 \\
E+00 \\
E+00 \\
E+00 \\
E+00 \\
E+00 \\
E+00\end{array}$ & $\begin{array}{l}4.22 \\
4.3 \\
4.48 \\
4.6 \\
4.7 \\
5.1 \\
5.4 \\
5.8\end{array}$ & $\begin{array}{l}3.6 \\
3.1 \\
2.8 \\
2.6 \\
2.4 \\
2.2 \\
2.0 \\
1.9\end{array}$ & $\begin{array}{l}1.62 \\
2.36 \\
3.19 \\
4.10 \\
5.08 \\
7.29 \\
9.52 \\
1.1\end{array}$ & $\begin{array}{l}6 . \\
7 . \\
8 . \\
1: \\
1: \\
1:\end{array}$ & $\begin{array}{l}0.0 \\
0.0 \\
0.0 \\
0.0 \\
0.0 \\
0.0 \\
0.0 \\
0.0\end{array}$ & $\begin{array}{l}-0.1 \\
-0.1 \\
-0.1 \\
-0.1 \\
-0.1 \\
-0.1 \\
-0.1 \\
-0.1\end{array}$ & $\begin{array}{l}3 \\
9 \\
5 \\
2 \\
0 \\
6 \\
3 \\
0\end{array}$ & $\begin{array}{l}0.153 \\
0.148 \\
0.145 \\
0.142 \\
0.139 \\
0.135 \\
0.132 \\
0.129\end{array}$ \\
\hline $\begin{array}{l}0.4000 \\
0.4500 \\
0.5000 \\
0.5500 \\
0.6000 \\
0.7000 \\
0.8000 \\
0.9000\end{array}$ & $\begin{array}{l}1.90 \\
1.84 \\
1.80 \\
1.76 \\
1.74 \\
1.70 \\
1.68 \\
1.66\end{array}$ & $\begin{array}{l}6.31 \\
6.75 \\
7.22 \\
7.70 \\
8.21 \\
9.25 \\
1.03 \\
1.1\end{array}$ & $\begin{array}{l}1.9 \\
1.8 \\
1.8 \\
1.7 \\
1.7 \\
1.7 \\
1.6\end{array}$ & $\begin{array}{l}1.4 \\
1.7 \\
1.9 \\
2.2 \\
2.5 \\
3.1 \\
3.7 \\
4.3\end{array}$ & $\begin{array}{l}2 . \\
2 . \\
2 . \\
2 . \\
2 . \\
3 .\end{array}$ & $\begin{array}{l}0.0 \\
0.0 \\
0.0 \\
0.0 \\
0.0 \\
0.0 \\
0.0 \\
0.0\end{array}$ & $\begin{array}{l}-0.1 \\
-0.1 \\
-0.1 \\
-0.1 \\
-0.1 \\
-0.1 \\
-0.1 \\
-0.1\end{array}$ & $\begin{array}{l}0.128 \\
0.126 \\
0.125 \\
0.123 \\
0.122 \\
0.120 \\
0.118 \\
0.116\end{array}$ & $\begin{array}{l}0.127 \\
0.125 \\
0.123 \\
0.122 \\
0.120 \\
0.1118 \\
0.1115 \\
0.114\end{array}$ \\
\hline $\begin{array}{l}1.0000 \\
1.2500 \\
1.5000 \\
1.7500 \\
2.0000 \\
2.5000 \\
3.0000 \\
3.5000\end{array}$ & $\begin{array}{l}61 E+00 \\
55 E+00 \\
61 E+00 \\
72 E+00 \\
84 E+00 \\
12 E+00 \\
40 E+00 \\
766 E+00\end{array}$ & $\begin{array}{l}1.271 \mathrm{E}-02 \\
1.588 \mathrm{E}-02 \\
1.927 \mathrm{E}-02 \\
2.284 \mathrm{E}-02 \\
2.656 \mathrm{E}-02 \\
3.437 \mathrm{E}-02 \\
4.260 \mathrm{E}-02 \\
5.115 \mathrm{E}-02\end{array}$ & $\begin{array}{l}1.674 E+00 \\
1.671 E+00 \\
1.680 E+00 \\
1.694 E+00 \\
1.711 E+00 \\
1.747 E+00 \\
1.783 E+00 \\
1.817 E+00\end{array}$ & $\begin{array}{l}4.912 \mathrm{E}-0 \\
6.408 \mathrm{E}-0 \\
7.900 \mathrm{E}-0 \\
9.382 \mathrm{E}-0 \\
1.085 \mathrm{E}+0 \\
1.374 \mathrm{E}+0 \\
1.658 \mathrm{E}+0 \\
1.935 \mathrm{E}+0\end{array}$ & $\begin{array}{l}3 . \\
4 . \\
5 . \\
6 . \\
7 . \\
9 . \\
1 .\end{array}$ & $\begin{array}{l}0.0 \\
0.0 \\
0.0 \\
0.0 \\
0.0 \\
0.0 \\
0.0 \\
0.0\end{array}$ & $\begin{array}{l}-0.10 \\
-0.10 \\
-0.09 \\
-0.09 \\
-0.09 \\
-0.09 \\
-0.09 \\
-0.08\end{array}$ & $\begin{array}{l}0.1 \\
0.1 \\
0.1 \\
0.1 \\
0.1 \\
0.1\end{array}$ & $\begin{array}{l}0.112 \\
0.108 \\
0.106 \\
0.103 \\
0.101 \\
0.098 \\
0.095\end{array}$ \\
\hline $\begin{array}{l}4.0000 \\
4.5000 \\
5.0000 \\
5.5000 \\
6.0000 \\
7.0000 \\
8.0000 \\
9.0000\end{array}$ & $\begin{array}{l}1.790 E+00 \\
1.812 E+00 \\
1.833 E+00 \\
1.852 E+00 \\
1.870 E+00 \\
1.902 E+00 \\
1.931 E+00 \\
1.956 E+00\end{array}$ & $\begin{array}{l}5.999 E-02 \\
6.908 E-02 \\
7.838 E-02 \\
8.787 E-02 \\
9.754 E-02 \\
1.173 E-01 \\
1.376 E-01 \\
1.584 E-01\end{array}$ & $\begin{array}{l}1.8 \\
1.8 \\
1.9 \\
1.9 \\
1.9 \\
2.0 \\
2.0 \\
2.1\end{array}$ & $\begin{array}{l}2.2 \\
2.4 \\
2.7 \\
2.9 \\
3.2 \\
3.7 \\
4.2 \\
4.7\end{array}$ & $\begin{array}{l}1.58 \\
1.79 \\
2.00 \\
2.21 \\
2.42 \\
2\end{array}$ & $\begin{array}{l}0.0 \\
0.0 \\
0.0 \\
0.0 \\
0.0 \\
0.0 \\
0.0 \\
0.0\end{array}$ & $\begin{array}{l}-0.0 \\
-0.0 \\
-0.0 \\
-0.0 \\
-0.0 \\
-0.0 \\
-0.0 \\
-0.0\end{array}$ & $\begin{array}{l}0 . \\
0 . \\
0 . \\
0 . \\
0 . \\
0 . \\
0 .\end{array}$ & $\begin{array}{l}0.091 \\
0.089 \\
0.088 \\
0.086 \\
0.085 \\
0.083 \\
0.081\end{array}$ \\
\hline $\begin{array}{l}10.0000 \\
12.5000 \\
15.0000 \\
17.5000 \\
20.0000 \\
25.0000 \\
30.0000 \\
35.0000\end{array}$ & $\begin{array}{l}1.979 E+00 \\
2.029 E+00 \\
2.069 E+00 \\
2.104 E+00 \\
2.134 E+00 \\
2.185 E+00 \\
2.226 E+00 \\
2.257 E+00\end{array}$ & $\begin{array}{l}2.33 \\
2.89 \\
3.46 \\
4.04 \\
5.21 \\
6.41 \\
7.63\end{array}$ & $\begin{array}{l}0 \\
0 \\
0 \\
0 \\
0 \\
0 \\
0\end{array}$ & $\begin{array}{l}5.1 \\
6.3 \\
7.4 \\
8.4 \\
9.4 \\
1.1 \\
1.3 \\
1.4\end{array}$ & 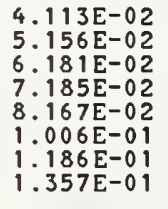 & $\begin{array}{l}0.0 \\
0.0 \\
0.0 \\
0.0 \\
0.0 \\
0.0 \\
7.636 \mathrm{E}-03 \\
5.984 \mathrm{E}-02\end{array}$ & $\begin{array}{l}-0.0 \\
-0.8 \\
-0.2 \\
-0.2 \\
-0.2 \\
-0.8 \\
-0.8 \\
-0.2\end{array}$ & & $\begin{array}{l}0.075 \\
0.072 \\
0.070 \\
0.068 \\
0.065 \\
0.062\end{array}$ \\
\hline $\begin{array}{l}40.0000 \\
45.0000 \\
50.0000 \\
55.0000 \\
60.0000 \\
70.0000 \\
80.0000 \\
90.0000\end{array}$ & $\begin{array}{l}302 E+00 \\
319 E+00 \\
334 E+00 \\
347 E+00 \\
369 E+00 \\
387 E+00 \\
403 E+00\end{array}$ & $\begin{array}{ll}0 & 1 \\
0 & 0 \\
0 & 0 \\
0 & 0 \\
0 & 0 \\
0 & 0 \\
0 & 0 \\
0 & 0\end{array}$ & $\begin{array}{l}0 \\
0 \\
0 \\
0 \\
0 \\
0\end{array}$ & $\begin{array}{l}1.8 \\
1.8 \\
2.0 \\
2.2 \\
2 .\end{array}$ & $\begin{array}{l}1 \\
1 \\
1 \\
1 \\
1 \\
1\end{array}$ & $\begin{array}{l}1.32 \\
2.2 \\
3.1 \\
4.1 \\
5.0 \\
6.7 \\
8.3 \\
9.8\end{array}$ & $\begin{array}{l}-0 . \\
-0 . \\
-0 . \\
-0 . \\
-0 . \\
-0 . \\
-0 . \\
-0 .\end{array}$ & $\begin{array}{l}0 . \\
0 . \\
0 . \\
0 . \\
0 . \\
0 .\end{array}$ & $\begin{array}{l}0 . \\
0 . \\
0 . \\
0 . \\
0 . \\
0 .\end{array}$ \\
\hline $\begin{array}{l}0 \\
0 \\
0 \\
0 \\
0 \\
0\end{array}$ & $\begin{array}{l}E+00 \\
E+00 \\
E+00 \\
E+00 \\
E+00 \\
E+00 \\
E+00 \\
E+00\end{array}$ & $\begin{array}{l}3.0 \\
3.7 \\
4.3 \\
5.0 \\
6.3 \\
7.6 \\
8 .\end{array}$ & 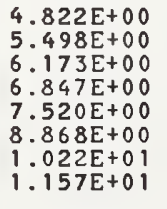 & 4 & & $\begin{array}{l}1 . \\
1 . \\
1 . \\
1 . \\
2 . \\
2 . \\
2 .\end{array}$ & $\begin{array}{l}-0 \\
-0 \\
-0 \\
-0 \\
-0 \\
-0 \\
-0 \\
-0\end{array}$ & & \\
\hline $\begin{array}{l}0.0000 \\
0.0000 \\
0.0000 \\
0.0000 \\
0.0000 \\
0.00000 \\
0.0000\end{array}$ & $\begin{array}{l}2.582 E+00 \\
2.595 E+00 \\
2.606 E+00 \\
2.616 E+00 \\
2.625 E+00 \\
2.641 E+00 \\
2.653 E+00 \\
2.664 E+00\end{array}$ & $\begin{array}{l}1.033 \mathrm{E} \\
1.167 \mathrm{E} \\
1.301 \mathrm{E} \\
1.435 \mathrm{E} \\
1.569 \mathrm{E} \\
1.838 \mathrm{E} \\
2.107 \mathrm{E} \\
2.376 \mathrm{E}\end{array}$ & 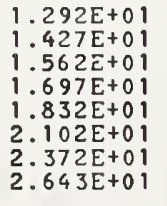 & 1 & $\begin{array}{l}1 \\
1 \\
1 \\
1 \\
1 \\
1\end{array}$ & $\begin{array}{l}.302 E+00 \\
.472 E+00 \\
.628 E+00 \\
.772 E+00 \\
.034 E+00 \\
.267 E+00 \\
.477 E+00\end{array}$ & $\begin{array}{l}-0.0 \\
-0.0 \\
-0.0 \\
-0.0 \\
-0.0 \\
-0.0 \\
-0.0 \\
-0.0\end{array}$ & $\begin{array}{l}0 . \\
0 . \\
0 . \\
0 . \\
0 . \\
0 . \\
0 . \\
0 .\end{array}$ & $\begin{array}{l}7 \\
0 \\
5 \\
5 \\
4\end{array}$ \\
\hline & $674 E+00$ & 646 & $13 E+01$ & $329 E+01$ & $95 \mathrm{E}-0$ & $668 \mathrm{E}$ & 0.005 & 016 &. \\
\hline
\end{tabular}


ELECTRONS IN ALUMINUM OXIDE

$I=145.2 \mathrm{eV} \quad$ DENSITY $=3.970 \mathrm{E}+00 \mathrm{~g} / \mathrm{cm}^{3}$

\begin{tabular}{|c|c|c|c|c|c|c|c|c|c|}
\hline VERGY & COLLISION & $\begin{array}{l}\text { OPPING POWE } \\
\text { RADIATIVE }\end{array}$ & TOTAL & $\begin{array}{l}\text { CSDA } \\
\text { RANGE }\end{array}$ & $\begin{array}{l}\text { DIATION } \\
\text { YIELD }\end{array}$ & $\begin{array}{l}\text { DEHS.EFF. } \\
\text { CORR. } \\
\text { (DELTA) }\end{array}$ & $\begin{array}{l}\text { de } \\
\operatorname{col} L \\
\text { Loss }\end{array}$ & $\begin{array}{l}\text { Jd ( } 1 \\
\text { SDA } \\
\text { ANGE }\end{array}$ & $\begin{array}{l}\text { I ) } \\
\text { RAD } \\
\text { YIELD }\end{array}$ \\
\hline $\mathrm{MeV}$ & $\mathrm{MeV} \mathrm{cm}^{2} / \mathrm{g}$ & $\mathrm{eV} \mathrm{cm}^{2} / \mathrm{g}$ & $\mathrm{eV} \mathrm{cm}^{2} / \mathrm{g}$ & $\mathrm{g} / \mathrm{cm}^{2}$ & & & & & \\
\hline $\begin{array}{l}0.0100 \\
0.0125 \\
0.0150 \\
0.0175 \\
0.0200 \\
0.0250 \\
0.0300 \\
0.0350\end{array}$ & $\begin{array}{l}1.731 E+01 \\
1.465 E+01 \\
1.278 E+01 \\
1.138 E+01 \\
1.029 E+01 \\
8.709 E+00 \\
7.605 E+00 \\
6.789 E+00\end{array}$ & $\begin{array}{l}-03 \\
-03 \\
-03 \\
-03 \\
-03 \\
-03 \\
-03 \\
-03\end{array}$ & $\begin{array}{l}1.7 \\
1.4 \\
1.2 \\
1.1 \\
1.0 \\
8.7 \\
7.6 \\
6.7\end{array}$ & $\begin{array}{l}E-04 \\
E-04 \\
E-04 \\
E-04 \\
E-03 \\
E-03 \\
E-03 \\
E-03\end{array}$ & $\begin{array}{l}714 E-04 \\
068 E-04 \\
407 E-04 \\
734 E-04 \\
050 E-04 \\
656 E-04 \\
231 E-04 \\
781 E-04\end{array}$ & $\begin{array}{l}0.0 \\
0.0 \\
0.0 \\
0.0 \\
0.0 \\
0.0 \\
0.0 \\
0.0\end{array}$ & $\begin{array}{l}-0.229 \\
-0.218 \\
-0.210 \\
-0.203 \\
-0.198 \\
-0.190 \\
-0.184 \\
-0.179\end{array}$ & $\begin{array}{l}0.270 \\
0.255 \\
0.244 \\
0.235 \\
0.228 \\
0.217 \\
0.208 \\
0.202\end{array}$ & $\begin{array}{l}0.266 \\
0.251 \\
0.241 \\
0.232 \\
0.225 \\
0.215 \\
0.207 \\
0.201\end{array}$ \\
\hline $\begin{array}{l}0.0400 \\
0.0450 \\
0.0500 \\
0.0550 \\
0.0600 \\
0.0700 \\
0.0800 \\
0.0900\end{array}$ & $\begin{array}{l}6.160 \mathrm{E}+00 \\
5.658 \mathrm{E}+00 \\
5.249 \mathrm{E}+00 \\
4.909 \mathrm{E}+00 \\
4.621 \mathrm{E}+00 \\
4.161 \mathrm{E}+00 \\
3.809 \mathrm{E}+00 \\
3.530 \mathrm{E}+00\end{array}$ & $\begin{array}{l}5.83 \\
5.85 \\
5.87 \\
5.89 \\
5.92 \\
5.96 \\
6.01 \\
6.06\end{array}$ & $\begin{array}{l}6.165 \mathrm{E}+00 \\
5.664 \mathrm{E}+00 \\
5.255 \mathrm{E}+00 \\
4.915 \mathrm{E}+00 \\
4.627 \mathrm{E}+00 \\
4.167 \mathrm{E}+00 \\
3.815 \mathrm{E}+00 \\
3.536 \mathrm{E}+00\end{array}$ & $\begin{array}{l}3.731 \mathrm{E}-03 \\
4.578 \mathrm{E}-03 \\
5.495 \mathrm{E}-03 \\
6.480 \mathrm{E}-03 \\
7.529 \mathrm{E}-03 \\
9.811 \mathrm{E}-03 \\
1.232 \mathrm{E}-02 \\
1.505 \mathrm{E}-02\end{array}$ & $\begin{array}{l}5.310 \mathrm{E}-04 \\
5.820 \mathrm{E}-04 \\
6.315 \mathrm{E}-04 \\
6.795 \mathrm{E}-04 \\
7.262 \mathrm{E}-04 \\
8.162 \mathrm{E}-04 \\
9.023 \mathrm{E}-04 \\
9.850 \mathrm{E}-04\end{array}$ & $\begin{array}{l}0.0 \\
0.0 \\
0.0 \\
0.0 \\
0.0 \\
0.0 \\
0.0 \\
0.0\end{array}$ & $\begin{array}{l}-0.175 \\
-0.171 \\
-0.169 \\
-0.166 \\
-0.164 \\
-0.160 \\
-0.157 \\
-0.154\end{array}$ & $\begin{array}{l}0.197 \\
0.192 \\
0.189 \\
0.185 \\
0.182 \\
0.178 \\
0.174 \\
0.170\end{array}$ & $\begin{array}{l}0.195 \\
0.191 \\
0.188 \\
0.184 \\
0.182 \\
0.177 \\
0.173 \\
0.170\end{array}$ \\
\hline $\begin{array}{l}0.1000 \\
0.1250 \\
0.1500 \\
0.1750 \\
0.2000 \\
0.2500 \\
0.3000 \\
0.3500\end{array}$ & $\begin{array}{l}3.305 E+00 \\
2.892 \mathrm{E}+00 \\
2.613 \mathrm{E}+00 \\
2.412 \mathrm{E}+00 \\
2.262 \mathrm{E}+00 \\
2.052 \mathrm{E}+00 \\
1.912 \mathrm{E}+00 \\
1.815 \mathrm{E}+00\end{array}$ & $\begin{array}{l}6.126 \mathrm{E}-03 \\
6.285 \mathrm{E}-03 \\
6.464 \mathrm{E}-03 \\
6.661 \mathrm{E}-03 \\
6.871 \mathrm{E}-03 \\
7.330 \mathrm{E}-03 \\
7.834 \mathrm{E}-03 \\
8.376 \mathrm{E}-03\end{array}$ & $\begin{array}{l}3.311 \mathrm{E}+00 \\
2.898 \mathrm{E}+00 \\
2.619 \mathrm{E}+00 \\
2.419 \mathrm{E}+00 \\
2.269 \mathrm{E}+00 \\
2.059 \mathrm{E}+00 \\
1.920 \mathrm{E}+00 \\
1.823 \mathrm{E}+00\end{array}$ & $\begin{array}{l}1.797 \mathrm{E}-02 \\
2.608 \mathrm{E}-02 \\
3.517 \mathrm{E}-02 \\
4.512 \mathrm{E}-02 \\
5.581 \mathrm{E}-02 \\
7.902 \mathrm{E}-02 \\
1.042 \mathrm{E}-01 \\
1.310 \mathrm{E}-01\end{array}$ & $\begin{array}{l}1.065 \mathrm{E}-03 \\
1.254 \mathrm{E}-03 \\
1.432 \mathrm{E}-03 \\
1.600 \mathrm{E}-03 \\
1.762 \mathrm{E}-03 \\
2.068 \mathrm{E}-03 \\
2.360 \mathrm{E}-03 \\
2.643 \mathrm{E}-03\end{array}$ & $\begin{array}{l}0.0 \\
0.0 \\
0.0 \\
0.0 \\
0.0 \\
1.094 \mathrm{E}-03 \\
2.440 \mathrm{E}-02 \\
4.961 \mathrm{E}-02\end{array}$ & $\begin{array}{l}-0.152 \\
-0.147 \\
-0.143 \\
-0.140 \\
-0.138 \\
-0.125 \\
-0.113 \\
-0.107\end{array}$ & $\begin{array}{l}0.167 \\
0.162 \\
0.157 \\
0.154 \\
0.151 \\
0.146 \\
0.139 \\
0.133\end{array}$ & $\begin{array}{l}0.167 \\
0.161 \\
0.157 \\
0.153 \\
0.150 \\
0.145 \\
0.137 \\
0.131\end{array}$ \\
\hline $\begin{array}{l}0.4000 \\
0.4500 \\
0.5000 \\
0.5500 \\
0.6000 \\
0.7000 \\
0.8000 \\
0.9000\end{array}$ & $\begin{array}{l}1.744 E+00 \\
1.691 E+00 \\
1.650 E+00 \\
1.618 E+00 \\
1.593 E+00 \\
1.557 E+00 \\
1.533 E+00 \\
1.517 E+00\end{array}$ & $\begin{array}{l}9.5631 \\
1.0201 \\
1.0861 \\
1.1551 \\
1.2981 \\
1.449 \\
1.6061\end{array}$ & $\begin{array}{l}1.753 \mathrm{E}+00 \\
1.700 \mathrm{E}+00 \\
1.660 \mathrm{E}+00 \\
1.629 \mathrm{E}+00 \\
1.605 \mathrm{E}+00 \\
1.570 \mathrm{E}+00 \\
1.547 \mathrm{E}+00 \\
1.533 \mathrm{E}+00\end{array}$ & $\begin{array}{l}1.590 \mathrm{E}-01 \\
1.879 \mathrm{E}-01 \\
2.177 \mathrm{E}-01 \\
2.481 \mathrm{E}-01 \\
2.791 \mathrm{E}-01 \\
3.421 \mathrm{E}-01 \\
4.063 \mathrm{E}-01 \\
4.712 \mathrm{E}-01\end{array}$ & $\begin{array}{l}3.191 \\
3.460 \\
3.728 \\
3.995 \\
4.529 \\
5.064 \\
5.603\end{array}$ & $\begin{array}{l}7.60 \\
1.03 \\
1.31 \\
1.59 \\
1.88 \\
2.45 \\
3.02 \\
3.58\end{array}$ & $\begin{array}{l}-0.102 \\
-0.097 \\
-0.094 \\
-0.090 \\
-0.087 \\
-0.082 \\
-0.078 \\
-0.074\end{array}$ & $\begin{array}{l}88 \\
3 \\
9 \\
6 \\
3 \\
8 \\
3 \\
9\end{array}$ & $\begin{array}{l}0.125 \\
0.120 \\
0.1116 \\
0.112 \\
0.109 \\
0.103 \\
0.097 \\
0.093\end{array}$ \\
\hline $\begin{array}{l}1.0000 \\
1.2500 \\
1.5000 \\
1.7500 \\
2.0000 \\
2.5000 \\
3.0000 \\
3.5000\end{array}$ & $\begin{array}{l}1.507 E+00 \\
1.494 E+00 \\
1.492 E+00 \\
1.495 E+00 \\
1.501 E+00 \\
1.514 E+00 \\
1.528 E+00 \\
1.541 E+00\end{array}$ & $\begin{array}{l}1.769 \mathrm{E}-02 \\
2.201 \mathrm{E}-02 \\
2.662 \mathrm{E}-02 \\
3.148 \mathrm{E}-02 \\
3.653 \mathrm{E}-02 \\
4.714 \mathrm{E}-02 \\
5.829 \mathrm{E}-02 \\
6.987 \mathrm{E}-02\end{array}$ & $\begin{array}{l}1.524 E+00 \\
1.516 E+00 \\
1.519 E+00 \\
1.527 E+00 \\
1.537 E+00 \\
1.561 E+00 \\
1.586 E+00 \\
1.611 E+00\end{array}$ & $\begin{array}{l}5.367 E-01 \\
7.013 \mathrm{E}-01 \\
8.661 \mathrm{E}-01 \\
1.030 \mathrm{E}+00 \\
1.193 \mathrm{E}+00 \\
1.516 \mathrm{E}+00 \\
1.834 \mathrm{E}+00 \\
2.147 \mathrm{E}+00\end{array}$ & $\begin{array}{l}6.147 \mathrm{E}-03 \\
7.528 \mathrm{E}-03 \\
8.942 \mathrm{E}-03 \\
1.039 \mathrm{E}-02 \\
1.186 \mathrm{E}-02 \\
1.488 \mathrm{E}-02 \\
1.798 \mathrm{E}-02 \\
2.113 \mathrm{E}-02\end{array}$ & $\begin{array}{l}4.143 \mathrm{E}-c \\
5.479 \mathrm{E}-\mathrm{C} \\
6.744 \mathrm{E}-\mathrm{C} \\
7.938 \mathrm{E}-\mathrm{C} \\
9.069 \mathrm{E}-\mathrm{c} \\
1.116 \mathrm{E}+\mathrm{c} \\
1.306 \mathrm{E}+\mathrm{C} \\
1.479 \mathrm{E}+\mathrm{C}\end{array}$ & $\begin{array}{l}-0.0 \\
-0.0 \\
-0.0 \\
-0.0 \\
-0.0 \\
-0.0 \\
-0.0 \\
-0.0\end{array}$ & & $\begin{array}{l}0.089 \\
0.081 \\
0.075 \\
0.070 \\
0.065 \\
0.058 \\
0.053 \\
0.048\end{array}$ \\
\hline $\begin{array}{l}4.0000 \\
4.5000 \\
5.0000 \\
5.5000 \\
6.0000 \\
7.0000 \\
8.0000 \\
9.0000\end{array}$ & $\begin{array}{l}1.553 E+00 \\
1.564 E+00 \\
1.575 E+00 \\
1.584 E+00 \\
1.593 E+00 \\
1.609 E+00 \\
1.622 E+00 \\
1.634 E+00\end{array}$ & $\begin{array}{l}8.181 \mathrm{E}-02 \\
9.407 \mathrm{E}-02 \\
1.066 \mathrm{E}-01 \\
1.194 \mathrm{E}-01 \\
1.324 \mathrm{E}-01 \\
1.590 \mathrm{E}-01 \\
1.862 \mathrm{E}-01 \\
2.140 \mathrm{E}-01\end{array}$ & $\begin{array}{l}1.635 E+00 \\
1.658 E+00 \\
1.681 E+00 \\
1.704 E+00 \\
1.725 E+00 \\
1.767 E+00 \\
1.808 E+00 \\
1.848 E+00\end{array}$ & $\begin{array}{l}2.455 \mathrm{E}+00 \\
2.759 \mathrm{E}+00 \\
3.058 \mathrm{E}+00 \\
3.353 \mathrm{E}+00 \\
3.645 \mathrm{E}+00 \\
4.218 \mathrm{E}+00 \\
4.777 \mathrm{E}+00 \\
5.324 \mathrm{E}+00\end{array}$ & $\begin{array}{l}2.433 \mathrm{E}-02 \\
2.756 \mathrm{E}-02 \\
3.081 \mathrm{E}-02 \\
3.408 \mathrm{E}-02 \\
3.735 \mathrm{E}-02 \\
4.392 \mathrm{E}-02 \\
5.049 \mathrm{E}-02 \\
5.704 \mathrm{E}-02\end{array}$ & $\begin{array}{l}1.638 E+00 \\
1.785 E+00 \\
1.921 E+00 \\
2.048 E+00 \\
2.167 E+00 \\
2.384 E+00 \\
2.578 E+00 \\
2.754 E+00\end{array}$ & $\begin{array}{l}-0.033 \\
-0.031 \\
-0.029 \\
-0.027 \\
-0.026 \\
-0.024 \\
-0.022 \\
-0.020\end{array}$ & $\begin{array}{l}0.054 \\
0.051 \\
0.049 \\
0.047 \\
0.044 \\
0.041 \\
0.039\end{array}$ & $\begin{array}{l}0.045 \\
0.041 \\
0.039 \\
0.037 \\
0.035 \\
0.031 \\
0.029 \\
0.027\end{array}$ \\
\hline $\begin{array}{l}10.0000 \\
12.5000 \\
15.0000 \\
17.5000 \\
20.0000 \\
25.0000 \\
30.0000 \\
35.0000\end{array}$ & $\begin{array}{l}1.645 E+00 \\
1.667 E+00 \\
1.685 E+00 \\
1.700 E+00 \\
1.713 E+00 \\
1.734 E+00 \\
1.750 E+00 \\
1.764 E+00\end{array}$ & $\begin{array}{l}2.422 \mathrm{E}-01 \\
3.145 \mathrm{E}-01 \\
3.886 \mathrm{E}-01 \\
4.642 \mathrm{E}-01 \\
5.409 \mathrm{E}-01 \\
6.968 \mathrm{E}-01 \\
8.552 \mathrm{E}-01 \\
1.015 \mathrm{E}+00\end{array}$ & $\begin{array}{l}1.887 E+00 \\
1.982 E+00 \\
2.074 E+00 \\
2.164 E+00 \\
2.254 E+00 \\
2.431 E+00 \\
2.606 E+00 \\
2.780 E+00\end{array}$ & $\begin{array}{l}5.859 E+00 \\
7.152 E+00 \\
8.385 E+00 \\
9.565 E+00 \\
1.070 E+01 \\
1.283 E+01 \\
1.482 E+01 \\
1.668 E+01\end{array}$ & $\begin{array}{l}6.354 \mathrm{E}-02 \\
7.956 \mathrm{E}-02 \\
9.516 \mathrm{E}-02 \\
1.103 \mathrm{E}-01 \\
1.249 \mathrm{E}-01 \\
1.527 \mathrm{E}-01 \\
1.786 \mathrm{E}-01 \\
2.026 \mathrm{E}-01\end{array}$ & $\begin{array}{l}2.914 E+00 \\
3.263 E+00 \\
3.557 E+00 \\
3.812 E+00 \\
4.038 E+00 \\
4.424 E+00 \\
4.747 E+00 \\
5.025 E+00\end{array}$ & $\begin{array}{l}-0.019 \\
-0.0117 \\
-0.0115 \\
-0.013 \\
-0.0112 \\
-0.0110 \\
-0.008 \\
-0.007\end{array}$ & & $\begin{array}{l}0.025 \\
0.021 \\
0.019 \\
0.017 \\
0.015 \\
0.013 \\
0.011 \\
0.010\end{array}$ \\
\hline $\begin{array}{l}40.0000 \\
45.0000 \\
50.0000 \\
55.0000 \\
60.0000 \\
70.0000 \\
80.00000 \\
90.0000\end{array}$ & $\begin{array}{l}1.776 E+00 \\
1.786 E+00 \\
1.795 E+00 \\
1.803 E+00 \\
1.810 E+00 \\
1.822 E+00 \\
1.833 E+00 \\
1.842 E+00\end{array}$ & $\begin{array}{l}1.340 E+00 \\
1.504 E+00 \\
1.669 E+00 \\
1.835 E+00 \\
2.168 E+00 \\
2.503 E+00 \\
2.841 E+00\end{array}$ & $\begin{array}{l}2.95 \\
3.12 \\
3.29 \\
3.47 \\
3.64 \\
3.99 \\
4.33 \\
4.68\end{array}$ & $\begin{array}{l}1.0 \\
2.0 \\
2.1 \\
2.3 \\
2.4\end{array}$ & $\begin{array}{l}2.2 \\
2.4 \\
2.6 \\
2.8 \\
3.0 \\
3.3 \\
3.6 \\
3.8\end{array}$ & $\begin{array}{l}5.269 E+00 \\
5.487 E+00 \\
5.684 E+00 \\
5.864 E+00 \\
6.029 E+00 \\
6.323 E+00 \\
6.581 E+00 \\
6.809 E+00\end{array}$ & $\begin{array}{l}-0.006 \\
-0.005 \\
-0.005 \\
-0.004 \\
-0.004 \\
-0.003 \\
-0.002 \\
-0.002\end{array}$ & & $\begin{array}{l}0.008 \\
0.008 \\
0.007 \\
0.006 \\
0.006 \\
0.005 \\
0.004 \\
0.003\end{array}$ \\
\hline $\begin{array}{l}.0000 \\
.0000 \\
.0000 \\
.0000 \\
.0000 \\
.0000 \\
.0000 \\
.0000\end{array}$ & $\begin{array}{l}1.851 \\
1.868 \\
1.882 \\
1.894 \\
1.904 \\
1.921 \\
1.935 \\
1.947\end{array}$ & $\begin{array}{l}3.17 \\
4.03 \\
4.88 \\
5.74 \\
6.61 \\
8.34 \\
1.00 \\
1.18\end{array}$ & $\begin{array}{l}5.898 \mathrm{E}+00 \\
6.769 \mathrm{E}+00 \\
7.641 \mathrm{E}+00 \\
8.515 \mathrm{E}+00 \\
1.027 \mathrm{E}+01 \\
1.202 \mathrm{E}+01 \\
1.378 \mathrm{E}+01\end{array}$ & $\begin{array}{l}3.839 E+01 \\
4.235 E+01 \\
4.582 E+01 \\
4.892 E+01 \\
5.426 E+01 \\
5.876 E+01 \\
6.264 E+01\end{array}$ & $\begin{array}{l}2.0 \\
5.3 \\
5.6 \\
6.0\end{array}$ & $\begin{array}{l}7.015 E+00 \\
7.452 E+00 \\
7.812 E+00 \\
8.117 E+00 \\
8.382 E+00 \\
8.825 E+00 \\
9.188 E+00 \\
9.495 E+00\end{array}$ & $\begin{array}{l}-0.0 \\
-0.0 \\
-0.0 \\
-0.0 \\
-0.0 \\
-0.0 \\
-0.0\end{array}$ & & $\begin{array}{l}0.003 \\
0.002 \\
0.002 \\
0.002 \\
0.001 \\
0.001 \\
0.001 \\
0.001\end{array}$ \\
\hline $\begin{array}{l}0.0000 \\
0.0000 \\
0.0000 \\
0.0000 \\
0.0000 \\
0.0000 \\
0.00000 \\
0.0000\end{array}$ & $\begin{array}{l}1.957 E+00 \\
1.966 E+00 \\
1.974 E+00 \\
1.981 E+00 \\
1.988 E+00 \\
1.999 E+00 \\
2.009 E+00 \\
2.018 E+00\end{array}$ & $\begin{array}{l}1.358 E+01 \\
1.533 E+01 \\
1.709 E+01 \\
1.885 E+01 \\
2.060 E+01 \\
2.412 E+01 \\
2.765 E+01 \\
3.117 E+01\end{array}$ & $\begin{array}{l}1.554 E+01 \\
1.730 E+01 \\
1.906 E+01 \\
2.083 E+01 \\
2.259 E+01 \\
2.612 E+01 \\
2.966 E+01 \\
3.319 E+01\end{array}$ & $\begin{array}{l}605 E+01 \\
910 E+01 \\
185 E+01 \\
436 E+01 \\
667 E+01 \\
078 E+01 \\
437 E+01 \\
756 E+01\end{array}$ & $\begin{array}{l}6.990 \mathrm{E}-01 \\
7.191 \mathrm{E}-01 \\
7.364 \mathrm{E}-01 \\
7.513 \mathrm{E}-01 \\
7.644 \mathrm{E}-01 \\
7.864 \mathrm{E}-01 \\
8.0411 \mathrm{E}-01 \\
8.187 \mathrm{E}-01\end{array}$ & $\begin{array}{l}9.761 \mathrm{E}+00 \\
9.996 \mathrm{E}+00 \\
1.021 \mathrm{E}+01 \\
1.040 \mathrm{E}+01 \\
1.057 \mathrm{E}+01 \\
1.088 \mathrm{E}+01 \\
1.115 \mathrm{E}+01 \\
1.138 \mathrm{E}+01\end{array}$ & $\begin{array}{l}-0.000 \\
-0.000 \\
-0.000 \\
-0.000 \\
-0.000 \\
-0.000 \\
-0.000 \\
-0.000\end{array}$ & & $\begin{array}{l}0.001 \\
0.001 \\
0.001 \\
0.000 \\
0.000 \\
0.000 \\
0.000 \\
0.000\end{array}$ \\
\hline .0000 & $2.026 E+00$ & $.470 E+01$ & $673 E+01$ & $.042 E+01$ & $311 E-01$ & $159 E+01$ & -0.0 & 4 & 000 \\
\hline
\end{tabular}




\begin{tabular}{|c|c|c|c|c|c|c|c|c|c|}
\hline \multirow{2}{*}{$\begin{array}{c}\text { ENERGY } \\
\mathrm{MeV}\end{array}$} & \multicolumn{2}{|c|}{$\begin{array}{l}\text { STOPPING POWER } \\
\text { COLLISION RADIATIVE }\end{array}$} & TOTAL & \multirow{2}{*}{$\begin{array}{l}\text { CSDA } \\
\text { RANGE } \\
\mathrm{g} / \mathrm{cm}^{2}\end{array}$} & \multirow[t]{2}{*}{$\begin{array}{l}\text { RADIATION } \\
\text { YIELD }\end{array}$} & \multirow[t]{2}{*}{$\begin{array}{l}\text { DENS.EFF. } \\
\text { CORR } \\
\text { (DELTA) }\end{array}$} & \multirow[t]{2}{*}{$\begin{array}{l}d(10 \\
\text { coll } \\
\text { LOSS }\end{array}$} & \multirow[t]{2}{*}{$\begin{array}{l}\text { )/d( I } \\
\text { CSDA } \\
\text { RANGE }\end{array}$} & \multirow[t]{2}{*}{$\begin{array}{l}\text { OgI ) } \\
\text { RAD } \\
\text { YIELD }\end{array}$} \\
\hline & $\mathrm{MeV} \mathrm{cm}^{2} / \mathrm{g}$ & $\mathrm{MeV} \mathrm{cm}^{2} / \mathrm{g}$ & $\mathrm{eV} \mathrm{cm}^{2} / \mathrm{g}$ & & & & & & \\
\hline $\begin{array}{l}0.0100 \\
0.0125 \\
0.0150 \\
0.0175 \\
0.0200 \\
0.0250 \\
0.0300 \\
0.0350\end{array}$ & $\begin{array}{l}1.756 \\
1.525 \\
1.354 \\
1.222 \\
1.030 \\
8.967 \\
7.986\end{array}$ & $\begin{array}{l}4.5 \\
4.6 \\
4.6 \\
4.7 \\
4.7 \\
4.7 \\
4.8\end{array}$ & $\begin{array}{l}1.5 \\
1.3 \\
1.2 \\
1.8 \\
8 .\end{array}$ & $\begin{array}{l}E-04 \\
E-04 \\
E-04 \\
-04 \\
E-04 \\
E-03 \\
E-03 \\
E-03\end{array}$ & $\begin{array}{l}4 \\
4 \\
4 \\
4 \\
4 \\
4 \\
4\end{array}$ & $\begin{array}{l}0.0 \\
0.0 \\
0.0 \\
0.0 \\
0.0 \\
0.0 \\
0.0 \\
0.0\end{array}$ & $\begin{array}{l}4 \\
6 \\
9 \\
4 \\
9 \\
3 \\
8 \\
4\end{array}$ & $\begin{array}{l}0.235 \\
0.223 \\
0.215 \\
0.208 \\
0.202 \\
0.194 \\
0.187 \\
0.182\end{array}$ & $\begin{array}{l}0.232 \\
0.221 \\
0.213 \\
0.206 \\
0.201 \\
0.192 \\
0.186 \\
0.181\end{array}$ \\
\hline $\begin{array}{l}0.0400 \\
0.0450 \\
0.0500 \\
0.0550 \\
0.0600 \\
0.0700 \\
0.0800 \\
0.0900\end{array}$ & $\begin{array}{l}7.23 \\
6.63 \\
6.14 \\
5.73 \\
5.39 \\
4.85 \\
4.43 \\
4.10\end{array}$ & $\begin{array}{l}4.8 \\
4.9 \\
4.9 \\
4.9 \\
5.0 \\
5.0\end{array}$ & $\begin{array}{l}7.237 E+00 \\
6.637 E+00 \\
6.150 E+00 \\
5.744 E+00 \\
5.402 E+00 \\
4.855 E+00 \\
4.438 E+00 \\
4.109 E+00\end{array}$ & $\begin{array}{l}4 . \\
5: \\
6 . \\
8 .\end{array}$ & $\begin{array}{l}4 . \\
4 . \\
4 . \\
5 \\
5 . \\
6 . \\
7 .\end{array}$ & $\begin{array}{l}0.0 \\
0.0 \\
0.0 \\
0.0 \\
0.0 \\
0.0 \\
0.0 \\
0.0\end{array}$ & $\begin{array}{l}-0 \\
-0 \\
-0 \\
-0 \\
-0 \\
-0 \\
-0\end{array}$ & $\begin{array}{l}0.178 \\
0.174 \\
0.171 \\
0.168 \\
0.166 \\
0.162 \\
0.159 \\
0.156\end{array}$ & $\begin{array}{l}0.1 \\
0.1 \\
0.1\end{array}$ \\
\hline $\begin{array}{l}0.1000 \\
0.1250 \\
0.1500 \\
0.1750 \\
0.2000 \\
0.2500 \\
0.7500 \\
0 . \quad 500\end{array}$ & $\begin{array}{l}3.837 E+00 \\
3.350 E+00 \\
3.021 E+00 \\
2.785 E+00 \\
2.600 E+00 \\
2.36 ? 2+00 \\
2.201 E+00 \\
2.030 E+00\end{array}$ & $\begin{array}{l}5.4 \\
5.6 \\
5.8 \\
6.2\end{array}$ & $\begin{array}{l}E+00 \\
E+00 \\
+00 \\
+00 \\
E+00 \\
E+00 \\
E+00 \\
+00 \\
+00\end{array}$ & $\begin{array}{l}-02 \\
-02 \\
-02 \\
-02 \\
-02 \\
-02 \\
-02 \\
-01\end{array}$ & & $\begin{array}{l}0.0 \\
0.0 \\
0.0 \\
0.0 \\
0.0 \\
0.0 \\
0.0 \\
4.066 \mathrm{E}-0\end{array}$ & $\begin{array}{l}-0 \\
-0 \\
-0 \\
-0 \\
-0 \\
-0\end{array}$ & & \\
\hline $\begin{array}{l}0.4000 \\
0.4500 \\
0.5000 \\
0.5500 \\
0.6000 \\
0.7000 \\
0.8000 \\
0.9000\end{array}$ & $\begin{array}{l}2.0 \\
1.9 \\
1.8 \\
1.8 \\
1.8 \\
1.7 \\
1.7 \\
1.7\end{array}$ & 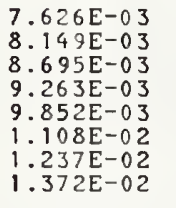 & $\begin{array}{l}013 E+00 \\
950 E+00 \\
902 E+00 \\
864 E+00 \\
835 E+00 \\
791 E+00 \\
762 E+00 \\
743 E+00\end{array}$ & $\begin{array}{l}378 \mathrm{E}-01 \\
631 \mathrm{E}-01 \\
891 \mathrm{E}-01 \\
156 \mathrm{E}-01 \\
427 \mathrm{E}-01 \\
979 \mathrm{E}-01 \\
542 \mathrm{E}-01 \\
113 \mathrm{E}-01\end{array}$ & & $\begin{array}{l}2.9 \\
5.8 \\
8.9 \\
1.2 \\
1.5 \\
2.2 \\
2.9 \\
3.5\end{array}$ & $\begin{array}{l}-0.0 \\
-0.08 \\
-0.08 \\
-0.0 \\
-0.0 \\
-0.06 \\
-0.06 \\
-0.0\end{array}$ & & $\begin{array}{l}0 . \\
0 . \\
0 .\end{array}$ \\
\hline $\begin{array}{l}1.0000 \\
1.2500 \\
1.5000 \\
1.7500 \\
2.0000 \\
2.5000 \\
3.0000 \\
3.5000\end{array}$ & $\begin{array}{l}1.715 \mathrm{E}+00 \\
1.697 \mathrm{E}+00 \\
1.691 \mathrm{E}+00 \\
1.691 \mathrm{E}+00 \\
1.695 \mathrm{E}+00 \\
1.706 \mathrm{E}+00 \\
1.719 \mathrm{E}+00 \\
1.733 \mathrm{E}+00\end{array}$ & $\begin{array}{l}1.8 \\
2.2 \\
2.8 \\
3.9 \\
4 .\end{array}$ & $\begin{array}{l}1.731 \mathrm{E}+00 \\
1.715 \mathrm{E}+00 \\
1.714 \mathrm{E}+00 \\
1.718 \mathrm{E}+00 \\
1.726 \mathrm{E}+00 \\
1.747 \mathrm{E}+00 \\
1.769 \mathrm{E}+00 \\
1.793 \mathrm{E}+00\end{array}$ & & & $\begin{array}{l}4.2 \\
5.8 \\
7.3 \\
8.7 \\
9.9\end{array}$ & & & \\
\hline $\begin{array}{l}4.0000 \\
4.5000 \\
5.0000 \\
5.5000 \\
6.0000 \\
7.0000 \\
8.0000 \\
9.0000\end{array}$ & $\begin{array}{l}1.745 E+00 \\
1.757 E+00 \\
1.768 E+00 \\
1.773 E+00 \\
1.788 E+00 \\
1.806 E+00 \\
1.821 E+00 \\
1.834 E+00\end{array}$ & $\begin{array}{l}2 \\
2 \\
2 \\
1 \\
1\end{array}$ & $\begin{array}{l}+00 \\
+00 \\
+00 \\
+00 \\
+00 \\
+00 \\
+00 \\
+00\end{array}$ & $\begin{array}{l}+00 \\
+00 \\
+00 \\
+00 \\
+00 \\
+00 \\
+00 \\
+00\end{array}$ & $\begin{array}{l}2 \\
2 \\
2 \\
2\end{array}$ & $\begin{array}{l}+00 \\
+00 \\
+00 \\
+00 \\
+00 \\
+00 \\
+00 \\
+00\end{array}$ & & & \\
\hline $\begin{array}{l}10.0 \\
12.5 \\
15.0 \\
17.5 \\
20.0 \\
25.0 \\
30.0 \\
35.0\end{array}$ & $\begin{array}{l}1.8 \\
1.8 \\
1.9 \\
1.9 \\
1.9 \\
1.9 \\
1.9\end{array}$ & $\begin{array}{l}2 . \\
3 . \\
4 .\end{array}$ & $\begin{array}{l}0 \\
0 \\
0 \\
0 \\
0 \\
0 \\
0\end{array}$ & $\begin{array}{l}0 \\
0 \\
0 \\
0 \\
0 \\
1 \\
1 \\
1\end{array}$ & & $\begin{array}{l}0 \\
0 \\
0 \\
0 \\
0 \\
0 \\
0 \\
0\end{array}$ & & & \\
\hline $\begin{array}{l}45.00000 \\
50.00000 \\
55.00000 \\
60.0000 \\
70.0000 \\
80.0000\end{array}$ & $\begin{array}{l}1.99 \\
2.00 \\
2.01 \\
2.02 \\
2.03 \\
2.04 \\
2.05\end{array}$ & $\begin{array}{l}1.3 \\
1.4 \\
1.5 \\
1.8\end{array}$ & $\begin{array}{l}0 \\
0 \\
0 \\
0 \\
0 \\
0 \\
0 \\
0\end{array}$ & $\begin{array}{l}1 \\
1 \\
1 \\
1 \\
1 \\
1 \\
1 \\
1\end{array}$ & & $\begin{array}{l}0 \\
0 \\
0 \\
0 \\
0 \\
0 \\
0\end{array}$ & & & \\
\hline $\begin{array}{l}0 \\
0 \\
0\end{array}$ & 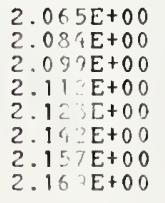 & & & & & & & & \\
\hline $\begin{array}{llll}0 & 0 & 0 & 0 \\
0 & 0 & 0 & 0 \\
0 & 0 & 0 & 0 \\
0 & 0 & 0 & 0 \\
0 & 0 & 0 & 0 \\
0 & 0 & 0 & 0 \\
0 & 0 & 0 & 0 \\
0 & 0 & 0 & 0\end{array}$ & $\begin{array}{l}2.15 E+00 \\
2.206 E+00 \\
2.213 E+00 \\
2.226 E+00 \\
2.237 E+00 \\
2.2 \div 6 E+00\end{array}$ & $2.718 E+01$ & $\begin{array}{l}1.554 E+01 \\
1.708 E+01 \\
1.862 E+01 \\
2.016 E+01 \\
2.325 E+01 \\
2.634 E+01 \\
2.943 E+01\end{array}$ & $\begin{array}{l}7.112 E+01 \\
7.418 E+01 \\
7.699 E+01 \\
7.957 E+01 \\
8.418 E+01 \\
8.822 E+01 \\
9.181 E+01\end{array}$ & & $\begin{array}{l}1.011 \mathrm{E}+01 \\
1.032 \mathrm{E}+01 \\
1.051 \mathrm{E}+01 \\
1.069 \mathrm{E}+01 \\
1.099 \mathrm{E}+01 \\
1.126 \mathrm{E}+01 \\
1.150 \mathrm{E}+01\end{array}$ & & & \\
\hline .000 & $2.255 E+00$ & $.026 E+01$ & $.252 E+01$ & $.504 E+01$ & $8.010 \mathrm{E}-01$ & $1.171 E+01$ & -0.000 & 0.003 & .000 \\
\hline
\end{tabular}


ELECTRONS IN BONE, COMPACT (ICRU)

$I=91.9 \mathrm{eV} \quad$ DENSITY $=1.850 \mathrm{E}+00 \mathrm{~g} / \mathrm{cm}^{3}$

\begin{tabular}{|c|c|c|c|c|c|c|c|c|c|}
\hline \multirow{2}{*}{$\begin{array}{l}\text { ENERGY } \\
\mathrm{MeV}\end{array}$} & COLLISION & STOPPING POWER & TOTAL & \multirow{2}{*}{$\begin{array}{l}\text { CSDA } \\
\text { RANGE } \\
\mathrm{g} / \mathrm{cm}^{2}\end{array}$} & \multirow[t]{2}{*}{$\begin{array}{l}\text { RADIATION } \\
\text { YIELD }\end{array}$} & \multirow[t]{2}{*}{$\begin{array}{l}\text { DEIS.EFF. } \\
\text { CORR } \\
\text { (DELTA) }\end{array}$} & \multirow[t]{2}{*}{$\begin{array}{l}d(109 \\
\text { coLL } \\
\text { LOSS }\end{array}$} & \multirow[t]{2}{*}{$\begin{array}{l}\text { J } / d(1) \\
\text { CSDA } \\
\text { RAHGE }\end{array}$} & \multirow[t]{2}{*}{$\begin{array}{l}\circ g \text { I ) } \\
\text { RAD } \\
\text { YIELD }\end{array}$} \\
\hline & $\mathrm{MeV} \mathrm{cm}^{2} / \mathrm{g}$ & $\mathrm{MeV} \mathrm{cm}^{2} / \mathrm{g}$ & $\mathrm{MeV} \mathrm{cm}^{2} / \mathrm{g}$ & & & & & & \\
\hline $\begin{array}{l}0.0100 \\
0.0125 \\
0.0150 \\
0.0175 \\
0.0200 \\
0.0250 \\
0.0300 \\
0.0350\end{array}$ & $\begin{array}{l}2.068 E+01 \\
1.742 E+01 \\
1.514 E+01 \\
1.344 E+01 \\
1.213 E+01 \\
1.023 E+01 \\
8.912 E+00 \\
7.939 E+00\end{array}$ & $\begin{array}{l}4.793 \mathrm{E}-03 \\
4.880 \mathrm{E}-03 \\
4.942 \mathrm{E}-03 \\
4.989 \mathrm{E}-03 \\
5.026 \mathrm{E}-03 \\
5.080 \mathrm{E}-03 \\
5.121 \mathrm{E}-03 \\
5.155 \mathrm{E}-03\end{array}$ & $\begin{array}{l}2.068 E+01 \\
1.742 E+01 \\
1.514 E+01 \\
1.345 E+01 \\
1.214 E+01 \\
1.024 E+01 \\
8.917 E+00 \\
7.944 E+00\end{array}$ & $\begin{array}{l}2.761 \mathrm{E}-04 \\
4.084 \mathrm{E}-04 \\
5.628 \mathrm{E}-04 \\
7.383 \mathrm{E}-04 \\
9.343 \mathrm{E}-04 \\
1.385 \mathrm{E}-03 \\
1.910 \mathrm{E}-03 \\
2.505 \mathrm{E}-03\end{array}$ & $\begin{array}{l}1.236 \mathrm{E}-04 \\
1.501 \mathrm{E}-04 \\
1.756 \mathrm{E}-04 \\
2.004 \mathrm{E}-04 \\
2.244 \mathrm{E}-04 \\
2.706 \mathrm{E}-04 \\
3.148 \mathrm{E}-04 \\
3.572 \mathrm{E}-04\end{array}$ & $\begin{array}{l}0.0 \\
0.0 \\
0.0 \\
0.0 \\
0.0 \\
0.0 \\
0.0 \\
0.0\end{array}$ & $\begin{array}{l}-0.207 \\
-0.198 \\
-0.191 \\
-0.186 \\
-0.182 \\
-0.175 \\
-0.169 \\
-0.165\end{array}$ & $\begin{array}{l}0.239 \\
0.227 \\
0.218 \\
0.211 \\
0.205 \\
0.196 \\
0.190 \\
0.184\end{array}$ & $\begin{array}{l}0.236 \\
0.224 \\
0.216 \\
0.209 \\
0.203 \\
0.195 \\
0.188 \\
0.183\end{array}$ \\
\hline $\begin{array}{l}0.0400 \\
0.0450 \\
0.0500 \\
0.0550 \\
0.0500 \\
0.0700 \\
0.0800 \\
0.0500\end{array}$ & $\begin{array}{l}7.190 E+00 \\
6.596 E+00 \\
6.112 E+00 \\
5.709 E+00 \\
5.370 E+00 \\
4.827 E+00 \\
4.412 E+00 \\
4.055 E+00\end{array}$ & $\begin{array}{l}5.184 \mathrm{E}-03 \\
5.211 \mathrm{E}-03 \\
5.237 \mathrm{E}-03 \\
5.263 \mathrm{E}-03 \\
5.288 \mathrm{E}-03 \\
5.338 \mathrm{E}-03 \\
5.390 \mathrm{E}-03 \\
5.445 \mathrm{E}-03\end{array}$ & $\begin{array}{l}7.196 E+00 \\
6.601 E+00 \\
6.117 E+00 \\
5.715 E+00 \\
5.375 E+00 \\
4.832 E+00 \\
4.417 E+00 \\
4.090 E+00\end{array}$ & $\begin{array}{l}3.168 E-03 \\
3.894 E-03 \\
4.682 E-03 \\
5.528 E-03 \\
6.431 E-03 \\
8.397 E-03 \\
1.056 E-02 \\
1.292 E-02\end{array}$ & $\begin{array}{l}3.982 E-04 \\
4.379 E-04 \\
4.764 E-04 \\
5.139 E-04 \\
5.504 E-04 \\
6.210 E-04 \\
6.887 E-04 \\
7.540 E-04\end{array}$ & $\begin{array}{l}0.0 \\
0.0 \\
0.0 \\
0.0 \\
0.0 \\
0.0 \\
0.0 \\
0.0\end{array}$ & $\begin{array}{l}-0.162 \\
-0.159 \\
-0.156 \\
-0.154 \\
-0.152 \\
-0.149 \\
-0.146 \\
-0.144\end{array}$ & $\begin{array}{l}0.180 \\
0.176 \\
0.173 \\
0.170 \\
0.168 \\
0.164 \\
0.160 \\
0.158\end{array}$ & $\begin{array}{l}0.179 \\
0.175 \\
0.172 \\
0.170 \\
0.167 \\
0.163 \\
0.160 \\
0.157\end{array}$ \\
\hline $\begin{array}{l}0.1000 \\
0.1250 \\
0.1520 \\
0.1750 \\
0.2000 \\
0.2500 \\
0.3000 \\
0.3500\end{array}$ & $\begin{array}{ll}3.82 & E+00 \\
3.3 .6 & E+00 \\
3.01 & E+00 \\
2.77 & E+00 \\
2.5 & E+00 \\
2.3 & E+00 \\
2.1 & E+00 \\
2.0 & E+00\end{array}$ & $\begin{array}{l}5.502 E-03 \\
5.657 E-03 \\
5.835 E-03 \\
6.007 E-03 \\
6.200 E-03 \\
6.618 E-03 \\
7.075 E-03 \\
7.557 E-03\end{array}$ & $\begin{array}{l}3.825 E+00 \\
3.342 E+00 \\
3.015 E+00 \\
2.781 E+00 \\
2.605 E+00 \\
2.361 E+00 \\
2.201 E+00 \\
2.087 E+00\end{array}$ & $\begin{array}{l}1.545 E-02 \\
2.247 E-02 \\
3.037 E-02 \\
3.901 E-02 \\
4.832 E-02 \\
6.855 E-02 \\
9.053 E-02 \\
1.139 E-01\end{array}$ & $\begin{array}{l}8.171 \mathrm{E}-04 \\
9.670 \mathrm{E}-04 \\
1.108 \mathrm{E}-03 \\
1.242 \mathrm{E}-03 \\
1.371 \mathrm{E}-03 \\
1.615 \mathrm{E}-03 \\
1.848 \mathrm{E}-03 \\
2.072 \mathrm{E}-03\end{array}$ & $\begin{array}{l}0.0 \\
0.0 \\
0.0 \\
0.0 \\
0.0 \\
0.0 \\
0.0 \\
2.843 \mathrm{E}-02\end{array}$ & $\begin{array}{l}-0.142 \\
-0.138 \\
-0.135 \\
-0.132 \\
-0.130 \\
-0.126 \\
-0.118 \\
-0.096\end{array}$ & $\begin{array}{l}0.155 \\
0.150 \\
0.147 \\
0.143 \\
0.141 \\
0.137 \\
0.134 \\
0.127\end{array}$ & $\begin{array}{l}0.155 \\
0.150 \\
0.146 \\
0.143 \\
0.140 \\
0.136 \\
0.133 \\
0.126\end{array}$ \\
\hline $\begin{array}{l}0.4000 \\
0.4500 \\
0.5000 \\
0.5500 \\
0.6000 \\
0.7000 \\
0.3000 \\
0.3000\end{array}$ & $\begin{array}{l}1.92 E+00 \\
1.932 E+00 \\
1.833 E+00 \\
1.815 E+00 \\
1.815 E+00 \\
1.770 E+00 \\
1.740 E+00 \\
1.719 E+00\end{array}$ & $\begin{array}{l}8.050 E-03 \\
8.643 E-03 \\
9.221 E-03 \\
9.823 E-03 \\
1.045 E-02 \\
1.175 E-02 \\
1.311 E-02 \\
1.454 E-02\end{array}$ & $\begin{array}{l}2.004 E+00 \\
1.941 E+00 \\
1.893 E+00 \\
1.855 E+00 \\
1.825 E+00 \\
1.782 E+00 \\
1.753 E+00 \\
1.734 E+00\end{array}$ & $\begin{array}{l}1.384 E-01 \\
1.637 E-01 \\
1.898 E-01 \\
2.165 E-01 \\
2.437 E-01 \\
2.992 E-01 \\
3.558 E-01 \\
4.132 E-01\end{array}$ & $\begin{array}{l}2.292 E-03 \\
2.509 E-03 \\
2.724 E-03 \\
2.939 E-03 \\
3.153 E-03 \\
3.582 E-03 \\
4.014 E-03 \\
4.449 E-03\end{array}$ & $\begin{array}{l}6.250 \mathrm{E}-02 \\
9.798 \mathrm{E}-02 \\
1.342 \mathrm{E}-01 \\
1.710 \mathrm{E}-01 \\
2.079 \mathrm{E}-01 \\
2.817 \mathrm{E}-01 \\
3.548 \mathrm{E}-01 \\
4.265 \mathrm{E}-01\end{array}$ & $\begin{array}{l}-0.089 \\
-0.084 \\
-0.079 \\
-0.075 \\
-0.071 \\
-0.066 \\
-0.061 \\
-0.057\end{array}$ & $\begin{array}{l}0.1121 \\
0.1116 \\
0.111 \\
0.1107 \\
0.103 \\
0.096 \\
0.091 \\
0.086\end{array}$ & $\begin{array}{l}0.119 \\
0.112 \\
0.107 \\
0.102 \\
0.098 \\
0.091 \\
0.084 \\
0.079\end{array}$ \\
\hline $\begin{array}{l}1.0000 \\
1.2500 \\
1.5000 \\
1.7500 \\
2.0000 \\
2.5000 \\
3.0000 \\
3.5000\end{array}$ & $\begin{array}{l}1.705 E+00 \\
1.656 E+00 \\
1.680 E+00 \\
1.681 E+00 \\
1.624 E+00 \\
1.635 E+00 \\
1.709 E+00 \\
1.722 E+00\end{array}$ & $\begin{array}{l}1.602 E-02 \\
1.995 E-02 \\
2.414 E-02 \\
2.854 E-02 \\
3.313 E-02 \\
4.277 E-02 \\
5.289 E-02 \\
6.341 E-02\end{array}$ & $\begin{array}{l}1.721 E+00 \\
1.706 E+00 \\
1.705 E+00 \\
1.709 E+00 \\
1.718 E+00 \\
1.739 E+00 \\
1.762 E+00 \\
1.786 E+00\end{array}$ & $\begin{array}{l}4.711 E-01 \\
6.171 E-01 \\
7.638 E-01 \\
9.103 E-01 \\
1.056 E+00 \\
1.346 E+00 \\
1.631 E+00 \\
1.913 E+00\end{array}$ & $\begin{array}{l}4.889 E-03 \\
6.009 E-03 \\
7.161 E-03 \\
8.341 E-03 \\
9.547 E-03 \\
1.202 E-02 \\
1.457 E-02 \\
1.717 E-02\end{array}$ & $\begin{array}{l}4.964 E-01 \\
6.626 E-01 \\
8.160 E-01 \\
9.575 E-01 \\
1.088 E+00 \\
1.323 E+00 \\
1.527 E+00 \\
1.709 E+00\end{array}$ & $\begin{array}{l}-0.054 \\
-0.048 \\
-0.044 \\
-0.041 \\
-0.039 \\
-0.035 \\
-0.033 \\
-0.031\end{array}$ & $\begin{array}{l}0.083 \\
0.075 \\
0.069 \\
0.065 \\
0.061 \\
0.056 \\
0.052 \\
0.049\end{array}$ & $\begin{array}{l}0.075 \\
0.066 \\
0.060 \\
0.055 \\
0.051 \\
0.046 \\
0.042 \\
0.039\end{array}$ \\
\hline $\begin{array}{l}4.0000 \\
4.5000 \\
5.0000 \\
5.5000 \\
6.0000 \\
7.0000 \\
8.0000 \\
9.0000\end{array}$ & $\begin{array}{l}1.735 E+00 \\
1.747 E+00 \\
1.753 E+00 \\
1.768 E+00 \\
1.775 E+00 \\
1.755 E+00 \\
1.810 E+00 \\
1.823 E+00\end{array}$ & $\begin{array}{l}7.427 \mathrm{E}-02 \\
8.541 \mathrm{E}-02 \\
9.680 \mathrm{E}-02 \\
1.084 \mathrm{E}-01 \\
1.202 \mathrm{E}-01 \\
1.444 \mathrm{E}-01 \\
1.692 \mathrm{E}-01 \\
1.945 \mathrm{E}-01\end{array}$ & $\begin{array}{l}1.809 E+00 \\
1.832 E+00 \\
1.855 E+00 \\
1.877 E+00 \\
1.898 E+00 \\
1.939 E+00 \\
1.979 E+00 \\
2.018 E+00\end{array}$ & $\begin{array}{l}2.191 E+00 \\
2.466 E+00 \\
2.737 E+00 \\
3.005 E+00 \\
3.270 E+00 \\
3.791 E+00 \\
4.301 E+00 \\
4.802 E+00\end{array}$ & $\begin{array}{l}1.981 E-02 \\
2.248 E-02 \\
2.517 E-02 \\
2.788 E-02 \\
3.060 E-02 \\
3.607 E-02 \\
4.156 E-02 \\
4.705 E-02\end{array}$ & $\begin{array}{l}1.872 E+00 \\
2.020 E+00 \\
2.156 E+00 \\
2.282 E+00 \\
2.399 E+00 \\
2.611 E+00 \\
2.801 E+00 \\
2.972 E+00\end{array}$ & $\begin{array}{l}-0.030 \\
-0.029 \\
-0.027 \\
-0.026 \\
-0.025 \\
-0.024 \\
-0.022 \\
-0.021\end{array}$ & $\begin{array}{l}0.046 \\
0.044 \\
0.043 \\
0.041 \\
0.040 \\
0.037 \\
0.035 \\
0.034\end{array}$ & $\begin{array}{l}0.037 \\
0.035 \\
0.033 \\
0.032 \\
0.031 \\
0.028 \\
0.027 \\
0.025\end{array}$ \\
\hline $\begin{array}{l}10.0000 \\
12.5000 \\
15.0000 \\
17.5000 \\
20.0000 \\
25.0000 \\
30.0000 \\
35.0000\end{array}$ & $\begin{array}{l}1.835 E+00 \\
1.860 E+00 \\
1.879 E+00 \\
1.896 E+00 \\
1.909 E+00 \\
1.931 E+00 \\
1.949 E+00 \\
1.963 E+00\end{array}$ & $\begin{array}{l}2.202 E-01 \\
2.860 E-01 \\
3.537 E-01 \\
4.226 E-01 \\
4.926 E-01 \\
6.351 E-01 \\
7.799 E-01 \\
9.264 E-01\end{array}$ & $\begin{array}{l}2.055 E+00 \\
2.146 E+00 \\
2.233 E+00 \\
2.318 E+00 \\
2.402 E+00 \\
2.566 E+00 \\
2.729 E+00 \\
2.890 E+00\end{array}$ & $\begin{array}{l}5.293 E+00 \\
6.483 E+00 \\
7.625 E+00 \\
8.724 E+00 \\
9.783 E+00 \\
1.180 E+01 \\
1.369 E+01 \\
1.547 E+01\end{array}$ & $\begin{array}{l}5.252 E-02 \\
6.608 E-02 \\
7.939 E-02 \\
9.240 E-02 \\
1.051 E-01 \\
1.294 E-01 \\
1.523 E-01 \\
1.739 E-01\end{array}$ & $\begin{array}{l}3.129 E+00 \\
3.473 E+00 \\
3.765 E+00 \\
4.021 E+00 \\
4.247 E+00 \\
4.637 E+00 \\
4.965 E+00 \\
5.247 E+00\end{array}$ & $\begin{array}{l}-0.019 \\
-0.017 \\
-0.014 \\
-0.0112 \\
-0.011 \\
-0.008 \\
-0.007 \\
-0.006\end{array}$ & $\begin{array}{l}0.032 \\
0.029 \\
0.027 \\
0.025 \\
0.023 \\
0.021 \\
0.018 \\
0.017\end{array}$ & $\begin{array}{l}0.024 \\
0.021 \\
0.019 \\
0.017 \\
0.0115 \\
0.012 \\
0.010 \\
0.009\end{array}$ \\
\hline $\begin{array}{l}40.0000 \\
45.0000 \\
50.0000 \\
55.0000 \\
60.0000 \\
70.0000 \\
80.0000 \\
90.0000\end{array}$ & $\begin{array}{l}1.976 E+00 \\
1.986 E+00 \\
1.996 E+00 \\
2.004 E+00 \\
2.012 E+00 \\
2.025 E+00 \\
2.037 E+00 \\
2.047 E+00\end{array}$ & $\begin{array}{l}1.074 E+00 \\
1.224 E+00 \\
1.374 E+00 \\
1.525 E+00 \\
1.676 E+00 \\
1.982 E+00 \\
2.289 E+00 \\
2.598 E+00\end{array}$ & $\begin{array}{l}3.050 E+00 \\
3.210 E+00 \\
3.369 E+00 \\
3.529 E+00 \\
3.688 E+00 \\
4.007 E+00 \\
4.326 E+00 \\
4.645 E+00\end{array}$ & $\begin{array}{l}1.715 E+01 \\
1.875 E+01 \\
2.027 E+01 \\
2.172 E+01 \\
2.310 E+01 \\
2.570 E+01 \\
2.811 E+01 \\
3.034 E+01\end{array}$ & $\begin{array}{l}1.943 \mathrm{E}-01 \\
2.134 \mathrm{E}-01 \\
2.316 \mathrm{E}-01 \\
2.487 \mathrm{E}-01 \\
2.649 \mathrm{E}-01 \\
2.949 \mathrm{E}-01 \\
3.221 \mathrm{E}-01 \\
3.468 \mathrm{E}-01\end{array}$ & $\begin{array}{l}5.495 E+00 \\
5.716 E+00 \\
5.915 E+00 \\
6.097 E+00 \\
6.263 E+00 \\
6.559 E+00 \\
6.817 E+00 \\
7.046 E+00\end{array}$ & $\begin{array}{l}-0.005 \\
-0.004 \\
-0.004 \\
-0.003 \\
-0.003 \\
-0.003 \\
-0.002 \\
-0.002\end{array}$ & $\begin{array}{l}0.016 \\
0.014 \\
0.0114 \\
0.0113 \\
0.0112 \\
0.011 \\
0.0110 \\
0.010\end{array}$ & $\begin{array}{l}0.008 \\
0.007 \\
0.006 \\
0.005 \\
0.005 \\
0.004 \\
0.004 \\
0.003\end{array}$ \\
\hline $\begin{array}{l}100.0000 \\
125.0000 \\
150.0000 \\
175.0000 \\
200.0000 \\
250.0000 \\
300.0000 \\
350.0000\end{array}$ & $\begin{array}{l}2.056 E+00 \\
2.075 E+00 \\
2.090 E+00 \\
2.103 E+00 \\
2.114 E+00 \\
2.133 E+00 \\
2.148 E+00 \\
2.160 E+00\end{array}$ & $\begin{array}{l}2.909 E+00 \\
3.690 E+00 \\
4.476 E+00 \\
5.266 E+00 \\
6.059 E+00 \\
7.652 E+00 \\
9.251 E+00 \\
1.086 E+01\end{array}$ & $\begin{array}{l}4.965 E+00 \\
5.765 E+00 \\
6.566 E+00 \\
7.369 E+00 \\
8.173 E+00 \\
9.784 E+00 \\
1.140 E+01 \\
1.302 E+01\end{array}$ & $\begin{array}{l}3.242 E+01 \\
3.709 E+01 \\
4.115 E+01 \\
4.474 E+01 \\
4.796 E+01 \\
5.354 E+01 \\
5.827 E+01 \\
6.238 E+01\end{array}$ & $\begin{array}{l}3.694 \mathrm{E}-01 \\
4.184 \mathrm{E}-01 \\
4.590 \mathrm{E}-01 \\
4.932 \mathrm{E}-01 \\
5.226 \mathrm{E}-01 \\
5.707 \mathrm{E}-01 \\
6.085 \mathrm{E}-01 \\
6.392 \mathrm{E}-01\end{array}$ & $\begin{array}{l}7.251 E+00 \\
7.688 E+00 \\
8.046 E+00 \\
8.350 E+00 \\
8.614 E+00 \\
9.057 E+00 \\
9.419 E+00 \\
9.726 E+00\end{array}$ & $\begin{array}{l}-0.0022 \\
-0.001 \\
-0.001 \\
-0.001 \\
-0.001 \\
-0.001 \\
-0.001 \\
-0.0000\end{array}$ & $\begin{array}{l}0.009 \\
0.008 \\
0.007 \\
0.007 \\
0.006 \\
0.006 \\
0.005 \\
0.005\end{array}$ & $\begin{array}{l}0.003 \\
0.002 \\
0.002 \\
0.001 \\
0.001 \\
0.001 \\
0.001 \\
0.001\end{array}$ \\
\hline $\begin{array}{l}400.0000 \\
450.0000 \\
500.0000 \\
550.0000 \\
600.0000 \\
700.0000 \\
800.0000 \\
900.0000\end{array}$ & $\begin{array}{l}2.171 \mathrm{E}+00 \\
2.181 \mathrm{E}+00 \\
2.189 \mathrm{E}+00 \\
2.197 \mathrm{E}+00 \\
2.204 \mathrm{E}+00 \\
2.217 \mathrm{E}+00 \\
2.228 \mathrm{E}+00 \\
2.237 \mathrm{E}+00\end{array}$ & $\begin{array}{l}1.246 E+01 \\
1.407 E+01 \\
1.569 E+01 \\
1.730 E+01 \\
1.892 E+01 \\
2.216 E+01 \\
2.540 E+01 \\
2.864 E+01\end{array}$ & $\begin{array}{l}1.464 E+01 \\
1.626 E+01 \\
1.788 E+01 \\
1.950 E+01 \\
2.112 E+01 \\
2.437 E+01 \\
2.763 E+01 \\
3.088 E+01\end{array}$ & $\begin{array}{l}6.600 E+01 \\
6.924 E+01 \\
7.217 E+01 \\
7.485 E+01 \\
7.731 E+01 \\
8.171 E+01 \\
8.556 E+01 \\
8.898 E+01\end{array}$ & $\begin{array}{l}6.647 \mathrm{E}-01 \\
6.863 \mathrm{E}-01 \\
7.048 \mathrm{E}-01 \\
7.210 \mathrm{E}-01 \\
7.352 \mathrm{E}-01 \\
7.591 \mathrm{E}-01 \\
7.785 \mathrm{E}-01 \\
7.947 \mathrm{E}-01\end{array}$ & $\begin{array}{l}9.991 E+00 \\
1.023 E+01 \\
1.044 E+01 \\
1.063 E+01 \\
1.080 E+01 \\
1.111 E+01 \\
1.137 E+01 \\
1.161 E+01\end{array}$ & $\begin{array}{l}-0.000 \\
-0.000 \\
-0.000 \\
-0.000 \\
-0.000 \\
-0.000 \\
-0.000 \\
-0.000\end{array}$ & $\begin{array}{l}0.005 \\
0.004 \\
0.004 \\
0.004 \\
0.004 \\
0.004 \\
0.004 \\
0.003\end{array}$ & $\begin{array}{l}0.001 \\
0.001 \\
0.000 \\
0.000 \\
0.000 \\
0.000 \\
0.000 \\
0.000\end{array}$ \\
\hline 000 & $2.246 E+00$ & $3.189 E+01$ & $3.414 E+01$ & $.206 E+01$ & $.083 \mathrm{E}-01$ & 1.182 & -0.000 & 0.003 & .000 \\
\hline
\end{tabular}




\begin{tabular}{|c|c|c|c|c|c|c|c|c|c|}
\hline \multirow{2}{*}{$\begin{array}{l}\text { ENERGY } \\
\text { MeV }\end{array}$} & \multicolumn{3}{|c|}{$\begin{array}{l}\text { STOPPING POWER } \\
\text { COLLISION }\end{array}$} & \multirow{2}{*}{$\begin{array}{l}\text { CSDA } \\
\text { RANGE } \\
g^{\prime} \mathrm{cm}^{2}\end{array}$} & \multirow[t]{2}{*}{$\begin{array}{l}\text { RADIATION } \\
\text { YIELD }\end{array}$} & \multirow[t]{2}{*}{$\begin{array}{l}\text { DEHS.EFF. } \\
\text { CORR. } \\
\text { (DELTA) }\end{array}$} & \multirow{2}{*}{\multicolumn{3}{|c|}{$\begin{array}{l}d(\log ) / d(\log I) \\
\text { COLL CSDA RAD } \\
\text { LOSS RANGE YIELD }\end{array}$}} \\
\hline & $\mathrm{MeV} \mathrm{cm}^{2} / \mathrm{g}$ & $\mathrm{MeV} \mathrm{cm}^{2} / \mathrm{g}$ & $\mathrm{MeV} \mathrm{cm}^{2} / \mathrm{g}$ & & & & & & \\
\hline $\begin{array}{l}0.0100 \\
0.0125 \\
0.0150 \\
0.0175 \\
0.0200 \\
0.0250 \\
0.0300 \\
0.0350\end{array}$ & $\begin{array}{l}1.971 E+01 \\
1.663 \mathrm{E}+01 \\
1.447 \mathrm{E}+01 \\
1.286 \mathrm{E}+01 \\
1.161 \mathrm{E}+01 \\
9.804 \mathrm{E}+00 \\
8.54 \mathrm{E}+00 \\
7.618 \mathrm{E}+00\end{array}$ & $\begin{array}{l}5.5 \\
5.6 \\
5.7 \\
5.7 \\
5.8 \\
5.9\end{array}$ & $\begin{array}{l}1 \\
1 \\
1 \\
1 \\
1 \\
0 \\
0 \\
0 \\
0\end{array}$ & $\begin{array}{l}-04 \\
-04 \\
-04 \\
-04 \\
-04 \\
-03 \\
-03 \\
-03\end{array}$ & $\begin{array}{l}1.468 \mathrm{E}-04 \\
1.787 \mathrm{E}-04 \\
2.095 \mathrm{E}-04 \\
2.393 \mathrm{E}-04 \\
2.683 \mathrm{E}-04 \\
3.242 \mathrm{E}-04 \\
3.775 \mathrm{E}-04 \\
4.287 \mathrm{E}-04\end{array}$ & $\begin{array}{l}0.0 \\
0.0 \\
0.0 \\
0.0 \\
0.0 \\
0.0 \\
0.0 \\
0.0\end{array}$ & $\begin{array}{l}0.214 \\
0.2204 \\
0.197 \\
0.191 \\
0.187 \\
0.179 \\
0.174 \\
0.169\end{array}$ & $\begin{array}{l}248 \\
235 \\
226 \\
218 \\
212 \\
202 \\
195 \\
190\end{array}$ & $\begin{array}{l}244 \\
232 \\
223 \\
216 \\
210 \\
200 \\
194\end{array}$ \\
\hline $\begin{array}{l}0.0400 \\
0.0450 \\
0.0500 \\
0.0550 \\
0.0600 \\
0.0700 \\
0.0800 \\
0.0900\end{array}$ & $\begin{array}{l}3 E+00 \\
5 E+00 \\
2 E+00 \\
3 E+00 \\
3 E+00 \\
3 E+00 \\
6 E+00 \\
2 E+00\end{array}$ & $\begin{array}{l}0 . \\
6 . \\
6 . \\
6 . \\
6 . \\
6 .\end{array}$ & $\begin{array}{l}5.8 \\
5.4 \\
5.1 \\
4.6 \\
4.2 \\
3.9\end{array}$ & $\begin{array}{l}E-03 \\
E-03 \\
E-03 \\
E-03 \\
E-03 \\
E-03 \\
E-02 \\
E-02\end{array}$ & $\begin{array}{l}-04 \\
-04 \\
-04 \\
-04 \\
-04 \\
-04 \\
-04 \\
-04\end{array}$ & $\begin{array}{l}0.0 \\
0.0 \\
0.0 \\
0.0 \\
0.0 \\
0.0 \\
0.0 \\
0.0\end{array}$ & $\begin{array}{l}-0.166 \\
-0.163 \\
-0.160 \\
-0.158 \\
-0.156 \\
-0.152 \\
-0.149 \\
-0.147\end{array}$ & & $\begin{array}{l}0.184 \\
0.180 \\
0.177 \\
0.174 \\
0.171 \\
0.167 \\
0.164 \\
0.161\end{array}$ \\
\hline $\begin{array}{l}0.1000 \\
0.1250 \\
0.1500 \\
0.1750 \\
0.2000 \\
0.2500 \\
0.3000 \\
0.3500\end{array}$ & $\begin{array}{l}3.21 \\
2.90 \\
2.67 \\
2.50 \\
2.27 \\
2.11\end{array}$ & $\begin{array}{l}6 . \\
6: \\
6 . \\
7 \\
7 . \\
8 .\end{array}$ & $\begin{array}{l}3.685 \mathrm{E}+00 \\
3.221 \mathrm{E}+00 \\
2.908 \mathrm{E}+00 \\
2.683 \mathrm{E}+00 \\
2.514 \mathrm{E}+00 \\
2.280 \mathrm{E}+00 \\
2.127 \mathrm{E}+00 \\
2.020 \mathrm{E}+00\end{array}$ & $\begin{array}{l}2.336 \mathrm{E}-02 \\
3.155 \mathrm{E}-02 \\
4.051 \mathrm{E}-02 \\
5.015 \mathrm{E}-02 \\
7.111 \mathrm{E}-02 \\
9.386 \mathrm{E}-02 \\
1.180 \mathrm{E}-01\end{array}$ & $\begin{array}{l}1.161 \mathrm{E}-03 \\
1.329 \mathrm{E}-03 \\
1.489 \mathrm{E}-03 \\
1.641 \mathrm{E}-03 \\
1.931 \mathrm{E}-03 \\
2.206 \mathrm{E}-03 \\
2.471 \mathrm{E}-03\end{array}$ & $\begin{array}{l}0.0 \\
0.0 \\
0.0 \\
0.0 \\
0.0 \\
0.0 \\
0.0 \\
8.920 \mathrm{E}-03\end{array}$ & & & $\begin{array}{l}0.158 \\
0.153 \\
0.149 \\
0.146 \\
0.143 \\
0.139 \\
0.136 \\
0.131\end{array}$ \\
\hline $\begin{array}{l}0.4000 \\
0.4500 \\
0.5000 \\
0.5500 \\
0.6000 \\
0.7000 \\
0.8000 \\
0.9000\end{array}$ & $\begin{array}{l}931 E+00 \\
871 E+00 \\
825 E+00 \\
789 E+00 \\
760 E+00 \\
713 E+00 \\
690 E+00 \\
671 E+00\end{array}$ & $\begin{array}{l}9 . \\
1: \\
1: \\
1:\end{array}$ & $\begin{array}{l}1.5 \\
1: 5 \\
1: 2 \\
1: 2 \\
1:\end{array}$ & $\begin{array}{l}1 \\
1 \\
1 \\
1 \\
1 \\
1 \\
1 \\
1\end{array}$ & & $\begin{array}{l}3.4 \\
6.2 \\
9.1 \\
1.2 \\
1.5 \\
2.1 \\
2.8 \\
3.4\end{array}$ & $\begin{array}{l}-0 \\
-0 \\
-0 \\
-0 \\
-0\end{array}$ & & \\
\hline $\begin{array}{l}1.0000 \\
1.2500 \\
1.5000 \\
1.7500 \\
2.0000 \\
2.5000 \\
3.0000 \\
3.5000\end{array}$ & $\begin{array}{l}1.64 \\
1.63 \\
1.63 \\
1.64 \\
1.65 \\
1.67 \\
1.68\end{array}$ & $\begin{array}{l}2.2 \\
2.7 \\
3.2 \\
3.7 \\
4.8 \\
5.8 \\
7.1\end{array}$ & $\begin{array}{l}1.2 \\
1.8 \\
1.8 \\
1.5 \\
1.2 \\
1.2\end{array}$ & $\begin{array}{l}1 \\
1 \\
1 \\
0 \\
0 \\
0 \\
0\end{array}$ & & $\begin{array}{l}4.0 \\
5.5 \\
6.9 \\
8.3 \\
9.5 \\
1.1 \\
1.3 \\
1.5\end{array}$ & & & $\begin{array}{l}82 \\
73 \\
66 \\
61 \\
57 \\
51 \\
46\end{array}$ \\
\hline $\begin{array}{l}4.00 \\
4.50 \\
5.00 \\
5.50 \\
6.00 \\
7.00 \\
8.00 \\
9.00\end{array}$ & $\begin{array}{l}1.70 \\
1.72 \\
1.73 \\
1.74 \\
1.75 \\
1.77\end{array}$ & $\begin{array}{l}9 . \\
1: \\
1: \\
1 .\end{array}$ & i. & 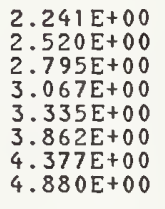 & $\begin{array}{l}2 \\
2 \\
3 \\
3 \\
4\end{array}$ & 1.8 & & & \\
\hline $\begin{array}{l}10.0000 \\
12.5000 \\
15.0000 \\
17.5000 \\
20.0000 \\
25.0000 \\
30.0000 \\
35.0000\end{array}$ & $\begin{array}{l}1.82 \\
1.84 \\
1.86 \\
1.87 \\
1.89 \\
1.91 \\
1.92\end{array}$ & $\begin{array}{l}3.2 \\
3.9 \\
4.7 \\
5.5 \\
7.1 \\
8.7 \\
1.0\end{array}$ & $\begin{array}{l}2.1 \\
2.2 \\
2.3 \\
2.4 \\
2.6 \\
2.7 \\
2.9\end{array}$ & $\begin{array}{l}0 \\
0 \\
0 \\
0 \\
0 \\
1 \\
1 \\
1\end{array}$ & 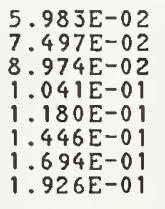 & 3.5 & $\begin{array}{l}- \\
- \\
- \\
- \\
- \\
-\end{array}$ & & \\
\hline $\begin{array}{l}45.0 \\
50.0 \\
55.0 \\
60.0 \\
70.0 \\
80.0\end{array}$ & $\begin{array}{l}1.9 \\
1.9 \\
1.9 \\
1.9 \\
1.9 \\
2.0 \\
2.0\end{array}$ & $\begin{array}{l}1 \\
1 \\
1 \\
1 \\
2\end{array}$ & $\begin{array}{l}0 \\
0 \\
0 \\
0 \\
0 \\
0 \\
0\end{array}$ & $\begin{array}{l}1 \\
1 \\
1 \\
1 \\
1\end{array}$ & & $\begin{array}{l}5 . \\
5 . \\
5\end{array}$ & & & \\
\hline $\begin{array}{l}0 \\
0 \\
0 \\
0 \\
0 \\
0 \\
0\end{array}$ & $\begin{array}{l}2.04 \\
2.05 \\
2.06 \\
2.08 \\
2.09 \\
2.11 \\
2.12\end{array}$ & $\begin{array}{l}4.1 \\
4.9 \\
5.8 \\
6.7 \\
8.5\end{array}$ & $\begin{array}{lll}0 & 0 \\
0 & 0 \\
0 & 0 \\
0 & 0 \\
0 & 0 \\
0 & 1 \\
0 & 1 \\
0 & 1\end{array}$ & & & $\begin{array}{l}0 \\
0 \\
0 \\
0 \\
0 \\
0\end{array}$ & & & \\
\hline $\begin{array}{l}450.0000 \\
500.0000 \\
550.0000 \\
600.00000 \\
700.0000 \\
800.00000 \\
900.0000\end{array}$ & $\begin{array}{l}2.13 \\
2.14 \\
2.15 \\
2.16 \\
2.16 \\
2.18 \\
2.18 \\
2.20\end{array}$ & $\begin{array}{l}1.5 \\
1.7 \\
1.92 \\
2.1 \\
2.4\end{array}$ & $\begin{array}{l}+01 \\
+01 \\
+01 \\
+01 \\
+01 \\
+01 \\
+01 \\
+01\end{array}$ & $\begin{array}{l}1 \\
1 \\
1 \\
1 \\
1 \\
1\end{array}$ & $\begin{array}{l}7.9 \\
7.2 \\
7.5 \\
7.5 \\
7.2\end{array}$ & $\begin{array}{l}0 \\
1 \\
1 \\
1 \\
1 \\
1 \\
1\end{array}$ & & & $\begin{array}{l}0 \\
0 \\
0\end{array}$ \\
\hline & $2.210 E+00$ & $.553 E+01$ & $774 E+01$ & $.636 E+01$ & $.235 E-01$ & $.151 E+01$ & -0.000 & 0.004 & 0.000 \\
\hline
\end{tabular}


ELECTRONS IN C-552 AIR-EQUIVALENT PLASTIC

$I=86.8 \mathrm{eV} \quad$ DENSITY $=1.760 \mathrm{E}+00 \mathrm{~g} / \mathrm{cm}^{3}$

\begin{tabular}{|c|c|c|c|c|c|c|c|c|c|}
\hline ENERGY & $\begin{array}{l}\text { COLLISION } \\
\mathrm{MeV} \mathrm{Cm}^{2} / \mathrm{g}\end{array}$ & $\begin{array}{l}\text { DPPING POWE } \\
\text { RADIATIVE } \\
\text { MeV } \mathrm{cm}^{2} / \mathrm{g}\end{array}$ & 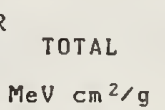 & $\begin{array}{l}\text { CSDA } \\
\text { RAMGE } \\
\mathrm{g} / \mathrm{Cm}^{2}\end{array}$ & $\begin{array}{l}\text { RADIATION } \\
\text { YIELD }\end{array}$ & $\begin{array}{l}\text { DEHS.EFF. } \\
\text { CORR } \\
\text { (DELTA) }\end{array}$ & $\begin{array}{l}d(1 \\
\text { COLL } \\
\text { LOSS }\end{array}$ & $\begin{array}{l}\mathrm{Jd} \\
\text { CSDA } \\
\text { RANGE }\end{array}$ & $\begin{array}{l}\circ \text { I ) } \\
\text { RAD } \\
\text { YIELD }\end{array}$ \\
\hline $\begin{array}{l}0.0100 \\
0.0125 \\
0.0150 \\
0.0175 \\
0.0200 \\
0.0250 \\
0.0300 \\
0.0350\end{array}$ & $\begin{array}{l}1.972 E+01 \\
1.660 E+01 \\
1.442 E+01 \\
1.281 E+01 \\
1.156 E+01 \\
9.741 E+00 \\
8.482 E+00 \\
7.554 E+00\end{array}$ & $\begin{array}{l}3.767 \mathrm{E}-03 \\
3.794 \mathrm{E}-03 \\
3.812 \mathrm{E}-03 \\
3.824 \mathrm{E}-03 \\
3.834 \mathrm{E}-03 \\
3.848 \mathrm{E}-03 \\
3.860 \mathrm{E}-03 \\
3.871 \mathrm{E}-03\end{array}$ & $\begin{array}{l}1.972 E+01 \\
1.661 E+01 \\
1.443 E+01 \\
1.281 E+01 \\
1.156 E+01 \\
9.745 E+00 \\
8.486 E+00 \\
7.558 E+00\end{array}$ & $\begin{array}{l}2.890 \mathrm{E}-04 \\
4.277 \mathrm{E}-04 \\
5.897 \mathrm{E}-04 \\
7.739 \mathrm{E}-04 \\
9.797 \mathrm{E}-04 \\
1.453 \mathrm{E}-03 \\
2.004 \mathrm{E}-03 \\
2.630 \mathrm{E}-03\end{array}$ & $\begin{array}{l}1.046 \mathrm{E}-04 \\
1.257 \mathrm{E}-04 \\
1.458 \mathrm{E}-04 \\
1.652 \mathrm{E}-04 \\
1.839 \mathrm{E}-04 \\
2.199 \mathrm{E}-04 \\
2.541 \mathrm{E}-04 \\
2.869 \mathrm{E}-04\end{array}$ & $\begin{array}{l}0.0 \\
0.0 \\
0.0 \\
0.0 \\
0.0 \\
0.0 \\
0.0 \\
0.0\end{array}$ & $\begin{array}{l}-0.205 \\
-0.196 \\
-0.189 \\
-0.184 \\
-0.180 \\
-0.173 \\
-0.168 \\
-0.164\end{array}$ & $\begin{array}{l}0.235 \\
0.224 \\
0.215 \\
0.208 \\
0.203 \\
0.194 \\
0.188 \\
0.182\end{array}$ & $\begin{array}{l}0.233 \\
0.222 \\
0.214 \\
0.207 \\
0.202 \\
0.193 \\
0.187 \\
0.182\end{array}$ \\
\hline $\begin{array}{l}0.0400 \\
0.0450 \\
0.0500 \\
0.0550 \\
0.0600 \\
0.0700 \\
0.0800 \\
0.0900\end{array}$ & $\begin{array}{l}6.841 E+00 \\
6.274 E+00 \\
5.813 E+00 \\
5.429 E+00 \\
5.106 E+00 \\
4.539 E+00 \\
4.194 E+00 \\
3.882 E+00\end{array}$ & $\begin{array}{l}3.883 \mathrm{E}-03 \\
3.896 \mathrm{E}-03 \\
3.909 \mathrm{E}-03 \\
3.924 \mathrm{E}-03 \\
3.939 \mathrm{E}-03 \\
3.973 \mathrm{E}-03 \\
4.010 \mathrm{E}-03 \\
4.050 \mathrm{E}-03\end{array}$ & $\begin{array}{l}6.845 E+00 \\
6.278 E+00 \\
5.816 E+00 \\
5.433 E+00 \\
5.110 E+00 \\
4.593 E+00 \\
4.198 E+00 \\
3.886 E+00\end{array}$ & $\begin{array}{l}3.326 \mathrm{E}-03 \\
4.090 \mathrm{E}-03 \\
4.918 \mathrm{E}-03 \\
5.809 \mathrm{E}-03 \\
6.758 \mathrm{E}-03 \\
8.827 \mathrm{E}-03 \\
1.111 \mathrm{E}-02 \\
1.359 \mathrm{E}-02\end{array}$ & $\begin{array}{l}3.185 \mathrm{E}-04 \\
3.491 \mathrm{E}-04 \\
3.789 \mathrm{E}-04 \\
4.078 \mathrm{E}-04 \\
4.360 \mathrm{E}-04 \\
4.907 \mathrm{E}-04 \\
5.431 \mathrm{E}-04 \\
5.938 \mathrm{E}-04\end{array}$ & $\begin{array}{l}0.0 \\
0.0 \\
0.0 \\
0.0 \\
0.0 \\
0.0 \\
0.0 \\
0.0\end{array}$ & $\begin{array}{l}-0.160 \\
-0.158 \\
-0.155 \\
-0.153 \\
-0.151 \\
-0.148 \\
-0.145 \\
-0.143\end{array}$ & $\begin{array}{l}0.178 \\
0.174 \\
0.171 \\
0.169 \\
0.166 \\
0.162 \\
0.159 \\
0.156\end{array}$ & $\begin{array}{l}0.178 \\
0.174 \\
0.171 \\
0.168 \\
0.166 \\
0.162 \\
0.159 \\
0.156\end{array}$ \\
\hline $\begin{array}{l}0.1000 \\
0.1250 \\
0.1500 \\
0.1750 \\
0.2000 \\
0.2500 \\
0.3000 \\
0.3500\end{array}$ & $\begin{array}{l}3.630 \mathrm{E}+00 \\
3.170 \mathrm{E}+00 \\
2.859 \mathrm{E}+00 \\
2.635 \mathrm{E}+00 \\
2.468 \mathrm{E}+00 \\
2.235 \mathrm{E}+00 \\
2.083 \mathrm{E}+00 \\
1.977 \mathrm{E}+00\end{array}$ & $\begin{array}{l}4.093 \mathrm{E}-03 \\
4.214 \mathrm{E}-03 \\
4.347 \mathrm{E}-03 \\
4.490 \mathrm{E}-03 \\
4.643 \mathrm{E}-03 \\
4.974 \mathrm{E}-03 \\
5.336 \mathrm{E}-03 \\
5.723 \mathrm{E}-03\end{array}$ & $\begin{array}{l}3.634 E+00 \\
3.174 E+00 \\
2.863 \mathrm{E}+00 \\
2.640 \mathrm{E}+00 \\
2.473 \mathrm{E}+00 \\
2.240 \mathrm{E}+00 \\
2.088 \mathrm{E}+00 \\
1.983 \mathrm{E}+00\end{array}$ & $\begin{array}{l}1.625 \mathrm{E}-02 \\
2.364 \mathrm{E}-02 \\
3.196 \mathrm{E}-02 \\
4.107 \mathrm{E}-02 \\
5.086 \mathrm{E}-02 \\
7.218 \mathrm{E}-02 \\
9.535 \mathrm{E}-02 \\
1.200 \mathrm{E}-01\end{array}$ & $\begin{array}{l}6.429 \mathrm{E}-04 \\
7.599 \mathrm{E}-04 \\
8.705 \mathrm{E}-04 \\
9.762 \mathrm{E}-04 \\
1.078 \mathrm{E}-03 \\
1.272 \mathrm{E}-03 \\
1.458 \mathrm{E}-03 \\
1.639 \mathrm{E}-03\end{array}$ & $\begin{array}{l}0.0 \\
0.0 \\
0.0 \\
0.0 \\
0.0 \\
0.0 \\
0.0 \\
0.0\end{array}$ & $\begin{array}{l}-0.141 \\
-0.137 \\
-0.133 \\
-0.131 \\
-0.129 \\
-0.125 \\
-0.122 \\
-0.121\end{array}$ & $\begin{array}{l}0.154 \\
0.149 \\
0.145 \\
0.142 \\
0.140 \\
0.136 \\
0.133 \\
0.130\end{array}$ & $\begin{array}{l}0.153 \\
0.149 \\
0.145 \\
0.142 \\
0.139 \\
0.135 \\
0.132 \\
0.129\end{array}$ \\
\hline $\begin{array}{l}0.4000 \\
0.4500 \\
0.5000 \\
0.5500 \\
0.6000 \\
0.7000 \\
0.8000 \\
0.9000\end{array}$ & $\begin{array}{l}1.899 \mathrm{E}+00 \\
1.840 \mathrm{E}+00 \\
1.794 \mathrm{E}+00 \\
1.758 \mathrm{E}+00 \\
1.729 \mathrm{E}+00 \\
1.687 \mathrm{E}+00 \\
1.659 \mathrm{E}+00 \\
1.639 \mathrm{E}+00\end{array}$ & $\begin{array}{l}6.134 \mathrm{E}-03 \\
6.567 \mathrm{E}-03 \\
7.020 \mathrm{E}-03 \\
7.491 \mathrm{E}-03 \\
7.977 \mathrm{E}-03 \\
8.995 \mathrm{E}-03 \\
1.006 \mathrm{E}-02 \\
1.118 \mathrm{E}-02\end{array}$ & $\begin{array}{l}1.906 \mathrm{E}+00 \\
1.847 \mathrm{E}+00 \\
1.801 \mathrm{E}+00 \\
1.765 \mathrm{E}+00 \\
1.737 \mathrm{E}+00 \\
1.696 \mathrm{E}+00 \\
1.669 \mathrm{E}+00 \\
1.650 \mathrm{E}+00\end{array}$ & $\begin{array}{l}1.457 \mathrm{E}-01 \\
1.724 \mathrm{E}-01 \\
1.998 \mathrm{E}-01 \\
2.279 \mathrm{E}-01 \\
2.564 \mathrm{E}-01 \\
3.147 \mathrm{E}-01 \\
3.742 \mathrm{E}-01 \\
4.345 \mathrm{E}-01\end{array}$ & $\begin{array}{l}1.815 \mathrm{E}-03 \\
1.990 \mathrm{E}-03 \\
2.164 \mathrm{E}-03 \\
2.337 \mathrm{E}-03 \\
2.510 \mathrm{E}-03 \\
2.858 \mathrm{E}-03 \\
3.209 \mathrm{E}-03 \\
3.564 \mathrm{E}-03\end{array}$ & $\begin{array}{l}1.229 \mathrm{E}-02 \\
3.896 \mathrm{E}-02 \\
6.802 \mathrm{E}-02 \\
9.881 \mathrm{E}-02 \\
1.308 \mathrm{E}-01 \\
1.972 \mathrm{E}-01 \\
2.651 \mathrm{E}-01 \\
3.331 \mathrm{E}-01\end{array}$ & $\begin{array}{l}-0.097 \\
-0.091 \\
-0.085 \\
-0.080 \\
-0.076 \\
-0.069 \\
-0.063 \\
-0.059\end{array}$ & $\begin{array}{l}0.127 \\
0.122 \\
0.117 \\
0.113 \\
0.109 \\
0.102 \\
0.096 \\
0.091\end{array}$ & $\begin{array}{l}0.125 \\
0.1119 \\
0.114 \\
0.109 \\
0.104 \\
0.096 \\
0.089 \\
0.084\end{array}$ \\
\hline $\begin{array}{l}1.0000 \\
1.2500 \\
1.5000 \\
1.7500 \\
2.0000 \\
2.5000 \\
3.0000 \\
3.5000\end{array}$ & $\begin{array}{l}1.626 \mathrm{E}+00 \\
1.608 \mathrm{E}+00 \\
1.602 \mathrm{E}+00 \\
1.602 \mathrm{E}+00 \\
1.605 \mathrm{E}+00 \\
1.615 \mathrm{E}+00 \\
1.627 \mathrm{E}+00 \\
1.639 \mathrm{E}+00\end{array}$ & $\begin{array}{l}1.234 \mathrm{E}-02 \\
1.542 \mathrm{E}-02 \\
1.871 \mathrm{E}-02 \\
2.218 \mathrm{E}-02 \\
2.581 \mathrm{E}-02 \\
3.342 \mathrm{E}-02 \\
4.144 \mathrm{E}-02 \\
4.978 \mathrm{E}-02\end{array}$ & $\begin{array}{l}1.638 \mathrm{E}+00 \\
1.623 \mathrm{E}+00 \\
1.620 \mathrm{E}+00 \\
1.624 \mathrm{E}+00 \\
1.630 \mathrm{E}+00 \\
1.648 \mathrm{E}+00 \\
1.668 \mathrm{E}+00 \\
1.689 \mathrm{E}+00\end{array}$ & $\begin{array}{l}4.953 \mathrm{E}-01 \\
6.488 \mathrm{E}-01 \\
8.030 \mathrm{E}-01 \\
9.572 \mathrm{E}-01 \\
1.111 \mathrm{E}+00 \\
1.416 \mathrm{E}+00 \\
1.717 \mathrm{E}+00 \\
2.015 \mathrm{E}+00\end{array}$ & $\begin{array}{l}3.923 \mathrm{E}-03 \\
4.840 \mathrm{E}-03 \\
5.787 \mathrm{E}-03 \\
6.760 \mathrm{E}-03 \\
7.758 \mathrm{E}-03 \\
9.814 \mathrm{E}-03 \\
1.194 \mathrm{E}-02 \\
1.411 \mathrm{E}-02\end{array}$ & $\begin{array}{l}4.005 \mathrm{E}-01 \\
5.636 \mathrm{E}-01 \\
7.168 \mathrm{E}-01 \\
8.594 \mathrm{E}-01 \\
9.920 \mathrm{E}-01 \\
1.231 \mathrm{E}+00 \\
1.441 \mathrm{E} \div 00 \\
1.627 \mathrm{E}+00\end{array}$ & $\begin{array}{l}-0.055 \\
-0.048 \\
-0.043 \\
-0.040 \\
-0.037 \\
-0.034 \\
-0.031 \\
-0.029\end{array}$ & $\begin{array}{l}0.087 \\
0.078 \\
0.072 \\
0.067 \\
0.063 \\
0.057 \\
0.052 \\
0.049\end{array}$ & $\begin{array}{l}0.079 \\
0.069 \\
0.062 \\
0.056 \\
0.052 \\
0.045 \\
0.041 \\
0.038\end{array}$ \\
\hline $\begin{array}{l}4.0000 \\
4.5000 \\
5.0000 \\
5.5000 \\
6.0000 \\
7.0000 \\
8.0000 \\
9.0000\end{array}$ & $\begin{array}{l}1.650 E+00 \\
1.661 E+00 \\
1.671 E+00 \\
1.681 E+00 \\
1.690 E+00 \\
1.705 E+00 \\
1.719 E+00 \\
1.731 E+00\end{array}$ & $\begin{array}{l}5.840 E-02 \\
6.726 \mathrm{E}-02 \\
7.633 \mathrm{E}-02 \\
8.559 \mathrm{E}-02 \\
9.501 \mathrm{E}-02 \\
1.143 \mathrm{E}-01 \\
1.341 \mathrm{E}-01 \\
1.543 \mathrm{E}-01\end{array}$ & $\begin{array}{l}1.709 E+00 \\
1.729 E+00 \\
1.748 E+00 \\
1.766 E+00 \\
1.785 E+00 \\
1.320 E+00 \\
1.853 E+00 \\
1.886 E+00\end{array}$ & $\begin{array}{l}2.310 E+0 \\
2.601 E+0 \\
2.888 E+0 \\
3.173 E+0 \\
3.454 E+0 \\
4.009 E+0 \\
4.554 E+0 \\
5.089 E+0\end{array}$ & $\begin{array}{l}1.6 \\
1.8 \\
2.0 \\
2.3 \\
2.5 \\
3.0 \\
3.4 \\
3.9\end{array}$ & $\begin{array}{l}1.79 \\
1.92 \\
2.08 \\
2.21 \\
2.33 \\
2.55 \\
2.74 \\
2.92\end{array}$ & $\begin{array}{l}-0.028 \\
-0.027 \\
-0.026 \\
-0.025 \\
-0.024 \\
-0.022 \\
-0.021 \\
-0.020\end{array}$ & $\begin{array}{l}0.046 \\
0.044 \\
0.042 \\
0.041 \\
0.039 \\
0.037 \\
0.035 \\
0.033\end{array}$ & $\begin{array}{l}0.035 \\
0.033 \\
0.032 \\
0.030 \\
0.029 \\
0.027 \\
0.025 \\
0.024\end{array}$ \\
\hline $\begin{array}{l}10.0000 \\
12.5000 \\
15.0000 \\
17.5000 \\
20.0000 \\
25.0000 \\
30.0000 \\
35.0000\end{array}$ & $\begin{array}{l}1.742 E+00 \\
1.765 E+00 \\
1.783 E+00 \\
1.799 E+00 \\
1.811 E+00 \\
1.832 E+00 \\
1.848 E+00 \\
1.862 E+00\end{array}$ & $\begin{array}{l}1.748 \mathrm{E}-01 \\
2.275 \mathrm{E}-01 \\
2.817 \mathrm{E}-01 \\
3.370 \mathrm{E}-01 \\
3.931 \mathrm{E}-01 \\
5.073 \mathrm{E}-01 \\
6.235 \mathrm{E}-01 \\
7.412 \mathrm{E}-01\end{array}$ & $\begin{array}{l}1.917 E+00 \\
1.993 E+00 \\
2.065 E+00 \\
2.135 E+00 \\
2.204 E+00 \\
2.339 E+00 \\
2.472 E+00 \\
2.603 E+00\end{array}$ & $\begin{array}{l}5.614 E+00 \\
6.893 \mathrm{E}+00 \\
8.125 \mathrm{E}+00 \\
9.316 \mathrm{E}+00 \\
1.047 \mathrm{E}+01 \\
1.267 \mathrm{E}+01 \\
1.475 \mathrm{E}+01 \\
1.672 \mathrm{E}+01\end{array}$ & $\begin{array}{l}4.419 \mathrm{E}-02 \\
5.590 \mathrm{E}-02 \\
6.748 \mathrm{E}-02 \\
7.886 \mathrm{E}-02 \\
9.002 \mathrm{E}-02 \\
1.116 \mathrm{E}-01 \\
1.321 \mathrm{E}-01 \\
1.516 \mathrm{E}-01\end{array}$ & $\begin{array}{l}3.080 E+00 \\
3.428 E+00 \\
3.724 E+00 \\
3.981 E+00 \\
4.210 E+00 \\
4.602 E+00 \\
4.932 E+00 \\
5.216 E+00\end{array}$ & $\begin{array}{l}-0.018 \\
-0.016 \\
-0.014 \\
-0.012 \\
-0.010 \\
-0.008 \\
-0.006 \\
-0.005\end{array}$ & $\begin{array}{l}0.032 \\
0.029 \\
0.026 \\
0.024 \\
0.023 \\
0.020 \\
0.018 \\
0.016\end{array}$ & $\begin{array}{l}0.023 \\
0.020 \\
0.018 \\
0.016 \\
0.014 \\
0.012 \\
0.010 \\
0.008\end{array}$ \\
\hline $\begin{array}{l}.0000 \\
.0000 \\
.0000 \\
.0000 \\
.0000 \\
.0000 \\
.0000\end{array}$ & $\begin{array}{l}1.873 E+00 \\
1.883 E+00 \\
1.892 E+00 \\
1.900 E+00 \\
1.907 E+00 \\
1.919 E+00 \\
1.930 E+00 \\
1.939 E+00\end{array}$ & $\begin{array}{l}8.601 E-01 \\
9.799 E-01 \\
1.101 E+00 \\
1.222 E+00 \\
1.344 E+00 \\
1.589 E+00 \\
1.836 E+00 \\
2.085 E+00\end{array}$ & $\begin{array}{l}2.733 \mathrm{E}+0 \\
2.863 \mathrm{E}+0 \\
2.992 \mathrm{E}+0 \\
3.122 \mathrm{E}+0 \\
3.251 \mathrm{E}+0 \\
3.509 \mathrm{E}+0 \\
3.766 \mathrm{E}+0 \\
4.024 \mathrm{E}+0\end{array}$ & $\begin{array}{l}1.859 E+01 \\
2.038 E+01 \\
2.209 E+01 \\
2.372 E+01 \\
2.529 E+01 \\
2.825 E+01 \\
3.100 E+01 \\
3.357 E+01\end{array}$ & $\begin{array}{l}1.70 \\
1.8 \\
2.0 \\
2.20 \\
2.3 \\
2.6 \\
2.8 \\
3.1\end{array}$ & $\begin{array}{l}5.4 \\
5.6 \\
5.8 \\
6.0 \\
6.2 \\
6.5 \\
6.8 \\
7.0\end{array}$ & $\begin{array}{l}-0.004 \\
-0.003 \\
-0.003 \\
-0.002 \\
-0.002 \\
-0.002 \\
-0.001 \\
-0.001\end{array}$ & $\begin{array}{l}0.015 \\
0.014 \\
0.013 \\
0.0112 \\
0.0111 \\
0.0110 \\
0.0110 \\
0.009\end{array}$ & \\
\hline $\begin{array}{l}0000 \\
00000 \\
00000 \\
0000\end{array}$ & $\begin{array}{l}1.94 \\
1.96 \\
1.97 \\
1.99 \\
2.00 \\
2.01 \\
2.03 \\
2.04\end{array}$ & $\begin{array}{l}2.335 E+00 \\
2.962 E+00 \\
3.595 E+00 \\
4.230 E+00 \\
4.869 E+00 \\
6.151 E+00 \\
7.438 E+00 \\
8.730 E+00\end{array}$ & $\begin{array}{l}28 E+00 \\
74 E+00 \\
22 E+00 \\
70 E+00 \\
69 E+00 \\
71 E+00 \\
77 E+01\end{array}$ & $\begin{array}{l}4.142 E+01 \\
4.619 E+01 \\
5.043 E+01 \\
5.425 E+01 \\
6.092 E+01 \\
6.660 E+01 \\
7.154 E+01\end{array}$ & $\begin{array}{l}3.831 \mathrm{E}-01 \\
4.233 \mathrm{E}-01 \\
4.575 \mathrm{E}-01 \\
4.872 \mathrm{E}-01 \\
5.361 \mathrm{E}-01 \\
5.751 \mathrm{E}-01 \\
6.070 \mathrm{E}-01\end{array}$ & $\begin{array}{l}7.239 \mathrm{E}+00 \\
7.680 \mathrm{E}+00 \\
8.042 \mathrm{E}+00 \\
8.348 \mathrm{E}+00 \\
8.613 \mathrm{E}+00 \\
9.058 \mathrm{E}+00 \\
9.421 \mathrm{E}+00 \\
9.729 \mathrm{E}+00\end{array}$ & $\begin{array}{l}-0.001 \\
-0.001 \\
-0.000 \\
-0.000 \\
-0.000 \\
-0.000 \\
-0.000 \\
-0.000\end{array}$ & & $\begin{array}{l}0.002 \\
0.002 \\
0.001 \\
0.001 \\
0.001 \\
0.001 \\
0.001 \\
0.001\end{array}$ \\
\hline $\begin{array}{l}00000 \\
00000 \\
0000 \\
00000 \\
0000 \\
0000 \\
0000 \\
00000\end{array}$ & $\begin{array}{l}2.055 E+00 \\
2.064 E+00 \\
2.072 E+00 \\
2.080 E+00 \\
2.086 E+00 \\
2.098 E+00 \\
2.108 E+00 \\
2.117 E+00\end{array}$ & $\begin{array}{l}1.002 E+01 \\
1.132 E+01 \\
1.262 E+01 \\
1.392 E+01 \\
1.522 E+01 \\
1.783 E+01 \\
2.044 E+01 \\
2.305 E+01\end{array}$ & $\begin{array}{l}1.208 E+01 \\
1.339 E+01 \\
1.469 E+01 \\
1.600 E+01 \\
1.731 E+01 \\
1.993 E+01 \\
2.255 E+01 \\
2.517 E+01\end{array}$ & $\begin{array}{l}7.592 E+01 \\
7.985 \mathrm{E}+01 \\
8.342 \mathrm{E}+01 \\
8.668 \mathrm{E}+01 \\
8.968 \mathrm{E}+01 \\
9.506 \mathrm{E}+01 \\
9.978 \mathrm{E}+01 \\
1.040 \mathrm{E}+02\end{array}$ & $\begin{array}{l}6.337 \mathrm{E}- \\
6.564 \mathrm{E}- \\
6.760 \mathrm{E}- \\
6.931 \mathrm{E}- \\
7.083 \mathrm{E}- \\
7.339 \mathrm{E}- \\
7.548 \mathrm{E}- \\
7.722 \mathrm{E}-\end{array}$ & $\begin{array}{l}9.995 E+00 \\
1.023 E+01 \\
1.044 E+01 \\
1.063 E+01 \\
1.080 E+01 \\
1.111 E+01 \\
1.138 E+01 \\
1.161 E+01\end{array}$ & $\begin{array}{l}-0.000 \\
-0.000 \\
-0.000 \\
-0.000 \\
-0.000 \\
-0.000 \\
-0.000 \\
-0.000\end{array}$ & $\begin{array}{l}0.004 \\
0.004 \\
0.004 \\
0.004 \\
0.003 \\
0.003 \\
0.003 \\
0.003\end{array}$ & $\begin{array}{l}0.000 \\
0.000 \\
0.000 \\
0.000 \\
0.000 \\
0.000 \\
0.000 \\
0.000\end{array}$ \\
\hline 0000 & $2.126 E+00$ & $2.567 E+01$ & $2.779 E+01$ & $.078 E+02$ & $869 \mathrm{E}-01$ & $1.183 E+01$ & -0.000 & 0.003 & 0.000 \\
\hline
\end{tabular}


ELECTRONS IN CALCIUM FLUORIDE

\begin{tabular}{|c|c|c|c|c|c|c|c|c|c|}
\hline ENERGY & & & & & RADIATION & & & & \\
\hline $\mathrm{MeV}$ & $\mathrm{MeV} \mathrm{cm}^{2} / \mathrm{g}$ & $\mathrm{MeV} \mathrm{cm}^{2} / \mathrm{g}$ & $\mathrm{MeV} \mathrm{cm}^{2} / \mathrm{g}$ & $\mathrm{g} / \mathrm{cm}^{2}$ & & & & & \\
\hline $\begin{array}{l}0.0100 \\
0.0125 \\
0.0150 \\
0.0175 \\
0.0200 \\
0.0250 \\
0.0300 \\
0.0350\end{array}$ & $\begin{array}{l}1.666 E+01 \\
1.412 E+01 \\
1.233 E+01 \\
1.099 E+01 \\
9.945 E+0 \\
8.424 E+0 \\
7.363 E+00 \\
6.577 E+00\end{array}$ & $\begin{array}{l}7.284 \mathrm{E}-03 \\
7.499 \mathrm{E}-03 \\
7.657 \mathrm{E}-03 \\
7.778 \mathrm{E}-03 \\
7.874 \mathrm{E}-03 \\
8.016 \mathrm{E}-03 \\
8.118 \mathrm{E}-03 \\
8.197 \mathrm{E}-03\end{array}$ & $\begin{array}{l}1.667 E+01 \\
1.413 E+01 \\
1.23 E E+01 \\
1.099 E+01 \\
9.95 E E+00 \\
8.432 E+00 \\
7.371 E+00 \\
6.585 E+00\end{array}$ & $\begin{array}{l}3.503 E-04 \\
5.139 \mathrm{E}-04 \\
7.039 \mathrm{E}-04 \\
9.190 \mathrm{E}-04 \\
1.158 \mathrm{E}-03 \\
1.706 \mathrm{E}-03 \\
2.343 \mathrm{E}-03 \\
3.062 \mathrm{E}-03\end{array}$ & $\begin{array}{l}2.300 \mathrm{E}-04 \\
2.809 \mathrm{E}-04 \\
3.3011 \mathrm{E}-04 \\
3.778 \mathrm{E}-04 \\
4.243 \mathrm{E}-04 \\
5.138 \mathrm{E}-04 \\
5.993 \mathrm{E}-04 \\
6.813 \mathrm{E}-04\end{array}$ & $\begin{array}{l}0.0 \\
0.0 \\
0.0 \\
0.0 \\
0.0 \\
0.0 \\
0.0 \\
0.0\end{array}$ & $\begin{array}{l}-0.236 \\
-0.224 \\
-0.216 \\
-0.209 \\
-0.203 \\
-0.195 \\
-0.188 \\
-0.183\end{array}$ & $\begin{array}{l}0.281 \\
0.265 \\
0.253 \\
0.243 \\
0.235 \\
0.224 \\
0.215 \\
0.208\end{array}$ & $\begin{array}{l}0.275 \\
0.259 \\
0.248 \\
0.239 \\
0.231 \\
0.220 \\
0.212 \\
0.205\end{array}$ \\
\hline $\begin{array}{l}0.0400 \\
0.0450 \\
0.0500 \\
0.0550 \\
0.0600 \\
0.0700 \\
0.0800 \\
0.0900\end{array}$ & $\begin{array}{l}5.970 E+00 \\
5.487 E+00 \\
5.093 E+00 \\
4.764 E+00 \\
4.436 E+00 \\
4.041 E+00 \\
3.701 E+00 \\
3.432 E+00\end{array}$ & $\begin{array}{l}8.263 \mathrm{E}-03 \\
8.319 \mathrm{E}-03 \\
8.370 \mathrm{E}-03 \\
8.416 \mathrm{E}-03 \\
8.453 \mathrm{E}-03 \\
8.541 \mathrm{E}-03 \\
8.621 \mathrm{E}-03 \\
8.704 \mathrm{E}-03\end{array}$ & $\begin{array}{l}5.979 E+00 \\
5.495 E+00 \\
5.1010+00 \\
4.773 E+00 \\
4.495 E+00 \\
4.050 E+00 \\
3.709 E+00 \\
3.440 E+00\end{array}$ & $\begin{array}{l}3.860 \mathrm{E}-03 \\
4.733 \mathrm{E}-03 \\
5.678 \mathrm{E}-03 \\
6.693 \mathrm{E}-03 \\
7.773 \mathrm{E}-03 \\
1.012 \mathrm{E}-02 \\
1.271 \mathrm{E}-02 \\
1.551 \mathrm{E}-02\end{array}$ & $\begin{array}{l}7.604 \mathrm{E}-04 \\
8.368 \mathrm{E}-04 \\
9.109 \mathrm{E}-04 \\
9.829 \mathrm{E}-04 \\
1.053 \mathrm{E}-03 \\
1.188 \mathrm{E}-03 \\
1.316 \mathrm{E}-03 \\
1.440 \mathrm{E}-03\end{array}$ & $\begin{array}{l}0.0 \\
0.0 \\
0.0 \\
0.0 \\
0.0 \\
0.0 \\
0.0 \\
0.0\end{array}$ & $\begin{array}{l}-0.179 \\
-0.175 \\
-0.172 \\
-0.170 \\
-0.167 \\
-0.163 \\
-0.160 \\
-0.157\end{array}$ & $\begin{array}{l}0.202 \\
0.198 \\
0.194 \\
0.190 \\
0.187 \\
0.182 \\
0.178 \\
0.174\end{array}$ & $\begin{array}{l}0.200 \\
0.196 \\
0.192 \\
0.189 \\
0.186 \\
0.181 \\
0.1777 \\
0.173\end{array}$ \\
\hline $\begin{array}{l}0.1000 \\
0.1250 \\
0.1500 \\
0.1750 \\
0.2000 \\
0.2500 \\
0.3000 \\
0.3500\end{array}$ & $\begin{array}{l}3.213 E+00 \\
2.814 E+00 \\
2.544 E+00 \\
2.345 E+00 \\
2.203 E+00 \\
2.000 E+00 \\
1.867 E+00 \\
1.774 E+00\end{array}$ & $\begin{array}{l}8.788 \mathrm{E}-03 \\
9.016 \mathrm{E}-03 \\
9.265 \mathrm{E}-03 \\
9.534 \mathrm{E}-03 \\
9.821 \mathrm{E}-03 \\
1.045 \mathrm{E}-02 \\
1.113 \mathrm{E}-02 \\
1.187 \mathrm{E}-02\end{array}$ & $\begin{array}{l}3.222 \mathrm{E}+00 \\
2.823 \mathrm{E}+00 \\
2.553 \mathrm{E}+00 \\
2.359 \mathrm{E}+00 \\
2.213 \mathrm{E}+00 \\
2.011 \mathrm{E}+00 \\
1.878 \mathrm{E}+00 \\
1.786 \mathrm{E}+00\end{array}$ & $\begin{array}{l}1.851 \mathrm{E}-02 \\
2.684 \mathrm{E}-02 \\
3.6117 \mathrm{E}-02 \\
4.638 \mathrm{E}-02 \\
5.733 \mathrm{E}-02 \\
8.1111 \mathrm{E}-02 \\
1.069 \mathrm{E}-01 \\
1.342 \mathrm{E}-01\end{array}$ & $\begin{array}{l}1.559 E-03 \\
1.840 E-03 \\
2.102 E-03 \\
2.350 E-03 \\
2.586 E-03 \\
3.033 E-03 \\
3.455 E-03 \\
3.859 E-03\end{array}$ & $\begin{array}{l}0.0 \\
0.0 \\
0.0 \\
0.0 \\
0.0 \\
0.0 \\
5.037 \mathrm{E}-03 \\
1.737 \mathrm{E}-02\end{array}$ & $\begin{array}{l}-0.155 \\
-0.150 \\
-0.146 \\
-0.143 \\
-0.140 \\
-0.136 \\
-0.125 \\
-0.119\end{array}$ & $\begin{array}{l}0.171 \\
0.165 \\
0.161 \\
0.157 \\
0.154 \\
0.149 \\
0.145 \\
0.140\end{array}$ & $\begin{array}{l}0.170 \\
0.164 \\
0.160 \\
0.156 \\
0.153 \\
0.148 \\
0.143 \\
0.138\end{array}$ \\
\hline $\begin{array}{l}0.4000 \\
0.4500 \\
0.5000 \\
0.5500 \\
0.6000 \\
0.7000 \\
0.8000 \\
0.9000\end{array}$ & $\begin{array}{l}1.706 E+00 \\
1.656 E+00 \\
1.617 E+00 \\
1.587 E+00 \\
1.563 E+00 \\
1.528 E+00 \\
1.506 E+00 \\
1.491 E+00\end{array}$ & $\begin{array}{l}1.266 \mathrm{E}-02 \\
1.348 \mathrm{E}-02 \\
1.435 \mathrm{E}-02 \\
1.525 \mathrm{E}-02 \\
1.618 \mathrm{E}-02 \\
1.812 \mathrm{E}-02 \\
2.016 \mathrm{E}-02 \\
2.229 \mathrm{E}-02\end{array}$ & $\begin{array}{l}1.719 E+00 \\
1.669 E+00 \\
1.631 E+00 \\
1.602 E+00 \\
1.579 E+00 \\
1.547 E+00 \\
1.526 E+00 \\
1.513 E+00\end{array}$ & $\begin{array}{l}1.628 \mathrm{E}-01 \\
1.923 \mathrm{E}-01 \\
2.226 \mathrm{E}-01 \\
2.536 \mathrm{E}-01 \\
2.850 \mathrm{E}-01 \\
3.491 \mathrm{E}-01 \\
4.142 \mathrm{E}-01 \\
4.800 \mathrm{E}-01\end{array}$ & $\begin{array}{l}4.25 \\
4.63 \\
5.01 \\
5.39 \\
5.76 \\
6.51 \\
7.25 \\
8.00\end{array}$ & $\begin{array}{l}3.256 \mathrm{E} \\
5.026 \mathrm{E} \\
7.003 \mathrm{E} \\
9.144 \mathrm{E} \\
1.141 \mathrm{E} \\
1.623 \mathrm{E} \\
2.128 \mathrm{E} \\
2.645 \mathrm{E}\end{array}$ & $\begin{array}{l}-0.113 \\
-0.0107 \\
-0.003 \\
-0.098 \\
-0.094 \\
-0.087 \\
-0.081 \\
-0.076\end{array}$ & $\begin{array}{l}0.135 \\
0.131 \\
0.128 \\
0.124 \\
0.121 \\
0.115 \\
0.110 \\
0.106\end{array}$ & $\begin{array}{l}0.133 \\
0.129 \\
0.125 \\
0.121 \\
0.117 \\
0.110 \\
0.104 \\
0.099\end{array}$ \\
\hline $\begin{array}{l}1.0000 \\
1.2500 \\
1.5000 \\
1.7500 \\
2.0000 \\
2.5000 \\
3.0000 \\
3.5000\end{array}$ & $\begin{array}{l}1.481 E+00 \\
1.470 E+00 \\
1.468 E+00 \\
1.471 E+00 \\
1.477 E+00 \\
1.491 E+00 \\
1.506 E+00 \\
1.520 E+00\end{array}$ & $\begin{array}{l}2.450 \mathrm{E}-02 \\
3.034 \mathrm{E}-02 \\
3.658 \mathrm{E}-02 \\
4.313 \mathrm{E}-02 \\
4.995 \mathrm{E}-02 \\
6.423 \mathrm{E}-02 \\
7.922 \mathrm{E}-02 \\
9.476 \mathrm{E}-02\end{array}$ & $\begin{array}{l}1.505 E+00 \\
1.500 E+00 \\
1.505 E+00 \\
1.515 E+00 \\
1.527 E+00 \\
1.555 E+00 \\
1.585 E+00 \\
1.615 E+00\end{array}$ & $\begin{array}{l}5.463 \mathrm{E}-01 \\
7.128 \mathrm{E}-01 \\
8.793 \mathrm{E}-01 \\
1.045 \mathrm{E}+00 \\
1.209 \mathrm{E}+00 \\
1.534 \mathrm{E}+00 \\
1.852 \mathrm{E}+00 \\
2.165 \mathrm{E}+00\end{array}$ & $\begin{array}{l}8.752 \mathrm{E}-03 \\
1.065 \mathrm{E}-02 \\
1.258 \mathrm{E}-02 \\
1.456 \mathrm{E}-02 \\
1.656 \mathrm{E}-02 \\
2.065 \mathrm{E}-02 \\
2.481 \mathrm{E}-02 \\
2.903 \mathrm{E}-02\end{array}$ & $\begin{array}{l}3.167 \mathrm{E}-01 \\
4.459 \mathrm{E}-01 \\
5.700 \mathrm{E}-01 \\
6.875 \mathrm{E}-01 \\
7.980 \mathrm{E}-01 \\
9.994 \mathrm{E}-01 \\
1.178 \mathrm{E}+00 \\
1.338 \mathrm{E}+00\end{array}$ & $\begin{array}{l}-0.072 \\
-0.064 \\
-0.059 \\
-0.054 \\
-0.051 \\
-0.047 \\
-0.044 \\
-0.041\end{array}$ & $\begin{array}{l}0.093 \\
0.087 \\
0.082 \\
0.078 \\
0.071 \\
0.066 \\
0.063\end{array}$ & $\begin{array}{l}0.095 \\
0.085 \\
0.078 \\
0.072 \\
0.067 \\
0.060 \\
0.055 \\
0.051\end{array}$ \\
\hline $\begin{array}{l}4.0000 \\
4.5000 \\
5.0000 \\
5.5000 \\
6.0000 \\
7.0000 \\
8.0000 \\
9.0000\end{array}$ & $\begin{array}{l}1.533 E+00 \\
1.545 E+00 \\
1.557 E+00 \\
1.567 E+00 \\
1.577 E+00 \\
1.594 E+00 \\
1.610 E+00 \\
1.623 E+00\end{array}$ & $\begin{array}{l}1.108 \mathrm{E}-01 \\
1.272 \mathrm{E}-01 \\
1.439 \mathrm{E}-01 \\
1.610 \mathrm{E}-01 \\
1.783 \mathrm{E}-01 \\
2.137 \mathrm{E}-01 \\
2.499 \mathrm{E}-01 \\
2.868 \mathrm{E}-01\end{array}$ & $\begin{array}{l}1.644 E+00 \\
1.673 E+00 \\
1.701 E+00 \\
1.728 E+00 \\
1.755 E+00 \\
1.808 E+00 \\
1.859 E+00 \\
1.910 E+00\end{array}$ & $\begin{array}{l}2.472 E+00 \\
2.773 E+00 \\
3.070 E+00 \\
3.361 E+00 \\
3.649 E+00 \\
4.210 E+00 \\
4.755 E+00 \\
5.286 E+00\end{array}$ & $\begin{array}{l}E-02 \\
E-02 \\
E-02 \\
E-02 \\
E-02 \\
E-02 \\
E-02 \\
E-02\end{array}$ & $\begin{array}{l}1.482 \\
1.614 \\
1.735 \\
1.848 \\
1.953 \\
2.144 \\
2.316 \\
2.472\end{array}$ & $\begin{array}{l}-0.039 \\
-0.038 \\
-0.037 \\
-0.035 \\
-0.034 \\
-0.0332 \\
-0.030 \\
-0.028\end{array}$ & $\begin{array}{l}.060 \\
.057 \\
.055 \\
.053 \\
.051 \\
.048 \\
.046 \\
.044\end{array}$ & $\begin{array}{l}0.048 \\
0.045 \\
0.043 \\
0.041 \\
0.040 \\
0.037 \\
0.035 \\
0.033\end{array}$ \\
\hline $\begin{array}{l}10.0000 \\
12.5000 \\
15.0000 \\
17.5000 \\
20.0000 \\
25.0000 \\
30.0000 \\
35.0000\end{array}$ & $\begin{array}{l}1.635 E+00 \\
1.660 E+00 \\
1.679 E+00 \\
1.695 E+00 \\
1.709 E+00 \\
1.731 E+00 \\
1.748 E+00 \\
1.762 E+00\end{array}$ & $\begin{array}{l}3.243 \mathrm{E}-01 \\
4.199 \mathrm{E}-01 \\
5.180 \mathrm{E}-01 \\
6.177 \mathrm{E}-01 \\
7.189 \mathrm{E}-01 \\
9.243 \mathrm{E}-01 \\
1.133 \mathrm{E}+00 \\
1.344 \mathrm{E}+00\end{array}$ & $\begin{array}{l}1.959 \mathrm{E}+ \\
2.079 \mathrm{E}+ \\
2.197 \mathrm{E}+ \\
2.313 \mathrm{E}+ \\
2.428 \mathrm{E}+ \\
2.655 \mathrm{E}+ \\
2.881 \mathrm{E}+ \\
3.106 \mathrm{E}+\end{array}$ & $\begin{array}{l}5.803 E+0 \\
7.041 E+0 \\
8.210 E+0 \\
9.319 E+0 \\
1.037 E+0 \\
1.234 E+0 \\
1.415 E+0 \\
1.582 E+0\end{array}$ & & $\begin{array}{l}2.615 \\
2.931 \\
3.203 \\
3.442 \\
3.656 \\
4.026 \\
4.338 \\
4.609\end{array}$ & & & $\begin{array}{l}0.024 \\
0.022 \\
0.019 \\
0.016 \\
0.014 \\
0.012\end{array}$ \\
\hline $\begin{array}{l}40.0000 \\
45.0000 \\
50.0000 \\
55.0000 \\
60.0000 \\
70.0000 \\
80.0000 \\
90.0000\end{array}$ & $\begin{array}{l}1.774 E+00 \\
1.784 E+00 \\
1.793 E+00 \\
1.802 E+00 \\
1.809 E+00 \\
1.822 E+00 \\
1.833 E+00 \\
1.843 E+00\end{array}$ & $\begin{array}{l}1.557 E+00 \\
1.771 E+00 \\
1.987 E+00 \\
2.204 E+00 \\
2.422 E+00 \\
2.860 E+00 \\
3.301 E+00 \\
3.744 E+00\end{array}$ & $\begin{array}{l}3.331 E+00 \\
3.555 E+00 \\
3.780 E+00 \\
4.005 E+00 \\
4.231 E+00 \\
4.682 E+00 \\
5.134 E+00 \\
5.587 E+00\end{array}$ & $\begin{array}{l}1.738 \mathrm{E}+0 \\
1.883 \mathrm{E}+0 \\
2.019 \mathrm{E}+0 \\
2.148 \mathrm{E}+0 \\
2.269 \mathrm{E}+0 \\
2.494 \mathrm{E}+0 \\
2.698 \mathrm{E}+0 \\
2.884 \mathrm{E}+0\end{array}$ & $\begin{array}{l}2.974 \mathrm{E}-0 \\
3.189 \mathrm{E}-0 \\
3.388 \mathrm{E}-0 \\
3.574 \mathrm{E}-0 \\
3.909 \mathrm{E}-0 \\
4.205 \mathrm{E}-0 \\
4.468 \mathrm{E}-0\end{array}$ & $\begin{array}{l}5.061 E+00 \\
5.253 E+00 \\
5.429 E+00 \\
5.590 E+00 \\
5.878 E+00 \\
6.130 E+00 \\
6.353 E+00\end{array}$ & & & $\begin{array}{l}0.007 \\
0.006 \\
0.005 \\
0.004\end{array}$ \\
\hline $\begin{array}{l}0.00 \\
0.00\end{array}$ & $\begin{array}{l}1.851 E+00 \\
1.869 E+00 \\
1.884 E+00 \\
1.896 E+00 \\
1.906 E+00 \\
1.923 E+00 \\
1.937 E+00 \\
1.949 E+00\end{array}$ & $\begin{array}{l}4.18 \\
5.3 \\
6.4 \\
7.5 \\
8.6 \\
1.0 \\
1.3 \\
1.5\end{array}$ & $\begin{array}{l}6.040 E+0 \\
7.177 E+0 \\
8.317 E+0 \\
9.460 E+0 \\
1.061 E+0 \\
1.290 E+0 \\
1.520 E+0 \\
1.750 E+0\end{array}$ & $\begin{array}{l}3 . \\
3 \\
4 . \\
4\end{array}$ & .92 & $\begin{array}{l}6.554 \mathrm{E}+0 \\
6.983 \mathrm{E}+0 \\
7.336 \mathrm{E}+0 \\
7.636 \mathrm{E}+0 \\
7.898 \mathrm{E}+0 \\
8.337 \mathrm{E}+0 \\
8.697 \mathrm{E}+0 \\
9.003 \mathrm{E}+0\end{array}$ & & & $\begin{array}{l}0.001 \\
0.001\end{array}$ \\
\hline $\begin{array}{l}0.0000 \\
0.0000 \\
0.0000 \\
0.0000 \\
0.0000\end{array}$ & $\begin{array}{l}1.959 E+00 \\
1.968 E+00 \\
1.976 E+00 \\
1.983 E+00 \\
1.990 E+00 \\
2.001 E+00 \\
2.011 E+00 \\
2.020 E+00\end{array}$ & $\begin{array}{l}1.785 E+01 \\
2.015 E+01 \\
2.246 E+01 \\
2.476 E+01 \\
2.707 E+01 \\
3.169 E+01 \\
3.631 E+01 \\
4.094 E+0\end{array}$ & $\begin{array}{l}296 E+01 \\
296+01\end{array}$ & $\begin{array}{l}5.887 E+01 \\
6.102 E+01 \\
6.297 E+01 \\
6.477 E+01 \\
6.796 E+01 \\
7.074 E+01 \\
7.320 E+01\end{array}$ & $\begin{array}{l}7 \\
7 \\
7 \\
8 \\
8\end{array}$ & $\begin{array}{l}9.502 E+00 \\
9.712 E+00 \\
9.902 E+00 \\
1.008 E+01 \\
1.038 E+01 \\
1.065 E+01 \\
1.088 E+01\end{array}$ & $\begin{array}{l}-0.001 \\
-0.000 \\
-0.000 \\
-0.000 \\
-0.000 \\
-0.000 \\
-0.000 \\
-0.000\end{array}$ & $\begin{array}{l}.007 \\
.007 \\
.007 \\
.006 \\
.006 \\
.006 \\
.006\end{array}$ & 000 \\
\hline 000 & $.028 \mathrm{E}+00$ & $4.557 E+01$ & $160 E+01$ & $542 E+01$ & & $09 \mathrm{E}+01$ & & 005 & \\
\hline
\end{tabular}




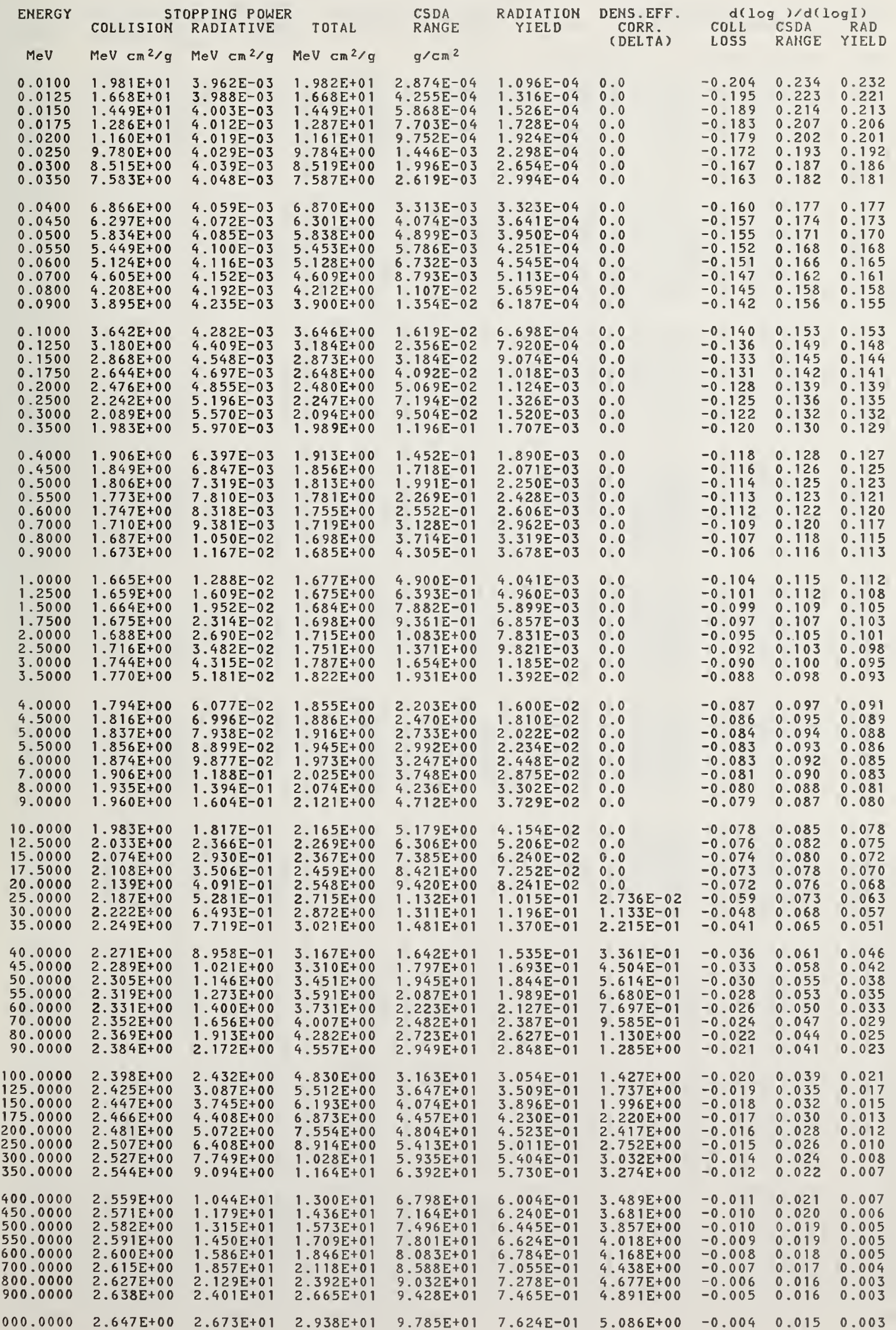




\begin{tabular}{|c|c|c|c|c|c|c|c|c|c|}
\hline ENERGY & 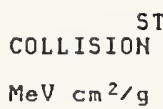 & 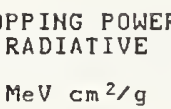 & $\begin{array}{c}\text { TOTAL } \\
\mathrm{MeV} \mathrm{cm}^{2} / \mathrm{g}\end{array}$ & $\begin{array}{l}\text { CSDA } \\
\text { RAKGE } \\
\mathrm{g} / \mathrm{Cm}^{2}\end{array}$ & $\begin{array}{l}\text { RADIATION } \\
\text { YIELD }\end{array}$ & $\begin{array}{l}\text { DENS. EFF. } \\
\text { CORR. } \\
\text { (DELTA) }\end{array}$ & $\begin{array}{l}d(1 \\
\text { coLL } \\
\text { LOSS }\end{array}$ & $\begin{array}{l}\text { ) } / d(1 \\
\text { CSDA } \\
\text { RAIGE }\end{array}$ & $\begin{array}{l}\text { gI ) } \\
\text { RAD } \\
\text { YIELD }\end{array}$ \\
\hline $\begin{array}{l}0.0100 \\
0.0125 \\
0.0150 \\
0.0175 \\
0.0200 \\
0.0250 \\
0.0300 \\
0.0350\end{array}$ & $\begin{array}{l}2.028 E+01 \\
1.708 E+01 \\
1.434 E+01 \\
1.317 E+01 \\
1.189 E+01 \\
1.002 E+01 \\
8.725 E+00 \\
7.771 E+00\end{array}$ & $\begin{array}{l}3.800 E-03 \\
3.824 E-03 \\
3.837 E-03 \\
3.847 E-03 \\
3.853 E-03 \\
3.863 E-03 \\
3.872 E-03 \\
3.882 E-03\end{array}$ & $\begin{array}{l}2.029 \mathrm{E}+01 \\
1.708 \mathrm{E}+01 \\
1.484 \mathrm{E}+01 \\
1.318 \mathrm{E}+01 \\
1.189 \mathrm{E}+01 \\
1.002 \mathrm{E}+01 \\
8.729 \mathrm{E}+00 \\
7.775 \mathrm{E}+00\end{array}$ & $\begin{array}{l}2.809 \mathrm{E}-04 \\
4.158 \mathrm{E}-04 \\
5.733 \mathrm{E}-04 \\
7.524 \mathrm{E}-04 \\
9.524 \mathrm{E}-04 \\
1.412 \mathrm{E}-03 \\
1.949 \mathrm{E}-03 \\
2.557 \mathrm{E}-03\end{array}$ & $\begin{array}{l}1.029 E-04 \\
1.234 E-04 \\
1.431 E-04 \\
1.620 E-04 \\
1.802 E-04 \\
2.152 E-04 \\
2.485 E-04 \\
2.803 E-04\end{array}$ & $\begin{array}{l}0.0 \\
0.0 \\
0.0 \\
0.0 \\
0.0 \\
0.0 \\
0.0 \\
0.0\end{array}$ & $\begin{array}{l}-0.205 \\
-0.196 \\
-0.189 \\
-0.184 \\
-0.180 \\
-0.173 \\
-0.168 \\
-0.164\end{array}$ & $\begin{array}{l}0.236 \\
0.224 \\
0.215 \\
0.209 \\
0.203 \\
0.194 \\
0.188 \\
0.182\end{array}$ & $\begin{array}{l}0.234 \\
0.222 \\
0.214 \\
0.207 \\
0.202 \\
0.193 \\
0.187 \\
0.182\end{array}$ \\
\hline $\begin{array}{l}0.0400 \\
0.0450 \\
0.0500 \\
0.0550 \\
0.0600 \\
0.0700 \\
0.0800 \\
0.0900\end{array}$ & $\begin{array}{l}7.037 E+00 \\
6.454 E+00 \\
5.930 E+00 \\
5.585 E+00 \\
5.252 E+00 \\
4.721 E+00 \\
4.314 E+00 \\
3.994 E+00\end{array}$ & $\begin{array}{l}3.893 \mathrm{E}-03 \\
3.906 \mathrm{E}-03 \\
3.919 \mathrm{E}-03 \\
3.934 \mathrm{E}-03 \\
3.950 \mathrm{E}-03 \\
3.985 \mathrm{E}-03 \\
4.024 \mathrm{E}-03 \\
4.066 \mathrm{E}-03\end{array}$ & $\begin{array}{l}7.041 \mathrm{E}+00 \\
6.458 \mathrm{E}+00 \\
5.984 \mathrm{E}+00 \\
5.589 \mathrm{E}+00 \\
5.256 \mathrm{E}+00 \\
4.725 \mathrm{E}+00 \\
4.318 \mathrm{E}+00 \\
3.998 \mathrm{E}+00\end{array}$ & $\begin{array}{l}3.234 \mathrm{E}-03 \\
3.976 \mathrm{E}-03 \\
4.781 \mathrm{E}-03 \\
5.647 \mathrm{E}-03 \\
6.570 \mathrm{E}-03 \\
8.580 \mathrm{E}-03 \\
1.080 \mathrm{E}-02 \\
1.321 \mathrm{E}-02\end{array}$ & $\begin{array}{l}3.111 \mathrm{E}-04 \\
3.409 \mathrm{E}-04 \\
3.698 \mathrm{E}-04 \\
3.979 \mathrm{E}-04 \\
4.254 \mathrm{E}-04 \\
4.786 \mathrm{E}-04 \\
5.298 \mathrm{E}-04 \\
5.792 \mathrm{E}-04\end{array}$ & $\begin{array}{l}0.0 \\
0.0 \\
0.0 \\
0.0 \\
0.0 \\
0.0 \\
0.0 \\
0.0\end{array}$ & $\begin{array}{l}-0.160 \\
-0.158 \\
-0.155 \\
-0.153 \\
-0.151 \\
-0.148 \\
-0.145 \\
-0.143\end{array}$ & $\begin{array}{l}0.178 \\
0.175 \\
0.171 \\
0.169 \\
0.166 \\
0.162 \\
0.159 \\
0.156\end{array}$ & $\begin{array}{l}0.178 \\
0.174 \\
0.171 \\
0.168 \\
0.166 \\
0.162 \\
0.159 \\
0.156\end{array}$ \\
\hline $\begin{array}{l}0.1000 \\
0.1250 \\
0.1500 \\
0.1750 \\
0.2000 \\
0.2500 \\
0.3000 \\
0.3500\end{array}$ & $\begin{array}{l}3.734 \mathrm{E}+00 \\
3.261 \mathrm{E}+00 \\
2.941 \mathrm{E}+00 \\
2.711 \mathrm{E}+00 \\
2.539 \mathrm{E}+00 \\
2.299 \mathrm{E}+00 \\
2.143 \mathrm{E}+00 \\
2.034 \mathrm{E}+00\end{array}$ & $\begin{array}{l}4.111 \mathrm{E}-03 \\
4.235 \mathrm{E}-03 \\
4.370 \mathrm{E}-03 \\
4.515 \mathrm{E}-03 \\
4.669 \mathrm{E}-03 \\
4.999 \mathrm{E}-03 \\
5.361 \mathrm{E}-03 \\
5.749 \mathrm{E}-03\end{array}$ & $\begin{array}{l}3.739 E+00 \\
3.265 E+00 \\
2.945 E+00 \\
2.716 E+00 \\
2.544 E+00 \\
2.304 E+00 \\
2.148 E+00 \\
2.040 E+00\end{array}$ & $\begin{array}{l}1.580 \mathrm{E}-02 \\
2.298 \mathrm{E}-02 \\
3.106 \mathrm{E}-02 \\
3.992 \mathrm{E}-02 \\
4.944 \mathrm{E}-02 \\
7.016 \mathrm{E}-02 \\
9.268 \mathrm{E}-02 \\
1.166 \mathrm{E}-01\end{array}$ & $\begin{array}{l}6.271 \mathrm{E}-04 \\
7.416 \mathrm{E}-04 \\
8.499 \mathrm{E}-04 \\
9.533 \mathrm{E}-04 \\
1.053 \mathrm{E}-03 \\
1.243 \mathrm{E}-03 \\
1.425 \mathrm{E}-03 \\
1.601 \mathrm{E}-03\end{array}$ & $\begin{array}{l}0.0 \\
0.0 \\
0.0 \\
0.0 \\
0.0 \\
0.0 \\
0.0 \\
0.0\end{array}$ & $\begin{array}{l}-0.141 \\
-0.137 \\
-0.134 \\
-0.131 \\
-0.129 \\
-0.125 \\
-0.122 \\
-0.120\end{array}$ & $\begin{array}{l}0.154 \\
0.149 \\
0.145 \\
0.142 \\
0.140 \\
0.136 \\
0.133 \\
0.130\end{array}$ & $\begin{array}{l}0.154 \\
0.149 \\
0.145 \\
0.142 \\
0.139 \\
0.135 \\
0.132 \\
0.129\end{array}$ \\
\hline $\begin{array}{l}0.4000 \\
0.4500 \\
0.5000 \\
0.5500 \\
0.6000 \\
0.7000 \\
0.8000 \\
0.9000\end{array}$ & $\begin{array}{l}1.956 E+00 \\
1.896 E+00 \\
1.850 E+00 \\
1.813 E+00 \\
1.783 E+00 \\
1.741 E+00 \\
1.712 E+00 \\
1.692 E+00\end{array}$ & $\begin{array}{l}6.162 \mathrm{E}-03 \\
6.597 \mathrm{E}-03 \\
7.054 \mathrm{E}-03 \\
7.530 \mathrm{E}-03 \\
8.022 \mathrm{E}-03 \\
9.050 \mathrm{E}-03 \\
1.013 \mathrm{E}-02 \\
1.126 \mathrm{E}-02\end{array}$ & $\begin{array}{l}1.962 E+00 \\
1.903 E+00 \\
1.857 E+00 \\
1.820 E+00 \\
1.792 E+00 \\
1.750 E+00 \\
1.722 E+00 \\
1.703 E+00\end{array}$ & $\begin{array}{l}1.416 E-01 \\
1.675 \mathrm{E}-01 \\
1.941 \mathrm{E}-01 \\
2.213 \mathrm{E}-01 \\
2.490 \mathrm{E}-01 \\
3.056 \mathrm{E}-01 \\
3.632 \mathrm{E}-01 \\
4.216 \mathrm{E}-01\end{array}$ & $\begin{array}{l}1.773 \mathrm{E}-03 \\
1.943 \mathrm{E}-03 \\
2.112 \mathrm{E}-03 \\
2.281 \mathrm{E}-03 \\
2.449 \mathrm{E}-03 \\
2.789 \mathrm{E}-03 \\
3.131 \mathrm{E}-03 \\
3.477 \mathrm{E}-03\end{array}$ & $\begin{array}{l}0.0 \\
8.343 \mathrm{E}-03 \\
3.198 \mathrm{E}-02 \\
5.810 \mathrm{E}-02 \\
8.611 \mathrm{E}-02 \\
1.460 \mathrm{E}-01 \\
2.090 \mathrm{E}-01 \\
2.734 \mathrm{E}-01\end{array}$ & $\begin{array}{l}-0.118 \\
-0.096 \\
-0.091 \\
-0.085 \\
-0.080 \\
-0.072 \\
-0.066 \\
-0.061\end{array}$ & $\begin{array}{l}0.128 \\
0.125 \\
0.121 \\
0.1117 \\
0.1113 \\
0.106 \\
0.100 \\
0.095\end{array}$ & $\begin{array}{l}0.127 \\
0.124 \\
0.118 \\
0.1114 \\
0.1109 \\
0.101 \\
0.093 \\
0.087\end{array}$ \\
\hline $\begin{array}{l}1.0000 \\
1.2500 \\
1.5000 \\
1.7500 \\
2.0000 \\
2.5000 \\
3.0000 \\
3.5000\end{array}$ & $\begin{array}{l}1.678 \mathrm{E}+00 \\
1.660 \mathrm{E}+00 \\
1.654 \mathrm{E}+00 \\
1.654 \mathrm{E}+00 \\
1.657 \mathrm{E}+00 \\
1.667 \mathrm{E}+00 \\
1.680 \mathrm{E}+00 \\
1.692 \mathrm{E}+00\end{array}$ & $\begin{array}{l}1.244 \mathrm{E}-02 \\
1.554 \mathrm{E}-02 \\
1.887 \mathrm{E}-02 \\
2.237 \mathrm{E}-02 \\
2.602 \mathrm{E}-02 \\
3.368 \mathrm{E}-02 \\
4.176 \mathrm{E}-02 \\
5.016 \mathrm{E}-02\end{array}$ & $\begin{array}{l}1.691 \mathrm{E}+00 \\
1.675 \mathrm{E}+00 \\
1.673 \mathrm{E}+00 \\
1.676 \mathrm{E}+00 \\
1.683 \mathrm{E}+00 \\
1.701 \mathrm{E}+00 \\
1.721 \mathrm{E}+00 \\
1.742 \mathrm{E}+00\end{array}$ & $\begin{array}{l}4.806 E-01 \\
6.293 E-01 \\
7.787 E-01 \\
9.280 E-01 \\
1.077 E+00 \\
1.372 E+00 \\
1.665 E+00 \\
1.953 E+00\end{array}$ & $\begin{array}{l}3.828 \mathrm{E}-03 \\
4.724 \mathrm{E}-03 \\
5.649 \mathrm{E}-03 \\
6.600 \mathrm{E}-03 \\
7.575 \mathrm{E}-03 \\
9.584 \mathrm{E}-03 \\
1.166 \mathrm{E}-02 \\
1.378 \mathrm{E}-02\end{array}$ & $\begin{array}{l}3.380 \mathrm{E}-01 \\
4.966 \mathrm{E}-01 \\
6.473 \mathrm{E}-01 \\
7.886 \mathrm{E}-01 \\
9.206 \mathrm{E}-01 \\
1.159 \mathrm{E}+00 \\
1.368 \mathrm{E}+00 \\
1.554 \mathrm{E}+00\end{array}$ & $\begin{array}{l}-0.056 \\
-0.049 \\
-0.044 \\
-0.040 \\
-0.037 \\
-0.033 \\
-0.031 \\
-0.029\end{array}$ & $\begin{array}{l}0.090 \\
0.081 \\
0.074 \\
0.069 \\
0.065 \\
0.058 \\
0.054 \\
0.050\end{array}$ & $\begin{array}{l}0.082 \\
0.071 \\
0.064 \\
0.058 \\
0.053 \\
0.046 \\
0.041 \\
0.038\end{array}$ \\
\hline $\begin{array}{l}4.00000 \\
4.50000 \\
5.00000 \\
5.50000 \\
6.00000 \\
7.00000 \\
8.00000 \\
9.0000\end{array}$ & $\begin{array}{l}1.704 \mathrm{E}+00 \\
1.715 \mathrm{E}+00 \\
1.726 \mathrm{E}+00 \\
1.735 \mathrm{E}+00 \\
1.745 \mathrm{E}+00 \\
1.761 \mathrm{E}+00 \\
1.775 \mathrm{E}+00 \\
1.788 \mathrm{E}+00\end{array}$ & $\begin{array}{l}5.883 \mathrm{E}-02 \\
6.775 \mathrm{E}-02 \\
7.688 \mathrm{E}-02 \\
8.620 \mathrm{E}-02 \\
9.569 \mathrm{E}-02 \\
1.151 \mathrm{E}-01 \\
1.351 \mathrm{E}-01 \\
1.554 \mathrm{E}-01\end{array}$ & $\begin{array}{l}1.763 \mathrm{E}+00 \\
1.783 \mathrm{E}+00 \\
1.803 \mathrm{E}+00 \\
1.822 \mathrm{E}+00 \\
1.840 \mathrm{E}+00 \\
1.876 \mathrm{E}+00 \\
1.910 \mathrm{E}+00 \\
1.944 \mathrm{E}+00\end{array}$ & $\begin{array}{l}2.239 \mathrm{E}+00 \\
2.521 \mathrm{E}+00 \\
2.800 \mathrm{E}+00 \\
3.076 \mathrm{E}+00 \\
3.349 \mathrm{E}+00 \\
3.887 \mathrm{E}+00 \\
4.415 \mathrm{E}+00 \\
4.934 \mathrm{E}+00\end{array}$ & $\begin{array}{l}1.594 \mathrm{E}-02 \\
1.814 \mathrm{E}-02 \\
2.035 \mathrm{E}-02 \\
2.259 \mathrm{E}-02 \\
2.485 \mathrm{E}-02 \\
2.940 \mathrm{E}-02 \\
3.398 \mathrm{E}-02 \\
3.857 \mathrm{E}-02\end{array}$ & $\begin{array}{l}1.721 E+00 \\
1.873 E+00 \\
2.012 E+00 \\
2.140 E+00 \\
2.258 E+00 \\
2.473 E+00 \\
2.663 E+00 \\
2.835 E+00\end{array}$ & $\begin{array}{l}-0.028 \\
-0.027 \\
-0.026 \\
-0.025 \\
-0.024 \\
-0.023 \\
-0.022 \\
-0.021\end{array}$ & $\begin{array}{l}0.047 \\
0.045 \\
0.043 \\
0.041 \\
0.040 \\
0.037 \\
0.035 \\
0.034\end{array}$ & $\begin{array}{l}0.035 \\
0.033 \\
0.032 \\
0.030 \\
0.029 \\
0.027 \\
0.026 \\
0.024\end{array}$ \\
\hline $\begin{array}{l}10.0000 \\
12.5000 \\
15.0000 \\
17.5000 \\
20.0000 \\
25.0000 \\
30.0000 \\
35.0000\end{array}$ & $\begin{array}{l}1.800 E+00 \\
1.824 E+00 \\
1.843 E+00 \\
1.859 E+00 \\
1.873 E+00 \\
1.895 E+00 \\
1.912 E+00 \\
1.926 E+00\end{array}$ & $\begin{array}{l}1.762 \mathrm{E}-01 \\
2.294 \mathrm{E}-01 \\
2.842 \mathrm{E}-01 \\
3.401 \mathrm{E}-01 \\
3.969 \mathrm{E}-01 \\
5.126 \mathrm{E}-01 \\
6.303 \mathrm{E}-01 \\
7.495 \mathrm{E}-01\end{array}$ & $\begin{array}{l}1.976 \mathrm{E}+00 \\
2.053 \mathrm{E}+00 \\
2.127 \mathrm{E}+00 \\
2.199 \mathrm{E}+00 \\
2.270 \mathrm{E}+00 \\
2.407 \mathrm{E}+00 \\
2.542 \mathrm{E}+00 \\
2.676 \mathrm{E}+00\end{array}$ & $\begin{array}{l}5.444 \mathrm{E}+00 \\
6.685 \mathrm{E}+00 \\
7.881 \mathrm{E}+00 \\
9.036 \mathrm{E}+00 \\
1.016 \mathrm{E}+01 \\
1.229 \mathrm{E}+01 \\
1.431 \mathrm{E}+01 \\
1.623 \mathrm{E}+01\end{array}$ & $\begin{array}{l}4.317 \mathrm{E}-02 \\
5.464 \mathrm{E}-02 \\
6.599 \mathrm{E}-02 \\
7.716 \mathrm{E}-02 \\
8.812 \mathrm{E}-02 \\
1.093 \mathrm{E}-01 \\
1.295 \mathrm{E}-01 \\
1.488 \mathrm{E}-01\end{array}$ & $\begin{array}{l}2.991 E+00 \\
3.331 E+00 \\
3.619 E+00 \\
3.870 E+00 \\
4.093 E+00 \\
4.478 E+00 \\
4.802 E+00 \\
5.083 E+00\end{array}$ & $\begin{array}{l}-0.020 \\
-0.017 \\
-0.015 \\
-0.013 \\
-0.012 \\
-0.009 \\
-0.007 \\
-0.006\end{array}$ & $\begin{array}{l}0.032 \\
0.029 \\
0.027 \\
0.025 \\
0.024 \\
0.021 \\
0.019 \\
0.017\end{array}$ & $\begin{array}{l}0.023 \\
0.021 \\
0.019 \\
0.017 \\
0.0116 \\
0.013 \\
0.011 \\
0.009\end{array}$ \\
\hline $\begin{array}{l}40.0000 \\
45.00000 \\
50.0000 \\
55.00000 \\
60.00000 \\
70.0000 \\
80.0000 \\
90.0000\end{array}$ & $\begin{array}{l}1.938 E+00 \\
1.948 E+00 \\
1.958 E+00 \\
1.966 E+00 \\
1.973 E+00 \\
1.986 E+00 \\
1.997 E+00 \\
2.007 E+00\end{array}$ & $\begin{array}{l}8.699 \mathrm{E}-01 \\
9.913 \mathrm{E}-01 \\
1.113 \mathrm{E}+00 \\
1.236 \mathrm{E}+00 \\
1.360 \mathrm{E}+00 \\
1.609 \mathrm{E}+00 \\
1.859 \mathrm{E}+00 \\
2.111 \mathrm{E}+00\end{array}$ & $\begin{array}{l}2.808 \mathrm{E}+00 \\
2.940 \mathrm{E}+00 \\
3.071 \mathrm{E}+00 \\
3.202 \mathrm{E}+00 \\
3.333 \mathrm{E}+00 \\
3.595 \mathrm{E}+00 \\
3.856 \mathrm{E}+00 \\
4.118 \mathrm{E}+00\end{array}$ & $\begin{array}{l}1.805 E+01 \\
1.980 E+01 \\
2.146 E+01 \\
2.305 E+01 \\
2.458 E+01 \\
2.747 E+01 \\
3.016 E+01 \\
3.267 E+01\end{array}$ & $\begin{array}{l}1.671 \mathrm{E}-01 \\
1.845 \mathrm{E}-01 \\
2.010 \mathrm{E}-01 \\
2.168 \mathrm{E}-01 \\
2.318 \mathrm{E}-01 \\
2.599 \mathrm{E}-01 \\
2.856 \mathrm{E}-01 \\
3.091 \mathrm{E}-01\end{array}$ & $\begin{array}{l}5.330 \mathrm{E}+00 \\
5.552 \mathrm{E}+00 \\
5.751 \mathrm{E}+00 \\
5.934 \mathrm{E}+00 \\
6.101 \mathrm{E}+00 \\
6.399 \mathrm{E}+00 \\
6.660 \mathrm{E}+00 \\
6.891 \mathrm{E}+00\end{array}$ & $\begin{array}{l}-0.005 \\
-0.004 \\
-0.003 \\
-0.003 \\
-0.002 \\
-0.002 \\
-0.001 \\
-0.001\end{array}$ & $\begin{array}{l}0.016 \\
0.0115 \\
0.014 \\
0.013 \\
0.0112 \\
0.0111 \\
0.0110 \\
0.009\end{array}$ & $\begin{array}{l}0.008 \\
0.007 \\
0.006 \\
0.006 \\
0.005 \\
0.004 \\
0.004 \\
0.003\end{array}$ \\
\hline $\begin{array}{l}100.0000 \\
125.0000 \\
150.0000 \\
175.0000 \\
200.0000 \\
250.0000 \\
300.0000 \\
350.0000\end{array}$ & $\begin{array}{l}2.015 E+00 \\
2.033 E+00 \\
2.048 E+00 \\
2.060 E+00 \\
2.071 E+00 \\
2.089 E+00 \\
2.103 E+00 \\
2.115 E+00\end{array}$ & $\begin{array}{l}2.364 E+00 \\
3.000 E+00 \\
3.641 E+00 \\
4.285 E+00 \\
4.932 E+00 \\
6.232 E+00 \\
7.537 E+00 \\
8.846 E+00\end{array}$ & $\begin{array}{l}4.379 \mathrm{E}+00 \\
5.034 \mathrm{E}+00 \\
5.689 \mathrm{E}+00 \\
6.346 \mathrm{E}+00 \\
7.003 \mathrm{E}+00 \\
8.320 \mathrm{E}+00 \\
9.640 \mathrm{E}+00 \\
1.096 \mathrm{E}+01\end{array}$ & $\begin{array}{l}3.502 E+01 \\
4.034 E+01 \\
4.501 E+01 \\
4.917 E+01 \\
5.292 E+01 \\
5.946 E+01 \\
6.504 E+01 \\
6.990 E+01\end{array}$ & $\begin{array}{l}3.309 \mathrm{E}-01 \\
3.785 \mathrm{E}-01 \\
4.186 \mathrm{E}-01 \\
4.528 \mathrm{E}-01 \\
4.825 \mathrm{E}-01 \\
5.316 \mathrm{E}-01 \\
5.707 \mathrm{E}-01 \\
6.027 \mathrm{E}-01\end{array}$ & $\begin{array}{l}7.098 \mathrm{E}+00 \\
7.538 \mathrm{E}+00 \\
7.899 \mathrm{E}+00 \\
8.205 \mathrm{E}+00 \\
8.471 \mathrm{E}+00 \\
8.915 \mathrm{E}+00 \\
9.279 \mathrm{E}+00 \\
9.586 \mathrm{E}+00\end{array}$ & $\begin{array}{l}-0.0001 \\
-0.001 \\
-0.0000 \\
-0.0000 \\
-0.0000 \\
-0.0000 \\
-0.0000 \\
-0.000\end{array}$ & $\begin{array}{l}0.009 \\
0.008 \\
0.007 \\
0.006 \\
0.006 \\
0.005 \\
0.005 \\
0.005\end{array}$ & $\begin{array}{l}0.0003 \\
0.0002 \\
0.0002 \\
0.0001 \\
0.0001 \\
0.0001 \\
0.0001 \\
0.001\end{array}$ \\
\hline $\begin{array}{l}400.00000 \\
450.0000 \\
500.0000 \\
550.0000 \\
600.00000 \\
700.0000 \\
800.0000 \\
900.00000\end{array}$ & $\begin{array}{l}2.126 \mathrm{E}+00 \\
2.135 \mathrm{E}+00 \\
2.143 \mathrm{E}+00 \\
2.151 \mathrm{E}+00 \\
2.158 \mathrm{E}+00 \\
2.170 \mathrm{E}+00 \\
2.181 \mathrm{E}+00 \\
2.190 \mathrm{E}+00\end{array}$ & $\begin{array}{l}1.016 E+01 \\
1.147 E+01 \\
1.279 E+01 \\
1.411 E+01 \\
1.543 E+01 \\
1.807 E+01 \\
2.072 E+01 \\
2.337 E+01\end{array}$ & $\begin{array}{l}1.228 \mathrm{E}+01 \\
1.361 \mathrm{E}+01 \\
1.493 \mathrm{E}+01 \\
1.626 \mathrm{E}+01 \\
1.759 \mathrm{E}+01 \\
2.024 \mathrm{E}+01 \\
2.290 \mathrm{E}+01 \\
2.556 \mathrm{E}+01\end{array}$ & $\begin{array}{l}7.421 \mathrm{E}+01 \\
7.807 \mathrm{E}+01 \\
8.158 \mathrm{E}+01 \\
8.479 \mathrm{E}+01 \\
8.774 \mathrm{E}+01 \\
9.304 \mathrm{E}+01 \\
9.768 \mathrm{E}+01 \\
1.018 \mathrm{E}+02\end{array}$ & $\begin{array}{l}6.296 \mathrm{E}-01 \\
6.524 \mathrm{E}-01 \\
6.722 \mathrm{E}-01 \\
6.895 \mathrm{E}-01 \\
7.047 \mathrm{E}-01 \\
7.305 \mathrm{E}-01 \\
7.516 \mathrm{E}-01 \\
7.692 \mathrm{E}-01\end{array}$ & $\begin{array}{l}.853 \mathrm{E}+00 \\
.009 \mathrm{E}+01 \\
.030 \mathrm{E}+01 \\
.049 \mathrm{E}+01 \\
.066 \mathrm{E}+01 \\
.097 \mathrm{E}+01 \\
.124 \mathrm{E}+01 \\
.147 \mathrm{E}+01\end{array}$ & $\begin{array}{l}-0.0000 \\
-0.000 \\
-0.0000 \\
-0.000 \\
-0.000 \\
-0.000 \\
-0.000 \\
-0.000\end{array}$ & $\begin{array}{l}0.004 \\
0.004 \\
0.004 \\
0.004 \\
0.004 \\
0.003 \\
0.003 \\
0.003\end{array}$ & $\begin{array}{l}0.0000 \\
0.0000 \\
0.0000 \\
0.0000 \\
0.0000 \\
0.0000 \\
0.0000 \\
0.0000\end{array}$ \\
\hline 0.0000 & $2.198 E+00$ & $2.602 \mathrm{E}+01$ & $2.822 E+01$ & $1.055 E+02$ & $7.841 \mathrm{E}-01$ & $1.168 E+01$ & -0.000 & 0.003 & 0.000 \\
\hline
\end{tabular}


ELECTRONS IN CERIC SULFATE DOSIMETER SOLUTION

$I=76.7 \mathrm{eV}$

$\begin{array}{cc}\text { ENERGY } & \begin{array}{c}5 T O \\ \text { MeV }\end{array} \\ & \text { MeV cOLISION } \\ & \\ 0.0100 & 2.237 E+01 \\ 0.0125 & 1.831 E+01 \\ 0.0150 & 1.633 E+01 \\ 0.0175 & 1.449 E+01 \\ 0.0200 & 1.307 E+01 \\ 0.0250 & 1.101 E+01 \\ 0.0300 & 9.577 E+00 \\ 0.0350 & 8.525 E+00 \\ 0.0400 & 7.717 E+00\end{array}$

$0.0400 \quad 7.717 \mathrm{E}+00$

$\begin{aligned} & 0.0450 \\ & 0.0500\end{aligned} 6.553 \mathrm{E}+00$

$0.0550 \quad 6.119 \mathrm{E}+00$

$0.0600 \quad 5.753 \mathrm{E}+00$

$0.0700 \quad 5.169 \mathrm{E}+00$

$0.0800 \quad 4.722 \mathrm{E}+00$

$0.1000 \quad 4.085 E+00$

$0.1250 \quad 3.566 \mathrm{E}+00$

0.1500

0.1750

0.2000

0.3000

0.3500

0.4000

0.4500

0.5000

0.5500

0.700

0.800

0.9000

$3.566 \mathrm{E}+00$
$3.215 \mathrm{E}+00$

2. $963 \mathrm{E}+00$

2. $773 \mathrm{E}+00$

2. $511 \mathrm{E}+00$

2. $219 \mathrm{E}+00$

2. $133 \mathrm{E}+00$

2. $069 \mathrm{E}+00$

$2.021 \mathrm{E}+00$
$1.981 \mathrm{E}+00$

1. $950 \mathrm{E}+00$

$1.904 E+00$

$1.873 E+00$

$1.0000 \quad 1.837 \mathrm{E}+00$

$1.2500 \quad 1.816 \mathrm{E}+00$

$1.5000 \quad 1.810 \mathrm{E}+00$

$1.7500 \quad 1.809 \mathrm{E}+00$

$2.0000 \quad 1.812 E+00$

3.0000

$3.5000 \quad 1.847 \mathrm{E}+00$

4.0000

4. 5000

$1.847 \mathrm{E}+00$

$1.881 \mathrm{E}+00$

1. $891 \mathrm{E}+00$

1. $900 \mathrm{E}+00$

$1.917 \mathrm{E}+00$

$1.932 \mathrm{E}+00$
$1.945 \mathrm{E}+00$

9.0000

10.0000 12.5000 15.000 20.0000

25.000

30.0000

35.0000

40.0000

45.0000

50.000

60.000

60.0000

80.0000

90.0000

1. $957 \mathrm{E}+00$

1. $982 \mathrm{E}+00$

2. $003 E+00$

2. $020 \mathrm{E}+0$

$2.059 \mathrm{E}+00$

$2.078 E+00$

2. $093 E+00$

2. $106 \mathrm{E}+00$

2. $118 \mathrm{E}+00$

$.128 E+00$

2. $145 \mathrm{E}+00$

$2.159 E+00$

$2.171 E+00$

2. $181 \mathrm{E}+00$

100.000

125.0000

175.000

200.0000

250.0000

250.0000
300.0000

350.0000

2. $191 \mathrm{E}+00$

2. $210 E+00$

$2.226 E+00$

2. $240 E+00$

$2.270 \mathrm{E}+00$

2. $286 E+00$

2. $299 \mathrm{E}+00$

400.0000

500.0000

550.0000

600.0000

700.0000

800.0000

900.0000

2. $310 \mathrm{E}+00$

2. $320 E+00$

2. $329 \mathrm{E}+00$

2. $337 \mathrm{E}+00$

2. $358 \mathrm{E}+00$

2. $358 E+00$
2. $369 E+00$

$2.369 E+00$
$2.379 E+00$

1000.0000

2. $388 \mathrm{E}+00$
OPP ING POWER

$\mathrm{MeV} \mathrm{cm}^{2} / \mathrm{g} \mathrm{MeV} \mathrm{cm}^{2} / \mathrm{g}$

3. $996 E-03 \quad 2.237 E+01$

$4.031 \mathrm{E}-03 \quad 1.882 \mathrm{E}+01$

4.052E-03 1.633E+01

$.067 \mathrm{E}-03$

$094 \mathrm{E}-03 \quad 1.101 \mathrm{E}+01$

4. $108 \mathrm{E}-03 \quad 9.581 \mathrm{E}+00$

$4.120 E-03$

$8.529 \mathrm{E}+00$

$4.134 E-03 \quad 7.721 E+00$

$4.148 E-03 \quad 7.079 E+00$

4. $164 \mathrm{E}-03 \quad 6.557 \mathrm{E}+00$

4.180E-03 6.123E+00

$4.198 \mathrm{E}-03 \quad 5.757 \mathrm{E}+00$

$4.236 \mathrm{E}-03 \quad 5.173 \mathrm{E}+00$

$\begin{array}{ll}4.278 E-03 & 4.727 E+00 \\ 4.324 E-03 & 4.374 E+00\end{array}$

$4.373 E-03 \quad 4.090 E+00$

4.5 E-03 3.570E+00

$4.803 \mathrm{E}-03 \quad 2.967 \mathrm{E}+00$

$4.966 \mathrm{E}-03 \quad 2.778 \mathrm{E}+00$

$5.316 \mathrm{E}-03 \quad 2.516 \mathrm{E}+00$

$6.700 \mathrm{E}-03$

2. $344 \mathrm{E}+00$

$6.548 \mathrm{E}-03 \quad 2.140 \mathrm{E}+00$

$7.009 \mathrm{E}-03 \quad 2.076 \mathrm{E}+00$

$7.492 \mathrm{E}-03 \quad 2.028 \mathrm{E}+00$

$7.995 \mathrm{E}-03 \quad 1.989 \mathrm{E}+00$

$8.515 \mathrm{E}-03 \quad 1.959 \mathrm{E}+00$

$\begin{array}{ll}1.602 \mathrm{E}-03 & 1.914 \mathrm{E}+00 \\ 1.074 \mathrm{E}-02 & 1.884 \mathrm{E}+00\end{array}$

$1.194 \mathrm{E}-02 \quad 1.863 \mathrm{E}+00$

1. $318 \mathrm{E}-02$

1. $646 \mathrm{E}-02$

1. $997 \mathrm{E}-02$

2. $752 \mathrm{E}-02$

$3.561 \mathrm{E}-02$

$4.413 \mathrm{E}-02$

$6.214 \mathrm{E}-02$

7. $154 \mathrm{E}-02$

$8.117 \mathrm{E}-02$

1.010E-01

1.215E-01

$1.425 \mathrm{E}-01$

1. $858 \mathrm{E}-01$

$2.418 \mathrm{E}-01$

2. $994 \mathrm{E}-01$

3. $583 \mathrm{E}-01$

$5.397 \mathrm{E}-01$

$6.635 \mathrm{E}-01$

9. $155 \mathrm{E}-01$

1. $043 \mathrm{E}+00$

1. $172 \mathrm{E}+00$

$.301 \mathrm{E}+00$

$1.431 \mathrm{E}+00$
$1.693 \mathrm{E}+00$

1. $956 \mathrm{E}+00$

2. $221 \mathrm{E}+00$

1. $850 \mathrm{E}+00$

1. $833 E+00$

$1.830 \mathrm{E}+00$

$1.839 \mathrm{E}+00$

$1.858 \mathrm{E}+00$

$1.879 \mathrm{E}+00$

$1.900 \mathrm{E}+00$

1. $921 E+00$

1. $942 \mathrm{E}+00$

1. $982 \mathrm{E}+0$

2. $001 \mathrm{E}+00$

2. $038 \mathrm{E}+00$

2. $143 \mathrm{E}+00$

2. $224 \mathrm{E}+00$

2. $378 E+00$

2. $453 \mathrm{E}+00$

2. $598 \mathrm{E}+00$

$2.741 \mathrm{E}+00$

2. $882 \mathrm{E}+00$

$3.022 \mathrm{E}+00$

$3.161 \mathrm{E}+00$

3. $300 \mathrm{E}+00$

3. $852 \mathrm{E}+00$

$3.852 \mathrm{E}+00$
$4.127 \mathrm{E}+00$

$4.127 \mathrm{E}+00$
$4.402 \mathrm{E}+00$

2. $487 E+00$

3. $830 \mathrm{E}+00$

$4.507 \mathrm{E}+00$

4.507E+00

$6.553 \mathrm{E}+00$

7. $926 \mathrm{E}+00$

$9.302 \mathrm{E}+00$

5. $367 \mathrm{E}+00$

$6.056 \mathrm{E}+00$

6. $747 \mathrm{E}+00$

$7.438 \mathrm{E}+00$

$8.824 E+00$

$1.021 \mathrm{E}+01$

$1.068 \mathrm{E}+01$

1. $206 E+01$

1. $345 E+01$

$1.622 \mathrm{E}+01$

1. $900 \mathrm{E}+01$

2. $178 \mathrm{E}+01$
$2.457 \mathrm{E}+01$

1. $299 \mathrm{E}+01$

1. $438 \mathrm{E}+0$

1. $578 \mathrm{E}+01$

1. $717 \mathrm{E}+0$

1. $857 \mathrm{E}+0$

$2.415 E+01$
$2.695 E+01$

2. $736 \mathrm{E}+01$

2. $975 \mathrm{E}+01$
$4.649 \mathrm{E}-03 \quad 3.219 \mathrm{E}+00$

2. $074 \mathrm{E}+00$

$3.438 \mathrm{E}+00$

$4.678 \mathrm{E}+00$

2. $136 \mathrm{E}+0$

DENSITY $=1.030 \mathrm{E}+00 \mathrm{~g} / \mathrm{cm}^{3}$

$\begin{array}{lcc}\text { CSDA } & \text { RADIATION } & \text { DEYS.EFF. } \\ \text { RANGE } & \text { YIELD } & \text { CORR. } \\ & & \text { (DELTA) }\end{array}$

$\mathrm{g} / \mathrm{cm}^{2}$

2. $538 E-04$ 3.762E-04

$.193 \mathrm{E}-04$

$6.821 \mathrm{E}-04$

8. $640 \mathrm{E}-04$

1. $283 \mathrm{E}-03$

$1.771 \mathrm{E}-03$
$2.325 \mathrm{E}-03$

2. $942 \mathrm{E}-03$

$3.620 \mathrm{E}-03$

4. $354 \mathrm{E}-03$

. $144 \mathrm{E}-03$

$5.987 \mathrm{E}-03$
$7.823 \mathrm{E}-03$

$9.848 \mathrm{E}-03$

9. $703 \mathrm{E}-05 \quad 0.0$

$1.169 E-04 \quad 0.0$

$1.360 \mathrm{E}-04 \quad 0.0$

$\begin{array}{ll}.543 \mathrm{E}-04 & 0.0 \\ 1.721 \mathrm{E}-04 & 0.0\end{array}$

$2.061 \mathrm{E}-04 \quad 0.0$

$\begin{array}{ll}2.385 \mathrm{E}-04 & 0.0 \\ 2.696 \mathrm{E}-04 & 0.0\end{array}$

$2.996 \mathrm{E}-04 \quad 0.0$

$3.286 \mathrm{E}-04 \quad 0.0$

$3.568 \mathrm{E}-04 \quad 0.0$

$3.843 E-04 \quad 0.0$

$4.111 E-04 \quad 0.0$

$\begin{array}{ll}4.630 E-04 & 0.0 \\ 5.129 E-04 & 0.0\end{array}$

$5.611 E-04 \quad 0.0$
0

$1.442 \mathrm{E}-02 \quad 6.079 \mathrm{E}-04 \quad 0.0$

$.099 E-02 \quad 7.196 E-04 \quad 0.0$

.838E-02 8.253E-04 0.0

$3.648 \mathrm{E}-02 \quad 9.263 \mathrm{E}-04 \quad 0.0$

$\begin{array}{lll}4.520 \mathrm{E}-02 & 1.023 \mathrm{E}-03 & 0.0 \\ 6.418 \mathrm{E}-02 & 1.209 \mathrm{E}-03 & 0.0\end{array}$

$\begin{array}{lll}8.418 E-02 & 1.209 E-03 & 0.0 \\ 8.481 E-02 & 1.386 E-03 & 0.0\end{array}$

$1.067 \mathrm{E}-01 \quad 1.558 \mathrm{E}-03 \quad 0.0$

$1.297 \mathrm{E}-01 \quad 1.726 \mathrm{E}-0.3 \quad 0.0$

$\begin{array}{lll}1.534 \mathrm{E}-01 & 1.892 \mathrm{E}-03 & 0.0\end{array}$

$\begin{array}{lll}778 E-01 & 2.056 E-03 & 0.0\end{array}$

$2.220 E-03 \quad 1.324 E-02$

$\begin{array}{lll}2.280 E-01 & 2.383 E-03 & 3.214 E-02\end{array}$

3. $324 \mathrm{E}-01 \quad 2.712 \mathrm{E}-03 \quad 7.786 \mathrm{E}-02$

$3.858 \mathrm{E}-01 \quad 3.377 \mathrm{E}-03 \quad 1.876 \mathrm{E}-01$

4. $397 \mathrm{E}-01$

$\begin{array}{lll}5.756 \mathrm{E}-01 & 4.582 \mathrm{E}-03 & 3.983 \mathrm{E}-01 \\ 7.121 \mathrm{E}-01 & 5.476 \mathrm{E}-03 & 5.470 \mathrm{E}-01\end{array}$

2. $470 \mathrm{E}-01$

$8.487 \mathrm{E}-01 \quad 5.476 \mathrm{E}-03 \quad 5.470 \mathrm{E}-01$

$9.849 \mathrm{E}-01 \quad 7.337 \mathrm{E}-03 \quad 8.234 \mathrm{E}-01$

$1.523 E+00 \quad 1.129 E-02 \quad 1.287 E+00$

2. $049 \mathrm{E}+00$

2. $308 \mathrm{E}+00$

2. $564 \mathrm{E}+00$

. $818 \mathrm{E}+00$

3. $564 \mathrm{E}+00$

4. $050 \mathrm{E}+00$

$4.999 \mathrm{E}+00$

6. $144 \mathrm{E}+00$

$7.249 \mathrm{E}+00$

$317 \mathrm{E}+00$

. $352 \mathrm{E}+00$

1. $133 \mathrm{E}+01$

1. $498 \mathrm{E}+0$

$1.334 \mathrm{E}-02$

$1.287 \mathrm{E}+00$
$1.481 \mathrm{E}+00$ 


\begin{tabular}{|c|c|c|c|c|c|c|c|c|c|}
\hline ENERGY & $\begin{array}{l}\text { COLLISION } \\
\mathrm{MeV} \mathrm{Cm}^{2} / \mathrm{g}\end{array}$ & $\begin{array}{l}\text { ING PO } \\
\text { DIAT IV }\end{array}$ & TOTAL & $\begin{array}{l}\text { CSDA } \\
\text { RANGE }\end{array}$ & $\begin{array}{l}\text { ADIATION } \\
\text { YIELD }\end{array}$ & $\begin{array}{c}\text { DENS.EFF . } \\
\text { CORR. } \\
\text { (DELTA) }\end{array}$ & $\begin{array}{l}d(1 \\
\text { coLL } \\
\text { LOSS }\end{array}$ & SD & $\begin{array}{l}\text { gI ) } \\
\text { RAD } \\
\text { YIELD }\end{array}$ \\
\hline $\begin{array}{l}0.0100 \\
0.0125 \\
0.0150 \\
0.0175 \\
0.0200 \\
0.0250 \\
0.0300 \\
0.0350\end{array}$ & $\begin{array}{l}+01 \\
+00 \\
+00 \\
+00 \\
+00 \\
+00 \\
+00 \\
+00\end{array}$ & $\begin{array}{l}1.8 \\
1.9 \\
2.0 \\
2.1 \\
2.2 \\
2.3 \\
2.4\end{array}$ & $\begin{array}{l}8.8 \\
7.8 \\
7.8 \\
6.4 \\
5.5 \\
4.8\end{array}$ & $\begin{array}{l}4 \\
4 \\
3 \\
3 \\
3 \\
3 \\
3 \\
3 \\
3\end{array}$ & $\begin{array}{l}8.286 \mathrm{E}-04 \\
1.039 \mathrm{E}-03 \\
1.247 \mathrm{E}-03 \\
1.454 \mathrm{E}-03 \\
1.658 \mathrm{E}-03 \\
2.060 \mathrm{E}-03 \\
2.454 \mathrm{E}-03 \\
2.839 \mathrm{E}-03\end{array}$ & $\begin{array}{l}0.0 \\
0.0 \\
0.0 \\
0.0 \\
0.0 \\
0.0 \\
0.0 \\
0.0\end{array}$ & $\begin{array}{l}-0.330 \\
-0.307 \\
-0.291 \\
-0.279 \\
-0.269 \\
-0.254 \\
-0.243 \\
-0.235\end{array}$ & $\begin{array}{l}0.469 \\
0.424 \\
0.392 \\
0.368 \\
0.350 \\
0.322 \\
0.303 \\
0.288\end{array}$ & $\begin{array}{l}0.419 \\
0.382 \\
0.356 \\
0.337 \\
0.322 \\
0.300\end{array}$ \\
\hline $\begin{array}{l}0.0400 \\
0.0450 \\
0.0500 \\
0.0550 \\
0.0600 \\
0.0700 \\
0.0800 \\
0.0900\end{array}$ & $\begin{array}{l}+00 \\
+00 \\
+00 \\
+00 \\
+00 \\
+00 \\
+00 \\
+00\end{array}$ & $\begin{array}{l}2.6 \\
2.6 \\
2.7 \\
2.8 \\
2.8 \\
2.9\end{array}$ & $\begin{array}{l}+00 \\
+00 \\
+00 \\
+00 \\
+00 \\
+00 \\
+00 \\
+00\end{array}$ & $\begin{array}{l}7.2 \\
8.6 \\
1.0 \\
1.1 \\
1.5 \\
1.8\end{array}$ & $\begin{array}{l}-03 \\
-033 \\
-03 \\
-03 \\
-03 \\
-03 \\
-03 \\
-03 \\
-03 \\
-03\end{array}$ & $\begin{array}{l}0.0 \\
0.0 \\
0.0 \\
0.0 \\
0.0 \\
0.0 \\
0.0 \\
0.0\end{array}$ & $\begin{array}{l}-0.228 \\
-0.222 \\
-0.218 \\
-0.213 \\
-0.210 \\
-0.203 \\
-0.198 \\
-0.194\end{array}$ & $\begin{array}{l}0.277 \\
0.267 \\
0.259 \\
0.253 \\
0.247 \\
0.237 \\
0.230 \\
0.223\end{array}$ & $\begin{array}{l}0.263 \\
0.255 \\
0.248 \\
0.242 \\
0.237 \\
0.229 \\
0.227\end{array}$ \\
\hline $\begin{array}{l}0.1000 \\
0.1250 \\
0.1500 \\
0.1750 \\
0.2000 \\
0.2500 \\
0.3000 \\
0.3500\end{array}$ & $\begin{array}{l}2.233 E+00 \\
1.970 E+00 \\
1.791 E+00 \\
1.661 E+00 \\
1.564 E+00 \\
1.429 E+00 \\
1.340 E+00 \\
1.279 E+00\end{array}$ & $\begin{array}{l}3.1 \\
3.3 \\
3.4 \\
3.5 \\
3.8 \\
4.0 \\
4.3\end{array}$ & $\begin{array}{l}2.2 \\
2.0 \\
1.8 \\
1.6 \\
1.6 \\
1.4 \\
1.3 \\
1.3\end{array}$ & $\begin{array}{l}1.1 \\
1.4 \\
1.8\end{array}$ & $\begin{array}{l}7.2 \\
8.7 \\
1.0 \\
1.1 \\
1.2 \\
1.5 \\
1.7 \\
1.9\end{array}$ & $\begin{array}{l}0.0 \\
0.0 \\
0.0 \\
0.0 \\
0.0 \\
2.448 E-04 \\
4.939 \mathrm{E}-03 \\
1.113 \mathrm{E}-02\end{array}$ & $\begin{array}{l}-0.1 \\
-0.1 \\
-0.1 \\
-0.1 \\
-0.1 \\
-0.1 \\
-0.1 \\
-0.1\end{array}$ & $\begin{array}{l}0 \\
0 \\
4 \\
9 \\
1 \\
4 \\
9\end{array}$ & $\begin{array}{l}0.212 \\
0.202 \\
0.195 \\
0.189 \\
0.185 \\
0.177 \\
0.170 \\
0.165\end{array}$ \\
\hline $\begin{array}{l}0.4000 \\
0.4500 \\
0.5000 \\
0.5500 \\
0.6000 \\
0.7000 \\
0.8000 \\
0.9000\end{array}$ & $\begin{array}{l}1.23 \\
1.20 \\
1.17 \\
1.15 \\
1.14 \\
1.12 \\
1.11 \\
1.10\end{array}$ & $\begin{array}{l}4.8 \\
5.1 \\
5.4 \\
5.7\end{array}$ & $\begin{array}{l}1.281 E+0 \\
1.251 E+0 \\
1.229 E+0 \\
1.214 E+0 \\
1.202 E+0 \\
1.189 E+0 \\
1.184 E+0 \\
1.184 E+0\end{array}$ & $\begin{array}{l}2.24 \\
2.64 \\
3.04 \\
3.45 \\
3.87 \\
4.70 \\
5.55 \\
6.39\end{array}$ & $\begin{array}{l}2.1 \\
2.2 \\
2.4 \\
2.6 \\
2.8\end{array}$ & $\begin{array}{l}1.84 \\
2.64 \\
3.49 \\
4.37 \\
5.27 \\
7.12 \\
9.02 \\
1.09\end{array}$ & $\begin{array}{l}-0.143 \\
-0.139 \\
-0.135 \\
-0.132 \\
-0.129 \\
-0.124 \\
-0.120 \\
-0.116\end{array}$ & $\begin{array}{l}0 \\
6 \\
2 \\
9 \\
4 \\
0 \\
6\end{array}$ & $\begin{array}{l}0 . \\
0 . \\
0 . \\
0 . \\
0 . \\
0 .\end{array}$ \\
\hline $\begin{array}{l}1.0000 \\
1.2500 \\
1.5000 \\
1.7500 \\
2.0000 \\
2.5000 \\
3.0000 \\
3.5000\end{array}$ & $\left\{\begin{array}{l}0 \\
0 \\
0 \\
0 \\
0 \\
0 \\
0\end{array}\right.$ & $\begin{array}{l}1.1 \\
1.3 \\
1.5 \\
1.9 \\
2.3\end{array}$ & $\begin{array}{l}1.2 \\
1.2 \\
1.2 \\
1.2 \\
1.3 \\
1.4\end{array}$ & b & $\begin{array}{l}2 \\
2 \\
2 \\
2 \\
2 \\
2 \\
2 \\
1\end{array}$ & $\begin{array}{l}1.2 \\
1.7 \\
2.2 \\
2.7 \\
3.1 \\
4.0 \\
4.9 \\
5.7\end{array}$ & $\begin{array}{l}-0 . \\
-0 . \\
-0 . \\
-0 . \\
-0 . \\
-0 . \\
-0\end{array}$ & & 0. \\
\hline $\begin{array}{l}4.0000 \\
4.5000 \\
5.0000 \\
5.5000 \\
6.0000 \\
7.0000 \\
8.0000 \\
9.0000\end{array}$ & $\begin{array}{l}+\infty 00 \\
+00 \\
+00 \\
+00 \\
+00 \\
+00 \\
+00 \\
+00\end{array}$ & $\begin{array}{l}3.7 \\
4.1 \\
4.6 \\
5.0 \\
6.0\end{array}$ & $\begin{array}{l}1.5 \\
1.6 \\
1.7 \\
1.7 \\
1.8 \\
1.9 \\
2.9\end{array}$ & $\begin{array}{l}3.2 \\
3.5 \\
3.8 \\
4.1\end{array}$ & $\begin{array}{l}1 \\
1 \\
1 \\
1\end{array}$ & $\begin{array}{l}6.59 \\
7.35 \\
8.07 \\
8.75 \\
9.41 \\
1.06 \\
1.17 \\
1.28\end{array}$ & $\begin{array}{l}-0.0 \\
-0.0 \\
-0.0 \\
-0.0 \\
-0.0 \\
-0.0 \\
-0.0 \\
-0.0\end{array}$ & $\begin{array}{l}6 \\
3 \\
0 \\
8 \\
3 \\
0 \\
7\end{array}$ & \\
\hline $\begin{array}{l}12.50 \\
15.00 \\
17.50 \\
20.00 \\
25.00 \\
30.00 \\
35.00\end{array}$ & $\begin{array}{l}0 \\
0 \\
0 \\
0\end{array}$ & $\begin{array}{l}1 \\
1 \\
1 \\
0\end{array}$ & $\begin{array}{l}2 \\
2 \\
3 \\
3\end{array}$ & $\begin{array}{l}.194 E+00 \\
.052 E+00 \\
.836 E+00 \\
.122 E+01 \\
.242 E+01 \\
.348 E+01\end{array}$ & 4.9 & $\begin{array}{l}1.381 E+0 \\
1.602 E+0 \\
1.798 E+0 \\
1.973 E+0 \\
2.134 E+0 \\
2.420 E+0 \\
2.668 E+0 \\
2.888 E+0\end{array}$ & & $\begin{array}{l}0.059 \\
0.054 \\
0.051 \\
0.048 \\
0.044 \\
0.040 \\
0.038\end{array}$ & \\
\hline $\begin{array}{l}40.00 \\
45.00 \\
50.00 \\
55.00 \\
60.00 \\
70.00 \\
80.00 \\
90.00\end{array}$ & $\begin{array}{l}0 \\
0 \\
0 \\
0 \\
0\end{array}$ & $\begin{array}{l}5 . \\
5 . \\
6 .\end{array}$ & $\begin{array}{l}6.1 \\
6.7 \\
7.2 \\
7.8 \\
9.0\end{array}$ & 1 & $\begin{array}{l}5.4 \\
5.6 \\
5.8\end{array}$ & $\begin{array}{l}0 \\
0 \\
0 \\
0 \\
0\end{array}$ & & & \\
\hline $\begin{array}{l}.00 \\
.00 \\
.00 \\
.00\end{array}$ & $\begin{array}{l}0 \\
0 \\
0\end{array}$ & $\begin{array}{l}1 \\
1 \\
1 \\
1 \\
1 \\
1\end{array}$ & & & & $\begin{array}{l}0 \\
0 \\
0 \\
0 \\
0 \\
0\end{array}$ & & & \\
\hline $\begin{array}{l}0.0000 \\
0.0000 \\
0.0000 \\
0.0000 \\
0.0000 \\
0.0000 \\
0.0000 \\
0.0000\end{array}$ & $\begin{array}{l}1.657 E+00 \\
1.665 E+00 \\
1.672 E+00 \\
1.679 E+00 \\
1.685 E+00 \\
1.695 E+00 \\
1.704 E+00 \\
1.712 E+00\end{array}$ & $\begin{array}{l}4.627 E+01 \\
5.220 E+01 \\
5.813 E+01 \\
6.407 E+01 \\
7.002 E+01 \\
8.192 E+01 \\
9.383 E+01 \\
1.057 E+02\end{array}$ & $\begin{array}{l}4.792 \mathrm{E}+01 \\
5.386 \mathrm{E}+01 \\
5.981 \mathrm{E}+01 \\
6.575 \mathrm{E}+01 \\
7.171 \mathrm{E}+01 \\
8.361 \mathrm{E}+01 \\
9.553 \mathrm{E}+01 \\
1.075 \mathrm{E}+02\end{array}$ & $\begin{array}{l}3.381 \mathrm{E}+01 \\
3.469 \mathrm{E}+01 \\
3.549 \mathrm{E}+01 \\
3.622 \mathrm{E}+01 \\
3.751 \mathrm{E}+01 \\
3.863 \mathrm{E}+01 \\
3.961 \mathrm{E}+01\end{array}$ & & $\begin{array}{l}7.342 \mathrm{E}+00 \\
7.545 \mathrm{E}+00 \\
7.729 \mathrm{E}+00 \\
7.898 \mathrm{E}+00 \\
8.198 \mathrm{E}+00 \\
8.459 \mathrm{E}+00 \\
8.690 \mathrm{E}+00\end{array}$ & & & \\
\hline & $E+00$ & $.177 E+02$ & $1.194 E+02$ & $50 E+01$ & $389 \mathrm{E}-01$ & $.897 E+00$ & -0.001 & 0.013 & 0.001 \\
\hline
\end{tabular}


ELECTRONS IN ETHYLENE

$I=50.7 \mathrm{eV} \quad$ DENSITY $=1.175 \mathrm{E}-03 \mathrm{~g} / \mathrm{cm}^{3}\left(20^{\circ} \mathrm{C}\right)$

\begin{tabular}{|c|c|c|c|c|c|c|c|c|c|}
\hline \multirow{2}{*}{$\begin{array}{c}\text { ENERGY } \\
\mathrm{MeV}\end{array}$} & \multicolumn{3}{|c|}{$\begin{array}{l}\text { STOPPING POWER } \\
\text { ON RADIATIVE TOTAL }\end{array}$} & \multirow{2}{*}{$\begin{array}{l}\text { CSDA } \\
\text { RANGE } \\
\mathrm{g} / \mathrm{cm}^{2}\end{array}$} & \multirow[t]{2}{*}{$\begin{array}{l}\text { RADIATION } \\
\text { YIELD }\end{array}$} & \multirow[t]{2}{*}{$\begin{array}{c}\text { DENS.EFF. } \\
\text { CORR. } \\
\text { (DELTA) }\end{array}$} & \multirow[t]{2}{*}{$\begin{array}{l}\quad d(10 \\
\text { coll } \\
\text { LOSS }\end{array}$} & \multirow[t]{2}{*}{$\begin{array}{l}\text { CSDA } \\
\text { RAHGE }\end{array}$} & \multirow[t]{2}{*}{$\begin{array}{l}\circ g \text { I ) } \\
\text { RAD } \\
\text { YIELD }\end{array}$} \\
\hline & $\mathrm{MeV} \mathrm{cm}^{2} / \mathrm{g}$ & $\mathrm{MeV} \mathrm{cm}^{2} / \mathrm{g}$ & $\mathrm{MeV} \mathrm{cm}^{2} / \mathrm{g}$ & & & & & & \\
\hline $\begin{array}{l}0.0100 \\
0.0125 \\
0.0150 \\
0.0175 \\
0.0200 \\
0.0250 \\
0.0300 \\
0.0350\end{array}$ & $\begin{array}{l}2.499 E+01 \\
2.095 E+01 \\
1.814 E+01 \\
1.607 E+01 \\
1.447 E+01 \\
1.215 E+01 \\
1.056 E+01 \\
9.382 E+00\end{array}$ & $\begin{array}{l}2.837 \mathrm{E}-03 \\
2.847 \mathrm{E}-03 \\
2.854 \mathrm{E}-03 \\
2.860 \mathrm{E}-03 \\
2.864 \mathrm{E}-03 \\
2.873 \mathrm{E}-03 \\
2.883 \mathrm{E}-03 \\
2.894 \mathrm{E}-03\end{array}$ & $\begin{array}{l}2.0 \\
1.8 \\
1.6 \\
1.5 \\
1.2 \\
1.0 \\
9.3\end{array}$ & $\begin{array}{l}2.24 \\
3.34 \\
4.63 \\
6.09 \\
7.74 \\
1.15 \\
1.59 \\
2.05\end{array}$ & $\begin{array}{l}6.226 \mathrm{E}-05 \\
7.476 \mathrm{E}-05 \\
8.675 \mathrm{E}-05 \\
9.831 \mathrm{E}-05 \\
1.095 \mathrm{E}-04 \\
1.311 \mathrm{E}-04 \\
1.517 \mathrm{E}-04 \\
1.716 \mathrm{E}-04\end{array}$ & $\begin{array}{l}0.0 \\
0.0 \\
0.0 \\
0.0 \\
0.0 \\
0.0 \\
0.0 \\
0.0\end{array}$ & $\begin{array}{l}-0.184 \\
-0.177 \\
-0.172 \\
-0.167 \\
-0.164 \\
-0.158 \\
-0.154 \\
-0.151\end{array}$ & $\begin{array}{l}0.208 \\
0.199 \\
0.192 \\
0.187 \\
0.182 \\
0.175 \\
0.170 \\
0.166\end{array}$ & $\begin{array}{l}0.207 \\
0.198 \\
0.191 \\
0.186 \\
0.182 \\
0.175 \\
0.170 \\
0.165\end{array}$ \\
\hline $\begin{array}{l}0.0400 \\
0.0450 \\
0.0500 \\
0.0550 \\
0.0600 \\
0.0700 \\
0.0800 \\
0.0900\end{array}$ & $\begin{array}{l}8.482 E+00 \\
7.768 E+00 \\
7.188 E+00 \\
6.707 E+00 \\
6.301 E+00 \\
5.654 E+00 \\
5.160 E+00 \\
4.771 E+00\end{array}$ & $\begin{array}{l}2.905 \mathrm{E}-03 \\
2.918 \mathrm{E}-03 \\
2.931 \mathrm{E}-03 \\
2.945 \mathrm{E}-03 \\
2.960 \mathrm{E}-03 \\
2.992 \mathrm{E}-03 \\
3.025 \mathrm{E}-03 \\
3.061 \mathrm{E}-03\end{array}$ & $\begin{array}{l}8.485 \mathrm{E}+00 \\
7.771 \mathrm{E}+00 \\
7.191 \mathrm{E}+00 \\
6.710 \mathrm{E}+00 \\
6.304 \mathrm{E}+00 \\
5.657 \mathrm{E}+00 \\
5.163 \mathrm{E}+00 \\
4.774 \mathrm{E}+00\end{array}$ & $\begin{array}{l}2.660 \mathrm{E}-03 \\
3.277 \mathrm{E}-03 \\
3.946 \mathrm{E}-03 \\
4.667 \mathrm{E}-03 \\
5.436 \mathrm{E}-03 \\
7.114 \mathrm{E}-03 \\
8.967 \mathrm{E}-03 \\
1.098 \mathrm{E}-02\end{array}$ & $\begin{array}{l}1.908 \mathrm{E}-04 \\
2.095 \mathrm{E}-04 \\
2.277 \mathrm{E}-04 \\
2.455 \mathrm{E}-04 \\
2.629 \mathrm{E}-04 \\
2.967 \mathrm{E}-04 \\
3.293 \mathrm{E}-04 \\
3.609 \mathrm{E}-04\end{array}$ & $\begin{array}{l}0.0 \\
0.0 \\
0.0 \\
0.0 \\
0.0 \\
0.0 \\
0.0 \\
0.0\end{array}$ & $\begin{array}{l}-0.148 \\
-0.145 \\
-0.143 \\
-0.141 \\
-0.140 \\
-0.137 \\
-0.135 \\
-0.133\end{array}$ & $\begin{array}{l}0.162 \\
0.159 \\
0.157 \\
0.154 \\
0.152 \\
0.149 \\
0.146 \\
0.144\end{array}$ & $\begin{array}{l}0.162 \\
0.159 \\
0.156 \\
0.154 \\
0.152 \\
0.149 \\
0.146 \\
0.144\end{array}$ \\
\hline $\begin{array}{l}0.1000 \\
0.1250 \\
0.1500 \\
0.1750 \\
0.2000 \\
0.2500 \\
0.3000 \\
0.3500\end{array}$ & $\begin{array}{l}4.457 E+00 \\
3.884 E+00 \\
3.497 E+00 \\
3.220 E+00 \\
3.012 E+00 \\
2.723 E+00 \\
2.534 E+00 \\
2.402 E+00\end{array}$ & $\begin{array}{l}3.099 \mathrm{E}-03 \\
3.201 \mathrm{E}-03 \\
3.312 \mathrm{E}-03 \\
3.429 \mathrm{E}-03 \\
3.553 \mathrm{E}-03 \\
3.820 \mathrm{E}-03 \\
4.110 \mathrm{E}-03 \\
4.420 \mathrm{E}-03\end{array}$ & $\begin{array}{l}4.460 \mathrm{E}+00 \\
3.887 \mathrm{E}+00 \\
3.501 \mathrm{E}+00 \\
3.223 \mathrm{E}+00 \\
3.015 \mathrm{E}+00 \\
2.727 \mathrm{E}+00 \\
2.538 \mathrm{E}+00 \\
2.407 \mathrm{E}+00\end{array}$ & $\begin{array}{l}1.315 \mathrm{E}-02 \\
1.918 \mathrm{E}-02 \\
2.598 \mathrm{E}-02 \\
3.343 \mathrm{E}-02 \\
4.146 \mathrm{E}-02 \\
5.896 \mathrm{E}-02 \\
7.801 \mathrm{E}-02 \\
9.827 \mathrm{E}-02\end{array}$ & $\begin{array}{l}3.916 \mathrm{E}-04 \\
4.652 \mathrm{E}-04 \\
5.352 \mathrm{E}-04 \\
6.024 \mathrm{E}-04 \\
6.672 \mathrm{E}-04 \\
7.918 \mathrm{E}-04 \\
9.116 \mathrm{E}-04 \\
1.028 \mathrm{E}-03\end{array}$ & $\begin{array}{l}0.0 \\
0.0 \\
0.0 \\
0.0 \\
0.0 \\
0.0 \\
0.0 \\
0.0\end{array}$ & $\begin{array}{l}-0.131 \\
-0.1127 \\
-0.125 \\
-0.122 \\
-0.120 \\
-0.117 \\
-0.115 \\
-0.113\end{array}$ & $\begin{array}{l}0.142 \\
0.138 \\
0.135 \\
0.132 \\
0.130 \\
0.127 \\
0.124 \\
0.122\end{array}$ & $\begin{array}{l}0.142 \\
0.137 \\
0.134 \\
0.132 \\
0.129 \\
0.126 \\
0.123 \\
0.121\end{array}$ \\
\hline $\begin{array}{l}0.4000 \\
0.4500 \\
0.5000 \\
0.5500 \\
0.6000 \\
0.7000 \\
0.8000 \\
0.9000\end{array}$ & $\begin{array}{l}2.307 E+00 \\
2.236 E+00 \\
2.182 E+00 \\
2.141 E+00 \\
2.108 E+00 \\
2.061 E+00 \\
2.032 E+00 \\
2.013 E+00\end{array}$ & $\begin{array}{l}4.750 \mathrm{E}-03 \\
5.098 \mathrm{E}-03 \\
5.462 \mathrm{E}-03 \\
5.841 \mathrm{E}-03 \\
6.233 \mathrm{E}-03 \\
7.053 \mathrm{E}-03 \\
7.915 \mathrm{E}-03 \\
8.816 \mathrm{E}-03\end{array}$ & $\begin{array}{l}2.312 \mathrm{E}+00 \\
2.241 \mathrm{E}+00 \\
2.188 \mathrm{E}+00 \\
2.146 \mathrm{E}+00 \\
2.114 \mathrm{E}+00 \\
2.068 \mathrm{E}+00 \\
2.040 \mathrm{E}+00 \\
2.022 \mathrm{E}+00\end{array}$ & $\begin{array}{l}1.195 \mathrm{E}-01 \\
1.415 \mathrm{E}-01 \\
1.641 \mathrm{E}-01 \\
1.871 \mathrm{E}-01 \\
2.106 \mathrm{E}-01 \\
2.585 \mathrm{E}-01 \\
3.072 \mathrm{E}-01 \\
3.565 \mathrm{E}-01\end{array}$ & $\begin{array}{l}1.143 \mathrm{E}-03 \\
1.256 \mathrm{E}-03 \\
1.369 \mathrm{E}-03 \\
1.482 \mathrm{E}-03 \\
1.595 \mathrm{E}-03 \\
1.821 \mathrm{E}-03 \\
2.049 \mathrm{E}-03 \\
2.279 \mathrm{E}-03\end{array}$ & $\begin{array}{l}0.0 \\
0.0 \\
0.0 \\
0.0 \\
0.0 \\
0.0 \\
0.0 \\
0.0\end{array}$ & $\begin{array}{l}-0.111 \\
-0.109 \\
-0.108 \\
-0.107 \\
-0.105 \\
-0.103 \\
-0.1102 \\
-0.100\end{array}$ & & $\begin{array}{l}0.1119 \\
0.1117 \\
0.1116 \\
0.1114 \\
0.1113 \\
0.1111 \\
0.1109 \\
0.107\end{array}$ \\
\hline $\begin{array}{l}1.0000 \\
1.2500 \\
1.5000 \\
1.7500 \\
2.0000 \\
2.5000 \\
3.0000 \\
3.5000\end{array}$ & $\begin{array}{l}2.002 E+00 \\
1.992 E+00 \\
1.996 E+00 \\
2.007 E+00 \\
2.021 E+00 \\
2.051 E+00 \\
2.082 E+00 \\
2.111 E+00\end{array}$ & $\begin{array}{l}9.754 \mathrm{E}-03 \\
1.224 \mathrm{E}-02 \\
1.490 \mathrm{E}-02 \\
1.770 \mathrm{E}-02 \\
2.062 \mathrm{E}-02 \\
2.678 \mathrm{E}-02 \\
3.327 \mathrm{E}-02 \\
4.004 \mathrm{E}-02\end{array}$ & $\begin{array}{l}2.011 E+00 \\
2.004 E+00 \\
2.011 E+00 \\
2.024 E+00 \\
2.041 E+00 \\
2.078 E+00 \\
2.115 E+00 \\
2.151 E+00\end{array}$ & $\begin{array}{l}4.061 \mathrm{E}-01 \\
5.307 \mathrm{E}-01 \\
6.553 \mathrm{E}-01 \\
7.792 \mathrm{E}-01 \\
9.022 \mathrm{E}-01 \\
1.145 \mathrm{E}+00 \\
1.383 \mathrm{E}+00 \\
1.618 \mathrm{E}+00\end{array}$ & $\begin{array}{l}2.511 \mathrm{E}-03 \\
3.104 \mathrm{E}-03 \\
3.712 \mathrm{E}-03 \\
4.335 \mathrm{E}-03 \\
4.971 \mathrm{E}-03 \\
6.275 \mathrm{E}-03 \\
7.613 \mathrm{E}-03 \\
8.977 \mathrm{E}-03\end{array}$ & $\begin{array}{l}0.0 \\
0.0 \\
0.0 \\
0.0 \\
0.0 \\
0.0 \\
0.0 \\
0.0\end{array}$ & $\begin{array}{l}-0.099 \\
-0.096 \\
-0.094 \\
-0.092 \\
-0.091 \\
-0.088\end{array}$ & & $\begin{array}{l}0.106 \\
0.103 \\
0.100 \\
0.098 \\
0.096 \\
0.093 \\
0.091 \\
0.089\end{array}$ \\
\hline $\begin{array}{l}4.0000 \\
4.5000 \\
5.0000 \\
5.5000 \\
6.0000 \\
7.0000 \\
8.0000 \\
9.0000\end{array}$ & $\begin{array}{l}2.16 \\
2.18 \\
2.20 \\
2.22 \\
2.26 \\
2.29 \\
2.32\end{array}$ & $\begin{array}{l}5.4 \\
6.1 \\
6.9 \\
7.6 \\
9.2 \\
1.0 \\
1.2\end{array}$ & $\begin{array}{l}2.218 \mathrm{E} \\
2.249 \mathrm{E} \\
2.278 \mathrm{E} \\
2.306 \mathrm{E} \\
2.358 \mathrm{E} \\
2.407 \mathrm{E} \\
2.453 \mathrm{E}\end{array}$ & $\begin{array}{l}1.84 \\
2.07 \\
2.29 \\
2.52 \\
2.73 \\
3.16 \\
3.58 \\
3.99\end{array}$ & $\begin{array}{l}1.036 \mathrm{E}-02 \\
1.177 \mathrm{E}-02 \\
1.318 \mathrm{E}-02 \\
1.461 \mathrm{E}-02 \\
1.604 \mathrm{E}-02 \\
1.894 \mathrm{E}-02 \\
2.185 \mathrm{E}-02 \\
2.477 \mathrm{E}-02\end{array}$ & $\begin{array}{l}0.0 \\
0.0 \\
0.0 \\
0.0 \\
0.0 \\
0.0 \\
0.0 \\
0.0\end{array}$ & $\begin{array}{l}-0.0 \\
-0.0 \\
-0.0 \\
-0.0 \\
-0.0 \\
-0.0 \\
-0.0 \\
-0.0\end{array}$ & $\begin{array}{l}91 \\
90 \\
39 \\
38 \\
36 \\
35 \\
34\end{array}$ & $\begin{array}{l}0.087 \\
0.086 \\
0.085 \\
0.083 \\
0.082 \\
0.080 \\
0.079 \\
0.077\end{array}$ \\
\hline $\begin{array}{l}10.0000 \\
12.5000 \\
15.0000 \\
17.5000 \\
20.0000 \\
25.0000 \\
30.0000 \\
35.0000\end{array}$ & $\begin{array}{l}2.354 \mathrm{E}+00 \\
2.410 \mathrm{E}+00 \\
2.456 \mathrm{E}+00 \\
2.496 \mathrm{E}+00 \\
2.529 \mathrm{E}+00 \\
2.577 \mathrm{E}+00 \\
2.612 \mathrm{E}+00 \\
2.639 \mathrm{E}+00\end{array}$ & $\begin{array}{l}1.85 \\
2.30 \\
2.75 \\
3.22 \\
4.16 \\
5.12 \\
6.10\end{array}$ & $\begin{array}{l}2.5 \\
2.6 \\
2.7 \\
2.8 \\
2.9\end{array}$ & $\begin{array}{l}4.402 E+00 \\
5.384 E+00 \\
6.331 E+00 \\
7.247 \mathrm{E}+00 \\
8.136 \mathrm{E}+00 \\
9.846 \mathrm{E}+00 \\
1.148 \mathrm{E}+01 \\
1.305 \mathrm{E}+01\end{array}$ & $\begin{array}{l}2.770 \mathrm{E}-02 \\
3.500 \mathrm{E}-02 \\
4.227 \mathrm{E}-02 \\
4.945 \mathrm{E}-02 \\
5.655 \mathrm{E}-02 \\
7.047 \mathrm{E}-02 \\
8.401 \mathrm{E}-02 \\
9.717 \mathrm{E}-02\end{array}$ & $\begin{array}{l}0.0 \\
0.0 \\
0.0 \\
0.0 \\
1.778 \mathrm{E}-02 \\
1.288 \mathrm{E}-01 \\
2.760 \mathrm{E}-01 \\
4.292 \mathrm{E}-01\end{array}$ & $\begin{array}{l}-0.075 \\
-0.073 \\
-0.071 \\
-0.070 \\
-0.059 \\
-0.044 \\
-0.036 \\
-0.031\end{array}$ & $\begin{array}{l}0.082 \\
0.080 \\
0.078 \\
0.076 \\
0.074 \\
0.069 \\
0.064 \\
0.060\end{array}$ & $\begin{array}{l}0.076 \\
0.074 \\
0.077 \\
0.069 \\
0.067 \\
0.059 \\
0.051 \\
0.045\end{array}$ \\
\hline $\begin{array}{l}45.0000 \\
50.0000 \\
55.0000 \\
60.0000 \\
70.00000 \\
80.0000 \\
90.0000\end{array}$ & $\begin{array}{l}2.6 \\
2.6 \\
2.7 \\
2.7 \\
2.7 \\
2.7\end{array}$ & $\begin{array}{l}8.088 \\
9.092 \\
1.019 \\
1.112 \\
1.316 \\
1.522 \\
1.730\end{array}$ & $\begin{array}{l}3 . \\
3 . \\
3 . \\
3 . \\
3 . \\
4 . \\
4 .\end{array}$ & $\begin{array}{l}1 \\
1 \\
1 \\
1 \\
2 \\
2 \\
2\end{array}$ & & & $\begin{array}{l}-0.027 \\
-0.025 \\
-0.023 \\
-0.022 \\
-0.021 \\
-0.019 \\
-0.018 \\
-0.017\end{array}$ & & $\begin{array}{l}0.040 \\
0.036 \\
0.033 \\
0.030 \\
0.028 \\
0.024 \\
0.022 \\
0.020\end{array}$ \\
\hline $\begin{array}{l}5.00000 \\
0.00000 \\
5.00000 \\
0.00000 \\
0.00000\end{array}$ & $\begin{array}{l}2.82 \\
2.84 \\
2.86 \\
2.88 \\
2.90\end{array}$ & $\begin{array}{l}2.9 \\
2.9 \\
3.5 \\
5.0 \\
5.1\end{array}$ & $\begin{array}{l}5.4 \\
5.8 \\
6.9 \\
8.0\end{array}$ & $\begin{array}{l}1 \\
1 \\
1 \\
1 \\
1 \\
1 \\
1\end{array}$ & & & $\begin{array}{l}-0 . \\
-0 \\
-0 \\
-0 \\
-0 \\
-0 \\
-0 \\
-0 .\end{array}$ & $\begin{array}{l}0 . \\
0 \\
0 \\
0 \\
0 . \\
0 . \\
0 .\end{array}$ & $\begin{array}{l}0.018 \\
0.015 \\
0.013 \\
0.012 \\
0.011 \\
0.009 \\
0.008 \\
0.007\end{array}$ \\
\hline $\begin{array}{l}.0000 \\
.0000 \\
.0000 \\
.0000 \\
.0000 \\
.0000 \\
.0000\end{array}$ & $\begin{array}{l}2.974 \mathrm{E}+00 \\
2.985 \mathrm{E}+00 \\
2.995 \mathrm{E}+00 \\
3.004 \mathrm{E}+00 \\
3.019 \mathrm{E}+00 \\
3.031 \mathrm{E}+00 \\
3.042 \mathrm{E}+00\end{array}$ & $\begin{array}{l}165 E+01 \\
75 E+01 \\
+94 E+01 \\
13 E+01 \\
33 E+01\end{array}$ & $\begin{array}{l}E+01 \\
E+01 \\
E+01 \\
E+01 \\
E+01 \\
E+01 \\
E+01\end{array}$ & $\begin{array}{l}336 E+01 \\
721 E+01 \\
076 E+01 \\
405 E+01 \\
000 E+01 \\
525 E+01 \\
995 E+01\end{array}$ & $\begin{array}{l}5.479 \mathrm{E}-01 \\
5.702 \mathrm{E}-01 \\
5.900 \mathrm{E}-01 \\
6.077 \mathrm{E}-01 \\
6.382 \mathrm{E}-01 \\
6.635 \mathrm{E}-01 \\
6.850 \mathrm{E}-01\end{array}$ & $\begin{array}{l}0 \\
0 \\
0 \\
0 \\
0 \\
0 \\
0\end{array}$ & $\begin{array}{l}-0 \\
-0 \\
-0 \\
-0 \\
-0 \\
-0\end{array}$ & $\begin{array}{l}17 \\
16 \\
15 \\
15 \\
14 \\
3 \\
13\end{array}$ & $\begin{array}{l}0.006 \\
0.005 \\
0.005 \\
0.004 \\
0.004 \\
0.003 \\
0.003 \\
0.002\end{array}$ \\
\hline 0.0000 & $3.052 E+00$ & $.153 E+01$ & $58 \mathrm{E}+01$ & $42 E+02$ & $035 \mathrm{E}-$ & $.751 \mathrm{E}+00$ & 0.0 & .012 & .002 \\
\hline
\end{tabular}




\begin{tabular}{|c|c|c|c|c|c|c|c|c|c|}
\hline NERGY & COLLISION & $\begin{array}{l}\text { OPP ING POWE } \\
\text { RADIATIVE }\end{array}$ & TOTAL & $\begin{array}{l}\text { CSDA } \\
\text { RAHGE }\end{array}$ & סN & $\begin{array}{l}\text { DENS.EFF. } \\
\text { CORR. } \\
\text { (DELTA) }\end{array}$ & $\begin{array}{l}\operatorname{dol}(1 \\
\operatorname{coLL} \\
\text { LOSS }\end{array}$ & 9 CSD & RA \\
\hline $\mathrm{MeV}$ & $\mathrm{MeV} \mathrm{cm}^{2} / \mathrm{g}$ & $\mathrm{MeV} \mathrm{cm} 2 / \mathrm{g}$ & $\mathrm{MeV} \mathrm{cm} 2 / \mathrm{g}$ & $\mathrm{g} / \mathrm{cm}^{2}$ & & & & & \\
\hline $\begin{array}{l}0.0125 \\
0.0150 \\
0.0175 \\
0.0200 \\
0.0250 \\
0.0300 \\
0.0350\end{array}$ & $\begin{array}{l}1.88 \\
1.63 \\
1.45 \\
1.30 \\
1.10 \\
9.59 \\
8.54\end{array}$ & $\begin{array}{l}4.0 \\
4.0 \\
4.0 \\
4.0 \\
4.0 \\
4.0\end{array}$ & $\begin{array}{l}1.6 \\
1.49 \\
1.35 \\
1.10 \\
9.5 \\
8.5\end{array}$ & $\begin{array}{l}4 \\
4 \\
4 \\
4 \\
4 \\
3 \\
3 \\
3\end{array}$ & $\begin{array}{l}9.615 \mathrm{E}-05 \\
1.158 \mathrm{E}-04 \\
1.346 \mathrm{E}-04 \\
1.527 \mathrm{E}-04 \\
1.702 \mathrm{E}-04 \\
2.037 \mathrm{E}-04 \\
2.356 \mathrm{E}-04 \\
2.662 \mathrm{E}-04\end{array}$ & $\begin{array}{l}0.0 \\
0.0 \\
0.0 \\
0.0 \\
0.0 \\
0.0 \\
0.0 \\
0.0\end{array}$ & $\begin{array}{l}-0.200 \\
-0.191 \\
-0.185 \\
-0.180 \\
-0.176 \\
-0.169 \\
-0.164 \\
-0.160\end{array}$ & $\begin{array}{l}0.228 \\
0.217 \\
0.209 \\
0.203 \\
0.197 \\
0.189 \\
0.183 \\
0.178\end{array}$ & $\begin{array}{l}0.226 \\
0.216 \\
0.208 \\
0.202\end{array}$ \\
\hline $\begin{array}{l}0.0400 \\
0.0450 \\
0.0500 \\
0.0550 \\
0.0600 \\
0.0700 \\
0.0800\end{array}$ & $\begin{array}{l}7.73 \\
7.08 \\
6.56 \\
6.12 \\
5.76 \\
5.17 \\
4.73 \\
4.37\end{array}$ & $\begin{array}{l}4.08 \\
4.09 \\
4.10 \\
4.12 \\
4.10 \\
4.17 \\
4.21 \\
4.26\end{array}$ & $\begin{array}{l}7.734 \\
7.099 \\
6.568 \\
6.134 \\
5.767 \\
5.181 \\
4.734 \\
4.381\end{array}$ & $\begin{array}{l}2.9 \\
3.6 \\
4.3 \\
5.1 \\
5.9 \\
7.8\end{array}$ & $\begin{array}{l}\text { E- } 04 \\
E-04 \\
E-04 \\
E-04 \\
E-04 \\
E-04 \\
E-04 \\
E-04\end{array}$ & $\begin{array}{l}0.0 \\
0.0 \\
0.0 \\
0.0 \\
0.0 \\
0.0 \\
0.0 \\
0.0\end{array}$ & $\begin{array}{l}-0.157 \\
-0.154 \\
-0.152 \\
-0.150 \\
-0.148 \\
-0.145 \\
-0.142 \\
-0.140\end{array}$ & $\begin{array}{l}0.100 \\
0.165 \\
0.163 \\
0.159 \\
0.156 \\
0.153\end{array}$ & \\
\hline $\begin{array}{l}0.1000 \\
0.1250 \\
0.1500 \\
0.1750 \\
0.2000 \\
0.2500 \\
0.3000 \\
0.3500\end{array}$ & $\begin{array}{l}4.092 E+00 \\
3.571 E+00 \\
3.220 E+00 \\
2.967 E+00 \\
2.778 E+00 \\
2.514 E+00 \\
2.342 E+00 \\
2.223 E+00\end{array}$ & $\begin{array}{l}4.307 \mathrm{E}-03 \\
4.436 \mathrm{E}-03 \\
4.577 \mathrm{E}-03 \\
4.728 \mathrm{E}-03 \\
4.888 \mathrm{E}-03 \\
5.233 \mathrm{E}-03 \\
5.611 \mathrm{E}-03 \\
6.017 \mathrm{E}-03\end{array}$ & $\begin{array}{l}4.096 E+00 \\
3.576 E+00 \\
3.224 E+00 \\
2.972 E+00 \\
2.783 E+00 \\
2.520 E+00 \\
2.348 E+00 \\
2.229 E+00\end{array}$ & $\begin{array}{l}1.439 \mathrm{E}-02 \\
2.095 \mathrm{E}-02 \\
2.833 \mathrm{E}-02 \\
3.643 \mathrm{E}-02 \\
4.513 \mathrm{E}-02 \\
6.408 \mathrm{E}-02 \\
8.468 \mathrm{E}-02\end{array}$ & $\begin{array}{l}E-04 \\
E-04 \\
E-04 \\
E-04 \\
E-03 \\
E-03 \\
E-03 \\
2 E-03\end{array}$ & $\begin{array}{l}0.0 \\
0.0 \\
0.0 \\
0.0 \\
0.0 \\
0.0 \\
0.0 \\
0.0\end{array}$ & $\begin{array}{l}-0.138 \\
-0.134 \\
-0.131 \\
-0.129 \\
-0.127 \\
-0.123 \\
-0.120 \\
-0.118\end{array}$ & $\begin{array}{l}0.151 \\
0.146 \\
0.143 \\
0.140 \\
0.137 \\
0.134 \\
0.131 \\
0.128\end{array}$ & 15 \\
\hline $\begin{array}{l}0.4000 \\
0.4500 \\
0.5000 \\
0.5500 \\
0.6000 \\
0.70000 \\
0.8000 \\
0.9000\end{array}$ & $\begin{array}{l}2.0 \\
1: 9 \\
1: 9 \\
1: 9 \\
1: 8 \\
1.8\end{array}$ & $\begin{array}{l}7.90 \\
7.38 \\
7.87 \\
8.39 \\
9.46 \\
1.06 \\
1.17\end{array}$ & $\begin{array}{l}2.1 \\
2.0 \\
2.0 \\
1.9 \\
1.9 \\
1.9 \\
1.8 \\
1.8\end{array}$ & $\begin{array}{l}2.0 \\
2.2 \\
2.7 \\
3.3 \\
3.8\end{array}$ & $\begin{array}{l}-03 \\
-03 \\
-03 \\
-03 \\
-03 \\
-03 \\
-03 \\
-03\end{array}$ & $\begin{array}{l}0.0 \\
0.0 \\
0.0 \\
1.262 \mathrm{E}-0 \\
3.136 \mathrm{E}-0 \\
7.687 \mathrm{E}-0 \\
1.295 \mathrm{E}-0 \\
1.864 \mathrm{E}-0\end{array}$ & $\begin{array}{l}-0.116 \\
-0.114 \\
-0.110 \\
-0.094 \\
-0.088 \\
-0.078 \\
-0.070 \\
-0.064\end{array}$ & $\begin{array}{l}0.12 \\
0.12 \\
0.11\end{array}$ & \\
\hline $\begin{array}{l}1.0000 \\
1.2500 \\
1.5000 \\
1.7500 \\
2.0000 \\
2.5000 \\
3.0000 \\
3.5000\end{array}$ & $\begin{array}{l}1.8 \\
1.8 \\
1.8 \\
1.8 \\
1.8\end{array}$ & $\begin{array}{l}1.3 \\
1.6 \\
1.9 \\
2.3 \\
2.7 \\
3.5 \\
4.3 \\
5.2\end{array}$ & $\begin{array}{l}1.8 \\
1.8 \\
1.8 \\
1.8 \\
1.8 \\
1.8 \\
1.8\end{array}$ & $\begin{array}{l}1 \\
1 \\
0\end{array}$ & $\begin{array}{l}3 \\
3 \\
3 \\
3 \\
2 \\
2\end{array}$ & $\begin{array}{l}6.8 \\
8.2 \\
1.0 \\
1.2 \\
1.2\end{array}$ & $\begin{array}{l}-0.059 \\
-0.055 \\
-0.043 \\
-0.039 \\
-0.035 \\
-0.031 \\
-0.028 \\
-0.026\end{array}$ & $\begin{array}{l}.067 \\
.059 \\
.054 \\
.050\end{array}$ & \\
\hline $\begin{array}{l}4.0000 \\
4.5000 \\
5.0000 \\
5.5000 \\
6.0000 \\
7.0000 \\
8.0000 \\
9.0000\end{array}$ & $\begin{array}{l}1.8 \\
1.89 \\
1.9 \\
1.9 \\
1.9\end{array}$ & & $\begin{array}{l}1.923 E+00 \\
1.944 E+00 \\
1.964 E+00 \\
1.983 E+00 \\
2.002 E+00 \\
2.040 E+00 \\
2.075 E+00 \\
2.110 E+00\end{array}$ & & $\begin{array}{l}E-02 \\
E-02 \\
E-02 \\
E-02 \\
E-02 \\
E-02 \\
E-02 \\
E-02\end{array}$ & $\begin{array}{l}1.657 \mathrm{E}+00 \\
1.816 \mathrm{E}+00 \\
1.962 \mathrm{E}+00 \\
2.096 \mathrm{E}+00 \\
2.220 \mathrm{E}+00 \\
2.445 \mathrm{E}+00 \\
2.643 \mathrm{E}+00 \\
2.821 \mathrm{E}+00\end{array}$ & $\begin{array}{l}-0.024 \\
-0.023 \\
-0.0222 \\
-0.0222 \\
-0.021 \\
-0.020 \\
-0.019 \\
-0.018\end{array}$ & $\begin{array}{l}2 \\
0 \\
8\end{array}$ & \\
\hline $\begin{array}{l}15.00 \\
17.50 \\
20.00 \\
25.00 \\
30.00 \\
35.00\end{array}$ & & $\begin{array}{l}5.3 \\
6.5 \\
7.8\end{array}$ & $\begin{array}{l}2 . \\
2 . \\
2 . \\
2 .\end{array}$ & & $\begin{array}{l}02 \\
02 \\
02 \\
02 \\
02 \\
01 \\
01\end{array}$ & & $\begin{array}{l}-0.018 \\
-0.016 \\
-0.015 \\
-0.014 \\
-0.013 \\
-0.010 \\
-00.009 \\
-0.007\end{array}$ & $\begin{array}{l}.025 \\
.024 \\
.022 \\
.020 \\
.018 \\
.017\end{array}$ & \\
\hline $\begin{array}{l}45.0 \\
50.0 \\
55.0 \\
60.0\end{array}$ & $\begin{array}{l}2.130 \\
2.139 \\
2.147 \\
2.161 \\
2.173 \\
2.184\end{array}$ & & $\begin{array}{l}3.0 \\
3: 1 \\
3: 2 \\
3: 4 \\
3.5 \\
3: 8 \\
4: 1 \\
4.3\end{array}$ & & $\begin{array}{l}1.7 \\
1.9 \\
2.1 \\
2.2 \\
2.5\end{array}$ & & & & \\
\hline 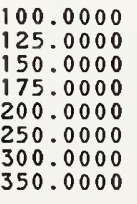 & $\begin{array}{l}2 . \\
2 . \\
2 . \\
2 . \\
2 . \\
2 . \\
2 .\end{array}$ & & $\frac{6}{7}$ & & & & & & \\
\hline $\begin{array}{l}b_{0} \\
b \\
0 \\
b \\
0 \\
0\end{array}$ & $\begin{array}{l}2.323 \mathrm{E} \\
2.3332 \mathrm{E} \\
2.340 \mathrm{E} \\
2.347 \mathrm{E} \\
2.361 \mathrm{E} \\
2.372 \mathrm{E} \\
2.382 \mathrm{E}\end{array}$ & $\begin{array}{l}1 \\
1 \\
1 \\
1 \\
2 .\end{array}$ & $4 E+01$ & $0 \mathrm{E}+01$ & $\begin{array}{l}.444 \mathrm{E}-01 \\
.644 \mathrm{E}-01 \\
.819 \mathrm{E}-01 \\
.974 \mathrm{E}-01 \\
.236 \mathrm{E}-01 \\
.451 \mathrm{E}-01 \\
.630 \mathrm{E}-01\end{array}$ & $\begin{array}{l}.026 \mathrm{E}+01 \\
.045 \mathrm{E}+01 \\
.062 \mathrm{E}+01 \\
.093 \mathrm{E}+01 \\
.120 \mathrm{E}+01 \\
.143 \mathrm{E}+01\end{array}$ & $\begin{array}{l}-0.000 \\
-0.000 \\
-0.000 \\
-0.000 \\
-0.000 \\
-0.000 \\
-0.000 \\
-0.000\end{array}$ & $\begin{array}{l}04 \\
04 \\
04 \\
04 \\
03 \\
03 \\
03 \\
03\end{array}$ & \\
\hline & $91 E+00$ & $713 E+01$ & $2 E+01$ & $56 E+01$ & $E-01$ & $44 E+01$ & & & \\
\hline
\end{tabular}




\begin{tabular}{|c|c|c|c|c|c|c|c|c|c|}
\hline ;Y & OLLISIOI & T AT TUE & TOTAL & A & N & $F$. & $\begin{array}{l}d(I \\
\text { COLL } \\
\text { LOSS }\end{array}$ & $\begin{array}{l}\text { ) /d ( } 1 \\
\text { CSDA } \\
\text { RANGE }\end{array}$ & $\begin{array}{l}\text { I) } \\
\text { RAD } \\
\text { YIELD }\end{array}$ \\
\hline $\mathrm{MeV}$ & $\mathrm{MeV} \mathrm{cm}^{2} / \mathrm{g}$ & $\mathrm{MeV} \mathrm{cm}^{2} / \mathrm{g}$ & $\mathrm{MeV} \mathrm{cm}^{2} / \mathrm{g}$ & $\mathrm{g} / \mathrm{cm}^{2}$ & & & & & \\
\hline $\begin{array}{l}0.0100 \\
0.0125 \\
0.0150 \\
0.0175 \\
0.0200 \\
0.0250 \\
0.0300 \\
0.0350\end{array}$ & $\begin{array}{ll}0 & 1 \\
0 & 1 \\
0 & 1 \\
0 & 1 \\
0 & 1 \\
0 & 0 \\
0 & 0 \\
0 & 0\end{array}$ & $\begin{array}{l}5.4 \\
5.4 \\
5.5 \\
5.5 \\
5.6 \\
5.6\end{array}$ & $\begin{array}{l}1 . \\
1 . \\
1 . \\
1 . \\
1 . \\
8 . \\
7 . \\
6 .\end{array}$ & $\begin{array}{l}3.2 \\
4.7 \\
6.5 \\
8.5 \\
1.0 \\
1.5 \\
2.1\end{array}$ & $\begin{array}{l}04 \\
04 \\
04 \\
04 \\
04 \\
04 \\
04 \\
04\end{array}$ & $\begin{array}{l}0.0 \\
0.0 \\
0.0 \\
0.0 \\
0.0 \\
0.0 \\
0.0 \\
0.0\end{array}$ & $\begin{array}{l}-0.225 \\
-0.214 \\
-0.206 \\
-0.200 \\
-0.195 \\
-0.187 \\
-0.181 \\
-0.176\end{array}$ & $\begin{array}{l}0.264 \\
0.250 \\
0.239 \\
0.230 \\
0.223 \\
0.213 \\
0.205 \\
0.199\end{array}$ & $\begin{array}{l}0.260 \\
0.246 \\
0.236 \\
0.228 \\
0.221 \\
0.211 \\
0.203 \\
0.197\end{array}$ \\
\hline $\begin{array}{l}0.0400 \\
0.0450 \\
0.0500 \\
0.0550 \\
0.0600 \\
0.0700 \\
0.0800 \\
0.0900\end{array}$ & $\begin{array}{l}6.331 E+00 \\
5.814 E+00 \\
5.393 E+00 \\
5.042 E+00 \\
4.746 E+00 \\
4.272 E+00 \\
3.909 E+00 \\
3.622 E+00\end{array}$ & $\begin{array}{l}5.7 \\
5.8 \\
5.8 \\
5.8 \\
5.8 \\
5.9\end{array}$ & $\begin{array}{l}5 . \\
5 . \\
5 . \\
4 . \\
4 . \\
3 . \\
3 .\end{array}$ & $\begin{array}{l}4.4 \\
5.3 \\
6.3 \\
7.3 \\
9.5 \\
1.1 \\
1.4\end{array}$ & $\begin{array}{l}04 \\
044 \\
044 \\
04 \\
04 \\
04 \\
04 \\
04\end{array}$ & $\begin{array}{l}0.0 \\
0.0 \\
0.0 \\
0.0 \\
0.0 \\
0.0 \\
0.0 \\
0.0\end{array}$ & $\begin{array}{l}-0.1 \\
-0.1 \\
-0.1 \\
-0.1 \\
-0.1 \\
-0.1 \\
-0.1 \\
-0.1\end{array}$ & $\begin{array}{l}0.194 \\
0.189 \\
0.186 \\
0.182 \\
0.180 \\
0.175 \\
0.171 \\
0.168\end{array}$ & $\begin{array}{l}0.192 \\
0.188 \\
0.185 \\
0.182 \\
0.179 \\
0.174 \\
0.170 \\
0.167\end{array}$ \\
\hline $\begin{array}{l}0.1000 \\
0.1250 \\
0.1500 \\
0.1750 \\
0.2000 \\
0.2500 \\
0.3000 \\
0.3500\end{array}$ & $\begin{array}{l}2.96 \\
2.67 \\
2.47 \\
2.31 \\
2.10 \\
1.96 \\
1.86\end{array}$ & $\begin{array}{l}6 \cdot 2 \\
6 \cdot 3 \\
6 \cdot 5 \\
6 \cdot 7 \\
7 \cdot 2 \\
7 \cdot 7 \\
8 \cdot 7\end{array}$ & $\begin{array}{l}2 . \\
2 . \\
2 . \\
2 . \\
2 . \\
1 .\end{array}$ & $\begin{array}{l}2.5 \\
3.4 \\
4.3 \\
5.4 \\
7.7 \\
1.0 \\
1.2\end{array}$ & $\begin{array}{l}-03 \\
-03 \\
-03 \\
-03 \\
-03 \\
-03 \\
-03 \\
-03\end{array}$ & $\begin{array}{l}0.0 \\
0.0 \\
0.0 \\
0.0 \\
0.0 \\
0.0 \\
0.0 \\
0.0\end{array}$ & $\begin{array}{l}-0.1 \\
-0.1 \\
-0.1 \\
-0.1 \\
-0.1 \\
-0.1 \\
-0.1 \\
-0.1\end{array}$ & $\begin{array}{l}0.165 \\
0.159 \\
0.155 \\
0.152 \\
0.149 \\
0.145 \\
0.141 \\
0.138\end{array}$ & $\begin{array}{l}0.164 \\
0.159 \\
0.155 \\
0.151 \\
0.148 \\
0.144 \\
0.140 \\
0.137\end{array}$ \\
\hline $\begin{array}{l}0.4000 \\
0.4500 \\
0.5000 \\
0.5500 \\
0.6000 \\
0.7000 \\
0.8000 \\
0.9000\end{array}$ & $\begin{array}{l}1.793 E+00 \\
1.739 E+00 \\
1.698 E+00 \\
1.665 E+00 \\
1.640 E+00 \\
1.603 E+00 \\
1.579 E+00 \\
1.563 E+00\end{array}$ & $\begin{array}{l}9.4 \\
1.0 \\
1.0 \\
1.1 \\
1.2 \\
1.4 \\
1.5\end{array}$ & $\begin{array}{l}1 . \\
1 . \\
1 . \\
1 . \\
1 . \\
1 .\end{array}$ & $\begin{array}{l}1.8 \\
2.1 \\
2.4 \\
2.7 \\
3.3 \\
3.9 \\
4.5\end{array}$ & $\begin{array}{l}-03 \\
-03 \\
-03 \\
-03 \\
-03 \\
-03 \\
-03 \\
-03\end{array}$ & $\begin{array}{l}8.985 \mathrm{E}-0 \\
2.665 \mathrm{E}-0 \\
4.637 \mathrm{E}-0 \\
6.760 \mathrm{E}-0 \\
8.997 \mathrm{E}-0 \\
1.371 \mathrm{E}-0 \\
1.860 \mathrm{E}-0 \\
2.359 \mathrm{E}-0\end{array}$ & $\begin{array}{l}-0.1 \\
-0.1 \\
-0.1 \\
-0.0 \\
-0.0 \\
-0.0 \\
-0.0 \\
-0.0\end{array}$ & $\begin{array}{l}0.1 \\
0.1 \\
0.1 \\
0.1 \\
0.1\end{array}$ & $\begin{array}{l}0.133 \\
0.128 \\
0.124 \\
0.1119 \\
0.1115 \\
0.1109 \\
0.1103 \\
0.098\end{array}$ \\
\hline $\begin{array}{l}1.0000 \\
1.2500 \\
1.5000 \\
1.7500 \\
2.0000 \\
2.5000 \\
3.0000 \\
3.5000\end{array}$ & $\begin{array}{l}1.552 E+00 \\
1.540 E+00 \\
1.538 E+00 \\
1.541 E+00 \\
1.547 E+00 \\
1.561 E+00 \\
1.576 E+00 \\
1.590 E+00\end{array}$ & $\begin{array}{l}2.1 \\
2.6 \\
3.1 \\
3.6 \\
4.6 \\
5.7 \\
6.9\end{array}$ & $\begin{array}{l}1.570 E+00 \\
1.561 E+00 \\
1.564 E+00 \\
1.572 E+00 \\
1.583 E+00 \\
1.608 E+00 \\
1.634 E+00 \\
1.660 E+00\end{array}$ & $\begin{array}{l}6.818 E-01 \\
8.418 E-01 \\
1.001 E+00 \\
1.160 E+00 \\
1.473 E+00 \\
1.782 E+00 \\
2.085 E+00\end{array}$ & $\begin{array}{l}913 E-03 \\
240 E-03 \\
599 E-03 \\
990 E-03 \\
141 E-02 \\
431 E-02 \\
729 E-02 \\
032 E-02\end{array}$ & $\begin{array}{l}2.859 \mathrm{E}-0 \\
4.090 \mathrm{E}-0 \\
5.271 \mathrm{E}-0 \\
6.390 \mathrm{E}-0 \\
7.448 \mathrm{E}-0 \\
9.392 \mathrm{E}-0 \\
1.114 \mathrm{E}+0 \\
1.273 \mathrm{E}+0\end{array}$ & $\begin{array}{l}-0.0 \\
-0.0 \\
-0.0 \\
-0.0 \\
-0.0 \\
-0.0 \\
-0.0 \\
-0.0\end{array}$ & $\begin{array}{l}0.1 \\
0.0 \\
0.0 \\
0.0 \\
0.0 \\
0.0 \\
0.0 \\
0.0\end{array}$ & $\begin{array}{l}93 \\
84 \\
77 \\
72 \\
67 \\
60 \\
55 \\
51\end{array}$ \\
\hline $\begin{array}{l}4.0000 \\
4.5000 \\
5.0000 \\
5.5000 \\
6.0000 \\
7.0000 \\
8.0000 \\
9.0000\end{array}$ & $\begin{array}{l}1.604 E+00 \\
1.616 E+00 \\
1.627 E+00 \\
1.637 E+00 \\
1.647 E+00 \\
1.664 E+00 \\
1.678 E+00 \\
1.691 E+00\end{array}$ & $\begin{array}{l}9.3 \\
1.0 \\
1.1 \\
1.3 \\
1.5 \\
1.8\end{array}$ & $\begin{array}{l}1.685 E+00 \\
1.709 E+00 \\
1.733 E+00 \\
1.756 E+00 \\
1.778 E+00 \\
1.821 E+00 \\
1.863 E+00 \\
1.903 E+00\end{array}$ & $\begin{array}{l}2 . \\
2 . \\
3 \\
3 . \\
4 .\end{array}$ & $\begin{array}{l}-02 \\
-02 \\
-02 \\
-02 \\
-02 \\
-02 \\
-02 \\
-02\end{array}$ & $\begin{array}{l}1.4 \\
1.5 \\
1.6 \\
1.7 \\
1.9 \\
2.1 \\
2.2 \\
2.4\end{array}$ & $\begin{array}{l}-0.0 \\
-0.0 \\
-0.0 \\
-0.0 \\
-0.0 \\
-0.0 \\
-0.0 \\
-0.0\end{array}$ & $\begin{array}{l}0.0 \\
0.0 \\
0.0 \\
0.0 \\
0.0 \\
0.0 \\
0.0\end{array}$ & $\begin{array}{l}0.045 \\
0.042 \\
0.040 \\
0.038 \\
0.035 \\
0.032 \\
0.030\end{array}$ \\
\hline $\begin{array}{l}12.5000 \\
15.0000 \\
17.5000 \\
20.0000 \\
25.0000\end{array}$ & $\begin{array}{l}1.72 \\
1.74 \\
1.76 \\
1.77 \\
1.79 \\
1.81 \\
1.82\end{array}$ & $\begin{array}{l}3.1 \\
3.8 \\
4.5 \\
5.3 \\
6.9\end{array}$ & $\begin{array}{l}2 \\
2 \\
2 \\
2 \\
2 \\
2 \\
2\end{array}$ & $\begin{array}{l}6 . \\
8 . \\
9 . \\
1 . \\
1 .\end{array}$ & $\begin{array}{l}-02 \\
-02 \\
-02 \\
-01 \\
-01 \\
-01 \\
01 \\
-01 \\
-01\end{array}$ & $\begin{array}{l}2.9 \\
3.2 \\
3.4 \\
3.6 \\
4.0 \\
4.3 \\
4.6\end{array}$ & $\begin{array}{l}-0 . \\
-0 . \\
-0 . \\
-0 . \\
-0 . \\
-0 . \\
-0 . \\
-0 .\end{array}$ & $\begin{array}{l}0.0 \\
0.0 \\
0.0 \\
0.0 \\
0.0 \\
0.0\end{array}$ & $\begin{array}{l}0.028 \\
0.024 \\
0.021 \\
0.019 \\
0.017 \\
0.0114 \\
0.012 \\
0.011\end{array}$ \\
\hline $\begin{array}{l}45.00000 \\
50.0000 \\
55.0000 \\
60.0000 \\
70.0000 \\
80.0000\end{array}$ & $\begin{array}{l}+00 \\
+00 \\
+00 \\
+00 \\
+00 \\
+00 \\
+00 \\
+00\end{array}$ & $\begin{array}{l}1 . \\
1 . \\
1 . \\
1 . \\
2 .\end{array}$ & & $\begin{array}{l}1 \\
1 \\
1 \\
1\end{array}$ & $\begin{array}{ll}-0 & 1 \\
0 & 1 \\
0 & 1 \\
0 & 1 \\
0 & 1 \\
0 & 1 \\
0 & 1 \\
0 & 1 \\
0 & 1 \\
0 & 1\end{array}$ & & $\begin{array}{l}-0 . \\
-0 . \\
-0 . \\
-0 . \\
-0 . \\
-0 . \\
-0 . \\
-0 .\end{array}$ & $\begin{array}{l}0 . \\
0 . \\
0 . \\
0 . \\
0 .\end{array}$ & $\begin{array}{l}0.010 \\
0.009 \\
0.008 \\
0.007 \\
0.006 \\
0.005 \\
0.005 \\
0.004\end{array}$ \\
\hline $\begin{array}{l}00 \\
00 \\
0\end{array}$ & $\begin{array}{l}918 E+00 \\
936 E+00 \\
950 E+00 \\
963 E+00 \\
973 E+00 \\
990 E+00 \\
004 E+00 \\
016 E+00\end{array}$ & & & & & $\begin{array}{l}6 . \\
7 . \\
7 . \\
7 . \\
7 . \\
8 .\end{array}$ & $\begin{array}{l}-0 \\
-0 \\
-0 \\
-0 \\
-0 \\
-0 \\
-0 \\
-0\end{array}$ & & $\begin{array}{l}04 \\
03 \\
02 \\
02 \\
02 \\
02 \\
01 \\
01 \\
01\end{array}$ \\
\hline $\begin{array}{l}.0000 \\
.0000 \\
.0000 \\
.0000 \\
.0000 \\
.0000\end{array}$ & $\begin{array}{l}2.027 E+00 \\
2.036 E+00 \\
2.044 E+00 \\
2.051 E+00 \\
2.058 E+00 \\
2.069 E+00 \\
2.080 E+00 \\
2.089 E+00\end{array}$ & $\begin{array}{lll}0 & 1 \\
0 & 1 \\
0 & 1 \\
0 & 1 \\
0 & 1 \\
0 & 1 \\
0 & 1\end{array}$ & $\begin{array}{l}1 \\
1 \\
1 \\
1 \\
1 \\
1\end{array}$ & $\begin{array}{l}1 \\
1 \\
1\end{array}$ & $\begin{array}{l}119 \mathrm{E}-01 \\
.295 \mathrm{E}-01 \\
.447 \mathrm{E}-01 \\
.581 \mathrm{E}-01 \\
.804 \mathrm{E}-01 \\
.985 \mathrm{E}-01 \\
.135 \mathrm{E}-01\end{array}$ & $\begin{array}{ll}E+0 & 0 \\
E+0 & 0 \\
E+0 & 0 \\
E+0 & 1 \\
E+0 & 1 \\
E+0 & 1 \\
E+0 & 1\end{array}$ & $\begin{array}{l}-0 \\
-0 \\
-0 \\
-0 \\
-0 \\
-0 \\
-0 \\
-0\end{array}$ & $\begin{array}{l}0 . \\
0 . \\
0 . \\
0 . \\
0 . \\
0 .\end{array}$ & 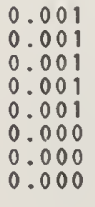 \\
\hline 00 & $097 \mathrm{E}+00$ & .445 & $4 E+0$ & $78 E+0$ & $1 \mathrm{E}$ & 91 & -0.0 & .004 & .00 \\
\hline
\end{tabular}




\begin{tabular}{|c|c|c|c|c|c|c|c|c|c|}
\hline ENERGY & 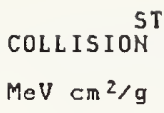 & $\begin{array}{l}\text { PPING POWE } \\
\text { RADI AT IVE } \\
\mathrm{MeV} \mathrm{cm}^{2} / \mathrm{g}\end{array}$ & $\begin{array}{l}\text { TOTAL } \\
\mathrm{MeV} \mathrm{cm}^{2} / \mathrm{g}\end{array}$ & $\begin{array}{l}\text { CSDA } \\
\text { RANGE } \\
\mathrm{g} / \mathrm{cm}^{2}\end{array}$ & YIEL & $\begin{array}{l}\text { ENS.EFF. } \\
\text { COPR. } \\
\text { (DELTA) }\end{array}$ & \multicolumn{3}{|c|}{$\begin{array}{ll} & d(\log ) / d(\log I) \\
\text { COLL } & \text { CSDA } \\
\text { LOSS } & \text { RANGE } \\
\text { RIELD }\end{array}$} \\
\hline $\begin{array}{l}0.0100 \\
0.0125 \\
0.0150 \\
0.0175 \\
0.0200 \\
0.0250 \\
0.0300 \\
0.0350\end{array}$ & $\begin{array}{l}2.059 \mathrm{E}+01 \\
1.732 \mathrm{E}+01 \\
1.504 \mathrm{E}+01 \\
1.335 \mathrm{E}+01 \\
1.204 \mathrm{E}+01 \\
1.014 \mathrm{E}+01 \\
8.828 \mathrm{E}+00 \\
7.860 \mathrm{E}+00\end{array}$ & $\begin{array}{l}3.368 \mathrm{E}-03 \\
3.383 \mathrm{E}-03 \\
3.392 \mathrm{E}-03 \\
3.399 \mathrm{E}-03 \\
3.404 \mathrm{E}-03 \\
3.413 \mathrm{E}-03\end{array}$ & $\begin{array}{l}2.059 E+01 \\
1.733 E+01 \\
1.504 E+01 \\
1.335 E+01 \\
1.204 E+01 \\
1.015 E+01 \\
8.832 E+00 \\
7.864 E+00\end{array}$ & $\begin{array}{l}2.76 \\
4.08 \\
5.64 \\
7.41 \\
9.38 \\
1.39 \\
1.92 \\
2.52\end{array}$ & $\begin{array}{l}9.020 \mathrm{E}-05 \\
1.081 \mathrm{E}-04 \\
1.251 \mathrm{E}-04 \\
1.416 \mathrm{E}-04 \\
1.574 \mathrm{E}-04 \\
1.879 \mathrm{E}-04 \\
2.169 \mathrm{E}-04 \\
2.448 \mathrm{E}-04\end{array}$ & $\begin{array}{l}0.0 \\
0.0 \\
0.0 \\
0.0 \\
0.0 \\
0.0 \\
0.0 \\
0.0\end{array}$ & $\begin{array}{l}-0.201 \\
-0.193 \\
-0.186 \\
-0.181 \\
-0.177 \\
-0.170 \\
-0.165 \\
-0.161\end{array}$ & $\begin{array}{l}1 \\
9 \\
1 \\
5 \\
9 \\
1 \\
4 \\
9\end{array}$ & $\begin{array}{l}0.229 \\
0.218 \\
0.210 \\
0.204 \\
0.198 \\
0.190 \\
0.184 \\
0.179\end{array}$ \\
\hline $\begin{array}{l}0.0400 \\
0.0450 \\
0.0500 \\
0.0550 \\
0.0600 \\
0.0700 \\
0.0800 \\
0.0900\end{array}$ & $\begin{array}{l}6.5 \\
6.0 \\
5.6 \\
5.3 \\
4.7 \\
4.3\end{array}$ & $\begin{array}{l}-03 \\
-03 \\
-03 \\
-03 \\
-03 \\
-03 \\
-03 \\
-03 \\
-03\end{array}$ & $\begin{array}{l}6.5 \\
6.5 \\
5.5 \\
5 . \\
4 . \\
4 . \\
4 .\end{array}$ & $\begin{array}{l}3.9 \\
4.7 \\
5.5 \\
6.4 \\
8.4 \\
1.0 \\
1.3\end{array}$ & $\begin{array}{l}2.9 \\
3.2 \\
3.4 \\
3.7 \\
4.1 \\
4.6 \\
5.0\end{array}$ & $\begin{array}{l}.0 \\
.0 \\
.0 \\
.0 \\
.0 \\
.0 \\
.0 \\
.0\end{array}$ & $\begin{array}{l}-0 . \\
-0 . \\
-0 . \\
-0 . \\
-0 . \\
-0 . \\
-0 .\end{array}$ & $\begin{array}{l}5 \\
2 \\
9 \\
6 \\
4 \\
0 \\
7 \\
4\end{array}$ & $\begin{array}{l}75 \\
71 \\
68 \\
66 \\
64 \\
60 \\
57 \\
54\end{array}$ \\
\hline $\begin{array}{l}0.1000 \\
0.1250 \\
0.1500 \\
0.1750 \\
0.2000 \\
0.2500 \\
0.3000 \\
0.3500\end{array}$ & $\begin{array}{l}3.290 E+00 \\
2.967 E+00 \\
2.735 E+00 \\
2.560 E+00 \\
2.318 E+00 \\
2.159 E+00 \\
2.049 E+00\end{array}$ & $\begin{array}{l}03 \\
03 \\
03 \\
03 \\
03 \\
03 \\
03 \\
03\end{array}$ & $\begin{array}{l}3.29 \\
2.97 \\
2.73 \\
2.56 \\
2.32 \\
2.16 \\
2.05\end{array}$ & $\begin{array}{l}-02 \\
-02 \\
-02 \\
-02 \\
-02 \\
-02 \\
-02 \\
-01\end{array}$ & $\begin{array}{l}6.5 \\
7.4 \\
8.3 \\
9.2 \\
1.0 \\
1.2 \\
1.4\end{array}$ & $\begin{array}{l}0 \\
0 \\
0 \\
0 \\
0 \\
0 \\
0\end{array}$ & $\begin{array}{l}-0 . \\
-0 . \\
-0 . \\
-0 . \\
-0 . \\
-0 . \\
-0 .\end{array}$ & $\begin{array}{l}0 \\
0 \\
0 \\
0 \\
0 \\
0\end{array}$ & \\
\hline $\begin{array}{l}0.4000 \\
0.4500 \\
0.5000 \\
0.5500 \\
0.6000 \\
0.7000 \\
0.8000 \\
0.9000\end{array}$ & $\begin{array}{l}1.906 \mathrm{E} \\
1.859 \mathrm{E} \\
1.821 \mathrm{E} \\
1.791 \mathrm{E} \\
1.747 \mathrm{E} \\
1.717 \mathrm{E} \\
1.696 \mathrm{E}\end{array}$ & $\begin{array}{l}03 \\
03 \\
03 \\
03 \\
03 \\
03 \\
03 \\
02\end{array}$ & $\begin{array}{l}1.974 E+00 \\
1.912 E+00 \\
1.865 E+00 \\
1.828 E+00 \\
1.798 E+00 \\
1.755 E+00 \\
1.726 E+00 \\
1.707 E+00\end{array}$ & $\begin{array}{l}1.6 \\
1.9 \\
2.1 \\
2.4 \\
3.0 \\
3.6 \\
4.1\end{array}$ & $\begin{array}{l}1.7 \\
1.8 \\
2.0 \\
2.1 \\
2.4 \\
2.7\end{array}$ & $\begin{array}{l}4.1 \\
7.1 \\
1.0 \\
1.3 \\
2.0 \\
2.7 \\
3.4\end{array}$ & $\begin{array}{l}-0 . \\
-0 . \\
-0 . \\
-0 . \\
-0 . \\
-0 . \\
-0 . \\
-0 .\end{array}$ & $\begin{array}{l}0 . \\
0 \\
0 \\
0 . \\
0 . \\
0 .\end{array}$ & \\
\hline $\begin{array}{l}1.0000 \\
1.2500 \\
1.5000 \\
1.7500 \\
2.0000 \\
2.5000 \\
3.0000 \\
3.5000\end{array}$ & $\begin{array}{l}1.663 \mathrm{E}+00 \\
1.657 \mathrm{E}+00 \\
1.657 \mathrm{E}+00 \\
1.660 \mathrm{E}+00 \\
1.670 \mathrm{E}+00\end{array}$ & $\begin{array}{l}1 \cdot 3 \\
1 \cdot 7 \\
2.0 \\
2.3 \\
3 \cdot 0 \\
3 \cdot 7 \\
4.5\end{array}$ & $\begin{array}{l}1.5 \\
1 . \\
1 . \\
1 . \\
1 . \\
1 . \\
1 .\end{array}$ & $\begin{array}{ll}0 & 1 \\
0 & 1 \\
0 & 1 \\
0 & 1 \\
0 & 0 \\
0 & 0 \\
0 & 0 \\
0 & 0\end{array}$ & $\begin{array}{l}4.2 \\
5.0 \\
5.9 \\
6.8 \\
8.6 \\
1.0 \\
1.2\end{array}$ & $\begin{array}{l}5.7 \\
7.2 \\
8.7 \\
1.0 \\
1.2 \\
1.4 \\
1.6\end{array}$ & $\begin{array}{l}-0.0 \\
-0.0 \\
-0.0 \\
-0.0 \\
-0.0 \\
-0.0 \\
-0.0 \\
-0.0\end{array}$ & $\begin{array}{l}0 \\
0 \\
0 \\
0 \\
0 \\
0\end{array}$ & \\
\hline $\begin{array}{l}4.0000 \\
4.5000 \\
5.0000 \\
5.5000 \\
6.0000 \\
7.0000 \\
8.0000 \\
9.0000\end{array}$ & $\begin{array}{l}1.719 \mathrm{E}+ \\
1.730 \mathrm{E}+ \\
1.740 \mathrm{E}+ \\
1.749 \mathrm{E}+ \\
1.765 \mathrm{E}+ \\
1.780 \mathrm{E}+\end{array}$ & $\begin{array}{l}02 \\
02 \\
02 \\
02 \\
02 \\
02 \\
01 \\
01 \\
01\end{array}$ & $\begin{array}{l}1: \\
1: \\
1.2 \\
1 . \\
1 . \\
1 .\end{array}$ & $\begin{array}{l}2.2 \\
2.5 \\
2.7 \\
3.0 \\
3.3 \\
3.8 \\
4.4 \\
4.9\end{array}$ & $\begin{array}{l}1.439 \mathrm{E}-02 \\
1.639 \mathrm{E}-02 \\
1.840 \mathrm{E}-02 \\
2.044 \mathrm{E}-02 \\
2.249 \mathrm{E}-02 \\
2.664 \mathrm{E}-02 \\
3.082 \mathrm{E}-02 \\
3.503 \mathrm{E}-02\end{array}$ & $\begin{array}{l}1.788 \mathrm{E}+00 \\
1.936 \mathrm{E}+00 \\
2.072 \mathrm{E}+00 \\
2.197 \mathrm{E}+00 \\
2.314 \mathrm{E}+00 \\
2.526 \mathrm{E}+00 \\
2.715 \mathrm{E}+00 \\
2.886 \mathrm{E}+00\end{array}$ & $\begin{array}{l}-0.029 \\
-0.028 \\
-0.027 \\
-0.026 \\
-0.025 \\
-0.024 \\
-0.022 \\
-0.021\end{array}$ & $\begin{array}{l}0.04 \\
0.04 \\
0.04 \\
0.04 \\
0.03 \\
0.0 \\
0.0\end{array}$ & $\begin{array}{l}34 \\
33 \\
32 \\
30 \\
29 \\
27\end{array}$ \\
\hline $\begin{array}{l}10.0000 \\
12.5000 \\
15.0000 \\
17.5000 \\
20.0000 \\
25.0000 \\
30.0000 \\
35.0000\end{array}$ & $\begin{array}{l}1.828 \mathrm{Et} \\
1.847 \mathrm{Et} \\
1.862 \mathrm{Et} \\
1.875 \mathrm{E}+ \\
1.896 \mathrm{Et} \\
1.913 \mathrm{Et}\end{array}$ & $\begin{array}{l}599 \mathrm{E}-01 \\
084 \mathrm{E}-01 \\
582 \mathrm{E}-01 \\
091 \mathrm{E}-01 \\
509 \mathrm{E}-01 \\
664 \mathrm{E}-01 \\
737 \mathrm{E}-01 \\
324 \mathrm{E}-01\end{array}$ & $\begin{array}{l}2 . \\
2 . \\
2 . \\
2 . \\
2 . \\
2 .\end{array}$ & $\begin{array}{l}5.4 \\
6.7 \\
7.9 \\
9.0 \\
1.0 \\
1.2 \\
1.4 \\
1.6\end{array}$ & $\begin{array}{l}.924 \mathrm{E}-02 \\
.978 \mathrm{E}-02 \\
.024 \mathrm{E}-02 \\
.057 \mathrm{E}-02 \\
.074 \mathrm{E}-02 \\
.005 \mathrm{E}-01 \\
195 \mathrm{E}-01 \\
.376 \mathrm{E}-01\end{array}$ & $\begin{array}{l}3.043 \mathrm{E} \\
3.387 \mathrm{E} \\
3.681 \mathrm{E} \\
3.939 \mathrm{E} \\
4.168 \mathrm{E} \\
4.564 \mathrm{E} \\
4.897 \mathrm{E} \\
5.184 \mathrm{E}\end{array}$ & $\begin{array}{l}-0.0 \\
-0.0 \\
-0.0 \\
-0.0 \\
-0.0 \\
-0.0 \\
-0.0 \\
-0.0\end{array}$ & $\begin{array}{l}0 . \\
0 . \\
0 . \\
0 . \\
0 . \\
0 .\end{array}$ & $\begin{array}{l}24 \\
21 \\
19 \\
17 \\
15 \\
12 \\
10 \\
08\end{array}$ \\
\hline $\begin{array}{l}40.00 \\
45.00 \\
50.00 \\
55.00 \\
60.00 \\
70.00 \\
80.00 \\
90.00\end{array}$ & .00 & $\begin{array}{l}9 \\
1 \\
1 \\
1 \\
1 \\
1 \\
1\end{array}$ & $\begin{array}{l}2 . \\
2 . \\
3 \\
3 .\end{array}$ & $\begin{array}{l}1 \\
2 . \\
2 . \\
2 . \\
2 . \\
2 . \\
3 . \\
3 .\end{array}$ & $\begin{array}{l}1 \\
1 \\
1 \\
2 \\
2\end{array}$ & $\begin{array}{l}5 \\
5 \\
6\end{array}$ & $\begin{array}{l}-0 \\
-0 \\
-0 \\
-0 \\
-0 \\
-0 \\
-0 \\
-0\end{array}$ & $\frac{4}{3}$ & $\begin{array}{l}06 \\
05 \\
05 \\
04 \\
04 \\
03 \\
3\end{array}$ \\
\hline $\begin{array}{l}0.00 \\
5.00 \\
0.00 \\
0.00\end{array}$ & $\begin{array}{l}0 \\
0 \\
0 \\
0\end{array}$ & $\begin{array}{l}2.7 \\
3.3 \\
3.9 \\
4.5 \\
5.6 \\
6.8 \\
8.0\end{array}$ & $\begin{array}{l}4 . \\
5 . \\
5 . \\
6 . \\
7 .\end{array}$ & $\begin{array}{l}4 . \\
4 . \\
5 . \\
5 . \\
6 . \\
6 . \\
7 .\end{array}$ & int & $\begin{array}{l}7 . \\
8 . \\
8 . \\
8 .\end{array}$ & $\begin{array}{l}-0 \\
-0 \\
-0 \\
-0 \\
-0 \\
-0 \\
-0\end{array}$ & & $\begin{array}{l}02 \\
02 \\
01 \\
01 \\
01 \\
01 \\
01 \\
01\end{array}$ \\
\hline $\begin{array}{l}0.0000 \\
0.0000 \\
0.0000 \\
0.0000 \\
0.0000 \\
0.0000 \\
0.0000\end{array}$ & $\begin{array}{l}0 \\
0\end{array}$ & $\begin{array}{l}9.28 \\
1.04 \\
1.17 \\
1.29 \\
1.41 \\
1.65 \\
1.89\end{array}$ & 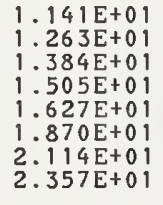 & $\begin{array}{l}7 \\
8 \\
8 \\
8 \\
9 \\
9 \\
1\end{array}$ & $\begin{array}{l}1 \\
1 \\
1 \\
1 \\
1 \\
1 \\
1\end{array}$ & $\begin{array}{c}0 \\
1 \\
1 \\
1 \\
1 \\
1 \\
1 \\
1\end{array}$ & $\begin{array}{l}0 \\
0 \\
0 \\
0 \\
0 \\
0 \\
0 \\
0\end{array}$ & $\begin{array}{l}4 \\
4 \\
3 \\
3 \\
3 \\
3 \\
3\end{array}$ & $\begin{array}{ll}0 & 0 \\
0 & 0 \\
0 & 0 \\
0 & 0 \\
0 & 0 \\
0 & 0\end{array}$ \\
\hline .000 & $6 \mathrm{E}$ & 38 & $1 E+01$ & $15 E+0$ & $16 \mathrm{E}-0$ & $181 \mathrm{E}$ & 00 & 0.003 & $\pi$ \\
\hline
\end{tabular}




\begin{tabular}{|c|c|c|c|c|c|c|c|c|c|}
\hline \multirow{3}{*}{$\begin{array}{l}\text { ENERGY } \\
\text { MeV }\end{array}$} & \multirow{2}{*}{\multicolumn{3}{|c|}{$\begin{array}{l}\text { STOPPING POWER } \\
\text { COLLISION RADIATIVE }\end{array}$}} & \multirow{3}{*}{$\begin{array}{l}\text { CSDA } \\
\text { RANGE } \\
g^{\prime} / \mathrm{Cm}^{2}\end{array}$} & \multirow{3}{*}{$\begin{array}{l}\text { RADIATION } \\
\text { YIELD }\end{array}$} & \multirow{3}{*}{$\begin{array}{l}\text { DENS.EFF } \\
\text { CORR. } \\
\text { (DELTA) }\end{array}$} & \multicolumn{3}{|c|}{$d(\log ) / d(\log I)$} \\
\hline & & & TOTAL & & & & & & RAD \\
\hline & $\mathrm{MeV} \mathrm{cm}^{2} / \mathrm{g}$ & $\mathrm{MeV} \mathrm{cm} 2 / \mathrm{g}$ & $\mathrm{leV} \mathrm{cm}^{2} / \mathrm{g}$ & & & & & & \\
\hline $\begin{array}{l}0.0100 \\
0.0125 \\
0.0150 \\
0.0175 \\
0.0200 \\
0.0250 \\
0.0300 \\
0.0350\end{array}$ & $\begin{array}{l}1.315 \\
1.168 \\
1.055 \\
8.894 \\
7.748\end{array}$ & $\begin{array}{l}.03 \\
-03 \\
-03 \\
.03 \\
-03 \\
-03 \\
-03 \\
.03\end{array}$ & $\begin{array}{l}1.3 \\
1.1 \\
1.0 \\
8.8 \\
7.7 \\
6.9\end{array}$ & $\begin{array}{l}181 E-04 \\
704 E-04 \\
480 E-04 \\
501 E-04 \\
076 E-03 \\
594 E-03 \\
198 E-03 \\
883 E-03\end{array}$ & $\begin{array}{l}E-04 \\
E-04 \\
E-04 \\
E-04 \\
E-04 \\
E-04 \\
E-04 \\
E-04\end{array}$ & $\begin{array}{l}0.0 \\
0.0 \\
0.0 \\
0.0 \\
0.0 \\
0.0 \\
0.0 \\
0.0\end{array}$ & $\begin{array}{l}-0.208 \\
-0.199 \\
-0.192 \\
-0.187 \\
-0.182 \\
-0.175 \\
-0.170 \\
-0.166\end{array}$ & $\begin{array}{l}0.240 \\
0.228 \\
0.219 \\
0.212 \\
0.206 \\
0.197 \\
0.190 \\
0.185\end{array}$ & $\begin{array}{l}0.238 \\
0.226 \\
0.217 \\
0.211 \\
0.205 \\
0.196 \\
0.190 \\
0.184\end{array}$ \\
\hline $\begin{array}{l}0.0400 \\
0.0450 \\
0.0500 \\
0.0550 \\
0.0600 \\
0.0700 \\
0.0800 \\
0.0900\end{array}$ & $\begin{array}{l}6.252 E+00 \\
5.736 E+00 \\
5.315 E+00 \\
4.965 E+00 \\
4.670 E+00 \\
4.198 E+00 \\
3.838 E+00 \\
3.553 E+00\end{array}$ & $\begin{array}{l}3.815 \mathrm{E}-03 \\
3.827 \mathrm{E}-03 \\
3.840 \mathrm{E}-03 \\
3.853 \mathrm{E}-03 \\
3.867 \mathrm{E}-03 \\
3.898 \mathrm{E}-03 \\
3.932 \mathrm{E}-03 \\
3.970 \mathrm{E}-03\end{array}$ & $\begin{array}{l}6.256 \mathrm{E}+00 \\
5.739 \mathrm{E}+00 \\
5.319 \mathrm{E}+00 \\
4.969 \mathrm{E}+00 \\
4.674 \mathrm{E}+00 \\
4.202 \mathrm{E}+00 \\
3.842 \mathrm{E}+00 \\
3.557 \mathrm{E}+00\end{array}$ & $\begin{array}{l}545 E-03 \\
480 E-03 \\
386 E-03 \\
359 E-03 \\
397 E-03 \\
359 E-03 \\
215 E-02 \\
486 E-02\end{array}$ & $\begin{array}{l}-04 \\
-04 \\
-04 \\
-04 \\
-04 \\
-04 \\
-04 \\
-04\end{array}$ & $\begin{array}{l}0.0 \\
0.0 \\
0.0 \\
0.0 \\
0.0 \\
0.0 \\
0.0 \\
0.0\end{array}$ & $\begin{array}{l}-0.162 \\
-0.160 \\
-0.157 \\
-0.155 \\
-0.153 \\
-0.150 \\
-0.147 \\
-0.144\end{array}$ & $\begin{array}{l}181 \\
177 \\
174 \\
171 \\
169 \\
164 \\
161 \\
158\end{array}$ & $\begin{array}{l}0.180 \\
0.176 \\
0.177 \\
0.171 \\
0.168 \\
0.164 \\
0.161 \\
0.158\end{array}$ \\
\hline $\begin{array}{l}0.1000 \\
0.1250 \\
0.1500 \\
0.1750 \\
0.2000 \\
0.2500 \\
0.3000 \\
0.3500\end{array}$ & $\begin{array}{l}3.323 \mathrm{E}+00 \\
2.903 \mathrm{E}+00 \\
2.619 \mathrm{E}+00 \\
2.415 \mathrm{E}+00 \\
2.261 \mathrm{E}+00 \\
2.048 \mathrm{E}+00 \\
1.907 \mathrm{E}+00 \\
1.809 \mathrm{E}+00\end{array}$ & $\begin{array}{l}4.125 \\
4.253 \\
4.392 \\
4.540 \\
4.863 \\
5.215 \\
5.592\end{array}$ & $\begin{array}{l}3.327 E+00 \\
2.907 E+00 \\
2.623 E+00 \\
2.419 E+00 \\
2.266 E+00 \\
2.053 E+00 \\
1.912 E+00 \\
1.814 E+00\end{array}$ & $\begin{array}{l}E-02 \\
E-02 \\
E-02 \\
E-02 \\
E-02 \\
E-02 \\
E-01 \\
E-01\end{array}$ & $\begin{array}{l}E-04 \\
E-04 \\
E-04 \\
E-03 \\
E-03 \\
E-03 \\
E-03 \\
E-03\end{array}$ & $\begin{array}{l}0.0 \\
0.0 \\
0.0 \\
0.0 \\
0.0 \\
6.058 \mathrm{E}-03 \\
2.136 \mathrm{E}-02 \\
3.978 \mathrm{E}-02\end{array}$ & $\begin{array}{l}-0.142 \\
-0.138 \\
-0.135 \\
-0.132 \\
-0.130 \\
-0.118 \\
-0.112 \\
-0.106\end{array}$ & $\begin{array}{l}.156 \\
.151 \\
.147 \\
.144 \\
.141 \\
.136 \\
.131 \\
.126\end{array}$ & $\begin{array}{l}0.155 \\
0.150 \\
0.147 \\
0.143 \\
0.141 \\
0.135 \\
0.130 \\
0.125\end{array}$ \\
\hline $\begin{array}{l}0.4000 \\
0.4500 \\
0.5000 \\
0.5500 \\
0.6000 \\
0.7000 \\
0.8000 \\
0.9000\end{array}$ & $\begin{array}{l}1.737 E+00 \\
1.683 E+00 \\
1.642 E+00 \\
1.609 E+00 \\
1.583 E+00 \\
1.546 E+00 \\
1.521 E+00 \\
1.503 E+00\end{array}$ & $\begin{array}{l}5.992 \mathrm{E}-03 \\
6.412 \mathrm{E}-03 \\
6.852 \mathrm{E}-03 \\
7.308 \mathrm{E}-03 \\
7.779 \mathrm{E}-03 \\
8.765 \mathrm{E}-03 \\
9.800 \mathrm{E}-03 \\
1.088 \mathrm{E}-02\end{array}$ & $\begin{array}{l}1.743 \mathrm{E}+00 \\
1.690 \mathrm{E}+00 \\
1.649 \mathrm{E}+00 \\
1.617 \mathrm{E}+00 \\
1.591 \mathrm{E}+00 \\
1.555 \mathrm{E}+00 \\
1.530 \mathrm{E}+00 \\
1.514 \mathrm{E}+00\end{array}$ & $\begin{array}{l}1.591 E-01 \\
1.883 \mathrm{E}-01 \\
2.183 \mathrm{E}-01 \\
2.489 \mathrm{E}-01 \\
2.801 \mathrm{E}-01 \\
3.437 \mathrm{E}-01 \\
4.086 \mathrm{E}-01 \\
4.743 \mathrm{E}-01\end{array}$ & $\begin{array}{l}1.939 \mathrm{E}-03 \\
2.125 \mathrm{E}-03 \\
2.310 \mathrm{E}-03 \\
2.495 \mathrm{E}-03 \\
2.679 \mathrm{E}-03 \\
3.048 \mathrm{E}-03 \\
3.419 \mathrm{E}-03 \\
3.794 \mathrm{E}-03\end{array}$ & $\begin{array}{l}6.095 \mathrm{E}-02 \\
8.445 \mathrm{E}-02 \\
1.098 \mathrm{E}-01 \\
1.367 \mathrm{E}-01 \\
1.648 \mathrm{E}-01 \\
2.236 \mathrm{E}-01 \\
2.846 \mathrm{E}-01 \\
3.467 \mathrm{E}-01\end{array}$ & $\begin{array}{l}-0.100 \\
-0.095 \\
-0.090 \\
-0.086 \\
-0.082 \\
-0.075 \\
-0.070 \\
-0.065\end{array}$ & $\begin{array}{l}.122 \\
.118 \\
.115 \\
112 \\
.108 \\
.103 \\
098 \\
.094\end{array}$ & $\begin{array}{l}0.120 \\
0.116 \\
0.112 \\
0.108 \\
0.104 \\
0.098 \\
0.092 \\
0.087\end{array}$ \\
\hline $\begin{array}{l}1.0000 \\
1.25000 \\
1.5000 \\
1.7500 \\
2.0000 \\
2.5000 \\
3.0000 \\
3.5000\end{array}$ & $\begin{array}{l}1.491 E+00 \\
1.476 E+00 \\
1.471 E+00 \\
1.471 E+00 \\
1.474 E+00 \\
1.483 E+00 \\
1.493 E+00 \\
1.503 E+00\end{array}$ & $\begin{array}{l}1.200 \mathrm{E}-02 \\
1.499 \mathrm{E}-02 \\
1.818 \mathrm{E}-02 \\
2.154 \mathrm{E}-02 \\
2.505 \mathrm{E}-02 \\
3.244 \mathrm{E}-02 \\
4.021 \mathrm{E}-02 \\
4.830 \mathrm{E}-02\end{array}$ & $\begin{array}{l}1.504 E+00 \\
1.491 E+00 \\
1.489 E+00 \\
1.493 E+00 \\
1.499 E+00 \\
1.515 E+00 \\
1.533 E+00 \\
1.552 E+00\end{array}$ & $\begin{array}{l}5.406 E-01 \\
7.077 E-01 \\
8.756 E-01 \\
1.043 E+00 \\
1.210 E+00 \\
1.542 E+00 \\
1.870 E+00 \\
2.194 E+00\end{array}$ & $\begin{array}{l}4.173 \mathrm{E}-03 \\
5.141 \mathrm{E}-03 \\
6.138 \mathrm{E}-03 \\
7.163 \mathrm{E}-03 \\
8.214 \mathrm{E}-03 \\
1.038 \mathrm{E}-02 \\
1.262 \mathrm{E}-02 \\
1.491 \mathrm{E}-02\end{array}$ & $\begin{array}{l}4.093 \mathrm{E}-01 \\
5.644 \mathrm{E}-01 \\
7.142 \mathrm{E}-01 \\
8.568 \mathrm{E}-01 \\
9.917 \mathrm{E}-01 \\
1.240 \mathrm{E}+00 \\
1.461 \mathrm{E}+00 \\
1.660 \mathrm{E}+00\end{array}$ & $\begin{array}{l}-0.060 \\
-0.052 \\
-0.046 \\
-0.041 \\
-0.037 \\
-0.032 \\
-0.028 \\
-0.026\end{array}$ & $\begin{array}{l}0.082 \\
0.075 \\
0.070 \\
0.066 \\
0.059 \\
0.054 \\
0.049\end{array}$ & $\begin{array}{l}0.083 \\
0.073 \\
0.066 \\
0.059 \\
0.054 \\
0.047 \\
0.041 \\
0.037\end{array}$ \\
\hline $\begin{array}{l}4.0000 \\
4.5000 \\
5.0000 \\
5.5000 \\
6.0000 \\
7.0000 \\
8.0000 \\
9.0000\end{array}$ & $\begin{array}{l}1.513 E+00 \\
1.523 E+00 \\
1.531 E+00 \\
1.539 E+00 \\
1.547 E+00 \\
1.560 E+00 \\
1.572 E+00 \\
1.583 E+00\end{array}$ & $\begin{array}{l}5.666 \mathrm{E}-02 \\
6.524 \mathrm{E}-02 \\
7.402 \mathrm{E}-02 \\
8.298 \mathrm{E}-02 \\
9.211 \mathrm{E}-02 \\
1.108 \mathrm{E}-01 \\
1.299 \mathrm{E}-01 \\
1.494 \mathrm{E}-01\end{array}$ & $\begin{array}{l}1.570 E+00 \\
1.588 E+00 \\
1.605 E+00 \\
1.622 E+00 \\
1.639 E+00 \\
1.671 E+00 \\
1.702 E+00 \\
1.732 E+00\end{array}$ & $\begin{array}{l}2.515 E+00 \\
2.832 E+00 \\
3.145 E+00 \\
3.455 E+00 \\
3.761 E+00 \\
4.365 E+00 \\
4.958 E+00 \\
5.541 E+00\end{array}$ & $\begin{array}{l}1.725 \mathrm{E}-02 \\
1.962 \mathrm{E}-02 \\
2.202 \mathrm{E}-02 \\
2.444 \mathrm{E}-02 \\
2.687 \mathrm{E}-02 \\
3.178 \mathrm{E}-02 \\
3.672 \mathrm{E}-02 \\
4.168 \mathrm{E}-02\end{array}$ & $\begin{array}{l}1.839 \mathrm{E} \\
2.003 \mathrm{E} \\
2.154 \mathrm{E} \\
2.293 \mathrm{E} \\
2.422 \mathrm{E} \\
2.655 \mathrm{E} \\
2.861 \mathrm{E} \\
3.046 \mathrm{E}\end{array}$ & $\begin{array}{l}-0.0 \\
-0.0 \\
-0.0 \\
-0.0 \\
-0.0 \\
-0.0 \\
-0.0 \\
-0.0\end{array}$ & & $\begin{array}{l}0.034 \\
0.031 \\
0.029 \\
0.027 \\
0.026 \\
0.024 \\
0.022 \\
0.020\end{array}$ \\
\hline $\begin{array}{l}10.0000 \\
12.5000 \\
15.0000 \\
17.5000 \\
20.0000 \\
25.00000 \\
30.0000 \\
35.0000\end{array}$ & $\begin{array}{l}1.592 E+00 \\
1.612 E+00 \\
1.629 E+00 \\
1.642 E+00 \\
1.654 E+00 \\
1.673 E+00 \\
1.688 E+00 \\
1.700 E+00\end{array}$ & $\begin{array}{l}1.693 \mathrm{E}-08 \\
2.201 \mathrm{E}-01 \\
2.723 \mathrm{E}-01 \\
3.256 \mathrm{E}-01 \\
3.797 \mathrm{E}-01 \\
4.896 \mathrm{E}-01 \\
6.014 \mathrm{E}-01 \\
7.146 \mathrm{E}-01\end{array}$ & $\begin{array}{l}1.761 E+00 \\
1.832 E+00 \\
1.901 E+00 \\
1.968 E+00 \\
2.034 E+00 \\
2.163 E+00 \\
2.289 E+00 \\
2.415 E+00\end{array}$ & $\begin{array}{l}7.504 E+00 \\
8.844 E+00 \\
1.014 E+01 \\
1.139 E+01 \\
1.377 E+01 \\
1.602 E+01 \\
1.814 E+01\end{array}$ & & $\begin{array}{l}3.214 E+00 \\
3.577 E+00 \\
3.881 E+00 \\
4.143 E+00 \\
4.374 E+00 \\
4.769 E+00 \\
5.099 E+00 \\
5.383 E+00\end{array}$ & $\begin{array}{l}-0 . \\
-0 . \\
-0 . \\
-0 \\
-0 \\
-0 . \\
-0 . \\
-0 .\end{array}$ & $\begin{array}{l}9 \\
6 \\
4 \\
2 \\
1 \\
8 \\
7 \\
5\end{array}$ & $\begin{array}{l}0.019 \\
0.017 \\
0.015 \\
0.014 \\
0.013 \\
0.0111 \\
0.009 \\
0.008\end{array}$ \\
\hline $\begin{array}{l}40.0000 \\
45.0000 \\
50.0000 \\
55.0000 \\
60.0000 \\
70.0000\end{array}$ & $\begin{array}{l}1.71 \\
1.72 \\
1.72 \\
1.73 \\
1.74 \\
1.75 \\
1.76 \\
1.77\end{array}$ & $\begin{array}{l}9.441 \mathrm{E}-01 \\
1.060 \mathrm{E}+00 \\
1.177 \mathrm{E}+00 \\
1.294 \mathrm{E}+00 \\
1.530 \mathrm{E}+00 \\
1.767 \mathrm{E}+0 \\
2.006 \mathrm{E}+0\end{array}$ & $\begin{array}{l}2.540 E+00 \\
2.664 E+00 \\
2.788 E+00 \\
2.912 E+00 \\
3.036 E+00 \\
3.283 E+00 \\
3.531 E+00 \\
3.778 E+00\end{array}$ & $\begin{array}{l}.208 E+01 \\
.392 E+01 \\
.567 E+01 \\
.735 E+01 \\
.052 E+01 \\
.346 E+01 \\
.619 E+01\end{array}$ & $\begin{array}{l}1.776 \mathrm{E}-01 \\
1.957 \mathrm{E}-01 \\
2.129 \mathrm{E}-01 \\
2.292 \mathrm{E}-01 \\
2.447 \mathrm{E}-01 \\
2.735 \mathrm{E}-01 \\
2.998 \mathrm{E}-01 \\
3.238 \mathrm{E}-01\end{array}$ & $\begin{array}{l}5.633 \mathrm{E}+ \\
5.856 \mathrm{E}+ \\
6.057 \mathrm{E}+ \\
6.240 \mathrm{E}+ \\
6.408 \mathrm{E}+ \\
6.707 \mathrm{E}+ \\
6.968 \mathrm{E}+ \\
7.199 \mathrm{E}+\end{array}$ & $\begin{array}{l}-0.0 \\
-0.0 \\
-0.0 \\
-0.0 \\
-0.0 \\
-0.0 \\
-0.0 \\
-0.0\end{array}$ & & \\
\hline $\begin{array}{l}0.0000 \\
5.0000 \\
0.0000 \\
5.0000 \\
0.0000 \\
0.0000 \\
0.0000 \\
0.0000\end{array}$ & $\begin{array}{l}1.780 E+00 \\
1.796 E+00 \\
1.809 E+00 \\
1.820 E+00 \\
1.830 E+00 \\
1.846 E+00 \\
1.859 E+00 \\
1.870 E+00\end{array}$ & $\begin{array}{l}2.245 E+00 \\
2.848 E+00 \\
3.455 E+00 \\
4.065 E+00 \\
4.677 E+00 \\
5.907 E+00 \\
7.142 E+00 \\
8.381 E+00\end{array}$ & $\begin{array}{l}4.025 \mathrm{E}+00 \\
4.644 \mathrm{E}+00 \\
5.264 \mathrm{E}+00 \\
5.885 \mathrm{E}+00 \\
6.507 \mathrm{E}+00 \\
7.753 \mathrm{E}+00 \\
9.001 \mathrm{E}+00 \\
1.025 \mathrm{E}+01\end{array}$ & $\begin{array}{l}1 \\
1 \\
1 \\
1 \\
1 \\
1 \\
1\end{array}$ & $\begin{array}{l}3.45 \\
3.94 \\
4.34 \\
4.68 \\
4.98 \\
5.46 \\
5.85 \\
6.1\end{array}$ & $\begin{array}{l}7.406 E+00 \\
7.847 E+00 \\
8.209 E+00 \\
8.515 E+00 \\
8.780 E+00 \\
9.225 E+00 \\
9.588 E+00 \\
9.896 E+00\end{array}$ & $\begin{array}{l}-0 . \\
-0 . \\
-0 . \\
-0 . \\
-0 . \\
-0 . \\
-0 .\end{array}$ & & \\
\hline $\begin{array}{l}.0000 \\
.0000 \\
.0000 \\
.0000\end{array}$ & $\begin{array}{l}1.888 \mathrm{E}+00 \\
1.895 \mathrm{E}+00 \\
1.902 \mathrm{E}+00 \\
1.908 \mathrm{E}+00 \\
1.919 \mathrm{E}+00 \\
1.929 \mathrm{E}+00\end{array}$ & $\begin{array}{l}1.08 \\
1.28 \\
1.33 \\
1.46 \\
1.71 \\
1.96\end{array}$ & $\begin{array}{l}1.4 \\
1.5 \\
1.6 \\
1.9 \\
2.9\end{array}$ & $\begin{array}{l}1 \\
1 \\
1 \\
1 \\
2\end{array}$ & $\begin{array}{l}6.656 E-01 \\
6.849 E-01 \\
7.018 E-01 \\
7.166 E-01 \\
7.417 E-01 \\
7.621 E-01 \\
7.791 E-01\end{array}$ & $\begin{array}{l}1.016 E+01 \\
1.040 E+01 \\
1.061 E+01 \\
1.080 E+01 \\
1.097 E+01 \\
1.128 E+01 \\
1.155 E+01 \\
1.178 E+01\end{array}$ & $\begin{array}{l}-0.000 \\
-0.000 \\
-0.000 \\
-0.000 \\
-0.000 \\
-0.000 \\
-0.000\end{array}$ & $\begin{array}{l}4 \\
4 \\
4 \\
3 \\
3 \\
3 \\
3 \\
3\end{array}$ & $\begin{array}{l}0 \\
0 \\
0 \\
0 \\
0 \\
0 \\
0 \\
0\end{array}$ \\
\hline .000 & $1.945 E+00$ & $.462 E+01$ & $2.656 E+01$ & $1.143 E+02$ & $7.935 E-01$ & $1.199 E+01$ & 0.000 & 0.003 & 000 \\
\hline
\end{tabular}




\begin{tabular}{|c|c|c|c|c|c|c|c|c|c|}
\hline ENERGY & COLLISION & $\begin{array}{l}\text { ING POW } \\
\text { DIAT IVE }\end{array}$ & TOTAL & $\begin{array}{l}\text { CSDA } \\
\text { RANGE }\end{array}$ & YIE & DENS.EFF . & $\begin{aligned} & d(1 \\
& \operatorname{coL} L \\
& \text { Loss }\end{aligned}$ & CSDA & $\begin{array}{l}\text { I ) } \\
\text { RAD } \\
\text { IEL D }\end{array}$ \\
\hline $\mathrm{MeV}$ & 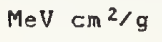 & $\mathrm{leV} \mathrm{cm}^{2} / \mathrm{g}$ & leV $\mathrm{cm}^{2} / \mathrm{g}$ & $g / c$ & & & & & \\
\hline $\begin{array}{l}0.0100 \\
0.0125 \\
0.0150 \\
0.0175 \\
0.0200 \\
0.0250 \\
0.0300 \\
0.0350\end{array}$ & $\begin{array}{l}1.58 \\
1.37 \\
1.22 \\
1.10 \\
9.31 \\
8.11\end{array}$ & $\begin{array}{l}3 \cdot 5 \\
3 \cdot 5 \\
3 \cdot 5 \\
3 \cdot 5 \\
3 \cdot 6 \\
3 \cdot 6\end{array}$ & $\begin{array}{l}1.5 \\
1 . \\
1 . \\
1 . \\
9 . \\
8 .\end{array}$ & $\begin{array}{l}4.4 \\
6.1 \\
8.1 \\
1.0 \\
1.5 \\
2.1\end{array}$ & $\begin{array}{l}1 . \\
1 . \\
1 . \\
1 . \\
2 . \\
2 .\end{array}$ & $\begin{array}{l}0.0 \\
0.0 \\
0.0 \\
0.0 \\
0.0 \\
0.0 \\
0.0 \\
0.0\end{array}$ & $\begin{array}{l}-0 \\
-0 \\
-0 \\
-0 \\
-0 \\
-0 \\
-0 \\
-0\end{array}$ & $\begin{array}{l}0.2 \\
0.2 \\
0.2 \\
0.2 \\
0.2 \\
0.1 \\
0.1\end{array}$ & $\begin{array}{l}0.239 \\
0.227 \\
0.218 \\
0.211 \\
0.206 \\
0.197 \\
0.190 \\
0.185\end{array}$ \\
\hline $\begin{array}{l}0.0400 \\
0.0450 \\
0.0500 \\
0.0550 \\
0.0600 \\
0.0700 \\
0.0800 \\
0.0900\end{array}$ & $\begin{array}{l}.546 \mathrm{E}+00 \\
.005 \mathrm{E}+00 \\
.565 \mathrm{E}+00 \\
.199 \mathrm{E}+00 \\
.890 \mathrm{E}+00 \\
.396 \mathrm{E}+00 \\
.019 \mathrm{E}+00 \\
.721 \mathrm{E}+00\end{array}$ & $\begin{array}{l}3 \cdot 6 \\
3 \cdot 6 \\
3.6 \\
3.6 \\
3.7 \\
3.7\end{array}$ & $\begin{array}{l}6.009 \\
5.569 \\
5.203 \\
4.893 \\
4.400 \\
4.022 \\
3.725\end{array}$ & $\begin{array}{l}4.28 \\
5.1 \\
6.07 \\
7.08 \\
9.22 \\
1.16 \\
1.4\end{array}$ & $\begin{array}{l}3.4 \\
3.7 \\
3.9 \\
4.2 \\
4.8 \\
5.3 \\
5.8\end{array}$ & $\begin{array}{l}0.0 \\
0.0 \\
0.0 \\
0.0 \\
0.0 \\
0.0 \\
0.0\end{array}$ & $\begin{array}{l}-0 . \\
-0 \\
-0 . \\
-0 . \\
-0 . \\
-0 \\
-0 . \\
-0 .\end{array}$ & $\begin{array}{l}0.1 \\
0.1 \\
0.1 \\
0.1 \\
0.1 \\
0.1 \\
0.1 \\
0.1\end{array}$ & $\begin{array}{l}0.180 \\
0.177 \\
0.174 \\
0.171 \\
0.168 \\
0.164 \\
0.161 \\
0.158\end{array}$ \\
\hline $\begin{array}{l}0.1000 \\
0.1250 \\
0.1500 \\
0.1750 \\
0.2000 \\
0.2500 \\
0.3000 \\
0.3500\end{array}$ & $\begin{array}{l}3.480 E+00 \\
3.040 E+00 \\
2.742 E+00 \\
2.528 E+00 \\
2.368 E+00 \\
2.145 E+00 \\
1.999 E+00 \\
1.895 E+00\end{array}$ & $\begin{array}{l}3.9 \\
4.0 \\
4.2 \\
4.3 \\
4.6 \\
5.0\end{array}$ & $\begin{array}{l}3.043 E+0 \\
2.746 E+0 \\
2.533 E+0 \\
2.373 E+0 \\
2.150 E+0 \\
2.004 E+0 \\
1.900 E+0\end{array}$ & $\begin{array}{l}2.468 \\
3.335 \\
4.285 \\
5.306 \\
7.527 \\
9.941 \\
1.251\end{array}$ & $\begin{array}{l}.04 \\
04 \\
.04 \\
.04 \\
.03 \\
03 \\
03 \\
03\end{array}$ & $\begin{array}{l}0.0 \\
0.0 \\
0.0 \\
0.0 \\
0.0 \\
0.0 \\
8.258 \mathrm{E}-03 \\
3.457 \mathrm{E}-02\end{array}$ & $\begin{array}{l}-0.1 \\
-0.1 \\
-0.1 \\
-0.1 \\
-0.1 \\
-0.1 \\
-0.1 \\
-0.0\end{array}$ & $\begin{array}{l}0 \\
0 \\
0 \\
0 \\
0 \\
0 \\
0\end{array}$ & $\begin{array}{l}0.156 \\
0.151 \\
0.147 \\
0.144 \\
0.141 \\
0.137 \\
0.132 \\
0.125\end{array}$ \\
\hline $\begin{array}{l}0.4000 \\
0.4500 \\
0.5000 \\
0.5500 \\
0.6000 \\
0.7000 \\
0.8000 \\
0.9000\end{array}$ & $\begin{array}{l}1.819 E+00 \\
1.762 E+00 \\
1.717 E+00 \\
1.683 E+00 \\
1.655 E+00 \\
1.615 E+00 \\
1.588 E+00\end{array}$ & $\begin{array}{l}6.171 \\
6.598 \\
7.044 \\
7.504 \\
8.467 \\
9.479 \\
1.054\end{array}$ & $\begin{array}{l}1.7 \\
1.7 \\
1.6 \\
1.6 \\
1.6 \\
1.5 \\
1.5\end{array}$ & $\begin{array}{ll} & 01 \\
-01 & 1 \\
0 & 1 \\
-01 & 1 \\
-01 \\
-01 \\
-01 \\
-01\end{array}$ & $\begin{array}{l}-03 \\
-03 \\
-03 \\
-03 \\
-03 \\
-03 \\
-03 \\
-03\end{array}$ & $\begin{array}{l}9.6 \\
1.2 \\
1.6 \\
1.9 \\
2.6 \\
3.3 \\
4.0\end{array}$ & $\begin{array}{l}-0.1 \\
-0 . \\
-0.2 \\
-0.2 \\
-0.1 \\
-0.1 \\
-0 . \\
-0 .\end{array}$ & $\begin{array}{l}0.1 \\
0.1 \\
0.1 \\
0.1 \\
0.1 \\
0.0 \\
0.0\end{array}$ & $\begin{array}{l}0.1119 \\
0.113 \\
0.108 \\
0.104 \\
0.100 \\
0.092 \\
0.087 \\
0.081\end{array}$ \\
\hline $\begin{array}{l}1.0000 \\
1.2500 \\
1.5000 \\
1.7500 \\
2.0000 \\
2.5000 \\
3.0000 \\
3.5000\end{array}$ & $\begin{array}{l}1.540 \\
1.534 \\
1.535 \\
1.538 \\
1.548 \\
1.560 \\
1.572\end{array}$ & $\begin{array}{l}-02 \\
-02 \\
-02 \\
-02 \\
-02 \\
-02 \\
-02\end{array}$ & $\begin{array}{l}1: \\
1: \\
1: \\
1:\end{array}$ & $\begin{array}{l}-01 \\
-01 \\
-01 \\
-01 \\
+00 \\
+00 \\
+00\end{array}$ & $\begin{array}{l}3.8 \\
4.7 \\
5.6 \\
6.6 \\
7.6 \\
9.6 \\
1.1 \\
1.3\end{array}$ & $\begin{array}{l}4.7 \\
6.3 \\
7.9 \\
9.3 \\
1.0 \\
1.3 \\
1.5 \\
1.7\end{array}$ & $\begin{array}{l}-0.1 \\
-0.1 \\
-0.2 \\
-0.1 \\
-0.1 \\
-0.1 \\
-0.2 \\
-0.1\end{array}$ & $\begin{array}{l}0 . \\
0 . \\
0 . \\
0 . \\
0 . \\
0 . \\
0 .\end{array}$ & $\begin{array}{l}0 . \\
0 . \\
0 . \\
0 . \\
0 . \\
0 . \\
0 .\end{array}$ \\
\hline $\begin{array}{l}4.0000 \\
4.5000 \\
5.0000 \\
5.5000 \\
6.0000 \\
7.0000 \\
8.0000 \\
9.0000\end{array}$ & $\begin{array}{l}1.583 E+00 \\
1.593 E+00 \\
1.603 E+00 \\
1.612 E+00 \\
1.621 E+00 \\
1.636 E+00 \\
1.649 E+00 \\
1.661 E+00\end{array}$ & $\begin{array}{l}6.34 \\
7.19 \\
8.06 \\
8.95 \\
1.07 \\
1.26\end{array}$ & $\begin{array}{l}1 . \\
1: \\
1 . \\
1: \\
1: \\
1 .\end{array}$ & $\begin{array}{l}0 \\
0 \\
0 \\
0 \\
0 \\
0\end{array}$ & $\begin{array}{l}1 . \\
1 . \\
2 . \\
2 . \\
2 . \\
2 . \\
3 . \\
3 .\end{array}$ & $\begin{array}{l}1.8 \\
2.0 \\
2.1 \\
2.2 \\
2.4 \\
2.6 \\
2.8 \\
3.0\end{array}$ & $\begin{array}{l}-0.1 \\
-0.1 \\
-0.2 \\
-0.2 \\
-0.2 \\
-0.1 \\
-0.2 \\
-0 .\end{array}$ & $\begin{array}{l}0 . \\
0 . \\
0 . \\
0 . \\
0 . \\
0 . \\
0 . \\
0 .\end{array}$ & $\begin{array}{l}0 . \\
0 . \\
0 . \\
0 . \\
0 . \\
0 . \\
0 .\end{array}$ \\
\hline $\begin{array}{l}10.0000 \\
12.5000 \\
15.0000 \\
17.5000 \\
20.0000 \\
25.0000 \\
30.0000 \\
35.0000\end{array}$ & $\begin{array}{l}1.671 \mathrm{E}+00 \\
1.693 \mathrm{E}+00 \\
1.710 \mathrm{E}+00 \\
1.725 \mathrm{E}+00 \\
1.737 \mathrm{E}+00 \\
1.757 \mathrm{E}+00 \\
1.772 \mathrm{E}+00 \\
1.785 \mathrm{E}+00\end{array}$ & $\begin{array}{l}2.1 \\
2.6 \\
3.1 \\
3.7\end{array}$ & $\begin{array}{l}836 E+00 \\
908 E+00 \\
976 E+00 \\
043 E+00 \\
109 E+00 \\
237 E+00 \\
362 E+00 \\
487 E+00\end{array}$ & $\begin{array}{l}0 \\
0 \\
0 \\
1 \\
1\end{array}$ & $\begin{array}{l}4.349 E-02 \\
5.505 E-02 \\
6.648 E-02 \\
7.774 E-02 \\
8.878 E-02 \\
1.102 E-01 \\
1.305 E-01 \\
1.499 E-01\end{array}$ & $\begin{array}{l}3 . \\
3 . \\
3 . \\
4 . \\
4 . \\
4 .\end{array}$ & $\begin{array}{l}-0.01 \\
-0.01 \\
-0.01 \\
-0.01 \\
-0.01 \\
-0.00 \\
-0.00 \\
-0.00\end{array}$ & $\begin{array}{l}0 . \\
0 . \\
0 . \\
0 . \\
0 . \\
0 .\end{array}$ & \\
\hline $\begin{array}{l}40.0000 \\
45.0000 \\
50.00000 \\
55.0000 \\
60.0000 \\
70.00000 \\
80.0000 \\
90.00000\end{array}$ & $\begin{array}{l}1.796 E+00 \\
1.806 E+00 \\
1.814 E+00 \\
1.822 E+00 \\
1.829 E+00 \\
1.841 E+00 \\
1.851 E+00 \\
1.860 E+00\end{array}$ & $\begin{array}{l}9 \\
1 \\
1 \\
1 \\
1 \\
1 .\end{array}$ & $\begin{array}{l}0 \\
0 \\
0 \\
0 \\
0 \\
0 \\
0\end{array}$ & $\begin{array}{l}1 \\
1 \\
1 \\
1 \\
1\end{array}$ & $\begin{array}{l}1 . \\
1 . \\
2 . \\
2 . \\
2 . \\
2 . \\
2 .\end{array}$ & $\begin{array}{l}2 . \\
5 . \\
6 . \\
6 . \\
6 .\end{array}$ & $\begin{array}{l}-0 \\
-0 \\
-0 \\
-0 \\
-0 \\
-0 \\
-0 \\
-0\end{array}$ & $\begin{array}{l}0 . \\
0 . \\
0 . \\
0 . \\
0 . \\
0 . \\
0 .\end{array}$ & \\
\hline 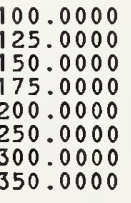 & $\begin{array}{l}0 \\
0 \\
0 \\
0 \\
0 \\
0\end{array}$ & $\begin{array}{l}2 . \\
3 . \\
4 . \\
4 .\end{array}$ & & 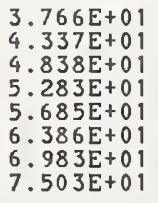 & $\begin{array}{l}3 . \\
4 . \\
4 . \\
4 . \\
5 .\end{array}$ & 8 & $\begin{array}{l}-0 \\
-0 \\
-0 \\
-0 \\
-0 \\
-0 \\
-0\end{array}$ & & \\
\hline $\begin{array}{l}.0000 \\
.00000 \\
.00000 \\
.00000 \\
.00000 \\
.00000 \\
.00000\end{array}$ & $\begin{array}{l}.989 E+00 \\
.996 E+00 \\
.002 E+00 \\
.014 E+00 \\
.024 E+00 \\
.033 E+00\end{array}$ & $\begin{array}{l}1.074 E+01 \\
1.197 E+01 \\
1.320 E+01 \\
1.444 E+01 \\
1.691 E+01 \\
1.939 E+01 \\
2.187 E+01\end{array}$ & $\begin{array}{l}E+01 \\
E+01 \\
E+01 \\
E+01 \\
E+01 \\
E+01 \\
E+01 \\
E+01\end{array}$ & $\begin{array}{l}1 \\
1 \\
1 \\
1 \\
1 \\
1 \\
1\end{array}$ & $\begin{array}{l}6.313 \mathrm{E}-01 \\
6.541 \mathrm{E}-01 \\
6.738 \mathrm{E}-01 \\
6.911 \mathrm{E}-01 \\
7.063 \mathrm{E}-01 \\
7.320 \mathrm{E}-01 \\
7.530 \mathrm{E}-01 \\
7.704 \mathrm{E}-01\end{array}$ & $\begin{array}{l}1 \\
1 \\
1 \\
1 \\
1 \\
1 \\
1\end{array}$ & $\begin{array}{l}-0 \\
-0 \\
-0 \\
-0 \\
-0 \\
-0 \\
-0 \\
-0\end{array}$ & $\begin{array}{l}0 . \\
0 . \\
0 . \\
0 . \\
0 . \\
0 . \\
0 .\end{array}$ & $\begin{array}{l}.000 \\
.000 \\
.000 \\
.000 \\
.000 \\
.000 \\
.000 \\
.000\end{array}$ \\
\hline 00 & $.040 \mathrm{E}+00$ & $.435 E+01$ & $639 E+01$ & $132 E+02$ & $.853 E-01$ & $.195 \mathrm{E}+01$ & -0.000 & 0.003 & 000 \\
\hline
\end{tabular}


ELECTRONS IN METHANE

$I=41.7 \mathrm{eV} \quad$ DENSITY $=6.672 \mathrm{E}-04 \mathrm{~g} / \mathrm{cm}^{3}\left(20^{\circ} \mathrm{C}\right)$

\begin{tabular}{|c|c|c|c|c|c|c|c|c|c|}
\hline \multirow{3}{*}{$\begin{array}{c}\text { ENERGY } \\
\mathrm{MeV}\end{array}$} & \multicolumn{3}{|c|}{ STOPP ING POWER } & \multirow{3}{*}{$\begin{array}{l}\text { CSDA } \\
\text { RANGE } \\
\mathrm{g} / \mathrm{cm}^{2}\end{array}$} & \multirow{3}{*}{$\begin{array}{l}\text { RADIATION } \\
\text { YIELD }\end{array}$} & \multirow{3}{*}{$\begin{array}{l}\text { DEHS.EFF. } \\
\text { COPR } \\
\text { (DELTA) }\end{array}$} & \multicolumn{3}{|c|}{$d(\log ) / d(\log I)$} \\
\hline & OLLISIO & RADIATIVE & TOTAL & & & & COLL & CSDA & $\begin{array}{l}\text { RAD } \\
\text { YIELD }\end{array}$ \\
\hline & $\mathrm{MeV} \mathrm{cm}^{2} / \mathrm{g}$ & $\mathrm{MeV} \mathrm{cm}^{2} / \mathrm{g}$ & $\mathrm{MeV} \mathrm{cm}{ }^{2} / \mathrm{g}$ & & & & & & \\
\hline $\begin{array}{l}0.0100 \\
0.0125 \\
0.0150 \\
0.0175 \\
0.0200 \\
0.0250 \\
0.0300 \\
0.0350\end{array}$ & $\begin{array}{l}2.829 E+01 \\
2.369 E+01 \\
2.049 E+01 \\
1.813 E+01 \\
1.631 E+01 \\
1.369 E+01 \\
1.188 E+01 \\
1.055 E+01\end{array}$ & $\begin{array}{l}2.603 \mathrm{E}-03 \\
2.612 \mathrm{E}-03 \\
2.620 \mathrm{E}-03 \\
2.625 \mathrm{E}-03 \\
2.630 \mathrm{E}-03 \\
2.640 \mathrm{E}-03 \\
2.651 \mathrm{E}-03 \\
2.662 \mathrm{E}-03\end{array}$ & $\begin{array}{l}2.829 E+01 \\
2.369 E+01 \\
2.049 E+01 \\
1.813 E+01 \\
1.632 E+01 \\
1.369 E+01 \\
1.188 E+01 \\
1.056 E+01\end{array}$ & $\begin{array}{l}7 E-04 \\
7 E-04 \\
5 E-04 \\
5 E-04 \\
0 E-04 \\
0 E-03 \\
3 E-03 \\
1 E-03\end{array}$ & $\begin{array}{l}5.026 \mathrm{E}-05 \\
6.045 \mathrm{E}-05 \\
7.022 \mathrm{E}-05 \\
7.967 \mathrm{E}-05 \\
8.884 \mathrm{E}-05 \\
1.065 \mathrm{E}-04 \\
1.234 \mathrm{E}-04 \\
1.397 \mathrm{E}-04\end{array}$ & $\begin{array}{l}0.0 \\
0.0 \\
0.0 \\
0.0 \\
0.0 \\
0.0 \\
0.0 \\
0.0\end{array}$ & $\begin{array}{l}-0.178 \\
-0.171 \\
-0.166 \\
-0.162 \\
-0.159 \\
-0.154 \\
-0.149 \\
-0.146\end{array}$ & $\begin{array}{l}0.200 \\
0.191 \\
0.185 \\
0.180 \\
0.176 \\
0.169 \\
0.164 \\
0.160\end{array}$ & $\begin{array}{l}0.199 \\
0.191 \\
0.184 \\
0.179 \\
0.175 \\
0.169 \\
0.164 \\
0.160\end{array}$ \\
\hline $\begin{array}{l}0.0400 \\
0.0450 \\
0.0500 \\
0.0550 \\
0.0600 \\
0.0700 \\
0.0800 \\
0.0900\end{array}$ & $\begin{array}{l}9.536 E+00 \\
8.730 E+00 \\
8.075 E+00 \\
7.532 E+00 \\
7.074 E+00 \\
6.344 E+00 \\
5.788 E+00 \\
5.349 E+00\end{array}$ & $\begin{array}{l}2.674 \mathrm{E}-03 \\
2.687 \mathrm{E}-03 \\
2.700 \mathrm{E}-03 \\
2.714 \mathrm{E}-03 \\
2.728 \mathrm{E}-03 \\
2.759 \mathrm{E}-03 \\
2.791 \mathrm{E}-03 \\
2.826 \mathrm{E}-03\end{array}$ & $\begin{array}{l}9.539 E+00 \\
8.733 \mathrm{E}+00 \\
8.078 \mathrm{E}+00 \\
7.534 \mathrm{E}+00 \\
7.077 \mathrm{E}+00 \\
6.347 \mathrm{E}+00 \\
5.790 \mathrm{E}+00 \\
5.352 \mathrm{E}+00\end{array}$ & $\begin{array}{l}2.360 \mathrm{E}-03 \\
2.908 \mathrm{E}-03 \\
3.504 \mathrm{E}-03 \\
4.146 \mathrm{E}-03 \\
4.831 \mathrm{E}-03 \\
6.326 \mathrm{E}-03 \\
7.978 \mathrm{E}-03 \\
9.777 \mathrm{E}-03\end{array}$ & $\begin{array}{l}1.556 \mathrm{E}-04 \\
1.710 \mathrm{E}-04 \\
1.860 \mathrm{E}-04 \\
2.006 \mathrm{E}-04 \\
2.150 \mathrm{E}-04 \\
2.429 \mathrm{E}-04 \\
2.698 \mathrm{E}-04 \\
2.960 \mathrm{E}-04\end{array}$ & $\begin{array}{l}0.0 \\
0.0 \\
0.0 \\
0.0 \\
0.0 \\
0.0 \\
0.0 \\
0.0\end{array}$ & $\begin{array}{l}-0.144 \\
-0.141 \\
-0.139 \\
-0.138 \\
-0.136 \\
-0.133 \\
-0.131 \\
-0.129\end{array}$ & $\begin{array}{l}0.157 \\
0.154 \\
0.152 \\
0.150 \\
0.148 \\
0.145 \\
0.142 \\
0.140\end{array}$ & $\begin{array}{l}0.157 \\
0.154 \\
0.152 \\
0.150 \\
0.148 \\
0.145 \\
0.142 \\
0.140\end{array}$ \\
\hline $\begin{array}{l}0.1000 \\
0.1250 \\
0.1500 \\
0.1750 \\
0.2000 \\
0.2500 \\
0.3000 \\
0.3500\end{array}$ & $\begin{array}{l}4.995 E+00 \\
4.350 E+00 \\
3.915 E+00 \\
3.603 E+00 \\
3.369 E+00 \\
3.044 E+00 \\
2.831 E+00 \\
2.683 E+00\end{array}$ & $\begin{array}{l}2.862 \mathrm{E}-03 \\
2.960 \mathrm{E}-03 \\
3.066 \mathrm{E}-03 \\
3.178 \mathrm{E}-03 \\
3.297 \mathrm{E}-03 \\
3.550 \mathrm{E}-03 \\
3.826 \mathrm{E}-03 \\
4.121 \mathrm{E}-03\end{array}$ & $\begin{array}{l}4.998 \mathrm{E}+00 \\
4.353 \mathrm{E}+00 \\
3.918 \mathrm{E}+00 \\
3.606 \mathrm{E}+00 \\
3.372 \mathrm{E}+00 \\
3.047 \mathrm{E}+00 \\
2.835 \mathrm{E}+00 \\
2.687 \mathrm{E}+00\end{array}$ & $\begin{array}{l}1.171 \mathrm{E}-02 \\
1.709 \mathrm{E}-02 \\
2.316 \mathrm{E}-02 \\
2.983 \mathrm{E}-02 \\
3.701 \mathrm{E}-02 \\
5.266 \mathrm{E}-02 \\
6.971 \mathrm{E}-02 \\
8.785 \mathrm{E}-02\end{array}$ & $\begin{array}{l}3.214 E-04 \\
3.825 E-04 \\
4.407 E-04 \\
4.966 E-04 \\
5.508 E-04 \\
6.550 E-04 \\
7.554 E-04 \\
8.534 E-04\end{array}$ & $\begin{array}{l}0.0 \\
0.0 \\
0.0 \\
0.0 \\
0.0 \\
0.0 \\
0.0 \\
0.0\end{array}$ & $\begin{array}{l}-0.128 \\
-0.124 \\
-0.122 \\
-0.119 \\
-0.118 \\
-0.115 \\
-0.1112 \\
-0.1110\end{array}$ & $\begin{array}{l}0.138 \\
0.134 \\
0.131 \\
0.129 \\
0.127 \\
0.124 \\
0.121 \\
0.119\end{array}$ & $\begin{array}{l}0.138 \\
0.134 \\
0.131 \\
0.128 \\
0.126 \\
0.123 \\
0.120 \\
0.118\end{array}$ \\
\hline $\begin{array}{l}0.4000 \\
0.4500 \\
0.5000 \\
0.5500 \\
0.6000 \\
0.7000 \\
0.8000 \\
0.9000\end{array}$ & $\begin{array}{l}2.576 E+00 \\
2.496 E+00 \\
2.435 E+00 \\
2.388 E+00 \\
2.351 E+00 \\
2.298 E+00 \\
2.264 E+00 \\
2.243 E+00\end{array}$ & $\begin{array}{l}4.434 E-03 \\
4.764 E-03 \\
5.109 E-03 \\
5.467 E-03 \\
5.839 E-03 \\
6.616 E-03 \\
7.432 E-03 \\
8.287 E-03\end{array}$ & $\begin{array}{l}2.580 \mathrm{E}+00 \\
2.501 \mathrm{E}+00 \\
2.440 \mathrm{E}+00 \\
2.393 \mathrm{E}+00 \\
2.357 \mathrm{E}+00 \\
2.305 \mathrm{E}+00 \\
2.272 \mathrm{E}+00 \\
2.251 \mathrm{E}+00\end{array}$ & $\begin{array}{l}1.069 \mathrm{E}-01 \\
1.266 \mathrm{E}-01 \\
1.468 \mathrm{E}-01 \\
1.675 \mathrm{E}-01 \\
1.886 \mathrm{E}-01 \\
2.315 \mathrm{E}-01 \\
2.752 \mathrm{E}-01 \\
3.195 \mathrm{E}-01\end{array}$ & $\begin{array}{l}9.500 \mathrm{E}-04 \\
1.046 \mathrm{E}-03 \\
1.141 \mathrm{E}-03 \\
1.236 \mathrm{E}-03 \\
1.332 \mathrm{E}-03 \\
1.523 \mathrm{E}-03 \\
1.717 \mathrm{E}-03 \\
1.912 \mathrm{E}-03\end{array}$ & $\begin{array}{l}0.0 \\
0.0 \\
0.0 \\
0.0 \\
0.0 \\
0.0 \\
0.0 \\
0.0\end{array}$ & $\begin{array}{l}-0.109 \\
-0.107 \\
-0.106 \\
-0.104 \\
-0.103 \\
-0.101 \\
-0.100 \\
-0.098\end{array}$ & $\begin{array}{l}0.1117 \\
0.1116 \\
0.1114 \\
0.1113 \\
0.1112 \\
0.1110 \\
0.109 \\
0.107\end{array}$ & $\begin{array}{l}0.1116 \\
0.1114 \\
0.113 \\
0.1112 \\
0.1110 \\
0.108 \\
0.107 \\
0.105\end{array}$ \\
\hline $\begin{array}{l}1.0000 \\
1.2500 \\
1.5000 \\
1.7500 \\
2.0000 \\
2.5000 \\
3.0000 \\
3.5000\end{array}$ & $\begin{array}{l}2.230 E+00 \\
2.217 E+00 \\
2.221 E+00 \\
2.232 E+00 \\
2.247 E+00 \\
2.280 E+00 \\
2.314 E+00 \\
2.345 E+00\end{array}$ & $\begin{array}{l}9.176 E-03 \\
1.153 \mathrm{E}-02 \\
1.405 \mathrm{E}-02 \\
1.671 \mathrm{E}-02 \\
1.949 \mathrm{E}-02 \\
2.534 \mathrm{E}-02 \\
3.152 \mathrm{E}-02 \\
3.795 \mathrm{E}-02\end{array}$ & $\begin{array}{l}2.239 \mathrm{E}+00 \\
2.229 \mathrm{E}+00 \\
2.235 \mathrm{E}+00 \\
2.249 \mathrm{E}+00 \\
2.267 \mathrm{E}+00 \\
2.306 \mathrm{E}+00 \\
2.345 \mathrm{E}+00 \\
2.383 \mathrm{E}+00\end{array}$ & $\begin{array}{l}3.640 E-01 \\
4.760 E-01 \\
5.881 E-01 \\
6.996 E-01 \\
8.103 E-01 \\
1.029 E+00 \\
1.244 E+00 \\
1.456 E+00\end{array}$ & $\begin{array}{l}2.110 \mathrm{E}-03 \\
2.614 \mathrm{E}-03 \\
3.133 \mathrm{E}-03 \\
3.665 \mathrm{E}-03 \\
4.208 \mathrm{E}-03 \\
5.325 \mathrm{E}-03 \\
6.473 \mathrm{E}-03 \\
7.645 \mathrm{E}-03\end{array}$ & $\begin{array}{l}0.0 \\
0.0 \\
0.0 \\
0.0 \\
0.0 \\
0.0 \\
0.0 \\
0.0\end{array}$ & $\begin{array}{l}-0.097 \\
-0.094 \\
-0.092 \\
-0.090 \\
-0.089 \\
-0.087 \\
-0.085 \\
-0.083\end{array}$ & $\begin{array}{l}0.1 \\
0.1 \\
0.1 \\
0.1 \\
0.0 \\
0.0 \\
0.0\end{array}$ & $\begin{array}{l}0.104 \\
0.101 \\
0.098 \\
0.096 \\
0.094 \\
0.092 \\
0.089 \\
0.088\end{array}$ \\
\hline $\begin{array}{l}4.0000 \\
4.5000 \\
5.0000 \\
5.5000 \\
6.0000 \\
7.0000 \\
8.0000 \\
9.0000\end{array}$ & $\begin{array}{l}2.375 E+00 \\
2.403 E+00 \\
2.428 E+00 \\
2.452 E+00 \\
2.474 E+00 \\
2.514 E+00 \\
2.549 E+00 \\
2.581 E+00\end{array}$ & $\begin{array}{l}4.462 E-02 \\
5.148 E-02 \\
5.852 E-02 \\
6.571 E-02 \\
7.303 E-02 \\
8.805 E-02 \\
1.035 E-01 \\
1.193 E-01\end{array}$ & $\begin{array}{l}2.420 E+00 \\
2.454 E+00 \\
2.487 E+00 \\
2.517 E+00 \\
2.547 E+00 \\
2.602 E+00 \\
2.653 E+00 \\
2.700 E+00\end{array}$ & $\begin{array}{l}1.664 E+00 \\
1.869 E+00 \\
2.071 E+00 \\
2.271 E+00 \\
2.469 E+00 \\
2.857 E+00 \\
3.238 E+00 \\
3.611 E+00\end{array}$ & $\begin{array}{l}8.8 \\
1.8 \\
1: \\
1: 2 \\
1: \\
1.8 \\
2 .\end{array}$ & $\begin{array}{l}0.0 \\
0.0 \\
0.0 \\
0.0 \\
0.0 \\
0.0 \\
0.0 \\
0.0\end{array}$ & $\begin{array}{l}-0 . \\
-0 . \\
-0 . \\
-0 \\
-0 . \\
-0 . \\
-0 \\
-0 .\end{array}$ & $\begin{array}{l}0.091 \\
0.090 \\
0.088 \\
0.087 \\
0.087 \\
0.085 \\
0.084 \\
0.082\end{array}$ & $\begin{array}{l}0.086 \\
0.085 \\
0.083 \\
0.082 \\
0.081 \\
0.079 \\
0.078 \\
0.077\end{array}$ \\
\hline $\begin{array}{l}10.0000 \\
12.5000 \\
15.0000 \\
17.5000 \\
20.0000 \\
25.0000 \\
30.0000 \\
35.0000\end{array}$ & $\begin{array}{l}2.610 \mathrm{E}+00 \\
2.671 \mathrm{E}+00 \\
2.722 \mathrm{E}+00 \\
2.765 \mathrm{E}+00 \\
2.803 \mathrm{E}+00 \\
2.863 \mathrm{E}+00 \\
2.904 \mathrm{E}+00 \\
2.935 \mathrm{E}+00\end{array}$ & $\begin{array}{l}1.354 E-01 \\
1.767 E-01 \\
2.194 E-01 \\
2.629 E-01 \\
3.073 E-01 \\
3.977 E-01 \\
4.899 E-01 \\
5.835 E-01\end{array}$ & $\begin{array}{l}2.745 E+00 \\
2.848 E+00 \\
2.941 E+00 \\
3.028 E+00 \\
3.110 E+00 \\
3.261 E+00 \\
3.394 E+00 \\
3.519 E+00\end{array}$ & $\begin{array}{l}3.978 E+00 \\
4.872 E+00 \\
5.736 E+00 \\
6.573 E+00 \\
7.388 E+00 \\
8.957 E+00 \\
1.046 E+01 \\
1.191 E+01\end{array}$ & $\begin{array}{l}2.385 \mathrm{E}-02 \\
3.022 \mathrm{E}-02 \\
3.657 \mathrm{E}-02 \\
4.288 \mathrm{E}-02 \\
4.912 \mathrm{E}-02 \\
6.139 \mathrm{E}-02 \\
7.336 \mathrm{E}-02 \\
8.505 \mathrm{E}-02\end{array}$ & $\begin{array}{l}0.0 \\
0.0 \\
0.0 \\
0.0 \\
0.0 \\
3.726 \mathrm{E}-02 \\
1.461 \mathrm{E}-01 \\
2.797 \mathrm{E}-01\end{array}$ & $\begin{array}{l}-0.0 \\
-0.0 \\
-0.0 \\
-0.0 \\
-0.0 \\
-0.0 \\
-0.0 \\
-0.0\end{array}$ & $\begin{array}{l}0 . \\
0 . \\
0 . \\
0 . \\
0 . \\
0 . \\
0 .\end{array}$ & $\begin{array}{l}0.075 \\
0.073 \\
0.071 \\
0.069 \\
0.068 \\
0.063 \\
0.056 \\
0.049\end{array}$ \\
\hline $\begin{array}{l}40.0000 \\
45.0000 \\
50.0000 \\
55.0000 \\
60.0000 \\
70.0000 \\
80.0000 \\
90.0000\end{array}$ & $\begin{array}{l}2.960 E+00 \\
2.981 E+00 \\
2.998 E+00 \\
3.014 E+00 \\
3.027 E+00 \\
3.051 E+00 \\
3.070 E+00 \\
3.087 E+00\end{array}$ & $\begin{array}{l}6.7 \\
7.7 \\
8.6 \\
9.6 \\
1.0 \\
1.2 \\
1.4 \\
1.6\end{array}$ & $\begin{array}{l}3.638 E+00 \\
3.754 E+00 \\
3.868 E+00 \\
3.980 E+00 \\
4.091 E+00 \\
4.311 E+00 \\
4.528 E+00 \\
4.744 E+00\end{array}$ & $\begin{array}{l}1.330 E+01 \\
1.466 E+01 \\
1.597 E+01 \\
1.724 E+01 \\
1.848 E+01 \\
2.086 E+01 \\
2.312 E+01 \\
2.528 E+01\end{array}$ & $\begin{array}{l}9.644 \mathrm{E}-02 \\
1.075 \mathrm{E}-01 \\
1.183 \mathrm{E}-01 \\
1.288 \mathrm{E}-01 \\
1.391 \mathrm{E}-01 \\
1.587 \mathrm{E}-01 \\
1.773 \mathrm{E}-01 \\
1.949 \mathrm{E}-01\end{array}$ & $\begin{array}{l}4.187 E-0 \\
5.553 E-0 \\
6.865 E-0 \\
8.112 E-0 \\
9.293 E-0 \\
1.146 E+0 \\
1.341 E+0 \\
1.517 E+0\end{array}$ & $\begin{array}{l}-0 . \\
-0 \\
-0 \\
-0 \\
-0 \\
-0 \\
-0 \\
-0 .\end{array}$ & $\begin{array}{l}0.058 \\
0.055 \\
0.052 \\
0.049 \\
0.047 \\
0.043 \\
0.040 \\
0.037\end{array}$ & $\begin{array}{l}0.044 \\
0.039 \\
0.036 \\
0.032 \\
0.030 \\
0.026 \\
0.023 \\
0.020\end{array}$ \\
\hline $\begin{array}{l}.0000 \\
.0000 \\
.0000 \\
.0000 \\
.0000 \\
.0000 \\
.0000\end{array}$ & $\begin{array}{l}3.102 E+00 \\
3.133 E+00 \\
3.158 E+00 \\
3.178 E+00 \\
3.196 E+00 \\
3.224 E+00 \\
3.247 E+00 \\
3.266 E+00\end{array}$ & $\begin{array}{l}1.857 E+00 \\
2.361 E+00 \\
2.869 E+00 \\
3.380 E+00 \\
3.893 E+00 \\
4.926 E+00 \\
5.964 E+00 \\
7.005 E+00\end{array}$ & $\begin{array}{l}4.959 E+00 \\
5.494 E+00 \\
6.027 E+00 \\
6.558 E+00 \\
7.089 E+00 \\
8.150 E+00 \\
9.211 E+00 \\
1.027 E+01\end{array}$ & $\begin{array}{l}2.734 E+01 \\
3.213 E+01 \\
3.647 E+01 \\
4.045 E+01 \\
4.411 E+01 \\
5.069 E+01 \\
5.645 E+01 \\
6.159 E+01\end{array}$ & $\begin{array}{l}2.116 \mathrm{E}-01 \\
2.499 \mathrm{E}-01 \\
2.838 \mathrm{E}-01 \\
3.142 \mathrm{E}-01 \\
3.415 \mathrm{E}-01 \\
3.888 \mathrm{E}-01 \\
4.284 \mathrm{E}-01 \\
4.623 \mathrm{E}-01\end{array}$ & $\begin{array}{l}1.677 E+00 \\
2.023 E+00 \\
2.312 E+00 \\
2.560 E+00 \\
2.778 E+00 \\
3.148 E+00 \\
3.457 E+00 \\
3.724 E+00\end{array}$ & $\begin{array}{l}-0.014 \\
-0.013 \\
-0.012 \\
-0.011 \\
-0.011 \\
-0.0110 \\
-0.008 \\
-0.008\end{array}$ & $\begin{array}{l}0.035 \\
0.031 \\
0.028 \\
0.026 \\
0.024 \\
0.022 \\
0.020 \\
0.019\end{array}$ & $\begin{array}{l}0.018 \\
0.015 \\
0.013 \\
0.011 \\
0.010 \\
0.008 \\
0.007 \\
0.006\end{array}$ \\
\hline $\begin{array}{l}0.0000 \\
0.0000 \\
0.0000 \\
0.0000 \\
0.0000 \\
0.0000 \\
0.0000 \\
0.0000\end{array}$ & $\begin{array}{l}3.282 E+00 \\
3.295 E+00 \\
3.307 E+00 \\
3.318 E+00 \\
3.328 E+00 \\
3.344 E+00 \\
3.358 E+00 \\
3.370 E+00\end{array}$ & $\begin{array}{l}8.051 E+00 \\
9.099 E+00 \\
1.015 E+01 \\
1.120 E+01 \\
1.225 E+01 \\
1.436 E+01 \\
1.648 E+01 \\
1.859 E+01\end{array}$ & $\begin{array}{l}1.133 E+01 \\
1.239 E+01 \\
1.346 E+01 \\
1.452 E+01 \\
1.558 E+01 \\
1.771 E+01 \\
1.983 E+01 \\
2.196 E+01\end{array}$ & $\begin{array}{l}7.044 E+01 \\
7.431 E+01 \\
7.789 E+01 \\
8.121 E+01 \\
8.723 E+01 \\
9.256 E+01 \\
9.735 E+01\end{array}$ & $\begin{array}{l}5.1 \\
5.4 \\
5.6 \\
5.7 \\
6.1 \\
6.3 \\
6.5\end{array}$ & $\begin{array}{l}3.959 E+00 \\
4.169 E+00 \\
4.360 E+00 \\
4.535 E+00 \\
4.696 E+00 \\
4.985 E+00 \\
5.238 E+00 \\
5.464 E+00\end{array}$ & $\begin{array}{l}-0 . \\
-0 \\
-0 \\
-0 \\
-0 \\
-0 \\
-0 \\
-0 \\
-0\end{array}$ & $\begin{array}{l}0.017 \\
0.0116 \\
0.0116 \\
0.015 \\
0.014 \\
0.0114 \\
0.013 \\
0.012\end{array}$ & $\begin{array}{l}0.006 \\
0.005 \\
0.005 \\
0.004 \\
0.004 \\
0.0003 \\
0.003 \\
0.002\end{array}$ \\
\hline 0 & $3.381 E+00$ & $2.071 E+01$ & $2.409 \mathrm{E}+01$ & $.017 E+02$ & $6.784 E-01$ & $5.668 E+00$ & -0.002 & 0.018 & 0.002 \\
\hline
\end{tabular}


ELECTRONS IN MUSCLE, SKELETAL (ICRP)

$I=75.3 \mathrm{eV} \quad$ DENSITY $=1.040 \mathrm{E}+00 \mathrm{~g} / \mathrm{cm}^{3}$

\begin{tabular}{|c|c|c|c|c|c|c|c|c|c|}
\hline GY & OLLISIOH & PING POL & TOTAL & $\begin{array}{l}\text { CSDA } \\
\text { RANGE }\end{array}$ & N & F. & $\begin{array}{c}d(J) \\
\text { COLL }\end{array}$ & CSD & $\begin{array}{l}\text { I) } \\
\text { RAD } \\
\text { IELD }\end{array}$ \\
\hline $\mathrm{MeV}$ & $\mathrm{MeV} \mathrm{cm}^{2} / \mathrm{g}$ & $\mathrm{MeV} \mathrm{cm}^{2} / \mathrm{g}$ & $\mathrm{MeV} \mathrm{cm}^{2} / \mathrm{g}$ & $g / \mathrm{cm}^{2}$ & & & & & \\
\hline $\begin{array}{l}0.0100 \\
0.0125 \\
0.0150 \\
0.0175 \\
0.0200 \\
0.0250 \\
0.0300 \\
0.0350\end{array}$ & $\begin{array}{l}2.2 \\
1.8 \\
1.6 \\
1.4 \\
1.3 \\
1.0 \\
9.5 \\
8.4\end{array}$ & $\begin{array}{l}3.8 \\
3.8 \\
3.8 \\
3.8 \\
3.8 \\
3.9 \\
3.9\end{array}$ & $\begin{array}{l}1 . \\
1 . \\
1 . \\
1 . \\
9 .\end{array}$ & $\begin{array}{l}2.5 \\
3.7 \\
5.2 \\
6.8 \\
8.6 \\
1.2 \\
1.7 \\
2.3\end{array}$ & $\begin{array}{l}9 . \\
1 . \\
1 . \\
1 . \\
1 . \\
1 . \\
2 .\end{array}$ & $\begin{array}{l}0.0 \\
0.0 \\
0.0 \\
0.0 \\
0.0 \\
0.0 \\
0.0 \\
0.0\end{array}$ & $\begin{array}{l}-0 \\
-0 \\
-0 \\
-0 \\
-0 \\
-0 \\
-0\end{array}$ & $\begin{array}{l}.228 \\
.217 \\
.209 \\
.202 \\
.197 \\
.189 \\
.183 \\
.178\end{array}$ & $\begin{array}{l}0.226 \\
0.215 \\
0.207 \\
0.201 \\
0.196 \\
0.188 \\
0.182 \\
0.177\end{array}$ \\
\hline $\begin{array}{l}0.0400 \\
0.0450 \\
0.0500 \\
0.0550 \\
0.0600 \\
0.0700 \\
0.0800 \\
0.0900\end{array}$ & $\begin{array}{l}+00 \\
+00 \\
+00 \\
+00 \\
+00 \\
+00 \\
+00 \\
+00\end{array}$ & $\begin{array}{l}3.9 \\
3.9 \\
3.9 \\
4.0 \\
4.0 \\
4.0\end{array}$ & $\begin{array}{l}7 . \\
6 . \\
6 . \\
5 . \\
5 . \\
4 .\end{array}$ & $\begin{array}{l}.03 \\
03 \\
03 \\
03 \\
03 \\
03 \\
03 \\
03 \\
03 \\
02\end{array}$ & $\begin{array}{l}04 \\
04 \\
04 \\
04 \\
04 \\
04 \\
04\end{array}$ & $\begin{array}{l}0.0 \\
0.0 \\
0.0 \\
0.0 \\
0.0 \\
0.0 \\
0.0\end{array}$ & $\begin{array}{l}-0 \\
-0 \\
-0 \\
-0 \\
-0 \\
-0 \\
-0 \\
-0\end{array}$ & $\begin{array}{l}4 \\
0 \\
7 \\
5 \\
2 \\
9 \\
5\end{array}$ & $\begin{array}{l}0.173 \\
0.170 \\
0.167 \\
0.164 \\
0.162 \\
0.158 \\
0.155\end{array}$ \\
\hline $\begin{array}{l}0.1000 \\
0.1250 \\
0.1500 \\
0.1750 \\
0.2000 \\
0.2500 \\
0.3000 \\
0.3500\end{array}$ & $\begin{array}{l}4.071 E+00 \\
3.552 E+00 \\
3.203 E+00 \\
2.951 E+00 \\
2.763 E+00 \\
2.501 E+00 \\
2.329 E+00 \\
2.211 E+00\end{array}$ & $\begin{array}{l}-03 \\
-03 \\
-03 \\
-03 \\
-03 \\
-03 \\
-03 \\
-03\end{array}$ & $\begin{array}{l}3 . \\
3 . \\
2 . \\
2 . \\
2 . \\
2 .\end{array}$ & $\begin{array}{l}1.4 \\
2.1 \\
2.8 \\
3.6 \\
4.5 \\
6.4 \\
8.5 \\
1.0\end{array}$ & $\begin{array}{l}6 . \\
7 . \\
8 . \\
9 . \\
1 . \\
1 .\end{array}$ & $\begin{array}{l}0.0 \\
0.0 \\
0.0 \\
0.0 \\
0.0 \\
0.0 \\
0.0 \\
0.0\end{array}$ & $\begin{array}{l}-0.1 \\
-0.1 \\
-0.1 \\
-0.1 \\
-0.1 \\
-0.1 \\
-0.1 \\
-0.1\end{array}$ & $\begin{array}{l}0.1 \\
0.1 \\
0.1 \\
0.1 \\
0.1 \\
0.1\end{array}$ & $\begin{array}{l}0.145 \\
0.142 \\
0.139 \\
0.137 \\
0.133 \\
0.129\end{array}$ \\
\hline $\begin{array}{l}0.4000 \\
0.4500 \\
0.5000 \\
0.5500 \\
0.6000 \\
0.7000 \\
0.8000 \\
0.9000\end{array}$ & $\begin{array}{l}2.125 E+00 \\
2.061 E+00 \\
2.012 E+00 \\
1.972 E+00 \\
1.941 E+00 \\
1.895 E+00 \\
1.863 E+00 \\
1.842 E+00\end{array}$ & $\begin{array}{l}-03 \\
-03 \\
-03 \\
-03 \\
-03 \\
-03 \\
-02 \\
-02\end{array}$ & $\begin{array}{l}2.0 \\
2.0 \\
1.9 \\
1.9 \\
1.9 \\
1.8 \\
1.8\end{array}$ & $\begin{array}{l}1.3 \\
1.5 \\
1.7 \\
2.0 \\
2.2 \\
2.8 \\
3.3 \\
3.8\end{array}$ & $\begin{array}{l}1.8 \\
1 . \\
2 . \\
2 . \\
2 . \\
2 . \\
3 .\end{array}$ & $\begin{array}{l}0.0 \\
0.0 \\
2.181 \mathrm{E}-03 \\
2.071 \mathrm{E}-02 \\
4.241 \mathrm{E}-02 \\
9.262 \mathrm{E}-02 \\
1.488 \mathrm{E}-01 \\
2.084 \mathrm{E}-01\end{array}$ & $\begin{array}{l}-0 \\
-0 \\
-0 \\
-0 \\
-0 \\
-0 \\
-0 \\
-0\end{array}$ & $\begin{array}{l}0.1 \\
0.1 \\
0.1 \\
0.1 \\
0.1\end{array}$ & $\begin{array}{l}0 \\
0 \\
0 \\
0 \\
0 \\
0\end{array}$ \\
\hline $\begin{array}{l}1.0000 \\
1.2500 \\
1.5000 \\
1.7500 \\
2.0000 \\
2.5000 \\
3.0000 \\
3.5000\end{array}$ & $\begin{array}{l}1.827 E+00 \\
1.806 E+00 \\
1.799 E+00 \\
1.799 E+00 \\
1.801 E+00 \\
1.812 E+00 \\
1.824 E+00 \\
1.836 E+00\end{array}$ & $\begin{array}{l}1.262 \\
1.578 \\
1.916 \\
2.271 \\
2.642 \\
3.421 \\
4.241 \\
5.095\end{array}$ & $\begin{array}{l}1.839 \mathrm{E}+0 \\
1.822 \mathrm{E}+0 \\
1.818 \mathrm{E}+0 \\
1.821 \mathrm{E}+0 \\
1.828 \mathrm{E}+0 \\
1.846 \mathrm{E}+0 \\
1.866 \mathrm{E}+0 \\
1.887 \mathrm{E}+0\end{array}$ & $\begin{array}{l}7.15 \\
8.53 \\
9.90 \\
1.26 \\
1.53 \\
1.79\end{array}$ & $\begin{array}{l}4.408 \\
5.272 \\
6.162 \\
7.074 \\
8.956 \\
1.090 \\
1.289\end{array}$ & $\begin{array}{l}2.6 \\
4.2 \\
5.7 \\
7.1 \\
8.5 \\
1.0 \\
1.3 \\
1.5\end{array}$ & & $\begin{array}{l}0.1 \\
0.0 \\
0.2 \\
0.0 \\
0.0 \\
0.8 \\
0.8 \\
0.0\end{array}$ & $\begin{array}{l}0 \\
0 \\
0 \\
0 \\
0 \\
0 \\
0\end{array}$ \\
\hline $\begin{array}{l}4.00000 \\
4.5000 \\
5.0000 \\
5.50000 \\
6.00000 \\
7.00000 \\
8.0000 \\
9.00000\end{array}$ & $\begin{array}{l}1.860 \\
1.870 \\
1.880 \\
1.889 \\
1.906\end{array}$ & $\begin{array}{l}6.8 \\
7.8 \\
8.7 \\
9.7 \\
1.1 \\
1.3 \\
1.5\end{array}$ & $\begin{array}{l}1.5 \\
1.5 \\
1.5 \\
1.5 \\
2.0\end{array}$ & $\begin{array}{l}2.3 \\
2.5 \\
2.8 \\
3.0 \\
3.5 \\
4.0 \\
4.5\end{array}$ & $\begin{array}{l}-02 \\
-02 \\
-02 \\
-02 \\
-02 \\
-02 \\
-02 \\
-02 \\
-02\end{array}$ & $\begin{array}{l}1.6 \\
1.8 \\
1.9 \\
2.1 \\
2.2 \\
2.4 \\
2.6 \\
2.8\end{array}$ & $\begin{array}{l}-0 . \\
-0 . \\
-0 . \\
-0 . \\
-0 . \\
-0 . \\
-0 . \\
-0 .\end{array}$ & $\begin{array}{l}0 . \\
0 . \\
0 . \\
0 .\end{array}$ & \\
\hline $\begin{array}{l}10.0000 \\
12.5000 \\
15.0000 \\
17.5000 \\
20.0000 \\
25.0000\end{array}$ & $\begin{array}{l}.946 E+00 \\
.971 E+00 \\
.992 E+00 \\
.009 E+00 \\
.023 E+00 \\
.047 E+00 \\
.066 E+00 \\
.081 E+00\end{array}$ & $\begin{array}{l}2.3 \\
2.8 \\
3.4 \\
4.0 \\
5.2 \\
6.4 \\
7.6\end{array}$ & $\begin{array}{l}2 . \\
2 . \\
2 . \\
2 . \\
2 . \\
2 .\end{array}$ & $\begin{array}{l}6.1 \\
7.3 \\
8.3 \\
9.4 \\
1.1\end{array}$ & $\begin{array}{l}\dot{E}-02 \\
-02 \\
-02 \\
-02 \\
-02 \\
-02 \\
-02 \\
-0 \\
-01 \\
-01 \\
-01 \\
-01\end{array}$ & $\begin{array}{l}3.3 \\
3.6 \\
3.8 \\
4.1 \\
4.4\end{array}$ & $\begin{array}{l}-0 . \\
-0 . \\
-0 . \\
-0 . \\
-0 . \\
-0 . \\
-0 . \\
-0 .\end{array}$ & $\begin{array}{l}0.1 \\
0.5 \\
0.1 \\
0.1 \\
0.1\end{array}$ & \\
\hline $\begin{array}{l}.0000 \\
.0000 \\
.0000 \\
.0000 \\
.0000 \\
.0000\end{array}$ & $\begin{array}{ll}+ & 0 \\
+ & 0 \\
+0 & 0 \\
+0 & 0 \\
+ & 0 \\
+0 & 0 \\
+ & 0 \\
+ & 0\end{array}$ & $\begin{array}{l}1 \\
1 \\
1 \\
1 \\
1 \\
1 \\
2\end{array}$ & $\begin{array}{l}0 \\
0 \\
0 \\
0 \\
0 \\
0\end{array}$ & $\begin{array}{l}1.8 \\
2.0 \\
2.1 \\
2.3 \\
2.5 \\
2.8 \\
3.0\end{array}$ & $\begin{array}{ll}0 & 1 \\
0 & 1 \\
0 & 1 \\
0 & 1 \\
0 & 1 \\
0 & 1 \\
01 & 1 \\
0 & 1\end{array}$ & $\begin{array}{l}5 . \\
5 . \\
5 . \\
5 . \\
6 . \\
6 .\end{array}$ & $\begin{array}{l}-0 . \\
-0 . \\
-0 . \\
-0 . \\
-0 . \\
-0 . \\
-0 . \\
-0 .\end{array}$ & $\begin{array}{l}0 . \\
0 . \\
0 . \\
0 . \\
0 . \\
0 .\end{array}$ & \\
\hline $\begin{array}{ll}0 & 0 \\
0 & 0\end{array}$ & $\begin{array}{l}177 \mathrm{E}+00 \\
197 \mathrm{E}+00 \\
212 \mathrm{E}+00 \\
226 \mathrm{E}+00 \\
237 \mathrm{E}+00 \\
256 \mathrm{E}+00 \\
271 \mathrm{E}+00 \\
.284 \mathrm{E}+00\end{array}$ & $\begin{array}{l}0 \\
0 \\
0 \\
0 \\
0 \\
0 \\
0 \\
0\end{array}$ & & $\begin{array}{l}1 \\
1 \\
1 \\
1 \\
1 \\
1\end{array}$ & $\begin{array}{l}1 \\
1 \\
1 \\
1 \\
1 \\
1 \\
1 \\
1\end{array}$ & $\begin{array}{l}7 . \\
7 . \\
7.8 \\
8 . \\
8 . \\
8 . \\
9 .\end{array}$ & $\begin{array}{l}-0 . \\
-0 . \\
-0 . \\
-0 . \\
-0 . \\
-0 . \\
-0 .\end{array}$ & $\begin{array}{l}9 \\
8 \\
7 \\
6 \\
6 \\
5 \\
5 \\
4\end{array}$ & \\
\hline $\begin{array}{llll}0 & 0 & 0 & 0 \\
0 & 0 & 0 & 0 \\
0 & 0 & 0 & 0 \\
0 & 0 & 0 & 0\end{array}$ & $\begin{array}{l}2.296 E+00 \\
2.306 E+00 \\
2.315 E+00 \\
2.323 E+00 \\
2.330 E+00 \\
2.343 E+00 \\
2.354 E+00 \\
2.364 E+00\end{array}$ & 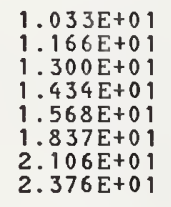 & $\begin{array}{l}397 E+01 \\
532 E+01 \\
667 E+01 \\
801 E+01 \\
072 E+01 \\
342 E+01 \\
612 E+01\end{array}$ & $\begin{array}{l}7.8 \\
8.1 \\
8.4 \\
8.9 \\
9.3\end{array}$ & $\begin{array}{lll}0 & 1 \\
0 & 1 \\
0 & 1 \\
0 & 1 \\
0 & 1 \\
0 & 1 \\
0 & 1 \\
0 & 1\end{array}$ & $\begin{array}{ll}0 & 0 \\
0 & 1 \\
0 & 1 \\
0 & 1 \\
0 & 1 \\
0 & 1 \\
01 & 1 \\
01 & 1 \\
0 & 1\end{array}$ & $\begin{array}{l}-0 . \\
-0 . \\
-0 . \\
-0 . \\
-0 . \\
-0 . \\
-0 .\end{array}$ & $\begin{array}{l}0 . \\
0 . \\
0 . \\
0 . \\
0 . \\
0 . \\
0 .\end{array}$ & $\begin{array}{ll} & 1 \\
0 & 0 \\
0 & 0 \\
0 & 0 \\
0 & 0 \\
0 & 0 \\
0 & 0 \\
0 & 0\end{array}$ \\
\hline 20 & $E+00$ & $645 E+01$ & $883 E+01$ & +02 & $56 \mathrm{E}-01$ & $168 E+01$ & 000 & 0.003 & 0.000 \\
\hline
\end{tabular}




\begin{tabular}{|c|c|c|c|c|c|c|c|c|c|}
\hline ENERGY & COLLISION & $\begin{array}{l}\text { PING POW } \\
\text { RADIAT IVE }\end{array}$ & TOTAL & $\begin{array}{l}\text { CSDA } \\
\text { RANGE }\end{array}$ & $\begin{array}{l}\text { RADIATION } \\
\text { YIELD }\end{array}$ & $\begin{array}{c}\text { DEHS.EFF . } \\
\text { CORR. } \\
\text { (DELTA) }\end{array}$ & $\begin{array}{l}d(1 \\
\text { COLL } \\
\text { LOSS }\end{array}$ & $\begin{array}{l}\text { I)dd( } \\
\text { CSDA } \\
\text { RAliGE }\end{array}$ & $\begin{array}{l}\circ \text { I I } \\
\text { RAD } \\
\text { YIELD }\end{array}$ \\
\hline $\mathrm{MeV}$ & $\mathrm{MeV} \mathrm{cm}^{2} / \mathrm{g}$ & $\mathrm{MeV} \mathrm{cm} 2 / \mathrm{g}$ & $\mathrm{MeV} \mathrm{cm}^{2} / \mathrm{g}$ & $\mathrm{g} / \mathrm{cm}^{2}$ & & & & & \\
\hline $\begin{array}{l}0.0100 \\
0.0125 \\
0.0150 \\
0.0175 \\
0.0200 \\
0.0250 \\
0.0300 \\
0.0350\end{array}$ & $\begin{array}{l}2.237 E+01 \\
1.881 E+01 \\
1.633 E+01 \\
1.449 E+01 \\
1.306 E+01 \\
1.100 E+01 \\
9.571 E+00 \\
8.519 E+00\end{array}$ & $\begin{array}{l}3.816 \mathrm{E}-03 \\
3.844 \mathrm{E}-03 \\
3.862 \mathrm{E}-03 \\
3.873 \mathrm{E}-03 \\
3.882 \mathrm{E}-03 \\
3.894 \mathrm{E}-03 \\
3.905 \mathrm{E}-03 \\
3.916 \mathrm{E}-03\end{array}$ & $\begin{array}{l}2.238 E+01 \\
1.882 E+01 \\
1.633 E+01 \\
1.449 E+01 \\
1.307 E+01 \\
1.100 E+01 \\
9.575 E+00 \\
8.523 E+00\end{array}$ & $\begin{array}{l}2.536 E-04 \\
3.759 E-04 \\
5.190 E-04 \\
6.818 E-04 \\
8.638 E-04 \\
1.283 E-03 \\
1.771 E-03 \\
2.326 E-03\end{array}$ & $\begin{array}{l}9.293 \mathrm{E}-05 \\
1.119 \mathrm{E}-04 \\
1.300 \mathrm{E}-04 \\
1.474 \mathrm{E}-04 \\
1.642 \mathrm{E}-04 \\
1.965 \mathrm{E}-04 \\
2.273 \mathrm{E}-04 \\
2.568 \mathrm{E}-04\end{array}$ & $\begin{array}{l}0.0 \\
0.0 \\
0.0 \\
0.0 \\
0.0 \\
0.0 \\
0.0 \\
0.0\end{array}$ & $\begin{array}{l}-0.199 \\
-0.190 \\
-0.184 \\
-0.179 \\
-0.175 \\
-0.169 \\
-0.164 \\
-0.160\end{array}$ & $\begin{array}{l}0.227 \\
0.216 \\
0.208 \\
0.202 \\
0.197 \\
0.188 \\
0.182 \\
0.177\end{array}$ & $\begin{array}{l}0.225 \\
0.215 \\
0.207 \\
0.201 \\
0.196 \\
0.188 \\
0.182 \\
0.177\end{array}$ \\
\hline $\begin{array}{l}0.0400 \\
0.0450 \\
0.0500 \\
0.0550 \\
0.0600 \\
0.0700 \\
0.0800 \\
0.0900\end{array}$ & $\begin{array}{l}7.711 E+00 \\
7.069 E+00 \\
6.547 E+00 \\
6.113 E+00 \\
5.747 E+00 \\
5.163 E+00 \\
4.717 E+00 \\
4.365 E+00\end{array}$ & $\begin{array}{l}3.928 \mathrm{E}-03 \\
3.941 \mathrm{E}-03 \\
3.955 \mathrm{E}-03 \\
3.970 \mathrm{E}-03 \\
3.986 \mathrm{E}-03 \\
4.022 \mathrm{E}-03 \\
4.061 \mathrm{E}-03 \\
4.104 \mathrm{E}-03\end{array}$ & $\begin{array}{l}7.715 E+00 \\
7.073 E+00 \\
6.551 E+00 \\
6.117 E+00 \\
5.751 E+00 \\
5.167 E+00 \\
4.721 E+00 \\
4.369 E+00\end{array}$ & $\begin{array}{l}2.944 E-03 \\
3.621 E-03 \\
4.356 E-03 \\
5.147 E-03 \\
5.991 E-03 \\
7.829 E-03 \\
9.857 E-03 \\
1.206 E-02\end{array}$ & $\begin{array}{l}2.853 E-04 \\
3.128 E-04 \\
3.396 E-04 \\
3.657 E-04 \\
3.911 E-04 \\
4.404 E-04 \\
4.878 E-04 \\
5.336 E-04\end{array}$ & $\begin{array}{l}0.0 \\
0.0 \\
0.0 \\
0.0 \\
0.0 \\
0.0 \\
0.0 \\
0.0\end{array}$ & $\begin{array}{l}-0.157 \\
-0.154 \\
-0.152 \\
-0.150 \\
-0.148 \\
-0.145 \\
-0.142 \\
-0.140\end{array}$ & $\begin{array}{l}0.173 \\
0.170 \\
0.167 \\
0.164 \\
0.162 \\
0.158 \\
0.155 \\
0.153\end{array}$ & $\begin{array}{l}0.173 \\
0.169 \\
0.167 \\
0.164 \\
0.162 \\
0.158 \\
0.155 \\
0.152\end{array}$ \\
\hline $\begin{array}{l}0.1000 \\
0.1250 \\
0.1500 \\
0.1750 \\
0.2000 \\
0.2500 \\
0.3000 \\
0.3500\end{array}$ & $\begin{array}{l}4.080 E+00 \\
3.561 E+00 \\
3.210 E+00 \\
2.958 E+00 \\
2.769 E+00 \\
2.506 E+00 \\
2.335 E+00 \\
2.215 E+00\end{array}$ & $\begin{array}{l}4.150 \mathrm{E}-03 \\
4.275 \mathrm{E}-03 \\
4.412 \mathrm{E}-03 \\
4.558 \mathrm{E}-03 \\
4.714 \mathrm{E}-03 \\
5.048 \mathrm{E}-03 \\
5.415 \mathrm{E}-03 \\
5.808 \mathrm{E}-03\end{array}$ & $\begin{array}{l}4.084 E+00 \\
3.565 E+00 \\
3.214 E+00 \\
2.962 E+00 \\
2.774 E+00 \\
2.511 E+00 \\
2.340 E+00 \\
2.221 E+00\end{array}$ & $\begin{array}{l}1.443 \mathrm{E}-02 \\
2.101 \mathrm{E}-02 \\
2.841 \mathrm{E}-02 \\
3.653 \mathrm{E}-02 \\
4.526 \mathrm{E}-02 \\
6.427 \mathrm{E}-02 \\
8.494 \mathrm{E}-02 \\
1.069 \mathrm{E}-01\end{array}$ & $\begin{array}{l}5.780 \mathrm{E}-04 \\
6.841 \mathrm{E}-04 \\
7.845 \mathrm{E}-04 \\
8.805 \mathrm{E}-04 \\
9.728 \mathrm{E}-04 \\
1.149 \mathrm{E}-03 \\
1.318 \mathrm{E}-03 \\
1.482 \mathrm{E}-03\end{array}$ & $\begin{array}{l}0.0 \\
0.0 \\
0.0 \\
0.0 \\
0.0 \\
0.0 \\
0.0 \\
0.0\end{array}$ & $\begin{array}{l}-0.138 \\
-0.134 \\
-0.131 \\
-0.128 \\
-0.126 \\
-0.123 \\
-0.120 \\
-0.118\end{array}$ & $\begin{array}{l}0.150 \\
0.146 \\
0.142 \\
0.139 \\
0.137 \\
0.133 \\
0.130 \\
0.128\end{array}$ & $\begin{array}{l}0.150 \\
0.145 \\
0.142 \\
0.139 \\
0.136 \\
0.132 \\
0.129 \\
0.127\end{array}$ \\
\hline $\begin{array}{l}0.4000 \\
0.4500 \\
0.5000 \\
0.5500 \\
0.6000 \\
0.7000 \\
0.8000 \\
0.9000\end{array}$ & $\begin{array}{l}2.129 E+00 \\
2.065 E+00 \\
2.016 E+00 \\
1.976 E+00 \\
1.945 E+00 \\
1.898 E+00 \\
1.866 E+00 \\
1.845 E+00\end{array}$ & $\begin{array}{l}6.226 \mathrm{E}-03 \\
6.666 \mathrm{E}-03 \\
7.129 \mathrm{E}-03 \\
7.610 \mathrm{E}-03 \\
8.108 \mathrm{E}-03 \\
9.148 \mathrm{E}-03 \\
1.024 \mathrm{E}-02 \\
1.139 \mathrm{E}-02\end{array}$ & $\begin{array}{l}2.136 E+00 \\
2.072 E+00 \\
2.023 E+00 \\
1.984 E+00 \\
1.953 E+00 \\
1.907 E+00 \\
1.877 E+00 \\
1.856 E+00\end{array}$ & $\begin{array}{l}1.299 \mathrm{E}-01 \\
1.537 \mathrm{E}-01 \\
1.781 \mathrm{E}-01 \\
2.031 \mathrm{E}-01 \\
2.285 \mathrm{E}-01 \\
2.803 \mathrm{E}-01 \\
3.332 \mathrm{E}-01 \\
3.868 \mathrm{E}-01\end{array}$ & $\begin{array}{l}1.642 \mathrm{E}-03 \\
1.801 \mathrm{E}-03 \\
1.957 \mathrm{E}-03 \\
2.114 \mathrm{E}-03 \\
2.271 \mathrm{E}-03 \\
2.585 \mathrm{E}-03 \\
2.903 \mathrm{E}-03 \\
3.224 \mathrm{E}-03\end{array}$ & $\begin{array}{l}0.0 \\
0.0 \\
4.240 \mathrm{E}-03 \\
2.378 \mathrm{E}-02 \\
4.634 \mathrm{E}-02 \\
9.798 \mathrm{E}-02 \\
1.553 \mathrm{E}-01 \\
2.158 \mathrm{E}-01\end{array}$ & $\begin{array}{l}-0.116 \\
-0.114 \\
-0.098 \\
-0.090 \\
-0.084 \\
-0.075 \\
-0.068 \\
-0.062\end{array}$ & $\begin{array}{l}0.126 \\
0.124 \\
0.122 \\
0.1118 \\
0.1115 \\
0.1108 \\
0.102 \\
0.097\end{array}$ & $\begin{array}{l}0.125 \\
0.123 \\
0.120 \\
0.116 \\
0.1122 \\
0.104 \\
0.096 \\
0.090\end{array}$ \\
\hline $\begin{array}{l}1.0000 \\
1.2500 \\
1.5000 \\
1.7500 \\
2.0000 \\
2.5000 \\
3.0000 \\
3.5000\end{array}$ & $\begin{array}{l}1.830 E+00 \\
1.809 E+00 \\
1.802 E+00 \\
1.801 E+00 \\
1.804 E+00 \\
1.814 E+00 \\
1.826 E+00 \\
1.839 E+00\end{array}$ & $\begin{array}{l}1.257 E-02 \\
1.572 E-02 \\
1.908 E-02 \\
2.263 E-02 \\
2.632 E-02 \\
3.408 E-02 \\
4.225 E-02 \\
5.076 E-02\end{array}$ & $\begin{array}{l}1.842 E+00 \\
1.825 E+00 \\
1.821 E+00 \\
1.824 E+00 \\
1.830 E+00 \\
1.848 E+00 \\
1.869 E+00 \\
1.890 E+00\end{array}$ & $\begin{array}{l}4.409 \mathrm{E}-01 \\
5.774 \mathrm{E}-01 \\
7.146 \mathrm{E}-01 \\
8.518 \mathrm{E}-01 \\
9.886 \mathrm{E}-01 \\
1.261 \mathrm{E}+00 \\
1.530 \mathrm{E}+00 \\
1.796 \mathrm{E}+00\end{array}$ & $\begin{array}{l}3.550 \mathrm{E}-03 \\
4.382 \mathrm{E}-03 \\
5.242 \mathrm{E}-03 \\
6.127 \mathrm{E}-03 \\
7.035 \mathrm{E}-03 \\
8.907 \mathrm{E}-03 \\
1.084 \mathrm{E}-02 \\
1.283 \mathrm{E}-02\end{array}$ & $\begin{array}{l}2.778 \mathrm{E}-01 \\
4.339 \mathrm{E}-01 \\
5.852 \mathrm{E}-01 \\
7.289 \mathrm{E}-01 \\
8.641 \mathrm{E}-01 \\
1.110 \mathrm{E}+00 \\
1.327 \mathrm{E}+00 \\
1.521 \mathrm{E}+00\end{array}$ & $\begin{array}{l}-0.057 \\
-0.048 \\
-0.042 \\
-0.038 \\
-0.035 \\
-0.031 \\
-0.028 \\
-0.026\end{array}$ & $\begin{array}{l}0.092 \\
0.083 \\
0.075 \\
0.070 \\
0.065 \\
0.058 \\
0.053 \\
0.049\end{array}$ & $\begin{array}{l}0.084 \\
0.073 \\
0.064 \\
0.057 \\
0.052 \\
0.045 \\
0.040 \\
0.036\end{array}$ \\
\hline $\begin{array}{l}4.0000 \\
4.5000 \\
5.0000 \\
5.5000 \\
6.0000 \\
7.0000 \\
8.0000 \\
9.0000\end{array}$ & $\begin{array}{l}1.851 E+00 \\
1.862 E+00 \\
1.873 E+00 \\
1.883 E+00 \\
1.892 E+00 \\
1.909 E+00 \\
1.924 E+00 \\
1.937 E+00\end{array}$ & $\begin{array}{l}5.954 \mathrm{E}-02 \\
6.857 \mathrm{E}-02 \\
7.782 \mathrm{E}-02 \\
8.725 \mathrm{E}-02 \\
9.686 \mathrm{E}-02 \\
1.165 \mathrm{E}-01 \\
1.367 \mathrm{E}-01 \\
1.574 \mathrm{E}-01\end{array}$ & $\begin{array}{l}1.910 E+00 \\
1.931 E+00 \\
1.951 E+00 \\
1.970 E+00 \\
1.989 E+00 \\
2.026 E+00 \\
2.061 E+00 \\
2.094 E+00\end{array}$ & $\begin{array}{l}2.059 \mathrm{E}+00 \\
2.319 \mathrm{E}+00 \\
2.577 \mathrm{E}+00 \\
2.832 \mathrm{E}+00 \\
3.084 \mathrm{E}+00 \\
3.583 \mathrm{E}+00 \\
4.072 \mathrm{E}+00 \\
4.553 \mathrm{E}+00\end{array}$ & $\begin{array}{l}1.485 \mathrm{E}-02 \\
1.690 \mathrm{E}-02 \\
1.898 \mathrm{E}-02 \\
2.108 \mathrm{E}-02 \\
2.320 \mathrm{E}-02 \\
2.747 \mathrm{E}-02 \\
3.178 \mathrm{E}-02 \\
3.611 \mathrm{E}-02\end{array}$ & $\begin{array}{l}1.695 \mathrm{E}+00 \\
1.853 \mathrm{E}+00 \\
1.998 \mathrm{E}+00 \\
2.131 \mathrm{E}+00 \\
2.255 \mathrm{E}+00 \\
2.478 \mathrm{E}+00 \\
2.675 \mathrm{E}+00 \\
2.852 \mathrm{E}+00\end{array}$ & $\begin{array}{l}-0.024 \\
-0.023 \\
-0.023 \\
-0.022 \\
-0.021 \\
-0.020 \\
-0.019 \\
-0.018\end{array}$ & $\begin{array}{l}0.046 \\
0.043 \\
0.041 \\
0.039 \\
0.038 \\
0.035 \\
0.033 \\
0.032\end{array}$ & $\begin{array}{l}0.033 \\
0.031 \\
0.029 \\
0.028 \\
0.027 \\
0.025 \\
0.023 \\
0.022\end{array}$ \\
\hline $\begin{array}{l}10.0000 \\
12.5000 \\
15.0000 \\
17.5000 \\
20.0000 \\
25.0000 \\
30.0000 \\
35.0000\end{array}$ & $\begin{array}{l}1.949 E+00 \\
1.974 E+00 \\
1.995 E+00 \\
2.012 E+00 \\
2.026 E+00 \\
2.050 E+00 \\
2.068 E+00 \\
2.084 E+00\end{array}$ & $\begin{array}{l}1.784 E-01 \\
2.323 E-01 \\
2.877 E-01 \\
3.443 E-01 \\
4.018 E-01 \\
5.189 E-01 \\
6.381 E-01 \\
7.588 E-01\end{array}$ & $\begin{array}{l}2.127 E+00 \\
2.206 E+00 \\
2.282 E+00 \\
2.356 E+00 \\
2.428 E+00 \\
2.569 E+00 \\
2.706 E+00 \\
2.842 E+00\end{array}$ & $\begin{array}{l}5.027 E+00 \\
6.181 E+00 \\
7.295 E+00 \\
8.373 E+00 \\
9.418 E+00 \\
1.142 E+01 \\
1.332 E+01 \\
1.512 E+01\end{array}$ & $\begin{array}{l}4.045 \mathrm{E}-02 \\
5.128 \mathrm{E}-02 \\
6.202 \mathrm{E}-02 \\
7.261 \mathrm{E}-02 \\
8.302 \mathrm{E}-02 \\
1.032 \mathrm{E}-01 \\
1.225 \mathrm{E}-01 \\
1.410 \mathrm{E}-01\end{array}$ & $\begin{array}{l}3.013 E+00 \\
3.361 E+00 \\
3.652 E+00 \\
3.905 E+00 \\
4.128 E+00 \\
4.511 E+00 \\
4.833 E+00 \\
5.111 E+00\end{array}$ & $\begin{array}{l}-0.018 \\
-0.016 \\
-0.015 \\
-0.013 \\
-0.012 \\
-0.010 \\
-0.008 \\
-0.007\end{array}$ & $\begin{array}{l}0.030 \\
0.027 \\
0.025 \\
0.024 \\
0.022 \\
0.020 \\
0.018 \\
0.016\end{array}$ & $\begin{array}{l}0.021 \\
0.0119 \\
0.017 \\
0.0116 \\
0.0115 \\
0.012 \\
0.0111 \\
0.009\end{array}$ \\
\hline $\begin{array}{l}40.0000 \\
45.0000 \\
50.0000 \\
55.0000 \\
60.0000 \\
70.0000 \\
80.0000 \\
90.0000\end{array}$ & $\begin{array}{l}2.097 \mathrm{E}+00 \\
2.108 \mathrm{E}+00 \\
2.118 \mathrm{E}+00 \\
2.126 \mathrm{E}+00 \\
2.134 \mathrm{E}+00 \\
2.148 \mathrm{E}+00 \\
2.160 \mathrm{E}+00 \\
2.171 \mathrm{E}+00\end{array}$ & $\begin{array}{l}8.807 E-01 \\
1.004 E+00 \\
1.127 E+00 \\
1.252 E+00 \\
1.377 E+00 \\
1.629 E+00 \\
1.883 E+00 \\
2.138 E+00\end{array}$ & $\begin{array}{l}2.977 E+00 \\
3.111 E+00 \\
3.245 E+00 \\
3.378 E+00 \\
3.512 E+00 \\
3.777 E+00 \\
4.043 E+00 \\
4.308 E+00\end{array}$ & $\begin{array}{l}1.684 E+01 \\
1.848 E+01 \\
2.005 E+01 \\
2.156 E+01 \\
2.301 E+01 \\
2.576 E+01 \\
2.832 E+01 \\
3.071 E+01\end{array}$ & $\begin{array}{l}1.585 \mathrm{E}-01 \\
1.753 \mathrm{E}-01 \\
1.913 \mathrm{E}-01 \\
2.065 \mathrm{E}-01 \\
2.211 \mathrm{E}-01 \\
2.484 \mathrm{E}-01 \\
2.735 \mathrm{E}-01 \\
2.965 \mathrm{E}-01\end{array}$ & $\begin{array}{l}5.356 \mathrm{E}+00 \\
5.576 \mathrm{E}+00 \\
5.774 \mathrm{E}+00 \\
5.955 \mathrm{E}+00 \\
6.121 \mathrm{E}+00 \\
6.418 \mathrm{E}+00 \\
6.677 \mathrm{E}+00 \\
6.907 \mathrm{E}+00\end{array}$ & $\begin{array}{l}-0.005 \\
-0.005 \\
-0.004 \\
-0.003 \\
-0.003 \\
-0.002 \\
-0.002 \\
-0.001\end{array}$ & $\begin{array}{l}0.0115 \\
0.014 \\
0.013 \\
0.0113 \\
0.0112 \\
0.011 \\
0.010 \\
0.009\end{array}$ & $\begin{array}{l}0.008 \\
0.007 \\
0.007 \\
0.006 \\
0.005 \\
0.004 \\
0.004 \\
0.003\end{array}$ \\
\hline $\begin{array}{l}00.0000 \\
25.0000 \\
50.0000 \\
75.0000 \\
00.0000 \\
50.0000 \\
00.0000 \\
50.0000\end{array}$ & $\begin{array}{l}2.180 E+00 \\
2.199 E+00 \\
2.215 E+00 \\
2.228 E+00 \\
2.240 E+00 \\
2.259 E+00 \\
2.274 E+00 \\
2.287 E+00\end{array}$ & $\begin{array}{l}2.394 E+00 \\
3.039 E+00 \\
3.688 E+00 \\
4.341 E+00 \\
4.996 E+00 \\
6.313 E+00 \\
7.635 E+00 \\
8.962 E+00\end{array}$ & $\begin{array}{l}4.574 E+00 \\
5.238 E+00 \\
5.903 E+00 \\
6.569 E+00 \\
7.236 E+00 \\
8.571 E+00 \\
9.909 E+00 \\
1.125 E+01\end{array}$ & $\begin{array}{l}3.297 \mathrm{E}+01 \\
3.807 \mathrm{E}+01 \\
4.256 \mathrm{E}+01 \\
4.658 \mathrm{E}+01 \\
5.020 \mathrm{E}+01 \\
5.654 \mathrm{E}+01 \\
6.196 \mathrm{E}+01 \\
6.670 \mathrm{E}+01\end{array}$ & $\begin{array}{l}3.179 \mathrm{E}-01 \\
3.649 \mathrm{E}-01 \\
4.046 \mathrm{E}-01 \\
4.388 \mathrm{E}-01 \\
4.684 \mathrm{E}-01 \\
5.177 \mathrm{E}-01 \\
5.571 \mathrm{E}-01 \\
5.895 \mathrm{E}-01\end{array}$ & $\begin{array}{l}7.113 \mathrm{E}+00 \\
7.553 \mathrm{E}+00 \\
7.913 \mathrm{E}+00 \\
8.219 \mathrm{E}+00 \\
8.484 \mathrm{E}+00 \\
8.928 \mathrm{E}+00 \\
9.291 \mathrm{E}+00 \\
9.598 \mathrm{E}+00\end{array}$ & $\begin{array}{l}-0.001 \\
-0.001 \\
-0.001 \\
-0.0000 \\
-0.000 \\
-0.000 \\
-0.000 \\
-0.000\end{array}$ & $\begin{array}{l}0.009 \\
0.008 \\
0.007 \\
0.006 \\
0.006 \\
0.005 \\
0.005 \\
0.004\end{array}$ & $\begin{array}{l}0.0003 \\
0.0002 \\
0.0002 \\
0.0001 \\
0.0001 \\
0.001 \\
0.001 \\
0.001\end{array}$ \\
\hline $\begin{array}{l}00.0000 \\
50.0000 \\
00.0000 \\
50.0000 \\
00.0000 \\
00.0000 \\
00.0000 \\
00.0000\end{array}$ & $\begin{array}{l}2.299 E+00 \\
2.309 E+00 \\
2.317 E+00 \\
2.325 E+00 \\
2.333 E+00 \\
2.346 E+00 \\
2.357 E+00 \\
2.367 E+00\end{array}$ & $\begin{array}{l}1.029 E+01 \\
1.162 E+01 \\
1.296 E+01 \\
1.430 E+01 \\
1.563 E+01 \\
1.831 E+01 \\
2.099 E+01 \\
2.368 E+01\end{array}$ & $\begin{array}{l}1.259 E+01 \\
1.393 E+01 \\
1.528 E+01 \\
1.662 E+01 \\
1.797 E+01 \\
2.066 E+01 \\
2.335 E+01 \\
2.605 E+01\end{array}$ & $\begin{array}{l}7.089 E+01 \\
7.467 E+01 \\
7.809 E+01 \\
8.123 E+01 \\
8.412 E+01 \\
8.931 E+01 \\
9.386 E+01 \\
9.791 E+01\end{array}$ & $\begin{array}{l}6.168 \mathrm{E}-01 \\
6.400 \mathrm{E}-01 \\
6.602 \mathrm{E}-01 \\
6.778 \mathrm{E}-01 \\
6.935 \mathrm{E}-01 \\
7.199 \mathrm{E}-01 \\
7.416 \mathrm{E}-01 \\
7.596 \mathrm{E}-01\end{array}$ & $\begin{array}{l}9.865 \mathrm{E}+00 \\
1.010 \mathrm{E}+01 \\
1.031 \mathrm{E}+01 \\
1.050 \mathrm{E}+01 \\
1.067 \mathrm{E}+01 \\
1.098 \mathrm{E}+01 \\
1.125 \mathrm{E}+01 \\
1.148 \mathrm{E}+01\end{array}$ & $\begin{array}{l}-0.000 \\
-0.000 \\
-0.000 \\
-0.000 \\
-0.000 \\
-0.000 \\
-0.000 \\
-0.000\end{array}$ & $\begin{array}{l}0.004 \\
0.004 \\
0.004 \\
0.004 \\
0.003 \\
0.003 \\
0.003 \\
0.003\end{array}$ & $\begin{array}{l}0.001 \\
0.0000 \\
0.0000 \\
0.0000 \\
0.0000 \\
0.0000 \\
0.0000 \\
0.000\end{array}$ \\
\hline 0.0000 & $2.376 E+00$ & $2.637 E+01$ & $74 E+01$ & 16 & $50 E-01$ & 70 & -0.000 & 003 & \\
\hline
\end{tabular}




\begin{tabular}{|c|c|c|c|c|c|c|c|c|c|}
\hline \multirow{3}{*}{$\begin{array}{l}\text { ENERGY } \\
\mathrm{MeV}\end{array}$} & \multicolumn{3}{|r|}{ 政 } & \multirow{3}{*}{$\begin{array}{l}\text { CSDA } \\
\text { RANGE } \\
\mathrm{g} / \mathrm{cm}^{2}\end{array}$} & \multirow{3}{*}{$\begin{array}{l}\text { RADIATION } \\
\text { YIELD }\end{array}$} & \multirow{3}{*}{$\begin{array}{l}\text { DENS.EFF. } \\
\text { CORR } \\
\text { (DELTA) }\end{array}$} & \multicolumn{3}{|c|}{$d(\log ) / d(\log I)$} \\
\hline & LL IS I 0 & & TOTAL & & & & & & D \\
\hline & $\mathrm{MeV} \mathrm{cm}^{2} / \mathrm{g}$ & $\mathrm{MeV} \mathrm{cm}^{2} / \mathrm{g}$ & $\mathrm{MeV} \mathrm{cm}^{2} / \mathrm{g}$ & & & & & & \\
\hline $\begin{array}{l}0.010 \\
0.012 \\
0.01 \\
0.01 \\
0.02 \\
0.02 \\
0.03\end{array}$ & $\begin{array}{l}01 \\
01 \\
01 \\
01 \\
01 \\
01 \\
01 \\
01 \\
00 \\
00\end{array}$ & $\begin{array}{l}3.7 \\
3.7 \\
3.7 \\
3.8 \\
3.8\end{array}$ & 8. & 4 & & $\begin{array}{l}0.0 \\
0.0 \\
0.0 \\
0.0 \\
0.0 \\
0.0 \\
0.0 \\
0.0\end{array}$ & & $\begin{array}{l}27 \\
16 \\
8 \\
22 \\
96 \\
38 \\
32 \\
7\end{array}$ & $\begin{array}{l}.22 \\
.21 \\
.20 \\
.20 \\
.19 \\
\end{array}$ \\
\hline $\begin{array}{l}0.0400 \\
0.0450 \\
0.0500 \\
0.0550 \\
0.0600 \\
0.0700 \\
0.0800 \\
0.0900\end{array}$ & $\begin{array}{l}+00 \\
+00 \\
+00 \\
+00 \\
+00 \\
+00 \\
+00 \\
+00\end{array}$ & $\begin{array}{l}3.8 \\
3.8 \\
3.8 \\
3 . \\
3 . \\
3 .\end{array}$ & $\begin{array}{l}+\infty 0 \\
+\infty 0 \\
+00\end{array}$ & $\begin{array}{l}-03 \\
-03 \\
-03\end{array}$ & & $\begin{array}{l}0.0 \\
0.0 \\
0.0 \\
0.0 \\
0.0 \\
0.0 \\
0.0 \\
0.0\end{array}$ & & & $\begin{array}{l}0.164 \\
0.162 \\
0.158\end{array}$ \\
\hline $\begin{array}{l}0.1000 \\
0.1250 \\
0.1500 \\
0.1750 \\
0.2000 \\
0.2500 \\
0.3000 \\
0.3500\end{array}$ & $\begin{array}{l}4.070 \mathrm{E}+00 \\
3.552 \mathrm{E}+00 \\
3.202 \mathrm{E}+00 \\
2.950 \mathrm{E}+00 \\
2.762 \mathrm{E}+00 \\
2.500 \mathrm{E}+00 \\
2.329 \mathrm{E}+00 \\
2.210 \mathrm{E}+00\end{array}$ & $\begin{array}{l}4.190 \mathrm{E}-03 \\
4.325 \mathrm{E}-03 \\
4.469 \mathrm{E}-03 \\
4.622 \mathrm{E}-03 \\
4.951 \mathrm{E}-03 \\
5.311 \mathrm{E}-03 \\
5.698 \mathrm{E}-03\end{array}$ & $\begin{array}{l}.955 \mathrm{E}+00 \\
.767 \mathrm{E}+00 \\
.505 \mathrm{E}+00 \\
.334 \mathrm{E}+00 \\
.215 \mathrm{E}+00\end{array}$ & 2 & & $\begin{array}{l}0.0 \\
0.0 \\
0.0 \\
0.0 \\
0.0 \\
0.0 \\
0.0 \\
0.0\end{array}$ & & & $\begin{array}{l}0.142 \\
0.139 \\
0.136 \\
0.132 \\
0.129 \\
0.127\end{array}$ \\
\hline $\begin{array}{l}0.4000 \\
0.4500 \\
0.5000 \\
0.5500 \\
0.6000 \\
0.7000 \\
0.8000 \\
0.9000\end{array}$ & $\begin{array}{l}E+00 \\
E+00 \\
E+00 \\
E+00 \\
E+00 \\
E+00 \\
E+00 \\
E+00\end{array}$ & $\begin{array}{l}03 \\
03 \\
03 \\
03 \\
03 \\
02\end{array}$ & 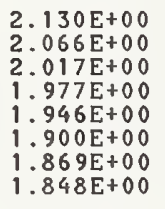 & $\begin{array}{l}1 \\
1 \\
1 \\
1 \\
1 \\
1 \\
1\end{array}$ & $\begin{array}{l}3 \\
3 \\
3 \\
3 \\
3 \\
3 \\
3 \\
3 \\
3\end{array}$ & $\begin{array}{l}0.0 \\
0.0 \\
1.519 \mathrm{E}-02 \\
3.828 \mathrm{E}-02 \\
6.393 \mathrm{E}-0 \\
1.207 \mathrm{E}-0 \\
1.821 \mathrm{E}-0 \\
2.460 \mathrm{E}-0\end{array}$ & & & $\begin{array}{l}23 \\
19 \\
14 \\
09 \\
01 \\
01\end{array}$ \\
\hline $\begin{array}{l}1.0000 \\
1.2500 \\
1.5000 \\
1.7500 \\
2.0000 \\
2.5000 \\
3.0000 \\
3.5000\end{array}$ & 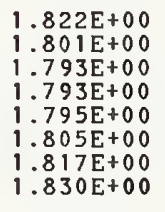 & $\begin{array}{l}02 \\
02 \\
02 \\
02 \\
02 \\
02 \\
02 \\
02 \\
02\end{array}$ & $\begin{array}{ll}0 & 0 \\
0 & 0 \\
0 & 0 \\
0 & 0 \\
0 & 0 \\
0 & 0 \\
0 & 0\end{array}$ & $\begin{array}{l}i \\
1 \\
1 \\
0 \\
0\end{array}$ & & $\begin{array}{l}7.7 \\
9: \\
1:\end{array}$ & & & $\begin{array}{l}71 \\
62 \\
56 \\
51 \\
44\end{array}$ \\
\hline $\begin{array}{l}4.5 \\
5.0 \\
5.5 \\
6.0 \\
7.0 \\
8.0 \\
9.0\end{array}$ & $\begin{array}{l}0 \\
0 \\
0 \\
0 \\
0 \\
0 \\
0\end{array}$ & $\begin{array}{l}7.6 \\
8.6 \\
9.5\end{array}$ & $\begin{array}{l}0 \\
0 \\
0 \\
0 \\
0\end{array}$ & $\begin{array}{l}0 \\
0 \\
0 \\
0\end{array}$ & $\begin{array}{l}2 \\
2 \\
2 \\
2 \\
2 \\
2 \\
2 \\
2\end{array}$ & $\begin{array}{l}0 \\
0 \\
0\end{array}$ & & & \\
\hline $\begin{array}{l}0 \\
0 \\
0 \\
0 \\
0 \\
0\end{array}$ & $\begin{array}{l}00 \\
00 \\
00 \\
00 \\
00 \\
00 \\
.00 \\
00 \\
00\end{array}$ & 1 & $\begin{array}{l}0 \\
0 \\
0 \\
0 \\
0\end{array}$ & $\begin{array}{l}0 \\
0 \\
0 \\
0 \\
1\end{array}$ & & 3. & & & \\
\hline 0 & $\begin{array}{ll}0 & 0 \\
0 & 0 \\
0 & 0 \\
0 & 0 \\
0 & 0\end{array}$ & & & & & & & & \\
\hline 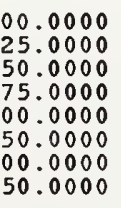 & $\begin{array}{l}262 E+00 \\
275 E+00\end{array}$ & C. & & & 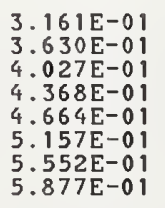 & $\begin{array}{l}0 \\
0 \\
0\end{array}$ & & & \\
\hline 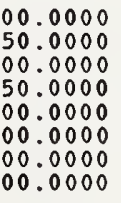 & $354 E+00$ & 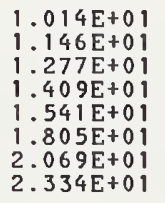 & & & & $\begin{array}{l}1 \\
1 \\
1 \\
1 \\
1 \\
1\end{array}$ & & & \\
\hline & $E+00$ & $9 E+01$ & $5 E+01$ & $2+02$ & L & $77 E+01$ & 0.000 & .003 & . \\
\hline
\end{tabular}




\begin{tabular}{|c|c|c|c|c|c|c|c|c|c|}
\hline \multirow{2}{*}{$\begin{array}{c}\text { ENERGY } \\
\mathrm{MeV}\end{array}$} & LISION & STOPPING POWER & TOTAL & \multirow{2}{*}{$\begin{array}{l}\text { CSDA } \\
\text { RANGE } \\
\mathrm{g} / \mathrm{cm}^{2}\end{array}$} & \multirow[t]{2}{*}{$\begin{array}{l}\text { RADIATION } \\
\text { YIELD }\end{array}$} & \multirow[t]{2}{*}{$\begin{array}{c}\text { DENS.EFF. } \\
\text { CORR. } \\
\text { (DELTA) }\end{array}$} & \multirow[t]{2}{*}{$\begin{array}{l}d(100 \\
\text { COLL } \\
\text { LOSS }\end{array}$} & \multirow[t]{2}{*}{$\begin{array}{l}9 \text { ) } / d(1 \\
\text { CSDA } \\
\text { RAMGE }\end{array}$} & \multirow[t]{2}{*}{$\begin{array}{l}\circ \text { gI ) } \\
\text { RAD } \\
\text { YIELD }\end{array}$} \\
\hline & $\mathrm{MeV} \mathrm{cm}^{2} / \mathrm{g}$ & $\mathrm{MeV} \mathrm{cm}^{2} / \mathrm{g}$ & $\mathrm{MeV} \mathrm{cm} 2 / g$ & & & & & & \\
\hline $\begin{array}{l}0.0100 \\
0.0125 \\
0.0150 \\
0.0175 \\
0.0200 \\
0.0250 \\
0.0300 \\
0.0350\end{array}$ & $\begin{array}{l}2.241 E+01 \\
1.884 E+01 \\
1.635 E+01 \\
1.451 E+01 \\
1.308 E+01 \\
1.102 E+01 \\
9.584 E+00 \\
8.530 E+00\end{array}$ & $\begin{array}{l}3.777 \mathrm{E}-03 \\
3.803 \mathrm{E}-03 \\
3.818 \mathrm{E}-03 \\
3.829 \mathrm{E}-03 \\
3.836 \mathrm{E}-03 \\
3.847 \mathrm{E}-03 \\
3.857 \mathrm{E}-03\end{array}$ & $\begin{array}{l}2.241 \mathrm{E} \\
1.885 \mathrm{E} \\
1.636 \mathrm{E} \\
1.451 \mathrm{E} \\
1.309 \mathrm{E} \\
1.102 \mathrm{E} \\
9.588 \mathrm{E} \\
8.534 \mathrm{E}\end{array}$ & $\begin{array}{l}2.531 E-04 \\
3.753 \mathrm{E}-04 \\
5.181 \mathrm{E}-04 \\
6.807 \mathrm{E}-04 \\
8.625 \mathrm{E}-04 \\
1.281 \mathrm{E}-03 \\
1.769 \mathrm{E}-03 \\
2.323 \mathrm{E}-03\end{array}$ & $\begin{array}{l}9.194 \mathrm{E}-05 \\
1.106 \mathrm{E}-04 \\
1.285 \mathrm{E}-04 \\
1.456 \mathrm{E}-04 \\
1.623 \mathrm{E}-04 \\
1.941 \mathrm{E}-04 \\
2.244 \mathrm{E}-04 \\
2.535 \mathrm{E}-04\end{array}$ & $\begin{array}{l}0.0 \\
0.0 \\
0.0 \\
0.0 \\
0.0 \\
0.0 \\
0.0 \\
0.0\end{array}$ & $\begin{array}{l}-0.198 \\
-0.190 \\
-0.184 \\
-0.179 \\
-0.175 \\
-0.168 \\
-0.164 \\
-0.160\end{array}$ & $\begin{array}{l}0.227 \\
0.216 \\
0.208 \\
0.202 \\
0.196 \\
0.188 \\
0.182 \\
0.177\end{array}$ & $\begin{array}{l}0.225 \\
0.214 \\
0.207 \\
0.200 \\
0.195 \\
0.187 \\
0.181 \\
0.177\end{array}$ \\
\hline $\begin{array}{l}0.0400 \\
0.0450 \\
0.0500 \\
0.0550 \\
0.0600 \\
0.0700 \\
0.0800 \\
0.0900\end{array}$ & $\begin{array}{l}7.0 \\
6.5 \\
6.1 \\
5.7 \\
5.1 \\
4.7\end{array}$ & $\begin{array}{l}3.8 \\
3.8 \\
3.9 \\
3.9 \\
3.9 \\
3.9 \\
4.0\end{array}$ & $\begin{array}{l}7 . \\
7 . \\
6 . \\
6 \\
5 . \\
5 . \\
4 . \\
4 .\end{array}$ & $\begin{array}{l}2.9 \\
3.6 \\
4.3 \\
5.1 \\
5.9 \\
7.8 \\
9.8\end{array}$ & $\begin{array}{l}-04 \\
-04 \\
-04 \\
-04 \\
-04 \\
-04 \\
-04 \\
-04\end{array}$ & $\begin{array}{l}0.0 \\
0.0 \\
0.0 \\
0.0 \\
0.0 \\
0.0 \\
0.0 \\
0.0\end{array}$ & $\begin{array}{l}-0.1 \\
-0.1 \\
-0.1 \\
-0.1 \\
-0.1 \\
-0.1 \\
-0.1 \\
-0.1\end{array}$ & $\begin{array}{l}3 \\
0 \\
7 \\
4 \\
2 \\
8 \\
5\end{array}$ & $\begin{array}{l}0.173 \\
0.169 \\
0.166 \\
0.164 \\
0.162 \\
0.158 \\
0.155 \\
0.152\end{array}$ \\
\hline $\begin{array}{l}0.1000 \\
0.1250 \\
0.1500 \\
0.1750 \\
0.2000 \\
0.2500 \\
0.3000 \\
0.3500\end{array}$ & $\begin{array}{l}3.5 \\
3.2 \\
2.9 \\
2.7 \\
2.5 \\
2.3\end{array}$ & $\begin{array}{l}4.0 \\
4.2 \\
4.3 \\
4.5 \\
4.6 \\
4.9 \\
5.3 \\
5.7\end{array}$ & $\begin{array}{l}4 \\
3 \\
3 \\
2 \\
2 \\
2 \\
2 \\
2\end{array}$ & $\begin{array}{l}-02 \\
-02 \\
-02 \\
-02 \\
-02 \\
-02 \\
-02\end{array}$ & $\begin{array}{l}.04 \\
.04 \\
.04 \\
04 \\
04 \\
03\end{array}$ & $\begin{array}{l}0.0 \\
0.0 \\
0.0 \\
0.0 \\
0.0 \\
0.0 \\
0.0 \\
0.0\end{array}$ & $\begin{array}{l}-0 . \\
-0 . \\
-0 . \\
-0 . \\
-0 . \\
-0 . \\
-0 . \\
-0 .\end{array}$ & $\begin{array}{l}0 \\
0 \\
0 \\
0 \\
0 .\end{array}$ & $\begin{array}{l}0 \\
5 \\
2 \\
9 \\
6 \\
2 \\
9 \\
7\end{array}$ \\
\hline $\begin{array}{l}0.4000 \\
0.4500 \\
0.5000 \\
0.5500 \\
0.6000 \\
0.7000 \\
0.8000 \\
0.9000\end{array}$ & $\begin{array}{l}31 E+00 \\
57 E+00 \\
18 E+00 \\
8 E+00 \\
46 E+00 \\
99 E+00 \\
57 E+00 \\
45 E+00\end{array}$ & $\begin{array}{l}6.1 \\
6.5 \\
7.0 \\
7.5 \\
8.0 \\
9.0 \\
1.0 \\
1 .\end{array}$ & $\begin{array}{l}2 . \\
2 . \\
2 . \\
1 . \\
1 . \\
1 . \\
1 . \\
1 .\end{array}$ & $\begin{array}{ll}-0 & 1 \\
-0 & 1 \\
-0 & 1 \\
-0 & 1 \\
-0 & 1 \\
-0 & 1 \\
-0 & 1 \\
-0 & 1\end{array}$ & $\begin{array}{l}-03 \\
-03 \\
-03 \\
-03 \\
-03 \\
-03\end{array}$ & $\begin{array}{l}0.0 \\
0.0 \\
8.567 \mathrm{E}-0 \\
2.960 \mathrm{E}-0 \\
5.348 \mathrm{E}-0 \\
1.074 \mathrm{E}-0 \\
1.666 \mathrm{E}-0 \\
2.287 \mathrm{E}-0\end{array}$ & $\begin{array}{l}-0 . \\
-0 . \\
-0 . \\
-0 . \\
-0 . \\
-0 . \\
-0 . \\
-0 .\end{array}$ & $\begin{array}{l}0 \\
0 \\
0 \\
0 \\
0 \\
0\end{array}$ & $\begin{array}{l}4 \\
3 \\
0 \\
5 \\
1 \\
2 \\
5\end{array}$ \\
\hline $\begin{array}{l}1.0000 \\
1.2500 \\
1.5000 \\
1.7500 \\
2.0000 \\
2.5000 \\
3.0000 \\
3.5000\end{array}$ & $\begin{array}{l}1.80 \\
1.80 \\
1.80 \\
1.80 \\
1.81 \\
1.82 \\
1.83\end{array}$ & $\begin{array}{l}-02 \\
-02 \\
-02 \\
-02 \\
-02 \\
-02 \\
-02 \\
-02 \\
-02\end{array}$ & & $\begin{array}{l}E-01 \\
E-01 \\
E-01 \\
E-01 \\
E-01 \\
E+00 \\
E+00 \\
E+00\end{array}$ & $\begin{array}{l}.03 \\
.03 \\
03 \\
.03 \\
.03 \\
.03\end{array}$ & $\begin{array}{l}2.9 \\
4.5 \\
6.0 \\
7.4 \\
8.8 \\
1.1 \\
1.3 \\
1.5\end{array}$ & $\begin{array}{l}-0.0 \\
-0.0 \\
-0.0 \\
-0.0 \\
-0.0 \\
-0.0 \\
-0.0 \\
-0.0\end{array}$ & & $\begin{array}{l}0 . \\
0 . \\
0 . \\
0 . \\
0 . \\
0 . \\
0 . \\
0 .\end{array}$ \\
\hline $\begin{array}{l}4.0000 \\
4.5000 \\
5.0000 \\
5.5000 \\
6.0000 \\
7.0000 \\
8.0000 \\
9.0000\end{array}$ & $\begin{array}{l}1.8 \\
1.8 \\
1.8 \\
1.8 \\
1.9 \\
1.9 \\
1.9\end{array}$ & $\begin{array}{l}6.7 \\
7.7 \\
8.6 \\
9.5 \\
1.1 \\
1.3 \\
1.5\end{array}$ & $\begin{array}{l}1.9 \\
1.9 \\
1.9 \\
1.9 \\
1.9 \\
2.0 \\
2.0 \\
2.0\end{array}$ & $\begin{array}{l}E+00 \\
E+00 \\
E+00 \\
B+00 \\
E+00 \\
E+00 \\
E+00 \\
+E+00 \\
5 E+00\end{array}$ & $\begin{array}{l}-02 \\
-02 \\
-02 \\
-02 \\
-02 \\
-02 \\
-02 \\
-02\end{array}$ & $\begin{array}{l}1.72 \\
1.88 \\
2.02 \\
2.16 \\
2.28 \\
2.51 \\
2.70 \\
2.88\end{array}$ & $\begin{array}{l}-0.0 \\
-0.0 \\
-0.0 \\
-0.0 \\
-0.0 \\
-0.0 \\
-0.0 \\
-0.0\end{array}$ & $\begin{array}{l}0.045 \\
0.042 \\
0.040 \\
0.039 \\
0.037 \\
0.035 \\
0.033 \\
0.031\end{array}$ & $\begin{array}{l}0.033 \\
0.030 \\
0.029 \\
0.027 \\
0.026 \\
0.024 \\
0.023 \\
0.022\end{array}$ \\
\hline $\begin{array}{l}0.0000 \\
2.5000 \\
5.0000 \\
7.5000 \\
0.0000 \\
5.0000 \\
0.0000 \\
5.0000\end{array}$ & $\begin{array}{l}8 E+00 \\
3 E+00 \\
3 E+00 \\
0 E+00 \\
5 E+00 \\
8 E+00 \\
7 E+00 \\
2 E+00\end{array}$ & $\begin{array}{l}1 \\
2 \\
2 \\
3 \\
3 \\
5 \\
6\end{array}$ & & $\begin{array}{ll}0 & 0 \\
0 & 0 \\
0 & 0 \\
0 & 0 \\
0 & 0 \\
0 & 1 \\
01 \\
01\end{array}$ & $\begin{array}{l}6 \\
7 \\
8 \\
1 \\
1\end{array}$ & $\begin{array}{l}3 . \\
3 . \\
3 . \\
3 . \\
4 . \\
4 . \\
4 . \\
5 .\end{array}$ & $\begin{array}{l}-0 . \\
-0 . \\
-0 . \\
-0 . \\
-0 . \\
-0 . \\
-0 . \\
-0 .\end{array}$ & $\begin{array}{l}0 \\
7 \\
5 \\
3 \\
2 \\
0 \\
8 \\
6\end{array}$ & \\
\hline $\begin{array}{ll}0 & 0 \\
0 & 0 \\
0 & 0 \\
0 & 0 \\
0 & 0 \\
0 & 0 \\
0 & 0 \\
0 & 0\end{array}$ & $\begin{array}{ll}+ & 0 \\
+ & 0 \\
+ & 0 \\
+0 & 0 \\
+ & 0 \\
+ & 0 \\
+ & 0 \\
+ & 0 \\
+0 & 0 \\
+0 & 0 \\
+ & 0\end{array}$ & $\begin{array}{l}9 \\
1 \\
1 \\
1 \\
1 \\
1 \\
2\end{array}$ & & $\begin{array}{l}+01 \\
+01 \\
+01 \\
+01 \\
+01 \\
+01 \\
+01 \\
+01\end{array}$ & $\begin{array}{l}1 \\
1 \\
1 \\
1 \\
1 \\
1\end{array}$ & & $\begin{array}{l}-0 \\
-0 \\
-0 \\
-0 \\
-0 \\
-0 \\
-0 \\
-0\end{array}$ & & \\
\hline $\begin{array}{l}00 \\
00\end{array}$ & $\begin{array}{l}2.1 \\
2.2 \\
2.2 \\
2.2 \\
2.2 \\
2.2 \\
2.2\end{array}$ & $\begin{array}{l}3 \\
3 \\
4 \\
4 \\
6 \\
7 \\
8\end{array}$ & & & $\begin{array}{l}-01 \\
-011 \\
-01 \\
-01 \\
-01 \\
-01 \\
-01 \\
-01\end{array}$ & $\begin{array}{l}7 . \\
7 . \\
7 . \\
8 . \\
8 . \\
8 . \\
9 .\end{array}$ & $\begin{array}{l}-0 \\
-0 \\
-0 \\
-0 \\
-0 \\
-0\end{array}$ & & \\
\hline 00 & $\begin{array}{l}2.306 E+00 \\
2.315 E+00 \\
2.323 E+00 \\
2.331 E+00 \\
2.344 E+00 \\
2.355 E+00 \\
2.365 E+00\end{array}$ & $\begin{array}{l}1 \\
1 \\
1 \\
1 \\
1 \\
2 \\
2\end{array}$ & $\begin{array}{l}1 \\
1 \\
1 \\
1 \\
1 \\
1 \\
1\end{array}$ & $\begin{array}{l}1 \\
1 \\
1 \\
1 \\
1 \\
1\end{array}$ & 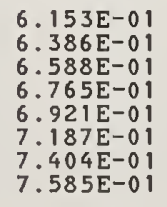 & 9. & $\begin{array}{l}0 \\
0 \\
0 \\
0 \\
0 \\
0\end{array}$ & & $\begin{array}{ll}0 & 0 \\
0 & 0 \\
0 & 0 \\
0 & 0 \\
0 & 0 \\
0 & 0\end{array}$ \\
\hline & $3 / 4 t+00$ & $.615 E+01$ & $52 F+01$ & $F+0$ & $9 F-0$ & $1.174 E+0$ & 00 & & \\
\hline
\end{tabular}




\begin{tabular}{|c|c|c|c|c|c|c|c|c|c|}
\hline \multirow{2}{*}{$\begin{array}{c}\text { ENERGY } \\
\text { MeV }\end{array}$} & \multicolumn{3}{|c|}{ STOPP ING POWER } & \multirow{2}{*}{$\begin{array}{l}\text { CSDA } \\
\text { RANGE } \\
\mathrm{g} / \mathrm{cm}^{2}\end{array}$} & \multirow[t]{2}{*}{$\begin{array}{l}\text { RADIATION } \\
\text { YIELD }\end{array}$} & \multirow[t]{2}{*}{$\begin{array}{c}\text { DENS.EFF } \\
\text { CORR } \\
\text { (DELTA) }\end{array}$} & \multirow[t]{2}{*}{$\begin{array}{l}\text { dC } 10 \\
\text { COLL } \\
\text { LOSS }\end{array}$} & \multirow[t]{2}{*}{$\begin{array}{l}\text { ) } / d(1 \\
\text { CSDA } \\
\text { RANGE }\end{array}$} & \multirow[t]{2}{*}{$\begin{array}{l}\circ g \text { I ) } \\
\text { RAD } \\
\text { YIELD }\end{array}$} \\
\hline & $\mathrm{MeV} \mathrm{cm}^{2} / \mathrm{g}$ & $\mathrm{MeV} \mathrm{cm}^{2} / \mathrm{g}$ & $\mathrm{MeV} \mathrm{cm}^{2} / \mathrm{g}$ & & & & & & \\
\hline $\begin{array}{l}0.0100 \\
0.0125 \\
0.0150 \\
0.0175 \\
0.0200 \\
0.0250 \\
0.0300 \\
0.0350\end{array}$ & $\begin{array}{ll}0 & 1 \\
0 & 1 \\
0 & 1 \\
01 & 1 \\
0 & 1 \\
0 & 1 \\
0 & 1 \\
0 & 0 \\
0 & 0\end{array}$ & $\begin{array}{l}3.1 \\
3 \cdot 1 \\
3 \cdot 1 \\
3 \cdot 1 \\
3 \cdot 1 \\
3.2 \\
3.2\end{array}$ & $\begin{array}{l}2 . \\
1 . \\
1 . \\
1 . \\
1 . \\
9 .\end{array}$ & $\begin{array}{l}4 \\
4 \\
4 \\
4 \\
4 \\
3 \\
3 \\
3\end{array}$ & $\begin{array}{l}7.5 \\
9.0 \\
1.0 \\
1.1 \\
1.3\end{array}$ & $\begin{array}{l}0.0 \\
0.0 \\
0.0 \\
0.0 \\
0.0 \\
0.0 \\
0.0 \\
0.0\end{array}$ & $\begin{array}{l}3 \\
5 \\
9 \\
4 \\
0 \\
4 \\
0 \\
6\end{array}$ & $\begin{array}{l}0 . \\
0 . \\
0 . \\
0 . \\
0 . \\
0 .\end{array}$ & $\begin{array}{l}0.218 \\
0.208 \\
0.201 \\
0.195\end{array}$ \\
\hline $\begin{array}{l}0.0400 \\
0.0450 \\
0.0500 \\
0.0550 \\
0.0600 \\
0.0700 \\
0.0800 \\
0.0900\end{array}$ & 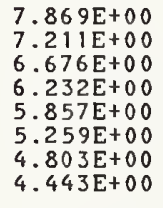 & $\begin{array}{l}3 \cdot 2 \\
3 \cdot 2 \\
3 \cdot 2 \\
3 \cdot 2 \\
3 \cdot 2 \\
3 \cdot 3\end{array}$ & $\begin{array}{l}7 . \\
6 . \\
6 . \\
5 . \\
5 . \\
4 . \\
4 .\end{array}$ & $\begin{array}{l}03 \\
03 \\
03 \\
03 \\
03 \\
03 \\
03 \\
03\end{array}$ & $\begin{array}{l}.04 \\
.04 \\
.04 \\
.04 \\
.04 \\
.04 \\
.04\end{array}$ & $\begin{array}{l}0.0 \\
0.0 \\
0.0 \\
0.0 \\
0.0 \\
0.0 \\
0.0 \\
0.0\end{array}$ & $\begin{array}{l}-0 . \\
-0 . \\
-0 . \\
-0 . \\
-0 . \\
-0 . \\
-0 . \\
-0 .\end{array}$ & $\begin{array}{l}0 \\
0 \\
0 \\
0 \\
0 \\
0\end{array}$ & $\begin{array}{l}0.168 \\
0.165 \\
0.162 \\
0.160 \\
0.158 \\
0.154 \\
0.151\end{array}$ \\
\hline $\begin{array}{l}0.1000 \\
0.1250 \\
0.1500 \\
0.1750 \\
0.2000 \\
0.2500 \\
0.3000 \\
0.3500\end{array}$ & 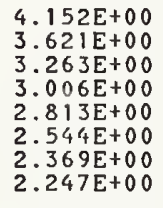 & $\begin{array}{l}3 \cdot 5 \\
3 \cdot 6 \\
3 \cdot 7 \\
3 \cdot 9 \\
4 \cdot 2 \\
4 \cdot 5 \\
4 \cdot 8\end{array}$ & $\begin{array}{l}4 . \\
3 . \\
3 . \\
3 . \\
2 . \\
2 . \\
2 . \\
2 .\end{array}$ & $\begin{array}{l}-02 \\
-02 \\
-02 \\
-02 \\
-02 \\
-02 \\
-02\end{array}$ & $\begin{array}{l}.04 \\
.04 \\
.04 \\
.04 \\
.04 \\
.04 \\
.03 \\
.03\end{array}$ & $\begin{array}{l}0.0 \\
0.0 \\
0.0 \\
0.0 \\
0.0 \\
0.0 \\
0.0 \\
0.0\end{array}$ & $\begin{array}{l}-0 \\
-0 \\
-0 \\
-0 \\
-0 \\
-0 \\
-0\end{array}$ & $\begin{array}{l}0 . \\
0 . \\
0 . \\
0 . \\
0 . \\
0 . \\
0 .\end{array}$ & \\
\hline $\begin{array}{l}0.4000 \\
0.4500 \\
0.5000 \\
0.5500 \\
0.6000 \\
0.7000 \\
0.8000 \\
0.9000\end{array}$ & $\begin{array}{l}2.156 E+00 \\
2.086 E+00 \\
2.032 E+00 \\
1.990 E+00 \\
1.956 E+00 \\
1.906 E+00 \\
1.872 E+00 \\
1.849 E+00\end{array}$ & $\begin{array}{l}5.6 \\
5.9 \\
6.4 \\
6.8 \\
7.7 \\
8.6\end{array}$ & $\begin{array}{l}2.161 E+00 \\
2.092 E+00 \\
2.038 E+00 \\
1.996 E+00 \\
1.963 E+00 \\
1.914 E+00 \\
1.881 E+00 \\
1.859 E+00\end{array}$ & $\begin{array}{l}1.279 \mathrm{E}-01 \\
1.515 \mathrm{E}-01 \\
1.757 \mathrm{E}-01 \\
2.005 \mathrm{E}-01 \\
2.258 \mathrm{E}-01 \\
2.774 \mathrm{E}-01 \\
3.301 \mathrm{E}-01 \\
3.836 \mathrm{E}-01\end{array}$ & $\begin{array}{l}1.484 E-03 \\
1.617 E-03 \\
1.750 E-03 \\
1.883 E-03 \\
2.151 E-03 \\
2.422 E-03 \\
2.696 E-03\end{array}$ & $\begin{array}{l}3.157 \\
6.655 \\
1.032 \\
1.409 \\
1.793 \\
2.572 \\
3.350 \\
4.119\end{array}$ & $\begin{array}{l}-0.087 \\
-0.080 \\
-0.075 \\
-0.070 \\
-0.066 \\
-0.060 \\
-0.055 \\
-0.051\end{array}$ & $\begin{array}{l}0 . \\
0 . \\
0 . \\
0 . \\
0 . \\
0 .\end{array}$ & $\begin{array}{l}0 \\
0 \\
0 \\
0 \\
0 \\
0\end{array}$ \\
\hline $\begin{array}{l}1.0000 \\
1.2500 \\
1.5000 \\
1.7500 \\
2.0000 \\
2.5000 \\
3.0000 \\
3.5000\end{array}$ & $\begin{array}{l}1.832 \mathrm{E} \\
1.809 \mathrm{E} \\
1.801 \mathrm{E} \\
1.799 \mathrm{E} \\
1.802 \mathrm{E} \\
1.811 \mathrm{E} \\
1.823 \mathrm{E}\end{array}$ & $\begin{array}{l}1.3 \\
1.6 \\
1.9 \\
2.2 \\
2.9 \\
3.6 \\
4.3\end{array}$ & $\begin{array}{l}1.8 \\
1.8 \\
1.8 \\
1.8 \\
1.8 \\
1.8 \\
1.8 \\
1.8\end{array}$ & $\begin{array}{ll}0 & 1 \\
0 & 1 \\
0 & 1 \\
0 & 1 \\
0 & 1 \\
0 & 0 \\
0 & 0\end{array}$ & $\begin{array}{l}2.975 \mathrm{E}- \\
3.689 \mathrm{E}- \\
4.428 \mathrm{E}- \\
5.190 \mathrm{E}- \\
5.972 \mathrm{E}- \\
7.587 \mathrm{E}- \\
9.258 \mathrm{E}- \\
1.097 \mathrm{E}-\end{array}$ & $\begin{array}{l}4.8 \\
6.6 \\
8.3 \\
9.8 \\
1.1 \\
1.3 \\
1.5 \\
1.7\end{array}$ & $\begin{array}{l}-0 . \\
-0 . \\
-0 . \\
-0 . \\
-0 . \\
-0 . \\
-0 . \\
-0 .\end{array}$ & $\begin{array}{l}0 . \\
0 . \\
0 . \\
0 . \\
0 . \\
0 . \\
0 .\end{array}$ & $\begin{array}{l}70 \\
61 \\
54 \\
49 \\
46 \\
40 \\
36\end{array}$ \\
\hline $\begin{array}{l}4.0000 \\
4.5000 \\
5.0000 \\
5.5000 \\
6.0000 \\
7.0000 \\
8.0000 \\
9.0000\end{array}$ & $\begin{array}{l}1.859 \\
1.870 \\
1.880 \\
1.889 \\
1.906\end{array}$ & $\begin{array}{l}5.8 \\
6.6 \\
7.4 \\
8.3 \\
1.0 \\
1.1 \\
1.3\end{array}$ & $\begin{array}{l}1 . \\
1 . \\
1 . \\
1 . \\
2 .\end{array}$ & $\begin{array}{l}2.0 \\
2.3 \\
2.5 \\
2.8 \\
3.0 \\
3.5 \\
4.0 \\
4.5\end{array}$ & $\begin{array}{l}1 . \\
1 . \\
1 . \\
1 . \\
1 . \\
2 . \\
2 . \\
3 .\end{array}$ & $\begin{array}{l}1.9 \\
2.1 \\
2.2 \\
2.3 \\
2.5 \\
2.7 \\
2.9 \\
3.1\end{array}$ & $\begin{array}{l}-0 . \\
-0 . \\
-0 . \\
-0 . \\
-0 . \\
-0 . \\
-0 . \\
-0 .\end{array}$ & $\begin{array}{l}0 . \\
0 . \\
0 . \\
0 . \\
0 . \\
0 .\end{array}$ & $\begin{array}{l}0 \\
0 \\
0 \\
0 \\
0 \\
0 \\
0\end{array}$ \\
\hline $\begin{array}{l}10.0000 \\
12.5000 \\
15.0000 \\
17.5000 \\
20.0000 \\
25.0000 \\
30.0000 \\
35.0000\end{array}$ & $\begin{array}{l}1.970 \\
1.990 \\
2.006 \\
2.019 \\
2.041 \\
2.058\end{array}$ & $\begin{array}{l}2.0 \\
2.4 \\
2.9 \\
3.4 \\
4.4\end{array}$ & $\begin{array}{l}2.099 \mathrm{E}+ \\
2.170 \mathrm{E}+ \\
2.238 \mathrm{E}+ \\
2.303 \mathrm{E}+ \\
2.366 \mathrm{E}+ \\
2.490 \mathrm{E}+ \\
2.610 \mathrm{E}+ \\
2.729 \mathrm{E}+\end{array}$ & $\begin{array}{l}0 \\
0 \\
0 \\
0 \\
0 \\
1 \\
1 \\
1\end{array}$ & $\begin{array}{l}3 . \\
4 . \\
5 . \\
6 . \\
7 . \\
9 . \\
1 . \\
1 .\end{array}$ & $\begin{array}{l}3 . \\
3 . \\
3 . \\
4 . \\
4 . \\
4.8 \\
5 . \\
5 .\end{array}$ & $\begin{array}{l}-0 \\
-0 \\
-0 \\
-0 \\
-0 \\
-0 \\
-0 \\
-0\end{array}$ & $\begin{array}{l}0 . \\
0 . \\
0 . \\
0 . \\
0 .\end{array}$ & \\
\hline $\begin{array}{l}40.00 \\
45.00 \\
50.00 \\
55.00 \\
60.00 \\
70.00 \\
80.00 \\
90.00\end{array}$ & $\begin{array}{l}2.1 \\
2.1 \\
2.1\end{array}$ & $\begin{array}{l}1 \\
1 \\
0 \\
0 \\
0\end{array}$ & $\begin{array}{l}2 . \\
2 \\
3 \\
3\end{array}$ & $\begin{array}{l}1 \\
1 \\
1 \\
1 \\
1 \\
1\end{array}$ & $\begin{array}{l}1 \\
1 \\
1 \\
1 \\
1 \\
1 \\
1 \\
1\end{array}$ & $\begin{array}{l}5 . \\
5 . \\
6 . \\
6 . \\
6 . \\
6 . \\
7 . \\
7 .\end{array}$ & $\begin{array}{l}-0 \\
-0 \\
-0 \\
-0 \\
-0 \\
-0 \\
-0 \\
-0\end{array}$ & & \\
\hline $\begin{array}{l}.00 \\
.00 \\
.00 \\
.00\end{array}$ & $\begin{array}{l}2 . \\
2.2 \\
2.2 \\
2 .\end{array}$ & $\begin{array}{l}3 \\
3 \\
4 \\
5\end{array}$ & $\begin{array}{l}0 \\
0 \\
0 \\
0 \\
0\end{array}$ & $\begin{array}{l}1 \\
1 \\
1 \\
1 \\
1 \\
1 \\
1\end{array}$ & & $\begin{array}{l}7 . \\
7 . \\
8 . \\
8 . \\
8 . \\
9 .\end{array}$ & $\begin{array}{l}- \\
- \\
- \\
- \\
- \\
-\end{array}$ & & \\
\hline $\begin{array}{l}0.0000 \\
0.0000 \\
0.0000 \\
0.0000 \\
0.0000 \\
0.0000\end{array}$ & $\begin{array}{l}2.282 \mathrm{E}+00 \\
2.292 \mathrm{E}+00 \\
2.301 \mathrm{E}+00 \\
2.309 \mathrm{E}+00 \\
2.316 \mathrm{E}+00 \\
2.329 \mathrm{E}+00\end{array}$ & $\begin{array}{ll}0 & 1 \\
0 & 1 \\
0 & 1 \\
0 & 1 \\
0 & 1 \\
0 & 1 \\
0 & 1\end{array}$ & $\begin{array}{l}E+01 \\
E+01 \\
E+01 \\
E+01 \\
E+01 \\
E+01 \\
E+01\end{array}$ & $\begin{array}{l}1 \\
1 \\
1 \\
1 \\
1 \\
1 \\
2\end{array}$ & $\begin{array}{l}1 \\
1 \\
1 \\
1 \\
1 \\
1 \\
1 \\
1\end{array}$ & $\begin{array}{l}1 \\
1 \\
1 \\
1 \\
1 \\
1 \\
1 \\
1\end{array}$ & $\begin{array}{l}-0 . \\
-0 . \\
-0 . \\
-0 . \\
-0 .\end{array}$ & $\begin{array}{l}0 \\
0 \\
0 \\
0 \\
0 \\
0 \\
0\end{array}$ & $\begin{array}{l}.000 \\
.000 \\
.000 \\
.000 \\
.000 \\
.000 \\
.000 \\
.000\end{array}$ \\
\hline .0000 & $.359 E+00$ & $.303 E+01$ & $539 \mathrm{E}+01$ & $107 E+02$ & $.560 \mathrm{E}-01$ & $1.210 \mathrm{E}+01$ & -0.000 & 0.002 & 0.000 \\
\hline
\end{tabular}


ELECTRONS IN PARAFFIN WAX

$I=55.9 \mathrm{eV} \quad$ DENSITY $=9.300 \mathrm{E}-01 \mathrm{~g} / \mathrm{cm}^{3}$

\begin{tabular}{|c|c|c|c|c|c|c|c|c|c|}
\hline NERGY & OLLISION & $\begin{array}{l}\text { ING POh } \\
\text { DIATIVE }\end{array}$ & TOTAL & $\begin{array}{l}\text { CSDA } \\
\text { RANGE }\end{array}$ & YIELD & $\begin{array}{c}\text { DEHS.EFF . } \\
\text { COPR. } \\
\text { (DELTA) }\end{array}$ & $\begin{array}{l}d(1) \\
\text { coll } \\
\text { Los }\end{array}$ & CSDA & $\begin{array}{l}g \text { I ) } \\
\text { RAD } \\
\text { YIELD }\end{array}$ \\
\hline $\mathrm{MeV}$ & $\mathrm{MeV} \mathrm{cm}^{2} / \mathrm{g}$ & $\mathrm{leV} \mathrm{cm}^{2} / \mathrm{g}$ & $\mathrm{MeV} \mathrm{cm}^{2} / \mathrm{g}$ & $\mathrm{g} / \mathrm{cm}^{2}$ & & & & & \\
\hline $\begin{array}{l}0.0100 \\
0.0125 \\
0.0150 \\
0.0175 \\
0.0200 \\
0.0250 \\
0.0300 \\
0.0350\end{array}$ & $\begin{array}{l}2.464 E+01 \\
2.067 E+01 \\
1.791 E+01 \\
1.587 E+01 \\
1.429 E+01 \\
1.201 E+01 \\
1.044 E+01 \\
9.282 E+00\end{array}$ & $\begin{array}{l}826 E-03 \\
837 E-03 \\
844 E-03 \\
849 E-03 \\
854 E-03 \\
863 E-03 \\
873 E-03 \\
884 E-03\end{array}$ & $\begin{array}{l}2.464 \\
2.067 \\
1.791 \\
1.587 \\
1.430 \\
1.202 \\
1.044 \\
9.285\end{array}$ & $\begin{array}{l}2.28 \\
3.39 \\
4.70 \\
6.18 \\
7.84 \\
1.16 \\
1.61 \\
2.12\end{array}$ & $\begin{array}{l}6.305 E-05 \\
7.565 E-05 \\
8.772 E-05 \\
9.936 E-05 \\
1.106 E-04 \\
1.323 E-04 \\
1.531 E-04 \\
1.731 E-04\end{array}$ & $\begin{array}{l}0.0 \\
0.0 \\
0.0 \\
0.0 \\
0.0 \\
0.0 \\
0.0 \\
0.0\end{array}$ & $\begin{array}{l}-0.188 \\
-0.180 \\
-0.175 \\
-0.170 \\
-0.167 \\
-0.161 \\
-0.156 \\
-0.153\end{array}$ & $\begin{array}{l}0.213 \\
0.203 \\
0.196 \\
0.190 \\
0.186 \\
0.178 \\
0.173 \\
0.168\end{array}$ & $\begin{array}{l}0.212 \\
0.202 \\
0.195 \\
0.190 \\
0.185 \\
0.178 \\
0.172 \\
0.168\end{array}$ \\
\hline $\begin{array}{l}0.0400 \\
0.0450 \\
0.0500 \\
0.0550 \\
0.0600 \\
0.0700 \\
0.0800 \\
0.0900\end{array}$ & $\begin{array}{l}94 E+00 \\
89 E+00 \\
17 E+00 \\
41 E+00 \\
41 E+00 \\
01 E+00 \\
13 E+00 \\
29 E+00\end{array}$ & $\begin{array}{l}5 E-03 \\
8 E-03 \\
1 E-03 \\
5 E-03 \\
0 E-03 \\
1 E-03 \\
4 E-03 \\
0 E-03\end{array}$ & $\begin{array}{l}8 . \\
7 . \\
7 . \\
6 . \\
6 . \\
5 . \\
5 . \\
4 .\end{array}$ & $\begin{array}{l}2.6 \\
3.3 \\
3.9 \\
4.7 \\
5.4 \\
7.1 \\
9.0 \\
1.1\end{array}$ & $\begin{array}{l}1.9 \\
2.1 \\
2.2 \\
2.4 \\
2.6 \\
2.9 \\
3.3 \\
3.6\end{array}$ & $\begin{array}{l}0.0 \\
0.0 \\
0.0 \\
0.0 \\
0.0 \\
0.0 \\
0.0 \\
0.0\end{array}$ & $\begin{array}{l}-0.150 \\
-0.147 \\
-0.145 \\
-0.143 \\
-0.142 \\
-0.139 \\
-0.136 \\
-0.134\end{array}$ & $\begin{array}{l}0.1 \\
0.1 \\
0.1 \\
0.1 \\
0.1 \\
0.1 \\
0.1 \\
0.1\end{array}$ & $\begin{array}{l}0.165 \\
0.161 \\
0.159 \\
0.157 \\
0.155 \\
0.151 \\
0.148\end{array}$ \\
\hline $\begin{array}{l}0.1000 \\
0.1250 \\
0.1500 \\
0.1750 \\
0.2000 \\
0.2500 \\
0.3000 \\
0.3500\end{array}$ & 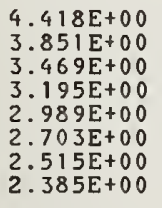 & $\begin{array}{l}-03 \\
-03 \\
-03 \\
-03 \\
-03 \\
03 \\
-03 \\
-03\end{array}$ & $\begin{array}{l}3.855 \mathrm{E}+0 \\
3.472 \mathrm{E}+0 \\
3.198 \mathrm{E}+0 \\
2.992 \mathrm{E}+0 \\
2.706 \mathrm{E}+0 \\
2.520 \mathrm{E}+0 \\
2.390 \mathrm{E}+0\end{array}$ & $\begin{array}{l}1.328 \\
1.936 \\
2.622 \\
3.373 \\
4.182 \\
5.945 \\
7.864 \\
9.905\end{array}$ & $\begin{array}{l}4.680 \\
5.382 \\
6.056 \\
6.707 \\
7.957 \\
9.159 \\
1.033\end{array}$ & $\begin{array}{l}0.0 \\
0.0 \\
0.0 \\
0.0 \\
0.0 \\
0.0 \\
0.0 \\
2.449 E-03\end{array}$ & $\begin{array}{l}-0.133 \\
-0.129 \\
-0.126 \\
-0.124 \\
-0.122 \\
-0.119 \\
-0.116 \\
-0.100\end{array}$ & $\begin{array}{l}0.1 \\
0.1 \\
0.1 \\
0.1 \\
0.1 \\
0.1 \\
0.1 \\
0.1\end{array}$ & $\begin{array}{l}0.144 \\
0.139 \\
0.136 \\
0.133 \\
0.131 \\
0.127 \\
0.125 \\
0.122\end{array}$ \\
\hline $\begin{array}{l}0.4000 \\
0.4500 \\
0.5000 \\
0.5500 \\
0.6000 \\
0.7000 \\
0.8000 \\
0.9000\end{array}$ & $\begin{array}{l}2.287 \mathrm{E}+00 \\
2.213 \mathrm{E}+00 \\
2.156 \mathrm{E}+00 \\
2.111 \mathrm{E}+00 \\
2.074 \mathrm{E}+00 \\
2.021 \mathrm{E}+00 \\
1.984 \mathrm{E}+00 \\
1.959 \mathrm{E}+00\end{array}$ & $\begin{array}{l}-03 \\
-03 \\
-03 \\
-03 \\
-03 \\
-03 \\
-03 \\
-03\end{array}$ & $\begin{array}{l}2 . \\
2 . \\
2 . \\
2 . \\
2 . \\
1 . \\
1 .\end{array}$ & $\begin{array}{l}1.2 \\
1.4 \\
1.6 \\
1.8 \\
2.1 \\
2.6\end{array}$ & $\begin{array}{l}1.1 \\
1.2 \\
1.3 \\
1.4 \\
1.6 \\
1.8 \\
2.0 \\
2.3\end{array}$ & $\begin{array}{l}3.4 \\
6.8 \\
1.0 \\
1.4 \\
1.8 \\
2.6 \\
3.4 \\
4.1\end{array}$ & $\begin{array}{l}-0.0 \\
-0.0 \\
-0.0 \\
-0.0 \\
-0.0 \\
-0.0 \\
-0.0 \\
-0.0\end{array}$ & $\begin{array}{l}0.1 \\
0.1 \\
0.1 \\
0.1 \\
0.0 \\
0.0 \\
0.0 \\
0.0\end{array}$ & $\begin{array}{l}0.115 \\
0.108 \\
0.103 \\
0.098 \\
0.093 \\
0.085 \\
0.078 \\
0.073\end{array}$ \\
\hline $\begin{array}{l}1.0000 \\
1.2500 \\
1.5000 \\
1.7500 \\
2.0000 \\
2.5000 \\
3.0000 \\
3.5000\end{array}$ & $\begin{array}{l}1.941 \mathrm{E}+00 \\
1.916 \mathrm{E}+00 \\
1.906 \mathrm{E}+00 \\
1.904 \mathrm{E}+00 \\
1.906 \mathrm{E}+00 \\
1.915 \mathrm{E}+00 \\
1.927 \mathrm{E}+00 \\
1.940 \mathrm{E}+00\end{array}$ & $\begin{array}{l}-03 \\
-02 \\
-02 \\
-02 \\
-02 \\
-02 \\
-02 \\
-02\end{array}$ & $\begin{array}{l}1 . \\
1: \\
1: \\
1: \\
1:\end{array}$ & $\begin{array}{l}5.4 \\
6.7 \\
8.0 \\
9.3 \\
1.1\end{array}$ & $\begin{array}{l}2.5 \\
3.1 \\
3.8 \\
4.4 \\
5.1 \\
6.5 \\
8.0 \\
9.5\end{array}$ & $\begin{array}{l}4.5 \\
6.7 \\
8.2 \\
1.0 \\
1.1 \\
1.2 \\
1.6 \\
1.8\end{array}$ & $\begin{array}{l}-0.0 \\
-0.0 \\
-0.0 \\
-0.0 \\
-0.0 \\
-0.0 \\
-0.0 \\
-0.0\end{array}$ & $\begin{array}{l}0 . \\
0 . \\
0 . \\
0 . \\
0 . \\
0 . \\
0 . \\
0 .\end{array}$ & $\begin{array}{l}0.068 \\
0.059 \\
0.053 \\
0.048 \\
0.044 \\
0.039 \\
0.035 \\
0.033\end{array}$ \\
\hline $\begin{array}{l}4.0000 \\
4.5000 \\
5.0000 \\
5.5000 \\
6.0000 \\
7.0000 \\
8.0000 \\
9.0000\end{array}$ & $\begin{array}{l}1.952 E+00 \\
1.964 E+00 \\
1.975 E+00 \\
1.985 E+00 \\
1.995 E+00 \\
2.012 E+00 \\
2.027 E+00 \\
2.040 E+00\end{array}$ & $\begin{array}{l}-02 \\
-02 \\
-02 \\
-02 \\
-02 \\
-02 \\
-02 \\
-01 \\
-01\end{array}$ & $\begin{array}{l}1.99 \\
2.01 \\
2.03 \\
2.05 \\
2.07 \\
2.10 \\
2.13 \\
2.16\end{array}$ & $\begin{array}{l}1.9 \\
2.2 \\
2.4 \\
2.6 \\
2.9 \\
3.4 \\
3.8 \\
4.3\end{array}$ & $\begin{array}{l}1.1 \\
1.2 \\
1.4 \\
1.5 \\
1.7 \\
2.0 \\
2.4 \\
2.7\end{array}$ & $\begin{array}{l}1.9 \\
2.1 \\
2.3 \\
2.4 \\
2.5 \\
2.7 \\
2.9 \\
3.1\end{array}$ & $\begin{array}{l}-0.0 \\
-0.0 \\
-0.0 \\
-0.0 \\
-0.0 \\
-0.0 \\
-0.0 \\
-0.0\end{array}$ & $\begin{array}{l}0 . \\
0 . \\
0 . \\
0 . \\
0 . \\
0 . \\
0 . \\
0 .\end{array}$ & $\begin{array}{l}0.031 \\
0.029 \\
0.028 \\
0.027 \\
0.026 \\
0.024 \\
0.022 \\
0.021\end{array}$ \\
\hline $\begin{array}{l}.0000 \\
.5000 \\
.0000 \\
.5000 \\
.0000 \\
.0000 \\
.0000 \\
.0000\end{array}$ & $\begin{array}{l}2.052 E+00 \\
2.077 E+00 \\
2.097 E+00 \\
2.113 E+00 \\
2.127 E+00 \\
2.149 E+00 \\
2.167 E+00 \\
2.181 E+00\end{array}$ & $\begin{array}{ll}-0 & 1 \\
-0 & 1 \\
-0 & 1 \\
-01 & 1 \\
-01 \\
-01\end{array}$ & $\begin{array}{l}2.262 \mathrm{E} \\
2.326 \mathrm{E} \\
2.388 \mathrm{E} \\
2.448 \mathrm{E} \\
2.565 \mathrm{E} \\
2.678 \mathrm{E} \\
2.791 \mathrm{E}\end{array}$ & $\begin{array}{l}5 . \\
7 . \\
8 . \\
9 . \\
1 .\end{array}$ & $\begin{array}{l}3.0 \\
3.9 \\
4.7 \\
5.6 \\
6.4 \\
8.1 \\
9.7 \\
1.1\end{array}$ & $\begin{array}{l}3.3 \\
3.8 \\
4.0 \\
4.2 \\
4.5 \\
4.5\end{array}$ & $\begin{array}{l}-0.015 \\
-0.012 \\
-0.010 \\
-0.008 \\
-0.007 \\
-0.005 \\
-0.004 \\
-0.003\end{array}$ & $\begin{array}{l}0.1 \\
0 . \\
0.5 \\
0.5 \\
0.5 \\
0.1 \\
0.5 \\
0.1\end{array}$ & $\begin{array}{l}0.020 \\
0.017 \\
0.015 \\
0.013 \\
0.012 \\
0.009 \\
0.007 \\
0.006\end{array}$ \\
\hline $\begin{array}{l}.0000 \\
.0000 \\
.0000 \\
.0000 \\
.0000 \\
.0000 \\
.0000\end{array}$ & $\begin{array}{l}2.194 \mathrm{E}+00 \\
2.205 \mathrm{E}+00 \\
2.214 \mathrm{E}+00 \\
2.223 \mathrm{E}+00 \\
2.231 \mathrm{E}+00 \\
2.245 \mathrm{E}+00 \\
2.257 \mathrm{E}+00 \\
2.268 \mathrm{E}+00\end{array}$ & $\begin{array}{l}8 . \\
9 . \\
1 . \\
1 .\end{array}$ & $\begin{array}{l}3 . \\
3 \\
3 \\
3\end{array}$ & $\begin{array}{l}1 \\
1 \\
1 \\
1 \\
1\end{array}$ & $\begin{array}{l}1.2 \\
1.4 \\
1.5 \\
1.6 \\
1.8 \\
2.0 \\
2.2\end{array}$ & $\begin{array}{l}.815 E+00 \\
.042 E+00 \\
.247 E+00 \\
.433 E+00 \\
.604 E+00 \\
.907 E+00 \\
.170 E+00 \\
.403 E+00\end{array}$ & $\begin{array}{l}-0.002 \\
-0.002 \\
-0.001 \\
-0.001 \\
-0.001 \\
-0.001 \\
-0.001 \\
-0.001\end{array}$ & $\begin{array}{l}0.012 \\
0.0111 \\
0.0110 \\
0.009 \\
0.009 \\
0.008 \\
0.007 \\
0.007\end{array}$ & $\begin{array}{l}0.005 \\
0.004 \\
0.004 \\
0.003 \\
0.003 \\
0.002 \\
0.002 \\
0.002\end{array}$ \\
\hline $\begin{array}{lll}0 & 0 & 0 \\
0 & 0 & 0 \\
0 & 0 & 0 \\
0 & 0 & 0\end{array}$ & $\begin{array}{l}2.277 E+00 \\
2.297 E+00 \\
2.313 E+00 \\
2.327 E+00 \\
2.339 E+00 \\
2.358 E+00 \\
2.374 E+00 \\
2.388 E+00\end{array}$ & $\begin{array}{l}+00 \\
+00 \\
+00 \\
+00 \\
+00 \\
+00\end{array}$ & $\begin{array}{l}4 \\
5 \\
5 \\
6\end{array}$ & $\begin{array}{l}1 \\
1 \\
1\end{array}$ & $\begin{array}{l}2.6 \\
3.1 \\
3.5 \\
3.8 \\
4.1\end{array}$ & $\begin{array}{l}0 \\
0 \\
0 \\
0 \\
0 \\
0 \\
0 \\
1\end{array}$ & $\begin{array}{l}-0.000 \\
-0.000 \\
-0.000 \\
-0.000 \\
-0.000 \\
-0.000 \\
-0.000\end{array}$ & $\begin{array}{l}6 \\
5 \\
5 \\
4 \\
4 \\
4 \\
3 \\
3\end{array}$ & $\begin{array}{l}0.0 \\
0.0 \\
0.0 \\
0.0 \\
0.0 \\
0.0 \\
0.0 \\
0.0\end{array}$ \\
\hline $\begin{array}{l}.00000 \\
.00000 \\
.00000 \\
.00000 \\
.00000 \\
.00000 \\
.00000\end{array}$ & $\begin{array}{l}2.400 \mathrm{E}+00 \\
2.410 \mathrm{E}+00 \\
2.419 \mathrm{E}+00 \\
2.428 \mathrm{E}+00 \\
2.435 \mathrm{E}+00 \\
2.449 \mathrm{E}+00 \\
2.461 \mathrm{E}+00 \\
2.471 \mathrm{E}+00\end{array}$ & $\begin{array}{l}+0 \\
+0 \\
+01 \\
+01 \\
+01 \\
+01 \\
+01 \\
+01 \\
+01\end{array}$ & $\begin{array}{l}1 \\
1 \\
1 \\
1 \\
1\end{array}$ & $\begin{array}{l}1 \\
1 \\
1 \\
1 \\
2\end{array}$ & $\begin{array}{l}5.900 E-01 \\
6.116 E-01 \\
6.306 E-01 \\
6.475 E-01 \\
6.763 E-01 \\
7.002 E-01 \\
7.202 E-01\end{array}$ & $\begin{array}{l}7 E+01 \\
1 E+01 \\
2 E+01 \\
1 E+01 \\
8 E+01 \\
9 E+01 \\
6 E+01 \\
9 E+01\end{array}$ & $\begin{array}{l}-0 . \\
-0 . \\
-0 . \\
-0 . \\
-0 . \\
-0 . \\
-0 . \\
-0 .\end{array}$ & $\begin{array}{l}.003 \\
.003 \\
.003 \\
.002 \\
.002 \\
.002 \\
.002 \\
.002\end{array}$ & $\begin{array}{l}0.000 \\
0.000 \\
0.0000 \\
0.0000 \\
0.0000 \\
0.0000 \\
0.0000\end{array}$ \\
\hline 0 & $.480 E+00$ & $149 E+01$ & $97 E+01$ & $0 E+02$ & $74 E-01$ & $20 E+0$ & -0.000 & .002 & \\
\hline
\end{tabular}




\begin{tabular}{|c|c|c|c|c|c|c|c|c|c|}
\hline \multirow{2}{*}{$\begin{array}{l}\text { ENERGY } \\
\text { MeV }\end{array}$} & \multicolumn{3}{|c|}{$\begin{array}{l}\text { STOPPING POWER } \\
\text { ST }\end{array}$} & \multirow{2}{*}{$\begin{array}{l}\text { CSDA } \\
\text { RANGE } \\
\mathrm{g} / \mathrm{CR}^{2}\end{array}$} & \multirow[t]{2}{*}{$\begin{array}{l}\text { RADIATION } \\
\text { YIELD }\end{array}$} & \multirow[t]{2}{*}{$\begin{array}{l}\text { DENS.EFF . } \\
\text { CORR. } \\
\text { (DELTA) }\end{array}$} & \multirow[t]{2}{*}{$\begin{array}{l}\text { dC } 100 \\
\text { coll } \\
\text { loss }\end{array}$} & \multirow[t]{2}{*}{$\begin{array}{l}\text { I } / \mathrm{d}(\mathrm{I} \\
\text { CSDA } \\
\text { RANGE }\end{array}$} & \multirow[t]{2}{*}{$\begin{array}{l}\circ \mathrm{OI}) \\
\text { RAD } \\
\text { YIELD }\end{array}$} \\
\hline & $\mathrm{MeV} \mathrm{cm}^{2} / \mathrm{g}$ & $\mathrm{MeV} \mathrm{cm}^{2} / \mathrm{g}$ & $\mathrm{MeV} \mathrm{Cm}^{2} / \mathrm{g}$ & & & & & & \\
\hline $\begin{array}{l}0.0100 \\
0.0125 \\
0.01150 \\
0.0175 \\
0.0200 \\
0.0250 \\
0.0300 \\
0.0350\end{array}$ & $\begin{array}{l}1.302 E+01 \\
1.114 E+01 \\
9.798 E+00 \\
8.782 E+00 \\
7.984 E+00 \\
6.810 E+00 \\
5.983 E+00 \\
5.366 E+00\end{array}$ & $\begin{array}{l}1.310 \mathrm{E}-02 \\
1.396 \mathrm{E}-02 \\
1.463 \mathrm{E}-02 \\
1.518 \mathrm{E}-02 \\
1.565 \mathrm{E}-02 \\
1.639 \mathrm{E}-02 \\
1.697 \mathrm{E}-02 \\
1.745 \mathrm{E}-02\end{array}$ & $\begin{array}{l}1.304 E+01 \\
1.116 E+01 \\
9.813 E+00 \\
8.797 E+00 \\
8.000 E+00 \\
6.827 E+00 \\
6.000 E+00 \\
5.383 E+00\end{array}$ & $\begin{array}{l}4.664 E-04 \\
6.745 E-04 \\
9.140 E-04 \\
1.184 E-03 \\
1.482 E-03 \\
2.161 E-03 \\
2.945 E-03 \\
3.826 E-03\end{array}$ & $\begin{array}{l}4.991 E-04 \\
6.250 E-04 \\
7.494 E-04 \\
8.722 E-04 \\
9.934 E-04 \\
1.231 E-03 \\
1.462 E-03 \\
1.687 E-03\end{array}$ & $\begin{array}{l}0.0 \\
0.0 \\
0.0 \\
0.0 \\
0.0 \\
0.0 \\
0.0 \\
0.0\end{array}$ & $\begin{array}{l}-0.282 \\
-0.266 \\
-0.253 \\
-0.244 \\
-0.237 \\
-0.225 \\
-0.216 \\
-0.210\end{array}$ & $\begin{array}{l}0.359 \\
0.332 \\
0.313 \\
0.298 \\
0.286 \\
0.269 \\
0.256 \\
0.246\end{array}$ & $\begin{array}{l}0.338 \\
0.315 \\
0.298 \\
0.285 \\
0.274 \\
0.258 \\
0.247 \\
0.238\end{array}$ \\
\hline $\begin{array}{l}0.0400 \\
0.0450 \\
0.0500 \\
0.0550 \\
0.0600 \\
0.0700 \\
0.0800 \\
0.0900\end{array}$ & $\begin{array}{l}4.887 E+00 \\
4.504 E+00 \\
4.130 E+00 \\
3.928 E+00 \\
3.706 E+00 \\
3.349 E+00 \\
3.075 E+00 \\
2.857 E+00\end{array}$ & $\begin{array}{l}1.786 \mathrm{E}-02 \\
1.822 \mathrm{E}-02 \\
1.854 \mathrm{E}-02 \\
1.884 \mathrm{E}-02 \\
1.910 \mathrm{E}-02 \\
1.959 \mathrm{E}-02 \\
2.003 \mathrm{E}-02 \\
2.044 \mathrm{E}-02\end{array}$ & $\begin{array}{l}4.905 E+00 \\
4.522 E+00 \\
4.209 E+00 \\
3.947 E+00 \\
3.725 E+00 \\
3.369 E+00 \\
3.095 E+00 \\
2.878 E+00\end{array}$ & $\begin{array}{l}4.801 E-03 \\
5.863 E-03 \\
7.011 E-03 \\
8.238 E-03 \\
9.543 E-03 \\
1.237 E-02 \\
1.547 E-02 \\
1.883 E-02\end{array}$ & $\begin{array}{l}1.906 E-03 \\
2.121 E-03 \\
2.330 E-03 \\
2.536 E-03 \\
2.737 E-03 \\
3.128 E-03 \\
3.506 E-03 \\
3.870 E-03\end{array}$ & $\begin{array}{l}0.0 \\
0.0 \\
0.0 \\
0.0 \\
0.0 \\
0.0 \\
0.0 \\
0.0\end{array}$ & $\begin{array}{l}-0.204 \\
-0.200 \\
-0.196 \\
-0.192 \\
-0.189 \\
-0.184 \\
-0.180 \\
-0.176\end{array}$ & $\begin{array}{l}0.238 \\
0.231 \\
0.225 \\
0.221 \\
0.216 \\
0.209 \\
0.204 \\
0.199\end{array}$ & $\begin{array}{l}0.231 \\
0.225 \\
0.220 \\
0.215 \\
0.211 \\
0.205 \\
0.200 \\
0.195\end{array}$ \\
\hline $\begin{array}{l}0.1000 \\
0.1250 \\
0.1500 \\
0.1750 \\
0.2000 \\
0.2500 \\
0.3000 \\
0.3500\end{array}$ & $\begin{array}{l}2.680 E+00 \\
2.356 E+00 \\
2.136 E+00 \\
1.978 E+00 \\
1.858 E+00 \\
1.693 E+00 \\
1.585 E+00 \\
1.509 E+00\end{array}$ & $\begin{array}{l}2.081 \mathrm{E}-02 \\
2.169 \mathrm{E}-02 \\
2.251 \mathrm{E}-02 \\
2.329 \mathrm{E}-02 \\
2.405 \mathrm{E}-02 \\
2.560 \mathrm{E}-02 \\
2.721 \mathrm{E}-02 \\
2.890 \mathrm{E}-02\end{array}$ & $\begin{array}{l}2.701 E+00 \\
2.378 E+00 \\
2.159 E+00 \\
2.001 E+00 \\
1.883 E+00 \\
1.718 E+00 \\
1.612 E+00 \\
1.537 E+00\end{array}$ & $\begin{array}{l}2.242 E-02 \\
3.232 E-02 \\
4.338 E-02 \\
5.543 E-02 \\
6.833 E-02 \\
9.622 E-02 \\
1.263 E-01 \\
1.581 E-01\end{array}$ & $\begin{array}{l}4.224 \mathrm{E}-03 \\
5.064 \mathrm{E}-03 \\
5.850 \mathrm{E}-03 \\
6.592 \mathrm{E}-03 \\
7.294 \mathrm{E}-03 \\
8.606 \mathrm{E}-03 \\
9.822 \mathrm{E}-03 \\
1.097 \mathrm{E}-02\end{array}$ & $\begin{array}{l}0.0 \\
0.0 \\
0.0 \\
0.0 \\
0.0 \\
0.0 \\
0.0 \\
1.408 \mathrm{E}-02\end{array}$ & $\begin{array}{l}-0.173 \\
-0.167 \\
-0.162 \\
-0.159 \\
-0.155 \\
-0.150 \\
-0.147 \\
-0.127\end{array}$ & $\begin{array}{l}0.195 \\
0.187 \\
0.181 \\
0.176 \\
0.172 \\
0.166 \\
0.161 \\
0.155\end{array}$ & $\begin{array}{l}0.191 \\
0.184 \\
0.178 \\
0.173 \\
0.170 \\
0.163 \\
0.159 \\
0.152\end{array}$ \\
\hline $\begin{array}{l}0.4000 \\
0.4500 \\
0.5000 \\
0.5500 \\
0.6000 \\
0.7000 \\
0.8000 \\
0.9000\end{array}$ & $\begin{array}{l}1.453 E+00 \\
1.412 E+00 \\
1.381 E+00 \\
1.356 E+00 \\
1.338 E+00 \\
1.311 E+00 \\
1.295 E+00 \\
1.284 E+00\end{array}$ & $\begin{array}{l}3.066 \mathrm{E}-02 \\
3.251 \mathrm{E}-02 \\
3.443 \mathrm{E}-02 \\
3.642 \mathrm{E}-02 \\
3.846 \mathrm{E}-02 \\
4.269 \mathrm{E}-02 \\
4.711 \mathrm{E}-02 \\
5.168 \mathrm{E}-02\end{array}$ & $\begin{array}{l}1.484 E+00 \\
1.444 E+00 \\
1.415 E+00 \\
1.393 E+00 \\
1.376 E+00 \\
1.354 E+00 \\
1.342 E+00 \\
1.336 E+00\end{array}$ & $\begin{array}{l}1.913 \mathrm{E}-01 \\
2.254 \mathrm{E}-01 \\
2.604 \mathrm{E}-01 \\
2.960 \mathrm{E}-01 \\
3.322 \mathrm{E}-01 \\
4.055 \mathrm{E}-01 \\
4.797 \mathrm{E}-01 \\
5.544 \mathrm{E}-01\end{array}$ & $\begin{array}{l}1.206 \mathrm{E}-02 \\
1.312 \mathrm{E}-02 \\
1.415 \mathrm{E}-02 \\
1.516 \mathrm{E}-02 \\
1.615 \mathrm{E}-02 \\
1.809 \mathrm{E}-02 \\
2.000 \mathrm{E}-02 \\
2.187 \mathrm{E}-02\end{array}$ & $\begin{array}{l}3.297 \mathrm{E}-02 \\
5.225 \mathrm{E}-02 \\
7.178 \mathrm{E}-02 \\
9.146 \mathrm{E}-02 \\
1.112 \mathrm{E}-01 \\
1.505 \mathrm{E}-01 \\
1.895 \mathrm{E}-01 \\
2.278 \mathrm{E}-01\end{array}$ & $\begin{array}{l}-0.121 \\
-0.117 \\
-0.113 \\
-0.109 \\
-0.106 \\
-0.101 \\
-0.097 \\
-0.094\end{array}$ & $\begin{array}{l}0.149 \\
0.144 \\
0.140 \\
0.136 \\
0.133 \\
0.127 \\
0.122 \\
0.118\end{array}$ & $\begin{array}{l}0.146 \\
0.141 \\
0.136 \\
0.132 \\
0.128 \\
0.122 \\
0.116 \\
0.112\end{array}$ \\
\hline $\begin{array}{l}1.0000 \\
1.2500 \\
1.5000 \\
1.7500 \\
2.0000 \\
2.5000 \\
3.0000 \\
3.5000\end{array}$ & $\begin{array}{l}1.278 E+00 \\
1.274 E+00 \\
1.278 E+00 \\
1.285 E+00 \\
1.294 E+00 \\
1.312 E+00 \\
1.331 E+00 \\
1.347 E+00\end{array}$ & $\begin{array}{l}5.640 \mathrm{E}-02 \\
6.874 \mathrm{E}-02 \\
8.177 \mathrm{E}-02 \\
9.534 \mathrm{E}-02 \\
1.094 \mathrm{E}-01 \\
1.385 \mathrm{E}-01 \\
1.687 \mathrm{E}-01 \\
1.999 \mathrm{E}-01\end{array}$ & $\begin{array}{l}1.335 E+00 \\
1.343 E+00 \\
1.360 E+00 \\
1.380 E+00 \\
1.403 E+00 \\
1.451 E+00 \\
1.499 E+00 \\
1.547 E+00\end{array}$ & $\begin{array}{l}6.293 \mathrm{E}-01 \\
8.162 \mathrm{E}-01 \\
1.001 \mathrm{E}+00 \\
1.184 \mathrm{E}+00 \\
1.363 \mathrm{E}+00 \\
1.714 \mathrm{E}+00 \\
2.053 \mathrm{E}+00 \\
2.381 \mathrm{E}+00\end{array}$ & $\begin{array}{l}2.373 E-02 \\
2.833 E-02 \\
3.289 E-02 \\
3.742 E-02 \\
4.193 E-02 \\
5.089 E-02 \\
5.974 E-02 \\
6.848 E-02\end{array}$ & $\begin{array}{l}2.652 \mathrm{E}-01 \\
3.551 \mathrm{E}-01 \\
4.394 \mathrm{E}-01 \\
5.186 \mathrm{E}-01 \\
5.933 \mathrm{E}-01 \\
7.310 \mathrm{E}-01 \\
8.560 \mathrm{E}-01 \\
9.707 \mathrm{E}-01\end{array}$ & $\begin{array}{l}-0.090 \\
-0.084 \\
-0.080 \\
-0.076 \\
-0.073 \\
-0.067 \\
-0.063 \\
-0.060\end{array}$ & $\begin{array}{l}0.114 \\
0.107 \\
0.102 \\
0.097 \\
0.094 \\
0.087 \\
0.083 \\
0.079\end{array}$ & $\begin{array}{l}0.108 \\
0.100 \\
0.093 \\
0.088 \\
0.084 \\
0.077 \\
0.072 \\
0.067\end{array}$ \\
\hline $\begin{array}{l}4.00000 \\
4.5000 \\
5.0000 \\
5.5000 \\
6.0000 \\
7.0000 \\
8.0000 \\
9.0000\end{array}$ & $\begin{array}{l}1.363 E+00 \\
1.377 E+00 \\
1.390 E+00 \\
1.401 E+00 \\
1.412 E+00 \\
1.431 E+00 \\
1.448 E+00 \\
1.462 E+00\end{array}$ & $\begin{array}{l}2.317 \mathrm{E}-01 \\
2.642 \mathrm{E}-01 \\
2.973 \mathrm{E}-01 \\
3.309 \mathrm{E}-01 \\
3.649 \mathrm{E}-01 \\
4.340 \mathrm{E}-01 \\
5.045 \mathrm{E}-01 \\
5.760 \mathrm{E}-01\end{array}$ & $\begin{array}{l}1.595 E+00 \\
1.641 E+00 \\
1.687 E+00 \\
1.732 E+00 \\
1.777 E+00 \\
1.865 E+00 \\
1.952 E+00 \\
2.038 E+00\end{array}$ & $\begin{array}{l}2.699 E+00 \\
3.008 E+00 \\
3.309 E+00 \\
3.601 E+00 \\
3.886 E+00 \\
4.436 E+00 \\
4.960 E+00 \\
5.461 E+00\end{array}$ & $\begin{array}{l}7.708 E-02 \\
8.554 E-02 \\
9.385 E-02 \\
1.020 E-01 \\
1.100 E-01 \\
1.256 E-01 \\
1.406 E-01 \\
1.551 E-01\end{array}$ & $\begin{array}{l}1.077 \mathrm{E}+00 \\
1.175 \mathrm{E}+00 \\
1.268 \mathrm{E}+00 \\
1.355 \mathrm{E}+00 \\
1.437 \mathrm{E}+00 \\
1.590 \mathrm{E}+00 \\
1.729 \mathrm{E}+00 \\
1.856 \mathrm{E}+00\end{array}$ & $\begin{array}{l}-0.057 \\
-0.054 \\
-0.052 \\
-0.050 \\
-0.048 \\
-0.045 \\
-0.043 \\
-0.040\end{array}$ & $\begin{array}{l}0.075 \\
0.072 \\
0.070 \\
0.068 \\
0.066 \\
0.062 \\
0.059 \\
0.056\end{array}$ & $\begin{array}{l}0.063 \\
0.060 \\
0.057 \\
0.055 \\
0.052 \\
0.049 \\
0.045 \\
0.042\end{array}$ \\
\hline $\begin{array}{l}10.0000 \\
12.5000 \\
15.0000 \\
17.5000 \\
20.0000 \\
25.0000 \\
30.0000 \\
35.0000\end{array}$ & $\begin{array}{l}1.475 E+00 \\
1.502 E+00 \\
1.523 E+00 \\
1.540 E+00 \\
1.555 E+00 \\
1.579 E+00 \\
1.598 E+00 \\
1.613 E+00\end{array}$ & $\begin{array}{l}6.485 E-01 \\
8.331 E-01 \\
1.022 E+00 \\
1.213 E+00 \\
1.407 E+00 \\
1.802 E+00 \\
2.202 E+00 \\
2.606 E+00\end{array}$ & $\begin{array}{l}2.123 E+00 \\
2.335 E+00 \\
2.545 E+00 \\
2.754 E+00 \\
2.963 E+00 \\
3.381 E+00 \\
3.800 E+00 \\
4.220 E+00\end{array}$ & $\begin{array}{l}5.942 E+00 \\
7.064 E+00 \\
8.089 E+00 \\
9.033 E+00 \\
9.909 E+00 \\
1.149 E+01 \\
1.288 E+01 \\
1.413 E+01\end{array}$ & $\begin{array}{l}1.690 E-01 \\
2.015 E-01 \\
2.312 E-01 \\
2.584 E-01 \\
2.834 E-01 \\
3.277 E-01 \\
3.659 E-01 \\
3.993 E-01\end{array}$ & $\begin{array}{l}1.975 E+00 \\
2.240 E+00 \\
2.471 E+00 \\
2.675 E+00 \\
2.858 E+00 \\
3.178 E+00 \\
3.451 E+00 \\
3.689 E+00\end{array}$ & $\begin{array}{l}-0.038 \\
-0.034 \\
-0.031 \\
-0.028 \\
-0.026 \\
-0.023 \\
-0.020 \\
-0.018\end{array}$ & $\begin{array}{l}0.054 \\
0.049 \\
0.046 \\
0.043 \\
0.040 \\
0.036 \\
0.033 \\
0.031\end{array}$ & $\begin{array}{l}0.040 \\
0.035 \\
0.031 \\
0.027 \\
0.025 \\
0.021 \\
0.018 \\
0.016\end{array}$ \\
\hline $\begin{array}{l}40.0000 \\
45.0000 \\
50.0000 \\
55.0000 \\
60.0000 \\
70.0000 \\
80.0000 \\
90.0000\end{array}$ & $\begin{array}{l}1.626 E+00 \\
1.638 E+00 \\
1.648 E+00 \\
1.657 E+00 \\
1.665 E+00 \\
1.678 E+00 \\
1.690 E+00 \\
1.700 E+00\end{array}$ & $\begin{array}{l}3.015 E+00 \\
3.426 E+00 \\
3.840 E+00 \\
4.256 E+00 \\
4.674 E+00 \\
5.513 E+00 \\
6.358 E+00 \\
7.206 E+00\end{array}$ & $\begin{array}{l}4.641 E+00 \\
5.064 E+00 \\
5.488 E+00 \\
5.913 E+00 \\
6.338 E+00 \\
7.192 E+00 \\
8.048 E+00 \\
8.906 E+00\end{array}$ & $\begin{array}{l}1.526 E+01 \\
1.629 E+01 \\
1.724 E+01 \\
1.812 E+01 \\
1.893 E+01 \\
2.041 E+01 \\
2.173 E+01 \\
2.291 E+01\end{array}$ & $\begin{array}{l}4.286 \mathrm{E}-01 \\
4.547 \mathrm{E}-01 \\
4.781 \mathrm{E}-01 \\
4.992 \mathrm{E}-01 \\
5.183 \mathrm{E}-01 \\
5.518 \mathrm{E}-01 \\
5.801 \mathrm{E}-01 \\
6.045 \mathrm{E}-01\end{array}$ & $\begin{array}{l}3.899 E+00 \\
4.089 E+00 \\
4.261 \mathrm{I}+00 \\
4.419 \mathrm{E}+00 \\
4.565 \mathrm{E}+00 \\
4.828 \mathrm{E}+00 \\
5.059 \mathrm{E}+00 \\
5.266 \mathrm{E}+00\end{array}$ & $\begin{array}{l}-0.0117 \\
-0.016 \\
-0.015 \\
-0.014 \\
-0.013 \\
-0.011 \\
-0.010 \\
-0.009\end{array}$ & $\begin{array}{l}0.029 \\
0.028 \\
0.027 \\
0.025 \\
0.025 \\
0.023 \\
0.022 \\
0.021\end{array}$ & $\begin{array}{l}0.0114 \\
0.0113 \\
0.0112 \\
0.0111 \\
0.0110 \\
0.008 \\
0.007 \\
0.007\end{array}$ \\
\hline $\begin{array}{l}100.00000 \\
125.0000 \\
150.0000 \\
175.0000 \\
200.00000 \\
250.00000 \\
300.0000 \\
350.0000\end{array}$ & $\begin{array}{l}1.709 E+00 \\
1.728 E+00 \\
1.743 E+00 \\
1.755 E+00 \\
1.766 E+00 \\
1.783 E+00 \\
1.797 E+00 \\
1.808 E+00\end{array}$ & $\begin{array}{l}8.056 E+00 \\
1.019 E+01 \\
1.234 E+01 \\
1.450 E+01 \\
1.666 E+01 \\
2.100 E+01 \\
2.535 E+01 \\
2.970 E+01\end{array}$ & $\begin{array}{l}66 E+00 \\
92 E+01 \\
09 E+01 \\
25 E+01 \\
43 E+01 \\
78 E+01 \\
14 E+01 \\
51 E+01\end{array}$ & $\begin{array}{l}2.398 E+01 \\
2.629 E+01 \\
2.822 E+01 \\
2.987 E+01 \\
3.131 E+01 \\
3.375 E+01 \\
3.576 E+01 \\
3.747 E+01\end{array}$ & $\begin{array}{l}6.258 E-01 \\
6.688 E-01 \\
7.017 E-01 \\
7.279 E-01 \\
7.492 E-01 \\
7.820 E-01 \\
8.064 E-01 \\
8.252 E-01\end{array}$ & $\begin{array}{l}5.453 \mathrm{E}+00 \\
5.855 \mathrm{E}+00 \\
6.190 \mathrm{E}+00 \\
6.476 \mathrm{E}+00 \\
6.726 \mathrm{E}+00 \\
7.147 \mathrm{E}+00 \\
7.495 \mathrm{E}+00 \\
7.791 \mathrm{E}+00\end{array}$ & $\begin{array}{l}-0.009 \\
-0.007 \\
-0.006 \\
-0.005 \\
-0.005 \\
-0.004 \\
-0.003 \\
-0.003\end{array}$ & $\begin{array}{l}0.020 \\
0.018 \\
0.017 \\
0.016 \\
0.0115 \\
0.014 \\
0.014 \\
0.013\end{array}$ & $\begin{array}{l}0.006 \\
0.005 \\
0.004 \\
0.003 \\
0.003 \\
0.002 \\
0.002 \\
0.002\end{array}$ \\
\hline $\begin{array}{l}400.00000 \\
450.0000 \\
500.00000 \\
550.00000 \\
600.00000 \\
700.00000 \\
800.00000 \\
900.00000\end{array}$ & $\begin{array}{l}1.818 E+00 \\
1.827 E+00 \\
1.835 E+00 \\
1.842 E+00 \\
1.848 E+00 \\
1.859 E+00 \\
1.869 E+00 \\
1.878 E+00\end{array}$ & $\begin{array}{l}3.407 E+01 \\
3.844 E+01 \\
4.281 E+01 \\
4.719 E+01 \\
5.157 E+01 \\
6.034 E+01 \\
6.912 E+01 \\
7.790 E+01\end{array}$ & $\begin{array}{l}3.589 E+01 \\
4.027 E+01 \\
4.465 E+01 \\
4.903 E+01 \\
5.342 E+01 \\
6.220 E+01 \\
7.099 E+01 \\
7.978 E+01\end{array}$ & $\begin{array}{l}3.895 \mathrm{E}+01 \\
4.027 \mathrm{E}+01 \\
4.145 \mathrm{E}+01 \\
4.251 \mathrm{E}+01 \\
4.349 \mathrm{E}+01 \\
4.522 \mathrm{E}+01 \\
4.673 \mathrm{E}+01 \\
4.806 \mathrm{E}+01\end{array}$ & $\begin{array}{l}8.404 \mathrm{E}-01 \\
8.528 \mathrm{E}-01 \\
8.632 \mathrm{E}-01 \\
8.720 \mathrm{E}-01 \\
8.797 \mathrm{E}-01 \\
8.923 \mathrm{E}-01 \\
9.023 \mathrm{E}-01 \\
9.104 \mathrm{E}-01\end{array}$ & $\begin{array}{l}8.048 E+00 \\
8.276 E+00 \\
8.481 E+00 \\
8.666 E+00 \\
8.836 E+00 \\
9.133 E+00 \\
9.401 E+00 \\
9.633 E+00\end{array}$ & $\begin{array}{l}-0.003 \\
-0.002 \\
-0.002 \\
-0.002 \\
-0.002 \\
-0.001 \\
-0.001 \\
-0.001\end{array}$ & $\begin{array}{l}0.0112 \\
0.0112 \\
0.012 \\
0.0111 \\
0.0111 \\
0.0111 \\
0.0110 \\
0.010\end{array}$ & $\begin{array}{l}0.002 \\
0.001 \\
0.001 \\
0.001 \\
0.001 \\
0.001 \\
0.001 \\
0.001\end{array}$ \\
\hline 0.0000 & $1.885 E+00$ & $8.669 E+01$ & $8.858 E+01$ & $924 \mathrm{E}+01$ & $9.171 \mathrm{E}-01$ & $.841 E+00$ & -0.001 & 0.010 & 001 \\
\hline
\end{tabular}




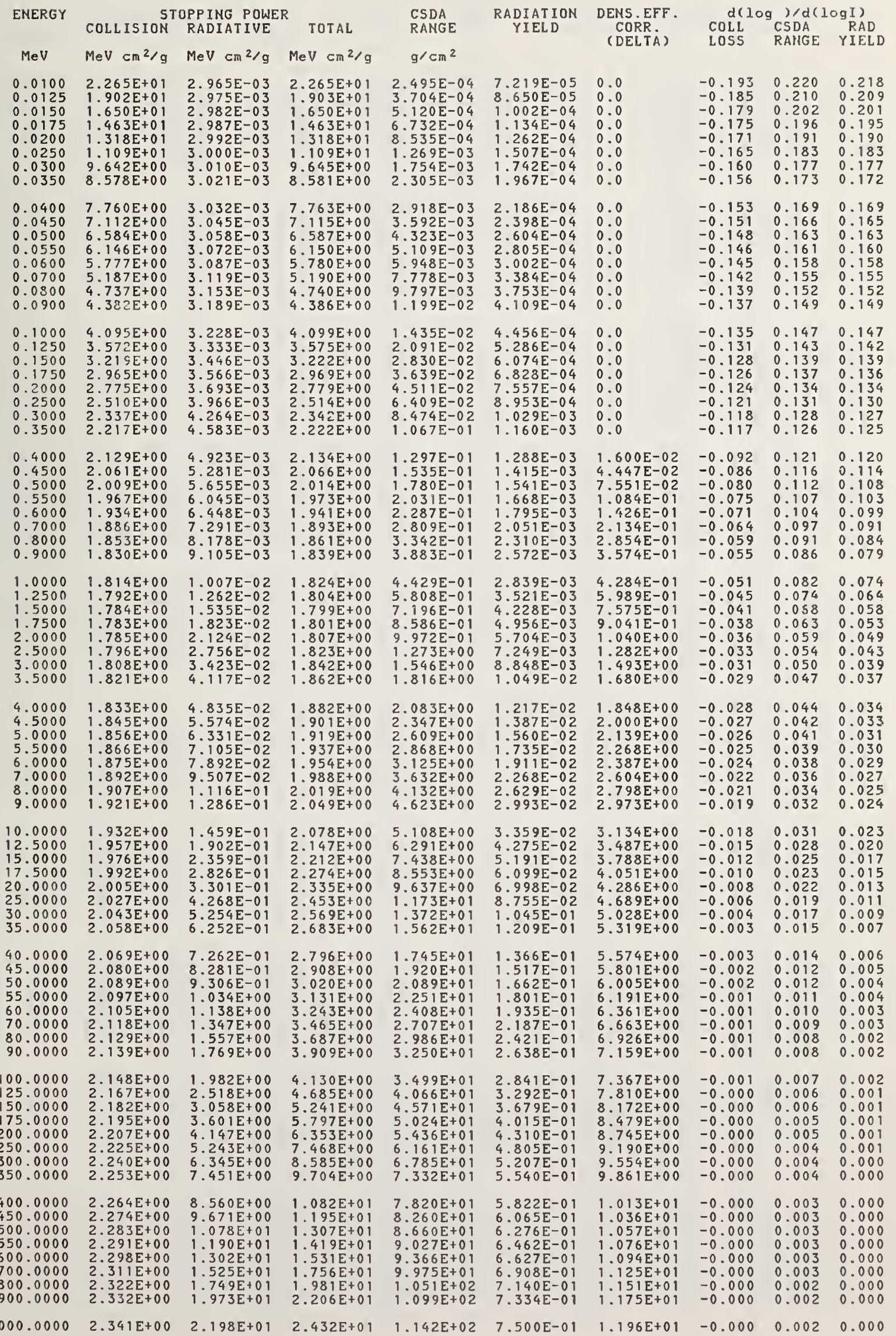


ELECTRONS IN POLYCARBONATE, "MAKROLON", "LEXAN"

$I=73.1 \mathrm{eV}$

ENERGY STOPPING POWER

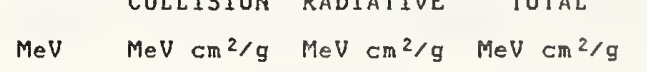

$0.0100 \quad 2.153 \mathrm{E}+01$

0.0125

0.0175

0.0200

0.0250

0.0300

$0.0400 \quad 7.412 E+00$

$0.0450 \quad 6.795 \mathrm{E}+00$

$0.055063 \mathrm{E}+00$

$0.0600-5.524 \mathrm{E}+00$

$0.0700 \quad 4.962 \mathrm{E}+00$

$0.0800 \quad 4.533 \mathrm{E}+00$

0.0900

0.1000

0.1250

0.1500

0.175

0.2000

0.2500

0.3500

0.4000

0.4500

0.5000

0.6000

0.7000

0.8000

0.9000

1.0000

1.2500

1.7500

2.000

2.5000

3.5000

4.0000

4.5000

5.0000

5.5000

6.0000

8.0000

9.0000

10.0000 12.500 15.0000 17.5000 20.0000 25.0000 30.0000 35.0000

40.0000 45.000 50.0000 55.0000 60.0000 70.0000 80.0000 90.0000

100.0000 125.0000 150.000 200.000

250.000

300.0000

350.0000

400.0000

450.0000

500.0000

550.0000
600.0000

700.0000

800.0000

900.0000

$4.194 \mathrm{E}+00$

$3.240 \mathrm{E}-03$

$3.254 \mathrm{E}-03$

$3.263 \mathrm{E}-03$
$3.270 \mathrm{E}-03$

. $393 \mathrm{E}+0$

$3.275 \mathrm{E}-03$

$3.284 \mathrm{E}-03$

$3.293 \mathrm{E}-03$
$3.304 \mathrm{E}-03$

$2.153 E+01$

$1.810 \mathrm{E}+01$

$1.571 E+01$

1. $394 E+01$

$1.257 \mathrm{E}+01$

$9.205 \mathrm{E}+00$

3. $315 \mathrm{E}-03$

.327E-03

$3.340 \mathrm{E}-03$

$3.355 \mathrm{E}-03$
$3.370 \mathrm{E}-03$

$3.403 \mathrm{E}-03$

$3.439 \mathrm{E}-03$
$3.477 \mathrm{E}-03$

$7.416 \mathrm{E}+00$

$6.798 \mathrm{E}+00$

$6.296 E+00$

$5.879 \mathrm{E}+00$

$5.527 E+00$

$4.965 \mathrm{E}+00$

$4.536 \mathrm{E}+00$

$3.920 \mathrm{E}+00$

$3.421 \mathrm{E}+00$

$3.518 \mathrm{E}-03$

3. $224 E+00$

$3.629 \mathrm{E}-03$

$3.749 \mathrm{E}-03$

$3.877 \mathrm{E}-03$

$4.013 E-03$

. $660 \mathrm{E}+00$

$2.407 \mathrm{E}+00$

$2.128 \mathrm{E}+00$
2.00

$4.623 \mathrm{E}-03$

$4.964 \mathrm{E}-03$

$3.425 E+00$

$3.088 \mathrm{E}+00$

$2.846 \mathrm{E}+00$

$2.664 \mathrm{E}+00$

$2.412 \mathrm{E}+00$

$2.247 E+00$

$2.044 \mathrm{E}+00$

$5.327 \mathrm{E}-03$

$5.710 \mathrm{E}-03$

$2.050 E+00$

$1.980 \mathrm{E}+00$

$1.891 \mathrm{E}+00$

$1.859 E+00$

$1.813 \mathrm{E}+00$

$1.782 \mathrm{E}+00$

$6.112 \mathrm{E}-03$

$6.529 \mathrm{E}-03$

$7.864 \mathrm{E}-03$

$8.813 \mathrm{E}-03$

1.986

$1.936 \mathrm{E}+00$

$.897 \mathrm{E}+00$

$1.866 E+00$

$1.821 \mathrm{E}+00$

$1.791 E+00$

$1.746 E+00$

$1.726 E+00$

$1.084 \mathrm{E}-02$

$1.357 \mathrm{E}-02$

$1.650 \mathrm{E}-02$

$1.718 \mathrm{E}+00$

$1.721 E+00$

$1.731 E+00$

$1.744 E+00$

$2.279 \mathrm{E}-02$

$2.955 \mathrm{E}-02$

$4.408 \mathrm{E}-02$

$1.769 \mathrm{E}+00$

$1.780 \mathrm{E}+00$

$1.791 \mathrm{E}+00$
$1.801 \mathrm{E}+00$

$1.811 \mathrm{E}+00$

$1.827 E+00$

$.842 \mathrm{E}+00$

$1.855 E+00$

$5.174 \mathrm{E}-02$

5. $962 \mathrm{E}-02$

$7.594 \mathrm{E}-02$

$8.434 \mathrm{E}-02$

$1.015 \mathrm{E}-01$

1. $192 \mathrm{E}-01$

$1.867 E+00$

$1.891 E+00$
$1.910 E+00$

$1.557 \mathrm{E}-01$

$2.029 \mathrm{E}-01$

2. $515 \mathrm{E}-01$

3. $011 \mathrm{E}-01$

3. $516 \mathrm{E}-01$

$4.544 \mathrm{E}-0$

$.939 \mathrm{E}+00$

$1.961 E+00$

$1.992 \mathrm{E}+00$

$5.591 \mathrm{E}-01$
$6.652 \mathrm{E}-01$

$2.003 E+00$

2. $014 \mathrm{E}+00$

$7.724 \mathrm{E}-01$

$8.805 \mathrm{E}-01$

$9.894 \mathrm{E}-01$

$1.099 \mathrm{E}+00$

.

$2.051 \mathrm{E}+00$

2.051E+00

$1.431 E+00$

$1.654 E+00$

$2.072 \mathrm{E}+00$

(1)

$2.099 \mathrm{E}+00$

$2.114 E+00$

2. $127 \mathrm{E}+00$

$2.138 \mathrm{E}+00$

$2.156 \mathrm{E}+00$

2. $171 \mathrm{E}+00$
$2.183 \mathrm{E}+00$

$2.194 \mathrm{E}+00$

$2.204 \mathrm{E}+00$

.

$2.227 \mathrm{E}+00$

$2.239 \mathrm{E}+00$

$2.250 \mathrm{E}+00$

$2.260 \mathrm{E}+00$

$2.673 E+00$

$3.820 \mathrm{E}+00$

$4.398 \mathrm{E}+00$

$5.560 \mathrm{E}+00$

$6.726 \mathrm{E}+00$

$9.071 E+00$

$1.025 \mathrm{E}+01$

$1.143 \mathrm{E}+01$

$1.379 E+01$

$1.615 \mathrm{E}+01$

$1.852 \mathrm{E}+01$
$2.089 \mathrm{E}+01$

$1.757 E+00$

1. $.739 E+00$

$1.738 \mathrm{E}+00$

$1.744 E+00$

$1.761 E+00$

$1.781 \mathrm{E}+00$

$1.821 E+00$

$1.840 \mathrm{E}+00$

$1.859 E+00$

$1.877 E+00$

$1.895 E+00$

$1.961 E+00$

$1.992 \mathrm{E}+00$

$2.022 E+00$

$2.094 \mathrm{E}+00$

2.1 $162 \mathrm{E}+00$

$2.291 E+00$

2. $415 \mathrm{E}+00$

$2.537 \mathrm{E}+00$

$2.657 \mathrm{E}+00$

$2.776 \mathrm{E}+00$

$2.894 E+00$

3. $012 E+00$

$3.247 E+00$

$3.247 \mathrm{E}+00$

$3.717 \mathrm{E}+00$

$3.951 E+00$

$4.185 E+00$

$4.772 E+00$

$5.359 \mathrm{E}+00$

$5.947 \mathrm{E}+00$

$6.536 \mathrm{E}+00$

$7.715 \mathrm{E}+00$
$8.897 \mathrm{E}+00$

$1.008 \mathrm{E}+01$

$1.127 \mathrm{E}+01$

$1.245 \mathrm{E}+0$

1. $364 E+01$

1. $683 \mathrm{E}+0$

$1.839 \mathrm{E}+01$

$2.077 \mathrm{E}+01$

$2.315 \mathrm{E}+01$

$2.327 \mathrm{E}+01$

DENSITY $=1.200 \mathrm{E}+00 \mathrm{~g} / \mathrm{cm}^{3}$

$\begin{array}{lcc}\text { CSDA } & \text { RADIATION } \\ \text { RANGE } & \text { YIELD } & \begin{array}{c}\text { DENS.EFF. } \\ \text { CORR } \\ \text { (DELTA) }\end{array} \\ {\mathrm{g} / \mathrm{Cm}^{2}} & & \end{array}$

2. $634 \mathrm{E}-04$

$.906 \mathrm{E}-04 \quad 9.938 \mathrm{E}-05 \quad 0.0$

$3.393 \mathrm{E}-04 \quad 1.151 \mathrm{E}-04 \quad 0.0$

.086E-04 1.303E-04 0.0

$1.333 \mathrm{E}-03 \quad 1.731 \mathrm{E}-04 \quad 0.0$

$1.842 \mathrm{E}-03 \quad 2.000 \mathrm{E}-04 \quad 0.0$

$2.419 \mathrm{E}-03 \quad 2.258 \mathrm{E}-04 \quad 0.0$

$3.061 E-03 \quad 2.507 E-04 \quad 0.0$

$3.766 \mathrm{E}-03 \quad 2.749 \mathrm{E}-04 \quad 0.0$

$\begin{array}{lll}4.531 E-03 & 2.984 E-04 & 0.0\end{array}$

$\begin{array}{lll}5.354 \mathrm{E}-03 & 3.213 \mathrm{E}-04 & 0.0\end{array}$

$6.231 \mathrm{E}-03 \quad 3.437 \mathrm{E}-04 \quad 0.0$

$8.144 \mathrm{E}-03 \quad 3.872 \mathrm{E}-04 \quad 0.0$

$\begin{array}{lll}1.025 \mathrm{E}-02 & 4.290 \mathrm{E}-04 & 0.0 \\ 1.255 \mathrm{E}-02 & 4.695 \mathrm{E}-04 & 0.0\end{array}$

$\begin{array}{lll}1.502 \mathrm{E}-02 & 5.088 \mathrm{E}-04 & 0.0\end{array}$

$\begin{array}{lll}.186 \mathrm{E}-02 & 6.028 \mathrm{E}-04 & 0.0\end{array}$

$2.957 \mathrm{E}-02 \quad 6.919 \mathrm{E}-04 \quad 0.0$

$\begin{array}{lll}3.802 \mathrm{E}-02 & 7.772 \mathrm{E}-04 & 0.0\end{array}$

$\begin{array}{lll}4.711 \mathrm{E}-02 & 8.594 \mathrm{E}-04 & 0.0 \\ 6.691 \mathrm{E}-02 & 1.017 \mathrm{E}-03 & 0.0\end{array}$

$\begin{array}{lll}6.691 \mathrm{E}-02 & 1.017 \mathrm{E}-03 & 0.0 \\ 8.843 \mathrm{E}-02 & 1.168 \mathrm{E}-03 & 0.0 \\ 1.113 \mathrm{E}-01 & 1.314 \mathrm{E}-03 & 0.0\end{array}$

$1.352 \mathrm{E}-01$

$1.600 \mathrm{E}-01$

$.855 \mathrm{E}-0$

2. $116 \mathrm{E}-01$

$2.925 \mathrm{E}-01$

$3.479 \mathrm{E}-01$

4.041E-0

$4.608 \mathrm{E}-01$
$6.039 \mathrm{E}-01$

$7.479 \mathrm{E}-0$

$8.919 E-01$

$1.036 \mathrm{E}+00$ 


\begin{tabular}{|c|c|c|c|c|c|c|c|c|c|}
\hline ENERGY & 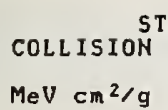 & $\begin{array}{l}\text { OPPING POW } \\
\text { RADIATIVE } \\
\mathrm{MeV} \mathrm{cm}^{2} / \mathrm{g}\end{array}$ & $\begin{array}{l}\text { TOTAL } \\
\mathrm{V} \mathrm{cm}^{2} / \mathrm{g}\end{array}$ & $\begin{array}{l}\text { CSDA } \\
\text { RANGE } \\
\mathrm{g} / \mathrm{cm}^{2}\end{array}$ & ADIATION & $\begin{array}{l}\text { DEIS.EFF. } \\
\text { CORR } \\
\text { (DELTA) }\end{array}$ & $\begin{array}{l}d c \\
\text { cold } \\
\text { LOSS }\end{array}$ & SD & $\begin{array}{l}\text { RI ) } \\
\text { RAD } \\
\text { YIELD }\end{array}$ \\
\hline $\begin{array}{l}0.0100 \\
0.0125 \\
0.0150 \\
0.0175 \\
0.0200 \\
0.0250 \\
0.0300 \\
0.0350\end{array}$ & $\begin{array}{l}2.441 \mathrm{E}+01 \\
2.049 \mathrm{E}+01 \\
1.775 \mathrm{E}+01 \\
1.573 \mathrm{E}+01 \\
1.417 \mathrm{E}+01 \\
1.191 \mathrm{E}+01 \\
1.035 \mathrm{E}+01 \\
9.206 \mathrm{E}+00\end{array}$ & $\begin{array}{l}2.847 \\
2.854 \\
2.860 \\
2.864 \\
2.873 \\
2.883 \\
2.894\end{array}$ & $\begin{array}{l}E+01 \\
E+01 \\
E+01 \\
E+01 \\
E+01 \\
E+01 \\
E+01 \\
E+00\end{array}$ & $\begin{array}{l}3.45 \\
4.74 \\
6.24 \\
7.92 \\
1.17 \\
1.63 \\
2.14\end{array}$ & $\begin{array}{l}\text { E-05 } \\
\text { E-05 } \\
\text { E-05 } \\
E-04 \\
\text { E-04 } \\
E-04 \\
\text { E-04 } \\
\text { E- } 04\end{array}$ & $\begin{array}{l}0.0 \\
0.0 \\
0.0 \\
0.0 \\
0.0 \\
0.0 \\
0.0 \\
0.0\end{array}$ & $\begin{array}{l}-0 \\
-0 \\
-0 \\
-0 \\
-0 \\
-0 \\
-0 \\
-0\end{array}$ & $\begin{array}{l}0.214 \\
0.204 \\
0.197 \\
0.191 \\
0.187 \\
0.179 \\
0.174 \\
0.169\end{array}$ & $\begin{array}{l}0.213 \\
0.203 \\
0.196 \\
0.191 \\
0.186 \\
0.179 \\
0.173 \\
0.169\end{array}$ \\
\hline $\begin{array}{l}0.0400 \\
0.0450 \\
0.0500 \\
0.0550 \\
0.0600 \\
0.0700 \\
0.0800 \\
0.0900\end{array}$ & $\begin{array}{l}8.325 \mathrm{E}+00 \\
7.627 \mathrm{E}+00 \\
7.060 \mathrm{E}+00 \\
6.589 \mathrm{E}+00 \\
6.191 \mathrm{E}+00 \\
5.557 \mathrm{E}+00 \\
5.074 \mathrm{E}+00 \\
4.692 \mathrm{E}+00\end{array}$ & $\begin{array}{l}2.918 \\
2.931 \\
2.945 \\
2.960 \\
2.992 \\
3.025 \\
3.061\end{array}$ & $\begin{array}{l}7.0 \\
6.5 \\
6.1 \\
5.5 \\
5.0 \\
4.6\end{array}$ & $\begin{array}{l}5 E-03 \\
3 E-03 \\
5 E-03 \\
8 E-03 \\
1 E-03 \\
9 E-03 \\
4 E-03\end{array}$ & $\begin{array}{l}E-04 \\
E-04 \\
E-04 \\
E-04 \\
E-04 \\
E-04 \\
E-04 \\
E-04\end{array}$ & $\begin{array}{l}0.0 \\
0.0 \\
0.0 \\
0.0 \\
0.0 \\
0.0 \\
0.0 \\
0.0\end{array}$ & $\begin{array}{l}-0.150 \\
-0.148 \\
-0.146 \\
-0.144 \\
-0.142 \\
-0.139 \\
-0.137 \\
-0.135\end{array}$ & $\begin{array}{l}0.166 \\
0.162 \\
0.160 \\
0.157 \\
0.155 \\
0.152 \\
0.149 \\
0.147\end{array}$ & $\begin{array}{l}0.165 \\
0.162 \\
0.160 \\
0.157 \\
0.155 \\
0.152 \\
0.149 \\
0.146\end{array}$ \\
\hline $\begin{array}{l}0.1000 \\
0.1250 \\
0.1500 \\
0.1750 \\
0.2000 \\
0.2500 \\
0.3000 \\
0.3500\end{array}$ & $\begin{array}{l}4.384 \mathrm{E}+00 \\
3.822 \mathrm{E}+00 \\
3.443 \mathrm{E}+00 \\
3.171 \mathrm{E}+00 \\
2.967 \mathrm{E}+00 \\
2.683 \mathrm{E}+00 \\
2.497 \mathrm{E}+00 \\
2.368 \mathrm{E}+00\end{array}$ & $\begin{array}{l}3.201 \\
3.312 \\
3.429 \\
3.553 \\
3.820 \\
4.110 \\
4.420\end{array}$ & $\begin{array}{l}4.387 \mathrm{E}+00 \\
3.825 \mathrm{E}+00 \\
3.446 \mathrm{E}+00 \\
3.174 \mathrm{E}+00 \\
2.970 \mathrm{E}+00 \\
2.687 \mathrm{E}+00 \\
2.501 \mathrm{E}+00 \\
2.373 \mathrm{E}+00\end{array}$ & $\begin{array}{l}-02 \\
-02 \\
-02 \\
-02 \\
-02 \\
-02 \\
-02 \\
-02\end{array}$ & $\begin{array}{l}E-04 \\
E-04 \\
E-04 \\
E-04 \\
E-04 \\
E-04 \\
E-04\end{array}$ & $\begin{array}{l}0.0 \\
0.0 \\
0.0 \\
0.0 \\
0.0 \\
0.0 \\
0.0 \\
0.0\end{array}$ & $\begin{array}{l}-0.1 \\
-0.1 \\
-0.1 \\
-0.1 \\
-0.1 \\
-0.1 \\
-0.1 \\
-0.1\end{array}$ & $\begin{array}{l}0.144 \\
0.140 \\
0.137 \\
0.134 \\
0.132 \\
0.129 \\
0.126 \\
0.124\end{array}$ & $\begin{array}{l}0.144 \\
0.140 \\
0.137 \\
0.134 \\
0.132 \\
0.128 \\
0.125 \\
0.123\end{array}$ \\
\hline $\begin{array}{l}0.4000 \\
0.4500 \\
0.5000 \\
0.5500 \\
0.6000 \\
0.7000 \\
0.8000 \\
0.9000\end{array}$ & $\begin{array}{l}2.272 \mathrm{E}+00 \\
2.199 \mathrm{E}+00 \\
2.142 \mathrm{E}+00 \\
2.097 \mathrm{E}+00 \\
2.061 \mathrm{E}+00 \\
2.008 \mathrm{E}+00 \\
1.972 \mathrm{E}+00 \\
1.947 \mathrm{E}+00\end{array}$ & $\begin{array}{l}5.05 \\
5.46 \\
5.84 \\
6.23 \\
7.05 \\
7.9\end{array}$ & $\begin{array}{l}2.204 \mathrm{E}+0 \\
2.147 \mathrm{E}+0 \\
2.103 \mathrm{E}+0 \\
2.068 \mathrm{E}+0 \\
2.016 \mathrm{E}+0 \\
1.980 \mathrm{E}+0 \\
1.956 \mathrm{E}+0\end{array}$ & $\begin{array}{l}1.213 \mathrm{E}-01 \\
1.437 \mathrm{E}-01 \\
1.667 \mathrm{E}-01 \\
1.902 \mathrm{E}-01 \\
2.142 \mathrm{E}-01 \\
2.632 \mathrm{E}-01 \\
3.133 \mathrm{E}-01 \\
3.641 \mathrm{E}-01\end{array}$ & $\begin{array}{l}1.160 \\
1.276 \\
1.391 \\
1.506 \\
1.622 \\
1.856 \\
2.092 \\
2.332\end{array}$ & $\begin{array}{l}2.626 \mathrm{E}-0 \\
5.906 \mathrm{E}-0 \\
9.409 \mathrm{E}-0 \\
1.307 \mathrm{E}-0 \\
1.683 \mathrm{E}-0 \\
2.453 \mathrm{E}-0 \\
3.231 \mathrm{E}-0 \\
4.002 \mathrm{E}-0\end{array}$ & $\begin{array}{l}-0.087 \\
-0.081 \\
-0.075 \\
-0.070 \\
-0.066 \\
-0.060 \\
-0.054 \\
-0.050\end{array}$ & $\begin{array}{l}3 \\
8 \\
4 \\
0 \\
3 \\
7 \\
2\end{array}$ & $\begin{array}{l}0.116 \\
0.110 \\
0.104 \\
0.099 \\
0.095 \\
0.087 \\
0.080 \\
0.074\end{array}$ \\
\hline $\begin{array}{l}1.0000 \\
1.2500 \\
1.5000 \\
1.7500 \\
2.0000 \\
2.5000 \\
3.0000 \\
3.5000\end{array}$ & $\begin{array}{l}1.930 \mathrm{E}+00 \\
1.905 \mathrm{E}+00 \\
1.895 \mathrm{E}+00 \\
1.893 \mathrm{E}+00 \\
1.895 \mathrm{E}+00 \\
1.905 \mathrm{E}+00 \\
1.917 \mathrm{E}+00 \\
1.930 \mathrm{E}+00\end{array}$ & $\begin{array}{l}9.754 \mathrm{E}-03 \\
1.224 \mathrm{E}-02 \\
1.490 \mathrm{E}-02 \\
1.770 \mathrm{E}-02 \\
2.062 \mathrm{E}-02 \\
2.678 \mathrm{E}-02 \\
3.327 \mathrm{E}-02 \\
4.004 \mathrm{E}-02\end{array}$ & $\begin{array}{l}1.917 \mathrm{E}+00 \\
1.910 \mathrm{E}+00 \\
1.911 \mathrm{E}+00 \\
1.916 \mathrm{E}+00 \\
1.932 \mathrm{E}+00 \\
1.950 \mathrm{E}+00 \\
1.970 \mathrm{E}+00\end{array}$ & $\begin{array}{l}4.155 \mathrm{E}-01 \\
5.452 \mathrm{E}-01 \\
6.759 \mathrm{E}-01 \\
8.068 \mathrm{E}-01 \\
9.375 \mathrm{E}-01 \\
1.197 \mathrm{E}+00 \\
1.455 \mathrm{E}+00 \\
1.710 \mathrm{E}+00\end{array}$ & $\begin{array}{l}2.575 \mathrm{E}-03 \\
3.200 \mathrm{E}-03 \\
3.848 \mathrm{E}-03 \\
4.516 \mathrm{E}-03 \\
5.203 \mathrm{E}-03 \\
6.623 \mathrm{E}-03 \\
8.095 \mathrm{E}-03 \\
9.608 \mathrm{E}-03\end{array}$ & $\begin{array}{l}4.759 \mathrm{E}- \\
6.568 \mathrm{E}- \\
8.243 \mathrm{E}- \\
9.785 \mathrm{E}- \\
1.121 \mathrm{E}+ \\
1.375 \mathrm{E}+ \\
1.596 \mathrm{E}+ \\
1.791 \mathrm{E}+\end{array}$ & $\begin{array}{l}-0.0 \\
-0.0 \\
-0.0 \\
-0.0 \\
-0.0 \\
-0.0 \\
-0.0 \\
-0.0\end{array}$ & $\begin{array}{l}0.078 \\
0.077 \\
0.064 \\
0.059 \\
0.055 \\
0.050 \\
0.046 \\
0.043\end{array}$ & $\begin{array}{l}0.069 \\
0.0660 \\
0.054 \\
0.049 \\
0.045 \\
0.039 \\
0.036 \\
0.033\end{array}$ \\
\hline $\begin{array}{l}4.0000 \\
4.5000 \\
5.0000 \\
5.5000 \\
6.0000 \\
7.0000 \\
8.0000 \\
9.0000\end{array}$ & $\begin{array}{l}1.942 E+00 \\
1.954 E+00 \\
1.965 E+00 \\
1.975 E+00 \\
1.984 E+00 \\
2.002 E+00 \\
2.017 E+00 \\
2.030 E+00\end{array}$ & $\begin{array}{l}4.7 \\
5.4 \\
6.1 \\
6.9 \\
7.6 \\
9.2\end{array}$ & $\begin{array}{l}1.989 \mathrm{E}+00 \\
2.008 \mathrm{E}+00 \\
2.026 \mathrm{E}+00 \\
2.044 \mathrm{E}+00 \\
2.061 \mathrm{E}+00 \\
2.094 \mathrm{E}+00 \\
2.126 \mathrm{E}+00 \\
2.156 \mathrm{E}+00\end{array}$ & $\begin{array}{l}1.9 \\
2.2 \\
2.4 \\
2.7 \\
2.9 \\
3.4 \\
3.9 \\
4.3\end{array}$ & $\begin{array}{l}1 . \\
1: \\
1: \\
1: \\
2 . \\
2 . \\
2 .\end{array}$ & $\begin{array}{l}1.9 \\
2.9 \\
2.2 \\
2.4 \\
2.5 \\
2.7 \\
2.9 \\
3.1\end{array}$ & $\begin{array}{l}-0 \\
-0 \\
-0 \\
-0 \\
-0 \\
-0 \\
-0 \\
-0\end{array}$ & $\begin{array}{l}1 \\
9 \\
7 \\
6 \\
5 \\
2 \\
1 \\
9\end{array}$ & $\begin{array}{l}0.031 \\
0.029 \\
0.028 \\
0.027 \\
0.026 \\
0.024 \\
0.023 \\
0.021\end{array}$ \\
\hline $\begin{array}{l}10.0000 \\
12.5000 \\
15.0000 \\
17.5000 \\
20.00000 \\
25.00000 \\
30.0000 \\
35.0000\end{array}$ & $\begin{array}{l}2.042 E+00 \\
2.067 E+00 \\
2.087 E+00 \\
2.103 E+00 \\
2.117 E+00 \\
2.139 E+00 \\
2.157 E+00 \\
2.171 E+00\end{array}$ & $\begin{array}{l}1.8 \\
2.3 \\
2.7 \\
3.2 \\
4.1\end{array}$ & $\begin{array}{l}2.2 \\
2.3 \\
2.3 \\
2.4 \\
2.5 \\
2.5\end{array}$ & $\begin{array}{l}.833 E+00 \\
.960 E+00 \\
.054 E+00 \\
119 E+00 \\
157 E+00 \\
116 E+01 \\
307 E+01\end{array}$ & $\begin{array}{l}3.102 \mathrm{E}-0 \\
3.956 \mathrm{E}-0 \\
4.811 \mathrm{E}-0 \\
5.661 \mathrm{E}-0 \\
6.503 \mathrm{E}-0 \\
8.156 \mathrm{E}-0 \\
9.759 \mathrm{E}-0 \\
1.131 \mathrm{E}-0\end{array}$ & $\begin{array}{l}3.298 E+00 \\
3.660 E+00 \\
3.967 E+00 \\
4.235 E+00 \\
4.473 E+00 \\
4.880 E+00 \\
5.221 E+00 \\
5.514 E+00\end{array}$ & & $\begin{array}{l}8 \\
5 \\
3 \\
1 \\
9 \\
7 \\
5 \\
3\end{array}$ & \\
\hline $\begin{array}{l}40.0000 \\
45.0000 \\
50.0000 \\
55.0000 \\
60.0000 \\
70.0000 \\
80.0000 \\
90.0000\end{array}$ & $\begin{array}{l}2.184 \\
2.195 \\
2.204 \\
2.213 \\
2.221 \\
2.235\end{array}$ & $\begin{array}{l}9.0 \\
1.0 \\
1.1 \\
1.3 \\
1.5 \\
1.7\end{array}$ & $\begin{array}{l}00 \\
00 \\
00 \\
00 \\
00 \\
00 \\
00 \\
00 \\
00\end{array}$ & & & $\begin{array}{l}5.7 \\
5.9 \\
6.2 \\
6 . \\
6.5 \\
6.8 \\
7 . \\
7 .\end{array}$ & $\begin{array}{l}-0 \\
-0 \\
-0 \\
-0 \\
-0 \\
-0 \\
-0 \\
-0\end{array}$ & & \\
\hline $\begin{array}{l}5.0000 \\
0.0000 \\
5.0000 \\
0.0000 \\
0.00000 \\
0.00000 \\
0.00000\end{array}$ & $\begin{array}{l}2.3 \\
2.3 \\
2.3 \\
2.3 \\
2.3 \\
2.3 \\
2.3\end{array}$ & $\begin{array}{l}2.4 \\
2.9 \\
3.5 \\
5.0 \\
5.1\end{array}$ & $\begin{array}{l}4 . \\
5 . \\
5 . \\
7 . \\
8 .\end{array}$ & & & $\begin{array}{l}0 \\
0 \\
0 \\
0 \\
0 \\
0\end{array}$ & & & \\
\hline $\begin{array}{l}0.0000 \\
0.0000 \\
0.0000 \\
0.0000\end{array}$ & $\begin{array}{l}2.39 \\
2.40 \\
2.41 \\
2.42 \\
2.43 \\
2.45 \\
2.46\end{array}$ & $\begin{array}{l}1 \\
1 \\
1 \\
1 \\
1 \\
1\end{array}$ & $\begin{array}{l}1 \\
1 \\
1 \\
1 \\
1\end{array}$ & $\begin{array}{l}1 \\
1 \\
1 \\
1 \\
1\end{array}$ & $\begin{array}{l}6.128 \mathrm{E}-01 \\
6.318 \mathrm{E}-01 \\
6.487 \mathrm{E}-01 \\
6.775 \mathrm{E}-01 \\
7.012 \mathrm{E}-01 \\
7.212 \mathrm{E}-01\end{array}$ & $\begin{array}{l}1.056 E+01 \\
1.077 E+01 \\
1.096 E+01 \\
1.114 E+01 \\
1.144 E+01 \\
1.171 E+01 \\
1.195 E+01\end{array}$ & $\begin{array}{l}-0 . \\
-0 . \\
-0 \\
-0 \\
-0 \\
-0 \\
-0 \\
-0 .\end{array}$ & $\begin{array}{l}0.003 \\
0.003 \\
0.003 \\
0.002 \\
0.002 \\
0.002 \\
0.002 \\
0.002\end{array}$ & $\begin{array}{l}0.000 \\
0.000 \\
0.000 \\
0.000 \\
0.000 \\
0.000 \\
0.000 \\
0.000\end{array}$ \\
\hline .000 & $2.469 \mathrm{E}+00$ & $2.153 E+01$ & $2.400 \mathrm{E}+01$ & $1.131 E+02$ & $7.383 \mathrm{E}-01$ & $1.216 E+01$ & -0.000 & 0.002 & 0.000 \\
\hline
\end{tabular}




\begin{tabular}{|c|c|c|c|c|c|c|c|c|c|}
\hline IERGY & 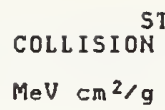 & $\begin{array}{l}\text { PING POW } \\
\text { ADIATIVE } \\
l \mathrm{eV} \mathrm{cm}^{2} / \mathrm{g}\end{array}$ & $\begin{array}{c}\text { TOTAL } \\
\mathrm{MeV} \mathrm{C \Omega}^{2} / \mathrm{g}\end{array}$ & $\begin{array}{l}\text { CSDA } \\
\text { RANGE }\end{array}$ & N & $\begin{array}{l}E F F . \\
R\end{array}$ & $\begin{array}{r}d s \\
\operatorname{col} L\end{array}$ & $\begin{array}{l}\text { CSDA } \\
\text { RANGE }\end{array}$ & $\begin{array}{l}\text { gI ) } \\
\text { RAD } \\
\text { YI ELD }\end{array}$ \\
\hline $\begin{array}{l}0.0100 \\
0.0125 \\
0.0150 \\
0.0175 \\
0.0200 \\
0.0250 \\
0.0300 \\
0.0350\end{array}$ & $\begin{array}{l}1.76 \\
1.53 \\
1.35 \\
1.22 \\
1.03 \\
8.97\end{array}$ & $\begin{array}{l}3.4 \\
3.4 \\
3.4 \\
3.4 \\
3.4 \\
3.4\end{array}$ & $\begin{array}{l}1.7 \\
1.5 \\
1.3 \\
1.2 \\
8.8\end{array}$ & $\begin{array}{l}4 \\
4 \\
4 \\
4 \\
4 \\
3 \\
3 \\
3\end{array}$ & $\begin{array}{l}9.0 \\
1.0 \\
1.2 \\
1.4 \\
1.5 \\
1.8 \\
2.1\end{array}$ & $\begin{array}{l}0.0 \\
0.0 \\
0.0 \\
0.0 \\
0.0 \\
0.0 \\
0.0\end{array}$ & $\begin{array}{l}1 \\
2 \\
6 \\
1 \\
7 \\
0 \\
5 \\
9\end{array}$ & $\begin{array}{l}30 \\
19 \\
11 \\
04 \\
99 \\
90 \\
84 \\
79\end{array}$ & $\begin{array}{l}0.228 \\
0.218 \\
0.210 \\
0.203 \\
0.198 \\
0.190 \\
0.183 \\
0.179\end{array}$ \\
\hline $\begin{array}{l}0.0400 \\
0.0450 \\
0.0500 \\
0.0550 \\
0.0600 \\
0.0700 \\
0.0800 \\
0.0900\end{array}$ & $\begin{array}{l}7.23 \\
6.63 \\
6.14 \\
5.73 \\
5.39 \\
4.84 \\
4.43\end{array}$ & $\begin{array}{l}3.52 \\
3.53 \\
3.55 \\
3.56 \\
3.60\end{array}$ & $\begin{array}{l}6.6 \\
6.1 \\
5.7 \\
5.3 \\
4.8 \\
4.8 \\
4 .\end{array}$ & $\begin{array}{l}4.0 \\
5.4 \\
6.3 \\
8.3\end{array}$ & $\begin{array}{l}2 . \\
3 \\
3 \\
3 \\
4\end{array}$ & $\begin{array}{l}0.0 \\
0.0 \\
0.0 \\
0.0 \\
0.0 \\
0.0\end{array}$ & $\begin{array}{l}-0 \\
-0 \\
-0 \\
-0 \\
-0 \\
-0\end{array}$ & & $\begin{array}{l}0.174 \\
0.171 \\
0.168 \\
0.166 \\
0.163 \\
0.159 \\
0.156\end{array}$ \\
\hline $\begin{array}{l}0.1000 \\
0.1250 \\
0.1500 \\
0.1750 \\
0.2000 \\
0.2500 \\
0.3000 \\
0.3500\end{array}$ & $\begin{array}{l}3.83 \\
3.34 \\
3.01 \\
2.78 \\
2.60 \\
2.35 \\
2.19 \\
2.08\end{array}$ & $\begin{array}{l}3.9 \\
4.0 \\
4.2 \\
4.5 \\
4.8\end{array}$ & $\begin{array}{l}3.3 \\
3.0 \\
2.7 \\
2.6 \\
2.3 \\
2.2 \\
2.0\end{array}$ & $\begin{array}{l}2 \\
2 \\
2 \\
2 \\
2 \\
2 \\
2 \\
1\end{array}$ & $\begin{array}{l}4 \\
4 \\
4 \\
4 \\
4 \\
3 \\
3 \\
3\end{array}$ & $\begin{array}{l}0.0 \\
0.0 \\
0.0 \\
0.0 \\
0.0 \\
0.0 \\
0.0\end{array}$ & & & \\
\hline $\begin{array}{l}0.4000 \\
0.4500 \\
0.5000 \\
0.5500 \\
0.6000 \\
0.7000 \\
0.8000 \\
0.9000\end{array}$ & $\begin{array}{l}+00 \\
+00 \\
+00 \\
+00 \\
+00 \\
+00 \\
+00 \\
+00\end{array}$ & $\begin{array}{l}0.0 \\
6.4 \\
6.8 \\
7.3 \\
8.2 \\
9.2 \\
1.0\end{array}$ & $\begin{array}{l}1.9 \\
1.8 \\
1.8 \\
1.8 \\
1.7 \\
1.7 \\
1.7\end{array}$ & $\begin{array}{ll}0 & 1 \\
0 & 1 \\
0 & 1 \\
0 & 1 \\
0 & 1 \\
0 & 1 \\
01 & 1\end{array}$ & $\begin{array}{l}0 \\
03 \\
03 \\
03 \\
03 \\
03 \\
03\end{array}$ & $\begin{array}{l}9.3 \\
3.7 \\
6.7 \\
9.8 \\
1.3 \\
1.9 \\
2.6 \\
3.3\end{array}$ & $\begin{array}{l}-0 . \\
-0 . \\
-0 \\
-0 .\end{array}$ & & $\begin{array}{l}18 \\
12 \\
07 \\
03 \\
94 \\
89\end{array}$ \\
\hline $\begin{array}{l}1.0000 \\
1.2500 \\
1.5000 \\
1.7500 \\
2.0000 \\
2.5000 \\
3.0000 \\
3.5000\end{array}$ & $\begin{array}{l}1.6 \\
1.6 \\
1.6 \\
1.6 \\
1.7\end{array}$ & $\begin{array}{l}1 . \\
2 . \\
2 . \\
3 . \\
3 . \\
4 .\end{array}$ & $\begin{array}{l}1.705 \mathrm{E} \\
1.701 \mathrm{E} \\
1.704 \mathrm{E} \\
1.710 \mathrm{E} \\
1.728 \mathrm{E} \\
1.748 \mathrm{E} \\
1.768 \mathrm{E}\end{array}$ & $\begin{array}{l}1 \\
1 \\
1 \\
0 \\
0\end{array}$ & $\begin{array}{l}3 \\
3 \\
3 \\
3 \\
3\end{array}$ & $\begin{array}{l}5 . \\
7 \\
8 . \\
1: \\
1: \\
1:\end{array}$ & 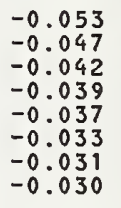 & & $\begin{array}{l}67 \\
60 \\
55 \\
51 \\
45 \\
41\end{array}$ \\
\hline $\begin{array}{l}4.0000 \\
4.5000 \\
5.0000 \\
5.5000 \\
6.0000 \\
7.0000 \\
8.0000 \\
9.0000\end{array}$ & $\begin{array}{l}+00 \\
+00 \\
+00 \\
+00 \\
+00 \\
+00 \\
+00 \\
+00\end{array}$ & $\begin{array}{l}6 . \\
7 . \\
7 . \\
8 . \\
1 . \\
1 . \\
1 .\end{array}$ & $\begin{array}{l}3: \\
1: \\
1: \\
1:\end{array}$ & $\begin{array}{l}0 \\
0 \\
0 \\
0 \\
0 \\
0\end{array}$ & 2. & $\begin{array}{l}1 . \\
1 . \\
2 . \\
2 . \\
2 . \\
2 . \\
2 . \\
2 .\end{array}$ & $\begin{array}{l}-0 \\
-0 \\
-0 \\
-0\end{array}$ & & \\
\hline $\begin{array}{l}.0 \\
.5 \\
.0 \\
.0\end{array}$ & $\begin{array}{l}1 . \\
1 . \\
1 . \\
1 .\end{array}$ & $\begin{array}{l}2 \\
2 \\
3 \\
3 \\
4 \\
5\end{array}$ & $\begin{array}{l}2 \\
2 \\
2 \\
2 \\
2 \\
2 \\
2\end{array}$ & & & $\begin{array}{l}3 . \\
3 \\
3 \\
4 \\
4 \\
4 \\
4 \\
5\end{array}$ & & & \\
\hline $\begin{array}{l}.0 \\
.0 \\
.0 \\
.0\end{array}$ & $\begin{array}{l}1.986 \mathrm{E}+0 \\
1.994 \mathrm{E}+0 \\
2.001 \mathrm{E}+0 \\
2.014 \mathrm{E}+0 \\
2.025 \mathrm{E}+0 \\
2.035 \mathrm{E}+0\end{array}$ & & & & & $\begin{array}{l}5 . \\
5 . \\
6 . \\
6 .\end{array}$ & & & \\
\hline $\begin{array}{l}.0 \\
.0 \\
.0 \\
.0 \\
0\end{array}$ & $\begin{array}{l}2 \\
2 \\
2 \\
2 \\
2 \\
2 \\
2\end{array}$ & $\begin{array}{l}2 \\
3 \\
3 \\
4 \\
5 \\
6\end{array}$ & & & & & & & \\
\hline .00 & $\begin{array}{l}2 \\
2 \\
2 \\
2 \\
2 \\
2 \\
2\end{array}$ & $\begin{array}{l}1 \\
1 \\
1 \\
1\end{array}$ & $\begin{array}{l}1 \\
1 \\
1 \\
2 \\
2\end{array}$ & $\begin{array}{l}1 \\
1 \\
1 \\
1 \\
1\end{array}$ & & 1 & & & $\begin{array}{ll}0 & 0 \\
0 & 0 \\
00 & 0 \\
0 & 0\end{array}$ \\
\hline & $.229 E+00$ & $E+01$ & $641 E+01$ & $99 \mathrm{E}+02$ & $717 \mathrm{E}-01$ & $183 E+01$ & 0.0 & .003 & 0.00 \\
\hline
\end{tabular}


ELECTRONS IN POLYMETHYL METHACRYLATE, "LUCITE", "PERSPEX", "PLEXIGLAS"

$I=74.0 \mathrm{eV} \quad$ DENSITY $=1.190 \mathrm{E}+00 \mathrm{~g} / \mathrm{cm}^{3}$

\begin{tabular}{|c|c|c|c|c|c|c|c|c|c|}
\hline $\mathrm{MeV}$ & $\begin{array}{l}\text { COLLISION } \\
\text { MeV } \mathrm{cm}^{2} / \mathrm{g}\end{array}$ & $\begin{array}{l}\text { PPPING POWE } \\
\text { RADIATIVE } \\
\mathrm{MeV} \mathrm{cm}^{2} / \mathrm{g}\end{array}$ & $\begin{array}{c}\text { TOTAL } \\
\mathrm{MeV} \mathrm{cm}^{2} / \mathrm{g}\end{array}$ & $\begin{array}{l}\text { CSDA } \\
\text { RANGE } \\
\mathrm{g} / \mathrm{cm}^{2}\end{array}$ & N & i) & $\begin{array}{l}d(1 \\
\operatorname{coll} \\
\text { Loss }\end{array}$ & $\begin{array}{l}\text { )/d ( } 1 \\
\text { CSDA } \\
\text { RANGE }\end{array}$ & $\begin{array}{l}\text { I ) } \\
\text { RAD } \\
\text { YIELD }\end{array}$ \\
\hline $\begin{array}{l}0.0100 \\
0.0125 \\
0.0150 \\
0.0175 \\
0.0200 \\
0.0250 \\
0.0300 \\
0.0350\end{array}$ & $\begin{array}{l}2.19 \\
1.84 \\
1.60 \\
1.42 \\
1.28 \\
1.08\end{array}$ & $\begin{array}{l}3 \cdot 3 \\
3 \cdot 3 \\
3 \cdot 3 \\
3 \cdot 3 \\
3 \cdot 3 \\
3 \cdot 3 \\
3 \cdot 3\end{array}$ & $\begin{array}{l}1.8 \\
1.2 \\
1.2 \\
1.2 \\
1.2 \\
9.8 \\
8.2\end{array}$ & $\begin{array}{l}04 \\
04 \\
04 \\
04 \\
04 \\
03 \\
03 \\
03\end{array}$ & $\begin{array}{l}9.9 \\
1.1 \\
1.3 \\
1.4 \\
1.7 \\
2.0\end{array}$ & $\begin{array}{l}0.0 \\
0.0 \\
0.0 \\
0.0 \\
0.0\end{array}$ & & $\begin{array}{l}7 \\
6 \\
8 \\
1 \\
6 \\
8 \\
2 \\
7\end{array}$ & $\begin{array}{l}0.225 \\
0.215 \\
0.207 \\
0.201 \\
0.195 \\
0.187 \\
0.181 \\
0.177\end{array}$ \\
\hline $\begin{array}{l}0.0400 \\
0.0450 \\
0.0500 \\
0.0550 \\
0.0600 \\
0.0700 \\
0.0800 \\
0.0900\end{array}$ & $\begin{array}{l}7.573 E+00 \\
6.942 E+00 \\
6.429 E+00 \\
6.003 E+00 \\
5.644 E+00 \\
5.070 E+00 \\
4.631 E+00\end{array}$ & $\begin{array}{l}3.4 \\
3.4 \\
3.4 \\
3.4 \\
3.5 \\
3.5\end{array}$ & $\begin{array}{l}6 . \\
6 . \\
6 . \\
5 . \\
5 . \\
4 .\end{array}$ & $\begin{array}{l}4.4 \\
5.2 \\
6.1 \\
7.9\end{array}$ & $\begin{array}{l}2 . \\
3 . \\
3 . \\
3 . \\
3 . \\
4 .\end{array}$ & $\begin{array}{l}0.0 \\
0.0 \\
0.0 \\
0.0 \\
0.0 \\
0.0\end{array}$ & & $\begin{array}{l}3 \\
0 \\
7 \\
4 \\
2 \\
8 \\
5 \\
2\end{array}$ & $\begin{array}{l}0.173 \\
0.169 \\
0.166 \\
0.164 \\
0.162 \\
0.158 \\
0.155\end{array}$ \\
\hline $\begin{array}{l}0.1000 \\
0.1250 \\
0.1500 \\
0.1750 \\
0.2000 \\
0.2500 \\
0.3000 \\
0.3500\end{array}$ & $\begin{array}{l}3.496 E+00 \\
3.152 E+00 \\
2.904 E+00 \\
2.719 E+00 \\
2.461 E+00 \\
2.292 E+00\end{array}$ & $\begin{array}{l}3.7 \\
3.8 \\
3.9 \\
4.1 \\
4.4 \\
4.7\end{array}$ & $\begin{array}{l}3 . \\
3 . \\
2 . \\
2 . \\
2 . \\
2 .\end{array}$ & $\begin{array}{l}-02 \\
-02 \\
-02 \\
-02 \\
-02 \\
-02 \\
-02 \\
-02 \\
01\end{array}$ & $\begin{array}{l}6 . \\
6 . \\
7 . \\
8 . \\
1 . \\
1 .\end{array}$ & $\begin{array}{l}0.0 \\
0.0 \\
0.0 \\
0.0 \\
0.0 \\
0.0 \\
0.0\end{array}$ & $\begin{array}{l}-0 \\
-0 \\
-0 \\
-0 \\
-0 \\
-0 \\
-0 \\
-0\end{array}$ & & $\begin{array}{l}0 . \\
0 . \\
0 . \\
0 . \\
0 . \\
0 .\end{array}$ \\
\hline $\begin{array}{l}0.4000 \\
0.4500 \\
0.5000 \\
0.5500 \\
0.6000 \\
0.7000 \\
0.8000 \\
0.9000\end{array}$ & $\begin{array}{l}2.090 E+00 \\
2.026 E+00 \\
1.975 E+00 \\
1.935 E+00 \\
1.903 E+00 \\
1.856 E+00 \\
1.825 E+00 \\
1.803 E+00\end{array}$ & $\begin{array}{l}6.2 \\
6.7 \\
7.1 \\
8.0 \\
9.0\end{array}$ & $\begin{array}{l}2 . \\
1 . \\
1 . \\
1 . \\
1 .\end{array}$ & $\begin{array}{ll}0 & 1 \\
0 & 1 \\
0 & 1 \\
0 & 1 \\
0 & 1 \\
0 & 1 \\
0 & 1 \\
0 & 1\end{array}$ & $\begin{array}{l}1 . \\
1 . \\
1: \\
1 . \\
2 . \\
2 . \\
2 . \\
2 .\end{array}$ & $\begin{array}{l}0.0 \\
1.466 \mathrm{E}-02 \\
4.112 \mathrm{E}-02 \\
6.992 \mathrm{E}-02 \\
1.005 \mathrm{E}-01 \\
1.650 \mathrm{E}-01 \\
2.321 \mathrm{E}-01 \\
3.001 \mathrm{E}-01\end{array}$ & $\begin{array}{l}-0 \\
-0 \\
-0 \\
-0 \\
-0 \\
-0 \\
-0 \\
-0\end{array}$ & & $\begin{array}{l}0.125 \\
0.120 \\
0.1115 \\
0.1110 \\
0.1105 \\
0.097 \\
0.090\end{array}$ \\
\hline $\begin{array}{l}1.0000 \\
1.2500 \\
1.5000 \\
1.7500 \\
2.0000 \\
2.5000 \\
3.0000 \\
3.5000\end{array}$ & $\begin{array}{l}1.788 E+00 \\
1.767 E+00 \\
1.760 E+00 \\
1.759 E+00 \\
1.762 E+00 \\
1.772 E+00 \\
1.784 E+00 \\
1.797 E+00\end{array}$ & $\begin{array}{l}1.39 \\
1.69 \\
2.00 \\
2.33 \\
3.03 \\
3.76\end{array}$ & $\begin{array}{l}1.7 \\
1.7 \\
1.7 \\
1.7 \\
1.8 \\
1.8\end{array}$ & $\begin{array}{l}1 \\
1 \\
1 \\
1 \\
0 \\
0 \\
0 \\
0\end{array}$ & $\begin{array}{l}3.1 \\
3.9 \\
4.7 \\
5.5 \\
6.3 \\
8.0 \\
9.8 \\
1.1\end{array}$ & $\begin{array}{l}5.3 \\
6.8 \\
8.3 \\
9.6 \\
1.2 \\
1.4 \\
1.6\end{array}$ & $\begin{array}{l}-0 \\
-0 \\
-0 \\
-0 \\
-0 \\
-0 \\
-0 \\
-0\end{array}$ & $\begin{array}{l}7 \\
8 \\
1 \\
6 \\
2 \\
6 \\
1 \\
8\end{array}$ & $\begin{array}{l}78 \\
58 \\
50 \\
55 \\
50 \\
44 \\
39\end{array}$ \\
\hline $\begin{array}{l}4.0000 \\
4.5000 \\
5.0000 \\
5.5000 \\
6.0000 \\
7.0000 \\
8.0000 \\
9.0000\end{array}$ & $\begin{array}{l}1.809 E+00 \\
1.821 E+00 \\
1.832 E+00 \\
1.842 E+00 \\
1.851 E+00 \\
1.868 E+00 \\
1.883 E+00\end{array}$ & $\begin{array}{l}6.115 \\
6.943 \\
7.788 \\
8.648 \\
1.041 \\
1.222\end{array}$ & $\begin{array}{l}1 . \\
1 . \\
1 . \\
1 .\end{array}$ & $\begin{array}{l}0 \\
0 \\
0 \\
0 \\
0 \\
0 \\
0 \\
0\end{array}$ & $\begin{array}{l}02 \\
02 \\
02 \\
02 \\
02 \\
02 \\
02\end{array}$ & $\begin{array}{l}1.7 \\
1 . \\
2 . \\
2 . \\
2 . \\
2 . \\
2 . \\
2 .\end{array}$ & $\begin{array}{l}-0 . \\
-0 . \\
-0 . \\
-0 . \\
-0 . \\
-0 . \\
-0 . \\
-0 .\end{array}$ & & $\begin{array}{l}0 . \\
0 . \\
0.1 \\
0 . \\
0.2 \\
0 .\end{array}$ \\
\hline $\begin{array}{l}10.00000 \\
12.5000 \\
15.0000 \\
17.5000 \\
20.0000 \\
25.0000\end{array}$ & $\begin{array}{l}1.932 E+00 \\
1.952 E+00 \\
1.968 E+00 \\
1.982 E+00 \\
2.004 E+00 \\
2.022 E+00 \\
2.036 E+00\end{array}$ & $\begin{array}{l}2.0 \\
2.5 \\
3.0 \\
3.6 \\
4.6\end{array}$ & $\begin{array}{l}0 \\
0 \\
0 \\
0 \\
0 \\
0\end{array}$ & $\begin{array}{l}6 \\
7 \\
8 \\
9 \\
1 \\
1\end{array}$ & $\begin{array}{l}4 . \\
5 . \\
6 \\
7 . \\
9 . \\
1 .\end{array}$ & $\begin{array}{l}3 . \\
3 . \\
3 \\
4 \\
4 . \\
4 .\end{array}$ & $\begin{array}{l}-0 \\
-0 \\
-0 \\
-0 \\
-0 \\
-0 \\
-0 \\
-0\end{array}$ & & \\
\hline $\begin{array}{l}45.0000 \\
50.0000 \\
55.0000 \\
60.0000\end{array}$ & $\begin{array}{l}2.049 E+00 \\
2.059 E+00 \\
2.069 E+00 \\
2.077 E+00 \\
2.085 E+00 \\
2.098 E+00 \\
2.109 E+00 \\
2.120 E+00\end{array}$ & $\begin{array}{l}9.0 \\
1.0 \\
1.1 \\
1.2 \\
1.4\end{array}$ & & $\begin{array}{l}1 . \\
2 . \\
2 . \\
2 .\end{array}$ & & $\begin{array}{l}5 . \\
5 . \\
6 . \\
6 . \\
6 . \\
6 .\end{array}$ & $\begin{array}{l}-0 \\
-0 \\
-0 \\
-0 \\
-0 \\
-0 \\
-0 \\
-0\end{array}$ & & \\
\hline $\begin{array}{l}0000 \\
0000 \\
0000\end{array}$ & $\begin{array}{l}0 \\
0 \\
0 \\
0 \\
0 \\
0 \\
0\end{array}$ & $\begin{array}{l}2 \\
3 \\
3 \\
4 \\
5\end{array}$ & & 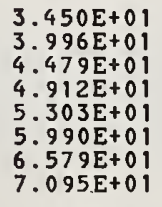 & & & & & \\
\hline $\begin{array}{l}0000 \\
0000 \\
0000 \\
0000 \\
0000\end{array}$ & $\begin{array}{l}2.263 \mathrm{E} \\
2.271 \mathrm{E} \\
2.278 \mathrm{E} \\
2.291 \mathrm{E}\end{array}$ & $\begin{array}{l}0 \\
01 \\
01 \\
01 \\
01 \\
01 \\
01 \\
01 \\
01\end{array}$ & $\begin{array}{l}1 \\
1 \\
1 \\
1 \\
1 \\
1\end{array}$ & $\begin{array}{l}1 \\
1 \\
1 \\
1 \\
1 \\
1\end{array}$ & & 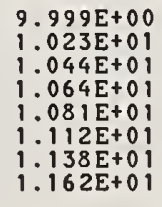 & $\begin{array}{l}0 \\
0 \\
0 \\
0 \\
0 \\
0 \\
0 \\
0\end{array}$ & & $\begin{array}{l}0 \\
0 \\
0 \\
0 \\
0 \\
0 \\
0 \\
0\end{array}$ \\
\hline 0000 & $20 E+00$ & 382 & $14 E+01$ & $92 E+0$ & 36 & $83 E+0$ & 0 . & 003 & \\
\hline
\end{tabular}




\begin{tabular}{|c|c|c|c|c|c|c|c|c|c|}
\hline $\mathrm{MeV}$ & 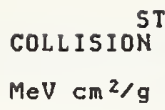 & $\begin{array}{l}\text { OPPING POWE } \\
\text { RADIATIVE } \\
M e V \mathrm{~cm}^{2} / \mathrm{g}\end{array}$ & $\begin{array}{c}\text { TOTAL } \\
\mathrm{MeV} \mathrm{cm}^{2} / \mathrm{g}\end{array}$ & $\begin{array}{l}\text { CSDA } \\
\text { RANGE } \\
\mathrm{g} / \mathrm{cm}^{2}\end{array}$ & $\begin{array}{l}\text { RADIATION } \\
\text { YIELD }\end{array}$ & $\begin{array}{l}\text { DENS.EFF. } \\
\text { CORR } \\
\text { (DELTA) }\end{array}$ & $\begin{array}{l}\text { de } \\
\text { COLL } \\
\text { LOSS }\end{array}$ & $\begin{array}{l}) / d(1 \\
S D A\end{array}$ & $\begin{array}{l}\text { gI ) } \\
\text { RAD } \\
\text { YIELD }\end{array}$ \\
\hline $\begin{array}{l}0.01100 \\
0.0125 \\
0.0150 \\
0.0175 \\
0.0200 \\
0.0250 \\
0.0300 \\
0.0350\end{array}$ & $\begin{array}{l}2.000 \\
1.733 \\
1.536 \\
1.384 \\
1.164 \\
1.011 \\
8.995\end{array}$ & $\begin{array}{l}2.88 \\
2.8 \\
2.9 \\
2.9 \\
2.9 \\
2.9 \\
2.9 \\
2.9\end{array}$ & $\begin{array}{l}+01 \\
+01 \\
+01 \\
+01 \\
+01 \\
+01 \\
+01 \\
+01 \\
+00\end{array}$ & $\begin{array}{l}3.5 \\
4.8 \\
6.3 \\
8.1 \\
1.2 \\
1.6\end{array}$ & $\begin{array}{l}7 . \\
9 \\
1: \\
1: \\
1: \\
1:\end{array}$ & $\begin{array}{l}0.0 \\
0.0 \\
0.0 \\
0.0 \\
0.0 \\
0.0 \\
0.0 \\
0.0\end{array}$ & $\begin{array}{l}-0 \\
-0 \\
-0 \\
-0 \\
-0 \\
-0 \\
-0\end{array}$ & $\begin{array}{l}15 \\
06 \\
98 \\
93 \\
88 \\
80 \\
75 \\
70\end{array}$ & $\begin{array}{l}0.214 \\
0.205 \\
0.198 \\
0.192 \\
0.187 \\
0.180 \\
0.174 \\
0.170\end{array}$ \\
\hline $\begin{array}{l}0.0400 \\
0.0450 \\
0.0500 \\
0.0550 \\
0.0600 \\
0.0700 \\
0.0800 \\
0.0900\end{array}$ & $\begin{array}{l}E+00 \\
E+00 \\
+00 \\
+00 \\
+00 \\
+00 \\
E+00 \\
E+00 \\
E+00\end{array}$ & $\begin{array}{l}2.9 \\
2.9 \\
2.9 \\
3.0 \\
3.0\end{array}$ & $\begin{array}{l}E+00 \\
E+00 \\
E+00 \\
E+00 \\
E+00 \\
E+00 \\
E+00 \\
E+00\end{array}$ & $\begin{array}{l}3.42 \\
4.12 \\
4.87 \\
5.67 \\
7.41 \\
9.34 \\
1.14\end{array}$ & $\begin{array}{l}2 . \\
2 . \\
2 . \\
2 . \\
3 . \\
3 .\end{array}$ & $\begin{array}{l}0.0 \\
0.0 \\
0.0 \\
0.0 \\
0.0 \\
0.0 \\
0.0 \\
0.0\end{array}$ & & $\begin{array}{l}56 \\
63 \\
61 \\
58 \\
56 \\
53 \\
50 \\
47\end{array}$ & $\begin{array}{l}0.166 \\
0.163 \\
0.160 \\
0.158 \\
0.156 \\
0.152 \\
0.149 \\
0.147\end{array}$ \\
\hline $\begin{array}{l}0.1000 \\
0.1250 \\
0.1500 \\
0.1750 \\
0.2000 \\
0.2500 \\
0.3000 \\
0.3500\end{array}$ & $\begin{array}{l}E+00 \\
E+00 \\
E+00 \\
+00 \\
+00 \\
+00 \\
+00 \\
+00 \\
E+00\end{array}$ & $\begin{array}{l}03 \\
03 \\
03 \\
03\end{array}$ & $\begin{array}{l}.290 \mathrm{E}+00 \\
.741 \mathrm{E}+00 \\
.370 \mathrm{E}+00 \\
.105 \mathrm{E}+00 \\
.905 \mathrm{E}+00 \\
.628 \mathrm{E}+00 \\
.447 \mathrm{E}+00 \\
.322 \mathrm{E}+00\end{array}$ & $\begin{array}{l}02 \\
02 \\
02 \\
02 \\
02 \\
02\end{array}$ & $\begin{array}{l}4 . \\
5 . \\
6 . \\
7 . \\
8 .\end{array}$ & $\begin{array}{l}0.0 \\
0.0 \\
0.0 \\
0.0 \\
0.0 \\
0.0 \\
0.0\end{array}$ & & & $\begin{array}{l}0.145 \\
0.140 \\
0.137 \\
0.134 \\
0.132 \\
0.128 \\
0.126\end{array}$ \\
\hline $\begin{array}{l}0.4000 \\
0.4500 \\
0.5000 \\
0.5500 \\
0.6000 \\
0.7000 \\
0.8000 \\
0.9000\end{array}$ & $\begin{array}{l}0 \\
00 \\
00 \\
00 \\
00 \\
00 \\
00 \\
00 \\
00 \\
00\end{array}$ & $\begin{array}{l}03 \\
03 \\
03 \\
03 \\
03 \\
03 \\
03\end{array}$ & $\begin{array}{l}2.1 \\
2.0 \\
2.0 \\
1.9\end{array}$ & $\begin{array}{l}1 \\
1 \\
1 \\
1 \\
1 \\
1\end{array}$ & $\begin{array}{l}1 . \\
1: \\
1: \\
1 . \\
0 .\end{array}$ & $\begin{array}{l}7.5 \\
1: \\
1: \\
2 . \\
2.8 \\
3 .\end{array}$ & & & $\begin{array}{l}19 \\
13 \\
08 \\
03 \\
98 \\
90 \\
83\end{array}$ \\
\hline $\begin{array}{l}1.0000 \\
1.2500 \\
1.5000 \\
1.7500 \\
2.0000 \\
2.5000 \\
3.0000 \\
3.5000\end{array}$ & $\begin{array}{l}.893 E+00 \\
.869 E+00 \\
.860 E+00 \\
.859 E+00 \\
.861 E+00 \\
.871 E+00 \\
.883 E+00 \\
.896 E+00\end{array}$ & $\begin{array}{l}02 \\
02 \\
02 \\
02 \\
02 \\
02 \\
02 \\
02\end{array}$ & $\begin{array}{l}0 \\
0 \\
0 \\
0 \\
0 \\
0 \\
0 \\
0\end{array}$ & $\begin{array}{l}1 \\
1 \\
1 \\
0\end{array}$ & $\begin{array}{l}2.6 \\
3.3 \\
3.5 \\
4.6\end{array}$ & $\begin{array}{l}4.3 \\
6.0 \\
7.6 \\
9.1 \\
1.0 \\
1.3 \\
1.5 \\
1.7\end{array}$ & & & $\begin{array}{l}72 \\
63 \\
56 \\
51 \\
47 \\
41 \\
37\end{array}$ \\
\hline $\begin{array}{l}4.0000 \\
4.5000 \\
5.0000 \\
5.5000 \\
6.0000 \\
7.0000 \\
8.0000 \\
9.0000\end{array}$ & $\begin{array}{l}100 \\
100 \\
000 \\
00 \\
00 \\
00 \\
00 \\
00 \\
00\end{array}$ & $\begin{array}{l}02 \\
02 \\
02 \\
02 \\
02 \\
02 \\
01\end{array}$ & $\left\{\begin{array}{l}0 \\
0 \\
0 \\
0 \\
0 \\
0\end{array}\right.$ & $\begin{array}{l}0 \\
0 \\
0 \\
0 \\
0\end{array}$ & $\begin{array}{l}2 \\
2 \\
2 \\
2 \\
2 \\
2 \\
2\end{array}$ & $\begin{array}{l}1 . \\
2 . \\
2 . \\
2 . \\
2 . \\
2 . \\
2 . \\
3 .\end{array}$ & & & $\begin{array}{l}31 \\
29 \\
28 \\
27 \\
25 \\
24\end{array}$ \\
\hline $\begin{array}{l}.5000 \\
.0000 \\
.5000 \\
.0000 \\
.0000 \\
.0000 \\
.0000\end{array}$ & $\begin{array}{l}+\infty \\
+00 \\
+00 \\
+00 \\
+00 \\
+00 \\
+00 \\
+00 \\
+00\end{array}$ & $\begin{array}{l}2 \\
2 \\
3\end{array}$ & $\begin{array}{l}0 \\
0 \\
0 \\
0 \\
0 \\
0\end{array}$ & $\begin{array}{l}0 \\
0 \\
0 \\
0 \\
1\end{array}$ & & $\begin{array}{l}3 . \\
3 . \\
3\end{array}$ & & & \\
\hline $\begin{array}{l}50.0 \\
55.0 \\
60.0 \\
70.0\end{array}$ & $\begin{array}{l}149 E+00 \\
160 E+00 \\
170 E+00 \\
178 E+00 \\
186 E+00 \\
200 E+00 \\
211 E+00 \\
222 E+00\end{array}$ & & & & & $\begin{array}{l}7.001 \mathrm{E}+ \\
7.233 \mathrm{E}+\end{array}$ & & & \\
\hline .0 & $\begin{array}{l}2.26 \\
2.28 \\
2.28\end{array}$ & $\begin{array}{l}3.0 \\
3.5 \\
4.0 \\
5.1 \\
6.2 \\
7.3\end{array}$ & & & & 8. & & & \\
\hline $\begin{array}{l}0 \\
0 \\
0\end{array}$ & $1 \mathrm{E}+00$ & $\begin{array}{l}1.284 E+01 \\
1.505 E+01 \\
1.726 E+01 \\
1.947 E+01\end{array}$ & $\begin{array}{c}\vdots \\
1 \\
1 \\
\vdots \\
\vdots\end{array}$ & & & & & & \\
\hline & +00 & $E+01$ & $2 E+01$ & $E+$ & $1<15-0$ & $3 E+01$ & 0.000 & 02 & 0.00 \\
\hline
\end{tabular}


ELECTRONS IN POLYSTYRENE

$I=68.7 \mathrm{eV} \quad$ DENSITY $=1.060 \mathrm{E}+00 \mathrm{~g} / \mathrm{cm}^{3}$

\begin{tabular}{|c|c|c|c|c|c|c|c|c|c|}
\hline ENERGY & OLLISIO & $\begin{array}{l}\text { PING POV } \\
\text { ADIATIVE }\end{array}$ & TOTAL & $\begin{array}{l}\text { CSDA } \\
\text { RANGE }\end{array}$ & $\begin{array}{l}\text { ADIATION } \\
\text { YIELD }\end{array}$ & $\begin{array}{l}\text { DENS.EFF. } \\
\text { CORR. } \\
\text { (DELTA) }\end{array}$ & $\begin{array}{l}d(1 \\
\operatorname{coLL} \\
\text { LOSS }\end{array}$ & $\begin{array}{l}\text { g }) / d(1 \\
\text { CSDA } \\
\text { RAHGE }\end{array}$ & $\begin{array}{l}\text { gI ) } \\
\text { RAD } \\
\text { YIELD }\end{array}$ \\
\hline $\mathrm{MeV}$ & $\mathrm{eV} \mathrm{cm}^{2} / \mathrm{g}$ & $\mathrm{MeV} \mathrm{cm}^{2} / \mathrm{g}$ & $\mathrm{MeV} \mathrm{cm}^{2} / \mathrm{g}$ & $\mathrm{g} / \mathrm{cm}^{2}$ & & & & & \\
\hline $\begin{array}{l}0.0100 \\
0.0125 \\
0.0150 \\
0.0175 \\
0.0200 \\
0.0250 \\
0.0300 \\
0.0350\end{array}$ & $\begin{array}{l}2.223 E+01 \\
1.868 E+01 \\
1.621 E+01 \\
1.437 E+01 \\
1.296 E+01 \\
1.091 E+01 \\
9.485 E+00 \\
8.440 E+00\end{array}$ & $\begin{array}{l}2.982 \mathrm{E}-03 \\
2.992 \mathrm{E}-03 \\
2.999 \mathrm{E}-03 \\
3.004 \mathrm{E}-03 \\
3.008 \mathrm{E}-03 \\
3.017 \mathrm{E}-03 \\
3.027 \mathrm{E}-03 \\
3.037 \mathrm{E}-03\end{array}$ & $\begin{array}{l}2.224 E+01 \\
1.869 \mathrm{E}+01 \\
1.6211 \mathrm{E}+01 \\
1.438 \mathrm{E}+01 \\
1.296 \mathrm{E}+01 \\
1.091 \mathrm{E}+01 \\
9.488 \mathrm{E}+00 \\
8.443 \mathrm{E}+00\end{array}$ & $\begin{array}{l}2.546 \mathrm{E}-04 \\
3.777 \mathrm{E}-04 \\
5.218 \mathrm{E}-04 \\
6.859 \mathrm{E}-04 \\
8.694 \mathrm{E}-04 \\
1.292 \mathrm{E}-03 \\
1.785 \mathrm{E}-03 \\
2.345 \mathrm{E}-03\end{array}$ & $\begin{array}{l}7.406 E-05 \\
8.869 E-05 \\
1.027 E-04 \\
1.162 E-04 \\
1.292 E-04 \\
1.543 E-04 \\
1.782 E-04 \\
2.013 E-04\end{array}$ & $\begin{array}{l}0.0 \\
0.0 \\
0.0 \\
0.0 \\
0.0 \\
0.0 \\
0.0 \\
0.0\end{array}$ & $\begin{array}{l}-0.195 \\
-0.187 \\
-0.181 \\
-0.176 \\
-0.172 \\
-0.166 \\
-0.162 \\
-0.158\end{array}$ & $\begin{array}{l}0.223 \\
0.212 \\
0.205 \\
0.198 \\
0.193 \\
0.185 \\
0.179 \\
0.175\end{array}$ & $\begin{array}{l}0.221 \\
0.211 \\
0.204 \\
0.198 \\
0.193 \\
0.185 \\
0.179 \\
0.174\end{array}$ \\
\hline $\begin{array}{l}0.0400 \\
0.0450 \\
0.0500 \\
0.0550 \\
0.0600 \\
0.0700 \\
0.0800 \\
0.0900\end{array}$ & $\begin{array}{l}7.637 E+00 \\
7.000 E+00 \\
6.481 E+00 \\
6.051 E+00 \\
5.688 E+00 \\
5.108 E+00 \\
4.666 E+00 \\
4.317 E+00\end{array}$ & $\begin{array}{l}3.048 \mathrm{E}-03 \\
3.061 \mathrm{E}-03 \\
3.074 \mathrm{E}-03 \\
3.088 \mathrm{E}-03 \\
3.103 \mathrm{E}-03 \\
3.135 \mathrm{E}-03 \\
3.169 \mathrm{E}-03 \\
3.206 \mathrm{E}-03\end{array}$ & $\begin{array}{l}7.640 E+00 \\
7.003 E+00 \\
6.484 E+00 \\
6.054 E+00 \\
5.691 E+00 \\
5.111 E+00 \\
4.669 E+00 \\
4.320 E+00\end{array}$ & $\begin{array}{l}2.968 \mathrm{E}-03 \\
3.653 \mathrm{E}-03 \\
4.395 \mathrm{E}-03 \\
5.194 \mathrm{E}-03 \\
6.047 \mathrm{E}-03 \\
7.905 \mathrm{E}-03 \\
9.955 \mathrm{E}-03 \\
1.218 \mathrm{E}-02\end{array}$ & $\begin{array}{l}2.235 \mathrm{E}-04 \\
2.452 \mathrm{E}-04 \\
2.662 \mathrm{E}-04 \\
2.867 \mathrm{E}-04 \\
3.068 \mathrm{E}-04 \\
3.458 \mathrm{E}-04 \\
3.834 \mathrm{E}-04 \\
4.197 \mathrm{E}-04\end{array}$ & $\begin{array}{l}0.0 \\
0.0 \\
0.0 \\
0.0 \\
0.0 \\
0.0 \\
0.0 \\
0.0\end{array}$ & $\begin{array}{l}-0.155 \\
-0.152 \\
-0.150 \\
-0.148 \\
-0.146 \\
-0.143 \\
-0.140 \\
-0.138\end{array}$ & $\begin{array}{l}0.171 \\
0.167 \\
0.165 \\
0.162 \\
0.160 \\
0.156 \\
0.153 \\
0.151\end{array}$ & $\begin{array}{l}0.170 \\
0.167 \\
0.164 \\
0.162 \\
0.160 \\
0.156 \\
0.153 \\
0.150\end{array}$ \\
\hline $\begin{array}{l}0.1000 \\
0.1250 \\
0.1500 \\
0.1750 \\
0.2000 \\
0.2500 \\
0.3000 \\
0.3500\end{array}$ & $\begin{array}{l}4.034 E+00 \\
3.520 E+00 \\
3.172 E+00 \\
2.923 E+00 \\
2.735 E+00 \\
2.475 E+00 \\
2.305 E+00 \\
2.187 E+00\end{array}$ & $\begin{array}{l}3.244 \mathrm{E}-03 \\
3.350 \mathrm{E}-03 \\
3.463 \mathrm{E}-03 \\
3.584 \mathrm{E}-03 \\
3.711 \mathrm{E}-03 \\
3.985 \mathrm{E}-03 \\
4.284 \mathrm{E}-03 \\
4.604 \mathrm{E}-03\end{array}$ & $\begin{array}{l}4.038 E+00 \\
3.523 E+00 \\
3.176 E+00 \\
2.926 E+00 \\
2.739 E+00 \\
2.479 E+00 \\
2.309 E+00 \\
2.192 E+00\end{array}$ & $\begin{array}{l}1.458 \mathrm{E}-02 \\
2.124 \mathrm{E}-02 \\
2.873 \mathrm{E}-02 \\
3.695 \mathrm{E}-02 \\
4.579 \mathrm{E}-02 \\
6.504 \mathrm{E}-02 \\
8.598 \mathrm{E}-02 \\
1.082 \mathrm{E}-01\end{array}$ & $\begin{array}{l}-04 \\
-04 \\
-04 \\
-04 \\
-04 \\
-04 \\
-04 \\
-03\end{array}$ & $\begin{array}{l}0.0 \\
0.0 \\
0.0 \\
0.0 \\
0.0 \\
0.0 \\
0.0 \\
0.0\end{array}$ & $\begin{array}{l}0.136 \\
0.132 \\
0.129 \\
0.127 \\
0.125 \\
0.122 \\
0.1119 \\
0.117\end{array}$ & $\begin{array}{l}0.148 \\
0.144 \\
0.140 \\
0.138 \\
0.135 \\
0.132 \\
0.129 \\
0.127\end{array}$ & $\begin{array}{l}0.148 \\
0.144 \\
0.140 \\
0.137 \\
0.135 \\
0.131 \\
0.128 \\
0.125\end{array}$ \\
\hline $\begin{array}{l}0.4000 \\
0.4500 \\
0.5000 \\
0.5500 \\
0.6000 \\
0.7000 \\
0.8000 \\
0.9000\end{array}$ & $\begin{array}{l}2.101 E+00 \\
2.035 E+00 \\
1.984 E+00 \\
1.943 E+00 \\
1.911 E+00 \\
1.864 E+00 \\
1.832 E+00 \\
1.810 E+00\end{array}$ & $\begin{array}{l}4.945 \mathrm{E}-03 \\
5.304 \mathrm{E}-03 \\
5.680 \mathrm{E}-03 \\
6.071 \mathrm{E}-03 \\
6.475 \mathrm{E}-03 \\
7.322 \mathrm{E}-03 \\
8.212 \mathrm{E}-03 \\
9.142 \mathrm{E}-03\end{array}$ & $\begin{array}{l}2.106 E+00 \\
2.040 E+00 \\
1.990 E+00 \\
1.950 E+00 \\
1.918 E+00 \\
1.871 E+00 \\
1.840 E+00 \\
1.819 E+00\end{array}$ & $\begin{array}{l}1.315 \mathrm{E}-01 \\
1.557 \mathrm{E}-01 \\
1.805 \mathrm{E}-01 \\
2.059 \mathrm{E}-01 \\
2.318 \mathrm{E}-01 \\
2.846 \mathrm{E}-01 \\
3.385 \mathrm{E}-01 \\
3.932 \mathrm{E}-01\end{array}$ & $\begin{array}{l}1.441 \mathrm{E}- \\
1.570 \mathrm{E}- \\
1.699 \mathrm{E}- \\
1.827 \mathrm{E}- \\
2.087 \mathrm{E}- \\
2.349 \mathrm{E}- \\
2.615 \mathrm{E}-\end{array}$ & $\begin{array}{l}2.729 \mathrm{E}-0 \\
2.688 \mathrm{E}-0 \\
5.420 \mathrm{E}-0 \\
8.383 \mathrm{E}-0 \\
1.152 \mathrm{E}-0 \\
1.810 \mathrm{E}-0 \\
2.492 \mathrm{E}-0 \\
3.179 \mathrm{E}-0\end{array}$ & $\begin{array}{l}-0.102 \\
-0.090 \\
-0.084 \\
-0.079 \\
-0.074 \\
-0.067 \\
-0.061 \\
-0.057\end{array}$ & $\begin{array}{l}0.124 \\
0.1119 \\
0.115 \\
0.1111 \\
0.107 \\
0.1100 \\
0.094 \\
0.089\end{array}$ & $\begin{array}{l}0.123 \\
0.1117 \\
0.112 \\
0.107 \\
0.102 \\
0.094 \\
0.088 \\
0.082\end{array}$ \\
\hline $\begin{array}{l}1.0000 \\
1.2500 \\
1.5000 \\
1.7500 \\
2.0000 \\
2.5000 \\
3.0000 \\
3.5000\end{array}$ & $\begin{array}{l}1.794 E+00 \\
1.773 E+00 \\
1.766 E+00 \\
1.765 E+00 \\
1.768 E+00 \\
1.778 E+00 \\
1.791 E+00 \\
1.804 E+00\end{array}$ & $\begin{array}{l}1.011 \mathrm{E}-02 \\
1.267 \mathrm{E}-02 \\
1.541 \mathrm{E}-02 \\
1.830 \mathrm{E}-02 \\
2.132 \mathrm{E}-02 \\
2.766 \mathrm{E}-02 \\
3.435 \mathrm{E}-02 \\
4.132 \mathrm{E}-02\end{array}$ & $\begin{array}{l}1.804 E+00 \\
1.786 E+00 \\
1.781 E+00 \\
1.783 E+00 \\
1.789 E+00 \\
1.806 E+00 \\
1.825 E+00 \\
1.845 E+00\end{array}$ & $\begin{array}{l}4.484 E-01 \\
5.878 E-01 \\
7.281 E-01 \\
8.684 E-01 \\
1.008 E+00 \\
1.287 E+00 \\
1.562 E+00 \\
1.835 E+00\end{array}$ & $\begin{array}{l}.885 \mathrm{E}-03 \\
.577 \mathrm{E}-03 \\
.293 \mathrm{E}-03 \\
.030 \mathrm{E}-03 \\
.788 \mathrm{E}-03 \\
.352 \mathrm{E}-03 \\
.970 \mathrm{E}-03 \\
.063 \mathrm{E}-02\end{array}$ & $\begin{array}{l}3.862 E-01 \\
5.515 E-01 \\
7.064 E-01 \\
8.501 E-01 \\
9.834 E-01 \\
1.222 E+00 \\
1.431 E+00 \\
1.616 E+00\end{array}$ & $\begin{array}{l}-0.053 \\
-0.046 \\
-0.042 \\
-0.039 \\
-0.037 \\
-0.034 \\
-0.031 \\
-0.030\end{array}$ & $\begin{array}{l}0.085 \\
0.077 \\
0.070 \\
0.065 \\
0.062 \\
0.056 \\
0.051 \\
0.048\end{array}$ & $\begin{array}{l}0.077 \\
0.067 \\
0.060 \\
0.055 \\
0.050 \\
0.045 \\
0.041 \\
0.038\end{array}$ \\
\hline $\begin{array}{l}4.0000 \\
4.5000 \\
5.0000 \\
5.5000 \\
6.0000 \\
7.0000 \\
8.0000 \\
9.0000\end{array}$ & $\begin{array}{l}1.816 \mathrm{E}+00 \\
1.828 \mathrm{E}+00 \\
1.839 \mathrm{E}+00 \\
1.849 \mathrm{E}+00 \\
1.859 \mathrm{E}+00 \\
1.876 \mathrm{E}+00 \\
1.891 \mathrm{E}+00 \\
1.904 \mathrm{E}+00\end{array}$ & $\begin{array}{l}4.852 \mathrm{E}-02 \\
5.593 \mathrm{E}-02 \\
6.353 \mathrm{E}-02 \\
7.129 \mathrm{E}-02 \\
7.919 \mathrm{E}-02 \\
9.539 \mathrm{E}-02 \\
1.120 \mathrm{E}-01 \\
1.290 \mathrm{E}-01\end{array}$ & $\begin{array}{l}1.865 E+00 \\
1.884 E+00 \\
1.902 E+00 \\
1.920 E+00 \\
1.938 E+00 \\
1.971 E+00 \\
2.003 E+00 \\
2.033 E+00\end{array}$ & $\begin{array}{l}2.104 E+00 \\
2.371 E+00 \\
2.635 E+00 \\
2.897 E+00 \\
3.156 E+00 \\
3.667 E+00 \\
4.171 E+00 \\
4.666 E+00\end{array}$ & $\begin{array}{l}1.233 \mathrm{E}-02 \\
1.405 \mathrm{E}-02 \\
1.580 \mathrm{E}-02 \\
1.757 \mathrm{E}-02 \\
1.936 \mathrm{E}-02 \\
2.297 \mathrm{E}-02 \\
2.662 \mathrm{E}-02 \\
3.029 \mathrm{E}-02\end{array}$ & $\begin{array}{l}1.78 \\
1.93 \\
2.07 \\
2.19 \\
2.31 \\
2.53 \\
2.72 \\
2.89\end{array}$ & $\begin{array}{l}-0.029 \\
-0.027 \\
-0.026 \\
-0.025 \\
-0.025 \\
-0.023 \\
-0.021 \\
-0.020\end{array}$ & $\begin{array}{l}0.046 \\
0.044 \\
0.042 \\
0.040 \\
0.039 \\
0.037 \\
0.035 \\
0.033\end{array}$ & $\begin{array}{l}0.035 \\
0.034 \\
0.032 \\
0.031 \\
0.030 \\
0.028 \\
0.026 \\
0.025\end{array}$ \\
\hline $\begin{array}{l}12.5000 \\
15.0000 \\
17.5000 \\
20.0000 \\
25.0000 \\
30.0000 \\
35.0000\end{array}$ & $\begin{array}{l}1.916 \mathrm{E}+00 \\
1.940 \mathrm{E}+00 \\
1.960 \mathrm{E}+00 \\
1.975 \mathrm{E}+00 \\
1.989 \mathrm{E}+00 \\
2.010 \mathrm{E}+00 \\
2.027 \mathrm{E}+00 \\
2.041 \mathrm{E}+00\end{array}$ & $\begin{array}{l}1.9 \\
2.3 \\
2.8 \\
3.3 \\
4.2 \\
5.2 \\
6.2\end{array}$ & $\begin{array}{l}2.0 \\
2.1 \\
2.1 \\
2.2 \\
2.3 \\
2.4 \\
2.5 \\
2.6\end{array}$ & $\begin{array}{l}5.1 \\
6.3 \\
7.5 \\
8.6 \\
9.7 \\
1.1 \\
1.9\end{array}$ & $\begin{array}{l}4 . \\
5 . \\
7 . \\
8 .\end{array}$ & $\begin{array}{l}3.054 \mathrm{E}+ \\
3.403 \mathrm{Et} \\
3.702 \mathrm{E}+ \\
3.963 \mathrm{E}+ \\
4.196 \mathrm{E}+ \\
4.596 \mathrm{Et} \\
4.933 \mathrm{E} \\
5.223 \mathrm{E}+\end{array}$ & $\begin{array}{l}-0.019 \\
-0.015 \\
-0.013 \\
-0.010 \\
-0.009 \\
-0.006 \\
-0.005 \\
-0.003\end{array}$ & $\begin{array}{l}0.03 \\
0.02 \\
0.02 \\
0.02 \\
0.02 \\
0.01 \\
0.01 \\
0.01\end{array}$ & $\begin{array}{l}0.023 \\
0.020 \\
0.018 \\
0.016 \\
0.014 \\
0.011 \\
0.009 \\
0.008\end{array}$ \\
\hline $\begin{array}{l}45.0000 \\
50.0000 \\
55.0000 \\
60.0000 \\
70.0000 \\
80.0000 \\
90.0000\end{array}$ & $\begin{array}{l}2.064 E+00 \\
2.073 \mathrm{E}+00 \\
2.081 \mathrm{E}+00 \\
2.089 \mathrm{E}+00 \\
2.102 \mathrm{E}+00 \\
2.113 \mathrm{E}+00 \\
2.123 \mathrm{E}+00\end{array}$ & $\begin{array}{l}8.3 \\
9.3 \\
1.0 \\
1.1 \\
1.35 \\
1.5 \\
1.7\end{array}$ & $\begin{array}{l}2.7 \\
2.8 \\
3.0 \\
3.1 \\
3.2 \\
3.4 \\
3.6 \\
3.8\end{array}$ & $\begin{array}{l}1.757 E+01 \\
1.933 E+01 \\
2.103 E+01 \\
2.266 E+01 \\
2.424 E+01 \\
2.723 E+01 \\
3.004 E+01 \\
3.268 E+01\end{array}$ & $\begin{array}{l}1.6 \\
1.8 \\
1.9 \\
2.2\end{array}$ & $\begin{array}{l}5.478 E+00 \\
5.704 E+00 \\
5.908 E+00 \\
6.093 E+00 \\
6.263 E+00 \\
6.565 E+00 \\
6.828 E+00 \\
7.060 E+00\end{array}$ & $\begin{array}{l}-0.003 \\
-0.002 \\
-0.002 \\
-0.002 \\
-0.001 \\
-0.001 \\
-0.001 \\
-0.001\end{array}$ & $\begin{array}{l}0.014 \\
0.013 \\
0.012 \\
0.011 \\
0.011 \\
0.010 \\
0.009 \\
0.008\end{array}$ & $\begin{array}{l}0.006 \\
0.005 \\
0.005 \\
0.004 \\
0.004 \\
0.003 \\
0.002 \\
0.002\end{array}$ \\
\hline $\begin{array}{l}0 \\
0 \\
0 \\
0 \\
0 \\
0\end{array}$ & $\begin{array}{l}2.132 E+00 \\
2.151 E+00 \\
2.166 E+00 \\
2.179 E+00 \\
2.190 E+00 \\
2.208 E+00 \\
2.223 E+00 \\
2.236 E+00\end{array}$ & $\begin{array}{l}1.98 \\
2.52 \\
3.06 \\
3.61 \\
4.15 \\
5.25 \\
6.36 \\
7.47\end{array}$ & $\begin{array}{l}4.1 \\
4.6 \\
5.2 \\
5.7 \\
6.3 \\
7.4 \\
8.5 \\
9.7\end{array}$ & $\begin{array}{l}3.518 E+01 \\
4.087 E+01 \\
4.592 E+01 \\
5.046 E+01 \\
5.458 E+01 \\
6.184 E+01 \\
6.808 E+01 \\
7.355 E+01\end{array}$ & $\begin{array}{l}3.3 \\
3.7 \\
4.0 \\
4.3 \\
4.8\end{array}$ & $\begin{array}{l}7 . \\
8 . \\
8 . \\
8 . \\
9 \\
9\end{array}$ & $\begin{array}{l}-0 \\
-0 \\
-0 \\
-0 \\
-0 \\
-0 \\
-0 \\
-0\end{array}$ & & $\begin{array}{l}0.002 \\
0.001 \\
0.001 \\
0.001 \\
0.001 \\
0.001 \\
0.000 \\
0.000\end{array}$ \\
\hline $\begin{array}{l}00000 \\
00000 \\
0000 \\
0000 \\
00000 \\
00000\end{array}$ & $\begin{array}{l}2.257 E+00 \\
2.266 E+00 \\
2.274 E+00 \\
2.281 E+00 \\
2.293 E+00 \\
2.304 E+00 \\
2.314 E+00\end{array}$ & $\begin{array}{l}8.583 \mathrm{E}+00 \\
9.697 \mathrm{E}+00 \\
1.081 \mathrm{E}+01 \\
1.193 \mathrm{E}+01 \\
1.305 \mathrm{E}+01 \\
1.529 \mathrm{E}+01 \\
1.754 \mathrm{E}+01 \\
1.978 \mathrm{E}+01\end{array}$ & $\begin{array}{l}1.083 E+01 \\
1.195 \mathrm{E}+01 \\
1.308 \mathrm{E}+01 \\
1.420 \mathrm{E}+01 \\
1.533 \mathrm{E}+01 \\
1.758 \mathrm{E}+01 \\
1.984 \mathrm{E}+01 \\
2.210 \mathrm{E}+01\end{array}$ & $\begin{array}{l}7.842 E+01 \\
8.282 E+01 \\
8.681 E+01 \\
9.048 E+01 \\
9.387 E+01 \\
9.996 E+01 \\
1.053 E+02 \\
1.101 E+02\end{array}$ & $\begin{array}{l}5.843 \mathrm{E}-01 \\
6.085 \mathrm{E}-01 \\
6.296 \mathrm{E}-01 \\
6.482 \mathrm{E}-01 \\
6.646 \mathrm{E}-01 \\
6.926 \mathrm{E}-01 \\
7.157 \mathrm{E}-01 \\
7.350 \mathrm{E}-01\end{array}$ & $\begin{array}{l}1.003 E+01 \\
1.026 E+01 \\
1.047 E+01 \\
1.066 E+01 \\
1.084 E+01 \\
1.115 E+01 \\
1.141 E+01 \\
1.165 E+01\end{array}$ & $\begin{array}{l}-0.0000 \\
-0.000 \\
-0.000 \\
-0.000 \\
-0.000 \\
-0.000 \\
-0.000 \\
-0.000\end{array}$ & $\begin{array}{l}0.003 \\
0.003 \\
0.003 \\
0.003 \\
0.003 \\
0.003 \\
0.003 \\
0.002\end{array}$ & $\begin{array}{l}0.000 \\
0.000 \\
0.000 \\
0.000 \\
0.000 \\
0.000 \\
0.000 \\
0.0000\end{array}$ \\
\hline .0000 & $2.323 E+00$ & $2.203 E+01$ & $2.436 E+01$ & $1.144 E+02$ & $7.515 \mathrm{E}-01$ & $1.186 E+01$ & -0.000 & 0.002 & 0.000 \\
\hline
\end{tabular}




\begin{tabular}{|c|c|c|c|c|c|c|c|c|c|}
\hline ERGY & COLLISIOS & $\begin{array}{l}\text { ING PO } \\
\text { DIATIV }\end{array}$ & TOTAL & $\begin{array}{l}\text { CSDA } \\
\text { RANGE }\end{array}$ & $\mathbf{N}$ & $\begin{array}{l}\text { DENS.EFF. } \\
\text { CORR. } \\
\text { (DELTA) }\end{array}$ & $\mathrm{COL}^{\mathrm{d}}$ & CSD & $\begin{array}{l}\text { gI ) } \\
\text { RAD } \\
\text { YIELD }\end{array}$ \\
\hline $\mathrm{MeV}$ & $\mathrm{MeV} \mathrm{cm} \mathrm{cm}^{2} / \mathrm{g}$ & $\mathrm{MeV} \mathrm{cm}^{2} / \mathrm{g}$ & $\mathrm{MeV} \mathrm{cm}^{2} / \mathrm{g}$ & $\mathrm{g} / \mathrm{cm}^{2}$ & & & & & \\
\hline $\begin{array}{l}0.01100 \\
0.0125 \\
0.0150 \\
0.0175 \\
0.0200 \\
0.0250 \\
0.0300 \\
0.0350\end{array}$ & $\begin{array}{l}1.8431 \\
1.5531 \\
1.351 \\
1.200 \\
1.084 \\
9.141 \\
7.065\end{array}$ & $\begin{array}{l}4 . \\
4 . \\
4: \\
4: \\
4 .\end{array}$ & $\begin{array}{l}1.5 \\
1.3 \\
1.2 \\
1.0 \\
9.1 \\
7.9\end{array}$ & $\begin{array}{l}4 \\
4 \\
4 \\
4 \\
3 \\
3 \\
3 \\
3\end{array}$ & $\begin{array}{l}249 E-04 \\
502 E-04 \\
743 E-04 \\
975 E-04 \\
199 E-04 \\
629 E-04 \\
037 E-04\end{array}$ & $\begin{array}{l}0.0 \\
0.0 \\
0.0 \\
0.0 \\
0.0 \\
0.0\end{array}$ & $\begin{array}{l}.210 \\
.201 \\
194 \\
189 \\
184 \\
177 \\
172\end{array}$ & & $\begin{array}{l}0.241 \\
0.229 \\
0.220 \\
0.213 \\
0.207 \\
0.198 \\
0.192 \\
0.186\end{array}$ \\
\hline $\begin{array}{l}0.0400 \\
0.0450 \\
0.0500 \\
0.0550 \\
0.0600 \\
0.0700 \\
0.0800 \\
0.0900\end{array}$ & 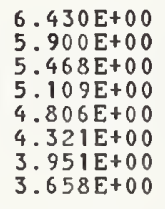 & $\begin{array}{l}4.3 \\
4.3 \\
4.3 \\
4.4 \\
4.4 \\
4.4\end{array}$ & $\begin{array}{l}5.9 \\
5.4 \\
5.1 \\
4.8 \\
4.3\end{array}$ & $\begin{array}{l}.03 \\
.03 \\
.03 \\
.03 \\
03 \\
.03 \\
.02\end{array}$ & $\begin{array}{l}-04 \\
-04 \\
04 \\
-04 \\
-04 \\
04 \\
-04 \\
-04\end{array}$ & $\begin{array}{l}0.0 \\
0.0 \\
0.0 \\
0.0 \\
0.0\end{array}$ & $\begin{array}{l}-0 \\
-0 \\
-0 \\
-0 \\
-0\end{array}$ & & $\begin{array}{l}0.18 \\
0.17 \\
0.17 \\
0.17 \\
0.17\end{array}$ \\
\hline $\begin{array}{l}0.1000 \\
0.1250 \\
0.1500 \\
0.1750 \\
0.2000 \\
0.2500 \\
0.3000\end{array}$ & $\begin{array}{l}2.90 \\
2.69 \\
2.38 \\
2.11 \\
1.96\end{array}$ & $\begin{array}{l}4.1 \\
4.8 \\
5.0 \\
5.1 \\
5.5 \\
5.9\end{array}$ & $\begin{array}{l}3.426 E+00 \\
2.994 E+00 \\
2.702 E+00 \\
2.492 E+00 \\
2.335 E+00 \\
2.117 E+00 \\
1.974 E+00 \\
1.875 E+00\end{array}$ & $\begin{array}{l}2.511 \mathrm{E}-02 \\
3.392 \mathrm{E}-02 \\
4.357 \mathrm{E}-02 \\
5.395 \mathrm{E}-02 \\
7.651 \mathrm{E}-02 \\
1.010 \mathrm{E}-01 \\
1.271 \mathrm{E}-01\end{array}$ & $\begin{array}{l}4 \\
3 \\
3 \\
3 \\
3 \\
3 \\
3\end{array}$ & $\begin{array}{l}0 \\
0 \\
0 \\
0 \\
0 \\
0\end{array}$ & $\begin{array}{l}-0 . \\
-0 . \\
-0 . \\
-0 . \\
-0 . \\
-0\end{array}$ & & $\begin{array}{l}0.13 \\
0.13 \\
0.13\end{array}$ \\
\hline $\begin{array}{l}0.4000 \\
0.4500 \\
0.5000 \\
0.5500 \\
0.6000 \\
0.7000 \\
0.8000 \\
0.9000\end{array}$ & $\begin{array}{l}1.797 E+00 \\
1.742 E+00 \\
1.699 E+00 \\
1.665 E+00 \\
1.639 E+00 \\
1.600 E+00 \\
1.573 E+00 \\
1.555 E+00\end{array}$ & $\begin{array}{l}7.775 \\
8.291 \\
8.823 \\
9.937 \\
1.1111 \\
1.233\end{array}$ & $\begin{array}{l}1.804 E+00 \\
1.749 E+00 \\
1.707 \mathrm{E}+00 \\
1.674 \mathrm{E}+00 \\
1.647 \mathrm{E}+00 \\
1.6100+00 \\
1.585 \mathrm{E}+00 \\
1.568 \mathrm{E}+00\end{array}$ & $\begin{array}{l}y \\
1 \\
1 \\
1 \\
1 \\
\vdots\end{array}$ & & $\begin{array}{l}2.29 \\
2.33 \\
4.75 \\
7.39 \\
1.02 \\
1.62 \\
2.25 \\
2.89\end{array}$ & $\begin{array}{l}-0 \\
-0 \\
-0 \\
-0\end{array}$ & & 12 \\
\hline $\begin{array}{l}1.0000 \\
1.2500 \\
1.5000 \\
1.7500 \\
2.0000 \\
2.5000 \\
3.0000 \\
3.5000\end{array}$ & $\begin{array}{l}1.522 \\
1.525 \\
1.535 \\
1.546 \\
1.558\end{array}$ & $\begin{array}{l}2.0 \\
2.4 \\
2.8 \\
3.6 \\
4.5 \\
5.4\end{array}$ & $\begin{array}{l}1.5 \\
1.5 \\
1.5 \\
1.5 \\
1.5 \\
1.5 \\
1.6\end{array}$ & $\begin{array}{l}1.4 \\
1.8 \\
2.1\end{array}$ & $\begin{array}{l}3 \\
3 \\
3 \\
2\end{array}$ & $\begin{array}{l}5 . \\
6 . \\
8 . \\
9 . \\
1 . \\
1 . \\
1 .\end{array}$ & $\begin{array}{l}-0 \\
-0 \\
-0 \\
-0\end{array}$ & & $\begin{array}{l}0.05 \\
0.05 \\
0.04\end{array}$ \\
\hline $\begin{array}{l}4.5000 \\
5.0000 \\
5.5000 \\
6.0000 \\
7.0000 \\
8.0000 \\
9.0000\end{array}$ & $\begin{array}{l}1.58 \\
1.50 \\
1.60 \\
1.62\end{array}$ & $\begin{array}{l}7 . \\
8 \\
9 \\
1 \\
1 . \\
1 . \\
1 .\end{array}$ & $\begin{array}{l}1.6 \\
1.6 \\
1.6 \\
1.7 \\
1.7 \\
1.7 \\
1.8\end{array}$ & $\begin{array}{l}0 \\
0 \\
0\end{array}$ & & $\begin{array}{l}1 . \\
2 . \\
2 . \\
2 . \\
2 . \\
2 . \\
2 .\end{array}$ & & & 0 \\
\hline $\begin{array}{l}.0000 \\
.5000 \\
.0000 \\
.0000 \\
.0000 \\
.0000\end{array}$ & $\begin{array}{l}+\infty 0 \\
+00 \\
+00 \\
+00 \\
+00 \\
+00 \\
+00 \\
+\infty\end{array}$ & $\begin{array}{l}2.4 \\
3.0 \\
3.6 \\
4.2 \\
5.5 \\
6.7\end{array}$ & $\begin{array}{l}1 . \\
2 . \\
2 . \\
2 . \\
2 . \\
2 . \\
2 .\end{array}$ & $\begin{array}{l}0 \\
0 \\
0 \\
0 \\
1 \\
1 \\
1\end{array}$ & $\begin{array}{l}2 \\
2 \\
2 \\
1 \\
1\end{array}$ & $\begin{array}{l}3 . \\
3 \\
3 \\
4 \\
4 . \\
4 . \\
4 .\end{array}$ & $\begin{array}{l}-0 \\
-0 \\
-0 \\
-0\end{array}$ & & 1 \\
\hline $\begin{array}{l}50.0 \\
55.0 \\
60.0 \\
70.0\end{array}$ & $\begin{array}{l}1 . \\
1 . \\
1 .\end{array}$ & & $\begin{array}{l}2 . \\
2 . \\
3 . \\
3 . \\
3 . \\
3 .\end{array}$ & $\begin{array}{l}2 \\
2 \\
2\end{array}$ & & $\begin{array}{l}5 . \\
5 . \\
5 .\end{array}$ & & & \\
\hline $\begin{array}{l}0 \\
0 \\
0 \\
0\end{array}$ & $\begin{array}{r}1.88 \\
1.89 \\
1.90 \\
1.92 \\
1.93\end{array}$ & $\begin{array}{l}3 . \\
5 . \\
5 . \\
8 . \\
9 .\end{array}$ & $\begin{array}{l}5 \\
6 \\
7 \\
8 . \\
9 \\
1\end{array}$ & $\begin{array}{l}1 \\
1 \\
1 \\
1 \\
1 \\
1\end{array}$ & & $\begin{array}{l}8 \\
8 \\
8 \\
0\end{array}$ & & & \\
\hline $\begin{array}{l}.0000 \\
.0000 \\
.0000\end{array}$ & $\begin{array}{l}1.960 E+00 \\
1.969 E+00 \\
1.977 E+00 \\
1.984 E+00 \\
1.990 E+00 \\
2.002 E+00 \\
2.012 E+00 \\
2.020 E+00\end{array}$ & $\begin{array}{l}\mathbf{1} \\
1 \\
1\end{array}$ & $\begin{array}{l}1 \\
1 \\
1 \\
1 \\
1 \\
1 \\
\vdots\end{array}$ & $\begin{array}{l}1 \\
1 \\
1 \\
1\end{array}$ & & $\begin{array}{l}0 \\
1 \\
1\end{array}$ & $\begin{array}{l}0 \\
0 \\
0 \\
0 \\
0 \\
0 \\
0\end{array}$ & & \\
\hline & $i+\infty 0$ & 01 & +01 & $1.044 E+02$ & & $1.174 E+01$ & -0.000 & & \\
\hline
\end{tabular}




\begin{tabular}{|c|c|c|c|c|c|c|c|c|c|}
\hline \multirow{2}{*}{$\begin{array}{l}\text { ENERGY } \\
\text { MeV }\end{array}$} & \multicolumn{3}{|c|}{$\begin{array}{l}\text { STOPPING POWER } \\
\text { COLLISION RADIATIVE }\end{array}$} & \multirow{2}{*}{$\begin{array}{l}\text { CSDA } \\
\text { RANGE } \\
g / \mathrm{cm}^{2}\end{array}$} & \multirow[t]{2}{*}{$\begin{array}{l}\text { RADIATION } \\
\text { YIELD }\end{array}$} & \multirow[t]{2}{*}{$\begin{array}{c}\text { DENS.EFF . } \\
\text { CORR. } \\
\text { (DELTA) }\end{array}$} & \multirow[t]{2}{*}{$\begin{array}{l}d{ }^{d(10} \\
\operatorname{coLt} \\
\text { loss }\end{array}$} & \multirow[t]{2}{*}{$\begin{array}{l}\text { g) } / d<1 \\
\text { CSDA } \\
\text { RANGE }\end{array}$} & \multirow[t]{2}{*}{$\begin{array}{l}\circ \text { Og I ) } \\
\text { RAD } \\
\text { YIELD }\end{array}$} \\
\hline & $\mathrm{MeV} \mathrm{cm}^{2} / \mathrm{g}$ & $\mathrm{MeV} \mathrm{cm}^{2} / \mathrm{g}$ & 10 & & & & & & \\
\hline $\begin{array}{l}0.0100 \\
0.0125 \\
0.0150 \\
0.0175 \\
0.0200 \\
0.0250 \\
0.0300 \\
0.0350\end{array}$ & $\begin{array}{l}1.930 E+01 \\
1.628 E+01 \\
1.416 E+01 \\
1.259 E+01 \\
1.137 E+01 \\
9.601 E+00 \\
8.370 E+00 \\
7.462 E+00\end{array}$ & $\begin{array}{l}-03 \\
-03 \\
-03 \\
-03 \\
-03 \\
-03 \\
-03 \\
-03\end{array}$ & $\begin{array}{l}1.6 \\
1.4 \\
1.2 \\
1.1 \\
9.6 \\
8.3 \\
7.4\end{array}$ & $\begin{array}{l}4.3 \\
6.0 \\
7.9 \\
1.0 \\
1.4 \\
2.0 \\
2.0\end{array}$ & $\begin{array}{l}1.6 \\
1.9 \\
2.3 \\
2.6 \\
2.9 \\
3.6 \\
4.2 \\
4.8\end{array}$ & $\begin{array}{l}0.0 \\
0.0 \\
0.0 \\
0.0 \\
0.0 \\
0.0 \\
0.0 \\
0.0\end{array}$ & & $\begin{array}{l}9 \\
6 \\
7 \\
9 \\
3 \\
3 \\
6 \\
6\end{array}$ & $\begin{array}{l}0.245 \\
0.232 \\
0.223 \\
0.216 \\
0.210 \\
0.201 \\
0.194 \\
0.189\end{array}$ \\
\hline $\begin{array}{l}0.0400 \\
0.0450 \\
0.0500 \\
0.0550 \\
0.0600 \\
0.0700 \\
0.0800 \\
0.0900\end{array}$ & $\begin{array}{l}6.762 E+00 \\
6.206 E+00 \\
5.753 E+00 \\
5.376 E+00 \\
5.053 E+00 \\
4.549 E+00 \\
4.160 E+00 \\
3.853 E+00\end{array}$ & $\begin{array}{l}-03 \\
-03 \\
-03 \\
-03 \\
-03 \\
-03 \\
-03 \\
-03 \\
-03 \\
-03 \\
-03\end{array}$ & $\begin{array}{l}6.2 \\
5.7 \\
5.3 \\
5.0 \\
4.5 \\
4.1 \\
3.8\end{array}$ & $\begin{array}{l}4.1 \\
4.9 \\
5.8 \\
6.8 \\
8.9 \\
1.9 \\
1.3\end{array}$ & $\begin{array}{l}5.3 \\
5.9 \\
6.9 \\
6.9 \\
7.4 \\
8.4 \\
9.3 \\
1.0\end{array}$ & $\begin{array}{l}0.0 \\
0.0 \\
0.0 \\
0.0 \\
0.0 \\
0.0 \\
0.0 \\
0.0\end{array}$ & $\begin{array}{l}66 \\
63 \\
61 \\
58 \\
56 \\
53 \\
50 \\
47\end{array}$ & $\begin{array}{l}36 \\
32 \\
8 \\
5 \\
3 \\
8 \\
5 \\
2\end{array}$ & $\begin{array}{l}0.184 \\
0.180 \\
0.177 \\
0.174 \\
0.172 \\
0.168 \\
0.164 \\
0.161\end{array}$ \\
\hline $\begin{array}{l}0.1000 \\
0.1250 \\
0.1500 \\
0.1750 \\
0.2000 \\
0.2500 \\
0.3000 \\
0.3500\end{array}$ & $\begin{array}{l}3.604 E+00 \\
3.150 E+00 \\
2.843 E+00 \\
2.623 E+00 \\
2.457 E+00 \\
2.227 E+00 \\
2.077 E+00 \\
1.972 E+00\end{array}$ & $\begin{array}{l}7.04 \\
7.22 \\
7.42 \\
7.64 \\
7.87 \\
8.38 \\
8.94 \\
9.55\end{array}$ & $\begin{array}{l}3.611 E+00 \\
3.157 E+00 \\
2.851 E+00 \\
2.630 E+00 \\
2.465 E+00 \\
2.235 E+00 \\
2.086 E+00 \\
1.982 E+00\end{array}$ & $\begin{array}{l}1.641 \mathrm{E}-0 \\
2.384 \mathrm{E}-0 \\
3.219 \mathrm{E}-0 \\
4.134 \mathrm{E}-0 \\
5.117 \mathrm{E}-0 \\
7.254 \mathrm{E}-0 \\
9.575 \mathrm{E}-0 \\
1.204 \mathrm{E}-0\end{array}$ & $\begin{array}{l}1.1 \\
1.3 \\
1.5 \\
1.6 \\
1.8 \\
2.1 \\
2.4 \\
2.7\end{array}$ & $\begin{array}{l}0.0 \\
0.0 \\
0.0 \\
0.0 \\
0.0 \\
0.0 \\
0.0 \\
0.0\end{array}$ & $\begin{array}{l}-0 . \\
-0 . \\
-0 . \\
-0 . \\
-0 . \\
-0 . \\
-0 .\end{array}$ & & $\begin{array}{l}0.159 \\
0.153 \\
0.149 \\
0.146 \\
0.144 \\
0.139 \\
0.136 \\
0.133\end{array}$ \\
\hline $\begin{array}{l}0.4000 \\
0.4500 \\
0.5000 \\
0.5500 \\
0.6000 \\
0.7000 \\
0.8000 \\
0.9000\end{array}$ & $\begin{array}{l}1.896 E+00 \\
1.838 E+00 \\
1.793 E+00 \\
1.758 E+00 \\
1.730 E+00 \\
1.690 E+00 \\
1.663 E+00 \\
1.645 E+00\end{array}$ & $\begin{array}{l}020 \mathrm{E}-02 \\
088 \mathrm{E}-02 \\
159 \mathrm{E}-02 \\
233 \mathrm{E}-02 \\
310 \mathrm{E}-02 \\
469 \mathrm{E}-02 \\
637 \mathrm{E}-02 \\
813 \mathrm{E}-02\end{array}$ & $\begin{array}{l}1.907 E+00 \\
1.849 E+00 \\
1.805 E+00 \\
1.770 E+00 \\
1.743 E+00 \\
1.704 E+00 \\
1.679 E+00 \\
1.663 E+00\end{array}$ & $\begin{array}{l}1.461 \mathrm{E}-01 \\
1.728 \mathrm{E}-01 \\
2.001 \mathrm{E}-01 \\
2.281 \mathrm{E}-01 \\
2.566 \mathrm{E}-01 \\
3.147 \mathrm{E}-01 \\
3.738 \mathrm{E}-01 \\
4.337 \mathrm{E}-01\end{array}$ & $\begin{array}{l}3.068 \mathrm{E}-03 \\
3.351 \mathrm{E}-03 \\
3.631 \mathrm{E}-03 \\
3.910 \mathrm{E}-03 \\
4.187 \mathrm{E}-03 \\
4.741 \mathrm{E}-03 \\
5.296 \mathrm{E}-03 \\
5.855 \mathrm{E}-03\end{array}$ & $\begin{array}{l}7.976 \mathrm{E}- \\
3.082 \mathrm{E}- \\
5.539 \mathrm{E}- \\
8.121 \mathrm{E}- \\
1.079 \mathrm{E}- \\
1.629 \mathrm{E}- \\
2.189 \mathrm{E}- \\
2.747 \mathrm{E}-\end{array}$ & & & $\begin{array}{l}0.129 \\
0.1124 \\
0.119 \\
0.1194 \\
0.110 \\
0.103 \\
0.097 \\
0.092\end{array}$ \\
\hline $\begin{array}{l}1.0000 \\
1.2500 \\
1.5000 \\
1.7500 \\
2.0000 \\
2.5000 \\
3.0000 \\
3.5000\end{array}$ & $\begin{array}{l}1.633 E+00 \\
1.618 E+00 \\
1.615 E+00 \\
1.618 E+00 \\
1.623 E+00 \\
1.638 E+00 \\
1.653 E+00 \\
1.669 E+00\end{array}$ & $\begin{array}{l}1.995 \mathrm{E}-02 \\
2.476 \mathrm{E}-02 \\
2.990 \mathrm{E}-02 \\
3.530 \mathrm{E}-02 \\
4.092 \mathrm{E}-02 \\
5.270 \mathrm{E}-02 \\
6.507 \mathrm{E}-02 \\
7.791 \mathrm{E}-02\end{array}$ & $\begin{array}{l}1.653 E+00 \\
1.643 E+00 \\
1.645 E+00 \\
1.653 E+00 \\
1.664 E+00 \\
1.690 E+00 \\
1.718 E+00 \\
1.747 E+00\end{array}$ & $\begin{array}{l}4.940 E-0 \\
6.458 E-0 \\
7.980 E-0 \\
9.496 E-0 \\
1.100 E+0 \\
1.399 E+0 \\
1.692 E+0 \\
1.981 E+0\end{array}$ & $\begin{array}{l}6.418 \mathrm{E}-03 \\
7.846 \mathrm{E}-03 \\
9.308 \mathrm{E}-03 \\
1.080 \mathrm{E}-02 \\
1.232 \mathrm{E}-02 \\
1.543 \mathrm{E}-02 \\
1.861 \mathrm{E}-02 \\
2.184 \mathrm{E}-02\end{array}$ & $\begin{array}{l}3.2 \\
4.62 \\
5.86 \\
7.01 \\
8.08 \\
1.08 \\
1.1 \\
1.3\end{array}$ & $\begin{array}{l}-0 \\
-0 \\
-0 \\
-0 \\
-0 \\
-0 \\
-0 \\
-0\end{array}$ & & $\begin{array}{l}0 . \\
0 . \\
0 . \\
0 . \\
0 . \\
0 . \\
0 .\end{array}$ \\
\hline $\begin{array}{l}4.00000 \\
4.5000 \\
5.0000 \\
5.5000 \\
6.0000 \\
7.0000 \\
8.0000 \\
9.0000\end{array}$ & $\begin{array}{l}683 E+00 \\
696 E+00 \\
708 E+00 \\
720 E+00 \\
730 E+00 \\
749 E+00 \\
765 E+00 \\
779 E+00\end{array}$ & $\begin{array}{l}1.0 \\
1.18 \\
1.3 \\
1.4 \\
1.7 \\
2.0 \\
2.3\end{array}$ & $\begin{array}{l}1.774 E+00 \\
1.801 E+00 \\
1.827 E+00 \\
1.853 E+00 \\
1.877 E+00 \\
1.925 E+00 \\
1.971 E+00 \\
2.016 E+00\end{array}$ & $\begin{array}{l}2.265 \mathrm{E}+00 \\
2.544 \mathrm{E}+00 \\
2.820 \mathrm{E}+00 \\
3.092 \mathrm{E}+00 \\
3.360 \mathrm{E}+00 \\
3.886 \mathrm{E}+00 \\
4.399 \mathrm{E}+00 \\
4.901 \mathrm{E}+00\end{array}$ & $\begin{array}{l}2.511 \mathrm{E}-0 \\
2.841 \mathrm{E}-0 \\
3.172 \mathrm{E}-\mathrm{d} \\
3.504 \mathrm{E}-\mathrm{c} \\
3.837 \mathrm{E}- \\
4.503 \mathrm{E}-\mathrm{c} \\
5.168 \mathrm{E}-\mathrm{c} \\
5.830 \mathrm{E}-\end{array}$ & $\begin{array}{l}1.458 \\
1.583 \\
1.699 \\
1.807 \\
1.908 \\
2.095 \\
2.264 \\
2.420\end{array}$ & & & $\begin{array}{l}48 \\
45 \\
44 \\
42 \\
40 \\
37 \\
35 \\
33\end{array}$ \\
\hline $\begin{array}{l}10.0000 \\
12.5000 \\
15.0000 \\
17.5000 \\
20.0000 \\
25.0000 \\
30.0000 \\
35.0000\end{array}$ & $\begin{array}{l}1.8 \\
1.8 \\
1.8 \\
1.8 \\
1.9\end{array}$ & $\begin{array}{l}3.479 \mathrm{E}-01 \\
4.295 \mathrm{E}-01 \\
5.127 \mathrm{E}-01 \\
5.971 \mathrm{E}-01 \\
7.686 \mathrm{E}-01 \\
9.428 \mathrm{E}-01 \\
1.119 \mathrm{E}+00\end{array}$ & $\begin{array}{l}2.165 E+00 \\
2.266 E+00 \\
2.366 E+00 \\
2.464 E+00 \\
2.657 E+00 \\
2.849 E+00 \\
3.040 E+00\end{array}$ & $\begin{array}{l}6.575 E+00 \\
7.704 E+00 \\
8.783 E+00 \\
9.819 E+00 \\
1.177 E+01 \\
1.359 E+01 \\
1.529 E+01\end{array}$ & $\begin{array}{l}6.486 \mathrm{E}-02 \\
8.101 \mathrm{E}-02 \\
9.672 \mathrm{E}-02 \\
1.119 \mathrm{E}-01 \\
1.267 \mathrm{E}-01 \\
1.546 \mathrm{E}-01 \\
1.806 \mathrm{E}-01 \\
2.048 \mathrm{E}-01\end{array}$ & $\begin{array}{l}2.888 \mathrm{E}+0 \\
3.168 \mathrm{E}+0 \\
3.415 \mathrm{E}+0 \\
3.636 \mathrm{E}+0 \\
4.017 \mathrm{E}+0 \\
4.337 \mathrm{E}+0 \\
4.613 \mathrm{E}+0\end{array}$ & $\begin{array}{l}-0.02 \\
-0.02 \\
-0.01 \\
-0.01 \\
-0.01 \\
-0.01 \\
-0.00 \\
-0.00\end{array}$ & & \\
\hline $\begin{array}{l}40.0000 \\
45.0000 \\
50.0000 \\
55.0000 \\
60.0000 \\
70.0000 \\
80.0000 \\
90.0000\end{array}$ & $\begin{array}{ll}0 & 0 \\
0 & 0 \\
0 & 0 \\
0 & 0\end{array}$ & $\begin{array}{l}1.4 \\
1.6 \\
1.8 \\
2.0 \\
2.3 \\
2.7 \\
3.1\end{array}$ & $\begin{array}{l}3 . \\
3 . \\
3 . \\
3 . \\
4\end{array}$ & & $\begin{array}{l}2 . \\
2 . \\
2 . \\
3 . \\
3 . \\
3 . \\
3 .\end{array}$ & $\begin{array}{l}5 . \\
5 . \\
5 . \\
5 . \\
6 . \\
6 .\end{array}$ & $\begin{array}{l}-0 \\
-0 \\
-0 \\
-0 \\
-0 \\
-0 \\
-0\end{array}$ & & \\
\hline .0 & $\begin{array}{l}2.0 \\
2.0\end{array}$ & $\begin{array}{l}4 \\
5 \\
6 \\
7\end{array}$ & & & & & & & \\
\hline .00 & $\begin{array}{l}0 \\
0 \\
0 \\
0 \\
0\end{array}$ & $\begin{array}{l}1 \\
1 \\
2 \\
2 \\
2 \\
3\end{array}$ & $\begin{array}{l}01 E+01 \\
95 E+01 \\
39 E+01 \\
34 E+01 \\
72 E+01 \\
61 E+01 \\
50 E+01\end{array}$ & $\begin{array}{l}08 E+01 \\
59 E+01 \\
87 E+01 \\
97 E+01 \\
71 E+01 \\
97 E+01 \\
87 E+01\end{array}$ & $\begin{array}{l}7.211 \mathrm{E}-01 \\
7.383 \mathrm{E}-01 \\
7.531 \mathrm{E}-01 \\
7.662 \mathrm{E}-01 \\
7.880 \mathrm{E}-01 \\
8.056 \mathrm{E}-01 \\
8.202 \mathrm{E}-01\end{array}$ & $\begin{array}{l}9.515 E+00 \\
9.725 E+00 \\
9.915 E+00 \\
1.009 E+01 \\
1.040 E+01 \\
1.066 E+01 \\
1.090 E+01\end{array}$ & $\begin{array}{l}-0.0000 \\
-0.0000 \\
-0.000 \\
-0.000 \\
-0.000 \\
-0.000 \\
-0.000 \\
-0.000\end{array}$ & $\begin{array}{l}0.006 \\
0.006 \\
0.006 \\
0.006 \\
0.005 \\
0.005 \\
0.005 \\
0.005\end{array}$ & \\
\hline .000 & $2.200 E+00$ & $3.820 \mathrm{E}+01$ & $4.040 E+01$ & $8.247 E+01$ & $8.324 E-01$ & $1.111 E+01$ & -0.000 & 0.005 & 0.000 \\
\hline
\end{tabular}


ELECTRONS IN PROPANE

$I=47.1 \mathrm{eV} \quad$ DENSITY $=1.879 \mathrm{E}-03 \mathrm{~g} / \mathrm{cm}^{3}\left(20^{\circ} \mathrm{C}\right)$

\begin{tabular}{|c|c|c|c|c|c|c|c|c|c|}
\hline ENERGY & $\begin{array}{l}\text { COLLISION } \\
\mathrm{MeV} \mathrm{Cm}^{2} / \mathrm{g}\end{array}$ & $\begin{array}{l}\text { PPING POWE } \\
\text { RADIATIVE } \\
\text { MeV } \mathrm{cm}^{2} / \mathrm{g}\end{array}$ & $\begin{array}{c}\text { TOTAL } \\
\mathrm{MeV} \mathrm{cm}^{2} / \mathrm{g}\end{array}$ & $\begin{array}{l}\text { CSDA } \\
\text { RANGE } \\
\mathrm{g} / \mathrm{Cm}^{2}\end{array}$ & $\begin{array}{l}\text { RADIATION } \\
\text { YIELD }\end{array}$ & $\begin{array}{l}\text { DENS.EFF. } \\
\text { CORR } \\
\text { (DELTA) }\end{array}$ & $\begin{array}{l}\text { de } 1 \\
\text { LoLL }\end{array}$ & $\begin{array}{l}\text { )/d(1 } \\
\text { CSDA } \\
\text { RANGE }\end{array}$ & $\begin{array}{l}\text { I I ) } \\
\text { RAD } \\
\text { YIELD }\end{array}$ \\
\hline $\begin{array}{l}0.0100 \\
0.0125 \\
0.0150 \\
0.0175 \\
0.0200 \\
0.0250 \\
0.0300 \\
0.0350\end{array}$ & $\begin{array}{l}2.618 E+01 \\
2.194 E+01 \\
1.899 E+01 \\
1.681 E+01 \\
1.513 E+01 \\
1.271 E+01 \\
1.103 E+01 \\
9.806 E+00\end{array}$ & $\begin{array}{l}2.752 \mathrm{E}-03 \\
2.762 \mathrm{E}-03 \\
2.769 \mathrm{E}-03 \\
2.774 \mathrm{E}-03 \\
2.779 \mathrm{E}-03 \\
2.789 \mathrm{E}-03 \\
2.799 \mathrm{E}-03 \\
2.810 \mathrm{E}-03\end{array}$ & $\begin{array}{l}2.618 E+01 \\
2.194 E+01 \\
1.899 E+01 \\
1.681 E+01 \\
1.514 E+01 \\
1.271 E+01 \\
1.104 E+01 \\
9.808 E+00\end{array}$ & $\begin{array}{l}2.142 \mathrm{E}-04 \\
3.189 \mathrm{E}-04 \\
4.418 \mathrm{E}-04 \\
5.820 \mathrm{E}-04 \\
7.390 \mathrm{E}-04 \\
1.101 \mathrm{E}-03 \\
1.525 \mathrm{E}-03 \\
2.006 \mathrm{E}-03\end{array}$ & $\begin{array}{l}5.755 E-05 \\
6.916 \mathrm{E}-05 \\
8.028 \mathrm{E}-05 \\
9.102 \mathrm{E}-05 \\
1.014 \mathrm{E}-04 \\
1.215 \mathrm{E}-04 \\
1.407 \mathrm{E}-04 \\
1.592 \mathrm{E}-04\end{array}$ & $\begin{array}{l}0.0 \\
0.0 \\
0.0 \\
0.0 \\
0.0 \\
0.0 \\
0.0 \\
0.0\end{array}$ & $\begin{array}{l}-0.182 \\
-0.175 \\
-0.170 \\
-0.165 \\
-0.162 \\
-0.156 \\
-0.152 \\
-0.149\end{array}$ & $\begin{array}{l}0.205 \\
0.196 \\
0.189 \\
0.184 \\
0.180 \\
0.173 \\
0.168 \\
0.164\end{array}$ & $\begin{array}{l}0.204 \\
0.195 \\
0.189 \\
0.184 \\
0.179 \\
0.173 \\
0.167 \\
0.163\end{array}$ \\
\hline $\begin{array}{l}0.0400 \\
0.0450 \\
0.0500 \\
0.0550 \\
0.0600 \\
0.0700 \\
0.0800 \\
0.0900\end{array}$ & $\begin{array}{l}8.863 E+00 \\
8.116 \mathrm{E}+00 \\
7.509 \mathrm{E}+00 \\
7.005 \mathrm{E}+00 \\
6.580 \mathrm{E}+00 \\
5.903 \mathrm{E}+00 \\
5.387 \mathrm{E}+00 \\
4.980 \mathrm{E}+00\end{array}$ & $\begin{array}{l}2.821 \mathrm{E}-03 \\
2.834 \mathrm{E}-03 \\
2.847 \mathrm{E}-03 \\
2.861 \mathrm{E}-03 \\
2.876 \mathrm{E}-03 \\
2.907 \mathrm{E}-03 \\
2.940 \mathrm{E}-03 \\
2.975 \mathrm{E}-03\end{array}$ & $\begin{array}{l}8.866 \mathrm{E}+00 \\
8.119 \mathrm{E}+00 \\
7.511 \mathrm{E}+00 \\
7.008 \mathrm{E}+00 \\
6.583 \mathrm{E}+00 \\
5.906 \mathrm{E}+00 \\
5.390 \mathrm{E}+00 \\
4.983 \mathrm{E}+00\end{array}$ & $\begin{array}{l}2.543 \mathrm{E}-03 \\
3.133 \mathrm{E}-03 \\
3.774 \mathrm{E}-03 \\
4.464 \mathrm{E}-03 \\
5.201 \mathrm{E}-03 \\
6.808 \mathrm{E}-03 \\
8.583 \mathrm{E}-03 \\
1.051 \mathrm{E}-02\end{array}$ & $\begin{array}{l}1.771 \mathrm{E}-04 \\
1.945 \mathrm{E}-04 \\
2.114 \mathrm{E}-04 \\
2.280 \mathrm{E}-04 \\
2.442 \mathrm{E}-04 \\
2.757 \mathrm{E}-04 \\
3.061 \mathrm{E}-04 \\
3.356 \mathrm{E}-04\end{array}$ & $\begin{array}{l}0.0 \\
0.0 \\
0.0 \\
0.0 \\
0.0 \\
0.0 \\
0.0 \\
0.0\end{array}$ & $\begin{array}{l}-0.146 \\
-0.144 \\
-0.142 \\
-0.140 \\
-0.138 \\
-0.136 \\
-0.133 \\
-0.131\end{array}$ & $\begin{array}{l}0.160 \\
0.157 \\
0.155 \\
0.153 \\
0.151 \\
0.147 \\
0.145 \\
0.142\end{array}$ & $\begin{array}{l}0.160 \\
0.157 \\
0.155 \\
0.152 \\
0.150 \\
0.147 \\
0.144 \\
0.142\end{array}$ \\
\hline $\begin{array}{l}0.1000 \\
0.1250 \\
0.1500 \\
0.1750 \\
0.2000 \\
0.2500 \\
0.3000 \\
0.3500\end{array}$ & $\begin{array}{l}4.652 E+00 \\
4.052 E+00 \\
3.648 E+00 \\
3.358 E+00 \\
3.141 E+00 \\
2.839 E+00 \\
2.641 E+00 \\
2.504 E+00\end{array}$ & $\begin{array}{l}3.013 \mathrm{E}-03 \\
3.114 \mathrm{E}-03 \\
3.222 \mathrm{E}-03 \\
3.338 \mathrm{E}-03 \\
3.460 \mathrm{E}-03 \\
3.722 \mathrm{E}-03 \\
4.007 \mathrm{E}-03 \\
4.311 \mathrm{E}-03\end{array}$ & $\begin{array}{l}4.655 \mathrm{E}+00 \\
4.056 \mathrm{E}+00 \\
3.652 \mathrm{E}+00 \\
3.362 \mathrm{E}+00 \\
3.144 \mathrm{E}+00 \\
2.842 \mathrm{E}+00 \\
2.645 \mathrm{E}+00 \\
2.508 \mathrm{E}+00\end{array}$ & $\begin{array}{l}1.259 \mathrm{E}-02 \\
1.837 \mathrm{E}-02 \\
2.488 \mathrm{E}-02 \\
3.203 \mathrm{E}-02 \\
3.973 \mathrm{E}-02 \\
5.651 \mathrm{E}-02 \\
7.479 \mathrm{E}-02 \\
9.423 \mathrm{E}-02\end{array}$ & $\begin{array}{l}3.643 \mathrm{E}-04 \\
4.330 \mathrm{E}-04 \\
4.984 \mathrm{E}-04 \\
5.612 \mathrm{E}-04 \\
6.219 \mathrm{E}-04 \\
7.386 \mathrm{E}-04 \\
8.509 \mathrm{E}-04 \\
9.603 \mathrm{E}-04\end{array}$ & $\begin{array}{l}0.0 \\
0.0 \\
0.0 \\
0.0 \\
0.0 \\
0.0 \\
0.0 \\
0.0\end{array}$ & $\begin{array}{l}-0.130 \\
-0.126 \\
-0.123 \\
-0.121 \\
-0.119 \\
-0.116 \\
-0.114 \\
-0.112\end{array}$ & $\begin{array}{l}0.140 \\
0.136 \\
0.133 \\
0.131 \\
0.129 \\
0.125 \\
0.123 \\
0.121\end{array}$ & $\begin{array}{l}0.140 \\
0.136 \\
0.133 \\
0.130 \\
0.128 \\
0.125 \\
0.122 \\
0.120\end{array}$ \\
\hline $\begin{array}{l}0.4000 \\
0.4500 \\
0.5000 \\
0.5500 \\
0.6000 \\
0.7000 \\
0.8000 \\
0.9000\end{array}$ & $\begin{array}{l}2.404 E+00 \\
2.330 \mathrm{E}+00 \\
2.274 \mathrm{E}+00 \\
2.230 \mathrm{E}+00 \\
2.196 \mathrm{E}+00 \\
2.147 \mathrm{E}+00 \\
2.116 \mathrm{E}+00 \\
2.096 \mathrm{E}+00\end{array}$ & $\begin{array}{l}4.635 \mathrm{E}-03 \\
4.976 \mathrm{E}-03 \\
5.334 \mathrm{E}-03 \\
5.705 \mathrm{E}-03 \\
6.089 \mathrm{E}-03 \\
6.894 \mathrm{E}-03 \\
7.739 \mathrm{E}-03 \\
8.623 \mathrm{E}-03\end{array}$ & $\begin{array}{l}2.409 \mathrm{E}+00 \\
2.335 \mathrm{E}+00 \\
2.279 \mathrm{E}+00 \\
2.236 \mathrm{E}+00 \\
2.202 \mathrm{E}+00 \\
2.154 \mathrm{E}+00 \\
2.124 \mathrm{E}+00 \\
2.105 \mathrm{E}+00\end{array}$ & $\begin{array}{l}1.146 \mathrm{E}-01 \\
1.357 \mathrm{E}-01 \\
1.574 \mathrm{E}-01 \\
1.795 \mathrm{E}-01 \\
2.021 \mathrm{E}-01 \\
2.480 \mathrm{E}-01 \\
2.948 \mathrm{E}-01 \\
3.421 \mathrm{E}-01\end{array}$ & $\begin{array}{l}1.068 \mathrm{E}-03 \\
1.174 \mathrm{E}-03 \\
1.281 \mathrm{E}-03 \\
1.386 \mathrm{E}-03 \\
1.492 \mathrm{E}-03 \\
1.705 \mathrm{E}-03 \\
1.920 \mathrm{E}-03 \\
2.137 \mathrm{E}-03\end{array}$ & $\begin{array}{l}0.0 \\
0.0 \\
0.0 \\
0.0 \\
0.0 \\
0.0 \\
0.0 \\
0.0\end{array}$ & $\begin{array}{l}-0.110 \\
-0.108 \\
-0.107 \\
-0.106 \\
-0.105 \\
-0.103 \\
-0.101 \\
-0.100\end{array}$ & $\begin{array}{l}0.119 \\
0.117 \\
0.116 \\
0.115 \\
0.114 \\
0.112 \\
0.110 \\
0.109\end{array}$ & $\begin{array}{l}0.1118 \\
0.1116 \\
0.1115 \\
0.1113 \\
0.112 \\
0.1110 \\
0.1108 \\
0.1106\end{array}$ \\
\hline $\begin{array}{l}1.0000 \\
1.2500 \\
1.5000 \\
1.7500 \\
2.0000 \\
2.5000 \\
3.0000 \\
3.5000\end{array}$ & $\begin{array}{l}2.084 E+00 \\
2.073 E+00 \\
2.078 E+00 \\
2.088 E+00 \\
2.103 E+00 \\
2.134 E+00 \\
2.166 E+00 \\
2.196 E+00\end{array}$ & $\begin{array}{l}9.544 \mathrm{E}-03 \\
1.198 \mathrm{E}-02 \\
1.459 \mathrm{E}-02 \\
1.734 \mathrm{E}-02 \\
2.021 \mathrm{E}-02 \\
2.626 \mathrm{E}-02 \\
3.263 \mathrm{E}-02 \\
3.928 \mathrm{E}-02\end{array}$ & $\begin{array}{l}2.094 \mathrm{E}+00 \\
2.085 \mathrm{E}+00 \\
2.092 \mathrm{E}+00 \\
2.106 \mathrm{E}+00 \\
2.123 \mathrm{E}+00 \\
2.160 \mathrm{E}+00 \\
2.199 \mathrm{E}+00 \\
2.235 \mathrm{E}+00\end{array}$ & $\begin{array}{l}3.898 \mathrm{E}-01 \\
5.095 \mathrm{E}-01 \\
6.293 \mathrm{E}-01 \\
7.484 \mathrm{E}-01 \\
8.666 \mathrm{E}-01 \\
1.100 \mathrm{E}+00 \\
1.330 \mathrm{E}+00 \\
1.555 \mathrm{E}+00\end{array}$ & $\begin{array}{l}2.356 \mathrm{E}-03 \\
2.914 \mathrm{E}-03 \\
3.488 \mathrm{E}-03 \\
4.075 \mathrm{E}-03 \\
4.675 \mathrm{E}-03 \\
5.907 \mathrm{E}-03 \\
7.171 \mathrm{E}-03 \\
8.462 \mathrm{E}-03\end{array}$ & $\begin{array}{l}0.0 \\
0.0 \\
0.0 \\
0.0 \\
0.0 \\
0.0 \\
0.0 \\
0.0\end{array}$ & $\begin{array}{l}-0.098 \\
-0.095 \\
-0.093 \\
-0.091 \\
-0.090 \\
-0.087 \\
-0.086 \\
-0.084\end{array}$ & $\begin{array}{l}0.107 \\
0.105 \\
0.103 \\
0.101 \\
0.099 \\
0.0997 \\
0.095 \\
0.093\end{array}$ & $\begin{array}{l}0.105 \\
0.102 \\
0.099 \\
0.097 \\
0.096 \\
0.093 \\
0.090 \\
0.088\end{array}$ \\
\hline $\begin{array}{l}4.0000 \\
4.5000 \\
5.0000 \\
5.5000 \\
6.0000 \\
7.0000 \\
8.0000 \\
9.0000\end{array}$ & $\begin{array}{l}2.224 E+00 \\
2.250 E+00 \\
2.274 E+00 \\
2.297 E+00 \\
2.318 E+00 \\
2.356 E+00 \\
2.389 E+00 \\
2.419 E+00\end{array}$ & $\begin{array}{l}4.616 \mathrm{E}-02 \\
5.323 \mathrm{E}-02 \\
6.049 \mathrm{E}-02 \\
6.790 \mathrm{E}-02 \\
7.546 \mathrm{E}-02 \\
9.094 \mathrm{E}-02 \\
1.068 \mathrm{E}-01 \\
1.231 \mathrm{E}-01\end{array}$ & $\begin{array}{l}2.270 \mathrm{E}+00 \\
2.304 \mathrm{E}+00 \\
2.335 \mathrm{E}+00 \\
2.365 \mathrm{E}+00 \\
2.393 \mathrm{E}+00 \\
2.447 \mathrm{E}+00 \\
2.496 \mathrm{E}+00 \\
2.542 \mathrm{E}+00\end{array}$ & $\begin{array}{l}1.777 E+00 \\
1.996 \mathrm{E}+00 \\
2.211 \mathrm{E}+00 \\
2.424 \mathrm{E}+00 \\
2.634 \mathrm{E}+00 \\
3.047 \mathrm{E}+00 \\
3.452 \mathrm{E}+00 \\
3.849 \mathrm{E}+00\end{array}$ & $\begin{array}{l}9.772 \mathrm{E}-03 \\
1.110 \mathrm{E}-02 \\
1.244 \mathrm{E}-02 \\
1.379 \mathrm{E}-02 \\
1.515 \mathrm{E}-02 \\
1.790 \mathrm{E}-02 \\
2.066 \mathrm{E}-02 \\
2.343 \mathrm{E}-02\end{array}$ & $\begin{array}{l}0.0 \\
0.0 \\
0.0 \\
0.0 \\
0.0 \\
0.0 \\
0.0 \\
0.0\end{array}$ & $\begin{array}{l}-0.083 \\
-0.081 \\
-0.080 \\
-0.080 \\
-0.079 \\
-0.077 \\
-0.076 \\
-0.075\end{array}$ & $\begin{array}{l}0.092 \\
0.091 \\
0.089 \\
0.088 \\
0.087 \\
0.086 \\
0.084 \\
0.083\end{array}$ & $\begin{array}{l}0.087 \\
0.085 \\
0.084 \\
0.083 \\
0.082 \\
0.080 \\
0.079 \\
0.077\end{array}$ \\
\hline $\begin{array}{l}10.0000 \\
12.5000 \\
15.0000 \\
17.5000 \\
20.0000 \\
25.0000 \\
30.00000 \\
35.0000\end{array}$ & $\begin{array}{l}2.446 E+00 \\
2.505 E+00 \\
2.551 E+00 \\
2.586 E+00 \\
2.613 E+00 \\
2.655 E+00 \\
2.685 E+00 \\
2.710 E+00\end{array}$ & $\begin{array}{l}1.397 \mathrm{E}-01 \\
1.823 \mathrm{E}-01 \\
2.262 \mathrm{E}-01 \\
2.710 \mathrm{E}-01 \\
3.167 \mathrm{E}-01 \\
4.097 \mathrm{E}-01 \\
5.045 \mathrm{E}-01 \\
6.007 \mathrm{E}-01\end{array}$ & $\begin{array}{l}2.586 E+00 \\
2.687 E+00 \\
2.777 E+00 \\
2.857 E+00 \\
2.930 E+00 \\
3.064 E+00 \\
3.190 E+00 \\
3.310 E+00\end{array}$ & $\begin{array}{l}4.239 E+00 \\
5.187 E+00 \\
6.102 E+00 \\
6.989 E+00 \\
7.853 E+00 \\
9.521 E+00 \\
1.112 E+01 \\
1.266 E+01\end{array}$ & $\begin{array}{l}2.621 \mathrm{E}-02 \\
3.316 \mathrm{E}-02 \\
4.007 \mathrm{E}-02 \\
4.695 \mathrm{E}-02 \\
5.376 \mathrm{E}-02 \\
6.721 \mathrm{E}-02 \\
8.035 \mathrm{E}-02 \\
9.314 \mathrm{E}-02\end{array}$ & $\begin{array}{l}0.0 \\
0.0 \\
1.733 \mathrm{E}-02 \\
8.510 \mathrm{E}-02 \\
1.770 \mathrm{E}-01 \\
3.825 \mathrm{E}-01 \\
5.859 \mathrm{E}-01 \\
7.756 \mathrm{E}-01\end{array}$ & $\begin{array}{l}-0.074 \\
-0.073 \\
-0.061 \\
-0.049 \\
-0.041 \\
-0.032 \\
-0.027 \\
-0.024\end{array}$ & $\begin{array}{l}0.082 \\
0.080 \\
0.077 \\
0.074 \\
0.070 \\
0.063 \\
0.058 \\
0.053\end{array}$ & $\begin{array}{l}0.076 \\
0.073 \\
0.070 \\
0.065 \\
0.059 \\
0.049 \\
0.042 \\
0.036\end{array}$ \\
\hline $\begin{array}{l}40.0000 \\
45.0000 \\
50.0000 \\
55.00000 \\
60.00000 \\
70.0000 \\
80.00000 \\
90.00000\end{array}$ & $\begin{array}{l}2.730 E+00 \\
2.747 E+00 \\
2.763 E+00 \\
2.776 E+00 \\
2.788 E+00 \\
2.809 E+00 \\
2.828 E+00 \\
2.843 E+00\end{array}$ & $\begin{array}{l}6.979 \mathrm{E}-01 \\
7.960 \mathrm{E}-01 \\
8.948 \mathrm{E}-01 \\
9.944 \mathrm{E}-01 \\
1.094 \mathrm{E}+00 \\
1.296 \mathrm{E}+00 \\
1.499 \mathrm{E}+00 \\
1.703 \mathrm{E}+00\end{array}$ & $\begin{array}{l}3.428 E+00 \\
3.543 E+00 \\
3.657 E+00 \\
3.770 E+00 \\
3.883 E+00 \\
4.105 E+00 \\
4.326 E+00 \\
4.546 E+00\end{array}$ & $\begin{array}{l}1.414 E+01 \\
1.558 E+01 \\
1.697 \mathrm{E}+01 \\
1.831 \mathrm{E}+01 \\
1.962 \mathrm{E}+01 \\
2.212 \mathrm{E}+01 \\
2.450 \mathrm{E}+01 \\
2.675 \mathrm{E}+01\end{array}$ & $\begin{array}{l}i .056 \mathrm{E}-01 \\
1.176 \mathrm{E}-01 \\
1.294 \mathrm{E}-01 \\
1.407 \mathrm{E}-01 \\
1.517 \mathrm{E}-01 \\
1.728 \mathrm{E}-01 \\
1.926 \mathrm{E}-01 \\
2.113 \mathrm{E}-01\end{array}$ & $\begin{array}{l}9.500 E-01 \\
1.110 E+00 \\
1.257 E+00 \\
1.393 E+00 \\
1.519 E+00 \\
1.746 E+00 \\
1.946 E+00 \\
2.125 E+00\end{array}$ & $\begin{array}{l}-0.022 \\
-0.020 \\
-0.019 \\
-0.018 \\
-0.017 \\
-0.016 \\
-0.015 \\
-0.015\end{array}$ & $\begin{array}{l}0.050 \\
0.047 \\
0.044 \\
0.042 \\
0.060 \\
0.037 \\
0.034 \\
0.032\end{array}$ & $\begin{array}{l}0.032 \\
0.029 \\
0.026 \\
0.024 \\
0.023 \\
0.020 \\
0.018 \\
0.016\end{array}$ \\
\hline $\begin{array}{l}.0000 \\
.0060 \\
.0000 \\
.0000 \\
.0000 \\
.0000 \\
.0000 \\
.0000\end{array}$ & $\begin{array}{l}2.857 E+00 \\
2.886 E+00 \\
2.909 E+00 \\
2.928 E+00 \\
2.944 E+00 \\
2.970 E+00 \\
2.991 E+00 \\
3.008 E+00\end{array}$ & $\begin{array}{l}1.909 E+00 \\
2.426 E+00 \\
2.947 \mathrm{E}+00 \\
3.471 \mathrm{E}+00 \\
3.998 \mathrm{E}+00 \\
5.056 \mathrm{E}+00 \\
6.121 \mathrm{E}+00 \\
7.189 \mathrm{E}+00\end{array}$ & $\begin{array}{l}4.766 E+00 \\
5.312 E+00 \\
5.856 E+00 \\
6.399 E+00 \\
6.942 E+00 \\
8.027 E+00 \\
9.111 E+00 \\
1.020 E+01\end{array}$ & $\begin{array}{l}2.890 \mathrm{E}+01 \\
3.386 \mathrm{E}+01 \\
3.835 \mathrm{E}+01 \\
4.243 \mathrm{E}+01 \\
4.618 \mathrm{E}+01 \\
5.287 \mathrm{E}+01 \\
5.871 \mathrm{E}+01 \\
6.390 \mathrm{E}+01\end{array}$ & $\begin{array}{l}2.289 \mathrm{E}-01 \\
2.690 \mathrm{E}-01 \\
3.043 \mathrm{E}-01 \\
3.356 \mathrm{E}-01 \\
3.636 \mathrm{E}-01 \\
4.117 \mathrm{E}-01 \\
4.517 \mathrm{E}-01 \\
4.856 \mathrm{E}-01\end{array}$ & $\begin{array}{l}2.287 E+00 \\
2.637 E+00 \\
2.928 E+00 \\
3.180 E+00 \\
3.402 E+00 \\
3.783 E+00 \\
4.103 E+00 \\
4.381 E+00\end{array}$ & $\begin{array}{l}-0.014 \\
-0.013 \\
-0.012 \\
-0.011 \\
-0.010 \\
-0.008 \\
-0.007 \\
-0.005\end{array}$ & $\begin{array}{l}0.030 \\
0.027 \\
0.025 \\
0.023 \\
0.021 \\
0.019 \\
0.017 \\
0.016\end{array}$ & $\begin{array}{l}0.015 \\
0.013 \\
0.011 \\
0.010 \\
0.009 \\
0.007 \\
0.006 \\
0.005\end{array}$ \\
\hline $\begin{array}{l}.0000 \\
.0000 \\
.0000 \\
.0000 \\
.0000 \\
.0000 \\
.0000 \\
.0000\end{array}$ & $\begin{array}{l}3.022 E+00 \\
3.034 E+00 \\
3.044 E+00 \\
3.054 E+00 \\
3.062 E+00 \\
3.077 E+00 \\
3.090 E+00 \\
3.101 E+00\end{array}$ & $\begin{array}{l}8.260 E+00 \\
9.334 E+00 \\
1.041 E+01 \\
1.149 E+01 \\
1.257 E+01 \\
1.473 E+01 \\
1.689 E+01 \\
1.906 E+01\end{array}$ & $\begin{array}{l}1.128 E+01 \\
1.237 E+01 \\
1.345 E+01 \\
1.454 E+01 \\
1.563 E+01 \\
1.781 E+01 \\
1.998 E+01 \\
2.216 E+01\end{array}$ & $\begin{array}{l}6.856 E+01 \\
7.279 E+01 \\
7.666 E+01 \\
8.024 E+01 \\
8.355 E+01 \\
8.954 E+01 \\
9.484 E+01 \\
9.959 E+01\end{array}$ & $\begin{array}{l}5.402 E-01 \\
5.626 \mathrm{E}-01 \\
5.826 \mathrm{E}-01 \\
6.005 \mathrm{E}-01 \\
6.313 \mathrm{E}-01 \\
6.569 \mathrm{E}-01 \\
6.787 \mathrm{E}-01\end{array}$ & $\begin{array}{l}4.626 E+00 \\
4.845 E+00 \\
5.044 E+00 \\
5.225 E+00 \\
5.392 E+00 \\
5.689 E+00 \\
5.949 E+00 \\
6.180 E+00\end{array}$ & $\begin{array}{l}-0.005 \\
-0.004 \\
-0.003 \\
-0.003 \\
-0.002 \\
-0.002 \\
-0.001 \\
-0.001\end{array}$ & $\begin{array}{l}0.015 \\
0.014 \\
0.014 \\
0.013 \\
0.013 \\
0.012 \\
0.011 \\
0.011\end{array}$ & $\begin{array}{l}0.005 \\
0.004 \\
0.004 \\
0.003 \\
0.003 \\
0.003 \\
0.002 \\
0.002\end{array}$ \\
\hline .0000 & $3.111 \mathrm{E}+00$ & $2.123 E+0 i$ & $2.434 E+01$ & $1.039 E+02$ & $6.975 E-01$ & $6.387 E+00$ & -0.001 & 0.010 & 0.002 \\
\hline
\end{tabular}


ELECTRONS IN SILICON DIOXIDE

$I=139.2 \mathrm{eV} \quad$ DENSITY $=2.320 \mathrm{E}+00 \mathrm{~g} / \mathrm{cm}^{3}$

\begin{tabular}{|c|c|c|c|c|c|c|c|c|c|}
\hline IERGY & $\begin{array}{l}\text { COLLISION } \\
\text { MEV } \mathrm{cm}^{2} / \mathrm{g}\end{array}$ & $\begin{array}{l}\text { OPPING POWE } \\
\text { RADIATIVE } \\
\text { MeV } \mathrm{cm}^{2} / \mathrm{g}\end{array}$ & $\begin{array}{c}\text { TOTAL } \\
\mathrm{MeV} \mathrm{cm}^{2} / \mathrm{g}\end{array}$ & $\begin{array}{l}\text { CSDA } \\
\text { RANGE } \\
\mathrm{g} / \mathrm{Cm}^{2}\end{array}$ & $\begin{array}{l}\text { DIATION } \\
\text { YIELD }\end{array}$ & $\begin{array}{l}\text { EHS.EFF. } \\
\text { CORR. } \\
\text { (DELTA) }\end{array}$ & $\begin{array}{l}\text { de } 1 \\
\text { coLL } \\
\text { Loss }\end{array}$ & $\begin{array}{l}) \text { Cd } 1 \\
\text { SDA } \\
\text { ANGE }\end{array}$ & $\begin{array}{l}\text { II ) } \\
\text { RAD } \\
\text { YIELD }\end{array}$ \\
\hline $\begin{array}{l}0.0100 \\
0.0125 \\
0.0150 \\
0.0175 \\
0.0200 \\
0.0250 \\
0.0300 \\
0.0350\end{array}$ & $\begin{array}{l}1.780 E+01 \\
1.506 E+01 \\
1.313 E+01 \\
1.169 E+01 \\
1.057 E+01 \\
8.939 E+00 \\
7.804 E+00 \\
6.965 E+00\end{array}$ & $\begin{array}{l}5.664 \mathrm{E}-03 \\
5.762 \mathrm{E}-03 \\
5.830 \mathrm{E}-03 \\
5.880 \mathrm{E}-03 \\
5.918 \mathrm{E}-03 \\
5.971 \mathrm{E}-03 \\
6.009 \mathrm{E}-03 \\
6.039 \mathrm{E}-03\end{array}$ & $\begin{array}{l}1.781 E+01 \\
1.506 E+01 \\
1.313 E+01 \\
1.169 E+01 \\
1.057 E+01 \\
8.945 E+00 \\
7.810 E+00 \\
6.971 E+00\end{array}$ & $\begin{array}{l}3.255 \mathrm{E}-04 \\
4.788 \mathrm{E}-04 \\
6.570 \mathrm{E}-04 \\
8.592 \mathrm{E}-04 \\
1.084 \mathrm{E}-03 \\
1.601 \mathrm{E}-03 \\
2.201 \mathrm{E}-03 \\
2.880 \mathrm{E}-03\end{array}$ & $\begin{array}{l}716 E-04 \\
074 E-04 \\
417 E-04 \\
749 E-04 \\
069 E-04 \\
684 E-04 \\
268 E-04 \\
827 E-04\end{array}$ & $\begin{array}{l}0.0 \\
0.0 \\
0.0 \\
0.0 \\
0.0 \\
0.0 \\
0.0 \\
0.0\end{array}$ & $\begin{array}{l}-0.227 \\
-0.216 \\
-0.208 \\
-0.202 \\
-0.196 \\
-0.188 \\
-0.182 \\
-0.177\end{array}$ & $\begin{array}{l}0.267 \\
0.252 \\
0.241 \\
0.232 \\
0.225 \\
0.215 \\
0.207 \\
0.200\end{array}$ & $\begin{array}{l}0.263 \\
0.248 \\
0.238 \\
0.230 \\
0.223 \\
0.213 \\
0.205 \\
0.199\end{array}$ \\
\hline $\begin{array}{l}0.0400 \\
0.0450 \\
0.0500 \\
0.0550 \\
0.0600 \\
0.0700 \\
0.0800 \\
0.0900\end{array}$ & $\begin{array}{l}6.318 \mathrm{E}+00 \\
5.803 \mathrm{E}+00 \\
5.383 \mathrm{E}+00 \\
5.033 \mathrm{E}+00 \\
4.738 \mathrm{E}+00 \\
4.265 \mathrm{E}+00 \\
3.904 \mathrm{E}+00 \\
3.618 \mathrm{E}+00\end{array}$ & $\begin{array}{l}6.065 \mathrm{E}-03 \\
6.089 \mathrm{E}-03 \\
6.112 \mathrm{E}-03 \\
6.135 \mathrm{E}-03 \\
6.159 \mathrm{E}-03 \\
6.207 \mathrm{E}-03 \\
6.259 \mathrm{E}-03 \\
6.315 \mathrm{E}-03\end{array}$ & $\begin{array}{l}6.324 E+00 \\
5.809 E+00 \\
5.389 E+00 \\
5.040 E+00 \\
4.744 E+00 \\
4.271 E+00 \\
3.910 E+00 \\
3.624 E+00\end{array}$ & $\begin{array}{l}3.634 \mathrm{E}-03 \\
4.460 \mathrm{E}-03 \\
5.355 \mathrm{E}-03 \\
6.315 \mathrm{E}-03 \\
7.338 \mathrm{E}-03 \\
9.564 \mathrm{E}-03 \\
1.201 \mathrm{E}-02 \\
1.467 \mathrm{E}-02\end{array}$ & $\begin{array}{l}.365 E-04 \\
.884 E-04 \\
.387 \mathrm{E}-04 \\
.876 \mathrm{E}-04 \\
.351 \mathrm{E}-04 \\
.267 \mathrm{E}-04 \\
.143 \mathrm{E}-04 \\
.985 \mathrm{E}-04\end{array}$ & $\begin{array}{l}0.0 \\
0.0 \\
0.0 \\
0.0 \\
0.0 \\
0.0 \\
0.0 \\
0.0\end{array}$ & $\begin{array}{l}-0.174 \\
-0.170 \\
-0.167 \\
-0.165 \\
-0.163 \\
-0.159 \\
-0.156 \\
-0.153\end{array}$ & $\begin{array}{l}0.195 \\
0.191 \\
0.187 \\
0.184 \\
0.181 \\
0.176 \\
0.172 \\
0.169\end{array}$ & $\begin{array}{l}0.194 \\
0.190 \\
0.186 \\
0.183 \\
0.180 \\
0.175 \\
0.172 \\
0.168\end{array}$ \\
\hline $\begin{array}{l}0.1000 \\
0.1250 \\
0.1500 \\
0.1750 \\
0.2000 \\
0.2500 \\
0.3000 \\
0.3500\end{array}$ & $\begin{array}{l}3.386 \mathrm{E}+00 \\
2.963 \mathrm{E}+00 \\
2.677 \mathrm{E}+00 \\
2.471 \mathrm{E}+00 \\
2.316 \mathrm{E}+00 \\
2.101 \mathrm{E}+00 \\
1.961 \mathrm{E}+00 \\
1.864 \mathrm{E}+00\end{array}$ & $\begin{array}{l}6.375 \mathrm{E}-03 \\
6.541 \mathrm{E}-03 \\
6.727 \mathrm{E}-03 \\
6.930 \mathrm{E}-03 \\
7.148 \mathrm{E}-03 \\
7.622 \mathrm{E}-03 \\
8.114 \mathrm{E}-03 \\
8.706 \mathrm{E}-03\end{array}$ & $\begin{array}{l}3.393 \mathrm{E}+00 \\
2.969 \mathrm{E}+00 \\
2.683 \mathrm{E}+00 \\
2.478 \mathrm{E}+00 \\
2.323 \mathrm{E}+00 \\
2.109 \mathrm{E}+00 \\
1.969 \mathrm{E}+00 \\
1.872 \mathrm{E}+00\end{array}$ & $\begin{array}{l}1.753 \mathrm{E}-02 \\
2.544 \mathrm{E}-02 \\
3.432 \mathrm{E}-02 \\
4.403 \mathrm{E}-02 \\
5.446 \mathrm{E}-02 \\
7.713 \mathrm{E}-02 \\
1.017 \mathrm{E}-01 \\
1.278 \mathrm{E}-01\end{array}$ & $\begin{array}{l}080 \mathrm{E}-03 \\
272 \mathrm{E}-03 \\
453 \mathrm{E}-03 \\
624 \mathrm{E}-03 \\
789 \mathrm{E}-03 \\
100 \mathrm{E}-03 \\
396 \mathrm{E}-03 \\
682 \mathrm{E}-03\end{array}$ & $\begin{array}{l}0.0 \\
0.0 \\
0.0 \\
0.0 \\
0.0 \\
0.0 \\
0.0 \\
0.0\end{array}$ & $\begin{array}{l}-0.151 \\
-0.146 \\
-0.142 \\
-0.140 \\
-0.137 \\
-0.133 \\
-0.130 \\
-0.125\end{array}$ & $\begin{array}{l}0.166 \\
0.160 \\
0.156 \\
0.153 \\
0.150 \\
0.145 \\
0.142 \\
0.139\end{array}$ & $\begin{array}{l}0.165 \\
0.160 \\
0.156 \\
0.152 \\
0.149 \\
0.144 \\
0.141 \\
0.138\end{array}$ \\
\hline $\begin{array}{l}0.4000 \\
0.4500 \\
0.5000 \\
0.5500 \\
0.6000 \\
0.7000 \\
0.8000 \\
0.9000\end{array}$ & $\begin{array}{l}1.792 E+00 \\
1.738 \mathrm{E}+00 \\
1.697 \mathrm{E}+00 \\
1.665 \mathrm{E}+00 \\
1.639 \mathrm{E}+00 \\
1.603 \mathrm{E}+00 \\
1.579 \mathrm{E}+00 \\
1.563 \mathrm{E}+00\end{array}$ & $\begin{array}{l}9.304 \mathrm{E}-03 \\
9.935 \mathrm{E}-03 \\
1.060 \mathrm{E}-02 \\
1.128 \mathrm{E}-02 \\
1.199 \mathrm{E}-02 \\
1.348 \mathrm{E}-02 \\
1.504 \mathrm{E}-02 \\
1.667 \mathrm{E}-02\end{array}$ & $\begin{array}{l}1.801 E+00 \\
1.748 E+00 \\
1.708 E+00 \\
1.676 E+00 \\
1.651 E+00 \\
1.616 E+00 \\
1.594 E+00 \\
1.579 E+00\end{array}$ & $\begin{array}{l}1.5 \\
1.8 \\
2.12 \\
2.4 \\
2.7 \\
3.3 \\
3.9 \\
4.5\end{array}$ & $\begin{array}{l}2.960 \mathrm{E}-03 \\
3.233 \mathrm{E}-03 \\
3.504 \mathrm{E}-03 \\
3.774 \mathrm{E}-03 \\
4.042 \mathrm{E}-03 \\
4.579 \mathrm{E}-03 \\
5.118 \mathrm{E}-03 \\
5.659 \mathrm{E}-03\end{array}$ & $\begin{array}{l}1.344 \mathrm{E}-02 \\
3.175 \mathrm{E}-02 \\
5.190 \mathrm{E}-02 \\
7.343 \mathrm{E}-02 \\
9.597 \mathrm{E}-02 \\
1.431 \mathrm{E}-01 \\
1.919 \mathrm{E}-01 \\
2.413 \mathrm{E}-01\end{array}$ & $\begin{array}{l}-0.110 \\
-0.104 \\
-0.100 \\
-0.096 \\
-0.092 \\
-0.086 \\
-0.081 \\
-0.076\end{array}$ & $\begin{array}{l}.135 \\
.130 \\
.126 \\
.123 \\
.119 \\
.114 \\
.109 \\
.104\end{array}$ & $\begin{array}{l}0.133 \\
0.1128 \\
0.1123 \\
0.119 \\
0.116 \\
0.109 \\
0.1103 \\
0.098\end{array}$ \\
\hline $\begin{array}{l}1.0000 \\
1.2500 \\
1.5000 \\
1.7500 \\
2.0000 \\
2.5000 \\
3.0000 \\
3.5000\end{array}$ & $\begin{array}{l}1.552 E+00 \\
1.540 E+00 \\
1.538 E+00 \\
1.542 E+00 \\
1.548 E+00 \\
1.563 E+00 \\
1.578 E+00 \\
1.593 E+00\end{array}$ & $\begin{array}{l}1.83 \\
2.28 \\
2.76 \\
3.26 \\
3.78 \\
4.88 \\
6.03 \\
7.23\end{array}$ & $\begin{array}{l}1.570 E+00 \\
1.563 E+00 \\
1.566 E+00 \\
1.575 E+00 \\
1.586 E+00 \\
1.612 E+00 \\
1.639 E+00 \\
1.665 E+00\end{array}$ & $\begin{array}{l}5.220 \mathrm{E} \\
6.817 \mathrm{E} \\
8.416 \mathrm{E} \\
1.001 \mathrm{E} \\
1.159 \mathrm{E} \\
1.472 \mathrm{E} \\
1.780 \mathrm{E} \\
2.082 \mathrm{E}\end{array}$ & $\begin{array}{l}9.01 \\
1.04 \\
1.19 \\
1.49 \\
1.80 \\
2.12\end{array}$ & $\begin{array}{l}2.9 \\
4.9 \\
5.2 \\
6.3 \\
7.4 \\
9.3 \\
1.1 \\
1.2\end{array}$ & $\begin{array}{l}-0 . \\
-0 . \\
-0 . \\
-0 \\
-0 . \\
-0 . \\
-0 . \\
-0 .\end{array}$ & & $\begin{array}{l}0.094 \\
0.085 \\
0.078 \\
0.073 \\
0.068 \\
0.061 \\
0.056 \\
0.052\end{array}$ \\
\hline $\begin{array}{l}4.0000 \\
4.5000 \\
5.0000 \\
5.5000 \\
6.0000 \\
7.0000 \\
8.0000 \\
9.0000\end{array}$ & $\begin{array}{l}1.606 E+00 \\
1.619 E+00 \\
1.630 E+00 \\
1.641 E+00 \\
1.650 E+00 \\
1.667 E+00 \\
1.682 E+00 \\
1.695 E+00\end{array}$ & $\begin{array}{l}8.475 \mathrm{E}-02 \\
9.743 \mathrm{E}-02 \\
1.104 \mathrm{E}-01 \\
1.236 \mathrm{E}-01 \\
1.371 \mathrm{E}-01 \\
1.646 \mathrm{E}-01 \\
1.927 \mathrm{E}-01 \\
2.214 \mathrm{E}-01\end{array}$ & $\begin{array}{l}1.691 E+00 \\
1.716 E+00 \\
1.741 E+00 \\
1.764 E+00 \\
1.787 E+00 \\
1.832 E+00 \\
1.875 E+00 \\
1.916 E+00\end{array}$ & $\begin{array}{l}2.6 \\
2.9 \\
3.2 \\
3.5 \\
4.0 \\
4.6 \\
5.1\end{array}$ & $\begin{array}{l}-02 \\
-02 \\
-02 \\
-02 \\
-02 \\
-02 \\
002 \\
002 \\
002 \\
-02\end{array}$ & $\begin{array}{l}1.53 \\
1.65 \\
1.77 \\
1.88 \\
2.08 \\
2.26 \\
2.43\end{array}$ & $\begin{array}{l}-0.038 \\
-0.036 \\
-0.034 \\
-0.032 \\
-0.030 \\
-0.028 \\
-0.025 \\
-0.024\end{array}$ & $\begin{array}{l}0.061 \\
0.058 \\
0.055 \\
0.053 \\
0.051 \\
0.048 \\
0.045 \\
0.043\end{array}$ & $\begin{array}{l}0.046 \\
0.043 \\
0.041 \\
0.039 \\
0.036 \\
0.033 \\
0.030\end{array}$ \\
\hline $\begin{array}{l}10.0000 \\
12.5000 \\
15.0000 \\
17.5000 \\
20.0000 \\
25.0000 \\
30.0000 \\
35.0000\end{array}$ & $\begin{array}{l}1.706 E+00 \\
1.730 E+00 \\
1.749 E+00 \\
1.765 E+00 \\
1.779 E+00 \\
1.801 E+00 \\
1.818 E+00 \\
1.833 E+00\end{array}$ & $\begin{array}{l}2.507 \mathrm{E}-01 \\
3.254 \mathrm{E}-01 \\
4.021 \mathrm{E}-01 \\
4.803 \mathrm{E}-01 \\
5.596 \mathrm{E}-01 \\
7.209 \mathrm{E}-01 \\
8.847 \mathrm{E}-01 \\
1.050 \mathrm{E}+00\end{array}$ & $\begin{array}{l}1.957 E+00 \\
2.056 E+00 \\
2.152 E+00 \\
2.246 E+00 \\
2.338 E+00 \\
2.522 E+00 \\
2.703 E+00 \\
2.883 E+00\end{array}$ & $\begin{array}{l}5.666 E+00 \\
6.912 E+00 \\
8.101 E+00 \\
9.238 E+00 \\
1.033 E+01 \\
1.239 E+01 \\
1.430 E+01 \\
1.609 E+01\end{array}$ & $\begin{array}{l}6.352 \mathrm{E}-02 \\
7.948 \mathrm{E}-02 \\
9.502 \mathrm{E}-02 \\
1.101 \mathrm{E}-01 \\
1.247 \mathrm{E}-01 \\
1.523 \mathrm{E}-01 \\
1.781 \mathrm{E}-01 \\
2.021 \mathrm{E}-01\end{array}$ & $\begin{array}{l}2.587 E+00 \\
2.921 E+00 \\
3.205 E+00 \\
3.452 E+00 \\
3.671 E+00 \\
4.047 E+00 \\
4.362 E+00 \\
4.635 E+00\end{array}$ & $\begin{array}{l}-0.022 \\
-0.019 \\
-0.017 \\
-0.015 \\
-0.014 \\
-0.012 \\
-0.010 \\
-0.009\end{array}$ & $\begin{array}{l}0.041 \\
0.036 \\
0.033 \\
0.031 \\
0.029 \\
0.025 \\
0.023 \\
0.021\end{array}$ & $\begin{array}{l}0.028 \\
0.025 \\
0.022 \\
0.019 \\
0.017 \\
0.015 \\
0.013 \\
0.011\end{array}$ \\
\hline $\begin{array}{l}40.0000 \\
45.0000 \\
50.0000 \\
55.0000 \\
60.0000 \\
70.00000 \\
80.0000 \\
90.0000\end{array}$ & $\begin{array}{l}1.845 E+00 \\
1.855 E+00 \\
1.865 E+00 \\
1.873 E+00 \\
1.880 E+00 \\
1.894 E+00 \\
1.905 E+00 \\
1.915 E+00\end{array}$ & $\begin{array}{l}1.21 \\
1.38 \\
1.55 \\
1.72 \\
1.89 \\
2.24 \\
2.59 \\
2.93\end{array}$ & $\begin{array}{l}E+00 \\
E+00 \\
E+00 \\
E+00 \\
E+00 \\
E+00 \\
E+00 \\
E+00\end{array}$ & $\begin{array}{l}E+01 \\
E+0 \\
E+0 \\
E+0 \\
E+01 \\
E+01 \\
E+01 \\
E+01\end{array}$ & $\begin{array}{l}2.4 \\
2.6 \\
2.8 \\
3.0 \\
3.3 \\
3.6 \\
3.8\end{array}$ & $\begin{array}{l}4.874 E+0 \\
5.089 E+0 \\
5.282 E+0 \\
5.459 E+0 \\
5.621 E+0 \\
5.911 E+0 \\
6.165 E+0 \\
6.391 E+0\end{array}$ & $\begin{array}{l}-0 . \\
-0 . \\
-0 \\
-0 . \\
-0 . \\
-0 \\
-0 . \\
-0 .\end{array}$ & & $\begin{array}{l}0.010 \\
0.009 \\
0.008 \\
0.007 \\
0.007 \\
0.006 \\
0.005 \\
0.004\end{array}$ \\
\hline $\begin{array}{l}0.0000 \\
5.0000 \\
0.0000 \\
5.0000 \\
0.0000 \\
0.0000 \\
0.0000\end{array}$ & $\begin{array}{l}1.923 E+00 \\
1.941 E+00 \\
1.956 E+00 \\
1.968 E+00 \\
1.978 E+00 \\
1.996 E+00 \\
2.010 E+00 \\
2.022 E+00\end{array}$ & $\begin{array}{l}3.28 \\
4.16 \\
5.05 \\
5.94 \\
6.83 \\
8.63 \\
1.04\end{array}$ & $\begin{array}{l}11 E+00 \\
13 E+00 \\
17 E+00 \\
63 E+01 \\
44 E+01 \\
26 E+01\end{array}$ & $\begin{array}{l}5 E+01 \\
6 E+01 \\
2 E+01 \\
1 E+01 \\
7 E+01 \\
1 E+01 \\
6 E+01\end{array}$ & $\begin{array}{l}4.5 \\
4.9 \\
5.3 \\
5.6 \\
6.0\end{array}$ & $\begin{array}{l}7.0 \\
7.3 \\
7.6 \\
7.9 \\
8.3 \\
8.7 \\
9.0\end{array}$ & $\begin{array}{l}-0 . \\
-0 \\
-0 \\
-0 \\
-0 \\
-0 \\
-0 \\
-0 .\end{array}$ & & $\begin{array}{l}0.004 \\
0.003 \\
0.002 \\
0.002 \\
0.002 \\
0.001 \\
0.001 \\
0.001\end{array}$ \\
\hline $\begin{array}{l}0.0000 \\
0.0000 \\
0.0000 \\
0.0000 \\
0.0000 \\
0.0000\end{array}$ & $\begin{array}{l}2.032 E+00 \\
2.041 E+00 \\
2.049 E+00 \\
2.057 E+00 \\
2.063 E+00 \\
2.075 E+00 \\
2.086 E+00 \\
2.095 E+00\end{array}$ & $\begin{array}{l}1.405 E+01 \\
1.586 E+01 \\
1.768 E+01 \\
1.950 E+01 \\
2.131 E+01 \\
2.496 E+01 \\
2.860 E+01 \\
3.225 E+01\end{array}$ & $\begin{array}{l}.608 E+01 \\
.790 E+01 \\
.973 E+01 \\
155 E+01 \\
338 E+01 \\
.703 E+01 \\
.069 E+01 \\
.434 E+01\end{array}$ & $\begin{array}{l}6.376 E+01 \\
6.670 E+01 \\
6.936 E+01 \\
7.179 E+01 \\
7.401 E+01 \\
7.799 E+01 \\
8.146 E+01 \\
8.454 E+01\end{array}$ & $\begin{array}{l}6.982 \mathrm{E}-01 \\
7.184 \mathrm{E}-01 \\
7.357 \mathrm{E}-01 \\
7.507 \mathrm{E}-01 \\
7.638 \mathrm{E}-01 \\
7.858 \mathrm{E}-01 \\
8.036 \mathrm{E}-01 \\
8.182 \mathrm{E}-01\end{array}$ & $\begin{array}{l}9.328 E+00 \\
9.563 E+00 \\
9.773 E+00 \\
9.963 E+00 \\
1.014 E+01 \\
1.044 E+01 \\
1.071 E+01 \\
1.095 E+01\end{array}$ & $\begin{array}{l}-0.0000 \\
-0.0000 \\
-0.000 \\
-0.000 \\
-0.000 \\
-0.000 \\
-0.000 \\
-0.000\end{array}$ & $\begin{array}{l}0.006 \\
0.006 \\
0.006 \\
0.006 \\
0.005 \\
0.005 \\
0.005 \\
0.005\end{array}$ & $\begin{array}{l}0.001 \\
0.001 \\
0.001 \\
0.001 \\
0.001 \\
0.000 \\
0.000 \\
0.000\end{array}$ \\
\hline 0.0000 & $2.103 E+00$ & $3.590 E+01$ & $3.800 \mathrm{E}+01$ & $8.730 E+01$ & $8.306 E-01$ & $1.116 E+01$ & -0.000 & 0.005 & 0.000 \\
\hline
\end{tabular}


ELECTRONS IN SODIUM IODIDE

$I=452.0 \mathrm{eV}$

\begin{tabular}{|c|c|c|c|c|c|c|c|c|c|}
\hline ENERGY & $\begin{array}{l}\text { COLLISION } \\
\mathrm{MeV} \mathrm{cm}^{2} / \mathrm{g}\end{array}$ & $\begin{array}{l}\text { ADIAIIVE } \\
\mathrm{eV} \mathrm{cm}^{2} / \mathrm{g}\end{array}$ & $\begin{array}{c}\text { TOTAL } \\
\mathrm{eV} \mathrm{cm}^{2} / \mathrm{g}\end{array}$ & $\begin{array}{l}\text { CSD } \\
\text { RAN } \\
g / C\end{array}$ & YIE & $\begin{array}{c}\text { DENS.EFF . } \\
\text { CORR. } \\
\text { (DELTA) }\end{array}$ & $\begin{array}{l}d(1 \\
\operatorname{col} L \\
\text { Loss }\end{array}$ & $\begin{array}{l}\text { SDA } \\
\text { ANGE }\end{array}$ & $\begin{array}{l}\text { I I ) } \\
\text { RAD } \\
\text { YIELD }\end{array}$ \\
\hline $\begin{array}{l}0.0100 \\
0.0125 \\
0.0150 \\
0.0175 \\
0.0200 \\
0.0250 \\
0.0300 \\
0.0350\end{array}$ & $\begin{array}{l}1.116 E+01 \\
9.603 E+00 \\
8.477 E+00 \\
7.622 E+00 \\
6.948 E+00 \\
5.949 E+00 \\
5.241 E+00 \\
4.711 E+00\end{array}$ & $\begin{array}{l}8 E-02 \\
2 E-02 \\
3 E-02 \\
8 E-02 \\
2 E-02 \\
7 E-02 \\
0 E-02 \\
0 E-02\end{array}$ & $\begin{array}{ll}0 & 1 \\
0 & 0 \\
0 & 0 \\
0 & 0 \\
0 & 0 \\
0 & 0 \\
0 & 0\end{array}$ & $\begin{array}{l}5.5 \\
8.0 \\
1.0 \\
1.3 \\
1.7 \\
2.5 \\
3.4\end{array}$ & $\begin{array}{l}.04 \\
.04 \\
.03 \\
.03 \\
-03 \\
-03 \\
03 \\
-03 \\
.03\end{array}$ & $\begin{array}{l}0.0 \\
0.0 \\
0.0 \\
0.0 \\
0.0 \\
0.0 \\
0.0\end{array}$ & $\begin{array}{l}-0 \\
-0 \\
-0 \\
-0 \\
-0 \\
-0\end{array}$ & $\begin{array}{l}16 \\
30 \\
55 \\
36 \\
20 \\
98 \\
31 \\
59\end{array}$ & $\begin{array}{l}0.382 \\
0.351 \\
0.330 \\
0.314 \\
0.301 \\
0.282 \\
0.268 \\
0.257\end{array}$ \\
\hline $\begin{array}{l}0.0400 \\
0.0450 \\
0.0500 \\
0.0550 \\
0.0600 \\
0.0700 \\
0.0800 \\
0.0900\end{array}$ & $\begin{array}{l}9 E+00 \\
8 E+00 \\
6 E+00 \\
9 E+00 \\
6 E+00 \\
5 E+00 \\
6 E+00 \\
6 E+00\end{array}$ & $\begin{array}{l}2 \cdot 2 \\
2 \cdot 2 \\
2 \cdot 3 \\
2 \cdot 3 \\
2 \cdot 4 \\
2 \cdot 5 \\
2\end{array}$ & $\begin{array}{l}+00 \\
+00 \\
+00 \\
+00 \\
+00 \\
+00 \\
+00 \\
+00\end{array}$ & $\begin{array}{l}6.7 \\
8.0 \\
9.4 \\
1.0 \\
1.4 \\
1.7\end{array}$ & $\begin{array}{l}03 \\
03 \\
03 \\
03 \\
03 \\
03 \\
03\end{array}$ & $\begin{array}{l}0 \\
0 \\
0 \\
0 \\
0 \\
0 \\
0 \\
0\end{array}$ & $\begin{array}{l}-0 \\
-0 \\
-0 \\
-0 \\
-0 \\
-0\end{array}$ & $\begin{array}{l}0 . \\
0 . \\
0 . \\
0 . \\
0 . \\
0 .\end{array}$ & $\begin{array}{l}49 \\
42 \\
36 \\
31 \\
26 \\
19 \\
13 \\
08\end{array}$ \\
\hline $\begin{array}{l}0.1000 \\
0.1250 \\
0.1500 \\
0.1750 \\
0.2000 \\
0.2500 \\
0.3000 \\
0.3500\end{array}$ & $\begin{array}{l}2.382 E+00 \\
2.098 E+00 \\
1.905 E+00 \\
1.766 E+00 \\
1.661 E+00 \\
1.516 E+00 \\
1.421 E+00 \\
1.355 E+00\end{array}$ & $\begin{array}{l}2.7 \\
2.8 \\
2.9 \\
3.0 \\
3.3 \\
3.5\end{array}$ & $\begin{array}{l}+00 \\
+00 \\
+00 \\
+00 \\
+00 \\
+00 \\
+00 \\
+00\end{array}$ & $\begin{array}{l}3.6 \\
4.8 \\
6.2 \\
7.6 \\
1.0 \\
1.4\end{array}$ & $\begin{array}{l}03 \\
03 \\
03 \\
03 \\
02 \\
02\end{array}$ & $\begin{array}{l}0.0 \\
0.0 \\
0.0 \\
0.0 \\
0.0 \\
0.0 \\
0.0 \\
1.129 E-03\end{array}$ & $\begin{array}{l}-0 \\
-0 \\
-0 \\
-0 \\
-0 \\
-0\end{array}$ & $\begin{array}{l}0 \\
0 \\
0 \\
0 \\
0 \\
0\end{array}$ & $\begin{array}{l}3 \\
95 \\
38 \\
33 \\
78 \\
71 \\
6\end{array}$ \\
\hline $\begin{array}{l}0.4000 \\
0.4500 \\
0.5000 \\
0.5500 \\
0.6000 \\
0.7000 \\
0.8000 \\
0.9000\end{array}$ & $\begin{array}{l}1.308 E+00 \\
1.273 E+00 \\
1.247 E+00 \\
1.227 E+00 \\
1.211 E+00 \\
1.190 E+00 \\
1.178 E+00 \\
1.170 E+00\end{array}$ & $\begin{array}{l}4.2 \\
4.4 \\
4.6 \\
4.9 \\
5.4 \\
6.0\end{array}$ & $\begin{array}{l}+00 \\
+00 \\
+00 \\
+00 \\
+00 \\
+00 \\
+00 \\
+00 \\
+00 \\
+00\end{array}$ & $\begin{array}{l}2.5 \\
2.8 \\
3.2 \\
3.6 \\
4.4 \\
5.2\end{array}$ & $\begin{array}{r}-02 \\
-02 \\
-02 \\
-02 \\
-02 \\
0 \\
0\end{array}$ & $\begin{array}{l}1 . \\
1 . \\
2 . \\
3 . \\
4 . \\
6 . \\
8 .\end{array}$ & $\begin{array}{l}-0 \\
-0 \\
-0 \\
-0 \\
-0 \\
-0\end{array}$ & & \\
\hline $\begin{array}{l}1.0000 \\
1.2500 \\
1.5000 \\
1.7500 \\
2.0000 \\
2.5000 \\
3.0000 \\
3.5000\end{array}$ & $\begin{array}{l}1.167 E+00 \\
1.167 E+00 \\
1.173 E+00 \\
1.182 E+00 \\
1.192 E+00 \\
1.212 E+00 \\
1.230 E+00 \\
1.247 E+00\end{array}$ & $\begin{array}{l}8.71 \\
1.03 \\
1.19 \\
1.36 \\
1.72 \\
2.09\end{array}$ & $\begin{array}{l}+00 \\
+00 \\
+00 \\
+00 \\
+00 \\
+00 \\
+00 \\
+00 \\
+00 \\
+00\end{array}$ & $\begin{array}{l}8.8 \\
1.0 \\
1.2 \\
1.4 \\
1.8 \\
2.1 \\
2.5\end{array}$ & $\begin{array}{l}02 \\
02 \\
02 \\
02 \\
02 \\
02 \\
02 \\
02\end{array}$ & $\begin{array}{l}1 . \\
2 . \\
2 . \\
3 . \\
4 . \\
5 . \\
6 .\end{array}$ & $\begin{array}{l}-0 \\
-0 \\
-0 \\
-0 \\
-0 \\
-0 \\
-0\end{array}$ & & $\begin{array}{l}1109 \\
109 \\
103 \\
098 \\
089 \\
082 \\
076\end{array}$ \\
\hline $\begin{array}{l}4.0000 \\
4.5000 \\
5.0000 \\
5.5000 \\
6.0000 \\
7.0000 \\
8.0000 \\
9.0000\end{array}$ & $\begin{array}{l}1.263 E+00 \\
1.277 E+00 \\
1.289 E+00 \\
1.301 E+00 \\
1.311 E+00 \\
1.330 E+00 \\
1.347 E+00 \\
1.361 E+00\end{array}$ & $\begin{array}{l}3.2 \\
3.6 \\
4.0 \\
4.4 \\
5.2 \\
6.1\end{array}$ & $\begin{array}{l}1.548 E+00 \\
1.601 E+00 \\
1.654 E+00 \\
1.706 E+00 \\
1.758 E+00 \\
1.860 E+00 \\
1.961 E+00 \\
2.062 E+00\end{array}$ & $\begin{array}{l}3.182 E+00 \\
3.489 E+00 \\
3.787 E+00 \\
4.075 E+00 \\
4.628 E+00 \\
5.152 E+00 \\
5.649 E+00\end{array}$ & 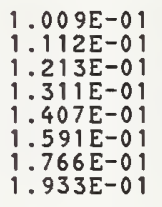 & $\begin{array}{l}7.184 E-0 \\
8.057 E-0 \\
8.881 E-0 \\
9.660 E-0 \\
1.040 E+0 \\
1.177 E+0 \\
1.301 E+0 \\
1.416 E+0\end{array}$ & $\begin{array}{l}-0 . \\
-0 . \\
-0 . \\
-0 . \\
-0 . \\
-0 . \\
-0 .\end{array}$ & & $\begin{array}{l}7 \\
1 \\
1 \\
8 \\
3 \\
9 \\
6\end{array}$ \\
\hline $\begin{array}{l}0.0000 \\
2.5000 \\
5.0000 \\
.5000 \\
0.0000 \\
5.0000 \\
0.0000 \\
5.0000\end{array}$ & $\begin{array}{l}374 E+00 \\
401 E+00 \\
423 E+00 \\
441 E+00 \\
456 E+00 \\
480 E+00 \\
499 E+00 \\
514 E+00\end{array}$ & $\begin{array}{l}1.00 \\
1.23 \\
1.46 \\
1.69 \\
2.16 \\
2.648 \\
3.13\end{array}$ & $\begin{array}{l}+00 \\
+00 \\
+00 \\
+00 \\
+00 \\
+00 \\
+00 \\
+00\end{array}$ & $\begin{array}{l}7.2 \\
8.2 \\
9.1 \\
9.9 \\
1.1 \\
1.2 \\
1.3\end{array}$ & & $\begin{array}{l}1 . \\
1 . \\
1 . \\
2 . \\
2 . \\
2 .\end{array}$ & $\begin{array}{l}-0 . \\
-0 . \\
-0 . \\
-0 . \\
-0 . \\
-0 . \\
-0 .\end{array}$ & & $\begin{array}{l}38 \\
34 \\
30 \\
28 \\
23 \\
20 \\
18\end{array}$ \\
\hline $\begin{array}{l}00 \\
00 \\
00 \\
00 \\
00\end{array}$ & $\begin{array}{l}9 E+00 \\
8 E+00 \\
7 E+00 \\
5 E+00 \\
9 E+00 \\
0 E+00 \\
0 E+00\end{array}$ & $\begin{array}{l}4.11 \\
4.60 \\
5.10 \\
5.60 \\
6.6\end{array}$ & $\begin{array}{l}+00 \\
+00 \\
+00 \\
+00 \\
+00 \\
+00 \\
+00 \\
+01\end{array}$ & $\begin{array}{l}1.5 \\
1.6 \\
1.7\end{array}$ & $\begin{array}{c}1 \\
1 \\
1 \\
1 \\
1\end{array}$ & & $\begin{array}{l}-0 . \\
-0 . \\
-0 . \\
-0 . \\
-0 . \\
-0 .\end{array}$ & & \\
\hline 0 & $\begin{array}{l}609 E+00 \\
627 E+00 \\
641 E+00 \\
653 E+00 \\
663 E+00 \\
680 E+00 \\
693 E+00\end{array}$ & $\begin{array}{l}1 . \\
1 . \\
1 . \\
1 . \\
2 . \\
3 . \\
3 .\end{array}$ & $\begin{array}{l}7 E+01 \\
5 E+01 \\
4 E+01 \\
3 E+01 \\
3 E+01 \\
4 E+01 \\
7 E+01 \\
0 E+01\end{array}$ & $\begin{array}{l}2 \\
2 \\
2 \\
2 \\
3\end{array}$ & 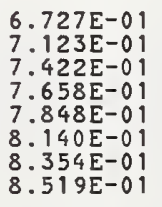 & & $\begin{array}{l}-0 . \\
-0 . \\
-0 . \\
-0 . \\
-0 . \\
-0 .\end{array}$ & & $\begin{array}{l}05 \\
04 \\
04 \\
04 \\
03 \\
03 \\
02\end{array}$ \\
\hline $\begin{array}{l}00 \\
00 \\
00\end{array}$ & $\begin{array}{l}722 E+00 \\
729 E+00 \\
736 E+00 \\
742 E+00 \\
753 E+00 \\
762 E+00 \\
770 E+00\end{array}$ & $\begin{array}{l}4.60 \\
5.13 \\
5.65 \\
6.17 \\
7.22 \\
8.28 \\
9.33\end{array}$ & $\begin{array}{l}01 \\
01 \\
01 \\
01 \\
01 \\
01 \\
01 \\
01 \\
01\end{array}$ & $\begin{array}{l}1 \\
1 \\
1 \\
1 \\
1\end{array}$ & $\begin{array}{l}1 \\
1 \\
1 \\
1 \\
1 \\
1\end{array}$ & $\begin{array}{l}7 . \\
7 . \\
7 . \\
8 . \\
8 .\end{array}$ & $\begin{array}{l}3 \\
3 \\
2 \\
2 \\
2 \\
2 \\
1\end{array}$ & & $\begin{array}{ll}02 \\
01 \\
01 \\
01 \\
01 \\
01 \\
01 \\
01\end{array}$ \\
\hline & $.777 E+00$ & .03 & $56 E+02$ & $96 E+0$ & 0 & $22 \mathrm{E}+00$ & 0.001 & 12 & 0.00 \\
\hline
\end{tabular}


ELECTRONS IN STILBENE

$I=67.7 \cdot V \quad$ DENSITY $=9.707 \mathrm{E}-01 \mathrm{~g} / \mathrm{cm}^{3}$

\begin{tabular}{|c|c|c|c|c|c|c|c|c|c|}
\hline \multirow{2}{*}{$\begin{array}{l}\text { ENERGY } \\
\text { MoV }\end{array}$} & \multicolumn{2}{|c|}{$\begin{array}{l}\text { STOPPING POWER } \\
\text { COLLISION RADIATIVE }\end{array}$} & TOTAL & \multirow{2}{*}{$\begin{array}{l}\text { CSDA } \\
\text { RANGE } \\
g / C \mathrm{C}^{2}\end{array}$} & \multirow[t]{2}{*}{$\begin{array}{l}\text { RADIATIOH } \\
\text { YIELD }\end{array}$} & \multirow[t]{2}{*}{$\begin{array}{l}\text { DENS. EFF } \\
\text { CORR. } \\
\text { (DELTA) }\end{array}$} & \multirow[t]{2}{*}{$\begin{array}{l}\text { d( } 100 \\
\text { coll } \\
\text { LOSS }\end{array}$} & \multirow[t]{2}{*}{$\begin{array}{l}\text { J/d ( } 1 \\
\text { CSDA } \\
\text { RANGE }\end{array}$} & \multirow[t]{2}{*}{$\begin{array}{l}\text { gI I } \\
\text { RAD } \\
\text { YIELD }\end{array}$} \\
\hline & $\mathrm{MeV} \mathrm{cm}^{2} / \mathrm{g}$ & $\mathrm{MeV} \mathrm{cm}^{2} / \mathrm{g}$ & MeV $\subset \mathrm{m}^{2} / g$ & & & & & & \\
\hline $\begin{array}{l}0.01100 \\
0.0125 \\
0.0150 \\
0.0175 \\
0.0200 \\
0.0250 \\
0.0300\end{array}$ & $\begin{array}{l}1.856 \\
1.610 \\
1.428 \\
1.287 \\
1.083 \\
9.418\end{array}$ & $\begin{array}{l}3.0 \\
3.0 \\
3.0 \\
3.0 \\
3.0 \\
3.0\end{array}$ & $\begin{array}{l}1.8 \\
1.6 \\
1.4 \\
1.2 \\
1.0 \\
9.4 \\
8.3\end{array}$ & $\begin{array}{l}2.5611 \\
3.801 \\
5.251 \\
6.904 \\
8.751 \\
1.300 \\
1.797\end{array}$ & $\begin{array}{l}E-05 \\
E-05 \\
E-04 \\
E-04 \\
E-04 \\
E-04 \\
E-04 \\
E-04\end{array}$ & $\begin{array}{l}0 \\
0 \\
0 \\
0 \\
0 \\
0 \\
0 \\
0\end{array}$ & $\begin{array}{l}0.195 \\
0.187 \\
0.181 \\
0.176 \\
0.172 \\
0.166 \\
0.161 \\
0.157\end{array}$ & $\begin{array}{l}2 \\
2 \\
4 \\
8 \\
3 \\
5 \\
9 \\
4\end{array}$ & $\begin{array}{l}0.221 \\
0.211 \\
0.203 \\
0.197 \\
0.192\end{array}$ \\
\hline $\begin{array}{l}0.0400 \\
0.0450 \\
0.0500 \\
0.0550 \\
0.0600 \\
0.0700 \\
0.0800 \\
0.0900\end{array}$ & $\begin{array}{l}6.94 \\
6.43 \\
6.00 \\
5.64 \\
5.07 \\
4.63 \\
4.78\end{array}$ & $\begin{array}{l}3.0 \\
3.0 \\
3.1 \\
3.1 \\
3.1 \\
3.1\end{array}$ & $\begin{array}{l}0 \\
0 \\
0 \\
0 \\
0 \\
0 \\
0 \\
0\end{array}$ & $\begin{array}{l}3.6 \\
4.8 \\
5.2 \\
6.8 \\
7.8 \\
1.8\end{array}$ & $\begin{array}{l}04 \\
04 \\
04 \\
04 \\
04 \\
04 \\
04 \\
04\end{array}$ & $\begin{array}{l}0 \\
0 \\
0 \\
0 \\
0 \\
0 \\
0 \\
0\end{array}$ & $\begin{array}{l}-0 \\
-0 \\
-0 \\
-0 \\
-0 \\
-0 \\
-0\end{array}$ & & \\
\hline $\begin{array}{l}0.1000 \\
0.1250 \\
0.1500 \\
0.1750 \\
0.2000 \\
0.2500 \\
0.3000 \\
0.3500\end{array}$ & $\begin{array}{l}3.493 \\
3.148 \\
2.90 \\
2.715 \\
2.456 \\
2.287\end{array}$ & $\begin{array}{l}3.3 \\
3.4 \\
3.6 \\
3.7 \\
4.0 \\
4.3\end{array}$ & $\begin{array}{ll}0 & 0 \\
0 & 0 \\
0 & 0 \\
0 & 0 \\
0 & 0 \\
0 & 0 \\
0 & 0 \\
0 & 0\end{array}$ & $\begin{array}{l}2 . \\
2 . \\
3 . \\
4 . \\
6 . \\
8 .\end{array}$ & $\begin{array}{l}04 \\
04 \\
04 \\
04 \\
04 \\
04 \\
03 \\
03\end{array}$ & $\begin{array}{l}0.0 \\
0.0 \\
0.0 \\
0.0 \\
0.0 \\
0.0 \\
0.0 \\
0.0\end{array}$ & $\begin{array}{l}-0 \\
-0 \\
-0 \\
-0 \\
-0 \\
-0 \\
-0\end{array}$ & & \\
\hline $\begin{array}{l}0.4000 \\
0.4500 \\
0.5000 \\
0.5500 \\
0.6000 \\
0.7000 \\
0.8000 \\
0.9000\end{array}$ & $\begin{array}{l}2.085 E+00 \\
2.020 E+00 \\
1.969 E+00 \\
1.930 E+00 \\
1.898 E+00 \\
1.851 E+00 \\
1.819 E+00 \\
1.797 E+00\end{array}$ & $\begin{array}{l}5 \cdot 3 \\
5.7 \\
6.1 \\
6.5 \\
7.3 \\
8.2\end{array}$ & $\begin{array}{ll}0 & 0 \\
0 & 0 \\
0 & 0 \\
0 & 0 \\
0 & 0 \\
0 & 0 \\
0 & 0 \\
0 & 0\end{array}$ & $\begin{array}{l}1 . \\
1 . \\
2 . \\
2 . \\
2 . \\
3 .\end{array}$ & $\begin{array}{l}03 \\
03 \\
03 \\
03 \\
03 \\
03 \\
03 \\
03\end{array}$ & $\begin{array}{l}0.0 \\
1.936 \mathrm{E}-02 \\
4.453 \mathrm{E}-02 \\
7.225 \mathrm{E}-02 \\
1.019 \mathrm{E}-01 \\
1.648 \mathrm{E}-01 \\
2.305 \mathrm{E}-01 \\
2.971 \mathrm{E}-01\end{array}$ & $\begin{array}{l}-0 \\
-0 \\
-0 \\
-0 \\
-0 \\
-0 \\
-0\end{array}$ & & $\begin{array}{l}0 \\
0 \\
0 \\
0\end{array}$ \\
\hline $\begin{array}{l}1.0000 \\
1.2500 \\
1.5000 \\
1.7500 \\
2.0000 \\
2.5000 \\
3.0000 \\
3.5000\end{array}$ & $\begin{array}{l}1.782 E+00 \\
1.761 E+00 \\
1.754 E+00 \\
1.753 E+00 \\
1.756 E+00 \\
1.767 E+00 \\
1.780 E+00\end{array}$ & $\begin{array}{l}1.017 \\
1.274 \\
1.550 \\
1.840 \\
2.143 \\
2.780 \\
3.452\end{array}$ & $\begin{array}{l}2 E+00 \\
4 E+00 \\
0 E+00 \\
2 E+00 \\
8 E+00 \\
5 E+00 \\
4 E+00 \\
4 E+00\end{array}$ & $\begin{array}{l}5 . \\
7 . \\
8 . \\
1 . \\
1 .\end{array}$ & $\begin{array}{l}03 \\
03 \\
03 \\
03 \\
03 \\
03 \\
03 \\
02\end{array}$ & $\begin{array}{l}3 . \\
5 . \\
6 . \\
8 . \\
9 . \\
1 . \\
1 .\end{array}$ & $\begin{array}{l}-0 \\
-0 \\
-0 \\
-0 \\
-0 \\
-0 .\end{array}$ & & \\
\hline $\begin{array}{l}4.0000 \\
4.5000 \\
5.0000 \\
5.5000 \\
6.0000 \\
7.0000 \\
8.0000 \\
9.0000\end{array}$ & $\begin{array}{l}1.81 \\
1.82 \\
1.83 \\
1.84 \\
1.86 \\
1.87 \\
1.89\end{array}$ & $\begin{array}{l}5.6 \\
6.38 \\
7.1 \\
7.9 \\
9.5 \\
1.1 \\
1.2\end{array}$ & $\begin{array}{l}00 \\
00 \\
00 \\
000 \\
000 \\
00 \\
00 \\
00\end{array}$ & $\begin{array}{l}2 . \\
2 . \\
2 . \\
3 . \\
3 . \\
4 .\end{array}$ & $\begin{array}{l}02 \\
02 \\
02 \\
02 \\
02 \\
02 \\
02 \\
02 \\
02\end{array}$ & & $\begin{array}{l}-0 \\
-0 \\
-0 \\
-0 \\
-0 \\
-0 \\
-0\end{array}$ & & \\
\hline $\begin{array}{l}10.0000 \\
12.5000 \\
15.0000 \\
17.5000 \\
20.0000 \\
25.0000 \\
30.0000 \\
35.0000\end{array}$ & $\begin{array}{l}1.904 \mathrm{E}+ \\
1.929 \mathrm{E}+ \\
1.948 \mathrm{E}+ \\
1.964 \mathrm{E}+ \\
1.978 \mathrm{E}+ \\
1.999 \mathrm{E}+ \\
2.016 \mathrm{E}+\end{array}$ & $\begin{array}{l}1.9 \\
2.3 \\
2.8 \\
3.3 \\
4.3 \\
5.2\end{array}$ & $\begin{array}{l}2 . \\
2 . \\
2 . \\
2 . \\
2 . \\
2 . \\
2 .\end{array}$ & $\begin{array}{l}6 . \\
7 . \\
8 . \\
9 . \\
1 . \\
1 .\end{array}$ & $\begin{array}{l}02 \\
02 \\
02 \\
02 \\
02 \\
02 \\
02 \\
01 \\
01\end{array}$ & $\begin{array}{l}3 . \\
3 . \\
3 . \\
3 . \\
4 . \\
4 .\end{array}$ & $\begin{array}{l}-0 \\
-0 \\
-0 \\
-0 \\
-0 \\
-0 \\
-0\end{array}$ & & $\begin{array}{l}0 . \\
0 . \\
0 . \\
0 . \\
0 . \\
0 . \\
0 .\end{array}$ \\
\hline $\begin{array}{l}40.0000 \\
45.0000 \\
50.0000 \\
55.0000 \\
60.0000 \\
70.0000 \\
80.0000\end{array}$ & $\begin{array}{l}2.05 \\
2.06 \\
2.06 \\
2.0 \\
2.0 \\
2.10\end{array}$ & $\begin{array}{l}8.3 \\
9.3 \\
1.0 \\
1.1 \\
1.3 \\
1.5\end{array}$ & $\begin{array}{l}00 \\
00 \\
00 \\
00 \\
00 \\
00 \\
00 \\
00 \\
00\end{array}$ & & a & & & & \\
\hline $\begin{array}{l}.0000 \\
.0000 \\
.0000 \\
.0000 \\
.0000\end{array}$ & $\begin{array}{l}2.15 \\
2.11 \\
2.11\end{array}$ & $\begin{array}{l}2 \\
3 \\
3 \\
4 \\
5\end{array}$ & $\begin{array}{l}+00 \\
+00 \\
+00 \\
+00 \\
+00 \\
+00 \\
+00 \\
+00\end{array}$ & & & & & & \\
\hline $\begin{array}{l}0.0000 \\
0.0000 \\
0.00000 \\
0.00000 \\
0.0000 \\
0.0000\end{array}$ & $\begin{array}{l}2.244 \\
2.252 \\
2.260 \\
2.267 \\
2.280 \\
2.291\end{array}$ & $\begin{array}{l}1 \\
1 \\
1 \\
1 \\
1\end{array}$ & $\begin{array}{l}01 \\
01 \\
01 \\
01 \\
01 \\
01 \\
01 \\
01 \\
01 \\
0 \\
1\end{array}$ & 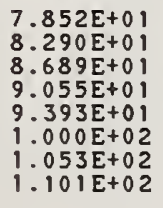 & $\begin{array}{ll}0 & 1 \\
0 & 1 \\
0 & 1 \\
01 \\
01 \\
01 \\
01 \\
01 \\
0 & 1\end{array}$ & 9 & $\begin{array}{l}0 \\
0 \\
0 \\
0 \\
0 \\
0\end{array}$ & & 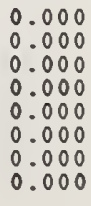 \\
\hline .0000 & $.309 E+00$ & $.211 E+01$ & $442 E+01$ & $144 E+02$ & $.530 E-01$ & $1.179 E+01$ & -0.000 & 00 & 0.000 \\
\hline
\end{tabular}




\begin{tabular}{|c|c|c|c|c|c|c|c|c|c|}
\hline ENERGY & $\begin{array}{l}\text { COLLISION } \\
\text { MeV } \mathrm{cm}^{2} / \mathrm{g}\end{array}$ & 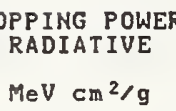 & 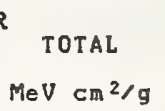 & $\begin{array}{l}\text { CSDA } \\
\text { RANGE } \\
\mathrm{g} / \mathrm{Cm}^{2}\end{array}$ & $\begin{array}{l}\text { RADIATION } \\
\text { YIELD }\end{array}$ & $\begin{array}{l}\text { DENS.EFF. } \\
\text { CORR . } \\
\text { (DELTA) }\end{array}$ & $\begin{array}{l}d(1 \\
\text { colL } \\
\text { Loss }\end{array}$ & $\begin{array}{l}\text { CSDA } \\
\text { RANGE }\end{array}$ & $\begin{array}{l}\text { gI ) } \\
\text { RAD } \\
\text { YIELD }\end{array}$ \\
\hline $\begin{array}{l}0.0100 \\
0.0125 \\
0.0150 \\
0.0175 \\
0.0200 \\
0.0250 \\
0.0300 \\
0.0350\end{array}$ & $\begin{array}{l}2.325 E+01 \\
1.952 E+01 \\
1.692 E+01 \\
1.500 E+01 \\
1.352 E+01 \\
1.137 E+01 \\
9.882 E+00 \\
8.789 E+00\end{array}$ & $\begin{array}{l}3.402 \mathrm{E}-03 \\
3.421 \mathrm{E}-03 \\
3.433 \mathrm{E}-03 \\
3.441 \mathrm{E}-03 \\
3.447 \mathrm{E}-03 \\
3.457 \mathrm{E}-03 \\
3.467 \mathrm{E}-03 \\
3.477 \mathrm{E}-03\end{array}$ & $\begin{array}{l}2.326 E+01 \\
1.953 E+01 \\
1.693 E+01 \\
1.500 E+01 \\
1.352 E+01 \\
1.137 E+01 \\
9.885 E+00 \\
8.792 E+00\end{array}$ & $\begin{array}{l}2.426 \mathrm{E}-04 \\
3.605 \mathrm{E}-04 \\
4.984 \mathrm{E}-04 \\
6.556 \mathrm{E}-04 \\
8.314 \mathrm{E}-04 \\
1.237 \mathrm{E}-03 \\
1.710 \mathrm{E}-03 \\
2.247 \mathrm{E}-03\end{array}$ & $\begin{array}{l}7.98 \\
9.60 \\
1.11 \\
1.26 \\
1.4 \\
1.68 \\
1.98 \\
2.2\end{array}$ & $\begin{array}{l}0.0 \\
0.0 \\
0.0 \\
0.0 \\
0.0 \\
0.0 \\
0.0 \\
0.0\end{array}$ & $\begin{array}{l}-0 . \\
-0 . \\
-0 \\
-0 \\
-0 \\
-0 \\
-0 \\
-0 .\end{array}$ & $\begin{array}{l}0.217 \\
0.207 \\
0.200 \\
0.194 \\
0.189 \\
0.181 \\
0.176 \\
0.171\end{array}$ & $\begin{array}{l}0.215 \\
0.206 \\
0.199 \\
0.193 \\
0.188 \\
0.181 \\
0.175 \\
0.171\end{array}$ \\
\hline $\begin{array}{l}0.0400 \\
0.0450 \\
0.0500 \\
0.0550 \\
0.0600 \\
0.0700 \\
0.0800 \\
0.0900\end{array}$ & $\begin{array}{l}7.950 E+00 \\
7.285 E+00 \\
6.743 E+00 \\
6.294 E+00 \\
5.915 E+00 \\
5.310 E+00 \\
4.849 E+00 \\
4.485 E+00\end{array}$ & $\begin{array}{l}3.488 \mathrm{E}-03 \\
3.501 \mathrm{E}-03 \\
3.514 \mathrm{E}-03 \\
3.529 \mathrm{E}-03 \\
3.544 \mathrm{E}-03 \\
3.578 \mathrm{E}-03 \\
3.614 \mathrm{E}-03 \\
3.654 \mathrm{E}-03\end{array}$ & $\begin{array}{l}7.953 E+00 \\
7.288 E+00 \\
6.747 E+00 \\
6.298 E+00 \\
5.919 E+00 \\
5.314 E+00 \\
4.853 E+00 \\
4.489 E+00\end{array}$ & $\begin{array}{l}2.846 E-03 \\
3.504 E-03 \\
4.217 E-03 \\
4.985 E-03 \\
5.805 E-03 \\
7.591 E-03 \\
9.564 E-03 \\
1.171 E-02\end{array}$ & $\begin{array}{l}2.452 \mathrm{E}-04 \\
2.690 \mathrm{E}-04 \\
2.922 \mathrm{E}-04 \\
3.148 \mathrm{E}-04 \\
3.368 \mathrm{E}-04 \\
3.796 \mathrm{E}-04 \\
4.208 \mathrm{E}-04 \\
4.607 \mathrm{E}-04\end{array}$ & $\begin{array}{l}0.0 \\
0.0 \\
0.0 \\
0.0 \\
0.0 \\
0.0 \\
0.0 \\
0.0\end{array}$ & $\begin{array}{l}-0.152 \\
-0.149 \\
-0.147 \\
-0.145 \\
-0.143 \\
-0.141 \\
-0.138 \\
-0.136\end{array}$ & $\begin{array}{l}0.167 \\
0.164 \\
0.161 \\
0.159 \\
0.157 \\
0.153 \\
0.150 \\
0.148\end{array}$ & $\begin{array}{l}0.167 \\
0.164 \\
0.161 \\
0.159 \\
0.157 \\
0.153 \\
0.150 \\
0.148\end{array}$ \\
\hline $\begin{array}{l}0.1000 \\
0.1250 \\
0.1500 \\
0.1750 \\
0.2000 \\
0.2500 \\
0.3000 \\
0.3500\end{array}$ & $\begin{array}{l}4.191 \mathrm{E}+00 \\
3.655 \mathrm{E}+00 \\
3.293 \mathrm{E}+00 \\
3.033 \mathrm{E}+00 \\
2.838 \mathrm{E}+00 \\
2.567 \mathrm{E}+00 \\
2.390 \mathrm{E}+00 \\
2.267 \mathrm{E}+00\end{array}$ & $\begin{array}{l}3.696 \\
3.812 \\
3.937 \\
4.071 \\
4.213 \\
4.518 \\
4.851\end{array}$ & $\begin{array}{l}4.195 E+00 \\
3.659 E+00 \\
3.297 E+00 \\
3.037 E+00 \\
2.842 E+00 \\
2.572 E+00 \\
2.395 E+00 \\
2.272 E+00\end{array}$ & $\begin{array}{l}1.40 \\
2.04 \\
2.76 \\
3.55 \\
4.40 \\
6.26 \\
8.28\end{array}$ & $\begin{array}{l}4.9 \\
5.9 \\
6.7 \\
7.6 \\
8.4 \\
9.9 \\
1.9\end{array}$ & $\begin{array}{l}0.0 \\
0.0 \\
0.0 \\
0.0 \\
0.0 \\
0.0 \\
0.0 \\
0.0\end{array}$ & $\begin{array}{l}-0.134 \\
-0.130 \\
-0.128 \\
-0.125 \\
-0.123 \\
-0.120 \\
-0.117 \\
-0.115\end{array}$ & $\begin{array}{l}0.146 \\
0.142 \\
0.138 \\
0.136 \\
0.133 \\
0.130 \\
0.127 \\
0.125\end{array}$ & $\begin{array}{l}0.146 \\
0.141 \\
0.138 \\
0.135 \\
0.133 \\
0.129 \\
0.126 \\
0.124\end{array}$ \\
\hline $\begin{array}{l}0.4000 \\
0.4500 \\
0.5000 \\
0.5500 \\
0.6000 \\
0.7000 \\
0.8000 \\
0.9000\end{array}$ & $\begin{array}{l}2.178 E+00 \\
2.112 E+00 \\
2.061 E+00 \\
2.022 E+00 \\
1.992 E+00 \\
1.949 E+00 \\
1.921 E+00 \\
1.904 E+00\end{array}$ & $\begin{array}{l}5.58 \\
5.98 \\
6.40 \\
6.82 \\
7.25 \\
8.25 \\
9.2 \\
1.02\end{array}$ & $\begin{array}{l}2.184 E+00 \\
2.118 E+00 \\
2.068 E+00 \\
2.029 E+00 \\
1.999 E+00 \\
1.957 E+00 \\
1.931 E+00 \\
1.914 E+00\end{array}$ & $\begin{array}{l}1.268 \\
1.500 \\
1.739 \\
1.983 \\
2.232 \\
2.738 \\
3.253 \\
3.773\end{array}$ & $\begin{array}{l}1.4 \\
1.5 \\
1.7 \\
1.8 \\
1.9 \\
2.2 \\
2.5 \\
2.8\end{array}$ & $\begin{array}{l}0.0 \\
0.0 \\
0.0 \\
0.0 \\
0.0 \\
0.0 \\
0.0 \\
0.0\end{array}$ & $\begin{array}{l}-0 . \\
-0 . \\
-0 \\
-0 \\
-0 \\
-0 \\
-0 \\
-0 .\end{array}$ & $\begin{array}{l}0.123 \\
0.121 \\
0.120 \\
0.1118 \\
0.117 \\
0.1115 \\
0.1113 \\
0.1112\end{array}$ & $\begin{array}{l}0.122 \\
0.120 \\
0.1118 \\
0.117 \\
0.115 \\
0.113 \\
0.111 \\
0.109\end{array}$ \\
\hline $\begin{array}{l}1.0000 \\
1.2500 \\
1.5000 \\
1.7500 \\
2.0000 \\
2.5000 \\
3.0000 \\
3.5000\end{array}$ & $\begin{array}{l}1.894 E+00 \\
1.886 E+00 \\
1.890 E+00 \\
1.901 E+00 \\
1.915 E+00 \\
1.945 E+00 \\
1.975 E+00 \\
2.003 E+00\end{array}$ & $\begin{array}{l}1.135 \mathrm{E}-02 \\
1.421 \mathrm{E}-02 \\
1.727 \mathrm{E}-02 \\
2.049 \mathrm{E}-02 \\
2.385 \mathrm{E}-02 \\
3.091 \mathrm{E}-02 \\
3.835 \mathrm{E}-02 \\
4.610 \mathrm{E}-02\end{array}$ & $\begin{array}{l}1.905 E+00 \\
1.900 E+00 \\
1.908 E+00 \\
1.922 E+00 \\
1.939 E+00 \\
1.976 E+00 \\
2.013 E+00 \\
2.049 E+00\end{array}$ & $\begin{array}{l}4.29 \\
5.61 \\
6.92 \\
8.23 \\
9.52 \\
1.20 \\
1.45 \\
1.70\end{array}$ & $\begin{array}{l}3.11 \\
3.83 \\
4.56 \\
5.32 \\
6.09 \\
7.66 \\
9.28 \\
1.09\end{array}$ & $\begin{array}{l}0.0 \\
0.0 \\
0.0 \\
0.0 \\
0.0 \\
0.0 \\
0.0 \\
0.0\end{array}$ & $\begin{array}{l}-0.101 \\
-0.098 \\
-0.096 \\
-0.094 \\
-0.092 \\
-0.089 \\
-0.087 \\
-0.086\end{array}$ & $\begin{array}{l}0.111 \\
0.108 \\
0.105 \\
0.104 \\
0.102 \\
0.099 \\
0.097 \\
0.095\end{array}$ & $\begin{array}{l}0.108 \\
0.105 \\
0.102 \\
0.100 \\
0.098 \\
0.095 \\
0.092 \\
0.090\end{array}$ \\
\hline $\begin{array}{l}4.0000 \\
4.5000 \\
5.0000 \\
5.5000 \\
6.0000 \\
7.0000 \\
8.0000 \\
9.0000\end{array}$ & $\begin{array}{l}2.030 \mathrm{E}+00 \\
2.054 \mathrm{E}+00 \\
2.077 \mathrm{E}+00 \\
2.098 \mathrm{E}+00 \\
2.117 \mathrm{E}+00 \\
2.153 \mathrm{E}+00 \\
2.184 \mathrm{E}+00 \\
2.212 \mathrm{E}+00\end{array}$ & $\begin{array}{l}5.411 \mathrm{E}-02 \\
6.234 \mathrm{E}-02 \\
7.077 \mathrm{E}-02 \\
7.938 \mathrm{E}-02 \\
8.815 \mathrm{E}-02 \\
1.061 \mathrm{E}-01 \\
1.246 \mathrm{E}-01 \\
1.434 \mathrm{E}-01\end{array}$ & $\begin{array}{l}2.084 E+00 \\
2.116 E+00 \\
2.147 E+00 \\
2.177 E+00 \\
2.205 E+00 \\
2.259 E+00 \\
2.308 E+00 \\
2.355 E+00\end{array}$ & $\begin{array}{l}1.94 \\
2.18 \\
2.42 \\
2.65 \\
2.87 \\
3.32 \\
3.76 \\
4.19\end{array}$ & $\begin{array}{l}1.25 \\
1.42 \\
1.59 \\
1.76 \\
1.93 \\
2.28 \\
2.62 \\
2.97\end{array}$ & $\begin{array}{l}0.0 \\
0.0 \\
0.0 \\
0.0 \\
0.0 \\
0.0 \\
0.0 \\
0.0\end{array}$ & $\begin{array}{l}-0.084 \\
-0.083 \\
-0.082 \\
-0.081 \\
-0.080 \\
-0.079 \\
-0.078 \\
-0.077\end{array}$ & $\begin{array}{l}0.094 \\
0.093 \\
0.091 \\
0.090 \\
0.089 \\
0.087 \\
0.086 \\
0.085\end{array}$ & $\begin{array}{l}0.089 \\
0.087 \\
0.086 \\
0.084 \\
0.083 \\
0.081 \\
0.080 \\
0.078\end{array}$ \\
\hline $\begin{array}{l}10.0000 \\
12.5000 \\
15.0000 \\
17.5000 \\
20.00000 \\
25.0000 \\
30.0000 \\
35.0000\end{array}$ & $\begin{array}{l}2.237 E+00 \\
2.292 E+00 \\
2.337 E+00 \\
2.375 E+00 \\
2.408 E+00 \\
2.462 E+00 \\
2.501 E+00 \\
2.530 E+00\end{array}$ & $\begin{array}{l}2.119 \mathrm{E}-01 \\
2.626 \mathrm{E}-01 \\
3.144 \mathrm{E}-01 \\
3.671 \mathrm{E}-01 \\
4.743 \mathrm{E}-01 \\
5.835 \mathrm{E}-01 \\
6.942 \mathrm{E}-01\end{array}$ & $\begin{array}{l}2.400 E+00 \\
2.504 E+00 \\
2.599 E+00 \\
2.689 E+00 \\
2.775 E+00 \\
2.937 E+00 \\
3.085 E+00 \\
3.225 E+00\end{array}$ & $\begin{array}{l}4.614 E+00 \\
5.634 E+00 \\
6.613 E+00 \\
7.559 E+00 \\
8.474 E+00 \\
1.022 E+01 \\
1.188 E+01 \\
1.347 E+01\end{array}$ & $\begin{array}{l}3.320 \mathrm{E}-02 \\
4.180 \mathrm{E}-02 \\
5.032 \mathrm{E}-02 \\
5.870 \mathrm{E}-02 \\
6.694 \mathrm{E}-02 \\
8.296 \mathrm{E}-02 \\
9.838 \mathrm{E}-02 \\
1.132 \mathrm{E}-01\end{array}$ & $\begin{array}{l}0.0 \\
0.0 \\
0.0 \\
0.0 \\
0.0 \\
1.908 \mathrm{E}-02 \\
1.033 \mathrm{E}-01 \\
2.153 \mathrm{E}-01\end{array}$ & $\begin{array}{l}-0.076 \\
-0.074 \\
-0.072 \\
-0.071 \\
-0.070 \\
-0.059 \\
-0.046 \\
-0.039\end{array}$ & $\begin{array}{l}0.083 \\
0.081 \\
0.079 \\
0.077 \\
0.075 \\
0.072 \\
0.068 \\
0.064\end{array}$ & $\begin{array}{l}0.074 \\
0.072 \\
0.070 \\
0.068 \\
0.064 \\
0.058 \\
0.051\end{array}$ \\
\hline $\begin{array}{l}40.00000 \\
45.00000 \\
50.0000 \\
55.00000 \\
60.00000 \\
70.0000 \\
80.0000 \\
90.0000\end{array}$ & $\begin{array}{l}2.554 E+00 \\
2.573 E+00 \\
2.590 E+00 \\
2.605 E+00 \\
2.618 E+00 \\
2.640 E+00 \\
2.658 E+00 \\
2.674 E+00\end{array}$ & $\begin{array}{l}9.188 \mathrm{E} \\
1.032 \mathrm{E} \\
1.147 \mathrm{E} \\
1.261 \mathrm{E} \\
1.493 \mathrm{E} \\
1.725 \mathrm{E} \\
1.960 \mathrm{E}\end{array}$ & $\begin{array}{l}3.360 E+00 \\
3.492 E+00 \\
3.622 E+00 \\
3.751 E+00 \\
3.879 E+00 \\
4.132 E+00 \\
4.384 E+00 \\
4.634 E+00\end{array}$ & $\begin{array}{l}1.499 \mathrm{E}+ \\
1.645 \mathrm{E}+ \\
1.785 \mathrm{E}+ \\
1.921 \mathrm{E}+ \\
2.052 \mathrm{E}+ \\
2.302 \mathrm{E}+ \\
2.537 \mathrm{E}+ \\
2.759 \mathrm{E}+\end{array}$ & $\begin{array}{l}1.27 \\
1.4 \\
1.5 \\
1.6 \\
1.7 \\
2.0 \\
2.2 \\
2.4\end{array}$ & $\begin{array}{l}3.356 \mathrm{E}-01 \\
4.562 \mathrm{E}-01 \\
5.735 \mathrm{E}-01 \\
6.861 \mathrm{E}-01 \\
7.934 \mathrm{E}-01 \\
9.924 \mathrm{E}-01 \\
1.172 \mathrm{E}+00 \\
1.336 \mathrm{E}+00\end{array}$ & $\begin{array}{l}-0.034 \\
-0.030 \\
-0.027 \\
-0.025 \\
-0.024 \\
-0.021 \\
-0.020 \\
-0.019\end{array}$ & & $\begin{array}{l}0.042 \\
0.038 \\
0.035 \\
0.032 \\
0.028 \\
0.025 \\
0.022\end{array}$ \\
\hline $\begin{array}{l}100.00000 \\
125.0000 \\
150.00000 \\
175.0000 \\
200.0000 \\
250.00000 \\
300.0000 \\
350.0000\end{array}$ & $\begin{array}{l}2.688 E+00 \\
2.717 E+00 \\
2.741 E+00 \\
2.760 E+00 \\
2.776 E+00 \\
2.803 E+00 \\
2.825 E+00 \\
2.843 E+00\end{array}$ & $\begin{array}{l}2.78 \\
3.38 \\
3.98 \\
4.58\end{array}$ & $\begin{array}{l}4.883 E+00 \\
5.505 E+00 \\
6.124 E+00 \\
6.743 E+00 \\
7.362 E+00 \\
8.599 E+00 \\
9.837 E+00 \\
1.107 E+01\end{array}$ & $\begin{array}{l}2.969 E+01 \\
3.451 E+01 \\
3.881 E+01 \\
4.270 E+01 \\
4.625 E+01 \\
5.252 E+01 \\
5.796 E+01 \\
6.274 E+01\end{array}$ & $\begin{array}{l}3.044 \mathrm{E}-01 \\
3.045 \mathrm{E}-01 \\
3.770 \mathrm{E}-01 \\
4.058 \mathrm{E}-01 \\
4.546 \mathrm{E}-01 \\
4.945 \mathrm{E}-01 \\
5.280 \mathrm{E}-01\end{array}$ & $\begin{array}{l}1.485 E+00 \\
1.809 E+00 \\
2.081 E+00 \\
2.315 E+00 \\
2.520 E+00 \\
2.870 E+00 \\
3.162 E+00 \\
3.414 E+00\end{array}$ & $\begin{array}{l}-0.018 \\
-0.016 \\
-0.015 \\
-0.014 \\
-0.014 \\
-0.012 \\
-0.011 \\
-0.010\end{array}$ & $\begin{array}{l}0.038 \\
0.034 \\
0.031 \\
0.029 \\
0.027 \\
0.024 \\
0.022 \\
0.021\end{array}$ & $\begin{array}{l}0 . \\
0.2 \\
0.2 \\
0.2 \\
0.2 \\
0.8 \\
0 .\end{array}$ \\
\hline $\begin{array}{l}0.0000 \\
0.0000 \\
0.00000 \\
0.00000 \\
0.0000 \\
0.00000 \\
0.00000\end{array}$ & $\begin{array}{l}2.871 E+00 \\
2.882 E+00 \\
2.892 E+00 \\
2.901 E+00 \\
2.917 E+00 \\
2.930 E+00 \\
2.941 E+00\end{array}$ & $\begin{array}{l}1.068 \mathrm{Et} \\
1.191 \mathrm{Et} \\
1.314 \mathrm{E}+ \\
1.437 \mathrm{Et} \\
1.683 \mathrm{E}+ \\
1.930 \mathrm{Et} \\
2.177 \mathrm{Et}\end{array}$ & $\begin{array}{l}1.355 \mathrm{E} \\
1.479 \mathrm{E} \\
1.603 \mathrm{E} \\
1.727 \mathrm{E} \\
1.975 \mathrm{E} \\
2.223 \mathrm{E} \\
2.472 \mathrm{E}\end{array}$ & $\begin{array}{l}6.702 E+01 \\
7.089 E+01 \\
7.442 E+01 \\
7.767 E+01 \\
8.067 E+01 \\
8.608 E+01 \\
9.085 E+01 \\
9.512 E+01\end{array}$ & $\begin{array}{l}5.565 \\
5.811 \\
6.027 \\
6.218 \\
6.388 \\
6.679 \\
6.920 \\
7.123\end{array}$ & $\begin{array}{l}3.836 \mathrm{E} \\
4.017 \mathrm{E} \\
4.184 \mathrm{E} \\
4.338 \mathrm{E} \\
4.614 \mathrm{E} \\
4.859 \mathrm{E} \\
5.077 \mathrm{E}\end{array}$ & $\begin{array}{l}-0 . \\
-0 . \\
-0 . \\
-0 . \\
-0 . \\
-0 . \\
-0 .\end{array}$ & $\begin{array}{l}0.020 \\
0.019 \\
0.018 \\
0.017 \\
0.017 \\
0.016 \\
0.015 \\
0.014\end{array}$ & $\begin{array}{l}0 . \\
0 . \\
0 .\end{array}$ \\
\hline 00 & $.951 E+00$ & $.425 E+01$ & $.720 E+01$ & $897 E+01$ & $7.297 \mathrm{E}-0$ & $.275 E+0$ & 0.003 & 0.014 & $\cdot v$ \\
\hline
\end{tabular}




\begin{tabular}{|c|c|c|c|c|c|c|c|c|c|}
\hline ENERGY & $\begin{array}{l}\text { COLLISION } \\
\text { MeV } \mathrm{cm}^{2} / \mathrm{g}\end{array}$ & $\begin{array}{l}\text { OPPING POWE } \\
\text { RADIATIVE } \\
\text { MeV } \mathrm{cm}^{2} / \mathrm{g}\end{array}$ & $\begin{array}{c}\text { TOTAL } \\
\mathrm{MeV} \mathrm{cm}^{2} / \mathrm{g}\end{array}$ & $\begin{array}{l}\text { CSDA } \\
\text { RANGE } \\
\mathrm{g} / \mathrm{cm}^{2}\end{array}$ & $\begin{array}{l}\text { ADIATION } \\
\text { YIELD }\end{array}$ & $\begin{array}{c}\text { DENS.EFF. } \\
\text { CORR } \\
\text { (DELTA) }\end{array}$ & $\begin{array}{l}d(1 \\
\text { coLL } \\
\text { Loss }\end{array}$ & $\begin{array}{l}\text { )/dCl } \\
\text { CSDA } \\
\text { RANGE }\end{array}$ & $\begin{array}{l}\text { gI ) } \\
\text { RAD } \\
\text { YIELD }\end{array}$ \\
\hline $\begin{array}{l}0.0100 \\
0.0125 \\
0.0150 \\
0.0175 \\
0.0200 \\
0.0250 \\
0.0300 \\
0.0350\end{array}$ & $\begin{array}{l}2.340 E+01 \\
1.964 E+01 \\
1.702 E+01 \\
1.509 E+01 \\
1.359 E+01 \\
1.143 E+01 \\
9.933 E+00 \\
8.834 E+00\end{array}$ & $\begin{array}{l}3.274 \mathrm{E}-03 \\
3.290 \mathrm{E}-03 \\
3.301 \mathrm{E}-03 \\
3.308 \mathrm{E}-03 \\
3.314 \mathrm{E}-03 \\
3.323 \mathrm{E}-03 \\
3.333 \mathrm{E}-03 \\
3.343 \mathrm{E}-03\end{array}$ & $\begin{array}{l}2.340 E+01 \\
1.964 E+01 \\
1.703 E+01 \\
1.509 E+01 \\
1.359 E+01 \\
1.143 E+01 \\
9.937 E+00 \\
8.837 E+00\end{array}$ & $\begin{array}{l}2.410 E-04 \\
3.581 E-04 \\
4.952 E-04 \\
6.516 E-04 \\
8.264 E-04 \\
1.229 E-03 \\
1.700 E-03 \\
2.235 E-03\end{array}$ & $\begin{array}{l}7.648 \mathrm{E}-05 \\
9.195 \mathrm{E}-05 \\
1.068 \mathrm{E}-04 \\
1.210 \mathrm{E}-04 \\
1.348 \mathrm{E}-04 \\
1.614 \mathrm{E}-04 \\
1.867 \mathrm{E}-04 \\
2.110 \mathrm{E}-04\end{array}$ & $\begin{array}{l}0.00 \\
0.0 \\
0.0 \\
0.0 \\
0.0 \\
0.0 \\
0.0 \\
0.0\end{array}$ & $\begin{array}{l}-0.190 \\
-0.182 \\
-0.177 \\
-0.172 \\
-0.168 \\
-0.162 \\
-0.158 \\
-0.154\end{array}$ & $\begin{array}{l}0.215 \\
0.206 \\
0.198 \\
0.193 \\
0.188 \\
0.180 \\
0.175 \\
0.170\end{array}$ & $\begin{array}{l}0.214 \\
0.205 \\
0.198 \\
0.192 \\
0.187 \\
0.180 \\
0.174 \\
0.170\end{array}$ \\
\hline $\begin{array}{l}0.0400 \\
0.0450 \\
0.0500 \\
0.0550 \\
0.0600 \\
0.0700 \\
0.0800 \\
0.0900\end{array}$ & $\begin{array}{l}7.990 \mathrm{E}+00 \\
7.321 \mathrm{E}+00 \\
6.776 \mathrm{E}+00 \\
6.325 \mathrm{E}+00 \\
5.944 \mathrm{E}+00 \\
5.335 \mathrm{E}+00 \\
4.872 \mathrm{E}+00 \\
4.506 \mathrm{E}+00\end{array}$ & $\begin{array}{l}3.355 \mathrm{E}-03 \\
3.367 \mathrm{E}-03 \\
3.381 \mathrm{E}-03 \\
3.395 \mathrm{E}-03 \\
3.410 \mathrm{E}-03 \\
3.443 \mathrm{E}-03 \\
3.479 \mathrm{E}-03 \\
3.518 \mathrm{E}-03\end{array}$ & $\begin{array}{l}7.993 E+00 \\
7.324 E+00 \\
6.780 E+00 \\
6.328 E+00 \\
5.947 E+00 \\
5.339 E+00 \\
4.875 E+00 \\
4.509 E+00\end{array}$ & $\begin{array}{l}2.830 \mathrm{E}-03 \\
3.485 \mathrm{E}-03 \\
4.195 \mathrm{E}-03 \\
4.959 \mathrm{E}-03 \\
5.775 \mathrm{E}-03 \\
7.553 \mathrm{E}-03 \\
9.516 \mathrm{E}-03 \\
1.165 \mathrm{E}-02\end{array}$ & $\begin{array}{l}2.345 \mathrm{E}-04 \\
2.573 \mathrm{E}-04 \\
2.795 \mathrm{E}-04 \\
3.012 \mathrm{E}-04 \\
3.223 \mathrm{E}-04 \\
3.633 \mathrm{E}-04 \\
4.029 \mathrm{E}-04 \\
4.411 \mathrm{E}-04\end{array}$ & $\begin{array}{l}0.0 \\
0.0 \\
0.0 \\
0.0 \\
0.0 \\
0.0 \\
0.0 \\
0.0\end{array}$ & $\begin{array}{l}-0.151 \\
-0.149 \\
-0.147 \\
-0.145 \\
-0.143 \\
-0.140 \\
-0.138 \\
-0.135\end{array}$ & $\begin{array}{l}0.167 \\
0.163 \\
0.161 \\
0.158 \\
0.156 \\
0.153 \\
0.150 \\
0.147\end{array}$ & $\begin{array}{l}0.166 \\
0.163 \\
0.160 \\
0.158 \\
0.156 \\
0.152 \\
0.150 \\
0.147\end{array}$ \\
\hline $\begin{array}{l}0.1000 \\
0.1250 \\
0.1500 \\
0.1750 \\
0.2000 \\
0.2500 \\
0.3000 \\
0.3500\end{array}$ & $\begin{array}{l}4.210 \mathrm{E}+00 \\
3.671 \mathrm{E}+00 \\
3.307 \mathrm{E}+00 \\
3.046 \mathrm{E}+00 \\
2.850 \mathrm{E}+00 \\
2.578 \mathrm{E}+00 \\
2.400 \mathrm{E}+00 \\
2.276 \mathrm{E}+00\end{array}$ & $\begin{array}{l}3.559 \mathrm{E}-03 \\
3.672 \mathrm{E}-03 \\
3.794 \mathrm{E}-03 \\
3.924 \mathrm{E}-03 \\
4.061 \mathrm{E}-03 \\
4.357 \mathrm{E}-03 \\
4.680 \mathrm{E}-03 \\
5.026 \mathrm{E}-03\end{array}$ & $\begin{array}{l}4.214 E+00 \\
3.675 E+00 \\
3.311 E+00 \\
3.050 E+00 \\
2.854 E+00 \\
2.582 E+00 \\
2.404 E+00 \\
2.281 E+00\end{array}$ & $\begin{array}{l}1.395 \mathrm{E}-02 \\
2.033 \mathrm{E}-02 \\
2.751 \mathrm{E}-02 \\
3.540 \mathrm{E}-02 \\
4.388 \mathrm{E}-02 \\
6.236 \mathrm{E}-02 \\
8.247 \mathrm{E}-02 \\
1.039 \mathrm{E}-01\end{array}$ & $\begin{array}{l}4.783 E-04 \\
5.672 E-04 \\
6.515 E-04 \\
7.322 E-04 \\
8.100 E-04 \\
9.592 E-04 \\
1.102 E-03 \\
1.241 E-03\end{array}$ & $\begin{array}{l}0.0 \\
0.0 \\
0.0 \\
0.0 \\
0.0 \\
0.0 \\
0.0 \\
0.0\end{array}$ & $\begin{array}{l}-0.134 \\
-0.130 \\
-0.127 \\
-0.125 \\
-0.123 \\
-0.1119 \\
-0.1117 \\
-0.115\end{array}$ & $\begin{array}{l}0.145 \\
0.141 \\
0.138 \\
0.135 \\
0.133 \\
0.129 \\
0.126 \\
0.124\end{array}$ & $\begin{array}{l}0.145 \\
0.1141 \\
0.137 \\
0.134 \\
0.132 \\
0.128 \\
0.126 \\
0.123\end{array}$ \\
\hline $\begin{array}{l}0.4000 \\
0.4500 \\
0.5000 \\
0.5500 \\
0.6000 \\
0.7000 \\
0.8000 \\
0.9000\end{array}$ & $\begin{array}{l}2.187 \mathrm{E}+00 \\
2.120 \mathrm{E}+00 \\
2.069 \mathrm{E}+00 \\
2.030 \mathrm{E}+00 \\
1.999 \mathrm{E}+00 \\
1.956 \mathrm{E}+00 \\
1.928 \mathrm{E}+00 \\
1.911 \mathrm{E}+00\end{array}$ & $\begin{array}{l}5.394 \mathrm{E}-03 \\
5.782 \mathrm{E}-03 \\
6.189 \mathrm{E}-03 \\
6.612 \mathrm{E}-03 \\
7.050 \mathrm{E}-03 \\
7.965 \mathrm{E}-03 \\
8.927 \mathrm{E}-03 \\
9.934 \mathrm{E}-03\end{array}$ & $\begin{array}{l}2.192 E+00 \\
2.126 E+00 \\
2.075 E+00 \\
2.037 E+00 \\
2.006 E+00 \\
1.964 E+00 \\
1.937 E+00 \\
1.921 E+00\end{array}$ & $\begin{array}{l}1.262 \mathrm{E}-01 \\
1.494 \mathrm{E}-01 \\
1.732 \mathrm{E}-01 \\
1.976 \mathrm{E}-01 \\
2.223 \mathrm{E}-01 \\
2.727 \mathrm{E}-01 \\
3.240 \mathrm{E}-01 \\
3.759 \mathrm{E}-01\end{array}$ & $\begin{array}{l}1.378 \mathrm{E}-03 \\
1.512 \mathrm{E}-03 \\
1.646 \mathrm{E}-03 \\
1.780 \mathrm{E}-03 \\
1.913 \mathrm{E}-03 \\
2.180 \mathrm{E}-03 \\
2.449 \mathrm{E}-03 \\
2.720 \mathrm{E}-03\end{array}$ & $\begin{array}{l}0.0 \\
0.0 \\
0.0 \\
0.0 \\
0.0 \\
0.0 \\
0.0 \\
0.0\end{array}$ & $\begin{array}{l}-0.1113 \\
-0.1111 \\
-0.1110 \\
-0.1108 \\
-0.1107 \\
-0.105 \\
-0.103 \\
-0.1102\end{array}$ & $\begin{array}{l}0.122 \\
0.121 \\
0.119 \\
0.118 \\
0.117 \\
0.115 \\
0.113 \\
0.112\end{array}$ & $\begin{array}{l}0.121 \\
0.119 \\
0.1118 \\
0.1116 \\
0.1115 \\
0.113 \\
0.111 \\
0.109\end{array}$ \\
\hline $\begin{array}{l}1.0000 \\
1.2500 \\
1.5000 \\
1.7500 \\
2.0000 \\
2.5000 \\
3.0000 \\
3.5000\end{array}$ & $\begin{array}{l}1.901 E+00 \\
1.892 E+00 \\
1.897 E+00 \\
1.908 E+00 \\
1.921 E+00 \\
1.951 E+00 \\
1.981 E+00 \\
2.010 E+00\end{array}$ & $\begin{array}{l}1.098 \mathrm{E}-02 \\
1.375 \mathrm{E}-02 \\
1.671 \mathrm{E}-02 \\
1.984 \mathrm{E}-02 \\
2.309 \mathrm{E}-02 \\
2.994 \mathrm{E}-02 \\
3.716 \mathrm{E}-02 \\
4.468 \mathrm{E}-02\end{array}$ & $\begin{array}{l}1.912 E+00 \\
1.906 E+00 \\
1.914 E+00 \\
1.927 E+00 \\
1.944 E+00 \\
1.981 E+00 \\
2.018 E+00 \\
2.054 E+00\end{array}$ & $\begin{array}{l}4.281 E-01 \\
5.592 E-01 \\
6.901 E-01 \\
8.203 E-01 \\
9.495 E-01 \\
1.204 E+00 \\
1.454 E+00 \\
1.700 E+00\end{array}$ & $\begin{array}{l}2.994 \\
3.690 \\
4.404 \\
5.133 \\
5.877 \\
7.399 \\
8.959 \\
1.055\end{array}$ & $\begin{array}{l}0.0 \\
0.0 \\
0.0 \\
0.0 \\
0.0 \\
0.0 \\
0.0 \\
0.0\end{array}$ & $\begin{array}{l}-0.100 \\
-0.098 \\
-0.095 \\
-0.093 \\
-0.092 \\
-0.089 \\
-0.087 \\
-0.086\end{array}$ & $\begin{array}{l}0.110 \\
0.107 \\
0.105 \\
0.103 \\
0.102 \\
0.099 \\
0.097 \\
0.095\end{array}$ & $\begin{array}{l}0.107 \\
0.104 \\
0.102 \\
0.099 \\
0.098 \\
0.095 \\
0.092 \\
0.090\end{array}$ \\
\hline $\begin{array}{l}4.0000 \\
4.5000 \\
5.0000 \\
5.5000 \\
6.0000 \\
7.0000 \\
8.0000 \\
9.0000\end{array}$ & $\begin{array}{l}2.036 E+00 \\
2.060 E+00 \\
2.083 E+00 \\
2.104 E+00 \\
2.123 E+00 \\
2.159 E+00 \\
2.190 E+00 \\
2.218 E+00\end{array}$ & $\begin{array}{l}5.245 \mathrm{E}-02 \\
6.044 \mathrm{E}-02 \\
6.862 \mathrm{E}-02 \\
7.698 \mathrm{E}-02 \\
8.550 \mathrm{E}-02 \\
1.029 \mathrm{E}-01 \\
1.208 \mathrm{E}-01 \\
1.392 \mathrm{E}-01\end{array}$ & $\begin{array}{l}2.088 E+00 \\
2.121 E+00 \\
2.152 E+00 \\
2.181 E+00 \\
2.209 E+00 \\
2.262 E+00 \\
2.311 E+00 \\
2.357 E+00\end{array}$ & $\begin{array}{l}1.941 E+00 \\
2.179 E+00 \\
2.413 E+00 \\
2.644 E+00 \\
2.871 E+00 \\
3.319 E+00 \\
3.756 E+00 \\
4.184 E+00\end{array}$ & $\begin{array}{l}1.216 \\
1.379 \\
1.543 \\
1.708 \\
1.874 \\
2.208 \\
2.543 \\
2.879\end{array}$ & $\begin{array}{l}0.0 \\
0.0 \\
0.0 \\
0.0 \\
0.0 \\
0.0 \\
0.0 \\
0.0\end{array}$ & $\begin{array}{l}-0.084 \\
-0.083 \\
-0.082 \\
-0.081 \\
-0.080 \\
-0.079 \\
-0.078 \\
-0.076\end{array}$ & $\begin{array}{l}0.094 \\
0.092 \\
0.091 \\
0.090 \\
0.089 \\
0.087 \\
0.086 \\
0.084\end{array}$ & $\begin{array}{l}0.088 \\
0.087 \\
0.085 \\
0.084 \\
0.083 \\
0.081 \\
0.080 \\
0.078\end{array}$ \\
\hline $\begin{array}{l}10.0000 \\
12.5000 \\
15.0000 \\
17.5000 \\
20.0000 \\
25.0000 \\
30.0000 \\
35.0000\end{array}$ & $\begin{array}{l}2.244 E+00 \\
2.298 E+00 \\
2.343 E+00 \\
2.381 E+00 \\
2.410 E+00 \\
2.454 E+00 \\
2.486 E+00 \\
2.512 E+00\end{array}$ & $\begin{array}{l}1.578 \mathrm{E}-01 \\
2.057 \mathrm{E}-01 \\
2.549 \mathrm{E}-01 \\
3.053 \mathrm{E}-01 \\
3.565 \mathrm{E}-01 \\
4.607 \mathrm{E}-01 \\
5.669 \mathrm{E}-01 \\
6.744 \mathrm{E}-01\end{array}$ & $\begin{array}{l}2.401 E+00 \\
2.504 E+00 \\
2.598 E+00 \\
2.686 E+00 \\
2.767 E+00 \\
2.915 E+00 \\
3.053 E+00 \\
3.186 E+00\end{array}$ & $\begin{array}{l}4.605 E+00 \\
5.624 E+00 \\
6.604 E+00 \\
7.550 E+00 \\
8.467 E+00 \\
1.023 E+01 \\
1.190 E+01 \\
1.350 E+01\end{array}$ & $\begin{array}{l}3.215 \mathrm{E}-02 \\
4.051 \mathrm{E}-02 \\
4.879 \mathrm{E}-02 \\
5.695 \mathrm{E}-02 \\
6.499 \mathrm{E}-02 \\
8.071 \mathrm{E}-02 \\
9.592 \mathrm{E}-02 \\
1.106 \mathrm{E}-01\end{array}$ & $\begin{array}{l}0.0 \\
0.0 \\
0.0 \\
7.537 \mathrm{E}-03 \\
5.064 \mathrm{E}-02 \\
1.907 \mathrm{E}-01 \\
3.525 \mathrm{E}-01 \\
5.139 \mathrm{E}-01\end{array}$ & $\begin{array}{l}-0.076 \\
-0.074 \\
-0.072 \\
-0.064 \\
-0.054 \\
-0.041 \\
-0.034 \\
-0.030\end{array}$ & $\begin{array}{l}0.083 \\
0.081 \\
0.079 \\
0.077 \\
0.074 \\
0.068 \\
0.063 \\
0.058\end{array}$ & $\begin{array}{l}0.077 \\
0.074 \\
0.072 \\
0.069 \\
0.065 \\
0.056 \\
0.049 \\
0.043\end{array}$ \\
\hline $\begin{array}{l}40.0000 \\
45.0000 \\
50.0000 \\
55.0000 \\
60.0000 \\
70.0000 \\
80.0000 \\
90.0000\end{array}$ & $\begin{array}{l}2.532 E+00 \\
2.550 E+00 \\
2.565 E+00 \\
2.578 E+00 \\
2.591 E+00 \\
2.612 E+00 \\
2.629 E+00 \\
2.645 E+00\end{array}$ & $\begin{array}{l}7.831 E-01 \\
8.928 E-01 \\
1.003 E+00 \\
1.114 E+00 \\
1.226 E+00 \\
1.451 E+00 \\
1.677 E+00 \\
1.905 E+00\end{array}$ & $\begin{array}{l}3.315 E+00 \\
3.443 E+00 \\
3.568 E+00 \\
3.693 E+00 \\
3.817 E+00 \\
4.062 E+00 \\
4.307 E+00 \\
4.550 E+00\end{array}$ & $\begin{array}{l}1.504 E+01 \\
1.652 E+01 \\
1.795 E+01 \\
1.933 E+01 \\
2.066 E+01 \\
2.320 E+01 \\
2.559 E+01 \\
2.785 E+01\end{array}$ & $\begin{array}{l}1.248 E-01 \\
1.385 E-01 \\
1.517 E-01 \\
1.644 E-01 \\
1.766 E-01 \\
1.999 E-01 \\
2.216 E-01 \\
2.419 E-01\end{array}$ & $\begin{array}{l}6.676 \mathrm{E}-01 \\
8.118 \mathrm{E}-01 \\
9.464 \mathrm{E}-01 \\
1.072 \mathrm{E}+00 \\
1.189 \mathrm{E}+00 \\
1.403 \mathrm{E}+00 \\
1.593 \mathrm{E}+00 \\
1.763 \mathrm{E}+00\end{array}$ & $\begin{array}{l}-0.027 \\
-0.024 \\
-0.023 \\
-0.021 \\
-0.020 \\
-0.019 \\
-0.018 \\
-0.017\end{array}$ & $\begin{array}{l}0.055 \\
0.052 \\
0.049 \\
0.046 \\
0.044 \\
0.041 \\
0.038 \\
0.036\end{array}$ & $\begin{array}{l}0.038 \\
0.034 \\
0.031 \\
0.029 \\
0.027 \\
0.023 \\
0.021 \\
0.019\end{array}$ \\
\hline $\begin{array}{l}100.0000 \\
125.00000 \\
150.0000 \\
175.0000 \\
200.0000 \\
250.0000 \\
300.0000 \\
350.0000\end{array}$ & $\begin{array}{l}2.658 E+00 \\
2.687 E+00 \\
2.709 E+00 \\
2.728 E+00 \\
2.744 E+00 \\
2.770 E+00 \\
2.791 E+00 \\
2.808 E+00\end{array}$ & $\begin{array}{l}2.134 E+00 \\
2.710 \mathrm{E}+00 \\
3.291 \mathrm{E}+00 \\
3.874 \mathrm{E}+00 \\
4.460 \mathrm{E}+00 \\
5.638 \mathrm{E}+00 \\
6.822 \mathrm{E}+00 \\
8.009 \mathrm{E}+00\end{array}$ & $\begin{array}{l}4.792 \mathrm{E}+00 \\
5.397 \mathrm{E}+00 \\
6.000 \mathrm{E}+00 \\
6.602 \mathrm{E}+00 \\
7.205 \mathrm{E}+00 \\
8.408 \mathrm{E}+00 \\
9.612 \mathrm{E}+00 \\
1.082 \mathrm{E}+01\end{array}$ & $\begin{array}{l}2.999 \mathrm{E}+01 \\
3.4900+01 \\
3.929 \mathrm{E}+01 \\
4.326 \mathrm{E}+01 \\
4.689 \mathrm{E}+01 \\
5.330 \mathrm{E}+01 \\
5.886 \mathrm{E}+01 \\
6.376 \mathrm{E}+01\end{array}$ & $\begin{array}{l}3.037 \mathrm{E} \\
3.408 \mathrm{E} \\
3.733 \mathrm{E} \\
4.020 \mathrm{E} \\
4.508 \mathrm{E} \\
4.909 \mathrm{E} \\
5.244 \mathrm{E}\end{array}$ & $\begin{array}{l}1.917 E+00 \\
2.251 E+00 \\
2.529 E+00 \\
2.769 E+00 \\
2.980 E+00 \\
3.341 E+00 \\
3.644 E+00 \\
3.907 E+00\end{array}$ & $\begin{array}{l}-0.017 \\
-0.015 \\
-0.014 \\
-0.013 \\
-0.012 \\
-0.011 \\
-0.010 \\
-0.008\end{array}$ & $\begin{array}{l}0.034 \\
0.030 \\
0.028 \\
0.026 \\
0.024 \\
0.022 \\
0.020 \\
0.019\end{array}$ & $\begin{array}{l}0.017 \\
0.015 \\
0.013 \\
0.011 \\
0.010 \\
0.008 \\
0.007 \\
0.006\end{array}$ \\
\hline $\begin{array}{l}.0000 \\
.0000 \\
.0000\end{array}$ & $\begin{array}{l}2.822 E+00 \\
2.834 E+00 \\
2.845 E+00 \\
2.854 E+00 \\
2.863 E+00 \\
2.877 E+00 \\
2.890 E+00 \\
2.901 E+00\end{array}$ & $\begin{array}{l}9.200 \mathrm{E}+00 \\
1.039 \mathrm{E}+01 \\
1.159 \mathrm{E}+01 \\
1.279 \mathrm{E}+01 \\
1.398 \mathrm{E}+01 \\
1.638 \mathrm{E}+01 \\
1.879 \mathrm{E}+01 \\
2.119 \mathrm{E}+01\end{array}$ & $\begin{array}{l}1.202 E+01 \\
1.323 \mathrm{E}+01 \\
1.443 \mathrm{E}+01 \\
1.564 \mathrm{E}+01 \\
1.685 \mathrm{E}+01 \\
1.926 \mathrm{E}+01 \\
2.168 \mathrm{E}+01 \\
2.409 \mathrm{E}+01\end{array}$ & $\begin{array}{l}6.814 E+01 \\
7.211 \mathrm{E}+01 \\
7.573 \mathrm{E}+01 \\
7.905 \mathrm{E}+01 \\
8.213 \mathrm{E}+01 \\
8.768 \mathrm{E}+01 \\
9.257 \mathrm{E}+01 \\
9.695 \mathrm{E}+01\end{array}$ & $\begin{array}{l}5.778 \\
5.994 \\
6.186 \\
6.357 \\
6.650 \\
6.893 \\
7.097\end{array}$ & $\begin{array}{l}4.140 E+00 \\
4.349 E+00 \\
4.539 E+00 \\
4.713 E+00 \\
4.873 E+00 \\
5.161 E+00 \\
5.414 E+00 \\
5.640 E+00\end{array}$ & $\begin{array}{l}-0.007 \\
-0.006 \\
-0.006 \\
-0.005 \\
-0.004 \\
-0.003 \\
-0.003 \\
-0.002\end{array}$ & $\begin{array}{l}0.018 \\
0.017 \\
0.016 \\
0.015 \\
0.015 \\
0.0114 \\
0.013 \\
0.013\end{array}$ & $\begin{array}{l}0.006 \\
0.005 \\
0.004 \\
0.004 \\
0.004 \\
0.003 \\
0.003 \\
0.002\end{array}$ \\
\hline & $2.910 E+00$ & $2.360 \mathrm{E}+01$ & $2.651 E+01$ & $.009 E+02$ & $7.273 E-01$ & $5.843 E+00$ & -0.002 & 0.012 & 0.002 \\
\hline
\end{tabular}


ELECTRONS IN TOLUENE

$I=62.5 \mathrm{eV} \quad$ DENSITY $=8.669 \mathrm{E}-01 \mathrm{~g} / \mathrm{cm}^{3}$

\begin{tabular}{|c|c|c|c|c|c|c|c|c|c|}
\hline $\mathrm{MeV}$ & $\mathrm{MeV} \mathrm{cm} 2 / \mathrm{g}$ & 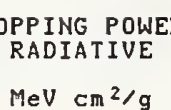 & $\begin{array}{l}\text { TOTAL } \\
\mathrm{V} \mathrm{cm}^{2} / \mathrm{g}\end{array}$ & $\begin{array}{l}\text { CSDA } \\
\text { RANGE }\end{array}$ & RADIAT & $\begin{array}{l}\text { DENS.EFF. } \\
\text { CORR. } \\
\text { (DELTA) }\end{array}$ & $\begin{array}{l}d(1) \\
\operatorname{coLL} \\
\text { Loss }\end{array}$ & $1 / d$ & $\begin{array}{l}\text { gI ) } \\
\text { RAD } \\
\text { YIELD }\end{array}$ \\
\hline $\begin{array}{l}0.01 \\
0.01 \\
0.01 \\
0.01 \\
0.02 \\
0.02 \\
0.03 \\
0.03\end{array}$ & $\begin{array}{ll}0 & 1 \\
0 & 1 \\
0 & 1 \\
0 & 1 \\
0 & 1 \\
0 & 1 \\
0 & 0 \\
0 & 0\end{array}$ & $\begin{array}{l}2.9 \\
2.9 \\
2.9 \\
2.9 \\
2.9 \\
3.0 \\
3.0\end{array}$ & $\begin{array}{l}1.6 \\
1.4 \\
1.3 \\
1.1 \\
9.7\end{array}$ & $\begin{array}{l}04 \\
04 \\
04 \\
04 \\
04 \\
03 \\
03\end{array}$ & $\begin{array}{l}7 . \\
8: \\
9 \\
1: \\
1: \\
1: \\
1: \\
1 .\end{array}$ & $\begin{array}{l}0.0 \\
0.0 \\
0.0 \\
0.0 \\
0.0 \\
0.0 \\
0.0 \\
0.0\end{array}$ & & $\begin{array}{l}8 \\
8 \\
1 \\
5 \\
0 \\
2 \\
6 \\
2\end{array}$ & $\begin{array}{l}0.194 \\
0.189 \\
0.182 \\
0.176\end{array}$ \\
\hline $\begin{array}{l}0.0400 \\
0.0450 \\
0.0500 \\
0.0550 \\
0.0600 \\
0.0700 \\
0.0800 \\
0.0900\end{array}$ & $\begin{array}{l}7.82 \\
7.16 \\
6.63 \\
6.19 \\
5.81 \\
5.22 \\
4.77 \\
4.41\end{array}$ & $\begin{array}{l}3.0 \\
3.0 \\
3.0 \\
3.0 \\
3.1 \\
3.1\end{array}$ & $\begin{array}{l}+00 \\
+00 \\
+00 \\
+00 \\
+00 \\
+00 \\
+00 \\
+00 \\
+00\end{array}$ & $\begin{array}{l}3.5 \\
4.2 \\
5.0 \\
5.9 \\
7.7 \\
9.7 \\
1.1\end{array}$ & $\begin{array}{l}2.1 \\
2.3 \\
2.5 \\
2.7 \\
2.9 \\
3.3 \\
3.7 \\
4.0\end{array}$ & $\begin{array}{l}0.0 \\
0.0 \\
0.0 \\
0.0 \\
0.0 \\
0.0 \\
0.0 \\
0.0\end{array}$ & & $\begin{array}{l}8 \\
5 \\
2 \\
0 \\
8 \\
4 \\
1 \\
8\end{array}$ & $\begin{array}{l}0.1 \\
0.1 \\
0.1\end{array}$ \\
\hline $\begin{array}{l}0.1000 \\
0.1250 \\
0.1500 \\
0.1750 \\
0.2000 \\
0.2500 \\
0.3000 \\
0.3500\end{array}$ & $\begin{array}{l}4.124 E+00 \\
3.597 E+00 \\
3.241 E+00 \\
2.985 E+00 \\
2.793 E+00 \\
2.527 E+00 \\
2.353 E+00 \\
2.232 E+00\end{array}$ & $\begin{array}{l}3.3 \\
3.4 \\
3.5 \\
3.6 \\
3.9 \\
4.2 \\
4.5\end{array}$ & $\begin{array}{l}4.127 E+00 \\
3.600 E+00 \\
3.244 E+00 \\
2.989 E+00 \\
2.797 E+00 \\
2.531 E+00 \\
2.357 E+00 \\
2.236 E+00\end{array}$ & $\begin{array}{l}2.0 \\
2.8 \\
3.6 \\
4.4 \\
6.3 \\
8.4 \\
1.0\end{array}$ & $\begin{array}{l}4.4 \\
5.2 \\
6.0 \\
6.7 \\
7.4 \\
8.8 \\
1.0 \\
1.1\end{array}$ & $\begin{array}{l}0.0 \\
0.0 \\
0.0 \\
0.0 \\
0.0 \\
0.0 \\
0.0 \\
0.0\end{array}$ & & & $\begin{array}{l}0 . \\
0 . \\
0 .\end{array}$ \\
\hline $\begin{array}{l}0.4000 \\
0.4500 \\
0.5000 \\
0.5500 \\
0.6000 \\
0.7000 \\
0.8000 \\
0.9000\end{array}$ & $\begin{array}{l}144 E+00 \\
077 E+00 \\
024 E+00 \\
983 E+00 \\
950 E+00 \\
901 E+00 \\
868 E+00 \\
845 E+00\end{array}$ & $\begin{array}{l}5.2 \\
5.6 \\
6.0 \\
6.4 \\
7.2 \\
8.1\end{array}$ & $\begin{array}{ll}0 & 0 \\
0 & 0 \\
0 & 0 \\
0 & 0 \\
0 & 0 \\
0 & 0 \\
0 & 0\end{array}$ & $\begin{array}{l}1.5 \\
1.7 \\
2.0 \\
2.2 \\
2.7\end{array}$ & $\begin{array}{l}1.403 \\
1.528 \\
1.65 \\
1.780 \\
2.033 \\
2.285 \\
2.54\end{array}$ & $\begin{array}{l}0.0 \\
2.097 \mathrm{E}-02 \\
4.725 \mathrm{E}-02 \\
7.602 \mathrm{E}-02 \\
1.066 \mathrm{E}-01 \\
1.715 \mathrm{E}-01 \\
2.389 \mathrm{E}-01 \\
3.071 \mathrm{E}-01\end{array}$ & $\begin{array}{l}-0.1 \\
-0.0 \\
-0.0 \\
-0.0 \\
-0.0 \\
-0.0 \\
-0.0 \\
-0.0\end{array}$ & $\begin{array}{l}3 \\
9 \\
4 \\
0 \\
6 \\
0 \\
4 \\
9\end{array}$ & $\begin{array}{l}0 . \\
0 .\end{array}$ \\
\hline $\begin{array}{l}1.0000 \\
1.2500 \\
1.5000 \\
1.7500 \\
2.0000 \\
2.5000 \\
3.0000 \\
3.5000\end{array}$ & $\begin{array}{l}1.830 E+00 \\
1.808 E+00 \\
1.800 E+00 \\
1.799 E+00 \\
1.801 E+00 \\
1.812 E+00 \\
1.824 E+00 \\
1.837 E+00\end{array}$ & $\begin{array}{l}1.2 \\
1.5 \\
2.1 \\
2.7 \\
3.4 \\
4.1\end{array}$ & $\begin{array}{l}.840 E+00 \\
.820 E+00 \\
.815 E+00 \\
.817 E+00 \\
.823 E+00 \\
.839 E+00 \\
.858 E+00 \\
.878 E+00\end{array}$ & $\begin{array}{l}4.395 \mathrm{E}-01 \\
5.762 \mathrm{E}-01 \\
7.138 \mathrm{E}-01 \\
8.515 \mathrm{E}-01 \\
9.889 \mathrm{E}-01 \\
1.262 \mathrm{E}+00\end{array}$ & $\begin{array}{l}2.812 \mathrm{E}-03 \\
3.488 \mathrm{E}-03 \\
4.187 \mathrm{E}-03 \\
4.908 \mathrm{E}-03 \\
5.648 \mathrm{E}-03 \\
7.177 \mathrm{E}-03 \\
8.760 \mathrm{E}-03 \\
1.039 \mathrm{E}-02\end{array}$ & $\begin{array}{l}3.75 \\
5.40 \\
6.95 \\
8.39 \\
9.73 \\
1.21 \\
1.42 \\
1.60\end{array}$ & & $\begin{array}{l}0.085 \\
0.076 \\
0.070 \\
0.065 \\
0.061 \\
0.055 \\
0.051 \\
0.048\end{array}$ & \\
\hline $\begin{array}{l}4.0000 \\
4.5000 \\
5.0000 \\
5.5000 \\
6.0000 \\
7.0000 \\
8.0000 \\
9.0000\end{array}$ & $\begin{array}{l}9 E+00 \\
1 E+00 \\
2 E+00 \\
2 E+00 \\
2 E+00 \\
9 E+00 \\
4 E+00 \\
7 E+00\end{array}$ & $\begin{array}{l}5.5 \\
6.3 \\
7.0 \\
7.8 \\
9.4\end{array}$ & $\begin{array}{l}1.917 \mathrm{E} \\
1.9353 \mathrm{E} \\
1.971 \mathrm{E} \\
2.004 \mathrm{E} \\
2.036 \mathrm{E} \\
2.066 \mathrm{E}\end{array}$ & $\begin{array}{l}2.327 \mathrm{E}+0 \\
2.587 \mathrm{E}+0 \\
2.844 \mathrm{E}+0 \\
3.099 \mathrm{E}+0 \\
3.602 \mathrm{E}+0 \\
4.097 \mathrm{E}+0 \\
4.585 \mathrm{E}+0\end{array}$ & $\begin{array}{l}1.37 \\
1.54 \\
1.71 \\
1.89 \\
2.24 \\
2.60 \\
2.96\end{array}$ & $\begin{array}{l}1.927 \mathrm{E} \\
2.066 \mathrm{E} \\
2.193 \mathrm{E} \\
2.312 \mathrm{E} \\
2.528 \mathrm{E} \\
2.720 \mathrm{E} \\
2.894 \mathrm{E}\end{array}$ & & $\begin{array}{l}5 \\
3 \\
1 \\
0 \\
8 \\
6 \\
4 \\
3\end{array}$ & \\
\hline $\begin{array}{l}10.0000 \\
12.5000 \\
15.0000 \\
17.5000 \\
20.0000 \\
25.0000 \\
30.0000 \\
35.0000\end{array}$ & $\begin{array}{l}1.94 \\
1.97 \\
1.99 \\
2.01 \\
2.02 \\
2.04 \\
2.06 \\
2.07\end{array}$ & $\begin{array}{l}2 . y \\
2.3 \\
3.2 \\
4.2\end{array}$ & $\begin{array}{l}2.1 \\
2.2 \\
2.2 \\
2.3 \\
2.4 \\
2.5 \\
2.7\end{array}$ & $\begin{array}{l}0 \\
0 \\
0 \\
0 \\
1\end{array}$ & $\begin{array}{l}5 . \\
6 . \\
6 . \\
8 . \\
1 . \\
1 .\end{array}$ & $\begin{array}{l}3.0 \\
3.4 \\
3.7 \\
3.9 \\
4.1 \\
4.5 \\
4.9 \\
5.2\end{array}$ & & $\begin{array}{l}0 . \\
0.8 \\
0.8 \\
0.8 \\
0 . \\
0 .\end{array}$ & \\
\hline $\begin{array}{l}40.0 \\
45.0 \\
50.0 \\
55.0 \\
60.0 \\
70.0 \\
80.0 \\
90.0\end{array}$ & $\begin{array}{l}2.0 \\
2.1 \\
2.1 \\
2.1 \\
2.1 \\
2.1\end{array}$ & $\begin{array}{l}8 . \\
9: \\
1: \\
1: \\
1:\end{array}$ & $\begin{array}{l}0 \\
0 \\
0 \\
0 \\
0 \\
0 \\
0\end{array}$ & & $\begin{array}{l}1 \\
1 \\
1 \\
1 \\
1 \\
1\end{array}$ & 7. & & & \\
\hline $\begin{array}{l}5.0000 \\
.0000 \\
.0000 \\
.0000 \\
.0000 \\
.0000 \\
.0000\end{array}$ & $\begin{array}{l}2.2 \\
2.2 \\
2.2 \\
2.2 \\
2.2\end{array}$ & $\begin{array}{l}3.0 \\
3.5 \\
4.1 \\
5.2\end{array}$ & $\begin{array}{l}0 \\
0 \\
0 \\
0 \\
0\end{array}$ & 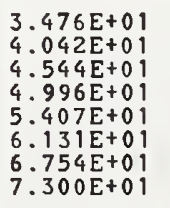 & $\begin{array}{l}3 . \\
3 . \\
3 \\
4 . \\
4 .\end{array}$ & 9: & & & \\
\hline $\begin{array}{l}0.0000 \\
0.0000 \\
0.0000 \\
0.00000 \\
0.00000 \\
0.0000 \\
0.0000 \\
0.0000\end{array}$ & $\begin{array}{l}2.284 E+00 \\
2.294 E+00 \\
2.303 E+00 \\
2.311 E+00 \\
2.318 E+00 \\
2.331 E+00 \\
2.342 E+00 \\
2.352 E+00\end{array}$ & $\begin{array}{l}8.552 \mathrm{Et}^{+} \\
9.662 \mathrm{Et} \\
1.077 \mathrm{E}+ \\
1.189 \mathrm{E}+ \\
1.300 \mathrm{E}+ \\
1.524 \mathrm{E}+ \\
1.747 \mathrm{Et} \\
1.972 \mathrm{E}+\end{array}$ & $\begin{array}{l}1 \\
1 \\
1 \\
1 \\
1 \\
1 \\
1\end{array}$ & $\begin{array}{l}1 \\
1 \\
1 \\
1 \\
1 \\
1 \\
2\end{array}$ & $\begin{array}{l}1 \\
1 \\
1 \\
1 \\
1 \\
1\end{array}$ & $\begin{array}{l}1.026 E+01 \\
1.047 E+01 \\
1.066 E+01 \\
1.084 E+01 \\
1.0114 E+01 \\
1.141 E+01 \\
1.165 E+01\end{array}$ & $\begin{array}{l}-0 \\
-0 \\
-0 \\
-0 \\
-0 \\
-0 \\
-0\end{array}$ & $\begin{array}{l}3 \\
3 \\
3 \\
3 \\
3 \\
3 \\
3 \\
2\end{array}$ & \\
\hline & $.360 E+00$ & $2.196 \mathrm{E}+01$ & $.432 E+01$ & $.139 E+02$ & $.485 E-01$ & $1.186 E+01$ & -0.000 & 0.002 & .000 \\
\hline
\end{tabular}


ELECTRONS IN WATER, LIQUID

$I=75.0 \mathrm{oV} \quad$ DENSITY $=1.000 \mathrm{E}+00 \mathrm{~g} / \mathrm{cm}^{3}$

\begin{tabular}{|c|c|c|c|c|c|c|c|c|c|}
\hline ENERGY & $\begin{array}{l}\text { COLLISION } \\
\text { MeV } \mathrm{cm}^{2} / \mathrm{g}\end{array}$ & $\begin{array}{l}\text { OPPING POWE } \\
\text { RADIAT IVE } \\
\text { MeV } \mathrm{cm}^{2} / \mathrm{g}\end{array}$ & $\begin{array}{c}\text { TOTAL } \\
\mathrm{MeV} \mathrm{cm}^{2} / \mathrm{g}\end{array}$ & $\begin{array}{l}\text { CSDA } \\
\text { RANGE } \\
\mathrm{g} / \mathrm{Cm}^{2}\end{array}$ & $\begin{array}{l}\text { RADIATION } \\
\text { YIELD }\end{array}$ & $\begin{array}{c}\text { DEHS.EFF. } \\
\text { CORR. } \\
\text { (DELTA) }\end{array}$ & $\begin{array}{l}d(x) \\
\text { coll } \\
\text { LOSS }\end{array}$ & $\begin{array}{l}9 \text { )/d(1 } \\
\text { CSDA } \\
\text { RANGE }\end{array}$ & $\begin{array}{l}\text { I) } \\
\text { RAD } \\
\text { IIELD }\end{array}$ \\
\hline $\begin{array}{l}0.0100 \\
0.0125 \\
0.0150 \\
0.0175 \\
0.0200 \\
0.0250 \\
0.0300 \\
0.0350\end{array}$ & $\begin{array}{l}2.256 E+01 \\
1.897 E+01 \\
1.647 E+01 \\
1.461 E+01 \\
1.317 E+01 \\
1.109 E+01 \\
9.653 E+00 \\
8.592 E+00\end{array}$ & $\begin{array}{l}3.898 \mathrm{E}-03 \\
3.927 \mathrm{E}-03 \\
3.944 \mathrm{E}-03 \\
3.955 \mathrm{E}-03 \\
3.963 \mathrm{E}-03 \\
3.974 \mathrm{E}-03 \\
3.984 \mathrm{E}-03 \\
3.994 \mathrm{E}-03\end{array}$ & $\begin{array}{l}2.257 E+01 \\
1.898 E+01 \\
1.647 E+01 \\
1.461 E+01 \\
1.318 E+01 \\
1.110 E+01 \\
9.657 E+00 \\
8.596 E+00\end{array}$ & $\begin{array}{l}2.515 \\
3.728 \\
5.147 \\
6.761 \\
8.566 \\
1.272 \\
1.756 \\
2.306\end{array}$ & $\begin{array}{l}9.408 E-05 \\
1.133 E-04 \\
1.316 E-04 \\
1.492 E-04 \\
1.663 E-04 \\
1.990 E-04 \\
2.301 E-04 \\
2.599 E-04\end{array}$ & $\begin{array}{l}0.0 \\
0.0 \\
0.0 \\
0.0 \\
0.0 \\
0.0 \\
0.0 \\
0.0\end{array}$ & $\begin{array}{l}-0.199 \\
-0.190 \\
-0.184 \\
-0.179 \\
-0.175 \\
-0.169 \\
-0.164 \\
-0.160\end{array}$ & $\begin{array}{l}0.227 \\
0.217 \\
0.208 \\
0.202 \\
0.197 \\
0.189 \\
0.182 \\
0.177\end{array}$ & $\begin{array}{l}0.225 \\
0.215 \\
0.207 \\
0.201 \\
0.196 \\
0.188 \\
0.182 \\
0.177\end{array}$ \\
\hline $\begin{array}{l}0.0400 \\
0.0450 \\
0.0500 \\
0.0550 \\
0.0600 \\
0.0700 \\
0.0800 \\
0.0900\end{array}$ & $\begin{array}{l}7.777 E+00 \\
7.130 E+00 \\
6.603 E+00 \\
6.166 E+00 \\
5.797 E+00 \\
5.207 E+00 \\
4.757 E+00 \\
4.402 E+00\end{array}$ & $\begin{array}{l}4.005 \mathrm{E}-03 \\
4.018 \mathrm{E}-03 \\
4.031 \mathrm{E}-03 \\
4.046 \mathrm{E}-03 \\
4.062 \mathrm{E}-03 \\
4.098 \mathrm{E}-03 \\
4.138 \mathrm{E}-03 \\
4.181 \mathrm{E}-03\end{array}$ & $\begin{array}{l}7.781 E+00 \\
7.134 E+00 \\
6.607 E+00 \\
6.170 E+00 \\
5.801 E+00 \\
5.211 E+00 \\
4.762 E+00 \\
4.407 E+00\end{array}$ & $\begin{array}{l}2.919 \mathrm{E}-03 \\
3.591 \mathrm{E}-03 \\
4.320 \mathrm{E}-03 \\
5.103 \mathrm{E}-03 \\
5.940 \mathrm{E}-03 \\
7.762 \mathrm{E}-03 \\
9.773 \mathrm{E}-03 \\
1.196 \mathrm{E}-02\end{array}$ & $\begin{array}{l}2.886 E-04 \\
3.165 E-04 \\
3.435 E-04 \\
3.698 E-04 \\
3.955 E-04 \\
4.452 E-04 \\
4.931 E-04 \\
5.393 E-04\end{array}$ & $\begin{array}{l}0.0 \\
0.0 \\
0.0 \\
0.0 \\
0.0 \\
0.0 \\
0.0\end{array}$ & $\begin{array}{l}-0.157 \\
-0.154 \\
-0.152 \\
-0.150 \\
-0.148 \\
-0.145 \\
-0.142 \\
-0.140\end{array}$ & $\begin{array}{l}0.173 \\
0.170 \\
0.167 \\
0.165 \\
0.162 \\
0.158 \\
0.155 \\
0.153\end{array}$ & $\begin{array}{l}0.173 \\
0.170 \\
0.167 \\
0.164 \\
0.162 \\
0.158 \\
0.155 \\
0.152\end{array}$ \\
\hline $\begin{array}{l}0.1000 \\
0.1250 \\
0.1500 \\
0.1750 \\
0.2000 \\
0.2500 \\
0.3000 \\
0.3500\end{array}$ & $\begin{array}{l}4.115 E+00 \\
3.591 E+00 \\
3.238 E+00 \\
2.984 E+00 \\
2.793 E+00 \\
2.528 E+00 \\
2.355 E+00 \\
2.235 E+00\end{array}$ & $\begin{array}{l}4.228 \mathrm{E}-03 \\
4.355 \mathrm{E}-03 \\
4.494 \mathrm{E}-03 \\
4.643 \mathrm{E}-03 \\
4.801 \mathrm{E}-03 \\
5.141 \mathrm{E}-03 \\
5.514 \mathrm{E}-03 \\
5.913 \mathrm{E}-03\end{array}$ & $\begin{array}{l}4.120 E+00 \\
3.596 E+00 \\
3.242 E+00 \\
2.988 E+00 \\
2.798 E+00 \\
2.533 E+00 \\
2.360 E+00 \\
2.241 E+00\end{array}$ & $\begin{array}{l}1.431 \mathrm{E}-02 \\
2.083 \mathrm{E}-02 \\
2.817 \mathrm{E}-02 \\
3.622 \mathrm{E}-02 \\
4.487 \mathrm{E}-02 \\
6.372 \mathrm{E}-02 \\
8.421 \mathrm{E}-02 \\
1.060 \mathrm{E}-01\end{array}$ & $\begin{array}{l}5.841 \mathrm{E}-04 \\
6.912 \mathrm{E}-04 \\
7.926 \mathrm{E}-04 \\
8.894 \mathrm{E}-04 \\
9.826 \mathrm{E}-04 \\
1.161 \mathrm{E}-03 \\
1.331 \mathrm{E}-03 \\
1.496 \mathrm{E}-03\end{array}$ & $\begin{array}{l}0.0 \\
0.0 \\
0.0 \\
0.0 \\
0.0 \\
0.0 \\
0.0 \\
0.0\end{array}$ & $\begin{array}{l}-0.138 \\
-0.134 \\
-0.131 \\
-0.128 \\
-0.126 \\
-0.123 \\
-0.120 \\
-0.118\end{array}$ & $\begin{array}{l}0.150 \\
0.146 \\
0.142 \\
0.139 \\
0.137 \\
0.133 \\
0.130 \\
0.128\end{array}$ & $\begin{array}{l}0.150 \\
0.145 \\
0.142 \\
0.139 \\
0.136 \\
0.132 \\
0.129 \\
0.127\end{array}$ \\
\hline $\begin{array}{l}0.4000 \\
0.4500 \\
0.5000 \\
0.5500 \\
0.6000 \\
0.7000 \\
0.8000 \\
0.9000\end{array}$ & $\begin{array}{l}2.148 E+00 \\
2.083 E+00 \\
2.034 E+00 \\
1.995 E+00 \\
1.963 E+00 \\
1.917 E+00 \\
1.886 E+00 \\
1.864 E+00\end{array}$ & $\begin{array}{l}6.339 \mathrm{E}-03 \\
6.787 \mathrm{E}-03 \\
7.257 \mathrm{E}-03 \\
7.747 \mathrm{E}-03 \\
8.254 \mathrm{E}-03 \\
9.312 \mathrm{E}-03 \\
1.043 \mathrm{E}-02 \\
1.159 \mathrm{E}-02\end{array}$ & $\begin{array}{l}2.154 E+00 \\
2.090 E+00 \\
2.041 E+00 \\
2.003 E+00 \\
1.972 E+00 \\
1.926 E+00 \\
1.896 E+00 \\
1.876 E+00\end{array}$ & $\begin{array}{l}1.288 \mathrm{E}-01 \\
1.523 \mathrm{E}-01 \\
1.766 \mathrm{E}-01 \\
2.013 \mathrm{E}-01 \\
2.265 \mathrm{E}-01 \\
2.778 \mathrm{E}-01 \\
3.302 \mathrm{E}-01 \\
3.832 \mathrm{E}-01\end{array}$ & $\begin{array}{l}1.6 \\
1.8 \\
1.9 \\
2.1 \\
2.2 \\
2.6 \\
2.9 \\
3.2\end{array}$ & $\begin{array}{l}0.0 \\
0.0 \\
0.0 \\
1.103 \mathrm{E}-02 \\
2.938 \mathrm{E}-02 \\
7.435 \mathrm{E}-02 \\
1.267 \mathrm{E}-01 \\
1.835 \mathrm{E}-01\end{array}$ & $\begin{array}{l}-0.1116 \\
-0.1114 \\
-0.1111 \\
-0.094 \\
-0.088 \\
-0.078 \\
-0.070 \\
-0.064\end{array}$ & $\begin{array}{l}0 . \\
0 . \\
0 . \\
0 . \\
0 . \\
0 .\end{array}$ & $\begin{array}{l}0.125 \\
0.123 \\
0.121 \\
0.118 \\
0.1114 \\
0.1106 \\
0.099 \\
0.093\end{array}$ \\
\hline $\begin{array}{l}1.0000 \\
1.2500 \\
1.5000 \\
1.7500 \\
2.0000 \\
2.5000 \\
3.0000 \\
3.5000\end{array}$ & $\begin{array}{l}1.849 E+00 \\
1.829 E+00 \\
1.822 E+00 \\
1.821 E+00 \\
1.824 E+00 \\
1.834 E+00 \\
1.846 E+00 \\
1.858 E+00\end{array}$ & $\begin{array}{l}1.280 \mathrm{E}-02 \\
1.600 \mathrm{E}-02 \\
1.942 \mathrm{E}-02 \\
2.303 \mathrm{E}-02 \\
2.678 \mathrm{E}-02 \\
3.468 \mathrm{E}-02 \\
4.299 \mathrm{E}-02 \\
5.164 \mathrm{E}-02\end{array}$ & $\begin{array}{l}1.862 E+00 \\
1.845 E+00 \\
1.841 E+00 \\
1.844 E+00 \\
1.850 E+00 \\
1.868 E+00 \\
1.889 E+00 \\
1.910 E+00\end{array}$ & $\begin{array}{l}4.367 E-01 \\
5.717 E-01 \\
7.075 E-01 \\
8.432 E-01 \\
9.785 E-01 \\
1.247 E+00 \\
1.514 E+00 \\
1.777 E+00\end{array}$ & $\begin{array}{l}3.57 \\
4.41 \\
5.28 \\
6.17 \\
7.08 \\
8.96 \\
1.09 \\
1.29\end{array}$ & $\begin{array}{l}2.42 \\
3.94 \\
5.43 \\
6.86 \\
8.21 \\
1.06 \\
1.28 \\
1.48\end{array}$ & $\begin{array}{l}-0.059 \\
-0.049 \\
-0.043 \\
-0.038 \\
-0.035 \\
-0.030 \\
-0.027 \\
-0.025\end{array}$ & $\begin{array}{l}0.095 \\
0.085 \\
0.077 \\
0.071 \\
0.066 \\
0.059 \\
0.054 \\
0.049\end{array}$ & $\begin{array}{l}0.087 \\
0.075 \\
0.066 \\
0.059 \\
0.053 \\
0.045 \\
0.040 \\
0.036\end{array}$ \\
\hline $\begin{array}{l}4.0000 \\
4.5000 \\
5.0000 \\
5.5000 \\
6.0000 \\
7.0000 \\
8.0000 \\
9.0000\end{array}$ & $\begin{array}{l}1.870 E+00 \\
1.882 E+00 \\
1.892 E+00 \\
1.902 E+00 \\
1.911 E+00 \\
1.928 E+00 \\
1.943 E+00 \\
1.956 E+00\end{array}$ & $\begin{array}{l}6.058 \mathrm{E}-02 \\
6.976 \mathrm{E}-02 \\
7.917 \mathrm{E}-02 \\
8.876 \mathrm{E}-02 \\
9.854 \mathrm{E}-02 \\
1.185 \mathrm{E}-01 \\
1.391 \mathrm{E}-01 \\
1.601 \mathrm{E}-01\end{array}$ & $\begin{array}{l}1.931 E+00 \\
1.951 E+00 \\
1.971 E+00 \\
1.991 E+00 \\
2.010 E+00 \\
2.047 E+00 \\
2.082 E+00 \\
2.116 E+00\end{array}$ & $\begin{array}{l}2.037 \mathrm{E}+00 \\
2.295 \mathrm{E}+00 \\
2.550 \mathrm{E}+00 \\
2.802 \mathrm{E}+00 \\
3.052 \mathrm{E}+00 \\
3.545 \mathrm{E}+00 \\
4.030 \mathrm{E}+00 \\
4.506 \mathrm{E}+00\end{array}$ & $\begin{array}{l}1.495 \mathrm{E}-02 \\
1.702 \mathrm{E}-02 \\
1.911 \mathrm{E}-02 \\
2.123 \mathrm{E}-02 \\
2.336 \mathrm{E}-02 \\
2.766 \mathrm{E}-02 \\
3.200 \mathrm{E}-02 \\
3.636 \mathrm{E}-02\end{array}$ & $\begin{array}{l}1.660 \mathrm{Et} \\
1.821 \mathrm{E}+ \\
1.967 \mathrm{Et} \\
2.102 \mathrm{E}+ \\
2.227 \mathrm{Et} \\
2.453 \mathrm{E}+ \\
2.652 \mathrm{E}+ \\
2.831 \mathrm{E}+\end{array}$ & $\begin{array}{l}-0.024 \\
-0.023 \\
-0.022 \\
-0.021 \\
-0.021 \\
-0.020 \\
-0.019 \\
-0.018\end{array}$ & & $\begin{array}{l}0.033 \\
0.031 \\
0.029 \\
0.027 \\
0.026 \\
0.024 \\
0.023 \\
0.021\end{array}$ \\
\hline $\begin{array}{l}10.0000 \\
12.5000 \\
15.0000 \\
17.5000 \\
20.0000 \\
25.0000 \\
30.0000 \\
35.0000\end{array}$ & $\begin{array}{l}1.968 E+00 \\
1.993 E+00 \\
2.014 E+00 \\
2.031 E+00 \\
2.046 E+00 \\
2.070 E+00 \\
2.089 E+00 \\
2.105 E+00\end{array}$ & $\begin{array}{l}1.814 \mathrm{E}-01 \\
2.362 \mathrm{E}-01 \\
2.926 \mathrm{E}-01 \\
3.501 \mathrm{E}-01 \\
4.086 \mathrm{E}-01 \\
5.277 \mathrm{E}-01 \\
6.489 \mathrm{E}-01 \\
7.716 \mathrm{E}-01\end{array}$ & $\begin{array}{l}2.149 \mathrm{E}+00 \\
2.230 \mathrm{E}+00 \\
2.306 \mathrm{E}+00 \\
2.381 \mathrm{E}+00 \\
2.454 \mathrm{E}+00 \\
2.598 \mathrm{E}+00 \\
2.738 \mathrm{E}+00 \\
2.876 \mathrm{E}+00\end{array}$ & $\begin{array}{l}4.975 E+00 \\
6.117 E+00 \\
7.219 E+00 \\
8.286 E+00 \\
9.320 E+00 \\
1.130 E+01 \\
1.317 E+01 \\
1.496 E+01\end{array}$ & $\begin{array}{l}4.072 \mathrm{E}-02 \\
5.163 \mathrm{E}-02 \\
6.243 \mathrm{E}-02 \\
7.309 \mathrm{E}-02 \\
8.355 \mathrm{E}-02 \\
1.039 \mathrm{E}-01 \\
1.233 \mathrm{E}-01 \\
1.418 \mathrm{E}-01\end{array}$ & $\begin{array}{l}3.34 \\
3.63 \\
3.88 \\
4.10 \\
4.48 \\
4.80 \\
5.08\end{array}$ & $\begin{array}{l}-0.018 \\
-0.016 \\
-0.015 \\
-0.014 \\
-0.013 \\
-0.010 \\
-0.009 \\
-0.007\end{array}$ & $\begin{array}{l}0 . \\
0 . \\
0 . \\
0 . \\
0 . \\
0 . \\
0 . \\
0 .\end{array}$ & $\begin{array}{l}0.020 \\
0.019 \\
0.017 \\
0.016 \\
0.015 \\
0.013 \\
0.0111 \\
0.010\end{array}$ \\
\hline $\begin{array}{l}40.00000 \\
45.0000 \\
50.0000 \\
55.0000 \\
60.0000 \\
70.0000 \\
80.0000 \\
90.0000\end{array}$ & $\begin{array}{l}118 E+00 \\
129 E+00 \\
139 E+00 \\
148 E+00 \\
156 E+00 \\
170 E+00 \\
182 E+00 \\
193 E+00\end{array}$ & $\begin{array}{l}8.955 E-01 \\
1.021 E+00 \\
1.146 E+00 \\
1.273 E+00 \\
1.400 E+00 \\
1.656 E+00 \\
1.914 E+00 \\
2.173 E+00\end{array}$ & $\begin{array}{l}3.013 E+00 \\
3.150 E+00 \\
3.286 E+00 \\
3.421 E+00 \\
3.556 E+00 \\
3.827 E+00 \\
4.096 E+00 \\
4.366 E+00\end{array}$ & $\begin{array}{l}1.665 E+01 \\
1.828 E+01 \\
1.983 E+01 \\
2.132 E+01 \\
2.276 E+01 \\
2.547 E+01 \\
2.799 E+01 \\
3.035 E+01\end{array}$ & $\begin{array}{l}1.594 \mathrm{E}-01 \\
1.762 \mathrm{E}-01 \\
1.923 \mathrm{E}-01 \\
2.076 \mathrm{E}-01 \\
2.222 \mathrm{E}-01 \\
2.496 \mathrm{E}-01 \\
2.747 \mathrm{E}-01 \\
2.978 \mathrm{E}-01\end{array}$ & $\begin{array}{l}5.326 E+00 \\
5.544 E+00 \\
5.741 E+00 \\
5.921 E+00 \\
6.087 E+00 \\
6.383 E+00 \\
6.641 E+00 \\
6.871 E+00\end{array}$ & & & \\
\hline $\begin{array}{l}0.0000 \\
5.0000 \\
0.0000 \\
5.0000 \\
0.0000 \\
0.0000 \\
0.0000 \\
0.0000\end{array}$ & $\begin{array}{l}2.202 E+00 \\
2.222 E+00 \\
2.238 E+00 \\
2.251 E+00 \\
2.263 E+00 \\
2.282 E+00 \\
2.297 E+00 \\
2.311 E+00\end{array}$ & $\begin{array}{l}2.434 E+00 \\
3.089 E+00 \\
3.749 E+00 \\
4.412 E+00 \\
5.078 E+00 \\
6.416 E+00 \\
7.760 E+00 \\
9.107 E+00\end{array}$ & $\begin{array}{l}4.636 E+00 \\
5.311 E+00 \\
5.987 E+00 \\
6.663 E+00 \\
7.341 E+00 \\
8.698 E+00 \\
1.006 E+01 \\
1.142 E+01\end{array}$ & $\begin{array}{l}258 E+01 \\
.761 E+01 \\
.204 E+01 \\
600 E+01 \\
957 E+01 \\
.582 E+01 \\
116 E+01 \\
.583 E+01\end{array}$ & $\begin{array}{ll}0 & 1 \\
0 & 1 \\
0 & 1 \\
01 & 1 \\
01 \\
01 \\
01 \\
01 \\
01\end{array}$ & $\begin{array}{l}7.077 E+00 \\
7.516 E+00 \\
7.876 E+00 \\
8.182 E+00 \\
8.447 E+00 \\
8.891 E+00 \\
9.254 E+00 \\
9.561 E+00\end{array}$ & $\begin{array}{l}-0 . \\
-0 \\
-0 \\
-0 \\
-0 \\
-0 \\
-0 \\
-0\end{array}$ & & \\
\hline .000 & $\begin{array}{l}349 E+00 \\
357 E+00 \\
370 E+00 \\
381 E+00 \\
391 E+00\end{array}$ & $\begin{array}{l}1.18 \\
1.31 \\
1.45 \\
1.58 \\
1.86 \\
2.13 \\
2.40\end{array}$ & $\begin{array}{l}E+01 \\
E+01 \\
E+01 \\
E+01 \\
E+01 \\
E+01 \\
E+01\end{array}$ & $\begin{array}{l}1 \\
1 \\
1 \\
1 \\
1 \\
1\end{array}$ & $\begin{array}{l}1 \\
1 \\
1 \\
1 \\
1 \\
1 \\
1\end{array}$ & $\begin{array}{l}1.8 \\
1.0 \\
1.0 \\
1.8 \\
1.8\end{array}$ & $\begin{array}{l}-0 . \\
-0 . \\
-0 \\
-0 \\
-0 \\
-0\end{array}$ & $\begin{array}{l}4 \\
4 \\
4 \\
4 \\
4 \\
3 \\
3 \\
3\end{array}$ & $\begin{array}{l}0.001 \\
0.000 \\
0.000 \\
0.000 \\
0.000 \\
0.000 \\
0.000 \\
0.000\end{array}$ \\
\hline 0.0000 & $00 E+00$ & $679 E+01$ & $2.919 E+01$ & $1.002 E+02$ & $.759 \mathrm{E}-01$ & $1.166 \mathrm{E}+01$ & -0.000 & 0.003 & 0.000 \\
\hline
\end{tabular}




\section{$I=75.0 \mathrm{eV} \quad$ DENSITY $=1.000 \mathrm{E}+00 \mathrm{~g} / \mathrm{cm}^{3}$}

\begin{tabular}{|c|c|c|c|c|c|c|c|c|c|}
\hline NERGY & $\begin{array}{l}\text { COLLISION } \\
\mathrm{MeV} \mathrm{cm}^{2} / \mathrm{g}\end{array}$ & $\begin{array}{l}\text { OPPING POWE } \\
\text { RADIATIVE } \\
\mathrm{MeV} \mathrm{cm}^{2} / \mathrm{g}\end{array}$ & $\begin{array}{c}\text { TOTAL } \\
\operatorname{MeV~} \mathrm{cm}^{2} / \mathrm{g}\end{array}$ & $\begin{array}{l}\text { CSDA } \\
\text { RANGE } \\
\mathrm{g} / \mathrm{Cm}^{2}\end{array}$ & $\begin{array}{l}\text { RADIATION } \\
\text { YIELD }\end{array}$ & $\begin{array}{l}\text { DENS.EFF. } \\
\text { CORR. } \\
\text { (DELTA) }\end{array}$ & $\begin{array}{l}d(1 \\
\operatorname{coLL} \\
\text { LOSS }\end{array}$ & $\begin{array}{l}\text { )/dC } 1 \\
\text { SDA } \\
\text { ANGE }\end{array}$ & $\begin{array}{l}\text { RI ) } \\
\text { RAD } \\
\text { YIELD }\end{array}$ \\
\hline $\begin{array}{l}0.0100 \\
0.0125 \\
0.0150 \\
0.0175 \\
0.0200 \\
0.0250 \\
0.0300 \\
0.0350\end{array}$ & $\begin{array}{l}2.256 \mathrm{E}+01 \\
1.897 \mathrm{E}+01 \\
1.647 \mathrm{E}+01 \\
1.461 \mathrm{E}+01 \\
1.317 \mathrm{E}+01 \\
1.109 \mathrm{E}+01 \\
9.653 \mathrm{E}+00 \\
8.592 \mathrm{E}+00\end{array}$ & $\begin{array}{l}3.898 \mathrm{E}-03 \\
3.927 \mathrm{E}-03 \\
3.944 \mathrm{E}-03 \\
3.955 \mathrm{E}-03 \\
3.963 \mathrm{E}-03 \\
3.974 \mathrm{E}-03 \\
3.984 \mathrm{E}-03 \\
3.994 \mathrm{E}-03\end{array}$ & $\begin{array}{l}2.257 \mathrm{E}+01 \\
1.898 \mathrm{E}+01 \\
1.647 \mathrm{E}+01 \\
1.461 \mathrm{E}+01 \\
1.318 \mathrm{E}+01 \\
1.110 \mathrm{E}+01 \\
9.657 \mathrm{E}+00 \\
8.596 \mathrm{E}+00\end{array}$ & $\begin{array}{l}3.72 \\
5.14 \\
6.76 \\
8.56 \\
1.27 \\
1.75 \\
2.30\end{array}$ & $\begin{array}{l}9.408 \mathrm{E}-05 \\
1.133 \mathrm{E}-04 \\
1.316 \mathrm{E}-04 \\
1.492 \mathrm{E}-04 \\
1.663 \mathrm{E}-04 \\
1.990 \mathrm{E}-04 \\
2.301 \mathrm{E}-04 \\
2.599 \mathrm{E}-04\end{array}$ & $\begin{array}{l}0.0 \\
0.0 \\
0.0 \\
0.0 \\
0.0 \\
0.0 \\
0.0 \\
0.0\end{array}$ & $\begin{array}{l}-0.199 \\
-0.190 \\
-0.184 \\
-0.179 \\
-0.175 \\
-0.169 \\
-0.164 \\
-0.160\end{array}$ & $\begin{array}{l}0.227 \\
0.217 \\
0.208 \\
0.202 \\
0.197 \\
0.189 \\
0.182 \\
0.177\end{array}$ & $\begin{array}{l}0.225 \\
0.215 \\
0.207 \\
0.201 \\
0.196 \\
0.188 \\
0.182 \\
0.177\end{array}$ \\
\hline $\begin{array}{l}0.0400 \\
0.0450 \\
0.0500 \\
0.0550 \\
0.0600 \\
0.0700 \\
0.0800 \\
0.0900\end{array}$ & $\begin{array}{l}7.777 E+00 \\
7.130 E+00 \\
6.603 E+00 \\
6.166 E+00 \\
5.797 E+00 \\
5.207 E+00 \\
4.757 E+00 \\
4.402 E+00\end{array}$ & $\begin{array}{l}4.005 \mathrm{E}-03 \\
4.018 \mathrm{E}-03 \\
4.031 \mathrm{E}-03 \\
4.046 \mathrm{E}-03 \\
4.062 \mathrm{E}-03 \\
4.098 \mathrm{E}-03 \\
4.138 \mathrm{E}-03 \\
4.181 \mathrm{E}-03\end{array}$ & $\begin{array}{l}7.781 E+00 \\
7.134 E+00 \\
6.607 E+00 \\
6.170 E+00 \\
5.801 E+00 \\
5.211 E+00 \\
4.762 E+00 \\
4.407 E+00\end{array}$ & $\begin{array}{l}2.919 \mathrm{E}-03 \\
3.591 \mathrm{E}-03 \\
4.320 \mathrm{E}-03 \\
5.103 \mathrm{E}-03 \\
5.940 \mathrm{E}-03 \\
7.762 \mathrm{E}-03 \\
9.773 \mathrm{E}-03 \\
1.196 \mathrm{E}-02\end{array}$ & $\begin{array}{l}2.886 \mathrm{E}-04 \\
3.165 \mathrm{E}-04 \\
3.435 \mathrm{E}-04 \\
3.698 \mathrm{E}-04 \\
3.955 \mathrm{E}-04 \\
4.452 \mathrm{E}-04 \\
4.931 \mathrm{E}-04 \\
5.393 \mathrm{E}-04\end{array}$ & $\begin{array}{l}0.0 \\
0.0 \\
0.0 \\
0.0 \\
0.0 \\
0.0 \\
0.0 \\
0.0\end{array}$ & $\begin{array}{l}-0.157 \\
-0.154 \\
-0.152 \\
-0.150 \\
-0.148 \\
-0.145 \\
-0.142 \\
-0.140\end{array}$ & $\begin{array}{l}0.173 \\
0.170 \\
0.167 \\
0.165 \\
0.162 \\
0.158 \\
0.155 \\
0.153\end{array}$ & $\begin{array}{l}0.173 \\
0.170 \\
0.167 \\
0.164 \\
0.162 \\
0.158 \\
0.155 \\
0.152\end{array}$ \\
\hline $\begin{array}{l}0.1000 \\
0.1250 \\
0.1500 \\
0.1750 \\
0.2000 \\
0.2500 \\
0.3000 \\
0.3500\end{array}$ & $\begin{array}{l}4.115 \mathrm{E}+00 \\
3.591 \mathrm{E}+00 \\
3.238 \mathrm{E}+00 \\
2.984 \mathrm{E}+00 \\
2.793 \mathrm{E}+00 \\
2.528 \mathrm{E}+00 \\
2.355 \mathrm{E}+00 \\
2.233 \mathrm{E}+00\end{array}$ & $\begin{array}{l}4.228 \mathrm{E}-03 \\
4.355 \mathrm{E}-03 \\
4.494 \mathrm{E}-03 \\
4.643 \mathrm{E}-03 \\
4.801 \mathrm{E}-03 \\
5.141 \mathrm{E}-03 \\
5.514 \mathrm{E}-03 \\
5.913 \mathrm{E}-03\end{array}$ & $\begin{array}{l}4.120 \mathrm{E}+00 \\
3.596 \mathrm{E}+00 \\
3.242 \mathrm{E}+00 \\
2.988 \mathrm{E}+00 \\
2.798 \mathrm{E}+00 \\
2.533 \mathrm{E}+00 \\
2.360 \mathrm{E}+00 \\
2.239 \mathrm{E}+00\end{array}$ & $\begin{array}{l}1.431 \mathrm{E}-02 \\
2.083 \mathrm{E}-02 \\
2.817 \mathrm{E}-02 \\
3.622 \mathrm{E}-02 \\
4.487 \mathrm{E}-02 \\
6.372 \mathrm{E}-02 \\
8.421 \mathrm{E}-02 \\
1.060 \mathrm{E}-01\end{array}$ & $\begin{array}{l}5.841 \mathrm{E}-04 \\
6.912 \mathrm{E}-04 \\
7.926 \mathrm{E}-04 \\
8.894 \mathrm{E}-04 \\
9.826 \mathrm{E}-04 \\
1.161 \mathrm{E}-03 \\
1.331 \mathrm{E}-03 \\
1.497 \mathrm{E}-03\end{array}$ & $\begin{array}{l}0.0 \\
0.0 \\
0.0 \\
0.0 \\
0.0 \\
0.0 \\
2.187 \mathrm{E}-03 \\
1.051 \mathrm{E}-02\end{array}$ & $\begin{array}{l}-0.138 \\
-0.134 \\
-0.131 \\
-0.128 \\
-0.126 \\
-0.123 \\
-0.120 \\
-0.118\end{array}$ & $\begin{array}{l}0.150 \\
0.146 \\
0.142 \\
0.139 \\
0.137 \\
0.133 \\
0.130 \\
0.128\end{array}$ & $\begin{array}{l}0.150 \\
0.145 \\
0.142 \\
0.139 \\
0.136 \\
0.132 \\
0.129 \\
0.127\end{array}$ \\
\hline $\begin{array}{l}0.4000 \\
0.4500 \\
0.5000 \\
0.5500 \\
0.6000 \\
0.7000 \\
0.8000 \\
0.9000\end{array}$ & $\begin{array}{l}2.145 \mathrm{E}+00 \\
2.079 \mathrm{E}+00 \\
2.028 \mathrm{E}+00 \\
1.988 \mathrm{E}+00 \\
1.956 \mathrm{E}+00 \\
1.910 \mathrm{E}+00 \\
1.879 \mathrm{E}+00 \\
1.858 \mathrm{E}+00\end{array}$ & $\begin{array}{l}6.339 \mathrm{E}-03 \\
6.787 \mathrm{E}-03 \\
7.257 \mathrm{E}-03 \\
7.747 \mathrm{E}-03 \\
8.254 \mathrm{E}-03 \\
9.312 \mathrm{E}-03 \\
1.043 \mathrm{E}-02 \\
1.159 \mathrm{E}-02\end{array}$ & $\begin{array}{l}2.152 E+00 \\
2.086 E+00 \\
2.035 E+00 \\
1.996 E+00 \\
1.965 E+00 \\
1.919 E+00 \\
1.890 E+00 \\
1.870 E+00\end{array}$ & $\begin{array}{l}1.288 \mathrm{E}-01 \\
1.524 \mathrm{E}-01 \\
1.767 \mathrm{E}-01 \\
2.015 \mathrm{E}-01 \\
2.268 \mathrm{E}-01 \\
2.783 \mathrm{E}-01 \\
3.308 \mathrm{E}-01 \\
3.841 \mathrm{E}-01\end{array}$ & $\begin{array}{l}1.659 \mathrm{E}-03 \\
1.819 \mathrm{E}-03 \\
1.978 \mathrm{E}-03 \\
2.137 \mathrm{E}-03 \\
2.295 \mathrm{E}-03 \\
2.614 \mathrm{E}-03 \\
2.935 \mathrm{E}-03 \\
3.260 \mathrm{E}-03\end{array}$ & $\begin{array}{l}2.188 \mathrm{E}-02 \\
3.663 \mathrm{E}-02 \\
5.424 \mathrm{E}-02 \\
7.393 \mathrm{E}-02 \\
9.538 \mathrm{E}-02 \\
1.422 \mathrm{E}-01 \\
1.925 \mathrm{E}-01 \\
2.448 \mathrm{E}-01\end{array}$ & $\begin{array}{l}-0 . \\
-0.0 \\
-0.0 \\
-0.0 \\
-0.0 \\
-0.0 \\
-0.0 \\
-0.0\end{array}$ & $\begin{array}{l}26 \\
24 \\
23 \\
20 \\
17 \\
10 \\
05 \\
99\end{array}$ & $\begin{array}{l}0.125 \\
0.123 \\
0.121 \\
0.1118 \\
0.1114 \\
0.106 \\
0.099 \\
0.093\end{array}$ \\
\hline $\begin{array}{l}1.0000 \\
1.2500 \\
1.5000 \\
1.7500 \\
2.0000 \\
2.5000 \\
3.0000 \\
3.5000\end{array}$ & $\begin{array}{l}1.844 E+00 \\
1.825 E+00 \\
1.820 E+00 \\
1.821 E+00 \\
1.825 E+00 \\
1.837 E+00 \\
1.850 E+00 \\
1.864 E+00\end{array}$ & $\begin{array}{l}1.280 \mathrm{E}-02 \\
1.600 \mathrm{E}-02 \\
1.942 \mathrm{E}-02 \\
2.303 \mathrm{E}-02 \\
2.678 \mathrm{E}-02 \\
3.468 \mathrm{E}-02 \\
4.299 \mathrm{E}-02 \\
5.164 \mathrm{E}-02\end{array}$ & $\begin{array}{l}1.857 E+00 \\
1.841 E+00 \\
1.839 E+00 \\
1.844 E+00 \\
1.851 E+00 \\
1.871 E+00 \\
1.893 E+00 \\
1.916 E+00\end{array}$ & $\begin{array}{l}4.378 \mathrm{E}-01 \\
5.731 \mathrm{E}-01 \\
7.090 \mathrm{E}-01 \\
8.448 \mathrm{E}-01 \\
9.801 \mathrm{E}-01 \\
1.249 \mathrm{E}+00 \\
1.514 \mathrm{E}+00 \\
1.777 \mathrm{E}+00\end{array}$ & $\begin{array}{l}3.588 \mathrm{E}-03 \\
4.428 \mathrm{E}-03 \\
5.293 \mathrm{E}-03 \\
6.183 \mathrm{E}-03 \\
7.094 \mathrm{E}-03 \\
8.973 \mathrm{E}-03 \\
1.091 \mathrm{E}-02 \\
1.290 \mathrm{E}-02\end{array}$ & $\begin{array}{l}2.983 E-01 \\
4.331 \mathrm{E}-01 \\
5.648 \mathrm{E}-01 \\
6.911 \mathrm{E}-01 \\
8.119 \mathrm{E}-01 \\
1.036 \mathrm{E}+00 \\
1.238 \mathrm{E}+00 \\
1.419 \mathrm{E}+00\end{array}$ & $\begin{array}{l}-0.059 \\
-0.049 \\
-0.043 \\
-0.038 \\
-0.035 \\
-0.030 \\
-0.027 \\
-0.025\end{array}$ & $\begin{array}{l}0.095 \\
0.085 \\
0.077 \\
0.071 \\
0.066 \\
0.059 \\
0.054 \\
0.049\end{array}$ & $\begin{array}{l}0.087 \\
0.075 \\
0.066 \\
0.059 \\
0.053 \\
0.045 \\
0.040 \\
0.036\end{array}$ \\
\hline $\begin{array}{l}4.0000 \\
4.5000 \\
5.0000 \\
5.5000 \\
6.0000 \\
7.0000 \\
8.0000 \\
9.0000\end{array}$ & $\begin{array}{l}1.877 E+00 \\
1.889 E+00 \\
1.900 E+00 \\
1.910 E+00 \\
1.919 E+00 \\
1.936 E+00 \\
1.951 E+00 \\
1.964 E+00\end{array}$ & $\begin{array}{l}6.058 \mathrm{E}-02 \\
6.976 \mathrm{E}-02 \\
7.917 \mathrm{E}-02 \\
8.876 \mathrm{E}-02 \\
9.854 \mathrm{E}-02 \\
1.185 \mathrm{E}-01 \\
1.391 \mathrm{E}-01 \\
1.601 \mathrm{E}-01\end{array}$ & $\begin{array}{l}1.937 E+00 \\
1.958 E+00 \\
1.979 E+00 \\
1.999 E+00 \\
2.018 E+00 \\
2.055 E+00 \\
2.090 E+00 \\
2.124 E+00\end{array}$ & $\begin{array}{l}2.037 \mathrm{E}+00 \\
2.293 \mathrm{E}+00 \\
2.547 \mathrm{E}+00 \\
2.799 \mathrm{E}+00 \\
3.048 \mathrm{E}+00 \\
3.539 \mathrm{E}+00 \\
4.021 \mathrm{E}+00 \\
4.496 \mathrm{E}+00\end{array}$ & $\begin{array}{l}1.493 \mathrm{E}-02 \\
1.698 \mathrm{E}-02 \\
1.907 \mathrm{E}-02 \\
2.117 \mathrm{E}-02 \\
2.329 \mathrm{E}-02 \\
2.757 \mathrm{E}-02 \\
3.189 \mathrm{E}-02 \\
3.623 \mathrm{E}-02\end{array}$ & $\begin{array}{l}1.585 \mathrm{E}+ \\
1.738 \mathrm{E}+ \\
1.880 \mathrm{E} \\
2.011 \mathrm{E}+ \\
2.133 \mathrm{E}+ \\
2.359 \mathrm{E}+ \\
2.555 \mathrm{E}+ \\
2.736 \mathrm{E}+\end{array}$ & $\begin{array}{l}-0.024 \\
-0.023 \\
-0.022 \\
-0.021 \\
-0.021 \\
-0.020 \\
-0.019 \\
-0.018\end{array}$ & $\begin{array}{l}0.046 \\
0.043 \\
0.041 \\
0.039 \\
0.038 \\
0.035 \\
0.033 \\
0.031\end{array}$ & $\begin{array}{l}0.033 \\
0.031 \\
0.029 \\
0.027 \\
0.026 \\
0.024 \\
0.023 \\
0.021\end{array}$ \\
\hline $\begin{array}{l}10.0000 \\
12.5000 \\
15.0000 \\
17.5000 \\
20.0000 \\
25.00000 \\
30.0000 \\
35.0000\end{array}$ & $\begin{array}{l}1.976 \mathrm{E}+00 \\
2.000 \mathrm{E}+00 \\
2.020 \mathrm{E}+00 \\
2.037 \mathrm{E}+00 \\
2.051 \mathrm{E}+00 \\
2.074 \mathrm{E}+00 \\
2.092 \mathrm{E}+00 \\
2.107 \mathrm{E}+00\end{array}$ & $\begin{array}{l}1.814 E-01 \\
2.362 E-01 \\
2.926 E-01 \\
3.501 E-01 \\
4.086 E-01 \\
5.277 E-01 \\
6.489 E-01 \\
7.716 E-01\end{array}$ & $\begin{array}{l}2.157 \mathrm{E}+00 \\
2.237 \mathrm{E}+00 \\
2.313 \mathrm{E}+00 \\
2.387 \mathrm{E}+00 \\
2.459 \mathrm{E}+00 \\
2.601 \mathrm{E}+00 \\
2.741 \mathrm{E}+00 \\
2.878 \mathrm{E}+00\end{array}$ & $\begin{array}{l}4.963 E+00 \\
6.101 E+00 \\
7.200 E+00 \\
8.264 E+00 \\
9.295 E+00 \\
1.127 E+01 \\
1.314 E+01 \\
1.492 E+01\end{array}$ & $\begin{array}{l}4.058 \mathrm{E}-02 \\
5.145 \mathrm{E}-02 \\
6.222 \mathrm{E}-02 \\
7.286 \mathrm{E}-02 \\
8.331 \mathrm{E}-02 \\
1.036 \mathrm{E}-01 \\
1.230 \mathrm{E}-01 \\
1.415 \mathrm{E}-01\end{array}$ & $\begin{array}{l}2.900 \mathrm{E}+00 \\
3.257 \mathrm{E}+00 \\
3.558 \mathrm{E}+00 \\
3.819 \mathrm{E}+00 \\
4.050 \mathrm{E}+00 \\
4.444 \mathrm{E}+00 \\
4.773 \mathrm{E}+00 \\
5.056 \mathrm{E}+00\end{array}$ & $\begin{array}{l}-0.018 \\
-0.016 \\
-0.015 \\
-0.014 \\
-0.013 \\
-0.010 \\
-0.009 \\
-0.007\end{array}$ & $\begin{array}{l}0.030 \\
0.027 \\
0.025 \\
0.024 \\
0.022 \\
0.020 \\
0.018 \\
0.017\end{array}$ & $\begin{array}{l}0.019 \\
0.017 \\
0.016 \\
0.015 \\
0.013 \\
0.011 \\
0.010\end{array}$ \\
\hline $\begin{array}{l}40.0000 \\
45.00000 \\
50.00000 \\
55.0000 \\
60.0000 \\
70.00000 \\
80.00000 \\
90.0000\end{array}$ & $\begin{array}{l}2.13 \\
2.14 \\
2.14 \\
2.15 \\
2.17 \\
2.18 \\
2.19\end{array}$ & $\begin{array}{l}8.955 E-01 \\
1.021 E+00 \\
1.146 E+00 \\
1.273 E+00 \\
1.400 E+00 \\
1.656 E+00 \\
1.914 E+00 \\
2.173 E+00\end{array}$ & $\begin{array}{l}3.151 \mathrm{E}+00 \\
3.287 \mathrm{E}+00 \\
3.422 \mathrm{E}+00 \\
3.558 \mathrm{E}+00 \\
3.828 \mathrm{E}+00 \\
4.097 \mathrm{E}+00 \\
4.367 \mathrm{E}+00\end{array}$ & $\begin{array}{l}1 \\
1 \\
1 \\
1 \\
1\end{array}$ & $\begin{array}{l}1.592 \mathrm{E}-01 \\
1.760 \mathrm{E}-01 \\
1.920 \mathrm{E}-01 \\
2.074 \mathrm{E}-01 \\
2.220 \mathrm{E}-01 \\
2.494 \mathrm{E}-01 \\
2.745 \mathrm{E}-01 \\
2.976 \mathrm{E}-01\end{array}$ & $\begin{array}{l}5.305 \mathrm{E}+00 \\
5.527 \mathrm{E}+00 \\
5.726 \mathrm{E}+00 \\
5.908 \mathrm{E}+00 \\
6.074 \mathrm{E}+00 \\
6.372 \mathrm{E}+00 \\
6.631 \mathrm{E}+00 \\
6.861 \mathrm{E}+00\end{array}$ & $\begin{array}{l}-0 . \\
-0 . \\
-0 \\
-0 . \\
-0 . \\
-0 . \\
-0 . \\
-0 .\end{array}$ & & \\
\hline $\begin{array}{l}.0000 \\
.0000 \\
.0000 \\
.0000 \\
.0000 \\
.0000 \\
.0000 \\
.0000\end{array}$ & $\begin{array}{l}2.203 E+00 \\
2.223 E+00 \\
2.239 E+00 \\
2.252 E+00 \\
2.264 E+00 \\
2.283 E+00 \\
2.298 E+00 \\
2.312 E+00\end{array}$ & $\begin{array}{l}3.08 \\
3.74 \\
4.41 \\
5.07 \\
6.41 \\
7.76 \\
9.10\end{array}$ & $\begin{array}{l}E+00 \\
E+00 \\
E+00 \\
E+00 \\
E+00 \\
E+00 \\
E+01 \\
E+01\end{array}$ & $\begin{array}{l}7 E+01 \\
0 E+01 \\
6 E+01 \\
3 E+01 \\
8 E+01 \\
2 E+01 \\
8 E+01\end{array}$ & $\begin{array}{ll}0 & 1 \\
0 & 1 \\
01 \\
01 \\
01 \\
01 \\
01\end{array}$ & $\begin{array}{l}7.864 \mathrm{E} \\
8.170 \mathrm{E} \\
8.434 \mathrm{E} \\
8.878 \mathrm{E} \\
9.243 \mathrm{E} \\
9.551 \mathrm{E}\end{array}$ & $\begin{array}{l}-0 . \\
-0 . \\
-0 . \\
-0 . \\
-0 . \\
-0 . \\
-0 . \\
-0 .\end{array}$ & $\begin{array}{l}09 \\
08 \\
07 \\
06 \\
06 \\
05 \\
05 \\
05\end{array}$ & $\begin{array}{l}02 \\
02 \\
0 \\
01 \\
01 \\
01 \\
01 \\
01 \\
01\end{array}$ \\
\hline $\begin{array}{l}0.0000 \\
0.00000 \\
0.00000 \\
0.00000 \\
0.0000 \\
0.0000 \\
0.00000 \\
0.0000\end{array}$ & $\begin{array}{l}2.323 E+00 \\
2.333 E+00 \\
2.342 E+00 \\
2.350 E+00 \\
2.357 E+00 \\
2.370 E+00 \\
2.382 E+00 \\
2.392 E+00\end{array}$ & $\begin{array}{l}1.046 E+01 \\
1.181 E+01 \\
1.317 E+01 \\
1.453 E+01 \\
1.589 E+01 \\
1.8611 E+01 \\
2.133 E+01 \\
2.406 E+01\end{array}$ & $\begin{array}{l}1.278 \mathrm{E}+01 \\
1.415 \mathrm{E}+01 \\
1.551 \mathrm{E}+01 \\
1.688 \mathrm{E}+01 \\
1.824 \mathrm{E}+01 \\
2.098 \mathrm{E}+01 \\
2.371 \mathrm{E}+01 \\
2.645 \mathrm{E}+01\end{array}$ & $\begin{array}{l}6.992 \mathrm{E}+01 \\
7.364 \mathrm{E}+01 \\
7.701 \mathrm{E}+01 \\
8.010 \mathrm{E}+01 \\
8.295 \mathrm{E}+01 \\
8.806 \mathrm{E}+01 \\
9.254 \mathrm{E}+01 \\
9.653 \mathrm{E}+01\end{array}$ & $\begin{array}{l}6.179 \mathrm{E}-01 \\
6.411 \mathrm{E}-01 \\
6.612 \mathrm{E}-01 \\
6.788 \mathrm{E}-01 \\
6.944 \mathrm{E}-01 \\
7.208 \mathrm{E}-01 \\
7.424 \mathrm{E}-01 \\
7.605 \mathrm{E}-01\end{array}$ & $\begin{array}{l}9.818 \mathrm{E}+00 \\
1.005 \mathrm{E}+01 \\
1.027 \mathrm{E}+01 \\
1.046 \mathrm{E}+01 \\
1.063 \mathrm{E}+01 \\
1.094 \mathrm{E}+01 \\
1.121 \mathrm{E}+01 \\
1.145 \mathrm{E}+01\end{array}$ & $\begin{array}{l}-0.000 \\
-0.000 \\
-0.000 \\
-0.000 \\
-0.000 \\
-0.000 \\
-0.000 \\
-0.000\end{array}$ & $\begin{array}{l}0.004 \\
0.004 \\
0.004 \\
0.004 \\
0.004 \\
0.003 \\
0.003 \\
0.003\end{array}$ & $\begin{array}{l}0.001 \\
0.000 \\
0.000 \\
0.000 \\
0.000 \\
0.000 \\
0.000 \\
0.000\end{array}$ \\
\hline .0000 & $2.400 E+00$ & $2.679 E+01$ & $2.919 E+01$ & $.001 E+02$ & $.758 \mathrm{E}-01$ & $1.166 E+01$ & -0.000 & 0.003 & 0.000 \\
\hline
\end{tabular}

Evaluated with the density-effect correction of Ashley (1982b). 


\begin{tabular}{|c|c|c|c|c|c|c|c|c|c|}
\hline NEROY & $\begin{array}{l}\text { COLLISION } \\
\text { MeV } \mathrm{cm}^{2} / g\end{array}$ & $\begin{array}{l}\text { OPPING POWE } \\
\text { RADIATIVE } \\
\text { MoV } \mathrm{cm}^{2} / \mathrm{g}\end{array}$ & $\begin{array}{c}\text { TOTAL } \\
\text { MoV } \mathrm{cm}^{2} / \mathrm{g}\end{array}$ & $\begin{array}{l}\text { CSDA } \\
\text { RANGE } \\
g / \mathrm{Cm}^{2}\end{array}$ & $\begin{array}{l}\text { ADIATION } \\
\text { YIELD }\end{array}$ & $\begin{array}{l}\text { DEHS.EFF. } \\
\text { CORR } \\
\text { (DELTA) }\end{array}$ & $\begin{array}{l}\text { d( } 1 \\
\text { coLL } \\
\text { Loss }\end{array}$ & $\begin{array}{l}2 / d(10 \\
\text { CSDA } \\
\text { RANGE }\end{array}$ & $\begin{array}{l}\text { gI) } \\
\text { RAD } \\
\text { YIELD }\end{array}$ \\
\hline $\begin{array}{l}0.0100 \\
0.0125 \\
0.0150 \\
0.0175 \\
0.0200 \\
0.0250 \\
0.0300 \\
0.0350\end{array}$ & $\begin{array}{l}2.277 E+01 \\
1.914 E+01 \\
1.661 E+01 \\
1.473 E+01 \\
1.328 E+01 \\
1.118 E+01 \\
9.726 E+00 \\
8.656 E+00\end{array}$ & $\begin{array}{l}3.898 \mathrm{E}-03 \\
3.927 \mathrm{E}-03 \\
3.944 \mathrm{E}-03 \\
3.955 \mathrm{E}-03 \\
3.963 \mathrm{E}-03 \\
3.974 \mathrm{E}-03 \\
3.984 \mathrm{E}-03 \\
3.994 \mathrm{E}-03\end{array}$ & $\begin{array}{l}2.277 E+01 \\
1.914 E+01 \\
1.661 E+01 \\
1.474 E+01 \\
1.329 E+01 \\
1.119 E+01 \\
9.730 E+00 \\
8.660 E+00\end{array}$ & $\begin{array}{l}2.489 \mathrm{E}-04 \\
3.691 \mathrm{E}-04 \\
5.097 \mathrm{E}-04 \\
6.699 \mathrm{E}-04 \\
8.488 \mathrm{E}-04 \\
1.261 \mathrm{E}-03 \\
1.742 \mathrm{E}-03 \\
2.287 \mathrm{E}-03\end{array}$ & $\begin{array}{l}9.311 E-05 \\
1.121 \mathrm{E}-04 \\
1.303 \mathrm{E}-04 \\
1.479 \mathrm{E}-04 \\
1.648 \mathrm{E}-04 \\
1.973 \mathrm{E}-04 \\
2.282 \mathrm{E}-04 \\
2.578 \mathrm{E}-04\end{array}$ & $\begin{array}{l}0.0 \\
0.0 \\
0.0 \\
0.0 \\
0.0 \\
0.0 \\
0.0 \\
0.0\end{array}$ & $\begin{array}{l}-0.197 \\
-0.189 \\
-0.183 \\
-0.178 \\
-0.174 \\
-0.167 \\
-0.163 \\
-0.159\end{array}$ & $\begin{array}{l}0.225 \\
0.214 \\
0.206 \\
0.200 \\
0.195 \\
0.187 \\
0.181 \\
0.176\end{array}$ & $\begin{array}{l}0.223 \\
0.213 \\
0.205 \\
0.199 \\
0.194 \\
0.186 \\
0.180 \\
0.175\end{array}$ \\
\hline $\begin{array}{l}0.0400 \\
0.0450 \\
0.0500 \\
0.0550 \\
0.0600 \\
0.0700 \\
0.0800 \\
0.0900\end{array}$ & $\begin{array}{l}7.834 E+00 \\
7.181 E+00 \\
6.650 E+00 \\
6.209 E+00 \\
5.836 E+00 \\
5.242 E+00 \\
4.789 E+00 \\
4.431 E+00\end{array}$ & $\begin{array}{l}4.005 E-03 \\
4.018 \mathrm{E}-03 \\
4.031 \mathrm{E}-03 \\
4.046 \mathrm{E}-03 \\
4.062 \mathrm{E}-03 \\
4.098 \mathrm{E}-03 \\
4.138 \mathrm{E}-03 \\
4.181 \mathrm{E}-03\end{array}$ & $\begin{array}{l}7.838 E+00 \\
7.185 E+00 \\
6.654 E+00 \\
6.213 E+00 \\
5.841 E+00 \\
5.246 E+00 \\
4.793 E+00 \\
4.435 E+00\end{array}$ & $\begin{array}{l}2.895 \mathrm{E}-03 \\
3.563 \mathrm{E}-03 \\
4.286 \mathrm{E}-03 \\
5.065 \mathrm{E}-03 \\
5.895 \mathrm{E}-03 \\
7.706 \mathrm{E}-03 \\
9.703 \mathrm{E}-03 \\
1.187 \mathrm{E}-02\end{array}$ & $\begin{array}{l}2.863 \mathrm{E}-04 \\
3.140 \mathrm{E}-04 \\
3.409 \mathrm{E}-04 \\
3.670 \mathrm{E}-04 \\
3.926 \mathrm{E}-04 \\
4.420 \mathrm{E}-04 \\
4.896 \mathrm{E}-04 \\
5.355 \mathrm{E}-04\end{array}$ & $\begin{array}{l}0.0 \\
0.0 \\
0.0 \\
0.0 \\
0.0 \\
0.0 \\
0.0 \\
0.0\end{array}$ & $\begin{array}{l}-0.156 \\
-0.153 \\
-0.151 \\
-0.149 \\
-0.147 \\
-0.144 \\
-0.141 \\
-0.139\end{array}$ & $\begin{array}{l}0.172 \\
0.169 \\
0.166 \\
0.163 \\
0.161 \\
0.157 \\
0.154 \\
0.152\end{array}$ & $\begin{array}{l}0.172 \\
0.168 \\
0.165 \\
0.163 \\
0.161 \\
0.157 \\
0.154 \\
0.151\end{array}$ \\
\hline $\begin{array}{l}0.1000 \\
0.1250 \\
0.1500 \\
0.1750 \\
0.2000 \\
0.2500 \\
0.3000 \\
0.3500\end{array}$ & $\begin{array}{l}4.142 E+00 \\
3.614 E+00 \\
3.257 \mathrm{E}+00 \\
3.001 \mathrm{E}+00 \\
2.809 \mathrm{E}+00 \\
2.542 \mathrm{E}+00 \\
2.368 \mathrm{E}+00 \\
2.247 \mathrm{E}+00\end{array}$ & $\begin{array}{l}4.228 \mathrm{E}-03 \\
4.355 \mathrm{E}-03 \\
4.494 \mathrm{E}-03 \\
4.643 \mathrm{E}-03 \\
4.801 \mathrm{E}-03 \\
5.141 \mathrm{E}-03 \\
5.514 \mathrm{E}-03 \\
5.913 \mathrm{E}-03\end{array}$ & $\begin{array}{l}4.146 E+00 \\
3.618 E+00 \\
3.262 E+00 \\
3.006 E+00 \\
2.814 E+00 \\
2.548 E+00 \\
2.374 E+00 \\
2.253 E+00\end{array}$ & $\begin{array}{l}1.421 \mathrm{E}-02 \\
2.069 \mathrm{E}-02 \\
2.799 \mathrm{E}-02 \\
3.598 \mathrm{E}-02 \\
4.459 \mathrm{E}-02 \\
6.333 \mathrm{E}-02 \\
8.370 \mathrm{E}-02 \\
1.054 \mathrm{E}-01\end{array}$ & $\begin{array}{l}5.801 \mathrm{E}-04 \\
6.866 \mathrm{E}-04 \\
7.874 \mathrm{E}-04 \\
8.837 \mathrm{E}-04 \\
9.765 \mathrm{E}-04 \\
1.154 \mathrm{E}-03 \\
1.323 \mathrm{E}-03 \\
1.488 \mathrm{E}-03\end{array}$ & $\begin{array}{l}0.0 \\
0.0 \\
0.0 \\
0.0 \\
0.0 \\
0.0 \\
0.0 \\
0.0\end{array}$ & $\begin{array}{l}-0.137 \\
-0.133 \\
-0.130 \\
-0.128 \\
-0.126 \\
-0.122 \\
-0.119 \\
-0.117\end{array}$ & $\begin{array}{l}0.149 \\
0.145 \\
0.141 \\
0.138 \\
0.136 \\
0.132 \\
0.130 \\
0.127\end{array}$ & $\begin{array}{l}0.149 \\
0.144 \\
0.141 \\
0.138 \\
0.136 \\
0.132 \\
0.129 \\
0.126\end{array}$ \\
\hline $\begin{array}{l}0.4000 \\
0.4500 \\
0.5000 \\
0.5500 \\
0.6000 \\
0.70000 \\
0.8000 \\
0.9000\end{array}$ & $\begin{array}{l}2.159 E+00 \\
2.094 E+00 \\
2.045 E+00 \\
2.007 E+00 \\
1.977 E+00 \\
1.934 E+00 \\
1.908 E+00 \\
1.891 E+00\end{array}$ & $\begin{array}{l}6.339 \mathrm{E}-03 \\
6.787 \mathrm{E}-03 \\
7.257 \mathrm{E}-03 \\
7.747 \mathrm{E}-03 \\
8.254 \mathrm{E}-03 \\
9.312 \mathrm{E}-03 \\
1.043 \mathrm{E}-02 \\
1.159 \mathrm{E}-02\end{array}$ & $\begin{array}{l}2.166 E+00 \\
2.101 E+00 \\
2.052 E+00 \\
2.014 E+00 \\
1.985 E+00 \\
1.944 E+00 \\
1.918 E+00 \\
1.903 E+00\end{array}$ & $\begin{array}{l}1.280 \mathrm{E}-01 \\
1.515 \mathrm{E}-01 \\
1.756 \mathrm{E}-01 \\
2.002 \mathrm{E}-01 \\
2.252 \mathrm{E}-01 \\
2.761 \mathrm{E}-01 \\
3.279 \mathrm{E}-01 \\
3.803 \mathrm{E}-01\end{array}$ & $\begin{array}{l}1.649 \mathrm{E}-03 \\
1.808 \mathrm{E}-03 \\
1.965 \mathrm{E}-03 \\
2.122 \mathrm{E}-03 \\
2.279 \mathrm{E}-03 \\
2.592 \mathrm{E}-03 \\
2.907 \mathrm{E}-03 \\
3.224 \mathrm{E}-03\end{array}$ & $\begin{array}{l}0.0 \\
0.0 \\
0.0 \\
0.0 \\
0.0 \\
0.0 \\
0.0 \\
0.0\end{array}$ & $\begin{array}{l}-0.115 \\
-0.114 \\
-0.112 \\
-0.111 \\
-0.109 \\
-0.107 \\
-0.105 \\
-0.104\end{array}$ & $\begin{array}{l}0.125 \\
0.123 \\
0.122 \\
0.121 \\
0.119 \\
0.117 \\
0.115 \\
0.114\end{array}$ & $\begin{array}{l}0.124 \\
0.122 \\
0.120 \\
0.119 \\
0.118 \\
0.115 \\
0.1113 \\
0.111\end{array}$ \\
\hline $\begin{array}{l}1.0000 \\
1.2500 \\
1.5000 \\
1.7500 \\
2.0000 \\
2.5000 \\
3.0000 \\
3.5000\end{array}$ & $\begin{array}{l}1.881 E+00 \\
1.874 E+00 \\
1.880 E+00 \\
1.891 E+00 \\
1.905 E+00 \\
1.936 E+00 \\
1.966 E+00 \\
1.995 E+00\end{array}$ & $\begin{array}{l}1.280 \mathrm{E}-02 \\
1.600 \mathrm{E}-02 \\
1.942 \mathrm{E}-02 \\
2.303 \mathrm{E}-02 \\
2.678 \mathrm{E}-02 \\
3.468 \mathrm{E}-02 \\
4.299 \mathrm{E}-02 \\
5.164 \mathrm{E}-02\end{array}$ & $\begin{array}{l}1.894 E+00 \\
1.890 E+00 \\
1.899 E+00 \\
1.914 E+00 \\
1.932 E+00 \\
1.970 E+00 \\
2.009 E+00 \\
2.047 E+00\end{array}$ & $\begin{array}{l}4.330 \mathrm{E}-01 \\
5.652 \mathrm{E}-01 \\
6.972 \mathrm{E}-01 \\
8.284 \mathrm{E}-01 \\
9.584 \mathrm{E}-01 \\
1.215 \mathrm{E}+00 \\
1.466 \mathrm{E}+00 \\
1.713 \mathrm{E}+00\end{array}$ & $\begin{array}{l}3.544 \mathrm{E}-03 \\
4.356 \mathrm{E}-03 \\
5.187 \mathrm{E}-03 \\
6.036 \mathrm{E}-03 \\
6.899 \mathrm{E}-03 \\
8.665 \mathrm{E}-03 \\
1.047 \mathrm{E}-02 \\
1.230 \mathrm{E}-02\end{array}$ & $\begin{array}{l}0.0 \\
0.0 \\
0.0 \\
0.0 \\
0.0 \\
0.0 \\
0.0 \\
0.0\end{array}$ & $\begin{array}{l}-0.102 \\
-0.099 \\
-0.097 \\
-0.095 \\
-0.093 \\
-0.091 \\
-0.089 \\
-0.087\end{array}$ & $\begin{array}{l}0.112 \\
0.110 \\
0.107 \\
0.105 \\
0.104 \\
0.101 \\
0.099 \\
0.097\end{array}$ & $\begin{array}{l}0.110 \\
0.106 \\
0.104 \\
0.101 \\
0.099 \\
0.096 \\
0.094 \\
0.091\end{array}$ \\
\hline $\begin{array}{l}4.0000 \\
4.5000 \\
5.0000 \\
5.5000 \\
6.0000 \\
7.0000 \\
8.0000 \\
9.0000\end{array}$ & $\begin{array}{l}2.022 E+00 \\
2.046 E+00 \\
2.069 E+00 \\
2.090 E+00 \\
2.110 E+00 \\
2.146 E+00 \\
2.178 E+00 \\
2.206 E+00\end{array}$ & $\begin{array}{l}6.058 \mathrm{E}-02 \\
6.976 \mathrm{E}-02 \\
7.917 \mathrm{E}-02 \\
8.876 \mathrm{E}-02 \\
9.854 \mathrm{E}-02 \\
1.185 \mathrm{E}-01 \\
1.391 \mathrm{E}-01 \\
1.601 \mathrm{E}-01\end{array}$ & $\begin{array}{l}2.082 E+00 \\
2.116 E+00 \\
2.148 E+00 \\
2.179 E+00 \\
2.209 E+00 \\
2.265 E+00 \\
2.317 E+00 \\
2.366 E+00\end{array}$ & $\begin{array}{l}1.955 \mathrm{E}+00 \\
2.193 \mathrm{E}+00 \\
2.427 \mathrm{E}+00 \\
2.658 \mathrm{E}+00 \\
2.886 \mathrm{E}+00 \\
3.333 \mathrm{E}+00 \\
3.770 \mathrm{E}+00 \\
4.197 \mathrm{E}+00\end{array}$ & $\begin{array}{l}1.416 \mathrm{E}-02 \\
1.604 \mathrm{E}-02 \\
1.792 \mathrm{E}-02 \\
1.982 \mathrm{E}-02 \\
2.172 \mathrm{E}-02 \\
2.555 \mathrm{E}-02 \\
2.938 \mathrm{E}-02 \\
3.321 \mathrm{E}-02\end{array}$ & $\begin{array}{l}0.0 \\
0.0 \\
0.0 \\
0.0 \\
0.0 \\
0.0 \\
0.0 \\
0.0\end{array}$ & $\begin{array}{l}-0.086 \\
-0.084 \\
-0.083 \\
-0.082 \\
-0.081 \\
-0.080 \\
-0.079 \\
-0.078\end{array}$ & $\begin{array}{l}0.095 \\
0.094 \\
0.093 \\
0.091 \\
0.090 \\
0.089 \\
0.087 \\
0.086\end{array}$ & $\begin{array}{l}0.090 \\
0.088 \\
0.087 \\
0.085 \\
0.084 \\
0.082 \\
0.080 \\
0.079\end{array}$ \\
\hline $\begin{array}{l}10.0000 \\
12.5000 \\
15.0000 \\
17.5000 \\
20.0000 \\
25.0000 \\
30.0000 \\
35.0000\end{array}$ & $\begin{array}{l}2.232 E+00 \\
2.286 E+00 \\
2.332 E+00 \\
2.370 E+00 \\
2.404 E+00 \\
2.460 E+00 \\
2.507 E+00 \\
2.544 E+00\end{array}$ & $\begin{array}{l}1.814 \mathrm{E}-01 \\
2.362 \mathrm{E}-01 \\
2.926 \mathrm{E}-01 \\
3.501 \mathrm{E}-01 \\
4.086 \mathrm{E}-01 \\
5.277 \mathrm{E}-01 \\
6.489 \mathrm{E}-01 \\
7.716 \mathrm{E}-01\end{array}$ & $\begin{array}{l}2.413 E+00 \\
2.523 E+00 \\
2.624 E+00 \\
2.721 E+00 \\
2.813 E+00 \\
2.988 E+00 \\
3.155 E+00 \\
3.316 E+00\end{array}$ & $\begin{array}{l}4.616 E+00 \\
5.628 E+00 \\
6.600 E+00 \\
7.535 E+00 \\
8.439 E+00 \\
1.016 E+01 \\
1.179 E+01 \\
1.334 E+01\end{array}$ & $\begin{array}{l}3.703 \mathrm{E}-02 \\
4.652 \mathrm{E}-02 \\
5.587 \mathrm{E}-02 \\
6.505 \mathrm{E}-02 \\
7.405 \mathrm{E}-02 \\
9.147 \mathrm{E}-02 \\
1.081 \mathrm{E}-01 \\
1.240 \mathrm{E}-01\end{array}$ & $\begin{array}{l}0.0 \\
0.0 \\
0.0 \\
0.0 \\
0.0 \\
0.0 \\
0.0 \\
1.481 E-02\end{array}$ & $\begin{array}{l}-0.077 \\
-0.075 \\
-0.073 \\
-0.072 \\
-0.071 \\
-0.069 \\
-0.068 \\
-0.058\end{array}$ & $\begin{array}{l}0.084 \\
0.082 \\
0.079 \\
0.077 \\
0.076 \\
0.073 \\
0.071 \\
0.068\end{array}$ & $\begin{array}{l}0.077 \\
0.074 \\
0.072 \\
0.070 \\
0.068 \\
0.065 \\
0.062 \\
0.059\end{array}$ \\
\hline $\begin{array}{l}40.0000 \\
45.0000 \\
50.0000 \\
55.0000 \\
60.0000 \\
70.0000 \\
80.0000 \\
90.0000\end{array}$ & $\begin{array}{l}2.574 E+00 \\
2.598 E+00 \\
2.617 E+00 \\
2.634 E+00 \\
2.649 E+00 \\
2.674 E+00 \\
2.694 E+00 \\
2.711 E+00\end{array}$ & $\begin{array}{l}8.955 E-01 \\
1.021 E+00 \\
1.146 E+00 \\
1.273 E+00 \\
1.400 E+00 \\
1.656 E+00 \\
1.914 E+00 \\
2.173 E+00\end{array}$ & $\begin{array}{l}3.469 E+00 \\
3.618 E+00 \\
3.764 E+00 \\
3.907 E+00 \\
4.049 E+00 \\
4.330 E+00 \\
4.608 E+00 \\
4.884 E+00\end{array}$ & $\begin{array}{l}1.481 E+01 \\
1.622 E+01 \\
1.758 E+01 \\
1.888 E+01 \\
2.014 E+01 \\
2.252 E+01 \\
2.476 E+01 \\
2.687 E+01\end{array}$ & $\begin{array}{l}1.392 \mathrm{E}-01 \\
1.537 \mathrm{E}-01 \\
1.677 \mathrm{E}-01 \\
1.811 \mathrm{E}-01 \\
1.940 \mathrm{E}-01 \\
2.184 \mathrm{E}-01 \\
2.410 \mathrm{E}-01 \\
2.620 \mathrm{E}-01\end{array}$ & $\begin{array}{l}6.811 E-02 \\
1.414 E-01 \\
2.242 E-01 \\
3.108 E-01 \\
3.984 E-01 \\
5.703 E-01 \\
7.335 E-01 \\
8.863 E-01\end{array}$ & $\begin{array}{l}-0.049 \\
-0.042 \\
-0.037 \\
-0.033 \\
-0.030 \\
-0.026 \\
-0.023 \\
-0.021\end{array}$ & $\begin{array}{l}0.065 \\
0.063 \\
0.060 \\
0.057 \\
0.055 \\
0.051 \\
0.048 \\
0.045\end{array}$ & $\begin{array}{l}0.055 \\
0.051 \\
0.047 \\
0.043 \\
0.040 \\
0.035 \\
0.031 \\
0.027\end{array}$ \\
\hline $\begin{array}{l}.0000 \\
.0000 \\
.0000 \\
.0000 \\
.0000 \\
.0000 \\
.0000\end{array}$ & $\begin{array}{l}2.726 E+00 \\
2.756 E+00 \\
2.779 E+00 \\
2.799 E+00 \\
2.815 E+00 \\
2.843 E+00 \\
2.865 E+00 \\
2.883 E+00\end{array}$ & $\begin{array}{l}2.434 E+00 \\
3.089 E+00 \\
3.749 E+00 \\
4.412 E+00 \\
5.078 E+00 \\
6.416 E+00 \\
7.760 E+00 \\
9.107 E+00\end{array}$ & $\begin{array}{l}5.159 E+00 \\
5.845 E+00 \\
6.528 E+00 \\
7.211 E+00 \\
7.894 E+00 \\
9.258 E+00 \\
1.062 E+01 \\
1.199 E+01\end{array}$ & $\begin{array}{l}2.886 E+01 \\
3.341 E+01 \\
3.746 E+01 \\
4.110 E+01 \\
4.441 E+01 \\
5.025 E+01 \\
5.529 E+01 \\
5.972 E+01\end{array}$ & $\begin{array}{l}2.817 \mathrm{E}-01 \\
3.256 \mathrm{E}-01 \\
3.634 \mathrm{E}-01 \\
3.963 \mathrm{E}-01 \\
4.252 \mathrm{E}-01 \\
4.741 \mathrm{E}-01 \\
5.138 \mathrm{E}-01 \\
5.469 \mathrm{E}-01\end{array}$ & $\begin{array}{l}1.029 E+00 \\
1.345 \mathrm{E}+00 \\
1.614 \mathrm{E}+00 \\
1.848 \mathrm{E}+00 \\
2.053 \mathrm{E}+00 \\
2.402 \mathrm{E}+00 \\
2.692 \mathrm{E}+00 \\
2.939 \mathrm{E}+00\end{array}$ & $\begin{array}{l}-0.019 \\
-0.017 \\
-0.015 \\
-0.014 \\
-0.014 \\
-0.013 \\
-0.012 \\
-0.011\end{array}$ & & $\begin{array}{l}0.020 \\
0.017 \\
0.014 \\
0.013 \\
0.010 \\
0.009 \\
0.008\end{array}$ \\
\hline $\begin{array}{l}0.0000 \\
0.0000 \\
0.0000 \\
0.0000 \\
0.0000 \\
0.00000 \\
0.0000 \\
0.0000\end{array}$ & $\begin{array}{l}2.899 \mathrm{E}+00 \\
2.912 \mathrm{E}+00 \\
2.924 \mathrm{E}+00 \\
2.935 \mathrm{E}+00 \\
2.945 \mathrm{E}+00 \\
2.962 \mathrm{E}+00 \\
2.977 \mathrm{E}+00 \\
2.989 \mathrm{E}+00\end{array}$ & $\begin{array}{l}1.046 E+01 \\
1.181 E+01 \\
1.317 E+01 \\
1.453 E+01 \\
1.589 E+01 \\
1.861 E+01 \\
2.133 E+01 \\
2.406 E+01\end{array}$ & $\begin{array}{l}336 E+01 \\
473 E+01 \\
609 E+01 \\
.746 E+01 \\
883 E+01 \\
.157 E+01 \\
.431 E+01 \\
.705 E+01\end{array}$ & $\begin{array}{l}367 E+01 \\
723 E+01 \\
048 E+01 \\
346 E+01 \\
622 E+01 \\
117 E+01 \\
554 E+01 \\
944 E+01\end{array}$ & $\left\{\begin{array}{l}1 \\
1 \\
1 \\
1\end{array}\right.$ & $\begin{array}{l}3.156 \mathrm{E}+00 \\
3.348 \mathrm{E}+00 \\
3.522 \mathrm{E}+00 \\
3.681 \mathrm{E}+00 \\
3.827 \mathrm{E}+00 \\
4.088 \mathrm{E}+00 \\
4.318 \mathrm{E}+00 \\
4.524 \mathrm{E}+00\end{array}$ & $\begin{array}{l}-0.011 \\
-0.011 \\
-0.010 \\
-0.010 \\
-0.009 \\
-0.008 \\
-0.008 \\
-0.007\end{array}$ & $\begin{array}{l}0.022 \\
0.021 \\
0.020 \\
0.020 \\
0.019 \\
0.018 \\
0.017 \\
0.016\end{array}$ & $\begin{array}{l}0.007 \\
0.006 \\
0.006 \\
0.005 \\
0.005 \\
0.004 \\
0.004 \\
0.003\end{array}$ \\
\hline .0000 & $3.000 E+00$ & $2.679 E+01$ & $2.979 E+01$ & $.296 E+01$ & $.435 E-01$ & $4.711 E+00$ & -0.006 & 0.016 & 0.003 \\
\hline
\end{tabular}




\begin{tabular}{|c|c|c|c|c|c|c|c|c|c|}
\hline NERGY & $\begin{array}{l}\text { COLLISION } \\
\mathrm{MeV} \mathrm{cm}^{2} / \mathrm{g}\end{array}$ & $\begin{array}{l}\text { OPPING POWE } \\
\text { RADIATIVE } \\
\text { MeV } \mathrm{cm}^{2} / \mathrm{g}\end{array}$ & $\begin{array}{c}\text { TOTAL } \\
\mathrm{MeV} \mathrm{cm}^{2} / \mathrm{g}\end{array}$ & $\begin{array}{l}\operatorname{CSDA} \\
\text { RANGE } \\
\mathrm{g} / \mathrm{Cm}^{2}\end{array}$ & $\begin{array}{l}\text { RADIATION } \\
\text { YIELD }\end{array}$ & $\begin{array}{l}\text { DENS.EFF. } \\
\text { CORR. } \\
\text { (DELTA) }\end{array}$ & $\begin{array}{l}d(s \\
\operatorname{col} L \\
\text { Loss }\end{array}$ & $\begin{array}{l}3 / 6 \\
\text { CSD }\end{array}$ & $\begin{array}{l}\circ \text { OgI ) } \\
\text { RAD } \\
\text { YIELD }\end{array}$ \\
\hline $\begin{array}{l}0.0100 \\
0.0125 \\
0.0150 \\
0.0175 \\
0.0200 \\
0.0250 \\
0.0300 \\
0.0350\end{array}$ & $\begin{array}{l}2.219 E+01 \\
1.857 E+01 \\
1.605 E+01 \\
1.419 E+01 \\
1.276 E+01 \\
1.069 E+01 \\
9.266 E+00 \\
8.218 E+00\end{array}$ & $\begin{array}{l}3.150 \mathrm{E}-03 \\
3.161 \mathrm{E}-03 \\
3.168 \mathrm{E}-03 \\
3.172 \mathrm{E}-03 \\
3.176 \mathrm{E}-03 \\
3.184 \mathrm{E}-03 \\
3.194 \mathrm{E}-03 \\
3.204 \mathrm{E}-03\end{array}$ & $\begin{array}{l}2.219 E+01 \\
1.857 E+01 \\
1.605 E+01 \\
1.420 E+01 \\
1.276 E+01 \\
1.070 E+01 \\
9.269 E+00 \\
8.221 E+00\end{array}$ & $\begin{array}{l}2.520 E-04 \\
3.757 E-04 \\
5.210 E-04 \\
6.869 E-04 \\
8.730 E-04 \\
1.303 E-03 \\
1.807 E-03 \\
2.381 E-03\end{array}$ & $\begin{array}{l}7.749 \mathrm{E}-05 \\
9.323 \mathrm{E}-05 \\
1.083 \mathrm{E}-04 \\
1.229 \mathrm{E}-04 \\
1.371 \mathrm{E}-04 \\
1.644 \mathrm{E}-04 \\
1.905 \mathrm{E}-04 \\
2.158 \mathrm{E}-04\end{array}$ & $\begin{array}{l}0.0 \\
0.0 \\
0.0 \\
0.0 \\
0.0 \\
0.0 \\
0.0 \\
0.0\end{array}$ & $\begin{array}{l}-0.182 \\
-0.175 \\
-0.170 \\
-0.166 \\
-0.163 \\
-0.158 \\
-0.154 \\
-0.151\end{array}$ & $\begin{array}{l}0.204 \\
0.196 \\
0.189 \\
0.184 \\
0.180 \\
0.173 \\
0.168 \\
0.164\end{array}$ & $\begin{array}{l}0.203 \\
0.195 \\
0.188 \\
0.183 \\
0.179 \\
0.173 \\
0.168 \\
0.164\end{array}$ \\
\hline $\begin{array}{l}0.0400 \\
0.0450 \\
0.0500 \\
0.0550 \\
0.0600 \\
0.0700 \\
0.0800 \\
0.0900\end{array}$ & $\begin{array}{l}7.414 E+00 \\
6.777 E+00 \\
6.260 E+00 \\
5.831 E+00 \\
5.469 E+00 \\
4.893 E+00 \\
4.453 E+00 \\
4.107 E+00\end{array}$ & $\begin{array}{l}3.215 \mathrm{E}-03 \\
3.228 \mathrm{E}-03 \\
3.241 \mathrm{E}-03 \\
3.255 \mathrm{E}-03 \\
3.270 \mathrm{E}-03 \\
3.303 \mathrm{E}-03 \\
3.337 \mathrm{E}-03 \\
3.375 \mathrm{E}-03\end{array}$ & $\begin{array}{l}7.417 E+00 \\
6.781 E+00 \\
6.263 E+00 \\
5.834 E+00 \\
5.473 E+00 \\
4.896 E+00 \\
4.456 E+00 \\
4.110 E+00\end{array}$ & $\begin{array}{l}3.02 \\
3.72 \\
4.4 \\
5.32 \\
6.2 \\
8.1 \\
1.02 \\
1.2\end{array}$ & $\begin{array}{l}2.403 \mathrm{E}-04 \\
2.641 \mathrm{E}-04 \\
2.874 \mathrm{E}-04 \\
3.101 \mathrm{E}-04 \\
3.324 \mathrm{E}-04 \\
3.758 \mathrm{E}-04 \\
4.179 \mathrm{E}-04 \\
4.587 \mathrm{E}-04\end{array}$ & $\begin{array}{l}0.0 \\
0.0 \\
0.0 \\
0.0 \\
0.0 \\
0.0 \\
0.0 \\
0.0\end{array}$ & $\begin{array}{l}-0.148 \\
-0.146 \\
-0.144 \\
-0.142 \\
-0.141 \\
-0.139 \\
-0.137 \\
-0.135\end{array}$ & $\begin{array}{l}0.161 \\
0.158 \\
0.156 \\
0.154 \\
0.152 \\
0.149 \\
0.147 \\
0.145\end{array}$ & $\begin{array}{l}0.161 \\
0.158 \\
0.156 \\
0.154 \\
0.152 \\
0.149 \\
0.147 \\
0.144\end{array}$ \\
\hline $\begin{array}{l}0.1000 \\
0.1250 \\
0.1500 \\
0.1750 \\
0.2000 \\
0.2500 \\
0.3000 \\
0.3500\end{array}$ & $\begin{array}{l}3.827 E+00 \\
3.317 E+00 \\
2.973 E+00 \\
2.726 E+00 \\
2.541 E+00 \\
2.280 E+00 \\
2.107 E+00 \\
1.986 E+00\end{array}$ & $\begin{array}{l}3.414 E-03 \\
3.523 \mathrm{E}-03 \\
3.640 \mathrm{E}-03 \\
3.764 \mathrm{E}-03 \\
3.896 \mathrm{E}-03 \\
4.179 \mathrm{E}-03 \\
4.489 \mathrm{E}-03 \\
4.820 \mathrm{E}-03\end{array}$ & $\begin{array}{l}3.830 E+00 \\
3.320 E+00 \\
2.977 E+00 \\
2.730 E+00 \\
2.545 E+00 \\
2.284 E+00 \\
2.111 E+00 \\
1.990 E+00\end{array}$ & $\begin{array}{l}1.515 E-02 \\
2.219 \mathrm{E}-02 \\
3.017 \mathrm{E}-02 \\
3.895 \mathrm{E}-02 \\
4.845 \mathrm{E}-02 \\
6.926 \mathrm{E}-02 \\
9.209 \mathrm{E}-02 \\
1.165 \mathrm{E}-01\end{array}$ & $\begin{array}{l}4.985 \mathrm{E}-04 \\
5.942 \mathrm{E}-04 \\
6.855 \mathrm{E}-04 \\
7.735 \mathrm{E}-04 \\
8.587 \mathrm{E}-04 \\
1.023 \mathrm{E}-03 \\
1.182 \mathrm{E}-03 \\
1.338 \mathrm{E}-03\end{array}$ & $\begin{array}{l}0.0 \\
0.0 \\
0.0 \\
0.0 \\
0.0 \\
3.446 \mathrm{E}-02 \\
7.681 \mathrm{E}-02 \\
1.205 \mathrm{E}-01\end{array}$ & $\begin{array}{l}-0.133 \\
-0.131 \\
-0.128 \\
-0.127 \\
-0.123 \\
-0.097 \\
-0.089 \\
-0.084\end{array}$ & $\begin{array}{l}0.143 \\
0.139 \\
0.137 \\
0.135 \\
0.133 \\
0.124 \\
0.116 \\
0.110\end{array}$ & $\begin{array}{l}0.143 \\
0.139 \\
0.136 \\
0.134 \\
0.132 \\
0.123 \\
0.1114 \\
0.108\end{array}$ \\
\hline $\begin{array}{l}0.4000 \\
0.4500 \\
0.5000 \\
0.5500 \\
0.6000 \\
0.7000 \\
0.8000 \\
0.9000\end{array}$ & $\begin{array}{l}1.897 E+00 \\
1.830 E+00 \\
1.778 E+00 \\
1.738 E+00 \\
1.705 E+00 \\
1.657 E+00 \\
1.624 E+00 \\
1.602 E+00\end{array}$ & $\begin{array}{l}5.173 E-03 \\
5.545 E-03 \\
5.935 E-03 \\
6.340 E-03 \\
6.759 E-03 \\
7.637 E-03 \\
8.559 E-03 \\
9.523 E-03\end{array}$ & $\begin{array}{l}1.902 E+00 \\
1.836 E+00 \\
1.784 E+00 \\
1.744 E+00 \\
1.712 E+00 \\
1.665 E+00 \\
1.633 E+00 \\
1.611 E+00\end{array}$ & $\begin{array}{l}1.422 \mathrm{E}-01 \\
1.690 \mathrm{E}-01 \\
1.967 \mathrm{E}-01 \\
2.250 \mathrm{E}-01 \\
2.540 \mathrm{E}-01 \\
3.133 \mathrm{E}-01 \\
3.740 \mathrm{E}-01 \\
4.356 \mathrm{E}-01\end{array}$ & $\begin{array}{l}1.492 \mathrm{E}-03 \\
1.645 \mathrm{E}-03 \\
1.798 \mathrm{E}-03 \\
1.951 \mathrm{E}-03 \\
2.104 \mathrm{E}-03 \\
2.413 \mathrm{E}-03 \\
2.726 \mathrm{E}-03 \\
3.042 \mathrm{E}-03\end{array}$ & $\begin{array}{l}1.650 \mathrm{E}-01 \\
2.097 \mathrm{E}-01 \\
2.543 \mathrm{E}-01 \\
2.986 \mathrm{E}-01 \\
3.424 \mathrm{E}-01 \\
4.282 \mathrm{E}-01 \\
5.111 \mathrm{E}-01 \\
5.909 \mathrm{E}-01\end{array}$ & $\begin{array}{l}-0.079 \\
-0.075 \\
-0.072 \\
-0.069 \\
-0.066 \\
-0.062 \\
-0.059 \\
-0.056\end{array}$ & $\begin{array}{l}0.105 \\
0.100 \\
0.096 \\
0.093 \\
0.090 \\
0.085 \\
0.081 \\
0.078\end{array}$ & $\begin{array}{l}0.102 \\
0.097 \\
0.093 \\
0.089 \\
0.086 \\
0.080 \\
0.076 \\
0.072\end{array}$ \\
\hline $\begin{array}{l}1.0000 \\
1.2500 \\
1.5000 \\
1.7500 \\
2.0000 \\
2.5000 \\
3.0000 \\
3.5000\end{array}$ & $\begin{array}{l}1.586 E+00 \\
1.563 E+00 \\
1.555 E+00 \\
1.553 E+00 \\
1.555 E+00 \\
1.564 E+00 \\
1.575 E+00 \\
1.587 E+00\end{array}$ & $\begin{array}{l}1.053 \mathrm{E}-02 \\
1.318 \mathrm{E}-02 \\
1.602 \mathrm{E}-02 \\
1.901 \mathrm{E}-02 \\
2.213 \mathrm{E}-02 \\
2.870 \mathrm{E}-02 \\
3.561 \mathrm{E}-02 \\
4.281 \mathrm{E}-02\end{array}$ & $\begin{array}{l}1.596 E+00 \\
1.576 E+00 \\
1.571 E+00 \\
1.572 E+00 \\
1.577 E+00 \\
1.593 E+00 \\
1.611 E+00 \\
1.630 E+00\end{array}$ & $\begin{array}{l}4.980 E-01 \\
6.558 E-01 \\
8.147 E-01 \\
9.739 E-01 \\
1.133 E+00 \\
1.448 E+00 \\
1.760 E+00 \\
2.069 E+00\end{array}$ & $\begin{array}{l}3.363 \mathrm{E}-03 \\
4.185 \mathrm{E}-03 \\
5.033 \mathrm{E}-03 \\
5.906 \mathrm{E}-03 \\
6.800 \mathrm{E}-03 \\
8.643 \mathrm{E}-03 \\
1.055 \mathrm{E}-02 \\
1.249 \mathrm{E}-02\end{array}$ & $\begin{array}{l}6.675 E-01 \\
8.458 E-01 \\
1.007 E+00 \\
1.153 E+00 \\
1.287 E+00 \\
1.524 E+00 \\
1.730 E+00 \\
1.913 E+00\end{array}$ & $\begin{array}{l}-0.054 \\
-0.050 \\
-0.047 \\
-0.045 \\
-0.043 \\
-0.040 \\
-0.038 \\
-0.036\end{array}$ & $\begin{array}{l}0.075 \\
0.069 \\
0.065 \\
0.062 \\
0.059 \\
0.055 \\
0.052 \\
0.050\end{array}$ & $\begin{array}{l}0.069 \\
0.063 \\
0.058 \\
0.055 \\
0.052 \\
0.048 \\
0.045 \\
0.042\end{array}$ \\
\hline $\begin{array}{l}4.0000 \\
4.5000 \\
5.0000 \\
5.5000 \\
6.0000 \\
7.0000 \\
8.0000 \\
9.0000\end{array}$ & $\begin{array}{l}1.598 \mathrm{E}+00 \\
1.609 \mathrm{E}+00 \\
1.619 \mathrm{E}+00 \\
1.628 \mathrm{E}+00 \\
1.637 \mathrm{E}+00 \\
1.652 \mathrm{E}+00 \\
1.665 \mathrm{E}+00 \\
1.677 \mathrm{E}+00\end{array}$ & $\begin{array}{l}5.026 \mathrm{E}-02 \\
5.792 \mathrm{E}-02 \\
6.576 \mathrm{E}-02 \\
7.378 \mathrm{E}-02 \\
8.193 \mathrm{E}-02 \\
9.865 \mathrm{E}-02 \\
1.158 \mathrm{E}-01 \\
1.334 \mathrm{E}-01\end{array}$ & $\begin{array}{l}1.649 E+00 \\
1.667 E+00 \\
1.685 E+00 \\
1.702 E+00 \\
1.719 E+00 \\
1.751 E+00 \\
1.781 E+00 \\
1.810 E+00\end{array}$ & $\begin{array}{l}2.374 E+00 \\
2.675 E+00 \\
2.974 E+00 \\
3.269 E+00 \\
3.561 E+00 \\
4.138 E+00 \\
4.704 E+00 \\
5.261 E+00\end{array}$ & $\begin{array}{l}1.448 \mathrm{E}-02 \\
1.649 \mathrm{E}-02 \\
1.853 \mathrm{E}-02 \\
2.059 \mathrm{E}-02 \\
2.267 \mathrm{E}-02 \\
2.686 \mathrm{E}-02 \\
3.109 \mathrm{E}-02 \\
3.534 \mathrm{E}-02\end{array}$ & $\begin{array}{l}2.077 E+00 \\
2.226 E+00 \\
2.364 E+00 \\
2.492 E+00 \\
2.612 E+00 \\
2.831 E+00 \\
3.029 E+00 \\
3.210 E+00\end{array}$ & $\begin{array}{l}-0.034 \\
-0.032 \\
-0.031 \\
-0.029 \\
-0.028 \\
-0.025 \\
-0.022 \\
-0.019\end{array}$ & $\begin{array}{l}0.048 \\
0.046 \\
0.044 \\
0.043 \\
0.042 \\
0.039 \\
0.037 \\
0.035\end{array}$ & $\begin{array}{l}0.040 \\
0.038 \\
0.037 \\
0.035 \\
0.034 \\
0.031 \\
0.029 \\
0.027\end{array}$ \\
\hline $\begin{array}{l}10.0000 \\
12.50000 \\
15.00000 \\
17.5000 \\
20.0000 \\
25.00000 \\
30.00000 \\
35.0000\end{array}$ & $\begin{array}{l}1.687 \mathrm{E}+00 \\
1.708 \mathrm{E}+00 \\
1.725 \mathrm{E}+00 \\
1.738 \mathrm{E}+00 \\
1.750 \mathrm{E}+00 \\
1.768 \mathrm{E}+00 \\
1.783 \mathrm{E}+00 \\
1.796 \mathrm{E}+00\end{array}$ & $\begin{array}{l}1.513 \mathrm{E}-01 \\
1.971 \mathrm{E}-01 \\
2.444 \mathrm{E}-01 \\
2.927 \mathrm{E}-01 \\
3.417 \mathrm{E}-01 \\
4.417 \mathrm{E}-01 \\
5.435 \mathrm{E}-01 \\
6.466 \mathrm{E}-01\end{array}$ & $\begin{array}{l}1.838 \mathrm{E}+00 \\
1.905 \mathrm{E}+00 \\
1.969 \mathrm{E}+00 \\
2.031 \mathrm{E}+00 \\
2.091 \mathrm{E}+00 \\
2.210 \mathrm{E}+00 \\
2.327 \mathrm{E}+00 \\
2.442 \mathrm{E}+00\end{array}$ & $\begin{array}{l}5.809 E+00 \\
7.145 E+00 \\
8.435 E+00 \\
9.686 E+00 \\
1.090 E+01 \\
1.322 E+01 \\
1.543 E+01 \\
1.753 E+01\end{array}$ & $\begin{array}{l}3.960 E-02 \\
5.027 E-02 \\
6.087 E-02 \\
7.134 E-02 \\
8.165 E-02 \\
1.017 E-01 \\
1.209 E-01 \\
1.393 E-01\end{array}$ & $\begin{array}{l}3.377 E+00 \\
3.745 E+00 \\
4.059 E+00 \\
4.334 E+00 \\
4.577 E+00 \\
4.993 E+00 \\
5.339 E+00 \\
5.636 E+00\end{array}$ & $\begin{array}{l}-0.017 \\
-0.013 \\
-0.010 \\
-0.008 \\
-0.006 \\
-0.004 \\
-0.003 \\
-0.002\end{array}$ & $\begin{array}{l}0.034 \\
0.030 \\
0.027 \\
0.024 \\
0.022 \\
0.019 \\
0.017 \\
0.015\end{array}$ & $\begin{array}{l}0.025 \\
0.021 \\
0.017 \\
0.015 \\
0.013 \\
0.010 \\
0.008 \\
0.006\end{array}$ \\
\hline $\begin{array}{l}40.0000 \\
45.00000 \\
50.00000 \\
55.00000 \\
60.00000 \\
70.0000 \\
80.00000 \\
90.0000\end{array}$ & $\begin{array}{l}1.806 \mathrm{E}+00 \\
1.816 \mathrm{E}+00 \\
1.824 \mathrm{E}+00 \\
1.832 \mathrm{E}+00 \\
1.838 \mathrm{E}+00 \\
1.850 \mathrm{E}+00 \\
1.861 \mathrm{E}+00 \\
1.870 \mathrm{E}+00\end{array}$ & $\begin{array}{l}7.508 E-01 \\
8.559 E-01 \\
9.617 E-01 \\
1.068 E+00 \\
1.175 E+00 \\
1.391 E+00 \\
1.608 E+00 \\
1.826 E+00\end{array}$ & $\begin{array}{l}2.557 \mathrm{E}+00 \\
2.672 \mathrm{E}+00 \\
2.786 \mathrm{E}+00 \\
2.900 \mathrm{E}+00 \\
3.014 \mathrm{E}+00 \\
3.241 \mathrm{E}+00 \\
3.469 \mathrm{E}+00 \\
3.696 \mathrm{E}+00\end{array}$ & $\begin{array}{l}1.953 E+01 \\
2.144 E+01 \\
2.327 E+01 \\
2.503 E+01 \\
2.672 E+01 \\
2.992 E+01 \\
3.290 E+01 \\
3.569 E+01\end{array}$ & $\begin{array}{l}1.568 \mathrm{E}-01 \\
1.735 \mathrm{E}-01 \\
1.894 \mathrm{E}-01 \\
2.046 \mathrm{E}-01 \\
2.192 \mathrm{E}-01 \\
2.465 \mathrm{E}-01 \\
2.715 \mathrm{E}-01 \\
2.946 \mathrm{E}-01\end{array}$ & $\begin{array}{l}5.894 E+00 \\
6.124 E+00 \\
6.330 E+00 \\
6.517 E+00 \\
6.688 E+00 \\
6.992 E+00 \\
7.256 E+00 \\
7.489 E+00\end{array}$ & $\begin{array}{l}-0.002 \\
-0.001 \\
-0.001 \\
-0.001 \\
-0.001 \\
-0.001 \\
-0.001 \\
-0.000\end{array}$ & $\begin{array}{l}0.0114 \\
0.012 \\
0.012 \\
0.011 \\
0.0110 \\
0.0009 \\
0.008 \\
0.008\end{array}$ & \\
\hline $\begin{array}{l}.0000 \\
.0000 \\
00000 \\
00000 \\
.0000 \\
0000 \\
0000 \\
.0000\end{array}$ & $\begin{array}{l}1.878 \mathrm{E}+00 \\
1.895 \mathrm{E}+00 \\
1.910 \mathrm{E}+00 \\
1.921 \mathrm{E}+00 \\
1.932 \mathrm{E}+00 \\
1.949 \mathrm{E}+00 \\
1.963 \mathrm{E}+00 \\
1.975 \mathrm{E}+00\end{array}$ & $\begin{array}{l}2.598 \mathrm{E}+00 \\
3.155 \mathrm{E}+00 \\
3.714 \mathrm{E}+00 \\
4.276 \mathrm{E}+00 \\
5.405 \mathrm{E}+00 \\
6.540 \mathrm{E}+00 \\
7.678 \mathrm{E}+00\end{array}$ & $\begin{array}{l}3.924 E+00 \\
4.494 E+00 \\
5.064 E+00 \\
5.636 E+00 \\
6.208 E+00 \\
7.354 E+00 \\
8.503 E+00 \\
9.653 E+00\end{array}$ & $\begin{array}{l}3.832 E+01 \\
4.427 E+01 \\
4.951 E+01 \\
5.418 E+01 \\
5.841 E+01 \\
6.580 E+01 \\
7.212 E+01 \\
7.763 E+01\end{array}$ & $\begin{array}{l}4.027 \mathrm{E}-01 \\
4.368 \mathrm{E}-01 \\
4.665 \mathrm{E}-01 \\
5.158 \mathrm{E}-01 \\
5.553 \mathrm{E}-01 \\
5.878 \mathrm{E}-01\end{array}$ & $\begin{array}{l}7.698 E+00 \\
8.141 E+00 \\
8.504 E+00 \\
8.811 E+00 \\
9.077 E+00 \\
9.522 E+00 \\
9.886 E+00 \\
1.019 E+01\end{array}$ & $\begin{array}{l}-0.000 \\
-0.000 \\
-0.000 \\
-0.000 \\
-0.000 \\
-0.000 \\
-0.000 \\
-0.000\end{array}$ & $\begin{array}{l}0.006 \\
0.006 \\
0.005 \\
0.005 \\
0.004 \\
0.004 \\
0.004\end{array}$ & $\begin{array}{l}0.001 \\
0.001 \\
0.001 \\
0.001 \\
0.000 \\
0.000 \\
0.000\end{array}$ \\
\hline $\begin{array}{l}0.0000 \\
0.00000 \\
0.00000 \\
0.00000 \\
0.00000 \\
0.00000 \\
0.00000 \\
0.0000\end{array}$ & $\begin{array}{l}1.985 \mathrm{E}+00 \\
1.994 \mathrm{E}+00 \\
2.002 \mathrm{E}+00 \\
2.009 \mathrm{E}+00 \\
2.016 \mathrm{E}+00 \\
2.028 \mathrm{E}+00 \\
2.038 \mathrm{E}+00 \\
2.047 \mathrm{E}+00\end{array}$ & $\begin{array}{l}8.820 E+00 \\
9.964 E+00 \\
1.111 E+01 \\
1.226 E+01 \\
1.340 E+01 \\
1.570 E+01 \\
1.801 E+01 \\
2.031 E+01\end{array}$ & $\begin{array}{l}1.080 E+01 \\
1.196 E+01 \\
1.311 E+01 \\
1.427 E+01 \\
1.542 E+01 \\
1.773 E+01 \\
2.005 E+01 \\
2.236 E+01\end{array}$ & $\begin{array}{l}8.253 E+01 \\
8.692 \mathrm{E}+01 \\
9.091 \mathrm{E}+01 \\
9.457 \mathrm{E}+01 \\
9.794 \mathrm{E}+01 \\
1.040 \mathrm{E}+02 \\
1.093 \mathrm{E}+02 \\
1.140 \mathrm{E}+02\end{array}$ & $\begin{array}{l}6.151 \mathrm{E}-01 \\
6.385 \mathrm{E}-01 \\
6.587 \mathrm{E}-01 \\
6.764 \mathrm{E}-01 \\
6.920 \mathrm{E}-01 \\
7.186 \mathrm{E}-01 \\
7.403 \mathrm{E}-01 \\
7.584 \mathrm{E}-01\end{array}$ & $\begin{array}{l}1.046 E+01 \\
1.070 E+01 \\
1.091 E+01 \\
1.110 E+01 \\
1.127 E+01 \\
1.158 E+01 \\
1.185 E+01 \\
1.208 E+01\end{array}$ & $\begin{array}{l}-0.000 \\
-0.000 \\
-0.000 \\
-0.000 \\
-0.000 \\
-0.000 \\
-0.000 \\
-0.000\end{array}$ & $\begin{array}{l}0.003 \\
0.003 \\
0.003 \\
0.003 \\
0.003 \\
0.003 \\
0.003 \\
0.002\end{array}$ & $\begin{array}{l}0.000 \\
0.000 \\
0.000 \\
0.000 \\
0.000 \\
0.000 \\
0.000 \\
0.000\end{array}$ \\
\hline .0000 & $2.055 E+00$ & $2.262 E+01$ & $2.468 E+01$ & $1.183 E+02$ & $7.739 \mathrm{E}-01$ & $1.229 E+01$ & -0.000 & 0.002 & 0.000 \\
\hline
\end{tabular}


POSITRONS IN CARBON (GRAPHITE)

$I=78.0 \mathrm{oV} \quad$ DENSITY $=1.700 \mathrm{E}+00 \mathrm{~g} / \mathrm{cm}^{3}$

\begin{tabular}{|c|c|c|c|c|c|c|c|c|c|}
\hline ENERGY & 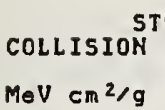 & $\begin{array}{l}\text { OPPING POWE } \\
\text { RADIATIVE } \\
\text { MeV } \mathrm{cm}^{2} / \mathrm{g}\end{array}$ & $\begin{array}{c}\text { TOTAL } \\
\mathrm{MeV} \mathrm{cm}^{2} / \mathrm{g}\end{array}$ & $\begin{array}{l}\text { CSDA } \\
\text { RANGE } \\
g^{\prime} \mathrm{Cm}^{2}\end{array}$ & $\begin{array}{l}\text { DDIATION } \\
\text { YIELD }\end{array}$ & $\begin{array}{l}\text { DEYIS.EFF. } \\
\text { CORR. } \\
\text { (DELTA) }\end{array}$ & $\begin{array}{l}d(1 \\
\operatorname{coLL} \\
\text { Loss }\end{array}$ & )$/ d(1$ & $\begin{array}{l}\text { RI ) } \\
\text { RAD } \\
\text { YIELD }\end{array}$ \\
\hline $\begin{array}{l}0.0100 \\
0.0125 \\
0.0150 \\
0.0175 \\
0.0200 \\
0.0250 \\
0.0300 \\
0.0350\end{array}$ & $\begin{array}{l}2.219 E+01 \\
1.856 E+01 \\
1.605 E+01 \\
1.419 E+01 \\
1.276 E+01 \\
1.069 E+01 \\
9.260 E+00 \\
8.212 E+00\end{array}$ & $\begin{array}{l}3.150 \mathrm{E}-03 \\
3.161 \mathrm{E}-03 \\
3.168 \mathrm{E}-03 \\
3.172 \mathrm{E}-03 \\
3.176 \mathrm{E}-03 \\
3.184 \mathrm{E}-03 \\
3.194 \mathrm{E}-03 \\
3.204 \mathrm{E}-03\end{array}$ & $\begin{array}{l}2.219 E+01 \\
1.857 E+01 \\
1.605 E+01 \\
1.419 E+01 \\
1.276 E+01 \\
1.069 E+01 \\
9.264 E+00 \\
8.215 E+00\end{array}$ & $\begin{array}{l}2.520 E-04 \\
3.758 E-04 \\
5.211 E-04 \\
6.871 E-04 \\
8.732 E-04 \\
1.303 E-03 \\
1.807 E-03 \\
2.382 E-03\end{array}$ & $\begin{array}{l}7.750 E-05 \\
9.324 E-05 \\
1.083 E-04 \\
1.229 E-04 \\
1.371 E-04 \\
1.644 E-04 \\
1.906 E-04 \\
2.159 E-04\end{array}$ & $\begin{array}{l}1.920 \mathrm{E}-03 \\
2.481 \mathrm{E}-03 \\
3.073 \mathrm{E}-03 \\
3.695 \mathrm{E}-03 \\
4.347 \mathrm{E}-03 \\
5.736 \mathrm{E}-03 \\
7.236 \mathrm{E}-03 \\
8.843 \mathrm{E}-03\end{array}$ & $\begin{array}{l}-0.182 \\
-0.175 \\
-0.170 \\
-0.165 \\
-0.162 \\
-0.157 \\
-0.153 \\
-0.149\end{array}$ & $\begin{array}{l}0.204 \\
0.195 \\
0.189 \\
0.184 \\
0.179 \\
0.173 \\
0.168 \\
0.164\end{array}$ & $\begin{array}{l}0.203 \\
0.194 \\
0.188 \\
0.183 \\
0.179 \\
0.172 \\
0.167 \\
0.163\end{array}$ \\
\hline $\begin{array}{l}0.0400 \\
0.0450 \\
0.0500 \\
0.0550 \\
0.0600 \\
0.0700 \\
0.0800 \\
0.0900\end{array}$ & $\begin{array}{l}7.408 E+00 \\
6.771 E+00 \\
6.254 E+00 \\
5.824 E+00 \\
5.462 E+00 \\
4.885 E+00 \\
4.445 E+00 \\
4.098 E+00\end{array}$ & $\begin{array}{l}3.215 \mathrm{E}-03 \\
3.228 \mathrm{E}-03 \\
3.241 \mathrm{E}-03 \\
3.255 \mathrm{E}-03 \\
3.270 \mathrm{E}-03 \\
3.303 \mathrm{E}-03 \\
3.337 \mathrm{E}-03 \\
3.375 \mathrm{E}-03\end{array}$ & $\begin{array}{l}7.412 E+00 \\
6.775 E+00 \\
6.257 E+00 \\
5.828 E+00 \\
5.465 E+00 \\
4.888 E+00 \\
4.448 E+00 \\
4.101 E+00\end{array}$ & $\begin{array}{l}3.024 \mathrm{E}-03 \\
3.730 \mathrm{E}-03 \\
4.499 \mathrm{E}-03 \\
5.328 \mathrm{E}-03 \\
6.214 \mathrm{E}-03 \\
8.153 \mathrm{E}-03 \\
1.030 \mathrm{E}-02 \\
1.265 \mathrm{E}-02\end{array}$ & $\begin{array}{l}2.404 \mathrm{E}-04 \\
2.642 \mathrm{E}-04 \\
2.875 \mathrm{E}-04 \\
3.103 \mathrm{E}-04 \\
3.327 \mathrm{E}-04 \\
3.762 \mathrm{E}-04 \\
4.183 \mathrm{E}-04 \\
4.593 \mathrm{E}-04\end{array}$ & $\begin{array}{l}1.055 \mathrm{E}-02 \\
1.236 \mathrm{E}-02 \\
1.425 \mathrm{E}-02 \\
1.624 \mathrm{E}-02 \\
1.832 \mathrm{E}-02 \\
2.271 \mathrm{E}-02 \\
2.740 \mathrm{E}-02 \\
3.237 \mathrm{E}-02\end{array}$ & $\begin{array}{l}-0.147 \\
-0.144 \\
-0.142 \\
-0.141 \\
-0.139 \\
-0.136 \\
-0.134 \\
-0.132\end{array}$ & $\begin{array}{l}0.160 \\
0.157 \\
0.155 \\
0.153 \\
0.151 \\
0.148 \\
0.145 \\
0.143\end{array}$ & $\begin{array}{l}0.160 \\
0.157 \\
0.155 \\
0.153 \\
0.151 \\
0.148 \\
0.145 \\
0.143\end{array}$ \\
\hline $\begin{array}{l}0.1000 \\
0.1250 \\
0.1500 \\
0.1750 \\
0.2000 \\
0.2500 \\
0.3000 \\
0.3500\end{array}$ & $\begin{array}{l}3.817 \mathrm{E}+00 \\
3.306 \mathrm{E}+00 \\
2.960 \mathrm{E}+00 \\
2.712 \mathrm{E}+00 \\
2.525 \mathrm{E}+00 \\
2.265 \mathrm{E}+00 \\
2.094 \mathrm{E}+00 \\
1.975 \mathrm{E}+00\end{array}$ & $\begin{array}{l}3.414 \mathrm{E}-03 \\
3.523 \mathrm{E}-03 \\
3.640 \mathrm{E}-03 \\
3.764 \mathrm{E}-03 \\
3.896 \mathrm{E}-03 \\
4.179 \mathrm{E}-03 \\
4.489 \mathrm{E}-03 \\
4.820 \mathrm{E}-03\end{array}$ & $\begin{array}{l}3.820 E+00 \\
3.309 E+00 \\
2.964 E+00 \\
2.716 E+00 \\
2.529 E+00 \\
2.270 E+00 \\
2.099 E+00 \\
1.979 E+00\end{array}$ & $\begin{array}{l}517 \mathrm{E}-02 \\
224 \mathrm{E}-02 \\
024 \mathrm{E}-02 \\
907 \mathrm{E}-02 \\
862 \mathrm{E}-02 \\
957 \mathrm{E}-02 \\
253 \mathrm{E}-02 \\
171 \mathrm{E}-01\end{array}$ & $\begin{array}{l}4.992 \mathrm{E}-04 \\
5.953 \mathrm{E}-04 \\
6.872 \mathrm{E}-04 \\
7.758 \mathrm{E}-04 \\
8.618 \mathrm{E}-04 \\
1.028 \mathrm{E}-03 \\
1.188 \mathrm{E}-03 \\
1.345 \mathrm{E}-03\end{array}$ & $\begin{array}{l}3.760 \mathrm{E}-02 \\
5.166 \mathrm{E}-02 \\
6.694 \mathrm{E}-02 \\
8.320 \mathrm{E}-02 \\
1.003 \mathrm{E}-01 \\
1.363 \mathrm{E}-01 \\
1.740 \mathrm{E}-01 \\
2.129 \mathrm{E}-01\end{array}$ & $\begin{array}{l}-0.130 \\
-0.126 \\
-0.123 \\
-0.121 \\
-0.118 \\
-0.1114 \\
-0.1110 \\
-0.106\end{array}$ & $\begin{array}{l}0.141 \\
0.137 \\
0.134 \\
0.131 \\
0.129 \\
0.125 \\
0.121 \\
0.118\end{array}$ & $\begin{array}{l}0.141 \\
0.136 \\
0.133 \\
0.130 \\
0.128 \\
0.124 \\
0.120 \\
0.117\end{array}$ \\
\hline $\begin{array}{l}0.4000 \\
0.4500 \\
0.5000 \\
0.5500 \\
0.5000 \\
0.7000 \\
0.8000 \\
0.9000\end{array}$ & $\begin{array}{l}1.887 E+00 \\
1.821 E+00 \\
1.770 E+00 \\
1.730 E+00 \\
1.698 E+00 \\
1.651 E+00 \\
1.619 E+00 \\
1.597 E+00\end{array}$ & $\begin{array}{l}5.173 E-03 \\
5.545 E-03 \\
5.935 E-03 \\
6.340 E-03 \\
6.759 E-03 \\
7.637 E-03 \\
8.559 E-03 \\
9.523 E-03\end{array}$ & $\begin{array}{l}1.892 E+00 \\
1.827 E+00 \\
1.776 E+00 \\
1.737 E+00 \\
1.705 E+00 \\
1.659 E+00 \\
1.628 E+00 \\
1.607 E+00\end{array}$ & $\begin{array}{l}1.430 \mathrm{E}-01 \\
1.699 \mathrm{E}-01 \\
1.976 \mathrm{E}-01 \\
2.261 \mathrm{E}-01 \\
2.552 \mathrm{E}-01 \\
3.147 \mathrm{E}-01 \\
3.756 \mathrm{E}-01 \\
4.374 \mathrm{E}-01\end{array}$ & $\begin{array}{l}1.500 \mathrm{E}-03 \\
1.654 \mathrm{E}-03 \\
1.807 \mathrm{E}-03 \\
1.961 \mathrm{E}-03 \\
2.114 \mathrm{E}-03 \\
2.424 \mathrm{E}-03 \\
2.737 \mathrm{E}-03 \\
3.054 \mathrm{E}-03\end{array}$ & $\begin{array}{l}2.524 E-01 \\
2.922 \mathrm{E}-01 \\
3.321 \mathrm{E}-01 \\
3.719 \mathrm{E}-01 \\
4.114 \mathrm{E}-01 \\
4.891 \mathrm{E}-01 \\
5.648 \mathrm{E}-01 \\
6.382 \mathrm{E}-01\end{array}$ & $\begin{array}{l}-0.102 \\
-0.099 \\
-0.096 \\
-0.094 \\
-0.091 \\
-0.086 \\
-0.082 \\
-0.079\end{array}$ & $\begin{array}{l}0.116 \\
0.113 \\
0.111 \\
0.109 \\
0.107 \\
0.104 \\
0.100 \\
0.098\end{array}$ & $\begin{array}{l}0.114 \\
0.112 \\
0.109 \\
0.107 \\
0.105 \\
0.100 \\
0.097 \\
0.093\end{array}$ \\
\hline $\begin{array}{l}1.0000 \\
1.2500 \\
1.5000 \\
1.7500 \\
2.0000 \\
2.5000 \\
3.0000 \\
3.5000\end{array}$ & $\begin{array}{l}1.582 E+00 \\
1.561 E+00 \\
1.553 E+00 \\
1.552 E+00 \\
1.555 E+00 \\
1.564 E+00 \\
1.576 E+00 \\
1.589 E+00\end{array}$ & $\begin{array}{l}1.053 \mathrm{E}-0 \\
1.318 \mathrm{E}-0 \\
1.602 \mathrm{E}-0 \\
1.901 \mathrm{E}-0 \\
2.213 \mathrm{E}-0 \\
2.870 \mathrm{E}-0 \\
3.561 \mathrm{E}-0 \\
4.281 \mathrm{E}-0\end{array}$ & $\begin{array}{l}1.592 \\
1.57 \\
1.56 \\
1.57 \\
1.57 \\
1.59 \\
1.61 \\
1.63\end{array}$ & $\begin{array}{l}5.0 \\
6.5 \\
8.1 \\
9.7 \\
1.1 \\
1.4 \\
1.7 \\
2.0\end{array}$ & $\begin{array}{l}3.3 \\
4.1 \\
5.0 \\
5.9 \\
6.8 \\
8.6 \\
1.0 \\
1.2\end{array}$ & $\begin{array}{l}7.091 E-01 \\
8.756 E-01 \\
1.028 E+00 \\
1.167 E+00 \\
1.295 E+00 \\
1.522 E+00 \\
1.720 E+00 \\
1.894 E+00\end{array}$ & $\begin{array}{l}-0.0 \\
-0.0 \\
-0.0 \\
-0.0 \\
-0.0 \\
-0.0 \\
-0.0 \\
-0.0\end{array}$ & $\begin{array}{l}5 \\
0 \\
5 \\
2 \\
9 \\
4 \\
0 \\
8 \\
8\end{array}$ & $\begin{array}{l}0.090 \\
0.084 \\
0.079 \\
0.075 \\
0.071 \\
0.066 \\
0.062 \\
0.059\end{array}$ \\
\hline $\begin{array}{l}4.0000 \\
4.5000 \\
5.0000 \\
5.5000 \\
6.0000 \\
7.0000 \\
8.0000 \\
9.0000\end{array}$ & $\begin{array}{l}1.600 E+00 \\
1.612 E+00 \\
1.622 E+00 \\
1.632 E+00 \\
1.641 E+00 \\
1.658 E+00 \\
1.672 E+00 \\
1.685 E+00\end{array}$ & $\begin{array}{l}5.026 \mathrm{E}-02 \\
5.792 \mathrm{E}-02 \\
6.576 \mathrm{E}-02 \\
7.378 \mathrm{E}-02 \\
8.193 \mathrm{E}-02 \\
9.865 \mathrm{E}-02 \\
1.158 \mathrm{E}-01 \\
1.334 \mathrm{E}-01\end{array}$ & $\begin{array}{l}1.651 E+00 \\
1.670 E+00 \\
1.688 E+00 \\
1.706 E+00 \\
1.723 E+00 \\
1.756 E+00 \\
1.788 E+00 \\
1.818 E+00\end{array}$ & $\begin{array}{l}2.376 E+00 \\
2.677 E+00 \\
2.975 E+00 \\
3.270 E+00 \\
3.561 E+00 \\
4.136 E+00 \\
4.700 E+00 \\
5.255 E+00\end{array}$ & $\begin{array}{l}1.4 \\
1.6 \\
1.8 \\
2.0 \\
2.2 \\
2.6 \\
3.1 \\
3.5\end{array}$ & $\begin{array}{l}2.051 \\
2.193 \\
2.323 \\
2.443 \\
2.555 \\
2.758 \\
2.939 \\
3.104\end{array}$ & $\begin{array}{l}-0 . \\
-0.0 \\
-0.0 \\
-0.2 \\
-0.2 \\
-0.0 \\
-0 . \\
-0 .\end{array}$ & $\begin{array}{l}0.065 \\
0.063 \\
0.061 \\
0.060 \\
0.058 \\
0.056 \\
0.054 \\
0.052\end{array}$ & $\begin{array}{l}0.057 \\
0.055 \\
0.053 \\
0.051 \\
0.050 \\
0.047 \\
0.045 \\
0.042\end{array}$ \\
\hline $\begin{array}{l}10.0000 \\
12.5000 \\
15.0000 \\
17.5000 \\
20.0000 \\
25.0000 \\
30.0000 \\
35.0000\end{array}$ & $\begin{array}{l}1.696 \mathrm{E}+00 \\
1.720 \mathrm{E}+00 \\
1.738 \mathrm{E}+00 \\
1.754 \mathrm{E}+00 \\
1.766 \mathrm{E}+00 \\
1.787 \mathrm{E}+00 \\
1.803 \mathrm{E}+00 \\
1.816 \mathrm{E}+00\end{array}$ & $\begin{array}{l}1.513 \mathrm{E}-01 \\
1.971 \mathrm{E}-01 \\
2.444 \mathrm{E}-01 \\
2.927 \mathrm{E}-01 \\
3.417 \mathrm{E}-01 \\
4.417 \mathrm{E}-01 \\
5.435 \mathrm{E}-01 \\
6.466 \mathrm{E}-01\end{array}$ & $\begin{array}{l}1.847 \mathrm{E}+00 \\
1.917 \mathrm{E}+00 \\
1.983 \mathrm{E}+00 \\
2.046 \mathrm{E}+00 \\
2.108 \mathrm{E}+00 \\
2.228 \mathrm{E}+00 \\
2.346 \mathrm{E}+00 \\
2.462 \mathrm{E}+00\end{array}$ & $\begin{array}{l}5.800 E+00 \\
7.129 E+00 \\
8.411 E+00 \\
9.652 E+00 \\
1.086 E+01 \\
1.316 E+01 \\
1.535 E+01 \\
1.743 E+01\end{array}$ & $\begin{array}{l}3.949 \mathrm{E}-02 \\
5.007 \mathrm{E}-02 \\
6.058 \mathrm{E}-02 \\
7.095 \mathrm{E}-02 \\
8.116 \mathrm{E}-02 \\
1.010 \mathrm{E}-01 \\
1.200 \mathrm{E}-01 \\
1.382 \mathrm{E}-01\end{array}$ & $\begin{array}{l}3.256 \mathrm{E}+00 \\
3.591 \mathrm{E}+00 \\
3.879 \mathrm{E}+00 \\
4.133 \mathrm{E}+00 \\
4.361 \mathrm{E}+00 \\
4.755 \mathrm{E}+00 \\
5.088 \mathrm{E}+00 \\
5.376 \mathrm{E}+00\end{array}$ & $\begin{array}{l}-0.033 \\
-0.028 \\
-0.023 \\
-0.019 \\
-0.016 \\
-0.011 \\
-0.008 \\
-0.006\end{array}$ & $\begin{array}{l}0.050 \\
0.046 \\
0.042 \\
0.039 \\
0.036 \\
0.033 \\
0.028 \\
0.026\end{array}$ & $\begin{array}{l}0.040 \\
0.036 \\
0.031 \\
0.028 \\
0.025 \\
0.020 \\
0.016 \\
0.013\end{array}$ \\
\hline $\begin{array}{l}40.0000 \\
45.0000 \\
50.0000 \\
55.0000 \\
60.0000 \\
70.0000 \\
80.0000 \\
90.0000\end{array}$ & $\begin{array}{l}1.827 E+00 \\
1.837 E+00 \\
1.845 E+00 \\
1.853 E+00 \\
1.860 E+00 \\
1.872 E+00 \\
1.882 E+00 \\
1.892 E+00\end{array}$ & $\begin{array}{l}7.508 \mathrm{E}-01 \\
8.559 \mathrm{E}-01 \\
9.617 \mathrm{E}-01 \\
1.068 \mathrm{E}+00 \\
1.175 \mathrm{E}+00 \\
1.391 \mathrm{E}+00 \\
1.608 \mathrm{E}+00 \\
1.826 \mathrm{E}+00\end{array}$ & $\begin{array}{l}2.578 \mathrm{E}+00 \\
2.692 \mathrm{E}+00 \\
2.807 \mathrm{E}+00 \\
2.921 \mathrm{E}+00 \\
3.035 \mathrm{E}+00 \\
3.263 \mathrm{E}+00 \\
3.490 \mathrm{E}+00 \\
3.718 \mathrm{E}+00\end{array}$ & $\begin{array}{l}1.941 E+01 \\
2.131 E+01 \\
2.313 E+01 \\
2.488 E+01 \\
2.655 E+01 \\
2.973 E+01 \\
3.269 E+01 \\
3.547 E+01\end{array}$ & $\begin{array}{l}1.556 E-01 \\
1.722 \mathrm{E}-01 \\
1.880 \mathrm{E}-01 \\
2.031 \mathrm{E}-01 \\
2.176 \mathrm{E}-01 \\
2.447 \mathrm{E}-01 \\
2.696 \mathrm{E}-01 \\
2.925 \mathrm{E}-01\end{array}$ & $\begin{array}{l}5.628 E+00 \\
5.854 E+00 \\
6.057 E+00 \\
6.241 E+00 \\
6.411 E+00 \\
6.712 E+00 \\
6.974 E+00 \\
7.206 E+00\end{array}$ & $\begin{array}{l}-0.005 \\
-0.004 \\
-0.003 \\
-0.003 \\
-0.002 \\
-0.002 \\
-0.001 \\
-0.001\end{array}$ & $\begin{array}{l}0.023 \\
0.022 \\
0.020 \\
0.019 \\
0.018 \\
0.016 \\
0.015 \\
0.013\end{array}$ & \\
\hline $\begin{array}{l}0000 \\
00000 \\
00000 \\
00000 \\
00000 \\
00000 \\
00000 \\
00000\end{array}$ & $\begin{array}{l}1.900 E+00 \\
1.917 E+00 \\
1.931 E+00 \\
1.943 E+00 \\
1.954 E+00 \\
1.971 E+00 \\
1.985 E+00 \\
1.997 E+00\end{array}$ & $\begin{array}{l}2.598 E+00 \\
3.155 E+00 \\
3.714 E+00 \\
4.276 E+00 \\
5.405 E+00 \\
6.540 E+00 \\
7.678 E+00\end{array}$ & $\begin{array}{l}3.946 \mathrm{E}+00 \\
4.516 \mathrm{E}+00 \\
5.086 \mathrm{E}+00 \\
5.658 \mathrm{E}+00 \\
6.230 \mathrm{E}+00 \\
7.376 \mathrm{E}+00 \\
8.525 \mathrm{E}+00 \\
9.675 \mathrm{E}+00\end{array}$ & $\begin{array}{l}08 E+01 \\
00 E+01 \\
21 E+01 \\
87 E+01 \\
08 E+01 \\
45 E+01 \\
75 E+01 \\
25 E+01\end{array}$ & $\begin{array}{l}3.607 \mathrm{E} \\
4.003 \mathrm{E} \\
4.344 \mathrm{E} \\
4.641 \mathrm{E} \\
5.135 \mathrm{E} \\
5.530 \mathrm{E} \\
5.856 \mathrm{E}\end{array}$ & $\begin{array}{l}8.219 \mathrm{E} \\
8.525 \mathrm{E} \\
8.791 \mathrm{E} \\
9.236 \mathrm{E} \\
9.599 \mathrm{E} \\
9.907 \mathrm{E}\end{array}$ & & $\begin{array}{l}0.013 \\
0.011 \\
0.010 \\
0.009 \\
0.008 \\
0.007 \\
0.007 \\
0.006\end{array}$ & \\
\hline $\begin{array}{l}.0000 \\
.0000 \\
.0000 \\
.0000 \\
.0000 \\
.0000 \\
.0000 \\
.0000\end{array}$ & $\begin{array}{l}2.007 E+00 \\
2.016 E+00 \\
2.024 E+00 \\
2.031 E+00 \\
2.038 E+00 \\
2.050 E+00 \\
2.060 E+00 \\
2.069 E+00\end{array}$ & $\begin{array}{l}8.820 E+00 \\
9.964 E+00 \\
1.111 E+01 \\
1.226 E+01 \\
1.340 E+01 \\
1.570 E+01 \\
1.801 E+01 \\
2.031 E+01\end{array}$ & $\begin{array}{l}1.083 E+01 \\
1.198 E+01 \\
1.313 E+01 \\
1.429 E+01 \\
1.544 E+01 \\
1.775 E+01 \\
2.007 E+01 \\
2.238 E+01\end{array}$ & $\begin{array}{l}8.213 \mathrm{E} \\
8.652 \mathrm{E} \\
9.050 \mathrm{E} \\
9.415 \mathrm{E} \\
9.752 \mathrm{E} \\
1.036 \mathrm{E} \\
1.088 \mathrm{E} \\
1.136 \mathrm{E}\end{array}$ & $\begin{array}{l}6 \cdot 1 \\
6 \cdot 3 \\
6 \cdot 5 \\
6 \cdot 7 \\
6.5 \\
7.9 \\
7.5\end{array}$ & $\begin{array}{l}1 \\
1 \\
1 \\
1 \\
1 \\
1\end{array}$ & $\begin{array}{l}-0.0000 \\
-0.000 \\
-0.000 \\
-0.000 \\
-0.0000 \\
-0.000 \\
-0.000 \\
-0.000\end{array}$ & $\begin{array}{l}06 \\
06 \\
05 \\
05 \\
0 \\
05 \\
5 \\
0\end{array}$ & $\begin{array}{l}0.0001 \\
0.0000 \\
0.000 \\
0.000 \\
0.0000 \\
0.0000 \\
0.0000 \\
0.0000\end{array}$ \\
\hline 0000 & $2.077 \mathrm{E}+00$ & $2.262 E+01$ & $.470 E+01$ & $1.178 E+02$ & $7.723 \mathrm{E}-01$ & $1.200 \mathrm{E}$ & -0.000 & 0.004 & .000 \\
\hline
\end{tabular}




\begin{tabular}{|c|c|c|c|c|c|c|c|c|c|}
\hline $\mathrm{MeV}$ & 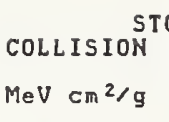 & $\begin{array}{l}\text { RADIATIVE } \\
\text { MeV } \mathrm{cm}^{2} / \mathrm{g}\end{array}$ & $\begin{array}{c}\text { TOTAL } \\
\mathrm{MeV} \mathrm{cm}^{2} / \mathrm{g}\end{array}$ & $\begin{array}{l}\text { CSD } \\
\text { RAN } \\
g / C\end{array}$ & $\mathrm{~N}$ & F. & $\begin{array}{l}d(1 \\
\text { COLL } \\
\text { LOSS }\end{array}$ & $\begin{array}{l}3 / d(1) \\
S D A\end{array}$ & $\begin{array}{l}\text { I ) } \\
\text { RAD }\end{array}$ \\
\hline $\begin{array}{l}0.0100 \\
0.0125 \\
0.0150 \\
0.0175 \\
0.0200 \\
0.0250 \\
0.0300 \\
0.0350\end{array}$ & $\begin{array}{l}1.846 \mathrm{E} \\
1.554 \mathrm{E} \\
1.349 \mathrm{E} \\
1.197 \mathrm{E} \\
1.080 \mathrm{E} \\
9.087 \mathrm{E}\end{array}$ & $\begin{array}{l}6.7 \\
6.7 \\
6.8 \\
6.9 \\
7.0 \\
7.0 \\
7.1\end{array}$ & $\begin{array}{l}1.8 \\
1.5 \\
1.3 \\
1.1 \\
1.0 \\
9.0 \\
7.9 \\
7.0\end{array}$ & $\begin{array}{l}3.0 \\
4.5 \\
6.3 \\
8.2 \\
1.0 \\
1.5\end{array}$ & $\begin{array}{l}E-04 \\
E-04 \\
E-04 \\
E-04 \\
E-04 \\
E-04 \\
E-04 \\
E-04\end{array}$ & $\begin{array}{l}0 \\
0 \\
0 \\
0 \\
0 \\
0 \\
0 \\
0\end{array}$ & $\begin{array}{l}1 \\
2 \\
5 \\
0 \\
6 \\
9 \\
4 \\
0\end{array}$ & $\begin{array}{l}3 \\
1 \\
2 \\
5 \\
9 \\
0 \\
4 \\
8\end{array}$ & $\begin{array}{l}0.239 \\
0.228 \\
0.219 \\
0.212 \\
0.207 \\
0.198 \\
0.192\end{array}$ \\
\hline $\begin{array}{l}0.0400 \\
0.0450 \\
0.0500 \\
0.0550 \\
0.0600 \\
0.0700 \\
0.0800 \\
0.0900\end{array}$ & $\begin{array}{l}5.8 \\
5.3 \\
5.0 \\
4.7 \\
4.2\end{array}$ & $\begin{array}{l}7.16 \\
7.15 \\
7.21 \\
7.25 \\
7.25 \\
7.35\end{array}$ & $\begin{array}{l}E+00 \\
E+00 \\
E+00 \\
E+00 \\
E+00 \\
E+00 \\
E+00 \\
E+00\end{array}$ & $\begin{array}{l}4.3 \\
5.2 \\
6.2 \\
7.2 \\
9.5 \\
1.1\end{array}$ & $\begin{array}{l}4 \\
4 \\
4 \\
4 \\
4 \\
4 \\
3 \\
3\end{array}$ & $\begin{array}{l}0.0 \\
0.0 \\
0.0 \\
0.0 \\
0.0 \\
0.0 \\
0.0 \\
0.0\end{array}$ & $\begin{array}{l}-0 \\
-0 \\
-0 \\
-0 \\
-0 \\
-0 \\
-0\end{array}$ & & $\begin{array}{l}179 \\
176 \\
174 \\
172\end{array}$ \\
\hline $\begin{array}{l}0.1000 \\
0.1250 \\
0.1500 \\
0.1750 \\
0.2000 \\
0.2500 \\
0.3000 \\
0.3500\end{array}$ & $\begin{array}{l}2.88 \\
2.58 \\
2.37 \\
2.22 \\
2.00 \\
1.85\end{array}$ & $\begin{array}{l}7.6 \\
7.8 \\
8.0 \\
8.3 \\
8.8 \\
9.4\end{array}$ & $\begin{array}{l}00 \\
00 \\
00 \\
00 \\
00 \\
00 \\
00 \\
00 \\
00\end{array}$ & $\begin{array}{l}2.5 \\
3.4 \\
4.4 \\
5.5 \\
7.9 \\
1.0 \\
1.3\end{array}$ & $\begin{array}{l}3 \\
3 \\
3 \\
3 \\
3 \\
3\end{array}$ & $\begin{array}{l}0.0 \\
0.0 \\
0.0 \\
0.0 \\
0.0 \\
0.0 \\
7.102 \mathrm{E}-03 \\
1.706 \mathrm{E}-02\end{array}$ & $\begin{array}{l}-0 \\
-0 \\
-0 \\
-0 \\
-0 \\
-0 \\
-0\end{array}$ & & $\begin{array}{l}0 . \\
0 . \\
0 . \\
0 . \\
0 . \\
0 .\end{array}$ \\
\hline $\begin{array}{l}0.4000 \\
0.4500 \\
0.5000 \\
0.5500 \\
0.6000 \\
0.7000 \\
0.8000 \\
0.9000\end{array}$ & $\begin{array}{l}1.627 \\
1.58 \\
1.55 \\
1.52 \\
1.49 \\
1.46 \\
1.45\end{array}$ & $\begin{array}{l}1.15 \\
1.23 \\
1.30 \\
1.35 \\
1.56 \\
1.7\end{array}$ & $\begin{array}{l}+00 \\
+00 \\
+00 \\
+00 \\
+00 \\
+00 \\
+00 \\
+00\end{array}$ & $\begin{array}{l}1 . \\
2 \\
2 \\
2 \\
3 \\
4 . \\
4 .\end{array}$ & $\begin{array}{l}3 \\
3 \\
3 \\
3 \\
3\end{array}$ & $\begin{array}{l}2.8 \\
4.1 \\
5.5 \\
6.9 \\
8.4 \\
1.1 \\
1.4 \\
1.7\end{array}$ & $\begin{array}{l}-0 \\
-0 \\
-0 \\
-0 \\
-0 \\
-0 \\
-0 \\
-0\end{array}$ & & $\begin{array}{l}0 . \\
0 . \\
0 . \\
0 . \\
0 . \\
0 . \\
0 .\end{array}$ \\
\hline $\begin{array}{l}1.0000 \\
1.2500 \\
1.5000 \\
1.7500 \\
2.0000 \\
2.5000 \\
3.0000 \\
3.5000\end{array}$ & $\begin{array}{l}1.43 \\
1.43 \\
1.44 \\
1.45 \\
1.47\end{array}$ & $\begin{array}{l}-02 \\
-02 \\
-02 \\
-02 \\
-02 \\
-02 \\
-02 \\
-02\end{array}$ & $\begin{array}{l}E+00 \\
E+00 \\
E+00 \\
E+00 \\
E+00 \\
E+00 \\
E+00 \\
E+00\end{array}$ & $\begin{array}{l}7 \\
8 \\
1 \\
1 \\
1 \\
1\end{array}$ & $\begin{array}{l}3 \\
3 \\
2 \\
2 \\
2 \\
2 \\
2\end{array}$ & $\begin{array}{l}2.0 \\
2.8 \\
3.6 \\
4.3 \\
5.1 \\
6.6 \\
8.1 \\
9.6\end{array}$ & $\begin{array}{l}-0.0 \\
-0.8 \\
-0.8 \\
-0.2 \\
-0.0 \\
-0.8 \\
-0.8 \\
-0 .\end{array}$ & & $\begin{array}{l}0 . \\
0 . \\
0 . \\
0 . \\
0 . \\
0 . \\
0 .\end{array}$ \\
\hline $\begin{array}{l}4.0000 \\
4.5000 \\
5.0000 \\
5.5000 \\
6.0000 \\
7.0000 \\
8.0000 \\
9.0000\end{array}$ & $\begin{array}{l}.513 \mathrm{E} \\
.523 \mathrm{E} \\
.533 \mathrm{E} \\
.541 \mathrm{E} \\
.556 \mathrm{E} \\
.570 \mathrm{E}\end{array}$ & $\begin{array}{l}1.1 \\
1.2 \\
1.4 \\
1.5 \\
1.8 \\
2.2 \\
2.5\end{array}$ & $\begin{array}{l}E+00 \\
+00 \\
+00 \\
+00 \\
E+00 \\
E+00 \\
E+00 \\
E+00\end{array}$ & $\begin{array}{l}2.8 \\
3.1 \\
3.4 \\
3.7 \\
4.3 \\
4.8\end{array}$ & $\begin{array}{l}3 \\
3 \\
4 \\
4\end{array}$ & $\begin{array}{l}1.1 \\
1.2 \\
1.3 \\
1.4 \\
1.6 \\
1.8 \\
2.0 \\
2.1\end{array}$ & $\begin{array}{l}-0 . \\
-0 . \\
-0 . \\
-0 . \\
-0 . \\
-0 . \\
-0 . \\
-0 .\end{array}$ & & $\begin{array}{l}54 \\
50 \\
46 \\
43 \\
38 \\
34\end{array}$ \\
\hline $\begin{array}{l}10.0000 \\
12.5000 \\
15.0000 \\
17.5000 \\
20.0000 \\
25.0000 \\
30.0000 \\
35.0000\end{array}$ & $\begin{array}{l}+00 \\
+00 \\
+00 \\
+00 \\
+00 \\
+00 \\
+00\end{array}$ & $\begin{array}{ll}0 & 1 \\
0 & 1 \\
0 & 1 \\
0 & 1 \\
0 & 1 \\
0 & 1 \\
0 & 0\end{array}$ & $\begin{array}{l}0 \\
0 \\
0 \\
0 \\
0 \\
0\end{array}$ & $\begin{array}{l}7 . \\
8 . \\
9 . \\
1 . \\
1 .\end{array}$ & 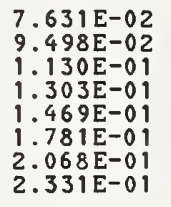 & $\begin{array}{l}2.3 \\
2.8 \\
2.8 \\
3.2 \\
3.9 \\
3.8 \\
4.9 \\
4.4\end{array}$ & $\begin{array}{l}-0 \\
-0 \\
-0 \\
-0 \\
-0 \\
-0 \\
-0 \\
-0\end{array}$ & & \\
\hline $\begin{array}{l}40.00 \\
45.00 \\
50.00 \\
55.00 \\
60.00 \\
70.00 \\
80.00 \\
90.00\end{array}$ & & $\begin{array}{l}1 \\
1 \\
1 \\
2 \\
2 \\
2 \\
3\end{array}$ & $\begin{array}{ll}0 & 0 \\
0 & 0 \\
0 & 0 \\
0 & 0 \\
0 & 0 \\
0 & 0 \\
0 & 0 \\
0 & 0\end{array}$ & & 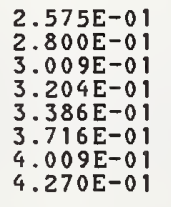 & $\begin{array}{l}4 . \\
4.8 \\
5.8 \\
5.2 \\
5 . \\
5 . \\
5 . \\
6 .\end{array}$ & $\begin{array}{l}-0 \\
-0 \\
-0 \\
-0 \\
-0 \\
-0 \\
-0 \\
-0\end{array}$ & & \\
\hline $\begin{array}{l}0 \\
0 \\
0 \\
0\end{array}$ & $\begin{array}{l}0 \\
0 \\
0\end{array}$ & & & & & & $\begin{array}{l}-0 \\
-0 \\
-0 \\
-0 \\
-0 \\
-0\end{array}$ & & \\
\hline $\begin{array}{l}.0000 \\
.00000 \\
.0000 \\
.0000\end{array}$ & $\begin{array}{l}0 \\
0\end{array}$ & $\begin{array}{l}1 \\
1 \\
1 \\
2 \\
2 \\
2 \\
3\end{array}$ & $\begin{array}{l}1 \\
1 \\
1 \\
1\end{array}$ & 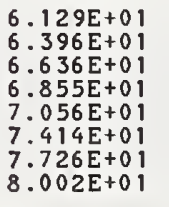 & 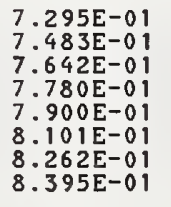 & $\begin{array}{l}0 \\
0 \\
0 \\
0 \\
0 \\
1\end{array}$ & $\begin{array}{l}-0 \\
-0 \\
-0\end{array}$ & & $\begin{array}{l}1 \\
1 \\
1 \\
1 \\
0 \\
0\end{array}$ \\
\hline 1.00 & $2 \mathrm{E}+0 \mathrm{O}$ & 0 & $9 E+0$ & 50 & 7 & 92 & 00 & 0.00 & \\
\hline
\end{tabular}




\begin{tabular}{|c|c|c|c|c|c|c|c|c|c|}
\hline $\mathrm{MeV}$ & $V \mathrm{~cm}^{2} / \mathrm{g}$ & $\mathrm{MeV} \mathrm{cm}^{2} / \mathrm{g}$ & $\begin{array}{l}\text { TOTAL } \\
\mathrm{eV} \mathrm{cm}^{2} / \mathrm{g}\end{array}$ & $\begin{array}{l}\text { CSDA } \\
\text { RANGE } \\
\mathrm{g} / \mathrm{Cm}^{2}\end{array}$ & ON & $\begin{array}{l}\text { DENS. EFF. } \\
\text { CORR } \\
\text { (DELTA) }\end{array}$ & $\begin{array}{r}d c \\
\text { coll } \\
\text { LOSS }\end{array}$ & C & $\begin{array}{l}\text { II ) } \\
\text { RAD } \\
\text { YIELD }\end{array}$ \\
\hline $\begin{array}{l}0.0100 \\
0.0125 \\
0.0150 \\
0.0175 \\
0.0200 \\
0.0250 \\
0.0300\end{array}$ & $\begin{array}{ll}0 & 1 \\
0 & 1 \\
0 & 1 \\
0 & 0 \\
0 & 0 \\
0 & 0 \\
0 & 0\end{array}$ & $\begin{array}{l}1.3 \\
1.3 \\
1.3 \\
1.4\end{array}$ & $\left\{\begin{array}{l}1 \\
1 \\
1 \\
0 \\
0 \\
0 \\
0 \\
0\end{array}\right.$ & $\begin{array}{l}04 \\
04 \\
04 \\
03 \\
03 \\
03 \\
03\end{array}$ & $\begin{array}{l}04 \\
04 \\
04 \\
04 \\
04 \\
04 \\
03\end{array}$ & $\begin{array}{l}0.0 \\
0.0 \\
0.0 \\
0.0 \\
0.0 \\
0.0 \\
0.0 \\
0.0\end{array}$ & & & $\begin{array}{l}0.283 \\
0.267 \\
0.255 \\
0.246 \\
0.239 \\
0.227 \\
0.219 \\
0.212\end{array}$ \\
\hline $\begin{array}{l}0.0400 \\
0.0450 \\
0.0500 \\
0.0550 \\
0.0600 \\
0.0700 \\
0.0800 \\
0.0900\end{array}$ & $\begin{array}{l}+00 \\
+00 \\
+00 \\
+00 \\
+00 \\
+00 \\
+00 \\
+00\end{array}$ & $\begin{array}{l}1.58 \\
1.60 \\
1.61 \\
1.64 \\
1.66\end{array}$ & $\begin{array}{l}4.5 \\
4.2 \\
3.8\end{array}$ & $\begin{array}{l}.03 \\
03 \\
03 \\
03 \\
03 \\
02 \\
02\end{array}$ & $\begin{array}{l}03 \\
03 \\
03 \\
03 \\
03 \\
03 \\
03 \\
03\end{array}$ & $\begin{array}{l}0.0 \\
0.0 \\
0.0 \\
0.0 \\
0.0 \\
0.0 \\
0.0 \\
0.0\end{array}$ & & $\begin{array}{l}1 \\
6 \\
2 \\
8 \\
5 \\
5 \\
0 \\
36 \\
3\end{array}$ & $\begin{array}{l}0.207 \\
0.203 \\
0.199 \\
0.196 \\
0.193 \\
0.188\end{array}$ \\
\hline $\begin{array}{l}0.1000 \\
0.1250 \\
0.1500 \\
0.1750 \\
0.2000 \\
0.2500 \\
0.3000\end{array}$ & $\begin{array}{ll}0 & 0 \\
0 & 0 \\
0 & 0 \\
0 & 0 \\
0 & 0 \\
0 & 0 \\
0 & 0 \\
0 & 0\end{array}$ & $\begin{array}{l}1.9 \\
1.8 \\
1.8 \\
2.9 \\
2.9\end{array}$ & $\begin{array}{l}0 \\
0 \\
0 \\
0 \\
0\end{array}$ & $\begin{array}{l}02 \\
02 \\
02 \\
02 \\
02 \\
02 \\
01\end{array}$ & $\begin{array}{l}3 \\
3 \\
3 \\
3 \\
3 \\
3 \\
3 \\
3\end{array}$ & $\begin{array}{l}0.0 \\
0.0 \\
2.607 \mathrm{E}-03 \\
2.460 \mathrm{E}-02 \\
4.545 \mathrm{E}-02 \\
3.470 \mathrm{E}-02 \\
1.217 \mathrm{E}-01\end{array}$ & & & $\begin{array}{l}0 . \\
0 . \\
0 . \\
0 .\end{array}$ \\
\hline $\begin{array}{l}0.4000 \\
0.4500 \\
0.5000 \\
0.5500 \\
0.6000 \\
0.7000 \\
0.8000 \\
0.9000\end{array}$ & $\begin{array}{l}00 \\
00 \\
00 \\
00 \\
00 \\
00 \\
00 \\
.00\end{array}$ & $\begin{array}{l}2.7 \\
2.9 \\
3.0 \\
3.4\end{array}$ & $\begin{array}{l}0 \\
0 \\
0 \\
0 \\
0\end{array}$ & $\begin{array}{l}1 \\
1 \\
1 \\
1 \\
1\end{array}$ & $\begin{array}{l}3 \\
2 \\
2 \\
2 \\
2 \\
2 \\
2\end{array}$ & $\begin{array}{l}1 \\
1 \\
1 \\
1 \\
1\end{array}$ & & & 0.10 \\
\hline $\begin{array}{l}1.0000 \\
1.2500 \\
1.5000 \\
1.7500 \\
2.0000 \\
2.5000 \\
3.0000\end{array}$ & $\begin{array}{l}1.225 \\
1.230 \\
1.236 \\
1.251 \\
1.266\end{array}$ & $\begin{array}{l}6.7 \\
7.8 \\
9.1 \\
1.1\end{array}$ & $\begin{array}{l}0 \\
0 \\
0 \\
0 \\
0 \\
0\end{array}$ & $\begin{array}{l}1 \\
0 \\
0 \\
0 \\
0\end{array}$ & $\begin{array}{l}2 \\
2 \\
2 \\
2 \\
2 \\
2 \\
2 \\
2\end{array}$ & 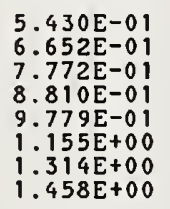 & $\begin{array}{l}-0 \\
-0 \\
-0 \\
-0 \\
-0 \\
-0 \\
-0\end{array}$ & & $\begin{array}{l}0.08 \\
0.08 \\
0.07 \\
0.08 \\
0.08\end{array}$ \\
\hline $\begin{array}{l}4.5000 \\
5.0000 \\
5.5000 \\
6.0000 \\
7.0000\end{array}$ & $\begin{array}{l}0 \\
0 \\
0 \\
0 \\
0\end{array}$ & $\begin{array}{l}2 \\
2 \\
2 \\
3 \\
3\end{array}$ & $\begin{array}{l}0 \\
0 \\
0 \\
0 \\
0\end{array}$ & 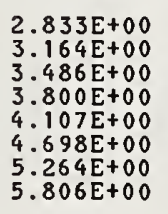 & & & & & $\begin{array}{l}0.0 \\
0.0 \\
0.0 \\
0.0\end{array}$ \\
\hline $\begin{array}{l}0 \\
0 \\
0 \\
0 \\
0\end{array}$ & $\begin{array}{l}0 \\
0 \\
0 \\
0 \\
0 \\
0 \\
0\end{array}$ & $\begin{array}{l}8 . \\
1 . \\
1 .\end{array}$ & $\begin{array}{l}0 \\
0 \\
0 \\
0 \\
0\end{array}$ & 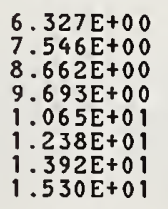 & & -7 & & & \\
\hline $\begin{array}{l}0 \\
0 \\
0 \\
0 \\
0\end{array}$ & & & $\begin{array}{l}0 \\
0 \\
0\end{array}$ & $\begin{array}{l}01 \\
01 \\
0 \\
0 \\
01 \\
01 \\
01 \\
01\end{array}$ & & & & & \\
\hline $\begin{array}{l}0 \\
0 \\
0 \\
0\end{array}$ & $\begin{array}{l}0 \\
0 \\
0 \\
0 \\
0\end{array}$ & & & & & & & & \\
\hline 0 & $\begin{array}{l}1 \\
1 \\
1 \\
1 \\
1\end{array}$ & $\begin{array}{l}3 \\
4\end{array}$ & 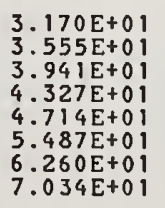 & $\begin{array}{l}318 E+01 \\
67 E+01 \\
501 E+01 \\
22 E+01 \\
32 E+01 \\
29 E+01\end{array}$ & & & & $\begin{array}{l}2 \\
1 \\
1 \\
1 \\
1 \\
10 \\
0 \\
10\end{array}$ & \\
\hline & e cora & 01 & $7.808 \mathrm{E}+01$ & $.485 E+01$ & $\begin{array}{l}y .1<4 t-01 \\
\end{array}$ & $14 E+01$ & -0.000 & .0 & 0.00 \\
\hline
\end{tabular}




\begin{tabular}{|c|c|c|c|c|c|c|c|c|c|}
\hline ENERGY & 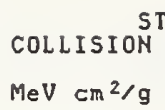 & 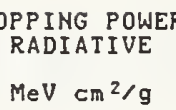 & 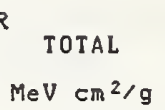 & $\begin{array}{l}\text { CSDA } \\
\text { RANGE } \\
\mathrm{g} / \mathrm{Cm}^{2}\end{array}$ & $\begin{array}{l}\text { RADIATION } \\
\text { YIELD }\end{array}$ & $\begin{array}{l}\text { DENS.EFF. } \\
\text { CORR. } \\
\text { (DELTA) }\end{array}$ & $\begin{array}{l}d c \\
\text { COLL } \\
\text { LOSS }\end{array}$ & $\begin{array}{l}\text { J'd } \\
\text { CSDA }\end{array}$ & $\begin{array}{l}\circ \text { Og } \\
\text { RAD } \\
\text { YIELD }\end{array}$ \\
\hline $\begin{array}{l}0.0100 \\
0.0125 \\
0.0150 \\
0.0175 \\
0.0200 \\
0.0250 \\
0.0300 \\
0.0350\end{array}$ & $\begin{array}{l}1.303 E+01 \\
1.110 E+01 \\
9.726 E+00 \\
8.690 E+00 \\
7.880 E+00 \\
6.689 E+00 \\
5.853 E+00 \\
5.231 E+00\end{array}$ & $\begin{array}{l}1.634 \mathrm{E}-02 \\
1.754 \mathrm{E}-02 \\
1.849 \mathrm{E}-02 \\
1.927 \mathrm{E}-02 \\
1.992 \mathrm{E}-02 \\
2.099 \mathrm{E}-02 \\
2.184 \mathrm{E}-02 \\
2.254 \mathrm{E}-02\end{array}$ & $\begin{array}{l}1.305 E+01 \\
1.112 E+01 \\
9.744 E+00 \\
8.709 E+00 \\
7.900 E+00 \\
6.710 E+00 \\
5.875 E+00 \\
5.254 E+00\end{array}$ & $\begin{array}{l}4.587 \mathrm{E}-04 \\
6.670 \mathrm{E}-04 \\
9.078 \mathrm{E}-04 \\
1.180 \mathrm{E}-03 \\
1.482 \mathrm{E}-03 \\
2.171 \mathrm{E}-03 \\
2.970 \mathrm{E}-03 \\
3.871 \mathrm{E}-03\end{array}$ & $\begin{array}{l}6.026 E-04 \\
7.651 E-04 \\
9.272 E-04 \\
1.088 E-03 \\
1.248 E-03 \\
1.564 E-03 \\
1.874 E-03 \\
2.178 E-03\end{array}$ & $\begin{array}{l}0.0 \\
0.0 \\
0.0 \\
0.0 \\
0.0 \\
0.0 \\
0.0 \\
0.0\end{array}$ & $\begin{array}{l}-0.270 \\
-0.256 \\
-0.245 \\
-0.237 \\
-0.230 \\
-0.220 \\
-0.212 \\
-0.206\end{array}$ & $\begin{array}{l}0.334 \\
0.312 \\
0.295 \\
0.282 \\
0.272 \\
0.257 \\
0.246 \\
0.237\end{array}$ & $\begin{array}{l}0.317 \\
0.296 \\
0.282 \\
0.270 \\
0.261 \\
0.248 \\
0.238 \\
0.230\end{array}$ \\
\hline $\begin{array}{l}0.0400 \\
0.0450 \\
0.0500 \\
0.0550 \\
0.0600 \\
0.0700 \\
0.0800 \\
0.0900\end{array}$ & $\begin{array}{l}4.749 E+00 \\
4.364 E+00 \\
4.049 E+00 \\
3.786 E+00 \\
3.564 E+00 \\
3.206 E+00 \\
2.932 E+00 \\
2.715 E+00\end{array}$ & $\begin{array}{l}2.314 \mathrm{E}-02 \\
2.367 \mathrm{E}-02 \\
2.414 \mathrm{E}-02 \\
2.458 \mathrm{E}-02 \\
2.497 \mathrm{E}-02 \\
2.569 \mathrm{E}-02 \\
2.634 \mathrm{E}-02 \\
2.693 \mathrm{E}-02\end{array}$ & $\begin{array}{l}4.773 E+00 \\
4.388 E+00 \\
4.073 E+00 \\
3.811 E+00 \\
3.589 E+00 \\
3.232 E+00 \\
2.958 E+00 \\
2.742 E+00\end{array}$ & $\begin{array}{l}4.872 \mathrm{E}-03 \\
5.965 \mathrm{E}-03 \\
7.149 \mathrm{E}-03 \\
8.419 \mathrm{E}-03 \\
9.772 \mathrm{E}-03 \\
1.271 \mathrm{E}-02 \\
1.595 \mathrm{E}-02 \\
1.947 \mathrm{E}-02\end{array}$ & $\begin{array}{l}2.477 \mathrm{E}-03 \\
2.771 \mathrm{E}-03 \\
3.060 \mathrm{E}-03 \\
3.345 \mathrm{E}-03 \\
3.625 \mathrm{E}-03 \\
4.172 \mathrm{E}-03 \\
4.704 \mathrm{E}-03 \\
5.222 \mathrm{E}-03\end{array}$ & $\begin{array}{l}0.0 \\
0.0 \\
0.0 \\
0.0 \\
0.0 \\
0.0 \\
0.0 \\
0.0\end{array}$ & $\begin{array}{l}-0.201 \\
-0.197 \\
-0.194 \\
-0.191 \\
-0.189 \\
-0.184 \\
-0.181 \\
-0.178\end{array}$ & $\begin{array}{l}0.230 \\
0.224 \\
0.219 \\
0.215 \\
0.211 \\
0.205 \\
0.200 \\
0.196\end{array}$ & $\begin{array}{l}0.224 \\
0.218 \\
0.214 \\
0.210 \\
0.207 \\
0.201 \\
0.197 \\
0.193\end{array}$ \\
\hline $\begin{array}{l}0.1000 \\
0.1250 \\
0.1500 \\
0.1750 \\
0.2000 \\
0.2500 \\
0.3000 \\
0.3500\end{array}$ & $\begin{array}{l}2.537 E+00 \\
2.210 E+00 \\
1.989 E+00 \\
1.829 E+00 \\
1.709 E+00 \\
1.542 E+00 \\
1.433 E+00 \\
1.357 E+00\end{array}$ & $\begin{array}{l}2.748 \mathrm{E}-02 \\
2.875 \mathrm{E}-02 \\
2.990 \mathrm{E}-02 \\
3.098 \mathrm{E}-02 \\
3.203 \mathrm{E}-02 \\
3.413 \mathrm{E}-02 \\
3.628 \mathrm{E}-02 \\
3.852 \mathrm{E}-02\end{array}$ & $\begin{array}{l}2.564 E+00 \\
2.239 E+00 \\
2.018 E+00 \\
1.860 E+00 \\
1.741 E+00 \\
1.576 E+00 \\
1.469 E+00 \\
1.395 E+00\end{array}$ & $\begin{array}{l}2.324 \mathrm{E}-02 \\
3.372 \mathrm{E}-02 \\
4.551 \mathrm{E}-02 \\
5.844 \mathrm{E}-02 \\
7.235 \mathrm{E}-02 \\
1.026 \mathrm{E}-01 \\
1.356 \mathrm{E}-01 \\
1.705 \mathrm{E}-01\end{array}$ & $\begin{array}{l}5.727 E-03 \\
6.940 \mathrm{E}-03 \\
8.090 \mathrm{E}-03 \\
9.183 \mathrm{E}-03 \\
1.023 \mathrm{E}-02 \\
1.219 \mathrm{E}-02 \\
1.403 \mathrm{E}-02 \\
1.576 \mathrm{E}-02\end{array}$ & $\begin{array}{l}5.406 \mathrm{E}-03 \\
2.558 \mathrm{E}-02 \\
4.392 \mathrm{E}-02 \\
6.093 \mathrm{E}-02 \\
7.694 \mathrm{E}-02 \\
1.068 \mathrm{E}-01 \\
1.348 \mathrm{E}-01 \\
1.613 \mathrm{E}-01\end{array}$ & $\begin{array}{l}-0.159 \\
-0.155 \\
-0.151 \\
-0.147 \\
-0.144 \\
-0.139 \\
-0.135 \\
-0.131\end{array}$ & $\begin{array}{l}0.191 \\
0.180 \\
0.172 \\
0.167 \\
0.162 \\
0.155 \\
0.150 \\
0.146\end{array}$ & $\begin{array}{l}0.188 \\
0.177 \\
0.169 \\
0.164 \\
0.159 \\
0.152 \\
0.147 \\
0.143\end{array}$ \\
\hline $\begin{array}{l}0.4000 \\
0.4500 \\
0.5000 \\
0.5500 \\
0.6000 \\
0.7000 \\
0.8000 \\
0.9000\end{array}$ & $\begin{array}{l}1.302 E+00 \\
1.261 E+00 \\
1.230 E+00 \\
1.206 E+00 \\
1.188 E+00 \\
1.161 E+00 \\
1.145 E+00 \\
1.135 E+00\end{array}$ & $\begin{array}{l}4.085 \mathrm{E}-02 \\
4.328 \mathrm{E}-02 \\
4.580 \mathrm{E}-02 \\
4.840 \mathrm{E}-02 \\
5.107 \mathrm{E}-02 \\
5.659 \mathrm{E}-02 \\
6.233 \mathrm{E}-02 \\
6.827 \mathrm{E}-02\end{array}$ & $\begin{array}{l}1.343 E+00 \\
1.304 E+00 \\
1.276 E+00 \\
1.255 E+00 \\
1.239 E+00 \\
1.218 E+00 \\
1.207 E+00 \\
1.203 E+00\end{array}$ & $\begin{array}{l}2.071 E-01 \\
2.449 E-01 \\
2.837 E-01 \\
3.232 E-01 \\
3.633 E-01 \\
4.448 E-01 \\
5.273 E-01 \\
6.104 E-01\end{array}$ & $\begin{array}{l}1.742 \mathrm{E}-02 \\
1.902 \mathrm{E}-02 \\
2.057 \mathrm{E}-02 \\
2.208 \mathrm{E}-02 \\
2.357 \mathrm{E}-02 \\
2.647 \mathrm{E}-02 \\
2.923 \mathrm{E}-02 \\
3.206 \mathrm{E}-02\end{array}$ & $\begin{array}{l}1.867 E-01 \\
2.112 E-01 \\
2.349 E-01 \\
2.580 E-01 \\
2.804 E-01 \\
3.237 E-01 \\
3.651 E-01 \\
4.048 E-01\end{array}$ & $\begin{array}{l}-0.128 \\
-0.125 \\
-0.123 \\
-0.120 \\
-0.118 \\
-0.115 \\
-0.111 \\
-0.108\end{array}$ & $\begin{array}{l}0.142 \\
0.139 \\
0.136 \\
0.134 \\
0.132 \\
0.128 \\
0.125 \\
0.122\end{array}$ & $\begin{array}{l}0.139 \\
0.136 \\
0.133 \\
0.131 \\
0.129 \\
0.125 \\
0.121 \\
0.118\end{array}$ \\
\hline $\begin{array}{l}1.0000 \\
1.2500 \\
1.5000 \\
1.7500 \\
2.0000 \\
2.5000 \\
3.0000 \\
3.5000\end{array}$ & $\begin{array}{l}1.129 E+00 \\
1.124 E+00 \\
1.127 E+00 \\
1.134 E+00 \\
1.142 E+00 \\
1.160 E+00 \\
1.177 E+00 \\
1.194 E+00\end{array}$ & $\begin{array}{l}7.439 \\
9.038 \\
1.072 \\
1.247 \\
1.428 \\
1.802 \\
2.190 \\
2.589\end{array}$ & $\begin{array}{l}1.203 \\
1.214 \\
1.234 \\
1.259 \\
1.285 \\
1.340 \\
1.396 \\
1.452\end{array}$ & $\begin{array}{l}6.93 \\
9.00 \\
1.10 \\
1.30 \\
1.50 \\
1.88 \\
2.24 \\
2.60\end{array}$ & $\begin{array}{l}3.4 \\
4.1 \\
4.7 \\
5.4 \\
6.0 \\
7.3 \\
8.5 \\
9.7\end{array}$ & $\begin{array}{l}4.4 \\
5.3 \\
6.1 \\
6.9 \\
7.6 \\
8.9 \\
1.0 \\
1.1\end{array}$ & $\begin{array}{l}-0.105 \\
-0.100 \\
-0.095 \\
-0.091 \\
-0.087 \\
-0.081 \\
-0.077 \\
-0.073\end{array}$ & $\begin{array}{l}0.1119 \\
0.1114 \\
0.1109 \\
0.105 \\
0.102 \\
0.096 \\
0.092 \\
0.088\end{array}$ & $\begin{array}{l}0.115 \\
0.108 \\
0.103 \\
0.098 \\
0.094 \\
0.087 \\
0.082 \\
0.077\end{array}$ \\
\hline $\begin{array}{l}4.0000 \\
4.5000 \\
5.0000 \\
5.5000 \\
6.0000 \\
7.0000 \\
8.0000 \\
9.0000\end{array}$ & $\begin{array}{l}1.208 E+00 \\
1.222 E+00 \\
1.234 E+00 \\
1.245 E+00 \\
1.256 E+00 \\
1.274 E+00 \\
1.290 E+00 \\
1.303 E+00\end{array}$ & $\begin{array}{l}2.99 \\
3.41 \\
3.83 \\
4.26 \\
4.69 \\
5.57 \\
6.47 \\
7.38\end{array}$ & $\begin{array}{l}1.508 E+00 \\
1.563 E+00 \\
1.618 E+00 \\
1.672 E+00 \\
1.725 E+00 \\
1.832 E+00 \\
1.937 E+00 \\
2.042 E+00\end{array}$ & $\begin{array}{l}2.937 E+00 \\
3.263 E+00 \\
3.577 E+00 \\
3.882 E+00 \\
4.176 E+00 \\
4.738 E+00 \\
5.269 E+00 \\
5.772 E+00\end{array}$ & $\begin{array}{l}1.1 \\
1.3 \\
1.4 \\
1.5 \\
1.7 \\
1.8 \\
2.0\end{array}$ & $\begin{array}{l}1.22 \\
1.32 \\
1.41 \\
1.49 \\
1.57 \\
1.72 \\
1.86 \\
1.98\end{array}$ & $\begin{array}{l}-0.070 \\
-0.066 \\
-0.064 \\
-0.061 \\
-0.058 \\
-0.054 \\
-0.050 \\
-0.046\end{array}$ & $\begin{array}{l}0.084 \\
0.081 \\
0.079 \\
0.076 \\
0.074 \\
0.070 \\
0.066 \\
0.063\end{array}$ & $\begin{array}{l}0.073 \\
0.069 \\
0.066 \\
0.063 \\
0.060 \\
0.055 \\
0.051 \\
0.047\end{array}$ \\
\hline $\begin{array}{l}10.0000 \\
12.5000 \\
15.0000 \\
17.5000 \\
20.0000 \\
25.00000 \\
30.0000 \\
35.0000\end{array}$ & $\begin{array}{l}1.315 E+00 \\
1.340 E+00 \\
1.360 E+00 \\
1.376 E+00 \\
1.390 E+00 \\
1.412 E+00 \\
1.429 E+00 \\
1.444 E+00\end{array}$ & $\begin{array}{l}8.305 \mathrm{E}-01 \\
1.065 \mathrm{E}+00 \\
1.304 \mathrm{E}+00 \\
1.547 \mathrm{E}+00 \\
1.794 \mathrm{E}+00 \\
2.293 \mathrm{E}+00 \\
2.800 \mathrm{E}+00 \\
3.313 \mathrm{E}+00\end{array}$ & $\begin{array}{l}2.146 E+00 \\
2.405 E+00 \\
2.664 E+00 \\
2.923 E+00 \\
3.183 E+00 \\
3.705 E+00 \\
4.230 E+00 \\
4.757 E+00\end{array}$ & $\begin{array}{l}6.250 E+00 \\
7.349 E+00 \\
8.336 E+00 \\
9.232 E+00 \\
1.005 E+01 \\
1.151 E+01 \\
1.277 E+01 \\
1.388 E+01\end{array}$ & $\begin{array}{l}2.23 \\
2.62 \\
2.96 \\
3.26 \\
3.54 \\
4.01 \\
4.41 \\
4.75\end{array}$ & $\begin{array}{l}2.110 E+00 \\
2.383 E+00 \\
2.6211 E+00 \\
2.833 E+00 \\
3.023 E+00 \\
3.352 E+00 \\
3.632 E+00 \\
3.874 E+00\end{array}$ & $\begin{array}{l}-0.043 \\
-0.037 \\
-0.033 \\
-0.030 \\
-0.028 \\
-0.024 \\
-0.022 \\
-0.020\end{array}$ & $\begin{array}{l}0.061 \\
0.055 \\
0.051 \\
0.047 \\
0.045 \\
0.040 \\
0.037 \\
0.035\end{array}$ & $\begin{array}{l}0.044 \\
0.038 \\
0.033 \\
0.029 \\
0.026 \\
0.021 \\
0.018 \\
0.016\end{array}$ \\
\hline $\begin{array}{l}40.0000 \\
45.0000 \\
50.0000 \\
55.0000 \\
60.0000 \\
70.00000 \\
80.0000 \\
90.0000\end{array}$ & $\begin{array}{l}1.46 \\
1.47 \\
1.48 \\
1.49 \\
1.50 \\
1.51 \\
1.52\end{array}$ & $\begin{array}{l}4.35 \\
4.87 \\
5.40 \\
5.93 \\
6.9 \\
8.00 \\
9.1\end{array}$ & $\begin{array}{l}5.2 \\
5.8 \\
6.3 \\
6.8 \\
7.4 \\
8.5 \\
9.5 \\
1.0\end{array}$ & $\begin{array}{l}1.4 \\
1.5 \\
1.6 \\
1.7 \\
1.8 \\
1.9 \\
2.0 \\
2.1\end{array}$ & $\begin{array}{l}5.3 \\
5.5 \\
5.7 \\
5.9 \\
6.2 \\
6.4 \\
6.7\end{array}$ & $\begin{array}{l}4.2 \\
4.4 \\
4.6 \\
4.7 \\
5.0 \\
5.2 \\
5.4\end{array}$ & $\begin{array}{l}-0.0 \\
-0.0 \\
-0.8 \\
-0.0 \\
-0.0 \\
-0.0 \\
-0.0\end{array}$ & $\begin{array}{l}0 . \\
0 . \\
0 . \\
0 . \\
0 . \\
0 .\end{array}$ & \\
\hline $\begin{array}{l}.0000 \\
.0000 \\
.0000 \\
.0000 \\
.0000 \\
0000 \\
.0000\end{array}$ & $\begin{array}{l}1.551 \mathrm{E}+00 \\
1.565 \mathrm{E}+00 \\
1.576 \mathrm{E}+00 \\
1.586 \mathrm{E}+00 \\
1.602 \mathrm{E}+00 \\
1.615 \mathrm{E}+00 \\
1.626 \mathrm{E}+00\end{array}$ & $\begin{array}{l}1.29 \\
1.56 \\
1.838 \\
2.11 \\
2.66 \\
3.21 \\
3.76\end{array}$ & $\begin{array}{l}1 . \\
1 . \\
1 . \\
2 . \\
2 . \\
3 .\end{array}$ & $\begin{array}{l}2.4 \\
2.5 \\
2.7 \\
2.8 \\
3.0 \\
3.1 \\
3.3\end{array}$ & $\begin{array}{l}7.5 \\
7.8 \\
7.9 \\
8.2 \\
8.4 \\
8.6\end{array}$ & $\begin{array}{l}6.4 \\
6.7 \\
6.9 \\
7.3 \\
7.7 \\
8.0\end{array}$ & $\begin{array}{l}-0.0 \\
-0.0 \\
-0.0 \\
-0.0 \\
-0.0 \\
-0.0 \\
-0.0 \\
-0.0\end{array}$ & $\begin{array}{l}3 \\
1 \\
0 \\
9 \\
8 \\
7 \\
6 \\
5\end{array}$ & $\begin{array}{l}0.006 \\
0.005 \\
0.004 \\
0.003 \\
0.003 \\
0.002 \\
0.002 \\
0.002\end{array}$ \\
\hline $\begin{array}{l}0.0000 \\
0.0000 \\
0.0000 \\
0.0000 \\
0.0000 \\
0.00000 \\
0.0000 \\
0.0000\end{array}$ & $\begin{array}{l}1.636 E+00 \\
1.644 E+00 \\
1.651 E+00 \\
1.658 E+00 \\
1.664 E+00 \\
1.675 E+00 \\
1.684 E+00 \\
1.692 E+00\end{array}$ & $\begin{array}{l}7.641 \mathrm{E}+01 \\
8.751 \mathrm{E}+01 \\
9.863 \mathrm{E}+01\end{array}$ & $\begin{array}{l}4.479 E+01 \\
5.033 \mathrm{E}+01 \\
5.588 \mathrm{E}+01 \\
6.142 \mathrm{E}+01 \\
6.697 \mathrm{E}+01 \\
7.808 \mathrm{E}+01 \\
8.920 \mathrm{E}+01 \\
1.003 \mathrm{E}+02\end{array}$ & $\begin{array}{l}4.068 E+0 \\
4.174 E+0\end{array}$ & $\begin{array}{l}8.745 \mathrm{E} \\
8.846 \mathrm{E} \\
9.002 \mathrm{E} \\
9.063 \mathrm{E} \\
9.164 \mathrm{E} \\
9.244 \mathrm{E} \\
9.308 \mathrm{E}\end{array}$ & $\begin{array}{l}8.299 E+00 \\
8.528 E+00 \\
8.735 E+00 \\
8.922 E+00 \\
9.093 E+00 \\
9.397 E+00 \\
9.661 E+00 \\
9.894 E+00\end{array}$ & $\begin{array}{l}-0.002 \\
-0.002 \\
-0.002 \\
-0.002 \\
-0.001 \\
-0.001 \\
-0.001 \\
-0.001\end{array}$ & $\begin{array}{l}0.015 \\
0.0114 \\
0.0114 \\
0.014 \\
0.013 \\
0.0113 \\
0.013 \\
0.012\end{array}$ & $\begin{array}{l}0.001 \\
0.001 \\
0.001 \\
0.001 \\
0.001 \\
0.001 \\
0.001 \\
0.001\end{array}$ \\
\hline .0000 & $.699 \mathrm{E}+00$ & $1.098 E+02$ & $1.114 E+02$ & $4.269 \mathrm{E}+01$ & $9.361 E-01$ & $1.010 E+01$ & -0.001 & 0.012 & 0.001 \\
\hline
\end{tabular}




\begin{tabular}{|c|c|c|c|c|c|c|c|c|c|}
\hline $\mathrm{MeV}$ & $\begin{array}{l}\text { ST } \\
\text { COLLISION } \\
\text { MeV } \mathrm{cm}^{2} / \mathrm{g}\end{array}$ & $\begin{array}{l}\text { OPPING POWER } \\
\text { RADIATIVE } \\
\mathrm{MeV} \mathrm{cm}^{2} / \mathrm{g}\end{array}$ & $\begin{array}{c}\text { TOTAL } \\
\mathrm{MeV} \mathrm{cm}^{2} / \mathrm{g}\end{array}$ & $\begin{array}{l}\text { CSDA } \\
\text { RANGE } \\
g / \mathrm{Cm}^{2}\end{array}$ & $\begin{array}{l}\text { DIATION } \\
\text { YIELD }\end{array}$ & $\begin{array}{l}\text { DENS.EFF. } \\
\text { CORR } \\
\text { (DELTA) }\end{array}$ & $\begin{array}{l}\operatorname{dil} \\
\operatorname{coll} \\
\operatorname{LosS}\end{array}$ & $\begin{array}{l}g \text { ) } / d(1 \\
\text { CSDA } \\
\text { RANGE }\end{array}$ & $\begin{array}{l}\text { I ) } \\
\text { RAD } \\
\text { (IELD }\end{array}$ \\
\hline $\begin{array}{l}0.0100 \\
0.0125 \\
0.0150 \\
0.0175 \\
0.0200 \\
0.0250 \\
0.0300 \\
0.0350\end{array}$ & $\begin{array}{l}1.005 E+01 \\
8.641 E+00 \\
7.623 E+00 \\
6.847 E+00 \\
6.236 E+00 \\
5.329 E+00 \\
4.685 E+00 \\
4.203 E+00\end{array}$ & $\begin{array}{l}2.045 \mathrm{E}-02 \\
2.251 \mathrm{E}-02 \\
2.421 \mathrm{E}-02 \\
2.566 \mathrm{E}-02 \\
2.693 \mathrm{E}-02 \\
2.908 \mathrm{E}-02 \\
3.086 \mathrm{E}-02 \\
3.240 \mathrm{E}-02\end{array}$ & $\begin{array}{l}1.007 E+01 \\
8.664 E+00 \\
7.647 E+00 \\
6.873 E+00 \\
6.263 E+00 \\
5.358 E+00 \\
4.716 E+00 \\
4.235 E+00\end{array}$ & $\begin{array}{l}6.238 \\
8.92 \\
1.20 \\
1.54 \\
1.92 \\
2.79 \\
3.79 \\
4.91\end{array}$ & $\begin{array}{l}9.433 \mathrm{E}-04 \\
1.217 \mathrm{E}-03 \\
1.495 \mathrm{E}-03 \\
1.774 \mathrm{E}-03 \\
2.055 \mathrm{E}-03 \\
2.617 \mathrm{E}-03 \\
3.178 \mathrm{E}-03 \\
3.738 \mathrm{E}-03\end{array}$ & $\begin{array}{l}0.0 \\
0.0 \\
0.0 \\
0.0 \\
0.0 \\
0.0 \\
0.0 \\
0.0\end{array}$ & $\begin{array}{l}318 \\
298 \\
284 \\
273 \\
264 \\
250 \\
241 \\
233\end{array}$ & $\begin{array}{l}0.432 \\
0.394 \\
0.367 \\
0.347 \\
0.331 \\
0.308 \\
0.291 \\
0.278\end{array}$ & $\begin{array}{l}0.387 \\
0.356 \\
0.335 \\
0.319 \\
0.306 \\
0.287 \\
0.273 \\
0.263\end{array}$ \\
\hline $\begin{array}{l}0.0400 \\
0.0450 \\
0.0500 \\
0.0550 \\
0.0600 \\
0.0700 \\
0.0800 \\
0.0900\end{array}$ & $\begin{array}{l}3.827 E+00 \\
3.526 E+00 \\
3.278 E+00 \\
3.071 E+00 \\
2.895 E+00 \\
2.611 E+00 \\
2.393 E+00 \\
2.220 E+00\end{array}$ & $\begin{array}{l}3.376 \mathrm{E}-02 \\
3.500 \mathrm{E}-02 \\
3.613 \mathrm{E}-02 \\
3.718 \mathrm{E}-02 \\
3.817 \mathrm{E}-02 \\
3.998 \mathrm{E}-02 \\
4.162 \mathrm{E}-02 \\
4.313 \mathrm{E}-02\end{array}$ & $\begin{array}{l}3.861 E+00 \\
3.561 E+00 \\
3.314 E+00 \\
3.108 E+00 \\
2.933 E+00 \\
2.651 E+00 \\
2.435 E+00 \\
2.263 E+00\end{array}$ & $\begin{array}{l}6.150 \mathrm{E}-03 \\
7.500 \mathrm{E}-03 \\
8.957 \mathrm{E}-03 \\
1.052 \mathrm{E}-02 \\
1.217 \mathrm{E}-02 \\
1.577 \mathrm{E}-02 \\
1.971 \mathrm{E}-02 \\
2.397 \mathrm{E}-02\end{array}$ & $\begin{array}{l}-03 \\
-03 \\
-03 \\
-03 \\
-03 \\
-03 \\
-03 \\
-03\end{array}$ & $\begin{array}{l}0.0 \\
0.0 \\
0.0 \\
0.0 \\
0.0 \\
0.0 \\
0.0 \\
0.0\end{array}$ & $\begin{array}{l}-0.227 \\
-0.222 \\
-0.218 \\
-0.214 \\
-0.211 \\
-0.206 \\
-0.201 \\
-0.198\end{array}$ & $\begin{array}{l}0.268 \\
0.260 \\
0.253 \\
0.247 \\
0.242 \\
0.234 \\
0.227 \\
0.221\end{array}$ & $\begin{array}{l}0.255 \\
0.248 \\
0.242 \\
0.237 \\
0.233 \\
0.225 \\
0.219 \\
0.215\end{array}$ \\
\hline $\begin{array}{l}0.1000 \\
0.1250 \\
0.1500 \\
0.1750 \\
0.2000 \\
0.2500 \\
0.3000 \\
0.3500\end{array}$ & $\begin{array}{l}2.079 E+00 \\
1.820 E+00 \\
1.644 E+00 \\
1.517 E+00 \\
1.421 E+00 \\
1.287 E+00 \\
1.200 E+00 \\
1.140 E+00\end{array}$ & $\begin{array}{l}4.454 \mathrm{E}-02 \\
4.772 \mathrm{E}-02 \\
5.054 \mathrm{E}-02 \\
5.312 \mathrm{E}-02 \\
5.555 \mathrm{E}-02 \\
6.015 \mathrm{E}-02 \\
6.460 \mathrm{E}-02 \\
6.900 \mathrm{E}-02\end{array}$ & $\begin{array}{l}2.123 E+00 \\
1.867 E+00 \\
1.694 E+00 \\
1.570 E+00 \\
1.476 E+00 \\
1.347 E+00 \\
1.265 E+00 \\
1.209 E+00\end{array}$ & $\begin{array}{l}2.854 \mathrm{E}-02 \\
4.114 \mathrm{E}-02 \\
5.523 \mathrm{E}-02 \\
7.059 \mathrm{E}-02 \\
8.703 \mathrm{E}-02 \\
1.226 \mathrm{E}-01 \\
1.610 \mathrm{E}-01 \\
2.015 \mathrm{E}-01\end{array}$ & $\begin{array}{l}1.072 \mathrm{E}-02 \\
1.324 \mathrm{E}-02 \\
1.565 \mathrm{E}-02 \\
1.796 \mathrm{E}-02 \\
2.019 \mathrm{E}-02 \\
2.439 \mathrm{E}-02 \\
2.831 \mathrm{E}-02 \\
3.199 \mathrm{E}-02\end{array}$ & $\begin{array}{l}0.0 \\
0.0 \\
0.0 \\
0.0 \\
7.387 \mathrm{E}-04 \\
8.466 \mathrm{E}-03 \\
1.693 \mathrm{E}-02 \\
2.596 \mathrm{E}-02\end{array}$ & $\begin{array}{l}-0.195 \\
-0.189 \\
-0.184 \\
-0.180 \\
-0.173 \\
-0.166 \\
-0.160 \\
-0.155\end{array}$ & $\begin{array}{l}0.217 \\
0.208 \\
0.201 \\
0.195 \\
0.191 \\
0.182 \\
0.176 \\
0.171\end{array}$ & $\begin{array}{l}0.210 \\
0.202 \\
0.196 \\
0.191 \\
0.186 \\
0.178 \\
0.172 \\
0.166\end{array}$ \\
\hline $\begin{array}{l}0.4000 \\
0.4500 \\
0.5000 \\
0.5500 \\
0.6000 \\
0.7000 \\
0.8000 \\
0.9000\end{array}$ & $\begin{array}{l}1.097 E+00 \\
1.065 E+00 \\
1.041 E+00 \\
1.022 E+00 \\
1.008 E+00 \\
9.887 E-01 \\
9.772 E-01 \\
9.707 E-01\end{array}$ & $\begin{array}{l}7.340 \\
7.781 \\
8.228 \\
8.677 \\
9.132 \\
1.005 \\
1.098 \\
1.193\end{array}$ & $\begin{array}{l}1.170 E+00 \\
1.142 E+00 \\
1.123 E+00 \\
1.109 E+00 \\
1.099 E+00 \\
1.089 E+00 \\
1.087 E+00 \\
1.090 E+00\end{array}$ & 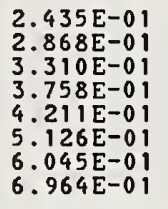 & $\begin{array}{l}.549 \mathrm{E}-02 \\
.881 \mathrm{E}-02 \\
.200 \mathrm{E}-02 \\
.507 \mathrm{E}-02 \\
.804 \mathrm{E}-02 \\
.371 \mathrm{E}-02 \\
.908 \mathrm{E}-02 \\
.421 \mathrm{E}-02\end{array}$ & $\begin{array}{l}3.545 \mathrm{E}-02 \\
4.530 \mathrm{E}-02 \\
5.543 \mathrm{E}-02 \\
6.577 \mathrm{E}-02 \\
7.627 \mathrm{E}-02 \\
9.756 \mathrm{E}-02 \\
1.191 \mathrm{E}-01 \\
1.406 \mathrm{E}-01\end{array}$ & $\begin{array}{l}-0.151 \\
-0.147 \\
-0.144 \\
-0.141 \\
-0.138 \\
-0.133 \\
-0.128 \\
-0.125\end{array}$ & $\begin{array}{l}0 . \\
0 . \\
0 . \\
0 . \\
0 . \\
0 . \\
0 . \\
0 .\end{array}$ & $\begin{array}{l}0.161 \\
0.157 \\
0.154 \\
0.150 \\
0.147 \\
0.142 \\
0.137 \\
0.132\end{array}$ \\
\hline $\begin{array}{l}1.0000 \\
1.2500 \\
1.5000 \\
1.7500 \\
2.0000 \\
2.5000 \\
3.0000 \\
3.5000\end{array}$ & $\begin{array}{l}9.675 \mathrm{E}-01 \\
9.676 \mathrm{E}-01 \\
9.737 \mathrm{E}-01 \\
9.822 \mathrm{E}-01 \\
9.916 \mathrm{E}-01 \\
1.011 \mathrm{E}+00 \\
1.028 \mathrm{E}+00 \\
1.044 \mathrm{E}+00\end{array}$ & $\begin{array}{l}1.290 \\
1.537 \\
1.792 \\
2.053 \\
2.319 \\
2.866 \\
3.427 \\
3.999\end{array}$ & $\begin{array}{l}1.096 E+00 \\
1.121 E+00 \\
1.153 E+00 \\
1.187 E+00 \\
1.224 E+00 \\
1.297 E+00 \\
1.371 E+00 \\
1.444 E+00\end{array}$ & $\begin{array}{l}7.879 E-01 \\
1.014 E+00 \\
1.233 E+00 \\
1.447 E+00 \\
1.655 E+00 \\
2.051 E+00 \\
2.426 E+00 \\
2.782 E+00\end{array}$ & $\begin{array}{l}6.915 \mathrm{E}-02 \\
8.081 \mathrm{E}-02 \\
9.173 \mathrm{E}-02 \\
1.021 \mathrm{E}-01 \\
1.120 \mathrm{E}-01 \\
1.307 \mathrm{E}-01 \\
1.482 \mathrm{E}-01 \\
1.647 \mathrm{E}-01\end{array}$ & $\begin{array}{l}1.621 \mathrm{E}-01 \\
2.153 \mathrm{E}-01 \\
2.673 \mathrm{E}-01 \\
3.183 \mathrm{E}-01 \\
3.683 \mathrm{E}-01 \\
4.653 \mathrm{E}-01 \\
5.586 \mathrm{E}-01 \\
6.481 \mathrm{E}-01\end{array}$ & $\begin{array}{l}-0.121 \\
-0.114 \\
-0.108 \\
-0.103 \\
-0.098 \\
-0.090 \\
-0.084 \\
-0.078\end{array}$ & $\begin{array}{l}0.135 \\
0.128 \\
0.122 \\
0.117 \\
0.113 \\
0.105 \\
0.099 \\
0.094\end{array}$ & $\begin{array}{l}0.129 \\
0.120 \\
0.113 \\
0.108 \\
0.102 \\
0.094 \\
0.086 \\
0.080\end{array}$ \\
\hline $\begin{array}{l}4.0000 \\
4.5000 \\
5.0000 \\
5.5000 \\
6.0000 \\
7.0000 \\
8.0000 \\
9.0000\end{array}$ & $\begin{array}{l}1.059 E+00 \\
1.072 E+00 \\
1.084 E+00 \\
1.095 E+00 \\
1.105 E+00 \\
1.123 E+00 \\
1.138 E+00 \\
1.151 E+00\end{array}$ & $\begin{array}{l}4.582 E-01 \\
5.174 E-01 \\
5.773 E-01 \\
6.379 E-01 \\
6.991 E-01 \\
8.233 E-01 \\
9.495 E-01 \\
1.077 E+00\end{array}$ & $\begin{array}{l}1.517 E+00 \\
1.589 E+00 \\
1.661 E+00 \\
1.733 E+00 \\
1.804 E+00 \\
1.946 E+00 \\
2.087 E+00 \\
2.228 E+00\end{array}$ & $\begin{array}{l}3.119 E+00 \\
3.441 E+00 \\
3.749 E+00 \\
4.044 E+00 \\
4.326 E+00 \\
4.860 E+00 \\
5.356 E+00 \\
5.820 E+00\end{array}$ & $\begin{array}{l}.803 \mathrm{E}-01 \\
.951 \mathrm{E}-01 \\
.093 \mathrm{E}-01 \\
.228 \mathrm{E}-01 \\
.357 \mathrm{E}-01 \\
.600 \mathrm{E}-01 \\
.824 \mathrm{E}-01 \\
.032 \mathrm{E}-01\end{array}$ & $\begin{array}{l}8.149 \\
8.926 \\
9.667 \\
1.038 \\
1.170 \\
1.293 \\
1.406\end{array}$ & $\begin{array}{l}-0.074 \\
-0.070 \\
-0.067 \\
-0.065 \\
-0.062 \\
-0.058 \\
-0.055 \\
-0.052\end{array}$ & & $\begin{array}{l}0.075 \\
0.070 \\
0.067 \\
0.063 \\
0.060 \\
0.054 \\
0.050 \\
0.046\end{array}$ \\
\hline $\begin{array}{l}10.0000 \\
12.5000 \\
15.0000 \\
17.5000 \\
20.0000 \\
25.0000 \\
30.0000 \\
35.0000\end{array}$ & $\begin{array}{l}1.163 E+00 \\
1.188 E+00 \\
1.208 E+00 \\
1.224 E+00 \\
1.238 E+00 \\
1.261 E+00 \\
1.279 E+00 \\
1.293 E+00\end{array}$ & $\begin{array}{l}1.206 E+00 \\
1.535 E+00 \\
1.870 E+00 \\
2.210 E+00 \\
2.554 E+00 \\
3.252 E+00 \\
3.9611 E+00 \\
4.678 E+00\end{array}$ & $\begin{array}{l}2.369 E+00 \\
2.723 E+00 \\
3.077 E+00 \\
3.434 E+00 \\
3.792 E+00 \\
4.513 E+00 \\
5.240 E+00 \\
5.972 E+00\end{array}$ & $\begin{array}{l}E+00 \\
E+00 \\
E+00 \\
E+00 \\
E+00 \\
E+01 \\
E+01 \\
E+01\end{array}$ & $\begin{array}{l}4.0 \\
4.0 \\
4.6 \\
5.0\end{array}$ & $\begin{array}{l}1.95 \\
2.13 \\
2.30 \\
2.58 \\
2.83 \\
3.04\end{array}$ & $\begin{array}{l}-0.050 \\
-0.045 \\
-0.041 \\
-0.038 \\
-0.036 \\
-0.032 \\
-0.029 \\
-0.027\end{array}$ & $\begin{array}{l}63 \\
57 \\
53 \\
50 \\
47 \\
43 \\
40 \\
38\end{array}$ & $\begin{array}{l}0.043 \\
0.037 \\
0.032 \\
0.029 \\
0.026 \\
0.022 \\
0.019 \\
0.016\end{array}$ \\
\hline $\begin{array}{l}40.0000 \\
45.0000 \\
50.0000 \\
55.0000 \\
60.0000 \\
70.0000 \\
80.0000 \\
90.0000\end{array}$ & $\begin{array}{l}1.306 E+00 \\
1.317 E+00 \\
1.326 E+00 \\
1.335 E+00 \\
1.342 E+00 \\
1.355 E+00 \\
1.366 E+00 \\
1.376 E+00\end{array}$ & $\begin{array}{l}5.402 E+00 \\
6.132 E+00 \\
6.865 E+00 \\
7.603 E+00 \\
8.345 E+00 \\
9.836 E+00 \\
1.134 E+01 \\
1.284 E+01\end{array}$ & $\begin{array}{l}.708 E+00 \\
.448 E+00 \\
.192 E+00 \\
.938 E+00 \\
.687 E+00 \\
.119 E+01 \\
.270 E+01 \\
.422 E+01\end{array}$ & $\begin{array}{l}1.348 E+01 \\
1.419 E+01 \\
1.483 E+01 \\
1.541 E+01 \\
1.595 E+01 \\
1.691 E+01 \\
1.775 E+01 \\
1.849 E+01\end{array}$ & $\begin{array}{l}.065 E-01 \\
.296 E-01 \\
.497 E-01 \\
.674 E-01 \\
.832 E-01 \\
.099 E-01 \\
.319 E-01 \\
.504 E-01\end{array}$ & $\begin{array}{l}3.241 E+00 \\
3.414 E+00 \\
3.574 E+00 \\
3.720 E+00 \\
3.857 E+00 \\
4.103 E+00 \\
4.322 E+00 \\
4.518 E+00\end{array}$ & $\begin{array}{l}-0.025 \\
-0.023 \\
-0.022 \\
-0.020 \\
-0.019 \\
-0.017 \\
-0.015 \\
-0.014\end{array}$ & & $\begin{array}{l}0.015 \\
0.013 \\
0.0112 \\
0.011 \\
0.010 \\
0.009 \\
0.008 \\
0.007\end{array}$ \\
\hline $\begin{array}{l}5.0000 \\
0.0000 \\
5.0000 \\
0.0000 \\
0.0000 \\
0.0000 \\
0.0000\end{array}$ & $\begin{array}{l}1.384 E+00 \\
1.401 E+00 \\
1.415 E+00 \\
1.426 E+00 \\
1.436 E+00 \\
1.452 E+00 \\
1.464 E+00 \\
1.475 E+00\end{array}$ & $\begin{array}{l}1.43 \\
1.8 \\
2.1 \\
2.5 \\
2.9 \\
3.7 \\
4.5 \\
5.28\end{array}$ & $\begin{array}{l}1.5 \\
1.5 \\
2.3 \\
2.7 \\
3.1 \\
3.8 \\
4.6\end{array}$ & $\begin{array}{l}+01 \\
+01 \\
+01 \\
+01 \\
+01 \\
+01 \\
+01 \\
+01\end{array}$ & $\begin{array}{l}7 . \\
7 . \\
8 . \\
8 . \\
8 . \\
8 . \\
9 .\end{array}$ & $\begin{array}{l}4.696 E+00 \\
5.080 E+00 \\
5.401 E+00 \\
5.677 E+00 \\
5.919 E+00 \\
6.329 E+00 \\
6.668 E+00 \\
6.959 E+00\end{array}$ & $\begin{array}{l}-0 \\
-0 \\
-0 \\
-0 \\
-0 \\
-0 \\
-0 \\
-0\end{array}$ & & $\begin{array}{l}0.006 \\
0.005 \\
0.004 \\
0.004 \\
0.003 \\
0.003 \\
0.002 \\
0.002\end{array}$ \\
\hline $\begin{array}{l}.0000 \\
.0000 \\
.0000 \\
.0000 \\
.0000\end{array}$ & $\begin{array}{l}1.491 E+00 \\
1.498 E+00 \\
1.505 E+00 \\
1.510 E+00 \\
1.520 E+00 \\
1.529 E+00 \\
1.536 E+00\end{array}$ & $\begin{array}{l}6.058 \mathrm{Et} \\
6.833 \mathrm{Et} \\
7.609 \mathrm{Et} \\
8.386 \mathrm{Et} \\
9.163 \mathrm{Et} \\
1.072 \mathrm{Et} \\
1.227 \mathrm{Et}\end{array}$ & $\begin{array}{l}E+01 \\
E+01 \\
E+01 \\
E+01 \\
E+02 \\
E+02 \\
E+02\end{array}$ & $\begin{array}{l}2.950 \mathrm{E} \\
3.011 \mathrm{E} \\
3.067 \mathrm{E} \\
3.167 \mathrm{E} \\
3.253 \mathrm{E}\end{array}$ & $\begin{array}{l}9.1 \\
9.2 \\
9.2 \\
9.3 \\
9.4 \\
9.4\end{array}$ & $\begin{array}{l}7.212 E+00 \\
7.437 E+00 \\
7.639 E+00 \\
7.823 E+00 \\
7.991 E+00 \\
8.290 E+00 \\
8.550 E+00 \\
8.780 E+00\end{array}$ & $\begin{array}{l}-0 . \\
-0 \\
-0 \\
-0 \\
-0 \\
-0 .\end{array}$ & $\begin{array}{l}8 \\
7 \\
7 \\
7 \\
6 \\
6 \\
5 \\
5\end{array}$ & $\begin{array}{l}0.002 \\
0.001 \\
0.001 \\
0.001 \\
0.001 \\
0.001 \\
0.001 \\
0.001\end{array}$ \\
\hline 0.0000 & $1.543 E+00$ & $1.539 E+02$ & $1.554 E+02$ & $3.396 E+01$ & $9.549 \mathrm{E}-01$ & $8.986 E+00$ & -0.002 & 0.015 & 0.001 \\
\hline
\end{tabular}


POSITRONS IN AIR, DRY (NEAR SEA LEVEL)

$I=85.7 \mathrm{eV} \quad$ DENSITY $=1.205 \mathrm{E}-03 \mathrm{~g} / \mathrm{cm}^{3}\left(20^{\circ} \mathrm{C}\right)$

\begin{tabular}{|c|c|c|c|c|c|c|c|c|c|}
\hline $\mathrm{MeV}$ & 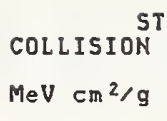 & $\mathrm{MeV} \mathrm{Cm}^{2} / \mathrm{g}$ & $\begin{array}{c}\text { TOTAL } \\
\mathrm{MeV} \mathrm{cm}^{2} / \mathrm{g}\end{array}$ & $\begin{array}{l}\text { CSDA } \\
\text { RANGE } \\
\mathrm{g} / \mathrm{Cm}^{2}\end{array}$ & $O N$ & $\begin{array}{l}\text { DENS.EFF. } \\
\text { CORR. } \\
\text { (DELTA) }\end{array}$ & $\begin{array}{l}\text { ded } \\
\text { coll } \\
\text { Loss }\end{array}$ & in & $\begin{array}{l}\text { I) } \\
\text { RAD } \\
\text { IELD }\end{array}$ \\
\hline $\begin{array}{l}0.0100 \\
0.0125 \\
0.0150 \\
0.0175 \\
0.0200 \\
0.0250 \\
0.0300 \\
0.0350\end{array}$ & $\begin{array}{l}1.82 \\
1.57 \\
1.39 \\
1.25 \\
1.05 \\
9.12\end{array}$ & $\begin{array}{l}3.9 \\
3.9 \\
3.9 \\
3.9 \\
3.9 \\
3.9 \\
3.9\end{array}$ & $\begin{array}{l}80 E+01 \\
25 E+01 \\
79 E+01 \\
97 E+01 \\
56 E+01 \\
53 E+01 \\
30 E+00 \\
00 E+00\end{array}$ & $\begin{array}{l}1 E-04 \\
0 E-04 \\
7 E-04 \\
5 E-04 \\
5 E-04 \\
5 E-03 \\
7 E-03 \\
9 E-03\end{array}$ & $\begin{array}{l}.658 E-05 \\
.167 E-04 \\
.359 E-04 \\
.545 E-04 \\
.725 E-04 \\
.072 E-04 \\
.404 E-04 \\
.723 E-04\end{array}$ & $\begin{array}{l}0.0 \\
0.0 \\
0.0 \\
0.0 \\
0.0 \\
0.0 \\
0.0 \\
0.0\end{array}$ & & $\begin{array}{l}.208 \\
.199 \\
193 \\
187 \\
183 \\
1176 \\
171 \\
167\end{array}$ & $\begin{array}{l}0.207 \\
0.198 \\
0.192 \\
0.186 \\
0.182 \\
0.176 \\
0.1771 \\
0.167\end{array}$ \\
\hline $\begin{array}{l}0.0400 \\
0.0450 \\
0.0500 \\
0.0550 \\
0.0600 \\
0.0700 \\
0.0800 \\
0.0900\end{array}$ & $\begin{array}{l}306 E+00 \\
680 E+00 \\
171 E+00 \\
749 E+00 \\
393 E+00 \\
826 E+00 \\
393 E+00 \\
052 E+00\end{array}$ & $\begin{array}{l}03 \\
03 \\
03 \\
03 \\
03 \\
03\end{array}$ & $\begin{array}{l}7.310 E+00 \\
6.684 E+00 \\
6.175 E+00 \\
5.753 E+00 \\
5.397 E+00 \\
4.830 E+00 \\
4.397 E+00\end{array}$ & $\begin{array}{l}E-03 \\
E-03 \\
-03 \\
-03 \\
-03 \\
-03 \\
-03 \\
-03 \\
-02 \\
-02\end{array}$ & $\begin{array}{l}.032 E-04 \\
.333 E-04 \\
.626 E-04 \\
.912 E-04 \\
.192 E-04 \\
.735 E-04 \\
.261 E-04\end{array}$ & $\begin{array}{l}0.0 \\
0.0 \\
0.0 \\
0.0 \\
0.0 \\
0.0 \\
0.0 \\
0.0\end{array}$ & & $\begin{array}{l}0.164 \\
0.161 \\
0.158 \\
0.156 \\
0.154 \\
0.151 \\
0.149 \\
0.147\end{array}$ & $\begin{array}{l}0.163 \\
0.160 \\
0.158 \\
0.156 \\
0.154 \\
0.151 \\
0.149\end{array}$ \\
\hline $\begin{array}{l}0.1000 \\
0.1250 \\
0.1500 \\
0.1750 \\
0.2000 \\
0.2500 \\
0.3000 \\
0.3500\end{array}$ & $\begin{array}{l}3.776 \mathrm{E}+00 \\
3.274 \mathrm{E}+00 \\
2.935 \mathrm{E}+00 \\
2.692 \mathrm{E}+00 \\
2.510 \mathrm{E}+00 \\
2.257 \mathrm{E}+00 \\
2.091 \mathrm{E}+00 \\
1.976 \mathrm{E}+00\end{array}$ & $\begin{array}{l}4.3 \\
4.4 \\
4.6 \\
4.7 \\
5.1 \\
5.4\end{array}$ & $\begin{array}{l}3.27 \\
2.94 \\
2.69 \\
2.51 \\
2.26 \\
2.09 \\
1.98\end{array}$ & $\begin{array}{l}2.2 \\
3.0 \\
3.9 \\
4.9 \\
7.0 \\
9.3\end{array}$ & $\begin{array}{l}7.4 \\
8.5 \\
9.6 \\
1.0 \\
1.2 \\
1.4 \\
1.6\end{array}$ & $\begin{array}{l}0.0 \\
0.0 \\
0.0 \\
0.0 \\
0.0 \\
0.0 \\
0.0 \\
0.0\end{array}$ & $\begin{array}{l}-0 . \\
-0 . \\
-0 . \\
-0 . \\
-0 . \\
-0 . \\
-0 . \\
-0 .\end{array}$ & & $\begin{array}{l}0.145 \\
0.141 \\
0.138 \\
0.136 \\
0.134 \\
0.131 \\
0.129 \\
0.127\end{array}$ \\
\hline $\begin{array}{l}0.4000 \\
0.4500 \\
0.5000 \\
0.5500 \\
0.6000 \\
0.7000 \\
0.8000 \\
0.9000\end{array}$ & $\begin{array}{l}1.831 \\
1.7841 \\
1.748 \\
1.719 \\
1.678 \\
1.652\end{array}$ & $\begin{array}{l}6.7 \\
7.2 \\
7.7 \\
8.2 \\
9.2 \\
1.0\end{array}$ & $\begin{array}{l}1.89 \\
1.83 \\
1.79 \\
1.75 \\
1.72 \\
1.68 \\
1.66 \\
1.64\end{array}$ & $\begin{array}{l}1.7 \\
1.9 \\
2.2 \\
2.5 \\
3.1 \\
3.7\end{array}$ & $\begin{array}{l}1.846 \\
2.030 \\
2.212 \\
2.394 \\
2.575 \\
2.939 \\
3.304 \\
3.671\end{array}$ & $\begin{array}{l}0.0 \\
0.0 \\
0.0 \\
0.0 \\
0.0 \\
0.0 \\
0.0 \\
0.0\end{array}$ & & & $\begin{array}{l}0.125 \\
0.123 \\
0.122 \\
0.121 \\
0.120 \\
0.118 \\
0.1116 \\
0.114\end{array}$ \\
\hline $\begin{array}{l}1.0000 \\
1.2500 \\
1.5000 \\
1.7500 \\
2.0000 \\
2.5000 \\
3.0000 \\
3.5000\end{array}$ & $\begin{array}{l}1.626 E+00 \\
1.617 E+00 \\
1.621 E+00 \\
1.630 E+00 \\
1.642 E+00 \\
1.668 E+00 \\
1.695 E+00 \\
1.720 E+00\end{array}$ & $\begin{array}{l}1.271 \mathrm{E}-02 \\
1.588 \mathrm{E}-02 \\
1.927 \mathrm{E}-02 \\
2.284 \mathrm{E}-02 \\
2.656 \mathrm{E}-02 \\
3.437 \mathrm{E}-02 \\
4.260 \mathrm{E}-02 \\
5.115 \mathrm{E}-02\end{array}$ & $\begin{array}{l}1.639 E+00 \\
1.633 E+00 \\
1.640 E+00 \\
1.653 E+00 \\
1.669 E+00 \\
1.703 E+00 \\
1.738 E+00 \\
1.772 E+00\end{array}$ & $\begin{array}{l}6.4 \\
8.0 \\
9.5 \\
1.1 \\
1.3 \\
1.6 \\
1.9\end{array}$ & $\begin{array}{l}4.0 \\
4.9 \\
5.9 \\
6.9 \\
7.9 \\
9.9 \\
1.2 \\
1.4\end{array}$ & $\begin{array}{l}0.0 \\
0.0 \\
0.0 \\
0.0 \\
0.0 \\
0.0 \\
0.0 \\
0.0\end{array}$ & $\begin{array}{l}-0 . \\
-0 . \\
-0 . \\
-0 . \\
-0.0 \\
-0 . \\
-0 . \\
-0 .\end{array}$ & & $\begin{array}{l}0.1113 \\
0.1110 \\
0.107 \\
0.105 \\
0.103 \\
0.100 \\
0.097 \\
0.095\end{array}$ \\
\hline $\begin{array}{l}4.0000 \\
4.5000 \\
5.0000 \\
5.5000 \\
6.0000 \\
7.0000 \\
8.0000 \\
9.0000\end{array}$ & $\begin{array}{l}744 E+00 \\
763 E+00 \\
786 E+00 \\
805 E+00 \\
823 E+00 \\
854 E+00 \\
883 E+00 \\
908 E+00\end{array}$ & $\begin{array}{l}0.908 \mathrm{E}-02 \\
7.838 \mathrm{E}-02 \\
8.787 \mathrm{E}-02 \\
9.754 \mathrm{E}-02 \\
1.173 \mathrm{E}-01 \\
1.376 \mathrm{E}-01 \\
1.584 \mathrm{E}-01\end{array}$ & $\begin{array}{l}1.835 \mathrm{E}+0 \\
1.865 \mathrm{E}+0 \\
1.893 \mathrm{E}+0 \\
1.920 \mathrm{E}+0 \\
1.972 \mathrm{E}+0 \\
2.020 \mathrm{E}+0 \\
2.066 \mathrm{E}+0\end{array}$ & $\begin{array}{l}2.5 \\
2.8 \\
3.0 \\
3.3 \\
3.8\end{array}$ & $\begin{array}{l}1.8 \\
2.8 \\
2.2 \\
2.4 \\
2.8\end{array}$ & $\begin{array}{l}0.0 \\
0.0 \\
0.0 \\
0.0 \\
0.0 \\
0.0 \\
0.0 \\
0.0\end{array}$ & & & $\begin{array}{l}0.0 \\
0.0 \\
0.0 \\
0.0 \\
0.0 \\
0.0 \\
0.0\end{array}$ \\
\hline $\begin{array}{l}.0000 \\
.5000 \\
.0000 \\
.5000 \\
.0000 \\
.0000 \\
.0000 \\
.0000\end{array}$ & $\begin{array}{l}931 E+00 \\
980 E+00 \\
020 E+00 \\
055 E+00 \\
085 E+00 \\
136 E+00 \\
176 E+00 \\
208 E+00\end{array}$ & 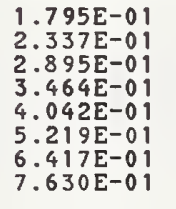 & $\begin{array}{l}2.3 \\
2.4 \\
2.4 \\
2.6 \\
2.8 \\
2.9\end{array}$ & $\begin{array}{l}6 . \\
7 . \\
9\end{array}$ & $\begin{array}{l}5 . \\
6 \\
7 . \\
8 . \\
1 . \\
1: \\
1 .\end{array}$ & $\begin{array}{l}0.0 \\
0.0 \\
0.0 \\
0.0 \\
0.0 \\
0.0 \\
7.636 \mathrm{E}-0 \\
5.984 \mathrm{E}-0\end{array}$ & & & $\begin{array}{l}0 . \\
0 . \\
0 . \\
0 . \\
0 .\end{array}$ \\
\hline $\begin{array}{l}0 \\
0 \\
0 \\
0 \\
0 \\
0 \\
0 \\
0\end{array}$ & $\begin{array}{l}252 E+00 \\
269 E+00 \\
284 E+00 \\
297 E+00 \\
319 E+00 \\
337 E+00 \\
353 E+00\end{array}$ & $\begin{array}{l}1.133 E+00 \\
1.258 E+00 \\
1.384 E+00 \\
1.637 E+00 \\
1.892 E+00 \\
2.148 E+00\end{array}$ & $\begin{array}{l}0 \\
0 \\
0 \\
0 \\
0 \\
0\end{array}$ & & & $\begin{array}{l}1 . \\
2 . \\
3 . \\
4\end{array}$ & & & \\
\hline $\begin{array}{ll}0 & 0 \\
0 & 0 \\
0 & 0\end{array}$ & $\begin{array}{l}2.5 \\
2.4 \\
2.4 \\
2.4 \\
2.4 \\
2.5\end{array}$ & $\begin{array}{l}3 . \\
3 . \\
5 . \\
6 .\end{array}$ & & & & $\begin{array}{l}2 . \\
2 . \\
2 . \\
2 .\end{array}$ & & & :. \\
\hline .0000 & $\begin{array}{l}2.545 \mathrm{E}+00 \\
2.556 \mathrm{E}+00 \\
2.566 \mathrm{E}+00 \\
2.575 \mathrm{E}+00 \\
2.590 \mathrm{E}+00 \\
2.603 \mathrm{E}+00 \\
2.614 \mathrm{E}+00\end{array}$ & $2.376 E+01$ & $\begin{array}{l}.286 E+01 \\
.422 E+01 \\
.557 E+01 \\
.692 E+01 \\
.827 E+01 \\
.097 E+01 \\
.367 E+01 \\
.638 E+01\end{array}$ & $\begin{array}{l}6.896 E+01 \\
7.266 E+01 \\
7.602 E+01 \\
7.910 E+01 \\
8.194 E+01 \\
8.705 E+01 \\
9.153 E+01 \\
9.553 E+01\end{array}$ & $\begin{array}{l}1 \\
1 \\
1 \\
1 \\
1 \\
1 \\
1\end{array}$ & $\begin{array}{l}3.116 E+00 \\
3.302 E+00 \\
3.472 E+00 \\
3.628 E+00 \\
3.772 E+00 \\
4.034 E+00 \\
4.267 E+00 \\
4.477 E+00\end{array}$ & & $\begin{array}{l}0.023 \\
0.022 \\
0.021 \\
0.021 \\
0.020 \\
0.019 \\
0.018 \\
0.017\end{array}$ & $\begin{array}{l}06 \\
06 \\
05 \\
05\end{array}$ \\
\hline & $2.624 E+00$ & $2.646 E+01$ & $308 E+01$ & $.914 E+01$ & $25 E-01$ & $.668 E+00$ & -0.005 & 0.017 & .00 \\
\hline
\end{tabular}


POSITRONS IN POLYMETHYL METHACRYLATE, "LUCITE", "PERSPEX", "PLEXIGLAS"

$I=74.0 \mathrm{eV} \quad$ DENSITY $=1.190 \mathrm{E}+00 \mathrm{~g} / \mathrm{cm}^{3}$

\begin{tabular}{|c|c|c|c|c|c|c|c|c|c|}
\hline $\mathrm{MeV}$ & $\begin{array}{l}\text { COLLISION } \\
\mathrm{M} O \mathrm{Cm} \mathrm{cm}^{2} / \mathrm{g}\end{array}$ & $\begin{array}{l}\text { OPPING POWE } \\
\text { RADIATIVE } \\
\text { MeV } \mathrm{cm}^{2} / \mathrm{g}\end{array}$ & $\begin{array}{c}\text { TOTAL } \\
\text { MeV } \mathrm{cm}^{2} / \mathrm{g}\end{array}$ & $\begin{array}{l}\text { CSDA } \\
\text { RANGE } \\
\mathrm{g} / \mathrm{cm}^{2}\end{array}$ & DIATION & $\begin{array}{l}E F F . \\
R \text { (A) }\end{array}$ & $\begin{array}{l}\text { d( } 1 \\
\text { COLL } \\
\text { LOSS }\end{array}$ & $\begin{array}{l}) / d(1 \\
\text { CSDA }\end{array}$ & $\begin{array}{l}\text { I ) } \\
\text { RAD } \\
\text { I ELD }\end{array}$ \\
\hline $\begin{array}{l}0.0100 \\
0.0125 \\
0.0150 \\
0.0175 \\
0.0200 \\
0.0250 \\
0.0300\end{array}$ & $\begin{array}{l}2.419 \mathrm{E}+01 \\
2.023 \mathrm{E}+01 \\
1.749 \mathrm{E}+01 \\
1.546 \mathrm{E}+01 \\
1.390 \mathrm{E}+01 \\
1.164 \mathrm{E}+01 \\
1.009 \mathrm{E}+01\end{array}$ & $\begin{array}{l}3.332 \mathrm{E} \\
3.349 \mathrm{E} \\
3.359 \mathrm{E} \\
3.366 \mathrm{E} \\
3.372 \mathrm{E} \\
3.382 \mathrm{E} \\
3.391 \mathrm{E}\end{array}$ & $\begin{array}{l}2.419 \\
2.024 \\
1.749 \\
1.546 \\
1.390 \\
1.164 \\
1.009 \\
8.047\end{array}$ & $\begin{array}{l}2.3 \\
3.4 \\
4.7 \\
6.3 \\
8.0 \\
1.1 \\
1.6 \\
2.1\end{array}$ & $\begin{array}{l}05 \\
05 \\
04 \\
04 \\
04 \\
04 \\
04 \\
04\end{array}$ & $\begin{array}{l}0.0 \\
0.0 \\
0.0 \\
0.0 \\
0.0 \\
0.0 \\
0.0 \\
0.0\end{array}$ & $\begin{array}{l}-0 \\
-0 \\
-0 \\
-0 \\
-0 \\
-0 \\
-0 \\
-0\end{array}$ & $\begin{array}{l}202 \\
194 \\
187 \\
182 \\
178 \\
172 \\
167 \\
163\end{array}$ & $\begin{array}{l}0.201 \\
0.193 \\
0.186 \\
0.182 \\
0.177 \\
0.171 \\
0.166 \\
0.163\end{array}$ \\
\hline $\begin{array}{l}0.0400 \\
0.0450 \\
0.0500 \\
0.0550 \\
0.0600 \\
0.0700 \\
0.0800\end{array}$ & $\begin{array}{l}7.374 E+00 \\
6.810 E+00 \\
6.343 E+00 \\
5.949 E+00 \\
5.321 E+00 \\
4.842 E+00\end{array}$ & $\begin{array}{l}3.42 \\
3.4 \\
3.45 \\
3.46 \\
3.5 \\
3.5\end{array}$ & $\begin{array}{l}8.0 \\
7.3 \\
6.8 \\
6.3 \\
5.9 \\
5.3 \\
4.8\end{array}$ & $\begin{array}{l}3.4 \\
4.1 \\
4.8 \\
5.7 \\
7.4 \\
9.4\end{array}$ & $\begin{array}{l}.04 \\
.04 \\
.04 \\
.04 \\
.04 \\
.04 \\
.04 \\
.04\end{array}$ & $\begin{array}{l}0.0 \\
0.0 \\
0.0 \\
0.0 \\
0.0 \\
0.0 \\
0.0\end{array}$ & $\begin{array}{l}-0 \\
-0 \\
-0 \\
-0 \\
-0 \\
-0 \\
-0 \\
-0\end{array}$ & $\begin{array}{l}0 \\
7 \\
5 \\
3 \\
1 \\
8 \\
6 \\
4\end{array}$ & $\begin{array}{l}0.159 \\
0.157 \\
0.155 \\
0.153 \\
0.151 \\
0.148\end{array}$ \\
\hline $\begin{array}{l}0.1250 \\
0.1500 \\
0.1750 \\
0.2000 \\
0.2500 \\
0.3000\end{array}$ & $\begin{array}{l}3.606 E+00 \\
3.232 E+00 \\
2.963 E+00 \\
2.762 E+00 \\
2.482 E+00 \\
2.300 E+00 \\
2.173 E+00\end{array}$ & $\begin{array}{l}3.7 \\
3.8 \\
3.9 \\
4.1 \\
4.4 \\
4.7 \\
5.1\end{array}$ & $\begin{array}{l}4.1 \\
3.6 \\
3.2 \\
2.9 \\
2.7 \\
2.4 \\
2.3 \\
2.1\end{array}$ & $\begin{array}{l}2.0 \\
2.7 \\
3.5 \\
4.4 \\
6.3 \\
8.4\end{array}$ & $\begin{array}{l}5 . \\
6 . \\
7 . \\
8 . \\
9 . \\
1 .\end{array}$ & $\begin{array}{l}0.0 \\
0.0 \\
0.0 \\
0.0 \\
0.0 \\
0.0 \\
0.0\end{array}$ & $\begin{array}{l}-0 \\
-0 \\
-0 \\
-0 \\
-0 \\
-0 \\
-0 \\
-0\end{array}$ & $\begin{array}{l}8 \\
6 \\
4 \\
2 \\
9 \\
9\end{array}$ & $\begin{array}{l}0 \\
0 \\
0 \\
0 \\
0 . \\
0 .\end{array}$ \\
\hline $\begin{array}{l}0.4500 \\
0.5000 \\
0.5500 \\
0.6000 \\
0.7000 \\
0.8000 \\
0.9000\end{array}$ & $\begin{array}{l}2.011 E+00 \\
1.956 E+00 \\
1.912 E+00 \\
1.877 E+00 \\
1.826 E+00 \\
1.791 E+00 \\
1.767 E+00\end{array}$ & $\begin{array}{l}5.867 \\
6.278 \\
6.707 \\
7.149 \\
8.076 \\
9.050 \\
1.007\end{array}$ & $\begin{array}{l}2.0 \\
1.9 \\
1.9 \\
1.8 \\
1.8 \\
1.8 \\
1.7\end{array}$ & $\begin{array}{l}1.5 \\
1.8 \\
2.0 \\
2.3 \\
2.8 \\
3.4 \\
3.9\end{array}$ & $\begin{array}{l}03 \\
03 \\
03 \\
03 \\
03 \\
03 \\
03 \\
03\end{array}$ & $\begin{array}{l}0.0 \\
1.466 \\
4.112 \\
6.992 \\
1.005 \\
1.650 \\
2.321 \\
3.001\end{array}$ & $\begin{array}{l}-0 . \\
-0 . \\
-0 . \\
-0 . \\
-0 . \\
-0 . \\
-0 . \\
-0 .\end{array}$ & $\begin{array}{l}0 \\
0 \\
0 \\
0 \\
0\end{array}$ & $\begin{array}{l}0 \\
0 \\
0 \\
0 \\
0 \\
0\end{array}$ \\
\hline $\begin{array}{l}1.0000 \\
1.2500 \\
1.5000 \\
1.7500 \\
2.0000 \\
2.5000 \\
3.0000 \\
3.5000\end{array}$ & $\begin{array}{l}1.750 E+00 \\
1.726 E+00 \\
1.716 E+00 \\
1.714 E+00 \\
1.716 E+00 \\
1.724 E+00 \\
1.736 E+00 \\
1.748 E+00\end{array}$ & $\begin{array}{l}1.3 \\
1.6 \\
2.0 \\
2.3 \\
3.0 \\
3.7\end{array}$ & $\begin{array}{l}1.740 \\
1.733 \\
1.734 \\
1.739 \\
1.755 \\
1.773 \\
1.793\end{array}$ & $\begin{array}{l}5.9 \\
7.4 \\
8.8 \\
1.0 \\
1.3 \\
1.5 \\
1.8\end{array}$ & $\begin{array}{l}03 \\
03 \\
03 \\
03 \\
03 \\
03 \\
02 \\
02\end{array}$ & $\begin{array}{l}5.3 \\
6.8 \\
8.3 \\
9.6 \\
1.2 \\
1.4 \\
1.6\end{array}$ & $\begin{array}{l}-0 . \\
-0 . \\
-0 . \\
-0 . \\
-0 . \\
-0 . \\
-0 . \\
-0 .\end{array}$ & $\begin{array}{l}0 \\
0 \\
0 \\
0 \\
0\end{array}$ & $\begin{array}{l}0 . \\
0 . \\
0 . \\
0 . \\
0 . \\
0 .\end{array}$ \\
\hline $\begin{array}{l}4.0000 \\
4.5000 \\
5.0000 \\
5.5000 \\
6.0000 \\
7.0000 \\
8.0000 \\
9.0000\end{array}$ & $\begin{array}{l}1.770 E+00 \\
1.781 E+00 \\
1.791 E+00 \\
1.800 E+00 \\
1.816 E+00 \\
1.831 E+00\end{array}$ & $\begin{array}{l}6.1 \\
6.9 \\
7.7 \\
8.6 \\
1.0 \\
1.2\end{array}$ & $\begin{array}{l}1.8 \\
1.8 \\
1.8 \\
1.8 \\
1.8 \\
1.9 \\
1.9\end{array}$ & $\begin{array}{l}2.4 \\
2.7 \\
2.9 \\
3.2 \\
3.7 \\
4.2\end{array}$ & $\begin{array}{l}1 . \\
1 . \\
1 . \\
2 . \\
2 .\end{array}$ & $\begin{array}{l}1.5 \\
2.5 \\
2.2 \\
2.3 \\
2.5 \\
2.7 \\
2.5\end{array}$ & $\begin{array}{l}-0 . \\
-0 . \\
-0 . \\
-0 . \\
-0 . \\
-0 . \\
-0 . \\
-0 .\end{array}$ & $\begin{array}{l}0 \\
0 \\
0 \\
0 \\
0 \\
0\end{array}$ & $\begin{array}{l}0 \\
0 \\
0 \\
0 \\
0 \\
0\end{array}$ \\
\hline $\begin{array}{l}0.0000 \\
2.5000 \\
5.0000 \\
7.5000 \\
0.0000 \\
5.0000\end{array}$ & $\begin{array}{l}1.880 E+00 \\
1.899 E+00 \\
1.915 E+00 \\
1.929 E+00 \\
1.951 E+00 \\
1.968 E+00\end{array}$ & $\begin{array}{l}2.0 \\
2.5 \\
3.0 \\
3.6 \\
4.6\end{array}$ & $\begin{array}{l}2 . \\
2 . \\
2.2 \\
2.2 \\
2.5\end{array}$ & $\begin{array}{l}5.2 \\
6.5 \\
7.6 \\
8.8 \\
9.9 \\
1.2\end{array}$ & $\begin{array}{l}4 . \\
5 . \\
6 \\
7 \\
9 . \\
1 .\end{array}$ & $\begin{array}{l}3.5 \\
3.9 \\
3.9 \\
4.2 \\
4.5\end{array}$ & $\begin{array}{l}-0 . \\
-0 . \\
-0 . \\
-0 . \\
-0 . \\
-0 . \\
-0 . \\
-0 .\end{array}$ & $\begin{array}{l}0.0 \\
0.0 \\
0.0 \\
0.0 \\
0.0 \\
0.0\end{array}$ & \\
\hline $\begin{array}{l}.0000 \\
.0000 \\
.0000 \\
.0000 \\
.0000\end{array}$ & $\begin{array}{l}2.00 \\
2.01 \\
2.02 \\
2.03 \\
2.04 \\
2\end{array}$ & $\begin{array}{l}9 \\
1 \\
1 \\
1 \\
1\end{array}$ & $\begin{array}{l}2 . \\
3 . \\
3 . \\
3\end{array}$ & 2.1 & & $\begin{array}{l}5 \\
5 . \\
6 . \\
6 . \\
6 .\end{array}$ & $\begin{array}{l}-0 \\
-0 . \\
-0 . \\
-0 . \\
-0 \\
-0 . \\
-0 . \\
-0 .\end{array}$ & & \\
\hline $\begin{array}{l}0 \\
000 \\
000 \\
000\end{array}$ & $\begin{array}{l}2.0 \\
2.11 \\
2.1 \\
2.1 \\
2.1\end{array}$ & $\begin{array}{l}2 \\
3 \\
3 \\
4 \\
5 \\
6\end{array}$ & $\begin{array}{l}4 \\
5 \\
6 \\
6 \\
7 \\
9\end{array}$ & $\begin{array}{l}1 \\
1 \\
1\end{array}$ & & & $\begin{array}{l}-0 \\
-0 \\
-0 \\
-0 \\
-0 \\
-0 \\
-0 \\
-0\end{array}$ & & \\
\hline 000 & $\begin{array}{l}2.200 E+00 \\
2.209 E+00 \\
2.217 E+00 \\
2.224 E+00 \\
2.237 E+00 \\
2.248 E+00 \\
2.257 E+00\end{array}$ & $\begin{array}{ll}0 & 1 \\
01 \\
01 \\
01 \\
01 \\
01 \\
01 \\
01 \\
01\end{array}$ & $\begin{array}{l}1 \\
1 \\
1 \\
1 \\
1 \\
1 \\
1 \\
1\end{array}$ & $\begin{array}{l}1 \\
1 \\
1 \\
1 \\
1 \\
1\end{array}$ & $\begin{array}{l}1 \\
1 \\
1 \\
1 \\
1 \\
1 \\
1 \\
1\end{array}$ & $\begin{array}{l}0 \\
1 \\
1 \\
1 \\
1 \\
1 \\
1\end{array}$ & $\begin{array}{l}-0 \\
-0 \\
-0 \\
-0 \\
-0 \\
-0\end{array}$ & $\begin{array}{l}4 \\
4 \\
3 \\
3 \\
3 \\
3 \\
3\end{array}$ & \\
\hline 0 & $66 E+00$ & 01 & $08 E+01$ & 03 & 72 & 83 & 0.000 & 0 & 000 \\
\hline
\end{tabular}




\begin{tabular}{|c|c|c|c|c|c|c|c|c|c|}
\hline \multirow{2}{*}{$\begin{array}{l}\text { ENERGY } \\
\text { MeV }\end{array}$} & \multicolumn{3}{|c|}{$\begin{array}{l}\text { STOPPING POWER } \\
\text { ON RADIATIVE } \\
\text { TOTAL }\end{array}$} & \multirow{2}{*}{$\begin{array}{l}\text { CSDA } \\
\text { RANGE } \\
\mathrm{g} / \mathrm{cm}^{2}\end{array}$} & \multirow[t]{2}{*}{$\begin{array}{l}\text { RADIATION } \\
\text { YIELD }\end{array}$} & \multirow[t]{2}{*}{$\begin{array}{l}\text { DENS.EFF. } \\
\text { CORR. } \\
\text { (DELTA) }\end{array}$} & \multirow[t]{2}{*}{$\begin{array}{l}d l^{d 0} \\
\text { CoLL } \\
\text { LOSS }\end{array}$} & \multirow[t]{2}{*}{$\begin{array}{l}9 \text { ) /d ( } 1 \\
\text { CSDA } \\
\text { RANGE }\end{array}$} & \multirow[t]{2}{*}{$\begin{array}{l}\text { OgI ) } \\
\text { RAD } \\
\text { YIELD }\end{array}$} \\
\hline & $\mathrm{MeV} \mathrm{cm}^{2} / \mathrm{g}$ & $\mathrm{MeV} \mathrm{cm}^{2} / \mathrm{g}$ & $\mathrm{leV} \mathrm{cm}^{2} / \mathrm{g}$ & & & & & & \\
\hline $\begin{array}{l}0.01100 \\
0.0125 \\
0.0150 \\
0.0175 \\
0.0200 \\
0.0250 \\
0.0300 \\
0.0350\end{array}$ & $\begin{array}{l}2.48 \\
2.077 \\
1.795 \\
1.587 \\
1.427 \\
1.196 \\
1.036 \\
9.18\end{array}$ & $\begin{array}{l}3.9 \\
3.9 \\
3.9 \\
3.9 \\
3.9 \\
3.9 \\
3.9\end{array}$ & $\begin{array}{l}1.7 \\
1.5 \\
1.4 \\
1.1 \\
9.0 \\
9.1\end{array}$ & $\begin{array}{l}4.3 \\
4.6 \\
6.1 \\
7.8 \\
1.1 \\
1.6\end{array}$ & & $\begin{array}{l}0.0 \\
0.0 \\
0.0 \\
0.0 \\
0.0 \\
0.0 \\
0.0\end{array}$ & & $\begin{array}{l}2 \\
4 \\
8 \\
3 \\
3 \\
9 \\
2 \\
7 \\
3\end{array}$ & $\begin{array}{l}0.201 \\
0.193 \\
0.187 \\
0.182 \\
0.178 \\
0.171 \\
0.167 \\
0.163\end{array}$ \\
\hline $\begin{array}{l}0.0400 \\
0.0450 \\
0.0500 \\
0.0550 \\
0.0600 \\
0.0700 \\
0.0800 \\
0.0900\end{array}$ & $\begin{array}{l}8.286 E+00 \\
7.574 E+00 \\
6.995 E+00 \\
6.516 E+00 \\
6.111 E+00 \\
5.466 E+00 \\
4.974 E+00 \\
4.587 E+00\end{array}$ & $\begin{array}{l}4 . \\
4.8 \\
4 . \\
4 . \\
4 . \\
4 . \\
4 .\end{array}$ & $\begin{array}{l}6.518 \mathrm{E}+0 \\
6.999 \mathrm{E}+0 \\
6.520 \mathrm{E}+0 \\
6.115 \mathrm{E}+0 \\
5.470 \mathrm{E}+0 \\
4.979 \mathrm{E}+0 \\
4.591 \mathrm{E}+0\end{array}$ & $\begin{array}{l}3.3 \\
4.0 \\
4.7 \\
5.5 \\
7.2 \\
9.2 \\
1.1\end{array}$ & & $\begin{array}{l}0.0 \\
0.0 \\
0.0 \\
0.0 \\
0.0 \\
0.0 \\
0.0\end{array}$ & & $\begin{array}{l}5 \\
7 \\
5 \\
3 \\
3 \\
1 \\
8 \\
6 \\
4\end{array}$ & $\begin{array}{l}0.160 \\
0.157 \\
0.155 \\
0.153 \\
0.151 \\
0.148 \\
0.146 \\
0.144\end{array}$ \\
\hline $\begin{array}{l}0.1000 \\
0.1250 \\
0.1500 \\
0.1750 \\
0.2000 \\
0.2500 \\
0.3000 \\
0.3500\end{array}$ & $\begin{array}{l}4.274 E+00 \\
3.704 E+00 \\
3.320 E+00 \\
3.044 E+00 \\
2.833 E+00 \\
2.551 E+00 \\
2.363 E+00 \\
2.233 E+00\end{array}$ & $\begin{array}{l}4 . \\
4 . \\
4.8 \\
5 .\end{array}$ & $\begin{array}{l}3.1 \\
3.3 \\
3.0 \\
2.8 \\
2.5 \\
2.3 \\
2.2\end{array}$ & $\begin{array}{l}1.3 \\
2.9 \\
3.4 \\
4.3 \\
6.1\end{array}$ & $\begin{array}{l}4 \\
4 \\
4 \\
4 \\
4 \\
3 \\
3 \\
7\end{array}$ & $\begin{array}{l}0.0 \\
0.0 \\
0.0 \\
0.0 \\
0.0 \\
0.0\end{array}$ & $\begin{array}{l}-0 \\
-0 \\
-0 \\
-0 \\
-0 \\
-0\end{array}$ & $\begin{array}{l}36 \\
39 \\
36 \\
34 \\
32 \\
29 \\
27 \\
25\end{array}$ & $\begin{array}{l}0.142 \\
0.138 \\
0.136 \\
0.133 \\
0.132 \\
0.129 \\
0.126\end{array}$ \\
\hline $\begin{array}{l}0.4000 \\
0.4500 \\
0.5000 \\
0.5500 \\
0.6000 \\
0.7000 \\
0.8000 \\
0.9000\end{array}$ & $\begin{array}{l}2.138 E+00 \\
2.068 E+00 \\
2.014 E+00 \\
1.971 E+00 \\
1.937 E+00 \\
1.886 E+00 \\
1.851 E+00 \\
1.827 E+00\end{array}$ & ك v & $\begin{array}{l}2.145 E+00 \\
2.075 E+00 \\
2.021 E+00 \\
1.979 E+00 \\
1.945 E+00 \\
1.895 E+00 \\
1.862 E+00 \\
1.839 E+00\end{array}$ & $\begin{array}{l}.507 \mathrm{E}-01 \\
.751 \mathrm{E}-01 \\
.001 \mathrm{E}-01 \\
.256 \mathrm{E}-01 \\
.777 \mathrm{E}-01 \\
.310 \mathrm{E}-01 \\
.851 \mathrm{E}-01\end{array}$ & $\begin{array}{l}1.638 \mathrm{E}-03 \\
1.802 \mathrm{E}-03 \\
1.965 \mathrm{E}-03 \\
2.128 \mathrm{E}-03 \\
2.290 \mathrm{E}-03 \\
2.617 \mathrm{E}-03 \\
2.947 \mathrm{E}-03 \\
3.280 \mathrm{E}-03\end{array}$ & $\begin{array}{l}0.0 \\
0.0 \\
0.0 \\
1.103 \mathrm{E}-02\end{array}$ & & & \\
\hline $\begin{array}{l}1.0000 \\
1.2500 \\
1.5000 \\
1.7500 \\
2.0000 \\
2.5000 \\
3.0000 \\
3.5000\end{array}$ & $\begin{array}{l}1.810 E+00 \\
1.786 E+00 \\
1.777 E+00 \\
1.775 E+00 \\
1.776 E+00 \\
1.785 E+00 \\
1.796 E+00 \\
1.808 E+00\end{array}$ & $\begin{array}{l}-02 \\
-02 \\
-02 \\
-02 \\
-02 \\
-02 \\
-02\end{array}$ & $\begin{array}{l}1.823 E+00 \\
1.802 E+00 \\
1.796 E+00 \\
1.798 E+00 \\
1.803 E+00 \\
1.820 E+00 \\
1.839 E+00 \\
1.859 E+00\end{array}$ & $\begin{array}{l}1 \\
1 \\
1 \\
1 \\
1 \\
0\end{array}$ & $\begin{array}{l}5.375 \mathrm{E}-03 \\
6.294 \mathrm{E}-03 \\
7.235 \mathrm{E}-03 \\
9.177 \mathrm{E}-03 \\
1.118 \mathrm{E}-02 \\
1.324 \mathrm{E}-02\end{array}$ & $\begin{array}{l}2.428 \\
3.944 \\
5.437 \\
6.866 \\
8.218 \\
1.069 \\
1.288 \\
1.484\end{array}$ & $\begin{array}{l}-0 \\
-0 \\
-0 \\
-0 \\
-0 \\
-0\end{array}$ & $\begin{array}{l}0.085 \\
0.078 \\
0.072 \\
0.067 \\
0.059 \\
0.054 \\
0.050\end{array}$ & $\begin{array}{l}7 \\
6 \\
7 \\
0 \\
4 \\
6 \\
1 \\
7\end{array}$ \\
\hline $\begin{array}{l}4.0000 \\
4.5000 \\
5.0000 \\
5.5000 \\
6.0000 \\
7.0000 \\
8.0000 \\
9.0000\end{array}$ & $\begin{array}{l}1.819 E+00 \\
1.830 E+00 \\
1.840 E+00 \\
1.849 E+00 \\
1.858 E+00 \\
1.875 E+00 \\
1.889 E+00 \\
1.902 E+00\end{array}$ & $\begin{array}{l}7.9 \\
8.8 \\
9.8 \\
1.1\end{array}$ & $\begin{array}{l}.879 E+00 \\
.899 E+00 \\
.919 E+00 \\
.938 E+00 \\
.957 E+00 \\
.993 E+00 \\
.028 E+00 \\
.062 E+00\end{array}$ & $\begin{array}{l}0 \\
0 \\
0 \\
0 \\
0\end{array}$ & $\begin{array}{l}1 . \\
1 . \\
1 . \\
2 . \\
2 . \\
3 .\end{array}$ & $\begin{array}{l}1.6 \\
1.8 \\
1.9 \\
2.1 \\
2.2 \\
2.4 \\
2.6 \\
2.8\end{array}$ & $\begin{array}{l}-0 \\
-0 \\
-0 \\
-0 \\
-0 \\
-0 \\
-0\end{array}$ & & \\
\hline $\begin{array}{l}12.5 \\
15.0 \\
17.5 \\
20.0 \\
25.0 \\
30.0 \\
35.0\end{array}$ & $\begin{array}{l}+00 \\
+00 \\
000 \\
00 \\
00 \\
000 \\
000 \\
00 \\
00\end{array}$ & $\begin{array}{l}2 . \\
2 . \\
4 . \\
5.2\end{array}$ & $\begin{array}{l}2.175 \mathrm{E}+ \\
2.252 \mathrm{E}+ \\
2.327 \mathrm{E}+ \\
2.400 \mathrm{E}+ \\
2.542 \mathrm{E}+ \\
2.683 \mathrm{E}+ \\
2.821 \mathrm{E}+\end{array}$ & $\begin{array}{l}0 \\
0 \\
0 \\
0 \\
1\end{array}$ & & & $\begin{array}{l}-0 \\
-0 \\
-0 \\
-0 \\
-0\end{array}$ & & \\
\hline $\begin{array}{l}40.00000 \\
45.00000 \\
50.0000 \\
55.0000 \\
60.0000 \\
70.0000\end{array}$ & $\begin{array}{l}0 \\
0 \\
0 \\
0 \\
0\end{array}$ & & $\begin{array}{l}3 \\
3 \\
3 \\
3 \\
4 \\
4 .\end{array}$ & 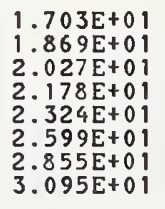 & & & & & \\
\hline 5.00 & $\begin{array}{l}0 \\
0\end{array}$ & & $\begin{array}{l}+00 \\
+00 \\
+00 \\
+00 \\
+00 \\
+00 \\
+01 \\
+01\end{array}$ & & & & $\begin{array}{l}-0 \\
-0 \\
-0\end{array}$ & & \\
\hline $\begin{array}{l}500.0000 \\
550.0000 \\
600.0000 \\
700.0000\end{array}$ & 2 & ! & $\begin{array}{l}\vdots \\
\vdots \\
\vdots \\
\vdots \\
\vdots \\
\vdots \\
\vdots\end{array}$ & $\begin{array}{l}1 \\
1 \\
1 \\
1 \\
1\end{array}$ & 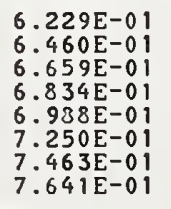 & $\begin{array}{l}1 \\
1 \\
1 \\
1 \\
1 \\
1\end{array}$ & $\begin{array}{l}0 \\
0 \\
0 \\
0 \\
0 \\
0 \\
0\end{array}$ & & $\begin{array}{l}01 \\
0 \\
0 \\
0 \\
0 \\
0 \\
0 \\
0 \\
0 \\
0 \\
0 \\
0\end{array}$ \\
\hline & $.345 E+00$ & $.679 E+01$ & $.914 E+01$ & $.012 E+02$. & $.793 \mathrm{E}-01$ & $.166 E+01$ & .0 .000 & 0.003 & $0.00 \mathrm{C}$ \\
\hline
\end{tabular}


MBS-114A IAt: V. 0.781

\begin{tabular}{|c|c|c|c|}
\hline $\begin{array}{l}\text { U.S. DEFT. OF COMM. } \\
\text { BIBLIOGRAPHIC DATA } \\
\text { SHEET }\end{array}$ & $\begin{array}{l}\text { 1. PUBL.ICATION OR REPORT NO. } \\
\text { NBSIR } 82-2550\end{array}$ & 2. Covt. Recestion Mo & 7. Reciptient's Accession No. \\
\hline 4. TITLE AND SUBTITLE & 4. TITLE AND SUBTITLE & & $\begin{array}{l}\text { 5. Publication Date: } \\
\text { August } 1982\end{array}$ \\
\hline \multicolumn{3}{|c|}{ STOPPING POWERS AND RANGES OF ELECTRONS AND POSITRONS } & 6. Performing Organization Code \\
\hline \multicolumn{3}{|c|}{ 7. AUTHORISI Martin J. Berger and Stephen M. Seltzer } & 8. Performing Organ. Report No. \\
\hline \multicolumn{3}{|c|}{ 9. PERFORMING ORGANIZATION NAME AND ADDRESS } & 10. Project/Task/Work Unit No. \\
\hline \multicolumn{3}{|c|}{$\begin{array}{l}\text { NATIONAL BUREAU OF STANDARDS } \\
\text { DEPARTMENT OF COMMERCE } \\
\text { WASHINGTON, DC } 20234\end{array}$} & 11. Contract/Grant No. \\
\hline \multirow{2}{*}{\multicolumn{3}{|c|}{$\begin{array}{l}\text { 12. SPONSORING ORGANIZATION NAME AND COMPLETE ADDRESS (sireet, city. State, zIP) } \\
\text { U.S. Dep. of Energy Office of Naval Research } \\
\text { Washington, D.C. }\end{array}$}} & 13. Type of Report \& Period Covered \\
\hline & & & C \\
\hline
\end{tabular}

15. SUPPLEMENTARY NOTES

Docurnent describes a computer program; SF-185, FIPS Software Summary, is attached.

16. ABSTRACT (A 200-wnot or less factual summary of most significant information. If document includes a significant bubliography or liserature survey, mention it here.)

Tables of stopping powers and related data are given for electrons in 37 elements and 60 compounds, and for positrons in 8 materials. The tables include (1) collision stopping powers (2) radiative stopping powers, (3) total stopping powers, (4) ranges (computed in the continuous-slowingdown approximation), (5) radiation yields (fraction of electron energy converted into bremsstrahlung), and (6) the logarithmic derivatives of all these quantities with respect to the mean excitation energy of the mediun. These results are given at 81 energies between $1000 \mathrm{MeV}$ and $10 \mathrm{keV}$. Restricted collision stopping powers are tabulated for selected materials, with cut-off energies of 1,10 and $100 \mathrm{keV}$. The principal new ingredients in the preparation of these tables were: (1) a revision and updating of the mean excitation energies which enter into the Bethe stopping-power formula, on the basis of the best available data from stopping-power measurements and analyses of experimental oscillator-strength distributions and dielectricresponse functions; (2) use of the general formulation of Sternheimer and Peierls for the density-effect correction to the collision stopping power; and (3) use of theoretical bremsstrahlung cross sections of Tseng and Pratt.

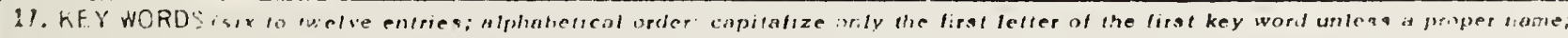

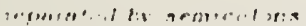

Colitision stopping power, electrons, positrons, radiation yield, radiative stopping power, range.

18. AVAILABILITY R Jnimited

For Offucial Districution. Do Hot Release to NTIS

Order From Sup. of Doc., U.S. Government Printing Office, Washington, DC 20402 , SD Sinc.k No. SNON3-003-

Oider From National Technicai information Service (NTIS), Springfield, VA. 22101

\begin{tabular}{|l|c|}
\hline $\begin{array}{l}\text { 19. SECURITY CLASS } \\
\text { (THIS REPORT) }\end{array}$ & $\begin{array}{c}\text { 21. NO. OF } \\
\text { PRINTED PAGES } \\
\text { UNCLASSIFIED }\end{array}$ \\
\hline $\begin{array}{l}\text { 20. SECURITY CLASS } \\
\text { (THIS PAGE) }\end{array}$ & $\begin{array}{c}\text { 22. PrICE } \\
\$ 15.00\end{array}$ \\
\hline
\end{tabular}




